\title{
Gini Coefficient: a New Way to Express Selectivity of Kinase Inhibitors Against a Family of Kinases
}

\author{
Piotr P. Graczyk
}

\section{Supporting information}

Calculation of Gini coefficient and hit rate for 85 kinase inhibitors

Calculation of example Lorenz curves for PD184352, AG1024 and Staurosporine

Calculation of Lorenz curves for clusters of Gini 0.417-0.460 and 0.553-0.595 at $10 \mu \mathrm{M}$ ATP 
Calculation of Gini coefficient and hit rate for $\mathbf{8 5}$ kinase inhibitors

Enter the value

Residual activity threshold for hit rate calculation [\%]

50 


\begin{tabular}{|c|c|c|c|c|c|c|c|c|c|}
\hline & $100 \mu \mathrm{M}$ ATP & & & & & & & & \\
\hline Kinase & AG1024@10 $\mu \mathrm{M}$ & Hit & sorted & normalized & position & cumulative sample fraction & inhibition\% & fraction of total inhibition & cumulative inhibition \\
\hline $\mathrm{Abl}(\mathrm{m})$ & 1 & 1 & 131 & 100 & 1 & 0.011764706 & 0 & 0 & 0 \\
\hline AMPK(r) & 84 & 0 & 118 & 100 & 2 & 0.023529412 & 0 & 0 & 0 \\
\hline $\operatorname{Arg}(m)$ & 6 & 1 & 116 & 100 & 3 & 0.035294118 & 0 & 0 & 0 \\
\hline Aurora-A(h) & 104 & 0 & 113 & 100 & 4 & 0.047058824 & 0 & 0 & 0 \\
\hline Axl(h) & 58 & 0 & 107 & 100 & 5 & 0.058823529 & 0 & 0 & 0 \\
\hline $\operatorname{Blk}(\mathrm{m})$ & 4 & 1 & 107 & 100 & 6 & 0.070588235 & 0 & 0 & 0 \\
\hline $\operatorname{Bmx}(\mathrm{h})$ & 84 & 0 & 105 & 100 & 7 & 0.082352941 & 0 & 0 & 0 \\
\hline СaMKII(r) & 89 & 0 & 105 & 100 & 8 & 0.094117647 & 0 & 0 & 0 \\
\hline CaMKIV(h) & 81 & 0 & 104 & 100 & 9 & 0.105882353 & 0 & 0 & 0 \\
\hline CDK1/cyclinB(h) & 67 & 0 & 104 & 100 & 10 & 0.117647059 & 0 & 0 & 0 \\
\hline $\mathrm{CDK} 2 /$ cyclinA(h) & 101 & 0 & 103 & 100 & 11 & 0.129411765 & 0 & 0 & 0 \\
\hline $\mathrm{CDK} 2 /$ cyclinE(h) & 95 & 0 & 101 & 100 & 12 & 0.141176471 & 0 & 0 & 0 \\
\hline CDK3/cyclinE(h) & 87 & 0 & 99 & 99 & 13 & 0.152941176 & 1 & 0.000503525 & 0.000503525 \\
\hline CDK5/p35(h) & 78 & 0 & 99 & 99 & 14 & 0.164705882 & 1 & 0.000503525 & 0.001007049 \\
\hline CDK6/cyclinD3(h) & 92 & 0 & 99 & 99 & 15 & 0.176470588 & 1 & 0.000503525 & 0.001510574 \\
\hline CDK7/cyclinH/MATI(h) & 93 & 0 & 98 & 98 & 16 & 0.188235294 & 2 & 0.001007049 & 0.002517623 \\
\hline CHK1(h) & 97 & 0 & 97 & 97 & 17 & 0.2 & 3 & 0.001510574 & 0.004028197 \\
\hline СHK2(h) & 88 & 0 & 96 & 96 & 18 & 0.211764706 & 4 & 0.002014099 & 0.006042296 \\
\hline CKI(y) & 87 & 0 & 96 & 96 & 19 & 0.223529412 & 4 & 0.002014099 & 0.008056395 \\
\hline СK2(h) & 96 & 0 & 95 & 95 & 20 & 0.235294118 & 5 & 0.002517623 & 0.010574018 \\
\hline $\mathrm{c}-\mathrm{RAF}(\mathrm{h})$ & 72 & 0 & 94 & 94 & 21 & 0.247058824 & 6 & 0.003021148 & 0.013595166 \\
\hline $\operatorname{CSK}(\mathrm{h})$ & 75 & 0 & 93 & 93 & 22 & 0.258823529 & 7 & 0.003524673 & 0.017119839 \\
\hline cSRC(h) & 6 & 1 & 93 & 93 & 23 & 0.270588235 & 7 & 0.003524673 & 0.020644512 \\
\hline Fes(h) & 70 & 0 & 93 & 93 & 24 & 0.282352941 & 7 & 0.003524673 & 0.024169184 \\
\hline FGFR3(h) & 91 & 0 & 92 & 92 & 25 & 0.294117647 & 8 & 0.004028197 & 0.028197382 \\
\hline $\mathrm{FH}$ t3(h) & 89 & 0 & 92 & 92 & 26 & 0.305882353 & 8 & 0.004028197 & 0.032225579 \\
\hline Fynn(h) & 5 & 1 & 91 & 91 & 27 & 0.317647059 & 9 & 0.004531722 & 0.036757301 \\
\hline GSK3a(h) & 89 & 0 & 91 & 91 & 28 & 0.329411765 & 9 & 0.004531722 & 0.041289023 \\
\hline GSK3B(h) & 52 & 0 & 91 & 91 & 29 & 0.341176471 & 9 & 0.004531722 & 0.045820745 \\
\hline IGF-1R(h) & 118 & 0 & 90 & 90 & 30 & 0.352941176 & 10 & 0.005035247 & 0.050855992 \\
\hline IKKa(h) & 82 & 0 & 90 & 90 & 31 & 0.364705882 & 10 & 0.005035247 & 0.055891239 \\
\hline IKКВ(h) & 93 & 0 & 90 & 90 & 32 & 0.376470588 & 10 & 0.005035247 & 0.060926485 \\
\hline $\mathbb{R}(\mathrm{h})$ & 105 & 0 & 89 & 89 & 33 & 0.388235294 & 11 & 0.005538771 & 0.066465257 \\
\hline JNKIal(h) & 79 & 0 & 89 & 89 & 34 & 0.4 & 11 & 0.005538771 & 0.072004028 \\
\hline JNK202(h) & 89 & 0 & 89 & 89 & 35 & 0.411764706 & 11 & 0.005538771 & 0.0775428 \\
\hline JNK3(r) & 60 & 0 & 89 & 89 & 36 & 0.423529412 & 11 & 0.005538771 & 0.083081571 \\
\hline Lck(h) & 24 & 1 & 89 & 89 & 37 & 0.435294118 & 11 & 0.005538771 & 0.088620342 \\
\hline Lyn(h) & 74 & 0 & 88 & 88 & 38 & 0.447058824 & 12 & 0.006042296 & 0.094662638 \\
\hline Lyn(m) & 5 & 1 & 88 & 88 & 39 & 0.458823529 & 12 & 0.006042296 & 0.100704935 \\
\hline MAPK1(h) & 85 & 0 & 88 & 88 & 40 & 0.470588235 & 12 & 0.006042296 & 0.106747231 \\
\hline MAPK2(h) & 113 & 0 & 88 & 88 & 41 & 0.482352941 & 12 & 0.006042296 & 0.112789527 \\
\hline MAPK2(m) & 93 & 0 & 88 & 88 & 42 & 0.494117647 & 12 & 0.006042296 & 0.118831823 \\
\hline MAPKAP-K2(h) & 90 & 0 & 87 & 87 & 43 & 0.505882353 & 13 & 0.006545821 & 0.125377644 \\
\hline MEK1(h) & 88 & 0 & 87 & 87 & 44 & 0.517647059 & 13 & 0.006545821 & 0.131923464 \\
\hline MKK4(m) & 88 & 0 & 87 & 87 & 45 & 0.529411765 & 13 & 0.006545821 & 0.138469285 \\
\hline MKK6(h) & 86 & 0 & 86 & 86 & 46 & 0.541176471 & 14 & 0.007049345 & 0.14551863 \\
\hline МKK $7($ (h) & 116 & 0 & 86 & 86 & 47 & 0.552941176 & 14 & 0.007049345 & 0.152567976 \\
\hline MSKI(h) & 92 & 0 & 85 & 85 & 48 & 0.564705882 & 15 & 0.00755287 & 0.160120846 \\
\hline $\mathrm{p} 7056 \mathrm{~K}(\mathrm{~h})$ & 36 & 1 & 84 & 84 & 49 & 0.576470588 & 16 & 0.008056395 & 0.168177241 \\
\hline PAK2(h) & 98 & 0 & 84 & 84 & 50 & 0.588235294 & 16 & 0.008056395 & 0.176233635 \\
\hline PDGFRa(h) & 88 & 0 & 84 & 84 & 51 & 0.6 & 16 & 0.008056395 & 0.18429003 \\
\hline PDGFRB(h) & 67 & 0 & 84 & 84 & 52 & 0.611764706 & 16 & 0.008056395 & 0.192346425 \\
\hline PDK1(h) & 84 & 0 & 82 & 82 & 53 & 0.623529412 & 18 & 0.009063444 & 0.201409869 \\
\hline PKA(b) & 74 & 0 & 81 & 81 & 54 & 0.635294118 & 19 & 0.009566969 & 0.210976838 \\
\hline PKA(h) & 107 & 0 & 79 & 79 & 55 & 0.647058824 & 21 & 0.010574018 & 0.221550856 \\
\hline PKBach) & 99 & 0 & 78 & 78 & 56 & 0.658823529 & 22 & 0.011077543 & 0.232628399 \\
\hline PKBB(h) & 90 & 0 & 78 & 78 & 57 & 0.670588235 & 22 & 0.011077543 & 0.243705942 \\
\hline $\mathrm{PKB}_{\gamma}(\mathrm{h})$ & 91 & 0 & 76 & 76 & 58 & 0.682352941 & 24 & 0.012084592 & 0.255790534 \\
\hline PKCu(h) & 87 & 0 & 75 & 75 & 59 & 0.694117647 & 25 & 0.012588117 & 0.268378651 \\
\hline РКСBI(h) & 99 & 0 & 74 & 74 & 60 & 0.705882353 & 26 & 0.013091641 & 0.281470292 \\
\hline PKCy(h) & 105 & 0 & 74 & 74 & 61 & 0.717647059 & 26 & 0.013091641 & 0.294561934 \\
\hline PKG(h) & 107 & 0 & 73 & 73 & 62 & 0.729411765 & 27 & 0.013595166 & 0.3081571 \\
\hline PKG:(h) & 78 & 0 & 72 & 72 & 63 & 0.741176471 & 28 & 0.014098691 & 0.322255791 \\
\hline PKCh(h) & 96 & 0 & 72 & 72 & 64 & 0.752941176 & 28 & 0.014098691 & 0.336354481 \\
\hline PKC (h) & 90 & 0 & 70 & 70 & 65 & 0.764705882 & 30 & 0.01510574 & 0.351460222 \\
\hline PKC $\mu(\mathrm{h})$ & 86 & 0 & 69 & 69 & 66 & 0.776470588 & 31 & 0.015609265 & 0.367069486 \\
\hline PKCO(h) & 91 & 0 & 67 & 67 & 67 & 0.788235294 & 33 & 0.016616314 & 0.383685801 \\
\hline PKD2(h) & 73 & 0 & 67 & 67 & 68 & 0.8 & 33 & 0.016616314 & 0.400302115 \\
\hline PRAK(h) & 76 & 0 & 67 & 67 & 69 & 0.811764706 & 33 & 0.016616314 & 0.416918429 \\
\hline PRK2(h) & 88 & 0 & 63 & 63 & 70 & 0.823529412 & 37 & 0.018630413 & 0.435548842 \\
\hline ROCK-II(h) & 23 & 1 & 60 & 60 & 71 & 0.835294118 & 40 & 0.020140987 & 0.455689829 \\
\hline ROCK-II(r) & 32 & 1 & 58 & 58 & 72 & 0.847058824 & 42 & 0.021148036 & 0.476837865 \\
\hline Rskl(h) & 89 & 0 & 58 & 58 & 73 & 0.858823529 & 42 & 0.021148036 & 0.497985901 \\
\hline Rskl(r) & 72 & 0 & 52 & 52 & 74 & 0.870588235 & 48 & 0.024169184 & 0.522155086 \\
\hline Rsk2(h) & 58 & 0 & 36 & 36 & 75 & 0.882352941 & 64 & 0.032225579 & 0.554380665 \\
\hline Rsk3(h) & 104 & 0 & 32 & 32 & 76 & 0.894117647 & 68 & 0.034239678 & 0.588620342 \\
\hline SAPK2a(h) & 99 & 0 & 24 & 24 & 77 & 0.905882353 & 76 & 0.038267875 & 0.626888218 \\
\hline SAPK2b(h) & 103 & 0 & 23 & 23 & 78 & 0.917647059 & 77 & 0.0387714 & 0.665659617 \\
\hline $\mathrm{SAPK} 3(\mathrm{~h})$ & 84 & 0 & 9 & 9 & 79 & 0.929411765 & 91 & 0.045820745 & 0.711480363 \\
\hline SAPK4(h) & 94 & 0 & 6 & 6 & 80 & 0.941176471 & 94 & 0.047331319 & 0.758811682 \\
\hline SGK(h) & 63 & 0 & 6 & 6 & 81 & 0.952941176 & 94 & 0.047331319 & 0.806143001 \\
\hline Syk(h) & 67 & 0 & 5 & 5 & 82 & 0.964705882 & 95 & 0.047834844 & 0.853977845 \\
\hline $\operatorname{TrkB(h)}$ & 131 & 0 & 5 & 5 & 83 & 0.976470588 & 95 & 0.047834844 & 0.901812689 \\
\hline Yes(h) & 9 & 1 & 4 & 4 & 84 & 0.988235294 & 96 & 0.048338369 & 0.950151057 \\
\hline $\mathrm{ZAP}-70(\mathrm{~h})$ & 69 & 0 & 1 & 1 & 85 & 1 & 99 & 0.049848943 & 1 \\
\hline & & & & & & Total inhibition & 1986 & & Total area \\
\hline & $\begin{array}{c}\text { Hit rate } \\
\text { Threshold }\end{array}$ & & & & & & & & Gini \\
\hline
\end{tabular}




\begin{tabular}{|c|c|c|c|c|c|c|c|c|c|}
\hline & $100 \mu \mathrm{M}$ ATP & & & & & & & & \\
\hline Kinase & AG1024@10 M & Hit & sorted & normalized & position & cumulative sample fraction & inhibition\% & fraction of total inhibition & cumulative inhibition \\
\hline $\mathrm{Abl(m)}$ & 10 & 1 & 128 & 100 & 1 & 0.011764706 & 0 & 0 & 0 \\
\hline AMPK(r) & 96 & 0 & 125 & 100 & 2 & 0.023529412 & 0 & 0 & 0 \\
\hline $\operatorname{Arg}(\mathrm{m})$ & 14 & 1 & 115 & 100 & 3 & 0.035294118 & 0 & 0 & 0 \\
\hline Aurora-A(h) & 78 & 0 & 109 & 100 & 4 & 0.047058824 & 0 & 0 & 0 \\
\hline Axl(h) & 78 & 0 & 107 & 100 & 5 & 0.058823529 & 0 & 0 & 0 \\
\hline $\mathrm{Blk}(\mathrm{m})$ & 7 & 1 & 107 & 100 & 6 & 0.070588235 & 0 & 0 & 0 \\
\hline $\mathrm{Bmx(h)}$ & 85 & 0 & 107 & 100 & 7 & 0.082352941 & 0 & 0 & 0 \\
\hline CaMKII(r) & 97 & 0 & 106 & 100 & 8 & 0.094117647 & 0 & 0 & 0 \\
\hline CaMKIV(h) & 91 & 0 & 106 & 100 & 9 & 0.105882353 & 0 & 0 & 0 \\
\hline CDK1/cyclinB(h) & 75 & 0 & 105 & 100 & 10 & 0.117647059 & 0 & 0 & 0 \\
\hline $\mathrm{CDK} 2 / \mathrm{cyclin} \mathrm{A}(\mathrm{h})$ & 97 & 0 & 105 & 100 & 11 & 0.129411765 & 0 & 0 & 0 \\
\hline $\mathrm{CDK} 2 /$ cyclinE(h) & 98 & 0 & 104 & 100 & 12 & 0.141176471 & 0 & 0 & 0 \\
\hline CDK3/cyclinE(h) & 93 & 0 & 104 & 100 & 13 & 0.152941176 & 0 & 0 & 0 \\
\hline CDK5/p35(h) & 105 & 0 & 104 & 100 & 14 & 0.164705882 & 0 & 0 & 0 \\
\hline CDK6/cyclinD3(h) & 91 & 0 & 103 & 100 & 15 & 0.176470588 & 0 & 0 & 0 \\
\hline CDK7/cyclinHMATI(h) & 99 & 0 & 101 & 100 & 16 & 0.188235294 & 0 & 0 & 0 \\
\hline CHK (hi) & 105 & 0 & 100 & 100 & 17 & 0.2 & 0 & 0 & 0 \\
\hline СHK2(h) & 99 & 0 & 99 & 99 & 18 & 0.211764706 & 1 & 0.000733138 & 0.000733138 \\
\hline $\mathrm{CKI}(\mathrm{y})$ & 104 & 0 & 99 & 99 & 19 & 0.223529412 & 1 & 0.000733138 & 0.001466276 \\
\hline СК2(h) & 99 & 0 & 99 & 99 & 20 & 0.235294118 & 1 & 0.000733138 & 0.002199413 \\
\hline$c-R A F(h)$ & 80 & 0 & 98 & 98 & 21 & 0.247058824 & 2 & 0.001466276 & 0.003665689 \\
\hline $\operatorname{CSK}(\mathrm{h})$ & 104 & 0 & 98 & 98 & 22 & 0.258823529 & 2 & 0.001466276 & 0.005131965 \\
\hline $\operatorname{cSRC}(\mathrm{h})$ & 44 & 1 & 97 & 97 & 23 & 0.270588235 & 3 & 0.002199413 & 0.007331378 \\
\hline Fes(h) & 93 & 0 & 97 & 97 & 24 & 0.282352941 & 3 & 0.002199413 & 0.009530792 \\
\hline FGFR3(h) & 90 & 0 & 97 & 97 & 25 & 0.294117647 & 3 & 0.002199413 & 0.011730205 \\
\hline $\mathrm{FH}$ (h) $(\mathrm{h})$ & 70 & 0 & 97 & 97 & 26 & 0.305882353 & 3 & 0.002199413 & 0.013929619 \\
\hline Fyn(h) & 33 & 1 & 97 & 97 & 27 & 0.317647059 & 3 & 0.002199413 & 0.016129032 \\
\hline GSK3u(h) & 90 & 0 & 97 & 97 & 28 & 0.329411765 & 3 & 0.002199413 & 0.018328446 \\
\hline GSK3B(h) & 90 & 0 & 96 & 96 & $\begin{array}{l}20 \\
29\end{array}$ & 0.341176471 & 4 & 0.002932551 & 0.021260997 \\
\hline $\mathrm{IGF}-1 \mathrm{R}(\mathrm{h})$ & 107 & 0 & 96 & 96 & 30 & 0.352941176 & 4 & 0.002932551 & 0.024193548 \\
\hline $\mathrm{IKK} \alpha(\mathrm{h})$ & 109 & 0 & 96 & 96 & 31 & 0.364705882 & 4 & 0.002932551 & 0.0271261 \\
\hline IKKB(h) & 96 & 0 & 96 & 96 & 32 & 0.376470588 & 4 & 0.002932551 & 0.030058651 \\
\hline $\mathrm{IR}(\mathrm{hl})$ & 103 & 0 & 95 & 95 & 33 & 0.388235294 & 5 & 0.003665689 & 0.03372434 \\
\hline JNKIal(h) & 96 & 0 & 94 & 94 & 34 & 0.4 & 6 & 0.004398827 & 0.038123167 \\
\hline JNK2a2(h) & 94 & 0 & 94 & 94 & 35 & 0.411764706 & 6 & 0.004398827 & 0.042521994 \\
\hline $\mathrm{JNK} 3(\mathrm{r})$ & 83 & 0 & 93 & 93 & 36 & 0.423529412 & 7 & 0.005131965 & 0.047653959 \\
\hline Lck(h) & 12 & 1 & 93 & 93 & 37 & 0.435294118 & 7 & 0.005131965 & 0.052785924 \\
\hline Lyn(h) & 73 & 0 & 93 & 93 & 38 & 0.447058824 & 7 & 0.005131965 & 0.057917889 \\
\hline Lyn(m) & 8 & 1 & 93 & 93 & 39 & 0.458823529 & 7 & 0.005131965 & 0.063049853 \\
\hline MAPKI(h) & $\begin{array}{l}8 \\
92\end{array}$ & 0 & 93 & 93 & 40 & 0.470588235 & 7 & 0.005131965 & 0.068181818 \\
\hline MAPK2(h) & 98 & 0 & 93 & 93 & 41 & 0.482352941 & 7 & 0.005131965 & 0.073313783 \\
\hline $\begin{array}{l}\text { MAAR L(n) } \\
\text { MAPK2(m) }\end{array}$ & $\begin{array}{l}50 \\
97\end{array}$ & 0 & 93 & 93 & 42 & 0.494117647 & 7 & 0.005131965 & 0.078445748 \\
\hline MAPKAP-K2(h) & 79 & 0 & 92 & 92 & 43 & 0.505882353 & 8 & 0.005865103 & 0.08431085 \\
\hline MEK1(h) & 88 & 0 & 92 & 92 & 44 & 0.517647059 & 8 & 0.005865103 & 0.090175953 \\
\hline MKK $4(\mathrm{~m})$ & 106 & 0 & 92 & 92 & 45 & 0.529411765 & 8 & 0.005865103 & 0.096041056 \\
\hline MKK $6(\mathrm{~h})$ & 81 & 0 & 91 & 91 & 46 & 0.541176471 & 9 & 0.00659824 & 0.102639296 \\
\hline MKK7(B(h) & 82 & 0 & 91 & 91 & 47 & 0.552941176 & 9 & 0.00659824 & 0.109237537 \\
\hline MSK1(h) & 97 & 0 & 90 & 90 & 48 & 0.564705882 & 10 & 0.007331378 & 0.116568915 \\
\hline $\mathrm{p} 70 \mathrm{~s} 6 \mathrm{~K}(\mathrm{~h})$ & 84 & 0 & 90 & 90 & $\begin{array}{l}48 \\
49\end{array}$ & 0.576470588 & 10 & 0.007331378 & 0.123900293 \\
\hline $\begin{array}{l}\text { PAK2(h) } \\
\text { (l) }\end{array}$ & 106 & 0 & 90 & 90 & 50 & 0.588235294 & 10 & 0.007331378 & 0.131231672 \\
\hline PDGFRa(h) & 125 & 0 & 90 & 90 & 51 & 0.6 & 10 & 0.007331378 & 0.13856305 \\
\hline PDGFRB(h) & 82 & 0 & 88 & 88 & 52 & 0.611764706 & 12 & 0.008797654 & 0.147360704 \\
\hline PDK $1(\mathrm{~h})$ & 92 & 0 & 88 & 88 & 53 & 0.623529412 & 12 & 0.008797654 & 0.156158358 \\
\hline PKA(b) & 88 & 0 & 88 & 88 & 54 & 0.635294118 & 12 & 0.008797654 & 0.164956012 \\
\hline $\begin{array}{l}\text { PRA(b) } \\
\text { PKA(h) }\end{array}$ & 84 & 0 & $\begin{array}{l}00 \\
87\end{array}$ & $\begin{array}{l}00 \\
87\end{array}$ & 55 & 0.647058824 & 13 & 0.009530792 & 0.174486804 \\
\hline PKBa(h) & 97 & 0 & 86 & 86 & 56 & 0.658823529 & 14 & 0.01026393 & 0.184750733 \\
\hline PKBB(h) & 87 & 0 & 85 & 85 & 57 & 0.670588235 & 15 & 0.010997067 & 0.195747801 \\
\hline PKB $\gamma(h)$ & 93 & 0 & 84 & 84 & 58 & 0.682352941 & 16 & 0.011730205 & 0.207478006 \\
\hline PKC(h) & 92 & 0 & 84 & 84 & 59 & 0.694117647 & 16 & 0.011730205 & 0.219208211 \\
\hline РКСBII(h) & 97 & 0 & 83 & 83 & 60 & 0.705882353 & 17 & 0.012463343 & 0.231671554 \\
\hline PKC (h) & 90 & 0 & 83 & 83 & 61 & 0.717647059 & 17 & 0.012463343 & 0.244134897 \\
\hline PKC\&h) & 115 & 0 & 82 & 82 & 62 & 0.729411765 & 18 & 0.013196481 & 0.257331378 \\
\hline 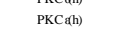 & 88 & 0 & 82 & 82 & 63 & 0.741176471 & 18 & 0.013196481 & 0.270527859 \\
\hline 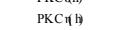 & 107 & 0 & 82 & 82 & 64 & 0.752941176 & 18 & 0.013196481 & 0.28372434 \\
\hline PKCl(h) & 86 & 0 & 81 & 81 & 65 & 0.764705882 & 19 & 0.013929619 & 0.297653959 \\
\hline PKCuhh & 94 & 0 & 80 & 80 & 66 & 0.776470588 & 20 & 0.014662757 & 0.312316716 \\
\hline $\begin{array}{l}P K C(n) \\
\text { PKCA }\end{array}$ & 96 & 0 & 80 & 80 & 67 & 0.788235294 & 20 & 0.014662757 & 0.326979472 \\
\hline $\begin{array}{l}\text { PKD2(h) } \\
\text { P. }\end{array}$ & 95 & 0 & 79 & 79 & 68 & 0.8 & 21 & 0.015395894 & 0.342375367 \\
\hline $\begin{array}{l}\text { PRAK(h) } \\
\text { Pall }\end{array}$ & $\begin{array}{l}75 \\
75\end{array}$ & 0 & 78 & 78 & 69 & 0.811764706 & 22 & 0.016129032 & 0.358504399 \\
\hline PRK2(h) & 101 & 0 & 78 & 78 & 70 & 0.823529412 & 22 & 0.016129032 & 0.374633431 \\
\hline $\begin{array}{l}\text { PRK(2) } \\
\text { ROCKII(h) }\end{array}$ & 52 & 0 & 75 & 75 & 71 & 0.835294118 & 25 & 0.018328446 & 0.392961877 \\
\hline ROCK-II(r) & 65 & 0 & 75 & 75 & 72 & 0.847058824 & 25 & 0.018328446 & 0.411290323 \\
\hline Rskl(h) & 82 & 0 & 73 & 73 & 73 & 0.858823529 & 27 & 0.019794721 & 0.431085044 \\
\hline Rskl(r) & 83 & 0 & 70 & 70 & 74 & 0.870588235 & 30 & 0.021994135 & 0.453079179 \\
\hline Rsk2(h) & 80 & 0 & 65 & 65 & 75 & 0.882352941 & 35 & 0.025659824 & 0.478739003 \\
\hline Rsk3(h) & 93 & 0 & 56 & 56 & 76 & 0.894117647 & 44 & 0.032258065 & 0.510997067 \\
\hline SAPK2a(h) & 93 & 0 & 53 & 53 & 77 & 0.905882353 & 47 & 0.034457478 & 0.545454545 \\
\hline SAPK2b(h) & $\begin{array}{l}50 \\
128\end{array}$ & 0 & 52 & 52 & 78 & 0.917647059 & 48 & 0.035190616 & 0.580645161 \\
\hline SAPK3(h) & 104 & 0 & 44 & 44 & 79 & 0.929411765 & 56 & 0.041055718 & 0.62170088 \\
\hline $\begin{array}{l}\mathrm{SAAR} \text { (h) } \\
\operatorname{SAPK} 4(\mathrm{~h})\end{array}$ & 107 & 0 & 33 & 33 & 80 & 0.941176471 & 67 & 0.049120235 & 0.670821114 \\
\hline SGK(h) & 56 & 0 & 14 & 14 & 81 & 0.952941176 & 86 & 0.063049853 & 0.733870968 \\
\hline Syk(h) & 100 & 0 & 12 & 12 & 82 & 0.964705882 & 88 & 0.064516129 & 0.798387097 \\
\hline $\mathrm{TrkB(h)}$ & 93 & 0 & 10 & 10 & 83 & 0.976470588 & 90 & 0.065982405 & 0.864369501 \\
\hline $\begin{array}{l}\text { IIrkB(h) } \\
\text { Yes(h) }\end{array}$ & 53 & 0 & 8 & 8 & 84 & 0.988235294 & 92 & 0.06744868 & 0.931818182 \\
\hline ZAP-70(h) & 93 & 0 & 7 & 7 & 85 & 1 & 93 & 0.068181818 & 1 \\
\hline & & & & & & Total inhibition & 1364 & & Total area \\
\hline & $\begin{array}{c}\text { Hit rate } \\
\text { Threshol }\end{array}$ & 5 & & & & & & & Gini \\
\hline
\end{tabular}




\begin{tabular}{|c|c|c|c|c|c|c|c|c|c|c|}
\hline & $10 \mu \mathrm{M}$ ATP & & & & & & & & & \\
\hline $\begin{array}{c}\text { Kinase } \\
\mathrm{Abl(m)}\end{array}$ & $\begin{array}{c}\text { AG1296@ @ } 10 \mu \mathrm{M} \\
72\end{array}$ & $\begin{array}{c}\mathrm{Hit} \\
0\end{array}$ & $\begin{array}{c}\text { sorted } \\
113\end{array}$ & $\begin{array}{c}\text { normalized } \\
100\end{array}$ & ${ }_{1}^{\text {position }}$ & $\begin{array}{c}\text { cumulative sample fraction } \\
0.011764706\end{array}$ & $\begin{array}{c}\text { inhibition\% } \\
0\end{array}$ & $\begin{array}{l}\text { fraction of total inhibition } \\
0\end{array}$ & $\begin{array}{c}\text { cumulative inhibition } \\
0\end{array}$ & area \\
\hline AMPK(r) & 88 & 0 & 110 & 100 & 2 & 0.023529412 & 0 & 0 & 0 & 0 \\
\hline $\operatorname{Arg}(\mathrm{m})$ & 92 & 0 & 109 & 100 & 3 & 0.035294118 & 0 & 0 & 0 & 0 \\
\hline Aurora-A(h) & 79 & 0 & 108 & 100 & 4 & 0.047058824 & 0 & 0 & 0 & 0 \\
\hline $\operatorname{Ax}(\mathrm{h})$ & 66 & 0 & 105 & 100 & 5 & 0.058823529 & 0 & 0 & 0 & 0 \\
\hline $\mathrm{Blk}(\mathrm{m})$ & 42 & 1 & 104 & 100 & 6 & 0.070588235 & 0 & 0 & 0 & 0 \\
\hline Bnxx(h) & 103 & 0 & 103 & 100 & 7 & 0.082352941 & 0 & 0 & 0 & 0 \\
\hline CaMKII(r) & 92 & 0 & 102 & 100 & 8 & 0.094117647 & 0 & 0 & 0 & 0 \\
\hline CaMKIV(h) & 94 & 0 & 102 & 100 & 9 & 0.105882353 & 0 & 0 & 0 & 0 \\
\hline 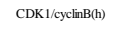 & 92 & 0 & 101 & 100 & 10 & 0.117647059 & 0 & 0 & 0 & 0 \\
\hline $\operatorname{CDK} 2 /$ cyclinA(h) & 90 & 0 & 100 & 100 & 11 & 0.129411765 & 0 & 0 & 0 & 0 \\
\hline $\mathrm{CDK} 2 /$ cyclinE(h) & 109 & 0 & 99 & 99 & 12 & 0.141176471 & 1 & 0.000739645 & 0.000739645 & $4.35085 \mathrm{E}-06$ \\
\hline CDK3/cyclinE(h) & 97 & 0 & 98 & 98 & 13 & 0.152941176 & 2 & 0.00147929 & 0.002218935 & $1.74034 \mathrm{E}-05$ \\
\hline CDK5/p35(h) & 93 & 0 & 98 & 98 & 14 & 0.164705882 & 2 & 0.00147929 & 0.003698225 & $3.48068 \mathrm{E}-05$ \\
\hline CDK6/lyclinD3(h) & 77 & 0 & 98 & $\begin{array}{l}50 \\
98\end{array}$ & 15 & 0.176470588 & 2 & 0.00147929 & 0.005177515 & 5.22102E-05 \\
\hline DKK7/cyclinH/MATI(h) & 87 & 0 & 97 & 97 & 16 & 0.188235294 & 3 & 0.002218935 & 0.00739645 & $7.39645 \mathrm{E}-05$ \\
\hline CHK1(h) & 94 & 0 & 96 & 96 & 17 & 0.2 & 4 & 0.00295858 & 0.01035503 & 0.00010442 \\
\hline СHK2(h) & 84 & 0 & 95 & 95 & 18 & 0.211764706 & 5 & 0.003698225 & 0.014053254 & 0.000143578 \\
\hline $\mathrm{CKI}(\mathrm{y})$ & 78 & 0 & 94 & 94 & 19 & 0.223529412 & 6 & 0.00443787 & 0.018491124 & 0.000191438 \\
\hline СК2(h) & 91 & 0 & 94 & 94 & 20 & 0.235294118 & 6 & 0.00443787 & 0.022928994 & 0.000243648 \\
\hline $\mathrm{c} \cdot \mathrm{RAF}(\mathrm{h})$ & 86 & 0 & 94 & 94 & 21 & 0.247058824 & 6 & 0.00443787 & 0.027366864 & 0.000295858 \\
\hline $\operatorname{CSK}(\mathrm{h})$ & 76 & 0 & 94 & 94 & 22 & 0.258823529 & 6 & 0.00443787 & 0.031804734 & 0.000348068 \\
\hline cSRC(h) & 78 & 0 & 94 & 94 & 23 & 0.270588235 & 6 & 0.00443787 & 0.036242604 & 0.000400278 \\
\hline Fes(h) & 87 & 0 & 94 & 94 & 24 & 0.282352941 & 6 & 0.00443787 & 0.040680473 & 0.000452489 \\
\hline FGFR3(h) & 84 & 0 & 93 & 93 & 25 & 0.294117647 & 7 & 0.005177515 & 0.045857988 & 0.00050905 \\
\hline $\mathrm{Fll}(\mathrm{h})$ & 14 & 1 & 93 & 93 & 26 & 0.305882353 & 7 & 0.005177515 & 0.051035503 & 0.000569962 \\
\hline Fynn(h) & 85 & 0 & 93 & 93 & 27 & 0.317647059 & 7 & 0.005177515 & 0.056213018 & 0.000630874 \\
\hline GSK3a(h) & 89 & 0 & 92 & 92 & 28 & 0.329411765 & 8 & 0.00591716 & 0.062130178 & 0.000696136 \\
\hline GSK3B(h) & 85 & 0 & 92 & 92 & 29 & 0.341176471 & 8 & 0.00591716 & 0.068047337 & 0.00076575 \\
\hline IGF-IR(h) & 102 & 0 & 92 & 92 & 30 & 0.352941176 & 8 & 0.00591716 & 0.073964497 & 0.000835364 \\
\hline $\mathrm{IKKa}(\mathrm{h})$ & 104 & 0 & 92 & 92 & 31 & 0.364705882 & 8 & 0.00591716 & 0.079881657 & 0.000904977 \\
\hline IKKB(h) & 99 & 0 & 92 & 92 & 32 & 0.376470588 & 8 & 0.00591716 & 0.085798817 & 0.000974591 \\
\hline $\mathrm{IR}(\mathrm{h})$ & 94 & 0 & 91 & 91 & 33 & 0.388235294 & 9 & 0.006656805 & 0.092455621 & 0.001048556 \\
\hline JNK1al(h) & 89 & 0 & 91 & 91 & 34 & 0.4 & 9 & 0.006656805 & 0.099112426 & 0.001126871 \\
\hline JNK2a2(h) & 88 & 0 & 90 & 90 & 35 & 0.411764706 & 10 & 0.00739645 & 0.106508876 & 0.001209537 \\
\hline $\mathrm{JNK} 3(\mathrm{r})$ & 92 & 0 & 89 & 89 & 36 & 0.423529412 & 11 & 0.008136095 & 0.11464497 & 0.001300905 \\
\hline Lck(h) & 64 & 0 & 89 & 89 & 37 & 0.435294118 & 11 & 0.008136095 & 0.122781065 & 0.001396624 \\
\hline Lyn(h) & 77 & 0 & 89 & 89 & 38 & 0.447058824 & 11 & 0.008136095 & 0.13091716 & 0.001492342 \\
\hline Lyn(m) & 58 & 0 & 89 & 89 & 39 & 0.458823529 & 11 & 0.008136095 & 0.139053254 & 0.001588061 \\
\hline MAPK1(h) & 86 & 0 & 89 & 89 & 40 & 0.470588235 & 11 & 0.008136095 & 0.147189349 & 0.00168378 \\
\hline MAPK2(h) & 85 & 0 & 89 & 89 & 41 & 0.482352941 & 11 & 0.008136095 & 0.155325444 & 0.001779499 \\
\hline MAPK2(m) & 86 & 0 & 89 & 89 & 42 & 0.494117647 & 11 & 0.008136095 & 0.163461538 & 0.001875218 \\
\hline MAPKAP-K2(h) & 86 & 0 & 88 & 88 & 43 & 0.505882353 & 12 & 0.00887574 & 0.172337278 & 0.001975287 \\
\hline MEK1(h) & 91 & 0 & 88 & 88 & 44 & 0.517647059 & 12 & 0.00887574 & 0.181213018 & 0.002079708 \\
\hline MKK4(m) & 77 & 0 & 88 & 88 & 45 & 0.529411765 & 12 & 0.00887574 & 0.190088757 & 0.002184128 \\
\hline MKK6(h) & 94 & 0 & 88 & 88 & 46 & 0.541176471 & 12 & 0.00887574 & 0.198964497 & 0.002288549 \\
\hline МКК77(h) & 110 & 0 & 87 & 87 & 47 & 0.552941176 & 13 & 0.009615385 & 0.208579882 & 0.00239732 \\
\hline MSKI(h) & 93 & 0 & 87 & 87 & 48 & 0.564705882 & 13 & 0.009615385 & 0.218195266 & 0.002510442 \\
\hline $\mathrm{p} 70 \mathrm{0S6K( \textrm {h } )}$ & 74 & 0 & 87 & 87 & 49 & 0.576470588 & 13 & 0.009615385 & 0.227810651 & 0.002623564 \\
\hline PAK2(h) & 95 & 0 & 86 & 86 & 50 & 0.588235294 & 14 & 0.01035503 & 0.23816568 & 0.002741037 \\
\hline PDGFRa(h) & 17 & 1 & 86 & 86 & 51 & 0.6 & 14 & 0.01035503 & 0.24852071 & 0.002862861 \\
\hline PDGFRB(h) & 47 & 1 & 86 & 86 & 52 & 0.611764706 & 14 & 0.01035503 & 0.25887574 & 0.002984685 \\
\hline PDK1(h) & 101 & 0 & 86 & 86 & 53 & 0.623529412 & 14 & 0.01035503 & 0.269230769 & 0.003106509 \\
\hline PKA(b) & 102 & 0 & 86 & 86 & 54 & 0.635294118 & 14 & 0.01035503 & 0.279585799 & 0.003228333 \\
\hline PKA(h) & 108 & 0 & 85 & 85 & 55 & 0.647058824 & 15 & 0.011094675 & 0.290680473 & 0.003354507 \\
\hline PKBar(h) & 89 & 0 & 85 & 85 & 56 & 0.658823529 & 15 & 0.011094675 & 0.301775148 & 0.003485033 \\
\hline PKBB(h) & 100 & 0 & 85 & 85 & 57 & 0.670588235 & 15 & 0.011094675 & 0.312869822 & 0.003615559 \\
\hline PKB $\times(h)$ & 98 & 0 & 84 & 84 & 58 & 0.682352941 & 16 & 0.01183432 & 0.324704142 & 0.003750435 \\
\hline PKC(h) & 88 & 0 & 84 & 84 & 59 & 0.694117647 & 16 & 0.01183432 & 0.336538462 & 0.003889662 \\
\hline PKCBII(h) & 93 & 0 & 81 & 81 & 60 & 0.705882353 & 19 & 0.014053254 & 0.350591716 & 0.004041942 \\
\hline PKC $($ (h) & 96 & 0 & 80 & 80 & 61 & 0.717647059 & 20 & 0.014792899 & 0.365384615 & 0.004211625 \\
\hline 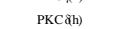 & 105 & 0 & 79 & 79 & 62 & 0.729411765 & 21 & 0.015532544 & 0.38091716 & 0.00439001 \\
\hline PKC \&h) & 89 & 0 & 79 & 79 & 63 & 0.741176471 & 21 & 0.015532544 & 0.396449704 & 0.004572746 \\
\hline PKCrit & 98 & 0 & 78 & 78 & 64 & 0.752941176 & 22 & 0.016272189 & 0.412721893 & 0.004759833 \\
\hline PKC, (h) & 89 & 0 & 78 & 78 & 65 & 0.764705882 & 22 & 0.016272189 & 0.428994083 & 0.00495127 \\
\hline PKC $\mu(\mathrm{h})$ & 89 & 0 & 78 & 78 & 66 & 0.776470588 & 22 & 0.016272189 & 0.445266272 & 0.005142708 \\
\hline PKCQ & 87 & 0 & 77 & 77 & 67 & 0.788235294 & 23 & 0.017011834 & 0.462278107 & 0.005338496 \\
\hline PKD2(h) & 80 & 0 & 77 & 77 & 68 & 0.8 & 23 & 0.017011834 & 0.479289941 & 0.005538636 \\
\hline PRAK(h) & 94 & 0 & 77 & 77 & 69 & 0.811764706 & 23 & 0.017011834 & 0.496301775 & 0.005738775 \\
\hline PRK2(h) & 81 & 0 & 76 & 76 & 70 & 0.823529412 & 24 & 0.017751479 & 0.514053254 & 0.005943265 \\
\hline ROCK-II(h) & 86 & 0 & 74 & 74 & 71 & 0.835294118 & 26 & 0.019230769 & 0.533284024 & 0.006160808 \\
\hline ROCK-IIIr) & 79 & 0 & 73 & 73 & 72 & 0.847058824 & 27 & 0.019970414 & 0.553254438 & 0.006391403 \\
\hline Rskl(h) & 73 & 0 & 72 & 72 & 73 & 0.858823529 & 28 & 0.020710059 & 0.573964497 & 0.0066307 \\
\hline Rskl(r) & 62 & 0 & 67 & 67 & 74 & 0.870588235 & 33 & 0.024408284 & 0.598372781 & 0.006896102 \\
\hline Rsk2(h) & 62 & 0 & 66 & 66 & 75 & 0.882352941 & 34 & 0.025147929 & 0.62352071 & 0.007187609 \\
\hline Rsk3(h) & 65 & 0 & 65 & 65 & 76 & 0.894117647 & 35 & 0.025887574 & 0.649408284 & 0.007487818 \\
\hline SAPK2a(h) & 88 & 0 & 64 & 64 & 77 & 0.905882353 & 36 & 0.026627219 & 0.676035503 & 0.007796728 \\
\hline SAPK2b(h) & 92 & 0 & 62 & 62 & 78 & 0.917647059 & 38 & 0.028106509 & 0.704142012 & 0.008118691 \\
\hline SAPK3(h) & 98 & 0 & 62 & 62 & 79 & 0.929411765 & 38 & 0.028106509 & 0.732248521 & 0.008449356 \\
\hline SAPK 4(h) & 94 & 0 & 60 & 60 & 80 & 0.941176471 & 40 & 0.029585799 & 0.76183432 & 0.008788723 \\
\hline SGK(h) & 78 & 0 & 58 & 58 & 81 & 0.952941176 & 42 & 0.031065089 & 0.792899408 & 0.009145493 \\
\hline Syk(h) & 89 & 0 & 47 & 47 & 82 & 0.964705882 & 53 & 0.039201183 & 0.832100592 & 0.009558824 \\
\hline TrkB(h) & 60 & 0 & 42 & 42 & 83 & 0.976470588 & 58 & 0.042899408 & 0.875 & 0.010041768 \\
\hline Yes(h) & 67 & 0 & 17 & 17 & 84 & 0.988235294 & 83 & 0.061390533 & 0.936390533 & 0.010655238 \\
\hline ZAP-70(h) & 113 & 0 & 14 & 14 & 85 & 1 & 86 & 0.063609467 & 1 & 0.011390533 \\
\hline & & & & & & Total inhibition & 1352 & & Total area & 0.251157327 \\
\hline & $\begin{array}{l}\text { Hit rate } \\
\text { Threshold }\end{array}$ & . & & & & & & & Gini & 0.497685346 \\
\hline
\end{tabular}




\begin{tabular}{|c|c|c|c|c|c|c|c|c|c|}
\hline & $100 \mu \mathrm{M}$ ATP & & & & & & & & \\
\hline Kinase & AG1296@ @ $10 \mu \mathrm{M}$ & Hit & sorted & normalized & position & cumulative sample fraction & inhibition\% & fraction of total inhibition & cumulative inhibition \\
\hline $\mathrm{Abl}(\mathrm{m})$ & 96 & 0 & 115 & 100 & 1 & 0.011764706 & 0 & 0 & 0 \\
\hline AMPK(r) & 101 & 0 & 114 & 100 & 2 & 0.023529412 & 0 & 0 & 0 \\
\hline $\operatorname{Arg}(\mathrm{m})$ & 94 & 0 & 108 & 100 & 3 & 0.035294118 & 0 & 0 & 0 \\
\hline Aurora-A(h) & 86 & 0 & 105 & 100 & 4 & 0.047058824 & 0 & 0 & 0 \\
\hline Axl(h) & 78 & 0 & 105 & 100 & 5 & 0.058823529 & 0 & 0 & 0 \\
\hline $\mathrm{Bk}(\mathrm{m})$ & 78 & 0 & 105 & 100 & 6 & 0.070588235 & 0 & 0 & 0 \\
\hline $\operatorname{Bmx}(\mathrm{h})$ & 97 & 0 & 104 & 100 & 7 & 0.082352941 & 0 & 0 & 0 \\
\hline CaMKII(r) & 100 & 0 & 104 & 100 & 8 & 0.094117647 & 0 & 0 & 0 \\
\hline CaMKIV(h) & 92 & 0 & 104 & 100 & 9 & 0.105882353 & 0 & 0 & 0 \\
\hline $\mathrm{CDK} 1 /$ cyclinB(h) & 88 & 0 & 102 & 100 & 10 & 0.117647059 & 0 & 0 & 0 \\
\hline $\mathrm{CDK} 2 /$ cyclinA(h) & 105 & 0 & 102 & 100 & 11 & 0.129411765 & 0 & 0 & 0 \\
\hline $\mathrm{CDK} 2 /$ cyclinE(h) & 97 & 0 & 102 & 100 & 12 & 0.141176471 & 0 & 0 & 0 \\
\hline CDK3/cyclinE(h) & 115 & 0 & 101 & 100 & 13 & 0.152941176 & 0 & 0 & 0 \\
\hline CDK5/p35(h) & 101 & 0 & 101 & 100 & 14 & 0.164705882 & 0 & 0 & 0 \\
\hline CDK6/cyclinD3(h) & 84 & 0 & 101 & 100 & 15 & 0.176470588 & 0 & 0 & 0 \\
\hline CDK7/cyclinHMATI(h) & 92 & 0 & 100 & 100 & 16 & 0.188235294 & 0 & 0 & 0 \\
\hline CHK1(h) & 91 & 0 & 100 & 100 & 17 & 0.2 & 0 & 0 & 0 \\
\hline СHK2(h) & 99 & 0 & 100 & 100 & 18 & 0.211764706 & 0 & 0 & 0 \\
\hline $\mathrm{CKI}(\mathrm{y})$ & 98 & 0 & 100 & 100 & 19 & 0.223529412 & 0 & 0 & 0 \\
\hline CK2(h) & 97 & 0 & 100 & 100 & 20 & 0.235294118 & 0 & 0 & 0 \\
\hline$c-\operatorname{RAF}(\mathrm{h})$ & 91 & 0 & 99 & 99 & 21 & 0.247058824 & 1 & 0.001142857 & 0.001142857 \\
\hline $\operatorname{CSK}(\mathrm{h})$ & 108 & 0 & 99 & 99 & 22 & 0.258823529 & 1 & 0.001142857 & 0.002285714 \\
\hline $\mathrm{cSRC}(\mathrm{h})$ & 83 & 0 & 98 & 98 & 23 & 0.270588235 & 2 & 0.002285714 & 0.004571429 \\
\hline Fes(h) & 102 & 0 & 97 & 97 & 24 & 0.282352941 & 3 & 0.003428571 & 0.008 \\
\hline FGFR3(h) & 70 & 0 & 97 & 97 & 25 & 0.294117647 & 3 & 0.003428571 & 0.011428571 \\
\hline Fll3(h) & 20 & 1 & 97 & 97 & 26 & 0.305882353 & 3 & 0.003428571 & 0.014857143 \\
\hline Fynn(h) & 92 & 0 & 97 & 97 & 27 & 0.317647059 & 3 & 0.003428571 & 0.018285714 \\
\hline GSK3a(h) & 94 & 0 & 96 & 96 & 28 & 0.329411765 & 4 & 0.004571429 & 0.022857143 \\
\hline GSK3B(h) & 84 & 0 & 95 & 95 & 29 & 0.341176471 & 5 & 0.005714286 & 0.028571429 \\
\hline IGF-IR(h) & 104 & 0 & 95 & 95 & 30 & 0.352941176 & 5 & 0.005714286 & 0.034285714 \\
\hline IKKa(h) & 90 & 0 & 95 & 95 & 31 & 0.364705882 & 5 & 0.005714286 & 0.04 \\
\hline IKKB(h) & 95 & 0 & 94 & 94 & 32 & 0.376470588 & 6 & 0.006857143 & 0.046857143 \\
\hline $\mathrm{IR}(\mathrm{h})$ & 104 & 0 & 94 & 94 & 33 & 0.388235294 & 6 & 0.006857143 & 0.053714286 \\
\hline JNKIal(h) & 92 & 0 & 94 & 94 & 34 & 0.4 & 6 & 0.006857143 & 0.060571429 \\
\hline JNK2a2(h) & 94 & 0 & 94 & 94 & 35 & 0.411764706 & 6 & 0.006857143 & 0.067428571 \\
\hline JNK3(r) & 86 & 0 & 94 & 94 & 36 & 0.423529412 & 6 & 0.006857143 & 0.074285714 \\
\hline Lck(h) & 80 & 0 & 93 & 93 & 37 & 0.435294118 & 7 & 0.008 & 0.082285714 \\
\hline Lyn(h) & 84 & 0 & 93 & 93 & 38 & 0.447058824 & 7 & 0.008 & 0.090285714 \\
\hline Lyn(m) & 90 & 0 & 93 & 93 & 39 & 0.458823529 & 7 & 0.008 & 0.098285714 \\
\hline MAPKI(h) & 84 & 0 & 92 & 92 & 40 & 0.470588235 & 8 & 0.009142857 & 0.107428571 \\
\hline MAPK2(h) & 105 & 0 & 92 & 92 & 41 & 0.482352941 & 8 & 0.009142857 & 0.116571429 \\
\hline MAPK2(m) & 94 & 0 & 92 & 92 & 42 & 0.494117647 & 8 & 0.009142857 & 0.125714286 \\
\hline MAPKAP-K2(h) & 93 & 0 & 92 & 92 & 43 & 0.505882353 & 8 & 0.009142857 & 0.134857143 \\
\hline MEK1(h) & 102 & 0 & 92 & 92 & 44 & 0.517647059 & 8 & 0.009142857 & 0.144 \\
\hline MKK4(m) & 88 & 0 & 92 & 92 & 45 & 0.529411765 & 8 & 0.009142857 & 0.153142857 \\
\hline MKKG(h) & 87 & 0 & 92 & 92 & 46 & 0.541176471 & 8 & 0.009142857 & 0.162285714 \\
\hline МKK 78 (h) & 77 & 0 & 92 & 92 & 47 & 0.552941176 & 8 & 0.009142857 & 0.171428571 \\
\hline MSK1(h) & 95 & 0 & 91 & 91 & 48 & 0.564705882 & 9 & 0.010285714 & 0.181714286 \\
\hline $\mathrm{p} 70 \mathrm{~S} 6 \mathrm{~K}(\mathrm{~h})$ & 93 & 0 & 91 & 91 & 49 & 0.576470588 & 9 & 0.010285714 & 0.192 \\
\hline PAK2(h) & 92 & 0 & 91 & 91 & 50 & 0.588235294 & 9 & 0.010285714 & 0.202285714 \\
\hline PDGFRo(h) & 44 & 1 & 90 & 90 & 51 & 0.6 & 10 & 0.011428571 & 0.213714286 \\
\hline PDGFRB(h) & 83 & 0 & 90 & 90 & 52 & 0.611764706 & 10 & 0.011428571 & 0.225142857 \\
\hline PDK1(h) & 97 & 0 & 90 & 90 & 53 & 0.623529412 & 10 & 0.011428571 & 0.236571429 \\
\hline PKA(b) & 83 & 0 & 89 & 89 & 54 & 0.635294118 & 11 & 0.012571429 & 0.249142857 \\
\hline PKA(h) & 100 & 0 & 88 & 88 & 55 & 0.647058824 & 12 & 0.013714286 & 0.262857143 \\
\hline PKBach) & 89 & 0 & 88 & 88 & 56 & 0.658823529 & 12 & 0.013714286 & 0.276571429 \\
\hline PKBB(h) & 100 & 0 & 88 & 88 & 57 & 0.670588235 & 12 & 0.013714286 & 0.290285714 \\
\hline PKB $\gamma(\mathrm{h})$ & 104 & 0 & 88 & 88 & 58 & 0.682352941 & 12 & 0.013714286 & 0.304 \\
\hline PKCadh) & 92 & 0 & 87 & 87 & 59 & 0.694117647 & 13 & 0.014857143 & 0.318857143 \\
\hline PKCBI(h) & 92 & 0 & 87 & 87 & 60 & 0.705882353 & 13 & 0.014857143 & 0.333714286 \\
\hline PKC (h(h) & 102 & 0 & 87 & 87 & 61 & 0.717647059 & 13 & 0.014857143 & 0.348571429 \\
\hline PKC (̧h) & 95 & 0 & 86 & 86 & 62 & 0.729411765 & 14 & 0.016 & 0.364571429 \\
\hline PKCa(h) & 88 & 0 & 86 & 86 & 63 & 0.741176471 & 14 & 0.016 & 0.380571429 \\
\hline PKCril & 105 & 0 & 86 & 86 & 64 & 0.752941176 & 14 & 0.016 & 0.396571429 \\
\hline PKC (h) & 90 & 0 & 85 & 85 & 65 & 0.764705882 & 15 & 0.017142857 & 0.413714286 \\
\hline $\mathrm{PKC \mu} \mu(\mathrm{h})$ & 94 & 0 & 85 & 85 & 66 & 0.776470588 & 15 & 0.017142857 & 0.430857143 \\
\hline PKCa & 85 & 0 & 84 & 84 & 67 & 0.788235294 & 16 & 0.018285714 & 0.449142857 \\
\hline PKD2(h) & 91 & 0 & 84 & 84 & 68 & 0.8 & 16 & 0.018285714 & 0.467428571 \\
\hline PRAK(h) & 86 & 0 & 84 & 84 & 69 & 0.811764706 & 16 & 0.018285714 & 0.485714286 \\
\hline PRK2(h) & 100 & 0 & 84 & 84 & 70 & 0.823529412 & 16 & 0.018285714 & 0.504 \\
\hline ROCK-II(h) & 87 & 0 & 83 & 83 & 71 & 0.835294118 & 17 & 0.019428571 & 0.523428571 \\
\hline ROCK-IIIr) & 88 & 0 & 83 & 83 & 72 & 0.847058824 & 17 & 0.019428571 & 0.542857143 \\
\hline Rskl(h) & 73 & 0 & 83 & 83 & 73 & 0.858823529 & 17 & 0.019428571 & 0.562285714 \\
\hline Rskl(r) & 73 & 0 & 80 & 80 & 74 & 0.870588235 & 20 & 0.022857143 & 0.585142857 \\
\hline Rsk2(h) & 85 & 0 & 79 & 79 & 75 & 0.882352941 & 21 & 0.024 & 0.609142857 \\
\hline Rsk3(h) & 77 & 0 & 78 & 78 & 76 & 0.894117647 & 22 & 0.025142857 & 0.634285714 \\
\hline SAPK2a(h) & 101 & 0 & 78 & 78 & 77 & 0.905882353 & 22 & 0.025142857 & 0.659428571 \\
\hline SAPK2b(h) & 114 & 0 & 77 & 77 & 78 & 0.917647059 & 23 & 0.026285714 & 0.685714286 \\
\hline SAPK3(h) & 100 & 0 & 77 & 77 & 79 & 0.929411765 & 23 & 0.026285714 & 0.712 \\
\hline SAPK4(h) & 93 & 0 & 73 & 73 & 80 & 0.941176471 & 27 & 0.030857143 & 0.742857143 \\
\hline $\mathrm{SGK}(\mathrm{h})$ & 92 & 0 & 73 & 73 & 81 & 0.952941176 & 27 & 0.030857143 & 0.773714286 \\
\hline Syk(h) & 99 & 0 & 70 & 70 & 82 & 0.964705882 & 30 & 0.034285714 & 0.808 \\
\hline TrkB(h) & 79 & 0 & 68 & 68 & 83 & 0.976470588 & 32 & 0.036571429 & 0.844571429 \\
\hline Yes(h) & 68 & 0 & 44 & 44 & 84 & 0.988235294 & 56 & 0.064 & 0.908571429 \\
\hline ZAP-70(h) & 87 & 0 & 20 & 20 & 85 & 1 & 80 & 0.091428571 & 1 \\
\hline & & & & & & Total inhibition & 875 & & Total area \\
\hline & $\begin{array}{l}\text { Hit rate } \\
\text { Threshold }\end{array}$ & & & & & & & & Gini \\
\hline
\end{tabular}




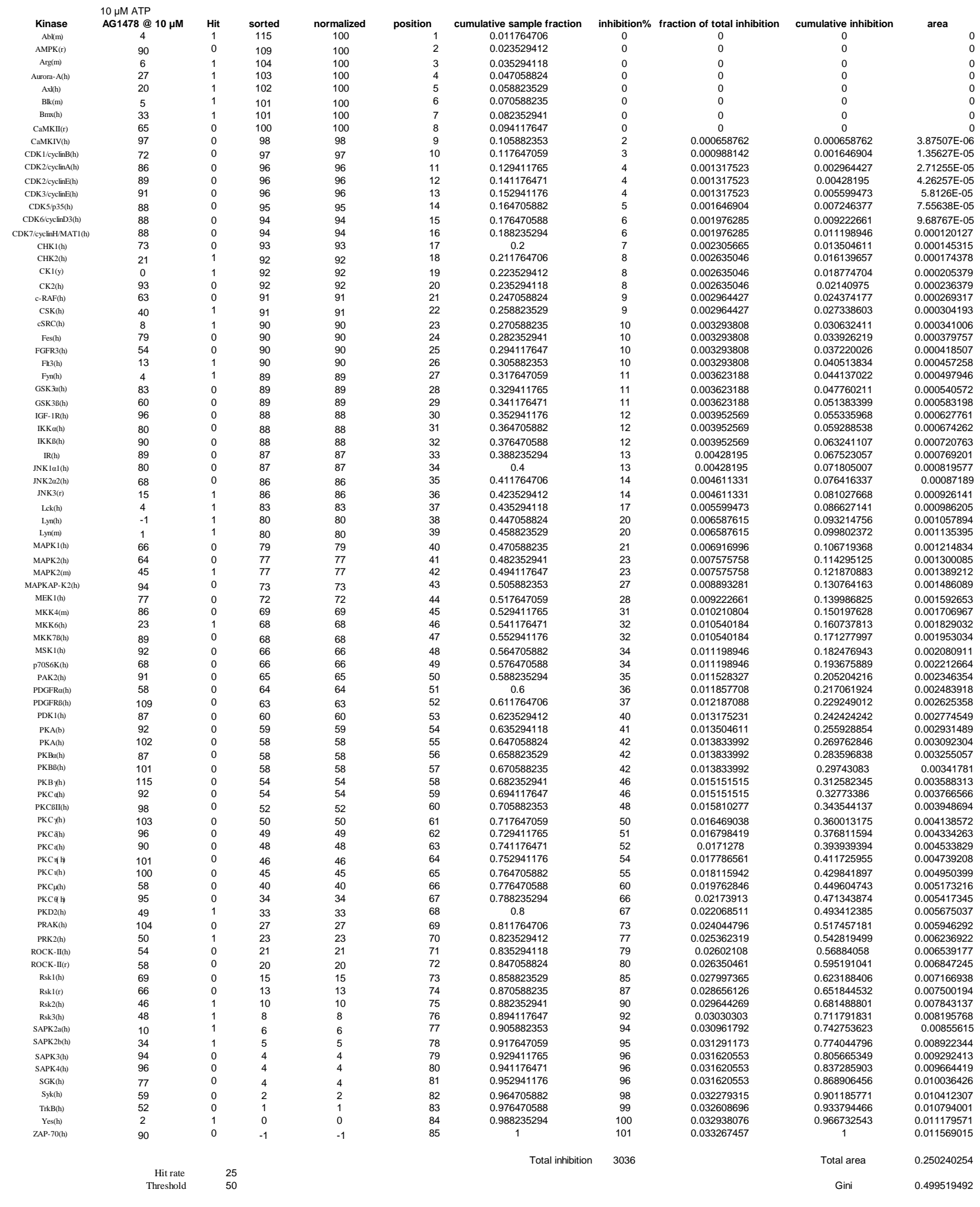




\begin{tabular}{|c|c|c|c|c|c|c|c|c|c|}
\hline & $100 \mu \mathrm{M}$ ATP & & & & & & & & \\
\hline Kinase & AG1478@ @ $10 \mu \mathrm{M}$ & Hit & sorted & normalized & position & cumulative sample fraction & inhibition\% & fraction of total inhibition & cumulative inhibition \\
\hline $\mathrm{Abl(m)}$ & 19 & 1 & 120 & 100 & 1 & 0.011764706 & 0 & 0 & 0 \\
\hline AMPK(r) & 101 & 0 & 113 & 100 & 2 & 0.023529412 & 0 & 0 & 0 \\
\hline $\operatorname{Arg}(\mathrm{m})$ & 16 & 1 & 111 & 100 & 3 & 0.035294118 & 0 & 0 & 0 \\
\hline Aurora-A(h) & 47 & 1 & 109 & 100 & 4 & 0.047058824 & 0 & 0 & 0 \\
\hline Axl(h) & 33 & 1 & 107 & 100 & 5 & 0.058823529 & 0 & 0 & 0 \\
\hline $\mathrm{Blk}(\mathrm{m})$ & 3 & 1 & 107 & 100 & 6 & 0.070588235 & 0 & 0 & 0 \\
\hline $\mathrm{Bmx(h)}$ & 59 & 0 & 106 & 100 & 7 & 0.082352941 & 0 & 0 & 0 \\
\hline CaMKII(r) & 97 & 0 & 106 & 100 & 8 & 0.094117647 & 0 & 0 & 0 \\
\hline CaMKIV(h) & 97 & 0 & 106 & 100 & 9 & 0.105882353 & 0 & 0 & 0 \\
\hline CDK1/cyclinB(h) & 86 & 0 & 106 & 100 & 10 & 0.117647059 & 0 & 0 & 0 \\
\hline $\mathrm{CDK} 2 / \mathrm{cyclin} \mathrm{A}(\mathrm{h})$ & 96 & 0 & 105 & 100 & 11 & 0.129411765 & 0 & 0 & 0 \\
\hline $\mathrm{CDK} 2 /$ cyclinE(h) & 100 & 0 & 104 & 100 & 12 & 0.141176471 & 0 & 0 & 0 \\
\hline CDK3/cyclinE(h) & 113 & 0 & 104 & 100 & 13 & 0.152941176 & 0 & 0 & 0 \\
\hline CDK5/p35(h) & 107 & 0 & 103 & 100 & 14 & 0.164705882 & 0 & 0 & 0 \\
\hline CDK6/cyclinD3(h) & 86 & 0 & 103 & 100 & 15 & 0.176470588 & 0 & 0 & 0 \\
\hline CDK7/cyclinHMATI(h) & 94 & 0 & 103 & 100 & 16 & 0.188235294 & 0 & 0 & 0 \\
\hline CHK (hi) & 92 & 0 & 103 & 100 & 17 & 0.2 & 0 & 0 & 0 \\
\hline СHK2(h) & 35 & 1 & 102 & 100 & 18 & 0.211764706 & 0 & 0 & 0 \\
\hline $\mathrm{CKI}(\mathrm{y})$ & 6 & 1 & 101 & 100 & 19 & 0.223529412 & 0 & 0 & 0 \\
\hline СК2(h) & $\begin{array}{l}0 \\
103\end{array}$ & 0 & 100 & 100 & 20 & 0.235294118 & 0 & 0 & 0 \\
\hline$c-R A F(h)$ & 84 & 0 & 100 & 100 & 21 & 0.247058824 & 0 & 0 & 0 \\
\hline CSK (h) & 93 & 0 & 100 & 100 & 22 & 0.258823529 & 0 & 0 & 0 \\
\hline $\operatorname{cSRC}(\mathrm{h})$ & 13 & 1 & 99 & 99 & 23 & 0.270588235 & 1 & 0.000481696 & 0.000481696 \\
\hline Fes(h) & 106 & 0 & 99 & 99 & 24 & 0.282352941 & 1 & 0.000481696 & 0.000963391 \\
\hline FGFR3(h) & 93 & 0 & 99 & 99 & 25 & 0.294117647 & 1 & 0.000481696 & 0.001445087 \\
\hline $\mathrm{FH}$ (h) $(\mathrm{h})$ & 21 & 1 & 99 & 99 & 26 & 0.305882353 & 1 & 0.000481696 & 0.001926782 \\
\hline Fyn(h) & 14 & 1 & 99 & 99 & 27 & 0.317647059 & 1 & 0.000481696 & 0.002408478 \\
\hline GSK3a(h) & 107 & 0 & 98 & 98 & 28 & 0.329411765 & 2 & 0.000963391 & 0.003371869 \\
\hline GSK3B(h) & 91 & 0 & 97 & $\begin{array}{l}50 \\
97\end{array}$ & $\begin{array}{l}20 \\
29\end{array}$ & 0.341176471 & 3 & 0.001445087 & 0.004816956 \\
\hline $\mathrm{IGF}-1 \mathrm{R}(\mathrm{h})$ & 106 & 0 & 97 & 97 & 30 & 0.352941176 & 3 & 0.001445087 & 0.006262042 \\
\hline IKKa(h) & 109 & 0 & 96 & 96 & 31 & 0.364705882 & 4 & 0.001926782 & 0.008188825 \\
\hline IKKB(h) & 100 & 0 & 96 & 96 & 32 & 0.376470588 & 4 & 0.001926782 & 0.010115607 \\
\hline $\mathrm{IR}(\mathrm{h})$ & 120 & 0 & 95 & 95 & 33 & 0.388235294 & $\begin{array}{l}4 \\
5\end{array}$ & 0.002408478 & 0.012524085 \\
\hline JNKIal(h) & 103 & 0 & 95 & 95 & 34 & 0.4 & 5 & 0.002408478 & 0.014932563 \\
\hline JNK2a2(h) & 99 & 0 & 94 & 94 & 35 & 0.411764706 & 6 & 0.002890173 & 0.017822736 \\
\hline $\mathrm{JNK} 3(\mathrm{r})$ & 69 & 0 & 94 & 94 & 36 & 0.423529412 & 6 & 0.002890173 & 0.020712909 \\
\hline Lck(h) & 3 & 1 & 93 & 93 & 37 & 0.435294118 & 7 & 0.003371869 & 0.024084778 \\
\hline Lyn(h) & 0 & 1 & 93 & 93 & 38 & 0.447058824 & 7 & 0.003371869 & 0.027456647 \\
\hline Lyn(m) & 2 & 1 & 93 & 93 & 39 & 0.458823529 & 7 & 0.003371869 & 0.030828516 \\
\hline MAPKI(h) & 64 & 0 & $\begin{array}{l}93 \\
92\end{array}$ & $\begin{array}{l}93 \\
92\end{array}$ & 40 & 0.470588235 & 8 & 0.003853565 & 0.034682081 \\
\hline MAPK2(h) & 73 & 0 & 92 & 92 & 41 & 0.482352941 & 8 & 0.003853565 & 0.038535645 \\
\hline $\begin{array}{l}\text { MAAR2 2(n) } \\
\text { MAPK2(m) }\end{array}$ & 63 & 0 & 92 & 92 & 42 & 0.494117647 & 8 & 0.003853565 & 0.04238921 \\
\hline MAPKAP-K2(h) & 106 & 0 & 91 & 91 & 43 & 0.505882353 & 9 & 0.00433526 & 0.04672447 \\
\hline MEK1(h) & 88 & 0 & 90 & 90 & 44 & 0.517647059 & 10 & 0.004816956 & 0.051541426 \\
\hline MKK 4(m) & 77 & 0 & 90 & 90 & 45 & 0.529411765 & 10 & 0.004816956 & 0.056358382 \\
\hline MKK $6(\mathrm{~h})$ & 18 & 1 & 88 & 88 & 46 & 0.541176471 & 12 & 0.005780347 & 0.062138728 \\
\hline MKK7(B(h) & 90 & 0 & 88 & 88 & 47 & 0.552941176 & 12 & 0.005780347 & 0.067919075 \\
\hline MSK1(h) & 104 & 0 & 87 & 87 & 48 & 0.564705882 & 13 & 0.006262042 & 0.074181118 \\
\hline $\mathrm{p} 70 \mathrm{~S} 6 \mathrm{~K}(\mathrm{~h})$ & 99 & 0 & 86 & 86 & 49 & 0.576470588 & 14 & 0.006743738 & 0.080924855 \\
\hline $\begin{array}{l}\text { PAK2(h) } \\
\text { (l) }\end{array}$ & 102 & 0 & 86 & 86 & 50 & 0.588235294 & 14 & 0.006743738 & 0.087668593 \\
\hline PDGFRa(h) & 76 & 0 & 85 & 85 & 51 & 0.6 & 15 & 0.007225434 & 0.094894027 \\
\hline PDGFRB(h) & 88 & 0 & 85 & 85 & 52 & 0.611764706 & 15 & 0.007225434 & 0.102119461 \\
\hline PDK $1(\mathrm{~h})$ & $\begin{array}{l}00 \\
99\end{array}$ & 0 & 84 & $\begin{array}{l}80 \\
84\end{array}$ & 53 & 0.623529412 & 16 & 0.007707129 & 0.10982659 \\
\hline PKA(b) & 104 & 0 & 80 & 80 & 54 & 0.635294118 & 20 & 0.009633911 & 0.119460501 \\
\hline $\begin{array}{l}\text { PRA(b) } \\
\text { PKA(h) }\end{array}$ & 93 & 0 & 77 & 77 & 55 & 0.647058824 & 23 & 0.011078998 & 0.130539499 \\
\hline PKBa(h) & 92 & 0 & 76 & 76 & 56 & 0.658823529 & 24 & 0.011560694 & 0.142100193 \\
\hline PKBB(h) & $\begin{array}{l}92 \\
111\end{array}$ & 0 & 74 & 74 & 57 & 0.670588235 & 26 & 0.012524085 & 0.154624277 \\
\hline PKB $\gamma(h)$ & 105 & 0 & 73 & 73 & 58 & 0.682352941 & $\begin{array}{l}20 \\
27\end{array}$ & 0.01300578 & 0.167630058 \\
\hline PKC(h) & 94 & 0 & 73 & 73 & 59 & 0.694117647 & 27 & 0.01300578 & 0.180635838 \\
\hline РКСBII(h) & 100 & 0 & 70 & 70 & 60 & 0.705882353 & 30 & 0.014450867 & 0.195086705 \\
\hline PKC (h) & 95 & 0 & 70 & 70 & 61 & 0.717647059 & 30 & 0.014450867 & 0.209537572 \\
\hline PKC\&h) & 87 & 0 & 69 & 69 & 62 & 0.729411765 & 31 & 0.014932563 & 0.224470135 \\
\hline 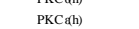 & 99 & 0 & 69 & 69 & 63 & 0.741176471 & 31 & 0.014932563 & 0.239402697 \\
\hline 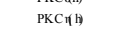 & 103 & 0 & 65 & 65 & 64 & 0.752941176 & 35 & 0.016859345 & 0.256262042 \\
\hline PKCl(h) & 96 & 0 & 64 & 64 & 65 & 0.764705882 & 36 & 0.01734104 & 0.273603083 \\
\hline PKC $\mu(\mathrm{h})$ & 70 & 0 & 63 & 63 & 66 & 0.776470588 & 37 & 0.017822736 & 0.291425819 \\
\hline $\begin{array}{l}P K C(n) \\
\text { PKCA }\end{array}$ & 90 & 0 & 61 & 61 & 67 & 0.788235294 & 39 & 0.018786127 & 0.310211946 \\
\hline $\begin{array}{l}\text { PKD2(h) } \\
\text { P. }\end{array}$ & 73 & 0 & 59 & 59 & 68 & 0.8 & 41 & 0.019749518 & 0.329961464 \\
\hline $\begin{array}{l}\text { PRAK(h) } \\
\text { Pall }\end{array}$ & $\begin{array}{l}13 \\
92\end{array}$ & 0 & $\begin{array}{l}59 \\
57\end{array}$ & $\begin{array}{l}59 \\
57\end{array}$ & 69 & 0.811764706 & 43 & 0.020712909 & 0.350674374 \\
\hline PRK2(h) & 70 & 0 & 47 & 47 & 70 & 0.823529412 & $\begin{array}{l}40 \\
53\end{array}$ & 0.025529865 & 0.376204239 \\
\hline $\begin{array}{l}\text { PRK(2) } \\
\text { ROCKII(h) }\end{array}$ & 80 & 0 & 35 & 35 & 71 & 0.835294118 & 65 & 0.031310212 & 0.407514451 \\
\hline ROCK-II(r) & 85 & 0 & 33 & 33 & 72 & 0.847058824 & 67 & 0.032273603 & 0.439788054 \\
\hline Rskl(h) & 85 & 0 & 21 & 21 & 73 & 0.858823529 & 79 & 0.03805395 & 0.477842004 \\
\hline Rskl(r) & 103 & 0 & 19 & 19 & 74 & 0.870588235 & 81 & 0.039017341 & 0.516859345 \\
\hline Rsk2(h) & 65 & 0 & 18 & 18 & 75 & 0.882352941 & 82 & 0.039499037 & 0.556358382 \\
\hline Rsk3(h) & 69 & 0 & 16 & 16 & 76 & 0.894117647 & 84 & 0.040462428 & 0.596820809 \\
\hline SAPK2a(h) & 16 & 1 & 16 & 16 & 77 & 0.905882353 & 84 & 0.040462428 & 0.637283237 \\
\hline SAPK2b(h) & 57 & 0 & 14 & 14 & 78 & 0.917647059 & 86 & 0.041425819 & 0.678709056 \\
\hline SAPK3(h) & 106 & 0 & 13 & 13 & 79 & 0.929411765 & 87 & 0.041907514 & 0.72061657 \\
\hline $\begin{array}{l}\mathrm{SAAR} \text { (h) } \\
\operatorname{SAPK} 4(\mathrm{~h})\end{array}$ & 99 & 0 & 6 & 6 & 80 & 0.941176471 & 94 & 0.045279383 & 0.765895954 \\
\hline SGK(h) & 95 & 0 & 6 & 6 & 81 & 0.952941176 & 94 & 0.045279383 & 0.811175337 \\
\hline Syk(h) & 61 & 0 & 3 & 3 & 82 & 0.964705882 & 97 & 0.04672447 & 0.857899807 \\
\hline $\mathrm{TrkB(h)}$ & 74 & 0 & 3 & 3 & 83 & 0.976470588 & 97 & 0.04672447 & 0.904624277 \\
\hline $\begin{array}{l}\text { IIrkB(h) } \\
\text { Yes(h) }\end{array}$ & $\begin{array}{l}74 \\
6\end{array}$ & 1 & 2 & $\begin{array}{l}3 \\
2\end{array}$ & 84 & 0.988235294 & $\begin{array}{l}97 \\
98\end{array}$ & 0.047206166 & 0.951830443 \\
\hline ZAP-70(h) & 98 & 0 & 0 & 0 & 85 & 1 & 100 & 0.048169557 & 1 \\
\hline & & & & & & Total inhibition & 2076 & & Total area \\
\hline & $\begin{array}{c}\text { Hit rate } \\
\text { Threshol }\end{array}$ & $\begin{array}{l}1 \\
5\end{array}$ & & & & & & & Gini \\
\hline
\end{tabular}




\begin{tabular}{|c|c|c|c|c|c|c|c|c|c|}
\hline & $10 \mu \mathrm{M}$ ATP & & & & & & & & \\
\hline Kinase & AG18@ $10 \mu \mathrm{M}$ & Hit & sorted & normalized & position & cumulative sample fraction & inhibition\% & fraction of total inhibition & cumulative inhibition \\
\hline $\mathrm{Ab}(\mathrm{m})$ & 98 & 0 & 122 & 100 & 1 & 0.011764706 & 0 & 0 & 0 \\
\hline AMPK(r) & 103 & 0 & 115 & 100 & 2 & 0.023529412 & 0 & 0 & 0 \\
\hline $\operatorname{Arg}(\mathrm{m})$ & 102 & 0 & 114 & 100 & 3 & 0.035294118 & 0 & 0 & 0 \\
\hline Aurora-A(h) & 80 & 0 & 110 & 100 & 4 & 0.047058824 & 0 & 0 & 0 \\
\hline Axl(h) & 96 & 0 & 109 & 100 & 5 & 0.058823529 & 0 & 0 & 0 \\
\hline $\mathrm{Blk}(\mathrm{m})$ & 76 & 0 & 109 & 100 & 6 & 0.070588235 & 0 & 0 & 0 \\
\hline Bmx(h) & 96 & 0 & 108 & 100 & 7 & 0.082352941 & 0 & 0 & 0 \\
\hline CaMKII(r) & 96 & 0 & 108 & 100 & 8 & 0.094117647 & 0 & 0 & 0 \\
\hline CaMKIV(h) & 103 & 0 & 107 & 100 & 9 & 0.105882353 & 0 & 0 & 0 \\
\hline CDK1/cyclinB(h) & 92 & 0 & 107 & 100 & 10 & 0.117647059 & 0 & 0 & 0 \\
\hline $\mathrm{CDK} 2 /$ cyclinA(h) & 97 & 0 & 105 & 100 & 11 & 0.129411765 & 0 & 0 & 0 \\
\hline $\mathrm{CDK} 2 / \mathrm{cyclin} E(\mathrm{~h})$ & 95 & 0 & 104 & 100 & 12 & 0.141176471 & 0 & 0 & 0 \\
\hline CDK3/cyclinE(h) & 97 & 0 & 104 & 100 & 13 & 0.152941176 & 0 & 0 & 0 \\
\hline CDK5/p35(h) & 97 & 0 & 104 & 100 & 14 & 0.164705882 & 0 & 0 & 0 \\
\hline CDK6/leyclinD3(h) & 100 & 0 & 104 & 100 & 15 & 0.176470588 & 0 & 0 & 0 \\
\hline CDK7/cyclinHMATI(h) & 98 & 0 & 104 & 100 & 16 & 0.188235294 & 0 & 0 & 0 \\
\hline $\begin{array}{l}\text { CHK } 1(\mathrm{~h}) \\
\text { (1) }\end{array}$ & 101 & 0 & 103 & 100 & 17 & 0.2 & 0 & 0 & 0 \\
\hline СHK2(h) & 95 & 0 & 103 & 100 & 18 & 0.211764706 & 0 & 0 & 0 \\
\hline CKI(y) & 99 & 0 & 103 & 100 & 19 & 0.223529412 & 0 & 0 & 0 \\
\hline СК2(h) & 15 & 1 & 102 & 100 & 20 & 0.235294118 & 0 & 0 & 0 \\
\hline c-RAF(h) & 94 & 0 & 102 & 100 & 21 & 0.247058824 & 0 & 0 & 0 \\
\hline $\operatorname{CSK}(\mathrm{h})$ & 56 & 0 & 102 & 100 & 22 & 0.258823529 & 0 & 0 & 0 \\
\hline cSRC(h) & 100 & 0 & 101 & 100 & 23 & 0.270588235 & 0 & 0 & 0 \\
\hline Fes(h) & 97 & 0 & 101 & 100 & 24 & 0.282352941 & 0 & 0 & 0 \\
\hline FGFR3(h) & 109 & 0 & 100 & 100 & 25 & 0.294117647 & 0 & 0 & 0 \\
\hline $\mathrm{FHt3( \textrm {h } )}$ & 91 & 0 & 100 & 100 & 26 & 0.305882353 & 0 & 0 & 0 \\
\hline Fyn(h) & 90 & 0 & 100 & 100 & 27 & 0.317647059 & 0 & 0 & 0 \\
\hline GSK3a(h) & 87 & 0 & 100 & 100 & 28 & 0.329411765 & 0 & 0 & 0 \\
\hline GSK3B(h) & 104 & 0 & 99 & 99 & 29 & 0.341176471 & 1 & 0.001639344 & 0.001639344 \\
\hline IGF-1R(h) & 114 & 0 & 99 & 99 & 30 & 0.352941176 & 1 & 0.001639344 & 0.003278689 \\
\hline IKKa(h) & 94 & 0 & 99 & 99 & 31 & 0.364705882 & 1 & 0.001639344 & 0.004918033 \\
\hline IKKB(h) & 107 & 0 & 99 & 99 & 32 & 0.376470588 & 1 & 0.001639344 & 0.006557377 \\
\hline $\mathrm{IR}(\mathrm{h})$ & 104 & 0 & 99 & 99 & 33 & 0.388235294 & 1 & 0.001639344 & 0.008196721 \\
\hline JNKIal(h) & 89 & 0 & 98 & 98 & 34 & $\begin{array}{l}0.4 \\
0.45\end{array}$ & 2 & 0.003278689 & 0.01147541 \\
\hline JNK2a2(h) & 107 & 0 & 98 & 98 & 35 & 0.411764706 & 2 & 0.003278689 & 0.014754098 \\
\hline $\mathrm{JNK} 3(\mathrm{r})$ & 101 & 0 & 98 & 98 & 36 & 0.423529412 & 2 & 0.003278689 & 0.018032787 \\
\hline Lck(h) & 79 & 0 & 98 & 98 & 37 & 0.435294118 & 2 & 0.003278689 & 0.021311475 \\
\hline Lyn(h) & 92 & 0 & 97 & 97 & 38 & 0.447058824 & 3 & 0.004918033 & 0.026229508 \\
\hline Lyn(m) & 80 & 0 & 97 & 97 & 39 & 0.458823529 & 3 & 0.004918033 & 0.031147541 \\
\hline MAPKI(h) & 99 & 0 & 97 & 97 & 40 & 0.470588235 & 3 & 0.004918033 & 0.036065574 \\
\hline MAPK $2(\mathrm{~h})$ & 108 & 0 & 97 & 97 & 41 & 0.482352941 & 3 & 0.004918033 & 0.040983607 \\
\hline MAPK2(m) & 105 & 0 & 97 & 97 & 42 & 0.494117647 & 3 & 0.004918033 & 0.045901639 \\
\hline MAPKAP-K2(h) & 88 & 0 & 97 & 97 & 43 & 0.505882353 & 3 & 0.004918033 & 0.050819672 \\
\hline MEKI(h) & $\begin{array}{l}102 \\
102\end{array}$ & 0 & 97 & 97 & 44 & 0.517647059 & 3 & 0.004918033 & 0.055737705 \\
\hline MKK $4(\mathrm{~m})$ & 108 & 0 & 96 & 96 & 45 & 0.529411765 & 4 & 0.006557377 & 0.062295082 \\
\hline MKK $6(\mathrm{~h})$ & 104 & 0 & 96 & 96 & 46 & 0.541176471 & 4 & 0.006557377 & 0.068852459 \\
\hline МKKᄀ7(h) & 93 & 0 & 96 & 96 & 47 & 0.552941176 & 4 & 0.006557377 & 0.075409836 \\
\hline MSKI(h) & 94 & 0 & 95 & 95 & 48 & 0.564705882 & 5 & 0.008196721 & 0.083606557 \\
\hline $\mathrm{p} 70 \mathrm{~S} 6 \mathrm{~K}(\mathrm{~h})$ & 81 & 0 & 95 & 95 & 49 & 0.576470588 & 5 & 0.008196721 & 0.091803279 \\
\hline PAK2(h) & 93 & 0 & 95 & 95 & 50 & 0.588235294 & 5 & 0.008196721 & 0.1 \\
\hline PDGFRa(h) & 102 & 0 & 95 & 95 & 51 & 0.6 & 5 & 0.008196721 & 0.108196721 \\
\hline PDGFRB(h) & 100 & 0 & 95 & 95 & 52 & 0.611764706 & 5 & 0.008196721 & 0.116393443 \\
\hline PDK1(h) & 97 & 0 & 94 & 94 & 53 & 0.623529412 & 6 & 0.009836066 & 0.126229508 \\
\hline PKA(b) & 99 & 0 & 94 & 94 & 54 & 0.635294118 & 6 & 0.009836066 & 0.136065574 \\
\hline PKA(h) & 109 & 0 & 94 & 94 & 55 & 0.647058824 & 6 & 0.009836066 & 0.145901639 \\
\hline PKBa(h) & 97 & 0 & 94 & 94 & 56 & 0.658823529 & 6 & 0.009836066 & 0.155737705 \\
\hline PKBB(h) & 97 & 0 & 93 & 93 & 57 & 0.670588235 & 7 & 0.01147541 & 0.167213115 \\
\hline PKB $\times(h)$ & 95 & 0 & 93 & 93 & 58 & 0.682352941 & 7 & 0.01147541 & 0.178688525 \\
\hline 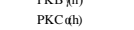 & 91 & 0 & 93 & 93 & 59 & 0.694117647 & 7 & 0.01147541 & 0.190163934 \\
\hline РКСBII(h) & 103 & 0 & 92 & 92 & 60 & 0.705882353 & 8 & 0.013114754 & 0.203278689 \\
\hline PKC $($ (h) & 104 & 0 & 92 & 92 & 61 & 0.717647059 & 8 & 0.013114754 & 0.216393443 \\
\hline PKC\&h) & 115 & 0 & 92 & 92 & 62 & 0.729411765 & 8 & 0.013114754 & 0.229508197 \\
\hline PKCah) & 57 & 0 & 91 & 91 & 63 & 0.741176471 & 9 & 0.014754098 & 0.244262295 \\
\hline PKCII & 122 & 0 & 91 & 91 & 64 & 0.752941176 & 9 & 0.014754098 & 0.259016393 \\
\hline PKC, (h) & 61 & 0 & 91 & 91 & 65 & 0.764705882 & 9 & 0.014754098 & 0.273770492 \\
\hline PKC $\mu(\mathrm{h})$ & 88 & 0 & 90 & 90 & 66 & 0.776470588 & 10 & 0.016393443 & 0.290163934 \\
\hline PKCA & 94 & 0 & 89 & 89 & 67 & 0.788235294 & 11 & 0.018032787 & 0.308196721 \\
\hline PKD2(h) & 95 & 0 & 89 & 89 & 68 & 0.8 & 11 & 0.018032787 & 0.326229508 \\
\hline PRAK(h) & 89 & 0 & 89 & 89 & 69 & 0.811764706 & 11 & 0.018032787 & 0.344262295 \\
\hline PRK2(h) & 98 & 0 & 89 & 89 & 70 & 0.823529412 & 11 & 0.018032787 & 0.362295082 \\
\hline ROCK-II(h) & 95 & 0 & 88 & 88 & 71 & 0.835294118 & 12 & 0.019672131 & 0.381967213 \\
\hline ROCK-II(r) & 88 & 0 & 88 & 88 & 72 & 0.847058824 & 12 & 0.019672131 & 0.401639344 \\
\hline Rskl(h) & 98 & 0 & 88 & 88 & 73 & 0.858823529 & 12 & 0.019672131 & 0.421311475 \\
\hline Rskl(r) & 92 & 0 & 88 & 88 & 74 & 0.870588235 & 12 & 0.019672131 & 0.440983607 \\
\hline Rsk2(h) & 89 & 0 & 87 & 87 & 75 & 0.882352941 & 13 & 0.021311475 & 0.462295082 \\
\hline Rsk3(h) & 87 & 0 & 87 & 87 & 76 & 0.894117647 & 13 & 0.021311475 & 0.483606557 \\
\hline SAPK2a(h) & 110 & 0 & 81 & 81 & 77 & 0.905882353 & 19 & 0.031147541 & 0.514754098 \\
\hline SAPK2b(h) & 100 & 0 & 80 & 80 & 78 & 0.917647059 & 20 & 0.032786885 & 0.547540984 \\
\hline $\mathrm{SAPK} 3(\mathrm{~h})$ & 99 & 0 & 80 & 80 & 79 & 0.929411765 & 20 & 0.032786885 & 0.580327869 \\
\hline SAPK4(h) & 93 & 0 & 79 & 79 & 80 & 0.941176471 & 21 & 0.03442623 & 0.614754098 \\
\hline $\mathrm{SGK}(\mathrm{h})$ & 99 & 0 & 76 & 76 & 81 & 0.952941176 & 24 & 0.039344262 & 0.654098361 \\
\hline Syk(h) & 91 & 0 & 61 & 61 & 82 & 0.964705882 & 39 & 0.063934426 & 0.718032787 \\
\hline $\operatorname{TrkB}(\mathrm{h})$ & 104 & 0 & 57 & 57 & 83 & 0.976470588 & 43 & 0.070491803 & 0.78852459 \\
\hline Yes(h) & 89 & 0 & 56 & 56 & 84 & 0.988235294 & 44 & 0.072131148 & 0.860655738 \\
\hline ZAP-70(h) & 88 & 0 & 15 & 15 & 85 & 1 & 85 & 0.139344262 & 1 \\
\hline & & & & & & Total inhibition & 610 & & Total area \\
\hline & $\begin{array}{c}\text { Hit rate } \\
\text { Threshold }\end{array}$ & 5 & & & & & & & Gini \\
\hline
\end{tabular}




\begin{tabular}{|c|c|c|c|c|c|c|c|c|c|}
\hline & $100 \mu \mathrm{M}$ ATP & & & & & & & & \\
\hline Kinase & AG18 @ $10 \mu \mathrm{M}$ & Hit & sorted & normalized & position & cumulative sample fraction & inhibition\% & fraction of total inhibition & cumulative inhibition \\
\hline $\mathrm{Ab}(\mathrm{m})$ & 110 & 0 & 137 & 100 & 1 & 0.011764706 & 0 & 0 & 0 \\
\hline $\mathrm{AMPK}(\mathrm{r})$ & 93 & 0 & 128 & 100 & 2 & 0.023529412 & 0 & 0 & 0 \\
\hline $\operatorname{Arg}(m)$ & 108 & 0 & 123 & 100 & 3 & 0.035294118 & 0 & 0 & 0 \\
\hline Aurora-A(h) & 85 & 0 & 121 & 100 & 4 & 0.047058824 & 0 & 0 & 0 \\
\hline $\operatorname{Ax}(\mathrm{h})$ & 96 & 0 & 119 & 100 & 5 & 0.058823529 & 0 & 0 & 0 \\
\hline $\mathrm{Bk}(\mathrm{m})$ & 90 & 0 & 112 & 100 & 6 & 0.070588235 & 0 & 0 & 0 \\
\hline Bnx(h) & 128 & 0 & 111 & 100 & 7 & 0.082352941 & 0 & 0 & 0 \\
\hline CaMKII(r) & 112 & 0 & 110 & 100 & 8 & 0.094117647 & 0 & 0 & 0 \\
\hline CaMKIV(h) & 105 & 0 & 110 & 100 & 9 & 0.105882353 & 0 & 0 & 0 \\
\hline 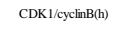 & 94 & 0 & 109 & 100 & 10 & 0.117647059 & 0 & 0 & 0 \\
\hline $\mathrm{CDK} 2 /$ cyclinA(h) & 109 & 0 & 108 & 100 & 11 & 0.129411765 & 0 & 0 & 0 \\
\hline $\mathrm{CDK} 2 /$ cyclinE(h) & 104 & 0 & 107 & 100 & 12 & 0.141176471 & 0 & 0 & 0 \\
\hline CDK3/cyclinE(h) & 99 & 0 & 107 & 100 & 13 & 0.152941176 & 0 & 0 & 0 \\
\hline CDK5/p35(h) & 97 & 0 & 107 & 100 & 14 & 0.164705882 & 0 & 0 & 0 \\
\hline CDK6/cyclinD3(h) & 96 & 0 & 106 & 100 & 15 & 0.176470588 & 0 & 0 & 0 \\
\hline CDK7/cyclinHMATI(h) & 95 & 0 & 106 & 100 & 16 & 0.188235294 & 0 & 0 & 0 \\
\hline СHK $1(\mathrm{hl})$ & 101 & 0 & 105 & 100 & 17 & 0.2 & 0 & 0 & 0 \\
\hline СнК2(h) & 97 & 0 & 105 & 100 & 18 & 0.211764706 & 0 & 0 & 0 \\
\hline $\mathrm{CK} 1(\mathrm{y})$ & 97 & 0 & 104 & 100 & 19 & 0.223529412 & 0 & 0 & 0 \\
\hline СК2(h) & 50 & 1 & 104 & 100 & 20 & 0.235294118 & 0 & 0 & 0 \\
\hline $\mathrm{c} \cdot \mathrm{RAF}(\mathrm{h})$ & 93 & 0 & 103 & 100 & 21 & 0.247058824 & 0 & 0 & 0 \\
\hline $\operatorname{CSK}(\mathrm{h})$ & 121 & 0 & 103 & 100 & 22 & 0.258823529 & 0 & 0 & 0 \\
\hline cSRC(h) & 90 & 0 & 102 & 100 & 23 & 0.270588235 & 0 & 0 & 0 \\
\hline Fes(h) & 96 & 0 & 102 & 100 & 24 & 0.282352941 & 0 & 0 & 0 \\
\hline FGFR3(h) & 119 & 0 & 101 & 100 & 25 & 0.294117647 & 0 & 0 & 0 \\
\hline $\mathrm{Fll}(\mathrm{h})$ & 99 & 0 & 101 & 100 & 26 & 0.305882353 & 0 & 0 & 0 \\
\hline Fynn(h) & 94 & 0 & 101 & 100 & 27 & 0.317647059 & 0 & 0 & 0 \\
\hline GSK3a(h) & 98 & 0 & 100 & 100 & 28 & 0.329411765 & 0 & 0 & 0 \\
\hline GSK3B(h) & 107 & 0 & 100 & 100 & 29 & 0.341176471 & 0 & 0 & 0 \\
\hline IGF-IR(h) & 97 & 0 & 99 & 99 & 30 & 0.352941176 & 1 & 0.002237136 & 0.002237136 \\
\hline $\mathrm{IKK} \alpha(\mathrm{h})$ & 92 & 0 & 99 & 99 & 31 & 0.364705882 & 1 & 0.002237136 & 0.004474273 \\
\hline IKKß(h) & 101 & 0 & 99 & 99 & 32 & 0.376470588 & 1 & 0.002237136 & 0.006711409 \\
\hline $\mathrm{IR}(\mathrm{h})$ & 97 & 0 & 99 & 99 & 33 & 0.388235294 & 1 & 0.002237136 & 0.008948546 \\
\hline JNK1al(h) & 91 & 0 & 99 & 99 & 34 & 0.4 & 1 & 0.002237136 & 0.011185682 \\
\hline JNK2a2(h) & 96 & 0 & 99 & 99 & 35 & 0.411764706 & 1 & 0.002237136 & 0.013422819 \\
\hline JNK3(r) & 107 & 0 & 98 & 98 & 36 & 0.423529412 & 2 & 0.004474273 & 0.017897092 \\
\hline Lck(h) & 90 & 0 & 98 & 98 & 37 & 0.435294118 & 2 & 0.004474273 & 0.022371365 \\
\hline Lyn(h) & 95 & 0 & 98 & 98 & 38 & 0.447058824 & 2 & 0.004474273 & 0.026845638 \\
\hline Lyn(m) & 102 & 0 & 98 & 98 & 39 & 0.458823529 & 2 & 0.004474273 & 0.031319911 \\
\hline MAPK1(h) & 99 & 0 & 97 & 97 & 40 & 0.470588235 & 3 & 0.006711409 & 0.03803132 \\
\hline MAPK2(h) & 105 & 0 & 97 & 97 & 41 & 0.482352941 & 3 & 0.006711409 & 0.044742729 \\
\hline MAPK2(m) & 96 & 0 & 97 & 97 & 42 & 0.494117647 & 3 & 0.006711409 & 0.051454139 \\
\hline MAPKAP-K2(h) & 89 & 0 & 97 & 97 & 43 & 0.505882353 & 3 & 0.006711409 & 0.058165548 \\
\hline MEK1(h) & 97 & 0 & 97 & 97 & 44 & 0.517647059 & 3 & 0.006711409 & 0.064876957 \\
\hline MKK4(m) & 89 & 0 & 97 & 97 & 45 & 0.529411765 & 3 & 0.006711409 & 0.071588367 \\
\hline MKK6(h) & 92 & 0 & 96 & 96 & 46 & 0.541176471 & 4 & 0.008948546 & 0.080536913 \\
\hline МКК77(h) & 91 & 0 & 96 & 96 & 47 & 0.552941176 & 4 & 0.008948546 & 0.089485459 \\
\hline MSKI(h) & 102 & 0 & 96 & 96 & 48 & 0.564705882 & 4 & 0.008948546 & 0.098434004 \\
\hline $\mathrm{p} 70 \mathrm{0S6K( \textrm {h } )}$ & 92 & 0 & 96 & 96 & 49 & 0.576470588 & 4 & 0.008948546 & 0.10738255 \\
\hline PAK2(h) & 94 & 0 & 96 & 96 & 50 & 0.588235294 & 4 & 0.008948546 & 0.116331096 \\
\hline PDGFRa(h) & 96 & 0 & 96 & 96 & 51 & 0.6 & 4 & 0.008948546 & 0.125279642 \\
\hline PDGFRB(h) & 96 & 0 & 96 & 96 & 52 & 0.611764706 & 4 & 0.008948546 & 0.134228188 \\
\hline PDK1(h) & 98 & 0 & 96 & 96 & 53 & 0.623529412 & 4 & 0.008948546 & 0.143176734 \\
\hline PKA(b) & 110 & 0 & 95 & 95 & 54 & 0.635294118 & 5 & 0.011185682 & 0.154362416 \\
\hline PKA(h) & 99 & 0 & 95 & 95 & 55 & 0.647058824 & 5 & 0.011185682 & 0.165548098 \\
\hline PKBar(h) & 96 & 0 & 95 & 95 & 56 & 0.658823529 & 5 & 0.011185682 & 0.176733781 \\
\hline PKBB(h) & 90 & 0 & 95 & 95 & 57 & 0.670588235 & 5 & 0.011185682 & 0.187919463 \\
\hline PKB $\times(h)$ & 100 & 0 & 94 & 94 & 58 & 0.682352941 & 6 & 0.013422819 & 0.201342282 \\
\hline PKC(a) & 106 & 0 & 94 & 94 & 59 & 0.694117647 & 6 & 0.013422819 & 0.214765101 \\
\hline PKCBII(h) & 99 & 0 & 94 & 94 & 60 & 0.705882353 & 6 & 0.013422819 & 0.228187919 \\
\hline PKC $($ (h) & 103 & 0 & 94 & 94 & 61 & 0.717647059 & 6 & 0.013422819 & 0.241610738 \\
\hline PKCæ̧h) & 137 & 0 & 93 & 93 & 62 & 0.729411765 & 7 & 0.015659955 & 0.257270694 \\
\hline PKC \&h) & 81 & 0 & 93 & 93 & 63 & 0.741176471 & 7 & 0.015659955 & 0.272930649 \\
\hline PKCrit & 92 & 0 & 92 & 92 & 64 & 0.752941176 & 8 & 0.017897092 & 0.29082774 \\
\hline PKC, (h) & 54 & 0 & 92 & 92 & 65 & 0.764705882 & 8 & 0.017897092 & 0.308724832 \\
\hline PKC $\mu(\mathrm{h})$ & 84 & 0 & 92 & 92 & 66 & 0.776470588 & 8 & 0.017897092 & 0.326621924 \\
\hline PKCQ & 92 & 0 & 92 & 92 & 67 & 0.788235294 & 8 & 0.017897092 & 0.344519016 \\
\hline PKD2(h) & 111 & 0 & 92 & 92 & 68 & 0.8 & 8 & 0.017897092 & 0.362416107 \\
\hline PRAK(h) & 95 & 0 & 92 & 92 & 69 & 0.811764706 & 8 & 0.017897092 & 0.380313199 \\
\hline PRK2(h) & 104 & 0 & 91 & 91 & 70 & 0.823529412 & 9 & 0.020134228 & 0.400447427 \\
\hline ROCK-II(h) & 94 & 0 & 91 & 91 & 71 & 0.835294118 & 9 & 0.020134228 & 0.420581655 \\
\hline ROCK-IIIr) & 95 & 0 & 90 & 90 & 72 & 0.847058824 & 10 & 0.022371365 & 0.44295302 \\
\hline Rskl(h) & 99 & 0 & 90 & 90 & 73 & 0.858823529 & 10 & 0.022371365 & 0.465324385 \\
\hline Rskl(r) & 92 & 0 & 90 & 90 & 74 & 0.870588235 & 10 & 0.022371365 & 0.487695749 \\
\hline Rsk2(h) & 101 & 0 & 90 & 90 & 75 & 0.882352941 & 10 & 0.022371365 & 0.510067114 \\
\hline Rsk3(h) & 98 & 0 & 89 & 89 & 76 & 0.894117647 & 11 & 0.024608501 & 0.534675615 \\
\hline SAPK2a(h) & 106 & 0 & 89 & 89 & 77 & 0.905882353 & 11 & 0.024608501 & 0.559284116 \\
\hline SAPK2b(h) & 123 & 0 & 86 & 86 & 78 & 0.917647059 & 14 & 0.031319911 & 0.590604027 \\
\hline SAPK3(h) & 107 & 0 & 85 & 85 & 79 & 0.929411765 & 15 & 0.033557047 & 0.624161074 \\
\hline SAPK 4(h) & 98 & 0 & 84 & 84 & 80 & 0.941176471 & 16 & 0.035794183 & 0.659955257 \\
\hline SGK(h) & 86 & 0 & 84 & 84 & 81 & 0.952941176 & 16 & 0.035794183 & 0.695749441 \\
\hline Syk(h) & 79 & 0 & 81 & 81 & 82 & 0.964705882 & 19 & 0.042505593 & 0.738255034 \\
\hline TrkB(h) & 103 & 0 & 79 & 79 & 83 & 0.976470588 & 21 & 0.046979866 & 0.785234899 \\
\hline Yes(h) & 84 & 0 & 54 & 54 & 84 & 0.988235294 & 46 & 0.102908277 & 0.888143177 \\
\hline ZAP-70(h) & 100 & 0 & 50 & 50 & 85 & 1 & 50 & 0.111856823 & 1 \\
\hline & & & & & & Total inhibition & 447 & & Total area \\
\hline & $\begin{array}{c}\text { Hit rate } \\
\text { Threshold }\end{array}$ & 5 & & & & & & & Gini \\
\hline
\end{tabular}




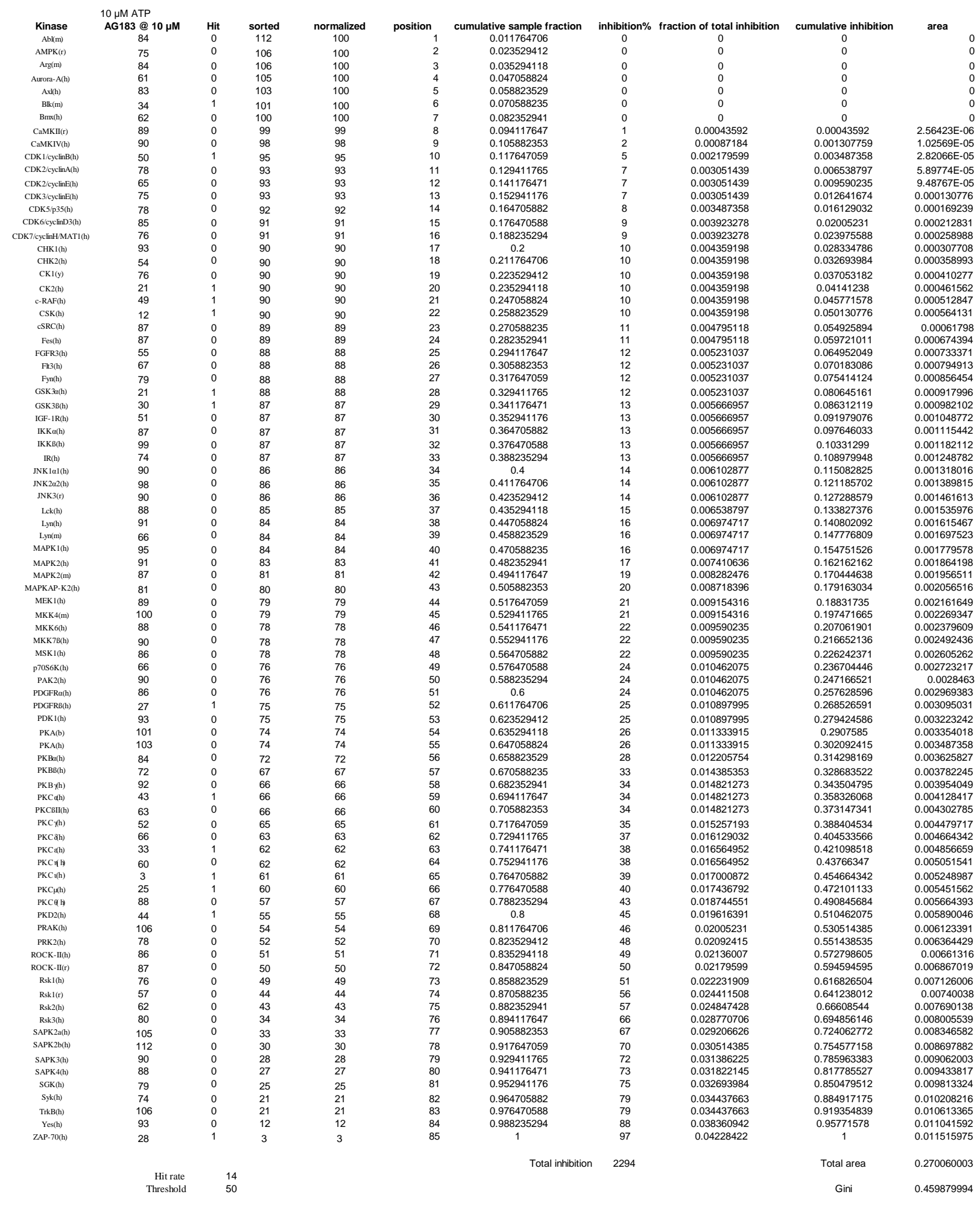




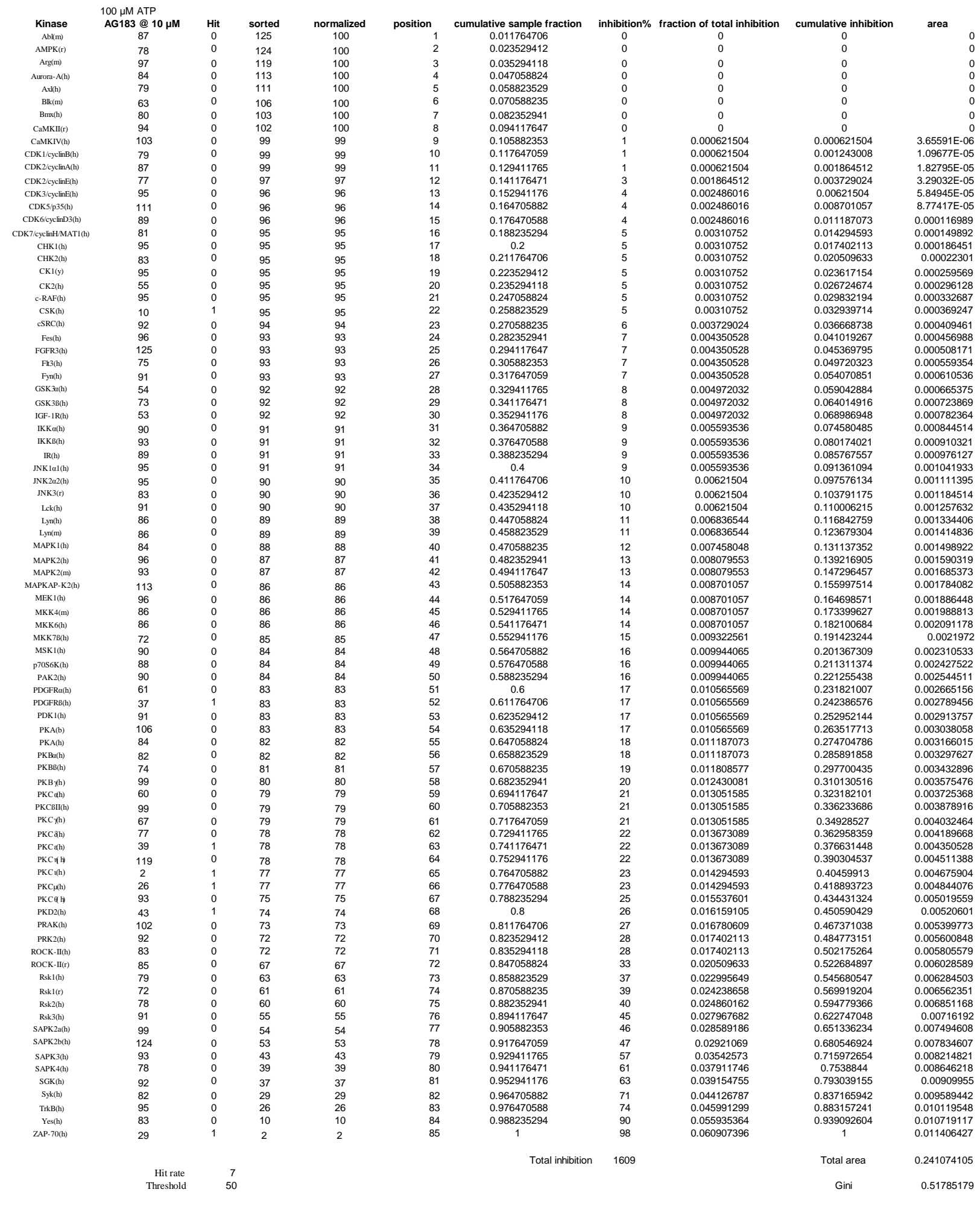




\begin{tabular}{|c|c|c|c|c|c|c|c|c|c|c|}
\hline Kinase & $\begin{array}{l}10 \mu \mathrm{M} \text { ATP } \\
\text { AG538@10 } \mu \mathrm{M}\end{array}$ & Hit & sorted & normalized & position & cumulative sample fraction & inhibition\% & fraction of total inhibition & cumulative inhibition & area \\
\hline $\mathrm{Ab}(\mathrm{m})$ & 16 & 1 & 148 & 100 & 1 & 0.011764706 & 0 & 0 & 0 & 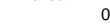 \\
\hline AMPK(r) & 9 & 1 & 115 & 100 & 2 & 0.023529412 & 0 & 0 & 0 & 0 \\
\hline $\operatorname{Arg}(\mathrm{m})$ & 50 & 1 & 115 & 100 & 3 & 0.035294118 & 0 & 0 & 0 & 0 \\
\hline Aurora-A(h) & 42 & 1 & 111 & 100 & 4 & 0.047058824 & 0 & 0 & 0 & 0 \\
\hline Axl(h) & 74 & 0 & 98 & 98 & 5 & 0.058823529 & 2 & 0.000805477 & 0.000805477 & $4.7381 \mathrm{E}-06$ \\
\hline $\mathrm{Blk}(\mathrm{m})$ & 26 & 1 & 97 & 97 & 6 & 0.070588235 & 3 & 0.001208216 & 0.002013693 & $1.65834 \mathrm{E}-05$ \\
\hline $\operatorname{Bmx}(\mathrm{h})$ & 82 & 0 & 96 & 96 & 7 & 0.082352941 & 4 & 0.001610954 & 0.003624648 & $3.31667 \mathrm{E}-05$ \\
\hline CaMKII(r) & 70 & 0 & 96 & 96 & 8 & 0.094117647 & 4 & 0.001610954 & 0.005235602 & $5.21191 E-05$ \\
\hline CaMKIV(h) & 88 & 0 & 93 & 93 & 9 & 0.105882353 & 7 & 0.00281917 & 0.008054772 & $7.81787 \mathrm{E}-05$ \\
\hline CDK1/cyclinB(h) & 57 & 0 & 93 & 93 & 10 & 0.117647059 & 7 & 0.00281917 & 0.010873943 & 0.000111345 \\
\hline $\mathrm{CDK} 2 /$ cyclinA(h) & 72 & 0 & 92 & 92 & 11 & 0.129411765 & 8 & 0.003221909 & 0.014095852 & 0.000146881 \\
\hline $\mathrm{CDK} 2 /$ cyclinE(h) & 85 & 0 & 90 & 90 & 12 & 0.141176471 & 10 & 0.004027386 & 0.018123238 & 0.000189524 \\
\hline CDK3/cyclinE(h) & 87 & 0 & 90 & 90 & 13 & 0.152941176 & 10 & 0.004027386 & 0.022150624 & 0.000236905 \\
\hline CDK5/p35(h) & 86 & 0 & 90 & 90 & 14 & 0.164705882 & 10 & 0.004027386 & 0.02617801 & 0.000284286 \\
\hline CDK6/cychin3(h) & 84 & 0 & 89 & 89 & 15 & 0.176470588 & 11 & 0.004430125 & 0.030608135 & 0.000334036 \\
\hline CDK7/cyclinHMATI(h) & 81 & 0 & 89 & 89 & 16 & 0.188235294 & 11 & 0.004430125 & 0.03503826 & 0.000386155 \\
\hline CHK1(h) & 84 & 0 & 88 & 88 & 17 & 0.2 & 12 & 0.004832863 & 0.039871124 & 0.000440643 \\
\hline СHK2(h) & 55 & 0 & 87 & 87 & 18 & 0.211764706 & 13 & 0.005235602 & 0.045106726 & 0.00049987 \\
\hline CKI(y) & 69 & 0 & 87 & 87 & 19 & 0.223529412 & 13 & 0.005235602 & 0.050342328 & 0.000561465 \\
\hline CK2(h) & 5 & 1 & 87 & 87 & 20 & 0.235294118 & 13 & 0.005235602 & 0.05557793 & 0.00062306 \\
\hline c-RAF(h) & 75 & 0 & 87 & 87 & 21 & 0.247058824 & 13 & 0.005235602 & 0.060813532 & 0.000684656 \\
\hline $\operatorname{CSK}(\mathrm{h})$ & 30 & 1 & 87 & 87 & 22 & 0.258823529 & 13 & 0.005235602 & 0.066049134 & 0.000746251 \\
\hline cSRC(h) & 58 & 0 & 86 & 86 & 23 & 0.270588235 & 14 & 0.005638341 & 0.071687475 & 0.000810215 \\
\hline Fes(h) & 84 & 0 & 86 & 86 & 24 & 0.282352941 & 14 & 0.005638341 & 0.077325816 & 0.000876549 \\
\hline FGFR3(h) & 77 & 0 & 86 & 86 & 25 & 0.294117647 & 14 & 0.005638341 & 0.082964156 & 0.000942882 \\
\hline Flls(h) & 31 & 1 & 85 & 85 & 26 & 0.305882353 & 15 & 0.006041079 & 0.089005236 & 0.001011585 \\
\hline Fynn(h) & 78 & 0 & 85 & 85 & 27 & 0.317647059 & 15 & 0.006041079 & 0.095046315 & 0.001082656 \\
\hline GSK3a(h) & 61 & 0 & 84 & 84 & 28 & 0.329411765 & 16 & 0.006443818 & 0.101490133 & 0.001156097 \\
\hline GSK3B(h) & 70 & 0 & 84 & 84 & 29 & 0.341176471 & 16 & 0.006443818 & 0.107933951 & 0.001231906 \\
\hline IGF-IR(h) & 148 & 0 & 84 & 84 & 30 & 0.352941176 & 16 & 0.006443818 & 0.114377769 & 0.001307716 \\
\hline IKKu(h) & 83 & 0 & 83 & 83 & 31 & 0.364705882 & 17 & 0.006846557 & 0.121224325 & 0.001385895 \\
\hline IKKB(h) & 75 & 0 & 83 & 83 & 32 & 0.376470588 & 17 & 0.006846557 & 0.128070882 & 0.001466442 \\
\hline $\mathrm{IR}(\mathrm{h})$ & 87 & 0 & 82 & 82 & 33 & 0.388235294 & 18 & 0.007249295 & 0.135320177 & 0.001549359 \\
\hline JNKIal(h) & 85 & 0 & 81 & 81 & 34 & 0.4 & 19 & 0.007652034 & 0.142972211 & 0.001637014 \\
\hline JNK202(h) & 89 & 0 & 81 & 81 & 35 & 0.411764706 & 19 & 0.007652034 & 0.150624245 & 0.001727038 \\
\hline $\mathrm{JNK} 3(\mathrm{r})$ & 81 & 0 & 81 & 81 & 36 & 0.423529412 & 19 & 0.007652034 & 0.158276279 & 0.001817062 \\
\hline Lck(h) & 98 & 0 & 81 & 81 & 37 & 0.435294118 & 19 & 0.007652034 & 0.165928313 & 0.001907086 \\
\hline Lyn(h) & 69 & 0 & 81 & 81 & 38 & 0.447058824 & 19 & 0.007652034 & 0.173580346 & 0.00199711 \\
\hline Lyn(m) & 43 & 1 & 81 & 81 & 39 & 0.458823529 & 19 & 0.007652034 & 0.18123238 & 0.002087134 \\
\hline MAPK I(h) & 81 & 0 & 80 & 80 & 40 & 0.470588235 & 20 & 0.008054772 & 0.189287153 & 0.002179527 \\
\hline MAPK $2(\mathrm{~h})$ & 86 & 0 & 80 & 80 & 41 & 0.482352941 & 20 & 0.008054772 & 0.197341925 & 0.002274289 \\
\hline MAPK2(m) & 59 & 0 & 80 & 80 & 42 & 0.494117647 & 20 & 0.008054772 & 0.205396698 & 0.002369051 \\
\hline MAPKAP-K $2(\mathrm{~h})$ & 80 & 0 & 78 & 78 & 43 & 0.505882353 & 22 & 0.00886025 & 0.214256947 & 0.002468551 \\
\hline MEKI(h) & 90 & 0 & 78 & 78 & 44 & 0.517647059 & 22 & 0.00886025 & 0.223117197 & 0.002572789 \\
\hline MKK4(m) & 80 & 0 & 77 & 77 & 45 & 0.529411765 & 23 & 0.009262988 & 0.232380185 & 0.002679396 \\
\hline MKK6(h) & 93 & 0 & 76 & 76 & 46 & 0.541176471 & 24 & 0.009665727 & 0.242045912 & 0.002790742 \\
\hline MKK7ß(h) & 60 & 0 & 76 & 76 & 47 & 0.552941176 & 24 & 0.009665727 & 0.251711639 & 0.002904456 \\
\hline MSKI(h) & 87 & 0 & 75 & 75 & 48 & 0.564705882 & 25 & 0.010068466 & 0.261780105 & 0.00302054 \\
\hline $\mathrm{p} 70 \mathrm{~S} 6 \mathrm{~K}(\mathrm{~h})$ & 70 & 0 & 75 & 75 & 49 & 0.576470588 & 25 & 0.010068466 & 0.27184857 & 0.003138992 \\
\hline PAK2(h) & 80 & 0 & 75 & 75 & 50 & 0.588235294 & 25 & 0.010068466 & 0.281917036 & 0.003257445 \\
\hline PDGFRa(h) & 81 & 0 & 74 & 74 & 51 & 0.6 & 26 & 0.010471204 & 0.29238824 & 0.003378266 \\
\hline PDGFRB(h) & 68 & 0 & 73 & 73 & 52 & 0.611764706 & 27 & 0.010873943 & 0.303262183 & 0.003503826 \\
\hline PDK1(h) & 93 & 0 & 72 & 72 & 53 & 0.623529412 & 28 & 0.011276681 & 0.314538864 & 0.003634124 \\
\hline PKA(b) & 86 & 0 & 70 & 70 & 54 & 0.635294118 & 30 & 0.012082159 & 0.326621023 & 0.003771529 \\
\hline PKA(h) & 96 & 0 & 70 & 70 & 55 & 0.647058824 & 30 & 0.012082159 & 0.338703182 & 0.003913672 \\
\hline PKBach) & 78 & 0 & 70 & 70 & 56 & 0.658823529 & 30 & 0.012082159 & 0.35078534 & 0.004055815 \\
\hline PKBB(h) & 76 & 0 & 69 & 69 & 57 & 0.670588235 & 31 & 0.012484897 & 0.363270238 & 0.004200327 \\
\hline PKB $\ngtr(\mathrm{h})$ & 87 & 0 & 69 & 69 & 58 & 0.682352941 & 31 & 0.012484897 & 0.375755135 & 0.004347208 \\
\hline PKC(h) & 54 & 0 & 68 & 68 & 59 & 0.694117647 & 32 & 0.012887636 & 0.388642771 & 0.004496458 \\
\hline PKCBII(h) & 50 & 1 & 68 & 68 & 60 & 0.705882353 & 32 & 0.012887636 & 0.401530407 & 0.004648078 \\
\hline PKC $>(\mathrm{h})$ & 97 & 0 & 61 & 61 & 61 & 0.717647059 & 39 & 0.015706806 & 0.417237213 & 0.00481628 \\
\hline PKC\&h) & 73 & 0 & 60 & 60 & 62 & 0.729411765 & 40 & 0.016109545 & 0.433346758 & 0.005003435 \\
\hline PKC æh) & 35 & 1 & 60 & 60 & 63 & 0.741176471 & 40 & 0.016109545 & 0.449456303 & 0.005192959 \\
\hline PKCiri & 60 & 0 & 59 & 59 & 64 & 0.752941176 & 41 & 0.016512284 & 0.465968586 & 0.005384852 \\
\hline PKC (h) & 21 & 1 & 58 & 58 & 65 & 0.764705882 & 42 & 0.016915022 & 0.482883609 & 0.005581483 \\
\hline PKC $\mu(\mathrm{h})$ & 32 & 1 & 57 & 57 & 66 & 0.776470588 & 43 & 0.017317761 & 0.500201369 & 0.005782853 \\
\hline PKCथ日 & 87 & 0 & 55 & 55 & 67 & 0.788235294 & 45 & 0.018123238 & 0.518324607 & 0.005991329 \\
\hline PKD2(h) & 25 & 1 & 54 & 54 & 68 & 0.8 & 46 & 0.018525977 & 0.536850584 & 0.006206913 \\
\hline PRAK(h) & 89 & 0 & 50 & 50 & 69 & 0.811764706 & 50 & 0.020136931 & 0.556987515 & 0.006434342 \\
\hline PRK2(h) & 92 & 0 & 50 & 50 & 70 & 0.823529412 & 50 & 0.020136931 & 0.577124446 & 0.006671247 \\
\hline ROCK-II(h) & 90 & 0 & 50 & 50 & 71 & 0.835294118 & 50 & 0.020136931 & 0.597261377 & 0.006908152 \\
\hline ROCK-II(r) & 81 & 0 & 47 & 47 & 72 & 0.847058824 & 53 & 0.021345147 & 0.618606524 & 0.007152164 \\
\hline Rskl(h) & 83 & 0 & 43 & 43 & 73 & 0.858823529 & 57 & 0.022956101 & 0.641562626 & 0.00741276 \\
\hline Rskl(r) & 68 & 0 & 42 & 42 & 74 & 0.870588235 & 58 & 0.02335884 & 0.664921466 & 0.007685201 \\
\hline Rsk2(h) & 76 & 0 & 38 & 38 & 75 & 0.882352941 & 62 & 0.024969795 & 0.689891261 & 0.007969487 \\
\hline Rsk3(h) & 81 & 0 & 35 & 35 & 76 & 0.894117647 & 65 & 0.02617801 & 0.716069271 & 0.008270356 \\
\hline SAPK2a(h) & 115 & 0 & 32 & 32 & 77 & 0.905882353 & 68 & 0.027386226 & 0.743455497 & 0.00858544 \\
\hline SAPK2b(h) & 115 & 0 & 31 & 31 & 78 & 0.917647059 & 69 & 0.027788965 & 0.771244462 & 0.00891 \\
\hline $\operatorname{SAPK} 3(\mathrm{~h})$ & 96 & 0 & 30 & 30 & 79 & 0.929411765 & 70 & 0.028191704 & 0.799436166 & 0.009239298 \\
\hline SAPK 4(h) & 90 & 0 & 26 & 26 & 80 & 0.941176471 & 74 & 0.029802658 & 0.829238824 & 0.009580441 \\
\hline SGK(h) & 75 & 0 & 25 & 25 & 81 & 0.952941176 & 75 & 0.030205397 & 0.859444221 & 0.00993343 \\
\hline Syk(h) & 47 & 1 & 21 & 21 & 82 & 0.964705882 & 79 & 0.031816351 & 0.891260572 & 0.010298263 \\
\hline TrkB(h) & 111 & 0 & 16 & 16 & 83 & 0.976470588 & 84 & 0.033830044 & 0.925090616 & 0.010684419 \\
\hline Yes(h) & 38 & 1 & 9 & 9 & 84 & 0.988235294 & 91 & 0.036649215 & 0.961739831 & 0.011099003 \\
\hline ZAP-70(h) & 50 & 1 & 5 & 5 & 85 & 1 & 95 & 0.038260169 & 1 & 0.011539646 \\
\hline & & & & & & Total inhibition & 2483 & & Total area & 0.291362441 \\
\hline & $\begin{array}{l}\text { Hit rate } \\
\text { Threshold }\end{array}$ & & & & & & & & Gini & 0.417275118 \\
\hline
\end{tabular}




\begin{tabular}{|c|c|c|c|c|c|c|c|c|c|}
\hline & 100 uM ATP & & & & & & & & \\
\hline Kinase & AG538@10 $\mu \mathrm{M}$ & Hit & sorted & normalized & position & cumulative sample fraction & inhibition\% & fraction of total inhibition & cumulative inhibition \\
\hline $\mathrm{Abl(m)}$ & 53 & 0 & 130 & 100 & 1 & 0.011764706 & 0 & 0 & 0 \\
\hline AMPK(r) & 13 & 1 & 128 & 100 & 2 & 0.023529412 & 0 & 0 & 0 \\
\hline $\operatorname{Arg}(\mathrm{m})$ & 77 & 0 & 111 & 100 & 3 & 0.035294118 & 0 & 0 & 0 \\
\hline Aurora-A(h) & 65 & 0 & 111 & 100 & 4 & 0.047058824 & 0 & 0 & 0 \\
\hline $\operatorname{Ax}(\mathrm{h})$ & 73 & 0 & 109 & 100 & 5 & 0.058823529 & 0 & 0 & 0 \\
\hline $\mathrm{Bk}(\mathrm{m})$ & 51 & 0 & 109 & 100 & 6 & 0.070588235 & 0 & 0 & 0 \\
\hline Bmx(h) & 88 & 0 & 109 & 100 & 7 & 0.082352941 & 0 & 0 & 0 \\
\hline CaMKII(r) & 87 & 0 & 104 & 100 & 8 & 0.094117647 & 0 & 0 & 0 \\
\hline CaMKIV(h) & 91 & 0 & 103 & 100 & 9 & 0.105882353 & 0 & 0 & 0 \\
\hline CDK1/cyclinB(h) & 76 & 0 & 101 & 100 & 10 & 0.117647059 & 0 & 0 & 0 \\
\hline $\mathrm{CDK} 2 /$ cyclinA(h) & 86 & 0 & 100 & 100 & 11 & 0.129411765 & 0 & 0 & 0 \\
\hline $\mathrm{CDK} 2 / \mathrm{cyclin} E(\mathrm{~h})$ & 95 & 0 & 97 & 97 & 12 & 0.141176471 & 3 & 0.001670379 & 0.001670379 \\
\hline CDK3/cyclinE(h) & 109 & 0 & 97 & 97 & 13 & 0.152941176 & 3 & 0.001670379 & 0.003340757 \\
\hline CDK5/p35(h) & 101 & 0 & 96 & 96 & 14 & 0.164705882 & 4 & 0.002227171 & 0.005567929 \\
\hline CDK $6 /$ cychinD3(h) & 91 & 0 & $\begin{array}{l}90 \\
96\end{array}$ & $\begin{array}{l}50 \\
96\end{array}$ & 15 & 0.176470588 & 4 & 0.002227171 & 0.0077951 \\
\hline CDK7/cyclinHMATI(h) & 91 & 0 & 95 & 95 & 16 & 0.188235294 & 5 & 0.002783964 & 0.010579065 \\
\hline CHK $1(\mathrm{~h})$ & 92 & 0 & 95 & 95 & 17 & 0.2 & 5 & 0.002783964 & 0.013363029 \\
\hline CHK2(h) & 74 & 0 & 94 & 94 & 18 & 0.211764706 & 6 & 0.003340757 & 0.016703786 \\
\hline $\mathrm{CKI}(\mathrm{y})$ & 96 & 0 & 94 & 94 & 19 & 0.223529412 & 6 & 0.003340757 & 0.020044543 \\
\hline СK2(h) & 1 & 1 & 92 & 92 & 20 & 0.235294118 & 8 & 0.004454343 & 0.024498886 \\
\hline$c \cdot \operatorname{RAF(h)}$ & 89 & 0 & 92 & 92 & 21 & 0.247058824 & 8 & 0.004454343 & 0.028953229 \\
\hline $\operatorname{CSK(h)}$ & 69 & 0 & 91 & 91 & 22 & 0.258823529 & 9 & 0.005011136 & 0.033964365 \\
\hline cSRC(h) & 76 & 0 & 91 & 91 & 23 & 0.270588235 & 9 & 0.005011136 & 0.038975501 \\
\hline Fes(h) & 100 & 0 & 91 & 91 & 24 & 0.282352941 & 9 & 0.005011136 & 0.043986637 \\
\hline FGFR3(h) & 91 & 0 & 91 & 91 & 25 & 0.294117647 & 9 & 0.005011136 & 0.048997773 \\
\hline Flls(h) & 63 & 0 & 91 & 91 & 26 & 0.305882353 & 9 & 0.005011136 & 0.054008909 \\
\hline Fynn(h) & 81 & 0 & 91 & 91 & 27 & 0.317647059 & 9 & 0.005011136 & 0.059020045 \\
\hline GSK3a(h) & 85 & 0 & 91 & 91 & 28 & 0.329411765 & 9 & 0.005011136 & 0.06403118 \\
\hline GSK3B(h) & 70 & 0 & 91 & 91 & 29 & 0.341176471 & 9 & 0.005011136 & 0.069042316 \\
\hline IGF- 1 R(h) & 128 & 0 & 90 & 90 & 30 & 0.352941176 & 10 & 0.005567929 & 0.074610245 \\
\hline $\mathrm{IKKa( \textrm {h } )}$ & 88 & 0 & 90 & 90 & 31 & 0.364705882 & 10 & 0.005567929 & 0.080178174 \\
\hline IKKB(h) & 64 & 0 & 90 & 90 & 32 & 0.376470588 & 10 & 0.005567929 & 0.085746102 \\
\hline $\mathrm{IR}(\mathrm{h})$ & 88 & 0 & 90 & 90 & 33 & 0.388235294 & 10 & 0.005567929 & 0.091314031 \\
\hline JNKIal(h) & 90 & 0 & 89 & 89 & 34 & 0.4 & 11 & 0.006124722 & 0.097438753 \\
\hline JNK202(h) & 94 & 0 & 88 & 88 & 35 & 0.411764706 & 12 & 0.006681514 & 0.104120267 \\
\hline $\mathrm{JNK} 3(\mathrm{r})$ & 87 & 0 & 88 & 88 & 36 & 0.423529412 & 12 & 0.006681514 & 0.110801782 \\
\hline Lck(h) & 85 & 0 & 88 & $\begin{array}{l}\circ 0 \\
88\end{array}$ & 37 & 0.435294118 & 12 & 0.006681514 & 0.117483296 \\
\hline Lyn(h) & 82 & 0 & 87 & 87 & 38 & 0.447058824 & 13 & 0.007238307 & 0.124721604 \\
\hline $\begin{array}{l}\text { Lyn(m) } \\
\text { Lynt }\end{array}$ & 45 & 1 & 87 & 87 & $\begin{array}{l}30 \\
39\end{array}$ & 0.458823529 & 13 & 0.007238307 & 0.131959911 \\
\hline MAPK1(h) & 81 & 0 & 87 & 87 & 40 & 0.470588235 & 13 & 0.007238307 & 0.139198218 \\
\hline MAPK2(h) & 91 & 0 & 87 & 87 & 41 & 0.482352941 & 13 & 0.007238307 & 0.146436526 \\
\hline MAPK2(m) & 62 & 0 & 86 & 86 & 42 & 0.494117647 & 14 & 0.0077951 & 0.154231626 \\
\hline MAPKAP-K2(h) & 78 & 0 & 86 & 86 & 43 & 0.505882353 & 14 & 0.0077951 & 0.162026726 \\
\hline MEK1(h) & 86 & 0 & 85 & 85 & 44 & 0.517647059 & 15 & 0.008351893 & 0.170378619 \\
\hline MKK4(m) & 69 & 0 & 85 & 85 & 45 & 0.529411765 & 15 & 0.008351893 & 0.178730512 \\
\hline $\begin{array}{l}\text { MKK } 4(\mathrm{~m}) \\
\text { MKK }\end{array}$ & 91 & 0 & 84 & 84 & 46 & 0.541176471 & 16 & 0.008908686 & 0.187639198 \\
\hline МKКㄱ(h) & 65 & 0 & 83 & 83 & 47 & 0.552941176 & 17 & 0.009465479 & 0.197104677 \\
\hline MSKI(h) & 91 & 0 & 82 & 82 & 48 & 0.564705882 & 18 & 0.010022272 & 0.207126949 \\
\hline $\mathrm{p} 70 \mathrm{~s} 6 \mathrm{~K}(\mathrm{~h})$ & 80 & 0 & 82 & 82 & 49 & 0.576470588 & 18 & 0.010022272 & 0.21714922 \\
\hline PAK2(h) & 78 & 0 & 81 & 81 & 50 & 0.588235294 & 19 & 0.010579065 & 0.227728285 \\
\hline PDGFRa(h) & 76 & 0 & 81 & 81 & 51 & 0.6 & 19 & 0.010579065 & 0.23830735 \\
\hline PDGFRB(h) & 78 & 0 & 81 & 81 & 52 & 0.611764706 & 19 & 0.010579065 & 0.248886414 \\
\hline PDK1(h) & 97 & 0 & 80 & 80 & 53 & 0.623529412 & 20 & 0.011135857 & 0.260022272 \\
\hline PKA(b) & 84 & 0 & 78 & 78 & 54 & 0.635294118 & 22 & 0.012249443 & 0.272271715 \\
\hline PKA(h) & 90 & 0 & 78 & 78 & 55 & 0.647058824 & 22 & 0.012249443 & 0.284521158 \\
\hline PKBr(h) & 81 & 0 & 78 & 78 & 56 & 0.658823529 & 22 & 0.012249443 & 0.296770601 \\
\hline $\begin{array}{l}\text { PKBB(h) } \\
\text { (h) }\end{array}$ & 74 & 0 & 77 & 77 & 57 & 0.670588235 & 23 & 0.012806236 & 0.309576837 \\
\hline PKB $\gamma($ hl) & 96 & 0 & 76 & 76 & 58 & 0.682352941 & 24 & 0.013363029 & 0.322939866 \\
\hline PKC(h) & 63 & 0 & 76 & 76 & 59 & 0.694117647 & 24 & 0.013363029 & 0.336302895 \\
\hline РКCBII(h) & 72 & 0 & 76 & 76 & 60 & 0.705882353 & 24 & 0.013363029 & 0.349665924 \\
\hline PKC $(\mathrm{h}(\mathrm{h})$ & 111 & 0 & 74 & 74 & 61 & 0.717647059 & 26 & 0.014476615 & 0.364142539 \\
\hline PKC\&h) & 109 & 0 & 74 & 74 & 62 & 0.729411765 & 26 & 0.014476615 & 0.378619154 \\
\hline PKCa(h) & 39 & 1 & 73 & 73 & 63 & 0.741176471 & 27 & 0.015033408 & 0.393652561 \\
\hline PKCII & 103 & 0 & 72 & 72 & 64 & 0.752941176 & 28 & 0.0155902 & 0.409242762 \\
\hline PKC, (h) & 18 & 1 & 70 & 70 & 65 & 0.764705882 & 30 & 0.016703786 & 0.425946548 \\
\hline PKC $\mu(\mathrm{h})$ & 25 & 1 & 69 & 69 & 66 & 0.776470588 & 31 & 0.017260579 & 0.443207127 \\
\hline 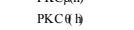 & 97 & 0 & 69 & 69 & 67 & 0.788235294 & 31 & 0.017260579 & 0.460467706 \\
\hline PKD2(h) & 20 & 1 & 69 & 69 & 68 & 0.8 & 31 & 0.017260579 & 0.477728285 \\
\hline PRAK(h) & 64 & 0 & 65 & 65 & 69 & 0.811764706 & 35 & 0.019487751 & 0.497216036 \\
\hline PRK2(h) & 109 & 0 & 65 & 65 & 70 & 0.823529412 & 35 & 0.019487751 & 0.516703786 \\
\hline ROCK-II(h) & 94 & 0 & 64 & 64 & 71 & 0.835294118 & 36 & 0.020044543 & 0.53674833 \\
\hline ROCK-II(r) & 90 & 0 & 64 & 64 & 72 & 0.847058824 & 36 & 0.020044543 & 0.556792873 \\
\hline Rskl(h) & 83 & 0 & 63 & 63 & 73 & 0.858823529 & 37 & 0.020601336 & 0.577394209 \\
\hline $\operatorname{Rskl(r)}$ & 69 & 0 & 63 & 63 & 74 & 0.870588235 & 37 & 0.020601336 & 0.597995546 \\
\hline $\begin{array}{l}\text { Rskk(t) } \\
\text { Rsk2(h) }\end{array}$ & 82 & 0 & 62 & 62 & 75 & 0.882352941 & 38 & 0.021158129 & 0.619153675 \\
\hline Rsk3(h) & 92 & 0 & 53 & 53 & 76 & 0.894117647 & 47 & 0.026169265 & 0.64532294 \\
\hline SAPK2a(h) & 111 & 0 & 51 & 51 & 77 & 0.905882353 & 49 & 0.027282851 & 0.672605791 \\
\hline SAPK2b(h) & 130 & 0 & 51 & 51 & 78 & 0.917647059 & 49 & 0.027282851 & 0.699888641 \\
\hline $\operatorname{SAPK} 3(\mathrm{~h})$ & 104 & 0 & 45 & 45 & 79 & 0.929411765 & 55 & 0.030623608 & 0.730512249 \\
\hline SAPK4(h) & 95 & 0 & 39 & 39 & 80 & 0.941176471 & 61 & 0.033964365 & 0.764476615 \\
\hline SGK(h) & 90 & 0 & 25 & 25 & 81 & 0.952941176 & 75 & 0.041759465 & 0.80623608 \\
\hline Syk(h) & 51 & 0 & 20 & 20 & 82 & 0.964705882 & 80 & 0.04454343 & 0.85077951 \\
\hline TrkB(h) & 91 & 0 & 18 & 18 & 83 & 0.976470588 & 82 & 0.045657016 & 0.896436526 \\
\hline Yes(h) & 87 & 0 & 13 & 13 & 84 & 0.988235294 & 87 & 0.04844098 & 0.944877506 \\
\hline ZAP-70(h) & 87 & 0 & 1 & 1 & 85 & 1 & 99 & 0.055122494 & 1 \\
\hline & & & & & & Total inhibition & 1796 & & Total area \\
\hline & $\begin{array}{l}\text { Hit rate } \\
\text { Threshold }\end{array}$ & 5 & & & & & & & Gini \\
\hline
\end{tabular}




\begin{tabular}{|c|c|c|c|c|c|c|c|c|c|}
\hline & $10 \mu \mathrm{M}$ ATP & & & & & & & & \\
\hline Kinase & Alsterpaullone @ 1 ㅆ & Hit & sorted & normalized & position & cumulative sample fraction & inhibition\% & fraction of total inhibition & cumulative inhibition \\
\hline $\mathrm{Abl(m)}$ & 34 & 1 & 112 & 100 & 1 & 0.011764706 & 0 & 0 & 0 \\
\hline AMPK(r) & 76 & 0 & 112 & 100 & 2 & 0.023529412 & 0 & 0 & 0 \\
\hline $\operatorname{Arg}(\mathrm{m})$ & 50 & 1 & 110 & 100 & 3 & 0.035294118 & 0 & 0 & 0 \\
\hline Aurora-A(h) & 102 & 0 & 110 & 100 & 4 & 0.047058824 & 0 & 0 & 0 \\
\hline Ax(l(h) & 79 & 0 & 109 & 100 & 5 & 0.058823529 & 0 & 0 & 0 \\
\hline $\operatorname{Blk}(\mathrm{m})$ & 31 & 1 & 109 & 100 & 6 & 0.070588235 & 0 & 0 & 0 \\
\hline $\operatorname{Bmx}(\mathrm{h})$ & 112 & 0 & 109 & 100 & 7 & 0.082352941 & 0 & 0 & 0 \\
\hline СамКII(r) & 95 & 0 & 107 & 100 & 8 & 0.094117647 & 0 & 0 & 0 \\
\hline CaMKIV(h) & 107 & 0 & 106 & 100 & 9 & 0.105882353 & 0 & 0 & 0 \\
\hline $\mathrm{CDK} 1 / \mathrm{cyclinB}(\mathrm{h})$ & 10 & 1 & 105 & 100 & 10 & 0.117647059 & 0 & 0 & 0 \\
\hline $\mathrm{CDK} 2 / \mathrm{cyclinA}(\mathrm{h})$ & 8 & 1 & 105 & 100 & 11 & 0.129411765 & 0 & 0 & 0 \\
\hline $\mathrm{CDK} 2 /$ cyclinE(h) & 4 & 1 & 104 & 100 & 12 & 0.141176471 & 0 & 0 & 0 \\
\hline CDK3/yclinE(h) & 18 & 1 & 104 & 100 & 13 & 0.152941176 & 0 & 0 & 0 \\
\hline CDK5/p35(h) & 8 & 1 & 104 & 100 & 14 & 0.164705882 & 0 & 0 & 0 \\
\hline $\mathrm{CDK} 6 / \mathrm{cyclin} \mathrm{D} 3$ (h) & 62 & 0 & 103 & 100 & 15 & 0.176470588 & 0 & 0 & 0 \\
\hline CDK7/cyclinH/MATI(h) & 96 & 0 & 103 & 100 & 16 & 0.188235294 & 0 & 0 & 0 \\
\hline CHK1(h) & 26 & 1 & 102 & 100 & 17 & 0.2 & 0 & 0 & 0 \\
\hline СHK2(h) & 6 & 1 & 102 & 100 & 18 & 0.211764706 & 0 & 0 & 0 \\
\hline $\mathrm{CK} 1(\mathrm{y})$ & 78 & 0 & 102 & 100 & 19 & 0.223529412 & 0 & 0 & 0 \\
\hline СК2(h) & $\begin{array}{l}10 \\
103\end{array}$ & 0 & 102 & 100 & 20 & 0.235294118 & 0 & 0 & 0 \\
\hline$c-\operatorname{RAF}(\mathrm{h})$ & 99 & 0 & 101 & 100 & 21 & 0.247058824 & 0 & 0 & 0 \\
\hline CSK(h) & 70 & 0 & 100 & 100 & 22 & 0.258823529 & 0 & 0 & 0 \\
\hline $\operatorname{csRC(h)}$ & 35 & 1 & 100 & 100 & 23 & 0.270588235 & 0 & 0 & 0 \\
\hline Fes(h) & 44 & 1 & 100 & 100 & 24 & 0.282352941 & 0 & 0 & 0 \\
\hline FGFR3(h) & 106 & 0 & 100 & 100 & 25 & 0.294117647 & 0 & 0 & 0 \\
\hline $\mathrm{Fll}(\mathrm{h}(\mathrm{h})$ & 14 & 1 & 100 & 100 & 26 & 0.305882353 & 0 & 0 & 0 \\
\hline Fyn(h) & 33 & 1 & 99 & 99 & 27 & 0.317647059 & 1 & 0.000431406 & 0.000431406 \\
\hline GSK3a(h) & 0 & 1 & 99 & 99 & 28 & 0.329411765 & 1 & 0.000431406 & 0.000862813 \\
\hline GSK3B(h) & 2 & 1 & 99 & 99 & 29 & 0.341176471 & 1 & 0.000431406 & 0.001294219 \\
\hline IGF-1R(h) & 109 & 0 & 98 & 98 & 30 & 0.352941176 & 2 & 0.000862813 & 0.002157032 \\
\hline IKKa(h) & 98 & 0 & 98 & 98 & 31 & 0.364705882 & 2 & 0.000862813 & 0.003019845 \\
\hline IKKB(h) & 97 & 0 & 97 & 97 & 32 & 0.376470588 & 3 & 0.001294219 & 0.004314064 \\
\hline $\mathbb{I R ( h )}$ & 109 & 0 & 96 & 96 & 33 & 0.388235294 & 4 & 0.001725626 & 0.006039689 \\
\hline JNKIal(h) & 100 & 0 & 96 & 96 & 34 & 0.4 & 4 & 0.001725626 & 0.007765315 \\
\hline JNK202(h) & 102 & 0 & 96 & 96 & $\begin{array}{l}34 \\
35\end{array}$ & 0.411764706 & 4 & 0.001725626 & 0.00949094 \\
\hline JNK3(r) & 110 & 0 & 95 & 95 & 36 & 0.423529412 & 5 & 0.002157032 & 0.011647972 \\
\hline Lck(h) & 31 & 1 & 95 & 95 & 37 & 0.435294118 & 5 & 0.002157032 & 0.013805004 \\
\hline Lynn(h) & 17 & 1 & 95 & 95 & 38 & 0.447058824 & 5 & 0.002157032 & 0.015962036 \\
\hline Lyy(m) & 8 & 1 & 94 & 94 & 39 & 0.458823529 & 6 & 0.002588438 & 0.018550475 \\
\hline MAPKI(h) & $\begin{array}{l}8 \\
92\end{array}$ & 0 & $\begin{array}{l}94 \\
94\end{array}$ & $\begin{array}{l}94 \\
94\end{array}$ & 40 & 0.470588235 & 6 & 0.002588438 & 0.021138913 \\
\hline MAPK2(h) & 102 & 0 & 93 & 93 & 41 & 0.482352941 & 7 & 0.003019845 & 0.024158758 \\
\hline $\begin{array}{l}\text { MAPKL(2) } \\
\text { MAPK2(m) }\end{array}$ & 79 & 0 & 92 & 92 & 42 & 0.494117647 & 8 & 0.003451251 & 0.027610009 \\
\hline MAPKAP-K2(h) & 63 & 0 & 89 & 89 & 43 & 0.505882353 & 11 & 0.00474547 & 0.032355479 \\
\hline MEK1(h) & 101 & 0 & 89 & 89 & 44 & 0.517647059 & 11 & 0.00474547 & 0.037100949 \\
\hline MKK4(m) & 104 & 0 & 88 & 88 & 45 & 0.529411765 & 12 & 0.005176877 & 0.042277826 \\
\hline MKK6(h) & 100 & 0 & 85 & 85 & 46 & 0.541176471 & 15 & 0.006471096 & 0.048748921 \\
\hline MKK & 93 & 0 & 83 & 83 & 47 & 0.552941176 & 17 & 0.007333909 & 0.05608283 \\
\hline MSKI(h) & 89 & 0 & 82 & 82 & 48 & 0.564705882 & 18 & 0.007765315 & 0.063848145 \\
\hline $\mathrm{p} 70 \mathrm{~s} 6 \mathrm{~K}(\mathrm{~h})$ & $\begin{array}{l}89 \\
104\end{array}$ & 0 & $\begin{array}{l}82 \\
81\end{array}$ & $\begin{array}{l}82 \\
81\end{array}$ & $\begin{array}{l}48 \\
49\end{array}$ & 0.576470588 & $\begin{array}{l}18 \\
19\end{array}$ & 0.008196721 & $\begin{array}{l}.0730484485 \\
0.07204466\end{array}$ \\
\hline $\begin{array}{l}\text { PAK2(h) } \\
\text { Pas }\end{array}$ & 30 & 1 & 79 & 79 & 50 & 0.588235294 & 21 & 0.009059534 & 0.0811044 \\
\hline PDGFRa(h) & 100 & 0 & 79 & 79 & 51 & 0.6 & 21 & 0.009059534 & 0.090163934 \\
\hline PDGFRB(h) & 100 & 0 & 78 & 78 & 52 & 0.611764706 & 22 & 0.00949094 & 0.099654875 \\
\hline PDK I(h) & 88 & 0 & 76 & 76 & 53 & 0.623529412 & 24 & 0.010353753 & 0.110008628 \\
\hline PKA(b) & 94 & 0 & 74 & 74 & 54 & 0.635294118 & 26 & 0.011216566 & 0.121225194 \\
\hline 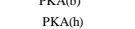 & $\begin{array}{l}94 \\
105\end{array}$ & 0 & $\begin{array}{l}74 \\
72\end{array}$ & $\begin{array}{l}14 \\
72\end{array}$ & $\begin{array}{l}54 \\
55\end{array}$ & 0.647058824 & $\begin{array}{l}20 \\
28\end{array}$ & 0.012079379 & 0.133304573 \\
\hline PKBa(h) & 98 & 0 & 70 & 70 & 56 & 0.658823529 & 30 & 0.012942192 & 0.146246764 \\
\hline PKBB(h) & 82 & 0 & 66 & 66 & 57 & 0.670588235 & 34 & 0.014667817 & 0.160914582 \\
\hline $\mathrm{PKB}_{\gamma}(\mathrm{h})$ & 99 & 0 & 65 & 65 & 58 & 0.682352941 & 35 & 0.015099223 & 0.176013805 \\
\hline PKCu(h) & 100 & 0 & 65 & 65 & 59 & 0.694117647 & 35 & 0.015099223 & 0.191113028 \\
\hline РКСBII(h) & 112 & 0 & 63 & 63 & 60 & 0.705882353 & 37 & 0.015962036 & 0.207075065 \\
\hline PKCY(h) & 102 & 0 & 62 & 62 & 61 & 0.717647059 & 38 & 0.016393443 & 0.223468507 \\
\hline РКС() & $\begin{array}{l}102 \\
105\end{array}$ & 0 & 60 & $\begin{array}{l}02 \\
60\end{array}$ & $\begin{array}{l}11 \\
62\end{array}$ & 0.729411765 & $\begin{array}{l}38 \\
40\end{array}$ & 0.017256255 & 0.240724763 \\
\hline $\begin{array}{l}\text { PKE:(h) } \\
\text { Pall }\end{array}$ & 94 & 0 & 59 & 59 & 63 & 0.741176471 & 41 & 0.017687662 & 0.258412425 \\
\hline $\begin{array}{l}\text { PKG (h) } \\
\text { (h) }\end{array}$ & 99 & 0 & 50 & 50 & 64 & 0.752941176 & 50 & 0.021570319 & 0.279982744 \\
\hline PKC (h) & 85 & 0 & 48 & 48 & 65 & 0.764705882 & 52 & 0.022433132 & 0.302415876 \\
\hline PKCu(h) & 104 & 0 & 44 & 44 & 66 & 0.776470588 & 56 & 0.024158758 & 0.326574633 \\
\hline PKCO(h) & 95 & 0 & 35 & 35 & 67 & 0.788235294 & 65 & 0.028041415 & 0.354616048 \\
\hline $\begin{array}{l}\text { PRC(f) } \\
\text { PKD2(h) }\end{array}$ & 95 & 0 & 34 & 34 & 68 & 0.8 & 66 & 0.028472821 & 0.38308887 \\
\hline PRAK(h) & 83 & 0 & $\begin{array}{l}34 \\
33\end{array}$ & $\begin{array}{l}34 \\
33\end{array}$ & 69 & 0.811764706 & 67 & 0.028904228 & 0.411993097 \\
\hline PRK2(h) & 65 & 0 & 31 & 31 & 70 & 0.823529412 & 69 & 0.029767041 & 0.441760138 \\
\hline $\begin{array}{l}\text { PRKL_(n) } \\
\text { ROCK-I(h) }\end{array}$ & 66 & 0 & 31 & 31 & 71 & 0.835294118 & $\begin{array}{l}69 \\
69\end{array}$ & 0.029767041 & 0.471527179 \\
\hline ROCK-IIIr) & 74 & 0 & 30 & 30 & 72 & 0.847058824 & 70 & 0.030198447 & 0.501725626 \\
\hline Rskl(h) & 81 & 0 & 26 & 26 & 73 & 0.858823529 & 74 & 0.031924072 & 0.533649698 \\
\hline Rskl(r) & 72 & 0 & 24 & 24 & 74 & 0.870588235 & 76 & 0.032786885 & 0.566436583 \\
\hline $\begin{array}{l}\text { Rskk2(h) } \\
\text { Rsher }\end{array}$ & 59 & 0 & 18 & 18 & 75 & 0.882352941 & 82 & 0.035375324 & 0.601811907 \\
\hline $\mathrm{Rsk} 3(\mathrm{~h})$ & 48 & 1 & 17 & 17 & 76 & 0.894117647 & 83 & 0.03580673 & 0.637618637 \\
\hline SAPK2a(h) & 110 & 0 & 14 & 14 & 77 & 0.905882353 & 86 & 0.037100949 & 0.674719586 \\
\hline SAPK2b(h) & 103 & 0 & 10 & 10 & 78 & 0.917647059 & 90 & 0.038826575 & 0.71354616 \\
\hline SAPK3(h) & 96 & 0 & 8 & 8 & 79 & 0.929411765 & 92 & 0.039689387 & 0.753235548 \\
\hline $\begin{array}{l}\mathrm{SARRS( \textrm {h } )} \\
\mathrm{SAPK} 4(\mathrm{~h})\end{array}$ & 89 & 0 & 8 & 8 & 80 & 0.941176471 & 92 & 0.039689387 & 0.792924935 \\
\hline SGK(h) & 96 & 0 & 8 & 8 & 81 & 0.952941176 & 92 & 0.039689387 & 0.832614323 \\
\hline Syk(h) & 60 & 0 & 6 & 6 & 82 & 0.964705882 & 94 & 0.0405522 & 0.873166523 \\
\hline TrkB(h) & 109 & 0 & 4 & 4 & 83 & 0.976470588 & 96 & 0.041415013 & 0.914581536 \\
\hline $\begin{array}{l}\text { IIkK(h) } \\
\text { Yes(h) }\end{array}$ & 24 & 1 & $\begin{array}{l}4 \\
2\end{array}$ & $\begin{array}{l}4 \\
2\end{array}$ & 84 & 0.988235294 & $\begin{array}{l}50 \\
98\end{array}$ & 0.042277826 & 0.9568593628 \\
\hline ZAP-70(h) & 65 & 0 & 0 & 0 & 85 & 1 & 100 & 0.043140638 & 1 \\
\hline & & & & & & Total inhibition & 2318 & & Total area \\
\hline & Hit rate & 5 & & & & & & & Gini \\
\hline
\end{tabular}




\begin{tabular}{|c|c|c|c|c|c|c|c|c|c|}
\hline & $100 \mu \mathrm{M}$ ATP & & & & & & & & \\
\hline Kinase & Alsterpaullone @ 1 ㅆ & Hit & sorted & normalized & position & cumulative sample fraction & inhibition\% & fraction of total inhibition & cumulative inhibition \\
\hline $\mathrm{Abl(m)}$ & 81 & 0 & 130 & 100 & 1 & 0.011764706 & 0 & 0 & 0 \\
\hline AMPK(r) & 76 & 0 & 124 & 100 & 2 & 0.023529412 & 0 & 0 & 0 \\
\hline $\operatorname{Arg}(\mathrm{m})$ & 75 & 0 & 117 & 100 & 3 & 0.035294118 & 0 & 0 & 0 \\
\hline Aurora-A(h) & 114 & 0 & 117 & 100 & 4 & 0.047058824 & 0 & 0 & 0 \\
\hline Ax(l(h) & 87 & 0 & 114 & 100 & 5 & 0.058823529 & 0 & 0 & 0 \\
\hline $\operatorname{Blk}(\mathrm{m})$ & 49 & 1 & 113 & 100 & 6 & 0.070588235 & 0 & 0 & 0 \\
\hline $\operatorname{Bmx}(\mathrm{h})$ & 96 & 0 & 112 & 100 & 7 & 0.082352941 & 0 & 0 & 0 \\
\hline CaMKII(r) & 113 & 0 & 109 & 100 & 8 & 0.094117647 & 0 & 0 & 0 \\
\hline CaMKIV(h) & 109 & 0 & 108 & 100 & 9 & 0.105882353 & 0 & 0 & 0 \\
\hline $\mathrm{CDK} 1 / \mathrm{cyclinB}(\mathrm{h})$ & 25 & 1 & 108 & 100 & 10 & 0.117647059 & 0 & 0 & 0 \\
\hline $\mathrm{CDK} 2 / \mathrm{cyclinA}(\mathrm{h})$ & 17 & 1 & 107 & 100 & 11 & 0.129411765 & 0 & 0 & 0 \\
\hline $\mathrm{CDK} 2 /$ cyclinE(h) & 7 & 1 & 105 & 100 & 12 & 0.141176471 & 0 & 0 & 0 \\
\hline CDK3/cyclinE(h) & 33 & 1 & 105 & 100 & 13 & 0.152941176 & 0 & 0 & 0 \\
\hline CDK5/p35(h) & 18 & 1 & 104 & 100 & 14 & 0.164705882 & 0 & 0 & 0 \\
\hline $\mathrm{CDK} 6 / \mathrm{cyclin} \mathrm{B} 3(\mathrm{~h})$ & 74 & 0 & 104 & 100 & 15 & 0.176470588 & 0 & 0 & 0 \\
\hline CDK $7 /$ cyclinHMATI(h) & 102 & 0 & 104 & 100 & 16 & 0.188235294 & 0 & 0 & 0 \\
\hline CHK 1 (h) & 45 & 1 & 104 & 100 & 17 & 0.2 & 0 & 0 & 0 \\
\hline СHK2(h) & 7 & 1 & 103 & 100 & 18 & 0.211764706 & 0 & 0 & 0 \\
\hline $\mathrm{CK} 1(\mathrm{y})$ & 95 & 0 & 103 & 100 & 19 & 0.223529412 & 0 & 0 & 0 \\
\hline СК2(h) & $\begin{array}{l}90 \\
104\end{array}$ & 0 & 102 & 100 & 20 & 0.235294118 & 0 & 0 & 0 \\
\hline$c-\operatorname{RAF}(\mathrm{h})$ & 91 & 0 & 102 & 100 & 21 & 0.247058824 & 0 & 0 & 0 \\
\hline CSK(h) & 90 & 0 & 102 & 100 & 22 & 0.258823529 & 0 & 0 & 0 \\
\hline $\operatorname{csRC(h)}$ & 54 & 0 & 102 & 100 & 23 & 0.270588235 & 0 & 0 & 0 \\
\hline Fes(h) & 62 & 0 & 101 & 100 & 24 & 0.282352941 & 0 & 0 & 0 \\
\hline FGFR3(h) & 98 & 0 & 101 & 100 & 25 & 0.294117647 & 0 & 0 & 0 \\
\hline $\mathrm{Fll}(\mathrm{h}(\mathrm{h})$ & 33 & 1 & 101 & 100 & 26 & 0.305882353 & 0 & 0 & 0 \\
\hline Fyn(h) & 60 & 0 & 100 & 100 & 27 & 0.317647059 & 0 & 0 & 0 \\
\hline GSK3a(h) & 6 & 1 & 100 & 100 & 28 & 0.329411765 & 0 & 0 & 0 \\
\hline GSK3B(h) & 8 & 1 & 100 & 100 & $\begin{array}{l}20 \\
29\end{array}$ & 0.341176471 & 0 & 0 & 0 \\
\hline IGF-IR(h) & 102 & 0 & 100 & 100 & 30 & 0.352941176 & 0 & 0 & 0 \\
\hline IKKa(h) & 99 & 0 & 99 & 99 & 31 & 0.364705882 & 1 & 0.000616903 & 0.000616903 \\
\hline IKKB(h) & 99 & 0 & 99 & 99 & 32 & 0.376470588 & 1 & 0.000616903 & 0.001233806 \\
\hline $\mathbb{I R ( h )}$ & 117 & 0 & 99 & 99 & 33 & 0.388235294 & 1 & 0.000616903 & 0.001850709 \\
\hline JNKIal(h) & 102 & 0 & 99 & 99 & 34 & 0.4 & 1 & 0.000616903 & 0.002467613 \\
\hline JNK202(h) & 104 & 0 & 98 & 98 & $\begin{array}{l}34 \\
35\end{array}$ & 0.411764706 & 2 & 0.001233806 & 0.003701419 \\
\hline JNK3(r) & 105 & 0 & 98 & 98 & 36 & 0.423529412 & 2 & 0.001233806 & 0.004935225 \\
\hline Lck(h) & 50 & 1 & 98 & 98 & 37 & 0.435294118 & 2 & 0.001233806 & 0.006169031 \\
\hline Lynn(h) & 20 & 1 & 97 & 97 & 38 & 0.447058824 & 3 & 0.001850709 & 0.008019741 \\
\hline Lyn(m) & 23 & 1 & 96 & 96 & 39 & 0.458823529 & 4 & 0.002467613 & 0.010487353 \\
\hline MAPKI(h) & $\begin{array}{l}23 \\
98\end{array}$ & 0 & $\begin{array}{l}90 \\
95\end{array}$ & $\begin{array}{l}90 \\
95\end{array}$ & 40 & 0.470588235 & 5 & 0.003084516 & 0.013571869 \\
\hline MAPK2(h) & 117 & 0 & 95 & 95 & 41 & 0.482352941 & 5 & 0.003084516 & 0.016656385 \\
\hline $\begin{array}{l}\text { MAPKL(2) } \\
\text { MAPK2(m) }\end{array}$ & 88 & 0 & 95 & 95 & 42 & 0.494117647 & 5 & 0.003084516 & 0.019740901 \\
\hline MAPKAP-K2(h) & 92 & 0 & 94 & 94 & 43 & 0.505882353 & 6 & 0.003701419 & 0.02344232 \\
\hline MEKI(h) & 93 & 0 & 93 & 93 & 44 & 0.517647059 & 7 & 0.004318322 & 0.027760642 \\
\hline MKK4(m) & 93 & 0 & 93 & 93 & 45 & 0.529411765 & 7 & 0.004318322 & 0.032078964 \\
\hline MKK6(h) & 101 & 0 & 93 & 93 & 46 & 0.541176471 & 7 & 0.004318322 & 0.036397286 \\
\hline MKK & 105 & 0 & 93 & 93 & 47 & 0.552941176 & 7 & 0.004318322 & 0.040715608 \\
\hline MSKI(h) & 91 & 0 & 92 & 92 & 48 & 0.564705882 & 8 & 0.004935225 & 0.045650833 \\
\hline $\mathrm{p} 70 \mathrm{~s} 6 \mathrm{~K}(\mathrm{~h})$ & 104 & 0 & 92 & $\begin{array}{l}92 \\
92\end{array}$ & $\begin{array}{l}48 \\
49\end{array}$ & 0.576470588 & $\begin{array}{l}8 \\
8\end{array}$ & $\begin{array}{l}.004932525 \\
0.004935225\end{array}$ & $\begin{array}{l}.0450505833 \\
0.050586058\end{array}$ \\
\hline $\begin{array}{l}\text { PAK2(h) } \\
\text { Pas }\end{array}$ & 51 & 0 & 92 & 92 & 50 & 0.588235294 & 8 & 0.004935225 & 0.055521283 \\
\hline PDGFRa(h) & 99 & 0 & 91 & 91 & 51 & 0.6 & 9 & 0.005552128 & 0.061073411 \\
\hline PDGFRB(h) & 93 & 0 & 91 & 91 & 52 & 0.611764706 & 9 & 0.005552128 & 0.06662554 \\
\hline PDK I(h) & 92 & 0 & 91 & 91 & 53 & 0.623529412 & 9 & 0.005552128 & 0.072177668 \\
\hline PKA(b) & 124 & 0 & 90 & 90 & 54 & 0.635294118 & 10 & 0.006169031 & 0.0783467 \\
\hline 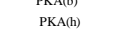 & $\begin{array}{l}104 \\
102\end{array}$ & 0 & 88 & 88 & $\begin{array}{l}54 \\
55\end{array}$ & 0.647058824 & 12 & 0.007402838 & 0.085749537 \\
\hline PKBa(h) & 100 & 0 & 88 & 88 & 56 & 0.658823529 & 12 & 0.007402838 & 0.093152375 \\
\hline PKBB(h) & 82 & 0 & 87 & 87 & 57 & 0.670588235 & 13 & 0.008019741 & 0.101172116 \\
\hline $\mathrm{PKB}_{\gamma}(\mathrm{h})$ & 103 & 0 & 86 & 86 & 58 & 0.682352941 & 14 & 0.008636644 & 0.10980876 \\
\hline PKCu(h) & 107 & 0 & 85 & 85 & 59 & 0.694117647 & 15 & 0.009253547 & 0.119062307 \\
\hline PKCBII(h) & 104 & 0 & 82 & 82 & 60 & 0.705882353 & 18 & 0.011104257 & 0.130166564 \\
\hline PKCY(h) & 101 & 0 & 81 & 81 & 61 & 0.717647059 & 19 & 0.01172116 & 0.141887724 \\
\hline РКС() & 100 & 0 & 78 & 78 & $\begin{array}{l}11 \\
62\end{array}$ & 0.729411765 & 22 & 0.013571869 & $\begin{array}{l}0.155459593 \\
0.1548\end{array}$ \\
\hline $\begin{array}{l}\text { PKE:(h) } \\
\text { Pall }\end{array}$ & 98 & 0 & 76 & 76 & 63 & 0.741176471 & 24 & 0.014805676 & 0.170265268 \\
\hline $\begin{array}{l}\text { PKG (h) } \\
\text { (h) }\end{array}$ & 91 & 0 & 75 & 75 & 64 & 0.752941176 & 25 & 0.015422579 & 0.185687847 \\
\hline PKC (h) & 95 & 0 & 74 & 74 & 65 & 0.764705882 & 26 & 0.016039482 & 0.201727329 \\
\hline PKCu(h) & 108 & 0 & 72 & 72 & 66 & 0.776470588 & 28 & 0.017273288 & 0.219000617 \\
\hline PKCO(h) & 95 & 0 & 66 & 66 & 67 & 0.788235294 & 34 & 0.020974707 & 0.239975324 \\
\hline PKD2(h) & 97 & 0 & 62 & 62 & 68 & 0.8 & 38 & 0.02344232 & 0.263417643 \\
\hline PRAK(h) & $\begin{array}{l}97 \\
108\end{array}$ & 0 & $\begin{array}{l}62 \\
60\end{array}$ & $\begin{array}{l}62 \\
60\end{array}$ & 69 & 0.811764706 & 40 & 0.024676126 & 0.288093769 \\
\hline PRK2(h) & 100 & 0 & 54 & 54 & 70 & 0.823529412 & 46 & 0.028377545 & 0.316471314 \\
\hline ROCK-II(h) & 92 & 0 & 51 & 51 & 71 & 0.835294118 & $\begin{array}{l}40 \\
49\end{array}$ & 0.030228254 & 0.346699568 \\
\hline ROCK-IIIr) & 88 & 0 & 50 & 50 & 72 & 0.847058824 & 50 & 0.030845157 & 0.377544725 \\
\hline Rskl(h) & 85 & 0 & 49 & 49 & 73 & 0.858823529 & 51 & 0.03146206 & 0.409006786 \\
\hline Rskl(r) & 78 & 0 & 45 & 45 & 74 & 0.870588235 & 55 & 0.033929673 & 0.442936459 \\
\hline $\begin{array}{l}\text { Rskk2(h) } \\
\text { Rsher }\end{array}$ & 100 & 0 & 33 & 33 & 75 & 0.882352941 & 67 & 0.041332511 & 0.48426897 \\
\hline Rsk3(h) & 72 & 0 & 33 & 33 & 76 & 0.894117647 & 67 & 0.041332511 & 0.525601481 \\
\hline SAPK2a(h) & 112 & 0 & 25 & 25 & 77 & 0.905882353 & 75 & 0.046267736 & 0.571869217 \\
\hline SAPK2b(h) & 130 & 0 & 23 & 23 & 78 & 0.917647059 & 77 & 0.047501542 & 0.619370759 \\
\hline SAPK3(h) & 99 & 0 & 20 & 20 & 79 & 0.929411765 & 80 & 0.049352252 & 0.66872301 \\
\hline $\begin{array}{l}\mathrm{SARRS( \textrm {h } )} \\
\mathrm{SAPK} 4(\mathrm{~h})\end{array}$ & 101 & 0 & 18 & 18 & 80 & 0.941176471 & 82 & 0.050586058 & 0.719309068 \\
\hline SGK(h) & 94 & 0 & 17 & 17 & 81 & 0.952941176 & 83 & 0.051202961 & 0.77051203 \\
\hline Syk(h) & 86 & 0 & 8 & 8 & 82 & 0.964705882 & 92 & 0.056755089 & 0.827267119 \\
\hline TrkB(h) & 93 & 0 & 7 & 7 & 83 & 0.976470588 & 93 & 0.057371993 & 0.884639112 \\
\hline Yes(h) & 66 & 0 & 7 & 7 & 84 & 0.988235294 & 93 & 0.057371993 & 0.942011104 \\
\hline ZAP-70(h) & 103 & 0 & 6 & 6 & 85 & 1 & 94 & 0.057988896 & 1 \\
\hline & & & & & & Total inhibition & 1621 & & Total area \\
\hline & Hit rate & 5 & & & & & & & Gini \\
\hline
\end{tabular}




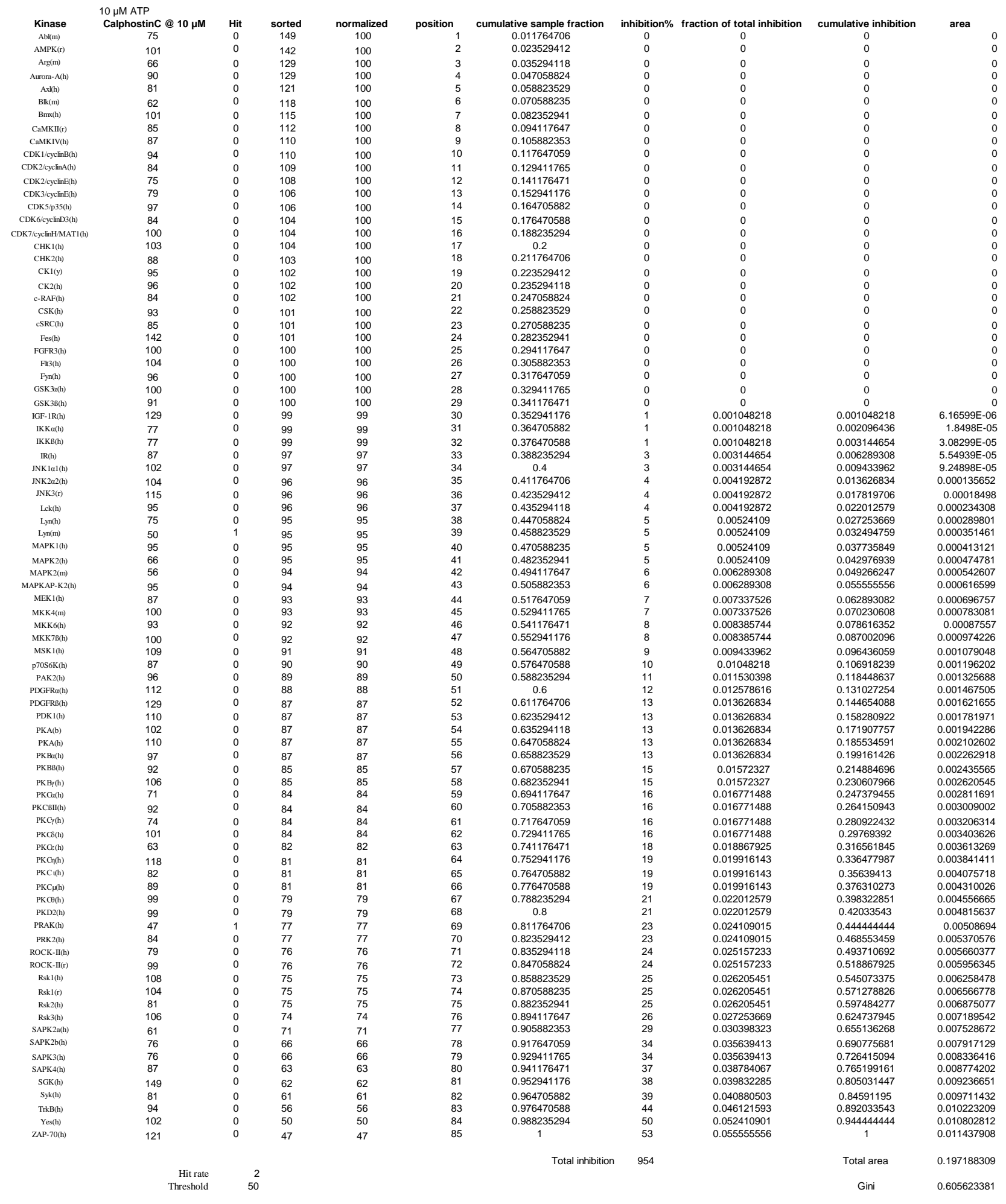




\begin{tabular}{|c|c|c|c|c|c|c|c|c|}
\hline & & & & & & & & \\
\hline Kinase & Calphostinc @ $10 \mu \mathrm{M}$ & Hit & sorted & normalized & position & cumulative sample fraction & inhibition\% & fraction of total inhibition \\
\hline $\mathrm{Abl}(\mathrm{m})$ & 62 & 0 & 140 & 100 & 1 & 0.011764706 & 0 & 0 \\
\hline AMPK(r) & 108 & 0 & 139 & 100 & 2 & 0.023529412 & 0 & 0 \\
\hline $\operatorname{Arg}(\mathrm{m})$ & 85 & 0 & 137 & 100 & 3 & 0.035294118 & 0 & 0 \\
\hline Aurora-A(h) & 81 & 0 & 136 & 100 & 4 & 0.047058824 & 0 & 0 \\
\hline $\operatorname{Ax}(\mathrm{h})$ & 86 & 0 & 131 & 100 & 5 & 0.058823529 & 0 & 0 \\
\hline 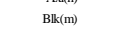 & 51 & 0 & 126 & 100 & 6 & 0.070588235 & 0 & 0 \\
\hline $\operatorname{Bnx}(\mathrm{h})$ & 92 & 0 & 124 & 100 & 7 & 0.082352941 & 0 & 0 \\
\hline CaMKII(r) & 80 & 0 & 124 & 100 & 8 & 0.094117647 & 0 & 0 \\
\hline CaMKIV(h) & 93 & 0 & 115 & 100 & 9 & 0.105882353 & 0 & 0 \\
\hline CDK1/cyclinB(h) & 89 & 0 & 113 & 100 & 10 & 0.117647059 & 0 & 0 \\
\hline CDK2/cyclinA(h) & 90 & 0 & 113 & 100 & 11 & 0.129411765 & 0 & 0 \\
\hline $\mathrm{CDK} 2 / \mathrm{cyclin} E(\mathrm{~h})$ & 78 & 0 & 113 & 100 & 12 & 0.141176471 & 0 & 0 \\
\hline CDK3/cyclinE(h) & 82 & 0 & 112 & 100 & 13 & 0.152941176 & 0 & 0 \\
\hline CDK5/(p35(h) & 112 & 0 & 112 & 100 & 14 & 0.164705882 & 0 & 0 \\
\hline CDKG/cyclinD3(h) & 98 & 0 & 111 & 100 & 15 & 0.176470588 & 0 & 0 \\
\hline CDK7//cyclinHMATI(h) & 90 & 0 & 109 & 100 & 16 & 0.188235294 & 0 & 0 \\
\hline CHK I(h) & 94 & 0 & 109 & 100 & 17 & 0.2 & 0 & 0 \\
\hline СНК2(h) & 92 & 0 & 109 & 100 & 18 & 0.211764706 & 0 & 0 \\
\hline $\mathrm{CK} 1(\mathrm{y})$ & 101 & 0 & 108 & 100 & 19 & 0.223529412 & 0 & 0 \\
\hline СК2(h) & 95 & 0 & 108 & 100 & 20 & 0.235294118 & 0 & 0 \\
\hline c-RAF(h) & 82 & 0 & 108 & 100 & 21 & 0.247058824 & 0 & 0 \\
\hline CSK(h) & 113 & 0 & 107 & 100 & 22 & 0.258823529 & 0 & 0 \\
\hline cSRC(h) & 101 & 0 & 106 & 100 & 23 & 0.270588235 & 0 & 0 \\
\hline Fes(h) & 140 & 0 & 106 & 100 & 24 & 0.282352941 & 0 & 0 \\
\hline FGFR3 (h) & 126 & 0 & 105 & 100 & 25 & 0.294117647 & 0 & 0 \\
\hline $\mathrm{FH} / 3(\mathrm{~h})$ & 99 & 0 & 105 & 100 & 26 & 0.305882353 & 0 & 0 \\
\hline Fynn(h) & 109 & 0 & 104 & 100 & 27 & 0.317647059 & 0 & 0 \\
\hline GSK3a(h) & 112 & 0 & 103 & 100 & 28 & 0.329411765 & 0 & 0 \\
\hline GSK3B(h) & 101 & 0 & 102 & 100 & 29 & 0.341176471 & 0 & 0 \\
\hline IGF-1R(h) & 131 & 0 & 101 & 100 & 30 & 0.352941176 & 0 & 0 \\
\hline IKKa(h) & 115 & 0 & 101 & 100 & 31 & 0.364705882 & 0 & 0 \\
\hline $\operatorname{IKKB(h)}$ & 88 & 0 & 101 & 100 & 32 & 0.376470588 & 0 & 0 \\
\hline $\mathrm{IR}(\mathrm{h})$ & 94 & 0 & 101 & 100 & 33 & 0.388235294 & 0 & 0 \\
\hline JNKIal(h) & 101 & 0 & 101 & 100 & 34 & 0.4 & 0 & 0 \\
\hline JNK202(h) & 106 & 0 & 101 & 100 & 35 & 0.411764706 & 0 & 0 \\
\hline JNK3(r) & 108 & 0 & 100 & 100 & 36 & 0.423529412 & 0 & 0 \\
\hline Lck(h) & 103 & 0 & 99 & 99 & 37 & 0.435294118 & 1 & 0.001156069 \\
\hline Lyn(h) & 69 & 0 & 98 & 98 & 38 & 0.447058824 & 2 & 0.002312139 \\
\hline $\operatorname{Lyn}(\mathrm{m})$ & 53 & 0 & 97 & 97 & 39 & 0.458823529 & 3 & 0.003468208 \\
\hline MAPK1(h) & 91 & 0 & 96 & 96 & 40 & 0.470588235 & 4 & 0.004624277 \\
\hline MAPK2(h) & 73 & 0 & 96 & 96 & 41 & 0.482352941 & 4 & 0.004624277 \\
\hline MAPK2(m) & 68 & 0 & 96 & 96 & 42 & 0.494117647 & 4 & 0.004624277 \\
\hline MAPKAP-K2(h) & 84 & 0 & 96 & 96 & 43 & 0.505882353 & 4 & 0.004624277 \\
\hline MEKI(h) & 96 & 0 & 95 & 95 & 44 & 0.517647059 & 5 & 0.005780347 \\
\hline MKK4(m) & 96 & 0 & 94 & 94 & 45 & 0.529411765 & 6 & 0.006936416 \\
\hline MKK6(h) & 100 & 0 & 94 & 94 & 46 & 0.541176471 & 6 & 0.006936416 \\
\hline MKКㄱ(h) & 107 & 0 & 94 & 94 & 47 & 0.552941176 & 6 & 0.006936416 \\
\hline MSK1(h) & 108 & 0 & 93 & 93 & 48 & 0.564705882 & 7 & 0.008092486 \\
\hline $\mathrm{p} 70 \mathrm{~S} 6 \mathrm{~K}(\mathrm{~h})$ & 102 & 0 & 93 & 93 & 49 & 0.576470588 & 7 & 0.008092486 \\
\hline PAK2(h) & 104 & 0 & 92 & 92 & 50 & 0.588235294 & 8 & 0.009248555 \\
\hline PDGFRa(h) & 124 & 0 & 92 & 92 & 51 & 0.6 & 8 & 0.009248555 \\
\hline PDGFRB(h) & 139 & 0 & 91 & 91 & 52 & 0.611764706 & 9 & 0.010404624 \\
\hline PDKI(h) & 88 & 0 & 90 & 90 & 53 & 0.623529412 & 10 & 0.011560694 \\
\hline PKA(b) & 105 & 0 & 90 & 90 & 54 & 0.635294118 & 10 & 0.011560694 \\
\hline PKA(h) & 97 & 0 & 89 & 89 & 55 & 0.647058824 & 11 & 0.012716763 \\
\hline PKBa(h) & 96 & 0 & 89 & 89 & 56 & 0.658823529 & 11 & 0.012716763 \\
\hline PKBB(h) & 96 & 0 & 88 & 88 & 57 & 0.670588235 & 12 & 0.013872832 \\
\hline PKB (hl) & 109 & 0 & 88 & 88 & 58 & 0.682352941 & 12 & 0.013872832 \\
\hline PKG(h) & 71 & 0 & 88 & 88 & 59 & 0.694117647 & 12 & 0.013872832 \\
\hline РКCBII(h) & 101 & 0 & 86 & 86 & 60 & 0.705882353 & 14 & 0.016184971 \\
\hline PKCy(h) & 66 & 0 & 85 & 85 & 61 & 0.717647059 & 15 & 0.01734104 \\
\hline РКБ(h) & 124 & 0 & 84 & 84 & 62 & 0.729411765 & 16 & 0.01849711 \\
\hline PKC:(h) & 71 & 0 & 83 & 83 & 63 & 0.741176471 & 17 & 0.019653179 \\
\hline PKG(h) & 137 & 0 & 82 & 82 & 64 & 0.752941176 & 18 & 0.020809249 \\
\hline PKC (1) & 105 & 0 & 82 & 82 & 65 & 0.764705882 & 18 & 0.020809249 \\
\hline PKC (h) & 93 & 0 & 82 & 82 & 66 & 0.776470588 & 18 & 0.020809249 \\
\hline 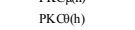 & 113 & 0 & 81 & 81 & 67 & 0.788235294 & 19 & 0.021965318 \\
\hline PKD2(h) & 106 & 0 & 80 & 80 & 68 & 0.8 & 20 & 0.023121387 \\
\hline PRAK(h) & 50 & 1 & 80 & 80 & 69 & 0.811764706 & 20 & 0.023121387 \\
\hline PRK2(h) & 68 & 0 & 79 & 79 & 70 & 0.823529412 & 21 & 0.024277457 \\
\hline ROCK-II(h) & 83 & 0 & 78 & 78 & 71 & 0.835294118 & 22 & 0.025433526 \\
\hline ROCK-II(r) & 82 & 0 & 73 & 73 & 72 & 0.847058824 & 27 & 0.031213873 \\
\hline Rskl(h) & 109 & 0 & 73 & 73 & 73 & 0.858823529 & 27 & 0.031213873 \\
\hline $\operatorname{Rskl(r)}$ & 136 & 0 & 72 & 72 & 74 & 0.870588235 & 28 & 0.032369942 \\
\hline $\begin{array}{l}\text { Rssk(t) } \\
\text { Rsk2(h) }\end{array}$ & 79 & 0 & 71 & 71 & 75 & 0.882352941 & 29 & 0.033526012 \\
\hline Rsk3(h) & 88 & 0 & 71 & 71 & 76 & 0.894117647 & 29 & 0.033526012 \\
\hline SAPK2a(h) & 72 & 0 & 69 & 69 & 77 & 0.905882353 & 31 & 0.03583815 \\
\hline SAPK2b(h) & 101 & 0 & 68 & 68 & 78 & 0.917647059 & 32 & 0.03699422 \\
\hline $\mathrm{SAPK} 3(\mathrm{~h})$ & 68 & 0 & 68 & 68 & 79 & 0.929411765 & 32 & 0.03699422 \\
\hline SAPK4(h) & 80 & 0 & 68 & 68 & 80 & 0.941176471 & 32 & 0.03699422 \\
\hline SGK(h) & 94 & 0 & 66 & 66 & 81 & 0.952941176 & 34 & 0.039306358 \\
\hline Syk(h) & 73 & 0 & 62 & $\begin{array}{l}60 \\
62\end{array}$ & 82 & 0.964705882 & 38 & 0.043930636 \\
\hline TrkB(h) & 89 & 0 & 53 & 53 & 83 & 0.976470588 & 47 & 0.05433526 \\
\hline Yes(h) & 113 & 0 & 51 & 51 & 84 & 0.988235294 & 49 & 0.056647399 \\
\hline ZAP-70(h) & 111 & 0 & 50 & 50 & 85 & 1 & 50 & 0.057803468 \\
\hline & & & & & & Total inhibition & 865 & \\
\hline & $\begin{array}{l}\text { Hit rate } \\
\end{array}$ & & & & & & & \\
\hline & Threshold & & & & & & & \\
\hline
\end{tabular}




\begin{tabular}{|c|c|c|c|c|c|c|c|c|}
\hline & $10 \mu \mathrm{M}$ ATP & & & & & & & \\
\hline Kinase & CDK2_5inh @ $10 \mu \mathrm{M}$ & Hit & sorted & normalized & position & cumulative sample fraction & inhibition\% & fraction of total inhibition \\
\hline $\mathrm{Ab}(\mathrm{m})$ & 75 & 0 & 233 & 100 & 1 & 0.011764706 & 0 & 0 \\
\hline AMPK(r) & 84 & 0 & 112 & 100 & 2 & 0.023529412 & 0 & 0 \\
\hline $\operatorname{Arg}(m)$ & 78 & 0 & 107 & 100 & 3 & 0.035294118 & 0 & 0 \\
\hline Aurora-A(h) & 78 & 0 & 106 & 100 & 4 & 0.047058824 & 0 & 0 \\
\hline Axl(h) & 98 & 0 & 104 & 100 & 5 & 0.058823529 & 0 & 0 \\
\hline $\mathrm{Blk}(\mathrm{m})$ & 59 & 0 & 104 & 100 & 6 & 0.070588235 & 0 & 0 \\
\hline Bmx(h) & 87 & 0 & 103 & 100 & 7 & 0.082352941 & 0 & 0 \\
\hline CaMKII(r) & 92 & 0 & 102 & 100 & 8 & 0.094117647 & 0 & 0 \\
\hline CaMKIV(h) & 99 & 0 & 101 & 100 & 9 & 0.105882353 & 0 & 0 \\
\hline $\mathrm{CDK} 1 / \mathrm{cyclin} B(\mathrm{~h})$ & 33 & 1 & 101 & 100 & 10 & 0.117647059 & 0 & 0 \\
\hline $\mathrm{CDK} 2 / \mathrm{cyc} \operatorname{lin}(\mathrm{h})$ & 19 & 1 & 100 & 100 & 11 & 0.129411765 & 0 & 0 \\
\hline $\mathrm{CDK} 2 / \mathrm{cyclin} E(\mathrm{~h})$ & 19 & 1 & 99 & 99 & 12 & 0.141176471 & 1 & 0.000704225 \\
\hline CDK3/cyclinE(h) & 47 & 1 & 99 & 99 & 13 & 0.152941176 & 1 & 0.000704225 \\
\hline $\mathrm{CDK} 5 / \mathrm{p} 35(\mathrm{~h})$ & 12 & 1 & 99 & 99 & 14 & 0.164705882 & 1 & 0.000704225 \\
\hline $\mathrm{CDK} /$ cypclinD3(h) & 92 & 0 & $\begin{array}{l}99 \\
99\end{array}$ & $\begin{array}{l}99 \\
99\end{array}$ & 15 & 0.176470588 & 1 & 0.000704225 \\
\hline CDK7/cyclinHMATI(h) & 82 & 0 & 99 & 99 & 16 & 0.188235294 & 1 & 0.000704225 \\
\hline CHK 1 (h) & 74 & 0 & 99 & 99 & 17 & 0.2 & 1 & 0.000704225 \\
\hline СHK2(h) & 80 & 0 & 98 & 98 & 18 & 0.211764706 & 2 & 0.001408451 \\
\hline $\mathrm{CKI}(\mathrm{y})$ & 92 & 0 & 98 & 98 & 19 & 0.223529412 & 2 & 0.001408451 \\
\hline CK2(h) & 99 & 0 & 98 & 98 & 20 & 0.235294118 & 2 & 0.001408451 \\
\hline$c \cdot \operatorname{RAF}(\mathrm{h})$ & 93 & 0 & 97 & 97 & 21 & 0.247058824 & 3 & 0.002112676 \\
\hline CSK(h) & 84 & 0 & 96 & 96 & 22 & 0.258823529 & 4 & 0.002816901 \\
\hline cSRC(h) & 77 & 0 & 95 & 95 & 23 & 0.270588235 & 5 & 0.003521127 \\
\hline Fes(h) & 77 & 0 & 94 & 94 & 24 & 0.282352941 & 6 & 0.004225352 \\
\hline FGFR3(h) & 99 & 0 & 94 & 94 & 25 & 0.294117647 & 6 & 0.004225352 \\
\hline $\begin{array}{c}\text { Furps(n) } \\
\text { Fllah) }\end{array}$ & 41 & 1 & 94 & 94 & 26 & 0.305882353 & 6 & 0.004225352 \\
\hline Fynn(h) & 68 & 0 & 94 & 94 & 27 & 0.317647059 & 6 & 0.004225352 \\
\hline GSK3a(h) & 92 & 0 & 93 & 93 & 28 & 0.329411765 & 7 & 0.004929577 \\
\hline GSK3B(h) & 98 & 0 & 93 & 93 & 29 & 0.341176471 & 7 & 0.004929577 \\
\hline IGF-1R(h) & 99 & 0 & 93 & 93 & 30 & 0.352941176 & 7 & 0.004929577 \\
\hline IKKa(h) & 89 & 0 & 93 & 93 & 31 & 0.364705882 & 7 & 0.004929577 \\
\hline IKKB(h) & 91 & 0 & 93 & 93 & 32 & 0.376470588 & 7 & 0.004929577 \\
\hline $\mathrm{IR}(\mathrm{h})$ & 99 & 0 & 92 & 92 & 33 & 0.388235294 & 8 & 0.005633803 \\
\hline JNKIal(h) & 81 & 0 & 92 & 92 & 34 & 0.4 & 8 & 0.005633803 \\
\hline JNK2a2(h) & 81 & 0 & 92 & 92 & 35 & 0.411764706 & 8 & 0.005633803 \\
\hline JNK3(r) & 71 & 0 & 92 & 92 & 36 & 0.423529412 & 8 & 0.005633803 \\
\hline Lck(l) & 72 & 0 & 92 & 92 & $\begin{array}{l}50 \\
37\end{array}$ & 0.435294118 & 8 & 0.005633803 \\
\hline Lyn(h) & 66 & 0 & 92 & 92 & 38 & 0.447058824 & 8 & 0.005633803 \\
\hline Lyn(m) & 74 & 0 & 91 & 91 & $\begin{array}{l}00 \\
39\end{array}$ & 0.458823529 & 9 & 0.006338028 \\
\hline MAPKI (h) & 102 & 0 & 91 & 91 & 40 & 0.470588235 & 9 & 0.006338028 \\
\hline MAPK2(h) & 94 & 0 & 91 & 91 & 41 & 0.482352941 & 9 & 0.006338028 \\
\hline MAPK2(m) & 88 & 0 & 90 & 90 & 42 & 0.494117647 & 10 & 0.007042254 \\
\hline MAPKAP-K2(h) & 95 & 0 & 89 & 89 & 43 & 0.505882353 & 11 & 0.007746479 \\
\hline MEKI(h) & 93 & 0 & $\begin{array}{l}89 \\
89\end{array}$ & $\begin{array}{l}89 \\
89\end{array}$ & 44 & 0.517647059 & 11 & 0.007746479 \\
\hline MKK4(m) & 93 & 0 & 89 & 89 & 45 & 0.529411765 & 11 & 0.007746479 \\
\hline $\begin{array}{l}\text { MKK } \\
\text { MKK } 6(\mathrm{~m})\end{array}$ & 93 & 0 & $\begin{array}{l}89 \\
89\end{array}$ & $\begin{array}{l}89 \\
89\end{array}$ & 46 & 0.541176471 & 11 & 0.007746479 \\
\hline MKK 7 (h) & 90 & 0 & 88 & 88 & 47 & 0.552941176 & 12 & 0.008450704 \\
\hline MSKI(h) & 89 & 0 & 88 & 88 & 48 & 0.564705882 & 12 & 0.008450704 \\
\hline $\mathrm{p} 70 \mathrm{~S} 6 \mathrm{~K}(\mathrm{~h})$ & 84 & 0 & 87 & 87 & 49 & 0.576470588 & 13 & 0.00915493 \\
\hline PAK2(h) & 97 & 0 & 87 & 87 & 50 & 0.588235294 & 13 & 0.00915493 \\
\hline PDGFRa(h) & 94 & 0 & 87 & 87 & 51 & 0.6 & 13 & 0.00915493 \\
\hline $\begin{array}{l}\text { PDGFRB(h) } \\
\text { Pats }\end{array}$ & 107 & 0 & 84 & 84 & 52 & 0.611764706 & 16 & 0.011267606 \\
\hline PDKI(h) & 87 & 0 & 84 & 84 & 53 & 0.623529412 & 16 & 0.011267606 \\
\hline PKA(b) & 103 & 0 & 84 & 84 & 54 & 0.635294118 & 16 & 0.011267606 \\
\hline PKA(h) & 92 & 0 & 83 & 83 & 55 & 0.647058824 & 17 & 0.011971831 \\
\hline PKBa(h) & 100 & 0 & 82 & 82 & 56 & 0.658823529 & 18 & 0.012676056 \\
\hline 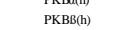 & 78 & 0 & $\begin{array}{l}82 \\
81\end{array}$ & $\begin{array}{l}82 \\
81\end{array}$ & 57 & 0.670588235 & 19 & 0.013380282 \\
\hline $\mathrm{PKB},(\mathrm{h})$ & 94 & 0 & 81 & 81 & 58 & 0.682352941 & 19 & 0.013380282 \\
\hline $\begin{array}{l}\text { PrBf(h) } \\
\text { PKGa(h) }\end{array}$ & 91 & 0 & 80 & 80 & $\begin{array}{l}00 \\
59\end{array}$ & 0.694117647 & 20 & 0.014084507 \\
\hline PKCBII(h) & 101 & 0 & 79 & 79 & 60 & 0.705882353 & 21 & 0.014788732 \\
\hline PKCy(h) & 101 & 0 & 79 & 79 & 61 & 0.717647059 & 21 & 0.014788732 \\
\hline PKC(h) & 104 & 0 & 78 & 78 & 62 & 0.729411765 & 22 & 0.015492958 \\
\hline PKE:(h) & 91 & 0 & 78 & 78 & 63 & 0.741176471 & 22 & 0.015492958 \\
\hline 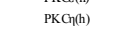 & 89 & 0 & 78 & 78 & 64 & 0.752941176 & 22 & 0.015492958 \\
\hline PKC(h) & 104 & 0 & 78 & 78 & 65 & 0.764705882 & 22 & 0.015492958 \\
\hline PKC $\mu(\mathrm{h})$ & 106 & 0 & 77 & 77 & 66 & 0.776470588 & 23 & 0.016197183 \\
\hline PKC $(\mathrm{h})$ & 96 & 0 & 77 & 77 & 67 & 0.788235294 & 23 & 0.016197183 \\
\hline PKD2(h) & 99 & 0 & 75 & 75 & 68 & 0.8 & 25 & 0.017605634 \\
\hline PRAK(h) & 112 & 0 & 74 & 74 & 69 & 0.811764706 & 26 & 0.018309859 \\
\hline PRK2(h) & 233 & 0 & 74 & 74 & 70 & 0.823529412 & 26 & 0.018309859 \\
\hline ROCK-III(h) & 67 & 0 & 72 & 72 & 71 & 0.835294118 & 28 & 0.01971831 \\
\hline ROCK-II(r) & 61 & 0 & 71 & 71 & 72 & 0.847058824 & 29 & 0.020422535 \\
\hline Rskl(h) & 89 & 0 & 68 & 68 & 73 & 0.858823529 & 32 & 0.022535211 \\
\hline Rsk1(r) & 79 & 0 & 67 & 67 & 74 & 0.870588235 & 33 & 0.023239437 \\
\hline $\begin{array}{l}\text { Rskit(t) } \\
\text { Rsk(h) }\end{array}$ & 87 & 0 & 66 & 66 & 75 & 0.882352941 & 34 & 0.023943662 \\
\hline Rsk3(h) & 88 & 0 & 66 & 66 & 76 & 0.894117647 & 34 & 0.023943662 \\
\hline SAPK2a(h) & 83 & 0 & 61 & 61 & 77 & 0.905882353 & 39 & 0.027464789 \\
\hline $\mathrm{SAPK} 2 \mathrm{~b}(\mathrm{~h})$ & 66 & 0 & 59 & 59 & 78 & 0.917647059 & 41 & 0.028873239 \\
\hline SAPK3(h) & 93 & 0 & 48 & 48 & 79 & 0.929411765 & 52 & 0.036619718 \\
\hline SAPK4(h) & 94 & 0 & 47 & 47 & 80 & 0.941176471 & 53 & 0.037323944 \\
\hline SGK(h) & 48 & 1 & 41 & 41 & 81 & 0.952941176 & 59 & 0.041549296 \\
\hline Syk(h) & $\begin{array}{l}40 \\
98\end{array}$ & 0 & $\begin{array}{l}41 \\
33\end{array}$ & $\begin{array}{l}41 \\
33\end{array}$ & 82 & 0.964705882 & 67 & 0.047183099 \\
\hline TTkB(h) & 92 & 0 & 19 & 19 & 83 & 0.976470588 & 81 & 0.057042254 \\
\hline Yes(h) & 78 & 0 & 19 & 19 & 84 & 0.988235294 & 81 & 0.057042254 \\
\hline ZAP-70(h) & 79 & 0 & 12 & 12 & 85 & 1 & 88 & 0.061971831 \\
\hline & & & & & & Total inhibition & 1420 & \\
\hline & $\begin{array}{r}\text { Hit rate } \\
\text { Threshold }\end{array}$ & 5 & & & & & & \\
\hline & Threshold & & & & & & & \\
\hline
\end{tabular}




\begin{tabular}{|c|c|c|c|c|c|c|c|c|c|}
\hline & $100 \mu \mathrm{M}$ ATP & & & & & & & & \\
\hline Kinase & CDK25inh@10 & Hit & sorted & normalized & position & cumulative sample fraction & inhibition\% & fraction of total inhibition & cumulative inhibition \\
\hline $\mathrm{Abl}(\mathrm{m})$ & 102 & 0 & 117 & 100 & 1 & 0.011764706 & 0 & 0 & 0 \\
\hline AMPK(r) & 89 & 0 & 112 & 100 & 2 & 0.023529412 & 0 & 0 & 0 \\
\hline $\operatorname{Arg}(\mathrm{m})$ & 87 & 0 & 112 & 100 & 3 & 0.035294118 & 0 & 0 & 0 \\
\hline Aurora-A(h) & 89 & 0 & 111 & 100 & 4 & 0.047058824 & 0 & 0 & 0 \\
\hline Axl(h) & 89 & 0 & 111 & 100 & 5 & 0.058823529 & 0 & 0 & 0 \\
\hline $\operatorname{Blk}(\mathrm{m})$ & 85 & 0 & 110 & 100 & 6 & 0.070588235 & 0 & 0 & 0 \\
\hline $\operatorname{Bmx}(\mathrm{h})$ & 91 & 0 & 108 & 100 & 7 & 0.082352941 & 0 & 0 & 0 \\
\hline CaMKII(r) & 94 & 0 & 107 & 100 & 8 & 0.094117647 & 0 & 0 & 0 \\
\hline CaMKIV(h) & 101 & 0 & 107 & 100 & 9 & 0.105882353 & 0 & 0 & 0 \\
\hline CDK1/cyclnBB(h) & 63 & 0 & 106 & 100 & 10 & 0.117647059 & 0 & 0 & 0 \\
\hline CDK2/cyclinA(h) & 48 & 1 & 106 & 100 & 11 & 0.129411765 & 0 & 0 & 0 \\
\hline $\mathrm{CDK} 2 /$ cyclnE(h) & 25 & 1 & 106 & 100 & 12 & 0.141176471 & 0 & 0 & 0 \\
\hline CDK3/yclinE(h) & 75 & 0 & 105 & 100 & 13 & 0.152941176 & 0 & 0 & 0 \\
\hline CDK5/p35(h) & 72 & 0 & 105 & 100 & 14 & 0.164705882 & 0 & 0 & 0 \\
\hline CDK6/cyclinD3(h) & 87 & 0 & 105 & 100 & 15 & 0.176470588 & 0 & 0 & 0 \\
\hline CDK $7 /$ cyclinHMATI(h) & 93 & 0 & 104 & 100 & 16 & 0.188235294 & 0 & 0 & 0 \\
\hline CHK1 (h) & 90 & 0 & 104 & 100 & 17 & 0.2 & 0 & 0 & 0 \\
\hline CHK2(h) & 97 & 0 & 104 & 100 & 18 & 0.211764706 & 0 & 0 & 0 \\
\hline CKI(y) & 112 & 0 & 102 & 100 & 19 & 0.223529412 & 0 & 0 & 0 \\
\hline СК2(h) & 104 & 0 & 102 & 100 & 20 & 0.235294118 & 0 & 0 & 0 \\
\hline c-RAF(h) & 91 & 0 & 102 & 100 & 21 & 0.247058824 & 0 & 0 & 0 \\
\hline $\operatorname{CSK}(\mathrm{h})$ & 93 & 0 & 101 & 100 & 22 & 0.258823529 & 0 & 0 & 0 \\
\hline $\operatorname{csRC(h)}$ & 89 & 0 & 101 & 100 & 23 & 0.270588235 & 0 & 0 & 0 \\
\hline Fess(h) & 105 & 0 & 101 & 100 & 24 & 0.282352941 & 0 & 0 & 0 \\
\hline FGFR3(h) & 112 & 0 & 101 & 100 & 25 & 0.294117647 & 0 & 0 & 0 \\
\hline $\mathrm{Fll}(\mathrm{h}(\mathrm{h})$ & 73 & 0 & 101 & 100 & 26 & 0.305882353 & 0 & 0 & 0 \\
\hline Fynn(h) & 88 & 0 & 101 & 100 & 27 & 0.317647059 & 0 & 0 & 0 \\
\hline GSK3a(h) & 101 & 0 & 100 & 100 & 28 & 0.329411765 & 0 & 0 & 0 \\
\hline GSK3B(h) & 98 & 0 & 100 & 100 & $\begin{array}{l}20 \\
29\end{array}$ & 0.341176471 & 0 & 0 & 0 \\
\hline IGF-IR(h) & 111 & 0 & 100 & 100 & 30 & 0.352941176 & 0 & 0 & 0 \\
\hline $\mathrm{IKKa(h)}$ & 97 & 0 & 99 & 99 & 31 & 0.364705882 & 1 & 0.001503759 & 0.001503759 \\
\hline IKKB(h) & 95 & 0 & 99 & 99 & 32 & 0.376470588 & 1 & 0.001503759 & 0.003007519 \\
\hline $\mathrm{IR}(\mathrm{h})$ & 105 & 0 & 99 & 99 & 33 & 0.388235294 & 1 & 0.001503759 & 0.004511278 \\
\hline JNKIal(h) & 96 & 0 & 99 & 99 & 34 & 0.4 & 1 & 0.001503759 & 0.006015038 \\
\hline JNK202(h) & 100 & 0 & 98 & 98 & $\begin{array}{l}54 \\
35\end{array}$ & 0.411764706 & 2 & 0.003007519 & 0.009022556 \\
\hline $\mathrm{JNK} 3(\mathrm{r})$ & 79 & 0 & 98 & 98 & 36 & 0.423529412 & 2 & 0.003007519 & 0.012030075 \\
\hline Lck(h) & 88 & 0 & 98 & 98 & 37 & 0.435294118 & 2 & 0.003007519 & 0.015037594 \\
\hline (Lekn) & 90 & 0 & 97 & 97 & 38 & 0.447058824 & 3 & 0.004511278 & 0.019548872 \\
\hline Lyy(m) & 96 & 0 & 97 & 97 & 39 & 0.458823529 & 3 & 0.004511278 & 0.02406015 \\
\hline MAPKI(h) & $\begin{array}{l}90 \\
105\end{array}$ & 0 & 97 & 97 & 40 & 0.470588235 & 3 & 0.004511278 & 0.028571429 \\
\hline MAPK2(h) & 108 & 0 & 97 & 97 & 41 & 0.482352941 & 3 & 0.004511278 & 0.033082707 \\
\hline $\begin{array}{l}\text { MAPKL(2) } \\
\text { MAPK2(m) }\end{array}$ & 101 & 0 & 96 & 96 & $\begin{array}{l}41 \\
42\end{array}$ & 0.494117647 & 4 & 0.006015038 & 0.039097744 \\
\hline MAPKAP-K2(h) & 96 & 0 & 96 & 96 & 43 & 0.505882353 & 4 & 0.006015038 & 0.045112782 \\
\hline MEK1(h) & 94 & 0 & 96 & 96 & 44 & 0.517647059 & 4 & 0.006015038 & 0.05112782 \\
\hline MKK4(m) & $\begin{array}{l}54 \\
110\end{array}$ & 0 & 96 & 96 & 45 & 0.529411765 & 4 & 0.006015038 & 0.057142857 \\
\hline MKK6(h) & 97 & 0 & 96 & 96 & 46 & 0.541176471 & 4 & 0.006015038 & 0.063157895 \\
\hline MKK & 101 & 0 & 96 & 96 & 47 & 0.552941176 & 4 & 0.006015038 & 0.069172932 \\
\hline MSKI(h) & 96 & 0 & 95 & 95 & 48 & 0.564705882 & 5 & 0.007518797 & 0.076691729 \\
\hline $\mathrm{p} 7056 \mathrm{~K}(\mathrm{~h})$ & $\begin{array}{l}90 \\
107\end{array}$ & 0 & 95 & 95 & $\begin{array}{l}48 \\
49\end{array}$ & 0.576470588 & $\begin{array}{l}5 \\
5\end{array}$ & 0.007518797 & 0.084210526 \\
\hline $\begin{array}{l}\text { PAK2(h) } \\
\text { Past }\end{array}$ & 92 & 0 & 94 & 94 & 50 & 0.588235294 & 6 & 0.009022556 & 0.093233083 \\
\hline PDGFRa(h) & 80 & 0 & 94 & 94 & 51 & 0.6 & 6 & 0.009022556 & 0.102255639 \\
\hline PDGFRB(h) & 98 & 0 & 94 & 94 & 52 & 0.611764706 & 6 & 0.009022556 & 0.111278195 \\
\hline PDKI(h) & 99 & 0 & 93 & $\begin{array}{l}94 \\
93\end{array}$ & 53 & 0.623529412 & 7 & 0.010526316 & 0.121804511 \\
\hline PKA(b) & 111 & 0 & 93 & 93 & 54 & 0.635294118 & 7 & 0.010526316 & 0.132330827 \\
\hline PKA(h) & 90 & 0 & 93 & 93 & 55 & 0.647058824 & 7 & 0.010526316 & 0.142857143 \\
\hline PKBa(h) & 99 & 0 & 92 & 92 & 56 & 0.658823529 & 8 & 0.012030075 & 0.154887218 \\
\hline PKBB(h) & 99 & 0 & 92 & 92 & 57 & 0.670588235 & 8 & 0.012030075 & 0.166917293 \\
\hline $\mathrm{PKB} \mathrm{B}_{(\mathrm{h})}$ & 106 & 0 & 91 & 91 & 58 & 0.682352941 & 9 & 0.013533835 & 0.180451128 \\
\hline PKCath) & 102 & 0 & 91 & 91 & 59 & 0.694117647 & 9 & 0.013533835 & 0.193984962 \\
\hline PKCBII(h) & 106 & 0 & 91 & 91 & 60 & 0.705882353 & 9 & 0.013533835 & 0.207518797 \\
\hline PKGy(h) & 104 & 0 & 90 & 90 & 61 & 0.717647059 & 10 & 0.015037594 & 0.222556391 \\
\hline РКФ(h) & 106 & 0 & 90 & 90 & 62 & 0.729411765 & 10 & 0.015037594 & 0.237593985 \\
\hline PKG:(h) & 100 & 0 & 90 & 90 & 63 & 0.741176471 & 10 & 0.015037594 & 0.252631579 \\
\hline $\begin{array}{l}\text { PKG (h) } \\
\text { (h) }\end{array}$ & 102 & 0 & 90 & 90 & 64 & 0.752941176 & 10 & 0.015037594 & 0.267669173 \\
\hline PKC (h) & 98 & 0 & 89 & 89 & 65 & 0.764705882 & 11 & 0.016541353 & 0.284210526 \\
\hline PKC $\mu(\mathrm{h})$ & 107 & 0 & 89 & 89 & 66 & 0.776470588 & 11 & 0.016541353 & 0.30075188 \\
\hline PKCO(h) & 93 & 0 & 89 & 89 & 67 & 0.788235294 & 11 & 0.016541353 & 0.317293233 \\
\hline PKD2(h) & 94 & 0 & 89 & 89 & 68 & 0.8 & 11 & 0.016541353 & 0.333834586 \\
\hline PRAK(h) & $\begin{array}{l}y_{4} \\
101\end{array}$ & 0 & $\begin{array}{l}89 \\
89\end{array}$ & $\begin{array}{l}89 \\
89\end{array}$ & 69 & 0.811764706 & 11 & 0.016541353 & 0.35037594 \\
\hline PRK2(h) & 100 & 0 & 88 & 88 & 70 & 0.823529412 & 12 & 0.018045113 & 0.368421053 \\
\hline $\begin{array}{l}\text { PRK_(n) } \\
\text { ROCK-II(h) }\end{array}$ & 96 & 0 & $\begin{array}{l}00 \\
88\end{array}$ & $\begin{array}{l}00 \\
88\end{array}$ & 71 & 0.835294118 & 12 & 0.018045113 & 0.386466165 \\
\hline ROCK-II(r) & 104 & 0 & 88 & 88 & 72 & 0.847058824 & 12 & 0.018045113 & 0.404511278 \\
\hline Rskl(h) & 90 & 0 & 87 & 87 & 73 & 0.858823529 & 13 & 0.019548872 & 0.42406015 \\
\hline Rsk1(r) & 85 & 0 & 87 & 87 & 74 & 0.870588235 & 13 & 0.019548872 & 0.443609023 \\
\hline $\begin{array}{l}\text { Rsksk2(h) } \\
\text { Rsk(h) }\end{array}$ & 101 & 0 & 85 & 85 & 75 & 0.882352941 & 15 & 0.022556391 & 0.466165414 \\
\hline Rsk3(h) & 89 & 0 & 85 & 85 & 76 & 0.894117647 & 15 & 0.022556391 & 0.488721805 \\
\hline SAPK2a(h) & 99 & 0 & 80 & 80 & 77 & 0.905882353 & 20 & 0.030075188 & 0.518796992 \\
\hline SAPK2b(h) & 88 & 0 & 79 & 79 & 78 & 0.917647059 & 21 & 0.031578947 & 0.55037594 \\
\hline SAPK3(h) & 92 & 0 & 75 & 75 & 79 & 0.929411765 & 25 & 0.037593985 & 0.587969925 \\
\hline SAPK4(h) & 96 & 0 & 73 & 73 & 80 & 0.941176471 & 27 & 0.040601504 & 0.628571429 \\
\hline SGK(h) & 45 & 1 & 72 & 72 & 81 & 0.952941176 & 28 & 0.042105263 & 0.670676692 \\
\hline Syk(h) & 117 & 0 & 63 & 63 & 82 & 0.964705882 & 37 & 0.055639098 & 0.726315789 \\
\hline TrkB(h) & 97 & 0 & 48 & 48 & 83 & 0.976470588 & 52 & 0.078195489 & 0.804511278 \\
\hline Yes(h) & 91 & 0 & 45 & 45 & 84 & 0.988235294 & 55 & 0.082706767 & 0.887218045 \\
\hline ZAP-70(h) & 95 & 0 & 25 & 25 & 85 & 1 & 75 & 0.112781955 & 1 \\
\hline & & & & & & Total inhibition & 665 & & Total area \\
\hline & Hit rate & & & & & & & & Gini \\
\hline
\end{tabular}




\begin{tabular}{|c|c|c|c|c|c|c|c|c|c|}
\hline & $10 \mu \mathrm{M}$ ATP & & & & & & & & \\
\hline Kinase & Curcumin @ $50 \mu \mathrm{M}$ & Hit & sorted & normalized & position & cumulative sample fraction & inhibition\% & fraction of total inhibition & cumulative inhibition \\
\hline $\mathrm{Ab}(\mathrm{m})$ & 8 & 1 & 147 & 100 & 1 & 0.011764706 & 0 & 0 & 0 \\
\hline $\mathrm{AMPK}(\mathrm{r})$ & 84 & 0 & 142 & 100 & 2 & 0.023529412 & 0 & 0 & 0 \\
\hline $\operatorname{Arg}(\mathrm{m})$ & 11 & 1 & 116 & 100 & 3 & 0.035294118 & 0 & 0 & 0 \\
\hline Aurora-A(h) & 45 & 1 & 112 & 100 & 4 & 0.047058824 & 0 & 0 & 0 \\
\hline Axl(h) & 18 & 1 & 105 & 100 & 5 & 0.058823529 & 0 & 0 & 0 \\
\hline $\operatorname{Blk}(\mathrm{m})$ & 13 & 1 & 105 & 100 & 6 & 0.070588235 & 0 & 0 & 0 \\
\hline Bnx(li) & 11 & 1 & 101 & 100 & 7 & 0.082352941 & 0 & 0 & 0 \\
\hline СамКII(r) & 11 & 1 & 101 & 100 & 8 & 0.094117647 & 0 & 0 & 0 \\
\hline CaMKIV(h) & 81 & 0 & 100 & 100 & 9 & 0.105882353 & 0 & 0 & 0 \\
\hline $\mathrm{CDK} 1 / \mathrm{cyclin} B(\mathrm{~h})$ & 5 & 1 & 100 & 100 & 10 & 0.117647059 & 0 & 0 & 0 \\
\hline $\operatorname{CDK} 2 /$ cyclinA(h) & 49 & 1 & 98 & 98 & 11 & 0.129411765 & 2 & 0.000507357 & 0.000507357 \\
\hline $\mathrm{CDK} 2 /$ cyclinE(h) & 80 & 0 & 97 & 97 & 12 & 0.141176471 & 3 & 0.000761035 & 0.001268392 \\
\hline CDK3/cyclinE(h) & 43 & 1 & 95 & 95 & 13 & 0.152941176 & 5 & 0.001268392 & 0.002536783 \\
\hline CDK5/p35(h) & 19 & 1 & 94 & 94 & 14 & 0.164705882 & 6 & 0.00152207 & 0.004058853 \\
\hline CDK6/cyclinD3(h) & 65 & 0 & 92 & 92 & 15 & 0.176470588 & 8 & 0.002029427 & 0.00608828 \\
\hline CDK7/cyclinHMATI(h) & 56 & 0 & 91 & 91 & 16 & 0.188235294 & 9 & 0.002283105 & 0.008371385 \\
\hline CHK1(h) & 77 & 0 & 89 & 89 & 17 & 0.2 & 11 & 0.002790462 & 0.011161847 \\
\hline CHK2(h) & 12 & 1 & 88 & 88 & 18 & 0.211764706 & 12 & 0.00304414 & 0.014205987 \\
\hline $\mathrm{CK} 1(\mathrm{y})$ & 20 & 1 & 88 & 88 & 19 & 0.223529412 & 12 & 0.00304414 & 0.017250127 \\
\hline CK2(h) & 50 & 1 & 86 & 86 & 20 & 0.235294118 & 14 & 0.003551497 & 0.020801624 \\
\hline$c-\operatorname{RAF}(\mathrm{h})$ & 62 & 0 & 86 & 86 & 21 & 0.247058824 & 14 & 0.003551497 & 0.02435312 \\
\hline $\operatorname{csk}(\mathrm{h})$ & 33 & 1 & 84 & 84 & 22 & 0.258823529 & 16 & 0.004058853 & 0.028411974 \\
\hline $\operatorname{cSRC(h)}$ & 17 & 1 & 84 & 84 & 23 & 0.270588235 & 16 & 0.004058853 & 0.032470827 \\
\hline Fes(h) & 50 & 1 & 81 & 81 & 24 & 0.282352941 & 19 & 0.004819888 & 0.037290715 \\
\hline FGFR3(h) & 76 & 0 & 80 & 80 & 25 & 0.294117647 & 20 & 0.005073567 & 0.042364282 \\
\hline Fll3(h) & 3 & 1 & 80 & 80 & 26 & 0.305882353 & 20 & 0.005073567 & 0.047437849 \\
\hline Fynn(h) & 48 & 1 & 77 & 77 & 27 & 0.317647059 & 23 & 0.005834602 & 0.053272451 \\
\hline GSK3a(h) & 24 & 1 & 76 & 76 & 28 & 0.329411765 & 24 & 0.00608828 & 0.059360731 \\
\hline GSK3B(h) & 19 & 1 & 75 & 75 & 29 & 0.341176471 & 25 & 0.006341958 & 0.065702689 \\
\hline IGF-1R(h) & 142 & 0 & 74 & 74 & 30 & 0.352941176 & 26 & 0.006595637 & 0.072298326 \\
\hline IKKu(h) & 56 & 0 & 71 & 71 & 31 & 0.364705882 & 29 & 0.007356672 & 0.079654997 \\
\hline IKKB(h) & 80 & 0 & 71 & 71 & 32 & 0.376470588 & 29 & 0.007356672 & 0.087011669 \\
\hline $\operatorname{IR(h)}$ & 75 & 0 & 68 & 68 & 33 & 0.388235294 & 32 & 0.008117707 & 0.095129376 \\
\hline JNKIal(h) & 92 & 0 & 67 & 67 & 34 & 0.4 & 33 & 0.008371385 & 0.103500761 \\
\hline JNK2a2(h) & 84 & 0 & 66 & 66 & 35 & 0.411764706 & 34 & 0.008625063 & 0.112125824 \\
\hline JNK3(r) & 67 & 0 & 66 & 66 & 36 & 0.423529412 & 34 & 0.008625063 & 0.120750888 \\
\hline Lck(h) & 22 & 1 & 65 & 65 & 37 & 0.435294118 & 35 & 0.008878742 & 0.12962963 \\
\hline Lyn(h) & -2 & 1 & 64 & 64 & 38 & 0.447058824 & 36 & 0.00913242 & 0.13876205 \\
\hline Lyn(m) & 2 & 1 & 62 & 62 & 39 & 0.458823529 & 38 & 0.009639777 & 0.148401826 \\
\hline MAPKI(h) & 71 & 0 & 60 & 60 & 40 & 0.470588235 & 40 & 0.010147133 & 0.15854896 \\
\hline MAPK2(h) & 112 & 0 & 58 & 58 & 41 & 0.482352941 & 42 & 0.01065449 & 0.16920345 \\
\hline MAPK2(m) & 101 & 0 & 58 & 58 & 42 & 0.494117647 & 42 & 0.01065449 & 0.17985794 \\
\hline MAPKAP-K2(h) & 68 & 0 & 58 & 58 & 43 & 0.505882353 & 42 & 0.01065449 & 0.19051243 \\
\hline MEK I(h) & 100 & 0 & 56 & 56 & 44 & 0.517647059 & 44 & 0.011161847 & 0.201674277 \\
\hline MKK4(m) & 116 & 0 & 56 & 56 & 45 & 0.529411765 & 44 & 0.011161847 & 0.212836124 \\
\hline MKK6(h) & 88 & 0 & 56 & 56 & 46 & 0.541176471 & 44 & 0.011161847 & 0.223997971 \\
\hline MKK $7 \mathrm{~B}(\mathrm{~h})$ & 74 & 0 & 51 & 51 & 47 & 0.552941176 & 49 & 0.012430238 & 0.236428209 \\
\hline MSKI(h) & 51 & 0 & 50 & 50 & 48 & 0.564705882 & 50 & 0.012683917 & 0.249112126 \\
\hline p7056K(h) & 9 & 1 & 50 & 50 & 49 & 0.576470588 & 50 & 0.012683917 & 0.261796043 \\
\hline PAK2(h) & 94 & 0 & 49 & 49 & 50 & 0.588235294 & 51 & 0.012937595 & 0.274733638 \\
\hline PDGFRa(h) & 48 & 1 & 49 & 49 & 51 & 0.6 & 51 & 0.012937595 & 0.287671233 \\
\hline PDGFRB(h) & 66 & 0 & 48 & 48 & 52 & 0.611764706 & 52 & 0.013191273 & 0.300862506 \\
\hline PDKI(h) & 43 & 1 & 48 & 48 & 53 & 0.623529412 & 52 & 0.013191273 & 0.31405378 \\
\hline PKA(b) & 60 & 0 & 45 & 45 & 54 & 0.635294118 & 55 & 0.013952308 & 0.328006088 \\
\hline PKA(h) & 58 & 0 & 45 & 45 & 55 & 0.647058824 & 55 & 0.013952308 & 0.341958397 \\
\hline PKBu(h) & 71 & 0 & 43 & 43 & 56 & 0.658823529 & 57 & 0.014459665 & 0.356418062 \\
\hline PKBB(h) & 58 & 0 & 43 & 43 & 57 & 0.670588235 & 57 & 0.014459665 & 0.370877727 \\
\hline $\mathrm{PKB}_{(}(\mathrm{h})$ & 58 & 0 & 33 & 33 & 58 & 0.682352941 & 67 & 0.016996449 & 0.387874176 \\
\hline PKCa(h) & 86 & 0 & 24 & 24 & 59 & 0.694117647 & 76 & 0.019279554 & 0.407153729 \\
\hline PKCBI(h) & 105 & 0 & 22 & 22 & 60 & 0.705882353 & 78 & 0.01978691 & 0.426940639 \\
\hline PKCy(h) & 91 & 0 & 20 & 20 & 61 & 0.717647059 & 80 & 0.020294267 & 0.447234906 \\
\hline PKC(h) & 98 & 0 & 19 & 19 & 62 & 0.729411765 & 81 & 0.020547945 & 0.467782851 \\
\hline PKC:(h) & 86 & 0 & 19 & 19 & 63 & 0.741176471 & 81 & 0.020547945 & 0.488330797 \\
\hline PKG (h) & 147 & 0 & 19 & 19 & 64 & 0.752941176 & 81 & 0.020547945 & 0.508878742 \\
\hline PKC (h) & 105 & 0 & 18 & 18 & 65 & 0.764705882 & 82 & 0.020801624 & 0.529680365 \\
\hline РКС山(h) & 64 & 0 & 17 & 17 & 66 & 0.776470588 & 83 & 0.021055302 & 0.550735667 \\
\hline 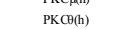 & 95 & 0 & 16 & 16 & 67 & 0.788235294 & 84 & 0.02130898 & 0.572044647 \\
\hline PKD2(h) & 66 & 0 & 13 & 13 & 68 & 0.8 & 87 & 0.022070015 & 0.594114663 \\
\hline PRAK(h) & 4 & 1 & 12 & 12 & 69 & 0.811764706 & 88 & 0.022323694 & 0.616438356 \\
\hline PRK2(h) & 49 & 1 & 11 & 11 & 70 & 0.823529412 & 89 & 0.022577372 & 0.639015728 \\
\hline ROCK-II(h) & 19 & 1 & 11 & 11 & 71 & 0.835294118 & 89 & 0.022577372 & 0.6615931 \\
\hline ROCK-II(r) & 16 & 1 & 11 & 11 & 72 & 0.847058824 & 89 & 0.022577372 & 0.684170472 \\
\hline Rskl(h) & 8 & 1 & 9 & 9 & 73 & 0.858823529 & 91 & 0.023084729 & 0.7072552 \\
\hline Rskl(r) & 1 & 1 & 8 & 8 & 74 & 0.870588235 & 92 & 0.023338407 & 0.730593607 \\
\hline Rsk2(h) & 5 & 1 & 8 & 8 & 75 & 0.882352941 & 92 & 0.023338407 & 0.753932014 \\
\hline Rsk3(h) & 6 & 1 & 6 & 6 & 76 & 0.894117647 & 94 & 0.023845764 & 0.777777778 \\
\hline SAPK2a(h) & 88 & 0 & 5 & 5 & 77 & 0.905882353 & 95 & 0.024099442 & 0.80187722 \\
\hline SAPK2b(h) & $\begin{array}{l}00 \\
89\end{array}$ & 0 & 5 & 5 & 78 & 0.917647059 & 95 & 0.024099442 & 0.825976662 \\
\hline SAPK3(h) & 100 & 0 & 4 & 4 & 79 & 0.929411765 & 96 & 0.02435312 & 0.850329782 \\
\hline SAPK $4(\mathrm{~h})$ & 101 & 0 & 4 & 4 & 80 & 0.941176471 & 96 & 0.02435312 & 0.874682902 \\
\hline SGK(h) & 45 & 1 & 3 & 3 & 81 & 0.952941176 & 97 & 0.024606799 & 0.899289701 \\
\hline Syk(h) & 56 & 0 & 2 & 2 & 82 & 0.964705882 & 98 & 0.024860477 & 0.924150178 \\
\hline TrkB(h) & 2 & 1 & 2 & 2 & 83 & 0.976470588 & 98 & 0.024860477 & 0.949010654 \\
\hline Yes(h) & 4 & 1 & 1 & 1 & 84 & 0.988235294 & 99 & 0.025114155 & 0.97412481 \\
\hline ZAP-70(h) & 97 & 0 & -2 & -2 & 85 & 1 & 102 & 0.02587519 & 1 \\
\hline & & & & & & Total inhibition & 3942 & & Total area \\
\hline & $\begin{array}{c}\text { Hit rate } \\
\text { Threshold }\end{array}$ & 5 & & & & & & & Gini \\
\hline
\end{tabular}




\begin{tabular}{|c|c|c|c|c|c|c|c|c|}
\hline & $100 \mu \mathrm{M}$ ATP & & & & & & & \\
\hline Kinase & Curcumin @ $50 \mu \mathrm{M}$ & Hit & sorted & normalized & position & cumulative sample fraction & inhibition\% & fraction of total inhibition \\
\hline $\mathrm{Abl}(\mathrm{m})$ & 37 & 1 & 143 & 100 & 1 & 0.011764706 & 0 & 0 \\
\hline AMPK(r) & 92 & 0 & 123 & 100 & 2 & 0.023529412 & 0 & 0 \\
\hline $\operatorname{Arg}(\mathrm{m})$ & 23 & 1 & 122 & 100 & 3 & 0.035294118 & 0 & 0 \\
\hline Aurora-A(h) & 75 & 0 & 113 & 100 & 4 & 0.047058824 & 0 & 0 \\
\hline Axl(h) & 33 & 1 & 112 & 100 & 5 & 0.058823529 & 0 & 0 \\
\hline $\operatorname{Blk}(\mathrm{m})$ & 5 & 1 & 111 & 100 & 6 & 0.070588235 & 0 & 0 \\
\hline Bmxx(h) & 22 & 1 & 109 & 100 & 7 & 0.082352941 & 0 & 0 \\
\hline СамKII(r) & 35 & 1 & 109 & 100 & 8 & 0.094117647 & 0 & 0 \\
\hline CaMKIV(h) & 91 & 0 & 108 & 100 & 9 & 0.105882353 & 0 & 0 \\
\hline CDK1/cyclnBB(h) & 21 & 1 & 108 & 100 & 10 & 0.117647059 & 0 & 0 \\
\hline $\mathrm{CDK} /$ /yclinA(h) & 66 & 0 & 106 & 100 & 11 & 0.129411765 & 0 & 0 \\
\hline $\mathrm{CDK} 2 /$ cyclinE(h) & 104 & 0 & 106 & 100 & 12 & 0.141176471 & 0 & 0 \\
\hline CDK3/cyclinE(h) & 75 & 0 & 104 & 100 & 13 & 0.152941176 & 0 & 0 \\
\hline CDK5/p35(h) & 65 & 0 & 104 & 100 & 14 & 0.164705882 & 0 & 0 \\
\hline CDK6/cyclinD3(h) & 73 & 0 & 102 & 100 & 15 & 0.176470588 & 0 & 0 \\
\hline CDK7/cyclinHMATI(h) & 81 & 0 & 102 & 100 & 16 & 0.188235294 & 0 & 0 \\
\hline СнК $1 \mathrm{~h})$ & 85 & 0 & 101 & 100 & 17 & 0.2 & 0 & 0 \\
\hline СHK2(h) & 9 & 1 & 101 & 100 & 18 & 0.211764706 & 0 & 0 \\
\hline $\mathrm{CK} 1(\mathrm{y})$ & 42 & 1 & 101 & 100 & 19 & 0.223529412 & 0 & 0 \\
\hline СК2(h) & 83 & 0 & 100 & 100 & 20 & 0.235294118 & 0 & 0 \\
\hline c-RAF(h) & 65 & 0 & 99 & 99 & 21 & 0.247058824 & 1 & 0.000361664 \\
\hline $\operatorname{CSK}(\mathrm{h})$ & 61 & 0 & 97 & 97 & 22 & 0.258823529 & 3 & 0.001084991 \\
\hline cSRC(h) & 38 & 1 & 96 & 96 & 23 & 0.270588235 & 4 & 0.001446655 \\
\hline Fes(h) & 76 & 0 & 94 & 94 & 24 & 0.282352941 & 6 & 0.002169982 \\
\hline FGFR3(h) & 84 & 0 & 94 & 94 & 25 & 0.294117647 & 6 & 0.002169982 \\
\hline Fill(h) & -2 & 1 & 93 & 93 & 26 & 0.305882353 & 7 & 0.002531646 \\
\hline Fyn(h) & 60 & 0 & 92 & 92 & 27 & 0.317647059 & 8 & 0.002893309 \\
\hline GSK3a(h) & 68 & 0 & 92 & 92 & 28 & 0.329411765 & 8 & 0.002893309 \\
\hline GSK3B(h) & 56 & 0 & 91 & 91 & 29 & 0.341176471 & 9 & 0.003254973 \\
\hline IGF-IR(h) & 92 & 0 & 89 & 89 & 30 & 0.352941176 & 11 & 0.0039783 \\
\hline $\mathrm{IKKa}(\mathrm{h})$ & 112 & 0 & 88 & 88 & 31 & 0.364705882 & 12 & 0.004339964 \\
\hline IKKB(h) & 83 & 0 & 85 & 85 & 32 & 0.376470588 & 15 & 0.005424955 \\
\hline $\mathbb{I R ( h )}$ & 89 & 0 & 84 & 84 & 33 & 0.388235294 & 16 & 0.005786618 \\
\hline JNKIal(h) & 100 & 0 & 84 & 84 & 34 & 0.4 & 16 & 0.005786618 \\
\hline JNK2202(h) & 101 & 0 & 83 & 83 & 35 & 0.411764706 & 17 & 0.006148282 \\
\hline JNK3(r) & 80 & 0 & 83 & 83 & 36 & 0.423529412 & 17 & 0.006148282 \\
\hline Lck(h) & 35 & 1 & 83 & 83 & 37 & 0.435294118 & 17 & 0.006148282 \\
\hline Lyn(h) & 4 & 1 & 83 & 83 & 38 & 0.447058824 & 17 & 0.006148282 \\
\hline $\operatorname{Lyn}(m)$ & 3 & 1 & 81 & 81 & 39 & 0.458823529 & 19 & 0.006871609 \\
\hline MAPK I (h) & 88 & 0 & 80 & 80 & 40 & 0.470588235 & 20 & 0.007233273 \\
\hline MAPK2(h) & 122 & 0 & 80 & 80 & 41 & 0.482352941 & 20 & 0.007233273 \\
\hline MAPK2(m) & 108 & 0 & 78 & 78 & 42 & 0.494117647 & 22 & 0.0079566 \\
\hline MAPKAP-K2(h) & 101 & 0 & 76 & 76 & 43 & 0.505882353 & 24 & 0.008679928 \\
\hline MEK1(h) & 96 & 0 & 75 & 75 & 44 & 0.517647059 & 25 & 0.009041591 \\
\hline MKK $4(\mathrm{~m})$ & 93 & 0 & 75 & 75 & 45 & 0.529411765 & 25 & 0.009041591 \\
\hline MKK6(h) & 97 & 0 & 75 & 75 & 46 & 0.541176471 & 25 & 0.009041591 \\
\hline MKK $7 \mathrm{B(h)}$ & 102 & 0 & 73 & 73 & 47 & 0.552941176 & 27 & 0.009764919 \\
\hline MSKI(h) & 66 & 0 & 73 & 73 & 48 & 0.564705882 & 27 & 0.009764919 \\
\hline p7056K(h) & 26 & 1 & 68 & 68 & 49 & 0.576470588 & 32 & 0.011573237 \\
\hline PAK2(h) & 94 & 0 & 67 & 67 & 50 & 0.588235294 & 33 & 0.011934901 \\
\hline PDGFRa(h) & 67 & 0 & 66 & 66 & 51 & 0.6 & 34 & 0.012296564 \\
\hline PDGFRB(h) & 61 & 0 & 66 & 66 & 52 & 0.611764706 & 34 & 0.012296564 \\
\hline PDKI(h) & 64 & 0 & 66 & 66 & 53 & 0.623529412 & 34 & 0.012296564 \\
\hline PKA(b) & 123 & 0 & 65 & 65 & 54 & 0.635294118 & 35 & 0.012658228 \\
\hline PKA(h) & 83 & 0 & 65 & 65 & 55 & 0.647058824 & 35 & 0.012658228 \\
\hline PKBa(h) & 80 & 0 & 64 & 64 & 56 & 0.658823529 & 36 & 0.013019892 \\
\hline PKB(h) & 60 & 0 & 61 & 61 & 57 & 0.670588235 & 39 & 0.014104882 \\
\hline $\mathrm{PKB}_{\gamma}(\mathrm{h})$ & 75 & 0 & 61 & 61 & 58 & 0.682352941 & 39 & 0.014104882 \\
\hline PKG(th) & 99 & 0 & 60 & 60 & 59 & 0.694117647 & 40 & 0.014466546 \\
\hline РКCBII(h) & 102 & 0 & 60 & 60 & 60 & 0.705882353 & 40 & 0.014466546 \\
\hline PKCy(h) & 104 & 0 & 56 & 56 & 61 & 0.717647059 & 44 & 0.015913201 \\
\hline PKБ(h) & 111 & 0 & 48 & 48 & 62 & 0.729411765 & 52 & 0.01880651 \\
\hline PKG:(h) & 94 & 0 & 47 & 47 & 63 & 0.741176471 & 53 & 0.019168174 \\
\hline PKG(h) & 143 & 0 & 42 & 42 & 64 & 0.752941176 & 58 & 0.020976492 \\
\hline PKC (h) & 108 & 0 & 38 & 38 & 65 & 0.764705882 & 62 & 0.022423146 \\
\hline PKC $\mu(\mathrm{h})$ & 78 & 0 & 37 & 37 & 66 & 0.776470588 & 63 & 0.02278481 \\
\hline PKCO(h) & 109 & 0 & 35 & 35 & 67 & 0.788235294 & 65 & 0.023508137 \\
\hline PKD2(h) & 84 & 0 & 35 & 35 & 68 & 0.8 & 65 & 0.023508137 \\
\hline PRAK(h) & 13 & 1 & 33 & 33 & 69 & 0.811764706 & 67 & 0.024231465 \\
\hline PRK2(h) & 83 & 0 & 26 & 26 & 70 & 0.823529412 & 74 & 0.02676311 \\
\hline ROCK-II(h) & 48 & 1 & 23 & 23 & 71 & 0.835294118 & 77 & 0.027848101 \\
\hline ROCK-II(r) & 47 & 1 & 23 & 23 & 72 & 0.847058824 & 77 & 0.027848101 \\
\hline Rskl(h) & 17 & 1 & 22 & 22 & 73 & 0.858823529 & 78 & 0.028209765 \\
\hline Rskl(r) & 23 & 1 & 21 & 21 & 74 & 0.870588235 & 79 & 0.028571429 \\
\hline Rsk2(h) & 19 & 1 & 20 & 20 & 75 & 0.882352941 & 80 & 0.028933092 \\
\hline Rsk3(h) & 20 & 1 & 19 & 19 & 76 & 0.894117647 & 81 & 0.029294756 \\
\hline SAPK2a(h) & 101 & 0 & 17 & 17 & 77 & 0.905882353 & 83 & 0.030018083 \\
\hline SAPK2b(h) & 113 & 0 & 13 & 13 & 78 & 0.917647059 & 87 & 0.031464738 \\
\hline $\mathrm{SAPK} 3(\mathrm{~h})$ & 106 & 0 & 9 & 9 & 79 & 0.929411765 & 91 & 0.032911392 \\
\hline SAPK4(h) & 109 & 0 & 9 & 9 & 80 & 0.941176471 & 91 & 0.032911392 \\
\hline SGK(h) & 66 & 0 & 7 & 7 & 81 & 0.952941176 & 93 & 0.03363472 \\
\hline Syk(h) & 73 & 0 & 5 & 5 & 82 & 0.964705882 & 95 & 0.034358047 \\
\hline TrkB(h) & 9 & 1 & 4 & 4 & 83 & 0.976470588 & 96 & 0.034719711 \\
\hline Yes(h) & 7 & 1 & 3 & 3 & 84 & 0.988235294 & 97 & 0.035081374 \\
\hline ZAP-70(h) & 106 & 0 & -2 & -2 & 85 & 1 & 102 & 0.036889693 \\
\hline & & & & & & Total inhibition & 2765 & \\
\hline & Hit rate & & & & & & & \\
\hline & Threshold & ( & & & & & & \\
\hline
\end{tabular}




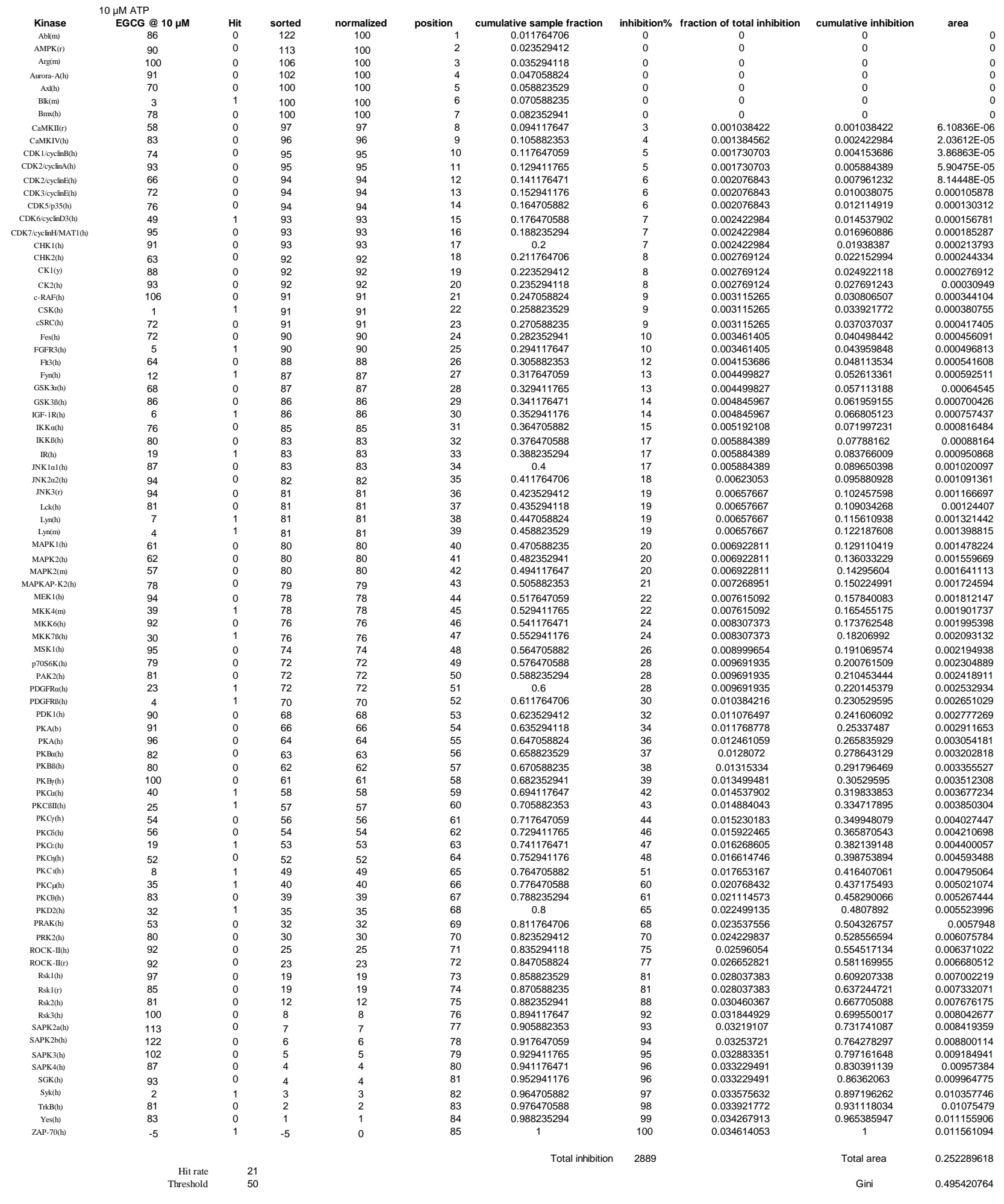




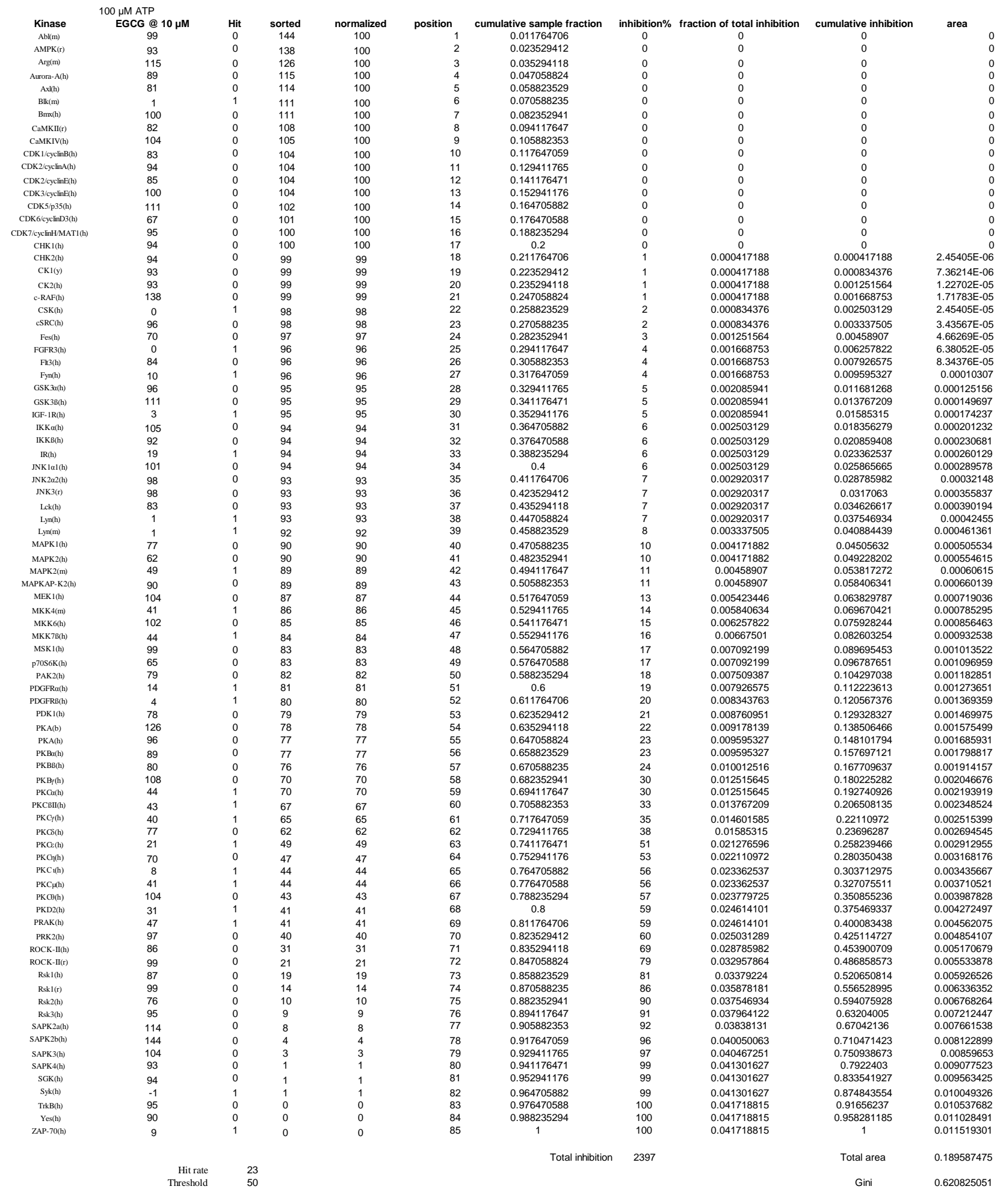




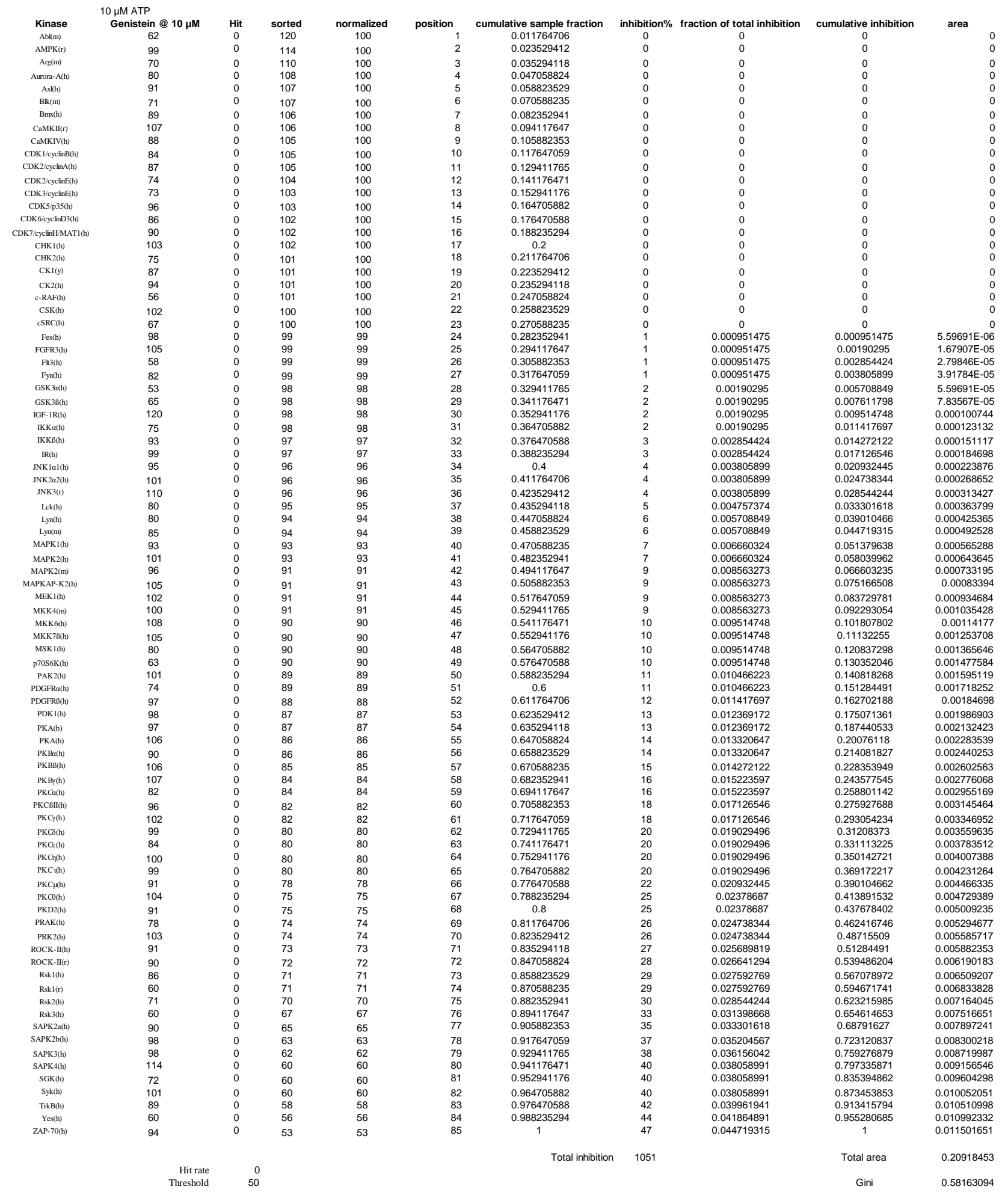




\begin{tabular}{|c|c|c|c|c|c|c|c|c|}
\hline & $100 \mu \mathrm{M}$ ATP & & & & & & & \\
\hline Kinase & Genistein @ $10 \mu \mathrm{M}$ & Hit & sorted & normalized & position & cumulative sample fraction & inhibition\% & fraction of total inhibition \\
\hline $\mathrm{Abl}(\mathrm{m})$ & 83 & 0 & 118 & 100 & 1 & 0.011764706 & 0 & 0 \\
\hline AMPK(r) & 102 & 0 & 117 & 100 & 2 & 0.023529412 & 0 & 0 \\
\hline $\operatorname{Arg}(\mathrm{m})$ & 91 & 0 & 115 & 100 & 3 & 0.035294118 & 0 & 0 \\
\hline Aurora-A(h) & 75 & 0 & 114 & 100 & 4 & 0.047058824 & 0 & 0 \\
\hline Axl(h) & 81 & 0 & 114 & 100 & 5 & 0.058823529 & 0 & 0 \\
\hline $\operatorname{Blk}(\mathrm{m})$ & 79 & 0 & 113 & 100 & 6 & 0.070588235 & 0 & 0 \\
\hline $\operatorname{Bmx}(\mathrm{h})$ & 94 & 0 & 112 & 100 & 7 & 0.082352941 & 0 & 0 \\
\hline СамKII(r) & 102 & 0 & 111 & 100 & 8 & 0.094117647 & 0 & 0 \\
\hline CaMKIV(h) & 112 & 0 & 109 & 100 & 9 & 0.105882353 & 0 & 0 \\
\hline CDK1/cyclnBB(h) & 104 & 0 & 108 & 100 & 10 & 0.117647059 & 0 & 0 \\
\hline $\mathrm{CDK} /$ /yclinA(h) & 96 & 0 & 107 & 100 & 11 & 0.129411765 & 0 & 0 \\
\hline $\mathrm{CDK} 2 /$ cyclnE(h) & 90 & 0 & 106 & 100 & 12 & 0.141176471 & 0 & 0 \\
\hline CDK3/cyclinE(h) & 85 & 0 & 106 & 100 & 13 & 0.152941176 & 0 & 0 \\
\hline CDK5/p35(h) & 96 & 0 & 106 & 100 & 14 & 0.164705882 & 0 & 0 \\
\hline CDK6/cyclinD3(h) & 95 & 0 & 105 & 100 & 15 & 0.176470588 & 0 & 0 \\
\hline CDK7/cyclinHMATI(h) & 93 & 0 & 105 & 100 & 16 & 0.188235294 & 0 & 0 \\
\hline СнК $1 \mathrm{~h})$ & 99 & 0 & 105 & 100 & 17 & 0.2 & 0 & 0 \\
\hline СHK2(h) & 98 & 0 & 104 & 100 & 18 & 0.211764706 & 0 & 0 \\
\hline $\mathrm{CK} 1(\mathrm{y})$ & 107 & 0 & 104 & 100 & 19 & 0.223529412 & 0 & 0 \\
\hline СК2(h) & 96 & 0 & 104 & 100 & 20 & 0.235294118 & 0 & 0 \\
\hline$c-R A F(h)$ & 79 & 0 & 104 & 100 & 21 & 0.247058824 & 0 & 0 \\
\hline $\operatorname{CSK}(\mathrm{h})$ & 114 & 0 & 103 & 100 & 22 & 0.258823529 & 0 & 0 \\
\hline cSRC(h) & 88 & 0 & 102 & 100 & 23 & 0.270588235 & 0 & 0 \\
\hline Fes(h) & 108 & 0 & 102 & 100 & 24 & 0.282352941 & 0 & 0 \\
\hline FGFR3(h) & 103 & 0 & 102 & 100 & 25 & 0.294117647 & 0 & 0 \\
\hline $\mathrm{FH}$ H(h) & 76 & 0 & 102 & 100 & 26 & 0.305882353 & 0 & 0 \\
\hline Fyn(h) & 97 & 0 & 101 & 100 & 27 & 0.317647059 & 0 & 0 \\
\hline GSK3a(h) & 90 & 0 & 101 & 100 & 28 & 0.329411765 & 0 & 0 \\
\hline GSK3B(h) & 84 & 0 & 100 & 100 & 29 & 0.341176471 & 0 & 0 \\
\hline IGF-IR(h) & 106 & 0 & 100 & 100 & 30 & 0.352941176 & 0 & 0 \\
\hline IKKa(h) & 97 & 0 & 100 & 100 & 31 & 0.364705882 & 0 & 0 \\
\hline IKKB(h) & 106 & 0 & 100 & 100 & 32 & 0.376470588 & 0 & 0 \\
\hline $\mathbb{I R ( h )}$ & 101 & 0 & 100 & 100 & 33 & 0.388235294 & 0 & 0 \\
\hline JNKIal(h) & 101 & 0 & 100 & 100 & 34 & 0.4 & 0 & 0 \\
\hline JNK2202(h) & 100 & 0 & 99 & 99 & 35 & 0.411764706 & 1 & 0.001901141 \\
\hline JNK3(r) & 91 & 0 & 99 & 99 & 36 & 0.423529412 & 1 & 0.001901141 \\
\hline Lck(h) & 100 & 0 & 98 & 98 & 37 & 0.435294118 & 2 & 0.003802281 \\
\hline Lyn(h) & 88 & 0 & 98 & 98 & 38 & 0.447058824 & 2 & 0.003802281 \\
\hline Lyy(m) & 79 & 0 & 98 & 98 & 39 & 0.458823529 & 2 & 0.003802281 \\
\hline MAPK I (h) & 102 & 0 & 98 & 98 & 40 & 0.470588235 & 2 & 0.003802281 \\
\hline MAPK2(h) & 97 & 0 & 97 & 97 & 41 & 0.482352941 & 3 & 0.005703422 \\
\hline MAPK2(m) & 95 & 0 & 97 & 97 & 42 & 0.494117647 & 3 & 0.005703422 \\
\hline MAPKAP-K2(h) & 100 & 0 & 97 & 97 & 43 & 0.505882353 & 3 & 0.005703422 \\
\hline MEK1(h) & 99 & 0 & 96 & 96 & 44 & 0.517647059 & 4 & 0.007604563 \\
\hline MKK $4(\mathrm{~m})$ & 92 & 0 & 96 & 96 & 45 & 0.529411765 & 4 & 0.007604563 \\
\hline MKK6(h) & 90 & 0 & 96 & 96 & 46 & 0.541176471 & 4 & 0.007604563 \\
\hline MKK7B(h) & 104 & 0 & 95 & 95 & 47 & 0.552941176 & 5 & 0.009505703 \\
\hline MSK1(h) & 88 & 0 & 95 & 95 & 48 & 0.564705882 & 5 & 0.009505703 \\
\hline p7056K(h) & 85 & 0 & 95 & 95 & 49 & 0.576470588 & 5 & 0.009505703 \\
\hline PAK2(h) & 118 & 0 & 95 & 95 & 50 & 0.588235294 & 5 & 0.009505703 \\
\hline PDGFRa(h) & 98 & 0 & 94 & 94 & 51 & 0.6 & 6 & 0.011406844 \\
\hline PDGFRB(h) & 90 & 0 & 94 & 94 & 52 & 0.611764706 & 6 & 0.011406844 \\
\hline PDK1(h) & 88 & 0 & 94 & 94 & 53 & 0.623529412 & 6 & 0.011406844 \\
\hline PKA(b) & 105 & 0 & 93 & 93 & 54 & 0.635294118 & 7 & 0.013307985 \\
\hline PKA(h) & 98 & 0 & 93 & 93 & 55 & 0.647058824 & 7 & 0.013307985 \\
\hline PKBa(h) & 95 & 0 & 92 & 92 & 56 & 0.658823529 & 8 & 0.015209125 \\
\hline PKB(h) & 111 & 0 & 91 & 91 & 57 & 0.670588235 & 9 & 0.017110266 \\
\hline $\mathrm{PKB}_{\gamma}(\mathrm{h})$ & 109 & 0 & 91 & 91 & 58 & 0.682352941 & 9 & 0.017110266 \\
\hline 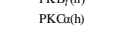 & 86 & 0 & 91 & 91 & 59 & 0.694117647 & 9 & 0.017110266 \\
\hline РКCBII(h) & 105 & 0 & 91 & 91 & 60 & 0.705882353 & 9 & 0.017110266 \\
\hline PKCy(h) & 85 & 0 & 90 & 90 & 61 & 0.717647059 & 10 & 0.019011407 \\
\hline PKБ(h) & 100 & 0 & 90 & 90 & 62 & 0.729411765 & 10 & 0.019011407 \\
\hline PKG:(h) & 95 & 0 & 90 & 90 & 63 & 0.741176471 & 10 & 0.019011407 \\
\hline PKCh(h) & 100 & 0 & 90 & 90 & 64 & 0.752941176 & 10 & 0.019011407 \\
\hline PKC (h) & 102 & 0 & 90 & 90 & 65 & 0.764705882 & 10 & 0.019011407 \\
\hline PKC $\mu(\mathrm{h})$ & 93 & 0 & 88 & 88 & 66 & 0.776470588 & 12 & 0.022813688 \\
\hline PKCO(h) & 114 & 0 & 88 & 88 & 67 & 0.788235294 & 12 & 0.022813688 \\
\hline PKD2(h) & 98 & 0 & 88 & 88 & 68 & 0.8 & 12 & 0.022813688 \\
\hline PRAK(h) & 90 & 0 & 88 & 88 & 69 & 0.811764706 & 12 & 0.022813688 \\
\hline PRK2(h) & 74 & 0 & 88 & 88 & 70 & 0.823529412 & 12 & 0.022813688 \\
\hline ROCK-II(h) & 85 & 0 & 86 & 86 & 71 & 0.835294118 & 14 & 0.02661597 \\
\hline ROCK-II(r) & 105 & 0 & 85 & 85 & 72 & 0.847058824 & 15 & 0.02851711 \\
\hline Rskl(h) & 94 & 0 & 85 & 85 & 73 & 0.858823529 & 15 & 0.02851711 \\
\hline Rskl(r) & 104 & 0 & 85 & 85 & 74 & 0.870588235 & 15 & 0.02851711 \\
\hline Rsk2(h) & 88 & 0 & 85 & 85 & 75 & 0.882352941 & 15 & 0.02851711 \\
\hline Rsk3(h) & 75 & 0 & 84 & 84 & 76 & 0.894117647 & 16 & 0.030418251 \\
\hline SAPK2a(h) & 100 & 0 & 83 & 83 & 77 & 0.905882353 & 17 & 0.032319392 \\
\hline SAPK2b(h) & 117 & 0 & 81 & 81 & 78 & 0.917647059 & 19 & 0.036121673 \\
\hline $\mathrm{SAPK} 3(\mathrm{~h})$ & 104 & 0 & 79 & 79 & 79 & 0.929411765 & 21 & 0.039923954 \\
\hline SAPK 4(h) & 91 & 0 & 79 & 79 & 80 & 0.941176471 & 21 & 0.039923954 \\
\hline SGK(h) & 91 & 0 & 79 & 79 & 81 & 0.952941176 & 21 & 0.039923954 \\
\hline Syk(h) & 115 & 0 & 76 & 76 & 82 & 0.964705882 & 24 & 0.045627376 \\
\hline TrkB(h) & 94 & 0 & 75 & 75 & 83 & 0.976470588 & 25 & 0.047528517 \\
\hline Yes(h) & 106 & 0 & 75 & 75 & 84 & 0.988235294 & 25 & 0.047528517 \\
\hline ZAP-70(h) & 113 & 0 & 74 & 74 & 85 & 1 & 26 & 0.049429658 \\
\hline & & & & & & Total inhibition & 526 & \\
\hline & Hit rate & & & & & & & \\
\hline & Threshold & & & & & & & \\
\hline
\end{tabular}




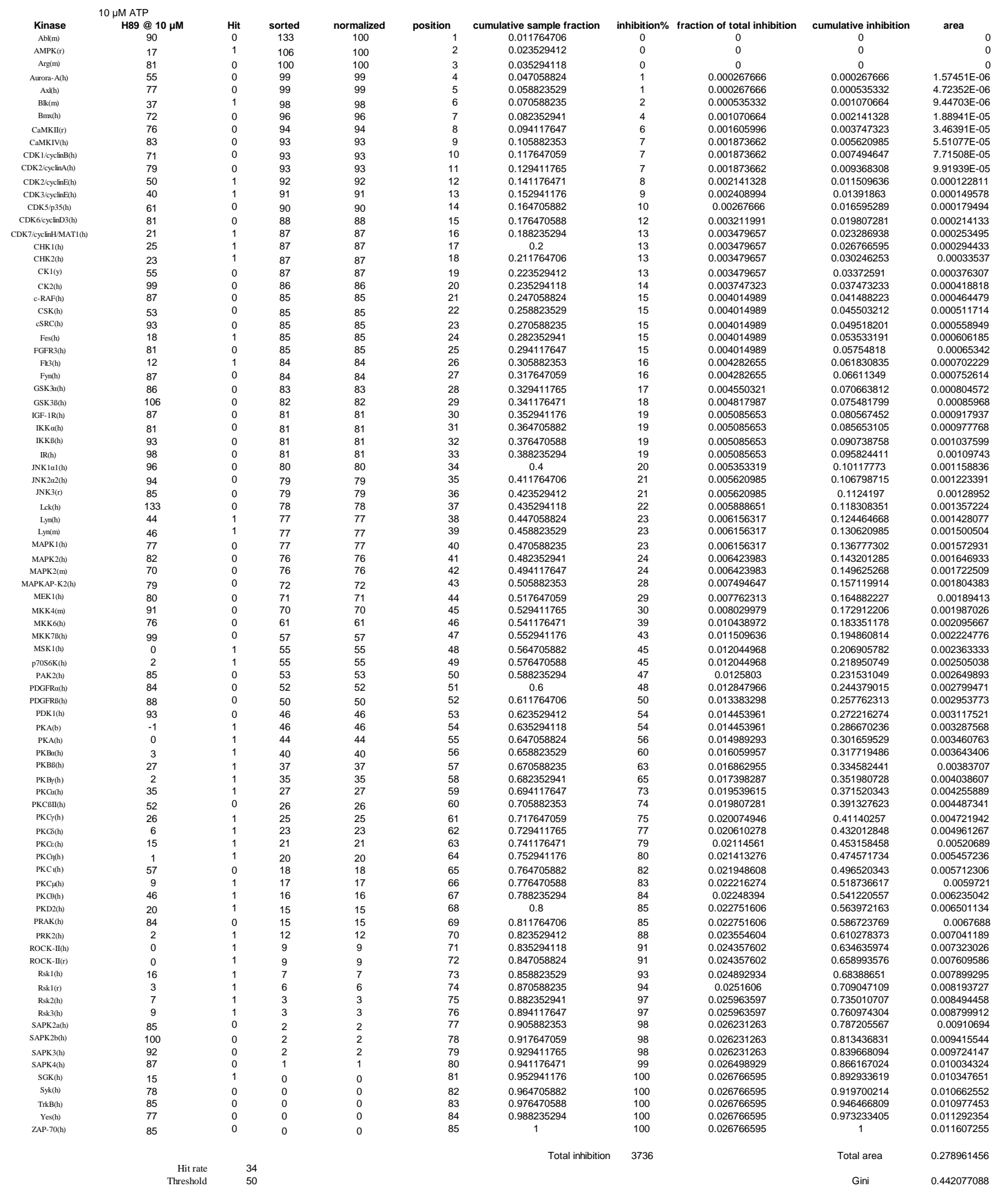


$100 \mu \mathrm{M}$ ATP

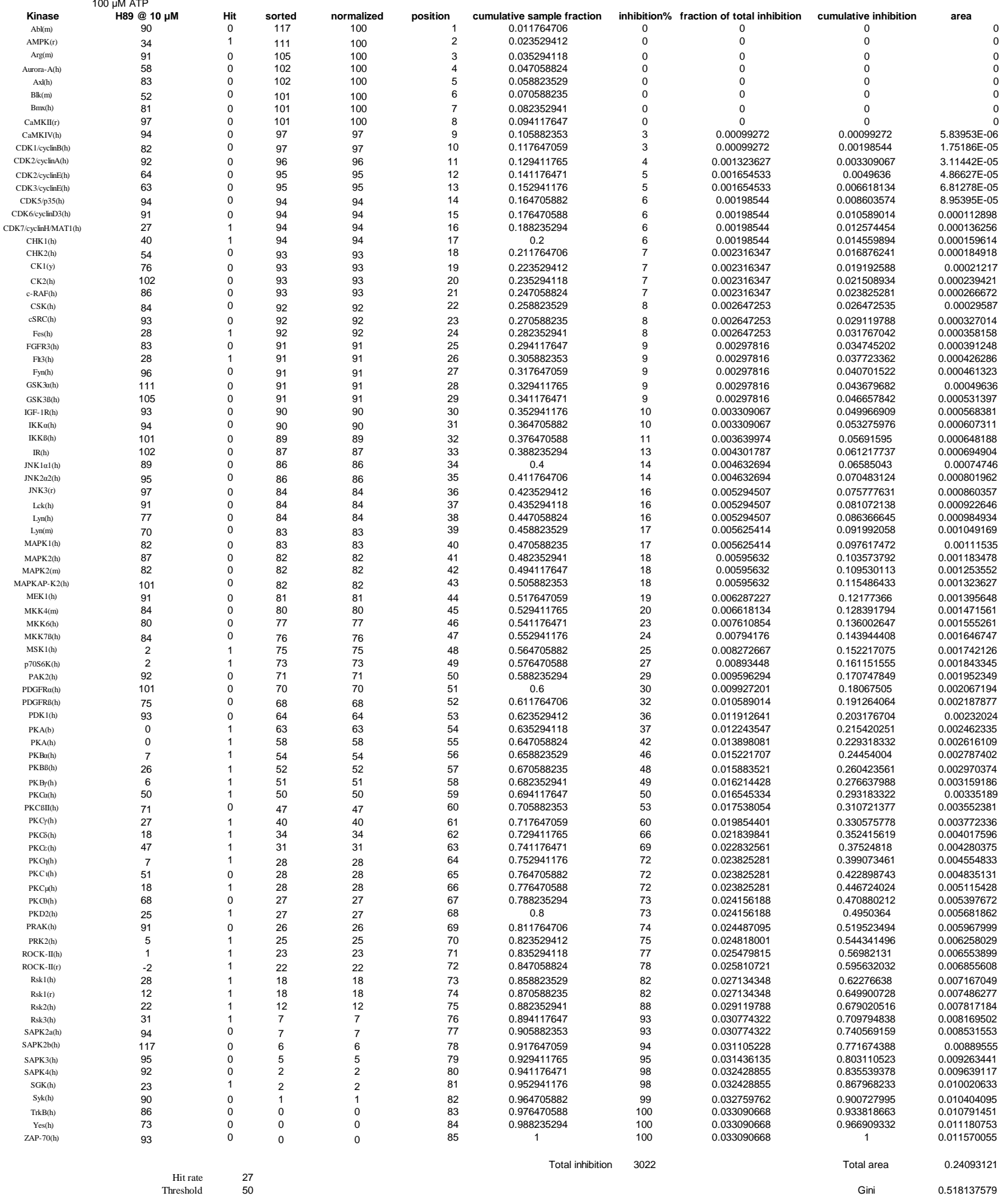




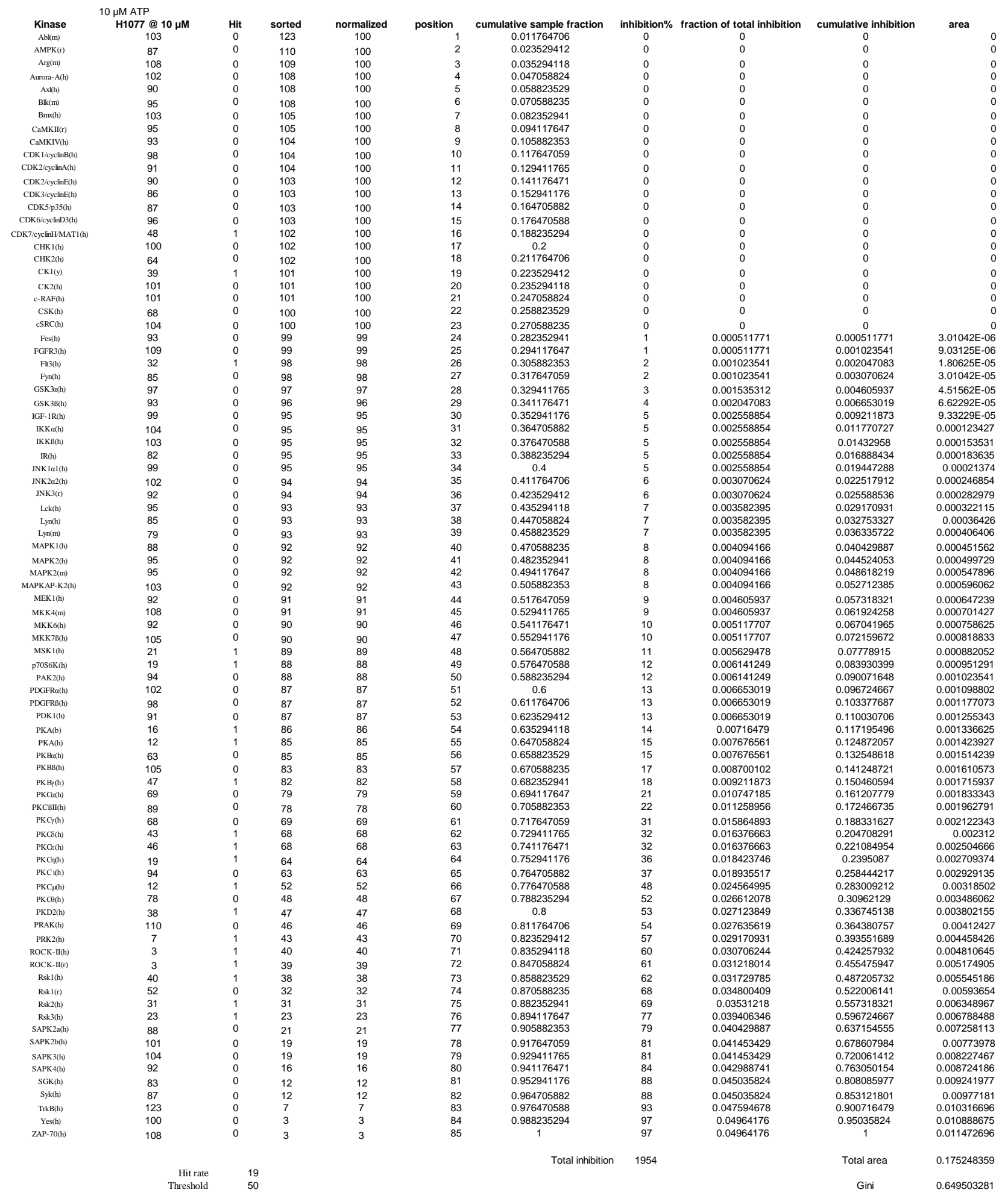




\begin{tabular}{|c|c|c|c|c|c|c|c|c|c|}
\hline & $100 \mu \mathrm{M}$ ATP & & & & & & & & \\
\hline Kinase & H1077@ $10 \mu \mathrm{M}$ & Hit & sorted & normalized & position & cumulative sample fraction & inhibition\% & fraction of total inhibition & cumulative inhibition \\
\hline $\mathrm{Abl}(\mathrm{m})$ & 99 & 0 & 128 & 100 & 1 & 0.011764706 & 0 & 0 & 0 \\
\hline AMPK(r) & 89 & 0 & 120 & 100 & 2 & 0.023529412 & 0 & 0 & 0 \\
\hline $\operatorname{Arg}(m)$ & 94 & 0 & 117 & 100 & 3 & 0.035294118 & 0 & 0 & 0 \\
\hline Aurora-A(h) & 104 & 0 & 115 & 100 & 4 & 0.047058824 & 0 & 0 & 0 \\
\hline $\mathrm{Ax}(\mathrm{h})$ & 94 & 0 & 113 & 100 & 5 & 0.058823529 & 0 & 0 & 0 \\
\hline $\operatorname{Blk}(\mathrm{m})$ & 93 & 0 & 112 & 100 & 6 & 0.070588235 & 0 & 0 & 0 \\
\hline Bmx(h) & 120 & 0 & 110 & 100 & 7 & 0.082352941 & 0 & 0 & 0 \\
\hline CaMKII(r) & 96 & 0 & 107 & 100 & 8 & 0.094117647 & 0 & 0 & 0 \\
\hline CaMKIV(h) & 113 & 0 & 107 & 100 & 9 & 0.105882353 & 0 & 0 & 0 \\
\hline CDK1/cyclinB(h) & 91 & 0 & 107 & 100 & 10 & 0.117647059 & 0 & 0 & 0 \\
\hline $\mathrm{CDK} 2 /$ cyclinA(h) & 102 & 0 & 106 & 100 & 11 & 0.129411765 & 0 & 0 & 0 \\
\hline $\mathrm{CDK} 2 / \mathrm{cyclin} E(\mathrm{~h})$ & 106 & 0 & 105 & 100 & 12 & 0.141176471 & 0 & 0 & 0 \\
\hline CDK3/ycycling(h) & 100 & 0 & 104 & 100 & 13 & 0.152941176 & 0 & 0 & 0 \\
\hline CDK5/335(h) & 93 & 0 & 104 & 100 & 14 & 0.164705882 & 0 & 0 & 0 \\
\hline CDK6/cyclinD3(h) & 95 & 0 & 103 & 100 & 15 & 0.176470588 & 0 & 0 & 0 \\
\hline CDK7/cyclinHAATI(h) & 57 & 0 & 103 & 100 & 16 & 0.188235294 & 0 & 0 & 0 \\
\hline CHKI(h) & 94 & 0 & 102 & 100 & 17 & 0.2 & 0 & 0 & 0 \\
\hline СHK2(h) & 74 & 0 & 102 & 100 & 18 & 0.211764706 & 0 & 0 & 0 \\
\hline $\mathrm{CKI}(\mathrm{y}) \mathrm{f}$ & 54 & 0 & 102 & 100 & 19 & 0.223529412 & 0 & 0 & 0 \\
\hline СK2(h) & 102 & 0 & 102 & 100 & 20 & 0.235294118 & 0 & 0 & 0 \\
\hline c-RAF(h) & 100 & 0 & 102 & 100 & 21 & 0.247058824 & 0 & 0 & 0 \\
\hline CSK (h) & 92 & 0 & 101 & 100 & 22 & 0.258823529 & 0 & 0 & 0 \\
\hline cSRC(h) & 96 & 0 & 101 & 100 & 23 & 0.270588235 & 0 & 0 & 0 \\
\hline Fes(h) & 112 & 0 & 101 & 100 & 24 & 0.282352941 & 0 & 0 & 0 \\
\hline FGFR3(h) & 80 & 0 & 100 & 100 & 25 & 0.294117647 & 0 & 0 & 0 \\
\hline Flls(h) & 73 & 0 & 100 & 100 & 26 & 0.305882353 & 0 & 0 & 0 \\
\hline Fynn(h) & 101 & 0 & 100 & 100 & 27 & 0.317647059 & 0 & 0 & 0 \\
\hline GSK3a(h) & 102 & 0 & 100 & 100 & 28 & 0.329411765 & 0 & 0 & 0 \\
\hline GSK3B(h) & 107 & 0 & 100 & 100 & 29 & 0.341176471 & 0 & 0 & 0 \\
\hline IGF-1R(h) & 99 & 0 & 99 & 99 & 30 & 0.352941176 & 1 & 0.000795545 & 0.000795545 \\
\hline $\mathrm{IKKa}(\mathrm{h})$ & 128 & 0 & 99 & 99 & 31 & 0.364705882 & 1 & 0.000795545 & 0.00159109 \\
\hline IKKB(h) & 101 & 0 & 99 & 99 & 32 & 0.376470588 & 1 & 0.000795545 & 0.002386635 \\
\hline $\mathrm{IR}(\mathrm{h})$ & 96 & 0 & 98 & 98 & 33 & 0.388235294 & 2 & 0.00159109 & 0.003977725 \\
\hline JNKIal(h) & 102 & 0 & 97 & 97 & 34 & 0.4 & 3 & 0.002386635 & 0.00636436 \\
\hline JNK2a2(h) & 96 & 0 & 96 & 96 & 35 & 0.411764706 & 4 & 0.00318218 & 0.009546539 \\
\hline $\mathrm{JNK} 3(\mathrm{r})$ & 79 & 0 & 96 & 96 & 36 & 0.423529412 & 4 & 0.00318218 & 0.012728719 \\
\hline Lck(h) & 95 & 0 & 96 & 96 & 37 & 0.435294118 & 4 & 0.00318218 & 0.015910899 \\
\hline Lyn(h) & 90 & 0 & 96 & 96 & 38 & 0.447058824 & 4 & 0.00318218 & 0.019093079 \\
\hline Lyn(m) & 90 & 0 & 96 & 96 & 39 & 0.458823529 & 4 & 0.00318218 & 0.022275259 \\
\hline MAPKI(h) & 88 & 0 & 96 & 96 & 40 & 0.470588235 & 4 & 0.00318218 & 0.025457438 \\
\hline MAPK2(h) & 96 & 0 & 95 & 95 & 41 & 0.482352941 & 5 & 0.003977725 & 0.029435163 \\
\hline MAPK2(m) & 100 & 0 & 95 & 95 & 42 & 0.494117647 & 5 & 0.003977725 & 0.033412888 \\
\hline MAPKAP-K2(h) & 110 & 0 & 95 & 95 & 43 & 0.505882353 & 5 & 0.003977725 & 0.037390613 \\
\hline MEK1(h) & 105 & 0 & 94 & 94 & 44 & 0.517647059 & 6 & 0.00477327 & 0.042163882 \\
\hline MKK $4(\mathrm{~m})$ & 98 & 0 & 94 & 94 & 45 & 0.529411765 & 6 & 0.00477327 & 0.046937152 \\
\hline MKK6(h) & 103 & 0 & 94 & 94 & 46 & 0.541176471 & 6 & 0.00477327 & 0.051710422 \\
\hline МKK $7($ (h) & 97 & 0 & 94 & 94 & 47 & 0.552941176 & 6 & 0.00477327 & 0.056483691 \\
\hline MSKI(h) & 32 & 1 & 93 & 93 & 48 & 0.564705882 & 7 & 0.005568815 & 0.062052506 \\
\hline $\mathrm{p} 70 \mathrm{~S} 6 \mathrm{~K}(\mathrm{~h})$ & 51 & 0 & 93 & 93 & 49 & 0.576470588 & 7 & 0.005568815 & 0.067621321 \\
\hline PAK2(h) & 101 & 0 & 92 & 92 & 50 & 0.588235294 & 8 & 0.00636436 & 0.07398568 \\
\hline PDGFRa(h) & 107 & 0 & 92 & 92 & 51 & 0.6 & 8 & 0.00636436 & 0.08035004 \\
\hline PDGFRB(h) & 83 & 0 & 91 & 91 & 52 & 0.611764706 & 9 & 0.007159905 & 0.087509944 \\
\hline PDK1(h) & 92 & 0 & 90 & 90 & 53 & 0.623529412 & 10 & 0.007955449 & 0.095465394 \\
\hline PKA(b) & 63 & 0 & 90 & 90 & 54 & 0.635294118 & 10 & 0.007955449 & 0.103420843 \\
\hline PKA(h) & 39 & 1 & 89 & 89 & 55 & 0.647058824 & 11 & 0.008750994 & 0.112171838 \\
\hline PKBach) & 81 & 0 & 88 & 88 & 56 & 0.658823529 & 12 & 0.009546539 & 0.121718377 \\
\hline PKBB(h) & 100 & 0 & 87 & 87 & 57 & 0.670588235 & 13 & 0.010342084 & 0.132060461 \\
\hline $\mathrm{PKB}_{\mathrm{y}(\mathrm{(h})}$ & 66 & 0 & 86 & 86 & 58 & 0.682352941 & 14 & 0.011137629 & 0.143198091 \\
\hline PKG(h) & 87 & 0 & 83 & 83 & 59 & 0.694117647 & 17 & 0.013524264 & 0.156722355 \\
\hline РКCBII(h) & 96 & 0 & 82 & 82 & 60 & 0.705882353 & 18 & 0.014319809 & 0.171042164 \\
\hline PKCy(h) & 82 & 0 & 81 & 81 & 61 & 0.717647059 & 19 & 0.015115354 & 0.186157518 \\
\hline РКడ(h) & 75 & 0 & 81 & 81 & 62 & 0.729411765 & 19 & 0.015115354 & 0.201272872 \\
\hline PKG:(h) & 77 & 0 & 80 & 80 & 63 & 0.741176471 & 20 & 0.015910899 & 0.217183771 \\
\hline PKG (h) & 55 & 0 & 79 & 79 & 64 & 0.752941176 & 21 & 0.016706444 & 0.233890215 \\
\hline PKC (h) & 99 & 0 & 77 & 77 & 65 & 0.764705882 & 23 & 0.018297534 & 0.252187749 \\
\hline PKC $\mu(\mathrm{h})$ & 56 & 0 & 75 & 75 & 66 & 0.776470588 & 25 & 0.019888624 & 0.272076372 \\
\hline PKCO(h) & 86 & 0 & 74 & 74 & 67 & 0.788235294 & 26 & 0.020684169 & 0.292760541 \\
\hline PKD2(h) & 57 & 0 & 73 & 73 & 68 & 0.8 & 27 & 0.021479714 & 0.314240255 \\
\hline PRAK(h) & 107 & 0 & 72 & 72 & 69 & 0.811764706 & 28 & 0.022275259 & 0.336515513 \\
\hline PRK2(h) & 23 & 1 & 68 & 68 & 70 & 0.823529412 & 32 & 0.025457438 & 0.361972951 \\
\hline ROCK-II(h) & 12 & 1 & 66 & 66 & 71 & 0.835294118 & 34 & 0.027048528 & 0.38902148 \\
\hline ROCK-II(r) & 17 & 1 & 63 & 63 & 72 & 0.847058824 & 37 & 0.029435163 & 0.418456643 \\
\hline Rskl(h) & 59 & 0 & 59 & 59 & 73 & 0.858823529 & 41 & 0.032617343 & 0.451073986 \\
\hline Rskl(r) & 57 & 0 & 57 & 57 & 74 & 0.870588235 & 43 & 0.034208433 & 0.485282418 \\
\hline Rsk2(h) & 68 & 0 & 57 & 57 & 75 & 0.882352941 & 43 & 0.034208433 & 0.519490851 \\
\hline Rsk3(h) & 72 & 0 & 57 & 57 & 76 & 0.894117647 & 43 & 0.034208433 & 0.553699284 \\
\hline SAPK2a(h) & 94 & 0 & 56 & 56 & 77 & 0.905882353 & 44 & 0.035003978 & 0.588703262 \\
\hline SAPK2b(h) & 117 & 0 & 55 & 55 & 78 & 0.917647059 & 45 & 0.035799523 & 0.624502784 \\
\hline $\mathrm{SAPK} 3(\mathrm{~h})$ & 102 & 0 & 54 & 54 & 79 & 0.929411765 & 46 & 0.036595068 & 0.661097852 \\
\hline SAPK4(h) & 103 & 0 & 51 & 51 & 80 & 0.941176471 & 49 & 0.038981702 & 0.700079554 \\
\hline SGK(h) & 95 & 0 & 39 & 39 & 81 & 0.952941176 & 61 & 0.048528242 & 0.748607796 \\
\hline Syk(h) & 115 & 0 & 32 & 32 & 82 & 0.964705882 & 68 & 0.054097056 & 0.802704853 \\
\hline $\operatorname{TrkB(h)}$ & 104 & 0 & 23 & 23 & 83 & 0.976470588 & 77 & 0.061256961 & 0.863961814 \\
\hline Yes(h) & 100 & 0 & 17 & 17 & 84 & 0.988235294 & 83 & 0.066030231 & 0.929992045 \\
\hline ZAP-70(h) & 81 & 0 & 12 & 12 & 85 & 1 & 88 & 0.070007955 & 1 \\
\hline & & & & & & Total inhibition & 1257 & & Total area \\
\hline & $\begin{array}{l}\text { Hit rate } \\
\text { Threshold }\end{array}$ & 5 & & & & & & & Gini \\
\hline
\end{tabular}




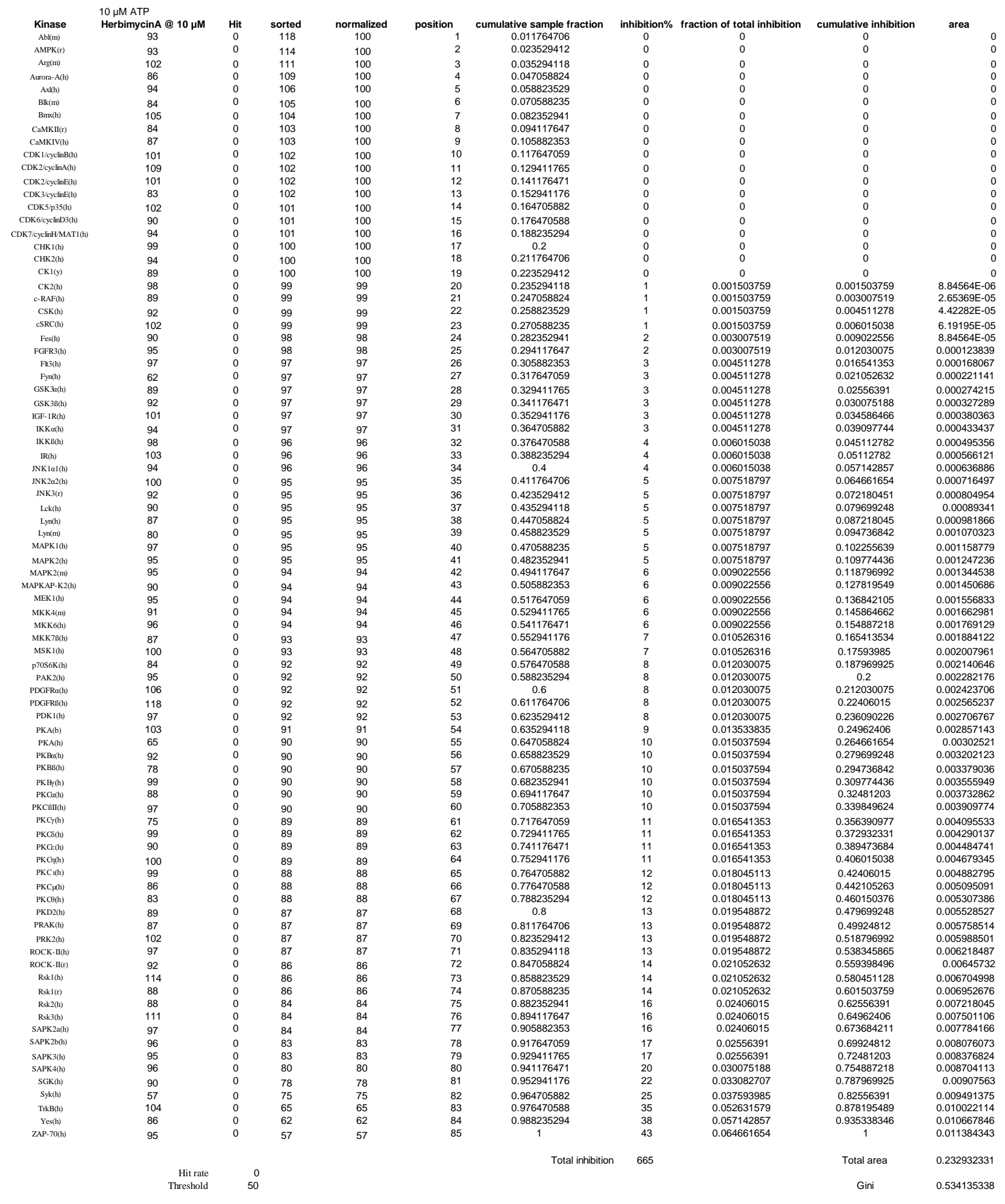




\begin{tabular}{|c|c|c|c|c|c|c|c|c|c|}
\hline & $100 \mu \mathrm{M}$ ATP & & & & & & & & \\
\hline Kinase & HerbimycinA@ @ $10 \mu \mathrm{M}$ & Hit & sorted & normalized & position & cumulative sample fraction & inhibition\% & fraction of total inhibition & cumulative inhibition \\
\hline $\mathrm{Abl}(\mathrm{m})$ & 101 & 0 & 129 & 100 & 1 & 0.011764706 & 0 & 0 & 0 \\
\hline AMPK(r) & 91 & 0 & 123 & 100 & 2 & 0.023529412 & 0 & 0 & 0 \\
\hline $\operatorname{Arg}(\mathrm{m})$ & 98 & 0 & 119 & 100 & 3 & 0.035294118 & 0 & 0 & 0 \\
\hline Aurora-A(h) & 99 & 0 & 115 & 100 & 4 & 0.047058824 & 0 & 0 & 0 \\
\hline Axl(h) & 88 & 0 & 114 & 100 & 5 & 0.058823529 & 0 & 0 & 0 \\
\hline $\operatorname{Blk}(\mathrm{m})$ & 86 & 0 & 113 & 100 & 6 & 0.070588235 & 0 & 0 & 0 \\
\hline $\operatorname{Bmx}(\mathrm{h})$ & 94 & 0 & 111 & 100 & 7 & 0.082352941 & 0 & 0 & 0 \\
\hline CaMKII(r) & 84 & 0 & 111 & 100 & 8 & 0.094117647 & 0 & 0 & 0 \\
\hline CaMKIV(h) & 81 & 0 & 109 & 100 & 9 & 0.105882353 & 0 & 0 & 0 \\
\hline CDK1/cyclnBB(h) & 113 & 0 & 108 & 100 & 10 & 0.117647059 & 0 & 0 & 0 \\
\hline CDK2/cyclinA(h) & 84 & 0 & 106 & 100 & 11 & 0.129411765 & 0 & 0 & 0 \\
\hline $\mathrm{CDK} 2 / \mathrm{cyclin} E(\mathrm{~h})$ & 104 & 0 & 106 & 100 & 12 & 0.141176471 & 0 & 0 & 0 \\
\hline CDK3/yclinE(h) & 115 & 0 & 106 & 100 & 13 & 0.152941176 & 0 & 0 & 0 \\
\hline CDK5/p35(h) & 111 & 0 & 105 & 100 & 14 & 0.164705882 & 0 & 0 & 0 \\
\hline CDK6/cyclinD3(h) & 83 & 0 & 105 & 100 & 15 & 0.176470588 & 0 & 0 & 0 \\
\hline CDK $7 /$ cyclinHMATI(h) & 96 & 0 & 105 & 100 & 16 & 0.188235294 & 0 & 0 & 0 \\
\hline CHK1 (h) & 99 & 0 & 104 & 100 & 17 & 0.2 & 0 & 0 & 0 \\
\hline CHK2(h) & 91 & 0 & 104 & 100 & 18 & 0.211764706 & 0 & 0 & 0 \\
\hline CKI(y) & 104 & 0 & 104 & 100 & 19 & 0.223529412 & 0 & 0 & 0 \\
\hline СК2(h) & 99 & 0 & 104 & 100 & 20 & 0.235294118 & 0 & 0 & 0 \\
\hline c-RAF(h) & 88 & 0 & 103 & 100 & 21 & 0.247058824 & 0 & 0 & 0 \\
\hline $\operatorname{CSK}(\mathrm{h})$ & 79 & 0 & 103 & 100 & 22 & 0.258823529 & 0 & 0 & 0 \\
\hline $\operatorname{csRC(h)}$ & 105 & 0 & 102 & 100 & 23 & 0.270588235 & 0 & 0 & 0 \\
\hline Fess(h) & 108 & 0 & 102 & 100 & 24 & 0.282352941 & 0 & 0 & 0 \\
\hline FGFR3(h) & 109 & 0 & 101 & 100 & 25 & 0.294117647 & 0 & 0 & 0 \\
\hline $\mathrm{Fll}(\mathrm{h}(\mathrm{h})$ & 98 & 0 & 101 & 100 & 26 & 0.305882353 & 0 & 0 & 0 \\
\hline Fynn(h) & 123 & 0 & 101 & 100 & 27 & 0.317647059 & 0 & 0 & 0 \\
\hline GSK3a(h) & 106 & 0 & 100 & 100 & 28 & 0.329411765 & 0 & 0 & 0 \\
\hline GSK $3 \mathrm{~B}(\mathrm{~h})$ & 84 & 0 & 100 & 100 & 29 & 0.341176471 & 0 & 0 & 0 \\
\hline IGF-IR(h) & 86 & 0 & 99 & 99 & 30 & 0.352941176 & 1 & 0.002024291 & 0.002024291 \\
\hline $\mathrm{IKKa(h)}$ & 97 & 0 & 99 & 99 & 31 & 0.364705882 & 1 & 0.002024291 & 0.004048583 \\
\hline IKKB(h) & 95 & 0 & 99 & 99 & 32 & 0.376470588 & 1 & 0.002024291 & 0.006072874 \\
\hline $\mathrm{IR}(\mathrm{h})$ & 102 & 0 & 99 & 99 & 33 & 0.388235294 & 1 & 0.002024291 & 0.008097166 \\
\hline JNKIal(h) & 97 & 0 & 99 & 99 & 34 & 0.4 & 1 & 0.002024291 & 0.010121457 \\
\hline JNK202(h) & 100 & 0 & 98 & 98 & $\begin{array}{l}54 \\
35\end{array}$ & 0.411764706 & 2 & 0.004048583 & 0.01417004 \\
\hline $\mathrm{JNK} 3(\mathrm{r})$ & 89 & 0 & 98 & 98 & 36 & 0.423529412 & 2 & 0.004048583 & 0.018218623 \\
\hline Lck(h) & 99 & 0 & 98 & 98 & 37 & 0.435294118 & 2 & 0.004048583 & 0.022267206 \\
\hline Lyn(h) & 94 & 0 & 98 & 98 & 38 & 0.447058824 & 2 & 0.004048583 & 0.026315789 \\
\hline Lyy(m) & 99 & 0 & 97 & 97 & 39 & 0.458823529 & 3 & 0.006072874 & 0.032388664 \\
\hline MAPKI(h) & 96 & 0 & 97 & 97 & 40 & 0.470588235 & 3 & 0.006072874 & 0.038461538 \\
\hline MAPK2(h) & 100 & 0 & 97 & 97 & 41 & 0.482352941 & 3 & 0.006072874 & 0.044534413 \\
\hline $\begin{array}{l}\text { MAPKL(2) } \\
\text { MAPK2(m) }\end{array}$ & 97 & 0 & 96 & 96 & $\begin{array}{l}41 \\
42\end{array}$ & 0.494117647 & 4 & 0.008097166 & 0.052631579 \\
\hline MAPKAP-K2(h) & 95 & 0 & 96 & 96 & 43 & 0.505882353 & 4 & 0.008097166 & 0.060728745 \\
\hline MEK1(h) & 90 & 0 & 96 & 96 & 44 & 0.517647059 & 4 & 0.008097166 & 0.068825911 \\
\hline MKK4(m) & 94 & 0 & 95 & 95 & 45 & 0.529411765 & $\begin{array}{l}4 \\
5\end{array}$ & 0.010121457 & 0.078947368 \\
\hline MKK6(h) & 94 & 0 & 95 & 95 & 46 & 0.541176471 & 5 & 0.010121457 & 0.089068826 \\
\hline MKK & 86 & 0 & 95 & 95 & 47 & 0.552941176 & 5 & 0.010121457 & 0.099190283 \\
\hline MSKI(h) & 104 & 0 & 95 & 95 & 48 & 0.564705882 & 5 & 0.010121457 & 0.109311741 \\
\hline $\mathrm{p} 7056 \mathrm{~K}(\mathrm{~h})$ & $\begin{array}{l}104 \\
105\end{array}$ & 0 & 94 & 94 & $\begin{array}{l}48 \\
49\end{array}$ & 0.576470588 & $\begin{array}{l}5 \\
6\end{array}$ & 0.012145749 & 0.12145749 \\
\hline $\begin{array}{l}\text { PAK2(h) } \\
\text { Past }\end{array}$ & 93 & 0 & 94 & 94 & 50 & 0.588235294 & 6 & 0.012145749 & 0.133603239 \\
\hline PDGFRa(h) & 85 & 0 & 94 & 94 & 51 & 0.6 & 6 & 0.012145749 & 0.145748988 \\
\hline PDGFRB(h) & 85 & 0 & 94 & 94 & 52 & 0.611764706 & 6 & 0.012145749 & 0.157894737 \\
\hline PDKI(h) & $\begin{array}{l}80 \\
91\end{array}$ & 0 & $\begin{array}{l}94 \\
94\end{array}$ & $\begin{array}{l}94 \\
94\end{array}$ & 53 & 0.623529412 & 6 & 0.012145749 & 0.170040486 \\
\hline PKA(b) & 94 & 0 & 94 & 94 & 54 & 0.635294118 & 6 & 0.012145749 & 0.182186235 \\
\hline PKA(h) & 111 & 0 & 94 & 94 & 55 & 0.647058824 & 6 & 0.012145749 & 0.194331984 \\
\hline PKBa(h) & 106 & 0 & 93 & 93 & 56 & 0.658823529 & 7 & 0.01417004 & 0.208502024 \\
\hline PKBB(h) & 98 & 0 & 93 & 93 & 57 & 0.670588235 & 7 & 0.01417004 & 0.222672065 \\
\hline $\mathrm{PKB} \mathrm{B}_{(\mathrm{h})}$ & 104 & 0 & 93 & 93 & 58 & 0.682352941 & 7 & 0.01417004 & 0.236842105 \\
\hline PKCath) & 102 & 0 & 92 & 92 & 59 & 0.694117647 & 8 & 0.016194332 & 0.253036437 \\
\hline PKCBII(h) & 103 & 0 & 92 & 92 & 60 & 0.705882353 & 8 & 0.016194332 & 0.269230769 \\
\hline PKCy(h) & 76 & 0 & 91 & 91 & 61 & 0.717647059 & 9 & 0.018218623 & 0.287449393 \\
\hline РКФ(h) & 101 & 0 & 91 & 91 & 62 & 0.729411765 & 9 & 0.018218623 & 0.305668016 \\
\hline PKG:(h) & 91 & 0 & 91 & 91 & 63 & 0.741176471 & 9 & 0.018218623 & 0.32388664 \\
\hline $\begin{array}{l}\text { PKG (h) } \\
\text { (h) }\end{array}$ & 105 & 0 & 91 & 91 & 64 & 0.752941176 & 9 & 0.018218623 & 0.342105263 \\
\hline PKC (h) & 93 & 0 & 90 & 90 & 65 & 0.764705882 & 10 & 0.020242915 & 0.362348178 \\
\hline PKC $\mu(\mathrm{h})$ & 106 & 0 & 89 & 89 & 66 & 0.776470588 & 11 & 0.022267206 & 0.384615385 \\
\hline PKCO(h) & 93 & 0 & 88 & 88 & 67 & 0.788235294 & 12 & 0.024291498 & 0.408906883 \\
\hline PKD2(h) & 94 & 0 & 88 & 88 & 68 & 0.8 & 12 & 0.024291498 & 0.433198381 \\
\hline PRAK(h) & $\begin{array}{l}54 \\
79\end{array}$ & 0 & 88 & 88 & 69 & 0.811764706 & 12 & 0.024291498 & 0.457489879 \\
\hline PRK2(h) & $\begin{array}{l}159 \\
129\end{array}$ & 0 & 87 & 87 & 70 & 0.823529412 & 13 & 0.026315789 & 0.483805668 \\
\hline $\begin{array}{l}\text { PRK_(n) } \\
\text { ROCK-II(h) }\end{array}$ & 103 & 0 & 86 & $\begin{array}{l}01 \\
86\end{array}$ & 71 & 0.835294118 & 14 & 0.028340081 & 0.512145749 \\
\hline ROCK-II(r) & 83 & 0 & 86 & 86 & 72 & 0.847058824 & 14 & 0.028340081 & 0.54048583 \\
\hline Rskl(h) & 101 & 0 & 86 & 86 & 73 & 0.858823529 & 14 & 0.028340081 & 0.568825911 \\
\hline Rskl(r) & 98 & 0 & 85 & 85 & 74 & 0.870588235 & 15 & 0.030364372 & 0.599190283 \\
\hline $\begin{array}{l}\text { Rsksk2(h) } \\
\text { Rsk(h) }\end{array}$ & 87 & 0 & 85 & 85 & 75 & 0.882352941 & 15 & 0.030364372 & 0.629554656 \\
\hline Rsk3(h) & 95 & 0 & 84 & 84 & 76 & 0.894117647 & 16 & 0.032388664 & 0.66194332 \\
\hline SAPK2a(h) & 95 & 0 & 84 & 84 & 77 & 0.905882353 & 16 & 0.032388664 & 0.694331984 \\
\hline SAPK2b(h) & 119 & 0 & 84 & 84 & 78 & 0.917647059 & 16 & 0.032388664 & 0.726720648 \\
\hline SAPK3(h) & 92 & 0 & 84 & 84 & 79 & 0.929411765 & 16 & 0.032388664 & 0.759109312 \\
\hline SAPK4(h) & 92 & 0 & 83 & 83 & 80 & 0.941176471 & 17 & 0.034412955 & 0.793522267 \\
\hline SGK(h) & 96 & 0 & 83 & 83 & 81 & 0.952941176 & 17 & 0.034412955 & 0.827935223 \\
\hline Syk(h) & 88 & 0 & 81 & 81 & 82 & 0.964705882 & 19 & 0.038461538 & 0.866396761 \\
\hline TrkB(h) & 94 & 0 & 79 & 79 & 83 & 0.976470588 & 21 & 0.042510121 & 0.908906883 \\
\hline $\begin{array}{l}\text { IIrkB(h) } \\
\text { Yesh(h) }\end{array}$ & $\begin{array}{l}94 \\
84\end{array}$ & 0 & 79 & 79 & 84 & 0.988235294 & 21 & 0.042510121 & 0.951417004 \\
\hline ZAP-70(h) & 114 & 0 & 76 & 76 & 85 & 1 & 24 & 0.048582996 & 1 \\
\hline & & & & & & Total inhibition & 494 & & Total area \\
\hline & $\begin{array}{r}\text { Hit rate } \\
\text {. }\end{array}$ & 5 & & & & & & & Gini \\
\hline
\end{tabular}




\begin{tabular}{|c|c|c|c|c|c|c|c|c|c|}
\hline & $10 \mu \mathrm{M}$ АTP & & & & & & & & \\
\hline Kinase & Hispidin @ $10 \mu \mathrm{M}$ & Hit & $\begin{array}{c}\text { sorted } \\
182\end{array}$ & normalized & position & cumulative sample fraction & inhibition\% & fraction of total inhibition & cumulative inhibition \\
\hline $\begin{array}{l}\text { Abl(m) } \\
\text { AMPK(r) }\end{array}$ & 93 & $\begin{array}{l}0 \\
0\end{array}$ & 182 & 100 & $\begin{array}{l}1 \\
2\end{array}$ & $\begin{array}{l}0.011764706 \\
0.023529412\end{array}$ & 0 & 0 & 0 \\
\hline $\operatorname{Arg}(\mathrm{m})$ & 107 & 0 & 145 & 100 & 3 & 0.035294118 & 0 & 0 & 0 \\
\hline Aurora-A(h) & 109 & 0 & 130 & 100 & 4 & 0.047058824 & 0 & 0 & 0 \\
\hline $\operatorname{Ax}(\mathrm{h})$ & 105 & 0 & 127 & 100 & 5 & 0.058823529 & 0 & 0 & 0 \\
\hline $\operatorname{Blk}(\mathrm{m})$ & 68 & 0 & 119 & 100 & 6 & 0.070588235 & 0 & 0 & 0 \\
\hline Bmx(h) & 127 & 0 & 115 & 100 & 7 & 0.082352941 & 0 & 0 & 0 \\
\hline CaMKII(r) & 103 & 0 & 114 & 100 & 8 & 0.094117647 & 0 & 0 & 0 \\
\hline CaMKIV(h) & 112 & 0 & 114 & 100 & 9 & 0.105882353 & 0 & 0 & 0 \\
\hline $\mathrm{CDK} 1 /$ cyclinB(h) & 90 & 0 & 113 & 100 & 10 & 0.117647059 & 0 & 0 & 0 \\
\hline $\mathrm{CDK} 2 /$ cyclinA(h) & 97 & 0 & 113 & 100 & 11 & 0.129411765 & 0 & 0 & 0 \\
\hline $\mathrm{CDK} 2 / \mathrm{cyclinE}$ (h) & 90 & 0 & 112 & 100 & 12 & 0.141176471 & 0 & 0 & 0 \\
\hline CDK3/cycling(h) & 104 & 0 & 112 & 100 & 13 & 0.152941176 & 0 & 0 & 0 \\
\hline CDK5/p35(h) & 113 & 0 & 112 & 100 & 14 & 0.164705882 & 0 & 0 & 0 \\
\hline CDK6/cyclinD33(h) & 103 & 0 & 111 & 100 & 15 & 0.176470588 & 0 & 0 & 0 \\
\hline CDK7/cyclinH/MATI(h) & 104 & 0 & 111 & 100 & 16 & 0.188235294 & 0 & 0 & 0 \\
\hline CHK1(h) & 105 & 0 & 110 & 100 & 17 & 0.2 & 0 & 0 & 0 \\
\hline CHK2(h) & 86 & 0 & 109 & 100 & 18 & 0.211764706 & 0 & 0 & 0 \\
\hline CKI(y) & 100 & 0 & 109 & 100 & 19 & 0.223529412 & 0 & 0 & 0 \\
\hline СK2(h) & 41 & 1 & 108 & 100 & 20 & 0.235294118 & 0 & 0 & 0 \\
\hline$c-\operatorname{RAF}(\mathrm{h})$ & 111 & 0 & 107 & 100 & 21 & 0.247058824 & 0 & 0 & 0 \\
\hline CSK (h) & 77 & 0 & 107 & 100 & 22 & 0.258823529 & 0 & 0 & 0 \\
\hline $\operatorname{csRC(h)}$ & 102 & 0 & 105 & 100 & 23 & 0.270588235 & 0 & 0 & 0 \\
\hline Fes(h) & 96 & 0 & 105 & 100 & 24 & 0.282352941 & 0 & 0 & 0 \\
\hline $\begin{array}{l}\text { Fes(h) } \\
\text { FGFR3(h) }\end{array}$ & $\begin{array}{l}90 \\
107\end{array}$ & 0 & 105 & 100 & $\begin{array}{l}24 \\
25\end{array}$ & 0.294117647 & 0 & 0 & 0 \\
\hline Fllts(h) & 101 & 0 & 105 & 100 & 26 & 0.305882353 & 0 & 0 & 0 \\
\hline Fyn(h) & 84 & 0 & 105 & 100 & 27 & 0.317647059 & 0 & 0 & 0 \\
\hline GSK3a(h) & 90 & 0 & 105 & 100 & 28 & 0.329411765 & 0 & 0 & 0 \\
\hline GSK3B(h) & 96 & 0 & 105 & 100 & $\begin{array}{l}28 \\
29\end{array}$ & 0.341176471 & 0 & 0 & 0 \\
\hline IGF-1R(h) & 163 & 0 & 104 & 100 & 30 & 0.352941176 & 0 & 0 & 0 \\
\hline IKKa(h) & 100 & 0 & 104 & 100 & 31 & 0.364705882 & 0 & 0 & 0 \\
\hline IKKB(h) & 101 & 0 & 104 & 100 & 32 & 0.376470588 & 0 & 0 & 0 \\
\hline $\operatorname{IR(h)}$ & 102 & 0 & 103 & 100 & 33 & 0.388235294 & 0 & 0 & 0 \\
\hline JNKIal(h) & 101 & 0 & 103 & 100 & 34 & 0.4 & 0 & 0 & 0 \\
\hline JNK202(h) & 105 & 0 & 103 & 100 & 35 & 0.411764706 & 0 & 0 & 0 \\
\hline JNK3(r) & $\begin{array}{l}105 \\
119\end{array}$ & 0 & 103 & 100 & 36 & 0.423529412 & 0 & 0 & 0 \\
\hline Lck(h) & 91 & 0 & 103 & 100 & 37 & 0.435294118 & 0 & 0 & 0 \\
\hline Lyn(h) & 96 & 0 & 103 & 100 & 38 & 0.447058824 & 0 & 0 & 0 \\
\hline Lyyn(m) & 78 & 0 & 102 & 100 & 39 & 0.458823529 & 0 & 0 & 0 \\
\hline MAPKI(h) & 111 & 0 & 102 & 100 & 40 & 0.470588235 & 0 & 0 & 0 \\
\hline MAPK2(h) & 110 & 0 & 102 & 100 & 41 & 0.482352941 & 0 & 0 & 0 \\
\hline $\operatorname{MAPK2(m)}$ & 92 & 0 & 101 & 100 & 42 & 0.494117647 & 0 & 0 & 0 \\
\hline MAPKAP-K2(h) & 98 & 0 & 101 & 100 & 43 & 0.505882353 & 0 & 0 & 0 \\
\hline MEKI(h) & 104 & 0 & 101 & 100 & 44 & 0.517647059 & 0 & 0 & 0 \\
\hline MKK $4(\mathrm{~m})$ & $\begin{array}{l}104 \\
103\end{array}$ & 0 & 101 & 100 & $\begin{array}{l}44 \\
45\end{array}$ & 0.529411765 & 0 & 0 & 0 \\
\hline MKK $6(\mathrm{hl})$ & 100 & 0 & 100 & 100 & 46 & 0.541176471 & 0 & 0 & 0 \\
\hline MKK $7($ (h) & 101 & 0 & 100 & 100 & 47 & 0.552941176 & 0 & 0 & 0 \\
\hline MSKI(h) & 102 & 0 & 100 & 100 & 48 & 0.564705882 & 0 & 0 & 0 \\
\hline $\mathrm{p} 70 \mathrm{~S} 6 \mathrm{~K}(\mathrm{~h})$ & 97 & 0 & 100 & 100 & 49 & 0.576470588 & 0 & 0 & 0 \\
\hline PAK2(h) & 109 & 0 & 99 & 99 & 50 & 0.588235294 & 1 & 0.002207506 & 0.002207506 \\
\hline $\begin{array}{l}\text { PAKL(a) } \\
\text { PDGFRa(h) }\end{array}$ & 96 & 0 & 98 & 98 & 51 & $\begin{array}{l}0.600 \\
0.6434\end{array}$ & 2 & 0.004415011 & 0.006622517 \\
\hline $\begin{array}{l}\text { PDGFRB(h) } \\
\text { (n) }\end{array}$ & 114 & 0 & 98 & 98 & 52 & 0.611764706 & 2 & 0.004415011 & 0.011037528 \\
\hline PDK 1(h) & 130 & 0 & 98 & 98 & 53 & 0.623529412 & 2 & 0.004415011 & 0.015452539 \\
\hline PKA(b) & 105 & 0 & 97 & 97 & 54 & 0.635294118 & 3 & 0.006622517 & 0.022075055 \\
\hline PKA(h) & 114 & 0 & 97 & 97 & 55 & 0.647058824 & 3 & 0.006622517 & 0.028697572 \\
\hline $\begin{array}{l}\text { PKBa(h) } \\
\text { PKB(t) }\end{array}$ & 98 & 0 & 97 & 97 & 56 & 0.658823529 & 3 & 0.006622517 & 0.035320088 \\
\hline PKBB(h) & 103 & 0 & 96 & 96 & 57 & 0.670588235 & 4 & 0.008830022 & 0.04415011 \\
\hline $\mathrm{PKB}_{(\gamma / 1)}$ & 113 & 0 & $\begin{array}{l}96 \\
96\end{array}$ & $\begin{array}{l}96 \\
96\end{array}$ & 58 & 0.682352941 & $\begin{array}{l}4 \\
4\end{array}$ & 0.008830022 & 0.052980132 \\
\hline PKCu(h) & 112 & 0 & 96 & 96 & 59 & 0.694117647 & 4 & 0.008830022 & 0.061810155 \\
\hline РКСВII(h) & 105 & 0 & 96 & 96 & 60 & 0.705882353 & 4 & 0.008830022 & 0.070640177 \\
\hline PKCy(h) & 182 & 0 & 96 & 96 & 61 & 0.717647059 & 4 & 0.008830022 & 0.079470199 \\
\hline PKG(h) & 145 & 0 & 96 & 96 & 62 & 0.729411765 & 4 & 0.008830022 & 0.088300221 \\
\hline PKE:(h) & 76 & 0 & 95 & 95 & 63 & 0.741176471 & 5 & 0.011037528 & 0.099337748 \\
\hline $\begin{array}{l}\text { PKG (h) } \\
\text { P(h) }\end{array}$ & 103 & 0 & 95 & 95 & 64 & 0.752941176 & 5 & 0.011037528 & 0.110375276 \\
\hline PKC (h) & 39 & 1 & 95 & 95 & 65 & 0.764705882 & 5 & 0.011037528 & 0.121412804 \\
\hline PKC $(\mathrm{h}(\mathrm{h})$ & 83 & 0 & 93 & 93 & 66 & 0.776470588 & 7 & 0.015452539 & 0.136865342 \\
\hline PKCO(h) & 95 & 0 & 92 & 92 & 67 & 0.788235294 & 8 & 0.017660044 & 0.154525386 \\
\hline PKD2(h) & 105 & 0 & 91 & 91 & 68 & 0.8 & 9 & 0.01986755 & 0.174392936 \\
\hline PRAK(h) & 95 & 0 & 91 & 91 & 69 & 0.811764706 & 9 & 0.01986755 & 0.194260486 \\
\hline PRK2(h) & 100 & 0 & 90 & 90 & 70 & 0.823529412 & 10 & 0.022075055 & 0.216335541 \\
\hline ROCK-II(h) & 96 & 0 & 90 & 90 & 71 & 0.835294118 & 10 & 0.022075055 & 0.238410596 \\
\hline ROCK-II(r) & 85 & 0 & 90 & 90 & 72 & 0.847058824 & 10 & 0.022075055 & 0.260485651 \\
\hline Rskl(h) & $\begin{array}{l}85 \\
108\end{array}$ & 0 & 88 & 88 & 73 & 0.858823529 & 12 & 0.026490066 & 0.286975717 \\
\hline Rskl(r) & 91 & 0 & $\begin{array}{l}00 \\
86\end{array}$ & $\begin{array}{l}00 \\
86\end{array}$ & 74 & 0.870588235 & 14 & 0.030905077 & 0.317880795 \\
\hline Rsk2(h) & 88 & 0 & 85 & 85 & 75 & 0.882352941 & 15 & 0.033112583 & 0.350993377 \\
\hline 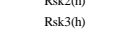 & $\begin{array}{l}00 \\
98\end{array}$ & 0 & 84 & 84 & 76 & 0.894117647 & 16 & 0.035320088 & 0.386313466 \\
\hline SAPK2a(h) & 99 & 0 & 83 & 83 & 77 & 0.905882353 & 17 & 0.037527594 & 0.42384106 \\
\hline SAPK2b(h) & $\begin{array}{l}99 \\
115\end{array}$ & 0 & $\begin{array}{l}83 \\
81\end{array}$ & $\begin{array}{l}83 \\
81\end{array}$ & 78 & 0.917647059 & 19 & 0.041942605 & 0.465783664 \\
\hline SAPK3(h) & 97 & 0 & 79 & 79 & 79 & 0.929411765 & 21 & 0.046357616 & 0.51214128 \\
\hline $\begin{array}{l}\text { SAARS3(h) } \\
\text { SAPK4(h) }\end{array}$ & 96 & 0 & 78 & 78 & 80 & 0.941176471 & 22 & 0.048565121 & 0.560706402 \\
\hline SGK(h) & 95 & 0 & 77 & 77 & 81 & 0.952941176 & 23 & 0.050772627 & 0.611479029 \\
\hline Syk(h) & 81 & 0 & 76 & 76 & 82 & 0.964705882 & 24 & 0.052980132 & 0.664459161 \\
\hline TrkB(h) & 105 & 0 & 68 & 68 & 83 & 0.976470588 & 32 & 0.070640177 & 0.735099338 \\
\hline Yes(h) & 112 & 0 & 41 & 41 & 84 & 0.988235294 & 59 & 0.130242826 & 0.865342163 \\
\hline ZAP-70(h) & 79 & 0 & 39 & 39 & 85 & 1 & 61 & 0.134657837 & 1 \\
\hline & & & & & & Total inhibition & 453 & & Total area \\
\hline & Hit rate & & & & & & & & Sini \\
\hline & Threshold & & & & & & & & Gini \\
\hline
\end{tabular}




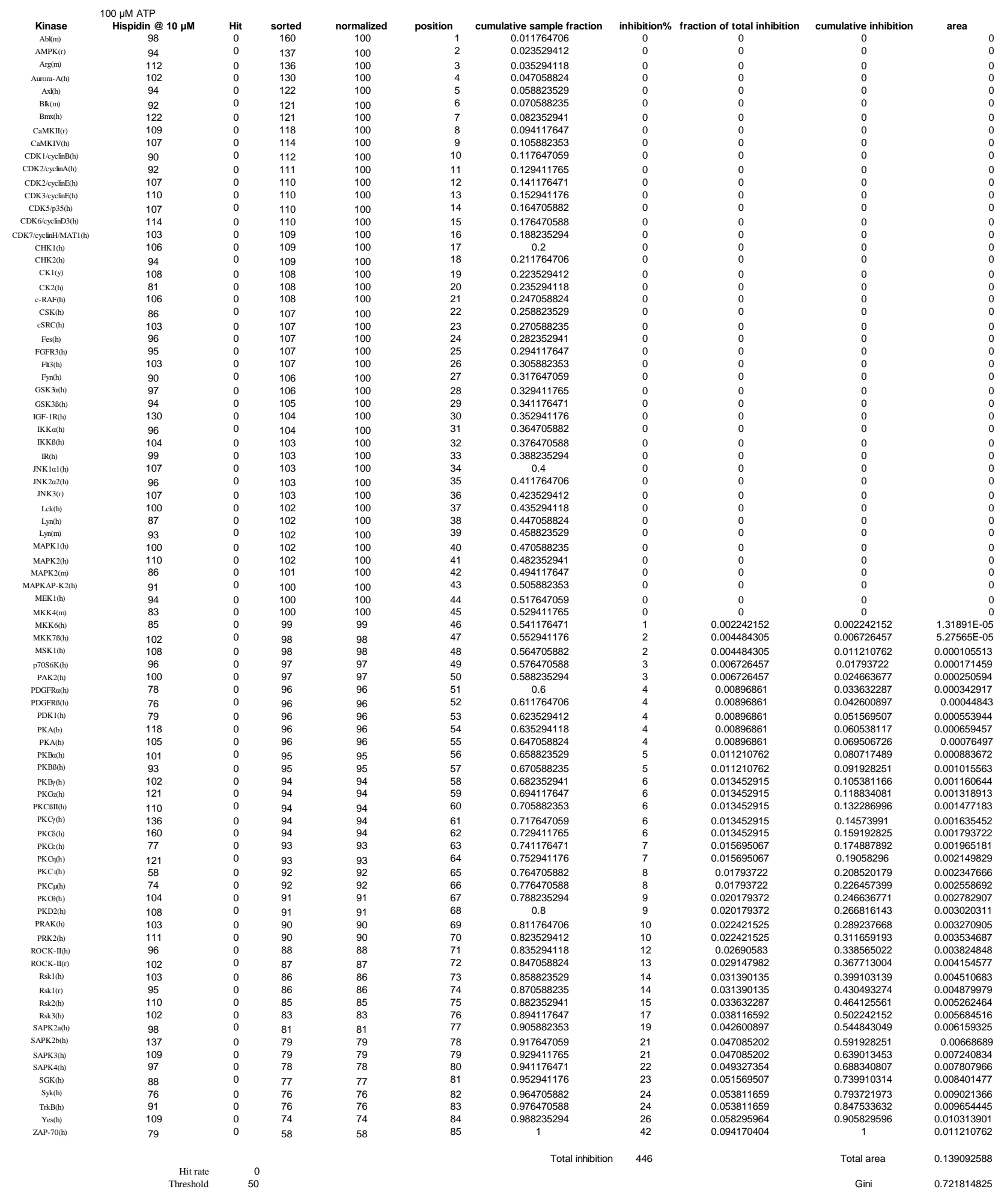




\begin{tabular}{|c|c|c|c|c|c|c|c|c|c|}
\hline & $10 \mu \mathrm{M}$ ATP & & & & & & & & \\
\hline Kinase & Indirubin@10 $\mu \mathrm{M}$ & Hit & sorted & normalized & position & cumulative sample fraction & inhibition\% & fraction of total inhibition & cumulative inhibition \\
\hline $\mathrm{Abl}(\mathrm{m})$ & 7 & 1 & 98 & 98 & 1 & 0.011764706 & 2 & 0.000366233 & 0.000366233 \\
\hline AMPK(r) & 8 & 1 & 92 & 92 & 2 & 0.023529412 & 8 & 0.001464933 & 0.001831166 \\
\hline $\operatorname{Arg}(\mathrm{m})$ & 8 & 1 & 91 & 91 & 3 & 0.035294118 & 9 & 0.00164805 & 0.003479216 \\
\hline Aurora-A(h) & 3 & 1 & 91 & 91 & 4 & 0.047058824 & 9 & 0.00164805 & 0.005127266 \\
\hline Axl(h) & 4 & 1 & 90 & 90 & 5 & 0.058823529 & 10 & 0.001831166 & 0.006958433 \\
\hline $\operatorname{Blk}(\mathrm{m})$ & 1 & 1 & 89 & 89 & 6 & 0.070588235 & 11 & 0.002014283 & 0.008972716 \\
\hline Bnxx(h) & 9 & 1 & 88 & 88 & 7 & 0.082352941 & 12 & 0.0021974 & 0.011170115 \\
\hline CaMKII(r) & 12 & 1 & 87 & 87 & 8 & 0.094117647 & 13 & 0.002380516 & 0.013550632 \\
\hline CaMKIV(h) & 30 & 1 & 86 & 86 & 9 & 0.105882353 & 14 & 0.002563633 & 0.016114265 \\
\hline CDK1/cyclinB(h) & 4 & 1 & 86 & 86 & 10 & 0.117647059 & 14 & 0.002563633 & 0.018677898 \\
\hline $\mathrm{CDK} /$ /cyclinA(h) & 6 & 1 & 84 & 84 & 11 & 0.129411765 & 16 & 0.002929866 & 0.021607764 \\
\hline $\mathrm{CDK} 2 /$ cyclinE(h) & 5 & 1 & 84 & 84 & 12 & 0.141176471 & 16 & 0.002929866 & 0.02453763 \\
\hline CDK3/cyclinE(h) & 3 & 1 & 81 & 81 & 13 & 0.152941176 & 19 & 0.003479216 & 0.028016847 \\
\hline CDK5/335(h) & 5 & 1 & 79 & 79 & 14 & 0.164705882 & 21 & 0.00384545 & 0.031862296 \\
\hline $\mathrm{CDK} 6 / \mathrm{cyclin} \mathrm{L} 3(\mathrm{~h})$ & 4 & 1 & 79 & 79 & 15 & 0.176470588 & 21 & 0.00384545 & 0.035707746 \\
\hline CDK7/cyclinHMATI(h) & 7 & 1 & 78 & 78 & 16 & 0.188235294 & 22 & 0.004028566 & 0.039736312 \\
\hline CHK I(h) & 12 & 1 & 77 & 77 & 17 & 0.2 & 23 & 0.004211683 & 0.043947995 \\
\hline СHK2(h) & 5 & 1 & 77 & 77 & 18 & 0.211764706 & 23 & 0.004211683 & 0.048159678 \\
\hline CKI(y) & 46 & 1 & 76 & 76 & 19 & 0.223529412 & 24 & 0.004394799 & 0.052554477 \\
\hline CK2(h) & 84 & 0 & 73 & 73 & 20 & 0.235294118 & 27 & 0.004944149 & 0.057498627 \\
\hline$c-\operatorname{RAF}(\mathrm{h})$ & 73 & 0 & 70 & 70 & 21 & 0.247058824 & 30 & 0.005493499 & 0.062992126 \\
\hline CSK(h) & 55 & 0 & 70 & 70 & 22 & 0.258823529 & 30 & 0.005493499 & 0.068485625 \\
\hline $\mathrm{cSRC(h)}$ & 3 & 1 & 69 & 69 & 23 & 0.270588235 & 31 & 0.005676616 & 0.074162241 \\
\hline Fes(h) & 40 & 1 & 69 & 69 & 24 & 0.282352941 & 31 & 0.005676616 & 0.079838857 \\
\hline FGFR3(h) & 2 & 1 & 68 & 68 & 25 & 0.294117647 & 32 & 0.005859733 & 0.08569859 \\
\hline Fllt3(h) & 1 & 1 & 62 & 62 & 26 & 0.305882353 & 38 & 0.006958433 & 0.092657023 \\
\hline Fyn(h) & 0 & 1 & 62 & 62 & 27 & 0.317647059 & 38 & 0.006958433 & 0.099615455 \\
\hline GSK3a(h) & 0 & 1 & 56 & 56 & 28 & 0.329411765 & 44 & 0.008057132 & 0.107672587 \\
\hline GSK3B(h) & 1 & 1 & 56 & 56 & 29 & 0.341176471 & 44 & 0.008057132 & 0.11572972 \\
\hline IGF-1R(h) & 8 & 1 & 56 & 56 & 30 & 0.352941176 & 44 & 0.008057132 & 0.123786852 \\
\hline IKKa(h) & 53 & 0 & 55 & 55 & 31 & 0.364705882 & 45 & 0.008240249 & 0.132027101 \\
\hline IKKB(h) & 86 & 0 & 55 & 55 & 32 & 0.376470588 & 45 & 0.008240249 & 0.14026735 \\
\hline $\mathrm{IR}(\mathrm{h})$ & 6 & 1 & 53 & 53 & 33 & 0.388235294 & 47 & 0.008606482 & 0.148873833 \\
\hline $\begin{array}{l}\text { INKIflal(h) } \\
\text { J }\end{array}$ & 4 & 1 & 50 & 50 & 34 & 0.4 & 50 & 0.009155832 & 0.158029665 \\
\hline JNK2a2(h) & 6 & 1 & 46 & 46 & 35 & 0.411764706 & 54 & 0.009888299 & 0.167917964 \\
\hline JNK3(r) & 2 & 1 & 40 & 40 & 36 & 0.423529412 & 60 & 0.010986999 & 0.178904962 \\
\hline Lck(h) & 2 & 1 & 40 & 40 & 37 & 0.435294118 & 60 & 0.010986999 & 0.189891961 \\
\hline Lyn(h) & -4 & 1 & 35 & 35 & 38 & 0.447058824 & 65 & 0.011902582 & 0.201794543 \\
\hline Lyn(m) & 1 & 1 & 33 & 33 & 39 & 0.458823529 & 67 & 0.012268815 & 0.214063358 \\
\hline MAPK 1 (h) & 91 & 0 & 30 & 30 & 40 & 0.470588235 & 70 & 0.012818165 & 0.226881524 \\
\hline MAPK2(h) & 98 & 0 & 22 & 22 & 41 & 0.482352941 & 78 & 0.014283098 & 0.241164622 \\
\hline MAPK2(m) & 90 & 0 & 21 & 21 & 42 & 0.494117647 & 79 & 0.014466215 & 0.255630837 \\
\hline MAPKAP-K2(h) & 69 & 0 & 17 & 17 & 43 & 0.505882353 & 83 & 0.015198682 & 0.270829518 \\
\hline MEK 1(h) & 56 & 0 & 16 & 16 & 44 & 0.517647059 & 84 & 0.015381798 & 0.286211317 \\
\hline MKK 4(m) & 9 & 1 & 12 & 12 & 45 & 0.529411765 & 88 & 0.016114265 & 0.302325581 \\
\hline MKK6(h) & 62 & 0 & 12 & 12 & 46 & 0.541176471 & 88 & 0.016114265 & 0.318439846 \\
\hline MKK $78($ (h) & 22 & 1 & 9 & 9 & 47 & 0.552941176 & 91 & 0.016663615 & 0.335103461 \\
\hline MSK I(h) & 21 & 1 & 9 & 9 & 48 & 0.564705882 & 91 & 0.016663615 & 0.351767076 \\
\hline p70s6K(h) & 9 & 1 & 9 & 9 & 49 & 0.576470588 & 91 & 0.016663615 & 0.36843069 \\
\hline PAK2(h) & 50 & 1 & 9 & 9 & 50 & 0.588235294 & 91 & 0.016663615 & 0.385094305 \\
\hline PDGFRa(h) & 35 & 1 & 9 & 9 & 51 & 0.6 & 91 & 0.016663615 & 0.40175792 \\
\hline PDGFRB(h) & 40 & 1 & 8 & 8 & 52 & 0.611764706 & 92 & 0.016846731 & 0.418604651 \\
\hline PDKI(h) & 9 & 1 & 8 & 8 & 53 & 0.623529412 & 92 & 0.016846731 & 0.435451383 \\
\hline PKA(b) & 70 & 0 & 8 & 8 & 54 & 0.635294118 & 92 & 0.016846731 & 0.452298114 \\
\hline PKA(h) & 69 & 0 & 7 & 7 & 55 & 0.647058824 & 93 & 0.017029848 & 0.469327962 \\
\hline PKBa(h) & 76 & 0 & 7 & 7 & 56 & 0.658823529 & 93 & 0.017029848 & 0.48635781 \\
\hline PKBB(h) & 79 & 0 & 7 & 7 & 57 & 0.670588235 & 93 & 0.017029848 & 0.503387658 \\
\hline $\mathrm{PKB}_{y}(\mathrm{~h})$ & 68 & 0 & 6 & 6 & 58 & 0.682352941 & 94 & 0.017212965 & 0.520600623 \\
\hline PKCa(h) & 77 & 0 & 6 & 6 & 59 & 0.694117647 & 94 & 0.017212965 & 0.537813587 \\
\hline PKCBII(h) & 92 & 0 & 6 & 6 & 60 & 0.705882353 & 94 & 0.017212965 & 0.555026552 \\
\hline PKCy(h) & 79 & 0 & 6 & 6 & 61 & 0.717647059 & 94 & 0.017212965 & 0.572239517 \\
\hline РКС(h) & 88 & 0 & 6 & 6 & 62 & 0.729411765 & 94 & 0.017212965 & 0.589452481 \\
\hline PKG:(h) & 84 & 0 & 6 & 6 & 63 & 0.741176471 & 94 & 0.017212965 & 0.606665446 \\
\hline PKG (h) & 89 & 0 & 5 & 5 & 64 & 0.752941176 & 95 & 0.017396081 & 0.624061527 \\
\hline PKC (h) & 77 & 0 & 5 & 5 & 65 & 0.764705882 & 95 & 0.017396081 & 0.641457608 \\
\hline $\mathrm{PKC}(\mathrm{hlh})$ & 62 & 0 & 5 & 5 & 66 & 0.776470588 & 95 & 0.017396081 & 0.65885369 \\
\hline PKCO(h) & 78 & 0 & 4 & 4 & 67 & 0.788235294 & 96 & 0.017579198 & 0.676432888 \\
\hline PKD2(h) & 70 & 0 & 4 & 4 & 68 & 0.8 & 96 & 0.017579198 & 0.694012086 \\
\hline PRAK(h) & 33 & 1 & 4 & 4 & 69 & 0.811764706 & 96 & 0.017579198 & 0.711591284 \\
\hline PRK2(h) & 56 & 0 & $\begin{array}{l}4 \\
4\end{array}$ & $\begin{array}{l}4 \\
4\end{array}$ & 70 & 0.823529412 & 96 & 0.017579198 & $\begin{array}{l}0.729170482 \\
0\end{array}$ \\
\hline ROCK-II(h) & 55 & 0 & 3 & 3 & 71 & 0.835294118 & 97 & 0.017762315 & 0.746932796 \\
\hline ROCK-II(r) & 17 & 1 & 3 & 3 & 72 & 0.847058824 & 97 & 0.017762315 & 0.764695111 \\
\hline Rskl(h) & 7 & 1 & 3 & 3 & 73 & 0.858823529 & 97 & 0.017762315 & 0.782457425 \\
\hline Rskl(r) & 6 & 1 & 2 & 2 & 74 & 0.870588235 & 98 & 0.017945431 & 0.800402857 \\
\hline Rsk2(h) & 9 & 1 & 2 & 2 & 75 & 0.882352941 & 98 & 0.017945431 & 0.818348288 \\
\hline Rsk3(h) & 6 & 1 & 2 & 2 & 76 & 0.894117647 & 98 & 0.017945431 & 0.836293719 \\
\hline SAPK2a(h) & 81 & 0 & 2 & 2 & 77 & 0.905882353 & 98 & 0.017945431 & 0.85423915 \\
\hline SAPK2b(h) & 91 & 0 & 1 & 1 & 78 & 0.917647059 & 99 & 0.018128548 & 0.872367698 \\
\hline $\mathrm{SAPK} 3(\mathrm{~h})$ & 86 & 0 & 1 & 1 & 79 & 0.929411765 & 99 & 0.018128548 & 0.890496246 \\
\hline SAPK4(h) & 56 & 0 & 1 & 1 & 80 & 0.941176471 & 99 & 0.018128548 & 0.908624794 \\
\hline SGK(h) & 16 & 1 & 1 & 1 & 81 & 0.952941176 & 99 & 0.018128548 & 0.926753342 \\
\hline Syk(h) & 6 & 1 & 0 & 0 & 82 & 0.964705882 & 100 & 0.018311665 & 0.945065006 \\
\hline TTkB(h) & 0 & 1 & 0 & 0 & 83 & 0.976470588 & 100 & 0.018311665 & 0.963376671 \\
\hline Yes(h) & 2 & 1 & 0 & 0 & 84 & 0.988235294 & 100 & 0.018311665 & 0.981688335 \\
\hline ZAP-70(h) & 87 & 0 & 0 & 0 & 85 & 1 & 100 & 0.018311665 & 1 \\
\hline & & & & & & Total inhibition & 5461 & & Total area \\
\hline & $\begin{array}{r}\text { Hit rate } \\
\text { Threshold }\end{array}$ & $\begin{array}{l}5 \\
5\end{array}$ & & & & & & & Gini \\
\hline
\end{tabular}




\begin{tabular}{|c|c|c|c|c|c|c|c|c|}
\hline & $100 \mu \mathrm{M}$ ATP & & & & & & & \\
\hline Kinase & Indirubin @ $10 \mu \mathrm{M}$ & Hit & sorted & normalized & position & cumulative sample fraction & inhibition\% & fraction of total inhibition \\
\hline $\mathrm{Abl}(\mathrm{m})$ & 35 & 1 & 116 & 100 & 1 & 0.011764706 & 0 & 0 \\
\hline AMPK(r) & 5 & 1 & 109 & 100 & 2 & 0.023529412 & 0 & 0 \\
\hline $\operatorname{Arg}(\mathrm{m})$ & 17 & 1 & 107 & 100 & 3 & 0.035294118 & 0 & 0 \\
\hline Aurora-A(h) & 5 & 1 & 104 & 100 & 4 & 0.047058824 & 0 & 0 \\
\hline Axl(h) & 3 & 1 & 103 & 100 & 5 & 0.058823529 & 0 & 0 \\
\hline $\operatorname{Blk}(\mathrm{m})$ & 2 & 1 & 100 & 100 & 6 & 0.070588235 & 0 & 0 \\
\hline Bmxx(h) & -6 & 1 & 98 & 98 & 7 & 0.082352941 & 2 & 0.000453001 \\
\hline CaMKII(r) & 44 & 1 & 98 & 98 & 8 & 0.094117647 & 2 & 0.000453001 \\
\hline CaMKIV(h) & 77 & 0 & 97 & 97 & 9 & 0.105882353 & 3 & 0.000679502 \\
\hline CDK1/cyclnBB(h) & 15 & 1 & 97 & 97 & 10 & 0.117647059 & 3 & 0.000679502 \\
\hline $\mathrm{CDK} /$ /yclinA(h) & 8 & 1 & 93 & 93 & 11 & 0.129411765 & 7 & 0.001585504 \\
\hline $\mathrm{CDK} 2 /$ cyclinE(h) & 3 & 1 & 93 & 93 & 12 & 0.141176471 & 7 & 0.001585504 \\
\hline CDK3/yclinE(h) & 15 & 1 & 92 & 92 & 13 & 0.152941176 & 8 & 0.001812005 \\
\hline CDK5/p35(h) & 2 & 1 & 91 & 91 & 14 & 0.164705882 & 9 & 0.002038505 \\
\hline CDK6/cyclinD3(h) & -5 & 1 & 91 & 91 & 15 & 0.176470588 & 9 & 0.002038505 \\
\hline CDK7/cyclinHMATI(h) & 17 & 1 & 91 & 91 & 16 & 0.188235294 & 9 & 0.002038505 \\
\hline СнК $1 \mathrm{~h})$ & 23 & 1 & 91 & 91 & 17 & 0.2 & 9 & 0.002038505 \\
\hline СHK2(h) & 4 & 1 & 90 & 90 & 18 & 0.211764706 & 10 & 0.002265006 \\
\hline $\mathrm{CK} 1(\mathrm{y})$ & 79 & 0 & 89 & 89 & 19 & 0.223529412 & 11 & 0.002491506 \\
\hline СК2(h) & 97 & 0 & 89 & 89 & 20 & 0.235294118 & 11 & 0.002491506 \\
\hline c-RAF(h) & 87 & 0 & 88 & 88 & 21 & 0.247058824 & 12 & 0.002718007 \\
\hline $\operatorname{CSK}(\mathrm{h})$ & 77 & 0 & 87 & 87 & 22 & 0.258823529 & 13 & 0.002944507 \\
\hline $\operatorname{csRCh}(\mathrm{h})$ & 2 & 1 & 87 & 87 & 23 & 0.270588235 & 13 & 0.002944507 \\
\hline Fess(h) & 69 & 0 & 83 & 83 & 24 & 0.282352941 & 17 & 0.00385051 \\
\hline FGFR3(h) & 13 & 1 & 83 & 83 & 25 & 0.294117647 & 17 & 0.00385051 \\
\hline $\mathrm{Fll}(\mathrm{h}(\mathrm{h})$ & -1 & 1 & 82 & 82 & 26 & 0.305882353 & 18 & 0.00407701 \\
\hline Fyn(h) & 2 & 1 & 81 & 81 & 27 & 0.317647059 & 19 & 0.004303511 \\
\hline GSK3a(h) & 5 & 1 & 80 & 80 & 28 & 0.329411765 & 20 & 0.004530011 \\
\hline GSK3B(h) & 4 & 1 & 79 & 79 & 29 & 0.341176471 & 21 & 0.004756512 \\
\hline IGF-IR(h) & 18 & 1 & 77 & 77 & 30 & 0.352941176 & 23 & 0.005209513 \\
\hline IKKa(h) & 89 & 0 & 77 & 77 & 31 & 0.364705882 & 23 & 0.005209513 \\
\hline IKKB(h) & 93 & 0 & 75 & 75 & 32 & 0.376470588 & 25 & 0.005662514 \\
\hline $\mathbb{I R ( h )}$ & 13 & 1 & 75 & 75 & 33 & 0.388235294 & 25 & 0.005662514 \\
\hline JNKIal(h) & 35 & 1 & 74 & 74 & 34 & 0.4 & 26 & 0.005889015 \\
\hline JNK2202(h) & 59 & 0 & 73 & 73 & 35 & 0.411764706 & 27 & 0.006115515 \\
\hline JNK3(r) & 9 & 1 & 69 & 69 & 36 & 0.423529412 & 31 & 0.007021518 \\
\hline Lck(h) & 3 & 1 & 60 & 60 & 37 & 0.435294118 & 40 & 0.009060023 \\
\hline Lyn(h) & 1 & 1 & 59 & 59 & 38 & 0.447058824 & 41 & 0.009286523 \\
\hline $\operatorname{Lyn}(m)$ & 4 & 1 & 59 & 59 & 39 & 0.458823529 & 41 & 0.009286523 \\
\hline MAPK1(h) & 88 & 0 & 58 & 58 & 40 & 0.470588235 & 42 & 0.009513024 \\
\hline MAPK2(h) & 100 & 0 & 45 & 45 & 41 & 0.482352941 & 55 & 0.012457531 \\
\hline MAPK2(m) & 103 & 0 & 44 & 44 & 42 & 0.494117647 & 56 & 0.012684032 \\
\hline MAPKAP-K2(h) & 92 & 0 & 43 & 43 & 43 & 0.505882353 & 57 & 0.012910532 \\
\hline MEK1(h) & 74 & 0 & 35 & 35 & 44 & 0.517647059 & 65 & 0.014722537 \\
\hline MKK $4(\mathrm{~m})$ & 23 & 1 & 35 & 35 & 45 & 0.529411765 & 65 & 0.014722537 \\
\hline MKK6(h) & 73 & 0 & 35 & 35 & 46 & 0.541176471 & 65 & 0.014722537 \\
\hline MKK $7 \mathrm{B(h)}$ & 27 & 1 & 34 & 34 & 47 & 0.552941176 & 66 & 0.014949037 \\
\hline MSKI(h) & 33 & 1 & 33 & 33 & 48 & 0.564705882 & 67 & 0.015175538 \\
\hline p7056K(h) & 34 & 1 & 31 & 31 & 49 & 0.576470588 & 69 & 0.015628539 \\
\hline PAK2(h) & 58 & 0 & 30 & 30 & 50 & 0.588235294 & 70 & 0.01585504 \\
\hline PDGFRa(h) & 59 & 0 & 27 & 27 & 51 & 0.6 & 73 & 0.016534541 \\
\hline PDGFRB(h) & 45 & 1 & 23 & 23 & 52 & 0.611764706 & 77 & 0.017440544 \\
\hline PDK I (h) & 35 & 1 & 23 & 23 & 53 & 0.623529412 & 77 & 0.017440544 \\
\hline PKA(b) & 109 & 0 & 22 & 22 & 54 & 0.635294118 & 78 & 0.017667044 \\
\hline PKA(h) & 75 & 0 & 21 & 21 & 55 & 0.647058824 & 79 & 0.017893545 \\
\hline PKBa(h) & 83 & 0 & 18 & 18 & 56 & 0.658823529 & 82 & 0.018573046 \\
\hline PKB(h) & 91 & 0 & 18 & 18 & 57 & 0.670588235 & 82 & 0.018573046 \\
\hline $\mathrm{PKB}_{\mathrm{y}(\mathrm{h})}$ & 90 & 0 & 17 & 17 & 58 & 0.682352941 & 83 & 0.018799547 \\
\hline PKCa(h) & 87 & 0 & 17 & 17 & 59 & 0.694117647 & 83 & 0.018799547 \\
\hline РКCBII(h) & 98 & 0 & 15 & 15 & 60 & 0.705882353 & 85 & 0.019252548 \\
\hline PKCy (h) & 89 & 0 & 15 & 15 & 61 & 0.717647059 & 85 & 0.019252548 \\
\hline PKБ(h) & 104 & 0 & 13 & 13 & 62 & 0.729411765 & 87 & 0.019705549 \\
\hline PKG:(h) & 91 & 0 & 13 & 13 & 63 & 0.741176471 & 87 & 0.019705549 \\
\hline PKG(h) & 98 & 0 & 12 & 12 & 64 & 0.752941176 & 88 & 0.01993205 \\
\hline PKC (h) & 97 & 0 & 10 & 10 & 65 & 0.764705882 & 90 & 0.020385051 \\
\hline PKC $\mu(\mathrm{h})$ & 91 & 0 & 9 & 9 & 66 & 0.776470588 & 91 & 0.020611552 \\
\hline PKCO(h) & 75 & 0 & 8 & 8 & 67 & 0.788235294 & 92 & 0.020838052 \\
\hline PKD2(h) & 80 & 0 & 5 & 5 & 68 & 0.8 & 95 & 0.021517554 \\
\hline PRAK(h) & 60 & 0 & 5 & 5 & 69 & 0.811764706 & 95 & 0.021517554 \\
\hline PRK2(h) & 83 & 0 & 5 & 5 & 70 & 0.823529412 & 95 & 0.021517554 \\
\hline ROCK-II(h) & 82 & 0 & 4 & 4 & 71 & 0.835294118 & 96 & 0.021744054 \\
\hline ROCK-II(r) & 43 & 1 & 4 & 4 & 72 & 0.847058824 & 96 & 0.021744054 \\
\hline Rskl(h) & 22 & 1 & 4 & 4 & 73 & 0.858823529 & 96 & 0.021744054 \\
\hline Rskl(r) & 21 & 1 & 3 & $\begin{array}{l}4 \\
3\end{array}$ & 74 & 0.870588235 & 97 & 0.021970555 \\
\hline Rsk2(h) & 31 & 1 & 3 & 3 & 75 & 0.882352941 & 97 & 0.021970555 \\
\hline Rsk3(h) & 10 & 1 & 3 & 3 & 76 & 0.894117647 & 97 & 0.021970555 \\
\hline SAPK2a(h) & 93 & 0 & 2 & 2 & 77 & 0.905882353 & 98 & 0.022197055 \\
\hline SAPK2b(h) & 107 & 0 & 2 & 2 & 78 & 0.917647059 & 98 & 0.022197055 \\
\hline $\mathrm{SAPK} 3(\mathrm{~h})$ & 91 & 0 & 2 & 2 & 79 & 0.929411765 & 98 & 0.022197055 \\
\hline SAPK4(h) & 81 & 0 & 2 & 2 & 80 & 0.941176471 & 98 & 0.022197055 \\
\hline SGK(h) & 30 & 1 & 1 & 1 & 81 & 0.952941176 & 99 & 0.022423556 \\
\hline Syk(h) & 18 & 1 & 0 & 0 & 82 & 0.964705882 & 100 & 0.022650057 \\
\hline TrkB(h) & 0 & 1 & -1 & -1 & 83 & 0.976470588 & 101 & 0.022876557 \\
\hline Yes(h) & 12 & 1 & -5 & -5 & 84 & 0.988235294 & 105 & 0.023782559 \\
\hline ZAP-70(h) & 116 & 0 & -6 & -6 & 85 & 1 & 106 & 0.02400906 \\
\hline & & & & & & Total inhibition & 4415 & \\
\hline & Hit rate & & & & & & & \\
\hline & Threshold & & & & & & & \\
\hline
\end{tabular}




\begin{tabular}{|c|c|c|c|c|c|c|c|c|c|}
\hline & $10 \mu \mathrm{M}$ ATP & & & & & & & & \\
\hline Kinase & JNK inh II @ 10 нM & Hit & sorted & normalized & position & cumulative sample fraction & inhibition\% & fraction of total inhibition & cumulative inhibition \\
\hline $\mathrm{Abl}(\mathrm{m})$ & 39 & 1 & 107 & 100 & 1 & 0.011764706 & 0 & 0 & 0 \\
\hline AMPK(r) & 25 & 1 & 103 & 100 & 2 & 0.023529412 & 0 & 0 & 0 \\
\hline $\operatorname{Arg}(\mathrm{m})$ & 97 & 0 & 102 & 100 & 3 & 0.035294118 & 0 & 0 & 0 \\
\hline Aurora-A(h) & 3 & 1 & 102 & 100 & 4 & 0.047058824 & 0 & 0 & 0 \\
\hline Axl(h) & 77 & 0 & 102 & 100 & 5 & 0.058823529 & 0 & 0 & 0 \\
\hline $\mathrm{Blk}(\mathrm{m})$ & 22 & 1 & 100 & 100 & 6 & 0.070588235 & 0 & 0 & 0 \\
\hline $\operatorname{Bmx}(\mathrm{h})$ & 47 & 1 & 97 & 97 & 7 & 0.082352941 & 3 & 0.000897398 & 0.000897398 \\
\hline CaMKII(r) & 86 & 0 & 97 & 97 & 8 & 0.094117647 & 3 & 0.000897398 & 0.001794795 \\
\hline CaMKIV(h) & 95 & 0 & 95 & 95 & 9 & 0.105882353 & 5 & 0.001495663 & 0.003290458 \\
\hline CDK1/cyclnBB(h) & 15 & 1 & 95 & 95 & 10 & 0.117647059 & 5 & 0.001495663 & 0.00478612 \\
\hline $\mathrm{CDK} 2 / \mathrm{cyclinA}(\mathrm{h})$ & 92 & 0 & 95 & 95 & 11 & 0.129411765 & 5 & 0.001495663 & 0.006281783 \\
\hline $\mathrm{CDK} 2 /$ cyclinE(h) & 75 & 0 & 94 & 94 & 12 & 0.141176471 & 6 & 0.001794795 & 0.008076578 \\
\hline CDK3/cyclinE(h) & 27 & 1 & 94 & 94 & 13 & 0.152941176 & 6 & 0.001794795 & 0.009871373 \\
\hline CDK5/p35(h) & 90 & 0 & 93 & 93 & 14 & 0.164705882 & 7 & 0.002093928 & 0.011965301 \\
\hline CDK6/cyclinD3(h) & 90 & 0 & 93 & 93 & 15 & 0.176470588 & 7 & 0.002093928 & 0.014059228 \\
\hline CDK7/cyclinH/MATI(h) & 23 & 1 & 92 & 92 & 16 & 0.188235294 & 8 & 0.00239306 & 0.016452288 \\
\hline CHK1(h) & 29 & 1 & 92 & 92 & 17 & 0.2 & 8 & 0.00239306 & 0.018845348 \\
\hline СHK2(h) & 76 & 0 & 92 & 92 & 18 & 0.211764706 & 8 & 0.00239306 & 0.021238409 \\
\hline $\mathrm{CKI}(\mathrm{y})$ & 44 & 1 & 91 & 91 & 19 & 0.223529412 & 9 & 0.002692193 & 0.023930601 \\
\hline CK2(h) & 44 & 1 & 91 & 91 & 20 & 0.235294118 & 9 & 0.002692193 & 0.026622794 \\
\hline $\mathrm{c}-\mathrm{RAF}(\mathrm{h})$ & 88 & 0 & 90 & 90 & 21 & 0.247058824 & 10 & 0.002991325 & 0.029614119 \\
\hline $\operatorname{CSK}(\mathrm{h})$ & 75 & 0 & 90 & 90 & 22 & 0.258823529 & 10 & 0.002991325 & 0.032605444 \\
\hline cSRC(h) & 107 & 0 & 89 & 89 & 23 & 0.270588235 & 11 & 0.003290458 & 0.035895902 \\
\hline Fes(h) & 32 & 1 & 89 & 89 & 24 & 0.282352941 & 11 & 0.003290458 & 0.03918636 \\
\hline FGFR3(h) & 71 & 0 & 88 & 88 & 25 & 0.294117647 & 12 & 0.00358959 & 0.04277595 \\
\hline $\mathrm{FH}$ H(h) & 8 & 1 & 86 & 86 & 26 & 0.305882353 & 14 & 0.004187855 & 0.046963805 \\
\hline Fyn(h) & 52 & 0 & 85 & 85 & 27 & 0.317647059 & 15 & 0.004486988 & 0.051450793 \\
\hline GSK3a(h) & 39 & 1 & 84 & 84 & 28 & 0.329411765 & 16 & 0.00478612 & 0.056236913 \\
\hline GSK3B(h) & 55 & 0 & 84 & 84 & 29 & 0.341176471 & 16 & 0.00478612 & 0.061023033 \\
\hline IGF-IR(h) & 97 & 0 & 83 & 83 & 30 & 0.352941176 & 17 & 0.005085253 & 0.066108286 \\
\hline IKKa(h) & 89 & 0 & 80 & 80 & 31 & 0.364705882 & 20 & 0.00598265 & 0.072090936 \\
\hline IKKB(h) & 89 & 0 & 79 & 79 & 32 & 0.376470588 & 21 & 0.006281783 & 0.078372719 \\
\hline $\operatorname{IR}(\mathrm{h})$ & 102 & 0 & 79 & 79 & 33 & 0.388235294 & 21 & 0.006281783 & 0.084654502 \\
\hline JNKIal(h) & 3 & 1 & 77 & 77 & 34 & 0.4 & 23 & 0.006880048 & 0.09153455 \\
\hline JNK202(h) & 77 & 0 & 77 & 77 & 35 & 0.411764706 & 23 & 0.006880048 & 0.098414598 \\
\hline $\mathrm{JNK} 3(\mathrm{r})$ & 3 & 1 & 77 & 77 & 36 & 0.423529412 & 23 & 0.006880048 & 0.105294646 \\
\hline Lck(h) & 91 & 0 & 77 & 77 & 37 & 0.435294118 & 23 & 0.006880048 & 0.112174693 \\
\hline Lyn(h) & 32 & 1 & 76 & 76 & 38 & 0.447058824 & 24 & 0.00717918 & 0.119353874 \\
\hline Lyn(m) & 36 & 1 & 76 & 76 & 39 & 0.458823529 & 24 & 0.00717918 & 0.126533054 \\
\hline MAPK1(h) & 94 & 0 & 76 & 76 & 40 & 0.470588235 & 24 & 0.00717918 & 0.133712235 \\
\hline MAPK2(h) & 100 & 0 & 75 & 75 & 41 & 0.482352941 & 25 & 0.007478313 & 0.141190547 \\
\hline MAPK2(m) & 51 & 0 & 75 & 75 & 42 & 0.494117647 & 25 & 0.007478313 & 0.14866886 \\
\hline MAPKAP-K2(h) & 92 & 0 & 73 & 73 & 43 & 0.505882353 & 27 & 0.008076578 & 0.156745438 \\
\hline MEK 1(h) & 50 & 1 & 71 & 71 & 44 & 0.517647059 & 29 & 0.008674843 & 0.165420281 \\
\hline MKK $4(\mathrm{~m})$ & 0 & 1 & 63 & 63 & 45 & 0.529411765 & 37 & 0.011067903 & 0.176488184 \\
\hline MKK6(h) & 84 & 0 & 56 & 56 & 46 & 0.541176471 & 44 & 0.013161831 & 0.189650015 \\
\hline МKK $78($ (h) & 14 & 1 & 55 & 55 & 47 & 0.552941176 & 45 & 0.013460963 & 0.203110978 \\
\hline MSKI(h) & $\begin{array}{l}14 \\
42\end{array}$ & 1 & 54 & 54 & 48 & 0.564705882 & 46 & 0.013760096 & 0.216871074 \\
\hline $\mathrm{p} 70 \mathrm{~S} 6 \mathrm{~K}(\mathrm{~h})$ & 23 & 1 & 52 & 52 & 49 & 0.576470588 & 48 & 0.014358361 & 0.231229435 \\
\hline 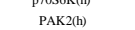 & 80 & 0 & 51 & 51 & 50 & 0.588235294 & $\begin{array}{l}40 \\
49\end{array}$ & 0.014657493 & 0.245886928 \\
\hline PDGFRa(h) & 94 & 0 & 50 & 50 & 51 & 0.6 & 50 & 0.014956626 & 0.260843554 \\
\hline PDGFRB(h) & 93 & 0 & 49 & 49 & 52 & 0.611764706 & 51 & 0.015255758 & 0.276099312 \\
\hline PDK I(h) & 37 & 1 & 48 & 48 & 53 & 0.623529412 & 52 & 0.015554891 & 0.291654203 \\
\hline PKA(b) & 79 & 0 & 47 & 47 & 54 & 0.635294118 & 53 & 0.015854023 & 0.307508226 \\
\hline PKA(h) & 79 & 0 & 47 & 47 & 55 & 0.647058824 & 53 & 0.015854023 & 0.323362249 \\
\hline PKBa(h) & 83 & 0 & 46 & 46 & 56 & 0.658823529 & 54 & 0.016153156 & 0.339515405 \\
\hline PKBB(h) & $\begin{array}{l}0.0 \\
102\end{array}$ & 0 & 44 & 44 & 57 & 0.670588235 & 56 & 0.016751421 & 0.356266826 \\
\hline $\mathrm{PKB} \mathrm{B}_{(\mathrm{h})}$ & 77 & 0 & 44 & 44 & 58 & 0.682352941 & 56 & 0.016751421 & 0.373018247 \\
\hline PKGa(h) & 56 & 0 & 42 & 42 & 59 & 0.694117647 & 58 & 0.017349686 & 0.390367933 \\
\hline РКСBII(h) & 91 & 0 & 41 & 41 & 60 & 0.705882353 & 59 & 0.017648818 & 0.408016751 \\
\hline PKCy(h) & 48 & 1 & 39 & 39 & 61 & 0.717647059 & 61 & 0.018247083 & 0.426263835 \\
\hline PKळ(h) & 77 & 0 & 39 & 39 & 62 & 0.729411765 & 61 & 0.018247083 & 0.444510918 \\
\hline PKG:(h) & 76 & 0 & 37 & 37 & 63 & 0.741176471 & 63 & 0.018845348 & 0.463356267 \\
\hline PKG(h) & 95 & 0 & 36 & 36 & 64 & 0.752941176 & 64 & 0.019144481 & 0.482500748 \\
\hline PKC (h) & 103 & 0 & 32 & 32 & 65 & 0.764705882 & 68 & 0.020341011 & 0.502841759 \\
\hline PKC $\mu(\mathrm{h})$ & 63 & 0 & 32 & 32 & 66 & 0.776470588 & 68 & 0.020341011 & 0.52318277 \\
\hline PKCO(h) & 85 & 0 & 30 & 30 & 67 & 0.788235294 & 70 & 0.020939276 & 0.544122046 \\
\hline PKD2(h) & 10 & 1 & 29 & 29 & 68 & 0.8 & 71 & 0.021238409 & 0.565360455 \\
\hline PRAK(h) & 25 & 1 & 29 & 29 & 69 & 0.811764706 & 71 & 0.021238409 & 0.586598863 \\
\hline PRK2(h) & 73 & 0 & 27 & 27 & 70 & 0.823529412 & 73 & 0.021836674 & 0.608435537 \\
\hline ROCK-II(h) & 49 & 1 & 25 & 25 & 71 & 0.835294118 & 75 & 0.022434939 & 0.630870476 \\
\hline ROCK-II(r) & 54 & 0 & 25 & 25 & 72 & 0.847058824 & 75 & 0.022434939 & 0.653305414 \\
\hline Rskl(h) & 47 & 1 & 23 & 23 & 73 & 0.858823529 & 77 & 0.023033204 & 0.676338618 \\
\hline Rskl(r) & 76 & 0 & 23 & 23 & 74 & 0.870588235 & 77 & 0.023033204 & 0.699371822 \\
\hline Rsk2(h) & 41 & 1 & 22 & 22 & 75 & 0.882352941 & 78 & 0.023332336 & 0.722704158 \\
\hline Rsk3(h) & 30 & 1 & 15 & 15 & 76 & 0.894117647 & 85 & 0.025426264 & 0.748130422 \\
\hline SAPK2a(h) & 92 & 0 & 15 & 15 & 77 & 0.905882353 & 85 & 0.025426264 & 0.773556686 \\
\hline SAPK2b(h) & 102 & 0 & 14 & 14 & 78 & 0.917647059 & 86 & 0.025725396 & 0.799282082 \\
\hline SAPK3(h) & 95 & 0 & 10 & 10 & 79 & 0.929411765 & 90 & 0.026921926 & 0.826204008 \\
\hline $\mathrm{SAPK} 4(\mathrm{~h})$ & 93 & 0 & 8 & 8 & 80 & 0.941176471 & 92 & 0.027520191 & 0.8537242 \\
\hline SGK(h) & 15 & 1 & 3 & 3 & 81 & 0.952941176 & 97 & 0.029015854 & 0.882740054 \\
\hline Syk(h) & 29 & 1 & 3 & 3 & 82 & 0.964705882 & 97 & 0.029015854 & 0.911755908 \\
\hline $\operatorname{TrkB}(\mathbf{h})$ & 2 & 1 & 3 & 3 & 83 & 0.976470588 & 97 & 0.029015854 & 0.940771762 \\
\hline Yes(h) & 46 & 1 & 2 & 2 & 84 & 0.988235294 & 98 & 0.029314987 & 0.970086748 \\
\hline ZAP-70(h) & 84 & 0 & 0 & 0 & 85 & 1 & 100 & 0.029913252 & 1 \\
\hline & & & & & & Total inhibition & 3343 & & Total area \\
\hline & $\begin{array}{r}\text { Hit rate } \\
\text { Threshold }\end{array}$ & & & & & & & & Gini \\
\hline
\end{tabular}




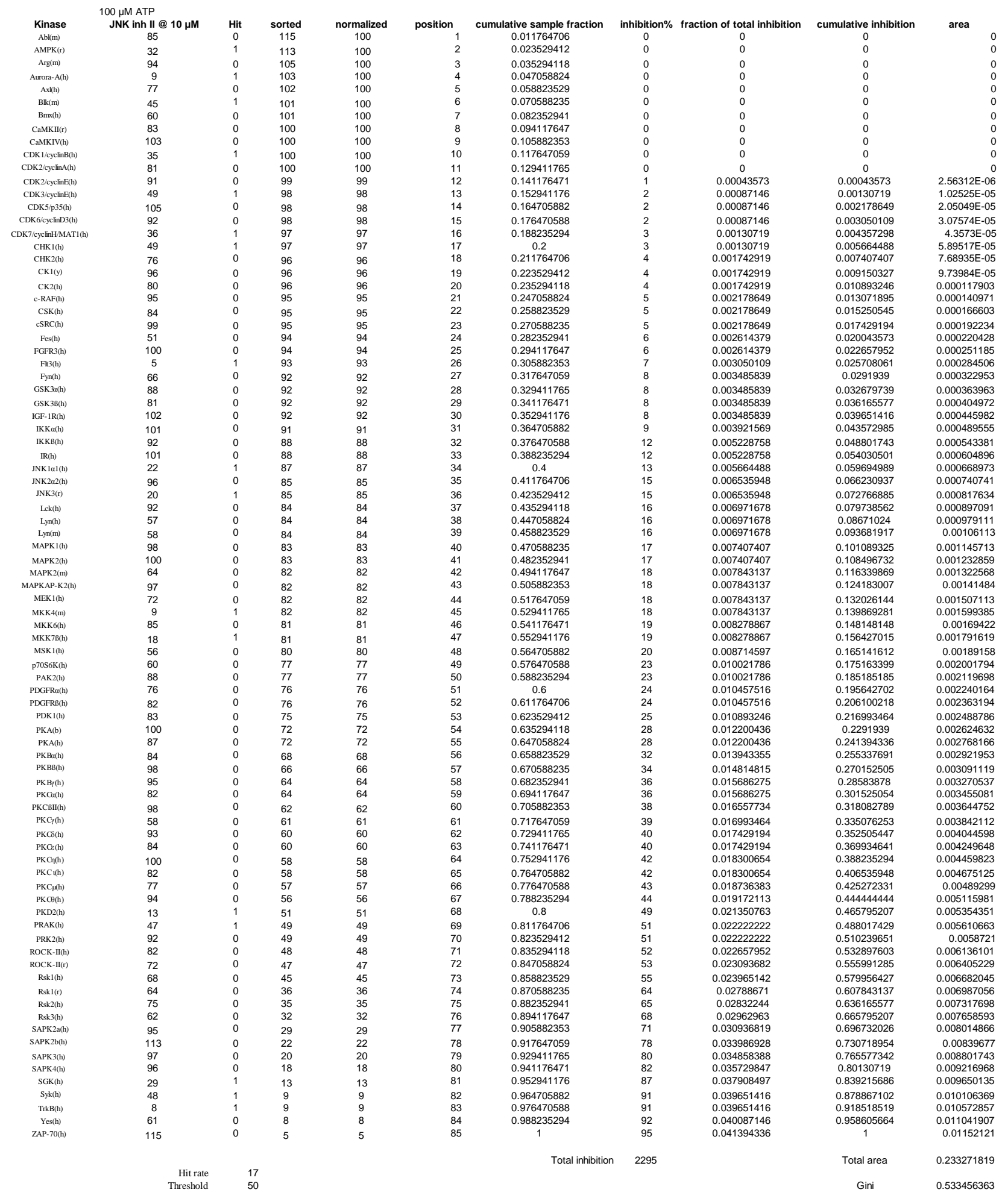




\begin{tabular}{|c|c|c|c|c|c|c|c|c|}
\hline & $0 \mu \mathrm{M}$ ATP & & & & & & & \\
\hline Kinase & K252c @ $10 \mu \mathrm{M}$ & Hit & sorted & normalized & position & cumulative sample fraction & inhibition\% & fraction of total inhibition \\
\hline $\mathrm{Abl}(\mathrm{m})$ & 72 & 0 & 97 & 97 & 1 & 0.011764706 & 3 & 0.000541908 \\
\hline AMPK(r) & 32 & 1 & 88 & 88 & 2 & 0.023529412 & 12 & 0.00216763 \\
\hline $\operatorname{Arg}(\mathrm{m})$ & 88 & 0 & 87 & 87 & 3 & 0.035294118 & 13 & 0.002348266 \\
\hline Aurora-A(h) & 6 & 1 & 85 & 85 & 4 & 0.047058824 & 15 & 0.002709538 \\
\hline $\operatorname{Ax}(\mathrm{h})$ & 11 & 1 & 84 & 84 & 5 & 0.058823529 & 16 & 0.002890173 \\
\hline $\operatorname{Blk}(\mathrm{m})$ & 14 & 1 & 78 & 78 & 6 & 0.070588235 & 22 & 0.003973988 \\
\hline Bnxx(h) & 49 & 1 & 74 & 74 & 7 & 0.082352941 & 26 & 0.004696532 \\
\hline CaMKII(r) & 3 & 1 & 74 & 74 & 8 & 0.094117647 & 26 & 0.004696532 \\
\hline CaMKIV(h) & 61 & 0 & 73 & 73 & 9 & 0.105882353 & 27 & 0.004877168 \\
\hline 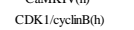 & 11 & 1 & 73 & 73 & 10 & 0.117647059 & 27 & 0.004877168 \\
\hline CDK2/cyclinA(h) & 14 & 1 & 72 & 72 & 11 & 0.129411765 & 28 & 0.005057803 \\
\hline $\mathrm{CDK} 2 /$ cyclinE(h) & 15 & 1 & 71 & 71 & 12 & 0.141176471 & 29 & 0.005238439 \\
\hline CDK3/cyclinE(h) & 31 & 1 & 64 & 64 & 13 & 0.152941176 & 36 & 0.00650289 \\
\hline CDK5 5335 (h) & 4 & 1 & 64 & 64 & 14 & 0.164705882 & 36 & 0.00650289 \\
\hline $\mathrm{CDK} 6 / \mathrm{cyclin} \mathrm{D} 3(\mathrm{~h})$ & 18 & 1 & 62 & 62 & 15 & 0.176470588 & 38 & 0.006864162 \\
\hline CDK7/cychinHMATI(h) & 73 & 0 & 61 & 61 & 16 & 0.188235294 & 39 & 0.007044798 \\
\hline CHKI(h) & 2 & 1 & 61 & 61 & 17 & 0.2 & 39 & 0.007044798 \\
\hline СHK2(h) & 5 & 1 & 61 & 61 & 18 & 0.211764706 & 39 & 0.007044798 \\
\hline CKI(y) & 87 & 0 & 61 & 61 & 19 & 0.223529412 & 39 & 0.007044798 \\
\hline СК2(b) & 41 & 1 & 59 & 59 & 20 & 0.235294118 & 41 & 0.007406069 \\
\hline$c \cdot \operatorname{RAF(h)}$ & 97 & 0 & 58 & 58 & 21 & 0.247058824 & 42 & 0.007586705 \\
\hline CSK(h) & 48 & 1 & 57 & 57 & 22 & 0.258823529 & 43 & 0.007767341 \\
\hline cSRC(h) & 61 & 0 & 57 & 57 & 23 & 0.270588235 & 43 & 0.007767341 \\
\hline Fes(h) & 62 & 0 & 54 & 54 & 24 & 0.282352941 & 46 & 0.008309249 \\
\hline FGFR3 (h) & 37 & 1 & 52 & 52 & 25 & 0.294117647 & 48 & 0.00867052 \\
\hline $\mathrm{FHt}(\mathrm{h}(\mathrm{h})$ & 4 & 1 & 52 & 52 & 26 & 0.305882353 & 48 & 0.00867052 \\
\hline Fynn(h) & 43 & 1 & 52 & 52 & 27 & 0.317647059 & 48 & 0.00867052 \\
\hline GSK3a(h) & 58 & 0 & 49 & 49 & 28 & 0.329411765 & 51 & 0.009212428 \\
\hline GSK3B(h) & 57 & 0 & 49 & 49 & 29 & 0.341176471 & 51 & 0.009212428 \\
\hline IGF-1R(h) & 59 & 0 & 48 & 48 & 30 & 0.352941176 & 52 & 0.009393064 \\
\hline IKKa(h) & 7 & 1 & 48 & 48 & 31 & 0.364705882 & 52 & 0.009393064 \\
\hline IKKB(h) & 49 & 1 & 47 & 47 & 32 & 0.376470588 & 53 & 0.009573699 \\
\hline $\mathrm{IR}(\mathrm{h})$ & 48 & 1 & 46 & 46 & 33 & 0.388235294 & 54 & 0.009754335 \\
\hline JNKIal(h) & 15 & 1 & 44 & 44 & 34 & 0.4 & 56 & 0.010115607 \\
\hline JNK202(h) & 27 & 1 & 43 & 43 & 35 & 0.411764706 & 57 & 0.010296243 \\
\hline $\mathrm{JNK} 3(\mathrm{r})$ & 7 & 1 & 42 & 42 & 36 & 0.423529412 & 58 & 0.010476879 \\
\hline Lck(1) & 64 & 0 & 42 & 42 & 37 & 0.435294118 & 58 & 0.010476879 \\
\hline Lyn(h) & 3 & 1 & 41 & 41 & 38 & 0.447058824 & 59 & 0.010657514 \\
\hline Lyn(m) & 2 & 1 & 40 & 40 & 39 & 0.458823529 & 60 & 0.01083815 \\
\hline MAPK1(h) & 42 & 1 & 40 & 40 & 40 & 0.470588235 & 60 & 0.01083815 \\
\hline MAPK2(h) & 20 & 1 & 37 & 37 & 41 & 0.482352941 & 63 & 0.011380058 \\
\hline MAPK2(m) & 18 & 1 & 35 & 35 & 42 & 0.494117647 & 65 & 0.011741329 \\
\hline MAPKAP-K2(h) & 40 & 1 & 35 & 35 & 43 & 0.505882353 & 65 & 0.011741329 \\
\hline MEK1(h) & 40 & 1 & 35 & $\begin{array}{l}35 \\
35\end{array}$ & 44 & 0.517647059 & 65 & 0.011741329 \\
\hline MKK4(m) & 14 & 1 & 32 & 32 & 45 & 0.529411765 & 68 & 0.012283237 \\
\hline $\begin{array}{l}\text { MKKK(m) } \\
\text { MKK } 6(\mathrm{~h})\end{array}$ & $\begin{array}{c}4 \\
6\end{array}$ & 1 & 31 & 31 & 46 & 0.541176471 & $\begin{array}{l}60 \\
69\end{array}$ & 0.012463873 \\
\hline MKКㄱ(h) & 13 & 1 & 28 & 28 & 47 & 0.552941176 & 72 & 0.01300578 \\
\hline MSK1(h) & 35 & 1 & 27 & 27 & 48 & 0.564705882 & 73 & 0.013186416 \\
\hline $\mathrm{p} 70 \mathrm{~s} 6 \mathrm{~K}(\mathrm{~h})$ & 7 & 1 & 20 & 20 & 49 & 0.576470588 & 80 & 0.014450867 \\
\hline $\begin{array}{l}\text { P/DSOK (h) } \\
\text { PAK(h) }\end{array}$ & 61 & 0 & 18 & 18 & 50 & 0.588235294 & 82 & 0.014812139 \\
\hline PDGFRa(h) & 35 & 1 & 18 & 18 & 51 & 0.6 & 82 & 0.014812139 \\
\hline PDGFRB(h) & 47 & 1 & 15 & 15 & 52 & 0.611764706 & 85 & 0.015354046 \\
\hline PDKI(h) & 2 & 1 & 15 & 15 & 53 & 0.623529412 & 85 & 0.015354046 \\
\hline PKA(b) & 46 & 1 & 14 & 14 & 54 & 0.635294118 & 86 & 0.015534682 \\
\hline PKA(h) & 52 & 0 & 14 & 14 & 55 & 0.647058824 & 86 & 0.015534682 \\
\hline PKBa(h) & 74 & 0 & 14 & 14 & 56 & 0.658823529 & 86 & 0.015534682 \\
\hline $\begin{array}{l}\text { PKBB(h) } \\
\text { (h) }\end{array}$ & $\begin{array}{l}74 \\
73\end{array}$ & 0 & $\begin{array}{l}14 \\
13\end{array}$ & $\begin{array}{l}14 \\
13\end{array}$ & 57 & 0.670588235 & 87 & 0.015715318 \\
\hline $\mathrm{PKB}_{\gamma}(\mathrm{h})$ & 71 & 0 & 13 & 13 & 58 & 0.682352941 & 87 & 0.015715318 \\
\hline PKG(h) & 28 & 1 & 11 & 11 & 59 & 0.694117647 & 89 & 0.01607659 \\
\hline РКCBII(h) & 54 & 0 & 11 & 11 & 60 & 0.705882353 & 89 & 0.01607659 \\
\hline PKCy(h) & 52 & 0 & 10 & 10 & 61 & 0.717647059 & 90 & 0.016257225 \\
\hline РКБ(h) & 64 & 0 & 10 & 10 & 62 & 0.729411765 & 90 & 0.016257225 \\
\hline PKC:(h) & 52 & 0 & 8 & 8 & 63 & 0.741176471 & 92 & 0.016618497 \\
\hline 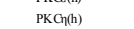 & 85 & 0 & 7 & 7 & 64 & 0.752941176 & 93 & 0.016799133 \\
\hline PKC (h) & 78 & 0 & 7 & 7 & 65 & 0.764705882 & 93 & 0.016799133 \\
\hline PKC (h) & 1 & 1 & 7 & 7 & 66 & 0.776470588 & 93 & 0.016799133 \\
\hline 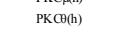 & 61 & 0 & 7 & 7 & 67 & 0.788235294 & 93 & 0.016799133 \\
\hline PKD2(h) & 3 & 1 & 7 & 7 & 68 & 0.8 & 93 & 0.016799133 \\
\hline PRAK(h) & 13 & 1 & 6 & 6 & 69 & 0.811764706 & 94 & 0.016979769 \\
\hline PRK2(h) & 3 & 1 & 6 & 6 & 70 & 0.823529412 & 94 & 0.016979769 \\
\hline ROCK-II(h) & 7 & 1 & 5 & 5 & 71 & 0.835294118 & 95 & 0.017160405 \\
\hline ROCK-II(r) & 10 & 1 & 5 & 5 & 72 & 0.847058824 & 95 & 0.017160405 \\
\hline Rskl(h) & 8 & 1 & 4 & 4 & 73 & 0.858823529 & 96 & 0.01734104 \\
\hline $\operatorname{Rskl(r)}$ & 2 & 1 & 4 & 4 & 74 & 0.870588235 & 96 & 0.01734104 \\
\hline $\begin{array}{l}\text { Rskit(t) } \\
\text { Rsk }\end{array}$ & 5 & 1 & 4 & $\begin{array}{l}4 \\
4\end{array}$ & 75 & 0.882352941 & 96 & 0.01734104 \\
\hline Rsk3(h) & 4 & 1 & 3 & 3 & 76 & 0.894117647 & 97 & 0.017521676 \\
\hline SAPK2a(h) & 74 & 0 & 3 & 3 & 77 & 0.905882353 & 97 & 0.017521676 \\
\hline SAPK2b(h) & 84 & 0 & 3 & 3 & 78 & 0.917647059 & 97 & 0.017521676 \\
\hline $\mathrm{SAPK} 3(\mathrm{~h})$ & 35 & 1 & 3 & 3 & 79 & 0.929411765 & 97 & 0.017521676 \\
\hline SAPK4(h) & 57 & 0 & 2 & 2 & 80 & 0.941176471 & 98 & 0.017702312 \\
\hline $\mathrm{SGK}(\mathrm{h})$ & 10 & 1 & 2 & 2 & 81 & 0.952941176 & 98 & 0.017702312 \\
\hline Syk(h) & 7 & 1 & 2 & 2 & 82 & 0.964705882 & 98 & 0.017702312 \\
\hline TrkB(h) & 0 & 1 & 2 & 2 & 83 & 0.976470588 & 98 & 0.017702312 \\
\hline Yes(h) & 42 & 1 & 1 & 1 & 84 & 0.988235294 & 99 & 0.017882948 \\
\hline ZAP-70(h) & 44 & 1 & 0 & 0 & 85 & 1 & 100 & 0.018063584 \\
\hline & & & & & & Total inhibition & 5536 & \\
\hline & $\begin{array}{l}\text { Hit rate } \\
\text { Threshold }\end{array}$ & & & & & & & \\
\hline & Threshold & & & & & & & \\
\hline
\end{tabular}




\begin{tabular}{|c|c|c|c|c|c|c|c|c|c|}
\hline & $100 \mu \mathrm{M}$ ATP & & & & & & & & \\
\hline Kinase & K252c @ $10 \mu \mathrm{M}$ & Hit & sorted & normalized & position & cumulative sample fraction & inhibition\% & fraction of total inhibition & cumulative inhibition \\
\hline $\mathrm{Abl}(\mathrm{m})$ & 93 & 0 & 133 & 100 & 1 & 0.011764706 & 0 & 0 & 0 \\
\hline AMPK(r) & 59 & 0 & 106 & 100 & 2 & 0.023529412 & 0 & 0 & 0 \\
\hline $\operatorname{Arg}(\mathrm{m})$ & 106 & 0 & 100 & 100 & 3 & 0.035294118 & 0 & 0 & 0 \\
\hline Aurora-A(h) & 10 & 1 & 99 & 99 & 4 & 0.047058824 & 1 & 0.000244081 & 0.000244081 \\
\hline Axl(h) & 19 & 1 & 99 & 99 & 5 & 0.058823529 & 1 & 0.000244081 & 0.000488162 \\
\hline $\operatorname{Blk}(\mathrm{m})$ & 31 & 1 & 96 & 96 & 6 & 0.070588235 & 4 & 0.000976324 & 0.001464486 \\
\hline Bnx(h) & 78 & 0 & 94 & 94 & 7 & 0.082352941 & 6 & 0.001464486 & 0.002928972 \\
\hline CaMKII(r) & 12 & 1 & 94 & 94 & 8 & 0.094117647 & 6 & 0.001464486 & 0.004393459 \\
\hline CaMKIV(h) & 99 & 0 & 93 & 93 & 9 & 0.105882353 & 7 & 0.001708567 & 0.006102026 \\
\hline $\mathrm{CDK} 1 /$ cyclinB(h) & 25 & 1 & 93 & 93 & 10 & 0.117647059 & 7 & 0.001708567 & 0.007810593 \\
\hline $\mathrm{CDK} /$ /cyclinA(h) & 33 & 1 & 90 & 90 & 11 & 0.129411765 & 10 & 0.00244081 & 0.010251403 \\
\hline $\mathrm{CDK} 2 /$ cyclinE(h) & 23 & 1 & 90 & 90 & 12 & 0.141176471 & 10 & 0.00244081 & 0.012692214 \\
\hline CDK $3 /$ cyclinE(h) & 58 & 0 & 89 & 89 & 13 & 0.152941176 & 11 & 0.002684891 & 0.015377105 \\
\hline CDK5/335(h) & 31 & 1 & 88 & 88 & 14 & 0.164705882 & 12 & 0.002928972 & 0.018306078 \\
\hline $\mathrm{CDK} 6 / \mathrm{cyclin} \mathrm{L} 3(\mathrm{~h})$ & 26 & 1 & 88 & 88 & 15 & 0.176470588 & 12 & 0.002928972 & 0.02123505 \\
\hline CDK7/cyclinHMATI(h) & 85 & 0 & 87 & 87 & 16 & 0.188235294 & 13 & 0.003173053 & 0.024408103 \\
\hline CHK I(h) & 4 & 1 & 86 & 86 & 17 & 0.2 & 14 & 0.003417134 & 0.027825238 \\
\hline СHK2(h) & 5 & 1 & 85 & 85 & 18 & 0.211764706 & 15 & 0.003661216 & 0.031486454 \\
\hline CKI(y) & 94 & 0 & 85 & 85 & 19 & 0.223529412 & 15 & 0.003661216 & 0.035147669 \\
\hline CK2(h) & 88 & 0 & 85 & 85 & 20 & 0.235294118 & 15 & 0.003661216 & 0.038808885 \\
\hline$c-\operatorname{RAF}(\mathrm{h})$ & 100 & 0 & 84 & 84 & 21 & 0.247058824 & 16 & 0.003905297 & 0.042714181 \\
\hline CSK(h) & 99 & 0 & 78 & 78 & 22 & 0.258823529 & 22 & 0.005369783 & 0.048083964 \\
\hline $\mathrm{cSRC(h)}$ & 73 & 0 & 78 & 78 & 23 & 0.270588235 & 22 & 0.005369783 & 0.053453747 \\
\hline Fes(h) & 64 & 0 & 75 & 75 & 24 & 0.282352941 & 25 & 0.006102026 & 0.059555773 \\
\hline FGFR3(h) & 70 & 0 & 74 & 74 & 25 & 0.294117647 & 26 & 0.006346107 & 0.065901879 \\
\hline Fllt3(h) & 2 & 1 & 74 & 74 & 26 & 0.305882353 & 26 & 0.006346107 & 0.072247986 \\
\hline Fyn(h) & 46 & 1 & 74 & 74 & 27 & 0.317647059 & 26 & 0.006346107 & 0.078594093 \\
\hline GSK3a(h) & 87 & 0 & 74 & 74 & 28 & 0.329411765 & 26 & 0.006346107 & 0.0849402 \\
\hline GSK3B(h) & 78 & 0 & 74 & 74 & 29 & 0.341176471 & 26 & 0.006346107 & 0.091286307 \\
\hline IGF-1R(h) & 73 & 0 & 73 & 73 & 30 & 0.352941176 & 27 & 0.006590188 & 0.097876495 \\
\hline IKKu(h) & 42 & 1 & 73 & 73 & 31 & 0.364705882 & 27 & 0.006590188 & 0.104466683 \\
\hline $\mathrm{IKKB(h)}$ & 75 & 0 & 73 & 73 & 32 & 0.376470588 & 27 & 0.006590188 & 0.111056871 \\
\hline $\operatorname{IR(h)}$ & 62 & 0 & 73 & 73 & 33 & 0.388235294 & 27 & 0.006590188 & 0.117647059 \\
\hline $\begin{array}{l}\text { INKIflal(h) } \\
\text { J }\end{array}$ & 67 & 0 & 72 & 72 & 34 & 0.4 & 28 & 0.006834269 & 0.124481328 \\
\hline JNK2a2(h) & 86 & 0 & 71 & 71 & 35 & 0.411764706 & 29 & 0.00707835 & 0.131559678 \\
\hline JNK3(r) & 20 & 1 & 70 & 70 & 36 & 0.423529412 & 30 & 0.007322431 & 0.138882109 \\
\hline Lck(h) & 72 & 0 & 67 & 67 & 37 & 0.435294118 & 33 & 0.008054674 & 0.146936783 \\
\hline Lyn(h) & 25 & 1 & 67 & 67 & 38 & 0.447058824 & 33 & 0.008054674 & 0.154991457 \\
\hline Lyn(m) & 5 & 1 & 64 & 64 & 39 & 0.458823529 & 36 & 0.008786917 & 0.163778374 \\
\hline MAPK 1 (h) & 61 & 0 & 62 & 62 & 40 & 0.470588235 & 38 & 0.009275079 & 0.173053454 \\
\hline MAPK2(h) & 31 & 1 & 61 & 61 & 41 & 0.482352941 & 39 & 0.00951916 & 0.182572614 \\
\hline MAPK2(m) & 30 & 1 & 60 & 60 & 42 & 0.494117647 & 40 & 0.009763241 & 0.192335856 \\
\hline MAPKAP-K2(h) & 67 & 0 & 59 & 59 & 43 & 0.505882353 & 41 & 0.010007322 & 0.202343178 \\
\hline MEK 1(h) & 74 & 0 & 58 & 58 & 44 & 0.517647059 & 42 & 0.010251403 & 0.212594581 \\
\hline MKK 4(m) & 46 & 1 & 46 & 46 & 45 & 0.529411765 & 54 & 0.013180376 & 0.225774957 \\
\hline MKK6(h) & 10 & 1 & 46 & 46 & 46 & 0.541176471 & 54 & 0.013180376 & 0.238955333 \\
\hline MKK $78($ (h) & 27 & 1 & 45 & 45 & 47 & 0.552941176 & 55 & 0.013424457 & 0.25237979 \\
\hline MSK I(h) & 45 & 1 & 45 & 45 & 48 & 0.564705882 & 55 & 0.013424457 & 0.265804247 \\
\hline p70s6K(h) & 28 & 1 & 44 & 44 & 49 & 0.576470588 & 56 & 0.013668538 & 0.279472785 \\
\hline PAK2(h) & 88 & 0 & 42 & 42 & 50 & 0.588235294 & 58 & 0.0141567 & 0.293629485 \\
\hline PDGFRa(h) & 71 & 0 & 37 & 37 & 51 & 0.6 & 63 & 0.015377105 & 0.30900659 \\
\hline PDGFRB(h) & 44 & 1 & 36 & 36 & 52 & 0.611764706 & 64 & 0.015621186 & 0.324627776 \\
\hline PDKI(h) & 4 & 1 & 33 & 33 & 53 & 0.623529412 & 67 & 0.016353429 & 0.340981206 \\
\hline PKA(b) & 96 & 0 & 31 & 31 & 54 & 0.635294118 & 69 & 0.016841591 & 0.357822797 \\
\hline PKA(h) & 85 & 0 & 31 & 31 & 55 & 0.647058824 & 69 & 0.016841591 & 0.374664389 \\
\hline PKBy(h) & 85 & 0 & 31 & 31 & 56 & 0.658823529 & 69 & 0.016841591 & 0.39150598 \\
\hline PKBB(h) & 73 & 0 & 30 & 30 & 57 & 0.670588235 & 70 & 0.017085672 & 0.408591652 \\
\hline $\mathrm{PKB}_{(y)}(\mathrm{h})$ & 93 & 0 & 28 & 28 & 58 & 0.682352941 & 72 & 0.017573835 & 0.426165487 \\
\hline $\mathrm{PKCu(h)}$ & 36 & 1 & 27 & 27 & 59 & 0.694117647 & 73 & 0.017817916 & 0.443983402 \\
\hline PKCBII(h) & 74 & 0 & 26 & 26 & 60 & 0.705882353 & 74 & 0.018061997 & 0.462045399 \\
\hline PKCy(h) & 74 & 0 & 25 & 25 & 61 & 0.717647059 & 75 & 0.018306078 & 0.480351477 \\
\hline РКС(h) & 94 & 0 & 25 & 25 & 62 & 0.729411765 & 75 & 0.018306078 & 0.498657554 \\
\hline PKC:(h) & 60 & 0 & 23 & 23 & 63 & 0.741176471 & 77 & 0.01879424 & 0.517451794 \\
\hline PKG (h) & 89 & 0 & 23 & 23 & 64 & 0.752941176 & 77 & 0.01879424 & 0.536246034 \\
\hline PKC (h) & 74 & 0 & 22 & 22 & 65 & 0.764705882 & 78 & 0.019038321 & 0.555284354 \\
\hline $\mathrm{PKC}(\mathrm{hlh})$ & 3 & 1 & 20 & 20 & 66 & 0.776470588 & 80 & 0.019526483 & 0.574810837 \\
\hline PKCO(h) & 73 & 0 & 19 & 19 & 67 & 0.788235294 & 81 & 0.019770564 & 0.594581401 \\
\hline PKD2(h) & 4 & 1 & 18 & 18 & 68 & 0.8 & 82 & 0.020014645 & 0.614596046 \\
\hline PRAK(h) & 37 & 1 & 17 & 17 & 69 & 0.811764706 & 83 & 0.020258726 & 0.634854772 \\
\hline PRK2(h) & $\begin{array}{r}51 \\
1\end{array}$ & 1 & 16 & 16 & 70 & 0.823529412 & 84 & 0.020502807 & 0.655357579 \\
\hline ROCK-II(h) & 22 & 1 & 13 & 13 & 71 & 0.835294118 & 87 & 0.02123505 & 0.676592629 \\
\hline ROCK-II(r) & 16 & 1 & 12 & 12 & 72 & 0.847058824 & 88 & 0.021479131 & 0.69807176 \\
\hline Rskl(h) & 17 & 1 & 10 & 10 & 73 & 0.858823529 & 90 & 0.021967293 & 0.720039053 \\
\hline Rskl(r) & 5 & 1 & 10 & 10 & 74 & 0.870588235 & 90 & 0.021967293 & 0.742006346 \\
\hline Rsk2(h) & 18 & 1 & 9 & 9 & 75 & 0.882352941 & 91 & 0.022211374 & 0.76421772 \\
\hline Rsk3(h) & 13 & 1 & 5 & 5 & 76 & 0.894117647 & 95 & 0.023187698 & 0.787405419 \\
\hline SAPK2a(h) & 74 & 0 & 5 & 5 & 77 & 0.905882353 & 95 & 0.023187698 & 0.810593117 \\
\hline SAPK2b(h) & 133 & 0 & 5 & 5 & 78 & 0.917647059 & 95 & 0.023187698 & 0.833780815 \\
\hline $\mathrm{SAPK} 3(\mathrm{~h})$ & 90 & 0 & 4 & 4 & 79 & 0.929411765 & 96 & 0.023431779 & 0.857212595 \\
\hline SAPK4(h) & 84 & 0 & 4 & 4 & 80 & 0.941176471 & 96 & 0.023431779 & 0.880644374 \\
\hline SGK(h) & 23 & 1 & 4 & 4 & 81 & 0.952941176 & 96 & 0.023431779 & 0.904076153 \\
\hline Syk(h) & 9 & 1 & 3 & 3 & 82 & 0.964705882 & 97 & 0.02367586 & 0.927752014 \\
\hline TrkB(h) & 1 & 1 & 2 & 2 & 83 & 0.976470588 & 98 & 0.023919941 & 0.951671955 \\
\hline Yes(h) & 45 & 1 & 1 & 1 & 84 & 0.988235294 & 99 & 0.024164022 & 0.975835978 \\
\hline ZAP-70(h) & 90 & 0 & 1 & 1 & 85 & 1 & 99 & 0.024164022 & 1 \\
\hline & & & & & & Total inhibition & 4097 & & Total area \\
\hline & $\begin{array}{l}\text { Hit rate } \\
\text { Threshold }\end{array}$ & $\begin{array}{l}4 \\
5\end{array}$ & & & & & & & Gini \\
\hline
\end{tabular}




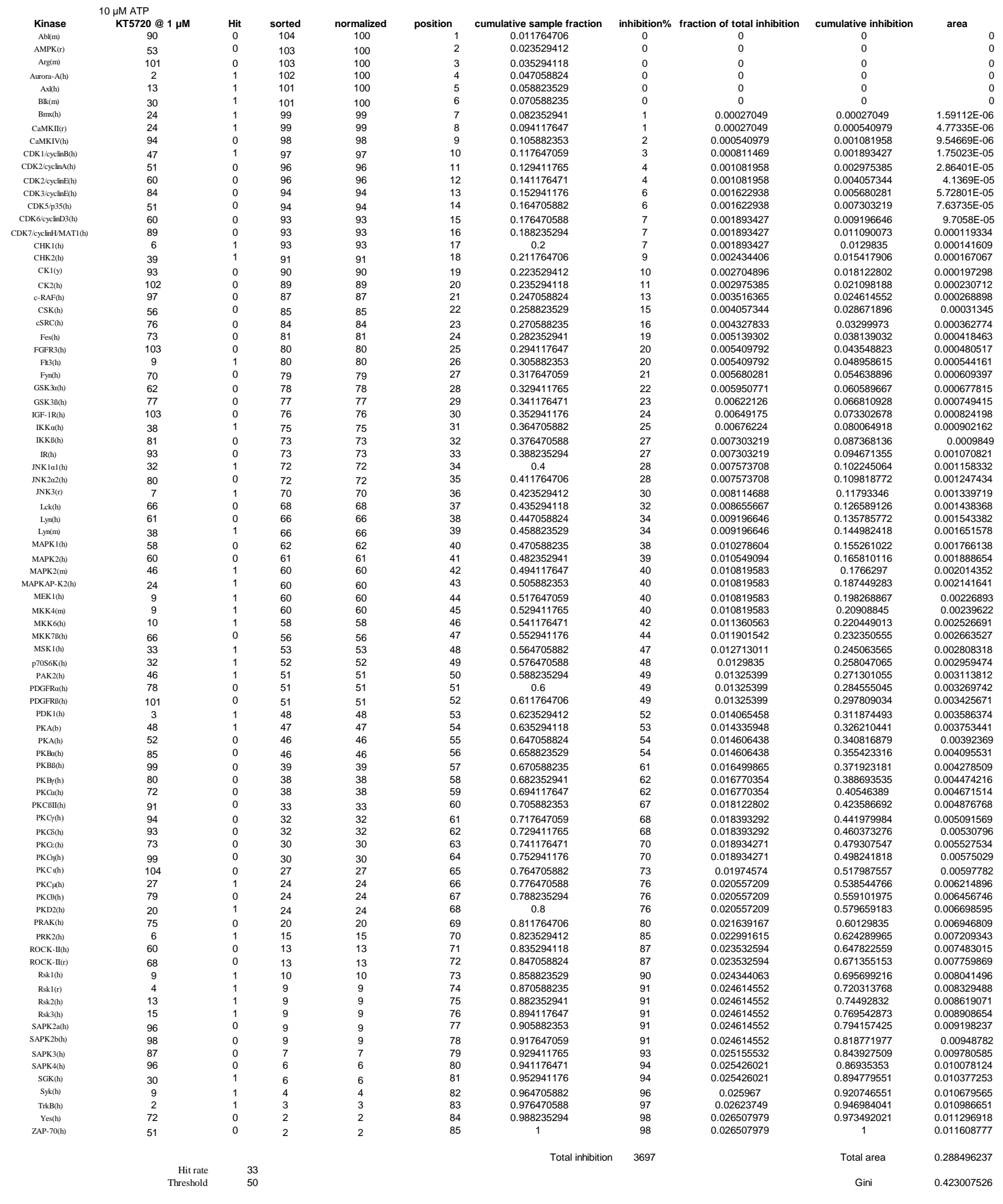




\begin{tabular}{|c|c|c|c|c|c|c|c|c|c|}
\hline & $100 \mu \mathrm{M}$ ATP & & & & & & & & \\
\hline Kinase & KT5720@ @ 1 нM & Hit & sorted & normalized & position & cumulative sample fraction & inhibition\% & fraction of total inhibition & cumulative inhibition \\
\hline $\mathrm{Abl}(\mathrm{m})$ & 93 & 0 & 124 & 100 & 1 & 0.011764706 & 0 & 0 & 0 \\
\hline AMPK(r) & 92 & 0 & 115 & 100 & 2 & 0.023529412 & 0 & 0 & 0 \\
\hline $\operatorname{Arg}(\mathrm{m})$ & 92 & 0 & 109 & 100 & 3 & 0.035294118 & 0 & 0 & 0 \\
\hline Aurora-A(h) & 13 & 1 & 106 & 100 & 4 & 0.047058824 & 0 & 0 & 0 \\
\hline $\mathrm{Ax}(\mathrm{h})$ & 28 & 1 & 106 & 100 & 5 & 0.058823529 & 0 & 0 & 0 \\
\hline $\operatorname{Blk}(\mathbf{m})$ & 40 & 1 & 102 & 100 & 6 & 0.070588235 & 0 & 0 & 0 \\
\hline Bmx(h) & 49 & 1 & 102 & 100 & 7 & 0.082352941 & 0 & 0 & 0 \\
\hline CaMKII(r) & 44 & 1 & 102 & 100 & 8 & 0.094117647 & 0 & 0 & 0 \\
\hline CaMKIV(h) & 115 & 0 & 102 & 100 & 9 & 0.105882353 & 0 & 0 & 0 \\
\hline CDK1/cyclinB(h) & 78 & 0 & 102 & 100 & 10 & 0.117647059 & 0 & 0 & 0 \\
\hline $\mathrm{CDK} 2 /$ cyclinA(h) & 67 & 0 & 101 & 100 & 11 & 0.129411765 & 0 & 0 & 0 \\
\hline $\mathrm{CDK} 2 / \mathrm{cyclin} E(\mathrm{~h})$ & 78 & 0 & 101 & 100 & 12 & 0.141176471 & 0 & 0 & 0 \\
\hline CDK3/ycycling(h) & 96 & 0 & 100 & 100 & 13 & 0.152941176 & 0 & 0 & 0 \\
\hline CDK5/335(h) & 109 & 0 & 100 & 100 & 14 & 0.164705882 & 0 & 0 & 0 \\
\hline CDK6/cyclinD33(h) & 81 & 0 & 97 & 97 & 15 & 0.176470588 & 3 & 0.001238135 & 0.001238135 \\
\hline CDK7/cyclinHAATI(h) & 93 & 0 & 97 & 97 & 16 & 0.188235294 & 3 & 0.001238135 & 0.002476269 \\
\hline CHK I(h) & 9 & 1 & 97 & 97 & 17 & 0.2 & 3 & 0.001238135 & 0.003714404 \\
\hline СHK2(h) & 54 & 0 & 97 & 97 & 18 & 0.211764706 & 3 & 0.001238135 & 0.004952538 \\
\hline $\mathrm{CKI}(\mathrm{y}) \mathrm{f}$ & 106 & 0 & 96 & 96 & 19 & 0.223529412 & 4 & 0.001650846 & 0.006603384 \\
\hline СK2(h) & 102 & 0 & 96 & 96 & 20 & 0.235294118 & 4 & 0.001650846 & 0.00825423 \\
\hline c-RAF(h) & 94 & 0 & 96 & 96 & 21 & 0.247058824 & 4 & 0.001650846 & 0.009905076 \\
\hline CSK (h) & 92 & 0 & 95 & 95 & 22 & 0.258823529 & 5 & 0.002063558 & 0.011968634 \\
\hline cSRC(h) & 84 & 0 & 94 & 94 & 23 & 0.270588235 & 6 & 0.002476269 & 0.014444903 \\
\hline Fes(h) & 100 & 0 & 94 & 94 & 24 & 0.282352941 & 6 & 0.002476269 & 0.016921172 \\
\hline FGFR3(h) & 88 & 0 & 93 & 93 & 25 & 0.294117647 & 7 & 0.002888981 & 0.019810153 \\
\hline Flls(h) & 15 & 1 & 93 & 93 & 26 & 0.305882353 & 7 & 0.002888981 & 0.022699133 \\
\hline Fyn(h) & 89 & 0 & 93 & 93 & 27 & 0.317647059 & 7 & 0.002888981 & 0.025588114 \\
\hline GSK3a(h) & 94 & 0 & 92 & 92 & 28 & 0.329411765 & 8 & 0.003301692 & 0.028889806 \\
\hline GSK3B(h) & 102 & 0 & 92 & 92 & 29 & 0.341176471 & 8 & 0.003301692 & 0.032191498 \\
\hline IGF-1R(h) & 106 & 0 & 92 & 92 & 30 & 0.352941176 & 8 & 0.003301692 & 0.03549319 \\
\hline $\mathrm{IKKa}(\mathrm{h})$ & 93 & 0 & 92 & 92 & 31 & 0.364705882 & 8 & 0.003301692 & 0.038794882 \\
\hline IKKB(h) & 97 & 0 & 92 & 92 & 32 & 0.376470588 & 8 & 0.003301692 & 0.042096574 \\
\hline $\mathbb{R}(\mathrm{h})$ & 102 & 0 & 89 & 89 & 33 & 0.388235294 & 11 & 0.004539827 & 0.046636401 \\
\hline JNKIal(h) & 74 & 0 & 88 & 88 & 34 & 0.4 & 12 & 0.004952538 & 0.051588939 \\
\hline JNK202(h) & 97 & 0 & 86 & 86 & 35 & 0.411764706 & 14 & 0.005777961 & 0.057366901 \\
\hline $\mathrm{JNK} 3(\mathrm{r})$ & 35 & 1 & 85 & 85 & 36 & 0.423529412 & 15 & 0.006190673 & 0.063557573 \\
\hline Lck(h) & 82 & 0 & 84 & 84 & 37 & 0.435294118 & 16 & 0.006603384 & 0.070160957 \\
\hline Lyn(h) & 69 & 0 & 83 & 83 & 38 & 0.447058824 & 17 & 0.007016096 & 0.077177053 \\
\hline Lyyn(m) & 69 & 0 & 83 & 83 & 39 & 0.458823529 & 17 & 0.007016096 & 0.084193149 \\
\hline MAPKI(h) & 63 & 0 & 82 & 82 & 40 & 0.470588235 & 18 & 0.007428807 & 0.091621956 \\
\hline MAPK2(h) & 72 & 0 & 81 & 81 & 41 & 0.482352941 & 19 & 0.007841519 & 0.099463475 \\
\hline MAPK2(m) & 65 & 0 & 80 & 80 & 42 & 0.494117647 & 20 & 0.00825423 & 0.107717705 \\
\hline MAPKAP-K2(h) & 76 & 0 & 78 & 78 & 43 & 0.505882353 & 22 & 0.009079653 & 0.116797359 \\
\hline MEKI(h) & 38 & 1 & 78 & 78 & 44 & 0.517647059 & 22 & 0.009079653 & 0.125877012 \\
\hline MKK $4(\mathrm{~m})$ & 73 & 0 & 78 & 78 & 45 & 0.529411765 & 22 & 0.009079653 & 0.134956665 \\
\hline MKK6(h) & 30 & 1 & 78 & 78 & 46 & 0.541176471 & 22 & 0.009079653 & 0.144036319 \\
\hline МKK $7($ (h) & 76 & 0 & 76 & 76 & 47 & 0.552941176 & 24 & 0.009905076 & 0.153941395 \\
\hline MSKI(h) & 44 & 1 & 76 & 76 & 48 & 0.564705882 & 24 & 0.009905076 & 0.163846471 \\
\hline $\mathrm{p} 70 \mathrm{~S} 6 \mathrm{~K}(\mathrm{~h})$ & 75 & 0 & 75 & 75 & 49 & 0.576470588 & 25 & 0.010317788 & 0.174164259 \\
\hline $\begin{array}{l}\text { PAK2(h) } \\
\text { PAK }\end{array}$ & 72 & 0 & 74 & 74 & 50 & 0.588235294 & 26 & 0.010730499 & 0.184894759 \\
\hline PDGFRa(h) & 72 & 0 & 74 & 74 & 51 & 0.6 & 26 & 0.010730499 & 0.195625258 \\
\hline PDGFRB(h) & 80 & 0 & 73 & 73 & 52 & 0.611764706 & 27 & 0.011143211 & 0.206768469 \\
\hline PDK I(h) & 8 & 1 & 72 & 72 & 53 & 0.623529412 & 28 & 0.011555922 & 0.218324391 \\
\hline PKA(b) & 78 & 0 & 72 & 72 & 54 & 0.635294118 & 28 & 0.011555922 & 0.229880314 \\
\hline PKA(h) & 74 & 0 & 72 & 72 & 55 & 0.647058824 & 28 & 0.011555922 & 0.241436236 \\
\hline PKBach) & 83 & 0 & 71 & 71 & 56 & 0.658823529 & 29 & 0.011968634 & 0.25340487 \\
\hline PKBB(h) & 92 & 0 & 70 & 70 & 57 & 0.670588235 & 30 & 0.012381345 & 0.265786215 \\
\hline $\mathrm{PKB}_{\mathrm{y}(\mathrm{(h})}$ & 86 & 0 & 69 & 69 & 58 & 0.682352941 & 31 & 0.012794057 & 0.278580272 \\
\hline PKG(h) & 96 & 0 & 69 & 69 & 59 & 0.694117647 & 31 & 0.012794057 & 0.291374329 \\
\hline РКCBII(h) & 100 & 0 & 67 & 67 & 60 & 0.705882353 & 33 & 0.01361948 & 0.304993809 \\
\hline PKCy(h) & 96 & 0 & 65 & 65 & 61 & 0.717647059 & 35 & 0.014444903 & 0.319438712 \\
\hline РКడ(h) & 102 & 0 & 63 & 63 & 62 & 0.729411765 & 37 & 0.015270326 & 0.334709038 \\
\hline PKG:(h) & 83 & 0 & 57 & 57 & 63 & 0.741176471 & 43 & 0.017746595 & 0.352455634 \\
\hline PKG(h) & 101 & 0 & 54 & 54 & 64 & 0.752941176 & 46 & 0.01898473 & 0.371440363 \\
\hline PKC (h) & 102 & 0 & 49 & 49 & 65 & 0.764705882 & 51 & 0.021048287 & 0.39248865 \\
\hline PKC $(\mathrm{h}(\mathrm{h})$ & 42 & 1 & 44 & 44 & 66 & 0.776470588 & 56 & 0.023111845 & 0.415600495 \\
\hline PKCO(h) & 92 & 0 & 44 & 44 & 67 & 0.788235294 & 56 & 0.023111845 & 0.43871234 \\
\hline PKD2(h) & 25 & 1 & 42 & 42 & 68 & 0.8 & 58 & 0.023937268 & 0.462649608 \\
\hline PRAK(h) & 70 & 0 & 40 & 40 & 69 & 0.811764706 & 60 & 0.024762691 & 0.487412299 \\
\hline PRK2(h) & 8 & 1 & 40 & 40 & 70 & 0.823529412 & 60 & 0.024762691 & 0.51217499 \\
\hline ROCK-II(h) & 85 & 0 & 38 & 38 & 71 & 0.835294118 & 62 & 0.025588114 & 0.537763104 \\
\hline ROCK-II(r) & 71 & 0 & 35 & 35 & 72 & 0.847058824 & 65 & 0.026826248 & 0.564589352 \\
\hline Rskl(h) & 23 & 1 & 31 & 31 & 73 & 0.858823529 & 69 & 0.028477095 & 0.593066447 \\
\hline Rskl(r) & 18 & 1 & 30 & 30 & 74 & 0.870588235 & 70 & 0.028889806 & 0.621956253 \\
\hline Rsk2(h) & 40 & 1 & 28 & 28 & 75 & 0.882352941 & 72 & 0.029715229 & 0.651671482 \\
\hline Rsk3(h) & 31 & 1 & 25 & 25 & 76 & 0.894117647 & 75 & 0.030953364 & 0.682624845 \\
\hline SAPK2a(h) & 97 & 0 & 25 & 25 & 77 & 0.905882353 & 75 & 0.030953364 & 0.713578209 \\
\hline SAPK2b(h) & 124 & 0 & 23 & 23 & 78 & 0.917647059 & 77 & 0.031778787 & 0.745356995 \\
\hline $\mathrm{SAPK} 3(\mathrm{~h})$ & 101 & 0 & 18 & 18 & 79 & 0.929411765 & 82 & 0.033842344 & 0.77919934 \\
\hline SAPK4(h) & 97 & 0 & 15 & 15 & 80 & 0.941176471 & 85 & 0.035080479 & 0.814279818 \\
\hline SGK(h) & 57 & 0 & 13 & 13 & 81 & 0.952941176 & 87 & 0.035905902 & 0.85018572 \\
\hline Syk(h) & 25 & 1 & 12 & 12 & 82 & 0.964705882 & 88 & 0.036318613 & 0.886504333 \\
\hline $\operatorname{TrkB(h)}$ & 12 & 1 & 9 & 9 & 83 & 0.976470588 & 91 & 0.037556748 & 0.924061081 \\
\hline Yes(h) & 78 & 0 & 8 & 8 & 84 & 0.988235294 & 92 & 0.037969459 & 0.962030541 \\
\hline ZAP-70(h) & 95 & 0 & 8 & 8 & 85 & 1 & 92 & 0.037969459 & 1 \\
\hline & & & & & & Total inhibition & 2423 & & Total area \\
\hline & $\begin{array}{l}\text { Hit rate } \\
\text { Threshold }\end{array}$ & $\begin{array}{l}2 \\
5\end{array}$ & & & & & & & Gini \\
\hline
\end{tabular}




\begin{tabular}{|c|c|c|c|c|c|c|c|c|c|}
\hline & $10 \mu \mathrm{M}$ ATP & & & & & & & & \\
\hline Kinase & Lavendustin A@1 1 M & Hit & sorted & normalized & position & cumulative sample fraction & inhibition\% & fraction of total inhibition & cumulative inhibition \\
\hline $\mathrm{Abl}(\mathrm{m})$ & 88 & 0 & 147 & 100 & 1 & 0.011764706 & 0 & 0 & 0 \\
\hline AMPK(r) & 106 & 0 & 120 & 100 & 2 & 0.023529412 & 0 & 0 & 0 \\
\hline $\operatorname{Arg}(\mathrm{m})$ & 99 & 0 & 118 & 100 & 3 & 0.035294118 & 0 & 0 & 0 \\
\hline Aurora-A(h) & 116 & 0 & 117 & 100 & 4 & 0.047058824 & 0 & 0 & 0 \\
\hline Axl(h) & 105 & 0 & 116 & 100 & 5 & 0.058823529 & 0 & 0 & 0 \\
\hline $\operatorname{Blk}(\mathrm{m})$ & 85 & 0 & 113 & 100 & 6 & 0.070588235 & 0 & 0 & 0 \\
\hline $\mathrm{Bmx(h)}$ & $\begin{array}{l}00 \\
110\end{array}$ & 0 & 112 & 100 & 7 & 0.082352941 & 0 & 0 & 0 \\
\hline CaMKII(r) & 92 & 0 & 111 & 100 & 8 & 0.094117647 & 0 & 0 & 0 \\
\hline CaMKIV(h) & 99 & 0 & 110 & 100 & 9 & 0.105882353 & 0 & 0 & 0 \\
\hline CDK1/cyclnBB(h) & 97 & 0 & 109 & 100 & 10 & 0.117647059 & 0 & 0 & 0 \\
\hline CDK2/cyclinA(h) & 100 & 0 & 108 & 100 & 11 & 0.129411765 & 0 & 0 & 0 \\
\hline $\mathrm{CDK} 2 / \mathrm{cyclin} \mathrm{E}(\mathrm{h})$ & 93 & 0 & 108 & 100 & 12 & 0.141176471 & 0 & 0 & 0 \\
\hline CDK3/cyclinE(h) & 118 & 0 & 108 & 100 & 13 & 0.152941176 & 0 & 0 & 0 \\
\hline CDK5/p35(h) & 111 & 0 & 108 & 100 & 14 & 0.164705882 & 0 & 0 & 0 \\
\hline CDKG/cyclinD3(h) & 103 & 0 & 108 & 100 & 15 & 0.176470588 & 0 & 0 & 0 \\
\hline CDK $7 /$ yclinHMATI(h) & 102 & 0 & 107 & 100 & 16 & 0.188235294 & 0 & 0 & 0 \\
\hline CHKI(h) & 147 & 0 & 107 & 100 & 17 & 0.2 & 0 & 0 & 0 \\
\hline CHK2(h) & 101 & 0 & 107 & 100 & 18 & 0.211764706 & 0 & 0 & 0 \\
\hline CKI(y) & 101 & 0 & 106 & 100 & 19 & 0.223529412 & 0 & 0 & 0 \\
\hline СК2(h) & 90 & 0 & 106 & 100 & 20 & 0.235294118 & 0 & 0 & 0 \\
\hline c-RAF(h) & 106 & 0 & 106 & 100 & 21 & 0.247058824 & 0 & 0 & 0 \\
\hline $\operatorname{CSK}(\mathrm{h})$ & 68 & 0 & 106 & 100 & 22 & 0.258823529 & 0 & 0 & 0 \\
\hline $\operatorname{csRC(h)}$ & 108 & 0 & 105 & 100 & 23 & 0.270588235 & 0 & 0 & 0 \\
\hline Fess(h) & 80 & 0 & 105 & 100 & 24 & 0.282352941 & 0 & 0 & 0 \\
\hline FGFR3(h) & 100 & 0 & 105 & 100 & 25 & 0.294117647 & 0 & 0 & 0 \\
\hline $\mathrm{Fll}(\mathrm{h}(\mathrm{h})$ & 101 & 0 & 104 & 100 & 26 & 0.305882353 & 0 & 0 & 0 \\
\hline Fyn(h) & 85 & 0 & 104 & 100 & 27 & 0.317647059 & 0 & 0 & 0 \\
\hline GSK3a(h) & 89 & 0 & 103 & 100 & 28 & 0.329411765 & 0 & 0 & 0 \\
\hline GSK3B(h) & 97 & 0 & 103 & 100 & $\begin{array}{l}20 \\
29\end{array}$ & 0.341176471 & 0 & 0 & 0 \\
\hline IGF-IR(h) & 106 & 0 & 103 & 100 & 30 & 0.352941176 & 0 & 0 & 0 \\
\hline IKKa(h) & 101 & 0 & 103 & 100 & 31 & 0.364705882 & 0 & 0 & 0 \\
\hline IKKB(h) & 102 & 0 & 102 & 100 & 32 & 0.376470588 & 0 & 0 & 0 \\
\hline $\mathrm{IR}(\mathrm{h})$ & 109 & 0 & 102 & 100 & 33 & 0.388235294 & 0 & 0 & 0 \\
\hline JNKIal(h) & 104 & 0 & 102 & 100 & 34 & 0.4 & 0 & 0 & 0 \\
\hline JNK202(h) & 81 & 0 & 101 & 100 & 35 & 0.411764706 & 0 & 0 & 0 \\
\hline JNK3(r) & 108 & 0 & 101 & 100 & 36 & 0.423529412 & 0 & 0 & 0 \\
\hline Lck(h) & 108 & 0 & 101 & 100 & 37 & 0.435294118 & 0 & 0 & 0 \\
\hline Lyn(h) & 105 & 0 & 101 & 100 & 38 & 0.447058824 & 0 & 0 & 0 \\
\hline Lyy(m) & 61 & 0 & 101 & 100 & 39 & 0.458823529 & 0 & 0 & 0 \\
\hline MAPKI(h) & $\begin{array}{l}61 \\
120\end{array}$ & 0 & 101 & 100 & 40 & 0.470588235 & 0 & 0 & 0 \\
\hline MAPK2(h) & 107 & 0 & 100 & 100 & 41 & 0.482352941 & 0 & 0 & 0 \\
\hline MAPK2(m) & 102 & 0 & 100 & 100 & 42 & 0.494117647 & 0 & 0 & 0 \\
\hline MAPKAP-K2(h) & 86 & 0 & 100 & 100 & 43 & 0.505882353 & 0 & 0 & 0 \\
\hline MEK1(h) & 100 & 0 & 100 & 100 & 44 & 0.517647059 & 0 & 0 & 0 \\
\hline MKK4(m) & 117 & 0 & 99 & 99 & 45 & 0.529411765 & 1 & 0.002673797 & 0.002673797 \\
\hline MKK6(h) & 96 & 0 & 99 & 99 & 46 & 0.541176471 & 1 & 0.002673797 & 0.005347594 \\
\hline MKK & 96 & 0 & 99 & 99 & 47 & 0.552941176 & 1 & 0.002673797 & 0.00802139 \\
\hline MSKI(h) & 103 & 0 & 99 & 99 & 48 & 0.564705882 & 1 & 0.002673797 & 0.010695187 \\
\hline $\mathrm{p} 70 \mathrm{~S} 6 \mathrm{~K}(\mathrm{~h})$ & 91 & 0 & 98 & 98 & 49 & 0.576470588 & 2 & 0.005347594 & 0.016042781 \\
\hline $\begin{array}{l}\text { PAK2(h) } \\
\text { Past }\end{array}$ & 100 & 0 & 97 & 97 & 50 & 0.588235294 & 3 & 0.00802139 & 0.024064171 \\
\hline PDGFRa(h) & 94 & 0 & 97 & 97 & 51 & 0.6 & 3 & 0.00802139 & 0.032085561 \\
\hline PDGFRB(h) & 94 & 0 & 97 & 97 & 52 & 0.611764706 & 3 & 0.00802139 & 0.040106952 \\
\hline PDKI(h) & 84 & 0 & 96 & 96 & 53 & 0.623529412 & 4 & 0.010695187 & 0.050802139 \\
\hline PKA(b) & 99 & 0 & 96 & 96 & 54 & 0.635294118 & 4 & 0.010695187 & 0.061497326 \\
\hline PKA(h) & 113 & 0 & 96 & 96 & 55 & 0.647058824 & 4 & 0.010695187 & 0.072192513 \\
\hline PKBa(h) & 101 & 0 & 96 & 96 & 56 & 0.658823529 & 4 & 0.010695187 & 0.082887701 \\
\hline PKB(h) & 101 & 0 & 96 & 96 & 57 & 0.670588235 & 4 & 0.010695187 & 0.093582888 \\
\hline $\mathrm{PKB}_{\mathrm{y}(\mathrm{h})}$ & 105 & 0 & 95 & 95 & 58 & 0.682352941 & $\begin{array}{l}4 \\
5\end{array}$ & 0.013368984 & 0.106951872 \\
\hline PKCath) & 94 & 0 & 95 & 95 & 59 & 0.694117647 & 5 & 0.013368984 & 0.120320856 \\
\hline PKCBII(h) & 107 & 0 & 95 & 95 & 60 & 0.705882353 & 5 & 0.013368984 & 0.13368984 \\
\hline PKCy(h) & 99 & 0 & 95 & 95 & 61 & 0.717647059 & 5 & 0.013368984 & 0.147058824 \\
\hline РКФ(h) & 108 & 0 & 94 & 94 & 62 & 0.729411765 & 6 & 0.016042781 & 0.163101604 \\
\hline PKG:(h) & 96 & 0 & 94 & 94 & 63 & 0.741176471 & 6 & 0.016042781 & 0.179144385 \\
\hline $\begin{array}{l}\text { PKG (h) } \\
\text { (h) }\end{array}$ & 108 & 0 & 94 & 94 & 64 & 0.752941176 & 6 & 0.016042781 & 0.195187166 \\
\hline PKC (h) & 95 & 0 & 94 & $\begin{array}{l}54 \\
94\end{array}$ & 65 & 0.764705882 & 6 & 0.016042781 & 0.211229947 \\
\hline PKC $\mu(\mathrm{h})$ & 103 & 0 & 93 & 93 & 66 & 0.776470588 & 7 & 0.018716578 & 0.229946524 \\
\hline PKCO(h) & 95 & 0 & 93 & 93 & 67 & 0.788235294 & 7 & 0.018716578 & 0.248663102 \\
\hline PKD2(h) & 88 & 0 & 92 & 92 & 68 & 0.8 & 8 & 0.021390374 & 0.270053476 \\
\hline PRAK(h) & $\begin{array}{l}88 \\
97\end{array}$ & 0 & $\begin{array}{l}92 \\
92\end{array}$ & $\begin{array}{l}92 \\
92\end{array}$ & 69 & 0.811764706 & 8 & 0.021390374 & 0.29144385 \\
\hline PRK2(h) & 104 & 0 & 91 & 91 & 70 & 0.823529412 & 9 & 0.024064171 & 0.315508021 \\
\hline $\begin{array}{l}\text { PRK_(n) } \\
\text { ROCK-II(h) }\end{array}$ & 96 & 0 & 91 & 91 & 71 & 0.835294118 & 9 & 0.024064171 & 0.339572193 \\
\hline ROCK-II(r) & 83 & 0 & 90 & 90 & 72 & 0.847058824 & 10 & 0.026737968 & 0.36631016 \\
\hline Rskl(h) & 85 & 0 & 89 & 89 & 73 & 0.858823529 & 11 & 0.029411765 & 0.395721925 \\
\hline Rskl(r) & 94 & 0 & 88 & 88 & 74 & 0.870588235 & 12 & 0.032085561 & 0.427807487 \\
\hline $\begin{array}{l}\text { Rsksk2(h) } \\
\text { Rsk(h) }\end{array}$ & 95 & 0 & 88 & 88 & 75 & 0.882352941 & 12 & 0.032085561 & 0.459893048 \\
\hline Rsk3(h) & 91 & 0 & 86 & 86 & 76 & 0.894117647 & 14 & 0.037433155 & 0.497326203 \\
\hline SAPK2a(h) & 95 & 0 & 85 & 85 & 77 & 0.905882353 & 15 & 0.040106952 & 0.537433155 \\
\hline SAPK2b(h) & 103 & 0 & 85 & 85 & 78 & 0.917647059 & 15 & 0.040106952 & 0.577540107 \\
\hline SAPK3(h) & 98 & 0 & 85 & 85 & 79 & 0.929411765 & 15 & 0.040106952 & 0.617647059 \\
\hline $\begin{array}{l}\mathrm{SARRS( \textrm {h } )} \\
\mathrm{SAPK} 4(\mathrm{~h})\end{array}$ & $\begin{array}{l}50 \\
93\end{array}$ & 0 & 84 & 84 & 80 & 0.941176471 & 16 & 0.042780749 & 0.660427807 \\
\hline SGK(h) & 106 & 0 & 83 & 83 & 81 & 0.952941176 & 17 & 0.045454545 & 0.705882353 \\
\hline Syk(h) & 112 & 0 & 81 & 81 & 82 & 0.964705882 & 19 & 0.050802139 & 0.756684492 \\
\hline TrkB(h) & 92 & 0 & 80 & 80 & 83 & 0.976470588 & 20 & 0.053475936 & 0.810160428 \\
\hline Yes(h) & 107 & 0 & 68 & 68 & 84 & 0.988235294 & 32 & 0.085561497 & 0.895721925 \\
\hline ZAP-70(h) & 96 & 0 & 61 & 61 & 85 & 1 & 39 & 0.104278075 & 1 \\
\hline & & & & & & Total inhibition & 374 & & Total area \\
\hline & Hit rate & 5 & & & & & & & Gini \\
\hline
\end{tabular}




\begin{tabular}{|c|c|c|c|c|c|c|c|c|c|}
\hline & $100 \mu \mathrm{M}$ ATP & & & & & & & & \\
\hline Kinase & Lavendustin A@1 $1 \mu \mathrm{M}$ & Hit & sorted & normalized & position & cumulative sample fraction & inhibition\% & fraction of total inhibition & cumulative inhibition \\
\hline $\mathrm{Abl}(\mathrm{m})$ & 101 & 0 & 137 & 100 & 1 & 0.011764706 & 0 & 0 & 0 \\
\hline AMPK(r) & 109 & 0 & 127 & 100 & 2 & 0.023529412 & 0 & 0 & 0 \\
\hline $\operatorname{Arg}(\mathrm{m})$ & 104 & 0 & 118 & 100 & 3 & 0.035294118 & 0 & 0 & 0 \\
\hline Aurora-A(h) & 115 & 0 & 118 & 100 & 4 & 0.047058824 & 0 & 0 & 0 \\
\hline $\operatorname{Axl}(\mathrm{h})$ & 89 & 0 & 116 & 100 & 5 & 0.058823529 & 0 & 0 & 0 \\
\hline $\mathrm{Blk}(\mathrm{m})$ & 94 & 0 & 116 & 100 & 6 & 0.070588235 & 0 & 0 & 0 \\
\hline Bmxx(h) & 118 & 0 & 116 & 100 & 7 & 0.082352941 & 0 & 0 & 0 \\
\hline CaMKII(r) & 102 & 0 & 115 & 100 & 8 & 0.094117647 & 0 & 0 & 0 \\
\hline CaMKIV(h) & 103 & 0 & 114 & 100 & 9 & 0.105882353 & 0 & 0 & 0 \\
\hline $\mathrm{CDK} 1 / \mathrm{cyclin} B(\mathrm{~h})$ & 85 & 0 & 113 & 100 & 10 & 0.117647059 & 0 & 0 & 0 \\
\hline $\mathrm{CDK} 2 /$ cyclinA(h) & 91 & 0 & 112 & 100 & 11 & 0.129411765 & 0 & 0 & 0 \\
\hline $\mathrm{CDK} 2 / \mathrm{cyclinE}(\mathrm{h})$ & 103 & 0 & 112 & 100 & 12 & 0.141176471 & 0 & 0 & 0 \\
\hline CDK3/cyclinE(h) & 137 & 0 & 111 & 100 & 13 & 0.152941176 & 0 & 0 & 0 \\
\hline CDK5/p35(h) & 103 & 0 & 111 & 100 & 14 & 0.164705882 & 0 & 0 & 0 \\
\hline CDK6/cyclinD3(h) & 99 & 0 & 109 & 100 & 15 & 0.176470588 & 0 & 0 & 0 \\
\hline CDK 7 /cyclinHMATI (h) & 104 & 0 & 109 & 100 & 16 & 0.188235294 & 0 & 0 & 0 \\
\hline CHK (h) & 107 & 0 & 108 & 100 & 17 & 0.2 & 0 & 0 & 0 \\
\hline СHK2(h) & 104 & 0 & 107 & 100 & 18 & 0.211764706 & 0 & 0 & 0 \\
\hline $\mathrm{CKI}(\mathrm{y})$ & 97 & 0 & 106 & 100 & 19 & 0.223529412 & 0 & 0 & 0 \\
\hline СК2(h) & 106 & 0 & 106 & 100 & 20 & 0.235294118 & 0 & 0 & 0 \\
\hline c-RAF(h) & 97 & 0 & 106 & 100 & 21 & 0.247058824 & 0 & 0 & 0 \\
\hline CSK(h) & 83 & 0 & 106 & 100 & 22 & 0.258823529 & 0 & 0 & 0 \\
\hline cSRC(h) & 105 & 0 & 105 & 100 & 23 & 0.270588235 & 0 & 0 & 0 \\
\hline Fes(h) & 99 & 0 & 105 & 100 & 24 & 0.282352941 & 0 & 0 & 0 \\
\hline FGFR3(h) & 104 & 0 & 104 & 100 & 25 & 0.294117647 & 0 & 0 & 0 \\
\hline $\mathrm{Flt3(h)}$ & 98 & 0 & 104 & 100 & 26 & 0.305882353 & 0 & 0 & 0 \\
\hline Fyn(h) & 93 & 0 & 104 & 100 & 27 & 0.317647059 & 0 & 0 & 0 \\
\hline GSK3a(h) & 99 & 0 & 104 & 100 & 28 & 0.329411765 & 0 & 0 & 0 \\
\hline GSK3B(h) & 101 & 0 & 104 & 100 & $\begin{array}{l}20 \\
29\end{array}$ & 0.341176471 & 0 & 0 & 0 \\
\hline IGF-IR(h) & 73 & 0 & 104 & 100 & 30 & 0.352941176 & 0 & 0 & 0 \\
\hline $\mathrm{IKK} a(\mathrm{~h})$ & 95 & 0 & 104 & 100 & 31 & 0.364705882 & 0 & 0 & 0 \\
\hline IKKB(h) & 109 & 0 & 103 & 100 & 32 & 0.376470588 & 0 & 0 & 0 \\
\hline $\mathrm{IR(h)}$ & 118 & 0 & 103 & 100 & 33 & 0.388235294 & 0 & 0 & 0 \\
\hline JNKIal(h) & 103 & 0 & 103 & 100 & 34 & 0.4 & 0 & 0 & 0 \\
\hline JNK202(h) & 104 & 0 & 103 & 100 & 35 & 0.411764706 & 0 & 0 & 0 \\
\hline JNK3(r) & 111 & 0 & 103 & 100 & 36 & 0.423529412 & 0 & 0 & 0 \\
\hline Lck(h) & 112 & 0 & 103 & 100 & 37 & 0.435294118 & 0 & 0 & 0 \\
\hline Lyn(h) & 103 & 0 & 102 & 100 & 38 & 0.447058824 & 0 & 0 & 0 \\
\hline Lyn(m) & 98 & 0 & 102 & 100 & 39 & 0.458823529 & 0 & 0 & 0 \\
\hline MAPKI(h) & $\begin{array}{c}98 \\
111\end{array}$ & 0 & $\begin{array}{l}102 \\
102\end{array}$ & 100 & 40 & 0.470588235 & 0 & 0 & 0 \\
\hline MAPK2(h) & 116 & 0 & 102 & 100 & 41 & 0.482352941 & 0 & 0 & 0 \\
\hline MAPK2(m) & 103 & 0 & 102 & 100 & 42 & 0.494117647 & 0 & 0 & 0 \\
\hline MAPKAP-K2(h) & 97 & 0 & 101 & 100 & 43 & 0.505882353 & 0 & 0 & 0 \\
\hline MEK1(h) & 93 & 0 & 101 & 100 & 44 & 0.517647059 & 0 & 0 & 0 \\
\hline MKK $4(\mathrm{~m})$ & 108 & 0 & 101 & 100 & 45 & 0.529411765 & 0 & 0 & 0 \\
\hline MKK $6(\mathrm{~h})$ & 100 & 0 & 101 & 100 & 46 & 0.541176471 & 0 & 0 & 0 \\
\hline MKK & 114 & 0 & 101 & 100 & 47 & 0.552941176 & 0 & 0 & 0 \\
\hline MSK1(h) & 102 & 0 & 100 & 100 & 48 & 0.564705882 & 0 & 0 & 0 \\
\hline $\mathrm{p} 70 \mathrm{~S} 6 \mathrm{~K}(\mathrm{~h})$ & 96 & 0 & 100 & 100 & 49 & 0.576470588 & 0 & 0 & 0 \\
\hline $\begin{array}{l}\text { PAK2(h) } \\
\text { Pasole }\end{array}$ & 104 & 0 & 100 & 100 & 50 & 0.588235294 & 0 & 0 & 0 \\
\hline $\begin{array}{l}\text { PARGRRa(h) } \\
\text { PDGFa }\end{array}$ & 75 & 0 & 99 & 99 & 51 & 0.6 & 1 & 0.003968254 & 0.003968254 \\
\hline PDGFRB(h) & 82 & 0 & 99 & 99 & 52 & 0.611764706 & 1 & 0.003968254 & 0.007936508 \\
\hline PDK I(h) & 113 & 0 & 99 & 99 & 53 & 0.623529412 & 1 & 0.003968254 & 0.011904762 \\
\hline PKA(b) & 112 & 0 & 99 & 99 & 54 & 0.635294118 & 1 & 0.003968254 & 0.015873016 \\
\hline $\begin{array}{l}\text { PKA(b) } \\
\text { PKA(h) }\end{array}$ & 101 & 0 & 99 & 99 & 55 & 0.647058824 & 1 & 0.003968254 & 0.01984127 \\
\hline PKBa(h) & 101 & 0 & 98 & 98 & 56 & 0.658823529 & 2 & 0.007936508 & 0.027777778 \\
\hline PKBB(h) & 95 & 0 & $\begin{array}{l}90 \\
98\end{array}$ & 98 & 57 & 0.670588235 & 2 & 0.007936508 & 0.035714286 \\
\hline PKB $\gamma(\mathfrak{h})$ & 106 & 0 & 98 & 98 & 58 & 0.682352941 & 2 & 0.007936508 & 0.043650794 \\
\hline PKC (h) & 101 & 0 & 97 & 97 & 59 & 0.694117647 & 3 & 0.011904762 & 0.055555556 \\
\hline РКСBI(h) & 102 & 0 & 97 & 97 & 60 & 0.705882353 & 3 & 0.011904762 & 0.067460317 \\
\hline PKC (h) & 93 & 0 & 97 & 97 & 61 & 0.717647059 & 3 & 0.011904762 & 0.079365079 \\
\hline РКC & $\begin{array}{c}93 \\
116\end{array}$ & 0 & 97 & 97 & $\begin{array}{l}11 \\
62\end{array}$ & 0.729411765 & 3 & 0.011904762 & 0.091269841 \\
\hline 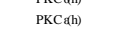 & 97 & 0 & 97 & 97 & 63 & 0.741176471 & 3 & 0.011904762 & 0.103174603 \\
\hline 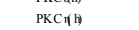 & 102 & 0 & 96 & 96 & 64 & 0.752941176 & 4 & 0.015873016 & 0.119047619 \\
\hline PKC I(h) & 92 & 0 & 96 & 96 & 65 & 0.764705882 & 4 & 0.015873016 & 0.134920635 \\
\hline PKC $\mu(\mathrm{h})$ & 96 & 0 & 96 & 96 & 66 & 0.776470588 & 4 & 0.015873016 & 0.150793651 \\
\hline РКС甘̈ & 104 & 0 & 95 & 95 & 67 & 0.788235294 & 5 & 0.01984127 & 0.170634921 \\
\hline $\begin{array}{l}\text { PKD2(h) } \\
\text { Pats }\end{array}$ & 105 & 0 & 95 & 95 & 68 & 0.8 & 5 & 0.01984127 & 0.19047619 \\
\hline PRAK(h) & 100 & 0 & 95 & 95 & 69 & 0.811764706 & 5 & 0.01984127 & 0.21031746 \\
\hline PRK2(h) & 106 & 0 & 94 & 94 & 70 & 0.823529412 & 6 & 0.023809524 & 0.234126984 \\
\hline ROCK-II(h) & 95 & 0 & 94 & 94 & 71 & 0.835294118 & 6 & 0.023809524 & 0.257936508 \\
\hline ROCK-II(r) & 99 & 0 & 93 & 93 & 72 & 0.847058824 & 7 & 0.027777778 & 0.285714286 \\
\hline Rskl(h) & $\begin{array}{l}99 \\
94\end{array}$ & 0 & $\begin{array}{l}93 \\
93\end{array}$ & $\begin{array}{l}93 \\
93\end{array}$ & 73 & 0.858823529 & 7 & 0.027777778 & 0.313492063 \\
\hline $\operatorname{Rskl(r)}$ & 85 & 0 & 93 & 93 & 74 & 0.870588235 & 7 & 0.027777778 & 0.341269841 \\
\hline $\begin{array}{l}\text { Rssk(t) } \\
\text { Rsk2(h) }\end{array}$ & 91 & 0 & 92 & 92 & 75 & 0.882352941 & 8 & 0.031746032 & 0.373015873 \\
\hline Rsk3(h) & 106 & 0 & 91 & 91 & 76 & 0.894117647 & 9 & 0.035714286 & 0.408730159 \\
\hline SAPK2a(h) & 97 & 0 & 91 & 91 & 77 & 0.905882353 & 9 & 0.035714286 & 0.444444444 \\
\hline SAPK2b(h) & 127 & 0 & 89 & 89 & 78 & 0.917647059 & 11 & 0.043650794 & 0.488095238 \\
\hline SAPK3(h) & 116 & 0 & 88 & 88 & 79 & 0.929411765 & 12 & 0.047619048 & 0.535714286 \\
\hline SAPK 4(h) & 100 & 0 & 85 & 85 & 80 & 0.941176471 & 15 & 0.05952381 & 0.595238095 \\
\hline $\begin{array}{c}\mathrm{SAAN}(\mathrm{h}) \\
\mathrm{SGK}(\mathrm{h})\end{array}$ & 96 & 0 & 85 & 85 & 81 & 0.952941176 & 15 & 0.05952381 & 0.654761905 \\
\hline Syk(h) & 88 & 0 & 83 & 83 & 82 & 0.964705882 & 17 & 0.067460317 & 0.722222222 \\
\hline TrkB(h) & 102 & 0 & 82 & 82 & 83 & 0.976470588 & 18 & 0.071428571 & 0.793650794 \\
\hline Yes(h) & 99 & 0 & 75 & 75 & 84 & 0.988235294 & 25 & 0.099206349 & 0.892857143 \\
\hline ZAP-70(h) & 98 & 0 & 73 & 73 & 85 & 1 & 27 & 0.107142857 & 1 \\
\hline & & & & & & Total inhibition & 252 & & Total area \\
\hline & Hit rate & 5 & & & & & & & Gini \\
\hline
\end{tabular}




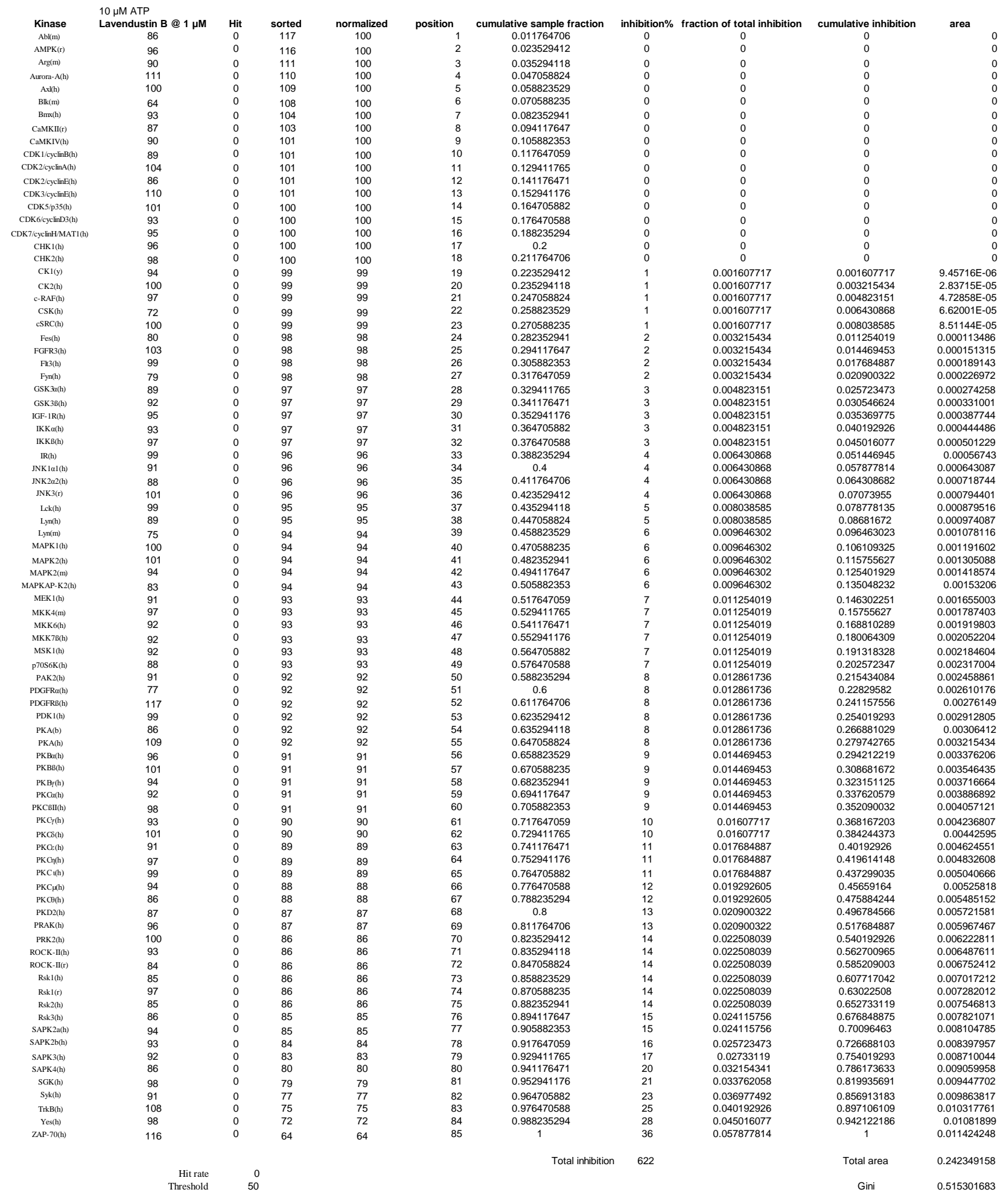




\begin{tabular}{|c|c|c|c|c|c|c|c|c|c|}
\hline & $100 \mu \mathrm{M}$ ATP & & & & & & & & \\
\hline Kinase & Lavendustin B @ $1 \mu \mathrm{M}$ & Hit & sorted & normalized & position & cumulative sample fraction & inhibition\% & fraction of total inhibition & cumulative inhibition \\
\hline $\mathrm{Abl}(\mathrm{m})$ & 90 & 0 & 131 & 100 & 1 & 0.011764706 & 0 & 0 & 0 \\
\hline AMPK(r) & 99 & 0 & 127 & 100 & 2 & 0.023529412 & 0 & 0 & 0 \\
\hline $\operatorname{Arg}(\mathrm{m})$ & 99 & 0 & 121 & 100 & 3 & 0.035294118 & 0 & 0 & 0 \\
\hline Aurora-A(h) & 101 & 0 & 119 & 100 & 4 & 0.047058824 & 0 & 0 & 0 \\
\hline Axl(h) & 84 & 0 & 113 & 100 & 5 & 0.058823529 & 0 & 0 & 0 \\
\hline $\operatorname{Blk}(\mathrm{m})$ & 93 & 0 & 113 & 100 & 6 & 0.070588235 & 0 & 0 & 0 \\
\hline $\mathrm{Bmx(h)}$ & 92 & 0 & 109 & 100 & 7 & 0.082352941 & 0 & 0 & 0 \\
\hline CaMKII(r) & 99 & 0 & 109 & 100 & 8 & 0.094117647 & 0 & 0 & 0 \\
\hline CaMKIV(h) & 104 & 0 & 108 & 100 & 9 & 0.105882353 & 0 & 0 & 0 \\
\hline CDK1/cyclnBB(h) & 87 & 0 & 108 & 100 & 10 & 0.117647059 & 0 & 0 & 0 \\
\hline $\mathrm{CDK} 2 /$ cyclinA(h) & 89 & 0 & 106 & 100 & 11 & 0.129411765 & 0 & 0 & 0 \\
\hline $\mathrm{CDK} 2 / \mathrm{cyclin} \mathrm{E}(\mathrm{h})$ & 100 & 0 & 105 & 100 & 12 & 0.141176471 & 0 & 0 & 0 \\
\hline CDK3/cyclinE(h) & 127 & 0 & 104 & 100 & 13 & 0.152941176 & 0 & 0 & 0 \\
\hline CDK5/p35(h) & 131 & 0 & 103 & 100 & 14 & 0.164705882 & 0 & 0 & 0 \\
\hline $\mathrm{CDK} 6 / \mathrm{cyclin} \mathrm{B} 3(\mathrm{~h})$ & 90 & 0 & 103 & 100 & 15 & 0.176470588 & 0 & 0 & 0 \\
\hline CDK $7 /$ yclinHMATI(h) & 100 & 0 & 102 & 100 & 16 & 0.188235294 & 0 & 0 & 0 \\
\hline CHKI(h) & 103 & 0 & 102 & 100 & 17 & 0.2 & 0 & 0 & 0 \\
\hline CHK2(h) & 108 & 0 & 101 & 100 & 18 & 0.211764706 & 0 & 0 & 0 \\
\hline CKI(y) & 93 & 0 & 101 & 100 & 19 & 0.223529412 & 0 & 0 & 0 \\
\hline СК2(h) & 102 & 0 & 101 & 100 & 20 & 0.235294118 & 0 & 0 & 0 \\
\hline c-RAF(h) & 99 & 0 & 100 & 100 & 21 & 0.247058824 & 0 & 0 & 0 \\
\hline $\operatorname{CSK}(\mathrm{h})$ & 101 & 0 & 100 & 100 & 22 & 0.258823529 & 0 & 0 & 0 \\
\hline $\operatorname{csRC(h)}$ & 99 & 0 & 100 & 100 & 23 & 0.270588235 & 0 & 0 & 0 \\
\hline Fess(h) & 89 & 0 & 99 & 99 & 24 & 0.282352941 & 1 & 0.001855288 & 0.001855288 \\
\hline FGFR3(h) & 88 & 0 & 99 & 99 & 25 & 0.294117647 & 1 & 0.001855288 & 0.003710575 \\
\hline $\mathrm{Fll}(\mathrm{h}(\mathrm{h})$ & 98 & 0 & 99 & 99 & 26 & 0.305882353 & 1 & 0.001855288 & 0.005565863 \\
\hline Fyn(h) & 93 & 0 & 99 & 99 & 27 & 0.317647059 & 1 & 0.001855288 & 0.00742115 \\
\hline GSK3a(h) & 93 & 0 & 99 & 99 & 28 & 0.329411765 & 1 & 0.001855288 & 0.009276438 \\
\hline GSK3B(h) & 98 & 0 & 99 & 99 & $\begin{array}{l}20 \\
29\end{array}$ & 0.341176471 & 1 & 0.001855288 & 0.011131725 \\
\hline IGF-IR(h) & 95 & 0 & 99 & 99 & 30 & 0.352941176 & 1 & 0.001855288 & 0.012987013 \\
\hline IKKa(h) & 96 & 0 & 98 & 98 & 31 & 0.364705882 & 2 & 0.003710575 & 0.016697588 \\
\hline IKKB(h) & 83 & 0 & 98 & 98 & 32 & 0.376470588 & 2 & 0.003710575 & 0.020408163 \\
\hline $\mathbb{I R}(\mathrm{h})$ & $\begin{array}{l}0.0 \\
113\end{array}$ & 0 & 98 & $\begin{array}{l}50 \\
98\end{array}$ & $\begin{array}{l}32 \\
33\end{array}$ & 0.388235294 & 2 & 0.003710575 & 0.024118738 \\
\hline JNKIal(h) & 92 & 0 & 98 & 98 & 34 & 0.4 & 2 & 0.003710575 & 0.027829314 \\
\hline JNK202(h) & 97 & 0 & 98 & 98 & $\begin{array}{l}54 \\
35\end{array}$ & 0.411764706 & 2 & 0.003710575 & 0.031539889 \\
\hline JNK3(r) & 106 & 0 & 97 & 97 & 36 & 0.423529412 & 3 & 0.005565863 & 0.037105751 \\
\hline Lck(h) & 87 & 0 & 97 & 97 & 37 & 0.435294118 & 3 & 0.005565863 & 0.042671614 \\
\hline Lyn(h) & 99 & 0 & 96 & 96 & 38 & 0.447058824 & 4 & 0.00742115 & 0.050092764 \\
\hline Lyn(m) & 96 & 0 & 96 & 96 & 39 & 0.458823529 & 4 & 0.00742115 & 0.057513915 \\
\hline MAPKI(h) & 94 & 0 & $\begin{array}{l}96 \\
95\end{array}$ & $\begin{array}{l}90 \\
95\end{array}$ & 40 & 0.470588235 & 5 & 0.009276438 & 0.066790353 \\
\hline MAPK2(h) & 99 & 0 & 95 & 95 & 41 & 0.482352941 & 5 & 0.009276438 & 0.07606679 \\
\hline MAPK2(m) & 93 & 0 & 95 & 95 & 42 & 0.494117647 & 5 & 0.009276438 & 0.085343228 \\
\hline MAPKAP-K2(h) & 95 & 0 & 95 & 95 & 43 & 0.505882353 & 5 & 0.009276438 & 0.094619666 \\
\hline MEK1(h) & 89 & 0 & 95 & 95 & 44 & 0.517647059 & 5 & 0.009276438 & 0.103896104 \\
\hline MKK4(m) & 109 & 0 & 94 & 94 & 45 & 0.529411765 & 6 & 0.011131725 & 0.115027829 \\
\hline MKK6(h) & 83 & 0 & 94 & 94 & 46 & 0.541176471 & 6 & 0.011131725 & 0.126159555 \\
\hline MKK & 92 & 0 & 94 & 94 & 47 & 0.552941176 & 6 & 0.011131725 & 0.13729128 \\
\hline MSKI(h) & 101 & 0 & 94 & 94 & 48 & 0.564705882 & 6 & 0.011131725 & 0.148423006 \\
\hline $\mathrm{p} 7056 \mathrm{~K}(\mathrm{~h})$ & 93 & 0 & $\begin{array}{l}94 \\
93\end{array}$ & $\begin{array}{l}94 \\
93\end{array}$ & $\begin{array}{l}48 \\
49\end{array}$ & 0.576470588 & $\begin{array}{l}0 \\
7\end{array}$ & 0.012987013 & $\begin{array}{l}.1464<3000 \\
0.161410019\end{array}$ \\
\hline $\begin{array}{l}\text { PAK2(h) } \\
\text { Past }\end{array}$ & 92 & 0 & 93 & 93 & 50 & 0.588235294 & 7 & 0.012987013 & 0.174397032 \\
\hline PDGFRa(h) & 79 & 0 & 93 & 93 & 51 & 0.6 & 7 & 0.012987013 & 0.187384045 \\
\hline PDGFRB(h) & 76 & 0 & 93 & 93 & 52 & 0.611764706 & 7 & 0.012987013 & 0.200371058 \\
\hline PDKI(h) & 102 & 0 & 93 & 93 & 53 & 0.623529412 & 7 & 0.012987013 & 0.213358071 \\
\hline PKA(b) & 100 & 0 & 93 & 93 & 54 & 0.635294118 & 7 & 0.012987013 & 0.226345083 \\
\hline PKA(h) & 119 & 0 & 92 & 92 & 55 & 0.647058824 & 8 & 0.014842301 & 0.241187384 \\
\hline PKBa(h) & 95 & 0 & 92 & 92 & 56 & 0.658823529 & 8 & 0.014842301 & 0.256029685 \\
\hline PKBB(h) & 88 & 0 & 92 & 92 & 57 & 0.670588235 & 8 & 0.014842301 & 0.270871985 \\
\hline $\mathrm{PKB}_{\mathrm{y}(\mathrm{h})}$ & 87 & 0 & 92 & 92 & 58 & 0.682352941 & 8 & 0.014842301 & 0.285714286 \\
\hline PKCath) & 92 & 0 & 92 & 92 & 59 & 0.694117647 & 8 & 0.014842301 & 0.300556586 \\
\hline PKCBII(h) & 98 & 0 & 91 & 91 & 60 & 0.705882353 & 9 & 0.016697588 & 0.317254174 \\
\hline PKCy(h) & 95 & 0 & 90 & 90 & 61 & 0.717647059 & 10 & 0.018552876 & 0.33580705 \\
\hline РКФ(h) & 108 & 0 & 90 & 90 & 62 & 0.729411765 & 10 & 0.018552876 & 0.354359926 \\
\hline PKG:(h) & 94 & 0 & 89 & 89 & 63 & 0.741176471 & 11 & 0.020408163 & 0.374768089 \\
\hline $\begin{array}{l}\text { PKG (h) } \\
\text { (h) }\end{array}$ & 105 & 0 & 89 & 89 & 64 & 0.752941176 & 11 & 0.020408163 & 0.395176252 \\
\hline PKC (h) & 85 & 0 & $\begin{array}{l}89 \\
89\end{array}$ & $\begin{array}{l}89 \\
89\end{array}$ & 65 & 0.764705882 & 11 & 0.020408163 & 0.415584416 \\
\hline PKC $\mu(\mathrm{h})$ & 88 & 0 & 89 & 89 & 66 & 0.776470588 & 11 & 0.020408163 & 0.435992579 \\
\hline PKCO(h) & 85 & 0 & 89 & 89 & 67 & 0.788235294 & 11 & 0.020408163 & 0.456400742 \\
\hline PKD2(h) & 109 & 0 & 88 & 88 & 68 & 0.8 & 12 & 0.022263451 & 0.478664193 \\
\hline PRAK(h) & 103 & 0 & $\begin{array}{l}88 \\
88\end{array}$ & $\begin{array}{l}88 \\
88\end{array}$ & 69 & 0.811764706 & 12 & 0.022263451 & 0.500927644 \\
\hline PRK2(h) & 113 & 0 & 88 & 88 & 70 & 0.823529412 & 12 & 0.022263451 & 0.523191095 \\
\hline $\begin{array}{l}\text { PRK_(n) } \\
\text { ROCK-II(h) }\end{array}$ & 91 & 0 & 87 & $\begin{array}{l}00 \\
87\end{array}$ & 71 & 0.835294118 & 13 & 0.024118738 & 0.547309833 \\
\hline ROCK-II(r) & 94 & 0 & 87 & 87 & 72 & 0.847058824 & 13 & 0.024118738 & 0.571428571 \\
\hline Rskl(h) & 95 & 0 & 87 & 87 & 73 & 0.858823529 & 13 & 0.024118738 & 0.59554731 \\
\hline Rskl(r) & 83 & 0 & 85 & 85 & 74 & 0.870588235 & 15 & 0.027829314 & 0.623376623 \\
\hline $\begin{array}{l}\text { Rsksk2(h) } \\
\text { Rsk(h) }\end{array}$ & 89 & 0 & 85 & 85 & 75 & 0.882352941 & 15 & 0.027829314 & 0.651205937 \\
\hline Rsk3(h) & 94 & 0 & 84 & 84 & 76 & 0.894117647 & 16 & 0.029684601 & 0.680890538 \\
\hline SAPK2a(h) & 97 & 0 & 84 & 84 & 77 & 0.905882353 & 16 & 0.029684601 & 0.710575139 \\
\hline SAPK2b(h) & 121 & 0 & 83 & 83 & 78 & 0.917647059 & 17 & 0.031539889 & 0.742115028 \\
\hline SAPK3(h) & 98 & 0 & 83 & 83 & 79 & 0.929411765 & 17 & 0.031539889 & 0.773654917 \\
\hline $\begin{array}{l}\mathrm{SARRS( \textrm {h } )} \\
\mathrm{SAPK} 4(\mathrm{~h})\end{array}$ & 98 & 0 & 83 & $\begin{array}{l}00 \\
83\end{array}$ & 80 & 0.941176471 & 17 & 0.031539889 & 0.805194805 \\
\hline SGK(h) & 82 & 0 & 82 & 82 & 81 & 0.952941176 & 18 & 0.033395176 & 0.838589981 \\
\hline Syk(h) & 84 & 0 & 79 & 79 & 82 & 0.964705882 & 21 & 0.038961039 & 0.87755102 \\
\hline TrkB(h) & $\begin{array}{l}84 \\
89\end{array}$ & 0 & 79 & 79 & 83 & 0.976470588 & 21 & 0.038961039 & 0.916512059 \\
\hline $\begin{array}{l}\text { IIrkB(h) } \\
\text { Yesh(h) }\end{array}$ & $\begin{array}{l}89 \\
79\end{array}$ & 0 & 79 & 79 & 84 & 0.988235294 & 21 & 0.038961039 & 0.955473098 \\
\hline ZAP-70(h) & 79 & 0 & 76 & 76 & 85 & 1 & 24 & 0.044526902 & 1 \\
\hline & & & & & & Total inhibition & 539 & & Total area \\
\hline & Hit rate & 5 & & & & & & & Gini \\
\hline
\end{tabular}




\begin{tabular}{|c|c|c|c|c|c|c|c|c|}
\hline & $10 \mu \mathrm{M}$ ATP & & & & & & & \\
\hline Kinase & LY294002@ @ 50 нM & Hit & sorted & normalized & position & cumulative sample fraction & inhibition\% & fraction of total inhibition \\
\hline $\mathrm{Abl}(\mathrm{m})$ & 91 & 0 & 133 & 100 & 1 & 0.011764706 & 0 & 0 \\
\hline AMPK(r) & 107 & 0 & 123 & 100 & 2 & 0.023529412 & 0 & 0 \\
\hline $\operatorname{Arg}(\mathrm{m})$ & 98 & 0 & 119 & 100 & 3 & 0.035294118 & 0 & 0 \\
\hline Aurora-A(h) & 108 & 0 & 117 & 100 & 4 & 0.047058824 & 0 & 0 \\
\hline Axl(h) & 89 & 0 & 115 & 100 & 5 & 0.058823529 & 0 & 0 \\
\hline $\mathrm{Blk}(\mathrm{m})$ & 52 & 0 & 113 & 100 & 6 & 0.070588235 & 0 & 0 \\
\hline Bmxx(h) & 62 & 0 & 112 & 100 & 7 & 0.082352941 & 0 & 0 \\
\hline СамKII(r) & 101 & 0 & 110 & 100 & 8 & 0.094117647 & 0 & 0 \\
\hline CaMKIV(h) & 105 & 0 & 109 & 100 & 9 & 0.105882353 & 0 & 0 \\
\hline CDK1/cyclnBB(h) & 68 & 0 & 108 & 100 & 10 & 0.117647059 & 0 & 0 \\
\hline $\mathrm{CDK} /$ /yclinA(h) & 79 & 0 & 107 & 100 & 11 & 0.129411765 & 0 & 0 \\
\hline $\mathrm{CDK} 2 /$ cyclinE(h) & 84 & 0 & 107 & 100 & 12 & 0.141176471 & 0 & 0 \\
\hline CDK3/cyclinE(h) & 85 & 0 & 107 & 100 & 13 & 0.152941176 & 0 & 0 \\
\hline CDK5/p35(h) & 75 & 0 & 106 & 100 & 14 & 0.164705882 & 0 & 0 \\
\hline CDK6/cyclinD3(h) & 107 & 0 & 106 & 100 & 15 & 0.176470588 & 0 & 0 \\
\hline CDK7//yclinH/MATI(h) & 72 & 0 & 105 & 100 & 16 & 0.188235294 & 0 & 0 \\
\hline СHK1(h) & 105 & 0 & 105 & 100 & 17 & 0.2 & 0 & 0 \\
\hline СHK2(h) & 109 & 0 & 105 & 100 & 18 & 0.211764706 & 0 & 0 \\
\hline $\mathrm{CK} 1(\mathrm{y})$ & 19 & 1 & 103 & 100 & 19 & 0.223529412 & 0 & 0 \\
\hline СК2(h) & 5 & 1 & 102 & 100 & 20 & 0.235294118 & 0 & 0 \\
\hline c-RAF(h) & 54 & 0 & 102 & 100 & 21 & 0.247058824 & 0 & 0 \\
\hline $\operatorname{CSK}(\mathrm{h})$ & 84 & 0 & 102 & 100 & 22 & 0.258823529 & 0 & 0 \\
\hline cSRC(h) & 93 & 0 & 101 & 100 & 23 & 0.270588235 & 0 & 0 \\
\hline Fes(h) & 94 & 0 & 101 & 100 & 24 & 0.282352941 & 0 & 0 \\
\hline FGFR3(h) & 106 & 0 & 101 & 100 & 25 & 0.294117647 & 0 & 0 \\
\hline Fill(h) & 42 & 1 & 100 & 100 & 26 & 0.305882353 & 0 & 0 \\
\hline Fyn(h) & 62 & 0 & 99 & 99 & 27 & 0.317647059 & 1 & 0.000731529 \\
\hline GSK3a(h) & 28 & 1 & 99 & 99 & 28 & 0.329411765 & 1 & 0.000731529 \\
\hline GSK3B(h) & 35 & 1 & 98 & 98 & 29 & 0.341176471 & 2 & 0.001463058 \\
\hline IGF-IR(h) & 97 & 0 & 98 & 98 & 30 & 0.352941176 & 2 & 0.001463058 \\
\hline $\mathrm{IKKa}(\mathrm{h})$ & 85 & 0 & 97 & 97 & 31 & 0.364705882 & 3 & 0.002194587 \\
\hline IKKB(h) & 77 & 0 & 97 & 97 & 32 & 0.376470588 & 3 & 0.002194587 \\
\hline $\mathbb{I R ( h )}$ & 101 & 0 & 96 & 96 & 33 & 0.388235294 & 4 & 0.002926116 \\
\hline JNKIal(h) & 105 & 0 & 95 & 95 & 34 & 0.4 & 5 & 0.003657644 \\
\hline JNK2202(h) & 99 & 0 & 95 & 95 & 35 & 0.411764706 & 5 & 0.003657644 \\
\hline JNK3(r) & 64 & 0 & 94 & 94 & 36 & 0.423529412 & 6 & 0.004389173 \\
\hline Lck(h) & 73 & 0 & 93 & 93 & 37 & 0.435294118 & 7 & 0.005120702 \\
\hline Lyn(h) & 83 & 0 & 92 & 92 & 38 & 0.447058824 & 8 & 0.005852231 \\
\hline Lyy(m) & 73 & 0 & 92 & 92 & 39 & 0.458823529 & 8 & 0.005852231 \\
\hline MAPK I (h) & 123 & 0 & 91 & 91 & 40 & 0.470588235 & 9 & 0.00658376 \\
\hline MAPK2(h) & 112 & 0 & 91 & 91 & 41 & 0.482352941 & 9 & 0.00658376 \\
\hline MAPK2(m) & 107 & 0 & 91 & 91 & 42 & 0.494117647 & 9 & 0.00658376 \\
\hline MAPKAP-K2(h) & 115 & 0 & 89 & 89 & 43 & 0.505882353 & 11 & 0.008046818 \\
\hline MEK1(h) & 97 & 0 & 88 & 88 & 44 & 0.517647059 & 12 & 0.008778347 \\
\hline MKK $4(\mathrm{~m})$ & 95 & 0 & 88 & 88 & 45 & 0.529411765 & 12 & 0.008778347 \\
\hline MKK6(h) & 88 & 0 & 88 & 88 & 46 & 0.541176471 & 12 & 0.008778347 \\
\hline MKK 7 (h) & 88 & 0 & 88 & 88 & 47 & 0.552941176 & 12 & 0.008778347 \\
\hline MSKI(h) & 86 & 0 & 87 & 87 & 48 & 0.564705882 & 13 & 0.009509876 \\
\hline p7056K(h) & 81 & 0 & 86 & 86 & 49 & 0.576470588 & 14 & 0.010241405 \\
\hline PAK2(h) & 102 & 0 & 86 & 86 & 50 & 0.588235294 & 14 & 0.010241405 \\
\hline PDGFRa(h) & 79 & 0 & 86 & 86 & 51 & 0.6 & 14 & 0.010241405 \\
\hline PDGFRB(h) & 110 & 0 & 85 & 85 & 52 & 0.611764706 & 15 & 0.010972933 \\
\hline PDKI(h) & 133 & 0 & 85 & 85 & 53 & 0.623529412 & 15 & 0.010972933 \\
\hline PKA(b) & 102 & 0 & 84 & 84 & 54 & 0.635294118 & 16 & 0.011704462 \\
\hline PKA(h) & 117 & 0 & 84 & 84 & 55 & 0.647058824 & 16 & 0.011704462 \\
\hline PKBa(h) & 75 & 0 & 83 & 83 & 56 & 0.658823529 & 17 & 0.012435991 \\
\hline PKB(h) & 98 & 0 & 83 & 83 & 57 & 0.670588235 & 17 & 0.012435991 \\
\hline $\mathrm{PKB}_{\gamma}(\mathrm{h})$ & 92 & 0 & 83 & 83 & 58 & 0.682352941 & 17 & 0.012435991 \\
\hline 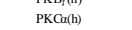 & 72 & 0 & 81 & 81 & 59 & 0.694117647 & 19 & 0.013899049 \\
\hline РКCBII(h) & 101 & 0 & 81 & 81 & 60 & 0.705882353 & 19 & 0.013899049 \\
\hline PKCy(h) & 92 & 0 & 79 & 79 & 61 & 0.717647059 & 21 & 0.015362107 \\
\hline PKБ(h) & 103 & 0 & 79 & 79 & 62 & 0.729411765 & 21 & 0.015362107 \\
\hline PKG:(h) & 59 & 0 & 79 & 79 & 63 & 0.741176471 & 21 & 0.015362107 \\
\hline PKG(h) & 76 & 0 & 77 & 77 & 64 & 0.752941176 & 23 & 0.016825165 \\
\hline PKC (h) & 113 & 0 & 76 & 76 & 65 & 0.764705882 & 24 & 0.017556693 \\
\hline PKC $\mu(h)$ & 86 & 0 & 75 & 75 & 66 & 0.776470588 & 25 & 0.018288222 \\
\hline PKCO(h) & 88 & 0 & 75 & 75 & 67 & 0.788235294 & 25 & 0.018288222 \\
\hline PKD2(h) & 87 & 0 & 73 & 73 & 68 & 0.8 & 27 & 0.01975128 \\
\hline PRAK(h) & 83 & 0 & 73 & 73 & 69 & 0.811764706 & 27 & 0.01975128 \\
\hline PRK2(h) & 83 & 0 & 72 & 72 & 70 & 0.823529412 & 28 & 0.020482809 \\
\hline ROCK-II(h) & 91 & 0 & 72 & 72 & 71 & 0.835294118 & 28 & 0.020482809 \\
\hline ROCK-II(r) & 106 & 0 & 70 & 70 & 72 & 0.847058824 & 30 & 0.021945867 \\
\hline Rskl(h) & 70 & 0 & 68 & 68 & 73 & 0.858823529 & 32 & 0.023408925 \\
\hline Rskl(r) & 79 & 0 & 64 & 64 & 74 & 0.870588235 & 36 & 0.02633504 \\
\hline Rsk2(h) & 86 & 0 & 62 & 62 & 75 & 0.882352941 & 38 & 0.027798098 \\
\hline Rsk3(h) & 91 & 0 & 62 & 62 & 76 & 0.894117647 & 38 & 0.027798098 \\
\hline SAPK2a(h) & 96 & 0 & 59 & 59 & 77 & 0.905882353 & 41 & 0.029992685 \\
\hline SAPK2b(h) & 102 & 0 & 54 & 54 & 78 & 0.917647059 & 46 & 0.033650329 \\
\hline $\mathrm{SAPK} 3(\mathrm{~h})$ & 100 & 0 & 52 & 52 & 79 & 0.929411765 & 48 & 0.035113387 \\
\hline SAPK4(h) & 99 & 0 & 42 & 42 & 80 & 0.941176471 & 58 & 0.042428676 \\
\hline SGK(h) & 95 & 0 & 35 & 35 & 81 & 0.952941176 & 65 & 0.047549378 \\
\hline Syk(h) & 88 & 0 & 30 & 30 & 82 & 0.964705882 & 70 & 0.051207023 \\
\hline TrkB(h) & 30 & 1 & 28 & 28 & 83 & 0.976470588 & 72 & 0.05267008 \\
\hline Yes(h) & 81 & 0 & 19 & 19 & 84 & 0.988235294 & 81 & 0.059253841 \\
\hline ZAP-70(h) & 119 & 0 & 5 & 5 & 85 & 1 & 95 & 0.069495245 \\
\hline & & & & & & Total inhibition & 1367 & \\
\hline & Hit rate & & & & & & & \\
\hline & Threshold & 5 & & & & & & \\
\hline
\end{tabular}




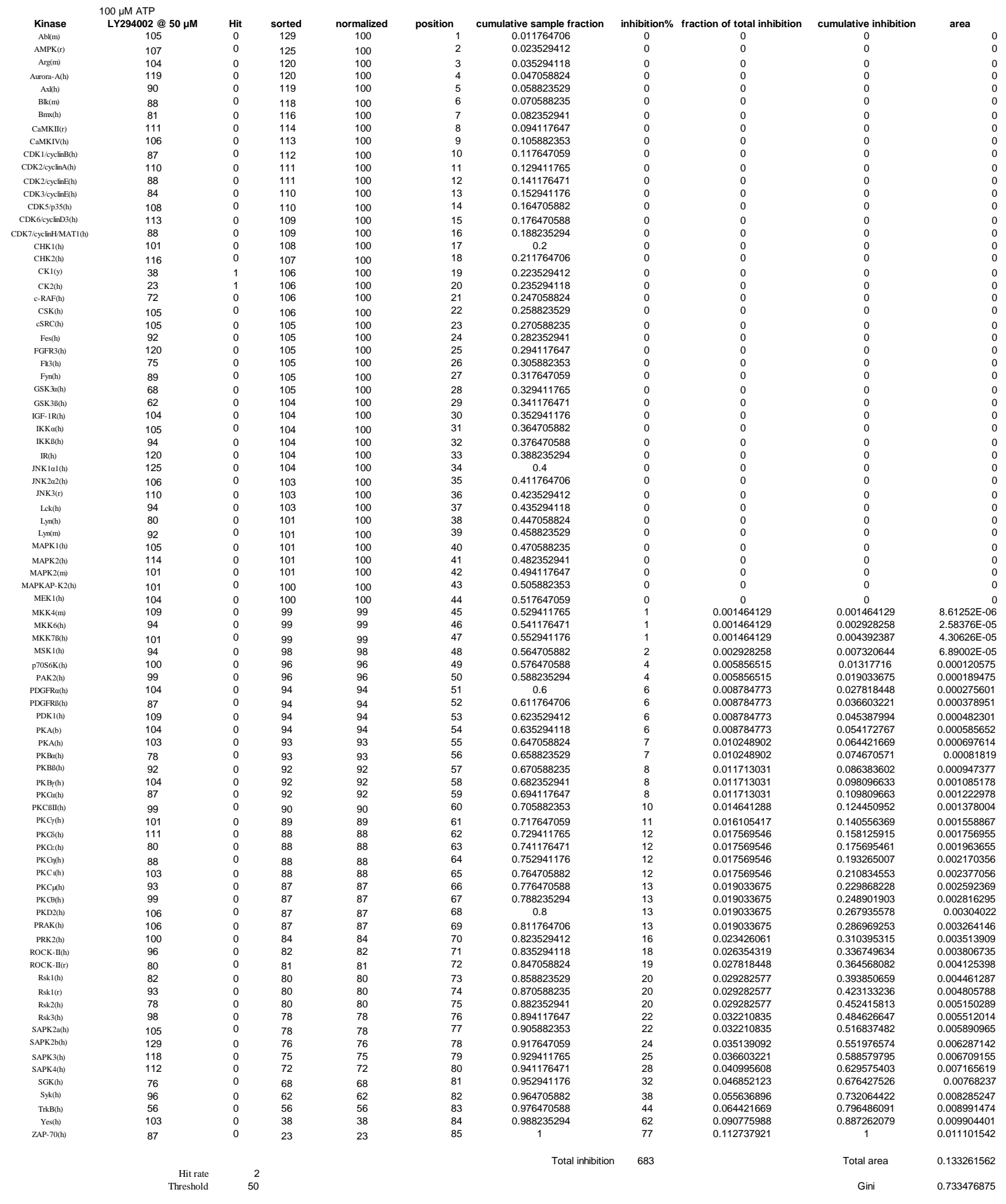




\begin{tabular}{|c|c|c|c|c|c|c|c|c|c|}
\hline & $10 \mu \mathrm{M}$ ATP & & & & & & & & \\
\hline Kinase & Olomoucin @ $50 \mu \mathrm{M}$ & Hit & sorted & normalized & position & cumulative sample fraction & inhibition\% & fraction of total inhibition & cumulative inhibition \\
\hline $\mathrm{Abl}(\mathrm{m})$ & 86 & 0 & 115 & 100 & 1 & 0.011764706 & 0 & 0 & 0 \\
\hline AMPK(r) & 95 & 0 & 111 & 100 & 2 & 0.023529412 & 0 & 0 & 0 \\
\hline $\operatorname{Arg}(\mathrm{m})$ & 90 & 0 & 111 & 100 & 3 & 0.035294118 & 0 & 0 & 0 \\
\hline Aurora-A(h) & 65 & 0 & 110 & 100 & 4 & 0.047058824 & 0 & 0 & 0 \\
\hline Axl(h) & 79 & 0 & 109 & 100 & 5 & 0.058823529 & 0 & 0 & 0 \\
\hline $\operatorname{Blk}(\mathrm{m})$ & 72 & 0 & 104 & 100 & 6 & 0.070588235 & 0 & 0 & 0 \\
\hline $\operatorname{Bmx}(\mathrm{h})$ & 102 & 0 & 103 & 100 & 7 & 0.082352941 & 0 & 0 & 0 \\
\hline CaMKII(r) & 95 & 0 & 103 & 100 & 8 & 0.094117647 & 0 & 0 & 0 \\
\hline CaMKIV(h) & 99 & 0 & 102 & 100 & 9 & 0.105882353 & 0 & 0 & 0 \\
\hline CDK1/cyclnBB(h) & 24 & 1 & 102 & 100 & 10 & 0.117647059 & 0 & 0 & 0 \\
\hline CDK2/cyclinA(h) & 14 & 1 & 102 & 100 & 11 & 0.129411765 & 0 & 0 & 0 \\
\hline $\mathrm{CDK} 2 /$ cyclinE(h) & 3 & 1 & 100 & 100 & 12 & 0.141176471 & 0 & 0 & 0 \\
\hline CDK3/cyclinE(h) & 20 & 1 & 99 & 99 & 13 & 0.152941176 & 1 & 0.000522739 & 0.000522739 \\
\hline CDK5/p35(h) & 8 & 1 & 99 & 99 & 14 & 0.164705882 & 1 & 0.000522739 & 0.001045478 \\
\hline $\mathrm{CDK} 6 / \mathrm{cyclin} \mathrm{B} 3(\mathrm{~h})$ & 89 & 0 & 99 & 99 & 15 & 0.176470588 & 1 & 0.000522739 & 0.001568217 \\
\hline CDK7/cyclinH/MATI(h) & 23 & 1 & 98 & 98 & 16 & 0.188235294 & 2 & 0.001045478 & 0.002613696 \\
\hline CHK1(h) & 97 & 0 & 97 & 97 & 17 & 0.2 & 3 & 0.001568217 & 0.004181913 \\
\hline СHK2(h) & 26 & 1 & 95 & 95 & 18 & 0.211764706 & 5 & 0.002613696 & 0.006795609 \\
\hline $\mathrm{CK} 1(\mathrm{y})$ & 63 & 0 & 95 & 95 & 19 & 0.223529412 & 5 & 0.002613696 & 0.009409305 \\
\hline СК2(h) & 89 & 0 & 95 & 95 & 20 & 0.235294118 & 5 & 0.002613696 & 0.012023001 \\
\hline c-RAF(h) & 94 & 0 & 95 & 95 & 21 & 0.247058824 & 5 & 0.002613696 & 0.014636696 \\
\hline $\operatorname{CSK}(\mathrm{h})$ & 55 & 0 & 94 & 94 & 22 & 0.258823529 & 6 & 0.003136435 & 0.017773131 \\
\hline $\operatorname{csRC(h)}$ & 99 & 0 & 94 & 94 & 23 & 0.270588235 & 6 & 0.003136435 & 0.020909566 \\
\hline Fess(h) & 38 & 1 & 93 & 93 & 24 & 0.282352941 & 7 & 0.003659174 & 0.02456874 \\
\hline FGFR3(h) & 88 & 0 & 93 & 93 & 25 & 0.294117647 & 7 & 0.003659174 & 0.028227914 \\
\hline $\mathrm{FH}$ H(h) & 67 & 0 & 93 & 93 & 26 & 0.305882353 & 7 & 0.003659174 & 0.031887088 \\
\hline Fyn(h) & 88 & 0 & 93 & 93 & 27 & 0.317647059 & 7 & 0.003659174 & 0.035546262 \\
\hline GSK3a(h) & 49 & 1 & 92 & 92 & 28 & 0.329411765 & 8 & 0.004181913 & 0.039728176 \\
\hline GSK3B(h) & 58 & 0 & 91 & 91 & $\begin{array}{l}20 \\
29\end{array}$ & 0.341176471 & $\begin{array}{l}\circ \\
9\end{array}$ & 0.004704652 & 0.044432828 \\
\hline IGF-IR(h) & 103 & 0 & 91 & 91 & 30 & 0.352941176 & 9 & 0.004704652 & 0.04913748 \\
\hline $\mathrm{IKKa}(\mathrm{h})$ & 84 & 0 & 91 & 91 & 31 & 0.364705882 & 9 & 0.004704652 & 0.053842133 \\
\hline IKKB(h) & $\begin{array}{l}84 \\
88\end{array}$ & 0 & 90 & 90 & 32 & 0.376470588 & 10 & 0.005227392 & 0.059069524 \\
\hline $\mathrm{IR}(\mathrm{h})$ & $\begin{array}{l}00 \\
100\end{array}$ & 0 & 90 & 90 & $\begin{array}{l}32 \\
33\end{array}$ & 0.388235294 & 10 & 0.005227392 & 0.064296916 \\
\hline JNKIal(h) & 55 & 0 & 90 & 90 & 34 & 0.4 & 10 & 0.005227392 & 0.069524307 \\
\hline JNK202(h) & 76 & 0 & 89 & 89 & 35 & 0.411764706 & 11 & 0.005750131 & 0.075274438 \\
\hline JNK3(r) & 41 & 1 & 89 & 89 & 36 & 0.423529412 & 11 & 0.005750131 & 0.081024569 \\
\hline Lck(h) & 94 & 0 & 89 & 89 & 37 & 0.435294118 & 11 & 0.005750131 & 0.086774699 \\
\hline Lyn(h) & 90 & 0 & 88 & 88 & 38 & 0.447058824 & 12 & 0.00627287 & 0.093047569 \\
\hline Lyy(m) & 75 & 0 & 88 & 88 & 39 & 0.458823529 & 12 & 0.00627287 & 0.099320439 \\
\hline MAPKI(h) & 44 & 1 & $\begin{array}{l}88 \\
88\end{array}$ & $\begin{array}{l}88 \\
88\end{array}$ & 40 & 0.470588235 & 12 & 0.00627287 & 0.105593309 \\
\hline MAPK2(h) & 33 & 1 & 88 & 88 & 41 & 0.482352941 & 12 & 0.00627287 & 0.111866179 \\
\hline $\begin{array}{l}\text { MAPKL(2) } \\
\text { MAPK2(m) }\end{array}$ & 26 & 1 & $\begin{array}{l}00 \\
88\end{array}$ & $\begin{array}{l}00 \\
88\end{array}$ & $\begin{array}{l}41 \\
42\end{array}$ & 0.494117647 & 12 & 0.00627287 & 0.118139049 \\
\hline MAPKAP-K2(h) & 95 & 0 & 87 & 87 & 43 & 0.505882353 & 13 & 0.006795609 & 0.124934658 \\
\hline MEK1(h) & 80 & 0 & 86 & 86 & 44 & 0.517647059 & 14 & 0.007318348 & 0.132253006 \\
\hline MKK4(m) & 82 & 0 & 85 & 85 & 45 & 0.529411765 & 15 & 0.007841087 & 0.140094093 \\
\hline MKK6(h) & 89 & 0 & 85 & 85 & 46 & 0.541176471 & 15 & 0.007841087 & 0.14793518 \\
\hline MKK & 87 & 0 & 84 & 84 & 47 & 0.552941176 & 16 & 0.008363826 & 0.156299007 \\
\hline MSKI(h) & 88 & 0 & 83 & 83 & 48 & 0.564705882 & 17 & 0.008886566 & 0.165185572 \\
\hline $\mathrm{p} 7056 \mathrm{~K}(\mathrm{~h})$ & 81 & 0 & $\begin{array}{l}83 \\
83\end{array}$ & $\begin{array}{l}83 \\
83\end{array}$ & $\begin{array}{l}48 \\
49\end{array}$ & 0.576470588 & 17 & 0.0088865666 & 0.174072138 \\
\hline $\begin{array}{l}\text { PAK2(h) } \\
\text { Past }\end{array}$ & 90 & 0 & 82 & 82 & 50 & 0.588235294 & 18 & 0.009409305 & 0.183481443 \\
\hline PDGFRa(h) & 78 & 0 & 82 & 82 & 51 & 0.6 & 18 & 0.009409305 & 0.192890748 \\
\hline PDGFRB(h) & 115 & 0 & 82 & 82 & 52 & 0.611764706 & 18 & 0.009409305 & 0.202300052 \\
\hline PDKI(h) & 110 & 0 & $\begin{array}{l}82 \\
82\end{array}$ & $\begin{array}{l}82 \\
82\end{array}$ & 53 & 0.623529412 & 18 & 0.009409305 & 0.211709357 \\
\hline PKA(b) & 111 & 0 & 82 & 82 & 54 & 0.635294118 & 18 & 0.009409305 & 0.221118662 \\
\hline 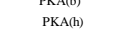 & 104 & 0 & 81 & 81 & $\begin{array}{l}54 \\
55\end{array}$ & 0.647058824 & $\begin{array}{l}10 \\
19\end{array}$ & 0.009932044 & 0.231050706 \\
\hline PKBa(h) & 91 & 0 & 80 & 80 & 56 & 0.658823529 & 20 & 0.010454783 & 0.241505489 \\
\hline PKBB(h) & 102 & 0 & 79 & 79 & 57 & 0.670588235 & 21 & 0.010977522 & 0.252483011 \\
\hline $\mathrm{PKB} \mathrm{B}_{(\mathrm{h})}$ & 111 & 0 & 79 & 79 & 58 & 0.682352941 & 21 & 0.010977522 & 0.263460533 \\
\hline PKCath) & 85 & 0 & 78 & 78 & 59 & 0.694117647 & 22 & 0.011500261 & 0.274960795 \\
\hline PKCBII(h) & 95 & 0 & 76 & 76 & 60 & 0.705882353 & 24 & 0.01254574 & 0.287506534 \\
\hline PKCy(h) & 93 & 0 & 75 & 75 & 61 & 0.717647059 & 25 & 0.013068479 & 0.300575013 \\
\hline РКФ(1) & 83 & 0 & $\begin{array}{l}15 \\
72\end{array}$ & $\begin{array}{l}15 \\
72\end{array}$ & $\begin{array}{l}61 \\
62\end{array}$ & 0.729411765 & $\begin{array}{l}20 \\
28\end{array}$ & 0.014636696 & 0.315211709 \\
\hline PKG:(h) & 93 & 0 & 72 & 72 & 63 & 0.741176471 & 28 & 0.014636696 & 0.329848406 \\
\hline $\begin{array}{l}\text { PKG (h) } \\
\text { (h) }\end{array}$ & 91 & 0 & 71 & 71 & 64 & 0.752941176 & 29 & 0.015159435 & 0.345007841 \\
\hline PKC (h) & 82 & 0 & 70 & 70 & 65 & 0.764705882 & 30 & 0.015682175 & 0.360690016 \\
\hline PKC $\mu(\mathrm{h})$ & 88 & 0 & 67 & 67 & 66 & 0.776470588 & 33 & 0.017250392 & 0.377940408 \\
\hline PKCO(h) & 93 & 0 & 65 & 65 & 67 & 0.788235294 & 35 & 0.01829587 & 0.396236278 \\
\hline PKD2(h) & 92 & 0 & 65 & 65 & 68 & 0.8 & 35 & 0.01829587 & 0.414532148 \\
\hline PRAK(h) & 109 & 0 & 63 & 63 & 69 & 0.811764706 & 37 & 0.019341349 & 0.433873497 \\
\hline PRK2(h) & 82 & 0 & 58 & 58 & 70 & 0.823529412 & 42 & 0.021955044 & 0.455828542 \\
\hline $\begin{array}{l}\text { PRK_(n) } \\
\text { ROCK-II(h) }\end{array}$ & 93 & 0 & 55 & 55 & 71 & 0.835294118 & 45 & 0.023523262 & 0.479351803 \\
\hline ROCK-II(r) & 102 & 0 & 55 & 55 & 72 & 0.847058824 & 45 & 0.023523262 & 0.502875065 \\
\hline Rskl(h) & 83 & 0 & 49 & 49 & 73 & 0.858823529 & 51 & 0.026659697 & 0.529534762 \\
\hline Rskl(r) & 65 & 0 & 44 & 44 & 74 & 0.870588235 & 56 & 0.029273393 & 0.558808155 \\
\hline $\begin{array}{l}\text { Rsksk2(h) } \\
\text { Rsk(h) }\end{array}$ & 71 & 0 & 41 & 41 & 75 & 0.882352941 & 59 & 0.03084161 & 0.589649765 \\
\hline Rsk3(h) & 72 & 0 & 38 & 38 & 76 & 0.894117647 & 62 & 0.032409827 & 0.622059592 \\
\hline SAPK2a(h) & 70 & 0 & 33 & 33 & 77 & 0.905882353 & 67 & 0.035023523 & 0.657083116 \\
\hline SAPK2b(h) & 85 & 0 & 26 & 26 & 78 & 0.917647059 & 74 & 0.038682697 & 0.695765813 \\
\hline SAPK3(h) & 103 & 0 & 26 & 26 & 79 & 0.929411765 & 74 & 0.038682697 & 0.73444851 \\
\hline SAPK4(h) & 98 & 0 & 24 & 24 & 80 & 0.941176471 & 76 & 0.039728176 & 0.774176686 \\
\hline SGK(h) & 82 & 0 & 23 & 23 & 81 & 0.952941176 & 77 & 0.040250915 & 0.814427601 \\
\hline Syk(h) & 82 & 0 & 20 & 20 & 82 & 0.964705882 & 80 & 0.041819132 & 0.856246733 \\
\hline TrkB(h) & 99 & 0 & 14 & 14 & 83 & 0.976470588 & 86 & 0.044955567 & 0.9012023 \\
\hline Yes(h) & 91 & 0 & 8 & 8 & 84 & 0.988235294 & 92 & 0.048092002 & 0.949294302 \\
\hline ZAP-70(h) & 79 & 0 & 3 & 3 & 85 & 1 & 97 & 0.050705698 & 1 \\
\hline & & & & & & Total inhibition & 1913 & & Total area \\
\hline & $\begin{array}{r}\text { Hit rate } \\
\text {. }\end{array}$ & & & & & & & & Gini \\
\hline
\end{tabular}




\begin{tabular}{|c|c|c|c|c|c|c|c|c|c|c|}
\hline & $100 \mu \mathrm{M}$ ATP & & & & & & & & & \\
\hline Kinase & Olomoucin @ $50 \mu \mathrm{M}$ & Hit & $\begin{array}{c}\text { sorted } \\
130\end{array}$ & normalized & position & cumulative sample fraction & inhibition\% & fraction of total inhibition & cumulative inhibition & area \\
\hline $\begin{array}{c}\mathrm{Abl}(\mathrm{m}) \\
\mathrm{AMPK}(\mathrm{r})\end{array}$ & $\begin{array}{c}92 \\
101\end{array}$ & $\begin{array}{l}0 \\
0\end{array}$ & $\begin{array}{l}130 \\
117\end{array}$ & $\begin{array}{l}100 \\
100\end{array}$ & $\begin{array}{l}1 \\
2\end{array}$ & $\begin{array}{l}0.011764706 \\
0.023529412\end{array}$ & $\begin{array}{l}0 \\
0\end{array}$ & 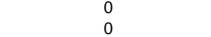 & $\begin{array}{l}0 \\
0\end{array}$ & $\begin{array}{l}0 \\
0\end{array}$ \\
\hline 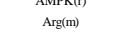 & $\begin{array}{l}101 \\
99\end{array}$ & 0 & 113 & 100 & 3 & 0.035294118 & 0 & 0 & 0 & 0 \\
\hline Aurora-A(h) & 93 & 0 & 113 & 100 & 4 & 0.047058824 & 0 & 0 & 0 & 0 \\
\hline Axl(h) & 77 & 0 & 112 & 100 & 5 & 0.058823529 & 0 & 0 & 0 & 0 \\
\hline $\mathrm{Blk}(\mathrm{m})$ & 89 & 0 & 112 & 100 & 6 & 0.070588235 & 0 & 0 & 0 & 0 \\
\hline $\operatorname{Bmx}(\mathrm{h})$ & 100 & 0 & 110 & 100 & 7 & 0.082352941 & 0 & 0 & 0 & 0 \\
\hline СамKII(r) & 112 & 0 & 108 & 100 & 8 & 0.094117647 & 0 & 0 & 0 & 0 \\
\hline CaMKIV(h) & 113 & 0 & 108 & 100 & 9 & 0.105882353 & 0 & 0 & 0 & 0 \\
\hline CDK1/cyclinB(h) & 44 & 1 & 107 & 100 & 10 & 0.117647059 & 0 & 0 & 0 & 0 \\
\hline CDK2/cyclinA(h) & 45 & 1 & 105 & 100 & 11 & 0.129411765 & 0 & 0 & 0 & 0 \\
\hline $\mathrm{CDK} 2 /$ cyclinE(h) & 13 & 1 & 104 & 100 & 12 & 0.141176471 & 0 & 0 & 0 & 0 \\
\hline CDK3/cyclinE(h) & 49 & 1 & 104 & 100 & 13 & 0.152941176 & 0 & 0 & 0 & 0 \\
\hline $\mathrm{CDK} 5 / \mathrm{p} 35(\mathrm{~h})$ & 71 & 0 & 103 & 100 & 14 & 0.164705882 & 0 & 0 & 0 & 0 \\
\hline CDK6/cyclinD3(h) & 92 & 0 & 102 & 100 & 15 & 0.176470588 & 0 & 0 & 0 & 0 \\
\hline CDK7/cyclinH/MATI(h) & 30 & 1 & 102 & 100 & 16 & 0.188235294 & 0 & 0 & 0 & 0 \\
\hline СHK1(h) & 100 & 0 & 102 & 100 & 17 & 0.2 & 0 & 0 & 0 & 0 \\
\hline СHK2(h) & 52 & 0 & 101 & 100 & 18 & 0.211764706 & 0 & 0 & 0 & 0 \\
\hline $\mathrm{CKI}(y)$ & 88 & 0 & 101 & 100 & 19 & 0.223529412 & 0 & 0 & 0 & 0 \\
\hline $\mathrm{CK} 2(\mathrm{~h})$ & 102 & 0 & 101 & 100 & 20 & 0.235294118 & 0 & 0 & 0 & 0 \\
\hline c-RAF(h) & 102 & 0 & 100 & 100 & 21 & 0.247058824 & 0 & 0 & 0 & 0 \\
\hline CSK(h) & 104 & 0 & 100 & 100 & 22 & 0.258823529 & 0 & 0 & 0 & 0 \\
\hline cSRC(h) & 88 & 0 & 100 & 100 & 23 & 0.270588235 & 0 & 0 & 0 & 0 \\
\hline Fes(h) & 60 & 0 & 99 & 99 & 24 & 0.282352941 & 1 & 0.000935454 & 0.000935454 & 5.50267E- 06 \\
\hline FGFR3(h) & 87 & 0 & 99 & 99 & 25 & 0.294117647 & 1 & 0.000935454 & 0.001870907 & $1.6508 \mathrm{E}-05$ \\
\hline $\mathrm{Fll}(\mathrm{h}(\mathrm{h})$ & 86 & 0 & 99 & 99 & 26 & 0.305882353 & 1 & 0.000935454 & 0.002806361 & 2.75133E- 05 \\
\hline Fynn(h) & 101 & 0 & 99 & 99 & 27 & 0.317647059 & 1 & 0.000935454 & 0.003741815 & 3.85187E-05 \\
\hline GSK3a(h) & 93 & 0 & 99 & 99 & 28 & 0.329411765 & 1 & 0.000935454 & 0.004677268 & 4.9524E-05 \\
\hline GSK3B(h) & 71 & 0 & 98 & 98 & 29 & 0.341176471 & 2 & 0.001870907 & 0.006548176 & 6.6032E- -05 \\
\hline IGF-1R(h) & 112 & 0 & 98 & 98 & 30 & 0.352941176 & 2 & 0.001870907 & 0.008419083 & 8.80427E-05 \\
\hline $\mathrm{IKKa}(\mathrm{h})$ & 99 & 0 & 98 & 98 & 31 & 0.364705882 & 2 & 0.001870907 & 0.010289991 & 0.000110053 \\
\hline $\mathrm{IKKB(h)}$ & 93 & 0 & 98 & 98 & 32 & 0.376470588 & 2 & 0.001870907 & 0.012160898 & 0.000132064 \\
\hline $\mathbb{I R ( h )}$ & 107 & 0 & 98 & 98 & 33 & 0.388235294 & 2 & 0.001870907 & 0.014031805 & 0.000154075 \\
\hline JNKIal(h) & 98 & 0 & 97 & 97 & 34 & 0.4 & 3 & 0.002806361 & 0.016838167 & 0.000181588 \\
\hline JNK2202(h) & 117 & 0 & 97 & 97 & 35 & 0.411764706 & 3 & 0.002806361 & 0.019644528 & 0.000214604 \\
\hline $\mathrm{JNK} 3(\mathrm{r})$ & 89 & 0 & 96 & 96 & 36 & 0.423529412 & 4 & 0.003741815 & 0.023386342 & 0.000253123 \\
\hline Lck(h) & 99 & 0 & 95 & 95 & 37 & 0.435294118 & 5 & 0.004677268 & 0.028063611 & 0.000302647 \\
\hline Lyn(h) & 94 & 0 & 95 & 95 & 38 & 0.447058824 & 5 & 0.004677268 & 0.032740879 & 0.000357673 \\
\hline Lyy(m) & 91 & 0 & 94 & 94 & 39 & 0.458823529 & 6 & 0.005612722 & 0.038353601 & 0.000418203 \\
\hline MAPK I (h) & 47 & 1 & 94 & 94 & 40 & 0.470588235 & 6 & 0.005612722 & 0.043966324 & 0.000484235 \\
\hline MAPK2(h) & 55 & 0 & 94 & 94 & 41 & 0.482352941 & 6 & 0.005612722 & 0.049579046 & 0.000550267 \\
\hline MAPK2(m) & 38 & 1 & 93 & 93 & 42 & 0.494117647 & 7 & 0.006548176 & 0.056127222 & 0.000621802 \\
\hline MAPKAP-K2(h) & 113 & 0 & 93 & 93 & 43 & 0.505882353 & 7 & 0.006548176 & 0.062675398 & 0.000698839 \\
\hline MEK1(h) & 75 & 0 & 93 & 93 & 44 & 0.517647059 & 7 & 0.006548176 & 0.069223573 & 0.000775876 \\
\hline MKK 4(m) & 87 & 0 & 93 & 93 & 45 & 0.529411765 & 7 & 0.006548176 & 0.075771749 & 0.000852914 \\
\hline MKK6(h) & 81 & 0 & 93 & 93 & 46 & 0.541176471 & 7 & 0.006548176 & 0.082319925 & 0.000929951 \\
\hline MKK 7 (h) & 89 & 0 & 92 & 92 & 47 & 0.552941176 & 8 & 0.00748363 & 0.089803555 & 0.001012491 \\
\hline MSK1(h) & 97 & 0 & 92 & 92 & 48 & 0.564705882 & 8 & 0.00748363 & 0.097287184 & 0.001100534 \\
\hline $\mathrm{p} 70 \mathrm{~S} 6 \mathrm{~K}(\mathrm{~h})$ & 102 & 0 & 92 & 92 & 49 & 0.576470588 & 8 & 0.00748363 & 0.104770814 & 0.001188576 \\
\hline PAK2(h) & 99 & 0 & 92 & 92 & 50 & 0.588235294 & 8 & 0.00748363 & 0.112254443 & 0.001276619 \\
\hline PDGFRa(h) & 110 & 0 & 91 & 91 & 51 & 0.6 & 9 & 0.008419083 & 0.120673527 & 0.001370165 \\
\hline PDGFRß(h) & 86 & 0 & 91 & 91 & 52 & 0.611764706 & 9 & 0.008419083 & 0.12909261 & 0.001469213 \\
\hline PDK1(h) & 100 & 0 & 89 & 89 & 53 & 0.623529412 & 11 & 0.010289991 & 0.139382601 & 0.001579266 \\
\hline PKA(b) & 94 & 0 & 89 & 89 & 54 & 0.635294118 & 11 & 0.010289991 & 0.149672591 & 0.001700325 \\
\hline PKA(h) & 98 & 0 & 89 & 89 & 55 & 0.647058824 & 11 & 0.010289991 & 0.159962582 & 0.001821383 \\
\hline PKBa(h) & 92 & 0 & 89 & 89 & 56 & 0.658823529 & 11 & 0.010289991 & 0.170252572 & 0.001942442 \\
\hline PKBB(h) & 99 & 0 & 89 & 89 & 57 & 0.670588235 & 11 & 0.010289991 & 0.180542563 & 0.002063501 \\
\hline $\mathrm{PKB}_{\gamma}(\mathrm{h})$ & 108 & 0 & 88 & 88 & 58 & 0.682352941 & 12 & 0.011225444 & 0.191768007 & 0.002190062 \\
\hline PKCa(h) & 95 & 0 & 88 & 88 & 59 & 0.694117647 & 12 & 0.011225444 & 0.202993452 & 0.002322126 \\
\hline РКСBI(h) & 98 & 0 & 88 & 88 & 60 & 0.705882353 & 12 & 0.011225444 & 0.214218896 & 0.00245419 \\
\hline PKCy(h) & 92 & 0 & 88 & 88 & 61 & 0.717647059 & 12 & 0.011225444 & 0.225444341 & 0.002586254 \\
\hline РКБ(h) & 85 & 0 & 87 & 87 & 62 & 0.729411765 & 13 & 0.012160898 & 0.237605239 & 0.002723821 \\
\hline PKG:(h) & 88 & 0 & 87 & 87 & 63 & 0.741176471 & 13 & 0.012160898 & 0.249766137 & 0.00286689 \\
\hline PKG(h) & 97 & 0 & 87 & 87 & 64 & 0.752941176 & 13 & 0.012160898 & 0.261927035 & 0.00300996 \\
\hline PKC 1 (h) & 86 & 0 & 86 & 86 & 65 & 0.764705882 & 14 & 0.013096352 & 0.275023386 & 0.003158532 \\
\hline PKC $\mu(\mathrm{h})$ & 96 & 0 & 86 & 86 & 66 & 0.776470588 & 14 & 0.013096352 & 0.288119738 & 0.003312607 \\
\hline PKCQ(h) & 89 & 0 & 86 & 86 & 67 & 0.788235294 & 14 & 0.013096352 & 0.30121609 & 0.003466681 \\
\hline PKD2(h) & 101 & 0 & 85 & 85 & 68 & 0.8 & 15 & 0.014031805 & 0.315247895 & 0.003626259 \\
\hline $\operatorname{PRAK(h)}$ & 130 & 0 & 81 & 81 & 69 & 0.811764706 & 19 & 0.01777362 & 0.333021515 & 0.003813349 \\
\hline PRK2(h) & 104 & 0 & 81 & 81 & 70 & 0.823529412 & 19 & 0.01777362 & 0.350795136 & 0.004022451 \\
\hline ROCK-II(h) & 93 & 0 & 79 & 79 & 71 & 0.835294118 & 21 & 0.019644528 & 0.370439663 & 0.004242558 \\
\hline ROCK-II(r) & 103 & 0 & 77 & 77 & 72 & 0.847058824 & 23 & 0.021515435 & 0.391955098 & 0.004484675 \\
\hline Rskl(h) & 89 & 0 & 75 & 75 & 73 & 0.858823529 & 25 & 0.023386342 & 0.415341441 & 0.004748803 \\
\hline Rskl(r) & 87 & 0 & 71 & 71 & 74 & 0.870588235 & 29 & 0.027128157 & 0.442469598 & 0.005045947 \\
\hline Rsk2(h) & 88 & 0 & 71 & 71 & 75 & 0.882352941 & 29 & 0.027128157 & 0.469597755 & 0.005365102 \\
\hline Rsk3(h) & 79 & 0 & 60 & 60 & 76 & 0.894117647 & 40 & 0.037418148 & 0.507015903 & 0.005744786 \\
\hline SAPK2a(h) & 98 & 0 & 55 & 55 & 77 & 0.905882353 & 45 & 0.042095416 & 0.549111319 & 0.006212513 \\
\hline SAPK2b(h) & 108 & 0 & 52 & 52 & 78 & 0.917647059 & 48 & 0.044901777 & 0.594013096 & 0.006724261 \\
\hline $\mathrm{SAPK} 3(\mathrm{~h})$ & 105 & 0 & 49 & 49 & 79 & 0.929411765 & 51 & 0.047708138 & 0.641721235 & 0.007269025 \\
\hline SAPK4(h) & 94 & 0 & 47 & 47 & 80 & 0.941176471 & 53 & 0.049579046 & 0.691300281 & 0.007841303 \\
\hline SGK(h) & 81 & 0 & 45 & 45 & 81 & 0.952941176 & 55 & 0.051449953 & 0.742750234 & 0.008435591 \\
\hline Syk(h) & 98 & 0 & 44 & 44 & 82 & 0.964705882 & 56 & 0.052385407 & 0.795135641 & 0.009046387 \\
\hline TrkB(h) & 91 & 0 & 38 & 38 & 83 & 0.976470588 & 62 & 0.057998129 & 0.85313377 & 0.009695702 \\
\hline Yes(h) & 93 & 0 & 30 & 30 & 84 & 0.988235294 & 70 & 0.065481759 & 0.918615529 & 0.010422055 \\
\hline ZAP-70(h) & 95 & 0 & 13 & 13 & 85 & 1 & 87 & 0.081384471 & 1 & 0.011285974 \\
\hline & & & & & & Total inhibition & 1069 & & Total area & 0.169971936 \\
\hline & $\begin{array}{r}\text { Hit rate } \\
\text { Threshold }\end{array}$ & 5 & & & & & & & Gini & 0.660056127 \\
\hline
\end{tabular}




\begin{tabular}{|c|c|c|c|c|c|c|c|c|c|}
\hline & $10 \mu \mathrm{M}$ ATP & & & & & & & & \\
\hline Kinase & PD153035@ $0.001 \mu \mathrm{M}$ & Hit & sorted & normalized & position & cumulative sample fraction & inhibition\% & fraction of total inhibition & cumulative inhibition \\
\hline $\mathrm{Abl}(\mathrm{m})$ & 94 & 0 & 118 & 100 & 1 & 0.011764706 & 0 & 0 & 0 \\
\hline AMPK(r) & 102 & 0 & 111 & 100 & 2 & 0.023529412 & 0 & 0 & 0 \\
\hline $\operatorname{Arg}(\mathrm{m})$ & 100 & 0 & 110 & 100 & 3 & 0.035294118 & 0 & 0 & 0 \\
\hline Aurora-A(h) & 91 & 0 & 107 & 100 & 4 & 0.047058824 & 0 & 0 & 0 \\
\hline Axl(h) & 92 & 0 & 107 & 100 & 5 & 0.058823529 & 0 & 0 & 0 \\
\hline $\operatorname{Blk}(\mathrm{m})$ & 76 & 0 & 107 & 100 & 6 & 0.070588235 & 0 & 0 & 0 \\
\hline $\mathrm{Bmx(h)}$ & 99 & 0 & 107 & 100 & 7 & 0.082352941 & 0 & 0 & 0 \\
\hline CaMKII(r) & 89 & 0 & 105 & 100 & 8 & 0.094117647 & 0 & 0 & 0 \\
\hline CaMKIV(h) & 105 & 0 & 105 & 100 & 9 & 0.105882353 & 0 & 0 & 0 \\
\hline CDK1/cyclnBB(h) & 97 & 0 & 105 & 100 & 10 & 0.117647059 & 0 & 0 & 0 \\
\hline CDK2/cyclinA(h) & 88 & 0 & 104 & 100 & 11 & 0.129411765 & 0 & 0 & 0 \\
\hline $\mathrm{CDK} 2 /$ cyclinE(h) & 96 & 0 & 104 & 100 & 12 & 0.141176471 & 0 & 0 & 0 \\
\hline CDK3/cyclinE(h) & 103 & 0 & $\begin{array}{l}103 \\
103\end{array}$ & 100 & 13 & 0.152941176 & 0 & 0 & 0 \\
\hline CDK5/p35(h) & 99 & 0 & 103 & 100 & 14 & 0.164705882 & 0 & 0 & 0 \\
\hline CDK6/cyclinD3(h) & 93 & 0 & 103 & 100 & 15 & 0.176470588 & 0 & 0 & 0 \\
\hline CDK $7 /$ cyclinHMATI(h) & 93 & 0 & 103 & 100 & 16 & 0.188235294 & 0 & 0 & 0 \\
\hline CHK1 (h) & 100 & 0 & 102 & 100 & 17 & 0.2 & 0 & 0 & 0 \\
\hline CHK2(h) & 98 & 0 & 102 & 100 & 18 & 0.211764706 & 0 & 0 & 0 \\
\hline CKI(y) & 99 & 0 & 102 & 100 & 19 & 0.223529412 & 0 & 0 & 0 \\
\hline СК2(h) & 97 & 0 & 102 & 100 & 20 & 0.235294118 & 0 & 0 & 0 \\
\hline c-RAF(h) & 95 & 0 & 101 & 100 & 21 & 0.247058824 & 0 & 0 & 0 \\
\hline $\operatorname{CSK}(\mathrm{h})$ & 62 & 0 & 101 & 100 & 22 & 0.258823529 & 0 & 0 & 0 \\
\hline $\operatorname{csRC(h)}$ & 111 & 0 & 101 & 100 & 23 & 0.270588235 & 0 & 0 & 0 \\
\hline Fess(h) & 98 & 0 & 100 & 100 & 24 & 0.282352941 & 0 & 0 & 0 \\
\hline FGFR3(h) & 92 & 0 & 100 & 100 & 25 & 0.294117647 & 0 & 0 & 0 \\
\hline $\mathrm{Fll}(\mathrm{h}(\mathrm{h})$ & 100 & 0 & 100 & 100 & 26 & 0.305882353 & 0 & 0 & 0 \\
\hline Fyn(h) & 57 & 0 & 99 & 99 & 27 & 0.317647059 & 1 & 0.001912046 & 0.001912046 \\
\hline GSK3a(h) & 87 & 0 & 99 & 99 & 28 & 0.329411765 & 1 & 0.001912046 & 0.003824092 \\
\hline GSK3B(h) & 93 & 0 & 99 & 99 & $\begin{array}{l}20 \\
29\end{array}$ & 0.341176471 & 1 & 0.001912046 & 0.005736138 \\
\hline IGF-IR(h) & 97 & 0 & 99 & 99 & 30 & 0.352941176 & 1 & 0.001912046 & 0.007648184 \\
\hline $\mathrm{IKKa(h)}$ & 98 & 0 & 99 & 99 & 31 & 0.364705882 & 1 & 0.001912046 & 0.009560229 \\
\hline IKKB(h) & 91 & 0 & 99 & 99 & 32 & 0.376470588 & 1 & 0.001912046 & 0.011472275 \\
\hline $\mathbb{I R}(\mathrm{h})$ & 104 & 0 & 99 & 99 & $\begin{array}{l}32 \\
33\end{array}$ & 0.388235294 & 1 & 0.001912046 & 0.013384321 \\
\hline JNKIal(h) & 94 & 0 & 98 & 98 & 34 & 0.4 & 2 & 0.003824092 & 0.017208413 \\
\hline JNK202(h) & 99 & 0 & 98 & 98 & $\begin{array}{l}54 \\
35\end{array}$ & 0.411764706 & 2 & 0.003824092 & 0.021032505 \\
\hline JNK3(r) & 107 & 0 & 98 & 98 & 36 & 0.423529412 & 2 & 0.003824092 & 0.024856597 \\
\hline Lck(h) & 105 & 0 & 98 & 98 & 37 & 0.435294118 & 2 & 0.003824092 & 0.028680688 \\
\hline Lyn(h) & 99 & 0 & 98 & 98 & 38 & 0.447058824 & 2 & 0.003824092 & 0.03250478 \\
\hline Lyy(m) & 73 & 0 & 97 & 97 & 39 & 0.458823529 & 3 & 0.005736138 & 0.038240918 \\
\hline MAPKI(h) & $\begin{array}{l}13 \\
94\end{array}$ & 0 & 97 & 97 & 40 & 0.470588235 & 3 & 0.005736138 & 0.043977055 \\
\hline MAPK2(h) & 97 & 0 & 97 & 97 & 41 & 0.482352941 & 3 & 0.005736138 & 0.049713193 \\
\hline $\begin{array}{l}\text { MAPKL(2) } \\
\text { MAPK2(m) }\end{array}$ & 89 & 0 & 97 & 97 & $\begin{array}{l}41 \\
42\end{array}$ & 0.494117647 & 3 & 0.005736138 & 0.055449331 \\
\hline MAPKAP-K2(h) & 105 & 0 & 96 & 96 & 43 & 0.505882353 & 4 & 0.007648184 & 0.063097514 \\
\hline MEK1(h) & 90 & 0 & 96 & 96 & 44 & 0.517647059 & 4 & 0.007648184 & 0.070745698 \\
\hline MKK4(m) & 80 & 0 & 96 & 96 & 45 & 0.529411765 & 4 & 0.007648184 & 0.078393881 \\
\hline MKK6(h) & 104 & 0 & 95 & 95 & 46 & 0.541176471 & 5 & 0.009560229 & 0.087954111 \\
\hline MKK & 89 & 0 & 95 & 95 & 47 & 0.552941176 & 5 & 0.009560229 & 0.09751434 \\
\hline MSKI(h) & 94 & 0 & 95 & 95 & 48 & 0.564705882 & 5 & 0.009560229 & 0.10707457 \\
\hline $\mathrm{p} 7056 \mathrm{~K}(\mathrm{~h})$ & $\begin{array}{l}94 \\
85\end{array}$ & 0 & 94 & 94 & $\begin{array}{l}48 \\
49\end{array}$ & 0.576470588 & $\begin{array}{l}5 \\
6\end{array}$ & 0.011472275 & 0.118546845 \\
\hline $\begin{array}{l}\text { PAK2(h) } \\
\text { Past }\end{array}$ & 99 & 0 & 94 & 94 & 50 & 0.588235294 & 6 & 0.011472275 & 0.13001912 \\
\hline PDGFRa(h) & 83 & 0 & 94 & 94 & 51 & 0.6 & 6 & 0.011472275 & 0.141491396 \\
\hline PDGFRB(h) & 118 & 0 & 94 & 94 & 52 & 0.611764706 & 6 & 0.011472275 & 0.152963671 \\
\hline PDKI(h) & 103 & 0 & 93 & 93 & 53 & 0.623529412 & 7 & 0.013384321 & 0.166347992 \\
\hline PKA(b) & 103 & 0 & 93 & 93 & 54 & 0.635294118 & 7 & 0.013384321 & 0.179732314 \\
\hline PKA(h) & 102 & 0 & 93 & 93 & 55 & 0.647058824 & 7 & 0.013384321 & 0.193116635 \\
\hline PKBa(h) & 96 & 0 & 93 & 93 & 56 & 0.658823529 & 7 & 0.013384321 & 0.206500956 \\
\hline PKBB(h) & 93 & 0 & 93 & 93 & 57 & 0.670588235 & 7 & 0.013384321 & 0.219885277 \\
\hline $\mathrm{PKB} \mathrm{B}_{(\mathrm{h})}$ & 92 & 0 & 92 & 92 & 58 & 0.682352941 & 8 & 0.015296367 & 0.235181644 \\
\hline PKCath) & 98 & 0 & 92 & 92 & 59 & 0.694117647 & 8 & 0.015296367 & 0.250478011 \\
\hline PKCBII(h) & 99 & 0 & 92 & 92 & 60 & 0.705882353 & 8 & 0.015296367 & 0.265774379 \\
\hline PKGy(h) & 101 & 0 & 92 & 92 & 61 & 0.717647059 & 8 & 0.015296367 & 0.281070746 \\
\hline РКФ(h) & 102 & 0 & 92 & 92 & 62 & 0.729411765 & 8 & 0.015296367 & 0.296367113 \\
\hline PKG:(h) & 96 & 0 & 91 & 91 & 63 & 0.741176471 & 9 & 0.017208413 & 0.313575526 \\
\hline $\begin{array}{l}\text { PKG (h) } \\
\text { (h) }\end{array}$ & 103 & 0 & 91 & 91 & 64 & 0.752941176 & 9 & 0.017208413 & 0.330783939 \\
\hline PKC (h) & 102 & 0 & 91 & 91 & 65 & 0.764705882 & 9 & 0.017208413 & 0.347992352 \\
\hline PKC $\mu(\mathrm{h})$ & 90 & 0 & 90 & 90 & 66 & 0.776470588 & 10 & 0.019120459 & 0.367112811 \\
\hline PKCO(h) & 95 & 0 & 90 & 90 & 67 & 0.788235294 & 10 & 0.019120459 & 0.38623327 \\
\hline PKD2(h) & 92 & 0 & 90 & 90 & 68 & 0.8 & 10 & 0.019120459 & 0.405353728 \\
\hline PRAK(h) & 107 & 0 & 90 & 90 & 69 & 0.811764706 & 10 & 0.019120459 & 0.424474187 \\
\hline PRK2(h) & 95 & 0 & 89 & 89 & 70 & 0.823529412 & 11 & 0.021032505 & 0.445506692 \\
\hline $\begin{array}{l}\text { PRK_(n) } \\
\text { ROCK-II(h) }\end{array}$ & 92 & 0 & 89 & $\begin{array}{l}89 \\
89\end{array}$ & 71 & 0.835294118 & 11 & 0.021032505 & 0.466539197 \\
\hline ROCK-II(r) & 110 & 0 & 89 & 89 & 72 & 0.847058824 & 11 & 0.021032505 & 0.487571702 \\
\hline Rskl(h) & 101 & 0 & 88 & 88 & 73 & 0.858823529 & 12 & 0.022944551 & 0.510516252 \\
\hline Rskl(r) & 87 & 0 & 88 & 88 & 74 & 0.870588235 & 12 & 0.022944551 & 0.533460803 \\
\hline $\begin{array}{l}\text { Rsksk2(h) } \\
\text { Rsk(h) }\end{array}$ & 91 & 0 & 87 & 87 & 75 & 0.882352941 & 13 & 0.024856597 & 0.5583174 \\
\hline Rsk3(h) & 101 & 0 & 87 & 87 & 76 & 0.894117647 & 13 & 0.024856597 & 0.583173996 \\
\hline SAPK2a(h) & 90 & 0 & 86 & 86 & 77 & 0.905882353 & 14 & 0.026768642 & 0.609942639 \\
\hline SAPK2b(h) & 93 & 0 & 85 & 85 & 78 & 0.917647059 & 15 & 0.028680688 & 0.638623327 \\
\hline SAPK3(h) & 86 & 0 & 83 & 83 & 79 & 0.929411765 & 17 & 0.03250478 & 0.671128107 \\
\hline SAPK4(h) & 98 & 0 & 80 & 80 & 80 & 0.941176471 & 20 & 0.038240918 & 0.709369025 \\
\hline SGK(h) & 90 & 0 & 80 & 80 & 81 & 0.952941176 & 20 & 0.038240918 & 0.747609943 \\
\hline Syk(h) & 80 & 0 & 76 & 76 & 82 & 0.964705882 & 24 & 0.045889101 & 0.793499044 \\
\hline TrkB(h) & 107 & 0 & 73 & 73 & 83 & 0.976470588 & 27 & 0.051625239 & 0.845124283 \\
\hline Yes(h) & 88 & 0 & 62 & 62 & 84 & 0.988235294 & 38 & 0.072657744 & 0.917782027 \\
\hline ZAP-70(h) & 107 & 0 & 57 & 57 & 85 & 1 & 43 & 0.082217973 & 1 \\
\hline & & & & & & Total inhibition & 523 & & Total area \\
\hline & Hit rate & & & & & & & & Gini \\
\hline
\end{tabular}




\begin{tabular}{|c|c|c|c|c|c|c|c|c|c|}
\hline & $100 \mu \mathrm{M}$ ATP & & & & & & & & \\
\hline Kinase & PD153035@ $0.001 \mu \mathrm{M}$ & Hit & sorted & normalized & position & cumulative sample fraction & inhibition\% & fraction of total inhibition & cumulative inhibition \\
\hline $\mathrm{Abl}(\mathrm{m})$ & 95 & 0 & 133 & 100 & 1 & 0.011764706 & 0 & 0 & 0 \\
\hline AMPK(r) & 100 & 0 & 122 & 100 & 2 & 0.023529412 & 0 & 0 & 0 \\
\hline $\operatorname{Arg}(\mathrm{m})$ & 95 & 0 & 122 & 100 & 3 & 0.035294118 & 0 & 0 & 0 \\
\hline Aurora-A(h) & 76 & 0 & 115 & 100 & 4 & 0.047058824 & 0 & 0 & 0 \\
\hline Axl(h) & 81 & 0 & 115 & 100 & 5 & 0.058823529 & 0 & 0 & 0 \\
\hline $\mathrm{Bl}(\mathrm{m})$ & 85 & 0 & 111 & 100 & 6 & 0.070588235 & 0 & 0 & 0 \\
\hline $\operatorname{Bmx}(\mathrm{h})$ & 99 & 0 & 110 & 100 & 7 & 0.082352941 & 0 & 0 & 0 \\
\hline CaMKII(r) & 106 & 0 & 110 & 100 & 8 & 0.094117647 & 0 & 0 & 0 \\
\hline CaMKIV(h) & 87 & 0 & 108 & 100 & 9 & 0.105882353 & 0 & 0 & 0 \\
\hline $\mathrm{CDK} 1 / \mathrm{cyclin} B(\mathrm{~h})$ & 87 & 0 & 108 & 100 & 10 & 0.117647059 & 0 & 0 & 0 \\
\hline $\operatorname{CDK} 2 /$ cyctina(h) & 91 & 0 & 107 & 100 & 11 & 0.129411765 & 0 & 0 & 0 \\
\hline $\mathrm{CDK} 2 /$ cyclinE(h) & 97 & 0 & 106 & 100 & 12 & 0.141176471 & 0 & 0 & 0 \\
\hline CDK3/cyclinE(h) & 108 & 0 & 106 & 100 & 13 & 0.152941176 & 0 & 0 & 0 \\
\hline CDK5/p35(h) & 115 & 0 & 105 & 100 & 14 & 0.164705882 & 0 & 0 & 0 \\
\hline $\mathrm{CDK} / \mathrm{cyc} \ln \mathrm{D} 3(\mathrm{~h})$ & 90 & 0 & 105 & 100 & 15 & 0.176470588 & 0 & 0 & 0 \\
\hline CDK7/cyclinHMATI(h) & 94 & 0 & 105 & 100 & 16 & 0.188235294 & 0 & 0 & 0 \\
\hline CHK1(h) & 102 & 0 & 104 & 100 & 17 & 0.2 & 0 & 0 & 0 \\
\hline CHK2(h) & 98 & 0 & 102 & 100 & 18 & 0.211764706 & 0 & 0 & 0 \\
\hline $\mathrm{CKI}(\mathrm{y})$ & 87 & 0 & 102 & 100 & 19 & 0.223529412 & 0 & 0 & 0 \\
\hline СК2(h) & 97 & 0 & 102 & 100 & 20 & 0.235294118 & 0 & 0 & 0 \\
\hline$c-\operatorname{RAF}(\mathrm{h})$ & 102 & 0 & 102 & 100 & 21 & 0.247058824 & 0 & 0 & 0 \\
\hline CSK(h) & 133 & 0 & 102 & 100 & 22 & 0.258823529 & 0 & 0 & 0 \\
\hline $\mathrm{cSRC(h)}$ & 94 & 0 & 102 & 100 & 23 & 0.270588235 & 0 & 0 & 0 \\
\hline Fes(h) & 105 & 0 & 101 & 100 & 24 & 0.282352941 & 0 & 0 & 0 \\
\hline FGFR3(h) & 87 & 0 & 101 & 100 & 25 & 0.294117647 & 0 & 0 & 0 \\
\hline Flls(h) & 97 & 0 & 100 & 100 & 26 & 0.305882353 & 0 & 0 & 0 \\
\hline Fyn(h) & 98 & 0 & 100 & 100 & 27 & 0.317647059 & 0 & 0 & 0 \\
\hline GSK3a $a(\mathrm{~h})$ & 100 & 0 & 100 & 100 & 28 & 0.329411765 & 0 & 0 & 0 \\
\hline GSK3B(h) & 85 & 0 & 100 & 100 & $\begin{array}{l}20 \\
29\end{array}$ & 0.341176471 & 0 & 0 & 0 \\
\hline IGF-IR(h) & 102 & 0 & 99 & 99 & 30 & 0.352941176 & 1 & 0.00234192 & 0.00234192 \\
\hline IKKu(h) & 105 & 0 & 99 & 99 & 31 & 0.364705882 & 1 & 0.00234192 & 0.004683841 \\
\hline IKKB(h) & 102 & 0 & 99 & 99 & 32 & 0.376470588 & 1 & 0.00234192 & 0.007025761 \\
\hline $\mathrm{IR}(\mathrm{h})$ & 115 & 0 & 99 & 99 & 33 & 0.388235294 & 1 & 0.00234192 & 0.009367681 \\
\hline JNKIal(h) & 104 & 0 & 98 & 98 & 34 & 0.4 & 2 & 0.004683841 & 0.014051522 \\
\hline JNK2a2(h) & 96 & 0 & 98 & 98 & 35 & 0.411764706 & 2 & 0.004683841 & 0.018735363 \\
\hline JNK3(r) & 106 & 0 & 98 & 98 & 36 & 0.423529412 & 2 & 0.004683841 & 0.023419204 \\
\hline Lck(h) & 99 & 0 & 98 & 98 & 37 & 0.435294118 & 2 & 0.004683841 & 0.028103044 \\
\hline 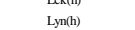 & 99 & 0 & 98 & 98 & 38 & 0.447058824 & 2 & 0.004683841 & 0.032786885 \\
\hline Lyn(m) & 90 & 0 & 98 & 98 & 39 & 0.458823529 & 2 & 0.004683841 & 0.037470726 \\
\hline MAPKI (h) & 97 & 0 & $\begin{array}{l}98 \\
98\end{array}$ & $\begin{array}{l}98 \\
98\end{array}$ & 40 & 0.470588235 & 2 & 0.004683841 & 0.042154567 \\
\hline MAPK2(h) & 110 & 0 & 98 & 98 & 41 & 0.482352941 & 2 & 0.004683841 & 0.046838407 \\
\hline $\begin{array}{l}\text { MAPKK (m) } \\
\text { MAP2(m) }\end{array}$ & 91 & 0 & $\begin{array}{l}50 \\
97\end{array}$ & 97 & 42 & 0.494117647 & 3 & 0.007025761 & 0.053864169 \\
\hline MAPKAP-K2(h) & 105 & 0 & 97 & 97 & 43 & 0.505882353 & 3 & 0.007025761 & 0.06088993 \\
\hline MEK 1(h) & 89 & 0 & 97 & 97 & 44 & 0.517647059 & 3 & 0.007025761 & 0.067915691 \\
\hline MKK 4(m) & 99 & 0 & 97 & 97 & 45 & 0.529411765 & 3 & 0.007025761 & 0.074941452 \\
\hline MKK $6(\mathrm{hl})$ & 87 & 0 & 97 & 97 & 46 & 0.541176471 & 3 & 0.007025761 & 0.081967213 \\
\hline MKK 7 B(h) & 77 & 0 & 97 & 97 & 47 & 0.552941176 & 3 & 0.007025761 & 0.088992974 \\
\hline MSK I(h) & 101 & 0 & 97 & 97 & 48 & 0.564705882 & 3 & 0.007025761 & 0.096018735 \\
\hline $\mathrm{p} 7056 \mathrm{~K}(\mathrm{~h})$ & 97 & 0 & 96 & 96 & $\begin{array}{l}48 \\
49\end{array}$ & 0.576470588 & 4 & 0.009367681 & 0.105386417 \\
\hline $\begin{array}{l}\text { PAK2(h) } \\
\text { Pal }\end{array}$ & 107 & 0 & 96 & 96 & 50 & 0.588235294 & 4 & 0.009367681 & 0.114754098 \\
\hline PDGFRa(h) & 77 & 0 & 96 & 96 & 51 & 0.6 & 4 & 0.009367681 & 0.12412178 \\
\hline PDGFRB(h) & 80 & 0 & 96 & 96 & 52 & 0.611764706 & 4 & 0.009367681 & 0.133489461 \\
\hline PDKI(h) & 101 & 0 & 95 & $\begin{array}{l}96 \\
95\end{array}$ & 53 & 0.623529412 & 5 & 0.011709602 & 0.145199063 \\
\hline PKA(b) & 92 & 0 & 95 & 95 & 54 & 0.635294118 & 5 & 0.011709602 & 0.156908665 \\
\hline $\begin{array}{l}\text { PRA(b) } \\
\text { PKA(h) }\end{array}$ & $\begin{array}{l}92 \\
92\end{array}$ & 0 & 95 & 95 & $\begin{array}{l}54 \\
55\end{array}$ & 0.647058824 & 5 & 0.011709602 & 0.168618267 \\
\hline PKBa(h) & 97 & 0 & 95 & 95 & 56 & 0.658823529 & 5 & 0.011709602 & 0.180327869 \\
\hline PKBB(h) & 93 & 0 & 95 & 95 & 57 & 0.670588235 & 5 & 0.011709602 & 0.192037471 \\
\hline $\mathrm{PKB}_{\gamma}(\mathrm{h})$ & 111 & 0 & 95 & 95 & 58 & 0.682352941 & 5 & 0.011709602 & 0.203747073 \\
\hline PKGa(h) & 102 & 0 & 94 & 94 & 59 & 0.694117647 & 6 & 0.014051522 & 0.217798595 \\
\hline РКСВII(h) & 98 & 0 & 94 & 94 & 60 & 0.705882353 & 6 & 0.014051522 & 0.231850117 \\
\hline PKC $\mathrm{y}(\mathrm{h})$ & 96 & 0 & 93 & 93 & 61 & 0.717647059 & 7 & 0.016393443 & 0.24824356 \\
\hline РКСऽ(h) & 95 & 0 & 93 & 93 & 62 & 0.729411765 & 7 & 0.016393443 & 0.264637002 \\
\hline PKG:(h) & 97 & 0 & 92 & 92 & 63 & 0.741176471 & 8 & 0.018735363 & 0.283372365 \\
\hline $\begin{array}{l}\text { PKCh(h) } \\
\text { (h) }\end{array}$ & 102 & 0 & 92 & 92 & 64 & 0.752941176 & 8 & 0.018735363 & 0.302107728 \\
\hline PKC (h) & 98 & 0 & 91 & 91 & 65 & 0.764705882 & 9 & 0.021077283 & 0.323185012 \\
\hline PKC $\mu(\mathrm{h})$ & 95 & 0 & 91 & 91 & 66 & 0.776470588 & 9 & 0.021077283 & 0.344262295 \\
\hline PKCO(h) & 93 & 0 & 91 & 91 & 67 & 0.788235294 & 9 & 0.021077283 & 0.365339578 \\
\hline PKD2(h) & 100 & 0 & 91 & 91 & 68 & 0.8 & 9 & 0.021077283 & 0.386416862 \\
\hline PRAK(h) & 108 & 0 & 90 & $\begin{array}{l}91 \\
90\end{array}$ & 69 & 0.811764706 & 10 & 0.023419204 & 0.409836066 \\
\hline PRK2(h) & 91 & 0 & 90 & 90 & 70 & 0.823529412 & 10 & 0.023419204 & 0.433255269 \\
\hline $\begin{array}{l}\text { PRK 2(n) } \\
\text { ROCK-II(h) }\end{array}$ & 98 & 0 & 90 & 90 & 71 & 0.835294118 & 10 & 0.023419204 & 0.456674473 \\
\hline ROCK-II(r) & 96 & 0 & 89 & 89 & 72 & 0.847058824 & 11 & 0.025761124 & 0.482435597 \\
\hline Rskl(h) & $\begin{array}{l}96 \\
110\end{array}$ & 0 & $\begin{array}{l}89 \\
87\end{array}$ & $\begin{array}{l}89 \\
87\end{array}$ & 73 & 0.858823529 & 13 & 0.030444965 & 0.512880562 \\
\hline Rskl(r) & 83 & 0 & 87 & 87 & 74 & 0.870588235 & 13 & 0.030444965 & 0.543325527 \\
\hline Rsk2(h) & 91 & 0 & 87 & 87 & 75 & 0.882352941 & 13 & 0.030444965 & 0.573770492 \\
\hline Rsk3(h) & 98 & 0 & 87 & 87 & 76 & 0.894117647 & 13 & 0.030444965 & 0.604215457 \\
\hline SAPK2a(h) & 96 & 0 & 87 & 87 & 77 & 0.905882353 & 13 & 0.030444965 & 0.634660422 \\
\hline SAPK2b(h) & $\begin{array}{l}90 \\
122\end{array}$ & 0 & $\begin{array}{l}01 \\
85\end{array}$ & $\begin{array}{l}01 \\
85\end{array}$ & 78 & 0.917647059 & 15 & 0.035128806 & 0.669789227 \\
\hline SAPK3(h) & 95 & 0 & 85 & 85 & 79 & 0.929411765 & 15 & 0.035128806 & 0.704918033 \\
\hline $\begin{array}{l}\text { SARSS(h) } \\
\text { SAPK4(h) }\end{array}$ & 98 & 0 & $\begin{array}{l}05 \\
83\end{array}$ & $\begin{array}{l}05 \\
83\end{array}$ & 80 & 0.941176471 & 17 & 0.039812646 & 0.744730679 \\
\hline SGK(h) & 100 & 0 & 81 & 81 & 81 & 0.952941176 & 19 & 0.044496487 & 0.789227166 \\
\hline Syk(h) & 90 & 0 & 80 & 80 & 82 & 0.964705882 & 20 & 0.046838407 & 0.836065574 \\
\hline TTkB(h) & 98 & 0 & 77 & 77 & 83 & 0.976470588 & 23 & 0.053864169 & 0.889929742 \\
\hline $\begin{array}{l}\text { IIkKB(h) } \\
\text { Yes(h) }\end{array}$ & $\begin{array}{l}98 \\
95\end{array}$ & 0 & 77 & 77 & $\begin{array}{l}83 \\
84\end{array}$ & 0.988235294 & 23 & $\begin{array}{l}.05380641169 \\
0.05386469\end{array}$ & 0.943793911 \\
\hline $\mathrm{ZAP}-70(\mathrm{~h})$ & 122 & 0 & 76 & 76 & 85 & 1 & 24 & 0.056206089 & 1 \\
\hline & & & & & & Total inhibition & 427 & & Total area \\
\hline & Hit rate & 5 & & & & & & & Gini \\
\hline
\end{tabular}




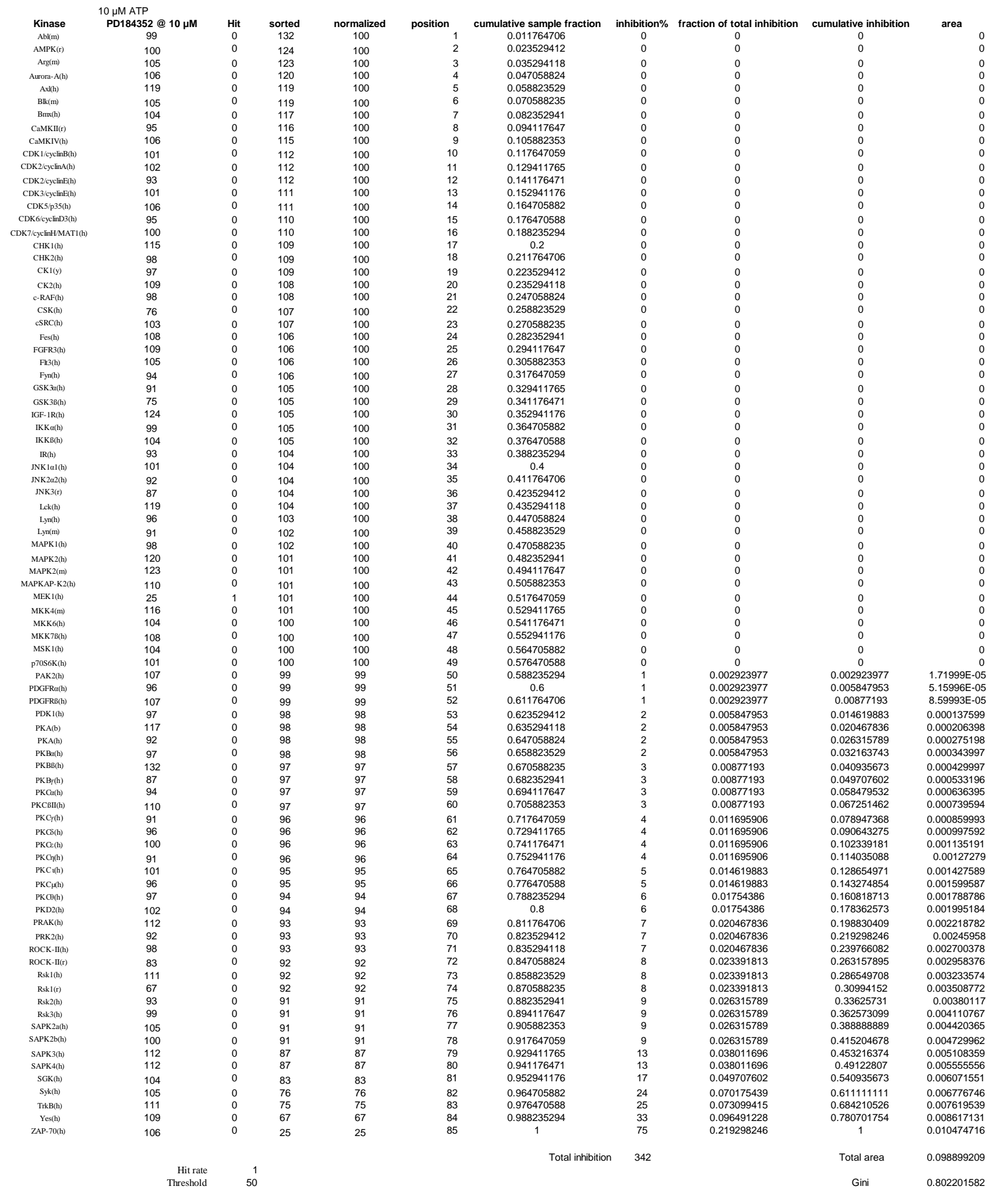




\begin{tabular}{|c|c|c|c|c|c|c|c|c|c|}
\hline & $100 \mu \mathrm{M}$ ATP & & & & & & & & \\
\hline Kinase & PD184352 @ $10 \mu \mathrm{M}$ & Hit & sorted & normalized & position & cumulative sample fraction & inhibition\% & fraction of total inhibition & cumulative inhibition \\
\hline $\mathrm{Abl}(\mathrm{m})$ & 102 & 0 & 136 & 100 & 1 & 0.011764706 & 0 & 0 & 0 \\
\hline AMPK(r) & 100 & 0 & 133 & 100 & 2 & 0.023529412 & 0 & 0 & 0 \\
\hline $\operatorname{Arg}(\mathrm{m})$ & 103 & 0 & 130 & 100 & 3 & 0.035294118 & 0 & 0 & 0 \\
\hline Aurora-A(h) & 86 & 0 & 126 & 100 & 4 & 0.047058824 & 0 & 0 & 0 \\
\hline Axl(h) & 103 & 0 & 123 & 100 & 5 & 0.058823529 & 0 & 0 & 0 \\
\hline $\operatorname{Blk}(\mathrm{m})$ & 90 & 0 & 123 & 100 & 6 & 0.070588235 & 0 & 0 & 0 \\
\hline Bmx(h) & 114 & 0 & 123 & 100 & 7 & 0.082352941 & 0 & 0 & 0 \\
\hline CaMKII(r) & 102 & 0 & 120 & 100 & 8 & 0.094117647 & 0 & 0 & 0 \\
\hline CaMKIV(h) & 119 & 0 & 119 & 100 & 9 & 0.105882353 & 0 & 0 & 0 \\
\hline $\mathrm{CDK} 1 /$ cyclinB(h) & 94 & 0 & 118 & 100 & 10 & 0.117647059 & 0 & 0 & 0 \\
\hline $\mathrm{CDK} 2 / \mathrm{cyclinA}(\mathrm{h})$ & 105 & 0 & 118 & 100 & 11 & 0.129411765 & 0 & 0 & 0 \\
\hline $\mathrm{CDK} 2 /$ cyclinE(h) & 110 & 0 & 118 & 100 & 12 & 0.141176471 & 0 & 0 & 0 \\
\hline CDK3/cyclinE(h) & 111 & 0 & 116 & 100 & 13 & 0.152941176 & 0 & 0 & 0 \\
\hline CDK5/p35(h) & 118 & 0 & 116 & 100 & 14 & 0.164705882 & 0 & 0 & 0 \\
\hline $\mathrm{CDK6/(cyclinD3(h)}$ & 100 & 0 & 116 & 100 & 15 & 0.176470588 & 0 & 0 & 0 \\
\hline CDK7/cyclinHMATI(h) & 100 & 0 & 115 & 100 & 16 & 0.188235294 & 0 & 0 & 0 \\
\hline CHKI(h) & 106 & 0 & 115 & 100 & 17 & 0.2 & 0 & 0 & 0 \\
\hline СHK2(h) & 99 & 0 & 114 & 100 & 18 & 0.211764706 & 0 & 0 & 0 \\
\hline $\mathrm{CKI}(\mathrm{y})$ & 101 & 0 & 114 & 100 & 19 & 0.223529412 & 0 & 0 & 0 \\
\hline CK2(h) & 107 & 0 & 113 & 100 & 20 & 0.235294118 & 0 & 0 & 0 \\
\hline c-RAF(h) & 95 & 0 & 112 & 100 & 21 & 0.247058824 & 0 & 0 & 0 \\
\hline CSK(h) & 102 & 0 & 112 & 100 & 22 & 0.258823529 & 0 & 0 & 0 \\
\hline $\mathrm{cSRC(h)}$ & 96 & 0 & 112 & 100 & 23 & 0.270588235 & 0 & 0 & 0 \\
\hline Fes(h) & 98 & 0 & 111 & 100 & 24 & 0.282352941 & 0 & 0 & 0 \\
\hline FGFR3(h) & 123 & 0 & 110 & 100 & 25 & 0.294117647 & 0 & 0 & 0 \\
\hline Fllt3(h) & 86 & 0 & 110 & 100 & 26 & 0.305882353 & 0 & 0 & 0 \\
\hline Fynn(h) & 107 & 0 & 110 & 100 & 27 & 0.317647059 & 0 & 0 & 0 \\
\hline GSK3a(h) & 100 & 0 & 109 & 100 & 28 & 0.329411765 & 0 & 0 & 0 \\
\hline GSK3B(h) & 102 & 0 & 109 & 100 & 29 & 0.341176471 & 0 & 0 & 0 \\
\hline IGF-1R(h) & 136 & 0 & 108 & 100 & 30 & 0.352941176 & 0 & 0 & 0 \\
\hline IKKu(h) & 133 & 0 & 107 & 100 & 31 & 0.364705882 & 0 & 0 & 0 \\
\hline IKKB(h) & 110 & 0 & 107 & 100 & 32 & 0.376470588 & 0 & 0 & 0 \\
\hline $\mathbb{I R ( h )}$ & 102 & 0 & 107 & 100 & 33 & 0.388235294 & 0 & 0 & 0 \\
\hline $\begin{array}{l}\text { INKIflal(h) } \\
\text { J }\end{array}$ & 112 & 0 & 106 & 100 & 34 & 0.4 & 0 & 0 & 0 \\
\hline JNK2a2(h) & 101 & 0 & 106 & 100 & 35 & 0.411764706 & 0 & 0 & 0 \\
\hline JNK3(r) & 81 & 0 & 105 & 100 & 36 & 0.423529412 & 0 & 0 & 0 \\
\hline Lck(h) & 112 & 0 & 105 & 100 & 37 & 0.435294118 & 0 & 0 & 0 \\
\hline Lyn(h) & 97 & 0 & 104 & 100 & 38 & 0.447058824 & 0 & 0 & 0 \\
\hline Lyn(m) & 104 & 0 & 104 & 100 & 39 & 0.458823529 & 0 & 0 & 0 \\
\hline MAPK 1 (h) & 101 & 0 & 104 & 100 & 40 & 0.470588235 & 0 & 0 & 0 \\
\hline MAPK2(h) & 104 & 0 & 104 & 100 & 41 & 0.482352941 & 0 & 0 & 0 \\
\hline MAPK2(m) & 120 & 0 & 103 & 100 & 42 & 0.494117647 & 0 & 0 & 0 \\
\hline MAPKAP-K2(h) & 114 & 0 & 103 & 100 & 43 & 0.505882353 & 0 & 0 & 0 \\
\hline MEKI(h) & 39 & 1 & 103 & 100 & 44 & 0.517647059 & 0 & 0 & 0 \\
\hline MKK4(m) & 115 & 0 & 103 & 100 & 45 & 0.529411765 & 0 & 0 & 0 \\
\hline MKK $6(\mathrm{hl})$ & 130 & 0 & 102 & 100 & 46 & 0.541176471 & 0 & 0 & 0 \\
\hline MKK 78 (h) & 123 & 0 & 102 & 100 & 47 & 0.552941176 & 0 & 0 & 0 \\
\hline MSKI(h) & $\begin{array}{l}108 \\
108\end{array}$ & 0 & 102 & 100 & 48 & 0.564705882 & 0 & 0 & 0 \\
\hline $\mathrm{p} 70 \mathrm{~S} 6 \mathrm{~K}(\mathrm{~h})$ & 112 & 0 & 102 & 100 & 49 & 0.576470588 & 0 & 0 & 0 \\
\hline $\begin{array}{l}\text { PATSOR(h) } \\
\text { PAK2(h) }\end{array}$ & 109 & 0 & 102 & 100 & 50 & 0.588235294 & 0 & 0 & 0 \\
\hline PDGFRa(h) & 116 & 0 & 102 & 100 & 51 & 0.6 & 0 & 0 & 0 \\
\hline PDGFRB(h) & 105 & 0 & 101 & 100 & 52 & 0.611764706 & 0 & 0 & 0 \\
\hline PDK I(h) & 90 & 0 & 101 & 100 & 53 & 0.623529412 & 0 & 0 & 0 \\
\hline PKA(b) & 118 & 0 & 101 & 100 & 54 & 0.635294118 & 0 & 0 & 0 \\
\hline PKA(h) & 98 & 0 & 101 & 100 & 55 & 0.647058824 & 0 & 0 & 0 \\
\hline PKBa(h) & 97 & 0 & 101 & 100 & 56 & 0.658823529 & 0 & 0 & 0 \\
\hline PKBB(h) & 118 & 0 & 101 & 100 & 57 & 0.670588235 & 0 & 0 & 0 \\
\hline $\mathrm{PKB}_{/}(\mathrm{h})$ & 106 & 0 & 101 & 100 & 58 & 0.682352941 & 0 & 0 & 0 \\
\hline PKCa(h) & 115 & 0 & 101 & 100 & 59 & 0.694117647 & 0 & 0 & 0 \\
\hline PKCBII(h) & 107 & 0 & 101 & 100 & 60 & 0.705882353 & 0 & 0 & 0 \\
\hline PKCy(h) & 101 & 0 & 100 & 100 & 61 & 0.717647059 & 0 & 0 & 0 \\
\hline PKФ(h) & 101 & 0 & 100 & 100 & 62 & 0.729411765 & 0 & 0 & 0 \\
\hline $\mathrm{PKC}_{(\mathrm{f})}$ & 100 & 0 & 100 & 100 & 63 & 0.741176471 & 0 & 0 & 0 \\
\hline PKCh(h) & 103 & 0 & 100 & 100 & 64 & 0.752941176 & 0 & 0 & 0 \\
\hline PKC (h) & 100 & 0 & 100 & 100 & 65 & 0.764705882 & 0 & 0 & 0 \\
\hline PKC $\mu$ (h) & 98 & 0 & 100 & 100 & 66 & 0.776470588 & 0 & 0 & 0 \\
\hline PKCO(h) & 95 & 0 & 99 & 99 & 67 & 0.788235294 & 1 & 0.005434783 & 0.005434783 \\
\hline PKD2(h) & 99 & 0 & 99 & 99 & 68 & 0.8 & 1 & 0.005434783 & 0.010869565 \\
\hline PRAK(h) & 104 & 0 & 98 & 98 & 69 & 0.811764706 & 2 & 0.010869565 & 0.02173913 \\
\hline PRK2(h) & 102 & 0 & $\begin{array}{l}50 \\
98\end{array}$ & $\begin{array}{l}90 \\
98\end{array}$ & 70 & 0.823529412 & 2 & 0.010869565 & 0.032608696 \\
\hline ROCK-II(h) & 101 & 0 & 98 & 98 & 71 & 0.835294118 & 2 & 0.010869565 & 0.043478261 \\
\hline ROCK-IIIr) & 109 & 0 & 98 & 98 & 72 & 0.847058824 & 2 & 0.010869565 & 0.054347826 \\
\hline Rskl(h) & 101 & 0 & 97 & 97 & 73 & 0.858823529 & 3 & 0.016304348 & 0.070652174 \\
\hline Rskl(r) & 126 & 0 & 97 & 97 & 74 & 0.870588235 & 3 & 0.016304348 & 0.086956522 \\
\hline Rsk2(h) & 101 & 0 & 96 & 96 & 75 & 0.882352941 & 4 & 0.02173913 & 0.108695652 \\
\hline Rsk3(h) & 103 & 0 & 95 & 95 & 76 & 0.894117647 & 5 & 0.027173913 & 0.135869565 \\
\hline SAPK2a(h) & 101 & 0 & 95 & 95 & 77 & 0.905882353 & 5 & 0.027173913 & 0.163043478 \\
\hline SAPK2b(h) & 123 & 0 & 94 & 94 & 78 & 0.917647059 & 6 & 0.032608696 & 0.195652174 \\
\hline SAPK3(h) & 110 & 0 & 90 & 90 & 79 & 0.929411765 & 10 & 0.054347826 & 0.25 \\
\hline SAPK4(h) & 116 & 0 & 90 & 90 & 80 & 0.941176471 & 10 & 0.054347826 & 0.304347826 \\
\hline SGK(h) & 113 & 0 & 86 & 86 & 81 & 0.952941176 & 14 & 0.076086957 & 0.380434783 \\
\hline Syk(h) & 98 & 0 & 86 & 86 & 82 & 0.964705882 & 14 & 0.076086957 & 0.456521739 \\
\hline TrkB(h) & 104 & 0 & 81 & 81 & 83 & 0.976470588 & 19 & 0.10326087 & 0.559782609 \\
\hline Yes(h) & 116 & 0 & 80 & 80 & 84 & 0.988235294 & 20 & 0.108695652 & 0.668478261 \\
\hline ZAP-70(h) & 80 & 0 & 39 & 39 & 85 & 1 & 61 & 0.331521739 & 1 \\
\hline & & & & & & Total inhibition & 184 & & Total area \\
\hline & $\begin{array}{r}\text { Hit rate } \\
\text { Threshold }\end{array}$ & 5 & & & & & & & Gini \\
\hline
\end{tabular}




\begin{tabular}{|c|c|c|c|c|c|c|c|c|c|}
\hline & $10 \mu \mathrm{M}$ ATP & & & & & & & & \\
\hline Kinase & PP1 analogue @ 1 нM & Hit & sorted & normalized & position & cumulative sample fraction & inhibition\% & fraction of total inhibition & cumulative inhibition \\
\hline $\mathrm{Abl}(\mathrm{m})$ & 48 & 1 & 127 & 100 & 1 & 0.011764706 & 0 & 0 & 0 \\
\hline AMPK(r) & 113 & 0 & 125 & 100 & 2 & 0.023529412 & 0 & 0 & 0 \\
\hline $\operatorname{Arg}(\mathrm{m})$ & 46 & 1 & 122 & 100 & 3 & 0.035294118 & 0 & 0 & 0 \\
\hline Aurora-A(h) & 125 & 0 & 117 & 100 & 4 & 0.047058824 & 0 & 0 & 0 \\
\hline Axl(h) & 114 & 0 & 117 & 100 & 5 & 0.058823529 & 0 & 0 & 0 \\
\hline $\mathrm{Blk}(\mathrm{m})$ & 17 & 1 & 115 & 100 & 6 & 0.070588235 & 0 & 0 & 0 \\
\hline Bnx(h) & 14 & 1 & 115 & 100 & 7 & 0.082352941 & 0 & 0 & 0 \\
\hline CaMKII(r) & 103 & 0 & 114 & 100 & 8 & 0.094117647 & 0 & 0 & 0 \\
\hline CaMKIV(h) & 108 & 0 & 114 & 100 & 9 & 0.105882353 & 0 & 0 & 0 \\
\hline CDK1/cyclinB(h) & 102 & 0 & 114 & 100 & 10 & 0.117647059 & 0 & 0 & 0 \\
\hline $\mathrm{CDK} 2 / \mathrm{cyclinA}(\mathrm{h})$ & 107 & 0 & 113 & 100 & 11 & 0.129411765 & 0 & 0 & 0 \\
\hline $\mathrm{CDK} 2 /$ cyclinE(h) & 96 & 0 & 113 & 100 & 12 & 0.141176471 & 0 & 0 & 0 \\
\hline CDK3/cyclinE(h) & 115 & 0 & 111 & 100 & 13 & 0.152941176 & 0 & 0 & 0 \\
\hline CDK5/p35(h) & 104 & 0 & 111 & 100 & 14 & 0.164705882 & 0 & 0 & 0 \\
\hline CDK6/cyclinD3(h) & 96 & 0 & 110 & 100 & 15 & 0.176470588 & 0 & 0 & 0 \\
\hline CDK7//yclinHMATI(h) & 97 & 0 & 109 & 100 & 16 & 0.188235294 & 0 & 0 & 0 \\
\hline СHK1(h) & 114 & 0 & 109 & 100 & 17 & 0.2 & 0 & 0 & 0 \\
\hline СHK2(h) & 84 & 0 & 109 & 100 & 18 & 0.211764706 & 0 & 0 & 0 \\
\hline CKI(y) & 21 & 1 & 108 & 100 & 19 & 0.223529412 & 0 & 0 & 0 \\
\hline СK2(h) & 110 & 0 & 108 & 100 & 20 & 0.235294118 & 0 & 0 & 0 \\
\hline $\mathrm{c}-\mathrm{RAF}(\mathrm{h})$ & 66 & 0 & 108 & 100 & 21 & 0.247058824 & 0 & 0 & 0 \\
\hline $\operatorname{CSK}(\mathrm{h})$ & 38 & 1 & 108 & 100 & 22 & 0.258823529 & 0 & 0 & 0 \\
\hline cSRC(h) & 11 & 1 & 108 & 100 & 23 & 0.270588235 & 0 & 0 & 0 \\
\hline Fes(h) & 77 & 0 & 107 & 100 & 24 & 0.282352941 & 0 & 0 & 0 \\
\hline FGFR3(h) & 76 & 0 & 107 & 100 & 25 & 0.294117647 & 0 & 0 & 0 \\
\hline Fllis(h) & 96 & 0 & 105 & 100 & 26 & 0.305882353 & 0 & 0 & 0 \\
\hline Fyn(h) & 30 & 1 & 105 & 100 & 27 & 0.317647059 & 0 & 0 & 0 \\
\hline GSK3a(h) & 102 & 0 & 104 & 100 & 28 & 0.329411765 & 0 & 0 & 0 \\
\hline GSK3B(h) & 109 & 0 & 104 & 100 & 29 & 0.341176471 & 0 & 0 & 0 \\
\hline IGF-IR(h) & 99 & 0 & 103 & 100 & 30 & 0.352941176 & 0 & 0 & 0 \\
\hline IKKa(h) & 98 & 0 & 103 & 100 & 31 & 0.364705882 & 0 & 0 & 0 \\
\hline IKKB(h) & 104 & 0 & 102 & 100 & 32 & 0.376470588 & 0 & 0 & 0 \\
\hline $\mathbb{I R ( h )}$ & 122 & 0 & 102 & 100 & 33 & 0.388235294 & 0 & 0 & 0 \\
\hline JNKIal(h) & 105 & 0 & 102 & 100 & 34 & 0.4 & 0 & 0 & 0 \\
\hline JNK202(h) & 98 & 0 & 102 & 100 & 35 & 0.411764706 & 0 & 0 & 0 \\
\hline $\mathrm{JNK} 3(\mathrm{r})$ & 75 & 0 & 101 & 100 & 36 & 0.423529412 & 0 & 0 & 0 \\
\hline Lck(h) & 21 & 1 & 101 & 100 & 37 & 0.435294118 & 0 & 0 & 0 \\
\hline Lyn(h) & 8 & 1 & 100 & 100 & 38 & 0.447058824 & 0 & 0 & 0 \\
\hline Lyn(m) & 1 & 1 & 100 & 100 & 39 & 0.458823529 & 0 & 0 & 0 \\
\hline MAPK I(h) & 108 & 0 & 100 & 100 & 40 & 0.470588235 & 0 & 0 & 0 \\
\hline MAPK2(h) & 113 & 0 & 99 & 99 & 41 & 0.482352941 & 1 & 0.000665779 & 0.000665779 \\
\hline MAPK2(m) & 101 & 0 & 99 & 99 & 42 & 0.494117647 & 1 & 0.000665779 & 0.001331558 \\
\hline MAPKAP-K2(h) & 107 & 0 & 98 & 98 & 43 & 0.505882353 & 2 & 0.001331558 & 0.002663116 \\
\hline MEK I(h) & 89 & 0 & 98 & 98 & 44 & 0.517647059 & 2 & 0.001331558 & 0.003994674 \\
\hline MKK4(m) & 98 & 0 & 98 & 98 & 45 & 0.529411765 & 2 & 0.001331558 & 0.005326232 \\
\hline MKK6(h) & 95 & 0 & 98 & 98 & 46 & 0.541176471 & 2 & 0.001331558 & 0.00665779 \\
\hline МKK $78($ (h) & 117 & 0 & 98 & 98 & 47 & 0.552941176 & 2 & 0.001331558 & 0.007989348 \\
\hline MSKI(h) & 99 & 0 & 98 & 98 & 48 & 0.564705882 & 2 & 0.001331558 & 0.009320905 \\
\hline $\mathrm{p} 70 \mathrm{~S} 6 \mathrm{~K}(\mathrm{~h})$ & 101 & 0 & 97 & 97 & 49 & 0.576470588 & 3 & 0.001997337 & 0.011318242 \\
\hline PAK2(h) & 85 & 0 & 96 & 96 & 50 & 0.588235294 & 4 & 0.002663116 & 0.013981358 \\
\hline PDGFRa(h) & 81 & 0 & 96 & 96 & 51 & 0.6 & 4 & 0.002663116 & 0.016644474 \\
\hline PDGFRB(h) & 92 & 0 & 96 & 96 & 52 & 0.611764706 & 4 & 0.002663116 & 0.01930759 \\
\hline PDK I(h) & 127 & 0 & 95 & 95 & 53 & 0.623529412 & 5 & 0.003328895 & 0.022636485 \\
\hline PKA(b) & 109 & 0 & 93 & 93 & 54 & 0.635294118 & 7 & 0.004660453 & 0.027296937 \\
\hline PKA(h) & 91 & 0 & 93 & 93 & 55 & 0.647058824 & 7 & 0.004660453 & 0.03195739 \\
\hline PKBa(h) & 98 & 0 & 92 & 92 & 56 & 0.658823529 & 8 & 0.005326232 & 0.037283622 \\
\hline PKBB(h) & 105 & 0 & 91 & 91 & 57 & 0.670588235 & 9 & 0.005992011 & 0.043275632 \\
\hline $\mathrm{PKB}_{\gamma}(\mathrm{h})$ & 100 & 0 & 89 & 89 & 58 & 0.682352941 & 11 & 0.007323569 & 0.050599201 \\
\hline PKGa(h) & 82 & 0 & 87 & 87 & 59 & 0.694117647 & 13 & 0.008655126 & 0.059254328 \\
\hline РКCBII(h) & 109 & 0 & 85 & 85 & 60 & 0.705882353 & 15 & 0.009986684 & 0.069241012 \\
\hline PKCy(h) & 87 & 0 & 84 & 84 & 61 & 0.717647059 & 16 & 0.010652463 & 0.079893475 \\
\hline PKG(h) & 100 & 0 & 84 & 84 & 62 & 0.729411765 & 16 & 0.010652463 & 0.090545939 \\
\hline PKG:(h) & 102 & 0 & 82 & 82 & 63 & 0.741176471 & 18 & 0.011984021 & 0.10252996 \\
\hline PKG (h) & 108 & 0 & 81 & 81 & 64 & 0.752941176 & 19 & 0.0126498 & 0.11517976 \\
\hline PKC (h) & 117 & 0 & 77 & 77 & 65 & 0.764705882 & 23 & 0.015312916 & 0.130492676 \\
\hline PKC $\mu(\mathrm{h})$ & 15 & 1 & 77 & 77 & 66 & 0.776470588 & 23 & 0.015312916 & 0.145805593 \\
\hline PKCO(h) & 98 & 0 & 76 & 76 & 67 & 0.788235294 & 24 & 0.015978695 & 0.161784288 \\
\hline PKD2(h) & 20 & 1 & 75 & 75 & 68 & 0.8 & 25 & 0.016644474 & 0.178428762 \\
\hline PRAK(h) & 115 & 0 & 70 & 70 & 69 & 0.811764706 & 30 & 0.019973369 & 0.19840213 \\
\hline PRK2(h) & 103 & 0 & 66 & 66 & 70 & 0.823529412 & 34 & 0.022636485 & 0.221038615 \\
\hline ROCK-II(h) & 93 & 0 & 48 & 48 & 71 & 0.835294118 & 52 & 0.034620506 & 0.255659121 \\
\hline ROCK-II(r) & 93 & 0 & 46 & 46 & 72 & 0.847058824 & 54 & 0.035952064 & 0.291611185 \\
\hline Rskl(h) & 108 & 0 & 38 & 38 & 73 & 0.858823529 & 62 & 0.041278296 & 0.332889481 \\
\hline Rskl(r) & 77 & 0 & 38 & 38 & 74 & 0.870588235 & 62 & 0.041278296 & 0.374167776 \\
\hline Rsk2(h) & 111 & 0 & 30 & 30 & 75 & 0.882352941 & 70 & 0.046604527 & 0.420772304 \\
\hline Rsk3(h) & 98 & 0 & 21 & 21 & 76 & 0.894117647 & 79 & 0.052596538 & 0.473368842 \\
\hline SAPK2a(h) & 38 & 1 & 21 & 21 & 77 & 0.905882353 & 79 & 0.052596538 & 0.525965379 \\
\hline SAPK2b(h) & 84 & 0 & 20 & 20 & 78 & 0.917647059 & 80 & 0.053262317 & 0.579227696 \\
\hline SAPK3(h) & 108 & 0 & 17 & 17 & 79 & 0.929411765 & 83 & 0.055259654 & 0.63448735 \\
\hline $\mathrm{SAPK} 4(\mathrm{~h})$ & 111 & 0 & 15 & 15 & 80 & 0.941176471 & 85 & 0.056591212 & 0.691078562 \\
\hline SGK(h) & 114 & 0 & 14 & 14 & 81 & 0.952941176 & 86 & 0.057256991 & 0.748335553 \\
\hline Syk(h) & 100 & 0 & 11 & 11 & 82 & 0.964705882 & 89 & 0.059254328 & 0.80758988 \\
\hline $\operatorname{TrkB}(\mathbf{h})$ & 70 & 0 & 8 & 8 & 83 & 0.976470588 & 92 & 0.061251664 & 0.868841545 \\
\hline Yes(h) & 2 & 1 & 2 & 2 & 84 & 0.988235294 & 98 & 0.065246338 & 0.934087883 \\
\hline ZAP-70(h) & 102 & 0 & 1 & 1 & 85 & 1 & 99 & 0.065912117 & 1 \\
\hline & & & & & & Total inhibition & 1502 & & Total area \\
\hline & $\begin{array}{l}\text { Hit rate } \\
\text { Threshold }\end{array}$ & ( & & & & & & & Gini \\
\hline
\end{tabular}




\begin{tabular}{|c|c|c|c|c|c|c|c|c|c|c|}
\hline Kinase & $\begin{array}{l}100 \mu \mathrm{M} \text { ATP } \\
\text { PP1 analogue @ } 1 \mu \mathrm{M}\end{array}$ & Hit & sorted & normalized & position & cumulative sample fraction & inhibition\% & fraction of total inhibition & & \\
\hline $\mathrm{Abl}(\mathrm{m})$ & 94 & 0 & 140 & 100 & 1 & 0.011764706 & 0 & 0 & 0 & ared \\
\hline $\mathrm{AMPK}(\mathrm{r})$ & 108 & 0 & 134 & 100 & 2 & 0.023529412 & 0 & 0 & 0 & 0 \\
\hline $\operatorname{Arg}(m)$ & 79 & 0 & 126 & 100 & 3 & 0.035294118 & 0 & 0 & 0 & 0 \\
\hline Aurora-A(h) & 108 & 0 & 122 & 100 & 4 & 0.047058824 & 0 & 0 & 0 & 0 \\
\hline Axl(h) & 101 & 0 & 120 & 100 & 5 & 0.058823529 & 0 & 0 & 0 & 0 \\
\hline $\mathrm{Blk}(\mathrm{m})$ & 28 & 1 & 117 & 100 & 6 & 0.070588235 & 0 & 0 & 0 & 0 \\
\hline $\operatorname{Bnx}(\mathrm{h})$ & 30 & 1 & 117 & 100 & 7 & 0.082352941 & 0 & 0 & 0 & 0 \\
\hline CaMKII(r) & 109 & 0 & 117 & 100 & 8 & 0.094117647 & 0 & 0 & 0 & 0 \\
\hline CaMKIV(h) & 113 & 0 & 117 & 100 & 9 & 0.105882353 & 0 & 0 & 0 & 0 \\
\hline CDK1/cyclinB(h) & 95 & 0 & 116 & 100 & 10 & 0.117647059 & 0 & 0 & 0 & 0 \\
\hline CDK2/cyclinA(h) & 103 & 0 & 116 & 100 & 11 & 0.129411765 & 0 & 0 & 0 & 0 \\
\hline $\mathrm{CDK} 2 / \mathrm{cyclin} E(\mathrm{~h})$ & 104 & 0 & 116 & 100 & 12 & 0.141176471 & 0 & 0 & 0 & 0 \\
\hline CDK3/cyclinE(h) & 113 & 0 & 115 & 100 & 13 & 0.152941176 & 0 & 0 & 0 & 0 \\
\hline CDK5/p35(h) & 112 & 0 & 114 & 100 & 14 & 0.164705882 & 0 & 0 & 0 & 0 \\
\hline CDK6/cyclinD3(h) & 102 & 0 & 113 & 100 & 15 & 0.176470588 & 0 & 0 & 0 & 0 \\
\hline CDK7/cyclinHMATI(h) & 112 & 0 & 113 & 100 & 16 & 0.188235294 & 0 & 0 & 0 & 0 \\
\hline СнК1(h) & 108 & 0 & 113 & 100 & 17 & 0.2 & 0 & 0 & 0 & 0 \\
\hline $\mathrm{CHK}_{2(\mathrm{~h})}$ & 93 & 0 & 113 & 100 & 18 & 0.211764706 & 0 & 0 & 0 & 0 \\
\hline CKI $(y)$ & 47 & 1 & 112 & 100 & 19 & 0.223529412 & 0 & 0 & 0 & 0 \\
\hline CK2(h) & 110 & 0 & 112 & 100 & 20 & 0.235294118 & 0 & 0 & 0 & 0 \\
\hline c-RAF(h) & 95 & 0 & 111 & 100 & 21 & 0.247058824 & 0 & 0 & 0 & 0 \\
\hline CSK(h) & 107 & 0 & 111 & 100 & 22 & 0.258823529 & 0 & 0 & 0 & 0 \\
\hline cSRC(h) & 20 & 1 & 111 & 100 & 23 & 0.270588235 & 0 & 0 & 0 & 0 \\
\hline Fes(h) & 71 & 0 & 111 & 100 & 24 & 0.282352941 & 0 & 0 & 0 & 0 \\
\hline FGFR3(h) & 86 & 0 & 110 & 100 & 25 & 0.294117647 & 0 & 0 & 0 & 0 \\
\hline $\mathrm{Flt3}(\mathrm{h})$ & 102 & 0 & 110 & 100 & 26 & 0.305882353 & 0 & 0 & 0 & 0 \\
\hline Fyn(h) & 42 & 1 & 109 & 100 & 27 & 0.317647059 & 0 & 0 & 0 & 0 \\
\hline GSK3a(h) & 101 & 0 & 109 & 100 & 28 & 0.329411765 & 0 & 0 & 0 & 0 \\
\hline GSK3B(h) & 116 & 0 & 109 & 100 & 29 & 0.341176471 & 0 & 0 & 0 & 0 \\
\hline IGF-1R(h) & 109 & 0 & 109 & 100 & 30 & 0.352941176 & 0 & 0 & 0 & 0 \\
\hline IKKa(h) & 105 & 0 & 108 & 100 & 31 & 0.364705882 & 0 & 0 & 0 & 0 \\
\hline IKKB(h) & 103 & 0 & 108 & 100 & 32 & 0.376470588 & 0 & 0 & 0 & 0 \\
\hline $\mathbb{I R ( h )}$ & 134 & 0 & 108 & 100 & 33 & 0.388235294 & 0 & 0 & 0 & 0 \\
\hline JNKlal(h) & 104 & 0 & 108 & 100 & 34 & 0.4 & 0 & 0 & 0 & 0 \\
\hline JNK2a2(h) & 117 & 0 & 108 & 100 & 35 & 0.411764706 & 0 & 0 & 0 & 0 \\
\hline JNK3(r) & 109 & 0 & 107 & 100 & 36 & 0.423529412 & 0 & 0 & 0 & 0 \\
\hline Lck(h) & 20 & 1 & 107 & 100 & 37 & 0.435294118 & 0 & 0 & 0 & 0 \\
\hline Lynn(h) & 7 & 1 & 107 & 100 & 38 & 0.447058824 & 0 & 0 & 0 & 0 \\
\hline Lyn(m) & 8 & 1 & 107 & 100 & 39 & 0.458823529 & 0 & 0 & 0 & 0 \\
\hline MAPKI(h) & 114 & 0 & 106 & 100 & 40 & 0.470588235 & 0 & 0 & 0 & 0 \\
\hline MAPK $2(\mathrm{~h})$ & 116 & 0 & 105 & 100 & 41 & 0.482352941 & 0 & 0 & 0 & 0 \\
\hline $\operatorname{MAPK} 2(\mathrm{~m})$ & 111 & 0 & 104 & 100 & 42 & 0.494117647 & 0 & 0 & 0 & 0 \\
\hline MAPKAP-K2(h) & 104 & 0 & 104 & 100 & 43 & 0.505882353 & 0 & 0 & 0 & 0 \\
\hline MEK1(h) & 110 & 0 & 104 & 100 & 44 & 0.517647059 & 0 & 0 & 0 & 0 \\
\hline MKK4(m) & 96 & 0 & 103 & 100 & 45 & 0.529411765 & 0 & 0 & 0 & 0 \\
\hline MKK6(h) & 113 & 0 & 103 & 100 & 46 & 0.541176471 & 0 & 0 & 0 & 0 \\
\hline MKK & 91 & 0 & 103 & 100 & 47 & 0.552941176 & 0 & 0 & 0 & 0 \\
\hline MSK1(h) & 102 & 0 & 103 & 100 & 48 & 0.564705882 & 0 & 0 & 0 & 0 \\
\hline p70S6K(h) & 122 & 0 & 102 & 100 & 49 & 0.576470588 & 0 & 0 & 0 & 0 \\
\hline PAK2(h) & 96 & 0 & 102 & 100 & 50 & 0.588235294 & 0 & 0 & 0 & 0 \\
\hline PDGFRa(h) & 94 & 0 & 102 & 100 & 51 & 0.6 & 0 & 0 & 0 & 0 \\
\hline PDGFRB(h) & 100 & 0 & 101 & 100 & 52 & 0.611764706 & 0 & 0 & 0 & 0 \\
\hline PDK1(h) & 111 & 0 & 101 & 100 & 53 & 0.623529412 & 0 & 0 & 0 & 0 \\
\hline PKA(b) & 140 & 0 & 101 & 100 & 54 & 0.635294118 & 0 & 0 & 0 & 0 \\
\hline PKA(h) & 108 & 0 & 100 & 100 & 55 & 0.647058824 & 0 & 0 & 0 & 0 \\
\hline PKBa(h) & 97 & 0 & 100 & 100 & 56 & 0.658823529 & 0 & 0 & 0 & 0 \\
\hline PKBB(h) & 111 & 0 & 97 & 97 & 57 & 0.670588235 & 3 & 0.003012048 & 0.003012048 & $1.77179 \mathrm{E}-05$ \\
\hline $\mathrm{PKB} /(\mathrm{h})$ & 107 & 0 & 97 & 97 & 58 & 0.682352941 & 3 & 0.003012048 & 0.006024096 & $5.31538 \mathrm{E}-05$ \\
\hline PKCa(h) & 96 & 0 & 97 & 97 & 59 & 0.694117647 & 3 & 0.003012048 & 0.009036145 & 8.85897E-05 \\
\hline РКСВI(h) & 113 & 0 & 96 & 96 & 60 & 0.705882353 & 4 & 0.004016064 & 0.013052209 & 0.000129931 \\
\hline PKCy(h) & 103 & 0 & 96 & 96 & 61 & 0.717647059 & 4 & 0.004016064 & 0.017068273 & 0.000177179 \\
\hline PKळ(h) & 120 & 0 & 96 & 96 & 62 & 0.729411765 & 4 & 0.004016064 & 0.021084337 & 0.000224427 \\
\hline PKG:(h) & 100 & 0 & 95 & 95 & 63 & 0.741176471 & 5 & 0.00502008 & 0.026104418 & 0.000277581 \\
\hline PKGh(h) & 107 & 0 & 95 & 95 & 64 & 0.752941176 & 5 & 0.00502008 & 0.031124498 & 0.000336641 \\
\hline PKC (h) & 109 & 0 & 94 & 94 & 65 & 0.764705882 & 6 & 0.006024096 & 0.037148594 & 0.000401606 \\
\hline PKC (h) $^{\prime}$ & 54 & 0 & 94 & 94 & 66 & 0.776470588 & 6 & 0.006024096 & 0.043172691 & 0.000472478 \\
\hline PKC (h) & 101 & 0 & 93 & 93 & 67 & 0.788235294 & 7 & 0.007028112 & 0.050200803 & 0.000549256 \\
\hline PKD2(h) & 41 & 1 & 92 & 92 & 68 & 0.8 & 8 & 0.008032129 & 0.058232932 & 0.000637845 \\
\hline PRAK(h) & 117 & 0 & 91 & 91 & 69 & 0.811764706 & 9 & 0.009036145 & 0.067269076 & 0.000738247 \\
\hline PRK2(h) & 111 & 0 & 86 & 86 & 70 & 0.823529412 & 14 & 0.014056225 & 0.081325301 & 0.000874085 \\
\hline ROCK-II(h) & 97 & 0 & 85 & 85 & 71 & 0.835294118 & 15 & 0.015060241 & 0.096385542 & 0.001045358 \\
\hline ROCK-IIIr) & 92 & 0 & 79 & 79 & 72 & 0.847058824 & 21 & 0.021084337 & 0.11746988 & 0.001257973 \\
\hline Rskl(h) & 107 & 0 & 71 & 71 & 73 & 0.858823529 & 29 & 0.029116466 & 0.146586345 & 0.001553272 \\
\hline $\operatorname{Rskl(r)}$ & 117 & 0 & 54 & 54 & 74 & 0.870588235 & 46 & 0.046184739 & 0.192771084 & 0.00199622 \\
\hline Rsk2(h) & 116 & 0 & 49 & 49 & 75 & 0.882352941 & 51 & 0.051204819 & 0.243975904 & 0.0025691 \\
\hline Rsk3(h) & 106 & 0 & 47 & 47 & 76 & 0.894117647 & 53 & 0.053212851 & 0.297188755 & 0.003183322 \\
\hline SAPK2a(h) & 49 & 1 & 42 & 42 & 77 & 0.905882353 & 58 & 0.058232932 & 0.355421687 & 0.003838885 \\
\hline SAPK2b(h) & 117 & 0 & 41 & 41 & 78 & 0.917647059 & 59 & 0.059236948 & 0.414658635 & 0.004529884 \\
\hline SAPK3(h) & 115 & 0 & 30 & 30 & 79 & 0.929411765 & 70 & 0.070281124 & 0.484939759 & 0.005291755 \\
\hline SAPK 4(h) & 126 & 0 & 28 & 28 & 80 & 0.941176471 & 72 & 0.072289157 & 0.557228916 & 0.006130404 \\
\hline SGK(h) & 108 & 0 & 20 & 20 & 81 & 0.952941176 & 80 & 0.080321285 & 0.637550201 & 0.007028112 \\
\hline Syk(h) & 103 & 0 & 20 & 20 & 82 & 0.964705882 & 80 & 0.080321285 & 0.717871486 & 0.007973069 \\
\hline TrkB(h) & 97 & 0 & 8 & 8 & 83 & 0.976470588 & 92 & 0.092369478 & 0.810240964 & 0.008988897 \\
\hline Yes(h) & 4 & 1 & 7 & 7 & 84 & 0.988235294 & 93 & 0.093373494 & 0.903614458 & 0.010081502 \\
\hline ZAP-70(h) & 85 & 0 & 4 & 4 & 85 & 1 & 96 & 0.096385542 & 1 & 0.011197732 \\
\hline & & & & & & Total inhibition & 996 & & Total area & 0.081644224 \\
\hline & $\begin{array}{r}\text { Hit rate } \\
\text { Threshold }\end{array}$ & 5 & & & & & & & Gini & 0.836711552 \\
\hline
\end{tabular}


$10 \mu \mathrm{M}$ ATP

\begin{tabular}{|c|c|c|c|c|c|c|c|c|c|}
\hline & & & & & & & & & \\
\hline$\underset{A b l(m)}{\text { Kinase }}$ & PP2 @ $\underset{9}{1} \mu \mathrm{MM}$ & $\begin{array}{c}\text { Hit } \\
1\end{array}$ & $\begin{array}{c}\text { sorted } \\
127\end{array}$ & $\begin{array}{l}\text { normalized } \\
100\end{array}$ & position $_{1}$ & $\begin{array}{c}\text { cumulative sample fraction } \\
0.011764706\end{array}$ & $\begin{array}{l}\text { inhibition\% } \\
0\end{array}$ & $\begin{array}{l}\text { fraction of total inhibition } \\
0\end{array}$ & $\begin{array}{l}\text { cumulative inhibition } \\
0\end{array}$ \\
\hline AMPK(r) & 112 & 0 & 123 & 100 & 2 & 0.023529412 & 0 & 0 & 0 \\
\hline $\operatorname{Arg}(\mathrm{m})$ & 11 & 1 & 117 & 100 & 3 & 0.035294118 & 0 & 0 & 0 \\
\hline Aurora-A(h) & 117 & 0 & 115 & 100 & 4 & 0.047058824 & 0 & 0 & 0 \\
\hline Axl(h) & 85 & 0 & 114 & 100 & 5 & 0.058823529 & 0 & 0 & 0 \\
\hline $\mathrm{Blk}(\mathrm{m})$ & 4 & 1 & 113 & 100 & 6 & 0.070588235 & 0 & 0 & 0 \\
\hline $\operatorname{Bmx}(\mathrm{h})$ & 3 & 1 & 112 & 100 & 7 & 0.082352941 & 0 & 0 & 0 \\
\hline CaMKII(r) & 99 & 0 & 112 & 100 & 8 & 0.094117647 & 0 & 0 & 0 \\
\hline CaMKIV(h) & 107 & 0 & 112 & 100 & 9 & 0.105882353 & 0 & 0 & 0 \\
\hline CDK1/cyclinB(h) & 98 & 0 & 110 & 100 & 10 & 0.117647059 & 0 & 0 & 0 \\
\hline $\mathrm{CDK} 2$ /cyclinA(h) & 107 & 0 & 110 & 100 & 11 & 0.129411765 & 0 & 0 & 0 \\
\hline $\mathrm{CDK} 2 / \mathrm{cyclin} E(\mathrm{~h})$ & 81 & 0 & 109 & 100 & 12 & 0.141176471 & 0 & 0 & 0 \\
\hline CDK3/cyclinE(h) & 104 & 0 & 109 & 100 & 13 & 0.152941176 & 0 & 0 & 0 \\
\hline CDK5/p35(h) & 101 & 0 & 109 & 100 & 14 & 0.164705882 & 0 & 0 & 0 \\
\hline CDN6/cyclinD3(h) & 99 & 0 & 108 & 100 & 15 & 0.176470588 & 0 & 0 & 0 \\
\hline CDK $7 /$ cyclinHMATI(h) & 92 & 0 & 108 & 100 & 16 & 0.188235294 & 0 & 0 & 0 \\
\hline CHK I(h) & 99 & 0 & 107 & 100 & 17 & 0.2 & 0 & 0 & 0 \\
\hline СHK2(h) & 97 & 0 & 107 & 100 & 18 & 0.211764706 & 0 & 0 & 0 \\
\hline CKI(y) & 31 & 1 & 107 & 100 & 19 & 0.223529412 & 0 & 0 & 0 \\
\hline СК2(h) & 110 & 0 & 106 & 100 & 20 & 0.235294118 & 0 & 0 & 0 \\
\hline$c-\operatorname{RAF(h)}$ & 22 & 1 & 105 & 100 & 21 & 0.247058824 & 0 & 0 & 0 \\
\hline $\operatorname{csk}(\mathrm{h})$ & 27 & 1 & 104 & 100 & 22 & 0.258823529 & 0 & 0 & 0 \\
\hline cSRC(h) & 3 & 1 & 103 & 100 & 23 & 0.270588235 & 0 & 0 & 0 \\
\hline Fes(h) & 85 & 0 & 103 & 100 & 24 & 0.282352941 & 0 & 0 & 0 \\
\hline FGFR3(h) & 91 & 0 & 102 & 100 & 25 & 0.294117647 & 0 & 0 & 0 \\
\hline Flls(h) & 81 & 0 & 101 & 100 & 26 & 0.305882353 & 0 & 0 & 0 \\
\hline Fyn(h) & 1 & 1 & 101 & 100 & 27 & 0.317647059 & 0 & 0 & 0 \\
\hline GSK3a(h) & 100 & 0 & 101 & 100 & 28 & 0.329411765 & 0 & 0 & 0 \\
\hline GSK3B(h) & 108 & 0 & 100 & 100 & 29 & 0.341176471 & 0 & 0 & 0 \\
\hline IGF-IR(h) & 123 & 0 & 100 & 100 & 30 & 0.352941176 & 0 & 0 & 0 \\
\hline $\mathrm{IKKa(h)}$ & 100 & 0 & 100 & 100 & 31 & 0.364705882 & 0 & 0 & 0 \\
\hline IKKB(h) & 109 & 0 & 100 & 100 & 32 & 0.376470588 & 0 & 0 & 0 \\
\hline $\mathbb{I R ( h )}$ & 110 & 0 & 99 & 99 & 33 & 0.388235294 & 1 & 0.000564334 & 0.000564334 \\
\hline JNKIal(h) & 109 & 0 & 99 & 99 & 34 & 0.4 & 1 & 0.000564334 & 0.001128668 \\
\hline JNK2a2(h) & 83 & 0 & 99 & 99 & 35 & 0.411764706 & 1 & 0.000564334 & 0.001693002 \\
\hline JNK3(r) & 84 & 0 & 99 & 99 & 36 & 0.423529412 & 1 & 0.000564334 & 0.002257336 \\
\hline Lck(h) & 2 & 1 & 98 & 98 & 37 & 0.435294118 & 2 & 0.001128668 & 0.003386005 \\
\hline Lyn(h) & -2 & 1 & 98 & 98 & 38 & 0.447058824 & 2 & 0.001128668 & 0.004514673 \\
\hline Lyn(m) & 1 & 1 & 97 & 97 & 39 & 0.458823529 & 3 & 0.001693002 & 0.006207675 \\
\hline MAPK1(h) & 112 & 0 & 97 & 97 & 40 & 0.470588235 & 3 & 0.001693002 & 0.007900677 \\
\hline MAPK2(h) & 94 & 0 & 96 & 96 & 41 & 0.482352941 & 4 & 0.002257336 & 0.010158014 \\
\hline $\operatorname{MAPK} 2(\mathrm{~m})$ & 97 & 0 & 96 & 96 & 42 & 0.494117647 & 4 & 0.002257336 & 0.01241535 \\
\hline MAPKAP-K2(h) & 107 & 0 & 95 & 95 & 43 & 0.505882353 & 5 & 0.00282167 & 0.01523702 \\
\hline MEK $1(\mathrm{~h})$ & 88 & 0 & 94 & 94 & 44 & 0.517647059 & 6 & 0.003386005 & 0.018623025 \\
\hline MKK $4(\mathrm{~m})$ & $\begin{array}{l}127 \\
127\end{array}$ & 0 & 94 & 94 & 45 & 0.529411765 & 6 & 0.003386005 & 0.022009029 \\
\hline MKK $6(\mathrm{~h})$ & 94 & 0 & 94 & 94 & 46 & 0.541176471 & 6 & 0.003386005 & 0.025395034 \\
\hline MKK $7($ (h) & 100 & 0 & 94 & 94 & 47 & 0.552941176 & 6 & 0.003386005 & 0.028781038 \\
\hline MSKI(h) & 74 & 0 & 93 & 93 & 48 & 0.564705882 & 7 & 0.003950339 & 0.032731377 \\
\hline p70s6K(h) & 89 & 0 & 93 & 93 & 49 & 0.576470588 & 7 & 0.003950339 & 0.036681716 \\
\hline PAK2(h) & 103 & 0 & 92 & 92 & 50 & 0.588235294 & 8 & 0.004514673 & 0.041196388 \\
\hline PDGFRa(h) & 53 & 0 & 91 & 91 & 51 & 0.6 & 9 & 0.005079007 & 0.046275395 \\
\hline PDGFRB(h) & 45 & 1 & 89 & 89 & 52 & 0.611764706 & 11 & 0.006207675 & 0.05248307 \\
\hline PDK1(h) & 112 & 0 & 89 & 89 & 53 & 0.623529412 & 11 & 0.006207675 & 0.058690745 \\
\hline PKA(b) & 66 & 0 & 88 & 88 & 54 & 0.635294118 & 12 & 0.006772009 & 0.065462754 \\
\hline PKA(h) & 72 & 0 & 88 & 88 & 55 & 0.647058824 & 12 & 0.006772009 & 0.072234763 \\
\hline PKBa(h) & 98 & 0 & 85 & 85 & 56 & 0.658823529 & 15 & 0.008465011 & 0.080699774 \\
\hline PKBB(h) & 114 & 0 & 85 & 85 & 57 & 0.670588235 & 15 & 0.008465011 & 0.089164786 \\
\hline $\mathrm{PKB}_{\gamma}(\mathrm{h})$ & 95 & 0 & 85 & 85 & 58 & 0.682352941 & 15 & 0.008465011 & 0.097629797 \\
\hline PKCa(h) & 72 & 0 & 84 & 84 & 59 & 0.694117647 & 16 & 0.009029345 & 0.106659142 \\
\hline РКСВП(h) & 100 & 0 & 83 & 83 & 60 & 0.705882353 & 17 & 0.009593679 & 0.116252822 \\
\hline $\mathrm{PKCy}(\mathrm{h})$ & 85 & 0 & 82 & 82 & 61 & 0.717647059 & 18 & 0.010158014 & 0.126410835 \\
\hline РКФ(h) & 105 & 0 & 82 & 82 & 62 & 0.729411765 & 18 & 0.010158014 & 0.136568849 \\
\hline PKG:(h) & 96 & 0 & 81 & 81 & 63 & 0.741176471 & 19 & 0.010722348 & 0.147291196 \\
\hline PKCh(h) & 93 & 0 & 81 & 81 & 64 & 0.752941176 & 19 & 0.010722348 & 0.158013544 \\
\hline PKC (h) & 103 & 0 & 74 & 74 & 65 & 0.764705882 & 26 & 0.014672686 & 0.17268623 \\
\hline PKC $\mu(\mathrm{h})$ & 93 & 0 & 72 & 72 & 66 & 0.776470588 & 28 & 0.015801354 & 0.188487585 \\
\hline PKCP(h) & 94 & 0 & 72 & 72 & 67 & 0.788235294 & 28 & 0.015801354 & 0.204288939 \\
\hline PKD2(h) & 82 & 0 & 66 & 66 & 68 & 0.8 & 34 & 0.019187359 & 0.223476298 \\
\hline PRAK(h) & 113 & 0 & 58 & 58 & 69 & 0.811764706 & 42 & 0.023702032 & 0.24717833 \\
\hline PRK2(h) & 108 & 0 & 53 & 53 & 70 & 0.823529412 & 47 & 0.026523702 & 0.273702032 \\
\hline $\begin{array}{l}\text { PRK (2) } \\
\text { ROCKI(h) }\end{array}$ & 94 & 0 & 53 & 53 & 71 & 0.835294118 & 47 & 0.026523702 & 0.300225734 \\
\hline ROCK-II(r) & 106 & 0 & 45 & 45 & 72 & 0.847058824 & 55 & 0.031038375 & 0.331264108 \\
\hline Rskl(h) & 88 & 0 & 31 & 31 & 73 & 0.858823529 & 69 & 0.038939052 & 0.37020316 \\
\hline $\operatorname{Rskl(r)}$ & 82 & 0 & 27 & 27 & 74 & 0.870588235 & 73 & 0.041196388 & 0.411399549 \\
\hline Rsk2(h) & 102 & 0 & 22 & 22 & 75 & 0.882352941 & 78 & 0.044018059 & 0.455417607 \\
\hline Rsk3(h) & 99 & 0 & 11 & 11 & 76 & 0.894117647 & 89 & 0.050225734 & 0.505643341 \\
\hline SAPK2a(h) & 58 & 0 & 9 & 9 & 77 & 0.905882353 & 91 & 0.051354402 & 0.556997743 \\
\hline SAPK2b(h) & 53 & 0 & 4 & 4 & 78 & 0.917647059 & 96 & 0.054176072 & 0.611173815 \\
\hline $\mathrm{SAPK} 3(\mathrm{~h})$ & 101 & 0 & 3 & 3 & 79 & 0.929411765 & 97 & 0.054740406 & 0.665914221 \\
\hline $\mathrm{SAPK} 4(\mathrm{~h})$ & 115 & 0 & 3 & 3 & 80 & 0.941176471 & 97 & 0.054740406 & 0.720654628 \\
\hline SGK(h) & 109 & 0 & 2 & 2 & 81 & 0.952941176 & 98 & 0.05530474 & 0.775959368 \\
\hline Syk(h) & 96 & 0 & 1 & 1 & 82 & 0.964705882 & 99 & 0.055869074 & 0.831828442 \\
\hline TrkB(h) & 89 & 0 & 1 & 1 & 83 & 0.976470588 & 99 & 0.055869074 & 0.887697517 \\
\hline Yes(h) & 1 & 1 & 1 & 1 & 84 & 0.988235294 & 99 & 0.055869074 & 0.943566591 \\
\hline ZAP-70(h) & 101 & 0 & 0 & 0 & 85 & 1 & 100 & 0.056433409 & 1 \\
\hline & & & & & & Total inhibition & 1772 & & Total area \\
\hline & & & & & & & & & Sini \\
\hline & & & & & & & & & Gini \\
\hline
\end{tabular}


$100 \mu \mathrm{M}$ ATP

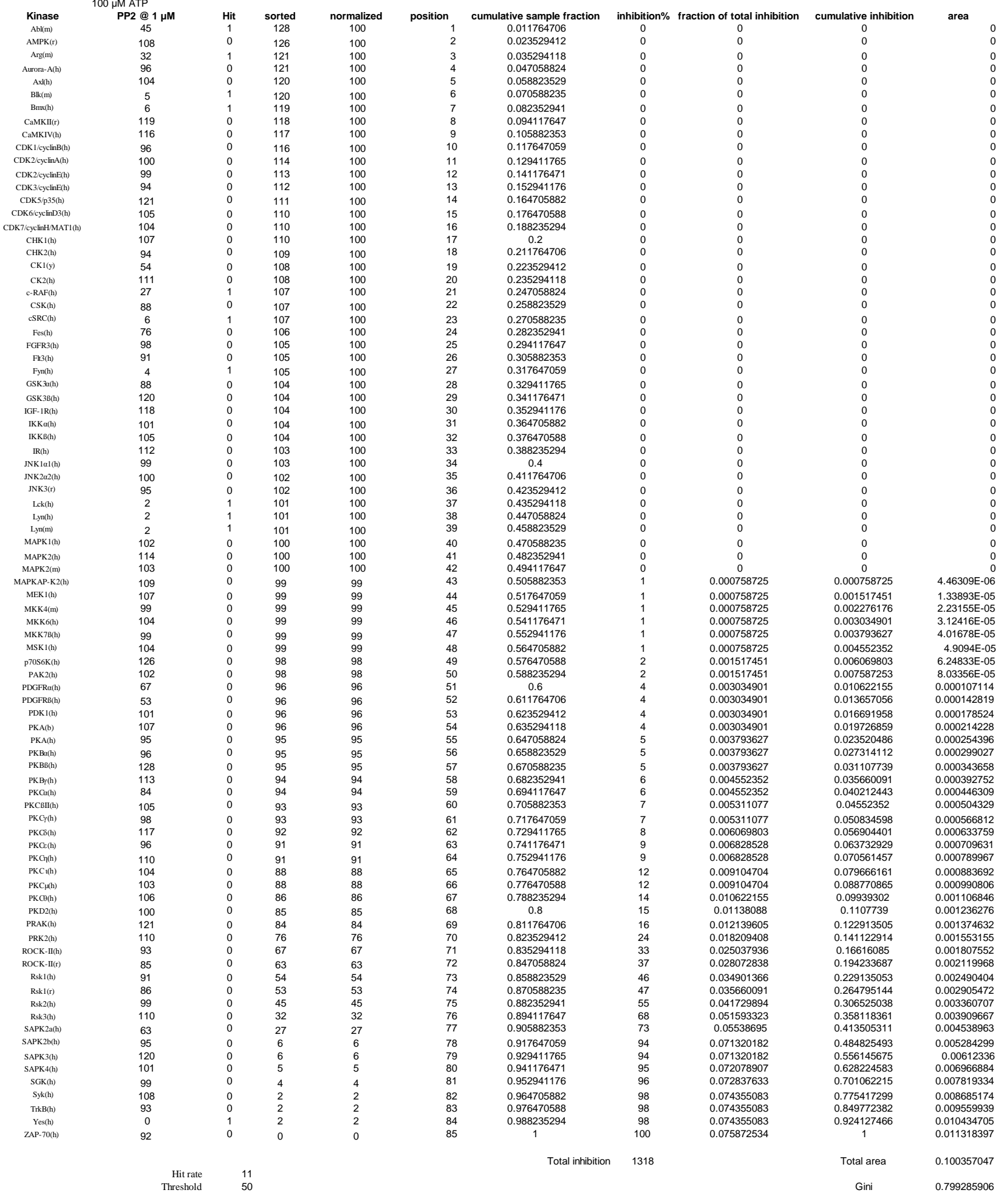




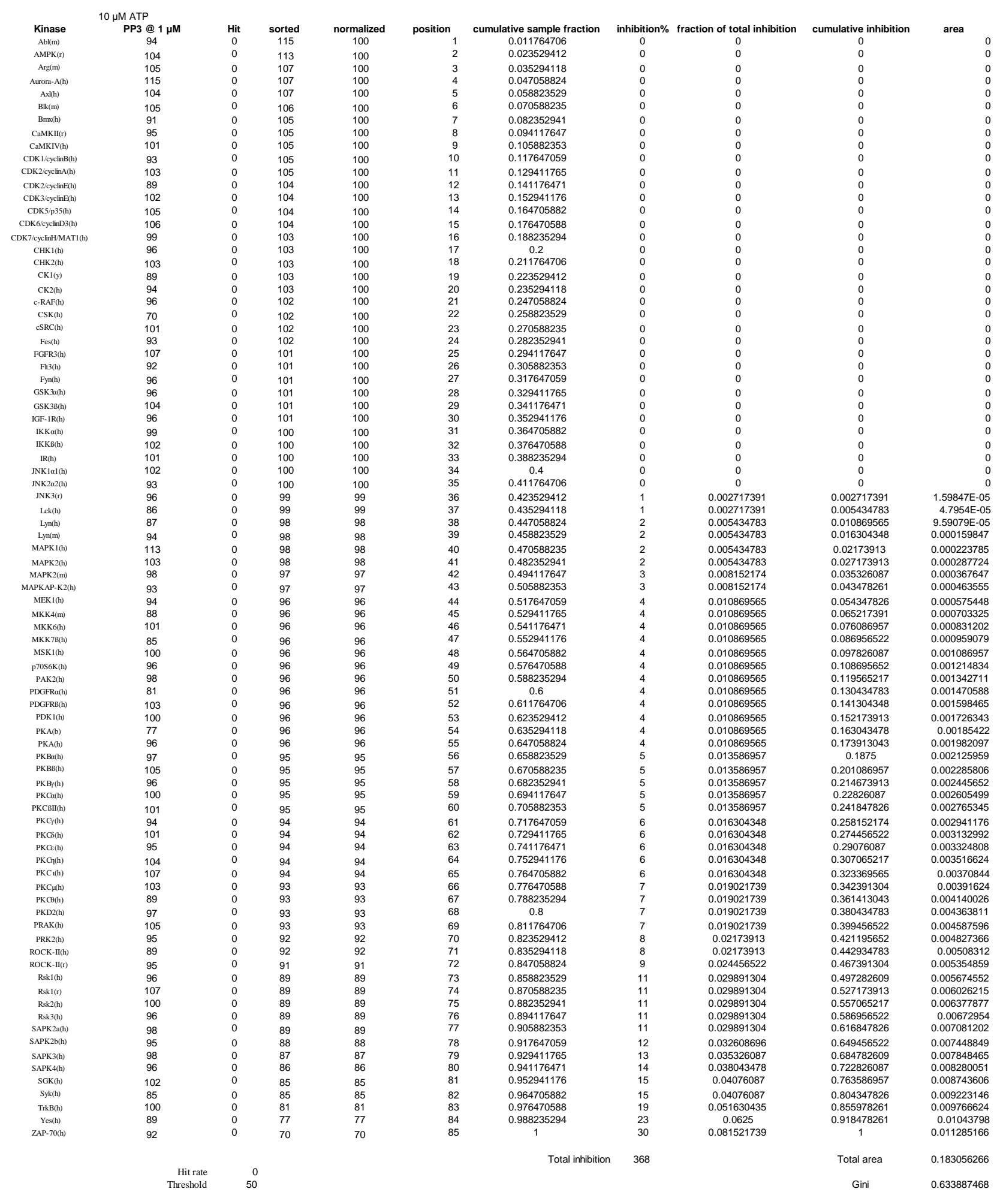


$100 \mu \mathrm{M}$ ATP

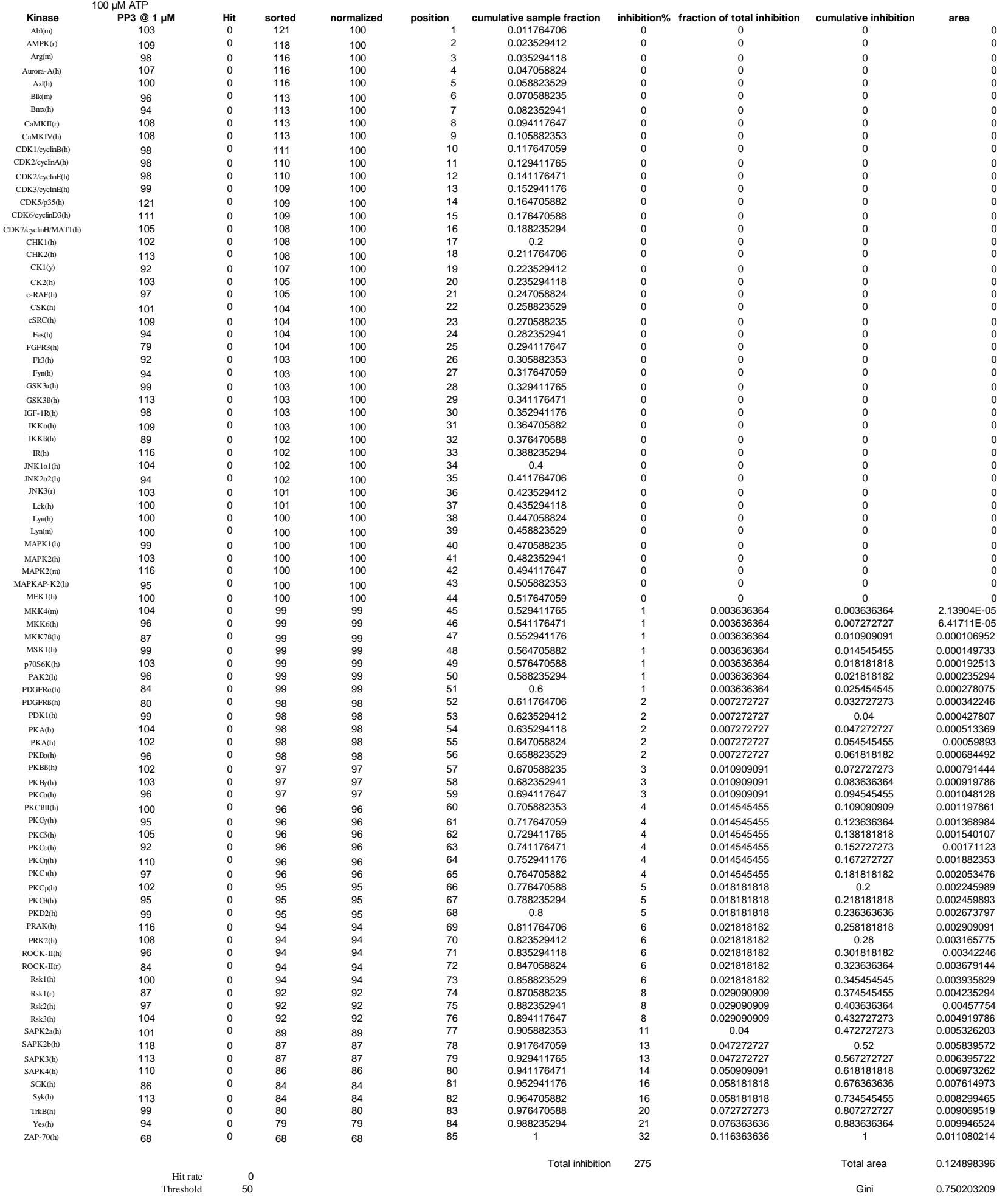




\begin{tabular}{|c|c|c|c|c|c|c|c|c|c|}
\hline & $10 \mu \mathrm{M}$ ATP & & & & & & & & \\
\hline Kinase & Ro31-8220 @ 1 нM & Hit & sorted & normalized & position & cumulative sample fraction & inhibition\% & fraction of total inhibition & cumulative inhibition \\
\hline $\mathrm{Abl}(\mathrm{m})$ & 85 & 0 & 112 & 100 & 1 & 0.011764706 & 0 & 0 & 0 \\
\hline AMPK(r) & 36 & 1 & 110 & 100 & 2 & 0.023529412 & 0 & 0 & 0 \\
\hline $\operatorname{Arg}(\mathrm{m})$ & 77 & 0 & 110 & 100 & 3 & 0.035294118 & 0 & 0 & 0 \\
\hline Aurora-A(h) & 83 & 0 & 108 & 100 & 4 & 0.047058824 & 0 & 0 & 0 \\
\hline Axl(h) & 31 & 1 & 104 & 100 & 5 & 0.058823529 & 0 & 0 & 0 \\
\hline $\mathrm{Blk}(\mathrm{m})$ & 31 & 1 & 104 & 100 & 6 & 0.070588235 & 0 & 0 & 0 \\
\hline Bnxx(h) & 52 & 0 & 102 & 100 & 7 & 0.082352941 & 0 & 0 & 0 \\
\hline CaMKII(r) & 87 & 0 & 100 & 100 & 8 & 0.094117647 & 0 & 0 & 0 \\
\hline CaMKIV(h) & 104 & 0 & 99 & 99 & 9 & 0.105882353 & 1 & 0.000234962 & 0.000234962 \\
\hline $\mathrm{CDK} 1 /$ cyclinB(h) & 1 & 1 & 99 & 99 & 10 & 0.117647059 & 1 & 0.000234962 & 0.000469925 \\
\hline $\mathrm{CDK} 2 / \mathrm{cyc} c \mathrm{in} A(\mathrm{~h})$ & 2 & 1 & 99 & 99 & 11 & 0.129411765 & 1 & 0.000234962 & 0.000704887 \\
\hline $\mathrm{CDK} 2 / \mathrm{cyclin} E(\mathrm{~h})$ & -4 & 1 & 95 & 95 & 12 & 0.141176471 & 5 & 0.001174812 & 0.001879699 \\
\hline CDK3/cyclinE(h) & 1 & 1 & 94 & 94 & 13 & 0.152941176 & 6 & 0.001409774 & 0.003289474 \\
\hline CDK5/p35(h) & 1 & 1 & 93 & 93 & 14 & 0.164705882 & 7 & 0.001644737 & 0.004934211 \\
\hline CDK6/cyclinD3(h) & 11 & 1 & 93 & 93 & 15 & 0.176470588 & 7 & 0.001644737 & 0.006578947 \\
\hline CDK7/cyclinHMATI(h) & 6 & 1 & 92 & 92 & 16 & 0.188235294 & 8 & 0.001879699 & 0.008458647 \\
\hline СHK1(h) & 25 & 1 & 89 & 89 & 17 & 0.2 & 11 & 0.002584586 & 0.011043233 \\
\hline CHK2(h) & 54 & 0 & 88 & 88 & 18 & 0.211764706 & 12 & 0.002819549 & 0.013862782 \\
\hline CKI(y) & 29 & 1 & 88 & 88 & 19 & 0.223529412 & 12 & 0.002819549 & 0.016682331 \\
\hline $\mathrm{CK} 2(\mathrm{~h})$ & 100 & 0 & 88 & 88 & 20 & 0.235294118 & 12 & 0.002819549 & 0.01950188 \\
\hline $\mathrm{c}-\mathrm{RAF}(\mathrm{h})$ & 108 & 0 & 88 & 88 & 21 & 0.247058824 & 12 & 0.002819549 & 0.022321429 \\
\hline $\operatorname{csK}(\mathrm{h})$ & 66 & 0 & 87 & 87 & 22 & 0.258823529 & 13 & 0.003054511 & 0.02537594 \\
\hline cSRCh) & 88 & 0 & 85 & 85 & 23 & 0.270588235 & 15 & 0.003524436 & 0.028900376 \\
\hline Fes(h) & 88 & 0 & 85 & 85 & 24 & 0.282352941 & 15 & 0.003524436 & 0.032424812 \\
\hline FGFR3(h) & 76 & 0 & 83 & 83 & 25 & 0.294117647 & 17 & 0.003994361 & 0.036419173 \\
\hline Flls(h) & 9 & 1 & 83 & 83 & 26 & 0.305882353 & 17 & 0.003994361 & 0.040413534 \\
\hline Fyn(h) & 78 & 0 & 82 & 82 & 27 & 0.317647059 & 18 & 0.004229323 & 0.044642857 \\
\hline GSK3a(h) & 2 & 1 & 80 & 80 & 28 & 0.329411765 & 20 & 0.004699248 & 0.049342105 \\
\hline GSK3B(h) & 2 & 1 & 78 & 78 & 29 & 0.341176471 & 22 & 0.005169173 & 0.054511278 \\
\hline IGF-IR(h) & 110 & 0 & 77 & 77 & 30 & 0.352941176 & 23 & 0.005404135 & 0.059915414 \\
\hline $\mathrm{IKKa(h)}$ & 65 & 0 & 76 & 76 & 31 & 0.364705882 & 24 & 0.005639098 & 0.065554511 \\
\hline IKKB(h) & 99 & 0 & 76 & 76 & 32 & 0.376470588 & 24 & 0.005639098 & 0.071193609 \\
\hline $\mathrm{IR}(\mathrm{h})$ & 95 & 0 & 74 & 74 & 33 & 0.388235294 & 26 & 0.006109023 & 0.077302632 \\
\hline JNK Ial(h) & 94 & 0 & 73 & 73 & 34 & 0.4 & 27 & 0.006343985 & 0.083646617 \\
\hline JNK202(h) & 93 & 0 & 73 & 73 & 35 & 0.411764706 & 27 & 0.006343985 & 0.089990602 \\
\hline JNK3(r) & 74 & 0 & 72 & 72 & 36 & 0.423529412 & 28 & 0.006578947 & 0.096569549 \\
\hline Lck(h) & 72 & 0 & 71 & 71 & 37 & 0.435294118 & 29 & 0.00681391 & 0.103383459 \\
\hline Lyn(h) & 57 & 0 & 71 & 71 & 38 & 0.447058824 & 29 & 0.00681391 & 0.110197368 \\
\hline Lyn(m) & 60 & 0 & 70 & 70 & 39 & 0.458823529 & 30 & 0.007048872 & 0.117246241 \\
\hline MAPK I (h) & 80 & 0 & 66 & 66 & 40 & 0.470588235 & 34 & 0.007988722 & 0.125234962 \\
\hline MAPK2(h) & 89 & 0 & 65 & 65 & 41 & 0.482352941 & 35 & 0.008223684 & 0.133458647 \\
\hline MAPK2(m) & 83 & 0 & 61 & 61 & 42 & 0.494117647 & 39 & 0.009163534 & 0.14262218 \\
\hline MAPKAP-K2(h) & 71 & 0 & 60 & 60 & 43 & 0.505882353 & 40 & 0.009398496 & 0.152020677 \\
\hline MEK1(h) & 82 & 0 & 57 & 57 & 44 & 0.517647059 & 43 & 0.010103383 & 0.16212406 \\
\hline $\mathrm{MKK} 4(\mathrm{~m})$ & 104 & 0 & 54 & 54 & 45 & 0.529411765 & 46 & 0.010808271 & 0.172932331 \\
\hline MKK6(h) & 92 & 0 & 52 & 52 & 46 & 0.541176471 & 48 & 0.011278195 & 0.184210526 \\
\hline MKK 7 (h) & 110 & 0 & 46 & 46 & 47 & 0.552941176 & 54 & 0.01268797 & 0.196898496 \\
\hline MSKI(h) & 1 & 1 & 42 & 42 & 48 & 0.564705882 & 58 & 0.01362782 & 0.210526316 \\
\hline $\mathrm{p} 70 \mathrm{~s} 6 \mathrm{~K}(\mathrm{~h})$ & 1 & 1 & 36 & 36 & 49 & 0.576470588 & 64 & 0.015037594 & 0.22556391 \\
\hline PAK2(h) & 85 & 0 & 31 & 31 & 50 & 0.588235294 & 69 & 0.016212406 & 0.241776316 \\
\hline PDGFRa(h) & 73 & 0 & 31 & 31 & 51 & 0.6 & 69 & 0.016212406 & 0.257988722 \\
\hline PDGFRB(h) & 70 & 0 & 31 & 31 & 52 & 0.611764706 & 69 & 0.016212406 & 0.274201128 \\
\hline PDK1(h) & 42 & 1 & 29 & 29 & 53 & 0.623529412 & 71 & 0.016682331 & 0.290883459 \\
\hline PKA(b) & 17 & 1 & 25 & 25 & 54 & 0.635294118 & 75 & 0.01762218 & 0.308505639 \\
\hline PKA(h) & 24 & 1 & 24 & 24 & 55 & 0.647058824 & 76 & 0.017857143 & 0.326362782 \\
\hline PKBa(h) & 31 & 1 & 22 & 22 & 56 & 0.658823529 & 78 & 0.018327068 & 0.34468985 \\
\hline PKBB(h) & 61 & 0 & 20 & 20 & 57 & 0.670588235 & 80 & 0.018796992 & 0.363486842 \\
\hline $\mathrm{PKB}_{\gamma}(\mathrm{h})$ & 4 & 1 & 17 & 17 & 58 & 0.682352941 & 83 & 0.01950188 & 0.382988722 \\
\hline PKCa(h) & 2 & 1 & 11 & 11 & 59 & 0.694117647 & 89 & 0.020911654 & 0.403900376 \\
\hline PKCBII(h) & 2 & 1 & 9 & 9 & 60 & 0.705882353 & 91 & 0.021381579 & 0.425281955 \\
\hline PKCy(h) & 5 & 1 & 9 & 9 & 61 & 0.717647059 & 91 & 0.021381579 & 0.446663534 \\
\hline PKळ(h) & 0 & 1 & 6 & 6 & 62 & 0.729411765 & 94 & 0.022086466 & 0.46875 \\
\hline PKG:(h) & 1 & 1 & 5 & 5 & 63 & 0.741176471 & 95 & 0.022321429 & 0.491071429 \\
\hline PKG (h) & 1 & 1 & 5 & 5 & 64 & 0.752941176 & 95 & 0.022321429 & 0.513392857 \\
\hline PKC (h) & 20 & 1 & 4 & 4 & 65 & 0.764705882 & 96 & 0.022556391 & 0.535949248 \\
\hline PKC & 22 & 1 & 3 & 3 & 66 & 0.776470588 & 97 & 0.022791353 & 0.558740602 \\
\hline PKCO(h) & 2 & 1 & 2 & 2 & 67 & 0.788235294 & 98 & 0.023026316 & 0.581766917 \\
\hline PKD2(h) & 46 & 1 & 2 & 2 & 68 & 0.8 & 98 & 0.023026316 & 0.604793233 \\
\hline PRAK(h) & 112 & 0 & 2 & 2 & 69 & 0.811764706 & 98 & 0.023026316 & 0.627819549 \\
\hline PRK2(h) & 3 & 1 & 2 & 2 & 70 & 0.823529412 & 98 & 0.023026316 & 0.650845865 \\
\hline ROCK-II(h) & 71 & 0 & 2 & 2 & 71 & 0.835294118 & 98 & 0.023026316 & 0.67387218 \\
\hline ROCK-II(r) & 76 & 0 & 2 & 2 & 72 & 0.847058824 & 98 & 0.023026316 & 0.696898496 \\
\hline Rskl(h) & 2 & 1 & 2 & 2 & 73 & 0.858823529 & 98 & 0.023026316 & 0.719924812 \\
\hline Rskl(r) & -2 & 1 & 1 & 1 & 74 & 0.870588235 & 99 & 0.023261278 & 0.74318609 \\
\hline Rsk2(h) & 1 & 1 & 1 & 1 & 75 & 0.882352941 & 99 & 0.023261278 & 0.766447368 \\
\hline Rsk3(h) & 0 & 1 & 1 & 1 & 76 & 0.894117647 & 99 & 0.023261278 & 0.789708647 \\
\hline SAPK2a(h) & 88 & 0 & 1 & 1 & 77 & 0.905882353 & 99 & 0.023261278 & 0.812969925 \\
\hline SAPK2b(h) & 93 & 0 & 1 & 1 & 78 & 0.917647059 & 99 & 0.023261278 & 0.836231203 \\
\hline $\mathrm{SAPK} 3(\mathrm{~h})$ & 102 & 0 & 1 & 1 & 79 & 0.929411765 & 99 & 0.023261278 & 0.859492481 \\
\hline SAPK4(h) & 99 & 0 & 1 & 1 & 80 & 0.941176471 & 99 & 0.023261278 & 0.882753759 \\
\hline SGK(h) & 9 & 1 & 1 & 1 & 81 & 0.952941176 & 99 & 0.023261278 & 0.906015038 \\
\hline Syk(h) & 88 & 0 & 0 & 0 & 82 & 0.964705882 & 100 & 0.023496241 & 0.929511278 \\
\hline $\operatorname{TrkB(h)}$ & 5 & 1 & 0 & 0 & 83 & 0.976470588 & 100 & 0.023496241 & 0.953007519 \\
\hline Yes(h) & 73 & 0 & -2 & 0 & 84 & 0.988235294 & 100 & 0.023496241 & 0.976503759 \\
\hline ZAP-70(h) & 99 & 0 & -4 & 0 & 85 & 1 & 100 & 0.023496241 & 1 \\
\hline & & & & & & Total inhibition & 4256 & & Total area \\
\hline & $\begin{array}{l}\text { Hit rate } \\
\text { Threshold }\end{array}$ & $\begin{array}{l}3 \\
5\end{array}$ & & & & & & & Gini \\
\hline
\end{tabular}




\begin{tabular}{|c|c|c|c|c|c|c|c|c|c|}
\hline & $100 \mu \mathrm{M}$ ATP & & & & & & & & \\
\hline Kinase & Ro31-8220@1 1 M & Hit & sorted & normalized & position & cumulative sample fraction & inhibition\% & fraction of total inhibition & cumulative inhibition \\
\hline $\mathrm{Abl}(\mathrm{m})$ & 92 & 0 & 122 & 100 & 1 & 0.011764706 & 0 & 0 & 0 \\
\hline AMPK(r) & 71 & 0 & 120 & 100 & 2 & 0.023529412 & 0 & 0 & 0 \\
\hline $\operatorname{Arg}(\mathrm{m})$ & 98 & 0 & 116 & 100 & 3 & 0.035294118 & 0 & 0 & 0 \\
\hline Aurora-A(h) & 110 & 0 & 113 & 100 & 4 & 0.047058824 & 0 & 0 & 0 \\
\hline Axl(h) & 43 & 1 & 113 & 100 & 5 & 0.058823529 & 0 & 0 & 0 \\
\hline $\mathrm{Blk}(\mathrm{m})$ & 55 & 0 & 110 & 100 & 6 & 0.070588235 & 0 & 0 & 0 \\
\hline Bnx(h) & 72 & 0 & 110 & 100 & 7 & 0.082352941 & 0 & 0 & 0 \\
\hline CaMKII(r) & 84 & 0 & 110 & 100 & 8 & 0.094117647 & 0 & 0 & 0 \\
\hline CaMKIV(h) & 106 & 0 & 107 & 100 & 9 & 0.105882353 & 0 & 0 & 0 \\
\hline CDK1/cyclnBB(h) & 4 & 1 & 107 & 100 & 10 & 0.117647059 & 0 & 0 & 0 \\
\hline CDK2/cyclinA(h) & 10 & 1 & 106 & 100 & 11 & 0.129411765 & 0 & 0 & 0 \\
\hline $\mathrm{CDK} 2 /$ cyclinE(h) & 1 & 1 & 106 & 100 & 12 & 0.141176471 & 0 & 0 & 0 \\
\hline CDK3/cyclinE(h) & 0 & 1 & 106 & 100 & 13 & 0.152941176 & 0 & 0 & 0 \\
\hline CDK5/p35(h) & 11 & 1 & 105 & 100 & 14 & 0.164705882 & 0 & 0 & 0 \\
\hline CDKG/cyclinD3(h) & 10 & 1 & 101 & 100 & 15 & 0.176470588 & 0 & 0 & 0 \\
\hline CDK7/cyclinHMATI(h) & 6 & 1 & 101 & 100 & 16 & 0.188235294 & 0 & 0 & 0 \\
\hline СнК 1 (h) & 39 & 1 & 100 & 100 & 17 & 0.2 & 0 & 0 & 0 \\
\hline CHK2(h) & 72 & 0 & 100 & 100 & 18 & 0.211764706 & 0 & 0 & 0 \\
\hline CKI(y) & 52 & 0 & 99 & 99 & 19 & 0.223529412 & 1 & 0.000286451 & 0.000286451 \\
\hline СK2(h) & 107 & 0 & 99 & 99 & 20 & 0.235294118 & 1 & 0.000286451 & 0.000572902 \\
\hline $\mathrm{c}-\mathrm{RAF}(\mathrm{h})$ & 116 & 0 & 98 & 98 & 21 & 0.247058824 & 2 & 0.000572902 & 0.001145803 \\
\hline $\operatorname{CSK}(\mathrm{h})$ & 94 & 0 & 97 & 97 & 22 & 0.258823529 & 3 & 0.000859353 & 0.002005156 \\
\hline cSRC(h) & 82 & 0 & 97 & 97 & 23 & 0.270588235 & 3 & 0.000859353 & 0.002864509 \\
\hline Fes(h) & 101 & 0 & 96 & 96 & 24 & 0.282352941 & 4 & 0.001145803 & 0.004010312 \\
\hline FGFR3(h) & 63 & 0 & 95 & 95 & 25 & 0.294117647 & 5 & 0.001432254 & 0.005442567 \\
\hline $\mathrm{FH}$ H(h) & 9 & 1 & 94 & 94 & 26 & 0.305882353 & 6 & 0.001718705 & 0.007161272 \\
\hline Fyn(h) & 100 & 0 & 92 & 92 & 27 & 0.317647059 & 8 & 0.002291607 & 0.009452879 \\
\hline GSK3a(h) & 6 & 1 & 92 & 92 & 28 & 0.329411765 & 8 & 0.002291607 & 0.011744486 \\
\hline GSK $3 B(h)$ & 5 & 1 & 92 & 92 & 29 & 0.341176471 & 8 & 0.002291607 & 0.014036093 \\
\hline IGF-IR(h) & 122 & 0 & 90 & 90 & 30 & 0.352941176 & 10 & 0.002864509 & 0.016900602 \\
\hline IKKa(h) & 107 & 0 & 89 & 89 & 31 & 0.364705882 & 11 & 0.00315096 & 0.020051561 \\
\hline IKKB(h) & 96 & 0 & 88 & 88 & 32 & 0.376470588 & 12 & 0.00343741 & 0.023488972 \\
\hline $\mathrm{IR}(\mathrm{h})$ & 106 & 0 & 87 & 87 & 33 & 0.388235294 & 13 & 0.003723861 & 0.027212833 \\
\hline JNKIal(h) & 100 & 0 & 84 & 84 & 34 & 0.4 & 16 & 0.004583214 & 0.031796047 \\
\hline JNK202(h) & 113 & 0 & 82 & 82 & 35 & 0.411764706 & 18 & 0.005156116 & 0.036952163 \\
\hline $\mathrm{JNK} 3(\mathrm{r})$ & 92 & 0 & 82 & 82 & 36 & 0.423529412 & 18 & 0.005156116 & 0.042108278 \\
\hline Lck(h) & 80 & 0 & 80 & 80 & 37 & 0.435294118 & 20 & 0.005729017 & 0.047837296 \\
\hline Lyn(h) & 69 & 0 & 80 & 80 & 38 & 0.447058824 & 20 & 0.005729017 & 0.053566313 \\
\hline Lyn(m) & 79 & 0 & 79 & 79 & 39 & 0.458823529 & 21 & 0.006015468 & 0.059581782 \\
\hline MAPK I(h) & 99 & 0 & 78 & 78 & 40 & 0.470588235 & 22 & 0.006301919 & 0.065883701 \\
\hline MAPK2(h) & 106 & 0 & 76 & 76 & 41 & 0.482352941 & 24 & 0.006874821 & 0.072758522 \\
\hline MAPK2(m) & 97 & 0 & 74 & 74 & 42 & 0.494117647 & 26 & 0.007447723 & 0.080206245 \\
\hline MAPKAP-K2(h) & 101 & 0 & 74 & 74 & 43 & 0.505882353 & 26 & 0.007447723 & 0.087653967 \\
\hline MEK 1(h) & 90 & 0 & 72 & 72 & 44 & 0.517647059 & 28 & 0.008020624 & 0.095674592 \\
\hline MKK $4(\mathrm{~m})$ & 95 & 0 & 72 & 72 & 45 & 0.529411765 & 28 & 0.008020624 & 0.103695216 \\
\hline MKK6(h) & 89 & 0 & 71 & 71 & 46 & 0.541176471 & 29 & 0.008307075 & 0.112002292 \\
\hline МKK $78($ (h) & 110 & 0 & 69 & 69 & 47 & 0.552941176 & 31 & 0.008879977 & 0.120882269 \\
\hline MSKI(h) & 2 & 1 & 66 & 66 & 48 & 0.564705882 & 34 & 0.00973933 & 0.130621598 \\
\hline $\mathrm{p} 7056 \mathrm{~K}(\mathrm{~h})$ & 4 & 1 & 65 & 65 & 49 & 0.576470588 & 35 & 0.010025781 & 0.140647379 \\
\hline PAK2(h) & 99 & 0 & 63 & 63 & 50 & 0.588235294 & 37 & 0.010598682 & 0.151246061 \\
\hline PDGFRa(h) & 97 & 0 & 59 & 59 & 51 & 0.6 & 41 & 0.011744486 & 0.162990547 \\
\hline PDGFRB(h) & 76 & 0 & 55 & 55 & 52 & 0.611764706 & 45 & 0.012890289 & 0.175880836 \\
\hline PDK1(h) & 66 & 0 & 52 & 52 & 53 & 0.623529412 & 48 & 0.013749642 & 0.189630478 \\
\hline PKA(b) & 74 & 0 & 43 & 43 & 54 & 0.635294118 & 57 & 0.0163277 & 0.205958178 \\
\hline PKA(h) & 59 & 0 & 43 & 43 & 55 & 0.647058824 & 57 & 0.0163277 & 0.222285878 \\
\hline PKBa(h) & 43 & 1 & 39 & 39 & 56 & 0.658823529 & 61 & 0.017473503 & 0.239759381 \\
\hline PKBB(h) & 74 & 0 & 34 & 34 & 57 & 0.670588235 & 66 & 0.018905758 & 0.258665139 \\
\hline $\mathrm{PKB}_{\gamma}(\mathrm{h})$ & 7 & 1 & 32 & 32 & 58 & 0.682352941 & 68 & 0.019478659 & 0.278143798 \\
\hline PKGa(h) & 1 & 1 & 29 & 29 & 59 & 0.694117647 & 71 & 0.020338012 & 0.29848181 \\
\hline РКСBII(h) & 8 & 1 & 26 & 26 & 60 & 0.705882353 & 74 & 0.021197365 & 0.319679175 \\
\hline PKCy(h) & 6 & 1 & 11 & 11 & 61 & 0.717647059 & 89 & 0.025494128 & 0.345173303 \\
\hline РКБ(h) & 2 & 1 & 10 & 10 & 62 & 0.729411765 & 90 & 0.025780579 & 0.370953881 \\
\hline PKG:(h) & 2 & 1 & 10 & 10 & 63 & 0.741176471 & 90 & 0.025780579 & 0.39673446 \\
\hline PKG (h) & 2 & 1 & 9 & 9 & 64 & 0.752941176 & 91 & 0.02606703 & 0.42280149 \\
\hline PKC (h) & 29 & 1 & 9 & 9 & 65 & 0.764705882 & 91 & 0.02606703 & 0.448868519 \\
\hline PKC $\mu(\mathrm{h})$ & 32 & 1 & 8 & 8 & 66 & 0.776470588 & 92 & 0.02635348 & 0.475221999 \\
\hline PKCO(h) & 7 & 1 & 7 & 7 & 67 & 0.788235294 & 93 & 0.026639931 & 0.501861931 \\
\hline PKD2(h) & 65 & 0 & 7 & 7 & 68 & 0.8 & 93 & 0.026639931 & 0.528501862 \\
\hline PRAK(h) & 113 & 0 & 7 & 7 & 69 & 0.811764706 & 93 & 0.026639931 & 0.555141793 \\
\hline PRK2(h) & 1 & 1 & 6 & 6 & 70 & 0.823529412 & 94 & 0.026926382 & 0.582068175 \\
\hline ROCK-II(h) & 87 & 0 & 6 & 6 & 71 & 0.835294118 & 94 & 0.026926382 & 0.608994557 \\
\hline ROCK-II(r) & 82 & 0 & 6 & 6 & 72 & 0.847058824 & 94 & 0.026926382 & 0.63592094 \\
\hline Rskl(h) & 9 & 1 & 5 & 5 & 73 & 0.858823529 & 95 & 0.027212833 & 0.663133773 \\
\hline Rskl(r) & 3 & 1 & 4 & 4 & 74 & 0.870588235 & 96 & 0.027499284 & 0.690633056 \\
\hline Rsk2(h) & 7 & 1 & 4 & 4 & 75 & 0.882352941 & 96 & 0.027499284 & 0.71813234 \\
\hline Rsk3(h) & 2 & 1 & 3 & 3 & 76 & 0.894117647 & 97 & 0.027785735 & 0.745918075 \\
\hline SAPK2a(h) & 105 & 0 & 2 & 2 & 77 & 0.905882353 & 98 & 0.028072186 & 0.773990261 \\
\hline SAPK2b(h) & 120 & 0 & 2 & 2 & 78 & 0.917647059 & 98 & 0.028072186 & 0.802062446 \\
\hline SAPK3(h) & 110 & 0 & 2 & 2 & 79 & 0.929411765 & 98 & 0.028072186 & 0.830134632 \\
\hline $\mathrm{SAPK} 4(\mathrm{~h})$ & 92 & 0 & 2 & 2 & 80 & 0.941176471 & 98 & 0.028072186 & 0.858206818 \\
\hline SGK(h) & 26 & 1 & 2 & 2 & 81 & 0.952941176 & 98 & 0.028072186 & 0.886279003 \\
\hline Syk(h) & 78 & 0 & 1 & 1 & 82 & 0.964705882 & 99 & 0.028358636 & 0.91463764 \\
\hline $\operatorname{TrkB}(\mathbf{h})$ & 34 & 1 & 1 & 1 & 83 & 0.976470588 & 99 & 0.028358636 & 0.942996276 \\
\hline Yes(h) & 80 & 0 & 1 & 1 & 84 & 0.988235294 & 99 & 0.028358636 & 0.971354913 \\
\hline ZAP-70(h) & 88 & 0 & 0 & 0 & 85 & 1 & 100 & 0.028645087 & 1 \\
\hline & & & & & & Total inhibition & 3491 & & Total area \\
\hline & $\begin{array}{l}\text { Hit rate } \\
\text { Threshold }\end{array}$ & $\begin{array}{l}3 \\
5\end{array}$ & & & & & & & Gini \\
\hline
\end{tabular}




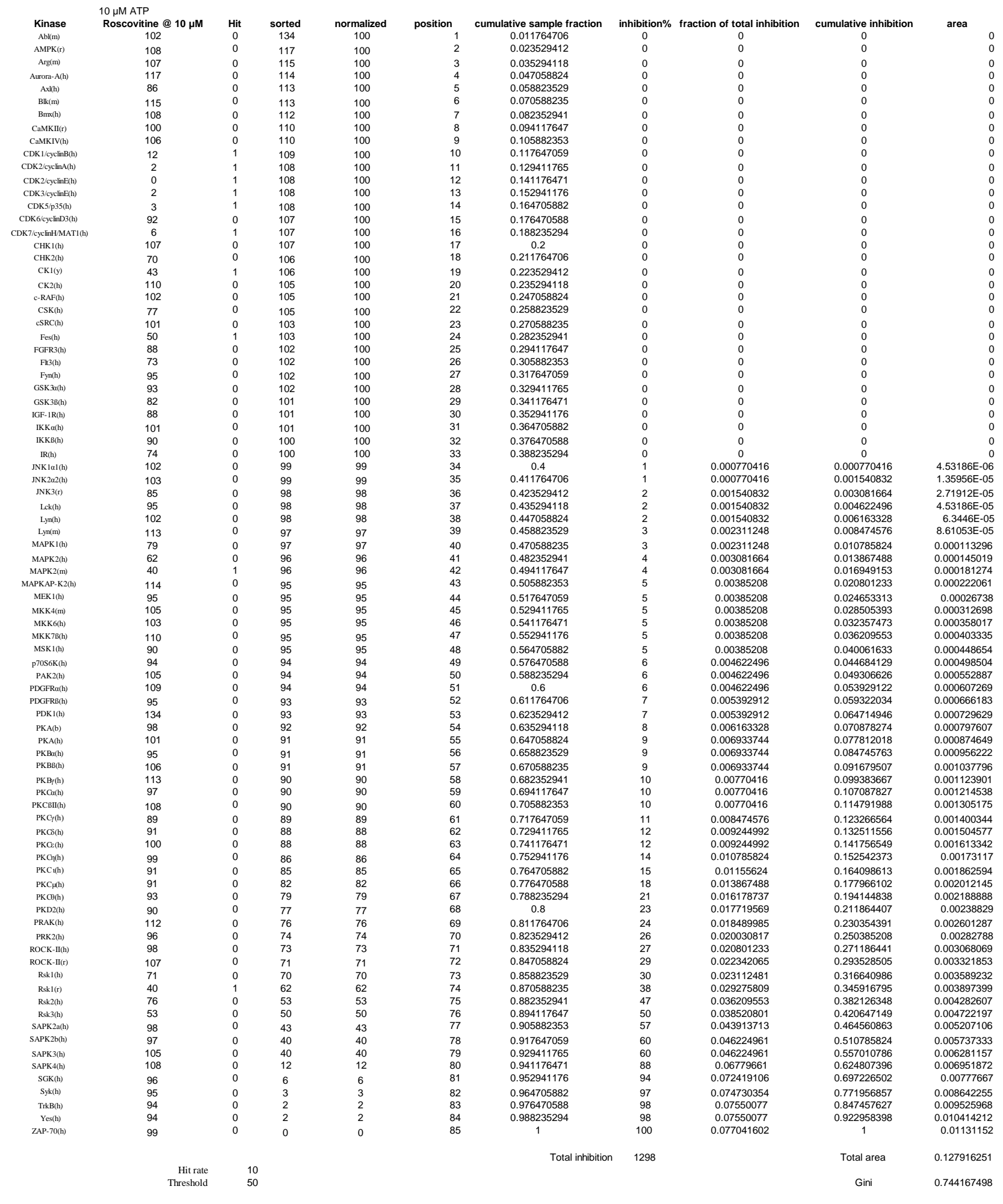




\begin{tabular}{|c|c|c|c|c|c|c|c|c|c|}
\hline & $100 \mu \mathrm{M}$ ATP & & & & & & & & \\
\hline Kinase & Roscovitine@10 $\mu \mathrm{M}$ & Hit & sorted & normalized & position & cumulative sample fraction & inhibition\% & fraction of total inhibition & cumulative inhibition \\
\hline $\mathrm{Abl}(\mathrm{m})$ & 97 & 0 & 138 & 100 & 1 & 0.011764706 & 0 & 0 & 0 \\
\hline AMPK(r) & 115 & 0 & 128 & 100 & 2 & 0.023529412 & 0 & 0 & 0 \\
\hline $\operatorname{Arg}(\mathrm{m})$ & 100 & 0 & 127 & 100 & 3 & 0.035294118 & 0 & 0 & 0 \\
\hline Aurora-A(h) & 121 & 0 & 121 & 100 & 4 & 0.047058824 & 0 & 0 & 0 \\
\hline Axl(h) & 99 & 0 & 121 & 100 & 5 & 0.058823529 & 0 & 0 & 0 \\
\hline $\operatorname{Blk}(\mathrm{m})$ & 105 & 0 & 118 & 100 & 6 & 0.070588235 & 0 & 0 & 0 \\
\hline $\mathrm{Bmx(h)}$ & 112 & 0 & 117 & 100 & 7 & 0.082352941 & 0 & 0 & 0 \\
\hline CaMKII(r) & 102 & 0 & 116 & 100 & 8 & 0.094117647 & 0 & 0 & 0 \\
\hline CaMKIV(h) & 114 & 0 & 115 & 100 & 9 & 0.105882353 & 0 & 0 & 0 \\
\hline CDK1/cyclnBB(h) & 24 & 1 & 115 & 100 & 10 & 0.117647059 & 0 & 0 & 0 \\
\hline CDK2/cyclinA(h) & 12 & 1 & 114 & 100 & 11 & 0.129411765 & 0 & 0 & 0 \\
\hline $\mathrm{CDK} 2 /$ cyclnE(h) & 0 & 1 & 112 & 100 & 12 & 0.141176471 & 0 & 0 & 0 \\
\hline CDK3/yclinE(h) & -3 & 1 & 112 & 100 & 13 & 0.152941176 & 0 & 0 & 0 \\
\hline CDK5/p35(h) & 13 & 1 & 111 & 100 & 14 & 0.164705882 & 0 & 0 & 0 \\
\hline CDKG/cyclinD3(h) & 128 & 0 & 111 & 100 & 15 & 0.176470588 & 0 & 0 & 0 \\
\hline CDK $7 /$ yclinHMATI(h) & 4 & 1 & 111 & 100 & 16 & 0.188235294 & 0 & 0 & 0 \\
\hline CHKI(h) & 99 & 0 & 111 & 100 & 17 & 0.2 & 0 & 0 & 0 \\
\hline СHK2(h) & 88 & 0 & 111 & 100 & 18 & 0.211764706 & 0 & 0 & 0 \\
\hline CKI(y) & 68 & 0 & 110 & 100 & 19 & 0.223529412 & 0 & 0 & 0 \\
\hline СК2(h) & $\begin{array}{l}10 \\
104\end{array}$ & 0 & 109 & 100 & 20 & 0.235294118 & 0 & 0 & 0 \\
\hline c-RAF(h) & 109 & 0 & 109 & 100 & 21 & 0.247058824 & 0 & 0 & 0 \\
\hline $\operatorname{CSK}(\mathrm{h})$ & 117 & 0 & 108 & 100 & 22 & 0.258823529 & 0 & 0 & 0 \\
\hline $\operatorname{csRC(h)}$ & 116 & 0 & 105 & 100 & 23 & 0.270588235 & 0 & 0 & 0 \\
\hline Fess(h) & 79 & 0 & 105 & 100 & 24 & 0.282352941 & 0 & 0 & 0 \\
\hline FGFR3(h) & 102 & 0 & 105 & 100 & 25 & 0.294117647 & 0 & 0 & 0 \\
\hline $\mathrm{Fll}(\mathrm{h}(\mathrm{h})$ & 94 & 0 & 105 & 100 & 26 & 0.305882353 & 0 & 0 & 0 \\
\hline Fynn(h) & 103 & 0 & 104 & 100 & 27 & 0.317647059 & 0 & 0 & 0 \\
\hline GSK3a(h) & 105 & 0 & 104 & 100 & 28 & 0.329411765 & 0 & 0 & 0 \\
\hline GSK $3 \mathrm{~B}(\mathrm{~h})$ & 112 & 0 & 104 & 100 & 29 & 0.341176471 & 0 & 0 & 0 \\
\hline IGF-IR(h) & 101 & 0 & 103 & 100 & 30 & 0.352941176 & 0 & 0 & 0 \\
\hline $\mathrm{IKKa}(\mathrm{h})$ & 118 & 0 & 103 & 100 & 31 & 0.364705882 & 0 & 0 & 0 \\
\hline IKKB(h) & 98 & 0 & 103 & 100 & 32 & 0.376470588 & 0 & 0 & 0 \\
\hline $\mathbb{I R}(\mathrm{h})$ & $\begin{array}{l}50 \\
88\end{array}$ & 0 & 103 & 100 & $\begin{array}{l}32 \\
33\end{array}$ & 0.388235294 & 0 & 0 & 0 \\
\hline JNK1a1(h) & 111 & 0 & 103 & 100 & 34 & 0.4 & 0 & 0 & 0 \\
\hline JNK202(h) & 99 & 0 & 102 & 100 & 35 & 0.411764706 & 0 & 0 & 0 \\
\hline $\mathrm{JNK} 3(\mathrm{r})$ & 85 & 0 & 102 & 100 & 36 & 0.423529412 & 0 & 0 & 0 \\
\hline Lck(h) & 99 & 0 & 102 & 100 & 37 & 0.435294118 & 0 & 0 & 0 \\
\hline Lyn(h) & 104 & 0 & 102 & 100 & $\begin{array}{l}31 \\
38\end{array}$ & 0.447058824 & 0 & 0 & 0 \\
\hline Lyy(m) & 111 & 0 & 101 & 100 & 39 & 0.458823529 & 0 & 0 & 0 \\
\hline MAPKI(h) & 85 & 0 & 101 & 100 & 40 & 0.470588235 & 0 & 0 & 0 \\
\hline MAPK2(h) & 74 & 0 & 101 & 100 & 41 & 0.482352941 & 0 & 0 & 0 \\
\hline $\begin{array}{l}\text { MAPKL(2) } \\
\text { MAPK2(m) }\end{array}$ & $\begin{array}{l}74 \\
52\end{array}$ & 0 & 100 & 100 & $\begin{array}{l}41 \\
42\end{array}$ & 0.494117647 & 0 & 0 & 0 \\
\hline MAPKAP-K2(h) & 101 & 0 & 99 & 99 & 43 & 0.505882353 & 1 & 0.001081081 & 0.001081081 \\
\hline MEK1(h) & 90 & 0 & 99 & 99 & 44 & 0.517647059 & 1 & 0.001081081 & 0.002162162 \\
\hline MKK4(m) & 91 & 0 & 99 & 99 & 45 & 0.529411765 & 1 & 0.001081081 & 0.003243243 \\
\hline MKK6(h) & 86 & 0 & 99 & 99 & 46 & 0.541176471 & 1 & 0.001081081 & 0.004324324 \\
\hline MKK & 90 & 0 & 99 & 99 & 47 & 0.552941176 & 1 & 0.001081081 & 0.005405405 \\
\hline MSKI(h) & 94 & 0 & 98 & 98 & 48 & 0.564705882 & 2 & 0.002162162 & 0.007567568 \\
\hline $\mathrm{p} 7056 \mathrm{~K}(\mathrm{~h})$ & $\begin{array}{l}94 \\
109\end{array}$ & 0 & 98 & 98 & $\begin{array}{l}48 \\
49\end{array}$ & 0.576470588 & 2 & 0.002162162 & 0.00972973 \\
\hline $\begin{array}{l}\text { PAK2(h) } \\
\text { Past }\end{array}$ & 111 & 0 & 97 & 97 & 50 & 0.588235294 & 3 & 0.003243243 & 0.012972973 \\
\hline PDGFRa(h) & 103 & 0 & 97 & 97 & 51 & 0.6 & 3 & 0.003243243 & 0.016216216 \\
\hline PDGFRB(h) & 88 & 0 & 96 & 96 & 52 & 0.611764706 & 4 & 0.004324324 & 0.020540541 \\
\hline PDKI(h) & 98 & 0 & 94 & 94 & 53 & 0.623529412 & 6 & 0.006486486 & 0.027027027 \\
\hline PKA(b) & 93 & 0 & 94 & 94 & 54 & 0.635294118 & 6 & 0.006486486 & 0.033513514 \\
\hline PKA(h) & 92 & 0 & 94 & 94 & 55 & 0.647058824 & 6 & 0.006486486 & 0.04 \\
\hline PKBa(h) & 97 & 0 & 94 & 94 & 56 & 0.658823529 & 6 & 0.006486486 & 0.046486486 \\
\hline PKBB(h) & 104 & 0 & 94 & 94 & 57 & 0.670588235 & 6 & 0.006486486 & 0.052972973 \\
\hline $\mathrm{PKB} \mathrm{B}_{(\mathrm{h})}$ & 111 & 0 & 94 & 94 & 58 & 0.682352941 & 6 & 0.006486486 & 0.059459459 \\
\hline PKCath) & 101 & 0 & 93 & 93 & 59 & 0.694117647 & 7 & 0.007567568 & 0.067027027 \\
\hline PKCBII(h) & 108 & 0 & 93 & 93 & 60 & 0.705882353 & 7 & 0.007567568 & 0.074594595 \\
\hline PKGy(h) & 99 & 0 & 92 & 92 & 61 & 0.717647059 & 8 & 0.008648649 & 0.083243243 \\
\hline РКФ(h) & 91 & 0 & 91 & 91 & 62 & 0.729411765 & 9 & 0.00972973 & 0.092972973 \\
\hline PKG:(h) & 94 & 0 & 91 & 91 & 63 & 0.741176471 & 9 & 0.00972973 & 0.102702703 \\
\hline $\begin{array}{l}\text { PKG (h) } \\
\text { (h) }\end{array}$ & 103 & 0 & 90 & 90 & 64 & 0.752941176 & 10 & 0.010810811 & 0.113513514 \\
\hline PKC (h) & 94 & 0 & 90 & 90 & 65 & 0.764705882 & 10 & 0.010810811 & 0.124324324 \\
\hline PKC $\mu(\mathrm{h})$ & 94 & 0 & 90 & 90 & 66 & 0.776470588 & 10 & 0.010810811 & 0.135135135 \\
\hline PKCO(h) & 105 & 0 & 89 & 89 & 67 & 0.788235294 & 11 & 0.011891892 & 0.147027027 \\
\hline PKD2(h) & 105 & 0 & 88 & 88 & 68 & 0.8 & 12 & 0.012972973 & 0.16 \\
\hline PRAK(h) & 110 & 0 & 88 & 88 & 69 & 0.811764706 & 12 & 0.012972973 & 0.172972973 \\
\hline PRK2(h) & 93 & 0 & 88 & 88 & 70 & 0.823529412 & 12 & 0.012972973 & 0.185945946 \\
\hline $\begin{array}{l}\text { PRK_(n) } \\
\text { ROCK-II(h) }\end{array}$ & 102 & 0 & $\begin{array}{l}10 \\
86\end{array}$ & $\begin{array}{l}00 \\
86\end{array}$ & 71 & 0.835294118 & 14 & 0.015135135 & 0.201081081 \\
\hline ROCK-II(r) & 121 & 0 & 86 & 86 & 72 & 0.847058824 & 14 & 0.015135135 & 0.216216216 \\
\hline Rskl(h) & 79 & 0 & 85 & 85 & 73 & 0.858823529 & 15 & 0.016216216 & 0.232432432 \\
\hline Rskl(r) & 86 & 0 & 85 & 85 & 74 & 0.870588235 & 15 & 0.016216216 & 0.248648649 \\
\hline $\begin{array}{l}\text { Rsksk2(h) } \\
\text { Rsk(h) }\end{array}$ & 90 & 0 & 79 & 79 & 75 & 0.882352941 & 21 & 0.022702703 & 0.271351351 \\
\hline Rsk3(h) & 89 & 0 & 79 & 79 & 76 & 0.894117647 & 21 & 0.022702703 & 0.294054054 \\
\hline SAPK2a(h) & 96 & 0 & 74 & 74 & 77 & 0.905882353 & 26 & 0.028108108 & 0.322162162 \\
\hline SAPK2b(h) & 138 & 0 & 68 & 68 & 78 & 0.917647059 & 32 & 0.034594595 & 0.356756757 \\
\hline SAPK3(h) & 111 & 0 & 52 & 52 & 79 & 0.929411765 & 48 & 0.051891892 & 0.408648649 \\
\hline SAPK4(h) & 94 & 0 & 24 & 24 & 80 & 0.941176471 & 76 & 0.082162162 & 0.490810811 \\
\hline SGK(h) & 103 & 0 & 13 & 13 & 81 & 0.952941176 & 87 & 0.094054054 & 0.584864865 \\
\hline Syk(h) & 127 & 0 & 12 & 12 & 82 & 0.964705882 & 88 & 0.095135135 & 0.68 \\
\hline TrkB(h) & 102 & 0 & 4 & 4 & 83 & 0.976470588 & 96 & 0.103783784 & 0.783783784 \\
\hline Yes(h) & 115 & 0 & $\begin{array}{l}4 \\
0\end{array}$ & 0 & 84 & 0.988235294 & 100 & 0.108108108 & 0.891891892 \\
\hline ZAP-70(h) & 103 & 0 & -3 & 0 & 85 & 1 & 100 & 0.108108108 & 1 \\
\hline & & & & & & Total inhibition & 925 & & Total area \\
\hline & $\begin{array}{r}\text { Hit rate } \\
\text {. }\end{array}$ & 5 & & & & & & & Gini \\
\hline
\end{tabular}




\begin{tabular}{|c|c|c|c|c|c|c|c|c|c|}
\hline & 10 यM ATP & & & & & & & & \\
\hline Kinase & Rottlerin @ $10 \mu \mathrm{M}$ & Hit & sorted & normalized & position & cumulative sample fraction & inhibition\% & fraction of total inhibition & cumulative inhibition \\
\hline $\mathrm{Abl}(\mathrm{m})$ & 75 & 0 & 133 & 100 & 1 & 0.011764706 & 0 & 0 & 0 \\
\hline AMPK(r) & 110 & 0 & 127 & 100 & 2 & 0.023529412 & 0 & 0 & 0 \\
\hline $\operatorname{Arg}(\mathrm{m})$ & 121 & 0 & 125 & 100 & 3 & 0.035294118 & 0 & 0 & 0 \\
\hline Aurora-A(h) & 62 & 0 & 121 & 100 & 4 & 0.047058824 & 0 & 0 & 0 \\
\hline Axl(h) & 58 & 0 & 118 & 100 & 5 & 0.058823529 & 0 & 0 & 0 \\
\hline $\operatorname{Blk}(\mathrm{m})$ & 46 & 1 & 117 & 100 & 6 & 0.070588235 & 0 & 0 & 0 \\
\hline $\operatorname{Bmx}(\mathrm{h})$ & 101 & 0 & 117 & 100 & 7 & 0.082352941 & 0 & 0 & 0 \\
\hline CaMKII(r) & 91 & 0 & 113 & 100 & 8 & 0.094117647 & 0 & 0 & 0 \\
\hline CaMKIV(h) & 92 & 0 & 113 & 100 & 9 & 0.105882353 & 0 & 0 & 0 \\
\hline CDK1/cyclnBB(h) & 76 & 0 & 113 & 100 & 10 & 0.117647059 & 0 & 0 & 0 \\
\hline CDK2/cyclinA(h) & 103 & 0 & 110 & 100 & 11 & 0.129411765 & 0 & 0 & 0 \\
\hline $\mathrm{CDK} 2 /$ cyclnE(h) & 97 & 0 & 110 & 100 & 12 & 0.141176471 & 0 & 0 & 0 \\
\hline CDK3/cyclinE(h) & 79 & 0 & 110 & 100 & 13 & 0.152941176 & 0 & 0 & 0 \\
\hline CDK5/p35(h) & 79 & 0 & 109 & 100 & 14 & 0.164705882 & 0 & 0 & 0 \\
\hline CDKG/cyclinD3(h) & 99 & 0 & 108 & 100 & 15 & 0.176470588 & 0 & 0 & 0 \\
\hline CDK $7 /$ yclinHMATI(h) & 117 & 0 & 107 & 100 & 16 & 0.188235294 & 0 & 0 & 0 \\
\hline CHKI(h) & 125 & 0 & 107 & 100 & 17 & 0.2 & 0 & 0 & 0 \\
\hline CHK2(h) & 57 & 0 & 107 & 100 & 18 & 0.211764706 & 0 & 0 & 0 \\
\hline CKI(y) & 82 & 0 & 105 & 100 & 19 & 0.223529412 & 0 & 0 & 0 \\
\hline СК2(h) & 98 & 0 & 104 & 100 & 20 & 0.235294118 & 0 & 0 & 0 \\
\hline c-RAF(h) & 101 & 0 & 103 & 100 & 21 & 0.247058824 & 0 & 0 & 0 \\
\hline $\operatorname{CSK}(\mathrm{h})$ & 92 & 0 & 101 & 100 & 22 & 0.258823529 & 0 & 0 & 0 \\
\hline $\operatorname{csRC(h)}$ & 107 & 0 & 101 & 100 & 23 & 0.270588235 & 0 & 0 & 0 \\
\hline Fess(h) & 113 & 0 & 101 & 100 & 24 & 0.282352941 & 0 & 0 & 0 \\
\hline FGFR3(h) & 108 & 0 & 99 & 99 & 25 & 0.294117647 & 1 & 0.000834725 & 0.000834725 \\
\hline $\mathrm{Fll}(\mathrm{h}(\mathrm{h})$ & 70 & 0 & 99 & 99 & 26 & 0.305882353 & 1 & 0.000834725 & 0.001669449 \\
\hline Fynn(h) & 81 & 0 & 98 & 98 & 27 & 0.317647059 & 2 & 0.001669449 & 0.003338898 \\
\hline GSK3a(h) & 65 & 0 & 98 & 98 & 28 & 0.329411765 & 2 & 0.001669449 & 0.005008347 \\
\hline GSK $3 \mathrm{~B}(\mathrm{~h})$ & 77 & 0 & 98 & 98 & 29 & 0.341176471 & 2 & 0.001669449 & 0.006677796 \\
\hline IGF-IR(h) & 127 & 0 & 98 & 98 & 30 & 0.352941176 & 2 & 0.001669449 & 0.008347245 \\
\hline $\mathrm{IKKa(h)}$ & 117 & 0 & 97 & 97 & 31 & 0.364705882 & 3 & 0.002504174 & 0.010851419 \\
\hline IKKB(h) & 84 & 0 & 97 & 97 & 32 & 0.376470588 & 3 & 0.002504174 & 0.013355593 \\
\hline $\mathbb{I R}(\mathrm{h})$ & $\begin{array}{l}04 \\
91\end{array}$ & 0 & 97 & 97 & $\begin{array}{l}32 \\
33\end{array}$ & 0.388235294 & 3 & 0.002504174 & 0.015859766 \\
\hline JNKIal(h) & 35 & 1 & 96 & 96 & 34 & 0.4 & 4 & 0.003338898 & 0.019198664 \\
\hline JNK202(h) & 92 & 0 & 96 & 96 & $\begin{array}{l}54 \\
35\end{array}$ & 0.411764706 & 4 & 0.003338898 & 0.022537563 \\
\hline JNK3(r) & 76 & 0 & 95 & 95 & 36 & 0.423529412 & 5 & 0.004173623 & 0.026711185 \\
\hline Lck(h) & 84 & 0 & 93 & 93 & 37 & 0.435294118 & 7 & 0.005843072 & 0.032554257 \\
\hline Lyn(h) & 83 & 0 & 92 & 92 & 38 & 0.447058824 & 8 & 0.006677796 & 0.039232053 \\
\hline Lyy(m) & 52 & 0 & 92 & 92 & 39 & 0.458823529 & 8 & 0.006677796 & 0.04590985 \\
\hline MAPKI(h) & 93 & 0 & $\begin{array}{l}92 \\
92\end{array}$ & $\begin{array}{l}92 \\
92\end{array}$ & 40 & 0.470588235 & 8 & 0.006677796 & 0.052587646 \\
\hline MAPK2(h) & 110 & 0 & 92 & 92 & 41 & 0.482352941 & 8 & 0.006677796 & 0.059265442 \\
\hline $\begin{array}{l}\text { MAPKL(2) } \\
\text { MAPK2(m) }\end{array}$ & 110 & 0 & 92 & 92 & $\begin{array}{l}41 \\
42\end{array}$ & 0.494117647 & 8 & 0.006677796 & 0.065943239 \\
\hline MAPKAP-K2(h) & 69 & 0 & 91 & 91 & 43 & 0.505882353 & 9 & 0.007512521 & 0.07345576 \\
\hline MEK1(h) & 101 & 0 & 91 & 91 & 44 & 0.517647059 & 9 & 0.007512521 & 0.08096828 \\
\hline MKK4(m) & 109 & 0 & 91 & 91 & 45 & 0.529411765 & 9 & 0.007512521 & 0.088480801 \\
\hline MKK6(h) & 98 & 0 & 91 & 91 & 46 & 0.541176471 & 9 & 0.007512521 & 0.095993322 \\
\hline MKK & 88 & 0 & 90 & 90 & 47 & 0.552941176 & 10 & 0.008347245 & 0.104340568 \\
\hline MSKI(h) & 92 & 0 & 90 & 90 & 48 & 0.564705882 & 10 & 0.008347245 & 0.112687813 \\
\hline $\mathrm{p} 7056 \mathrm{~K}(\mathrm{~h})$ & 70 & 0 & 90 & 90 & $\begin{array}{l}48 \\
49\end{array}$ & 0.576470588 & 10 & 0.008347245 & 0.121035058 \\
\hline $\begin{array}{l}\text { PAK2(h) } \\
\text { Past }\end{array}$ & 91 & 0 & 90 & 90 & 50 & 0.588235294 & 10 & 0.008347245 & 0.129382304 \\
\hline PDGFRa(h) & 107 & 0 & 89 & 89 & 51 & 0.6 & 11 & 0.00918197 & 0.138564274 \\
\hline PDGFRB(h) & 113 & 0 & 88 & 88 & 52 & 0.611764706 & 12 & 0.010016694 & 0.148580968 \\
\hline PDKI(h) & 72 & 0 & 86 & 86 & 53 & 0.623529412 & 14 & 0.011686144 & 0.160267112 \\
\hline PKA(b) & 52 & 0 & 84 & 84 & 54 & 0.635294118 & 16 & 0.013355593 & 0.173622705 \\
\hline PKA(h) & 86 & 0 & 84 & 84 & 55 & 0.647058824 & 16 & 0.013355593 & 0.186978297 \\
\hline PKBa(h) & 90 & 0 & 83 & 83 & 56 & 0.658823529 & 17 & 0.014190317 & 0.201168614 \\
\hline PKBB(h) & 75 & 0 & 83 & 83 & 57 & 0.670588235 & 17 & 0.014190317 & 0.215358932 \\
\hline $\mathrm{PKB} \mathrm{B}_{(\mathrm{h})}$ & 83 & 0 & 82 & 82 & 58 & 0.682352941 & 18 & 0.015025042 & 0.230383973 \\
\hline PKCath) & 96 & 0 & 81 & 81 & 59 & 0.694117647 & 19 & 0.015859766 & 0.24624374 \\
\hline PKCBII(h) & 95 & 0 & 79 & 79 & 60 & 0.705882353 & 21 & 0.017529215 & 0.263772955 \\
\hline PKGy(h) & 118 & 0 & 79 & 79 & 61 & 0.717647059 & 21 & 0.017529215 & 0.28130217 \\
\hline РКФ(h) & 97 & 0 & 77 & 77 & 62 & 0.729411765 & 23 & 0.019198664 & 0.300500835 \\
\hline PKG:(h) & 90 & 0 & 77 & 77 & 63 & 0.741176471 & 23 & 0.019198664 & 0.319699499 \\
\hline $\begin{array}{l}\text { PKG (h) } \\
\text { (h) }\end{array}$ & 113 & 0 & 76 & 76 & 64 & 0.752941176 & 24 & 0.020033389 & 0.339732888 \\
\hline PKC (h) & 77 & 0 & 76 & 76 & 65 & 0.764705882 & 24 & 0.020033389 & 0.359766277 \\
\hline PKC $\mu(\mathrm{h})$ & 90 & 0 & 75 & 75 & 66 & 0.776470588 & 25 & 0.020868114 & 0.380634391 \\
\hline PKCO(h) & 133 & 0 & 75 & 75 & 67 & 0.788235294 & 25 & 0.020868114 & 0.401502504 \\
\hline PKD2(h) & 91 & 0 & 72 & 72 & 68 & 0.8 & 28 & 0.023372287 & 0.424874791 \\
\hline PRAK(h) & 42 & 1 & 72 & 72 & 69 & 0.811764706 & 28 & 0.023372287 & 0.448247078 \\
\hline PRK2(h) & 97 & 0 & 70 & 70 & 70 & 0.823529412 & 30 & 0.025041736 & 0.473288815 \\
\hline $\begin{array}{l}\text { PRK_(n) } \\
\text { ROCK-II(h) }\end{array}$ & 98 & 0 & 70 & 70 & 71 & 0.835294118 & 30 & 0.025041736 & 0.498330551 \\
\hline ROCK-II(r) & 89 & 0 & 69 & 69 & 72 & 0.847058824 & 31 & 0.025876461 & 0.524207012 \\
\hline Rskl(h) & 90 & 0 & 68 & 68 & 73 & 0.858823529 & 32 & 0.026711185 & 0.550918197 \\
\hline Rsk1(r) & 92 & 0 & 65 & 65 & 74 & 0.870588235 & 35 & 0.029215359 & 0.580133556 \\
\hline $\begin{array}{l}\text { Rsksk2(h) } \\
\text { Rsk(h) }\end{array}$ & 68 & 0 & 65 & 65 & 75 & 0.882352941 & 35 & 0.029215359 & 0.609348915 \\
\hline Rsk3(h) & 96 & 0 & 65 & 65 & 76 & 0.894117647 & 35 & 0.029215359 & 0.638564274 \\
\hline SAPK2a(h) & 107 & 0 & 63 & 63 & 77 & 0.905882353 & 37 & 0.030884808 & 0.669449082 \\
\hline SAPK2b(h) & 104 & 0 & 62 & 62 & 78 & 0.917647059 & 38 & 0.031719533 & 0.701168614 \\
\hline SAPK3(h) & 105 & 0 & 58 & 58 & 79 & 0.929411765 & 42 & 0.035058431 & 0.736227045 \\
\hline SAPK4(h) & 98 & 0 & 57 & 57 & 80 & 0.941176471 & 43 & 0.035893155 & 0.7721202 \\
\hline SGK(h) & 65 & 0 & 52 & 52 & 81 & 0.952941176 & 48 & 0.040066778 & 0.812186978 \\
\hline Syk(h) & 72 & 0 & 52 & 52 & 82 & 0.964705882 & 48 & 0.040066778 & 0.852253756 \\
\hline TrkB(h) & 65 & 0 & 46 & 46 & 83 & 0.976470588 & 54 & 0.045075125 & 0.897328881 \\
\hline $\begin{array}{l}\text { IIrkB(h) } \\
\text { Yesh(h) }\end{array}$ & $\begin{array}{l}63 \\
63\end{array}$ & 0 & $\begin{array}{l}40 \\
42\end{array}$ & $\begin{array}{l}40 \\
42\end{array}$ & 84 & 0.988235294 & $\begin{array}{l}54 \\
58\end{array}$ & 0.048414023 & 0.945742905 \\
\hline ZAP-70(h) & 99 & 0 & 35 & 35 & 85 & 1 & 65 & 0.054257095 & 1 \\
\hline & & & & & & Total inhibition & 1198 & & Total area \\
\hline & Hit rate & 5 & & & & & & & Gini \\
\hline
\end{tabular}




\begin{tabular}{|c|c|c|c|c|c|c|c|c|}
\hline & $100 \mu \mathrm{M}$ ATP & & & & & & & \\
\hline Kinase & Rottlerin @ $10 \mu \mathrm{M}$ & Hit & sorted & normalized & position & cumulative sample fraction & inhibition\% & fraction of total inhibition \\
\hline $\mathrm{Abl}(\mathrm{m})$ & 90 & 0 & 151 & 100 & 1 & 0.011764706 & 0 & 0 \\
\hline AMPK(r) & 106 & 0 & 127 & 100 & 2 & 0.023529412 & 0 & 0 \\
\hline $\operatorname{Arg}(\mathrm{m})$ & 119 & 0 & 126 & 100 & 3 & 0.035294118 & 0 & 0 \\
\hline Aurora-A(h) & 54 & 0 & 123 & 100 & 4 & 0.047058824 & 0 & 0 \\
\hline Axl(h) & 75 & 0 & 122 & 100 & 5 & 0.058823529 & 0 & 0 \\
\hline $\mathrm{Blk}(\mathrm{m})$ & 35 & 1 & 119 & 100 & 6 & 0.070588235 & 0 & 0 \\
\hline Bmxx(h) & 106 & 0 & 116 & 100 & 7 & 0.082352941 & 0 & 0 \\
\hline СамKII(r) & 103 & 0 & 116 & 100 & 8 & 0.094117647 & 0 & 0 \\
\hline CaMKIV(h) & 99 & 0 & 112 & 100 & 9 & 0.105882353 & 0 & 0 \\
\hline CDK1/cyclnBB(h) & 89 & 0 & 111 & 100 & 10 & 0.117647059 & 0 & 0 \\
\hline $\mathrm{CDK} /$ /yclinA(h) & 88 & 0 & 110 & 100 & 11 & 0.129411765 & 0 & 0 \\
\hline $\mathrm{CDK} 2 /$ cyclinE(h) & 96 & 0 & 110 & 100 & 12 & 0.141176471 & 0 & 0 \\
\hline CDK3/cyclinE(h) & 93 & 0 & 109 & 100 & 13 & 0.152941176 & 0 & 0 \\
\hline CDK5/p35(h) & 99 & 0 & 106 & 100 & 14 & 0.164705882 & 0 & 0 \\
\hline CDK6/cyclinD3(h) & 99 & 0 & 106 & 100 & 15 & 0.176470588 & 0 & 0 \\
\hline CDK7/cyclinHMATI(h) & 116 & 0 & 106 & 100 & 16 & 0.188235294 & 0 & 0 \\
\hline СнК $1 \mathrm{~h})$ & 126 & 0 & 105 & 100 & 17 & 0.2 & 0 & 0 \\
\hline СHK2(h) & 77 & 0 & 105 & 100 & 18 & 0.211764706 & 0 & 0 \\
\hline $\mathrm{CK} 1(\mathrm{y})$ & 79 & 0 & 103 & 100 & 19 & 0.223529412 & 0 & 0 \\
\hline СК2(h) & 93 & 0 & 102 & 100 & 20 & 0.235294118 & 0 & 0 \\
\hline c-RAF(h) & 102 & 0 & 102 & 100 & 21 & 0.247058824 & 0 & 0 \\
\hline $\operatorname{CSK}(\mathrm{h})$ & 101 & 0 & 102 & 100 & 22 & 0.258823529 & 0 & 0 \\
\hline cSRC(h) & 100 & 0 & 102 & 100 & 23 & 0.270588235 & 0 & 0 \\
\hline Fes(h) & 89 & 0 & 101 & 100 & 24 & 0.282352941 & 0 & 0 \\
\hline FGFR3(h) & 97 & 0 & 101 & 100 & 25 & 0.294117647 & 0 & 0 \\
\hline Flts(h) & 87 & 0 & 101 & 100 & 26 & 0.305882353 & 0 & 0 \\
\hline Fynn(h) & 97 & 0 & 100 & 100 & 27 & 0.317647059 & 0 & 0 \\
\hline GSK3a(h) & 56 & 0 & 100 & 100 & 28 & 0.329411765 & 0 & 0 \\
\hline GSK3B(h) & 73 & 0 & 100 & 100 & 29 & 0.341176471 & 0 & 0 \\
\hline IGF-IR(h) & 127 & 0 & 99 & 99 & 30 & 0.352941176 & 1 & 0.001101322 \\
\hline IKKa(h) & 123 & 0 & 99 & 99 & 31 & 0.364705882 & 1 & 0.001101322 \\
\hline IKKB(h) & 98 & 0 & 99 & 99 & 32 & 0.376470588 & 1 & 0.001101322 \\
\hline $\mathbb{I R ( h )}$ & 86 & 0 & 98 & 98 & 33 & 0.388235294 & 2 & 0.002202643 \\
\hline JNKIal(h) & 76 & 0 & 97 & 97 & 34 & 0.4 & 3 & 0.003303965 \\
\hline JNK2202(h) & 95 & 0 & 97 & 97 & 35 & 0.411764706 & 3 & 0.003303965 \\
\hline JNK3(r) & 86 & 0 & 97 & 97 & 36 & 0.423529412 & 3 & 0.003303965 \\
\hline Lck(h) & 92 & 0 & 97 & 97 & 37 & 0.435294118 & 3 & 0.003303965 \\
\hline Lyn(h) & 75 & 0 & 96 & 96 & 38 & 0.447058824 & 4 & 0.004405286 \\
\hline Lyn(m) & 69 & 0 & 95 & 95 & 39 & 0.458823529 & 5 & 0.005506608 \\
\hline MAPK I (h) & 89 & 0 & 95 & 95 & 40 & 0.470588235 & 5 & 0.005506608 \\
\hline MAPK2(h) & 97 & 0 & 95 & 95 & 41 & 0.482352941 & 5 & 0.005506608 \\
\hline MAPK2(m) & 109 & 0 & 95 & 95 & 42 & 0.494117647 & 5 & 0.005506608 \\
\hline MAPKAP-K2(h) & 75 & 0 & 94 & 94 & 43 & 0.505882353 & 6 & 0.00660793 \\
\hline MEK1(h) & 102 & 0 & 94 & 94 & 44 & 0.517647059 & 6 & 0.00660793 \\
\hline MKK $4(\mathrm{~m})$ & 81 & 0 & 93 & 93 & 45 & 0.529411765 & 7 & 0.007709251 \\
\hline MKK6(h) & 97 & 0 & 93 & 93 & 46 & 0.541176471 & 7 & 0.007709251 \\
\hline MKK $7 \mathrm{B(h)}$ & 86 & 0 & 93 & 93 & 47 & 0.552941176 & 7 & 0.007709251 \\
\hline MSK1(h) & 95 & 0 & 92 & 92 & 48 & 0.564705882 & 8 & 0.008810573 \\
\hline p7056K(h) & 100 & 0 & 92 & 92 & 49 & 0.576470588 & 8 & 0.008810573 \\
\hline PAK2(h) & 102 & 0 & 90 & 90 & 50 & 0.588235294 & 10 & 0.011013216 \\
\hline PDGFRa(h) & 112 & 0 & 90 & 90 & 51 & 0.6 & 10 & 0.011013216 \\
\hline PDGFRB(h) & 110 & 0 & 90 & 90 & 52 & 0.611764706 & 10 & 0.011013216 \\
\hline PDK1(h) & 73 & 0 & 89 & 89 & 53 & 0.623529412 & 11 & 0.012114537 \\
\hline PKA(b) & 85 & 0 & 89 & 89 & 54 & 0.635294118 & 11 & 0.012114537 \\
\hline PKA(h) & 72 & 0 & 89 & 89 & 55 & 0.647058824 & 11 & 0.012114537 \\
\hline PKBa(h) & 101 & 0 & 89 & 89 & 56 & 0.658823529 & 11 & 0.012114537 \\
\hline PKB(h) & 78 & 0 & 88 & 88 & 57 & 0.670588235 & 12 & 0.013215859 \\
\hline $\mathrm{PKB}_{\gamma}(\mathrm{h})$ & 90 & 0 & 87 & 87 & 58 & 0.682352941 & 13 & 0.014317181 \\
\hline 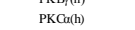 & 94 & 0 & 86 & 86 & 59 & 0.694117647 & 14 & 0.015418502 \\
\hline $\begin{array}{l}\text { PKCBII(h) } \\
\text { Р }\end{array}$ & 94 & 0 & 86 & 86 & 60 & 0.705882353 & 14 & 0.015418502 \\
\hline PKCy(h) & 92 & 0 & 86 & 86 & 61 & 0.717647059 & 14 & 0.015418502 \\
\hline PKБ(h) & 106 & 0 & 86 & 86 & 62 & 0.729411765 & 14 & 0.015418502 \\
\hline PKG:(h) & 82 & 0 & 85 & 85 & 63 & 0.741176471 & 15 & 0.016519824 \\
\hline PKG(h) & 111 & 0 & 85 & 85 & 64 & 0.752941176 & 15 & 0.016519824 \\
\hline PKC (h) & 70 & 0 & 82 & 82 & 65 & 0.764705882 & 18 & 0.019823789 \\
\hline PKC $\mu(\mathrm{h})$ & 95 & 0 & 81 & 81 & 66 & 0.776470588 & 19 & 0.02092511 \\
\hline PKCO(h) & 151 & 0 & 80 & 80 & 67 & 0.788235294 & 20 & 0.022026432 \\
\hline PKD2(h) & 105 & 0 & 79 & 79 & 68 & 0.8 & 21 & 0.023127753 \\
\hline PRAK(h) & 44 & 1 & 78 & 78 & 69 & 0.811764706 & 22 & 0.024229075 \\
\hline PRK2(h) & $\begin{array}{l}101 \\
101\end{array}$ & 0 & 77 & 77 & 70 & 0.823529412 & 23 & 0.025330396 \\
\hline ROCK-II(h) & 95 & 0 & 76 & 76 & 71 & 0.835294118 & 24 & 0.026431718 \\
\hline ROCK-II(r) & 93 & 0 & 75 & 75 & 72 & 0.847058824 & 25 & 0.02753304 \\
\hline Rskl(h) & 69 & 0 & 75 & 75 & 73 & 0.858823529 & 25 & 0.02753304 \\
\hline Rskl(r) & 116 & 0 & 75 & 75 & 74 & 0.870588235 & 25 & 0.02753304 \\
\hline Rsk2(h) & 80 & 0 & 74 & 74 & 75 & 0.882352941 & 26 & 0.028634361 \\
\hline Rsk3(h) & 89 & 0 & 73 & 73 & 76 & 0.894117647 & 27 & 0.029735683 \\
\hline SAPK2a(h) & 105 & 0 & 73 & 73 & 77 & 0.905882353 & 27 & 0.029735683 \\
\hline SAPK2b(h) & 122 & 0 & 72 & 72 & 78 & 0.917647059 & 28 & 0.030837004 \\
\hline $\mathrm{SAPK} 3(\mathrm{~h})$ & 110 & 0 & 70 & 70 & 79 & 0.929411765 & 30 & 0.033039648 \\
\hline SAPK 4(h) & 102 & 0 & 69 & 69 & 80 & 0.941176471 & 31 & 0.034140969 \\
\hline SGK(h) & 90 & 0 & 69 & 69 & 81 & 0.952941176 & 31 & 0.034140969 \\
\hline Syk(h) & 85 & 0 & 56 & 56 & 82 & 0.964705882 & 44 & 0.04845815 \\
\hline TrkB(h) & 86 & 0 & 54 & 54 & 83 & 0.976470588 & 46 & 0.050660793 \\
\hline Yes(h) & 74 & 0 & 44 & 44 & 84 & 0.988235294 & 56 & 0.061674009 \\
\hline ZAP-70(h) & 100 & 0 & 35 & 35 & 85 & 1 & 65 & 0.071585903 \\
\hline & & & & & & Total inhibition & 908 & \\
\hline & Hit rate & & & & & & & \\
\hline & Threshold & 5 & & & & & & \\
\hline
\end{tabular}




\begin{tabular}{|c|c|c|c|c|c|c|c|c|}
\hline & $10 \mu \mathrm{M}$ ATP & & & & & & & \\
\hline Kinase & SB202190@ @ $10 \mu \mathrm{M}$ & Hit & sorted & normalized & position & cumulative sample fraction & inhibition\% & fraction of total inhibition \\
\hline $\mathrm{Ab}(\mathrm{m})$ & 60 & 0 & 130 & 100 & 1 & 0.011764706 & 0 & 0 \\
\hline AMPK(r) & 117 & 0 & 117 & 100 & 2 & 0.023529412 & 0 & 0 \\
\hline $\operatorname{Arg}(\mathrm{m})$ & 48 & 1 & 115 & 100 & 3 & 0.035294118 & 0 & 0 \\
\hline Aurora-A(h) & 93 & 0 & 108 & 100 & 4 & 0.047058824 & 0 & 0 \\
\hline Ax(h) & 108 & 0 & 107 & 100 & 5 & 0.058823529 & 0 & 0 \\
\hline $\operatorname{Blk}(\mathrm{m})$ & 42 & 1 & 106 & 100 & 6 & 0.070588235 & 0 & 0 \\
\hline Bnx(h) & 45 & 1 & 105 & 100 & 7 & 0.082352941 & 0 & 0 \\
\hline CaMKII(r) & 102 & 0 & 104 & 100 & 8 & 0.094117647 & 0 & 0 \\
\hline CaMKIV(h) & 104 & 0 & 104 & 100 & 9 & 0.105882353 & 0 & 0 \\
\hline CDK1/cyclinB(h) & 96 & 0 & 104 & 100 & 10 & 0.117647059 & 0 & 0 \\
\hline $\operatorname{CDK} 2 /$ cyclinA(h) & 101 & 0 & 102 & 100 & 11 & 0.129411765 & 0 & 0 \\
\hline $\mathrm{CDK} 2 /$ cyclinE(h) & 89 & 0 & 102 & 100 & 12 & 0.141176471 & 0 & 0 \\
\hline CDK3/yclinE(h) & 100 & 0 & 102 & 100 & 13 & 0.152941176 & 0 & 0 \\
\hline CDK5/p35(h) & 106 & 0 & 102 & 100 & 14 & 0.164705882 & 0 & 0 \\
\hline CDK6/cyclinD3(h) & 95 & 0 & 101 & 100 & 15 & 0.176470588 & 0 & 0 \\
\hline CDK7/cyclinH/MATI(h) & 99 & 0 & 101 & 100 & 16 & 0.188235294 & 0 & 0 \\
\hline CHK1(h) & 104 & 0 & 100 & 100 & 17 & 0.2 & 0 & 0 \\
\hline СHK2(h) & 93 & 0 & 100 & 100 & 18 & 0.211764706 & 0 & 0 \\
\hline $\mathrm{CKI}(\mathrm{y})$ & 1 & 1 & 99 & 99 & 19 & 0.223529412 & 1 & 0.00034153 \\
\hline $\mathrm{CK} 2(\mathrm{~h})$ & 107 & 0 & 99 & 99 & 20 & 0.235294118 & 1 & 0.00034153 \\
\hline$c-\operatorname{RAF}(\mathrm{h})$ & 4 & 1 & 96 & 96 & 21 & 0.247058824 & 4 & 0.00136612 \\
\hline $\operatorname{CSK}(\mathrm{h})$ & 77 & 0 & 96 & 96 & 22 & 0.258823529 & 4 & 0.00136612 \\
\hline cSRCh) & 22 & 1 & 96 & 96 & 23 & 0.270588235 & 4 & 0.00136612 \\
\hline Fes(h) & 102 & 0 & 95 & 95 & 24 & 0.282352941 & 5 & 0.00170765 \\
\hline FGFR3(h) & 93 & 0 & 95 & 95 & 25 & 0.294117647 & 5 & 0.00170765 \\
\hline Flls(h) & 89 & 0 & 93 & 93 & 26 & 0.305882353 & 7 & 0.00239071 \\
\hline Fynn(h) & 26 & 1 & 93 & 93 & 27 & 0.317647059 & 7 & 0.00239071 \\
\hline GSK3a $a(\mathrm{~h})$ & 31 & 1 & 93 & 93 & 28 & 0.329411765 & 7 & 0.00239071 \\
\hline GSK3B(h) & 9 & 1 & 93 & 93 & 29 & 0.341176471 & 7 & 0.00239071 \\
\hline IGF-1R(h) & 99 & 0 & 92 & 92 & 30 & 0.352941176 & 8 & 0.00273224 \\
\hline IKKa(h) & 52 & 0 & 91 & 91 & 31 & 0.364705882 & 9 & 0.00307377 \\
\hline IKKB(h) & 102 & 0 & 90 & 90 & 32 & 0.376470588 & 10 & 0.003415301 \\
\hline $\mathrm{IR}(\mathrm{h})$ & 101 & 0 & 89 & 89 & 33 & 0.388235294 & 11 & 0.003756831 \\
\hline JNKIal(h) & 42 & 1 & 89 & 89 & 34 & 0.4 & 11 & 0.003756831 \\
\hline JNK2a2(h) & 8 & 1 & 89 & 89 & 35 & 0.411764706 & 11 & 0.003756831 \\
\hline JNK3(r) & 1 & 1 & 89 & 89 & 36 & 0.423529412 & 11 & 0.003756831 \\
\hline Lck(h) & 22 & 1 & 87 & 87 & 37 & 0.435294118 & 13 & 0.004439891 \\
\hline Lyn(h) & 15 & 1 & 87 & 87 & 38 & 0.447058824 & 13 & 0.004439891 \\
\hline Lyn(m) & 12 & 1 & 86 & 86 & 39 & 0.458823529 & 14 & 0.004781421 \\
\hline MAPK Ih) & 89 & 0 & 86 & 86 & 40 & 0.470588235 & 14 & 0.004781421 \\
\hline MAPK2(h) & 102 & 0 & 85 & 85 & 41 & 0.482352941 & 15 & 0.005122951 \\
\hline MAPK2(m) & 86 & 0 & 81 & 81 & 42 & 0.494117647 & 19 & 0.006489071 \\
\hline MAPKAP-K2(h) & 93 & 0 & 78 & 78 & 43 & 0.505882353 & 22 & 0.007513661 \\
\hline MEK 1(h) & 91 & 0 & 77 & 77 & 44 & 0.517647059 & 23 & 0.007855191 \\
\hline MKK4(m) & 2 & 1 & 77 & 77 & 45 & 0.529411765 & 23 & 0.007855191 \\
\hline MKK6(h) & 3 & 1 & 75 & 75 & 46 & 0.541176471 & 25 & 0.008538251 \\
\hline MKK 7 (h) & 73 & 0 & 73 & 73 & 47 & 0.552941176 & 27 & 0.009221311 \\
\hline MSK I(h) & 70 & 0 & 72 & 72 & 48 & 0.564705882 & 28 & 0.009562842 \\
\hline $\mathrm{p} 70 \mathrm{~S} 6 \mathrm{~K}(\mathrm{~h})$ & 34 & 1 & 70 & 70 & 49 & 0.576470588 & 30 & 0.010245902 \\
\hline PAK2(h) & 96 & 0 & 68 & 68 & 50 & 0.588235294 & 32 & 0.010928962 \\
\hline $\begin{array}{l}\text { PDARRa(h) } \\
\text { PDGFR(h) }\end{array}$ & 87 & 0 & 67 & 67 & 51 & 0.6 & 33 & 0.011270492 \\
\hline PDGFRB(h) & 96 & 0 & 66 & 66 & 52 & 0.611764706 & 34 & 0.011612022 \\
\hline PDK I(h) & $\begin{array}{l}96 \\
130\end{array}$ & 0 & 64 & 64 & 53 & 0.623529412 & 36 & 0.012295082 \\
\hline PKA(b) & 21 & 1 & 63 & 63 & 54 & 0.635294118 & 37 & 0.012636612 \\
\hline PKA(h) & 14 & 1 & 60 & 60 & 55 & 0.647058824 & 40 & 0.013661202 \\
\hline PKBa(h) & 63 & 0 & 52 & 52 & 56 & 0.658823529 & 48 & 0.016393443 \\
\hline PKBB(h) & 95 & 0 & 48 & 48 & 57 & 0.670588235 & 52 & 0.017759563 \\
\hline $\mathrm{PKB}_{\gamma}(\mathrm{h})$ & 75 & 0 & 45 & 45 & 58 & 0.682352941 & 55 & 0.018784153 \\
\hline PKGa(h) & 66 & 0 & 43 & 43 & 59 & 0.694117647 & 57 & 0.019467213 \\
\hline РКСВI(h) & 100 & 0 & 42 & 42 & 60 & 0.705882353 & 58 & 0.019808743 \\
\hline PKCy(h) & 85 & 0 & 42 & 42 & 61 & 0.717647059 & 58 & 0.019808743 \\
\hline PKC(h) & 78 & 0 & 42 & 42 & 62 & 0.729411765 & 58 & 0.019808743 \\
\hline PKG:(h) & 77 & 0 & 34 & 34 & 63 & 0.741176471 & 66 & 0.022540984 \\
\hline PKCh(h) & 64 & 0 & 31 & 31 & 64 & 0.752941176 & 69 & 0.023565574 \\
\hline PKC (h) & 89 & 0 & 30 & 30 & 65 & 0.764705882 & 70 & 0.023907104 \\
\hline PKC $\mu(\mathrm{h})$ & 27 & 1 & 30 & 30 & 66 & 0.776470588 & 70 & 0.023907104 \\
\hline PKC (h) & 86 & 0 & 27 & 27 & 67 & 0.788235294 & 73 & 0.024931694 \\
\hline PKD2(h) & 21 & 1 & 26 & 26 & 68 & 0.8 & 74 & 0.025273224 \\
\hline PRAK(h) & 104 & 0 & 25 & 25 & 69 & 0.811764706 & 75 & 0.025614754 \\
\hline PRK2(h) & 43 & 1 & 22 & 22 & 70 & 0.823529412 & 78 & 0.026639344 \\
\hline ROCK-II(h) & 30 & 1 & 22 & 22 & 71 & 0.835294118 & 78 & 0.026639344 \\
\hline ROCK-II(r) & 25 & 1 & 21 & 21 & 72 & 0.847058824 & 79 & 0.026980874 \\
\hline Rsk1(h) & 72 & 0 & 21 & 21 & 73 & 0.858823529 & 79 & 0.026980874 \\
\hline Rskl(r) & 42 & 1 & 15 & 15 & 74 & 0.870588235 & 85 & 0.029030055 \\
\hline Rsk2(h) & 81 & 0 & 14 & 14 & 75 & 0.882352941 & 86 & 0.029371585 \\
\hline Rsk3(h) & 67 & 0 & 12 & 12 & 76 & 0.894117647 & 88 & 0.030054645 \\
\hline SAPK2a(h) & 1 & 1 & 9 & 9 & 77 & 0.905882353 & 91 & 0.031079235 \\
\hline SAPK2b(h) & 0 & 1 & 8 & 8 & 78 & 0.917647059 & 92 & 0.031420765 \\
\hline SAPK3(h) & 68 & 0 & 4 & 4 & 79 & 0.929411765 & 96 & 0.032786885 \\
\hline SAPK4(h) & 90 & 0 & 3 & 3 & 80 & 0.941176471 & 97 & 0.033128415 \\
\hline SGK(h) & 87 & 0 & 2 & 2 & 81 & 0.952941176 & 98 & 0.033469945 \\
\hline Syk(h) & 115 & 0 & 1 & 1 & 82 & 0.964705882 & 99 & 0.033811475 \\
\hline TTkB(h) & 105 & 0 & 1 & 1 & 83 & 0.976470588 & 99 & 0.033811475 \\
\hline Yes(h) & 30 & 1 & 1 & 1 & 84 & 0.988235294 & 99 & 0.033811475 \\
\hline $\mathrm{ZAP}-70(\mathrm{~h})$ & 92 & 0 & 0 & 0 & 85 & 1 & 100 & 0.034153005 \\
\hline & & & & & & Total inhibition & 2928 & \\
\hline & Hit rate & 2 & & & & & & \\
\hline & Threshold & 5 & & & & & & \\
\hline
\end{tabular}




\begin{tabular}{|c|c|c|c|c|c|c|c|c|}
\hline & $100 \mu \mathrm{M}$ ATP & & & & & & & \\
\hline Kinase & SB202190@10 $\mu \mathrm{M}$ & Hit & sorted & normalized & position & cumulative sample fraction & inhibition\% & fraction of total inhibition \\
\hline $\mathrm{Abl}(\mathrm{m})$ & 97 & 0 & 130 & 100 & 1 & 0.011764706 & 0 & 0 \\
\hline AMPK(r) & 105 & 0 & 122 & 100 & 2 & 0.023529412 & 0 & 0 \\
\hline $\operatorname{Arg}(m)$ & 70 & 0 & 120 & 100 & 3 & 0.035294118 & 0 & 0 \\
\hline Aurora-A(h) & 105 & 0 & 119 & 100 & 4 & 0.047058824 & 0 & 0 \\
\hline Axl(h) & 102 & 0 & 118 & 100 & 5 & 0.058823529 & 0 & 0 \\
\hline $\mathrm{Blk}(\mathrm{m})$ & 67 & 0 & 115 & 100 & 6 & 0.070588235 & 0 & 0 \\
\hline $\operatorname{Bmx}(\mathrm{h})$ & 58 & 0 & 112 & 100 & 7 & 0.082352941 & 0 & 0 \\
\hline СамKII(r) & 111 & 0 & 111 & 100 & 8 & 0.094117647 & 0 & 0 \\
\hline CaMKIV(h) & 112 & 0 & 111 & 100 & 9 & 0.105882353 & 0 & 0 \\
\hline CDK1/cyclnBB(h) & 101 & 0 & 111 & 100 & 10 & 0.117647059 & 0 & 0 \\
\hline $\mathrm{CDK} /$ /yclinA(h) & 96 & 0 & 110 & 100 & 11 & 0.129411765 & 0 & 0 \\
\hline $\mathrm{CDK} 2 /$ cyclinE(h) & 115 & 0 & 108 & 100 & 12 & 0.141176471 & 0 & 0 \\
\hline CDK3/cyclinE(h) & 120 & 0 & 107 & 100 & 13 & 0.152941176 & 0 & 0 \\
\hline CDK5/p35(h) & 118 & 0 & 106 & 100 & 14 & 0.164705882 & 0 & 0 \\
\hline CDK6/cyclinD3(h) & 100 & 0 & 105 & 100 & 15 & 0.176470588 & 0 & 0 \\
\hline CDK7//yclinH/MATI(h) & 100 & 0 & 105 & 100 & 16 & 0.188235294 & 0 & 0 \\
\hline СHK1(h) & 108 & 0 & 105 & 100 & 17 & 0.2 & 0 & 0 \\
\hline СHK2(h) & 102 & 0 & 105 & 100 & 18 & 0.211764706 & 0 & 0 \\
\hline $\mathrm{CK} 1(\mathrm{y})$ & 2 & 1 & 105 & 100 & 19 & 0.223529412 & 0 & 0 \\
\hline СК2(h) & 110 & 0 & 104 & 100 & 20 & 0.235294118 & 0 & 0 \\
\hline c-RAF(h) & 6 & 1 & 103 & 100 & 21 & 0.247058824 & 0 & 0 \\
\hline $\operatorname{CSK}(\mathrm{h})$ & 107 & 0 & 103 & 100 & 22 & 0.258823529 & 0 & 0 \\
\hline cSRC(h) & 43 & 1 & 103 & 100 & 23 & 0.270588235 & 0 & 0 \\
\hline Fes(h) & 100 & 0 & 102 & 100 & 24 & 0.282352941 & 0 & 0 \\
\hline FGFR3(h) & 130 & 0 & 102 & 100 & 25 & 0.294117647 & 0 & 0 \\
\hline $\mathrm{FH}$ H(h) & 94 & 0 & 102 & 100 & 26 & 0.305882353 & 0 & 0 \\
\hline Fynn(h) & 50 & 1 & 102 & 100 & 27 & 0.317647059 & 0 & 0 \\
\hline GSK3a(h) & 80 & 0 & 102 & 100 & 28 & 0.329411765 & 0 & 0 \\
\hline GSK3B(h) & 34 & 1 & 101 & 100 & 29 & 0.341176471 & 0 & 0 \\
\hline IGF-IR(h) & 122 & 0 & 100 & 100 & 30 & 0.352941176 & 0 & 0 \\
\hline $\mathrm{IKKa}(\mathrm{h})$ & 119 & 0 & 100 & 100 & 31 & 0.364705882 & 0 & 0 \\
\hline IKKB(h) & 105 & 0 & 100 & 100 & 32 & 0.376470588 & 0 & 0 \\
\hline $\mathbb{I R ( h )}$ & 111 & 0 & 100 & 100 & 33 & 0.388235294 & 0 & 0 \\
\hline JNKIal(h) & 99 & 0 & 100 & 100 & 34 & 0.4 & 0 & 0 \\
\hline JNK2202(h) & 63 & 0 & 99 & 99 & 35 & 0.411764706 & 1 & 0.000591017 \\
\hline JNK3(r) & 6 & 1 & 98 & 98 & 36 & 0.423529412 & 2 & 0.001182033 \\
\hline Lck(h) & 28 & 1 & 98 & 98 & 37 & 0.435294118 & 2 & 0.001182033 \\
\hline Lyn(h) & 30 & 1 & 97 & 97 & 38 & 0.447058824 & 3 & 0.00177305 \\
\hline Lyy(m) & 38 & 1 & 97 & 97 & 39 & 0.458823529 & 3 & 0.00177305 \\
\hline MAPK1(h) & 100 & 0 & 96 & 96 & 40 & 0.470588235 & 4 & 0.002364066 \\
\hline MAPK2(h) & 102 & 0 & 96 & 96 & 41 & 0.482352941 & 4 & 0.002364066 \\
\hline MAPK2(m) & 102 & 0 & 95 & 95 & 42 & 0.494117647 & 5 & 0.002955083 \\
\hline MAPKAP-K2(h) & 111 & 0 & 95 & 95 & 43 & 0.505882353 & 5 & 0.002955083 \\
\hline MEK1(h) & 92 & 0 & 95 & 95 & 44 & 0.517647059 & 5 & 0.002955083 \\
\hline MKK $4(\mathrm{~m})$ & 59 & 0 & 94 & 94 & 45 & 0.529411765 & 6 & 0.003546099 \\
\hline MKK6(h) & 2 & 1 & 94 & 94 & 46 & 0.541176471 & 6 & 0.003546099 \\
\hline MKK7B(h) & 79 & 0 & 94 & 94 & 47 & 0.552941176 & 6 & 0.003546099 \\
\hline MSKI(h) & 84 & 0 & 92 & 92 & 48 & 0.564705882 & 8 & 0.004728132 \\
\hline p7056K(h) & 81 & 0 & 92 & 92 & 49 & 0.576470588 & 8 & 0.004728132 \\
\hline PAK2(h) & 100 & 0 & 92 & 92 & 50 & 0.588235294 & 8 & 0.004728132 \\
\hline PDGFRa(h) & 106 & 0 & 91 & 91 & 51 & 0.6 & 9 & 0.005319149 \\
\hline PDGFRB(h) & 95 & 0 & 88 & 88 & 52 & 0.611764706 & 12 & 0.007092199 \\
\hline PDK1(h) & 98 & 0 & 88 & 88 & 53 & 0.623529412 & 12 & 0.007092199 \\
\hline PKA(b) & 63 & 0 & 85 & 85 & 54 & 0.635294118 & 15 & 0.008865248 \\
\hline PKA(h) & 50 & 1 & 84 & 84 & 55 & 0.647058824 & 16 & 0.009456265 \\
\hline PKBa(h) & 69 & 0 & 83 & 83 & 56 & 0.658823529 & 17 & 0.010047281 \\
\hline PKB(h) & 92 & 0 & 81 & 81 & 57 & 0.670588235 & 19 & 0.011229314 \\
\hline $\mathrm{PKB}_{\gamma}(\mathrm{h})$ & 103 & 0 & 80 & 80 & 58 & 0.682352941 & 20 & 0.011820331 \\
\hline 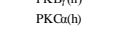 & 83 & 0 & 80 & 80 & 59 & 0.694117647 & 20 & 0.011820331 \\
\hline РКCBII(h) & 104 & 0 & 79 & 79 & 60 & 0.705882353 & 21 & 0.012411348 \\
\hline PKCy(h) & 95 & 0 & 74 & 74 & 61 & 0.717647059 & 26 & 0.01536643 \\
\hline PKБ(h) & 94 & 0 & 70 & 70 & 62 & 0.729411765 & 30 & 0.017730496 \\
\hline PKG:(h) & 88 & 0 & 69 & 69 & 63 & 0.741176471 & 31 & 0.018321513 \\
\hline PKG(h) & 88 & 0 & 68 & 68 & 64 & 0.752941176 & 32 & 0.01891253 \\
\hline PKC (h) & 92 & 0 & 68 & 68 & 65 & 0.764705882 & 32 & 0.01891253 \\
\hline PKC $\mu(\mathrm{h})$ & 68 & 0 & 67 & 67 & 66 & 0.776470588 & 33 & 0.019503546 \\
\hline PKCO(h) & 105 & 0 & 64 & 64 & 67 & 0.788235294 & 36 & 0.021276596 \\
\hline PKD2(h) & 33 & 1 & 63 & 63 & 68 & 0.8 & 37 & 0.021867612 \\
\hline PRAK(h) & 103 & 0 & 63 & 63 & 69 & 0.811764706 & 37 & 0.021867612 \\
\hline PRK2(h) & 85 & 0 & 59 & 59 & 70 & 0.823529412 & 41 & 0.024231678 \\
\hline ROCK-II(h) & 68 & 0 & 58 & 58 & 71 & 0.835294118 & 42 & 0.024822695 \\
\hline ROCK-II(r) & 74 & 0 & 50 & 50 & 72 & 0.847058824 & 50 & 0.029550827 \\
\hline Rskl(h) & 94 & 0 & 50 & 50 & 73 & 0.858823529 & 50 & 0.029550827 \\
\hline Rskl(r) & 95 & 0 & 43 & 43 & 74 & 0.870588235 & 57 & 0.033687943 \\
\hline Rsk2(h) & 102 & 0 & 38 & 38 & 75 & 0.882352941 & 62 & 0.036643026 \\
\hline Rsk3(h) & 96 & 0 & 34 & 34 & 76 & 0.894117647 & 66 & 0.039007092 \\
\hline SAPK2a(h) & -1 & 1 & 33 & 33 & 77 & 0.905882353 & 67 & 0.039598109 \\
\hline SAPK2b(h) & -1 & 1 & 30 & 30 & 78 & 0.917647059 & 70 & 0.041371158 \\
\hline $\mathrm{SAPK} 3(\mathrm{~h})$ & 97 & 0 & 28 & 28 & 79 & 0.929411765 & 72 & 0.042553191 \\
\hline SAPK 4(h) & 105 & 0 & 6 & 6 & 80 & 0.941176471 & 94 & 0.055555556 \\
\hline SGK(h) & 103 & 0 & 6 & 6 & 81 & 0.952941176 & 94 & 0.055555556 \\
\hline Syk(h) & 80 & 0 & 2 & 2 & 82 & 0.964705882 & 98 & 0.057919622 \\
\hline TrkB(h) & 98 & 0 & 2 & 2 & 83 & 0.976470588 & 98 & 0.057919622 \\
\hline Yes(h) & 64 & 0 & -1 & 0 & 84 & 0.988235294 & 100 & 0.059101655 \\
\hline ZAP-70(h) & 91 & 0 & -1 & 0 & 85 & 1 & 100 & 0.059101655 \\
\hline & & & & & & Total inhibition & 1692 & \\
\hline & Hit rate & & & & & & & \\
\hline & Threshold & & & & & & & \\
\hline
\end{tabular}




\begin{tabular}{|c|c|c|c|c|c|c|c|c|c|}
\hline & $10 \mu \mathrm{M}$ ATP & & & & & & & & \\
\hline Kinase & SB203580@ $10 \mu \mathrm{M}$ & Hit & sorted & normalized & position & cumulative sample fraction & inhibition\% & fraction of total inhibition & cumulative inhibition \\
\hline $\mathrm{Abl}(\mathrm{m})$ & 71 & 0 & 115 & 100 & 1 & 0.011764706 & 0 & 0 & 0 \\
\hline AMPK(r) & 101 & 0 & 108 & 100 & 2 & 0.023529412 & 0 & 0 & 0 \\
\hline $\operatorname{Arg}(\mathrm{m})$ & 55 & 0 & 105 & 100 & 3 & 0.035294118 & 0 & 0 & 0 \\
\hline Aurora-A(h) & 93 & 0 & 105 & 100 & 4 & 0.047058824 & 0 & 0 & 0 \\
\hline Axl(h) & 100 & 0 & 104 & 100 & 5 & 0.058823529 & 0 & 0 & 0 \\
\hline $\operatorname{Blk}(\mathrm{m})$ & 66 & 0 & 104 & 100 & 6 & 0.070588235 & 0 & 0 & 0 \\
\hline $\operatorname{Bmx}(\mathrm{h})$ & 51 & 0 & 104 & 100 & 7 & 0.082352941 & 0 & 0 & 0 \\
\hline CaMKII(r) & 98 & 0 & 103 & 100 & 8 & 0.094117647 & 0 & 0 & 0 \\
\hline CaMKIV(h) & 99 & 0 & 103 & 100 & 9 & 0.105882353 & 0 & 0 & 0 \\
\hline CDK1/cyclnBB(h) & 96 & 0 & 103 & 100 & 10 & 0.117647059 & 0 & 0 & 0 \\
\hline CDK2/cyclinA(h) & 103 & 0 & 102 & 100 & 11 & 0.129411765 & 0 & 0 & 0 \\
\hline $\mathrm{CDK} 2 /$ cyclnE(h) & 92 & 0 & 101 & 100 & 12 & 0.141176471 & 0 & 0 & 0 \\
\hline CDK3/cyclinE(h) & 93 & 0 & 101 & 100 & 13 & 0.152941176 & 0 & 0 & 0 \\
\hline CDK5/p35(h) & 105 & 0 & 100 & 100 & 14 & 0.164705882 & 0 & 0 & 0 \\
\hline CDKG/cyclinD3(h) & 99 & 0 & 100 & 100 & 15 & 0.176470588 & 0 & 0 & 0 \\
\hline CDK $7 /$ yclinHMATI(h) & 104 & 0 & 100 & 100 & 16 & 0.188235294 & 0 & 0 & 0 \\
\hline CHKI(h) & 96 & 0 & 99 & 99 & 17 & 0.2 & 1 & 0.000462963 & 0.000462963 \\
\hline СHK2(h) & 94 & 0 & 99 & 99 & 18 & 0.211764706 & 1 & 0.000462963 & 0.000925926 \\
\hline $\mathrm{CK} 1(\mathrm{y})$ & 0 & 1 & 99 & 99 & 19 & 0.223529412 & 1 & 0.000462963 & 0.001388889 \\
\hline СК2(h) & 105 & 0 & 99 & 99 & 20 & 0.235294118 & 1 & 0.000462963 & 0.001851852 \\
\hline c-RAF(h) & 5 & 1 & 98 & 98 & 21 & 0.247058824 & 2 & 0.000925926 & 0.002777778 \\
\hline CSK(h) & 93 & 0 & 98 & 98 & 22 & 0.258823529 & 2 & 0.000925926 & 0.003703704 \\
\hline $\operatorname{csRC(h)}$ & 34 & 1 & 97 & 97 & 23 & 0.270588235 & 3 & 0.001388889 & 0.005092593 \\
\hline Fess(h) & 103 & 0 & 97 & 97 & 24 & 0.282352941 & 3 & 0.001388889 & 0.006481481 \\
\hline FGFR3(h) & 101 & 0 & 96 & 96 & 25 & 0.294117647 & 4 & 0.001851852 & 0.008333333 \\
\hline Fill(h) & 66 & 0 & 96 & 96 & 26 & 0.305882353 & 4 & 0.001851852 & 0.010185185 \\
\hline Fyn(h) & 17 & 1 & 96 & 96 & 27 & 0.317647059 & 4 & 0.001851852 & 0.012037037 \\
\hline GSK3a(h) & 46 & 1 & 96 & 96 & 28 & 0.329411765 & 4 & 0.001851852 & 0.013888889 \\
\hline GSK3B(h) & 14 & 1 & 94 & 94 & $\begin{array}{l}20 \\
29\end{array}$ & 0.341176471 & $\begin{array}{l}4 \\
6\end{array}$ & 0.002777778 & 0.016666667 \\
\hline IGF-IR(h) & 96 & 0 & 94 & 94 & 30 & 0.352941176 & 6 & 0.002777778 & 0.019444444 \\
\hline $\mathrm{IKKa}(\mathrm{h})$ & 100 & 0 & 93 & 93 & 31 & 0.364705882 & 7 & 0.003240741 & 0.022685185 \\
\hline IKKB(h) & 94 & 0 & 93 & 93 & 32 & 0.376470588 & 7 & 0.003240741 & 0.025925926 \\
\hline $\mathrm{IR}(\mathrm{h})$ & 102 & 0 & 93 & 93 & 33 & 0.388235294 & 7 & 0.003240741 & 0.029166667 \\
\hline JNKIal(h) & 64 & 0 & 93 & 93 & 34 & 0.4 & 7 & 0.003240741 & 0.032407407 \\
\hline JNK202(h) & 24 & 1 & 93 & 93 & $\begin{array}{l}54 \\
35\end{array}$ & 0.411764706 & 7 & 0.003240741 & 0.035648148 \\
\hline JNK3(r) & 4 & 1 & 92 & 92 & 36 & 0.423529412 & 8 & 0.003703704 & 0.039351852 \\
\hline Lck(h) & 29 & 1 & 92 & 92 & 37 & 0.435294118 & 8 & 0.003703704 & 0.043055556 \\
\hline Lyn(h) & 23 & 1 & 92 & 92 & $\begin{array}{l}31 \\
38\end{array}$ & 0.447058824 & 8 & 0.003703704 & 0.046759259 \\
\hline Lyy(m) & 29 & 1 & 92 & 92 & 39 & 0.458823529 & 8 & 0.003703704 & 0.050462963 \\
\hline MAPKI(h) & $\begin{array}{l}29 \\
93\end{array}$ & 0 & 90 & 90 & 40 & 0.470588235 & 10 & 0.00462963 & 0.055092593 \\
\hline MAPK2(h) & 97 & 0 & 90 & 90 & 41 & 0.482352941 & 10 & 0.00462963 & 0.059722222 \\
\hline $\begin{array}{l}\text { MAPKL(2) } \\
\text { MAPK2(m) }\end{array}$ & 90 & 0 & 90 & 90 & $\begin{array}{l}41 \\
42\end{array}$ & 0.494117647 & 10 & 0.00462963 & 0.064351852 \\
\hline MAPKAP-K2(h) & 104 & 0 & 88 & 88 & 43 & 0.505882353 & 12 & 0.005555556 & 0.069907407 \\
\hline MEK1(h) & 98 & 0 & 87 & 87 & 44 & 0.517647059 & 13 & 0.006018519 & 0.075925926 \\
\hline MKK4(m) & 0 & 1 & 87 & 87 & 45 & 0.529411765 & 13 & 0.006018519 & 0.081944444 \\
\hline MKK6(h) & 5 & 1 & 87 & 87 & 46 & 0.541176471 & 13 & 0.006018519 & 0.087962963 \\
\hline MKK & 87 & 0 & 86 & 86 & 47 & 0.552941176 & 14 & 0.006481481 & 0.094444444 \\
\hline MSKI(h) & 87 & 0 & 86 & 86 & 48 & 0.564705882 & 14 & 0.006481481 & 0.100925926 \\
\hline $\mathrm{p} 7056 \mathrm{~K}(\mathrm{~h})$ & 82 & 0 & $\begin{array}{l}80 \\
85\end{array}$ & $\begin{array}{l}86 \\
85\end{array}$ & $\begin{array}{l}48 \\
49\end{array}$ & 0.576470588 & $\begin{array}{l}14 \\
15\end{array}$ & 0.006944444 & 0.10787037 \\
\hline $\begin{array}{l}\text { PAK2(h) } \\
\text { Past }\end{array}$ & 93 & 0 & 84 & 84 & 50 & 0.588235294 & 16 & 0.007407407 & 0.115277778 \\
\hline PDGFRa(h) & 90 & 0 & 84 & 84 & 51 & 0.6 & 16 & 0.007407407 & 0.122685185 \\
\hline PDGFRB(h) & 99 & 0 & 83 & 83 & 52 & 0.611764706 & 17 & 0.00787037 & 0.130555556 \\
\hline PDKI(h) & 104 & 0 & 82 & 82 & 53 & 0.623529412 & 18 & 0.008333333 & 0.138888889 \\
\hline PKA(b) & 99 & 0 & 81 & 81 & 54 & 0.635294118 & 19 & 0.008796296 & 0.147685185 \\
\hline 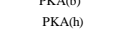 & 70 & 0 & 81 & 81 & $\begin{array}{l}54 \\
55\end{array}$ & 0.647058824 & 19 & 0.008796296 & 0.156481481 \\
\hline PKBa(h) & 78 & 0 & 78 & 78 & 56 & 0.658823529 & 22 & 0.010185185 & 0.166666667 \\
\hline PKBB(h) & 90 & 0 & 74 & 74 & 57 & 0.670588235 & 26 & 0.012037037 & 0.178703704 \\
\hline $\mathrm{PKB} \mathrm{B}_{(\mathrm{h})}$ & 84 & 0 & 73 & 73 & 58 & 0.682352941 & 27 & 0.0125 & 0.191203704 \\
\hline PKCath) & 73 & 0 & 71 & 71 & 59 & 0.694117647 & 29 & 0.013425926 & 0.20462963 \\
\hline PKCBII(h) & 92 & 0 & 70 & 70 & 60 & 0.705882353 & 30 & 0.013888889 & 0.218518519 \\
\hline PKCy(h) & 81 & 0 & 69 & 69 & 61 & 0.717647059 & 31 & 0.014351852 & 0.23287037 \\
\hline РКФ(h) & 87 & 0 & 68 & 68 & 62 & 0.729411765 & 32 & 0.014814815 & 0.247685185 \\
\hline PKG:(h) & 86 & 0 & 66 & 66 & 63 & 0.741176471 & 34 & 0.015740741 & 0.263425926 \\
\hline $\begin{array}{l}\text { PKG (h) } \\
\text { (h) }\end{array}$ & 74 & 0 & 66 & 66 & 64 & 0.752941176 & $\begin{array}{l}34 \\
34\end{array}$ & 0.015740741 & 0.279166667 \\
\hline PKC (h) & 97 & 0 & 64 & 64 & 65 & 0.764705882 & 36 & 0.016666667 & 0.295833333 \\
\hline PKC $\mu(\mathrm{h})$ & 53 & 0 & 55 & 55 & 66 & 0.776470588 & 45 & 0.020833333 & 0.316666667 \\
\hline PKCO(h) & 86 & 0 & 53 & 53 & 67 & 0.788235294 & 47 & 0.021759259 & 0.338425926 \\
\hline PKD2(h) & 43 & 1 & 51 & 51 & 68 & 0.8 & 49 & 0.022685185 & 0.361111111 \\
\hline PRAK(h) & 96 & 0 & 46 & 46 & 69 & 0.811764706 & 54 & 0.025 & 0.386111111 \\
\hline PRK2(h) & 88 & 0 & 43 & 43 & 70 & 0.823529412 & 57 & 0.026388889 & 0.4125 \\
\hline $\begin{array}{l}\text { PRK_(n) } \\
\text { ROCK-II(h) }\end{array}$ & 81 & 0 & $\begin{array}{l}43 \\
42\end{array}$ & $\begin{array}{l}43 \\
42\end{array}$ & 71 & 0.835294118 & 58 & 0.026851852 & 0.439351852 \\
\hline ROCK-II(r) & 69 & 0 & 34 & 34 & 72 & 0.847058824 & 66 & 0.030555556 & 0.469907407 \\
\hline Rskl(h) & 84 & 0 & 29 & 29 & 73 & 0.858823529 & 71 & 0.03287037 & 0.502777778 \\
\hline Rskl(r) & $\begin{array}{l}64 \\
68\end{array}$ & 0 & 29 & 29 & 74 & 0.870588235 & 71 & 0.03287037 & 0.535648148 \\
\hline $\begin{array}{l}\text { Rsksk2(h) } \\
\text { Rsk(h) }\end{array}$ & 92 & 0 & 24 & 24 & 75 & 0.882352941 & 76 & 0.035185185 & 0.570833333 \\
\hline $\mathrm{Rsk} 3(\mathrm{~h})$ & 85 & 0 & 23 & 23 & 76 & 0.894117647 & 77 & 0.035648148 & 0.606481481 \\
\hline SAPK2a(h) & 2 & 1 & 17 & 17 & 77 & 0.905882353 & 83 & 0.038425926 & 0.644907407 \\
\hline SAPK2b(h) & 3 & 1 & 14 & 14 & 78 & 0.917647059 & 86 & 0.039814815 & 0.684722222 \\
\hline SAPK $3(\mathrm{~h})$ & 83 & 0 & 5 & 5 & 79 & 0.929411765 & 95 & 0.043981481 & 0.728703704 \\
\hline $\begin{array}{l}\mathrm{SARRS( \textrm {h } )} \\
\mathrm{SAPK} 4(\mathrm{~h})\end{array}$ & $\begin{array}{l}00 \\
92\end{array}$ & 0 & 5 & 5 & 80 & 0.941176471 & 95 & 0.043981481 & 0.772685185 \\
\hline SGK(h) & 100 & 0 & 4 & 4 & 81 & 0.952941176 & 96 & 0.044444444 & 0.81712963 \\
\hline Syk(h) & 103 & 0 & 3 & 3 & 82 & 0.964705882 & 97 & 0.044907407 & 0.862037037 \\
\hline TrkB(h) & 108 & 0 & 2 & 2 & 83 & 0.976470588 & 98 & 0.04537037 & 0.907407407 \\
\hline $\begin{array}{l}\text { IIrkB(h) } \\
\text { Yesh(h) }\end{array}$ & 42 & 1 & 0 & 0 & 84 & 0.988235294 & $\begin{array}{l}98 \\
100\end{array}$ & 0.046296296 & 0.953703704 \\
\hline ZAP-70(h) & 115 & 0 & 0 & 0 & 85 & 1 & 100 & 0.046296296 & 1 \\
\hline & & & & & & Total inhibition & 2160 & & Total area \\
\hline & $\begin{array}{r}\text { Hit rate } \\
\text {. }\end{array}$ & 1 & & & & & & & Gini \\
\hline
\end{tabular}




\begin{tabular}{|c|c|c|c|c|c|c|c|c|c|}
\hline & $100 \mu \mathrm{M}$ ATP & & & & & & & & \\
\hline Kinase & SB203580@ @ $10 \mu \mathrm{M}$ & Hit & sorted & normalized & position & cumulative sample fraction & inhibition\% & fraction of total inhibition & cumulative inhibition \\
\hline $\mathrm{Abl}(\mathrm{m})$ & 98 & 0 & 117 & 100 & 1 & 0.011764706 & 0 & 0 & 0 \\
\hline AMPK(r) & 107 & 0 & 113 & 100 & 2 & 0.023529412 & 0 & 0 & 0 \\
\hline $\operatorname{Arg}(\mathrm{m})$ & 79 & 0 & 110 & 100 & 3 & 0.035294118 & 0 & 0 & 0 \\
\hline Aurora-A(h) & 89 & 0 & 110 & 100 & 4 & 0.047058824 & 0 & 0 & 0 \\
\hline Axl(h) & 95 & 0 & 110 & 100 & 5 & 0.058823529 & 0 & 0 & 0 \\
\hline $\operatorname{Blk}(\mathrm{m})$ & 82 & 0 & 109 & 100 & 6 & 0.070588235 & 0 & 0 & 0 \\
\hline $\operatorname{Bmx}(\mathrm{h})$ & 84 & 0 & 109 & 100 & 7 & 0.082352941 & 0 & 0 & 0 \\
\hline CаMKII(r) & 107 & 0 & 109 & 100 & 8 & 0.094117647 & 0 & 0 & 0 \\
\hline CaMKIV(h) & 104 & 0 & 107 & 100 & 9 & 0.105882353 & 0 & 0 & 0 \\
\hline CDK1/cyclnBB(h) & 105 & 0 & 107 & 100 & 10 & 0.117647059 & 0 & 0 & 0 \\
\hline CDK2/cyclinA(h) & 109 & 0 & 107 & 100 & 11 & 0.129411765 & 0 & 0 & 0 \\
\hline $\mathrm{CDK} 2 /$ cyclnE(h) & 107 & 0 & 107 & 100 & 12 & 0.141176471 & 0 & 0 & 0 \\
\hline CDK3/yclinE(h) & 117 & 0 & 107 & 100 & 13 & 0.152941176 & 0 & 0 & 0 \\
\hline CDK5/p35(h) & 109 & 0 & 107 & 100 & 14 & 0.164705882 & 0 & 0 & 0 \\
\hline CDKG/cyclinD3(h) & 83 & 0 & 106 & 100 & 15 & 0.176470588 & 0 & 0 & 0 \\
\hline CDK $7 /$ yclinHMATI(h) & 107 & 0 & 105 & 100 & 16 & 0.188235294 & 0 & 0 & 0 \\
\hline CHKI(h) & 100 & 0 & 105 & 100 & 17 & 0.2 & 0 & 0 & 0 \\
\hline CHK2(h) & 103 & 0 & 104 & 100 & 18 & 0.211764706 & 0 & 0 & 0 \\
\hline $\mathrm{CK} 1(\mathrm{y})$ & 1 & 1 & 104 & 100 & 19 & 0.223529412 & 0 & 0 & 0 \\
\hline СК2(h) & 110 & 0 & $\begin{array}{l}104 \\
104 \\
\end{array}$ & 100 & 20 & 0.235294118 & 0 & 0 & 0 \\
\hline c-RAF(h) & 8 & 1 & 103 & 100 & 21 & 0.247058824 & 0 & 0 & 0 \\
\hline $\operatorname{CSK}(\mathrm{h})$ & 110 & 0 & 103 & 100 & 22 & 0.258823529 & 0 & 0 & 0 \\
\hline $\operatorname{csRC(h)}$ & 57 & 0 & 102 & 100 & 23 & 0.270588235 & 0 & 0 & 0 \\
\hline Fess(h) & 102 & 0 & 102 & 100 & 24 & 0.282352941 & 0 & 0 & 0 \\
\hline FGFR3(h) & 102 & 0 & 102 & 100 & 25 & 0.294117647 & 0 & 0 & 0 \\
\hline $\mathrm{Fll}(\mathrm{h}(\mathrm{h})$ & 83 & 0 & 102 & 100 & 26 & 0.305882353 & 0 & 0 & 0 \\
\hline Fynn(h) & 63 & 0 & 101 & 100 & 27 & 0.317647059 & 0 & 0 & 0 \\
\hline GSK3a(h) & 81 & 0 & 101 & 100 & 28 & 0.329411765 & 0 & 0 & 0 \\
\hline GSK $3 \mathrm{~B}(\mathrm{~h})$ & 48 & 1 & 101 & 100 & 29 & 0.341176471 & 0 & 0 & 0 \\
\hline IGF-IR(h) & 102 & 0 & 100 & 100 & 30 & 0.352941176 & 0 & 0 & 0 \\
\hline $\mathrm{IKKa}(\mathrm{h})$ & 96 & 0 & 100 & 100 & 31 & 0.364705882 & 0 & 0 & 0 \\
\hline IKKB(h) & 100 & 0 & 100 & 100 & 32 & 0.376470588 & 0 & 0 & 0 \\
\hline $\mathrm{IR}(\mathrm{h})$ & 109 & 0 & 100 & 100 & 33 & 0.388235294 & 0 & 0 & 0 \\
\hline JNKIal(h) & 106 & 0 & 99 & 99 & 34 & 0.4 & 1 & 0.000742942 & 0.000742942 \\
\hline JNK202(h) & 77 & 0 & 99 & 99 & $\begin{array}{l}54 \\
35\end{array}$ & 0.411764706 & 1 & 0.000742942 & 0.001485884 \\
\hline $\mathrm{JNK} 3(\mathrm{r})$ & 19 & 1 & 98 & 98 & 36 & 0.423529412 & 2 & 0.001485884 & 0.002971768 \\
\hline Lck(h) & 35 & 1 & 98 & 98 & 37 & 0.435294118 & 2 & 0.001485884 & 0.004457652 \\
\hline Lyn(h) & 42 & 1 & 98 & 98 & 38 & 0.447058824 & 2 & 0.001485884 & 0.005943536 \\
\hline Lyy(m) & 45 & 1 & 97 & 97 & 39 & 0.458823529 & 3 & 0.002228826 & 0.008172363 \\
\hline MAPKI(h) & $\begin{array}{l}45 \\
99\end{array}$ & 0 & 97 & 97 & 40 & 0.470588235 & 3 & 0.002228826 & 0.010401189 \\
\hline MAPK2(h) & 100 & 0 & 97 & 97 & 41 & 0.482352941 & 3 & 0.002228826 & 0.012630015 \\
\hline $\begin{array}{l}\text { MAPKL(2) } \\
\text { MAPK2(m) }\end{array}$ & 101 & 0 & 97 & 97 & $\begin{array}{l}41 \\
42\end{array}$ & 0.494117647 & 3 & 0.002228826 & 0.014858841 \\
\hline MAPKAP-K2(h) & 97 & 0 & 97 & 97 & 43 & 0.505882353 & 3 & 0.002228826 & 0.017087667 \\
\hline MEK1(h) & 102 & 0 & 96 & 96 & 44 & 0.517647059 & 4 & 0.002971768 & 0.020059435 \\
\hline MKK4(m) & 70 & 0 & 96 & 96 & 45 & 0.529411765 & 4 & 0.002971768 & 0.023031204 \\
\hline MKK6(h) & 4 & 1 & 95 & 95 & 46 & 0.541176471 & 5 & 0.00371471 & 0.026745914 \\
\hline MKK & 82 & 0 & 95 & 95 & 47 & 0.552941176 & 5 & 0.00371471 & 0.030460624 \\
\hline MSKI(h) & 99 & 0 & 94 & 94 & 48 & 0.564705882 & 6 & 0.004457652 & 0.034918276 \\
\hline $\mathrm{p} 7056 \mathrm{~K}(\mathrm{~h})$ & $\begin{array}{l}107 \\
107\end{array}$ & 0 & $\begin{array}{l}94 \\
94\end{array}$ & $\begin{array}{l}94 \\
94\end{array}$ & $\begin{array}{l}48 \\
49\end{array}$ & 0.576470588 & $\begin{array}{l}0 \\
6\end{array}$ & 0.004457652 & 0.039375929 \\
\hline $\begin{array}{l}\text { PAK2(h) } \\
\text { Past }\end{array}$ & 97 & 0 & 94 & 94 & 50 & 0.588235294 & 6 & 0.004457652 & 0.043833581 \\
\hline PDGFRa(h) & 92 & 0 & 93 & 93 & 51 & 0.6 & 7 & 0.005200594 & 0.049034175 \\
\hline PDGFRB(h) & 101 & 0 & 92 & 92 & 52 & 0.611764706 & 8 & 0.005943536 & 0.054977712 \\
\hline PDKI(h) & 98 & 0 & 90 & $\begin{array}{l}92 \\
90\end{array}$ & 53 & 0.623529412 & 10 & 0.007429421 & 0.062407132 \\
\hline PKA(b) & 110 & 0 & 89 & 89 & 54 & 0.635294118 & 11 & 0.008172363 & 0.070579495 \\
\hline PKA(h) & 96 & 0 & 89 & 89 & 55 & 0.647058824 & 11 & 0.008172363 & 0.078751857 \\
\hline PKBa(h) & 82 & 0 & 87 & 87 & 56 & 0.658823529 & 13 & 0.009658247 & 0.088410104 \\
\hline PKBB(h) & 87 & 0 & 87 & 87 & 57 & 0.670588235 & 13 & 0.009658247 & 0.098068351 \\
\hline $\mathrm{PKB} \mathrm{B}_{(\mathrm{h})}$ & 97 & 0 & 87 & 87 & 58 & 0.682352941 & 13 & 0.009658247 & 0.107726597 \\
\hline PKCath) & 85 & 0 & 85 & 85 & 59 & 0.694117647 & 15 & 0.011144131 & 0.118870728 \\
\hline PKCBII(h) & 97 & 0 & 84 & 84 & 60 & 0.705882353 & 16 & 0.011887073 & 0.130757801 \\
\hline PKGy(h) & 94 & 0 & 83 & $\begin{array}{l}84 \\
83\end{array}$ & 61 & 0.717647059 & 17 & 0.012630015 & 0.143387816 \\
\hline РКФ(h) & 94 & 0 & 83 & 83 & 62 & 0.729411765 & 17 & 0.012630015 & 0.156017831 \\
\hline PKG:(h) & 89 & 0 & 82 & 82 & 63 & 0.741176471 & 18 & 0.013372957 & 0.169390788 \\
\hline $\begin{array}{l}\text { PKG (h) } \\
\text { (h) }\end{array}$ & 87 & 0 & 82 & 82 & 64 & 0.752941176 & 18 & 0.013372957 & 0.182763744 \\
\hline PKC (h) & 95 & 0 & 82 & 82 & 65 & 0.764705882 & 18 & 0.013372957 & 0.196136701 \\
\hline PKC $\mu(\mathrm{h})$ & 82 & 0 & 82 & 82 & 66 & 0.776470588 & 18 & 0.013372957 & 0.209509658 \\
\hline PKCO(h) & 87 & 0 & 81 & 81 & 67 & 0.788235294 & 19 & 0.014115899 & 0.223625557 \\
\hline PKD2(h) & 62 & 0 & 79 & 79 & 68 & 0.8 & 21 & 0.015601783 & 0.23922734 \\
\hline PRAK(h) & 100 & 0 & 78 & 78 & 69 & 0.811764706 & 22 & 0.016344725 & 0.255572065 \\
\hline PRK2(h) & 104 & 0 & 77 & 77 & 70 & 0.823529412 & 23 & 0.017087667 & 0.272659733 \\
\hline $\begin{array}{l}\text { PRK_(n) } \\
\text { ROCK-II(h) }\end{array}$ & 97 & 0 & 70 & 70 & 71 & 0.835294118 & 30 & 0.022288262 & 0.294947994 \\
\hline ROCK-II(r) & 78 & 0 & 63 & 63 & 72 & 0.847058824 & 37 & 0.027488856 & 0.32243685 \\
\hline Rskl(h) & 103 & 0 & 62 & 62 & 73 & 0.858823529 & 38 & 0.028231798 & 0.350668648 \\
\hline Rsk1(r) & 93 & 0 & 61 & 61 & 74 & 0.870588235 & 39 & 0.02897474 & 0.379643388 \\
\hline $\begin{array}{l}\text { Rsksk2(h) } \\
\text { Rsk(h) }\end{array}$ & 98 & 0 & 57 & 57 & 75 & 0.882352941 & 43 & 0.031946508 & 0.411589896 \\
\hline $\mathrm{Rsk} 3(\mathrm{~h})$ & 104 & 0 & 48 & 48 & 76 & 0.894117647 & 52 & 0.038632987 & 0.450222883 \\
\hline SAPK2a(h) & -1 & 1 & 45 & 45 & 77 & 0.905882353 & 55 & 0.040861813 & 0.491084695 \\
\hline SAPK2b(h) & 6 & 1 & 42 & 42 & 78 & 0.917647059 & 58 & 0.043090639 & 0.534175334 \\
\hline SAPK3(h) & 105 & 0 & 35 & 35 & 79 & 0.929411765 & 65 & 0.048291233 & 0.582466568 \\
\hline SAPK4(h) & 107 & 0 & 19 & 19 & 80 & 0.941176471 & 81 & 0.060178306 & 0.642644874 \\
\hline SGK(h) & 113 & 0 & 8 & 8 & 81 & 0.952941176 & 92 & 0.068350669 & 0.710995542 \\
\hline Syk(h) & 90 & 0 & 6 & 6 & 82 & 0.964705882 & 94 & 0.069836553 & 0.780832095 \\
\hline TrkB(h) & 101 & 0 & 4 & 4 & 83 & 0.976470588 & 96 & 0.071322437 & 0.852154532 \\
\hline Yes(h) & 61 & 0 & 1 & 1 & 84 & 0.988235294 & $\begin{array}{l}50 \\
99\end{array}$ & 0.073551263 & 0.925705795 \\
\hline ZAP-70(h) & 94 & 0 & -1 & 0 & 85 & 1 & 100 & 0.074294205 & 1 \\
\hline & & & & & & Total inhibition & 1346 & & Total area \\
\hline & $\begin{array}{r}\text { Hit rate } \\
\text {. }\end{array}$ & 5 & & & & & & & Gini \\
\hline
\end{tabular}




\begin{tabular}{|c|c|c|c|c|c|c|c|c|}
\hline & $\mu \mathrm{M}$ ATP & & & & & & & \\
\hline Kinase & ST638@ $10 \mu \mathrm{M}$ & Hit & sorted & normalized & position & cumulative sample fraction & inhibition\% & fraction of total inhibition \\
\hline $\mathrm{Abl}(\mathrm{m})$ & 54 & 0 & 126 & 100 & 1 & 0.011764706 & 0 & 0 \\
\hline AMPK(r) & 85 & 0 & 112 & 100 & 2 & 0.023529412 & 0 & 0 \\
\hline $\operatorname{Arg}(\mathrm{m})$ & 59 & 0 & 108 & 100 & 3 & 0.035294118 & 0 & 0 \\
\hline Aurora-A(h) & 8 & 1 & 107 & 100 & 4 & 0.047058824 & 0 & 0 \\
\hline Axl(h) & 56 & 0 & 104 & 100 & 5 & 0.058823529 & 0 & 0 \\
\hline $\mathrm{Blk}(\mathrm{m})$ & 18 & 1 & 103 & 100 & 6 & 0.070588235 & 0 & 0 \\
\hline Bmxx(h) & 65 & 0 & 102 & 100 & 7 & 0.082352941 & 0 & 0 \\
\hline СамKII(r) & 96 & 0 & 102 & 100 & 8 & 0.094117647 & 0 & 0 \\
\hline CaMKIV(h) & 81 & 0 & 102 & 100 & 9 & 0.105882353 & 0 & 0 \\
\hline CDK1/cyclnBB(h) & 81 & 0 & 102 & 100 & 10 & 0.117647059 & 0 & 0 \\
\hline $\mathrm{CDK} /$ /yclinA(h) & 93 & 0 & 101 & 100 & 11 & 0.129411765 & 0 & 0 \\
\hline $\mathrm{CDK} 2 /$ cyclinE(h) & 94 & 0 & 101 & 100 & 12 & 0.141176471 & 0 & 0 \\
\hline CDK3/cyclinE(h) & 98 & 0 & 101 & 100 & 13 & 0.152941176 & 0 & 0 \\
\hline $\mathrm{CDK} 5 / 235(\mathrm{~h})$ & 99 & 0 & 99 & 99 & 14 & 0.164705882 & 1 & 0.000506073 \\
\hline CDK6/cyclinD3(h) & 76 & 0 & 99 & 99 & 15 & 0.176470588 & 1 & 0.000506073 \\
\hline CDK7/cyclinHMATI(h) & 80 & 0 & 99 & 99 & 16 & 0.188235294 & 1 & 0.000506073 \\
\hline СнК $1 \mathrm{~h})$ & 107 & 0 & 98 & 98 & 17 & 0.2 & 2 & 0.001012146 \\
\hline СHK2(h) & 34 & 1 & 98 & 98 & 18 & 0.211764706 & 2 & 0.001012146 \\
\hline CKI(y) & 26 & 1 & 98 & 98 & 19 & 0.223529412 & 2 & 0.001012146 \\
\hline СК2(h) & 101 & 0 & 97 & 97 & 20 & 0.235294118 & 3 & 0.001518219 \\
\hline c-RAF(h) & 35 & 1 & 97 & 97 & 21 & 0.247058824 & 3 & 0.001518219 \\
\hline $\operatorname{CSK}(\mathrm{h})$ & 58 & 0 & 96 & 96 & 22 & 0.258823529 & 4 & 0.002024291 \\
\hline cSRCh) & 17 & 1 & 96 & 96 & 23 & 0.270588235 & 4 & 0.002024291 \\
\hline Fes(h) & 83 & 0 & 96 & 96 & 24 & 0.282352941 & 4 & 0.002024291 \\
\hline FGFR3(h) & 95 & 0 & 95 & 95 & 25 & 0.294117647 & 5 & 0.002530364 \\
\hline Fill(h) & 44 & 1 & 95 & 95 & 26 & 0.305882353 & 5 & 0.002530364 \\
\hline Fyn(h) & 27 & 1 & 95 & 95 & 27 & 0.317647059 & 5 & 0.002530364 \\
\hline GSK3a(h) & 44 & 1 & 95 & 95 & 28 & 0.329411765 & 5 & 0.002530364 \\
\hline GSK3B(h) & 59 & 0 & 94 & 94 & 29 & 0.341176471 & 6 & 0.003036437 \\
\hline IGF-IR(h) & 96 & 0 & 94 & 94 & 30 & 0.352941176 & 6 & 0.003036437 \\
\hline IKKa(h) & 81 & 0 & 94 & 94 & 31 & 0.364705882 & 6 & 0.003036437 \\
\hline IKKB(h) & 70 & 0 & 93 & 93 & 32 & 0.376470588 & 7 & 0.00354251 \\
\hline $\mathbb{I R ( h )}$ & 101 & 0 & 92 & 92 & 33 & 0.388235294 & 8 & 0.004048583 \\
\hline JNKIal(h) & 44 & 1 & 92 & 92 & 34 & 0.4 & 8 & 0.004048583 \\
\hline JNK2202(h) & 66 & 0 & 91 & 91 & 35 & 0.411764706 & 9 & 0.004554656 \\
\hline JNK3(r) & 51 & 0 & 91 & 91 & 36 & 0.423529412 & 9 & 0.004554656 \\
\hline Lck(h) & 10 & 1 & 90 & 90 & 37 & 0.435294118 & 10 & 0.005060729 \\
\hline Lyn(h) & 61 & 0 & 90 & 90 & 38 & 0.447058824 & 10 & 0.005060729 \\
\hline Lyy(m) & 39 & 1 & 89 & 89 & 39 & 0.458823529 & 11 & 0.005566802 \\
\hline MAPK I (h) & 88 & 0 & 88 & 88 & 40 & 0.470588235 & 12 & 0.006072874 \\
\hline MAPK2(h) & 108 & 0 & 88 & 88 & 41 & 0.482352941 & 12 & 0.006072874 \\
\hline MAPK2(m) & 102 & 0 & 88 & 88 & 42 & 0.494117647 & 12 & 0.006072874 \\
\hline MAPKAP-K2(h) & 101 & 0 & 85 & 85 & 43 & 0.505882353 & 15 & 0.007591093 \\
\hline MEK1(h) & 95 & 0 & 85 & 85 & 44 & 0.517647059 & 15 & 0.007591093 \\
\hline MKK $4(\mathrm{~m})$ & 103 & 0 & 85 & 85 & 45 & 0.529411765 & 15 & 0.007591093 \\
\hline MKK6(h) & 95 & 0 & 83 & 83 & 46 & 0.541176471 & 17 & 0.008603239 \\
\hline MKK $7 \mathrm{B(h)}$ & 99 & 0 & 82 & 82 & 47 & 0.552941176 & 18 & 0.009109312 \\
\hline MSKI(h) & 85 & 0 & 81 & 81 & 48 & 0.564705882 & 19 & 0.009615385 \\
\hline p7056K(h) & 78 & 0 & 81 & 81 & 49 & 0.576470588 & 19 & 0.009615385 \\
\hline $\begin{array}{l}\text { PAK2(h) } \\
\text { Past }\end{array}$ & 96 & 0 & 81 & 81 & 50 & 0.588235294 & 19 & 0.009615385 \\
\hline PDGFRa(h) & 89 & 0 & 81 & 81 & 51 & 0.6 & 19 & 0.009615385 \\
\hline PDGFRB(h) & 92 & 0 & 81 & 81 & 52 & 0.611764706 & 19 & 0.009615385 \\
\hline PDKI(h) & $\begin{array}{l}92 \\
126\end{array}$ & 0 & 80 & 80 & 53 & 0.623529412 & 20 & 0.010121457 \\
\hline PKA(b) & 91 & 0 & 78 & 78 & 54 & 0.635294118 & 22 & 0.011133603 \\
\hline PKA(h) & 97 & 0 & 78 & 78 & 55 & 0.647058824 & 22 & 0.011133603 \\
\hline PKBa(h) & 91 & 0 & 76 & 76 & 56 & 0.658823529 & 24 & 0.012145749 \\
\hline PKB(h) & 112 & 0 & 76 & 76 & 57 & 0.670588235 & 24 & 0.012145749 \\
\hline $\mathrm{PKB}_{\gamma}(\mathrm{h})$ & 98 & 0 & 75 & 75 & 58 & 0.682352941 & 25 & 0.012651822 \\
\hline PKG(th) & 78 & 0 & 70 & 70 & 59 & 0.694117647 & 30 & 0.015182186 \\
\hline РКCBII(h) & 95 & 0 & 68 & 68 & 60 & 0.705882353 & 32 & 0.016194332 \\
\hline PKCy (h) & 90 & 0 & 66 & 66 & 61 & 0.717647059 & 34 & 0.017206478 \\
\hline PKБ(h) & 102 & 0 & 65 & 65 & 62 & 0.729411765 & 35 & 0.017712551 \\
\hline PKG:(h) & 75 & 0 & 61 & 61 & 63 & 0.741176471 & 39 & 0.019736842 \\
\hline PKG(h) & 102 & 0 & 60 & 60 & 64 & 0.752941176 & 40 & 0.020242915 \\
\hline PKC (h) & 81 & 0 & 59 & $\begin{array}{l}50 \\
59\end{array}$ & 65 & 0.764705882 & 41 & 0.020748988 \\
\hline PKC $\mu(\mathrm{h})$ & 88 & 0 & 59 & 59 & 66 & 0.776470588 & 41 & 0.020748988 \\
\hline PKCO(h) & 102 & 0 & 58 & 58 & 67 & 0.788235294 & 42 & 0.021255061 \\
\hline PKD2(h) & 76 & 0 & 57 & 57 & 68 & 0.8 & 43 & 0.021761134 \\
\hline $\operatorname{PRAK}(\mathrm{h})$ & 97 & 0 & 56 & 56 & 69 & 0.811764706 & 44 & 0.022267206 \\
\hline PRK2(h) & 94 & 0 & 54 & 54 & 70 & 0.823529412 & 46 & 0.023279352 \\
\hline ROCK-II(h) & 85 & 0 & 51 & 51 & 71 & 0.835294118 & 49 & 0.024797571 \\
\hline ROCK-II(r) & 98 & 0 & 50 & 50 & 72 & 0.847058824 & 50 & 0.025303644 \\
\hline Rskl(h) & 92 & 0 & 44 & 44 & 73 & 0.858823529 & 56 & 0.028340081 \\
\hline Rskl(r) & 57 & 0 & 44 & 44 & 74 & 0.870588235 & 56 & 0.028340081 \\
\hline Rsk2(h) & 60 & 0 & 44 & 44 & 75 & 0.882352941 & 56 & 0.028340081 \\
\hline Rsk3(h) & 82 & 0 & 39 & 39 & 76 & 0.894117647 & 61 & 0.030870445 \\
\hline SAPK2a(h) & 50 & 1 & 35 & 35 & 77 & 0.905882353 & 65 & 0.032894737 \\
\hline SAPK2b(h) & 94 & 0 & 34 & 34 & 78 & 0.917647059 & 66 & 0.03340081 \\
\hline SAPK3(h) & 90 & 0 & 29 & 29 & 79 & 0.929411765 & 71 & 0.035931174 \\
\hline SAPK4(h) & 99 & 0 & 27 & 27 & 80 & 0.941176471 & 73 & 0.03694332 \\
\hline SGK(h) & 88 & 0 & 26 & 26 & 81 & 0.952941176 & 74 & 0.037449393 \\
\hline Syk(h) & 81 & 0 & 18 & 18 & 82 & 0.964705882 & 82 & 0.041497976 \\
\hline TrkB(h) & 68 & 0 & 17 & 17 & 83 & 0.976470588 & 83 & 0.042004049 \\
\hline Yes(h) & 29 & 1 & 10 & 10 & 84 & 0.988235294 & 90 & 0.045546559 \\
\hline ZAP-70(h) & 104 & 0 & 8 & 8 & 85 & 1 & 92 & 0.046558704 \\
\hline & & & & & & Total inhibition & 1976 & \\
\hline & $\begin{array}{l}\text { Hit rate } \\
\text {. }\end{array}$ & & & & & & & \\
\hline & Threshold & ( & & & & & & \\
\hline
\end{tabular}




\begin{tabular}{|c|c|c|c|c|c|c|c|c|c|c|}
\hline Kinase & $\begin{array}{l}100 \mu \mathrm{M} \text { ATP } \\
\text { ST638 @ } 10 \mu \mathrm{M}\end{array}$ & Hit & sorted & normalized & position & cumulative sample fraction & inhibition $\%$ & & & \\
\hline $\mathrm{Abl}(\mathrm{m})$ & 92 & 0 & 123 & 100 & 1 & 0.011764706 & 0 & 0 & 0 & area \\
\hline AMPK(r) & 102 & 0 & 113 & 100 & 2 & 0.023529412 & 0 & 0 & 0 & 0 \\
\hline $\operatorname{Arg}(\mathrm{m})$ & 81 & 0 & 112 & 100 & 3 & 0.035294118 & 0 & 0 & 0 & 0 \\
\hline Aurora-A(h) & 30 & 1 & 110 & 100 & 4 & 0.047058824 & 0 & 0 & 0 & 0 \\
\hline Axl(h) & 82 & 0 & 110 & 100 & 5 & 0.058823529 & 0 & 0 & 0 & 0 \\
\hline $\operatorname{Blk}(\mathrm{m})$ & 15 & 1 & 109 & 100 & 6 & 0.070588235 & 0 & 0 & 0 & 0 \\
\hline $\operatorname{Bmx}(\mathrm{h})$ & 94 & 0 & 108 & 100 & 7 & 0.082352941 & 0 & 0 & 0 & 0 \\
\hline CaMKII(r) & 94 & 0 & 107 & 100 & 8 & 0.094117647 & 0 & 0 & 0 & 0 \\
\hline CaMKIV(h) & 97 & 0 & 106 & 100 & 9 & 0.105882353 & 0 & 0 & 0 & 0 \\
\hline CDK1/cyclinB(h) & 90 & 0 & 105 & 100 & 10 & 0.117647059 & 0 & 0 & 0 & 0 \\
\hline $\mathrm{CDK} 2 /$ cyclinA(h) & 103 & 0 & 105 & 100 & 11 & 0.129411765 & 0 & 0 & 0 & 0 \\
\hline $\mathrm{CDK} 2 /$ cyclinE(h) & 94 & 0 & 104 & 100 & 12 & 0.141176471 & 0 & 0 & 0 & 0 \\
\hline CDK3/cycling(h) & 97 & 0 & 104 & 100 & 13 & 0.152941176 & 0 & 0 & 0 & 0 \\
\hline CDK5/p35(h) & 110 & 0 & 103 & 100 & 14 & 0.164705882 & 0 & 0 & 0 & 0 \\
\hline CDK6/cyclinD3(h) & 94 & 0 & 103 & 100 & 15 & 0.176470588 & 0 & 0 & 0 & 0 \\
\hline CDK7/cyclinHMATI(h) & 94 & 0 & 103 & 100 & 16 & 0.188235294 & 0 & 0 & 0 & 0 \\
\hline СHK1(h) & 105 & 0 & 102 & 100 & 17 & 0.2 & 0 & 0 & 0 & 0 \\
\hline СHK2(h) & 47 & 1 & 102 & 100 & 18 & 0.211764706 & 0 & 0 & 0 & 0 \\
\hline CKI(y) & 60 & 0 & 102 & 100 & 19 & 0.223529412 & 0 & 0 & 0 & 0 \\
\hline CK2(h) & 103 & 0 & 102 & 100 & 20 & 0.235294118 & 0 & 0 & 0 & 0 \\
\hline c-RAF(h) & 52 & 0 & 101 & 100 & 21 & 0.247058824 & 0 & 0 & 0 & \\
\hline $\operatorname{CSK}(\mathrm{h})$ & 94 & 0 & 99 & 99 & 22 & 0.258823529 & 1 & 0.000849618 & 0.000849618 & 4.99775E-06 \\
\hline cSRC(h) & 48 & 1 & 98 & 98 & 23 & 0.270588235 & 2 & 0.001699235 & 0.002548853 & $1.9991 \mathrm{E}-05$ \\
\hline Fes(h) & 104 & 0 & 97 & 97 & 24 & 0.282352941 & 3 & 0.002548853 & 0.005097706 & 4.49798E-05 \\
\hline FGFR3(h) & 91 & 0 & 97 & 97 & 25 & 0.294117647 & 3 & 0.002548853 & 0.007646559 & $7.49663 \mathrm{E}-05$ \\
\hline Flls(h) & 107 & 0 & 97 & 97 & 26 & 0.305882353 & 3 & 0.002548853 & 0.010195412 & 0.000104953 \\
\hline Fynn(h) & 59 & 0 & 97 & 97 & 27 & 0.317647059 & 3 & 0.002548853 & 0.012744265 & 0.000134939 \\
\hline GSK3a(h) & 93 & 0 & 97 & 97 & 28 & 0.329411765 & 3 & 0.002548853 & 0.015293118 & 0.000164926 \\
\hline GSK3B(h) & 87 & 0 & 96 & 96 & 29 & 0.341176471 & 4 & 0.003398471 & 0.018691589 & 0.00019991 \\
\hline IGF-1R(h) & 97 & 0 & 96 & 96 & 30 & 0.352941176 & 4 & 0.003398471 & 0.022090059 & 0.000239892 \\
\hline $\mathrm{IKKa}(\mathrm{h})$ & 103 & 0 & 96 & 96 & 31 & 0.364705882 & 4 & 0.003398471 & 0.02548853 & 0.000279874 \\
\hline IKKB(h) & 102 & 0 & 95 & 95 & 32 & 0.376470588 & 5 & 0.004248088 & 0.029736619 & 0.000324854 \\
\hline $\mathbb{I R ( h )}$ & 94 & 0 & 94 & 94 & 33 & 0.388235294 & 6 & 0.005097706 & 0.034834325 & 0.000379829 \\
\hline JNKIal(h) & 92 & 0 & 94 & 94 & 34 & 0.4 & 6 & 0.005097706 & 0.039932031 & 0.000439802 \\
\hline JNK202(h) & 88 & 0 & 94 & 94 & 35 & 0.411764706 & 6 & 0.005097706 & 0.045029737 & 0.000499775 \\
\hline JNK3(r) & 67 & 0 & 94 & 94 & 36 & 0.423529412 & 6 & 0.005097706 & 0.050127443 & 0.000559748 \\
\hline Lck(h) & 15 & 1 & 94 & 94 & 37 & 0.435294118 & 6 & 0.005097706 & 0.055225149 & 0.000619721 \\
\hline Lyn(h) & 66 & 0 & 94 & 94 & 38 & 0.447058824 & 6 & 0.005097706 & 0.060322855 & 0.000679694 \\
\hline Lyn(m) & 57 & 0 & 94 & 94 & 39 & 0.458823529 & 6 & 0.005097706 & 0.065420561 & 0.000739667 \\
\hline MAPK I(h) & 87 & 0 & 94 & 94 & 40 & 0.470588235 & 6 & 0.005097706 & 0.070518267 & 0.00079964 \\
\hline MAPK2(h) & 113 & 0 & 94 & 94 & 41 & 0.482352941 & 6 & 0.005097706 & 0.075615973 & 0.000859613 \\
\hline MAPK2(m) & 105 & 0 & 93 & 93 & 42 & 0.494117647 & 7 & 0.005947324 & 0.081563297 & 0.000924584 \\
\hline MAPKAP-K2(h) & 102 & 0 & 93 & 93 & 43 & 0.505882353 & 7 & 0.005947324 & 0.08751062 & 0.000994552 \\
\hline MEK1(h) & 96 & 0 & 93 & 93 & 44 & 0.517647059 & 7 & 0.005947324 & 0.093457944 & 0.001064521 \\
\hline MKK4(m) & 73 & 0 & 93 & 93 & 45 & 0.529411765 & 7 & 0.005947324 & 0.099405268 & 0.001134489 \\
\hline МKK6(h) & 93 & 0 & 92 & 92 & 46 & 0.541176471 & 8 & 0.006796941 & 0.106202209 & 0.001209456 \\
\hline MKK 7 (h) & 76 & 0 & 92 & 92 & 47 & 0.552941176 & 8 & 0.006796941 & 0.11299915 & 0.00128942 \\
\hline MSKI(h) & 88 & 0 & 92 & 92 & 48 & 0.564705882 & 8 & 0.006796941 & 0.119796092 & 0.001369384 \\
\hline $\mathrm{p} 70 \mathrm{~S} 6 \mathrm{~K}(\mathrm{~h})$ & 96 & 0 & 91 & 91 & 49 & 0.576470588 & 9 & 0.007646559 & 0.127442651 & 0.001454346 \\
\hline PAK2(h) & 93 & 0 & 90 & 90 & 50 & 0.588235294 & 10 & 0.008496177 & 0.135938828 & 0.001549303 \\
\hline PDGFRa(h) & 86 & 0 & 90 & 90 & 51 & 0.6 & 10 & 0.008496177 & 0.144435004 & 0.001649258 \\
\hline PDGFRB(h) & 87 & 0 & 90 & 90 & 52 & 0.611764706 & 10 & 0.008496177 & 0.152931181 & 0.001749213 \\
\hline PDK1(h) & 94 & 0 & 88 & 88 & 53 & 0.623529412 & 12 & 0.010195412 & 0.163126593 & 0.001859163 \\
\hline PKA(b) & 112 & 0 & 88 & 88 & 54 & 0.635294118 & 12 & 0.010195412 & 0.173322005 & 0.001979109 \\
\hline PKA(h) & 97 & 0 & 88 & 88 & 55 & 0.647058824 & 12 & 0.010195412 & 0.183517417 & 0.002099055 \\
\hline PKBach) & 90 & 0 & 88 & 88 & 56 & 0.658823529 & 12 & 0.010195412 & 0.193712829 & 0.002219001 \\
\hline PKBB(h) & 110 & 0 & 87 & 87 & 57 & 0.670588235 & 13 & 0.01104503 & 0.204757859 & 0.002343945 \\
\hline $\mathrm{PKB}_{\gamma}(\mathrm{h})$ & 99 & 0 & 87 & 87 & 58 & 0.682352941 & 13 & 0.01104503 & 0.215802889 & 0.002473887 \\
\hline PKGath) & 94 & 0 & 87 & 87 & 59 & 0.694117647 & 13 & 0.01104503 & 0.226847918 & 0.002603828 \\
\hline PKCBII(h) & 98 & 0 & 86 & 86 & 60 & 0.705882353 & 14 & 0.011894647 & 0.238742566 & 0.002738768 \\
\hline PKCy(h) & 90 & 0 & 86 & 86 & 61 & 0.717647059 & 14 & 0.011894647 & 0.250637213 & 0.002878705 \\
\hline РКФ(h) & 102 & 0 & 85 & 85 & 62 & 0.729411765 & 15 & 0.012744265 & 0.263381478 & 0.003023639 \\
\hline PKG:(h) & 86 & 0 & 85 & 85 & 63 & 0.741176471 & 15 & 0.012744265 & 0.276125743 & 0.003173572 \\
\hline PKG (h) & 104 & 0 & 82 & 82 & 64 & 0.752941176 & 18 & 0.015293118 & 0.291418862 & 0.003338498 \\
\hline PKC (h) & 92 & 0 & 82 & 82 & 65 & 0.764705882 & 18 & 0.015293118 & 0.30671198 & 0.003518417 \\
\hline PKC $\mu(\mathrm{h})$ & 95 & 0 & 81 & 81 & 66 & 0.776470588 & 19 & 0.016142736 & 0.322854715 & 0.003703333 \\
\hline PKCO(h) & 108 & 0 & 81 & 81 & 67 & 0.788235294 & 19 & 0.016142736 & 0.338997451 & 0.003893248 \\
\hline PKD2(h) & 88 & 0 & 80 & 80 & 68 & 0.8 & 20 & 0.016992353 & 0.355989805 & 0.00408816 \\
\hline PRAK(h) & 73 & 0 & 76 & 76 & 69 & 0.811764706 & 24 & 0.020390824 & 0.376380629 & 0.004308061 \\
\hline PRK2(h) & 85 & 0 & 76 & 76 & 70 & 0.823529412 & 24 & 0.020390824 & 0.396771453 & 0.004547953 \\
\hline ROCK-II(h) & 85 & 0 & 75 & 75 & 71 & 0.835294118 & 25 & 0.021240442 & 0.418011895 & 0.004792843 \\
\hline ROCK-II(r) & 82 & 0 & 73 & 73 & 72 & 0.847058824 & 27 & 0.022939677 & 0.440951572 & 0.005052726 \\
\hline Rskl(h) & 93 & 0 & 73 & 73 & 73 & 0.858823529 & 27 & 0.022939677 & 0.463891249 & 0.005322605 \\
\hline Rskl(r) & 109 & 0 & 67 & 67 & 74 & 0.870588235 & 33 & 0.028037383 & 0.491928632 & 0.00562247 \\
\hline Rsk2(h) & 76 & 0 & 66 & 66 & 75 & 0.882352941 & 34 & 0.028887001 & 0.520815633 & 0.005957319 \\
\hline Rsk3(h) & 81 & 0 & 60 & 60 & 76 & 0.894117647 & 40 & 0.033984707 & 0.55480034 & 0.006327153 \\
\hline SAPK2a(h) & 80 & 0 & 59 & 59 & 77 & 0.905882353 & 41 & 0.034834325 & 0.589634664 & 0.006731971 \\
\hline SAPK2b(h) & 123 & 0 & 57 & 57 & 78 & 0.917647059 & 43 & 0.03653356 & 0.626168224 & 0.007151782 \\
\hline $\mathrm{SAPK} 3(\mathrm{~h})$ & 97 & 0 & 53 & 53 & 79 & 0.929411765 & 47 & 0.039932031 & 0.666100255 & 0.007601579 \\
\hline SAPK4(h) & 96 & 0 & 52 & 52 & 80 & 0.941176471 & 48 & 0.040781648 & 0.706881903 & 0.008076366 \\
\hline SGK(h) & 101 & 0 & 48 & 48 & 81 & 0.952941176 & 52 & 0.044180119 & 0.751062022 & 0.008576141 \\
\hline Syk(h) & 106 & 0 & 47 & 47 & 82 & 0.964705882 & 53 & 0.045029737 & 0.796091759 & 0.009100905 \\
\hline $\operatorname{TrkB(h)}$ & 75 & 0 & 30 & 30 & 83 & 0.976470588 & 70 & 0.059473237 & 0.855564996 & 0.009715628 \\
\hline Yes(h) & 53 & 0 & 15 & 15 & 84 & 0.988235294 & 85 & 0.072217502 & 0.927782498 & 0.010490279 \\
\hline ZAP-70(h) & 88 & 0 & 15 & 15 & 85 & 1 & 85 & 0.072217502 & 1 & 0.011339897 \\
\hline & & & & & & Total inhibition & 1177 & & Total area & 0.188210305 \\
\hline & $\begin{array}{r}\text { Hit rate } \\
\text { Threshold }\end{array}$ & 5 & & & & & & & Gini & 0.623579389 \\
\hline
\end{tabular}




\begin{tabular}{|c|c|c|c|c|c|c|c|c|}
\hline & & & & & & & & \\
\hline Kinase & Staurosporine @ $1 \mu \mathrm{M}$ & Hit & sorted & normalized & position & cumulative sample fraction & inhibition\% & fraction of total inhibition \\
\hline $\mathrm{Abl}(\mathrm{m})$ & 9 & 1 & 96 & 96 & 1 & 0.011764706 & 4 & 0.000524246 \\
\hline AMPK(r) & 2 & 1 & 90 & 90 & 2 & 0.023529412 & 10 & 0.001310616 \\
\hline $\operatorname{Arg}(\mathrm{m})$ & 7 & 1 & 90 & 90 & 3 & 0.035294118 & 10 & 0.001310616 \\
\hline Aurora-A(h) & 0 & 1 & 88 & 88 & 4 & 0.047058824 & 12 & 0.001572739 \\
\hline Axl(h) & 3 & 1 & 61 & 61 & 5 & 0.058823529 & 39 & 0.005111402 \\
\hline $\operatorname{Blk}(\mathrm{m})$ & 0 & 1 & 61 & 61 & 6 & 0.070588235 & 39 & 0.005111402 \\
\hline Bmx(h) & 0 & 1 & 54 & 54 & 7 & 0.082352941 & 46 & 0.006028834 \\
\hline CaMKII(r) & 0 & 1 & 48 & 48 & 8 & 0.094117647 & 52 & 0.006815203 \\
\hline CaMKIV(h) & 2 & 1 & 40 & 40 & 9 & 0.105882353 & 60 & 0.007863696 \\
\hline CDK1/cyclinB(h) & 0 & 1 & 24 & 24 & 10 & 0.117647059 & 76 & 0.009960682 \\
\hline $\mathrm{CDK} 2 / \mathrm{cyclinA}(\mathrm{h})$ & 1 & 1 & 22 & 22 & 11 & 0.129411765 & 78 & 0.010222805 \\
\hline $\mathrm{CDK} 2 /$ cyclinE(h) & -4 & 1 & 18 & 18 & 12 & 0.141176471 & 82 & 0.010747051 \\
\hline CDK3/cyclinE(h) & 1 & 1 & 17 & 17 & 13 & 0.152941176 & 83 & 0.010878113 \\
\hline $\mathrm{CDK5} / \mathrm{p35( \textrm {h } )}$ & 0 & 1 & 14 & 14 & 14 & 0.164705882 & 86 & 0.011271298 \\
\hline $\mathrm{CDK} 6 /$ cyclinD3(h) & 17 & 1 & 13 & 13 & 15 & 0.176470588 & 87 & 0.011402359 \\
\hline CDK7//yclinHMATI(h) & 3 & 1 & 13 & 13 & 16 & 0.188235294 & 87 & 0.011402359 \\
\hline CHK $1(\mathrm{~h})$ & 1 & 1 & 12 & 12 & 17 & 0.2 & 88 & 0.011533421 \\
\hline СHK2(h) & 3 & 1 & 11 & 11 & 18 & 0.211764706 & 89 & 0.011664482 \\
\hline $\mathrm{CKI}(y)$ & 14 & 1 & 10 & 10 & 19 & 0.223529412 & 90 & 0.011795544 \\
\hline CK2(h) & 96 & 0 & 9 & 9 & 20 & 0.235294118 & 91 & 0.011926606 \\
\hline $\mathrm{c}-\mathrm{RAF}(\mathrm{h})$ & 90 & 0 & 9 & 9 & 21 & 0.247058824 & 91 & 0.011926606 \\
\hline $\operatorname{CSK}(\mathrm{h})$ & 10 & 1 & 7 & 7 & 22 & 0.258823529 & 93 & 0.012188729 \\
\hline cSRC(h) & 4 & 1 & 6 & 6 & 23 & 0.270588235 & 94 & 0.01231979 \\
\hline Fes(h) & 0 & 1 & 6 & 6 & 24 & 0.282352941 & 94 & 0.01231979 \\
\hline FGFR3(h) & 1 & 1 & 5 & 5 & 25 & 0.294117647 & 95 & 0.012450852 \\
\hline Fll3(h) & -1 & 1 & 4 & 4 & 26 & 0.305882353 & 96 & 0.012581913 \\
\hline Fyn(h) & 0 & 1 & 3 & 3 & 27 & 0.317647059 & 97 & 0.012712975 \\
\hline GSK3a(h) & 1 & 1 & 3 & 3 & 28 & 0.329411765 & 97 & 0.012712975 \\
\hline GSK3B(h) & 2 & 1 & 3 & 3 & 29 & 0.341176471 & 97 & 0.012712975 \\
\hline IGF-IR(h) & 11 & 1 & 3 & 3 & 30 & 0.352941176 & 97 & 0.012712975 \\
\hline IKKa(h) & 6 & 1 & 3 & 3 & 31 & 0.364705882 & 97 & 0.012712975 \\
\hline IKKB(h) & 40 & 1 & 3 & 3 & 32 & 0.376470588 & 97 & 0.012712975 \\
\hline $\mathrm{IR}(\mathrm{h})$ & 6 & 1 & 2 & 2 & 33 & 0.388235294 & 98 & 0.012844037 \\
\hline JNKIal(h) & 22 & 1 & 2 & 2 & 34 & 0.4 & 98 & 0.012844037 \\
\hline JNK202(h) & 54 & 0 & 2 & 2 & 35 & 0.411764706 & 98 & 0.012844037 \\
\hline $\mathrm{JNK} 3(\mathrm{r})$ & 18 & 1 & 2 & 2 & 36 & 0.423529412 & 98 & 0.012844037 \\
\hline Lck(h) & 0 & 1 & 2 & 2 & 37 & 0.435294118 & 98 & 0.012844037 \\
\hline Lyn(h) & -4 & 1 & 2 & 2 & 38 & 0.447058824 & 98 & 0.012844037 \\
\hline Lyn(m) & 0 & 1 & 2 & 2 & 39 & 0.458823529 & 98 & 0.012844037 \\
\hline MAPK I(h) & 61 & 0 & 1 & 1 & 40 & 0.470588235 & 99 & 0.012975098 \\
\hline MAPK2(h) & 61 & 0 & 1 & 1 & 41 & 0.482352941 & 99 & 0.012975098 \\
\hline MAPK2(m) & 48 & 1 & 1 & 1 & 42 & 0.494117647 & 99 & 0.012975098 \\
\hline MAPKAP-K2(h) & 1 & 1 & 1 & 1 & 43 & 0.505882353 & 99 & 0.012975098 \\
\hline MEK1(h) & -2 & 1 & 1 & 1 & 44 & 0.517647059 & 99 & 0.012975098 \\
\hline MKK $4(\mathrm{~m})$ & 1 & 1 & 1 & 1 & 45 & 0.529411765 & 99 & 0.012975098 \\
\hline MKK6(h) & 2 & 1 & 1 & 1 & 46 & 0.541176471 & 99 & 0.012975098 \\
\hline МKK $78($ (h) & 12 & 1 & 1 & 1 & 47 & 0.552941176 & 99 & 0.012975098 \\
\hline MSKI(h) & 1 & 1 & 1 & 1 & 48 & 0.564705882 & 99 & 0.012975098 \\
\hline $\mathrm{p} 70 \mathrm{~S} 6 \mathrm{~K}(\mathrm{~h})$ & 1 & 1 & 1 & 1 & 49 & 0.576470588 & 99 & 0.012975098 \\
\hline PAK2(h) & 0 & 1 & 0 & 0 & 50 & 0.588235294 & 100 & 0.01310616 \\
\hline PDGFRa(h) & 0 & 1 & 0 & 0 & 51 & 0.6 & 100 & 0.01310616 \\
\hline PDGFRB(h) & 2 & 1 & 0 & 0 & 52 & 0.611764706 & 100 & 0.01310616 \\
\hline PDK I(h) & -3 & 1 & 0 & 0 & 53 & 0.623529412 & 100 & 0.01310616 \\
\hline PKA(b) & -2 & 1 & 0 & 0 & 54 & 0.635294118 & 100 & 0.01310616 \\
\hline PKA(h) & 0 & 1 & 0 & 0 & 55 & 0.647058824 & 100 & 0.01310616 \\
\hline PKBa(h) & 2 & 1 & 0 & 0 & 56 & 0.658823529 & 100 & 0.01310616 \\
\hline PKBB(h) & 3 & 1 & 0 & 0 & 57 & 0.670588235 & 100 & 0.01310616 \\
\hline $\mathrm{PKB}_{\gamma}(\mathrm{h})$ & -1 & 1 & 0 & 0 & 58 & 0.682352941 & 100 & 0.01310616 \\
\hline PKCa(h) & 5 & 1 & 0 & 0 & 59 & 0.694117647 & 100 & 0.01310616 \\
\hline РКCBII(h) & 0 & 1 & 0 & 0 & 60 & 0.705882353 & 100 & 0.01310616 \\
\hline PKCy(h) & 3 & 1 & 0 & 0 & 61 & 0.717647059 & 100 & 0.01310616 \\
\hline PKG(h) & 0 & 1 & 0 & 0 & 62 & 0.729411765 & 100 & 0.01310616 \\
\hline PKG:(h) & -1 & 1 & 0 & 0 & 63 & 0.741176471 & 100 & 0.01310616 \\
\hline PKG (h) & -1 & 1 & 0 & 0 & 64 & 0.752941176 & 100 & 0.01310616 \\
\hline PKC (h) & 9 & 1 & 0 & 0 & 65 & 0.764705882 & 100 & 0.01310616 \\
\hline PKC $\mu(\mathrm{h})$ & 0 & 1 & 0 & 0 & 66 & 0.776470588 & 100 & 0.01310616 \\
\hline PKCO(h) & -1 & 1 & 0 & 0 & 67 & 0.788235294 & 100 & 0.01310616 \\
\hline PKD2(h) & 2 & 1 & 0 & 0 & 68 & 0.8 & 100 & 0.01310616 \\
\hline PRAK(h) & 13 & 1 & 0 & 0 & 69 & 0.811764706 & 100 & 0.01310616 \\
\hline PRK2(h) & 0 & 1 & 0 & 0 & 70 & 0.823529412 & 100 & 0.01310616 \\
\hline ROCK-II(h) & 0 & 1 & 0 & 0 & 71 & 0.835294118 & 100 & 0.01310616 \\
\hline ROCK-II(r) & -1 & 1 & -1 & 0 & 72 & 0.847058824 & 100 & 0.01310616 \\
\hline Rskl(h) & 0 & 1 & -1 & 0 & 73 & 0.858823529 & 100 & 0.01310616 \\
\hline Rskl(r) & 1 & 1 & -1 & 0 & 74 & 0.870588235 & 100 & 0.01310616 \\
\hline Rsk2(h) & 0 & 1 & -1 & 0 & 75 & 0.882352941 & 100 & 0.01310616 \\
\hline Rsk3(h) & 0 & 1 & -1 & 0 & 76 & 0.894117647 & 100 & 0.01310616 \\
\hline SAPK2a(h) & 88 & 0 & -1 & 0 & 77 & 0.905882353 & 100 & 0.01310616 \\
\hline SAPK2b(h) & 90 & 0 & -1 & 0 & 78 & 0.917647059 & 100 & 0.01310616 \\
\hline SAPK3(h) & 24 & 1 & -1 & 0 & 79 & 0.929411765 & 100 & 0.01310616 \\
\hline $\mathrm{SAPK} 4(\mathrm{~h})$ & 13 & 1 & -2 & 0 & 80 & 0.941176471 & 100 & 0.01310616 \\
\hline SGK(h) & 3 & 1 & -2 & 0 & 81 & 0.952941176 & 100 & 0.01310616 \\
\hline Syk(h) & -1 & 1 & -3 & 0 & 82 & 0.964705882 & 100 & 0.01310616 \\
\hline TrkB(h) & -1 & 1 & -4 & 0 & 83 & 0.976470588 & 100 & 0.01310616 \\
\hline Yes(h) & 0 & 1 & -4 & 0 & 84 & 0.988235294 & 100 & 0.01310616 \\
\hline ZAP-70(h) & -4 & 1 & -4 & 0 & 85 & 1 & 100 & 0.01310616 \\
\hline & & & & & & Total inhibition & 7630 & \\
\hline & $\begin{array}{c}\text { Hit rate } \\
\text { Threshold }\end{array}$ & & & & & & & \\
\hline & Threshold & & & & & & & \\
\hline
\end{tabular}




\begin{tabular}{|c|c|c|c|c|c|c|c|c|c|c|}
\hline & $100 \mu \mathrm{M}$ ATP & & & & & & & & & \\
\hline Kinase & Staurosporine @ $1 \mu \mathrm{M}$ & Hit & $\begin{array}{l}\text { sorted } \\
114\end{array}$ & $\begin{array}{l}\text { normalized } \\
100\end{array}$ & position $_{1}$ & $\begin{array}{c}\text { cumulative sample fraction } \\
0.011764706\end{array}$ & inhibition\% & $\begin{array}{c}\text { fraction of total inhibition } \\
0\end{array}$ & $\begin{array}{c}\text { cumulative inhibition } \\
0\end{array}$ & area \\
\hline $\begin{array}{l}\mathrm{Abl}(\mathrm{m}) \\
\mathrm{AMPK}(\mathrm{r})\end{array}$ & $\begin{array}{l}39 \\
-5\end{array}$ & 1 & 97 & 97 & 2 & 0.023529412 & 3 & 0.000424328 & 0.000424328 & $2.49605 \mathrm{E}-06$ \\
\hline $\operatorname{Arg}(\mathrm{m})$ & 17 & 1 & 92 & 92 & 3 & 0.035294118 & 8 & 0.001131542 & 0.00155587 & 1.16482E-05 \\
\hline Aurora-A(h) & 4 & 1 & 84 & 84 & 4 & 0.047058824 & 16 & 0.002263083 & 0.003818953 & 3.16166E-05 \\
\hline Axl(h) & 3 & 1 & 82 & 82 & 5 & 0.058823529 & 18 & 0.002545969 & 0.006364922 & $5.99052 \mathrm{E}-05$ \\
\hline $\operatorname{Blk}(\mathrm{m})$ & 0 & 1 & 80 & 80 & 6 & 0.070588235 & 20 & 0.002828854 & 0.009193777 & $9.15218 \mathrm{E}-05$ \\
\hline $\operatorname{Bmx}(\mathrm{h})$ & -4 & 1 & 71 & 71 & 7 & 0.082352941 & 29 & 0.004101839 & 0.013295615 & 0.000132291 \\
\hline CaMKII(r) & 0 & 1 & 69 & 69 & 8 & 0.094117647 & 31 & 0.004384724 & 0.017680339 & 0.000182211 \\
\hline CaMKIV(h) & 12 & 1 & 64 & 64 & 9 & 0.105882353 & 36 & 0.005091938 & 0.022772277 & 0.000237957 \\
\hline CDK $1 /$ cyclnin (h) & 1 & 1 & 59 & 59 & 10 & 0.117647059 & 41 & 0.005799151 & 0.028571429 & 0.000302022 \\
\hline $\mathrm{CDK} 2 /$ cyclinA(h) & -3 & 1 & 56 & 56 & 11 & 0.129411765 & 44 & 0.006223479 & 0.034794908 & 0.000372743 \\
\hline $\mathrm{CDK} 2 /$ cyclinE(h) & 1 & 1 & 54 & 54 & 12 & 0.141176471 & 46 & 0.006506365 & 0.041301273 & 0.000447625 \\
\hline CDK3/cyclinE(h) & -5 & 1 & 53 & 53 & 13 & 0.152941176 & 47 & 0.006647808 & 0.047949081 & 0.000525002 \\
\hline CDK5/p35(h) & 19 & 1 & 47 & 47 & 14 & 0.164705882 & 53 & 0.007496464 & 0.055445545 & 0.000608204 \\
\hline CDK6/cyclinD3(h) & $\begin{array}{l}19 \\
25\end{array}$ & 1 & $\begin{array}{l}47 \\
46\end{array}$ & $\begin{array}{l}47 \\
46\end{array}$ & 15 & 0.176470588 & 54 & 0.007637907 & 0.063083451 & 0.000697229 \\
\hline CDK 7/cyclinHMATI(h) & 5 & 1 & 39 & 39 & 16 & 0.188235294 & 61 & 0.008628006 & 0.071711457 & 0.000792911 \\
\hline CHK1 (h) & 0 & 1 & 34 & 34 & 17 & 0.2 & 66 & 0.009335219 & 0.081046676 & 0.000898577 \\
\hline СHK2(h) & 14 & 1 & 31 & 31 & 18 & 0.211764706 & 69 & 0.009759547 & 0.090806223 & 0.001010899 \\
\hline $\mathrm{CK} 1(\mathrm{y})$ & 27 & 1 & 27 & 27 & 19 & 0.223529412 & 73 & 0.010325318 & 0.101131542 & 0.001129046 \\
\hline СК2(h) & 97 & 0 & 25 & 25 & 20 & 0.235294118 & 75 & 0.010608204 & 0.111739745 & 0.001252184 \\
\hline c-RAF(h) & 92 & 0 & 22 & 22 & 21 & 0.247058824 & 78 & 0.011032532 & 0.122772277 & 0.001379482 \\
\hline CSK(h) & 46 & 1 & 19 & 19 & 22 & 0.258823529 & 81 & 0.01145686 & 0.134229137 & 0.001511773 \\
\hline $\operatorname{csRC(h)}$ & 2 & 1 & 17 & 17 & 23 & 0.270588235 & 83 & 0.011739745 & 0.145968883 & 0.001648224 \\
\hline Fes(h) & -2 & 1 & 17 & 17 & 24 & 0.282352941 & 83 & 0.011739745 & 0.157708628 & 0.001786338 \\
\hline FGFR3(h) & 0 & 1 & 15 & 15 & 25 & 0.294117647 & 85 & 0.012022631 & 0.169731259 & 0.001926117 \\
\hline Fllts(h) & 0 & 1 & 15 & 15 & 26 & 0.305882353 & 85 & 0.012022631 & 0.18175389 & 0.00206756 \\
\hline $\begin{array}{l}\text { Filis(n) } \\
\text { Fyn(h) }\end{array}$ & 1 & 1 & 14 & 14 & 27 & 0.317647059 & $\begin{array}{l}85 \\
86\end{array}$ & 0.012164074 & 0.193917963 & 0.002209834 \\
\hline GSK3ath & 5 & 1 & 12 & 12 & 28 & 0.329411765 & 88 & 0.012446959 & 0.206364922 & 0.002354605 \\
\hline GSK $3 \mathrm{~B}(\mathrm{~h})$ & 9 & 1 & 9 & 9 & 29 & 0.341176471 & 91 & 0.012871287 & 0.219236209 & 0.002503536 \\
\hline IGF-1R(h) & 31 & 1 & 8 & 8 & 30 & 0.352941176 & 92 & 0.01301273 & 0.232248939 & 0.002655795 \\
\hline $\mathrm{IKKa( \textrm {h } )}$ & 22 & 1 & 8 & 8 & 31 & 0.364705882 & 92 & 0.01301273 & 0.245261669 & 0.002808886 \\
\hline IKKB(h) & 47 & 1 & 7 & 7 & 32 & 0.376470588 & 93 & 0.013154173 & 0.258415842 & 0.002962809 \\
\hline $\mathbb{I R ( h )}$ & 17 & 1 & 6 & 6 & $\begin{array}{l}32 \\
33\end{array}$ & 0.388235294 & 94 & 0.013295615 & 0.271711457 & 0.003118396 \\
\hline JNKIal(h) & 69 & 0 & 5 & 5 & 34 & 0.4 & 95 & 0.013437058 & 0.285148515 & 0.003275647 \\
\hline JNK202(h) & 80 & 0 & 5 & 5 & 35 & 0.411764706 & 95 & 0.013437058 & 0.298585573 & 0.00343373 \\
\hline JNK3(r) & 53 & 0 & 4 & 4 & 36 & 0.423529412 & 96 & 0.013578501 & 0.312164074 & 0.003592645 \\
\hline Lck(h) & 0 & 1 & 4 & 4 & 37 & 0.435294118 & 96 & 0.013578501 & 0.325742574 & 0.003752392 \\
\hline (Lekn) & 0 & 1 & 4 & $\begin{array}{l}4 \\
4\end{array}$ & 38 & 0.447058824 & 96 & 0.013578501 & 0.339321075 & 0.003912139 \\
\hline Lyy(m) & -1 & 1 & 3 & 3 & 39 & 0.458823529 & 97 & 0.013719943 & 0.353041018 & 0.004072718 \\
\hline MAPKI(h) & $\begin{array}{l}-1 \\
71\end{array}$ & 0 & 3 & 3 & 40 & 0.470588235 & 97 & 0.013719943 & 0.366760962 & 0.004234129 \\
\hline MAPK2(h) & 82 & 0 & 3 & 3 & 41 & 0.482352941 & 97 & 0.013719943 & 0.380480905 & 0.00439554 \\
\hline MAPK2(m) & 59 & 0 & 3 & 3 & 42 & 0.494117647 & 97 & 0.013719943 & 0.394200849 & 0.004556951 \\
\hline MAPKAP-K2(h) & 6 & 1 & 2 & 2 & 43 & 0.505882353 & 98 & 0.013861386 & 0.408062235 & 0.004719195 \\
\hline 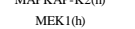 & $\begin{array}{l}6 \\
3\end{array}$ & 1 & 2 & 2 & 44 & 0.517647059 & 98 & 0.013861386 & 0.421923621 & 0.00488227 \\
\hline MKK4(m) & 64 & 0 & 2 & 2 & 45 & 0.529411765 & 98 & 0.013861386 & 0.435785007 & 0.005045345 \\
\hline MKKG(h) & 0 & 1 & 2 & 2 & 46 & 0.541176471 & 98 & 0.013861386 & 0.449646393 & 0.00520842 \\
\hline MKK 7 (h) & 15 & 1 & 1 & 1 & 47 & 0.552941176 & 99 & 0.014002829 & 0.463649222 & 0.005372327 \\
\hline MSKI(h) & 0 & 1 & 1 & 1 & 48 & 0.564705882 & 99 & 0.014002829 & 0.477652051 & 0.005537066 \\
\hline $\mathrm{p} 70 \mathrm{~S} 6 \mathrm{~K}(\mathrm{~h})$ & 0 & 1 & 1 & 1 & 49 & 0.576470588 & 99 & 0.014002829 & 0.49165488 & 0.005701805 \\
\hline $\begin{array}{l}\text { PAK2(h) } \\
\text { Past }\end{array}$ & 1 & 1 & 1 & 1 & 50 & 0.588235294 & 99 & 0.014002829 & 0.505657709 & 0.005866545 \\
\hline PDGFRa(h) & 1 & 1 & 1 & 1 & 51 & 0.6 & 99 & 0.014002829 & 0.519660537 & 0.006031284 \\
\hline PDGFRB(h) & -1 & 1 & 1 & 1 & 52 & 0.611764706 & 99 & 0.014002829 & 0.533663366 & 0.006196023 \\
\hline PDKI(h) & $\begin{array}{l}-1 \\
-2\end{array}$ & 1 & 1 & 1 & 53 & 0.623529412 & 99 & 0.014002829 & 0.547666195 & 0.006360762 \\
\hline PKA(b) & 2 & 1 & 1 & 1 & 54 & 0.635294118 & 99 & 0.014002829 & 0.561669024 & 0.006525501 \\
\hline 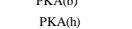 & 0 & 1 & 1 & 1 & $\begin{array}{l}54 \\
55\end{array}$ & 0.647058824 & $\begin{array}{l}99 \\
99\end{array}$ & 0.014002829 & 0.575671853 & 0.00669024 \\
\hline PKBa(h) & 2 & 1 & 0 & 0 & 56 & 0.658823529 & 100 & 0.014144272 & 0.589816124 & 0.006855812 \\
\hline PKBB(h) & 3 & 1 & 0 & 0 & 57 & 0.670588235 & 100 & 0.014144272 & 0.603960396 & 0.007022215 \\
\hline $\mathrm{PKB} \mathrm{B}_{(\mathrm{h})}$ & 0 & 1 & 0 & 0 & 58 & 0.682352941 & 100 & 0.014144272 & 0.618104668 & 0.007188618 \\
\hline PKCu(h) & 1 & 1 & 0 & 0 & 59 & 0.694117647 & 100 & 0.014144272 & 0.632248939 & 0.007355021 \\
\hline $\begin{array}{l}\text { PKCBII(h) } \\
\text { Р }\end{array}$ & 8 & 1 & 0 & 0 & 60 & 0.705882353 & 100 & 0.014144272 & 0.646393211 & 0.007521424 \\
\hline PKCy(h) & 3 & 1 & 0 & 0 & 61 & 0.717647059 & 100 & 0.014144272 & 0.660537482 & 0.007687828 \\
\hline РКФ(1) & 4 & 1 & 0 & 0 & $\begin{array}{l}61 \\
62\end{array}$ & 0.729411765 & 100 & 0.014144272 & 0.674681754 & 0.007854231 \\
\hline PKG:(h) & 0 & 1 & 0 & 0 & 63 & 0.741176471 & 100 & 0.014144272 & 0.688826025 & 0.008020634 \\
\hline $\begin{array}{l}\text { PKG (h) } \\
\text { (h) }\end{array}$ & 7 & 1 & 0 & 0 & 64 & 0.752941176 & 100 & 0.014144272 & 0.702970297 & 0.008187037 \\
\hline PKC (h) & 15 & 1 & 0 & 0 & 65 & 0.764705882 & 100 & 0.014144272 & 0.717114569 & 0.00835344 \\
\hline PKC $\mu(\mathrm{h})$ & 1 & 1 & 0 & 0 & 66 & 0.776470588 & 100 & 0.014144272 & 0.73125884 & 0.008519844 \\
\hline PKCO(h) & 4 & 1 & 0 & 0 & 67 & 0.788235294 & 100 & 0.014144272 & 0.745403112 & 0.008686247 \\
\hline PKD2(h) & 1 & 1 & 0 & 0 & 68 & 0.8 & 100 & 0.014144272 & 0.759547383 & 0.00885265 \\
\hline PRAK(h) & 56 & 0 & 0 & 0 & 69 & 0.811764706 & 100 & 0.014144272 & 0.773691655 & 0.009019053 \\
\hline PRK2(h) & -11 & 1 & 0 & 0 & 70 & 0.823529412 & 100 & 0.014144272 & 0.787835926 & 0.009185456 \\
\hline ROCK-II(h) & -2 & 1 & 0 & 0 & 71 & 0.835294118 & 100 & 0.014144272 & 0.801980198 & 0.00935186 \\
\hline ROCK-II(r) & -1 & 1 & 0 & 0 & 72 & 0.847058824 & 100 & 0.014144272 & 0.81612447 & 0.009518263 \\
\hline Rskl(h) & $\begin{array}{r}-1 \\
0\end{array}$ & 1 & $\begin{array}{c}0 \\
-1\end{array}$ & 0 & 73 & 0.858823529 & 100 & 0.014144272 & 0.830268741 & 0.009684666 \\
\hline Rskl(r) & 2 & 1 & -1 & 0 & 74 & 0.870588235 & 100 & 0.014144272 & 0.844413013 & 0.009851069 \\
\hline $\begin{array}{l}\text { Rsksk2(h) } \\
\text { Rsk(h) }\end{array}$ & -1 & 1 & -1 & 0 & 75 & 0.882352941 & 100 & 0.014144272 & 0.858557284 & 0.010017472 \\
\hline Rsk3(h) & 0 & 1 & -1 & 0 & 76 & 0.894117647 & 100 & 0.014144272 & 0.872701556 & 0.010183876 \\
\hline SAPK2a(h) & 84 & 0 & -2 & 0 & 77 & 0.905882353 & 100 & 0.014144272 & 0.886845827 & 0.010350279 \\
\hline SAPK2b(h) & 114 & 0 & $\begin{array}{l}-2 \\
-2\end{array}$ & 0 & 78 & 0.917647059 & 100 & 0.014144272 & 0.900990099 & 0.010516682 \\
\hline SAPK $3(\mathrm{~h})$ & 54 & 0 & -2 & 0 & 79 & 0.929411765 & 100 & 0.014144272 & 0.915134371 & 0.010683085 \\
\hline SAPK4(h) & 34 & 1 & -3 & 0 & 80 & 0.941176471 & 100 & 0.014144272 & 0.929278642 & 0.010849488 \\
\hline 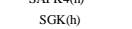 & $\begin{array}{c}04 \\
0\end{array}$ & 1 & -3 & 0 & 81 & 0.952941176 & 100 & 0.014144272 & 0.943422914 & 0.011015892 \\
\hline Syk(h) & -3 & 1 & -4 & 0 & 82 & 0.964705882 & 100 & 0.014144272 & 0.957567185 & 0.011182295 \\
\hline TrkB(h) & 0 & 1 & -5 & 0 & 83 & 0.976470588 & 100 & 0.014144272 & 0.971711457 & 0.011348698 \\
\hline Yes(h) & 1 & 1 & -5 & 0 & 84 & 0.988235294 & 100 & 0.014144272 & 0.985855728 & 0.011515101 \\
\hline ZAP-70(h) & 8 & 1 & -11 & 0 & 85 & 1 & 100 & 0.014144272 & 1 & 0.011681504 \\
\hline & & & & & & Total inhibition & 7070 & & Total area & 0.425141859 \\
\hline & Hit rate & 7 & & & & & & & Gini & 0149716283 \\
\hline
\end{tabular}




\begin{tabular}{|c|c|c|c|c|c|c|c|c|c|}
\hline & $10 \mu \mathrm{M}$ ATP & & & & & & & & \\
\hline Kinase & SU6656 @ 1 M & Hit & sorted & normalized & position & cumulative sample fraction & inhibition\% & fraction of total inhibition & cumulative inhibition \\
\hline $\mathrm{Abl}(\mathrm{m})$ & 18 & 1 & 115 & 100 & 1 & 0.011764706 & 0 & 0 & 0 \\
\hline AMPK(r) & 53 & 0 & 109 & 100 & 2 & 0.023529412 & 0 & 0 & 0 \\
\hline $\operatorname{Arg}(\mathrm{m})$ & 36 & 1 & 109 & 100 & 3 & 0.035294118 & 0 & 0 & 0 \\
\hline Aurora-A(h) & 6 & 1 & 108 & 100 & 4 & 0.047058824 & 0 & 0 & 0 \\
\hline Axl(h) & 2 & 1 & 107 & 100 & 5 & 0.058823529 & 0 & 0 & 0 \\
\hline $\mathrm{Blk}(\mathrm{m})$ & 0 & 1 & 107 & 100 & 6 & 0.070588235 & 0 & 0 & 0 \\
\hline Bnx(h) & 100 & 0 & 105 & 100 & 7 & 0.082352941 & 0 & 0 & 0 \\
\hline CaMKII(r) & 97 & 0 & 104 & 100 & 8 & 0.094117647 & 0 & 0 & 0 \\
\hline CaMKIV(h) & 94 & 0 & 104 & 100 & 9 & 0.105882353 & 0 & 0 & 0 \\
\hline CDK1/cyclnBB(h) & 22 & 1 & 104 & 100 & 10 & 0.117647059 & 0 & 0 & 0 \\
\hline $\mathrm{CDK} 2 / \mathrm{cyclinA}(\mathrm{h})$ & 61 & 0 & 102 & 100 & 11 & 0.129411765 & 0 & 0 & 0 \\
\hline $\mathrm{CDK} 2 /$ cyclinE(h) & 83 & 0 & 101 & 100 & 12 & 0.141176471 & 0 & 0 & 0 \\
\hline CDK3/cyclinE(h) & 90 & 0 & 101 & 100 & 13 & 0.152941176 & 0 & 0 & 0 \\
\hline CDK5/p35(h) & 34 & 1 & 101 & 100 & 14 & 0.164705882 & 0 & 0 & 0 \\
\hline CDKG/cyclinD3(h) & 95 & 0 & 100 & 100 & 15 & 0.176470588 & 0 & 0 & 0 \\
\hline CDK7/cyclinH/MATI(h) & 36 & 1 & 100 & 100 & 16 & 0.188235294 & 0 & 0 & 0 \\
\hline CHK1(h) & 52 & 0 & 100 & 100 & 17 & 0.2 & 0 & 0 & 0 \\
\hline СHK2(h) & 31 & 1 & 100 & 100 & 18 & 0.211764706 & 0 & 0 & 0 \\
\hline CKI(y) & 87 & 0 & 99 & 99 & 19 & 0.223529412 & 1 & 0.000406835 & 0.000406835 \\
\hline CK2(h) & 96 & 0 & 98 & 98 & 20 & 0.235294118 & 2 & 0.00081367 & 0.001220504 \\
\hline $\mathrm{c}-\mathrm{RAF}(\mathrm{h})$ & 102 & 0 & 98 & 98 & 21 & 0.247058824 & 2 & 0.00081367 & 0.002034174 \\
\hline $\operatorname{CSK}(\mathrm{h})$ & 71 & 0 & 98 & 98 & 22 & 0.258823529 & 2 & 0.00081367 & 0.002847844 \\
\hline cSRC(h) & 7 & 1 & 98 & 98 & 23 & 0.270588235 & 2 & 0.00081367 & 0.003661513 \\
\hline Fes(h) & 44 & 1 & 97 & 97 & 24 & 0.282352941 & 3 & 0.001220504 & 0.004882018 \\
\hline FGFR3(h) & 72 & 0 & 97 & 97 & 25 & 0.294117647 & 3 & 0.001220504 & 0.006102522 \\
\hline Fll3(h) & 10 & 1 & 96 & 96 & 26 & 0.305882353 & 4 & 0.001627339 & 0.007729862 \\
\hline Fyn(h) & 1 & 1 & 96 & 96 & 27 & 0.317647059 & 4 & 0.001627339 & 0.009357201 \\
\hline GSK3a(h) & 94 & 0 & 95 & 95 & 28 & 0.329411765 & 5 & 0.002034174 & 0.011391375 \\
\hline GSK3B(h) & 75 & 0 & 95 & 95 & 29 & 0.341176471 & 5 & 0.002034174 & 0.013425549 \\
\hline IGF-IR(h) & 105 & 0 & 95 & 95 & 30 & 0.352941176 & 5 & 0.002034174 & 0.015459723 \\
\hline IKKa(h) & 92 & 0 & 94 & 94 & 31 & 0.364705882 & 6 & 0.002441009 & 0.017900732 \\
\hline IKKB(h) & 100 & 0 & 94 & 94 & 32 & 0.376470588 & 6 & 0.002441009 & 0.020341741 \\
\hline $\mathbb{I R ( h )}$ & 104 & 0 & 94 & 94 & 33 & 0.388235294 & 6 & 0.002441009 & 0.02278275 \\
\hline JNKIal(h) & 93 & 0 & 93 & 93 & 34 & 0.4 & 7 & 0.002847844 & 0.025630594 \\
\hline JNK202(h) & 90 & 0 & 93 & 93 & 35 & 0.411764706 & 7 & 0.002847844 & 0.028478438 \\
\hline $\mathrm{JNK} 3(\mathrm{r})$ & 89 & 0 & 92 & 92 & 36 & 0.423529412 & 8 & 0.003254679 & 0.031733116 \\
\hline Lck(h) & 6 & 1 & 91 & 91 & 37 & 0.435294118 & 9 & 0.003661513 & 0.03539463 \\
\hline Lyn(h) & -1 & 1 & 91 & 91 & 38 & 0.447058824 & 9 & 0.003661513 & 0.039056143 \\
\hline Lyn(m) & 1 & 1 & 90 & 90 & 39 & 0.458823529 & 10 & 0.004068348 & 0.043124491 \\
\hline MAPK I(h) & 107 & 0 & 90 & 90 & 40 & 0.470588235 & 10 & 0.004068348 & 0.04719284 \\
\hline MAPK2(h) & 100 & 0 & 89 & 89 & 41 & 0.482352941 & 11 & 0.004475183 & 0.051668023 \\
\hline MAPK2(m) & 85 & 0 & 89 & 89 & 42 & 0.494117647 & 11 & 0.004475183 & 0.056143206 \\
\hline MAPKAP-K2(h) & 98 & 0 & 87 & 87 & 43 & 0.505882353 & 13 & 0.005288853 & 0.061432059 \\
\hline MEK 1(h) & 100 & 0 & 87 & 87 & 44 & 0.517647059 & 13 & 0.005288853 & 0.066720911 \\
\hline MKK $4(\mathrm{~m})$ & 83 & 0 & 86 & 86 & 45 & 0.529411765 & 14 & 0.005695688 & 0.072416599 \\
\hline MKK6(h) & 85 & 0 & 85 & 85 & 46 & 0.541176471 & 15 & 0.006102522 & 0.078519121 \\
\hline МKK $78($ (h) & 109 & 0 & 85 & 85 & 47 & 0.552941176 & 15 & 0.006102522 & 0.084621644 \\
\hline MSK I(h) & 98 & 0 & 83 & 83 & 48 & 0.564705882 & 17 & 0.006916192 & 0.091537836 \\
\hline $\mathrm{p} 7056 \mathrm{~K}(\mathrm{~h})$ & 76 & 0 & 83 & 83 & 49 & 0.576470588 & 17 & 0.006916192 & 0.098454028 \\
\hline PAK2(h) & 101 & 0 & 76 & 76 & 50 & 0.588235294 & 24 & 0.009764036 & 0.108218063 \\
\hline PDGFRa(h) & 54 & 0 & 75 & 75 & 51 & 0.6 & 25 & 0.010170871 & 0.118388934 \\
\hline PDGFRB(h) & 68 & 0 & 74 & 74 & 52 & 0.611764706 & 26 & 0.010577705 & 0.12896664 \\
\hline PDK I(h) & 115 & 0 & 72 & 72 & 53 & 0.623529412 & 28 & 0.011391375 & 0.140358015 \\
\hline PKA(b) & 74 & 0 & 71 & 71 & 54 & 0.635294118 & 29 & 0.01179821 & 0.152156225 \\
\hline PKA(h) & 108 & 0 & 71 & 71 & 55 & 0.647058824 & 29 & 0.01179821 & 0.163954434 \\
\hline PKBa(h) & 99 & 0 & 70 & 70 & 56 & 0.658823529 & 30 & 0.012205045 & 0.176159479 \\
\hline PKBB(h) & 107 & 0 & 68 & 68 & 57 & 0.670588235 & 32 & 0.013018714 & 0.189178194 \\
\hline $\mathrm{PKB}_{\gamma}(\mathrm{h})$ & 95 & 0 & 64 & 64 & 58 & 0.682352941 & 36 & 0.014646054 & 0.203824247 \\
\hline PKGa(h) & 91 & 0 & 61 & 61 & 59 & 0.694117647 & 39 & 0.015866558 & 0.219690806 \\
\hline РКСBII(h) & 91 & 0 & 58 & 58 & 60 & 0.705882353 & 42 & 0.017087063 & 0.236777868 \\
\hline PKCy(h) & 94 & 0 & 56 & 56 & 61 & 0.717647059 & 44 & 0.017900732 & 0.2546786 \\
\hline PKळ(h) & 104 & 0 & 55 & 55 & 62 & 0.729411765 & 45 & 0.018307567 & 0.272986168 \\
\hline PKG:(h) & 89 & 0 & 54 & 54 & 63 & 0.741176471 & 46 & 0.018714402 & 0.29170057 \\
\hline PKG (h) & 101 & 0 & 54 & 54 & 64 & 0.752941176 & 46 & 0.018714402 & 0.310414972 \\
\hline PKC (h) & 104 & 0 & 53 & 53 & 65 & 0.764705882 & 47 & 0.019121237 & 0.329536208 \\
\hline PKC $\mu(\mathrm{h})$ & 96 & 0 & 52 & 52 & 66 & 0.776470588 & 48 & 0.019528072 & 0.34906428 \\
\hline PKCO(h) & 87 & 0 & 44 & 44 & 67 & 0.788235294 & 56 & 0.02278275 & 0.37184703 \\
\hline PKD2(h) & 86 & 0 & 44 & 44 & 68 & 0.8 & 56 & 0.02278275 & 0.39462978 \\
\hline PRAK(h) & 98 & 0 & 36 & 36 & 69 & 0.811764706 & 64 & 0.026037429 & 0.420667209 \\
\hline PRK2(h) & 70 & 0 & 36 & 36 & 70 & 0.823529412 & 64 & 0.026037429 & 0.446704638 \\
\hline ROCK-II(h) & 54 & 0 & 34 & 34 & 71 & 0.835294118 & 66 & 0.026851098 & 0.473555736 \\
\hline ROCK-II(r) & 64 & 0 & 31 & 31 & 72 & 0.847058824 & 69 & 0.028071603 & 0.501627339 \\
\hline Rskl(h) & 56 & 0 & 22 & 22 & 73 & 0.858823529 & 78 & 0.031733116 & 0.533360456 \\
\hline Rskl(r) & 55 & 0 & 18 & 18 & 74 & 0.870588235 & 82 & 0.033360456 & 0.566720911 \\
\hline Rsk2(h) & 44 & 1 & 10 & 10 & 75 & 0.882352941 & 90 & 0.036615134 & 0.603336046 \\
\hline Rsk3(h) & 71 & 0 & 7 & 7 & 76 & 0.894117647 & 93 & 0.037835639 & 0.641171684 \\
\hline SAPK2a(h) & 98 & 0 & 6 & 6 & 77 & 0.905882353 & 94 & 0.038242474 & 0.679414158 \\
\hline SAPK2b(h) & 109 & 0 & 6 & 6 & 78 & 0.917647059 & 94 & 0.038242474 & 0.717656631 \\
\hline SAPK3(h) & 97 & 0 & 2 & 2 & 79 & 0.929411765 & 98 & 0.039869813 & 0.757526444 \\
\hline $\mathrm{SAPK} 4(\mathrm{~h})$ & 95 & 0 & 1 & 1 & 80 & 0.941176471 & 99 & 0.040276648 & 0.797803092 \\
\hline SGK(h) & 93 & 0 & 1 & 1 & 81 & 0.952941176 & 99 & 0.040276648 & 0.83807974 \\
\hline Syk(h) & 58 & 0 & 1 & 1 & 82 & 0.964705882 & 99 & 0.040276648 & 0.878356387 \\
\hline $\operatorname{TrkB}(\mathbf{h})$ & 1 & 1 & 1 & 1 & 83 & 0.976470588 & 99 & 0.040276648 & 0.918633035 \\
\hline Yes(h) & 1 & 1 & 0 & 0 & 84 & 0.988235294 & 100 & 0.040683483 & 0.959316517 \\
\hline ZAP-70(h) & 101 & 0 & -1 & 0 & 85 & 1 & 100 & 0.040683483 & 1 \\
\hline & & & & & & Total inhibition & 2458 & & Total area \\
\hline & $\begin{array}{c}\text { Hit rate } \\
\text { Threshold }\end{array}$ & . & & & & & & & Gini \\
\hline
\end{tabular}




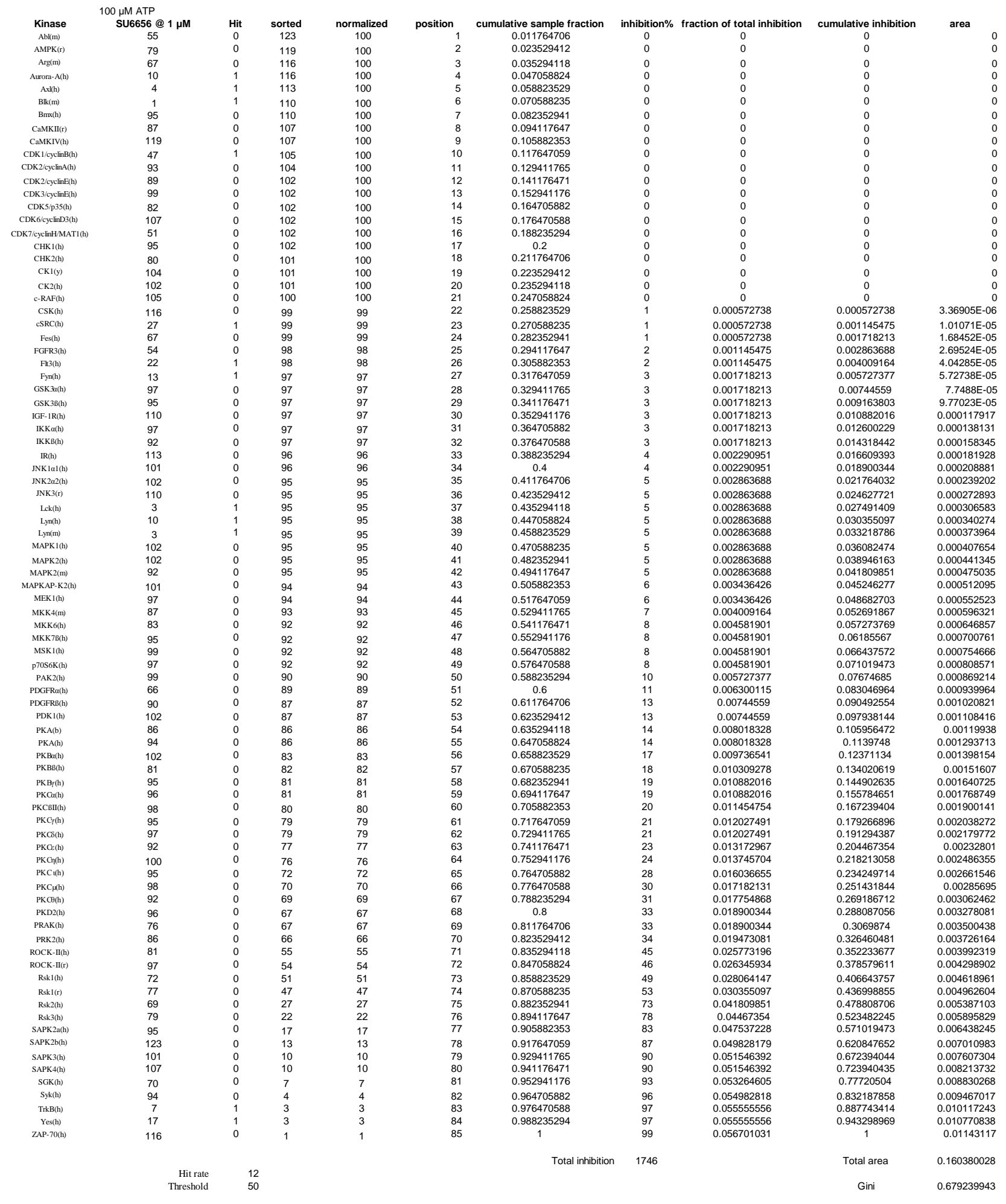




\begin{tabular}{|c|c|c|c|c|c|c|c|c|c|c|}
\hline Kinase & $\begin{array}{l}10 \mu \mathrm{M} \text { ATP } \\
\text { Wortmannin @ } 1 \mu \mathrm{M}\end{array}$ & Hit & sorted & normalized & position & cumulative sample fraction & inhibition\% & fraction of total inhibition & cumulative inhibition & area \\
\hline $\mathrm{Ab}(\mathrm{m})$ & 100 & 0 & 120 & 100 & 1 & 0.011764706 & 0 & 0 & 0 & 0 \\
\hline $\mathrm{AMPK}(\mathrm{r})$ & 105 & 0 & 118 & 100 & 2 & 0.023529412 & 0 & 0 & 0 & 0 \\
\hline $\operatorname{Arg}(\mathrm{m})$ & 93 & 0 & 118 & 100 & 3 & 0.035294118 & 0 & 0 & 0 & 0 \\
\hline Aurora-A(h) & 116 & 0 & 117 & 100 & 4 & 0.047058824 & 0 & 0 & 0 & 0 \\
\hline Axl(h) & 96 & 0 & 117 & 100 & 5 & 0.058823529 & 0 & 0 & 0 & 0 \\
\hline $\operatorname{Blk}(\mathrm{m})$ & 89 & 0 & 116 & 100 & 6 & 0.070588235 & 0 & 0 & 0 & 0 \\
\hline $\operatorname{Bmx}(\mathrm{l})$ & 120 & 0 & 115 & 100 & 7 & 0.082352941 & 0 & 0 & 0 & 0 \\
\hline CaMKII(r) & 107 & 0 & 113 & 100 & 8 & 0.094117647 & 0 & 0 & 0 & 0 \\
\hline CaMKIV(h) & 113 & 0 & 112 & 100 & 9 & 0.105882353 & 0 & 0 & 0 & 0 \\
\hline $\mathrm{CDK} 1 / \mathrm{cyclin} B(\mathrm{~h})$ & 99 & 0 & 111 & 100 & 10 & 0.117647059 & 0 & 0 & 0 & 0 \\
\hline $\operatorname{CDK} 2 /$ cyclinA(h) & 98 & 0 & 110 & 100 & 11 & 0.129411765 & 0 & 0 & 0 & 0 \\
\hline $\mathrm{CDK} 2 / \mathrm{cyclin} E(\mathrm{~h})$ & 99 & 0 & 110 & 100 & 12 & 0.141176471 & 0 & 0 & 0 & 0 \\
\hline CDK3/cyclinE(h) & 103 & 0 & 110 & 100 & 13 & 0.152941176 & 0 & 0 & 0 & 0 \\
\hline CDK5/335(h) & 102 & 0 & 109 & 100 & 14 & 0.164705882 & 0 & 0 & 0 & 0 \\
\hline CDK6/cyclinD3(h) & 100 & 0 & 109 & 100 & 15 & 0.176470588 & 0 & 0 & 0 & 0 \\
\hline CDK7/cyclinHMATI(h) & 96 & 0 & 108 & 100 & 16 & 0.188235294 & 0 & 0 & 0 & 0 \\
\hline СНК1(h) & 105 & 0 & 108 & 100 & 17 & 0.2 & 0 & 0 & 0 & 0 \\
\hline CHK2(h) & 99 & 0 & 108 & 100 & 18 & 0.211764706 & 0 & 0 & 0 & 0 \\
\hline CKI(y) & 97 & 0 & 108 & 100 & 19 & 0.223529412 & 0 & 0 & 0 & 0 \\
\hline CK2(h) & 102 & 0 & 107 & 100 & 20 & 0.235294118 & 0 & 0 & 0 & 0 \\
\hline$c-\operatorname{RAF(h)}$ & 105 & 0 & 107 & 100 & 21 & 0.247058824 & 0 & 0 & 0 & 0 \\
\hline $\operatorname{CSK(h)}$ & 72 & 0 & 107 & 100 & 22 & 0.258823529 & 0 & 0 & 0 & 0 \\
\hline $\mathrm{cSRC}(\mathrm{h})$ & 100 & 0 & 107 & 100 & 23 & 0.270588235 & 0 & 0 & 0 & 0 \\
\hline Fes(h) & 99 & 0 & 106 & 100 & 24 & 0.282352941 & 0 & 0 & 0 & 0 \\
\hline FGFR3(h) & 96 & 0 & 105 & 100 & 25 & 0.294117647 & 0 & 0 & 0 & 0 \\
\hline Flt3(h) & 102 & 0 & 105 & 100 & 26 & 0.305882353 & 0 & 0 & 0 & 0 \\
\hline Fynn(l) & 94 & 0 & 105 & 100 & 27 & 0.317647059 & 0 & 0 & 0 & 0 \\
\hline GSK3a(h) & 89 & 0 & 105 & 100 & 28 & 0.329411765 & 0 & 0 & 0 & 0 \\
\hline GSK3B(h) & 88 & 0 & 105 & 100 & 29 & 0.341176471 & 0 & 0 & 0 & 0 \\
\hline IGF-IR(h) & 110 & 0 & 105 & 100 & 30 & 0.352941176 & 0 & 0 & 0 & 0 \\
\hline IKKu(h) & 95 & 0 & 104 & 100 & 31 & 0.364705882 & 0 & 0 & 0 & 0 \\
\hline IKKB(h) & 104 & 0 & 104 & 100 & 32 & 0.376470588 & 0 & 0 & 0 & 0 \\
\hline $\mathbb{I R ( h )}$ & 101 & 0 & 103 & 100 & 33 & 0.388235294 & 0 & 0 & 0 & 0 \\
\hline JNKIal(h) & 102 & 0 & 103 & 100 & 34 & 0.4 & 0 & 0 & 0 & 0 \\
\hline JNK2a2(h) & 102 & 0 & 103 & 100 & 35 & 0.411764706 & 0 & 0 & 0 & 0 \\
\hline JNK3(r) & 109 & 0 & 102 & 100 & 36 & 0.423529412 & 0 & 0 & 0 & 0 \\
\hline Lck(h) & 107 & 0 & 102 & 100 & 37 & 0.435294118 & 0 & 0 & 0 & 0 \\
\hline Lyn(h) & 108 & 0 & 102 & 100 & 38 & 0.447058824 & 0 & 0 & 0 & 0 \\
\hline Lyn(m) & 89 & 0 & 102 & 100 & 39 & 0.458823529 & 0 & 0 & 0 & 0 \\
\hline MAPK 1(h) & 112 & 0 & 102 & 100 & 40 & 0.470588235 & 0 & 0 & 0 & 0 \\
\hline MAPK2(h) & 117 & 0 & 102 & 100 & 41 & 0.482352941 & 0 & 0 & 0 & 0 \\
\hline MAPK2(m) & 91 & 0 & 102 & 100 & 42 & 0.494117647 & 0 & 0 & 0 & 0 \\
\hline MAPKAP-K2(h) & 107 & 0 & 101 & 100 & 43 & 0.505882353 & 0 & 0 & 0 & 0 \\
\hline MEKI(h) & 100 & 0 & 101 & 100 & 44 & 0.517647059 & 0 & 0 & 0 & 0 \\
\hline MKK4(m) & 105 & 0 & 101 & 100 & 45 & 0.529411765 & 0 & 0 & 0 & 0 \\
\hline MKK6(h) & 91 & 0 & 100 & 100 & 46 & 0.541176471 & 0 & 0 & 0 & 0 \\
\hline MKK 7 (h) & 109 & 0 & 100 & 100 & 47 & 0.552941176 & 0 & 0 & 0 & 0 \\
\hline MSK1(h) & 101 & 0 & 100 & 100 & 48 & 0.564705882 & 0 & 0 & 0 & 0 \\
\hline $\mathrm{p} 70 \mathrm{~S} 6 \mathrm{~K}(\mathrm{~h})$ & 85 & 0 & 100 & 100 & 49 & 0.576470588 & 0 & 0 & 0 & 0 \\
\hline PAK2(h) & 110 & 0 & 99 & 99 & 50 & 0.588235294 & 1 & 0.003521127 & 0.003521127 & $2.07125 \mathrm{E}-05$ \\
\hline PDGFRa(h) & 62 & 0 & 99 & 99 & 51 & 0.6 & 1 & 0.003521127 & 0.007042254 & $6.21375 \mathrm{E}-05$ \\
\hline PDGFRB(h) & 99 & 0 & 99 & 99 & 52 & 0.611764706 & 1 & 0.003521127 & 0.01056338 & 0.000103563 \\
\hline PDK1(h) & 94 & 0 & 99 & 99 & 53 & 0.623529412 & 1 & 0.003521127 & 0.014084507 & 0.000144988 \\
\hline PKA(b) & 103 & 0 & 99 & 99 & 54 & 0.635294118 & 1 & 0.003521127 & 0.017605634 & 0.000186413 \\
\hline PKA(h) & 107 & 0 & 99 & 99 & 55 & 0.647058824 & 1 & 0.003521127 & 0.021126761 & 0.000227838 \\
\hline PKBa(h) & 97 & 0 & 98 & 98 & 56 & 0.658823529 & 2 & 0.007042254 & 0.028169014 & 0.000289975 \\
\hline PKBB(h) & 111 & 0 & 98 & 98 & 57 & 0.670588235 & 2 & 0.007042254 & 0.035211268 & 0.000372825 \\
\hline $\mathrm{PKB}_{\gamma}(\mathrm{h})$ & 117 & 0 & 98 & 98 & 58 & 0.682352941 & 2 & 0.007042254 & 0.042253521 & 0.000455675 \\
\hline PKGa(h) & 86 & 0 & 97 & 97 & 59 & 0.694117647 & 3 & 0.01056338 & 0.052816901 & 0.000559238 \\
\hline РКСBI(h) & 103 & 0 & 97 & 97 & 60 & 0.705882353 & 3 & 0.01056338 & 0.063380282 & 0.000683513 \\
\hline PKCy(h) & 92 & 0 & 96 & 96 & 61 & 0.717647059 & 4 & 0.014084507 & 0.077464789 & 0.0008285 \\
\hline РКФ((h) & 102 & 0 & 96 & 96 & 62 & 0.729411765 & 4 & 0.014084507 & 0.091549296 & 0.0009942 \\
\hline PKE:(h) & 86 & 0 & 96 & 96 & 63 & 0.741176471 & 4 & 0.014084507 & 0.105633803 & 0.001159901 \\
\hline PKG $(\mathrm{h})$ & 102 & 0 & 96 & 96 & 64 & 0.752941176 & 4 & 0.014084507 & 0.11971831 & 0.001325601 \\
\hline PKCi(h) & 105 & 0 & 95 & 95 & 65 & 0.764705882 & 5 & 0.017605634 & 0.137323944 & 0.001512013 \\
\hline PKC $\mu(\mathrm{h})$ & 101 & 0 & 95 & 95 & 66 & 0.776470588 & 5 & 0.017605634 & 0.154929577 & 0.001719138 \\
\hline PKCP(h) & 95 & 0 & 94 & 94 & 67 & 0.788235294 & 6 & 0.021126761 & 0.176056338 & 0.001946976 \\
\hline PKD2(h) & 99 & 0 & 94 & 94 & 68 & 0.8 & 6 & 0.021126761 & 0.197183099 & 0.002195526 \\
\hline PRAK(h) & 118 & 0 & 94 & 94 & 69 & 0.811764706 & 6 & 0.021126761 & 0.218309859 & 0.002444076 \\
\hline PRK2(h) & 88 & 0 & 93 & 93 & 70 & 0.823529412 & 7 & 0.024647887 & 0.242957746 & 0.002713339 \\
\hline ROCK-II(h) & 96 & 0 & 93 & 93 & 71 & 0.835294118 & 7 & 0.024647887 & 0.267605634 & 0.003003314 \\
\hline ROCK-II(r) & 110 & 0 & 92 & 92 & 72 & 0.847058824 & 8 & 0.028169014 & 0.295774648 & 0.003314002 \\
\hline Rskl(h) & 106 & 0 & 91 & 91 & 73 & 0.858823529 & 9 & 0.031690141 & 0.327464789 & 0.003666114 \\
\hline Rskl(r) & 84 & 0 & 91 & 91 & 74 & 0.870588235 & 9 & 0.031690141 & 0.35915493 & 0.00403894 \\
\hline Rsk2(h) & 93 & 0 & 89 & 89 & 75 & 0.882352941 & 11 & 0.038732394 & 0.397887324 & 0.00445319 \\
\hline Rsk3(h) & 105 & 0 & 89 & 89 & 76 & 0.894117647 & 11 & 0.038732394 & 0.436619718 & 0.004908865 \\
\hline SAPK2a(h) & 94 & 0 & 89 & 89 & 77 & 0.905882353 & 11 & 0.038732394 & 0.475352113 & 0.00536454 \\
\hline SAPK2b(h) & 115 & 0 & 88 & 88 & 78 & 0.917647059 & 12 & 0.042253521 & 0.517605634 & 0.005840928 \\
\hline SAPK3(h) & 104 & 0 & 88 & 88 & 79 & 0.929411765 & 12 & 0.042253521 & 0.559859155 & 0.006338028 \\
\hline SAPK 4 (h) & 108 & 0 & 86 & 86 & 80 & 0.941176471 & 14 & 0.049295775 & 0.60915493 & 0.006876553 \\
\hline SGK(h) & 98 & 0 & 86 & 86 & 81 & 0.952941176 & 14 & 0.049295775 & 0.658450704 & 0.007456504 \\
\hline Syk(h) & 108 & 0 & 85 & 85 & 82 & 0.964705882 & 15 & 0.052816901 & 0.711267606 & 0.008057167 \\
\hline TrkB(h) & 98 & 0 & 84 & 84 & 83 & 0.976470588 & 16 & 0.056338028 & 0.767605634 & 0.008699254 \\
\hline Yes(h) & 118 & 0 & 72 & 72 & 84 & 0.988235294 & 28 & 0.098591549 & 0.866197183 & 0.009610605 \\
\hline $\mathrm{ZAP}-70(\mathrm{~h})$ & 108 & 0 & 62 & 62 & 85 & 1 & 38 & 0.133802817 & 1 & 0.01097763 \\
\hline & & & & & & Total inhibition & 284 & & Total area & 0.112551781 \\
\hline & $\begin{array}{r}\text { Hit rate } \\
\text { Threshold }\end{array}$ & 5 & & & & & & & Gini & 0.774896437 \\
\hline
\end{tabular}




\begin{tabular}{|c|c|c|c|c|c|c|c|c|c|c|}
\hline & $100 \mu \mathrm{M}$ ATP & & & & & & & & & \\
\hline Kinase & Wortmannin @ $1 \mu \mathrm{M}$ & Hit & sorted & normalized & position & cumulative sample fraction & inhibition\% & fraction of total inhibition & cumulative inhibition & area \\
\hline $\begin{array}{c}\mathrm{Abl}(\mathrm{m}) \\
\mathrm{AMPK}(\mathrm{r})\end{array}$ & $\begin{array}{r}89 \\
108\end{array}$ & $\begin{array}{l}0 \\
0\end{array}$ & $\begin{array}{l}139 \\
121\end{array}$ & $\begin{array}{l}100 \\
100\end{array}$ & $\begin{array}{l}1 \\
2\end{array}$ & $\begin{array}{l}0.011764706 \\
0.023529412\end{array}$ & $\begin{array}{l}0 \\
0\end{array}$ & $\begin{array}{l}0 \\
0\end{array}$ & (2) & $\begin{array}{l}0 \\
0\end{array}$ \\
\hline 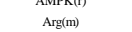 & $\begin{array}{l}108 \\
107\end{array}$ & 0 & 120 & $\begin{array}{l}100 \\
100\end{array}$ & $\begin{array}{l}2 \\
3\end{array}$ & $\begin{array}{l}0.023529412 \\
0.035294118\end{array}$ & 0 & 0 & 0 & 0 \\
\hline Aurora-A(h) & 98 & 0 & 119 & 100 & 4 & 0.047058824 & 0 & 0 & 0 & 0 \\
\hline Axl(h) & 92 & 0 & 119 & 100 & 5 & 0.058823529 & 0 & 0 & 0 & 0 \\
\hline $\mathrm{Blk}(\mathrm{m})$ & 73 & 0 & 116 & 100 & 6 & 0.070588235 & 0 & 0 & 0 & 0 \\
\hline $\operatorname{Bmx}(\mathrm{h})$ & 111 & 0 & 114 & 100 & 7 & 0.082352941 & 0 & 0 & 0 & 0 \\
\hline CaMKII(r) & 94 & 0 & 114 & 100 & 8 & 0.094117647 & 0 & 0 & 0 & 0 \\
\hline CaMKIV(h) & 102 & 0 & 114 & 100 & 9 & 0.105882353 & 0 & 0 & 0 & 0 \\
\hline CDK1/cyclinB(h) & 105 & 0 & 114 & 100 & 10 & 0.117647059 & 0 & 0 & 0 & 0 \\
\hline CDK2/cyclinA(h) & 104 & 0 & 113 & 100 & 11 & 0.129411765 & 0 & 0 & 0 & 0 \\
\hline $\mathrm{CDK} 2 /$ cyclinE(h) & 102 & 0 & 112 & 100 & 12 & 0.141176471 & 0 & 0 & 0 & 0 \\
\hline CDK3/cyclinE(h) & 101 & 0 & 111 & 100 & 13 & 0.152941176 & 0 & 0 & 0 & 0 \\
\hline CDK5/p35(h) & 139 & 0 & 111 & 100 & 14 & 0.164705882 & 0 & 0 & 0 & 0 \\
\hline CDK6/cyclinD3(h) & 106 & 0 & 111 & 100 & 15 & 0.176470588 & 0 & 0 & 0 & 0 \\
\hline CDK7/cyclinH/MATI(h) & 99 & 0 & 108 & 100 & 16 & 0.188235294 & 0 & 0 & 0 & 0 \\
\hline СHK1(h) & 101 & 0 & 108 & 100 & 17 & 0.2 & 0 & 0 & 0 & 0 \\
\hline СHK2(h) & 102 & 0 & 108 & 100 & 18 & 0.211764706 & 0 & 0 & 0 & 0 \\
\hline CKI(y) & 114 & 0 & 107 & 100 & 19 & 0.223529412 & 0 & 0 & 0 & 0 \\
\hline CK2(h) & 102 & 0 & 107 & 100 & 20 & 0.235294118 & 0 & 0 & 0 & 0 \\
\hline c-RAF(h) & 99 & 0 & 107 & 100 & 21 & 0.247058824 & 0 & 0 & 0 & 0 \\
\hline CSK(h) & 107 & 0 & 107 & 100 & 22 & 0.258823529 & 0 & 0 & 0 & 0 \\
\hline cSRC(h) & 111 & 0 & 107 & 100 & 23 & 0.270588235 & 0 & 0 & 0 & 0 \\
\hline Fes(h) & 114 & 0 & 107 & 100 & 24 & 0.282352941 & 0 & 0 & 0 & 0 \\
\hline FGFR3(h) & 114 & 0 & 106 & 100 & 25 & 0.294117647 & 0 & 0 & 0 & 0 \\
\hline $\mathrm{Fll}(\mathrm{h}(\mathrm{h})$ & 97 & 0 & 106 & 100 & 26 & 0.305882353 & 0 & 0 & 0 & 0 \\
\hline Fynn(h) & 98 & 0 & 106 & 100 & 27 & 0.317647059 & 0 & 0 & 0 & 0 \\
\hline GSK3a(h) & 98 & 0 & 106 & 100 & 28 & 0.329411765 & 0 & 0 & 0 & 0 \\
\hline GSK3B(h) & 95 & 0 & 106 & 100 & 29 & 0.341176471 & 0 & 0 & 0 & 0 \\
\hline IGF-IR(h) & 119 & 0 & 105 & 100 & 30 & 0.352941176 & 0 & 0 & 0 & 0 \\
\hline $\mathrm{IKKa(h)}$ & 113 & 0 & 105 & 100 & 31 & 0.364705882 & 0 & 0 & 0 & 0 \\
\hline $\mathrm{IKKB(h)}$ & 96 & 0 & 104 & 100 & 32 & 0.376470588 & 0 & 0 & 0 & 0 \\
\hline $\mathbb{I R ( h )}$ & 102 & 0 & 104 & 100 & 33 & 0.388235294 & 0 & 0 & 0 & 0 \\
\hline JNKIal(h) & 104 & 0 & 104 & 100 & 34 & 0.4 & 0 & 0 & 0 & 0 \\
\hline JNK2202(h) & 95 & 0 & 102 & 100 & 35 & 0.411764706 & 0 & 0 & 0 & 0 \\
\hline JNK3(r) & 102 & 0 & 102 & 100 & 36 & 0.423529412 & 0 & 0 & 0 & 0 \\
\hline Lck(h) & 107 & 0 & 102 & 100 & 37 & 0.435294118 & 0 & 0 & 0 & 0 \\
\hline Lyn(h) & 107 & 0 & 102 & 100 & 38 & 0.447058824 & 0 & 0 & 0 & 0 \\
\hline Lyn(m) & 85 & 0 & 102 & 100 & 39 & 0.458823529 & 0 & 0 & 0 & 0 \\
\hline MAPK I(h) & 94 & 0 & 102 & 100 & 40 & 0.470588235 & 0 & 0 & 0 & 0 \\
\hline MAPK2(h) & 97 & 0 & 102 & 100 & 41 & 0.482352941 & 0 & 0 & 0 & 0 \\
\hline MAPK2(m) & 95 & 0 & 101 & 100 & 42 & 0.494117647 & 0 & 0 & 0 & 0 \\
\hline MAPKAP-K2(h) & 108 & 0 & 101 & 100 & 43 & 0.505882353 & 0 & 0 & 0 & 0 \\
\hline MEK1(h) & 121 & 0 & 101 & 100 & 44 & 0.517647059 & 0 & 0 & 0 & 0 \\
\hline MKK4(m) & 92 & 0 & 101 & 100 & 45 & 0.529411765 & 0 & 0 & 0 & 0 \\
\hline MKK6(h) & 96 & 0 & 100 & 100 & 46 & 0.541176471 & 0 & 0 & 0 & 0 \\
\hline MKK 7 (h) & 102 & 0 & 100 & 100 & 47 & 0.552941176 & 0 & 0 & 0 & 0 \\
\hline MSK1(h) & 99 & 0 & 100 & 100 & 48 & 0.564705882 & 0 & 0 & 0 & 0 \\
\hline p7056K(h) & 100 & 0 & 99 & 99 & 49 & 0.576470588 & 1 & 0.003968254 & 0.003968254 & $2.33427 \mathrm{E}-05$ \\
\hline PAK2(h) & 114 & 0 & 99 & 99 & 50 & 0.588235294 & 1 & 0.003968254 & 0.007936508 & $7.0028 \mathrm{E}-05$ \\
\hline PDGFRa(h) & 87 & 0 & 99 & 99 & 51 & 0.6 & 1 & 0.003968254 & 0.011904762 & 0.000116713 \\
\hline PDGFRB(h) & 96 & 0 & 99 & 99 & 52 & 0.611764706 & 1 & 0.003968254 & 0.015873016 & 0.000163399 \\
\hline PDK1(h) & 88 & 0 & 98 & 98 & 53 & 0.623529412 & 2 & 0.007936508 & 0.023809524 & 0.000233427 \\
\hline PKA(b) & 111 & 0 & 98 & 98 & 54 & 0.635294118 & 2 & 0.007936508 & 0.031746032 & 0.000326797 \\
\hline PKA(h) & 92 & 0 & 98 & 98 & 55 & 0.647058824 & 2 & 0.007936508 & 0.03968254 & 0.000420168 \\
\hline PKBach) & 96 & 0 & 97 & 97 & 56 & 0.658823529 & 3 & 0.011904762 & 0.051587302 & 0.000536881 \\
\hline PKB(h) & 100 & 0 & 97 & 97 & 57 & 0.670588235 & 3 & 0.011904762 & 0.063492063 & 0.000676937 \\
\hline $\mathrm{PKB}_{\gamma}(\mathrm{h})$ & 116 & 0 & 97 & 97 & 58 & 0.682352941 & 3 & 0.011904762 & 0.075396825 & 0.000816993 \\
\hline PKCa(h) & 95 & 0 & 97 & 97 & 59 & 0.694117647 & 3 & 0.011904762 & 0.087301587 & 0.000957049 \\
\hline РКCBII(h) & 108 & 0 & 96 & 96 & 60 & 0.705882353 & 4 & 0.015873016 & 0.103174603 & 0.001120448 \\
\hline PKCy(h) & 91 & 0 & 96 & 96 & 61 & 0.717647059 & 4 & 0.015873016 & 0.119047619 & 0.00130719 \\
\hline РКБ(h) & 101 & 0 & 96 & 96 & 62 & 0.729411765 & 4 & 0.015873016 & 0.134920635 & 0.001493931 \\
\hline PKG:(h) & 88 & 0 & 96 & 96 & 63 & 0.741176471 & 4 & 0.015873016 & 0.150793651 & 0.001680672 \\
\hline PKG(h) & 97 & 0 & 95 & 95 & 64 & 0.752941176 & 5 & 0.01984127 & 0.170634921 & 0.001890756 \\
\hline PKC 1 (h) & 104 & 0 & 95 & 95 & 65 & 0.764705882 & 5 & 0.01984127 & 0.19047619 & 0.002124183 \\
\hline PKC $\mu(\mathrm{h})$ & 94 & 0 & 95 & 95 & 66 & 0.776470588 & 5 & 0.01984127 & 0.21031746 & 0.00235761 \\
\hline PKCO(h) & 107 & 0 & 95 & 95 & 67 & 0.788235294 & 5 & 0.01984127 & 0.23015873 & 0.002591036 \\
\hline PKD2(h) & 106 & 0 & 94 & 94 & 68 & 0.8 & 6 & 0.023809524 & 0.253968254 & 0.002847806 \\
\hline PRAK(h) & 106 & 0 & 94 & 94 & 69 & 0.811764706 & 6 & 0.023809524 & 0.277777778 & 0.003127918 \\
\hline PRK2(h) & 84 & 0 & 94 & 94 & 70 & 0.823529412 & 6 & 0.023809524 & 0.301587302 & 0.00340803 \\
\hline ROCK-II(h) & 87 & 0 & 93 & 93 & 71 & 0.835294118 & 7 & 0.027777778 & 0.329365079 & 0.003711485 \\
\hline ROCK-IIIr) & 112 & 0 & 93 & 93 & 72 & 0.847058824 & 7 & 0.027777778 & 0.357142857 & 0.004038282 \\
\hline Rskl(h) & 97 & 0 & 92 & 92 & 73 & 0.858823529 & 8 & 0.031746032 & 0.388888889 & 0.004388422 \\
\hline $\operatorname{Rskl(r)}$ & 99 & 0 & 92 & 92 & 74 & 0.870588235 & 8 & 0.031746032 & 0.420634921 & 0.004761905 \\
\hline Rsk2(h) & 93 & 0 & 92 & 92 & 75 & 0.882352941 & 8 & 0.031746032 & 0.452380952 & 0.005135387 \\
\hline Rsk3(h) & 90 & 0 & 91 & 91 & 76 & 0.894117647 & 9 & 0.035714286 & 0.488095238 & 0.005532213 \\
\hline SAPK2a(h) & 107 & 0 & 90 & 90 & 77 & 0.905882353 & 10 & 0.03968254 & 0.527777778 & 0.005975724 \\
\hline SAPK2b(h) & 120 & 0 & 89 & 89 & 78 & 0.917647059 & 11 & 0.043650794 & 0.571428571 & 0.00646592 \\
\hline $\mathrm{SAPK} 3(\mathrm{~h})$ & 106 & 0 & 88 & 88 & 79 & 0.929411765 & 12 & 0.047619048 & 0.619047619 & 0.007002801 \\
\hline $\operatorname{SAPK} 4(\mathrm{~h})$ & 105 & 0 & 88 & 88 & 80 & 0.941176471 & 12 & 0.047619048 & 0.666666667 & 0.007563025 \\
\hline SGK(h) & 100 & 0 & 87 & 87 & 81 & 0.952941176 & 13 & 0.051587302 & 0.718253968 & 0.008146592 \\
\hline Syk(h) & 101 & 0 & 87 & 87 & 82 & 0.964705882 & 13 & 0.051587302 & 0.76984127 & 0.008753501 \\
\hline $\operatorname{TrkB}(\mathrm{h})$ & 93 & 0 & 85 & 85 & 83 & 0.976470588 & 15 & 0.05952381 & 0.829365079 & 0.009407096 \\
\hline Yes(h) & 106 & 0 & 84 & 84 & 84 & 0.988235294 & 16 & 0.063492063 & 0.892857143 & 0.010130719 \\
\hline ZAP-70(h) & 119 & 0 & 73 & 73 & 85 & 1 & 27 & 0.107142857 & 1 & 0.011134454 \\
\hline & & & & & & Total inhibition & 252 & & Total area & 0.130438842 \\
\hline & Hit rate & 5 & & & & & & & Gini & 0739122316 \\
\hline
\end{tabular}




\begin{tabular}{|c|c|c|c|c|c|c|c|c|}
\hline & $0 \mu \mathrm{M}$ ATP & & & & & & & \\
\hline Kinase & 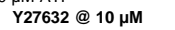 & Hit & sorted & normalized & position & cumulative sample fraction & inhibition\% & fraction of total inhibition \\
\hline $\mathrm{Abl}(\mathrm{m})$ & 96 & 0 & 116 & 100 & 1 & 0.011764706 & 0 & 0 \\
\hline AMPK(r) & 82 & 0 & 115 & 100 & 2 & 0.023529412 & 0 & 0 \\
\hline $\operatorname{Arg}(\mathrm{m})$ & 90 & 0 & 111 & 100 & 3 & 0.035294118 & 0 & 0 \\
\hline Aurora-A(h) & 91 & 0 & 108 & 100 & 4 & 0.047058824 & 0 & 0 \\
\hline Axl(h) & 96 & 0 & 107 & 100 & 5 & 0.058823529 & 0 & 0 \\
\hline $\mathrm{Blk}(\mathrm{m})$ & 65 & 0 & 106 & 100 & 6 & 0.070588235 & 0 & 0 \\
\hline Bmxx(h) & 94 & 0 & 106 & 100 & 7 & 0.082352941 & 0 & 0 \\
\hline СамKII(r) & 96 & 0 & 104 & 100 & 8 & 0.094117647 & 0 & 0 \\
\hline CaMKIV(h) & 101 & 0 & 103 & 100 & 9 & 0.105882353 & 0 & 0 \\
\hline CDK1/cyclnBB(h) & 88 & 0 & 103 & 100 & 10 & 0.117647059 & 0 & 0 \\
\hline $\mathrm{CDK} /$ /yclinA(h) & 76 & 0 & 102 & 100 & 11 & 0.129411765 & 0 & 0 \\
\hline $\mathrm{CDK} 2 /$ cyclinE(h) & 67 & 0 & 102 & 100 & 12 & 0.141176471 & 0 & 0 \\
\hline CDK3/cyclinE(h) & 88 & 0 & 101 & 100 & 13 & 0.152941176 & 0 & 0 \\
\hline CDK5/p35(h) & 73 & 0 & 101 & 100 & 14 & 0.164705882 & 0 & 0 \\
\hline CDK6/cyclinD3(h) & 89 & 0 & 101 & 100 & 15 & 0.176470588 & 0 & 0 \\
\hline CDK7/cyclinHMATI(h) & 82 & 0 & 101 & 100 & 16 & 0.188235294 & 0 & 0 \\
\hline СнК $1 \mathrm{~h})$ & 104 & 0 & 101 & 100 & 17 & 0.2 & 0 & 0 \\
\hline СHK2(h) & 91 & 0 & 100 & 100 & 18 & 0.211764706 & 0 & 0 \\
\hline $\mathrm{CK} 1(\mathrm{y})$ & 50 & 1 & 99 & 99 & 19 & 0.223529412 & 1 & 0.000570451 \\
\hline СК2(h) & 108 & 0 & 99 & 99 & 20 & 0.235294118 & 1 & 0.000570451 \\
\hline c-RAF(h) & 106 & 0 & 97 & 97 & 21 & 0.247058824 & 3 & 0.001711352 \\
\hline $\operatorname{CSK}(\mathrm{h})$ & 73 & 0 & 97 & 97 & 22 & 0.258823529 & 3 & 0.001711352 \\
\hline cSRCh) & 101 & 0 & 97 & 97 & 23 & 0.270588235 & 3 & 0.001711352 \\
\hline Fes(h) & 83 & 0 & 96 & 96 & 24 & 0.282352941 & 4 & 0.002281803 \\
\hline FGFR3(h) & 106 & 0 & 96 & 96 & 25 & 0.294117647 & 4 & 0.002281803 \\
\hline $\mathrm{Fll}(\mathrm{h}(\mathrm{h})$ & 81 & 0 & 96 & 96 & 26 & 0.305882353 & 4 & 0.002281803 \\
\hline Fyn(h) & 92 & 0 & 96 & 96 & 27 & 0.317647059 & 4 & 0.002281803 \\
\hline GSK3a(h) & 97 & 0 & 96 & 96 & 28 & 0.329411765 & 4 & 0.002281803 \\
\hline GSK3B(h) & 94 & 0 & 96 & 96 & 29 & 0.341176471 & 4 & 0.002281803 \\
\hline IGF-IR(h) & 90 & 0 & 95 & 95 & 30 & 0.352941176 & 5 & 0.002852253 \\
\hline IKKa(h) & 72 & 0 & 94 & 94 & 31 & 0.364705882 & 6 & 0.003422704 \\
\hline IKKB(h) & 75 & 0 & 94 & 94 & 32 & 0.376470588 & 6 & 0.003422704 \\
\hline $\mathbb{I R ( h )}$ & 96 & 0 & 94 & 94 & 33 & 0.388235294 & 6 & 0.003422704 \\
\hline JNKIal(h) & 99 & 0 & 92 & 92 & 34 & 0.4 & 8 & 0.004563605 \\
\hline JNK2202(h) & 103 & 0 & 92 & 92 & 35 & 0.411764706 & 8 & 0.004563605 \\
\hline JNK3(r) & 111 & 0 & 92 & 92 & 36 & 0.423529412 & 8 & 0.004563605 \\
\hline Lck(h) & 91 & 0 & 91 & 91 & 37 & 0.435294118 & 9 & 0.005134056 \\
\hline Lyn(h) & 107 & 0 & 91 & 91 & 38 & 0.447058824 & 9 & 0.005134056 \\
\hline $\operatorname{Lyn}(m)$ & 66 & 0 & 91 & 91 & 39 & 0.458823529 & 9 & 0.005134056 \\
\hline MAPK1(h) & 101 & 0 & 90 & 90 & 40 & 0.470588235 & 10 & 0.005704507 \\
\hline MAPK2(h) & 96 & 0 & 90 & 90 & 41 & 0.482352941 & 10 & 0.005704507 \\
\hline MAPK2(m) & 92 & 0 & 90 & 90 & 42 & 0.494117647 & 10 & 0.005704507 \\
\hline MAPKAP-K2(h) & 88 & 0 & 90 & 90 & 43 & 0.505882353 & 10 & 0.005704507 \\
\hline MEK1(h) & 95 & 0 & 90 & 90 & 44 & 0.517647059 & 10 & 0.005704507 \\
\hline MKK $4(\mathrm{~m})$ & 102 & 0 & 90 & 90 & 45 & 0.529411765 & 10 & 0.005704507 \\
\hline MKK6(h) & 87 & 0 & 89 & 89 & 46 & 0.541176471 & 11 & 0.006274957 \\
\hline MKK $7 \mathrm{B(h)}$ & 115 & 0 & 88 & 88 & 47 & 0.552941176 & 12 & 0.006845408 \\
\hline MSK1(h) & 32 & 1 & 88 & 88 & 48 & 0.564705882 & 12 & 0.006845408 \\
\hline p7056K(h) & 62 & 0 & 88 & 88 & 49 & 0.576470588 & 12 & 0.006845408 \\
\hline PAK2(h) & 96 & 0 & 88 & 88 & 50 & 0.588235294 & 12 & 0.006845408 \\
\hline PDGFRa(h) & 84 & 0 & 88 & 88 & 51 & 0.6 & 12 & 0.006845408 \\
\hline PDGFRB(h) & 94 & 0 & 87 & 87 & 52 & 0.611764706 & 13 & 0.007415859 \\
\hline PDK I(h) & 88 & 0 & 87 & 87 & 53 & 0.623529412 & 13 & 0.007415859 \\
\hline PKA(b) & 83 & 0 & 84 & 84 & 54 & 0.635294118 & 16 & 0.00912721 \\
\hline PKA(h) & 90 & 0 & 83 & 83 & 55 & 0.647058824 & 17 & 0.009697661 \\
\hline PKBa(h) & 76 & 0 & 83 & 83 & 56 & 0.658823529 & 17 & 0.009697661 \\
\hline PKB(h) & 88 & 0 & 82 & 82 & 57 & 0.670588235 & 18 & 0.010268112 \\
\hline $\mathrm{PKB}_{\gamma}(\mathrm{h})$ & 87 & 0 & 82 & 82 & 58 & 0.682352941 & 18 & 0.010268112 \\
\hline 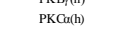 & 75 & 0 & 82 & 82 & 59 & 0.694117647 & 18 & 0.010268112 \\
\hline РКCBII(h) & 90 & 0 & 81 & 81 & 60 & 0.705882353 & 19 & 0.010838562 \\
\hline PKCy(h) & 99 & 0 & 76 & 76 & 61 & 0.717647059 & 24 & 0.013690816 \\
\hline PKБ(h) & 25 & 1 & 76 & 76 & 62 & 0.729411765 & 24 & 0.013690816 \\
\hline PKG:(h) & 11 & 1 & 75 & 75 & 63 & 0.741176471 & 25 & 0.014261266 \\
\hline PKG(h) & 4 & 1 & 75 & 75 & 64 & 0.752941176 & 25 & 0.014261266 \\
\hline PKC (h) & $\begin{array}{r}4 \\
82\end{array}$ & 0 & 73 & 73 & 65 & 0.764705882 & 27 & 0.015402168 \\
\hline PKC $\mu(\mathrm{h})$ & 70 & 0 & 73 & 73 & 66 & 0.776470588 & 27 & 0.015402168 \\
\hline PKCO(h) & 41 & 1 & 72 & 72 & 67 & 0.788235294 & 28 & 0.015972618 \\
\hline PKD2(h) & 90 & 0 & 70 & 70 & 68 & 0.8 & 30 & 0.01711352 \\
\hline PRAK(h) & 116 & 0 & 67 & 67 & 69 & 0.811764706 & 33 & 0.018824872 \\
\hline PRK2(h) & 1 & 1 & 66 & 66 & 70 & 0.823529412 & 34 & 0.019395322 \\
\hline ROCK-II(h) & 2 & 1 & 65 & 65 & 71 & 0.835294118 & 35 & 0.019965773 \\
\hline ROCK-II(r) & 2 & 1 & 62 & 62 & 72 & 0.847058824 & 38 & 0.021677125 \\
\hline Rskl(h) & 42 & 1 & 50 & 50 & 73 & 0.858823529 & 50 & 0.028522533 \\
\hline Rskl(r) & 24 & 1 & 42 & 42 & 74 & 0.870588235 & 58 & 0.033086138 \\
\hline Rsk2(h) & 32 & 1 & 41 & 41 & 75 & 0.882352941 & 59 & 0.033656589 \\
\hline Rsk3(h) & 20 & 1 & 32 & 32 & 76 & 0.894117647 & 68 & 0.038790645 \\
\hline SAPK2a(h) & 92 & 0 & 32 & 32 & 77 & 0.905882353 & 68 & 0.038790645 \\
\hline SAPK2b(h) & 100 & 0 & 25 & 25 & 78 & 0.917647059 & 75 & 0.042783799 \\
\hline $\mathrm{SAPK} 3(\mathrm{~h})$ & 101 & 0 & 24 & 24 & 79 & 0.929411765 & 76 & 0.04335425 \\
\hline SAPK4(h) & 97 & 0 & 20 & 20 & 80 & 0.941176471 & 80 & 0.045636052 \\
\hline SGK(h) & 90 & 0 & 11 & 11 & 81 & 0.952941176 & 89 & 0.050770108 \\
\hline Syk(h) & 102 & 0 & 4 & 4 & 82 & 0.964705882 & 96 & 0.054763263 \\
\hline TrkB(h) & 103 & 0 & $\begin{array}{l}4 \\
2\end{array}$ & 2 & 83 & 0.976470588 & 98 & 0.055904164 \\
\hline Yes(h) & 97 & 0 & 2 & 2 & 84 & 0.988235294 & 98 & 0.055904164 \\
\hline ZAP-70(h) & 101 & 0 & 1 & 1 & 85 & 1 & 99 & 0.056474615 \\
\hline & & & & & & Total inhibition & 1753 & \\
\hline & Hit rate & & & & & & & \\
\hline & Threshold & 5 & & & & & & \\
\hline
\end{tabular}




\begin{tabular}{|c|c|c|c|c|c|c|c|c|c|}
\hline & $100 \mu \mathrm{M}$ ATP & & & & & & & & \\
\hline Kinase & Y27632@ $10 \mu \mathrm{M}$ & Hit & sorted & normalized & position & cumulative sample fraction & inhibition\% & fraction of total inhibition & cumulative inhibition \\
\hline $\mathrm{Abl}(\mathrm{m})$ & 88 & 0 & 126 & 100 & 1 & 0.011764706 & 0 & 0 & 0 \\
\hline AMPK(r) & 90 & 0 & 124 & 100 & 2 & 0.023529412 & 0 & 0 & 0 \\
\hline $\operatorname{Arg}(\mathrm{m})$ & 106 & 0 & 116 & 100 & 3 & 0.035294118 & 0 & 0 & 0 \\
\hline Aurora-A(h) & 88 & 0 & 111 & 100 & 4 & 0.047058824 & 0 & 0 & 0 \\
\hline Axl(h) & 89 & 0 & 111 & 100 & 5 & 0.058823529 & 0 & 0 & 0 \\
\hline $\mathrm{Blk}(\mathrm{m})$ & 64 & 0 & 109 & 100 & 6 & 0.070588235 & 0 & 0 & 0 \\
\hline Bnx(h) & 98 & 0 & 108 & 100 & 7 & 0.082352941 & 0 & 0 & 0 \\
\hline CaMKIII(r) & 99 & 0 & 107 & 100 & 8 & 0.094117647 & 0 & 0 & 0 \\
\hline CaMKIV(h) & 94 & 0 & 106 & 100 & 9 & 0.105882353 & 0 & 0 & 0 \\
\hline CDK1/cyclnBB(h) & 97 & 0 & 106 & 100 & 10 & 0.117647059 & 0 & 0 & 0 \\
\hline CDK2/cyclinA(h) & 95 & 0 & 106 & 100 & 11 & 0.129411765 & 0 & 0 & 0 \\
\hline $\mathrm{CDK} 2 /$ cyclinE(h) & 72 & 0 & 105 & 100 & 12 & 0.141176471 & 0 & 0 & 0 \\
\hline CDK3/cyclinE(h) & 90 & 0 & 103 & 100 & 13 & 0.152941176 & 0 & 0 & 0 \\
\hline CDK5/p35(h) & 105 & 0 & 103 & 100 & 14 & 0.164705882 & 0 & 0 & 0 \\
\hline CDKG/cyclinD3(h) & 99 & 0 & 103 & 100 & 15 & 0.176470588 & 0 & 0 & 0 \\
\hline CDK 7/cyclinHMATl(h) & 74 & 0 & 103 & 100 & 16 & 0.188235294 & 0 & 0 & 0 \\
\hline CHKI(h) & 97 & 0 & 102 & 100 & 17 & 0.2 & 0 & 0 & 0 \\
\hline CHK2(h) & 89 & 0 & 102 & 100 & 18 & 0.211764706 & 0 & 0 & 0 \\
\hline CKI(y) & 68 & 0 & 102 & 100 & 19 & 0.223529412 & 0 & 0 & 0 \\
\hline СK2(h) & 101 & 0 & 101 & 100 & 20 & 0.235294118 & 0 & 0 & 0 \\
\hline $\mathrm{c}-\mathrm{RAF}(\mathrm{h})$ & 99 & 0 & 101 & 100 & 21 & 0.247058824 & 0 & 0 & 0 \\
\hline $\operatorname{csk}(\mathrm{h})$ & 102 & 0 & 100 & 100 & 22 & 0.258823529 & 0 & 0 & 0 \\
\hline cSRC(h) & 102 & 0 & 100 & 100 & 23 & 0.270588235 & 0 & 0 & 0 \\
\hline Fes(h) & 103 & 0 & 99 & 99 & 24 & 0.282352941 & 1 & 0.000820345 & 0.000820345 \\
\hline FGFR3(h) & 102 & 0 & 99 & 99 & 25 & 0.294117647 & 1 & 0.000820345 & 0.001640689 \\
\hline $\mathrm{FH}$ H(h) & 86 & 0 & 99 & 99 & 26 & 0.305882353 & 1 & 0.000820345 & 0.002461034 \\
\hline Fyn(h) & 103 & 0 & 99 & 99 & 27 & 0.317647059 & 1 & 0.000820345 & 0.003281378 \\
\hline GSK3a(h) & 108 & 0 & 99 & 99 & 28 & 0.329411765 & 1 & 0.000820345 & 0.004101723 \\
\hline GSK $3 B(h)$ & 96 & 0 & 98 & 98 & 29 & 0.341176471 & 2 & 0.001640689 & 0.005742412 \\
\hline IGF-IR(h) & 103 & 0 & 98 & 98 & 30 & 0.352941176 & 2 & 0.001640689 & 0.007383101 \\
\hline IKKa(h) & 94 & 0 & 98 & 98 & 31 & 0.364705882 & 2 & 0.001640689 & 0.00902379 \\
\hline IKKB(h) & 95 & 0 & 98 & 98 & 32 & 0.376470588 & 2 & 0.001640689 & 0.010664479 \\
\hline $\mathrm{IR}(\mathrm{h})$ & 111 & 0 & 98 & 98 & 33 & 0.388235294 & 2 & 0.001640689 & 0.012305168 \\
\hline JNKIal(h) & 95 & 0 & 97 & 97 & 34 & 0.4 & 3 & 0.002461034 & 0.014766202 \\
\hline JNK202(h) & 101 & 0 & 97 & 97 & 35 & 0.411764706 & 3 & 0.002461034 & 0.017227235 \\
\hline $\mathrm{JNK} 3(\mathrm{r})$ & 96 & 0 & 97 & 97 & 36 & 0.423529412 & 3 & 0.002461034 & 0.019688269 \\
\hline Lck(h) & 99 & 0 & 96 & 96 & 37 & 0.435294118 & 4 & 0.003281378 & 0.022969647 \\
\hline Lyn(h) & 109 & 0 & 96 & 96 & 38 & 0.447058824 & 4 & 0.003281378 & 0.026251025 \\
\hline Lyn(m) & 80 & 0 & 96 & 96 & 39 & 0.458823529 & 4 & 0.003281378 & 0.029532404 \\
\hline MAPK I(h) & 99 & 0 & 96 & 96 & 40 & 0.470588235 & 4 & 0.003281378 & 0.032813782 \\
\hline MAPK2(h) & 116 & 0 & 95 & 95 & 41 & 0.482352941 & 5 & 0.004101723 & 0.036915505 \\
\hline MAPK2(m) & 97 & 0 & 95 & 95 & 42 & 0.494117647 & 5 & 0.004101723 & 0.041017227 \\
\hline MAPKAP-K2(h) & 98 & 0 & 95 & 95 & 43 & 0.505882353 & 5 & 0.004101723 & 0.04511895 \\
\hline MEK 1(h) & 98 & 0 & 95 & 95 & 44 & 0.517647059 & 5 & 0.004101723 & 0.049220673 \\
\hline MKK $4(\mathrm{~m})$ & 100 & 0 & 94 & 94 & 45 & 0.529411765 & 6 & 0.004922067 & 0.05414274 \\
\hline MKK6(h) & 96 & 0 & 94 & 94 & 46 & 0.541176471 & 6 & 0.004922067 & 0.059064807 \\
\hline МKK $78($ (h) & 96 & 0 & 94 & 94 & 47 & 0.552941176 & 6 & 0.004922067 & 0.063986874 \\
\hline MSKI(h) & $\begin{array}{l}90 \\
46\end{array}$ & 1 & 94 & 94 & 48 & 0.564705882 & 6 & 0.004922067 & 0.068908942 \\
\hline $\mathrm{p} 7056 \mathrm{~K}(\mathrm{~h})$ & 88 & 0 & 94 & 94 & 49 & 0.576470588 & 6 & 0.004922067 & 0.073831009 \\
\hline PAK2(h) & 106 & 0 & 93 & 93 & 50 & 0.588235294 & 7 & 0.005742412 & 0.079573421 \\
\hline PDGFRa(h) & 85 & 0 & 92 & 92 & 51 & 0.6 & 8 & 0.006562756 & 0.086136177 \\
\hline PDGFRB(h) & 107 & 0 & 92 & 92 & 52 & 0.611764706 & 8 & 0.006562756 & 0.092698934 \\
\hline PDK I(h) & 86 & 0 & 90 & 90 & 53 & 0.623529412 & 10 & 0.008203445 & 0.100902379 \\
\hline PKA(b) & 111 & 0 & 90 & 90 & 54 & 0.635294118 & 10 & 0.008203445 & 0.109105824 \\
\hline PKA(h) & 90 & 0 & 90 & 90 & 55 & 0.647058824 & 10 & 0.008203445 & 0.11730927 \\
\hline PKBa(h) & 87 & 0 & 89 & 89 & 56 & 0.658823529 & 11 & 0.00902379 & 0.12633306 \\
\hline PKBB(h) & 83 & 0 & 89 & 89 & 57 & 0.670588235 & 11 & 0.00902379 & 0.13535685 \\
\hline $\mathrm{PKB}_{\gamma}(\mathrm{h})$ & 106 & 0 & 88 & 88 & 58 & 0.682352941 & 12 & 0.009844135 & 0.145200984 \\
\hline PKGa(h) & 92 & 0 & 88 & 88 & 59 & 0.694117647 & 12 & 0.009844135 & 0.155045119 \\
\hline РКСBII(h) & 100 & 0 & 88 & 88 & 60 & 0.705882353 & 12 & 0.009844135 & 0.164889253 \\
\hline PKCy(h) & 94 & 0 & 87 & 87 & 61 & 0.717647059 & 13 & 0.010664479 & 0.175553733 \\
\hline PKळ(h) & 46 & 1 & 87 & 87 & 62 & 0.729411765 & 13 & 0.010664479 & 0.186218212 \\
\hline PKG:(h) & 39 & 1 & 86 & 86 & 63 & 0.741176471 & 14 & 0.011484824 & 0.197703035 \\
\hline PKG (h) & 13 & 1 & 86 & 86 & 64 & 0.752941176 & 14 & 0.011484824 & 0.209187859 \\
\hline PKC (h) & 98 & 0 & 85 & 85 & 65 & 0.764705882 & 15 & 0.012305168 & 0.221493027 \\
\hline PKC $\mu(\mathrm{h})$ & 87 & 0 & 83 & 83 & 66 & 0.776470588 & 17 & 0.013945857 & 0.235438884 \\
\hline PKCO(h) & 79 & 0 & 82 & 82 & 67 & 0.788235294 & 18 & 0.014766202 & 0.250205086 \\
\hline PKD2(h) & 93 & 0 & 81 & 81 & 68 & 0.8 & 19 & 0.015586546 & 0.265791632 \\
\hline PRAK(h) & 98 & 0 & 80 & 80 & 69 & 0.811764706 & 20 & 0.016406891 & 0.282198523 \\
\hline PRK2(h) & -4 & 1 & 79 & 79 & 70 & 0.823529412 & 21 & 0.017227235 & 0.299425759 \\
\hline ROCK-II(h) & 2 & 1 & 74 & 74 & 71 & 0.835294118 & 26 & 0.021328958 & 0.320754717 \\
\hline ROCK-II(r) & 0 & 1 & 72 & 72 & 72 & 0.847058824 & 28 & 0.022969647 & 0.343724364 \\
\hline Rskl(h) & 66 & 0 & 68 & 68 & 73 & 0.858823529 & 32 & 0.026251025 & 0.36997539 \\
\hline Rskl(r) & 51 & 0 & 66 & 66 & 74 & 0.870588235 & 34 & 0.027891715 & 0.397867104 \\
\hline Rsk2(h) & 56 & 0 & 64 & 64 & 75 & 0.882352941 & 36 & 0.029532404 & 0.427399508 \\
\hline Rsk3(h) & 49 & 1 & 56 & 56 & 76 & 0.894117647 & 44 & 0.03609516 & 0.463494668 \\
\hline SAPK2a(h) & 94 & 0 & 51 & 51 & 77 & 0.905882353 & 49 & 0.040196883 & 0.50369155 \\
\hline SAPK2b(h) & 124 & 0 & 49 & 49 & 78 & 0.917647059 & 51 & 0.041837572 & 0.545529122 \\
\hline SAPK3(h) & 94 & 0 & 46 & 46 & 79 & 0.929411765 & 54 & 0.044298605 & 0.589827728 \\
\hline $\mathrm{SAPK} 4(\mathrm{~h})$ & 103 & 0 & 46 & 46 & 80 & 0.941176471 & 54 & 0.044298605 & 0.634126333 \\
\hline SGK(h) & 92 & 0 & 39 & 39 & 81 & 0.952941176 & 61 & 0.050041017 & 0.68416735 \\
\hline Syk(h) & 82 & 0 & 13 & 13 & 82 & 0.964705882 & 87 & 0.071369975 & 0.755537326 \\
\hline $\operatorname{TrkB}(\mathbf{h})$ & 95 & 0 & 2 & 2 & 83 & 0.976470588 & 98 & 0.080393765 & 0.835931091 \\
\hline Yes(h) & 81 & 0 & 0 & 0 & 84 & 0.988235294 & 100 & 0.082034454 & 0.917965546 \\
\hline ZAP-70(h) & 126 & 0 & -4 & 0 & 85 & 1 & 100 & 0.082034454 & 1 \\
\hline & & & & & & Total inhibition & 1219 & & Total area \\
\hline & $\begin{array}{l}\text { Hit rate } \\
\text { Threshold }\end{array}$ & ( & & & & & & & Gini \\
\hline
\end{tabular}




\begin{tabular}{|c|c|c|c|c|c|c|c|c|c|}
\hline & $10 \mu \mathrm{M}$ ATP & & & & & & & & \\
\hline Kinase & ZM336372@10 $\mu \mathrm{M}$ & Hit & sorted & normalized & position & cumulative sample fraction & inhibition\% & fraction of total inhibition & cumulative inhibition \\
\hline $\mathrm{Abl}(\mathrm{m})$ & 55 & 0 & 110 & 100 & 1 & 0.011764706 & 0 & 0 & 0 \\
\hline AMPK(r) & 87 & 0 & 102 & 100 & 2 & 0.023529412 & 0 & 0 & 0 \\
\hline $\operatorname{Arg}(\mathrm{m})$ & 58 & 0 & 101 & 100 & 3 & 0.035294118 & 0 & 0 & 0 \\
\hline Aurora-A(h) & 89 & 0 & 101 & 100 & 4 & 0.047058824 & 0 & 0 & 0 \\
\hline Axl(h) & 101 & 0 & 101 & 100 & 5 & 0.058823529 & 0 & 0 & 0 \\
\hline $\operatorname{Blk}(\mathrm{m})$ & 45 & 1 & 100 & 100 & 6 & 0.070588235 & 0 & 0 & 0 \\
\hline $\mathrm{Bmx(h)}$ & 86 & 0 & 100 & 100 & 7 & 0.082352941 & 0 & 0 & 0 \\
\hline CaMKII(r) & 97 & 0 & 100 & 100 & 8 & 0.094117647 & 0 & 0 & 0 \\
\hline CaMKIV(h) & 99 & 0 & 100 & 100 & 9 & 0.105882353 & 0 & 0 & 0 \\
\hline CDK1/cyclnBB(h) & 99 & 0 & 99 & 99 & 10 & 0.117647059 & 1 & 0.00059312 & 0.00059312 \\
\hline $\mathrm{CDK} 2 / \mathrm{cyclinA}(\mathrm{h})$ & 110 & 0 & 99 & 99 & 11 & 0.129411765 & 1 & 0.00059312 & 0.00118624 \\
\hline $\mathrm{CDK} 2 /$ cyclinE(h) & 95 & 0 & 99 & 99 & 12 & 0.141176471 & 1 & 0.00059312 & 0.001779359 \\
\hline CDK3/cyclinE(h) & 79 & 0 & 99 & 99 & 13 & 0.152941176 & 1 & 0.00059312 & 0.002372479 \\
\hline CDK5/p35(h) & 99 & 0 & 99 & 99 & 14 & 0.164705882 & 1 & 0.00059312 & 0.002965599 \\
\hline $\mathrm{CDK} 6 / \mathrm{cyclin} \mathrm{B} 3(\mathrm{~h})$ & 92 & 0 & 99 & 99 & 15 & 0.176470588 & 1 & 0.00059312 & 0.003558719 \\
\hline CDK7/cyclinHMATI(h) & 92 & 0 & 99 & 99 & 16 & 0.188235294 & 1 & 0.00059312 & 0.004151839 \\
\hline CHKI(h) & 98 & 0 & 99 & 99 & 17 & 0.2 & 1 & 0.00059312 & 0.004744958 \\
\hline СHK2(h) & 56 & 0 & 98 & 98 & 18 & 0.211764706 & 2 & 0.00118624 & 0.005931198 \\
\hline $\mathrm{CK} 1(\mathrm{y})$ & 89 & 0 & 98 & 98 & 19 & 0.223529412 & 2 & 0.00118624 & 0.007117438 \\
\hline СК2(h) & 100 & 0 & 98 & 98 & 20 & 0.235294118 & 2 & 0.00118624 & 0.008303677 \\
\hline c-RAF(h) & 1 & 1 & 98 & 98 & 21 & 0.247058824 & 2 & 0.00118624 & 0.009489917 \\
\hline CSK(h) & 54 & 0 & 97 & 97 & 22 & 0.258823529 & 3 & 0.001779359 & 0.011269276 \\
\hline $\operatorname{csRC(h)}$ & 87 & 0 & 95 & 95 & 23 & 0.270588235 & 5 & 0.002965599 & 0.014234875 \\
\hline Fess(h) & 91 & 0 & 95 & 95 & 24 & 0.282352941 & 5 & 0.002965599 & 0.017200474 \\
\hline FGFR3(h) & 101 & 0 & 95 & 95 & 25 & 0.294117647 & 5 & 0.002965599 & 0.020166074 \\
\hline $\mathrm{FH}$ H(h) & 94 & 0 & 95 & 95 & 26 & 0.305882353 & 5 & 0.002965599 & 0.023131673 \\
\hline Fyn(h) & 84 & 0 & 95 & 95 & 27 & 0.317647059 & 5 & 0.002965599 & 0.026097272 \\
\hline GSK3a(h) & 92 & 0 & 94 & 94 & 28 & 0.329411765 & 6 & 0.003558719 & 0.029655991 \\
\hline GSK $3 \mathrm{~B}(\mathrm{~h})$ & 98 & 0 & 94 & 94 & 29 & 0.341176471 & 6 & 0.003558719 & 0.033214709 \\
\hline IGF-IR(h) & 99 & 0 & 94 & 94 & 30 & 0.352941176 & 6 & 0.003558719 & 0.036773428 \\
\hline $\mathrm{IKKa( \textrm {h } )}$ & 88 & 0 & 94 & 94 & 31 & 0.364705882 & 6 & 0.003558719 & 0.040332147 \\
\hline IKKB(h) & 87 & 0 & 94 & 94 & 32 & 0.376470588 & 6 & 0.003558719 & 0.043890866 \\
\hline $\mathbb{I R}(\mathrm{h})$ & 100 & 0 & 94 & $\begin{array}{l}54 \\
94\end{array}$ & $\begin{array}{l}32 \\
33\end{array}$ & 0.388235294 & 6 & 0.003558719 & 0.047449585 \\
\hline JNKIal(h) & 81 & 0 & 93 & 93 & 34 & 0.4 & 7 & 0.004151839 & 0.051601423 \\
\hline JNK202(h) & 92 & 0 & 92 & 92 & 35 & 0.411764706 & 8 & 0.004744958 & 0.056346382 \\
\hline JNK3(r) & 91 & 0 & 92 & 92 & 36 & 0.423529412 & 8 & 0.004744958 & 0.06109134 \\
\hline Lck(h) & 5 & 1 & 92 & 92 & 37 & 0.435294118 & 8 & 0.004744958 & 0.065836299 \\
\hline Lynnh) & 16 & 1 & 92 & 92 & 38 & 0.447058824 & 8 & 0.004744958 & 0.070581257 \\
\hline Lyy(m) & 14 & 1 & 92 & 92 & 39 & 0.458823529 & 8 & 0.004744958 & 0.075326216 \\
\hline MAPKI(h) & $\begin{array}{l}14 \\
99\end{array}$ & 0 & $\begin{array}{l}92 \\
92\end{array}$ & $\begin{array}{l}92 \\
92\end{array}$ & 40 & 0.470588235 & 8 & 0.004744958 & 0.080071174 \\
\hline MAPK2(h) & 98 & 0 & 91 & 91 & 41 & 0.482352941 & 9 & 0.005338078 & 0.085409253 \\
\hline $\begin{array}{l}\text { MAPKL(2) } \\
\text { MAPK2(m) }\end{array}$ & 86 & 0 & 91 & 91 & $\begin{array}{l}41 \\
42\end{array}$ & 0.494117647 & 9 & 0.005338078 & 0.090747331 \\
\hline MAPKAP-K2(h) & 99 & 0 & 91 & 91 & 43 & 0.505882353 & 9 & 0.005338078 & 0.096085409 \\
\hline MEK1(h) & 88 & 0 & 91 & 91 & 44 & 0.517647059 & 9 & 0.005338078 & 0.101423488 \\
\hline MKK4(m) & 1 & 1 & 91 & 91 & 45 & 0.529411765 & 9 & 0.005338078 & 0.106761566 \\
\hline MKK6(h) & 12 & 1 & 91 & 91 & 46 & 0.541176471 & 9 & 0.005338078 & 0.112099644 \\
\hline MKK & 99 & 0 & 90 & 90 & 47 & 0.552941176 & 10 & 0.005931198 & 0.118030842 \\
\hline MSKI(h) & 92 & 0 & 89 & 89 & 48 & 0.564705882 & 11 & 0.006524318 & 0.12455516 \\
\hline $\mathrm{p} 7056 \mathrm{~K}(\mathrm{~h})$ & 84 & 0 & $\begin{array}{l}89 \\
89\end{array}$ & $\begin{array}{l}89 \\
89\end{array}$ & $\begin{array}{l}48 \\
49\end{array}$ & 0.576470588 & 11 & 0.006524318 & 0.131079478 \\
\hline $\begin{array}{l}\text { PAK2(h) } \\
\text { Past }\end{array}$ & 94 & 0 & 88 & 88 & 50 & 0.588235294 & 12 & 0.007117438 & 0.138196916 \\
\hline PDGFRa(h) & 5 & 1 & 88 & 88 & 51 & 0.6 & 12 & 0.007117438 & 0.145314353 \\
\hline PDGFRB(h) & 35 & 1 & 88 & 88 & 52 & 0.611764706 & 12 & 0.007117438 & 0.152431791 \\
\hline PDKI(h) & 94 & 0 & 88 & 88 & 53 & 0.623529412 & 12 & 0.007117438 & 0.159549229 \\
\hline PKA(b) & 91 & 0 & 88 & 88 & 54 & 0.635294118 & 12 & 0.007117438 & 0.166666667 \\
\hline PKA(h) & 101 & 0 & 87 & 87 & 55 & 0.647058824 & 13 & 0.007710558 & 0.174377224 \\
\hline PKBa(h) & 93 & 0 & 87 & 87 & 56 & 0.658823529 & 13 & 0.007710558 & 0.182087782 \\
\hline PKBB(h) & 98 & 0 & 87 & 87 & 57 & 0.670588235 & 13 & 0.007710558 & 0.189798339 \\
\hline $\mathrm{PKB} \mathrm{B}_{(\mathrm{h})}$ & 92 & 0 & 87 & 87 & 58 & 0.682352941 & 13 & 0.007710558 & 0.197508897 \\
\hline PKCath) & 88 & 0 & 86 & 86 & 59 & 0.694117647 & 14 & 0.008303677 & 0.205812574 \\
\hline PKCBII(h) & 87 & 0 & 86 & 86 & 60 & 0.705882353 & 14 & 0.008303677 & 0.214116251 \\
\hline PKCy(h) & 91 & 0 & 86 & 86 & 61 & 0.717647059 & 14 & 0.008303677 & 0.222419929 \\
\hline РКФ(1) & 95 & 0 & $\begin{array}{l}80 \\
84\end{array}$ & $\begin{array}{l}86 \\
84\end{array}$ & $\begin{array}{l}61 \\
62\end{array}$ & 0.729411765 & $\begin{array}{l}14 \\
16\end{array}$ & 0.009489917 & 0.231909846 \\
\hline PKG:(h) & 82 & 0 & 84 & 84 & 63 & 0.741176471 & 16 & 0.009489917 & 0.241399763 \\
\hline $\begin{array}{l}\text { PKG (h) } \\
\text { (h) }\end{array}$ & 100 & 0 & 83 & 83 & 64 & 0.752941176 & 17 & 0.010083037 & 0.2514828 \\
\hline PKC (h) & 100 & 0 & 82 & 82 & 65 & 0.764705882 & 18 & 0.010676157 & 0.262158956 \\
\hline PKC $\mu(\mathrm{h})$ & 86 & 0 & 81 & 81 & 66 & 0.776470588 & 19 & 0.011269276 & 0.273428233 \\
\hline PKCO(h) & 94 & 0 & 79 & 79 & 67 & 0.788235294 & 21 & 0.012455516 & 0.285883749 \\
\hline PKD2(h) & 99 & 0 & 79 & 79 & 68 & 0.8 & 21 & 0.012455516 & 0.298339265 \\
\hline PRAK(h) & 90 & 0 & 73 & 73 & 69 & 0.811764706 & 27 & 0.016014235 & 0.314353499 \\
\hline PRK2(h) & 94 & 0 & 69 & 69 & 70 & 0.823529412 & 31 & 0.018386714 & 0.332740214 \\
\hline $\begin{array}{l}\text { PRK_(n) } \\
\text { ROCK-II(h) }\end{array}$ & 73 & 0 & 58 & 58 & 71 & 0.835294118 & 42 & 0.024911032 & 0.357651246 \\
\hline ROCK-II(r) & 69 & 0 & 56 & 56 & 72 & 0.847058824 & 44 & 0.026097272 & 0.383748517 \\
\hline Rskl(h) & 95 & 0 & 55 & 55 & 73 & 0.858823529 & 45 & 0.026690391 & 0.410438909 \\
\hline Rskl(r) & 91 & 0 & 54 & 54 & 74 & 0.870588235 & 46 & 0.027283511 & 0.43772242 \\
\hline $\begin{array}{l}\text { Rsksk2(h) } \\
\text { Rsk(h) }\end{array}$ & 88 & 0 & 45 & 45 & 75 & 0.882352941 & 55 & 0.03262159 & 0.470344009 \\
\hline Rsk3(h) & 102 & 0 & 35 & 35 & 76 & 0.894117647 & 65 & 0.038552788 & 0.508896797 \\
\hline SAPK2a(h) & 4 & 1 & 16 & 16 & 77 & 0.905882353 & 84 & 0.049822064 & 0.558718861 \\
\hline SAPK2b(h) & 14 & 1 & 14 & 14 & 78 & 0.917647059 & 86 & 0.051008304 & 0.609727165 \\
\hline SAPK3(h) & 95 & 0 & 14 & 14 & 79 & 0.929411765 & 86 & 0.051008304 & 0.660735469 \\
\hline $\begin{array}{l}\mathrm{SARRS( \textrm {h } )} \\
\mathrm{SAPK} 4(\mathrm{~h})\end{array}$ & 95 & 0 & 12 & 12 & 80 & 0.941176471 & 88 & 0.052194543 & 0.712930012 \\
\hline SGK(h) & 83 & 0 & 5 & 5 & 81 & 0.952941176 & 95 & 0.056346382 & 0.769276394 \\
\hline Syk(h) & 79 & 0 & 5 & 5 & 82 & 0.964705882 & 95 & 0.056346382 & 0.825622776 \\
\hline TrkB(h) & 94 & 0 & 4 & 4 & 83 & 0.976470588 & 96 & 0.056939502 & 0.882562278 \\
\hline $\begin{array}{l}\text { IIrkB(h) } \\
\text { Yesh(h) }\end{array}$ & $\begin{array}{l}54 \\
91\end{array}$ & 0 & $\begin{array}{l}4 \\
1\end{array}$ & 1 & 84 & 0.988235294 & $\begin{array}{l}90 \\
99\end{array}$ & 0.058718861 & 0.941281139 \\
\hline ZAP-70(h) & 88 & 0 & 1 & 1 & 85 & 1 & 99 & 0.058718861 & 1 \\
\hline & & & & & & Total inhibition & 1686 & & Total area \\
\hline & $\begin{array}{r}\text { Hit rate } \\
\text {. }\end{array}$ & 5 & & & & & & & Gini \\
\hline
\end{tabular}




\begin{tabular}{|c|c|c|c|c|c|c|c|c|c|}
\hline & $10 \mu \mathrm{M}$ ATP & & & & & & & & \\
\hline Kinase & ZM336372@10 $\mu \mathrm{M}$ & Hit & sorted & normalized & position & cumulative sample fraction & inhibition\% & fraction of total inhibition & cumulative inhibition \\
\hline $\mathrm{Abl}(\mathrm{m})$ & 69 & 0 & 124 & 100 & 1 & 0.011764706 & 0 & 0 & 0 \\
\hline AMPK(r) & 101 & 0 & 116 & 100 & 2 & 0.023529412 & 0 & 0 & 0 \\
\hline $\operatorname{Arg}(\mathrm{m})$ & 58 & 0 & 114 & 100 & 3 & 0.035294118 & 0 & 0 & 0 \\
\hline Aurora-A(h) & 76 & 0 & 109 & 100 & 4 & 0.047058824 & 0 & 0 & 0 \\
\hline Axl(h) & 88 & 0 & 105 & 100 & 5 & 0.058823529 & 0 & 0 & 0 \\
\hline $\operatorname{Blk}(\mathrm{m})$ & 72 & 0 & 105 & 100 & 6 & 0.070588235 & 0 & 0 & 0 \\
\hline Bmxx(h) & 92 & 0 & 102 & 100 & 7 & 0.082352941 & 0 & 0 & 0 \\
\hline СамKII(r) & 95 & 0 & 102 & 100 & 8 & 0.094117647 & 0 & 0 & 0 \\
\hline CaMKIV(h) & 101 & 0 & 102 & 100 & 9 & 0.105882353 & 0 & 0 & 0 \\
\hline CDK1/cyclnBB(h) & 100 & 0 & 101 & 100 & 10 & 0.117647059 & 0 & 0 & 0 \\
\hline $\mathrm{CDK} /$ /yclinA(h) & 91 & 0 & 101 & 100 & 11 & 0.129411765 & 0 & 0 & 0 \\
\hline $\mathrm{CDK} 2 /$ cyclinE(h) & 98 & 0 & 100 & 100 & 12 & 0.141176471 & 0 & 0 & 0 \\
\hline CDK3/cyclinE(h) & 93 & 0 & 100 & 100 & 13 & 0.152941176 & 0 & 0 & 0 \\
\hline CDK5/p35(h) & 116 & 0 & 99 & 99 & 14 & 0.164705882 & 1 & 0.000684932 & 0.000684932 \\
\hline CDKG/cyclinD3(h) & 91 & 0 & 99 & 99 & 15 & 0.176470588 & 1 & 0.000684932 & 0.001369863 \\
\hline CDK7/cyclinHMATI(h) & 95 & 0 & 98 & 98 & 16 & 0.188235294 & 2 & 0.001369863 & 0.002739726 \\
\hline СнК $1 \mathrm{~h})$ & 93 & 0 & 98 & 98 & 17 & 0.2 & 2 & 0.001369863 & 0.004109589 \\
\hline СHK2(h) & 77 & 0 & 97 & 97 & 18 & 0.211764706 & 3 & 0.002054795 & 0.006164384 \\
\hline $\mathrm{CK} 1(\mathrm{y})$ & 87 & 0 & 97 & 97 & 19 & 0.223529412 & 3 & 0.002054795 & 0.008219178 \\
\hline СК2(h) & 100 & 0 & 97 & 97 & 20 & 0.235294118 & 3 & 0.002054795 & 0.010273973 \\
\hline c-RAF(h) & 3 & 1 & 95 & 95 & 21 & 0.247058824 & 5 & 0.003424658 & 0.01369863 \\
\hline $\operatorname{CSK}(\mathrm{h})$ & 87 & 0 & 95 & 95 & 22 & 0.258823529 & 5 & 0.003424658 & 0.017123288 \\
\hline cSRC(h) & 89 & 0 & 95 & 95 & 23 & 0.270588235 & 5 & 0.003424658 & 0.020547945 \\
\hline Fes(h) & 102 & 0 & 95 & 95 & 24 & 0.282352941 & 5 & 0.003424658 & 0.023972603 \\
\hline FGFR3(h) & 80 & 0 & 95 & 95 & 25 & 0.294117647 & 5 & 0.003424658 & 0.02739726 \\
\hline $\mathrm{Fll}(\mathrm{h}(\mathrm{h})$ & 97 & 0 & 94 & 94 & 26 & 0.305882353 & 6 & 0.004109589 & 0.031506849 \\
\hline Fyn(h) & 88 & 0 & 94 & 94 & 27 & 0.317647059 & 6 & 0.004109589 & 0.035616438 \\
\hline GSK3a(h) & 102 & 0 & 94 & 94 & 28 & 0.329411765 & 6 & 0.004109589 & 0.039726027 \\
\hline GSK3B(h) & 99 & 0 & 94 & 94 & 29 & 0.341176471 & 6 & 0.004109589 & 0.043835616 \\
\hline IGF-IR(h) & 95 & 0 & 94 & 94 & 30 & 0.352941176 & 6 & 0.004109589 & 0.047945205 \\
\hline IKKa(h) & 86 & 0 & 93 & 93 & 31 & 0.364705882 & 7 & 0.004794521 & 0.052739726 \\
\hline IKKB(h) & 99 & 0 & 93 & 93 & 32 & 0.376470588 & 7 & 0.004794521 & 0.057534247 \\
\hline $\mathbb{I R ( h )}$ & 102 & 0 & 93 & 93 & 33 & 0.388235294 & 7 & 0.004794521 & 0.062328767 \\
\hline JNKIal(h) & 94 & 0 & 93 & 93 & 34 & 0.4 & 7 & 0.004794521 & 0.067123288 \\
\hline JNK2202(h) & 82 & 0 & 92 & 92 & 35 & 0.411764706 & 8 & 0.005479452 & 0.07260274 \\
\hline JNK3(r) & 88 & 0 & 92 & 92 & 36 & 0.423529412 & 8 & 0.005479452 & 0.078082192 \\
\hline Lck(h) & 10 & 1 & 92 & 92 & 37 & 0.435294118 & 8 & 0.005479452 & 0.083561644 \\
\hline Lyn(h) & 44 & 1 & 92 & 92 & 38 & 0.447058824 & 8 & 0.005479452 & 0.089041096 \\
\hline Lyn(m) & 31 & 1 & 92 & 92 & 39 & 0.458823529 & 8 & 0.005479452 & 0.094520548 \\
\hline MAPK1(h) & 89 & 0 & 92 & 92 & 40 & 0.470588235 & 8 & 0.005479452 & 0.1 \\
\hline MAPK2(h) & 92 & 0 & 92 & 92 & 41 & 0.482352941 & 8 & 0.005479452 & 0.105479452 \\
\hline MAPK2(m) & 97 & 0 & 92 & 92 & 42 & 0.494117647 & 8 & 0.005479452 & 0.110958904 \\
\hline MAPKAP-K2(h) & 124 & 0 & 91 & 91 & 43 & 0.505882353 & 9 & 0.006164384 & 0.117123288 \\
\hline MEK1(h) & 92 & 0 & 91 & 91 & 44 & 0.517647059 & 9 & 0.006164384 & 0.123287671 \\
\hline MKK $4(\mathrm{~m})$ & 71 & 0 & 91 & 91 & 45 & 0.529411765 & 9 & 0.006164384 & 0.129452055 \\
\hline MKK6(h) & 9 & 1 & 91 & 91 & 46 & 0.541176471 & 9 & 0.006164384 & 0.135616438 \\
\hline MKK $7 \mathrm{B(h)}$ & 83 & 0 & 91 & 91 & 47 & 0.552941176 & 9 & 0.006164384 & 0.141780822 \\
\hline MSKI(h) & 98 & 0 & 90 & 90 & 48 & 0.564705882 & 10 & 0.006849315 & 0.148630137 \\
\hline p7056K(h) & 97 & 0 & 89 & 89 & 49 & 0.576470588 & 11 & 0.007534247 & 0.156164384 \\
\hline PAK2(h) & 92 & 0 & 89 & 89 & 50 & 0.588235294 & 11 & 0.007534247 & 0.16369863 \\
\hline PDGFRa(h) & 5 & 1 & 89 & 89 & 51 & 0.6 & 11 & 0.007534247 & 0.171232877 \\
\hline PDGFRB(h) & 40 & 1 & 88 & 88 & 52 & 0.611764706 & 12 & 0.008219178 & 0.179452055 \\
\hline PDKI(h) & $\begin{array}{l}40 \\
89\end{array}$ & 0 & 88 & 88 & 53 & 0.623529412 & 12 & 0.008219178 & 0.187671233 \\
\hline PKA(b) & 82 & 0 & 88 & 88 & 54 & 0.635294118 & 12 & 0.008219178 & 0.195890411 \\
\hline PKA(h) & 81 & 0 & 88 & 88 & 55 & 0.647058824 & 12 & 0.008219178 & 0.204109589 \\
\hline PKBa(h) & 91 & 0 & 88 & 88 & 56 & 0.658823529 & 12 & 0.008219178 & 0.212328767 \\
\hline PKB(h) & 93 & 0 & 87 & 87 & 57 & 0.670588235 & 13 & 0.00890411 & 0.221232877 \\
\hline $\mathrm{PKB}_{\gamma}(\mathrm{h})$ & 94 & 0 & 87 & 87 & 58 & 0.682352941 & 13 & 0.00890411 & 0.230136986 \\
\hline PKC(th) & 105 & 0 & 87 & 87 & 59 & 0.694117647 & 13 & 0.00890411 & 0.239041096 \\
\hline РКCBII(h) & 92 & 0 & 86 & 86 & 60 & 0.705882353 & 14 & 0.009589041 & 0.248630137 \\
\hline PKCy (h) & 92 & 0 & 86 & 86 & 61 & 0.717647059 & 14 & 0.009589041 & 0.258219178 \\
\hline PKБ(h) & 95 & 0 & 83 & 83 & 62 & 0.729411765 & 17 & 0.011643836 & 0.269863014 \\
\hline PKG:(h) & 92 & 0 & 83 & 83 & 63 & 0.741176471 & 17 & 0.011643836 & 0.281506849 \\
\hline PKG(h) & 114 & 0 & 82 & 82 & 64 & 0.752941176 & 18 & 0.012328767 & 0.293835616 \\
\hline PKC (h) & 88 & 0 & 82 & 82 & 65 & 0.764705882 & 18 & 0.012328767 & 0.306164384 \\
\hline PKC $\mu(\mathrm{h})$ & 92 & 0 & 81 & 81 & 66 & 0.776470588 & 19 & 0.013013699 & 0.319178082 \\
\hline PKCO(h) & 94 & 0 & 81 & 81 & 67 & 0.788235294 & 19 & 0.013013699 & 0.332191781 \\
\hline PKD2(h) & 91 & 0 & 80 & 80 & 68 & 0.8 & 20 & 0.01369863 & 0.345890411 \\
\hline PRAK(h) & 81 & 0 & 77 & 77 & 69 & 0.811764706 & 23 & 0.015753425 & 0.361643836 \\
\hline PRK2(h) & 87 & 0 & 77 & 77 & 70 & 0.823529412 & 23 & 0.015753425 & 0.37739726 \\
\hline ROCK-II(h) & 83 & 0 & 76 & 76 & 71 & 0.835294118 & 24 & 0.016438356 & 0.393835616 \\
\hline ROCK-II(r) & 73 & 0 & 73 & 73 & 72 & 0.847058824 & 27 & 0.018493151 & 0.412328767 \\
\hline Rskl(h) & 105 & 0 & 72 & 72 & 73 & 0.858823529 & 28 & 0.019178082 & 0.431506849 \\
\hline $\operatorname{Rskl(r)}$ & 77 & 0 & 71 & 71 & 74 & 0.870588235 & 29 & 0.019863014 & 0.451369863 \\
\hline Rsk2(h) & 88 & 0 & 69 & 69 & 75 & 0.882352941 & 31 & 0.021232877 & 0.47260274 \\
\hline Rsk3(h) & 90 & 0 & 58 & 58 & 76 & 0.894117647 & 42 & 0.028767123 & 0.501369863 \\
\hline SAPK2a(h) & 8 & 1 & 44 & 44 & 77 & 0.905882353 & 56 & 0.038356164 & 0.539726027 \\
\hline SAPK2b(h) & 22 & 1 & 40 & 40 & 78 & 0.917647059 & 60 & 0.04109589 & 0.580821918 \\
\hline $\mathrm{SAPK} 3(\mathrm{~h})$ & 93 & 0 & 31 & 31 & 79 & 0.929411765 & 69 & 0.047260274 & 0.628082192 \\
\hline SAPK4(h) & 94 & 0 & 22 & 22 & 80 & 0.941176471 & 78 & 0.053424658 & 0.681506849 \\
\hline SGK(h) & 91 & 0 & 10 & 10 & 81 & 0.952941176 & 90 & 0.061643836 & 0.743150685 \\
\hline Syk(h) & 109 & 0 & 9 & 9 & 82 & 0.964705882 & 91 & 0.062328767 & 0.805479452 \\
\hline TrkB(h) & 94 & 0 & 8 & 8 & 83 & 0.976470588 & 92 & 0.063013699 & 0.868493151 \\
\hline Yes(h) & 86 & 0 & 5 & 5 & 84 & 0.988235294 & 95 & 0.065068493 & 0.933561644 \\
\hline ZAP-70(h) & 95 & 0 & 3 & 3 & 85 & 1 & 97 & 0.066438356 & 1 \\
\hline & & & & & & Total inhibition & 1460 & & Total area \\
\hline & $\begin{array}{r}\text { Hit rate } \\
\text {. }\end{array}$ & 5 & & & & & & & Gini \\
\hline
\end{tabular}




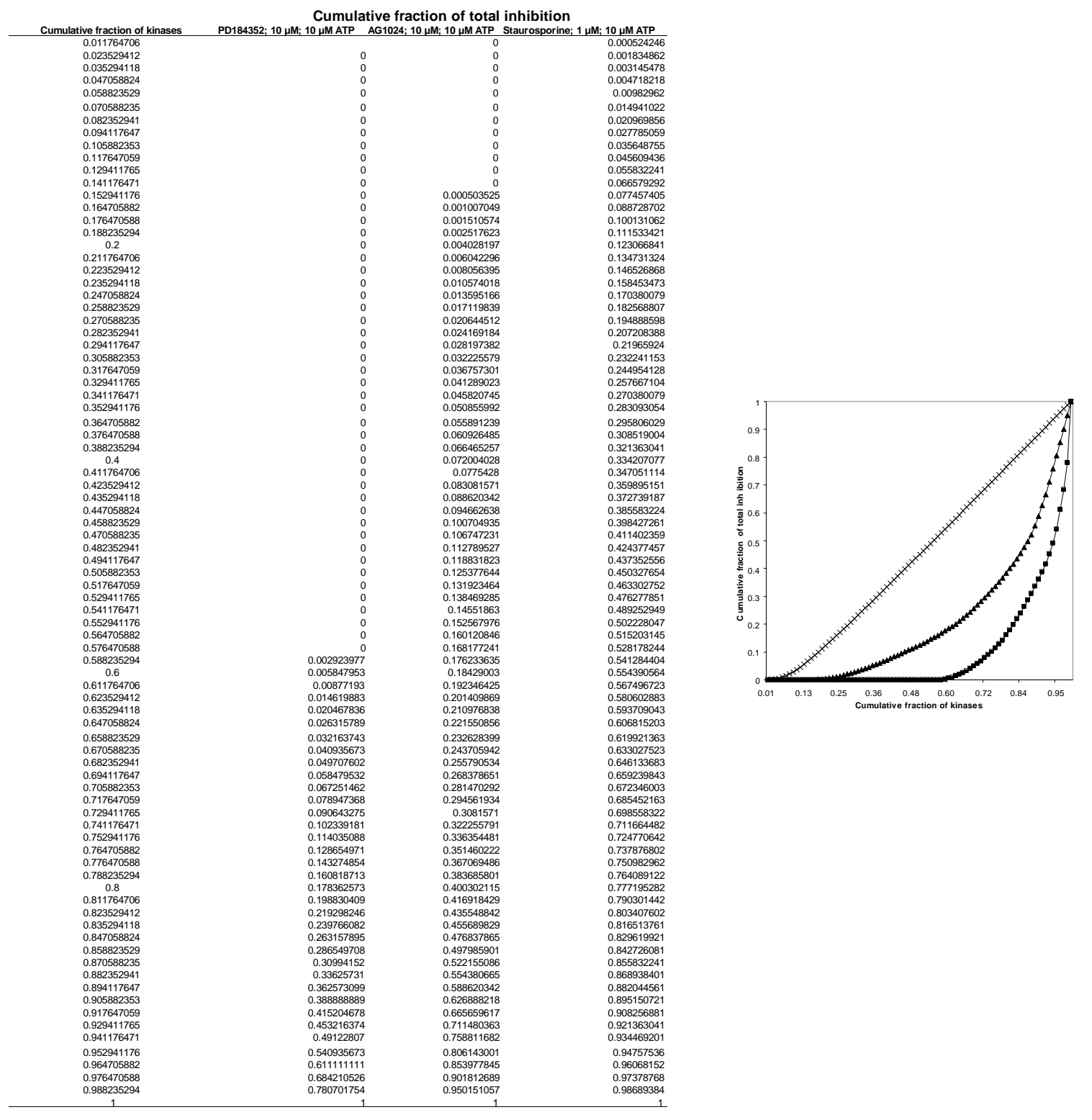




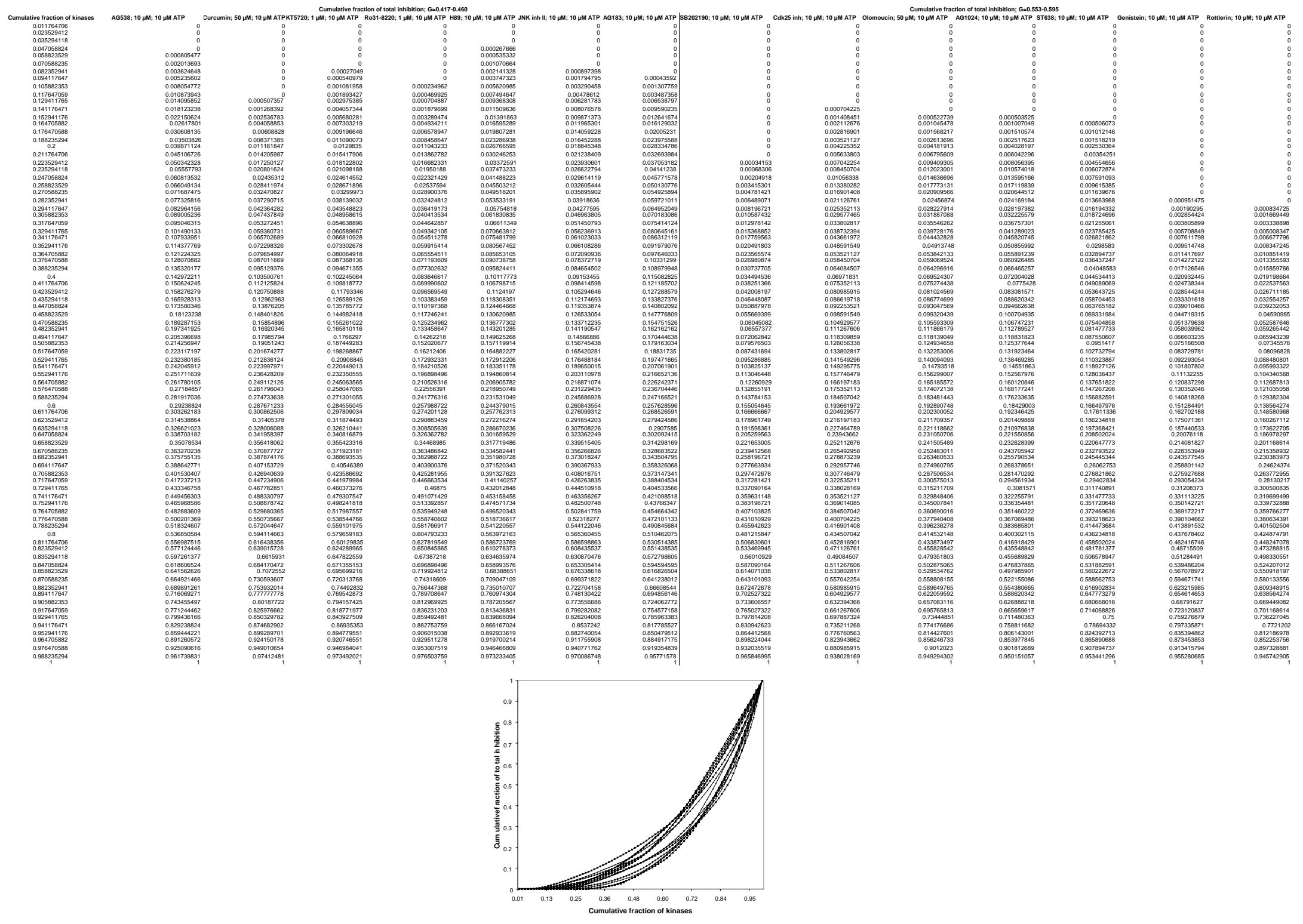




\begin{tabular}{|c|c|c|}
\hline Inhibitor & Gini at 10 & Gini at 100 \\
\hline AG1024 & 0.568 & 0.637 \\
\hline AG1296 & 0.498 & \\
\hline AG1478 & 0.5 & 0.649 \\
\hline AG18 & 0.68 & 0.652 \\
\hline AG183 & 0.46 & 0.518 \\
\hline AG538 & 0.417 & 0.5 \\
\hline Alsterpaullone & 0.633 & 0.704 \\
\hline $\begin{array}{l}\text { Calphostin C } \\
\text { Cdk2/5 inh }\end{array}$ & $\begin{array}{l}0.606 \\
0.555\end{array}$ & $\begin{array}{l}0.651 \\
0.678\end{array}$ \\
\hline Curcumin & 0.417 & 0.538 \\
\hline EGCG & 0.495 & 0.621 \\
\hline Genistein & 0.582 & \\
\hline H89 & 0.442 & 0.518 \\
\hline HA1077 & 0.65 & 0.677 \\
\hline $\begin{array}{l}\text { Herbimycin A } \\
\text { HHisidjin }\end{array}$ & 0.534 & $\begin{array}{r}0.59 \\
0.722\end{array}$ \\
\hline $\begin{array}{l}\text { Hispidin } \\
\text { Indirubin }\end{array}$ & $\begin{array}{r}0.79 \\
0.291\end{array}$ & $\begin{array}{l}0.722 \\
0.407\end{array}$ \\
\hline Indirubin & 0.445 & 0.533 \\
\hline $\mathrm{K} 252 \mathrm{c}$ & 0.236 & 0.384 \\
\hline KT5720 & 0.423 & 0.537 \\
\hline Lavendustin A & 0.726 & 0.779 \\
\hline Lavendustin B & 0.515 & 0.548 \\
\hline LY294002 & 0.619 & 0.733 \\
\hline Olomoucin & 0.556 & 0.66 \\
\hline PD153035 & 0.616 & 0.623 \\
\hline PD184352 & 0.802 & 0.905 \\
\hline PP1 analog & 0.758 & 0.837 \\
\hline & 0.722 & 0.799 \\
\hline PP3 & 0.634 & 0.75 \\
\hline $\begin{array}{l}\text { Ro31-8220 } \\
\text { Rospivite }\end{array}$ & 0.432 & 0.527 \\
\hline Roscovitine & 0.744 & 0.805 \\
\hline Rottlerin & 0.595 & 0.627 \\
\hline SB202190 & 0.553 & $\begin{array}{r}0.7 \\
0731\end{array}$ \\
\hline SB203580 & 0.621 & 0.731 \\
\hline ST638 & 0.568 & 0.624 \\
\hline Staurosporine & 0.093 & 0.15 \\
\hline sU6656 & 0.607 & 0.679 \\
\hline Wortmannin & $\begin{array}{l}0.775 \\
0.628\end{array}$ & $\begin{array}{l}0.739 \\
0.703\end{array}$ \\
\hline $\begin{array}{l}\text { Y727632 } \\
\text { ZM336372 }\end{array}$ & $\begin{array}{l}0.628 \\
0.635\end{array}$ & 0.609 \\
\hline
\end{tabular}

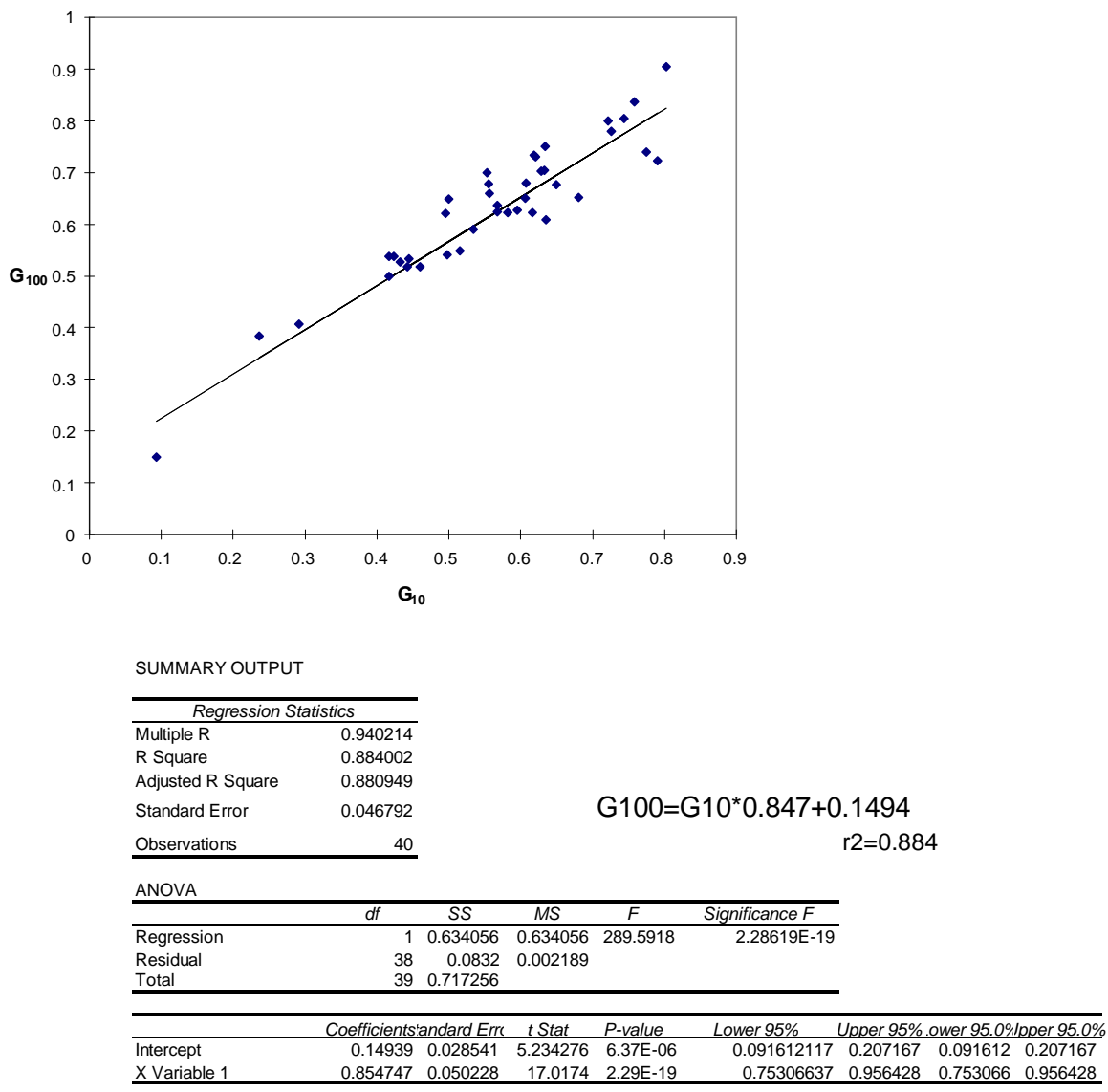

RESIDUAL OUTPUT

\begin{tabular}{|c|c|c|c|c|}
\hline \multirow[t]{40}{*}{ Observation } & \multicolumn{4}{|c|}{ 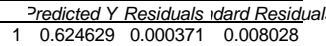 } \\
\hline & 2 & 0.564797 & -0.034797 & -0.753373 \\
\hline & 3 & 0.566506 & 0.070494 & 1.526229 \\
\hline & 4 & $\begin{array}{l}0.720361 \\
0532316\end{array}$ & $\begin{array}{l}-0.080361 \\
-0.026316\end{array}$ & $\begin{array}{l}-1.739861 \\
-0\end{array}$ \\
\hline & 5 & 0.532316 & -0.026316 & -0.569767 \\
\hline & 6 & 0.496417 & -0.007417 & -0.160584 \\
\hline & 7 & 0.681043 & 0.010957 & 0.237236 \\
\hline & 8 & 0.65711 & -0.01811 & -0.392083 \\
\hline & & 0.613517 & 0.053483 & 1.157929 \\
\hline & 10 & 0.495562 & 0.030438 & 0.658994 \\
\hline & 11 & 0.563087 & 0.045913 & 0.994036 \\
\hline & 12 & 0.636596 & -0.024596 & -0.53251 \\
\hline & 13 & 0.516931 & -0.010931 & -0.236663 \\
\hline & 14 & 0.694718 & -0.029718 & -0.643423 \\
\hline & 15 & 0.595568 & -0.016568 & -0.358702 \\
\hline & 16 & 0.815238 & -0.105238 & -2.278462 \\
\hline & 17 & 0.387864 & 0.007136 & 0.154495 \\
\hline & 18 & 0.519495 & 0.002505 & 0.054229 \\
\hline & 19 & 0.341708 & 0.030292 & 0.655844 \\
\hline & 20 & 0.500691 & 0.024309 & 0.526308 \\
\hline & 21 & 0.759679 & 0.008321 & 0.180149 \\
\hline & & & -0.044182 & -0.956574 \\
\hline & 23 & 0.668221 & 0.053779 & 1.164341 \\
\hline & 24 & 0.614372 & 0.033628 & 0.728062 \\
\hline & 25 & 0.666512 & -0.055512 & -1.201864 \\
\hline & 26 & 0.82464 & 0.06836 & 1.480034 \\
\hline & 27 & 0.787031 & 0.037969 & 0.822048 \\
\hline & 28 & 0.757115 & 0.030885 & 0.668678 \\
\hline & & 0.681043 & 0.056957 & 1.233164 \\
\hline & 30 & 0.508384 & 0.006616 & 0.14325 \\
\hline & 31 & 0.775065 & 0.017935 & 0.38831 \\
\hline & 32 & 0.647707 & -0.032707 & -0.708134 \\
\hline & 33 & 0.611808 & 0.077192 & 1.671254 \\
\hline & 34 & 0.669931 & 0.049069 & 1.062378 \\
\hline & 35 & 0.625484 & -0.013484 & -0.291935 \\
\hline & 36 & 0.218624 & -0.080624 & 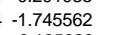 \\
\hline & 37 & 0.657964 & 0.009036 & 0.195628 \\
\hline & 38 & 0.801562 & -0.074562 & -1.614309 \\
\hline & 39 & 0.676769 & 0.014231 & 0.308115 \\
\hline & 40 & 0.682752 & -0.084752 & -1.834932 \\
\hline
\end{tabular}




\section{Calculation of the Gini coefficient for subsets of 10, 20, 40, 60 and 80 kinases}

Summary

Page

AG1024; $10 \mu \mathrm{M}$ at $10 \mu \mathrm{M}$ ATP

S87

Staurosporine; $1 \mu \mathrm{M}$ at $100 \mu \mathrm{M}$ ATP

S88-S93

PD184352; $10 \mu \mathrm{M}$ at $10 \mu \mathrm{M}$ ATP

S94-S99

S100-S105

\section{Method:}

For each inhibitor the observed percentage of inhibition values are randomized by sorting together with the RAND() function output in Excel. Then the top 10, 20, 40, 60 or 80 datapoints are used for calculation of the Gini coefficient using the standard approach described in the manuscript. Each sorting process is repeated 13 times to generate 13 Gini values calculated for each inhibitor and each sample size 
Calculation of Gini coefficient for subsets of 10, 20, 40,60 and 80 kinases for AG1024; $10 \mu \mathrm{M}$ at $10 \mu \mathrm{M}$ ATP, Staurosporine; $1 \mu \mathrm{M}$ at $100 \mu \mathrm{M}$ ATP and PD184352; $10 \mu \mathrm{M}$ at $10 \mu \mathrm{M}$ ATP

\begin{tabular}{|c|c|c|c|c|c|c|c|}
\hline & $\begin{array}{l}\text { \#kinases } \\
\text { fraction of panel }\end{array}$ & $\begin{array}{r}10 \\
12 \\
\end{array}$ & $\begin{array}{l}20 \\
24 \\
\end{array}$ & $\begin{array}{l}40 \\
47 \\
\end{array}$ & $\begin{array}{r}60 \\
71 \\
\end{array}$ & $\begin{array}{r}80 \\
94 \\
\end{array}$ & $\begin{array}{r}85 \\
100 \\
\end{array}$ \\
\hline \multirow[t]{15}{*}{ AG1024; $10 \mu \mathrm{M} ; 10 \mu \mathrm{M}$ ATP } & & 0.42941176 & 0.589535 & 0.599303 & 0.60457 & 0.572388 & 0.567976 \\
\hline & & 0.61906615 & 0.575553 & 0.531952 & 0.577966 & 0.571602 & \\
\hline & & 0.51 & 0.488176 & 0.533655 & 0.585512 & 0.572144 & \\
\hline & & 0.47557252 & 0.540339 & 0.488571 & 0.554745 & 0.557741 & \\
\hline & & 0.39838337 & 0.514025 & 0.525 & 0.579844 & 0.566771 & \\
\hline & & 0.63853211 & 0.578645 & 0.586852 & 0.54177 & 0.556234 & \\
\hline & & 0.51100324 & 0.504936 & 0.547286 & 0.578456 & 0.569769 & \\
\hline & & 0.5460177 & 0.544106 & 0.54312 & 0.567157 & 0.577508 & \\
\hline & & 0.65771429 & 0.574862 & 0.551049 & 0.565003 & 0.56345 & \\
\hline & & 0.56532258 & 0.56147 & 0.559742 & 0.554488 & 0.5724 & \\
\hline & & 0.3848 & 0.477394 & 0.59112 & 0.575232 & 0.573336 & \\
\hline & & 0.48709677 & 0.566229 & 0.593215 & 0.554966 & 0.557539 & \\
\hline & & 0.623 & 0.50506 & 0.571835 & 0.585565 & 0.573054 & \\
\hline & mean & 0.52660927 & 0.540025 & 0.555593 & 0.571175 & 0.567995 & 0.567976 \\
\hline & st. dev. & 0.09133939 & 0.037994 & 0.032352 & 0.016952 & 0.007022 & \\
\hline \multirow[t]{14}{*}{ Staurosporine; $1 \mu \mathrm{M} ; 100 \mu \mathrm{M}$ ATP } & & 0.29167905 & 0.224685 & 0.148671 & 0.142825 & 0.148402 & 0.149716 \\
\hline & & 0.03747368 & 0.139868 & 0.184498 & 0.174142 & 0.154354 & \\
\hline & & 0.15525 & 0.163243 & 0.144701 & 0.157726 & 0.156703 & \\
\hline & & $\begin{array}{l}0.14529201 \\
0.06307692\end{array}$ & $\begin{array}{l}0.177681 \\
0.136763\end{array}$ & $\begin{array}{l}0.142566 \\
0.117826\end{array}$ & $\begin{array}{r}0.131631 \\
0.14554\end{array}$ & $\begin{array}{l}0.149478 \\
0.145417\end{array}$ & \\
\hline & & 0.07538803 & 0.117588 & 0.107972 & 0.191178 & 0.147984 & \\
\hline & & 0.08290214 & 0.167166 & 0.148667 & 0.164552 & 0.157315 & \\
\hline & & 0.33930435 & 0.049469 & 0.125668 & 0.15348 & 0.143408 & \\
\hline & & 0.06818182 & 0.209987 & 0.159539 & 0.159036 & 0.156389 & \\
\hline & & 0.09394619 & 0.079624 & 0.103399 & 0.165024 & 0.145512 & \\
\hline & & 0.0374078 & 0.262006 & 0.133141 & 0.148134 & 0.14305 & \\
\hline & & 0.14073171 & 0.171259 & 0.158731 & 0.132833 & 0.145447 & \\
\hline & & 0.22166667 & 0.153509 & 0.105517 & 0.160927 & 0.148672 & \\
\hline & mean & 0.13479234 & 0.157911 & 0.136992 & 0.155925 & 0.149395 & 0.149716 \\
\hline & st. dev. & 0.09616582 & 0.057006 & 0.024277 & 0.01643 & 0.005129 & \\
\hline \multirow[t]{15}{*}{ PD184352; $10 \mu \mathrm{M} ; 10 \mu \mathrm{M}$ ATP } & & 0.54210526 & 0.747468 & 0.804108 & 0.826296 & 0.789839 & 0.802202 \\
\hline & & 0.816 & 0.709048 & 0.763108 & 0.779976 & 0.799027 & \\
\hline & & 0.65714286 & 0.718254 & 0.832398 & 0.822678 & 0.807899 & \\
\hline & & 0.68888889 & 0.831944 & 0.764306 & 0.752482 & 0.808269 & \\
\hline & & 0.81666667 & 0.833186 & 0.768439 & 0.805006 & 0.80262 & \\
\hline & & 0.9 & 0.8 & 0.755328 & 0.798023 & 0.799027 & \\
\hline & & 0.71666667 & 0.853333 & 0.828627 & 0.813927 & 0.805023 & \\
\hline & & 0.70869565 & 0.706364 & 0.858523 & 0.799716 & 0.813661 & \\
\hline & & 0.78275862 & 0.6775 & 0.696233 & 0.760987 & 0.761654 & \\
\hline & & 0.53225806 & 0.925 & 0.770041 & 0.82981 & 0.805661 & \\
\hline & & 0.6 & 0.709459 & 0.855874 & 0.823127 & 0.789839 & \\
\hline & & 0.61176471 & 0.768182 & 0.752311 & 0.803091 & 0.805023 & \\
\hline & & 0.77058824 & 0.739189 & 0.780607 & 0.807956 & 0.800933 & \\
\hline & mean & 0.70334889 & 0.770687 & 0.786916 & 0.801775 & 0.799113 & 0.802202 \\
\hline & st. dev. & 0.11251672 & 0.072925 & 0.046681 & 0.024264 & 0.013154 & \\
\hline
\end{tabular}

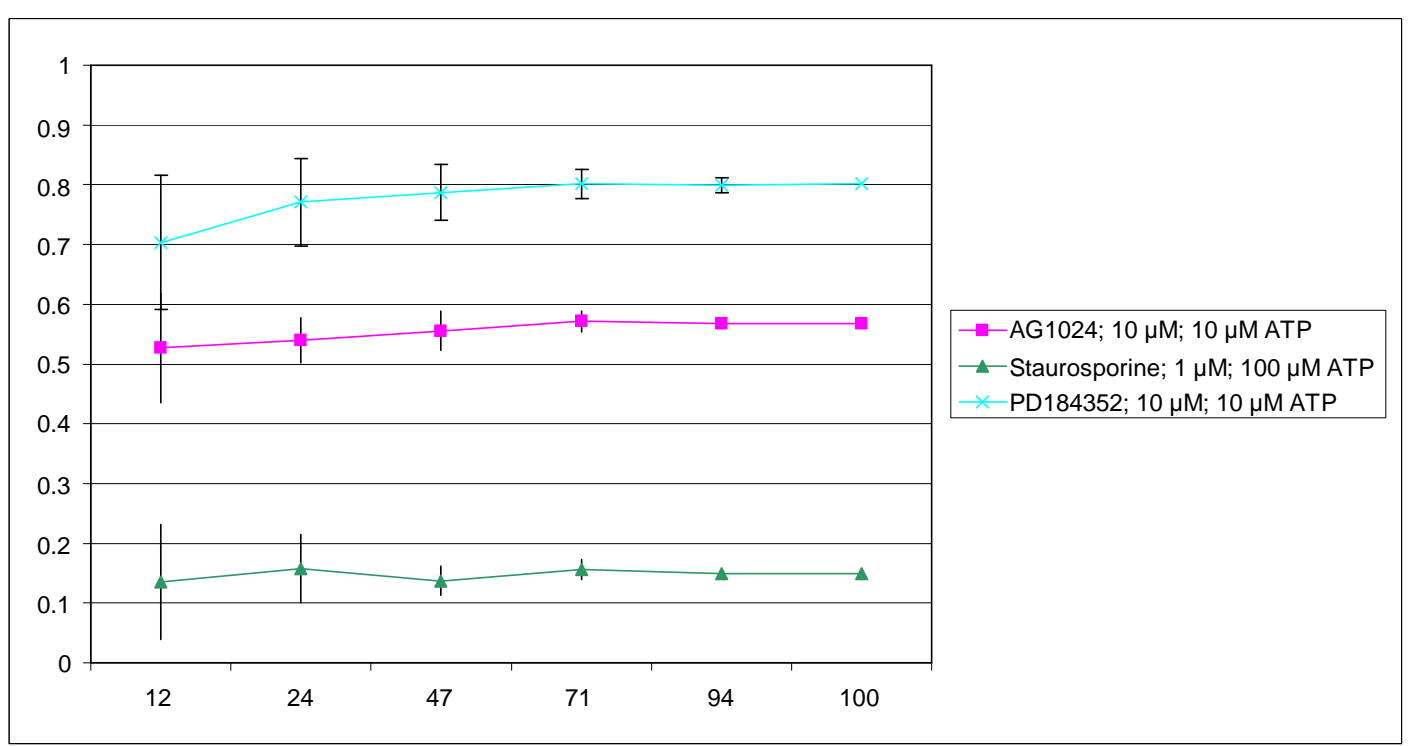




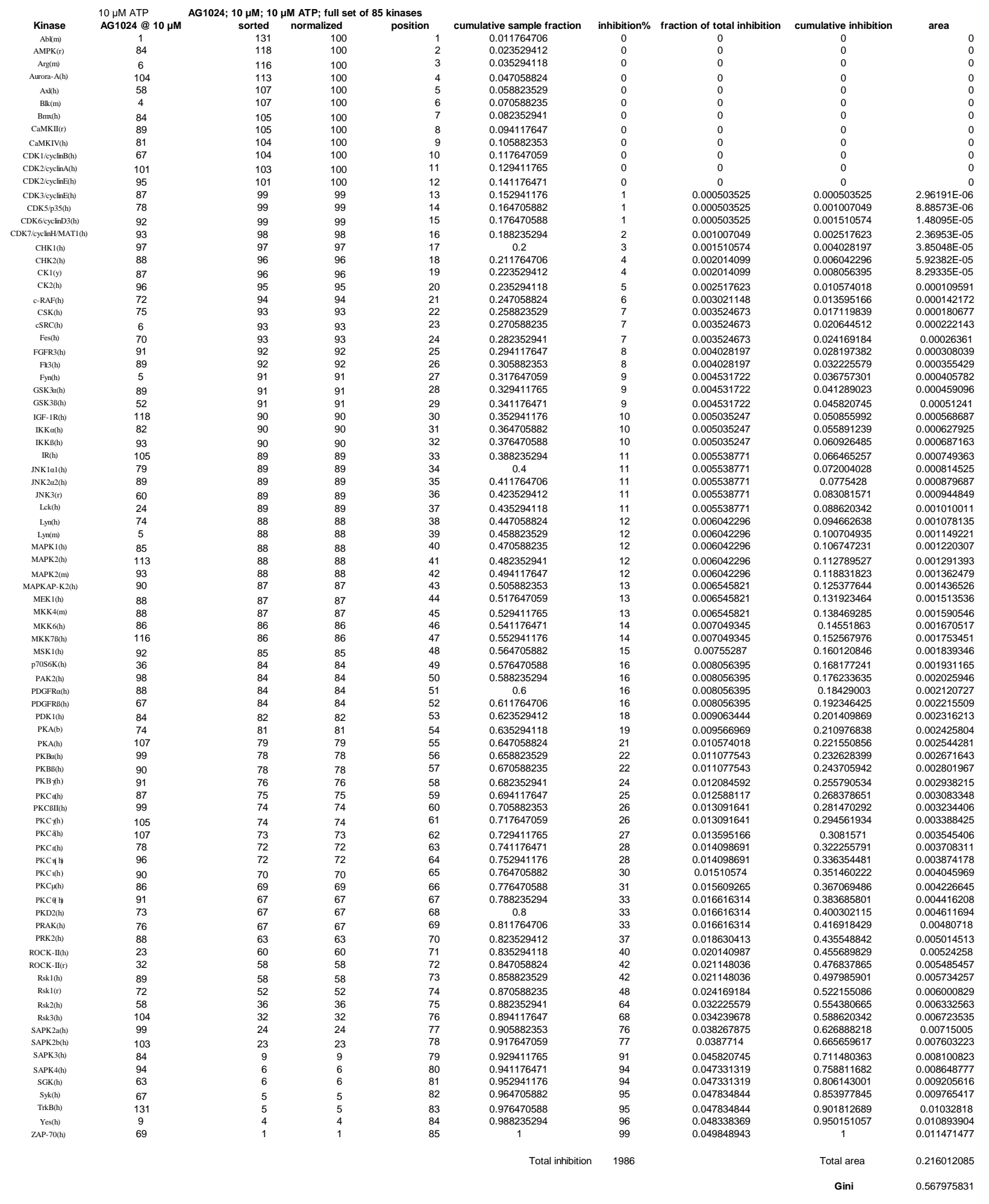




\begin{tabular}{|c|c|c|c|c|c|c|c|c|}
\hline $\begin{array}{l}\text { AG1024; } 10 \\
\text { position }\end{array}$ & $\begin{array}{l}\mu \mathrm{M} ; 10 \mu \mathrm{M} \text { ATF } \\
\text { inhibition } \%\end{array}$ & $\begin{array}{l}\text {; sample of } 80 \mathrm{~K} \\
\text { rand }\end{array}$ & $\begin{array}{l}\text { nases } \\
\text { cumulative sample fraction }\end{array}$ & sample inhibition & sorted & fraction of inhibition & cumulative fraction of inhibition & area \\
\hline 1 & 0 & 0.077629727 & 0.0125 & 0 & 0 & 0 & 0 & 0 \\
\hline 2 & 31 & 0.419946446 & 0.025 & 31 & 0 & 0 & 0 & 0 \\
\hline $\begin{array}{l}3 \\
4\end{array}$ & $\begin{array}{l}94 \\
12\end{array}$ & $\begin{array}{l}0.378781967 \\
0.469378385\end{array}$ & $\begin{array}{l}0.0375 \\
0.05\end{array}$ & $\begin{array}{l}94 \\
12\end{array}$ & $\begin{array}{l}0 \\
0\end{array}$ & $\begin{array}{l}0 \\
0\end{array}$ & $\begin{array}{l}0 \\
0\end{array}$ & $\begin{array}{l}0 \\
0\end{array}$ \\
\hline 5 & 21 & 0.668965996 & 0.0625 & 21 & 0 & 0 & 0 & 0 \\
\hline 6 & 4 & 0.336760074 & 0.075 & 4 & 0 & 0 & 0 & 0 \\
\hline 7 & 10 & 0.79340397 & 0.0875 & 10 & 0 & 0 & 0 & 0 \\
\hline 8 & 0 & 0.436107003 & 0.1 & 0 & 0 & 0 & 0 & 0 \\
\hline 9 & 14 & 0.785126405 & 0.1125 & 14 & 0 & 0 & 0 & 0 \\
\hline 10 & 25 & 0.060322178 & 0.125 & 25 & 0 & 0 & 0 & 0 \\
\hline 11 & 24 & 0.700969112 & 0.1375 & 24 & 0 & 0 & 0 & 0 \\
\hline 12 & 0 & 0.803330367 & 0.15 & 0 & 0 & 0 & 0 & 0 \\
\hline 13 & 33 & 0.95512897 & 0.1625 & 33 & 1 & 0.000548246 & 0.000548246 & $3.43 \mathrm{E}-06$ \\
\hline 14 & 0 & 0.712717741 & 0.175 & 0 & 1 & 0.000548246 & 0.001096491 & $1.03 \mathrm{E}-05$ \\
\hline 15 & 42 & 0.892751637 & 0.1875 & 42 & 1 & 0.000548246 & 0.001644737 & $1.71 \mathrm{E}-05$ \\
\hline 16 & 22 & 0.466286158 & 0.2 & 22 & 2 & 0.001096491 & 0.002741228 & $2.74 \mathrm{E}-05$ \\
\hline 17 & 16 & 0.468514737 & 0.2125 & 16 & 3 & 0.001644737 & 0.004385965 & 4. $45 \mathrm{E}-05$ \\
\hline 18 & 18 & 0.91539885 & 0.225 & 18 & 4 & 0.002192982 & 0.006578947 & $6.85 \mathrm{E}-05$ \\
\hline 19 & 3 & 0.732929847 & 0.2375 & 3 & 4 & 0.002192982 & 0.00877193 & $9.59 \mathrm{E}-05$ \\
\hline $\begin{array}{l}20 \\
21\end{array}$ & $\begin{array}{l}26 \\
10\end{array}$ & $\begin{array}{l}0.347931235 \\
0.458931872\end{array}$ & $\begin{array}{c}0.25 \\
0.2625\end{array}$ & $\begin{array}{l}26 \\
10\end{array}$ & $\begin{array}{l}5 \\
6\end{array}$ & $\begin{array}{l}0.002741228 \\
0.003289474\end{array}$ & $\begin{array}{l}0.011513158 \\
0.014802632\end{array}$ & $\begin{array}{l}0.000127 \\
0.000164\end{array}$ \\
\hline 22 & 13 & 0.906385436 & 0.275 & 13 & 7 & 0.003837719 & 0.018640351 & 0.000209 \\
\hline 23 & 0 & 0.56343597 & 0.2875 & 0 & 7 & 0.003837719 & 0.02247807 & 0.000257 \\
\hline 24 & 16 & 0.148224886 & 0.3 & 16 & 8 & 0.004385965 & 0.026864035 & 0.000308 \\
\hline 25 & 27 & 0.995920143 & 0.3125 & 27 & 8 & 0.004385965 & 0.03125 & 0.000363 \\
\hline 26 & 76 & 0.60288183 & 0.325 & 76 & 9 & 0.004934211 & 0.036184211 & 0.000421 \\
\hline 27 & 26 & 0.201781972 & 0.3375 & 26 & 9 & 0.004934211 & 0.041118421 & 0.000483 \\
\hline 28 & 12 & 0.085164709 & 0.35 & 12 & 9 & 0.004934211 & 0.046052632 & 0.000545 \\
\hline 29 & 68 & 0.020111818 & 0.3625 & 68 & 10 & 0.005482456 & 0.051535088 & 0.00061 \\
\hline 30 & 2 & 0.157041099 & 0.375 & 2 & 10 & 0.005482456 & 0.057017544 & 0.000678 \\
\hline 31 & 42 & 0.275788179 & 0.3875 & 42 & 10 & 0.005482456 & 0.0625 & 0.000747 \\
\hline 32 & 4 & 0.241604746 & 0.4 & 4 & 11 & 0.006030702 & 0.068530702 & 0.000819 \\
\hline 33 & 94 & 0.003789157 & 0.4125 & 94 & 11 & 0.006030702 & 0.074561404 & 0.000894 \\
\hline 34 & 13 & 0.986788055 & 0.425 & 13 & 11 & 0.006030702 & 0.080592105 & 0.00097 \\
\hline 35 & 0 & 0.831934791 & 0.4375 & 0 & 11 & 0.006030702 & 0.086622807 & 0.001045 \\
\hline 36 & 7 & 0.864149158 & 0.45 & 7 & 11 & 0.006030702 & 0.092653509 & 0.00112 \\
\hline 37 & 6 & 0.108597636 & 0.4625 & 6 & 12 & 0.006578947 & 0.099232456 & 0.001199 \\
\hline 38 & 28 & 0.21172699 & 0.475 & 28 & 12 & 0.006578947 & 0.105811404 & 0.001282 \\
\hline 39 & 64 & 0.88904823 & 0.4875 & 64 & 12 & 0.006578947 & 0.112390351 & 0.001364 \\
\hline 40 & 30 & 0.167968143 & 0.5 & 30 & 12 & 0.006578947 & 0.118969298 & 0.001446 \\
\hline 41 & 1 & 0.029603227 & 0.5125 & 1 & 12 & 0.006578947 & 0.125548246 & 0.001528 \\
\hline 42 & 11 & 0.462846173 & 0.525 & 11 & 13 & 0.007127193 & 0.132675439 & 0.001614 \\
\hline 43 & 37 & 0.75584603 & 0.5375 & 37 & 13 & 0.007127193 & 0.139802632 & 0.001703 \\
\hline 44 & 12 & 0.319608218 & 0.55 & 12 & 13 & 0.007127193 & 0.146929825 & 0.001792 \\
\hline 45 & 8 & 0.962024121 & 0.5625 & 8 & 14 & 0.007675439 & 0.154605263 & 0.001885 \\
\hline 46 & 0 & 0.184800261 & 0.575 & 0 & 14 & 0.007675439 & 0.162280702 & 0.001981 \\
\hline 47 & 40 & 0.806641854 & 0.5875 & 40 & 15 & 0.008223684 & 0.170504386 & 0.00208 \\
\hline 48 & 13 & 0.550265427 & 0.6 & 13 & 16 & 0.00877193 & 0.179276316 & 0.002186 \\
\hline 49 & 12 & 0.166554565 & 0.6125 & 12 & 16 & 0.00877193 & 0.188048246 & 0.002296 \\
\hline 50 & 0 & 0.600659562 & 0.625 & 0 & 18 & 0.009868421 & 0.197916667 & 0.002412 \\
\hline 51 & 15 & 0.531074555 & 0.6375 & 15 & 19 & 0.010416667 & 0.208333333 & 0.002539 \\
\hline 52 & 22 & 0.059222758 & 0.65 & 22 & 21 & 0.011513158 & 0.219846491 & 0.002676 \\
\hline $\begin{array}{l}53 \\
54\end{array}$ & $\begin{array}{c}0 \\
33\end{array}$ & $\begin{array}{l}0.971580681 \\
0.369969875\end{array}$ & $\begin{array}{c}0.6625 \\
0.675\end{array}$ & $\begin{array}{r}0 \\
33\end{array}$ & $\begin{array}{l}22 \\
22\end{array}$ & $\begin{array}{l}0.012061404 \\
0.012061404\end{array}$ & $\begin{array}{l}0.231907895 \\
0.243969298\end{array}$ & $\begin{array}{l}0.002823 \\
0.002974\end{array}$ \\
\hline 55 & 5 & 0.548840847 & 0.6875 & 5 & 24 & 0.013157895 & 0.257127193 & 0.003132 \\
\hline 56 & 0 & 0.995120885 & 0.7 & 0 & 25 & 0.01370614 & 0.270833333 & 0.0033 \\
\hline 57 & 9 & 0.502663614 & 0.7125 & 9 & 26 & 0.014254386 & 0.285087719 & 0.003475 \\
\hline 58 & 8 & 0.809978661 & 0.725 & 8 & 26 & 0.014254386 & 0.299342105 & 0.003653 \\
\hline 59 & 33 & 0.052515756 & 0.7375 & 33 & 27 & 0.014802632 & 0.314144737 & 0.003834 \\
\hline 60 & 9 & 0.382888468 & 0.75 & 9 & 28 & 0.015350877 & 0.329495614 & 0.004023 \\
\hline 61 & 10 & 0.887996208 & 0.7625 & 10 & 30 & 0.016447368 & 0.345942982 & 0.004221 \\
\hline 62 & 0 & 0.956385917 & 0.775 & 0 & 31 & 0.016995614 & 0.362938596 & 0.004431 \\
\hline 63 & 11 & 0.613729786 & 0.7875 & 11 & 33 & 0.018092105 & 0.381030702 & 0.00465 \\
\hline 64 & 48 & 0.310910314 & 0.8 & 48 & 33 & 0.018092105 & 0.399122807 & 0.004876 \\
\hline 65 & 1 & 0.175800999 & 0.8125 & 1 & 33 & 0.018092105 & 0.417214912 & 0.005102 \\
\hline 66 & 11 & 0.105931361 & 0.825 & 11 & 37 & 0.020285088 & 0.4375 & 0.005342 \\
\hline 67 & 91 & 0.635286664 & 0.8375 & 91 & 40 & 0.021929825 & 0.459429825 & 0.005606 \\
\hline 68 & 14 & 0.309601608 & 0.85 & 14 & 42 & 0.023026316 & 0.48245614 & 0.005887 \\
\hline 69 & 0 & 0.617643572 & 0.8625 & 0 & 42 & 0.023026316 & 0.505482456 & 0.006175 \\
\hline 70 & 11 & 0.909045657 & 0.875 & 11 & 48 & 0.026315789 & 0.531798246 & 0.006483 \\
\hline 71 & 7 & 0.145151281 & 0.8875 & 7 & 64 & 0.035087719 & 0.566885965 & 0.006867 \\
\hline 72 & 19 & 0.055913243 & 0.9 & 19 & 68 & 0.037280702 & 0.604166667 & 0.007319 \\
\hline 73 & 99 & 0.411113982 & 0.9125 & 99 & 76 & 0.041666667 & 0.645833333 & 0.007812 \\
\hline 74 & 9 & 0.731349757 & 0.925 & 9 & 77 & 0.042214912 & 0.688048246 & 0.008337 \\
\hline 75 & 95 & 0.28688297 & 0.9375 & 95 & 91 & 0.049890351 & 0.737938596 & 0.008912 \\
\hline 76 & 11 & 0.872043139 & 0.95 & 11 & 94 & 0.051535088 & 0.789473684 & 0.009546 \\
\hline 77 & 77 & 0.509813456 & 0.9625 & 77 & 94 & 0.051535088 & 0.841008772 & 0.010191 \\
\hline 78 & 12 & 0.730123189 & 0.975 & 12 & 95 & 0.052083333 & 0.893092105 & 0.010838 \\
\hline 79 & 1 & 0.676855339 & 0.9875 & 1 & 96 & 0.052631579 & 0.945723684 & 0.011493 \\
\hline 80 & 96 & 0.195492337 & 1 & 96 & 99 & 0.054276316 & 1 & 0.012161 \\
\hline 81 & 28 & 0.706749102 & & & & & & \\
\hline 82 & 7 & 0.755865443 & & total inhibition & 1824 & & Total area & 0.213473 \\
\hline 83 & 16 & 0.742438073 & & & & & & \\
\hline $\begin{array}{l}84 \\
85\end{array}$ & $\begin{array}{l}95 \\
16\end{array}$ & $\begin{array}{r}0.68451995 \\
0.010522127\end{array}$ & & & & & Gini & 0.573054 \\
\hline
\end{tabular}

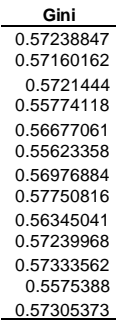

$\begin{aligned} \text { mean } & 0.567995007 \\ \text { st dev } & 0.007021662\end{aligned}$ 
AG1024; $10 \mu \mathrm{M} ; 10 \mu \mathrm{M}$ ATP; sample of 60 kinases

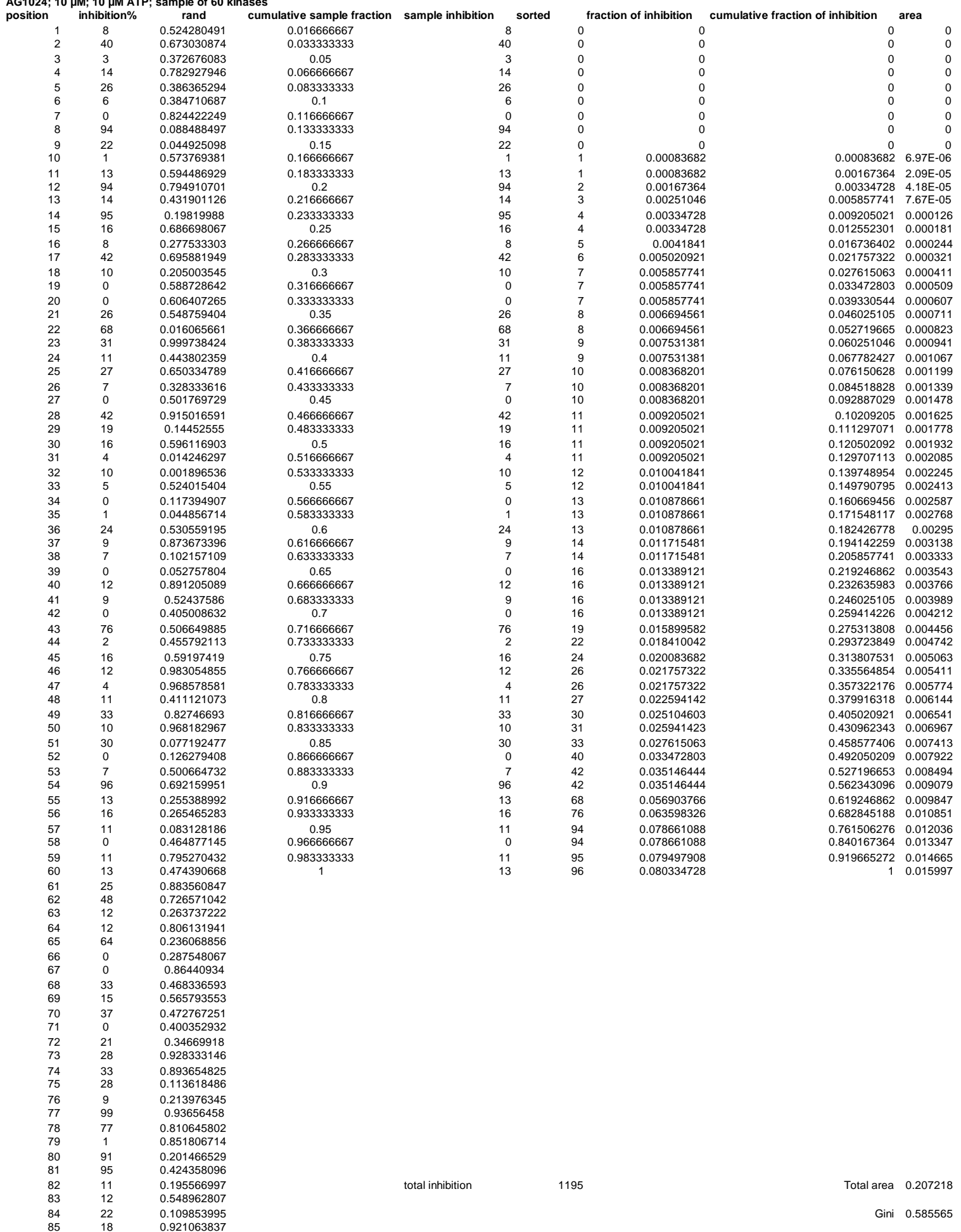

\begin{tabular}{c}
\multicolumn{1}{c}{ Gini } \\
\hline 0.6045695 \\
0.5779661 \\
0.58551215 \\
0.55474453 \\
0.57984364 \\
0.54177028 \\
0.57845583 \\
0.56715749 \\
0.5650026 \\
0.55448805 \\
0.57523163 \\
0.55496552 \\
0.58556485 \\
\hline
\end{tabular}

$\begin{array}{ll}\text { mean } & 0.571174782 \\ \text { st dev } & 0.016952346\end{array}$ 
AG1024; $10 \mu \mathrm{M} ; 10 \mu \mathrm{M}$ ATP; sample of 40 kinases

\begin{tabular}{|c|c|c|c|c|c|c|c|c|c|}
\hline position & inhibition\% & rand & cumulative sample fraction & sample inhibition & sorted & & fraction of inhibition & cumulative fraction of inhibition & area \\
\hline $\begin{array}{l}1 \\
2\end{array}$ & $\begin{array}{l}8 \\
9\end{array}$ & $\begin{array}{c}0.9814277 \\
0.594423443\end{array}$ & $\begin{array}{l}0.025 \\
0.05\end{array}$ & $\begin{array}{l}8 \\
9\end{array}$ & & $\begin{array}{l}0 \\
0\end{array}$ & $\begin{array}{l}0 \\
0\end{array}$ & $\begin{array}{ll}0 \\
0 & 0\end{array}$ & $\begin{array}{l}0 \\
0\end{array}$ \\
\hline 3 & 42 & 0.279516533 & 0.075 & 42 & & 0 & 0 & 0 & 0 \\
\hline 4 & 0 & 0.635739322 & 0.1 & 0 & & 0 & 0 & 0 & 0 \\
\hline 5 & 0 & 0.441714541 & 0.125 & 0 & & 0 & 0 & 0 & 0 \\
\hline 6 & 21 & 0.203727848 & 0.15 & 21 & & 0 & 0 & 0 & 0 \\
\hline 7 & 25 & 0.306353999 & 0.175 & 25 & & 0 & 0 & 0 & 0 \\
\hline 8 & 10 & 0.921181226 & 0.2 & 10 & & 0 & 0 & 0 & 0 \\
\hline 9 & 33 & 0.598105198 & 0.225 & 33 & & 1 & 0.001150748 & 0.001150748 & $1.44 \mathrm{E}-05$ \\
\hline 10 & 16 & 0.168872783 & 0.25 & 16 & & 1 & 0.001150748 & 0.002301496 & $4.32 \mathrm{E}-05$ \\
\hline 11 & 12 & 0.981446163 & 0.275 & 12 & & 7 & 0.008055236 & 0.010356732 & 0.000158 \\
\hline 12 & 26 & 0.654392677 & 0.3 & 26 & & 8 & 0.009205984 & 0.019562716 & 0.000374 \\
\hline 13 & 22 & 0.833577728 & 0.325 & 22 & & 8 & 0.009205984 & 0.0287687 & 0.000604 \\
\hline 14 & 11 & 0.492892528 & 0.35 & 11 & & 9 & 0.010356732 & 0.039125432 & 0.000849 \\
\hline 15 & 1 & 0.815057126 & 0.375 & 1 & & 9 & 0.010356732 & 0.049482163 & 0.001108 \\
\hline 16 & 1 & 0.254754544 & 0.4 & 1 & & 10 & 0.01150748 & 0.060989643 & 0.001381 \\
\hline 17 & 48 & 0.335489629 & 0.425 & 48 & & 11 & 0.012658228 & 0.073647871 & 0.001683 \\
\hline 18 & 42 & 0.572589812 & 0.45 & 42 & & 12 & 0.013808976 & 0.087456847 & 0.002014 \\
\hline 19 & 18 & 0.809412537 & 0.475 & 18 & & 12 & 0.013808976 & 0.101265823 & 0.002359 \\
\hline 20 & 16 & 0.379653545 & 0.5 & 16 & & 13 & 0.014959724 & 0.116225547 & 0.002719 \\
\hline 21 & 12 & 0.369473147 & 0.525 & 12 & & 14 & 0.016110472 & 0.132336018 & 0.003107 \\
\hline 22 & 68 & 0.374979896 & 0.55 & 68 & & 15 & 0.01726122 & 0.149597238 & 0.003524 \\
\hline 23 & 15 & 0.113709295 & 0.575 & 15 & & 16 & 0.018411968 & 0.168009206 & 0.00397 \\
\hline 24 & 0 & 0.281653088 & 0.6 & 0 & & 16 & 0.018411968 & 0.186421174 & 0.00443 \\
\hline 25 & 95 & 0.937187294 & 0.625 & 95 & & 18 & 0.020713464 & 0.207134638 & 0.004919 \\
\hline 26 & 13 & 0.736727239 & 0.65 & 13 & & 21 & 0.024165708 & 0.231300345 & 0.00548 \\
\hline 27 & 14 & 0.54455305 & 0.675 & 14 & & 22 & 0.025316456 & 0.256616801 & 0.006099 \\
\hline 28 & 0 & 0.007813617 & 0.7 & 0 & & 24 & 0.027617952 & 0.284234753 & 0.006761 \\
\hline 29 & 9 & 0.620265719 & 0.725 & 9 & & 25 & 0.0287687 & 0.313003452 & 0.007465 \\
\hline 30 & 28 & 0.645524147 & 0.75 & 28 & & 26 & 0.029919448 & 0.3429229 & 0.008199 \\
\hline 31 & 0 & 0.905698311 & 0.775 & 0 & & 27 & 0.031070196 & 0.373993096 & 0.008961 \\
\hline 32 & 94 & 0.580535073 & 0.8 & 94 & & 28 & 0.032220944 & 0.406214039 & 0.009753 \\
\hline 33 & 0 & 0.954902387 & 0.825 & 0 & & 33 & 0.037974684 & 0.444188723 & 0.01063 \\
\hline 34 & 8 & 0.849279154 & 0.85 & 8 & & 42 & 0.048331415 & 0.492520138 & 0.011709 \\
\hline 35 & 94 & 0.362525666 & 0.875 & 94 & & 42 & 0.048331415 & 0.540851554 & 0.012917 \\
\hline 36 & 24 & 0.985465002 & 0.9 & 24 & & 48 & 0.055235903 & 0.596087457 & 0.014212 \\
\hline 37 & 7 & 0.384759611 & 0.925 & 7 & & 68 & 0.078250863 & 0.67433832 & 0.01588 \\
\hline 38 & 0 & 0.328349908 & 0.95 & 0 & & 94 & 0.108170311 & 0.782508631 & 0.018211 \\
\hline 39 & 27 & 0.073836302 & 0.975 & 27 & & 94 & 0.108170311 & 0.890678941 & 0.020915 \\
\hline 40 & 0 & 0.629029862 & 1 & 0 & & 95 & 0.109321059 & 1 & 0.023633 \\
\hline 41 & 19 & 0.010747757 & & & & & & & \\
\hline 42 & 64 & 0.136130993 & & & & & & & \\
\hline 43 & 14 & 0.495414636 & & & & & & & \\
\hline 44 & 9 & 0.318070991 & & & & & & & \\
\hline 45 & 13 & 0.17631708 & & & & & & & \\
\hline 46 & 33 & 0.975137999 & & & & & & & \\
\hline 47 & 0 & 0.530547932 & & & & & & & \\
\hline 48 & 16 & 0.413223461 & & & & & & & \\
\hline 49 & 2 & 0.988111981 & & & & & & & \\
\hline 50 & 4 & 0.557142709 & & & & & & & \\
\hline 51 & 11 & 0.36497566 & & & & & & & \\
\hline 52 & 10 & 0.041483126 & & & & & & & \\
\hline 53 & 91 & 0.162229899 & & & & & & & \\
\hline 54 & 13 & 0.470897551 & & & & & & & \\
\hline $\begin{array}{l}55 \\
56\end{array}$ & $\begin{array}{l}11 \\
37\end{array}$ & $\begin{array}{r}0.299214999 \\
0.02421365\end{array}$ & & & & & & & \\
\hline 57 & 33 & $\begin{array}{l}0.261636248 \\
0.2638\end{array}$ & & & & & & & \\
\hline 58 & 7 & 0.078737999 & & & & & & & \\
\hline 59 & 7 & 0.313433066 & & & & & & & \\
\hline 60 & 22 & 0.602525634 & & & & & & & \\
\hline 61 & 5 & 0.186708624 & & & & & & & \\
\hline 62 & 12 & 0.919718622 & & & & & & & \\
\hline 63 & 0 & 0.278636729 & & & & & & & \\
\hline 64 & 11 & 0.746117949 & & & & & & & \\
\hline 65 & 16 & 0.26007038 & & & & & & & \\
\hline 66 & 26 & 0.24102349 & & & & & & & \\
\hline 67 & 31 & 0.08678736 & & & & & & & \\
\hline 68 & 4 & 0.063472498 & & & & & & & \\
\hline 69 & 77 & 0.081299636 & & & & & & & \\
\hline $\begin{array}{l}70 \\
71\end{array}$ & $\begin{array}{l}96 \\
30\end{array}$ & $\begin{array}{l}0.773385891 \\
0.994131377\end{array}$ & & & & & & & \\
\hline $\begin{array}{l}71 \\
72\end{array}$ & $\begin{array}{c}30 \\
0\end{array}$ & $\begin{array}{r}0.994131377 \\
0.64318656\end{array}$ & & & & & & & \\
\hline 73 & 11 & 0.008564912 & & & & & & & \\
\hline 74 & 76 & 0.043653558 & & & & & & & \\
\hline 75 & 99 & 0.074562167 & & & & & & & \\
\hline 76 & 0 & 0.368431312 & & & & & & & \\
\hline 77 & 40 & 0.596671627 & & & & & & & \\
\hline 78 & 12 & 0.764406764 & & & & & & & \\
\hline 79 & 28 & 0.127877385 & & & & & & & \\
\hline 80 & 1 & 0.656722679 & & & & & & & \\
\hline 81 & 6 & 0.53105631 & & & & & & & \\
\hline 82 & 10 & 0.262384443 & & total inhibition & & 869 & & Total area & 0.214082 \\
\hline 83 & 3 & 0.718325818 & & & & & & & \\
\hline 84 & 95 & 0.320108388 & & & & & & Gini & 0.571835 \\
\hline
\end{tabular}

$\begin{array}{r}\multicolumn{1}{c}{\text { Gini }} \\ \hline 0.59930341 \\ 0.53195212 \\ 0.53365522 \\ 0.48857143 \\ 0.525 \\ 0.58685226 \\ 0.5428648 \\ 0.54312039 \\ 0.55104878 \\ 0.5597424 \\ 0.59111958 \\ 0.59321503 \\ 0.57183544 \\ \hline\end{array}$

$\begin{array}{ll}\text { mean } & 0.555592503 \\ \text { st dev } & 0.032351616\end{array}$ 
AG1024; $10 \mu \mathrm{M} ; 10 \mu \mathrm{M}$ ATP; sample of 20 kinases

\begin{tabular}{|c|c|c|c|c|c|c|c|c|c|}
\hline position & inhibition\% & rand & cumulative sample fraction & sample inhibition & sorted & & fraction of inhibition & cumulative fraction of inhibition & area \\
\hline 1 & $\begin{array}{c}9 \\
13\end{array}$ & 0.706333623 & 0.05 & $\begin{array}{r}9 \\
13\end{array}$ & 9 & $\begin{array}{l}0 \\
0\end{array}$ & $\begin{array}{l}0 \\
0\end{array}$ & $\begin{array}{ll}0 \\
0\end{array}$ & $\begin{array}{l}0 \\
0\end{array}$ \\
\hline 2 & 13 & 0.92520082 & 0.1 & 13 & & 0 & 0 & 0 & 0 \\
\hline $\begin{array}{l}3 \\
4\end{array}$ & $\begin{array}{l}42 \\
11\end{array}$ & $\begin{array}{l}0.479771559 \\
0.537238812\end{array}$ & $\begin{array}{l}0.15 \\
0.2\end{array}$ & $\begin{array}{l}42 \\
11\end{array}$ & & $\begin{array}{l}0 \\
1\end{array}$ & $\begin{array}{r}0 \\
0.00297619\end{array}$ & $\begin{array}{r}0 \\
0.00297619\end{array}$ & $\begin{array}{r}0 \\
7.44 \mathrm{E}-05\end{array}$ \\
\hline 5 & 0 & 0.348829034 & 0.25 & 0 & b & 9 & 0.026785714 & 0.029761905 & 0.000818 \\
\hline 6 & 9 & 0.857955968 & 0.3 & 9 & 9 & 9 & 0.026785714 & 0.056547619 & 0.002158 \\
\hline 7 & 11 & 0.428912814 & 0.35 & 11 & & 9 & 0.026785714 & 0.083333333 & 0.003497 \\
\hline 8 & 37 & 0.160977438 & 0.4 & 37 & & 10 & 0.029761905 & 0.113095238 & 0.004911 \\
\hline 9 & 0 & 0.579877624 & 0.45 & 0 & o & 10 & 0.029761905 & 0.142857143 & 0.006399 \\
\hline 10 & 15 & 0.929684609 & 0.5 & 15 & & 11 & 0.032738095 & 0.175595238 & 0.007961 \\
\hline 11 & 10 & 0.034606601 & 0.55 & 10 & & 11 & 0.032738095 & 0.208333333 & 0.009598 \\
\hline 12 & 1 & 0.69776179 & 0.6 & 1 & 1 & 11 & 0.032738095 & 0.241071429 & 0.011235 \\
\hline 13 & 0 & 0.038247222 & 0.65 & 0 & b & 13 & 0.038690476 & 0.279761905 & 0.013021 \\
\hline 14 & 26 & 0.562322941 & 0.7 & 26 & & 15 & 0.044642857 & 0.324404762 & 0.015104 \\
\hline 15 & 26 & 0.04515349 & 0.75 & 26 & & 19 & 0.056547619 & 0.380952381 & 0.017634 \\
\hline 16 & 11 & 0.752555447 & 0.8 & 11 & & 26 & 0.077380952 & 0.458333333 & 0.020982 \\
\hline 17 & 77 & 0.237715726 & 0.85 & 77 & & 26 & 0.077380952 & 0.535714286 & 0.024851 \\
\hline 18 & 10 & 0.072828147 & 0.9 & 10 & & 37 & 0.110119048 & 0.645833333 & 0.029539 \\
\hline 19 & 19 & 0.755191603 & 0.95 & 19 & & 42 & 0.125 & 0.770833333 & 0.035417 \\
\hline $\begin{array}{l}20 \\
21\end{array}$ & $\begin{array}{c}9 \\
16\end{array}$ & $\begin{array}{l}0.382012981 \\
0.468529298\end{array}$ & 1 & 9 & 9 & 77 & 0.229166667 & 1 & 0.044271 \\
\hline 22 & 0 & 0.083817521 & & & & & & & \\
\hline 23 & 64 & 0.738075047 & & & & & & & \\
\hline 24 & 0 & 0.030162924 & & & & & & & \\
\hline 25 & 31 & 0.597393136 & & & & & & & \\
\hline 26 & 33 & 0.816410337 & & & & & & & \\
\hline 27 & 16 & 0.330045855 & & & & & & & \\
\hline 28 & 1 & 0.970586414 & & & & & & & \\
\hline 29 & 0 & 0.59312626 & & & & & & & \\
\hline 30 & 7 & 0.206084201 & & & & & & & \\
\hline 31 & 0 & 0.60736332 & & & & & & & \\
\hline 32 & 11 & 0.808938443 & & & & & & & \\
\hline 33 & 0 & 0.05101344 & & & & & & & \\
\hline 34 & 21 & 0.098050887 & & & & & & & \\
\hline 35 & 7 & 0.656113777 & & & & & & & \\
\hline 36 & 0 & 0.791262696 & & & & & & & \\
\hline $\begin{array}{l}37 \\
38\end{array}$ & $\begin{array}{l}16 \\
13\end{array}$ & $\begin{array}{l}0.473225136 \\
0.022038845\end{array}$ & & & & & & & \\
\hline 39 & 27 & 0.11702851 & & & & & & & \\
\hline $\begin{array}{l}40 \\
41\end{array}$ & $\begin{array}{c}8 \\
24\end{array}$ & 0.410255987 & & & & & & & \\
\hline $\begin{array}{l}41 \\
42\end{array}$ & $\begin{array}{l}24 \\
95\end{array}$ & $\begin{array}{l}0.064927326 \\
0.531494601\end{array}$ & & & & & & & \\
\hline 43 & 96 & 0.246083984 & & & & & & & \\
\hline 44 & 12 & 0.344052541 & & & & & & & \\
\hline 45 & 28 & 0.163597068 & & & & & & & \\
\hline 46 & 16 & 0.68201391 & & & & & & & \\
\hline 47 & 91 & 0.493510718 & & & & & & & \\
\hline 48 & 4 & 0.418839009 & & & & & & & \\
\hline 49 & 33 & 0.309259902 & & & & & & & \\
\hline 50 & 33 & 0.580385534 & & & & & & & \\
\hline 51 & 76 & 0.894519951 & & & & & & & \\
\hline 52 & 18 & 0.908898196 & & & & & & & \\
\hline $\begin{array}{l}53 \\
54\end{array}$ & $\begin{array}{l}2 \\
28\end{array}$ & $\begin{array}{l}0.530220467 \\
0.644995813\end{array}$ & & & & & & & \\
\hline $\begin{array}{l}55 \\
56\end{array}$ & $\begin{array}{l}99 \\
5\end{array}$ & $\begin{array}{l}0.788158986 \\
0.929391074\end{array}$ & & & & & & & \\
\hline 57 & 14 & 0.246253226 & & & & & & & \\
\hline 58 & 7 & 0.579468307 & & & & & & & \\
\hline 59 & 68 & 0.031369104 & & & & & & & \\
\hline 60 & 12 & 0.683115251 & & & & & & & \\
\hline 61 & 12 & 0.959353887 & & & & & & & \\
\hline 62 & 12 & 0.473016628 & & & & & & & \\
\hline 63 & 95 & 0.734749977 & & & & & & & \\
\hline 64 & 94 & 0.472811223 & & & & & & & \\
\hline 65 & 40 & 0.154707086 & & & & & & & \\
\hline 66 & 8 & 0.324124334 & & & & & & & \\
\hline 67 & 42 & 0.602112716 & & & & & & & \\
\hline 68 & 94 & 0.855407996 & & & & & & & \\
\hline 69 & 11 & 0.68506802 & & & & & & & \\
\hline 70 & 14 & 0.764211604 & & & & & & & \\
\hline $\begin{array}{l}71 \\
72\end{array}$ & $\begin{array}{c}4 \\
12\end{array}$ & $\begin{array}{l}0.162215629 \\
0.881059492\end{array}$ & & & & & & & \\
\hline 73 & 22 & 0.839915448 & & & & & & & \\
\hline 74 & 0 & 0.809710366 & & & & & & & \\
\hline 75 & 22 & 0.340182638 & & & & & & & \\
\hline 76 & 13 & 0.259972793 & & & & & & & \\
\hline 77 & 1 & 0.368525705 & & & & & & & \\
\hline 78 & 30 & 0.640262725 & & & & & & & \\
\hline 79 & 25 & 0.088760376 & & & & & & & \\
\hline 80 & 48 & 0.499125461 & & & & & & & \\
\hline 81 & 10 & 0.675426732 & & & & & & & \\
\hline 82 & 0 & 0.884243047 & & total inhibition & & 336 & & Total area & 0.24747 \\
\hline 83 & 0 & 0.724793148 & & & & & & & \\
\hline $\begin{array}{l}84 \\
85\end{array}$ & $\begin{array}{l}6 \\
3\end{array}$ & $\begin{array}{r}0.464748039 \\
0.96292189\end{array}$ & & & & & & Gini & 0.50506 \\
\hline
\end{tabular}

$\begin{array}{ll}\text { mean } & 0.540025335 \\ \text { st dev } & 0.037994245\end{array}$ 
AG1024; $10 \mu \mathrm{M} ; 10 \mu \mathrm{M}$ ATP; sample of 10 kinases

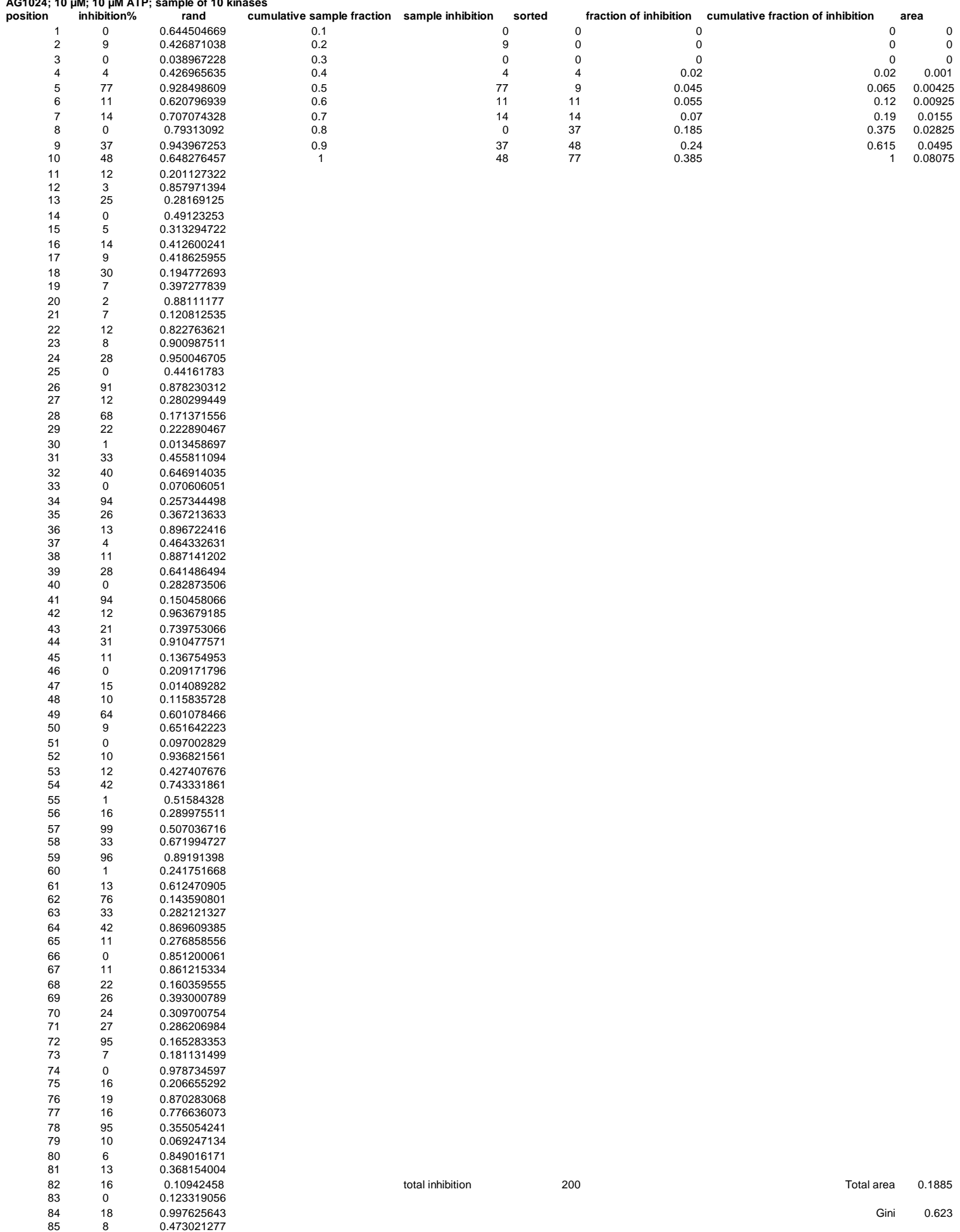

\begin{tabular}{rr}
\multicolumn{1}{c}{ Gini } & \\
\hline 0.42941176 & \\
0.61906615 & \\
0.51 & \\
0.47557252 & \\
0.39838337 & \\
0.63853211 & \\
0.51100324 & \\
0.5460177 & \\
0.65771429 & \\
0.56532258 & \\
0.3848 & \\
0.48709677 & \\
0.623 & \\
\hline mean & 0.526609268 \\
st dev & 0.09133939
\end{tabular}




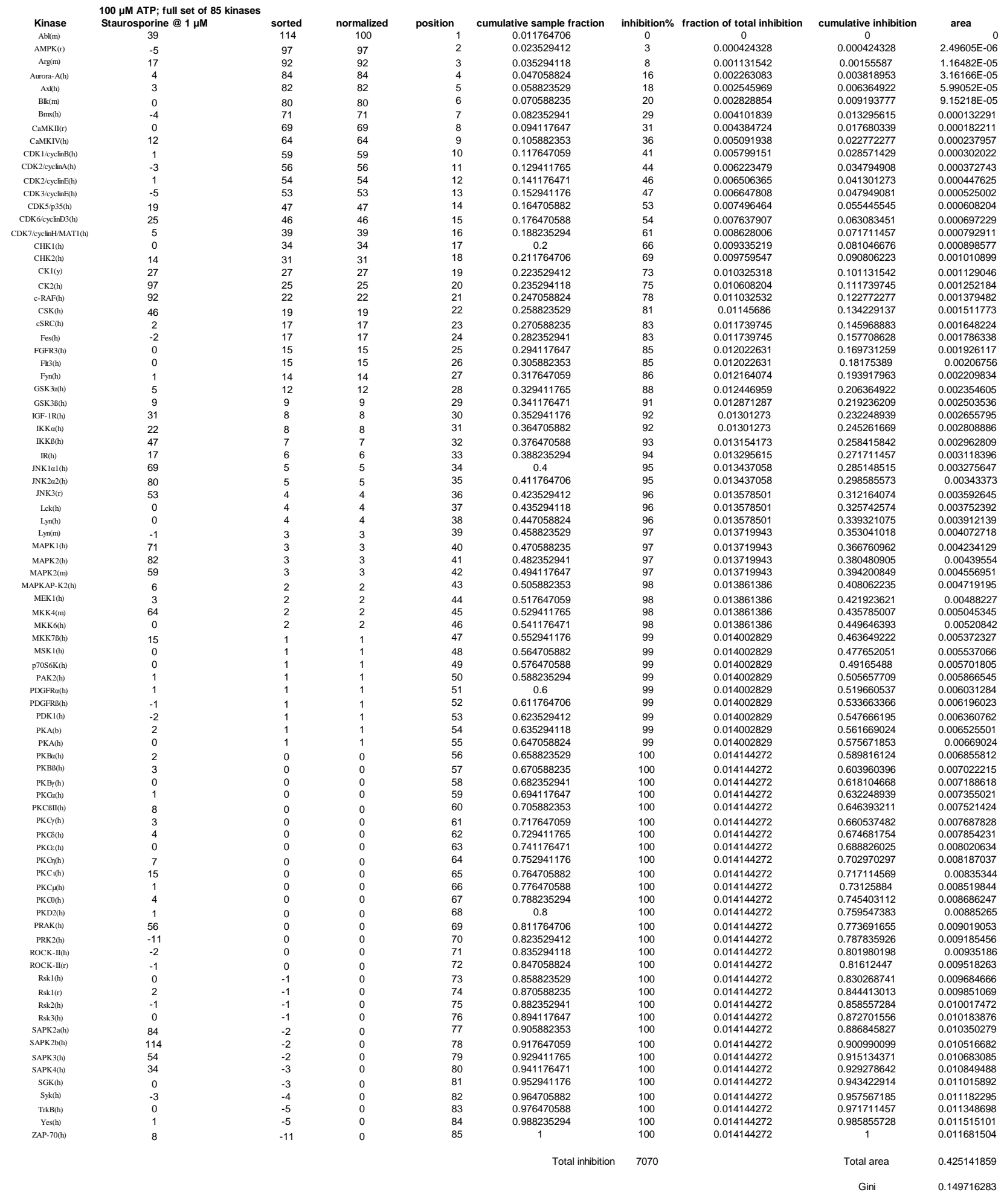




\begin{tabular}{|c|c|c|c|c|c|c|c|c|}
\hline $\begin{array}{l}\text { Staurospori } \\
\text { position }\end{array}$ & $\begin{array}{l}\text { e; } 1 \mu \mathrm{M} ; 100 \mathrm{r} \\
\text { inhibition\% }\end{array}$ & $\begin{array}{l}\text { ATP; sample } \\
\text { rand }\end{array}$ & $\begin{array}{l}\text { f } 80 \text { kinases } \\
\text { cumulative sample fraction }\end{array}$ & sample inhibition & sorted & fraction of inhibition & cumulative fraction of inhibition & area \\
\hline 1 & 96 & 0.392068886 & 0.0125 & 96 & 0 & 0 & 0 & 0 \\
\hline 2 & 81 & 0.081475466 & 0.025 & 81 & 3 & 0.000450924 & 0.000450924 & $2.82 \mathrm{E}-06$ \\
\hline $\begin{array}{l}3 \\
4\end{array}$ & $\begin{array}{l}53 \\
100\end{array}$ & $\begin{array}{l}0.449148537 \\
0.593999039\end{array}$ & $\begin{array}{c}0.0375 \\
0.05\end{array}$ & $\begin{array}{r}53 \\
100\end{array}$ & $\begin{array}{r}8 \\
16\end{array}$ & $\begin{array}{r}0.001202465 \\
0.00240493\end{array}$ & $\begin{array}{r}0.001653389 \\
0.00405832\end{array}$ & $\begin{array}{l}1.32 \mathrm{E}-05 \\
3.57 \mathrm{E}-05\end{array}$ \\
\hline $\begin{array}{l}5 \\
6\end{array}$ & $\begin{array}{c}98 \\
0\end{array}$ & $\begin{array}{l}0.616553696 \\
0.551817804\end{array}$ & $\begin{array}{c}0.0625 \\
0.075\end{array}$ & $\begin{array}{r}98 \\
0\end{array}$ & $\begin{array}{l}18 \\
29\end{array}$ & $\begin{array}{l}0.002705546 \\
0.004358936\end{array}$ & $\begin{array}{l}0.006763866 \\
0.011122802\end{array}$ & $\begin{array}{l}6.76 \mathrm{E}-05 \\
0.000112\end{array}$ \\
\hline $\begin{array}{l}6 \\
7\end{array}$ & 46 & 0.932524408 & 0.0875 & 46 & 31 & 0.004659552 & 0.015782354 & 0.000168 \\
\hline 8 & 100 & 0.22739756 & 0.1 & 100 & 36 & 0.005411093 & 0.021193447 & 0.000231 \\
\hline 9 & 100 & 0.885620781 & 0.1125 & 100 & 41 & 0.006162633 & 0.02735608 & 0.000303 \\
\hline 10 & 75 & 0.118583643 & 0.125 & 75 & 44 & 0.006613558 & 0.033969638 & 0.000383 \\
\hline 11 & 99 & 0.866312802 & 0.1375 & 99 & 46 & 0.006914174 & 0.040883812 & 0.000468 \\
\hline 12 & 99 & 0.189298612 & 0.15 & 99 & 47 & 0.007064482 & 0.047948294 & 0.000555 \\
\hline 13 & 100 & 0.302453694 & 0.1625 & 100 & 53 & 0.007966331 & 0.055914625 & 0.000649 \\
\hline 14 & 100 & 0.260493297 & 0.175 & 100 & 54 & 0.008116639 & 0.064031264 & 0.00075 \\
\hline 15 & 100 & 0.664209817 & 0.1875 & 100 & 61 & 0.009168796 & 0.07320006 & 0.000858 \\
\hline 16 & 100 & 0.831101344 & 0.2 & 100 & 66 & 0.009920337 & 0.083120397 & 0.000977 \\
\hline 17 & 100 & 0.80838981 & 0.2125 & 100 & 69 & 0.010371261 & 0.093491658 & 0.001104 \\
\hline 18 & 36 & 0.413908812 & 0.225 & 36 & 73 & 0.010972494 & 0.104464152 & 0.001237 \\
\hline 19 & 100 & 0.042124638 & 0.2375 & 100 & 75 & 0.01127311 & 0.115737261 & 0.001376 \\
\hline 20 & 95 & 0.754379906 & $\begin{array}{c}0.25 \\
0.2625\end{array}$ & $\begin{array}{l}95 \\
83\end{array}$ & $\begin{array}{l}78 \\
81\end{array}$ & & $\begin{array}{l}0.127461296 \\
0.139636254\end{array}$ & \\
\hline $\begin{array}{l}21 \\
22\end{array}$ & $\begin{array}{l}83 \\
98\end{array}$ & $\begin{array}{c}0.231230338 \\
0.61199062\end{array}$ & $\begin{array}{c}0.2625 \\
0.275\end{array}$ & $\begin{array}{l}83 \\
98\end{array}$ & $\begin{array}{l}81 \\
83\end{array}$ & $\begin{array}{l}0.012174959 \\
0.012475575\end{array}$ & $\begin{array}{l}0.139636254 \\
0.152111829\end{array}$ & $\begin{array}{l}0.001669 \\
0.001823\end{array}$ \\
\hline $\begin{array}{l}22 \\
23\end{array}$ & $\begin{array}{l}98 \\
99\end{array}$ & 0.058929881 & 0.2875 & $\begin{array}{l}98 \\
99\end{array}$ & $\begin{array}{l}83 \\
83\end{array}$ & 0.012475575 & 0.164587404 & 0.001979 \\
\hline 24 & 97 & 0.725868482 & 0.3 & 97 & 85 & 0.012776191 & 0.177363595 & 0.002137 \\
\hline 25 & 41 & 0.898248737 & 0.3125 & 41 & 85 & 0.012776191 & 0.190139787 & 0.002297 \\
\hline 26 & 100 & 0.563877354 & 0.325 & 100 & 86 & 0.012926499 & 0.203066286 & 0.002458 \\
\hline 27 & 100 & 0.327577545 & 0.3375 & 100 & 88 & 0.013227116 & 0.216293401 & 0.002621 \\
\hline 28 & 100 & 0.854771568 & 0.35 & 100 & 91 & 0.01367804 & 0.229971441 & 0.002789 \\
\hline 29 & 8 & 0.006762001 & 0.3625 & 8 & 92 & 0.013828348 & 0.24379979 & 0.002961 \\
\hline 30 & 29 & 0.268337378 & 0.375 & 29 & 92 & 0.013828348 & 0.257628138 & 0.003134 \\
\hline 31 & 99 & 0.287777444 & 0.3875 & 99 & 93 & 0.013978656 & 0.271606794 & 0.003308 \\
\hline 32 & 99 & 0.665495138 & 0.4 & 99 & 94 & 0.014128964 & 0.285735758 & 0.003483 \\
\hline 33 & 100 & 0.981715273 & 0.4125 & 100 & 95 & 0.014279273 & 0.300015031 & 0.003661 \\
\hline $\begin{array}{l}34 \\
35\end{array}$ & 97 & 0.288141502 & $\begin{array}{r}0.425 \\
0.4375\end{array}$ & 97 & 95 & 0.014279273 & 0.314294303 & 0.003839 \\
\hline $\begin{array}{l}35 \\
36\end{array}$ & $\begin{array}{l}94 \\
99\end{array}$ & $\begin{array}{l}0.478782068 \\
0.505883311\end{array}$ & $\begin{array}{c}0.4375 \\
0.45\end{array}$ & $\begin{array}{l}94 \\
99\end{array}$ & $\begin{array}{l}96 \\
96\end{array}$ & $\begin{array}{l}0.014429581 \\
0.014429581\end{array}$ & $\begin{array}{l}0.328723884 \\
0.343153465\end{array}$ & $\begin{array}{l}0.004019 \\
0.004199\end{array}$ \\
\hline 37 & 98 & 0.483428457 & 0.4625 & 98 & 96 & 0.014429581 & 0.357583045 & 0.00438 \\
\hline 38 & 100 & 0.777188384 & 0.475 & 100 & 97 & 0.014579889 & 0.372162934 & 0.004561 \\
\hline 39 & 73 & 0.011186005 & 0.4875 & 73 & 97 & 0.014579889 & 0.386742823 & 0.004743 \\
\hline 40 & 16 & 0.243326895 & 0.5 & 16 & 97 & 0.014579889 & 0.401322712 & 0.004925 \\
\hline 41 & 91 & 0.303972134 & 0.5125 & 91 & 98 & 0.014730197 & 0.416052908 & 0.005109 \\
\hline 42 & 96 & 0.005356681 & 0.525 & 96 & 98 & 0.014730197 & 0.430783105 & 0.005293 \\
\hline 43 & 100 & 0.099142489 & 0.5375 & 100 & 98 & 0.014730197 & 0.445513302 & 0.005477 \\
\hline 44 & 100 & 0.918484294 & 0.55 & 100 & 98 & 0.014730197 & 0.460243499 & 0.005661 \\
\hline 45 & 3 & 0.586615196 & 0.5625 & 3 & 99 & 0.014880505 & 0.475124004 & 0.005846 \\
\hline 46 & 100 & 0.27779259 & 0.575 & 100 & 99 & 0.014880505 & 0.490004509 & 0.006032 \\
\hline 47 & 31 & 0.99990586 & 0.5875 & 31 & 99 & 0.014880505 & 0.504885014 & 0.006218 \\
\hline 48 & 69 & 0.119319002 & 0.6 & 69 & 99 & 0.014880505 & 0.519765519 & 0.006404 \\
\hline 49 & 100 & 0.144026981 & 0.6125 & 100 & 99 & 0.014880505 & 0.534646024 & 0.00659 \\
\hline 50 & 100 & 0.378569885 & 0.625 & 100 & 99 & 0.014880505 & 0.549526529 & 0.006776 \\
\hline 51 & 100 & 0.045327797 & 0.6375 & 100 & 99 & 0.014880505 & 0.564407034 & 0.006962 \\
\hline 52 & 100 & 0.580596644 & 0.65 & 100 & 99 & 0.014880505 & 0.579287539 & 0.007148 \\
\hline $\begin{array}{l}53 \\
54\end{array}$ & $\begin{array}{l}99 \\
100\end{array}$ & $\begin{array}{l}0.653572834 \\
0.715368884\end{array}$ & $\begin{array}{c}0.6625 \\
0.675\end{array}$ & $\begin{array}{r}99 \\
100\end{array}$ & $\begin{array}{r}99 \\
100\end{array}$ & & $\begin{array}{l}0.594168044 \\
0.609198858\end{array}$ & 0.007334 \\
\hline 54 & & 0.715368884 & & 100 & 100 & 0.015030813 & 0.609198858 & 0.007521 \\
\hline 55 & 85 & 0.521373812 & 0.6875 & 85 & 100 & $\begin{array}{l}0.015030813 \\
0.015030813\end{array}$ & 0.624229671 & $\begin{array}{l}0.007709 \\
0.007897\end{array}$ \\
\hline 56 & 100 & 0.901709888 & 0.7 & 100 & 100 & 0.015030813 & 0.639260484 & \\
\hline 57 & 66 & 0.069160449 & 0.7125 & 66 & 100 & 0.015030813 & 0.654291297 & 0.008085 \\
\hline 58 & 86 & 0.833414826 & 0.725 & 86 & 100 & 0.015030813 & 0.66932211 & 0.008273 \\
\hline 59 & 100 & 0.218297748 & 0.7375 & 100 & 100 & 0.015030813 & 0.684352923 & 0.00846 \\
\hline 60 & 99 & 0.59733241 & 0.75 & 99 & 100 & 0.015030813 & 0.699383737 & 0.008648 \\
\hline 61 & 88 & 0.936727841 & 0.7625 & 88 & 100 & 0.015030813 & 0.71441455 & 0.008836 \\
\hline 62 & 83 & 0.678850976 & 0.775 & 83 & 100 & 0.015030813 & 0.729445363 & 0.009024 \\
\hline 63 & 99 & 0.616836936 & 0.7875 & 99 & 100 & 0.015030813 & 0.744476176 & 0.009212 \\
\hline 64 & 96 & 0.22613311 & 0.8 & 96 & 100 & 0.015030813 & 0.759506989 & 0.0094 \\
\hline 65 & 92 & 0.370585824 & 0.8125 & 92 & 100 & 0.015030813 & 0.774537802 & 0.009588 \\
\hline 66 & 85 & 0.712325079 & 0.825 & 85 & 100 & 0.015030813 & 0.789568616 & 0.009776 \\
\hline 67 & 47 & 0.32370636 & 0.8375 & 47 & 100 & 0.015030813 & 0.804599429 & 0.009964 \\
\hline 68 & 54 & 0.143118108 & 0.85 & 54 & 100 & 0.015030813 & 0.819630242 & 0.010151 \\
\hline 69 & 61 & 0.021061909 & 0.8625 & 61 & 100 & 0.015030813 & 0.834661055 & 0.010339 \\
\hline 70 & 95 & 0.358823376 & $\begin{array}{r}0.875 \\
0.8875\end{array}$ & 95 & 100 & 0.015030813 & $\begin{array}{l}0.849691868 \\
0.864722681\end{array}$ & 0.010527 \\
\hline 71 & 92 & 0.061442037 & 0.8875 & 92 & 100 & 0.015030813 & 0.864722681 & $\begin{array}{r}0.010715 \\
0.010903\end{array}$ \\
\hline $\begin{array}{l}72 \\
73\end{array}$ & $\begin{array}{l}44 \\
97\end{array}$ & $\begin{array}{l}0.264759124 \\
0.178464879\end{array}$ & $\begin{array}{c}0.9 \\
0.9125\end{array}$ & $\begin{array}{l}44 \\
97\end{array}$ & $\begin{array}{l}100 \\
100\end{array}$ & $\begin{array}{l}0.015030813 \\
0.015030813\end{array}$ & $\begin{array}{l}0.879753495 \\
0.894784308\end{array}$ & $\begin{array}{l}0.010903 \\
0.011091\end{array}$ \\
\hline 74 & 98 & 0.928303585 & 0.925 & 98 & 100 & 0.015030813 & 0.909815121 & 0.011279 \\
\hline 75 & 100 & 0.22842814 & 0.9375 & 100 & 100 & 0.015030813 & 0.924845934 & 0.011467 \\
\hline 76 & 100 & 0.500664264 & 0.95 & 100 & 100 & 0.015030813 & 0.939876747 & 0.011655 \\
\hline 77 & 18 & 0.684932447 & 0.9625 & 18 & 100 & 0.015030813 & 0.95490756 & 0.011842 \\
\hline 78 & 100 & 0.592118089 & 0.975 & 100 & 100 & 0.015030813 & 0.969938374 & 0.01203 \\
\hline 79 & 78 & 0.467967383 & 0.9875 & 78 & 100 & 0.015030813 & 0.984969187 & 0.012218 \\
\hline 80 & 93 & 0.687891365 & 1 & 93 & 100 & 0.015030813 & 1 & 0.012406 \\
\hline 81 & 100 & 0.958394417 & & & & & & \\
\hline $\begin{array}{l}82 \\
83\end{array}$ & $\begin{array}{l}100 \\
20\end{array}$ & $\begin{array}{l}0.211867758 \\
0.495347802\end{array}$ & & total inhibition & 6653 & & Total area & 0.425664 \\
\hline $\begin{array}{l}83 \\
84\end{array}$ & $\begin{array}{l}20 \\
100\end{array}$ & $\begin{array}{l}0.495347802 \\
0.732104901\end{array}$ & & & & & Gini & 0.148672 \\
\hline 85 & 97 & 0.730713404 & & & & & & \\
\hline
\end{tabular}

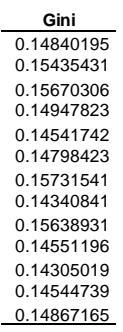

$\begin{array}{rr}\text { mean } & 0.149394886 \\ \text { st dev } & 0.005129498\end{array}$ 
Staurosporine; $1 \mu \mathrm{M} ; 100 \mu \mathrm{M}$ ATP; sample of 60 kinases

\begin{tabular}{|c|c|c|c|c|c|c|c|c|}
\hline position & inhibition\% & rand & cumulative sample fraction & sample inhibition & sorted & fraction of inhibition & cumulative fraction of inhibition & area \\
\hline $\begin{array}{l}1 \\
2\end{array}$ & $\begin{array}{l}99 \\
98\end{array}$ & $\begin{array}{l}0.418716804 \\
0.646050063\end{array}$ & $\begin{array}{l}0.016666667 \\
0.033333333\end{array}$ & $\begin{array}{r}99 \\
98\end{array}$ & $\begin{array}{l}0 \\
3\end{array}$ & $\begin{array}{r}0 \\
0.00061237\end{array}$ & $\begin{array}{r}0 \\
0.00061237\end{array}$ & $\begin{array}{r}0 \\
5.1 \mathrm{E}-06\end{array}$ \\
\hline $\begin{array}{l}3 \\
4\end{array}$ & $\begin{array}{l}100 \\
100\end{array}$ & $\begin{array}{l}0.819753646 \\
0.482260371\end{array}$ & $\begin{array}{c}0.05 \\
0.0666666667\end{array}$ & $\begin{array}{l}100 \\
100\end{array}$ & $\begin{array}{l}16 \\
18\end{array}$ & $\begin{array}{l}0.003265973 \\
0.003674219\end{array}$ & $\begin{array}{l}0.003878343 \\
0.007552562\end{array}$ & $\begin{array}{l}3.74 \mathrm{E}-05 \\
9.53 \mathrm{E}-05\end{array}$ \\
\hline $\begin{array}{l}5 \\
6\end{array}$ & $\begin{array}{l}100 \\
100\end{array}$ & $\begin{array}{c}0.597280046 \\
0.56721309\end{array}$ & $\begin{array}{c}0.083333333 \\
0.1\end{array}$ & $\begin{array}{l}100 \\
100\end{array}$ & $\begin{array}{l}20 \\
29\end{array}$ & $\begin{array}{l}0.004082466 \\
0.005919575\end{array}$ & $\begin{array}{l}0.011635028 \\
0.017554603\end{array}$ & $\begin{array}{r}0.00016 \\
0.000243\end{array}$ \\
\hline 7 & 92 & 0.797581071 & 0.116666667 & 92 & 31 & 0.006327822 & 0.023882425 & 0.000345 \\
\hline 8 & 75 & 0.343165632 & 0.133333333 & 75 & 41 & 0.008369055 & 0.03225148 & 0.000468 \\
\hline 9 & 100 & 0.184092311 & 0.15 & 100 & 46 & 0.009389671 & 0.041641151 & 0.000616 \\
\hline 10 & 69 & 0.26660319 & 0.166666667 & 69 & 47 & 0.009593795 & 0.051234946 & 0.000774 \\
\hline 11 & 97 & 0.560850724 & 0.183333333 & 97 & 54 & 0.011022658 & 0.062257604 & 0.000946 \\
\hline 12 & 16 & 0.094089295 & 0.2 & 16 & 66 & 0.013472137 & 0.075729741 & 0.00115 \\
\hline 13 & 100 & 0.43059915 & 0.216666667 & 100 & 69 & 0.014084507 & 0.089814248 & 0.00138 \\
\hline 14 & 100 & 0.886087035 & 0.233333333 & 100 & 73 & 0.014901 & 0.104715248 & 0.001621 \\
\hline 15 & 0 & 0.647934478 & 0.25 & 0 & 75 & 0.015309247 & 0.120024495 & 0.001873 \\
\hline 16 & 18 & 0.937644448 & 0.266666667 & 18 & 78 & 0.015921617 & 0.135946111 & 0.002133 \\
\hline 17 & 46 & 0.893533857 & 0.283333333 & 46 & 81 & 0.016533987 & 0.152480098 & 0.002404 \\
\hline 18 & 66 & 0.424884652 & 0.3 & 66 & 83 & 0.016942233 & 0.169422331 & 0.002683 \\
\hline 19 & 20 & 0.401163568 & 0.316666667 & 20 & 83 & 0.016942233 & 0.186364564 & 0.002965 \\
\hline $\begin{array}{l}20 \\
21\end{array}$ & $\begin{array}{l}100 \\
41\end{array}$ & $\begin{array}{l}0.458339749 \\
0.864518018\end{array}$ & $\begin{array}{c}0.333333333 \\
0.35\end{array}$ & $\begin{array}{r}100 \\
41\end{array}$ & $\begin{array}{l}85 \\
85\end{array}$ & $\begin{array}{l}0.01735048 \\
0.01735048\end{array}$ & $\begin{array}{l}0.203715044 \\
0.221065524\end{array}$ & $\begin{array}{r}0.003251 \\
0.00354\end{array}$ \\
\hline 22 & 97 & 0.144801071 & 0.366666667 & 97 & 86 & 0.017554603 & 0.238620127 & 0.003831 \\
\hline 23 & 86 & 0.813459683 & 0.383333333 & 86 & 88 & 0.01796285 & 0.256582976 & 0.004127 \\
\hline 24 & 73 & 0.955497452 & 0.4 & 73 & 92 & 0.018779343 & 0.275362319 & 0.004433 \\
\hline 25 & 96 & 0.101766094 & 0.416666667 & 96 & 92 & 0.018779343 & 0.294141662 & 0.004746 \\
\hline 26 & 100 & 0.368231358 & 0.433333333 & 100 & 93 & 0.018983466 & 0.313125128 & 0.005061 \\
\hline 27 & 100 & 0.876449365 & 0.45 & 100 & 94 & 0.019187589 & 0.332312717 & 0.005379 \\
\hline 28 & 100 & 0.580882377 & 0.466666667 & 100 & 96 & 0.019595836 & 0.351908553 & 0.005702 \\
\hline 29 & 99 & 0.524186794 & 0.483333333 & 99 & 96 & 0.019595836 & 0.371504389 & 0.006028 \\
\hline 30 & 99 & 0.301299001 & 0.5 & 99 & 97 & 0.019799959 & 0.391304348 & 0.006357 \\
\hline 31 & 54 & 0.098993124 & 0.516666667 & 54 & 97 & 0.019799959 & 0.411104307 & 0.006687 \\
\hline 32 & 3 & 0.414065238 & 0.533333333 & 3 & 97 & 0.019799959 & 0.430904266 & 0.007017 \\
\hline 33 & 31 & 0.141212101 & 0.55 & 31 & 98 & 0.020004082 & 0.450908349 & 0.007348 \\
\hline $\begin{array}{l}34 \\
35\end{array}$ & 94 & 0.667624077 & 0.5666666667 & 94 & 98 & 0.020004082 & 0.470912431 & 0.007682 \\
\hline $\begin{array}{l}35 \\
36\end{array}$ & $\begin{array}{c}96 \\
100\end{array}$ & $\begin{array}{l}0.385133343 \\
0.163942233\end{array}$ & $\begin{array}{c}0.583333333 \\
0.6\end{array}$ & $\begin{array}{r}96 \\
100\end{array}$ & $\begin{array}{l}99 \\
99\end{array}$ & $\begin{array}{l}0.020208206 \\
0.020208206\end{array}$ & $\begin{array}{l}0.491120637 \\
0.511328843\end{array}$ & $\begin{array}{l}0.00801 / \\
0.008354\end{array}$ \\
\hline $\begin{array}{l}37 \\
38\end{array}$ & $\begin{array}{l}78 \\
85\end{array}$ & $\begin{array}{l}0.673456959 \\
0.244108894\end{array}$ & $\begin{array}{l}0.616666667 \\
0.633333333\end{array}$ & $\begin{array}{l}78 \\
85\end{array}$ & $\begin{array}{l}99 \\
99\end{array}$ & $\begin{array}{l}0.020208206 \\
0.020208206\end{array}$ & $\begin{array}{l}0.531537048 \\
0.551745254\end{array}$ & $\begin{array}{l}0.008691 \\
0.009027\end{array}$ \\
\hline 39 & 100 & 0.467649969 & 0.65 & 100 & 99 & 0.020208206 & 0.57195346 & 0.009364 \\
\hline 40 & 93 & 0.753643079 & 0.666666667 & 93 & 99 & 0.020208206 & 0.592161666 & 0.009701 \\
\hline 41 & 98 & 0.021853338 & 0.683333333 & 98 & 99 & 0.020208206 & 0.612369871 & 0.010038 \\
\hline 42 & 83 & 0.190570128 & 0.7 & 83 & 99 & 0.020208206 & 0.632578077 & 0.010375 \\
\hline 43 & 99 & 0.137501001 & 0.7166666667 & 99 & 100 & 0.020412329 & 0.652990406 & 0.010713 \\
\hline 44 & 99 & 0.707737158 & 0.733333333 & 99 & 100 & 0.020412329 & 0.673402735 & 0.011053 \\
\hline 45 & 99 & 0.194709011 & 0.75 & 99 & 100 & 0.020412329 & 0.693815064 & 0.011393 \\
\hline 46 & 100 & 0.366834993 & 0.766666667 & 100 & 100 & 0.020412329 & 0.714227393 & 0.011734 \\
\hline 47 & 92 & 0.200748748 & 0.783333333 & 92 & 100 & 0.020412329 & 0.734639722 & 0.012074 \\
\hline 48 & 99 & 0.391521255 & 0.8 & 99 & 100 & 0.020412329 & 0.755052051 & 0.012414 \\
\hline 49 & 100 & 0.886496898 & 0.816666667 & 100 & 100 & 0.020412329 & 0.77546438 & 0.012754 \\
\hline 50 & 100 & 0.818979815 & 0.833333333 & 100 & 100 & 0.020412329 & 0.79587671 & 0.013095 \\
\hline 51 & 99 & 0.4395462 & 0.85 & 99 & 100 & 0.020412329 & 0.816289039 & 0.013435 \\
\hline 52 & 97 & 0.670528274 & 0.866666667 & 97 & 100 & 0.020412329 & 0.836701368 & 0.013775 \\
\hline $\begin{array}{l}53 \\
54\end{array}$ & $\begin{array}{l}85 \\
29\end{array}$ & $\begin{array}{l}0.514054022 \\
0.029604573\end{array}$ & $\begin{array}{c}0.883333333 \\
0.9\end{array}$ & $\begin{array}{l}85 \\
29\end{array}$ & $\begin{array}{l}100 \\
100\end{array}$ & $\begin{array}{l}0.020412329 \\
0.020412329\end{array}$ & $\begin{array}{l}0.857113697 \\
0.877526026\end{array}$ & $\begin{array}{l}0.014115 \\
0.014455\end{array}$ \\
\hline 55 & 100 & 0.365728991 & 0.9166666667 & 100 & 100 & 0.020412329 & 0.897938355 & 0.014796 \\
\hline 56 & 47 & 0.275029091 & 0.933333333 & 47 & 100 & 0.020412329 & 0.918350684 & 0.015136 \\
\hline 57 & 100 & 0.05466004 & 0.95 & 100 & 100 & 0.020412329 & 0.938763013 & 0.015476 \\
\hline 58 & 88 & 0.771440274 & 0.966666667 & 88 & 100 & 0.020412329 & 0.959175342 & 0.015816 \\
\hline 59 & 83 & 0.743537818 & 0.983333333 & 83 & 100 & 0.020412329 & 0.979587671 & 0.016156 \\
\hline 60 & 81 & 0.753784524 & 1 & 81 & 100 & 0.020412329 & 1 & 0.016497 \\
\hline 61 & 98 & 0.792080268 & & & & & & \\
\hline 62 & 100 & 0.371157499 & & & & & & \\
\hline 63 & 95 & 0.73131413 & & & & & & \\
\hline 64 & 36 & 0.971259909 & & & & & & \\
\hline 65 & 96 & 0.509425684 & & & & & & \\
\hline 66 & 98 & 0.297199134 & & & & & & \\
\hline 67 & 61 & 0.167078247 & & & & & & \\
\hline 68 & 100 & 0.196803222 & & & & & & \\
\hline 69 & 8 & 0.188380004 & & & & & & \\
\hline $\begin{array}{l}70 \\
71\end{array}$ & $\begin{array}{l}44 \\
100\end{array}$ & $\begin{array}{r}0.87096102 \\
0.952319978\end{array}$ & & & & & & \\
\hline 72 & 100 & 0.030305296 & & & & & & \\
\hline 73 & 99 & 0.128736753 & & & & & & \\
\hline 74 & 100 & 0.593825012 & & & & & & \\
\hline 75 & 53 & 0.782324231 & & & & & & \\
\hline 76 & 100 & 0.313070216 & & & & & & \\
\hline 77 & 97 & 0.869656262 & & & & & & \\
\hline 78 & 95 & 0.528155328 & & & & & & \\
\hline 79 & 100 & 0.435639928 & & & & & & \\
\hline 80 & 100 & 0.498784432 & & & & & & \\
\hline 81 & 91 & 0.622984511 & & & & & & \\
\hline 82 & 100 & 0.313868169 & & total inhibition & 4899 & & Total area & 0.419536 \\
\hline 83 & 100 & 0.258644047 & & & & & & \\
\hline 84 & 100 & 0.223340672 & & & & & Gini & 0.160927 \\
\hline
\end{tabular}

$\begin{array}{r}\multicolumn{1}{c}{\text { Gini }} \\ \hline 0.1428249 \\ 0.17414173 \\ 0.15772613 \\ 0.13163119 \\ 0.14553958 \\ 0.19117772 \\ 0.16455244 \\ 0.15347989 \\ 0.15903623 \\ 0.16502376 \\ 0.14813376 \\ 0.13283304 \\ 0.1609274 \\ \hline\end{array}$

$\begin{array}{rr}\text { mean } & 0.155925213 \\ \text { st dev } & 0.016430031\end{array}$ 
Staurosporine; $1 \mu \mathrm{M} ; 100 \mu \mathrm{M}$ ATP; sample of 40 kinases

\begin{tabular}{|c|c|c|c|c|c|c|c|c|}
\hline position & inhibition\% & rand & cumulative sample fraction & sample inhibition & sorted & fraction of inhibition & cumulative fraction of inhibition & area \\
\hline 1 & 100 & 0.165199278 & 0.025 & 100 & 18 & 0.005172414 & 0.005172414 & 6.47E-05 \\
\hline 2 & 73 & 0.391245504 & 0.05 & 73 & 41 & 0.011781609 & 0.016954023 & 0.000277 \\
\hline $\begin{array}{l}3 \\
4\end{array}$ & $\begin{array}{l}86 \\
100\end{array}$ & $\begin{array}{l}0.459484672 \\
0.049401928\end{array}$ & $\begin{array}{l}0.075 \\
0.1\end{array}$ & $\begin{array}{r}86 \\
100\end{array}$ & $\begin{array}{l}44 \\
54\end{array}$ & $\begin{array}{l}0.012643678 \\
0.015517241\end{array}$ & $\begin{array}{l}0.029597701 \\
0.045114943\end{array}$ & $\begin{array}{l}0.000582 \\
0.000934\end{array}$ \\
\hline $\begin{array}{l}4 \\
5\end{array}$ & 99 & 0.012589546 & 0.125 & 99 & 61 & 0.017528736 & 0.062643678 & 0.001347 \\
\hline 6 & 96 & 0.631510876 & 0.15 & 96 & 66 & 0.018965517 & 0.081609195 & 0.001803 \\
\hline 7 & 66 & 0.2270517 & 0.175 & 66 & 69 & 0.019827586 & 0.101436782 & 0.002288 \\
\hline 8 & 88 & 0.788327645 & 0.2 & 88 & 73 & 0.020977011 & 0.122413793 & 0.002798 \\
\hline 9 & 18 & 0.560679345 & 0.225 & 18 & 75 & 0.021551724 & 0.143965517 & 0.00333 \\
\hline 10 & 44 & 0.811792264 & 0.25 & 44 & 81 & 0.023275862 & 0.167241379 & 0.00389 \\
\hline 11 & 100 & 0.752190693 & 0.275 & 100 & 83 & 0.023850575 & 0.191091954 & 0.004479 \\
\hline 12 & 93 & 0.309046018 & 0.3 & 93 & 85 & 0.024425287 & 0.215517241 & 0.005083 \\
\hline 13 & 100 & 0.931741428 & 0.325 & 100 & 86 & 0.024712644 & 0.240229885 & 0.005697 \\
\hline 14 & 100 & 0.690007925 & 0.35 & 100 & 88 & 0.025287356 & 0.265517241 & 0.006322 \\
\hline 15 & 69 & 0.111839029 & 0.375 & 69 & 91 & 0.026149425 & 0.291666667 & 0.006965 \\
\hline 16 & 98 & 0.326942526 & 0.4 & 98 & 93 & 0.026724138 & 0.318390805 & 0.007626 \\
\hline 17 & 91 & 0.659401033 & 0.425 & 91 & 94 & 0.027011494 & 0.345402299 & 0.008297 \\
\hline 18 & 41 & 0.649848594 & 0.45 & 41 & 95 & 0.027298851 & 0.372701149 & 0.008976 \\
\hline 19 & 95 & 0.156110814 & 0.475 & 95 & 96 & 0.027586207 & 0.400287356 & 0.009662 \\
\hline 20 & 99 & $\begin{array}{l}0.513523739 \\
0.282452593\end{array}$ & $\begin{array}{l}0.5 \\
0.525\end{array}$ & $\begin{array}{r}99 \\
100\end{array}$ & $\begin{array}{l}96 \\
97\end{array}$ & 0.027586207 & 0.427873563 & $\begin{array}{l}0.010352 \\
0.011045\end{array}$ \\
\hline $\begin{array}{l}21 \\
22\end{array}$ & $\begin{array}{l}100 \\
100\end{array}$ & $\begin{array}{l}0.282452593 \\
0.144849466\end{array}$ & $\begin{array}{l}0.525 \\
0.55\end{array}$ & $\begin{array}{l}100 \\
100\end{array}$ & $\begin{array}{l}97 \\
98\end{array}$ & $\begin{array}{r}0.027873563 \\
0.02816092\end{array}$ & $\begin{array}{l}0.455747126 \\
0.483908046\end{array}$ & $\begin{array}{l}0.011045 \\
0.011746\end{array}$ \\
\hline 23 & 97 & 0.436662447 & 0.575 & 97 & 99 & 0.028448276 & $\begin{array}{l}0.483908046 \\
0.512356322\end{array}$ & 0.012453 \\
\hline 24 & 100 & 0.144157741 & 0.6 & 100 & 99 & 0.028448276 & 0.540804598 & 0.013165 \\
\hline 25 & 100 & 0.014211005 & 0.625 & 100 & 99 & 0.028448276 & 0.569252874 & 0.013876 \\
\hline 26 & 96 & 0.006074992 & 0.65 & 96 & 99 & 0.028448276 & 0.597701149 & 0.014587 \\
\hline 27 & 61 & 0.805637905 & 0.675 & 61 & 100 & 0.028735632 & 0.626436782 & 0.015302 \\
\hline 28 & 75 & 0.006061001 & 0.7 & 75 & 100 & 0.028735632 & 0.655172414 & 0.01602 \\
\hline 29 & 100 & 0.155851315 & 0.725 & 100 & 100 & 0.028735632 & 0.683908046 & 0.016739 \\
\hline 30 & 100 & 0.575696257 & 0.75 & 100 & 100 & 0.028735632 & 0.712643678 & 0.017457 \\
\hline 31 & 81 & 0.729350493 & 0.775 & 81 & 100 & 0.028735632 & 0.74137931 & 0.018175 \\
\hline 32 & 99 & 0.765120569 & 0.8 & 99 & 100 & 0.028735632 & 0.770114943 & 0.018894 \\
\hline 33 & 94 & 0.029171014 & 0.825 & 94 & 100 & 0.028735632 & 0.798850575 & 0.019612 \\
\hline 34 & 99 & 0.579656326 & 0.85 & 99 & 100 & 0.028735632 & 0.827586207 & $\begin{array}{r}0.02033 \\
0.021049\end{array}$ \\
\hline $\begin{array}{l}35 \\
36\end{array}$ & $\begin{array}{l}54 \\
100\end{array}$ & $\begin{array}{r}0.610486137 \\
0.49088893\end{array}$ & $\begin{array}{c}0.875 \\
0.9\end{array}$ & $\begin{array}{r}54 \\
100\end{array}$ & $\begin{array}{l}100 \\
100\end{array}$ & $\begin{array}{l}0.028735632 \\
0.028735632\end{array}$ & $\begin{array}{l}0.856321839 \\
0.885057471\end{array}$ & $\begin{array}{l}0.021049 \\
0.021767\end{array}$ \\
\hline 37 & 85 & 0.706846073 & 0.925 & 85 & 100 & 0.028735632 & 0.913793103 & 0.022486 \\
\hline 38 & 100 & 0.117263079 & 0.95 & 100 & 100 & 0.028735632 & 0.942528736 & 0.023204 \\
\hline 39 & 83 & 0.421752899 & 0.975 & 83 & 100 & 0.028735632 & 0.971264368 & 0.023922 \\
\hline 40 & 100 & 0.075669802 & 1 & 100 & 100 & 0.028735632 & 1 & 0.024641 \\
\hline 41 & 100 & 0.494656068 & & & & & & \\
\hline 42 & 100 & 0.614469637 & & & & & & \\
\hline 43 & 100 & 0.477182425 & & & & & & \\
\hline 44 & 99 & 0.067735963 & & & & & & \\
\hline 45 & 78 & 0.102850636 & & & & & & \\
\hline 46 & 85 & 0.970439081 & & & & & & \\
\hline 47 & 100 & 0.252490625 & & & & & & \\
\hline 48 & 99 & 0.095276157 & & & & & & \\
\hline 49 & 98 & 0.018820622 & & & & & & \\
\hline 50 & 92 & 0.650368381 & & & & & & \\
\hline 51 & 100 & 0.397720711 & & & & & & \\
\hline 52 & 92 & 0.501706777 & & & & & & \\
\hline 53 & 100 & 0.895179357 & & & & & & \\
\hline 54 & 100 & 0.223168446 & & & & & & \\
\hline 55 & 53 & 0.282787496 & & & & & & \\
\hline 56 & 20 & 0.624807323 & & & & & & \\
\hline 57 & 96 & 0.788295165 & & & & & & \\
\hline 58 & 99 & 0.991635886 & & & & & & \\
\hline 59 & 97 & 0.163526319 & & & & & & \\
\hline 60 & 97 & 0.947934736 & & & & & & \\
\hline 61 & 100 & 0.34173044 & & & & & & \\
\hline 62 & 100 & 0.683517872 & & & & & & \\
\hline 63 & 100 & 0.551699861 & & & & & & \\
\hline 64 & 29 & 0.586585794 & & & & & & \\
\hline 65 & 97 & 0.386324137 & & & & & & \\
\hline 66 & 99 & 0.535940992 & & & & & & \\
\hline 67 & 46 & 0.700463909 & & & & & & \\
\hline 68 & 0 & 0.266577371 & & & & & & \\
\hline 69 & 100 & 0.338283168 & & & & & & \\
\hline $\begin{array}{l}70 \\
71\end{array}$ & $\begin{array}{l}83 \\
16\end{array}$ & $\begin{array}{c}0.88709488 \\
0.916461532\end{array}$ & & & & & & \\
\hline 72 & 100 & 0.240322494 & & & & & & \\
\hline 73 & 100 & 0.137240502 & & & & & & \\
\hline 74 & 47 & 0.527968108 & & & & & & \\
\hline 75 & 100 & 0.816854743 & & & & & & \\
\hline 76 & 31 & 0.368449245 & & & & & & \\
\hline 77 & 100 & 0.952112408 & & & & & & \\
\hline 78 & 100 & 0.693312618 & & & & & & \\
\hline 79 & 8 & 0.009125692 & & & & & & \\
\hline 80 & 99 & 0.643517525 & & & & & & \\
\hline 81 & 36 & 0.932601778 & & & & & & \\
\hline 82 & $\begin{array}{l}95 \\
98\end{array}$ & 0.405500171 & & total inhibition & 3480 & & Total area & 0.447241 \\
\hline $\begin{array}{l}83 \\
84\end{array}$ & $\begin{array}{l}98 \\
98\end{array}$ & $\begin{array}{l}0.350898335 \\
0.026953814\end{array}$ & & & & & Gini & 0.105517 \\
\hline
\end{tabular}

\begin{tabular}{c} 
Gini \\
\hline 0.14867077 \\
0.18449765 \\
0.14470105 \\
0.14256637 \\
0.11782627 \\
0.10797205 \\
0.14866687 \\
0.12566822 \\
0.15953937 \\
0.10339875 \\
0.13314056 \\
0.15873052 \\
0.10551724 \\
\hline
\end{tabular}

$\begin{array}{ll}\text { mean } & 0.136991976 \\ \text { st dev } & 0.024277294\end{array}$ 
Staurosporine; $1 \mu \mathrm{M} ; 100 \mu \mathrm{M}$ ATP; sample of 20 kinases

\begin{tabular}{|c|c|c|c|c|c|c|c|c|}
\hline position & inhibition\% & rand & cumulative sample fraction & sample inhibition & sorted & fraction of inhibition & cumulative fraction of inhibition & area \\
\hline $\begin{array}{l}1 \\
2\end{array}$ & $\begin{array}{l}100 \\
100\end{array}$ & $\begin{array}{l}0.424829563 \\
0.964069122\end{array}$ & $\begin{array}{l}0.05 \\
0.1\end{array}$ & $\begin{array}{l}100 \\
100\end{array}$ & 0 & $\begin{array}{r}0 \\
0.021778584\end{array}$ & $\begin{array}{r}0 \\
0.021778584\end{array}$ & $\begin{array}{r}0 \\
0.000544\end{array}$ \\
\hline & & & & & 36 & 0.021778584 & & 0.000544 \\
\hline $\begin{array}{l}3 \\
4\end{array}$ & $\begin{array}{l}53 \\
54\end{array}$ & $\begin{array}{r}0.832084276 \\
0.34646106\end{array}$ & $\begin{array}{l}0.15 \\
0.2\end{array}$ & $\begin{array}{l}53 \\
54\end{array}$ & $\begin{array}{l}53 \\
54\end{array}$ & $\begin{array}{l}0.032062916 \\
0.032667877\end{array}$ & $\begin{array}{r}0.0538415 \\
0.086509377\end{array}$ & $\begin{array}{l}0.001891 \\
0.003509\end{array}$ \\
\hline $\begin{array}{l}5 \\
6\end{array}$ & $\begin{array}{l}100 \\
100\end{array}$ & $\begin{array}{l}0.763398475 \\
0.959716601\end{array}$ & $\begin{array}{l}0.25 \\
0.3\end{array}$ & $\begin{array}{l}100 \\
100\end{array}$ & $\begin{array}{l}61 \\
73\end{array}$ & $\begin{array}{l}0.036902601 \\
0.044162129\end{array}$ & $\begin{array}{l}0.123411978 \\
0.167574108\end{array}$ & $\begin{array}{l}0.005248 \\
0.007275\end{array}$ \\
\hline $\begin{array}{l}0 \\
7\end{array}$ & 91 & 0.029468399 & 0.35 & 91 & 91 & 0.055051422 & 0.222625529 & 0.009755 \\
\hline 8 & 96 & 0.496442276 & 0.4 & 96 & 92 & 0.055656382 & 0.278281912 & 0.012523 \\
\hline 9 & 100 & 0.813503207 & 0.45 & 100 & 96 & 0.058076225 & 0.336358137 & 0.015366 \\
\hline 10 & 99 & 0.576524591 & 0.5 & 99 & 99 & 0.059891107 & 0.396249244 & 0.018315 \\
\hline 11 & 100 & 0.542260057 & 0.55 & 100 & 99 & 0.059891107 & 0.456140351 & 0.02131 \\
\hline 12 & 92 & 0.941415459 & 0.6 & 92 & 99 & 0.059891107 & 0.516031458 & 0.024304 \\
\hline 13 & 36 & 0.794715884 & 0.65 & 36 & 100 & 0.060496068 & 0.576527526 & 0.027314 \\
\hline 14 & 61 & 0.033580117 & 0.7 & 61 & 100 & 0.060496068 & 0.637023593 & 0.030339 \\
\hline 15 & 73 & 0.282665581 & 0.75 & 73 & 100 & 0.060496068 & 0.697519661 & 0.033364 \\
\hline 16 & 99 & 0.081902686 & 0.8 & 99 & 100 & 0.060496068 & 0.758015729 & 0.036388 \\
\hline 17 & 0 & 0.121246342 & 0.85 & 0 & 100 & 0.060496068 & 0.818511797 & 0.039413 \\
\hline 18 & 99 & 0.420973807 & 0.9 & 99 & 100 & 0.060496068 & 0.879007864 & 0.042438 \\
\hline 19 & 100 & 0.697391345 & 0.95 & 100 & 100 & 0.060496068 & 0.939503932 & 0.045463 \\
\hline 20 & 100 & 0.157556086 & 1 & 100 & 100 & 0.060496068 & 1 & 0.048488 \\
\hline 22 & 100 & 0.300067668 & & & & & & \\
\hline 23 & 99 & 0.998526457 & & & & & & \\
\hline 24 & 100 & 0.052644735 & & & & & & \\
\hline 25 & 100 & 0.291577888 & & & & & & \\
\hline 26 & 41 & 0.325426477 & & & & & & \\
\hline 27 & 100 & 0.463389747 & & & & & & \\
\hline 28 & 44 & 0.873469266 & & & & & & \\
\hline 29 & 88 & 0.673457574 & & & & & & \\
\hline 30 & 100 & 0.057592732 & & & & & & \\
\hline 31 & 99 & 0.209356745 & & & & & & \\
\hline 32 & 47 & 0.678290008 & & & & & & \\
\hline 33 & 100 & 0.349930109 & & & & & & \\
\hline $\begin{array}{l}34 \\
35\end{array}$ & $\begin{array}{l}8 \\
98\end{array}$ & $\begin{array}{l}0.789124107 \\
0.337021098\end{array}$ & & & & & & \\
\hline 36 & 100 & 0.286052233 & & & & & & \\
\hline $\begin{array}{l}37 \\
38\end{array}$ & 100 & 0.436951102 & & & & & & \\
\hline 39 & 99 & 0.185990334 & & & & & & \\
\hline 40 & 96 & 0.259152263 & & & & & & \\
\hline 41 & 83 & 0.970695005 & & & & & & \\
\hline 42 & 95 & 0.239004253 & & & & & & \\
\hline 43 & 98 & 0.54222049 & & & & & & \\
\hline 44 & 99 & 0.658860985 & & & & & & \\
\hline 45 & 83 & 0.414725191 & & & & & & \\
\hline 46 & 85 & 0.669558758 & & & & & & \\
\hline 47 & 75 & 0.429302008 & & & & & & \\
\hline 48 & 100 & 0.46490984 & & & & & & \\
\hline 49 & 31 & 0.631336663 & & & & & & \\
\hline 50 & 92 & 0.325019458 & & & & & & \\
\hline 51 & 93 & 0.292959547 & & & & & & \\
\hline 52 & 100 & 0.347363193 & & & & & & \\
\hline $\begin{array}{l}53 \\
54\end{array}$ & $\begin{array}{c}94 \\
100\end{array}$ & $\begin{array}{c}0.434839497 \\
0.6055106\end{array}$ & & & & & & \\
\hline 55 & 16 & 0.320313294 & & & & & & \\
\hline 56 & 85 & 0.488975972 & & & & & & \\
\hline 57 & 46 & 0.33280249 & & & & & & \\
\hline 58 & 100 & 0.639264196 & & & & & & \\
\hline 59 & 96 & 0.69975316 & & & & & & \\
\hline 60 & 78 & 0.324425657 & & & & & & \\
\hline 61 & 20 & 0.45497707 & & & & & & \\
\hline 62 & 100 & 0.102547523 & & & & & & \\
\hline 63 & 100 & 0.471423678 & & & & & & \\
\hline 64 & 18 & 0.561730819 & & & & & & \\
\hline 65 & 100 & 0.389839583 & & & & & & \\
\hline 66 & 99 & 0.625808907 & & & & & & \\
\hline 67 & 99 & 0.859408518 & & & & & & \\
\hline 68 & 69 & 0.503340268 & & & & & & \\
\hline 69 & 98 & 0.502549776 & & & & & & \\
\hline $\begin{array}{l}70 \\
71\end{array}$ & 66 & 0.054915457 & & & & & & \\
\hline 72 & 86 & $\begin{array}{r}0.488351532 \\
0.06514676\end{array}$ & & & & & & \\
\hline 73 & 3 & 0.688030784 & & & & & & \\
\hline 74 & 98 & 0.742600539 & & & & & & \\
\hline 75 & 29 & 0.831439747 & & & & & & \\
\hline 76 & 95 & 0.425347572 & & & & & & \\
\hline 77 & 100 & 0.229212563 & & & & & & \\
\hline 78 & 100 & 0.020202881 & & & & & & \\
\hline 79 & 97 & 0.388015643 & & & & & & \\
\hline 80 & 97 & 0.339391942 & & & & & & \\
\hline 81 & 100 & 0.006600485 & & & & & & \\
\hline $\begin{array}{l}82 \\
83\end{array}$ & $\begin{array}{l}100 \\
97\end{array}$ & $\begin{array}{l}0.015154452 \\
0.253319382\end{array}$ & & total inhibition & 1653 & & Total area & 0.423246 \\
\hline 83 & 81 & $\begin{array}{l}0.253319382 \\
0.762039521\end{array}$ & & & & & Gini & 0.153509 \\
\hline
\end{tabular}

\begin{tabular}{c}
\multicolumn{1}{c}{ Gini } \\
\hline 0.22468522 \\
0.13986811 \\
0.16324341 \\
0.1776808 \\
0.13676294 \\
0.11758824 \\
0.16716604 \\
0.0494695 \\
0.20998699 \\
0.07962357 \\
0.26200575 \\
0.17125935 \\
0.15350877 \\
\hline
\end{tabular}

$\begin{array}{ll}\text { mean } & 0.157911437 \\ \text { st dev } & 0.057005928\end{array}$ 
Staurosporine; $1 \mu \mathrm{M} ; 100 \mu \mathrm{M}$ ATP; sample of 10 kinases

\begin{tabular}{|c|c|c|c|c|c|c|c|c|}
\hline position & inhibition $\%$ & rand & cumulative sample fraction & sample inhibition & sorted & fraction of inhibition & cumulative fraction of inhibition & area \\
\hline 1 & 44 & 0.012734736 & 0.1 & 44 & 3 & 0.004166667 & 0.004166667 & 0.000208 \\
\hline 2 & 88 & 0.362263291 & 0.2 & 88 & 41 & 0.056944444 & 0.061111111 & 0.003264 \\
\hline $\begin{array}{l}3 \\
4\end{array}$ & $\begin{array}{l}100 \\
78\end{array}$ & $\begin{array}{l}0.868295998 \\
0.009947931\end{array}$ & $\begin{array}{l}0.3 \\
0.4\end{array}$ & $\begin{array}{r}100 \\
78\end{array}$ & $\begin{array}{l}44 \\
78\end{array}$ & $\begin{array}{l}0.061111111 \\
0.108333333\end{array}$ & $\begin{array}{l}0.122222222 \\
0.230555556\end{array}$ & $\begin{array}{l}0.009167 \\
0.017639\end{array}$ \\
\hline 5 & 83 & 0.645811254 & 0.5 & 83 & 83 & 0.115277778 & 0.345833333 & 0.028819 \\
\hline 6 & 83 & 0.856742748 & 0.6 & 83 & 83 & 0.115277778 & 0.4611111111 & 0.040347 \\
\hline 7 & 3 & 0.322653105 & 0.7 & 3 & 88 & 0.122222222 & 0.583333333 & 0.052222 \\
\hline 8 & 41 & 0.915202818 & 0.8 & 41 & 100 & 0.138888889 & 0.722222222 & 0.065278 \\
\hline 9 & 100 & 0.319886625 & 0.9 & 100 & 100 & 0.138888889 & 0.861111111 & 0.079167 \\
\hline 10 & 100 & 0.320436658 & 1 & 100 & 100 & 0.138888889 & 1 & 0.093056 \\
\hline 11 & 100 & 0.178465451 & & & & & & \\
\hline 12 & 95 & 0.477640168 & & & & & & \\
\hline 13 & 100 & 0.830831887 & & & & & & \\
\hline 14 & 46 & 0.819474667 & & & & & & \\
\hline 15 & 98 & 0.657451602 & & & & & & \\
\hline 16 & 98 & 0.64685609 & & & & & & \\
\hline 17 & 81 & 0.121275967 & & & & & & \\
\hline 18 & 98 & 0.630958204 & & & & & & \\
\hline 19 & 100 & 0.711295584 & & & & & & \\
\hline 20 & 100 & 0.755238613 & & & & & & \\
\hline 21 & 96 & 0.378386979 & & & & & & \\
\hline $\begin{array}{l}22 \\
23\end{array}$ & $\begin{array}{l}100 \\
99\end{array}$ & $\begin{array}{c}0.284509632 \\
0.58436085\end{array}$ & & & & & & \\
\hline 24 & 91 & 0.974741424 & & & & & & \\
\hline 25 & 100 & 0.938513965 & & & & & & \\
\hline 26 & 100 & 0.76779241 & & & & & & \\
\hline 27 & 100 & 0.897924991 & & & & & & \\
\hline 28 & 47 & 0.431684939 & & & & & & \\
\hline 29 & 99 & 0.452220546 & & & & & & \\
\hline 30 & 100 & 0.945453073 & & & & & & \\
\hline 31 & 97 & 0.693883285 & & & & & & \\
\hline 32 & 99 & 0.099190983 & & & & & & \\
\hline 33 & 86 & 0.967477174 & & & & & & \\
\hline $\begin{array}{l}34 \\
35\end{array}$ & $\begin{array}{l}97 \\
100\end{array}$ & $\begin{array}{r}0.48435214 \\
0.837878659\end{array}$ & & & & & & \\
\hline 36 & 96 & 0.866622034 & & & & & & \\
\hline 37 & $\begin{array}{l}66 \\
95\end{array}$ & 0.700955975 & & & & & & \\
\hline 39 & 100 & 0.981674343 & & & & & & \\
\hline 40 & 100 & 0.305676883 & & & & & & \\
\hline 41 & 92 & 0.670154774 & & & & & & \\
\hline 42 & 98 & 0.825282637 & & & & & & \\
\hline 43 & 96 & 0.123464593 & & & & & & \\
\hline 44 & 100 & 0.587653381 & & & & & & \\
\hline 45 & 16 & 0.826243403 & & & & & & \\
\hline 46 & 100 & 0.202202528 & & & & & & \\
\hline 47 & 97 & 0.539229016 & & & & & & \\
\hline 48 & 18 & 0.209722636 & & & & & & \\
\hline 49 & 85 & 0.655535157 & & & & & & \\
\hline 50 & 85 & 0.588346225 & & & & & & \\
\hline 51 & 100 & 0.577201691 & & & & & & \\
\hline 52 & 100 & 0.510653708 & & & & & & \\
\hline $\begin{array}{l}53 \\
54\end{array}$ & $\begin{array}{l}100 \\
53\end{array}$ & $\begin{array}{l}0.942870956 \\
0.467420857\end{array}$ & & & & & & \\
\hline 55 & 99 & 0.576017973 & & & & & & \\
\hline 56 & 93 & 0.634345462 & & & & & & \\
\hline 57 & 36 & 0.704799659 & & & & & & \\
\hline 58 & 69 & 0.872243976 & & & & & & \\
\hline 59 & 75 & 0.374600074 & & & & & & \\
\hline 60 & 100 & 0.81895789 & & & & & & \\
\hline 61 & 97 & 0.248030762 & & & & & & \\
\hline 62 & 8 & 0.762490933 & & & & & & \\
\hline 63 & 94 & 0.919108327 & & & & & & \\
\hline 64 & 100 & 0.43810829 & & & & & & \\
\hline 65 & 100 & 0.744689816 & & & & & & \\
\hline 66 & 100 & 0.697365525 & & & & & & \\
\hline 67 & 99 & 0.448022127 & & & & & & \\
\hline 68 & 99 & 0.555008146 & & & & & & \\
\hline 69 & 92 & 0.936450596 & & & & & & \\
\hline $\begin{array}{l}70 \\
71\end{array}$ & $\begin{array}{c}29 \\
0\end{array}$ & $\begin{array}{l}0.114027946 \\
0.962201376\end{array}$ & & & & & & \\
\hline 72 & 61 & $\begin{array}{l}0.9622013 / 6 \\
0.979618715\end{array}$ & & & & & & \\
\hline 73 & 31 & 0.549398543 & & & & & & \\
\hline 74 & 54 & 0.632085507 & & & & & & \\
\hline 75 & 100 & 0.768487941 & & & & & & \\
\hline 76 & 100 & 0.112695219 & & & & & & \\
\hline 77 & 99 & 0.884226532 & & & & & & \\
\hline 78 & 100 & 0.584914513 & & & & & & \\
\hline 79 & 100 & 0.704132192 & & & & & & \\
\hline 80 & 99 & 0.917988288 & & & & & & \\
\hline 81 & 73 & 0.618265811 & & & & & & \\
\hline $\begin{array}{l}82 \\
83\end{array}$ & $\begin{array}{l}100 \\
20\end{array}$ & $\begin{array}{l}0.577398648 \\
0.261938355\end{array}$ & & total inhibition & 720 & & Total area & 0.3891 \\
\hline 84 & 99 & 0.004114625 & & & & & Gini & 0.221667 \\
\hline
\end{tabular}




\begin{tabular}{|c|c|c|c|c|c|c|c|c|c|}
\hline & $10 \mu \mathrm{M}$ ATP; full set of 85 kinases & & & & & & & & \\
\hline Kinase & PD184352@ @ $10 \mu \mathrm{M}$ & sorted & normalized & position & cumulative sample fraction & inhibition\% & fraction of total inhibition & cumulative inhibition & area \\
\hline $\mathrm{Ab}(\mathrm{m})$ & 99 & 132 & 100 & 1 & 0.011764706 & 0 & 0 & 0 & 0 \\
\hline AMPK(r) & 100 & 124 & 100 & 2 & 0.023529412 & 0 & 0 & 0 & 0 \\
\hline Arg(m) & 105 & 123 & 100 & 3 & 0.035294118 & 0 & 0 & 0 & 0 \\
\hline Aurora-A(h) & 106 & 120 & 100 & 4 & 0.047058824 & 0 & 0 & 0 & 0 \\
\hline Axl(h) & 119 & 119 & 100 & 5 & 0.058823529 & 0 & 0 & 0 & 0 \\
\hline $\operatorname{Blk}(\mathrm{m})$ & 105 & 119 & 100 & 6 & 0.070588235 & 0 & 0 & 0 & 0 \\
\hline Bmx(h) & 104 & 117 & 100 & 7 & 0.082352941 & 0 & 0 & 0 & 0 \\
\hline CaMKII(r) & 95 & 116 & 100 & 8 & 0.094117647 & 0 & 0 & 0 & 0 \\
\hline CaMKIV(h) & 106 & 115 & 100 & 9 & 0.105882353 & 0 & 0 & 0 & 0 \\
\hline $\mathrm{CDK1} /$ cyclinB(h) & 101 & 112 & 100 & 10 & 0.117647059 & 0 & 0 & 0 & 0 \\
\hline $\mathrm{CDK} 2 / \mathrm{cyclinA}(\mathrm{h})$ & 102 & 112 & 100 & 11 & 0.129411765 & 0 & 0 & 0 & 0 \\
\hline $\mathrm{CDK} 2 / \mathrm{cyclin}(\mathrm{h})$ & 93 & 112 & 100 & 12 & 0.141176471 & 0 & 0 & 0 & 0 \\
\hline CDK3/cyclinE(h) & 101 & 111 & 100 & 13 & 0.152941176 & 0 & 0 & 0 & 0 \\
\hline $\mathrm{CDK5} / 335(\mathrm{~h})$ & 106 & 111 & 100 & 14 & 0.164705882 & 0 & 0 & 0 & 0 \\
\hline CDK6/cyclinD3(h) & 95 & 110 & 100 & 15 & 0.176470588 & 0 & 0 & 0 & 0 \\
\hline CDK7/cyclinHMATI(h) & 100 & 110 & 100 & 16 & 0.188235294 & 0 & 0 & 0 & 0 \\
\hline СHK1(h) & 115 & 109 & 100 & 17 & 0.2 & 0 & 0 & 0 & 0 \\
\hline СHK2(h) & 98 & 109 & 100 & 18 & 0.211764706 & 0 & 0 & 0 & 0 \\
\hline $\mathrm{CKI}(\mathrm{y})$ & 97 & 109 & 100 & 19 & 0.223529412 & 0 & 0 & 0 & 0 \\
\hline СK2(h) & 109 & 108 & 100 & 20 & 0.235294118 & 0 & 0 & 0 & 0 \\
\hline$c \cdot \operatorname{RAF}(\mathrm{h})$ & 98 & 108 & 100 & 21 & 0.247058824 & 0 & 0 & 0 & 0 \\
\hline $\operatorname{CSK}(\mathrm{h})$ & 76 & 107 & 100 & 22 & 0.258823529 & 0 & 0 & 0 & 0 \\
\hline $\mathrm{cSRC(h)}$ & 103 & 107 & 100 & 23 & 0.270588235 & 0 & 0 & 0 & 0 \\
\hline Fes(h) & 108 & 106 & 100 & 24 & 0.282352941 & 0 & 0 & 0 & 0 \\
\hline FGFR3(h) & 109 & 106 & 100 & 25 & 0.294117647 & 0 & 0 & 0 & 0 \\
\hline Flls(h) & 105 & 106 & 100 & 26 & 0.305882353 & 0 & 0 & 0 & 0 \\
\hline Fynn(h) & 94 & 106 & 100 & 27 & 0.317647059 & 0 & 0 & 0 & 0 \\
\hline GSK3a(h) & 91 & 105 & 100 & 28 & 0.329411765 & 0 & 0 & 0 & 0 \\
\hline GSK3B(h) & 75 & 105 & 100 & 29 & 0.341176471 & 0 & 0 & 0 & 0 \\
\hline IGF-IR(h) & 124 & 105 & 100 & 30 & 0.352941176 & 0 & 0 & 0 & 0 \\
\hline IKKa(h) & 99 & 105 & 100 & 31 & 0.364705882 & 0 & 0 & 0 & 0 \\
\hline $\mathrm{IKKB}(\mathrm{h})$ & 104 & 105 & 100 & 32 & 0.376470588 & 0 & 0 & 0 & 0 \\
\hline $\operatorname{IR}(\mathrm{h})$ & 93 & 104 & 100 & 33 & 0.388235294 & 0 & 0 & 0 & 0 \\
\hline JNKIal(h) & 101 & 104 & 100 & 34 & 0.4 & 0 & 0 & 0 & 0 \\
\hline JNK202(h) & 92 & 104 & 100 & 35 & 0.411764706 & 0 & 0 & 0 & 0 \\
\hline JNK3(r) & 87 & 104 & 100 & 36 & 0.423529412 & 0 & 0 & 0 & 0 \\
\hline Lck(h) & 119 & 104 & 100 & 37 & 0.435294118 & 0 & 0 & 0 & 0 \\
\hline Lyn(h) & 96 & 103 & 100 & 38 & 0.447058824 & 0 & 0 & 0 & 0 \\
\hline Lyn(m) & 91 & 102 & 100 & 39 & 0.458823529 & 0 & 0 & 0 & 0 \\
\hline MAPK I(h) & 98 & 102 & 100 & 40 & 0.470588235 & 0 & 0 & 0 & 0 \\
\hline MAPK2(h) & 120 & 101 & 100 & 41 & 0.482352941 & 0 & 0 & 0 & 0 \\
\hline MAPK2(m) & 123 & 101 & 100 & 42 & 0.494117647 & 0 & 0 & 0 & 0 \\
\hline MAPKAP-K2(h) & 110 & 101 & 100 & 43 & 0.505882353 & 0 & 0 & 0 & 0 \\
\hline MEKI(h) & 25 & 101 & 100 & 44 & 0.517647059 & 0 & 0 & 0 & 0 \\
\hline MKK4(m) & 116 & 101 & 100 & 45 & 0.529411765 & 0 & 0 & 0 & 0 \\
\hline MKK6(h) & 104 & 100 & 100 & 46 & 0.541176471 & 0 & 0 & 0 & 0 \\
\hline МKK 7 (h) & 108 & 100 & 100 & 47 & 0.552941176 & 0 & 0 & 0 & 0 \\
\hline MSK1(h) & 104 & 100 & 100 & 48 & 0.564705882 & 0 & 0 & 0 & 0 \\
\hline $\mathrm{p} 70 \mathrm{~S} 6 \mathrm{~K}(\mathrm{~h})$ & 101 & 100 & 100 & 49 & 0.576470588 & 0 & 0 & 0 & 0 \\
\hline PAK2(h) & 107 & 99 & 99 & 50 & 0.588235294 & 1 & 0.002923977 & 0.002923977 & $1.71999 \mathrm{E}-05$ \\
\hline PDGFRa(h) & 96 & 99 & 99 & 51 & 0.6 & 1 & 0.002923977 & 0.005847953 & $5.15996 \mathrm{E}-05$ \\
\hline PDGFRB(h) & 107 & 99 & 99 & 52 & 0.611764706 & 1 & 0.002923977 & 0.00877193 & $8.59993 \mathrm{E}-05$ \\
\hline PDKI(h) & 97 & 98 & 98 & 53 & 0.623529412 & 2 & 0.005847953 & 0.014619883 & 0.000137599 \\
\hline PKA(b) & 117 & 98 & 98 & 54 & 0.635294118 & 2 & 0.005847953 & 0.020467836 & 0.000206398 \\
\hline PKA(h) & 92 & 98 & 98 & 55 & 0.647058824 & 2 & 0.005847953 & 0.026315789 & 0.000275198 \\
\hline PKBa(h) & 97 & 98 & 98 & 56 & 0.658823529 & 2 & 0.005847953 & 0.032163743 & 0.000343997 \\
\hline PKBB(h) & 132 & 97 & 97 & 57 & 0.670588235 & 3 & 0.00877193 & 0.040935673 & 0.000429997 \\
\hline $\mathrm{PKB}_{\gamma}(\mathrm{h})$ & 87 & 97 & 97 & 58 & 0.682352941 & 3 & 0.00877193 & 0.049707602 & 0.000533196 \\
\hline PKGa(h) & 94 & 97 & 97 & 59 & 0.694117647 & 3 & 0.00877193 & 0.058479532 & 0.000636395 \\
\hline PKCBII(h) & 110 & 97 & 97 & 60 & 0.705882353 & 3 & 0.00877193 & 0.067251462 & 0.000739594 \\
\hline PKCy(h) & 91 & 96 & 96 & 61 & 0.717647059 & 4 & 0.011695906 & 0.078947368 & 0.000859993 \\
\hline PKG(h) & 96 & 96 & 96 & 62 & 0.729411765 & 4 & 0.011695906 & 0.090643275 & 0.000997592 \\
\hline $\mathrm{PKC}$ (h) & 100 & 96 & 96 & 63 & 0.741176471 & 4 & 0.011695906 & 0.102339181 & 0.001135191 \\
\hline PKGYh(h) & 91 & 96 & 96 & 64 & 0.752941176 & 4 & 0.011695906 & 0.114035088 & 0.00127279 \\
\hline PKC, (h) & 101 & 95 & 95 & 65 & 0.764705882 & 5 & 0.014619883 & 0.128654971 & 0.001427589 \\
\hline PKC $\mu(\mathrm{h})$ & 96 & 95 & 95 & 66 & 0.776470588 & 5 & 0.014619883 & 0.143274854 & 0.001599587 \\
\hline PKC (h) & 97 & 94 & 94 & 67 & 0.788235294 & 6 & 0.01754386 & 0.160818713 & 0.001788786 \\
\hline PKD2(h) & 102 & 94 & 94 & 68 & 0.8 & 6 & 0.01754386 & 0.178362573 & 0.001995184 \\
\hline PRAK(h) & 112 & 93 & 93 & 69 & 0.811764706 & 7 & 0.020467836 & 0.198830409 & 0.002218782 \\
\hline PRK2(h) & 92 & 93 & 93 & 70 & 0.823529412 & 7 & 0.020467836 & 0.219298246 & 0.00245958 \\
\hline ROCK-II(h) & 98 & 93 & 93 & 71 & 0.835294118 & 7 & 0.020467836 & 0.239766082 & 0.002700378 \\
\hline ROCK-II(r) & 83 & 92 & 92 & 72 & 0.847058824 & 8 & 0.023391813 & 0.263157895 & 0.002958376 \\
\hline Rskl(h) & 111 & 92 & 92 & 73 & 0.858823529 & 8 & 0.023391813 & 0.286549708 & 0.003233574 \\
\hline Rskl(r) & 67 & 92 & 92 & 74 & 0.870588235 & 8 & 0.023391813 & 0.30994152 & 0.003508772 \\
\hline Rsk2(h) & 93 & 91 & 91 & 75 & 0.882352941 & 9 & 0.026315789 & 0.33625731 & 0.00380117 \\
\hline Rsk3(h) & 99 & 91 & 91 & 76 & 0.894117647 & 9 & 0.026315789 & 0.362573099 & 0.004110767 \\
\hline SAPK2a(h) & 105 & 91 & 91 & 77 & 0.905882353 & 9 & 0.026315789 & 0.388888889 & 0.004420365 \\
\hline SAPK2b(h) & 100 & 91 & 91 & 78 & 0.917647059 & 9 & 0.026315789 & 0.415204678 & 0.004729962 \\
\hline SAPK $3(\mathrm{~h})$ & 112 & 87 & 87 & 79 & 0.929411765 & 13 & 0.038011696 & 0.453216374 & 0.005108359 \\
\hline SAPK 4 (h) & 112 & 87 & 87 & 80 & 0.941176471 & 13 & 0.038011696 & 0.49122807 & 0.005555556 \\
\hline SGK(h) & 104 & 83 & 83 & 81 & 0.952941176 & 17 & 0.049707602 & 0.540935673 & 0.006071551 \\
\hline Syk(h) & 105 & 76 & 76 & 82 & 0.964705882 & 24 & 0.070175439 & 0.611111111 & 0.006776746 \\
\hline TrkB(h) & 111 & 75 & 75 & 83 & 0.976470588 & 25 & 0.073099415 & 0.684210526 & 0.007619539 \\
\hline Yes(h) & 109 & 67 & 67 & 84 & 0.988235294 & 33 & 0.096491228 & 0.780701754 & 0.008617131 \\
\hline ZAP-70(h) & 106 & 25 & 25 & 85 & 1 & 75 & 0.219298246 & 1 & 0.010474716 \\
\hline & & & & & Total inhibition & 342 & & Total area & 0.098899209 \\
\hline & & & & & & & & Gini & 0.802201582 \\
\hline
\end{tabular}


PD184352; $10 \mu \mathrm{M} ; 10 \mu \mathrm{M}$ ATP; sample of 80 kinases

\begin{tabular}{|c|c|c|c|c|c|c|c|c|}
\hline position & inhibition\% & rand & cumulative sample fraction & sample inhibition & sorted & fraction of inhibition & cumulative fraction of inhibition & area \\
\hline 1 & 0 & 0.412978523 & 0.0125 & 0 & 0 & 0 & 0 & 0 \\
\hline 2 & 0 & 0.82726357 & 0.025 & 0 & 0 & 0 & 0 & 0 \\
\hline $\begin{array}{l}3 \\
4\end{array}$ & $\begin{array}{l}7 \\
9\end{array}$ & $\begin{array}{l}0.515372326 \\
0.038765372\end{array}$ & $\begin{array}{c}0.0375 \\
0.05\end{array}$ & $\begin{array}{l}7 \\
9\end{array}$ & $\begin{array}{l}0 \\
0\end{array}$ & $\begin{array}{l}0 \\
0\end{array}$ & $\begin{array}{l}0 \\
0\end{array}$ & $\begin{array}{l}0 \\
0\end{array}$ \\
\hline 5 & 0 & 0.265396552 & 0.0625 & 0 & 0 & 0 & 0 & 0 \\
\hline 6 & 0 & 0.597864772 & 0.075 & 0 & 0 & 0 & 0 & 0 \\
\hline 7 & 0 & 0.758660253 & 0.0875 & 0 & 0 & 0 & 0 & 0 \\
\hline 8 & 0 & 0.586357964 & 0.1 & 0 & 0 & 0 & 0 & 0 \\
\hline 9 & 4 & 0.257230189 & 0.1125 & 4 & 0 & 0 & 0 & 0 \\
\hline 10 & 0 & 0.509743099 & 0.125 & 0 & 0 & 0 & 0 & 0 \\
\hline 11 & 0 & 0.065457061 & 0.1375 & 0 & 0 & 0 & 0 & 0 \\
\hline 12 & 0 & 0.60138962 & 0.15 & 0 & 0 & 0 & 0 & 0 \\
\hline 13 & 0 & 0.385545293 & 0.1625 & 0 & 0 & 0 & 0 & 0 \\
\hline 14 & 7 & 0.434944463 & 0.175 & 7 & 0 & 0 & 0 & 0 \\
\hline 15 & 0 & 0.890100791 & 0.1875 & 0 & 0 & 0 & 0 & 0 \\
\hline 16 & 33 & 0.812966858 & 0.2 & 33 & 0 & 0 & 0 & 0 \\
\hline 17 & 8 & 0.440007324 & 0.2125 & 8 & 0 & 0 & 0 & 0 \\
\hline 18 & 3 & 0.548999876 & 0.225 & 3 & 0 & 0 & 0 & 0 \\
\hline 19 & 13 & 0.239797236 & 0.2375 & 13 & 0 & 0 & 0 & 0 \\
\hline $\begin{array}{l}20 \\
21\end{array}$ & $\begin{array}{l}0 \\
0\end{array}$ & $\begin{array}{r}0.84181015 \\
0.253104804\end{array}$ & $\begin{array}{c}0.25 \\
0.2625\end{array}$ & $\begin{array}{l}0 \\
0\end{array}$ & $\begin{array}{l}0 \\
0\end{array}$ & $\begin{array}{l}0 \\
0\end{array}$ & $\begin{array}{l}0 \\
0\end{array}$ & $\begin{array}{l}0 \\
0\end{array}$ \\
\hline 22 & 0 & 0.746078438 & 0.275 & 0 & 0 & 0 & 0 & 0 \\
\hline 23 & 75 & 0.552269994 & 0.2875 & 75 & 0 & 0 & 0 & 0 \\
\hline 24 & 2 & 0.033630825 & 0.3 & 2 & 0 & 0 & 0 & 0 \\
\hline 25 & 0 & 0.67285856 & 0.3125 & 0 & 0 & 0 & 0 & 0 \\
\hline 26 & 0 & 0.427552475 & 0.325 & 0 & 0 & 0 & 0 & 0 \\
\hline 27 & 0 & 0.085408245 & 0.3375 & 0 & 0 & 0 & 0 & 0 \\
\hline 28 & 7 & 0.539439792 & 0.35 & 7 & 0 & 0 & 0 & 0 \\
\hline 29 & 0 & 0.981662745 & 0.3625 & 0 & 0 & 0 & 0 & 0 \\
\hline 30 & 6 & 0.641035219 & 0.375 & 6 & 0 & 0 & 0 & 0 \\
\hline 31 & 0 & 0.636364672 & 0.3875 & 0 & 0 & 0 & 0 & 0 \\
\hline 32 & 5 & 0.34464123 & 0.4 & 5 & 0 & 0 & 0 & 0 \\
\hline 33 & 0 & 0.862354928 & 0.4125 & 0 & 0 & 0 & 0 & 0 \\
\hline 34 & 0 & 0.135520965 & 0.425 & 0 & 0 & 0 & 0 & 0 \\
\hline 35 & 0 & 0.463017966 & 0.4375 & 0 & 0 & 0 & 0 & 0 \\
\hline 36 & 17 & 0.647962295 & 0.45 & 17 & 0 & 0 & 0 & 0 \\
\hline 37 & 4 & 0.178159999 & 0.4625 & 4 & 0 & 0 & 0 & 0 \\
\hline 38 & 0 & 0.176716326 & 0.475 & 0 & 0 & 0 & 0 & 0 \\
\hline 39 & 0 & 0.500848977 & 0.4875 & 0 & 0 & 0 & 0 & $\begin{array}{l}0 \\
0\end{array}$ \\
\hline 40 & 24 & $\begin{array}{l}0.581851868 \\
0.538287553\end{array}$ & $\begin{array}{l}0.5 \\
0.5125\end{array}$ & $\begin{array}{r}24 \\
0\end{array}$ & $\begin{array}{l}0 \\
0\end{array}$ & $\begin{array}{l}0 \\
0\end{array}$ & $\begin{array}{l}0 \\
0\end{array}$ & 0 \\
\hline $\begin{array}{l}41 \\
42\end{array}$ & $\begin{array}{l}0 \\
9\end{array}$ & $\begin{array}{l}0.53828 / 553 \\
0.892021603\end{array}$ & $\begin{array}{l}0.5125 \\
0.525\end{array}$ & $\begin{array}{l}0 \\
9\end{array}$ & $\begin{array}{l}0 \\
0\end{array}$ & $\begin{array}{l}0 \\
0\end{array}$ & $\begin{array}{l}0 \\
0\end{array}$ & 0 \\
\hline 43 & 3 & 0.578365298 & 0.5375 & 3 & 0 & 0 & 0 & 0 \\
\hline 44 & 1 & 0.743659059 & 0.55 & 1 & 0 & 0 & 0 & 0 \\
\hline 45 & 0 & 0.168636609 & 0.5625 & 0 & 0 & 0 & 0 & 0 \\
\hline 46 & 0 & 0.983813476 & 0.575 & 0 & 0 & 0 & 0 & 0 \\
\hline 47 & 0 & 0.325973778 & 0.5875 & 0 & 1 & 0.002985075 & 0.002985075 & $1.87 \mathrm{E}-05$ \\
\hline 48 & 0 & 0.608885301 & 0.6 & 0 & 1 & 0.002985075 & 0.005970149 & $5.6 \mathrm{E}-05$ \\
\hline 49 & 0 & 0.065560637 & 0.6125 & 0 & 1 & 0.002985075 & 0.008955224 & $9.33 \mathrm{E}-05$ \\
\hline 50 & 0 & 0.711054011 & 0.625 & 0 & 2 & 0.005970149 & 0.014925373 & 0.000149 \\
\hline 51 & 25 & 0.970227373 & 0.6375 & 25 & 2 & 0.005970149 & 0.020895522 & 0.000224 \\
\hline 52 & 0 & 0.602450602 & 0.65 & 0 & 2 & 0.005970149 & 0.026865672 & 0.000299 \\
\hline $\begin{array}{l}53 \\
54\end{array}$ & $\begin{array}{l}0 \\
2\end{array}$ & $\begin{array}{l}0.300139322 \\
0.459394254\end{array}$ & $\begin{array}{c}0.6625 \\
0.675\end{array}$ & $\begin{array}{l}0 \\
2\end{array}$ & $\begin{array}{l}3 \\
3\end{array}$ & $\begin{array}{l}0.008955224 \\
0.008955224\end{array}$ & $\begin{array}{l}0.035820896 \\
0.044776119\end{array}$ & $\begin{array}{l}0.000392 \\
0.000504\end{array}$ \\
\hline 55 & 0 & 0.994001453 & 0.6875 & 0 & 3 & 0.008955224 & 0.053731343 & 0.000616 \\
\hline 56 & 3 & 0.843034058 & 0.7 & 3 & 3 & 0.008955224 & 0.062686567 & 0.000728 \\
\hline 57 & 2 & 0.353902007 & 0.7125 & 2 & 4 & 0.011940299 & 0.074626866 & 0.000858 \\
\hline 58 & 0 & 0.562871223 & 0.725 & 0 & 4 & 0.011940299 & 0.086567164 & 0.001007 \\
\hline 59 & 0 & 0.815964915 & 0.7375 & 0 & 4 & 0.011940299 & 0.098507463 & 0.001157 \\
\hline 60 & 1 & 0.150863488 & 0.75 & 1 & 4 & 0.011940299 & 0.110447761 & 0.001306 \\
\hline 61 & 8 & 0.943522595 & 0.7625 & 8 & 5 & 0.014925373 & 0.125373134 & 0.001474 \\
\hline 62 & 0 & 0.036631983 & 0.775 & 0 & 6 & 0.017910448 & 0.143283582 & 0.001679 \\
\hline 63 & 3 & 0.471621229 & 0.7875 & 3 & 6 & 0.017910448 & 0.16119403 & 0.001903 \\
\hline 64 & 0 & 0.901151726 & 0.8 & 0 & 7 & 0.020895522 & 0.182089552 & 0.002146 \\
\hline 65 & 0 & 0.674662154 & 0.8125 & 0 & 7 & 0.020895522 & 0.202985075 & 0.002407 \\
\hline 66 & 8 & 0.051991507 & 0.825 & 8 & 7 & 0.020895522 & 0.223880597 & 0.002668 \\
\hline 67 & 0 & 0.085380951 & 0.8375 & 0 & 8 & 0.023880597 & 0.247761194 & 0.002948 \\
\hline 68 & 0 & 0.538137459 & 0.85 & 0 & 8 & 0.023880597 & 0.271641791 & 0.003246 \\
\hline 69 & 4 & 0.201635503 & 0.8625 & 4 & 8 & 0.023880597 & 0.295522388 & 0.003545 \\
\hline 70 & 1 & 0.760918663 & 0.875 & 1 & 9 & 0.026865672 & 0.32238806 & 0.003862 \\
\hline 71 & 0 & 0.961491361 & 0.8875 & 0 & 9 & 0.026865672 & 0.349253731 & 0.004198 \\
\hline 72 & 9 & 0.996711186 & 0.9 & 9 & 9 & 0.026865672 & 0.376119403 & 0.004534 \\
\hline 73 & 9 & 0.065913547 & 0.9125 & 9 & 9 & 0.026865672 & 0.402985075 & 0.004869 \\
\hline 74 & 6 & 0.719764044 & 0.925 & 6 & 13 & 0.03880597 & 0.441791045 & 0.00528 \\
\hline 75 & 0 & 0.292369661 & 0.9375 & 0 & 13 & 0.03880597 & 0.480597015 & 0.005765 \\
\hline 76 & 4 & 0.419212535 & 0.95 & 4 & 17 & 0.050746269 & 0.531343284 & 0.006325 \\
\hline 77 & 13 & 0.371995609 & 0.9625 & 13 & 24 & 0.071641791 & 0.602985075 & 0.00709 \\
\hline 78 & 0 & 0.970419409 & 0.975 & 0 & 25 & 0.074626866 & 0.67761194 & 0.008004 \\
\hline 79 & 0 & 0.161886531 & 0.9875 & 0 & 33 & 0.098507463 & 0.776119403 & 0.009086 \\
\hline 80 & 0 & 0.469937272 & 1 & 0 & 75 & 0.223880597 & 1 & 0.011101 \\
\hline 81 & 0 & 0.067378232 & & & & & & \\
\hline 82 & 2 & 0.81775136 & & total inhibition & 335 & & Total area & 0.099534 \\
\hline 83 & 0 & 0.852430875 & & & & & & \\
\hline
\end{tabular}

0.452063424

Gini $\quad 0.800933$

0.78983918

0.80789877

0.80826923

0.80261976

0.79902695

0.80502266

0.81366099

0.76165414

0.78983918

0.80502266

$\begin{array}{r}0.80502266 \\ \hline\end{array}$

$\begin{array}{rr}\text { mean } & 0.799113415 \\ \text { st dev } & 0.013153561\end{array}$ 
PD184352; $10 \mu \mathrm{M} ; 10 \mu \mathrm{M}$ ATP; sample of 60 kinases

\begin{tabular}{|c|c|c|c|c|c|c|c|c|c|}
\hline position & inhibition\% & rand & cumulative sample fraction & sample inhibition & sorted & & fraction of inhibition & cumulative fraction of inhibition & area \\
\hline 1 & 0 & 0.083175166 & 0.016666667 & & 0 & 0 & 0 & 0 & 0 \\
\hline 2 & 9 & 0.332699806 & 0.033333333 & & 9 & 0 & 0 & 0 & 0 \\
\hline $\begin{array}{l}3 \\
4\end{array}$ & $\begin{array}{l}0 \\
0\end{array}$ & 0.846929214 & 0.05 & & 0 & $\begin{array}{l}0 \\
0\end{array}$ & 0 & 0 & $\begin{array}{l}0 \\
0\end{array}$ \\
\hline $\begin{array}{l}4 \\
5\end{array}$ & $\begin{array}{l}0 \\
0\end{array}$ & $\begin{array}{c}0.167762972 \\
0.68133901\end{array}$ & $\begin{array}{l}0.066666667 \\
0.083333333\end{array}$ & & $\begin{array}{l}0 \\
0\end{array}$ & $\begin{array}{l}0 \\
0\end{array}$ & $\begin{array}{l}0 \\
0\end{array}$ & $\begin{array}{l}0 \\
0\end{array}$ & $\begin{array}{l}0 \\
0\end{array}$ \\
\hline $\begin{array}{l}5 \\
6\end{array}$ & 0 & 0.800091116 & 0.1 & & 0 & 0 & $\begin{array}{l}0 \\
0\end{array}$ & $\begin{array}{l}0 \\
0\end{array}$ & 0 \\
\hline 7 & 0 & 0.746233271 & 0.116666667 & & 0 & 0 & 0 & 0 & 0 \\
\hline 8 & 0 & 0.484216711 & 0.133333333 & & 0 & 0 & 0 & 0 & 0 \\
\hline 9 & 0 & 0.123904194 & 0.15 & & 0 & 0 & 0 & 0 & 0 \\
\hline 10 & 4 & 0.402980312 & 0.166666667 & & 4 & 0 & 0 & 0 & 0 \\
\hline 11 & 0 & 0.695270444 & 0.183333333 & & 0 & 0 & 0 & 0 & 0 \\
\hline 12 & 0 & 0.701729836 & 0.2 & & 0 & 0 & 0 & 0 & 0 \\
\hline 13 & 8 & 0.123251553 & 0.216666667 & & 8 & 0 & 0 & 0 & 0 \\
\hline 14 & 0 & 0.073489899 & 0.233333333 & & 0 & 0 & 0 & 0 & 0 \\
\hline 15 & 0 & 0.165090345 & 0.25 & & 0 & 0 & 0 & 0 & 0 \\
\hline 16 & 0 & 0.931728555 & 0.266666667 & & 0 & 0 & 0 & 0 & 0 \\
\hline 17 & 0 & 0.770216412 & 0.283333333 & & 0 & 0 & 0 & 0 & 0 \\
\hline 18 & 6 & 0.873296793 & 0.3 & & 6 & 0 & 0 & 0 & 0 \\
\hline 19 & 0 & 0.947176096 & 0.316666667 & & 0 & 0 & 0 & 0 & 0 \\
\hline 20 & 7 & 0.262419802 & 0.333333333 & & 7 & 0 & 0 & 0 & 0 \\
\hline 21 & 0 & 0.384518079 & 0.35 & & 0 & 0 & 0 & 0 & 0 \\
\hline 22 & 0 & 0.103768608 & 0.366666667 & & 0 & 0 & 0 & 0 & 0 \\
\hline 23 & 0 & 0.996866036 & 0.383333333 & & 0 & 0 & 0 & 0 & 0 \\
\hline 24 & 0 & 0.382167196 & 0.4 & & 0 & 0 & 0 & 0 & 0 \\
\hline 25 & 7 & 0.333125906 & 0.416666667 & & 7 & 0 & 0 & 0 & 0 \\
\hline 26 & 0 & 0.951746154 & 0.433333333 & & 0 & 0 & 0 & 0 & 0 \\
\hline 27 & 0 & 0.933592874 & 0.45 & & 0 & 0 & 0 & 0 & 0 \\
\hline 28 & 13 & 0.574718961 & 0.466666667 & & 13 & 0 & 0 & 0 & 0 \\
\hline 29 & 9 & 0.171653294 & 0.483333333 & & 9 & 0 & 0 & 0 & 0 \\
\hline 30 & 5 & 0.217847007 & 0.5 & & 5 & 0 & 0 & 0 & 0 \\
\hline 31 & 0 & 0.896432097 & 0.516666667 & & 0 & 0 & 0 & 0 & 0 \\
\hline 32 & 0 & 0.073313838 & 0.533333333 & & 0 & 0 & 0 & 0 & 0 \\
\hline 33 & 33 & 0.195409114 & 0.55 & & 33 & 0 & 0 & 0 & 0 \\
\hline 34 & 0 & 0.292048225 & 0.566666667 & & 0 & 0 & 0 & 0 & $\begin{array}{r}0 \\
29 E-05\end{array}$ \\
\hline 35 & 0 & 0.590381512 & 0.583333333 & & 0 & 1 & 0.003484321 & 0.003484321 & $\begin{array}{r}2.9 \mathrm{E}-05 \\
0.715-0\end{array}$ \\
\hline 36 & 0 & 0.283874068 & $\begin{array}{l}0.6 \\
0.6767\end{array}$ & & 0 & 1 & 0.003484321 & 0.006968641 & $\begin{array}{l}8.71 \mathrm{E}-05 \\
0.000145\end{array}$ \\
\hline $\begin{array}{l}37 \\
38\end{array}$ & $\begin{array}{l}2 \\
3\end{array}$ & $\begin{array}{l}0.001240722 \\
0.726031236\end{array}$ & $\begin{array}{l}0.616666667 \\
0.633333333\end{array}$ & & $\begin{array}{l}2 \\
3\end{array}$ & $\begin{array}{l}1 \\
2\end{array}$ & $\begin{array}{l}0.003484321 \\
0.006968641\end{array}$ & $\begin{array}{l}0.010452962 \\
0.017421603\end{array}$ & $\begin{array}{l}0.000145 \\
0.000232\end{array}$ \\
\hline 39 & 1 & 0.147323082 & 0.65 & & 1 & 2 & 0.006968641 & 0.024390244 & 0.000348 \\
\hline 40 & 1 & 0.787719248 & 0.666666667 & & 1 & 3 & 0.010452962 & 0.034843206 & 0.000494 \\
\hline 41 & 0 & 0.327976192 & 0.683333333 & & 0 & 3 & 0.010452962 & 0.045296167 & 0.000668 \\
\hline 42 & 3 & 0.452311962 & 0.7 & & 3 & 3 & 0.010452962 & 0.055749129 & 0.000842 \\
\hline 43 & 17 & 0.051851516 & 0.716666667 & & 17 & 4 & 0.013937282 & 0.069686411 & 0.001045 \\
\hline 44 & 0 & 0.932351573 & 0.733333333 & & 0 & 4 & 0.013937282 & 0.083623693 & 0.001278 \\
\hline 45 & 2 & 0.600344909 & 0.75 & & 2 & 5 & 0.017421603 & 0.101045296 & 0.001539 \\
\hline 46 & 25 & 0.797185748 & 0.766666667 & & 25 & 6 & 0.020905923 & 0.12195122 & 0.001858 \\
\hline 47 & 3 & 0.439008474 & 0.783333333 & & 3 & 7 & 0.024390244 & 0.146341463 & 0.002236 \\
\hline 48 & 0 & 0.088540341 & 0.8 & & 0 & 7 & 0.024390244 & 0.170731707 & 0.002642 \\
\hline 49 & 9 & 0.093947367 & 0.816666667 & & 9 & 7 & 0.024390244 & 0.195121951 & 0.003049 \\
\hline 50 & 0 & 0.177460456 & 0.833333333 & & 0 & 8 & 0.027874564 & 0.222996516 & 0.003484 \\
\hline 51 & 0 & 0.71111647 & 0.85 & & 0 & 9 & 0.031358885 & 0.254355401 & 0.003978 \\
\hline 52 & 24 & 0.744542546 & 0.866666667 & & 24 & 9 & 0.031358885 & 0.285714286 & 0.004501 \\
\hline $\begin{array}{l}53 \\
54\end{array}$ & $\begin{array}{l}9 \\
0\end{array}$ & $\begin{array}{l}0.844695316 \\
0.654014781\end{array}$ & $\begin{array}{c}0.883333333 \\
0.9\end{array}$ & & $\begin{array}{l}9 \\
0\end{array}$ & $\begin{array}{l}9 \\
9\end{array}$ & $\begin{array}{l}0.031358885 \\
0.031358885\end{array}$ & $\begin{array}{l}0.317073171 \\
0.348432056\end{array}$ & 0.005023 \\
\hline 54 & & 0.654014781 & 0.9 & & & 9 & 0.031358885 & 0.348432056 & 0.005546 \\
\hline 55 & 75 & 0.628727018 & 0.916666667 & & 75 & 13 & 0.045296167 & 0.393728223 & 0.006185 \\
\hline 56 & 4 & 0.084544834 & 0.933333333 & & 4 & 17 & 0.059233449 & 0.452961672 & 0.007056 \\
\hline 57 & 0 & 0.735691371 & 0.95 & & 0 & 24 & 0.083623693 & 0.536585366 & 0.008246 \\
\hline 58 & 0 & 0.812833059 & 0.966666667 & & 0 & 25 & 0.087108014 & 0.62369338 & 0.009669 \\
\hline 59 & 7 & 0.02143798 & 0.983333333 & & 7 & 33 & 0.114982578 & 0.738675958 & 0.011353 \\
\hline 60 & 1 & 0.033371299 & 1 & & 1 & 75 & 0.261324042 & 1 & 0.014489 \\
\hline 61 & 0 & 0.838705071 & & & & & & & \\
\hline 62 & 0 & 0.076015927 & & & & & & & \\
\hline 63 & 0 & 0.055112602 & & & & & & & \\
\hline 64 & 0 & 0.446210397 & & & & & & & \\
\hline 65 & 0 & 0.544675936 & & & & & & & \\
\hline 66 & 2 & 0.246667993 & & & & & & & \\
\hline 67 & 4 & 0.704310381 & & & & & & & \\
\hline 68 & 0 & 0.306148662 & & & & & & & \\
\hline 69 & 0 & 0.629155799 & & & & & & & \\
\hline $\begin{array}{l}70 \\
71\end{array}$ & 0 & 0.462907702 & & & & & & & \\
\hline $\begin{array}{l}11 \\
72\end{array}$ & $\begin{array}{l}0 \\
2\end{array}$ & $\begin{array}{l}0.686983275 \\
0.232284607\end{array}$ & & & & & & & \\
\hline 73 & 0 & 0.240373246 & & & & & & & \\
\hline 74 & 8 & 0.412709608 & & & & & & & \\
\hline 75 & 0 & 0.972116889 & & & & & & & \\
\hline 76 & 13 & 0.396699836 & & & & & & & \\
\hline 77 & 0 & 0.563641866 & & & & & & & \\
\hline 78 & 0 & 0.22205718 & & & & & & & \\
\hline 79 & 5 & 0.142833496 & & & & & & & \\
\hline 80 & 8 & 0.628879001 & & & & & & & \\
\hline 81 & 0 & 0.577220536 & & & & & & & \\
\hline 82 & 4 & $\begin{array}{r}0.480931585 \\
\end{array}$ & & total inhibition & & 287 & & Total area & 0.096022 \\
\hline $\begin{array}{l}83 \\
84\end{array}$ & $\begin{array}{l}0 \\
3\end{array}$ & $\begin{array}{l}0.797805614 \\
0.855917645\end{array}$ & & & & & & Gini & 0.807956 \\
\hline 84 & & & & & & & & & \\
\hline
\end{tabular}

$\begin{array}{r}\multicolumn{1}{c}{\text { Gini }} \\ \hline 0.8262963 \\ 0.77997602 \\ 0.8226776 \\ 0.75248227 \\ 0.80500589 \\ 0.7980226 \\ 0.81392694 \\ 0.79971559 \\ 0.76098696 \\ 0.8298103 \\ 0.82312661 \\ 0.8030914 \\ 0.80795587 \\ \hline\end{array}$

$\begin{array}{ll}\text { mean } & 0.801774949 \\ \text { st dev } & 0.024264309\end{array}$ 
PD184352; $10 \mu \mathrm{M} ; 10 \mu \mathrm{M}$ ATP; sample of 40 kinases

\begin{tabular}{|c|c|c|c|c|c|c|c|c|c|}
\hline position & inhibition\% & rand & cumulative sample fraction & sample inhibition & sorted & & fraction of inhibition & cumulative fraction of inhibition & area \\
\hline 1 & 0 & 0.993189897 & 0.025 & & 0 & 0 & 0 & 0 & 0 \\
\hline 2 & 9 & 0.030342844 & 0.05 & & 9 & 0 & 0 & 0 & 0 \\
\hline 3 & 0 & 0.911826371 & 0.075 & & 0 & 0 & 0 & 0 & 0 \\
\hline 4 & 0 & 0.565374719 & 0.1 & & 0 & 0 & 0 & 0 & 0 \\
\hline 5 & 0 & 0.078558881 & 0.125 & & 0 & 0 & 0 & 0 & 0 \\
\hline 6 & 7 & 0.367199334 & 0.15 & & 7 & 0 & 0 & 0 & 0 \\
\hline 7 & 0 & 0.857566559 & 0.175 & & 0 & 0 & 0 & 0 & 0 \\
\hline 8 & 2 & 0.753713856 & 0.2 & & 2 & 0 & 0 & 0 & 0 \\
\hline 9 & 0 & 0.586219665 & 0.225 & & 0 & 0 & 0 & 0 & 0 \\
\hline 10 & 5 & 0.213638634 & 0.25 & & 5 & 0 & 0 & 0 & 0 \\
\hline 11 & 0 & 0.863610848 & 0.275 & & 0 & 0 & 0 & 0 & 0 \\
\hline 12 & 0 & 0.653543357 & 0.3 & & 0 & 0 & 0 & 0 & 0 \\
\hline 13 & 0 & 0.100721726 & 0.325 & & 0 & 0 & 0 & 0 & 0 \\
\hline 14 & 0 & 0.993670044 & 0.35 & & 0 & 0 & 0 & 0 & 0 \\
\hline 15 & 3 & 0.95372548 & 0.375 & & 3 & 0 & 0 & 0 & 0 \\
\hline 16 & 3 & 0.48319424 & 0.4 & & 3 & 0 & 0 & 0 & 0 \\
\hline 17 & 0 & 0.217243761 & 0.425 & & 0 & 0 & 0 & 0 & 0 \\
\hline 18 & 0 & 0.837782195 & 0.45 & & 0 & 0 & 0 & 0 & 0 \\
\hline 19 & 0 & 0.981055604 & 0.475 & & 0 & 0 & 0 & 0 & 0 \\
\hline 20 & 0 & 0.561518504 & 0.5 & & 0 & 0 & 0 & 0 & 0 \\
\hline 21 & 8 & 0.459636175 & 0.525 & & 8 & 0 & 0 & 0 & 0 \\
\hline 22 & 0 & 0.744381979 & 0.55 & & 0 & 0 & 0 & 0 & 0 \\
\hline 23 & 0 & 0.642020618 & 0.575 & & 0 & 0 & 0 & 0 & 0 \\
\hline 24 & 7 & 0.407241939 & 0.6 & & 7 & 0 & 0 & 0 & 0 \\
\hline 25 & 1 & 0.189567925 & 0.625 & & 1 & 0 & 0 & 0 & 0 \\
\hline 26 & 0 & 0.946194394 & 0.65 & & 0 & 1 & 0.009345794 & 0.009345794 & 0.000117 \\
\hline 27 & 4 & 0.457390293 & 0.675 & & 4 & 2 & 0.018691589 & 0.028037383 & 0.000467 \\
\hline 28 & 2 & 0.014846331 & 0.7 & & 2 & 2 & 0.018691589 & 0.046728972 & 0.000935 \\
\hline 29 & 0 & 0.562878619 & 0.725 & & 0 & 3 & 0.028037383 & 0.074766355 & 0.001519 \\
\hline 30 & 6 & 0.196502806 & 0.75 & & 6 & 3 & 0.028037383 & 0.102803738 & 0.00222 \\
\hline 31 & 0 & 0.350029974 & 0.775 & & 0 & 4 & 0.037383178 & 0.140186916 & 0.003037 \\
\hline 32 & 0 & 0.189975909 & 0.8 & & 0 & 5 & 0.046728972 & 0.186915888 & 0.004089 \\
\hline 33 & 9 & 0.050552375 & 0.825 & & 9 & 6 & 0.056074766 & 0.242990654 & 0.005374 \\
\hline 34 & 17 & 0.364124035 & 0.85 & 17 & 17 & 7 & 0.065420561 & 0.308411215 & 0.006893 \\
\hline 35 & 0 & 0.038986554 & 0.875 & & 0 & 7 & 0.065420561 & 0.373831776 & 0.008528 \\
\hline 36 & 0 & 0.284340848 & 0.9 & & 0 & 8 & 0.074766355 & 0.448598131 & 0.01028 \\
\hline 37 & 24 & 0.684766482 & 0.925 & 24 & 24 & 9 & 0.08411215 & 0.53271028 & 0.012266 \\
\hline 38 & 0 & 0.475123894 & 0.95 & & 0 & 9 & 0.08411215 & 0.61682243 & 0.014369 \\
\hline 39 & 0 & 0.464199262 & 0.975 & & 0 & 17 & 0.158878505 & 0.775700935 & 0.017407 \\
\hline 40 & 0 & 0.458871153 & 1 & & 0 & 24 & 0.224299065 & 1 & 0.022196 \\
\hline 41 & 2 & 0.539011308 & & & & & & & \\
\hline 42 & 8 & 0.615780403 & & & & & & & \\
\hline 43 & 3 & 0.78717504 & & & & & & & \\
\hline 44 & 25 & 0.043759939 & & & & & & & \\
\hline 45 & 0 & 0.427494231 & & & & & & & \\
\hline 46 & 4 & 0.511015312 & & & & & & & \\
\hline 47 & 0 & 0.102370886 & & & & & & & \\
\hline 48 & 0 & 0.964645641 & & & & & & & \\
\hline 49 & 0 & 0.176554828 & & & & & & & \\
\hline 50 & 4 & 0.526158785 & & & & & & & \\
\hline 51 & 0 & 0.897894025 & & & & & & & \\
\hline 52 & 0 & 0.061068031 & & & & & & & \\
\hline $\begin{array}{l}53 \\
54\end{array}$ & $\begin{array}{l}0 \\
7\end{array}$ & $\begin{array}{l}0.797982332 \\
0.766017619\end{array}$ & & & & & & & \\
\hline 55 & 0 & 0.50876797 & & & & & & & \\
\hline 56 & 0 & 0.222561841 & & & & & & & \\
\hline 57 & 6 & 0.769305738 & & & & & & & \\
\hline 58 & 8 & 0.988852682 & & & & & & & \\
\hline 59 & 2 & 0.039128403 & & & & & & & \\
\hline 60 & 9 & 0.870520867 & & & & & & & \\
\hline 61 & 0 & 0.46510221 & & & & & & & \\
\hline 62 & 33 & 0.308107609 & & & & & & & \\
\hline 63 & 1 & 0.173721632 & & & & & & & \\
\hline 64 & 0 & 0.49728475 & & & & & & & \\
\hline 65 & 0 & 0.290013122 & & & & & & & \\
\hline 66 & 13 & 0.210835404 & & & & & & & \\
\hline 67 & 0 & 0.801653035 & & & & & & & \\
\hline 68 & 0 & 0.132492136 & & & & & & & \\
\hline 69 & 0 & 0.922023514 & & & & & & & \\
\hline 70 & 3 & 0.274903023 & & & & & & & \\
\hline 71 & 0 & 0.339287091 & & & & & & & \\
\hline 72 & 0 & 0.18104468 & & & & & & & \\
\hline 73 & 75 & 0.856899294 & & & & & & & \\
\hline 74 & 5 & 0.785844381 & & & & & & & \\
\hline 75 & 4 & 0.692822975 & & & & & & & \\
\hline 76 & 9 & 0.511671488 & & & & & & & \\
\hline 77 & 0 & 0.871447875 & & & & & & & \\
\hline 78 & 0 & 0.422559117 & & & & & & & \\
\hline 79 & 1 & 0.777954298 & & & & & & & \\
\hline 80 & 0 & 0.367578266 & & & & & & & \\
\hline 81 & 0 & 0.693141395 & & & & & & & \\
\hline 82 & 13 & 0.990681558 & & total inhibition & & 107 & & Total area & 0.109696 \\
\hline $\begin{array}{l}83 \\
84\end{array}$ & $\begin{array}{l}0 \\
0\end{array}$ & $\begin{array}{l}0.435779044 \\
0.804479341\end{array}$ & & & & & & & 0.780607 \\
\hline $\begin{array}{l}84 \\
85\end{array}$ & $\begin{array}{l}0 \\
0 \\
0\end{array}$ & $\begin{array}{l}0.804479341 \\
0\end{array}$ & & & & & & Gini & i 0.780607 \\
\hline
\end{tabular}


PD184352; $10 \mu \mathrm{M} ; 10 \mu \mathrm{M}$ ATP; sample of 20 kinases

\begin{tabular}{|c|c|c|c|c|c|c|c|c|c|}
\hline position & inhibition\% & rand & cumulative sample fraction & sample inhibition & sorted & & fraction of inhibition & cumulative fraction of inhibition & area \\
\hline $\begin{array}{l}1 \\
2\end{array}$ & $\begin{array}{l}9 \\
0\end{array}$ & $\begin{array}{l}0.551459358 \\
0.748941474\end{array}$ & 0.05 & & 9 & $\begin{array}{l}0 \\
0\end{array}$ & $\begin{array}{l}0 \\
0\end{array}$ & $\begin{array}{l}0 \\
0\end{array}$ & $\begin{array}{l}0 \\
0\end{array}$ \\
\hline $\begin{array}{l}2 \\
3\end{array}$ & 0 & 0.47236357 & 0.15 & & $\begin{array}{l}0 \\
0\end{array}$ & $\begin{array}{l}0 \\
0\end{array}$ & $\begin{array}{l}0 \\
0\end{array}$ & 0 & $\begin{array}{l}0 \\
0\end{array}$ \\
\hline $\begin{array}{l}3 \\
4\end{array}$ & 0 & 0.26131324 & 0.2 & & $\begin{array}{l}0 \\
0\end{array}$ & 0 & $\begin{array}{l}0 \\
0\end{array}$ & $\begin{array}{l}0 \\
0\end{array}$ & $\begin{array}{l}0 \\
0\end{array}$ \\
\hline 5 & 3 & 0.893143244 & 0.25 & & 3 & 0 & 0 & 0 & 0 \\
\hline 6 & 1 & 0.056560183 & 0.3 & & 1 & 0 & 0 & 0 & 0 \\
\hline 7 & 0 & 0.341895184 & 0.35 & & 0 & 0 & 0 & 0 & 0 \\
\hline 8 & 5 & 0.767017741 & 0.4 & & 5 & 0 & 0 & 0 & 0 \\
\hline 9 & 4 & 0.302583606 & 0.45 & & 4 & 0 & 0 & 0 & 0 \\
\hline 10 & 0 & 0.649413816 & 0.5 & & 0 & 0 & 0 & 0 & 0 \\
\hline 11 & 0 & 0.507794859 & 0.55 & & 0 & 0 & 0 & 0 & 0 \\
\hline 12 & 1 & 0.698864338 & 0.6 & & 1 & 0 & 0 & 0 & 0 \\
\hline 13 & 0 & 0.56818997 & 0.65 & & 0 & 1 & 0.027027027 & 0.027027027 & 0.000676 \\
\hline 14 & 0 & 0.355322949 & 0.7 & & 0 & 1 & 0.027027027 & 0.054054054 & 0.002027 \\
\hline 15 & 9 & 0.343590791 & 0.75 & & 9 & 3 & 0.081081081 & 0.135135135 & 0.00473 \\
\hline 16 & 0 & 0.772951441 & 0.8 & & 0 & 4 & 0.108108108 & 0.243243243 & 0.009459 \\
\hline 17 & 0 & 0.95601968 & 0.85 & & 0 & 5 & 0.135135135 & 0.378378378 & 0.015541 \\
\hline 18 & 0 & 0.005677661 & 0.9 & & 0 & 5 & 0.135135135 & 0.513513514 & 0.022297 \\
\hline 19 & 0 & 0.498406259 & 0.95 & & 0 & 9 & 0.243243243 & 0.756756757 & 0.031757 \\
\hline 20 & 5 & 0.28136698 & 1 & & 5 & 9 & 0.243243243 & 1 & 0.043919 \\
\hline $\begin{array}{l}21 \\
22\end{array}$ & $\begin{array}{l}8 \\
2\end{array}$ & $\begin{array}{l}0.747605424 \\
0.621107103\end{array}$ & & & & & & & \\
\hline $\begin{array}{l}22 \\
23\end{array}$ & $\begin{array}{l}2 \\
0\end{array}$ & $\begin{array}{l}0.621107103 \\
0.957073413\end{array}$ & & & & & & & \\
\hline 24 & 1 & 0.624001861 & & & & & & & \\
\hline 25 & 9 & 0.415275092 & & & & & & & \\
\hline 26 & 0 & 0.446256523 & & & & & & & \\
\hline 27 & 3 & 0.768577272 & & & & & & & \\
\hline 28 & 4 & 0.908462457 & & & & & & & \\
\hline 29 & 0 & 0.924209967 & & & & & & & \\
\hline 30 & 7 & 0.561367436 & & & & & & & \\
\hline 31 & 0 & 0.200303838 & & & & & & & \\
\hline 32 & 25 & 0.708698431 & & & & & & & \\
\hline 33 & 0 & 0.447198926 & & & & & & & \\
\hline 34 & 4 & 0.501482541 & & & & & & & \\
\hline $\begin{array}{l}35 \\
36\end{array}$ & $\begin{array}{l}0 \\
0\end{array}$ & $\begin{array}{l}0.782367278 \\
0.953646134\end{array}$ & & & & & & & \\
\hline 37 & 0 & 0.196402782 & & & & & & & \\
\hline 38 & 0 & 0.618218633 & & & & & & & \\
\hline $\begin{array}{l}39 \\
40\end{array}$ & $\begin{array}{c}0 \\
33\end{array}$ & $\begin{array}{l}0.533796274 \\
0.356439255\end{array}$ & & & & & & & \\
\hline 41 & 0 & 0.714641017 & & & & & & & \\
\hline 42 & 0 & 0.884622365 & & & & & & & \\
\hline 43 & 6 & 0.542983223 & & & & & & & \\
\hline 44 & 0 & 0.422987319 & & & & & & & \\
\hline 45 & 8 & 0.189343331 & & & & & & & \\
\hline 46 & 0 & 0.633309828 & & & & & & & \\
\hline 47 & 0 & 0.800404393 & & & & & & & \\
\hline 48 & 2 & 0.594136906 & & & & & & & \\
\hline 49 & 2 & 0.187839832 & & & & & & & \\
\hline 50 & 0 & 0.563922186 & & & & & & & \\
\hline 51 & 0 & 0.741050133 & & & & & & & \\
\hline 52 & 0 & 0.934892222 & & & & & & & \\
\hline $\begin{array}{l}53 \\
54\end{array}$ & $\begin{array}{l}9 \\
0\end{array}$ & $\begin{array}{l}0.651701518 \\
0.562563867\end{array}$ & & & & & & & \\
\hline 55 & 6 & 0.748737753 & & & & & & & \\
\hline 56 & 0 & 0.423917947 & & & & & & & \\
\hline 57 & 7 & 0.176517646 & & & & & & & \\
\hline 58 & 0 & 0.137203931 & & & & & & & \\
\hline 59 & 24 & 0.980238635 & & & & & & & \\
\hline 60 & 0 & 0.115911352 & & & & & & & \\
\hline 61 & 17 & 0.803582533 & & & & & & & \\
\hline 62 & 75 & 0.360169099 & & & & & & & \\
\hline 63 & 13 & 0.309360867 & & & & & & & \\
\hline 64 & 0 & 0.858769575 & & & & & & & \\
\hline 65 & 0 & 0.873498526 & & & & & & & \\
\hline 66 & 0 & 0.507404317 & & & & & & & \\
\hline 67 & 4 & 0.4257405 & & & & & & & \\
\hline 68 & 3 & 0.949817248 & & & & & & & \\
\hline 69 & 8 & 0.514036047 & & & & & & & \\
\hline 70 & 0 & 0.611910253 & & & & & & & \\
\hline 71 & 0 & 0.792132761 & & & & & & & \\
\hline $\begin{array}{l}72 \\
73\end{array}$ & $\begin{array}{l}0 \\
0\end{array}$ & $\begin{array}{c}0.4468681 \\
0.857833105\end{array}$ & & & & & & & \\
\hline 74 & 0 & 0.545694103 & & & & & & & \\
\hline 75 & 7 & 0.680568603 & & & & & & & \\
\hline 76 & 0 & 0.999504717 & & & & & & & \\
\hline 77 & 0 & 0.930869602 & & & & & & & \\
\hline 78 & 0 & 0.609270422 & & & & & & & \\
\hline 79 & 0 & 0.357418246 & & & & & & & \\
\hline 80 & 0 & 0.025520526 & & & & & & & \\
\hline 81 & 0 & 0.922343528 & & & & & & & \\
\hline 82 & 2 & 0.682339181 & & total inhibition & & 37 & & Total area & 0.130405 \\
\hline 83 & 13 & 0.192028406 & & & & & & & \\
\hline $\begin{array}{l}84 \\
85\end{array}$ & $\begin{array}{l}3 \\
0\end{array}$ & $\begin{array}{l}0.432871375 \\
0.528973186\end{array}$ & & & & & & Gini & 0.739189 \\
\hline
\end{tabular}


PD184352; $10 \mu \mathrm{M} ; 10 \mu \mathrm{M}$ ATP; sample of 10 kinases

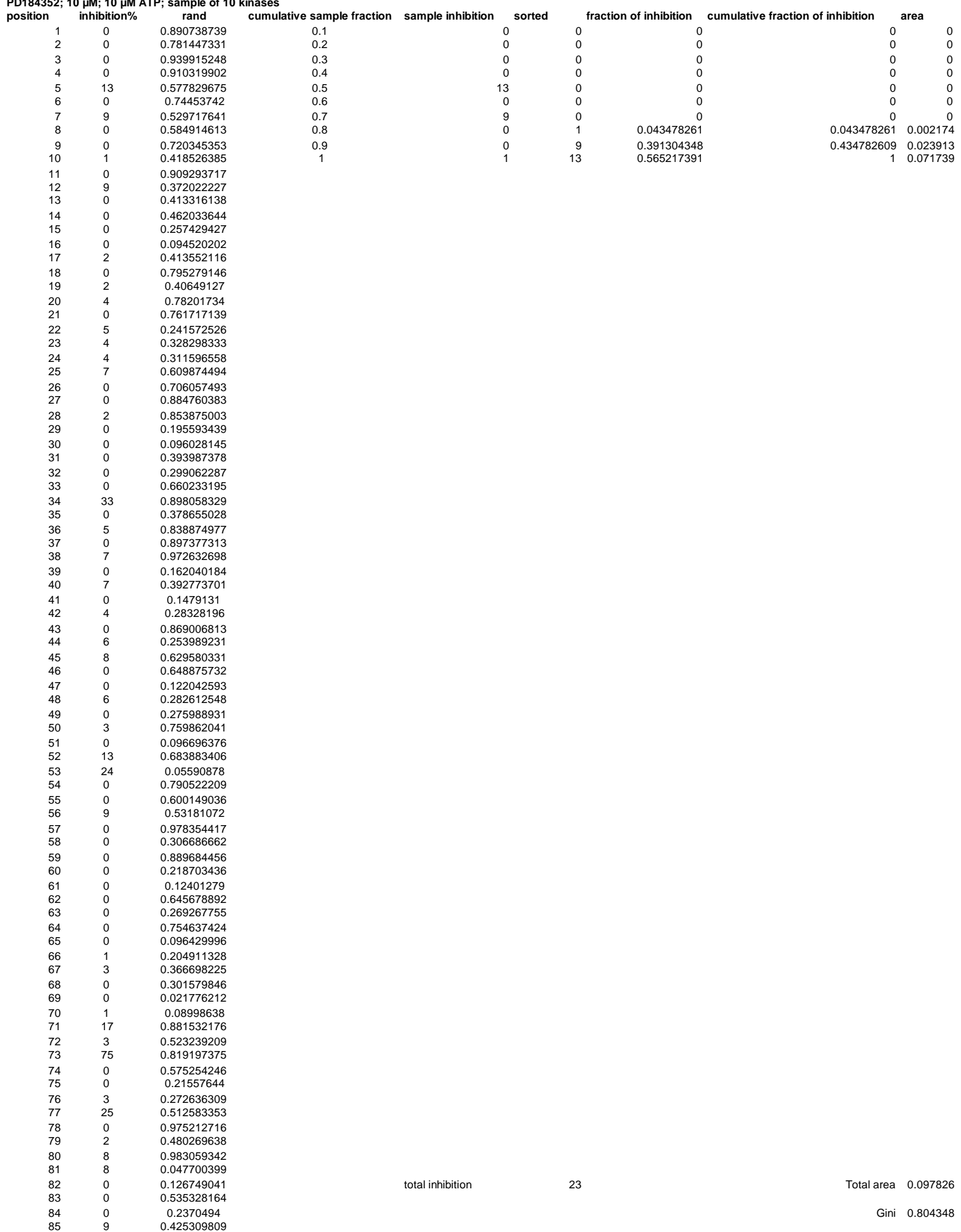

$\begin{array}{ll}\text { mean } & 0.703348894 \\ \text { st dev } & 0.112516724\end{array}$ 


\section{Calculation of the Gini coefficient for subsets of 50 , $100,200,300$ and 400 kinases}

$\begin{array}{ll}\text { Summary }(\text { Table 5) } & \text { Page } \\ \text { AG1024; } 10 \mu \mathrm{M} ; 10 \mu \mathrm{M} \text { ATP } 510 \text { kinases } & \mathrm{S} 107 \\ \text { AG1024; } 10 \mu \mathrm{M} ; 10 \mu \mathrm{M} \text { ATP } 400 \text { kinases } & \mathrm{S} 108-\mathrm{S} 113 \\ \text { AG1024; } 10 \mu \mathrm{M} ; 10 \mu \mathrm{M} \text { ATP } 300 \text { kinases } & \mathrm{S} 114-\mathrm{S} 119 \\ \text { AG1024; } 10 \mu \mathrm{M} ; 10 \mu \mathrm{M} \text { ATP } 200 \text { kinases } & \mathrm{S} 120-\mathrm{S} 125 \\ \text { AG1024; } 10 \mu \mathrm{M} ; 10 \mu \mathrm{M} \text { ATP } 100 \text { kinases } & \mathrm{S} 126-\mathrm{S} 131 \\ \text { AG1024; } 10 \mu \mathrm{M} ; 10 \mu \mathrm{M} \text { ATP } 50 \text { kinases } & \mathrm{S} 132-\mathrm{S} 137 \\ \text { Staurosporine; } 1 \mu \mathrm{M} ; 100 \mu \mathrm{M} \text { ATP } 510 \text { kinases } & \mathrm{S} 138-\mathrm{S} 143 \\ \text { Staurosporine; } 1 \mu \mathrm{M} ; 100 \mu \mathrm{M} \text { ATP } 400 \text { kinases } & \mathrm{S} 144-\mathrm{S} 149 \\ \text { Staurosporine; } 1 \mu \mathrm{M} ; 100 \mu \mathrm{M} \text { ATP } 300 \text { kinases } & \mathrm{S} 150-\mathrm{S} 155 \\ \text { Staurosporine; } 1 \mu \mathrm{M} ; 100 \mu \mathrm{M} \text { ATP } 200 \text { kinases } & \mathrm{S} 156-\mathrm{S} 161 \\ \text { Staurosporine; } 1 \mu \mathrm{M} ; 100 \mu \mathrm{M} \text { ATP } 100 \text { kinases } & \mathrm{S} 162-\mathrm{S} 167 \\ \text { Staurosporine; } 1 \mu \mathrm{M} ; 100 \mu \mathrm{M} \text { ATP } 50 \text { kinases } & \mathrm{S} 168-\mathrm{S} 173 \\ \text { PD184352; } 10 \mu \mathrm{M} ; 10 \mu \mathrm{M} \text { ATP } 510 \text { kinases } & \mathrm{S} 174-\mathrm{S} 179 \\ \text { PD184352; } 10 \mu \mathrm{M} ; 10 \mu \mathrm{M} \text { ATP } 400 \text { kinases } & \mathrm{S} 180-\mathrm{S} 185 \\ \text { PD184352; } 10 \mu \mathrm{M} ; 10 \mu \mathrm{M} \text { ATP } 300 \text { kinases } & \mathrm{S} 186-\mathrm{S} 191 \\ \text { PD184352; } 10 \mu \mathrm{M} ; 10 \mu \mathrm{M} \text { ATP } 200 \text { kinases } & \mathrm{S} 192-\mathrm{S} 197 \\ \text { PD184352; } 10 \mu \mathrm{M} ; 10 \mu \mathrm{M} \text { ATP } 100 \text { kinases } & \mathrm{S} 198-\mathrm{S} 203 \\ \text { PD184352; } 10 \mu \mathrm{M} ; 10 \mu \mathrm{M} \text { ATP } 50 \text { kinases } & \mathrm{S} 204-\mathrm{S} 209 \\ & \mathrm{~S} 210-\mathrm{S} 215\end{array}$

\section{Method:}

For each inhibitor the observed percentage of inhibition values are randomized by sorting together with the RAND() function output in Excel. Then the top 50,100, 200, 300 or 400 datapoints are used for calculation of the Gini coefficient using the standard approach described in the manuscript. Each sorting process is repeated 13 times to generate 13 Gini values calculated for each inhibitor and each sample size 
Table 5. Calculation of Gini coefficient for subsets of 50, 100, 200, 300 and 400 kinases for AG1024; $10 \mu \mathrm{M}$ at $10 \mu \mathrm{M}$ ATP, Staurosporine; $1 \mu \mathrm{M}$ at $100 \mu \mathrm{M}$ ATP and PD184352; $10 \mu \mathrm{M}$ at $10 \mu \mathrm{M}$ ATP

\begin{tabular}{|c|c|c|c|c|c|c|c|}
\hline & \#kinases & 50 & $\begin{array}{r}100 \\
20\end{array}$ & $\begin{array}{r}200 \\
39\end{array}$ & $\begin{array}{r}300 \\
59\end{array}$ & $\begin{array}{r}400 \\
78\end{array}$ & $\begin{array}{l}510 \\
100\end{array}$ \\
\hline \multirow[t]{15}{*}{ AG1024; $10 \mu \mathrm{M} ; 10 \mu \mathrm{M}$ ATP } & & 0.56054054 & 0.571458 & 0.550159 & 0.572816 & 0.560779 & 0.567976 \\
\hline & & 0.57759717 & 0.568643 & 0.562881 & 0.569889 & 0.568606 & \\
\hline & & 0.56310966 & 0.538383 & 0.551408 & 0.571576 & 0.564264 & \\
\hline & & 0.58657895 & 0.550868 & 0.580276 & 0.565694 & 0.567111 & \\
\hline & & 0.55951426 & 0.529636 & 0.565325 & 0.564264 & 0.56489 & \\
\hline & & 0.55940635 & 0.59178 & 0.575234 & 0.57501 & 0.561716 & \\
\hline & & 0.59538101 & 0.542294 & 0.566907 & 0.557886 & 0.567199 & \\
\hline & & 0.56102767 & 0.526777 & 0.566472 & 0.566185 & 0.56633 & \\
\hline & & 0.57673981 & 0.576709 & 0.556779 & 0.56667 & 0.565579 & \\
\hline & & 0.53333891 & 0.574894 & 0.570393 & 0.572536 & 0.565668 & \\
\hline & & 0.6418216 & 0.581033 & 0.574226 & 0.566206 & 0.564574 & \\
\hline & & 0.53410256 & 0.525031 & 0.59087 & 0.571274 & 0.559129 & \\
\hline & & 0.5043095 & 0.555028 & 0.567009 & 0.570341 & 0.568542 & \\
\hline & mean & 0.56565138 & 0.556349 & 0.567534 & 0.568488 & 0.564953 & 0.567976 \\
\hline & st. dev. & 0.03331947 & 0.02259 & 0.011274 & 0.004584 & 0.002904 & \\
\hline \multirow[t]{15}{*}{ Staurosporine; $1 \mu \mathrm{M} ; 100 \mu \mathrm{M}$ ATP } & & 0.16818866 & 0.144028 & 0.136376 & 0.148453 & 0.153074 & 0.149716 \\
\hline & & 0.15139406 & 0.19386 & 0.114858 & 0.141671 & 0.144666 & \\
\hline & & 0.1063508 & 0.156638 & 0.156114 & 0.15881 & 0.154248 & \\
\hline & & 0.09986456 & 0.140953 & 0.136411 & 0.146965 & 0.147273 & \\
\hline & & 0.10675787 & 0.155713 & 0.134264 & 0.144031 & 0.140082 & \\
\hline & & 0.17340583 & 0.159437 & 0.145877 & 0.147465 & 0.147098 & \\
\hline & & 0.13569852 & 0.115353 & 0.151697 & 0.147842 & 0.155566 & \\
\hline & & 0.18376207 & 0.128528 & 0.165388 & 0.134048 & 0.146925 & \\
\hline & & 0.19248105 & 0.142119 & 0.174144 & 0.149909 & 0.157495 & \\
\hline & & 0.19411646 & 0.135632 & 0.127796 & 0.143095 & 0.131666 & \\
\hline & & 0.14707846 & 0.144037 & 0.183178 & 0.157549 & 0.152169 & \\
\hline & & 0.17705868 & 0.133035 & 0.17084 & 0.149484 & 0.150623 & \\
\hline & & 0.12262287 & 0.122028 & 0.140797 & 0.136914 & 0.151718 & \\
\hline & mean & 0.15067538 & 0.143951 & 0.149057 & 0.146634 & 0.148662 & 0.149716 \\
\hline & st. dev. & 0.0338488 & 0.019931 & 0.020065 & 0.007004 & 0.006998 & \\
\hline \multirow[t]{15}{*}{ PD184352; $10 \mu \mathrm{M} ; 10 \mu \mathrm{M}$ ATP } & & 0.74580645 & 0.792992 & 0.767477 & 0.807306 & 0.799139 & 0.802202 \\
\hline & & 0.78184211 & 0.739096 & 0.800342 & 0.811173 & 0.804961 & \\
\hline & & 0.78380952 & 0.794414 & 0.813656 & 0.786352 & 0.801917 & \\
\hline & & 0.746875 & 0.788976 & 0.788789 & 0.794024 & 0.811373 & \\
\hline & & 0.84 & 0.80554 & 0.813657 & 0.785924 & 0.804287 & \\
\hline & & 0.88155251 & 0.773122 & 0.773619 & 0.802917 & 0.776983 & \\
\hline & & 0.80310559 & 0.802634 & 0.828124 & 0.80971 & 0.810688 & \\
\hline & & 0.78588957 & 0.815861 & 0.782902 & 0.805892 & 0.7993 & \\
\hline & & 0.81310345 & 0.848934 & 0.80092 & 0.786728 & 0.808882 & \\
\hline & & 0.81219512 & 0.858478 & 0.806832 & 0.813009 & 0.805915 & \\
\hline & & 0.75968912 & 0.792687 & 0.785075 & 0.803209 & 0.802737 & \\
\hline & & 0.77904 & 0.815812 & 0.800567 & 0.779831 & 0.797339 & \\
\hline & & 0.81921569 & 0.809935 & 0.773619 & 0.795529 & 0.803925 & \\
\hline & mean & 0.79631724 & 0.80296 & 0.795045 & 0.798585 & 0.802111 & 0.802202 \\
\hline & st. dev. & 0.038066 & 0.030311 & 0.018219 & 0.011151 & 0.008695 & \\
\hline
\end{tabular}

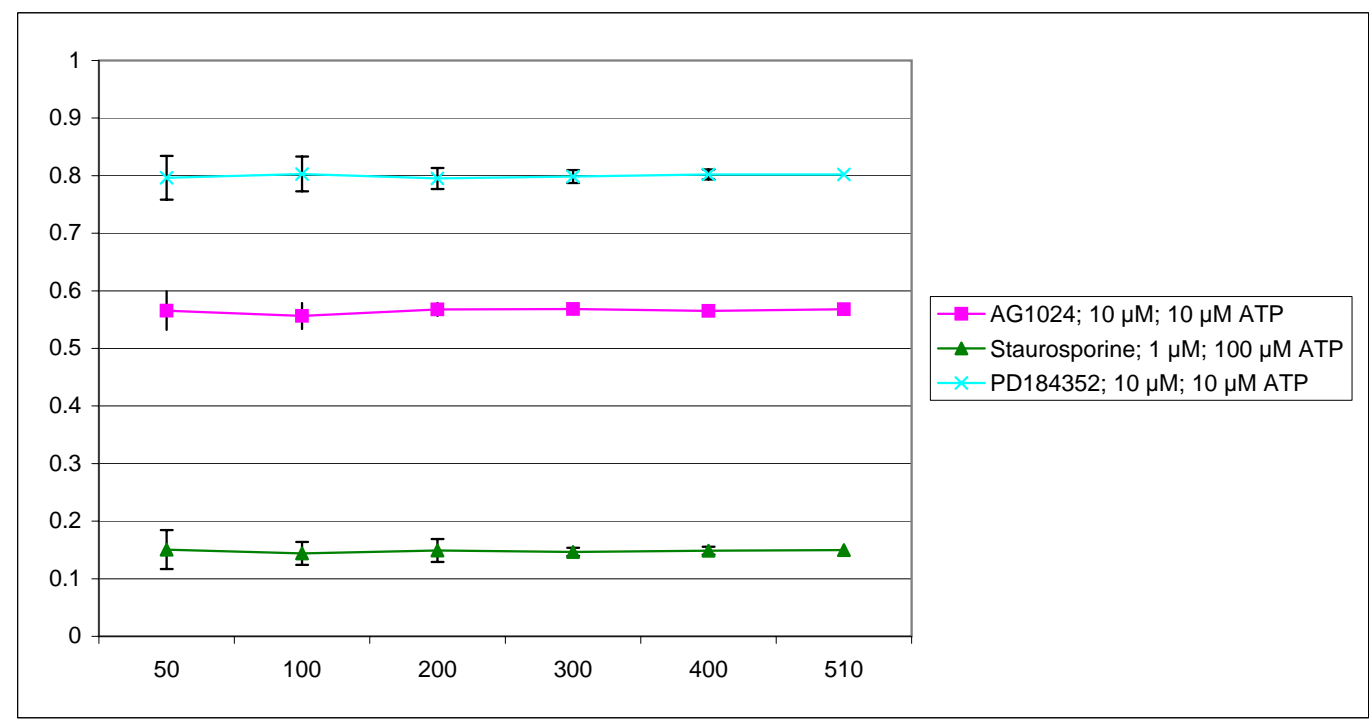




\begin{tabular}{|c|c|}
\hline & $10 \mu \mathrm{M}$ ATP \\
\hline Kinase & AG1024@ $10 \mu \mathrm{M}$ \\
\hline $\mathrm{Ab}(\mathrm{m})$ & 1 \\
\hline & 1 \\
\hline & 1 \\
\hline & 1 \\
\hline & 1 \\
\hline & 1 \\
\hline AMPK(r) & 84 \\
\hline & 84 \\
\hline & 84 \\
\hline & 84 \\
\hline & 84 \\
\hline & 84 \\
\hline $\operatorname{Arg}(\mathrm{m})$ & 6 \\
\hline & 6 \\
\hline & 6 \\
\hline & 6 \\
\hline & 6 \\
\hline & 6 \\
\hline Aurora-A(h) & 104 \\
\hline & 104 \\
\hline & 104 \\
\hline & 104 \\
\hline & 104 \\
\hline & 104 \\
\hline Axl(h) & 58 \\
\hline & 58 \\
\hline & 58 \\
\hline & 58 \\
\hline & 58 \\
\hline & 58 \\
\hline$B k(\mathrm{~m})$ & 4 \\
\hline & 4 \\
\hline & 4 \\
\hline & 4 \\
\hline & 4 \\
\hline & 4 \\
\hline Bnxx(h) & 84 \\
\hline & 84 \\
\hline & 84 \\
\hline & 84 \\
\hline & 84 \\
\hline & 84 \\
\hline CaMKII(r) & 89 \\
\hline & 89 \\
\hline & 89 \\
\hline & 89 \\
\hline & 89 \\
\hline & 89 \\
\hline CaMKIV(h) & 81 \\
\hline & 81 \\
\hline & 81 \\
\hline & 81 \\
\hline & 81 \\
\hline & 81 \\
\hline $\mathrm{CDK} 1 /$ cyclinB(h) & 67 \\
\hline & 67 \\
\hline & 67 \\
\hline & 67 \\
\hline & 67 \\
\hline & 67 \\
\hline $\operatorname{CDK} 2 / \operatorname{cyclinA}(\mathrm{h})$ & 101 \\
\hline & 101 \\
\hline & 101 \\
\hline & 101 \\
\hline & 101 \\
\hline & 101 \\
\hline CDK2/cyclinE(h) & 95 \\
\hline & 95 \\
\hline & 95 \\
\hline & 95 \\
\hline & 95 \\
\hline & 95 \\
\hline CDK3/cyclinE(h) & 87 \\
\hline & 87 \\
\hline & 87 \\
\hline & 87 \\
\hline & 87 \\
\hline & 87 \\
\hline $\operatorname{CDK} 5 / 335(\mathrm{~h})$ & 78 \\
\hline & 78 \\
\hline & 78 \\
\hline & 78 \\
\hline & 78 \\
\hline & 78 \\
\hline CDK6/cyclinD3(h) & 92 \\
\hline & 92 \\
\hline & 92 \\
\hline & 92 \\
\hline & 92 \\
\hline & 92 \\
\hline CDK7/cyclinH/MATI(h) & 93 \\
\hline & 93 \\
\hline & 93 \\
\hline & 93 \\
\hline & 93 \\
\hline
\end{tabular}

\begin{tabular}{|c|c|c|}
\hline position & cumulative sample fraction & inhibition\% \\
\hline 1 & 0.001960784 & 0 \\
\hline 2 & 0.003921569 & 0 \\
\hline 3 & 0.005882353 & 0 \\
\hline 4 & 0.007843137 & 0 \\
\hline 5 & 0.009803922 & 0 \\
\hline 6 & 0.011764706 & 0 \\
\hline 7 & 0.01372549 & 0 \\
\hline 8 & 0.015686275 & 0 \\
\hline 9 & 0.017647059 & 0 \\
\hline 10 & 0.019607843 & 0 \\
\hline 11 & 0.021568627 & 0 \\
\hline 12 & 0.023529412 & 0 \\
\hline 13 & 0.025490196 & 0 \\
\hline 14 & 0.02745098 & 0 \\
\hline 15 & 0.029411765 & 0 \\
\hline 16 & 0.031372549 & 0 \\
\hline 17 & 0.033333333 & 0 \\
\hline 18 & 0.035294118 & 0 \\
\hline 19 & 0.037254902 & 0 \\
\hline 20 & 0.039215686 & 0 \\
\hline 21 & 0.041176471 & 0 \\
\hline 22 & 0.043137255 & 0 \\
\hline 23 & 0.045098039 & 0 \\
\hline 24 & 0.047058824 & 0 \\
\hline 25 & 0.049019608 & 0 \\
\hline 26 & 0.050980392 & 0 \\
\hline 27 & 0.052941176 & 0 \\
\hline 28 & 0.054901961 & 0 \\
\hline 29 & 0.056862745 & 0 \\
\hline 30 & 0.058823529 & 0 \\
\hline 31 & 0.060784314 & 0 \\
\hline 32 & 0.062745098 & 0 \\
\hline 33 & 0.064705882 & 0 \\
\hline 34 & 0.066666667 & 0 \\
\hline 35 & 0.068627451 & 0 \\
\hline 36 & 0.070588235 & 0 \\
\hline 37 & 0.07254902 & 0 \\
\hline 38 & 0.074509804 & 0 \\
\hline 39 & 0.076470588 & 0 \\
\hline 40 & 0.078431373 & 0 \\
\hline 41 & 0.080392157 & 0 \\
\hline 42 & 0.082352941 & 0 \\
\hline 43 & 0.084313725 & 0 \\
\hline 44 & 0.08627451 & 0 \\
\hline 45 & 0.088235294 & 0 \\
\hline 46 & 0.090196078 & 0 \\
\hline 47 & 0.092156863 & 0 \\
\hline 48 & 0.094117647 & 0 \\
\hline 49 & 0.096078431 & 0 \\
\hline 50 & 0.098039216 & 0 \\
\hline 51 & 0.1 & 0 \\
\hline 52 & 0.101960784 & 0 \\
\hline 53 & 0.103921569 & 0 \\
\hline 54 & 0.105882353 & 0 \\
\hline 55 & 0.107843137 & 0 \\
\hline 56 & 0.109803922 & 0 \\
\hline 57 & 0.111764706 & 0 \\
\hline 58 & 0.11372549 & 0 \\
\hline 59 & 0.115686275 & 0 \\
\hline 60 & 0.117647059 & 0 \\
\hline 61 & 0.119607843 & 0 \\
\hline 62 & 0.121568627 & 0 \\
\hline 63 & 0.123529412 & 0 \\
\hline 64 & 0.125490196 & 0 \\
\hline 65 & 0.12745098 & 0 \\
\hline 66 & 0.129411765 & 0 \\
\hline 67 & 0.131372549 & 0 \\
\hline 68 & 0.133333333 & 0 \\
\hline 69 & 0.135294118 & 0 \\
\hline 70 & 0.137254902 & 0 \\
\hline 71 & 0.139215686 & 0 \\
\hline 72 & 0.141176471 & 0 \\
\hline 73 & 0.143137255 & 1 \\
\hline 74 & 0.145098039 & 1 \\
\hline 75 & 0.147058824 & 1 \\
\hline 76 & 0.149019608 & 1 \\
\hline 77 & 0.150980392 & 1 \\
\hline 78 & 0.152941176 & 1 \\
\hline 79 & 0.154901961 & 1 \\
\hline 80 & 0.156862745 & 1 \\
\hline 81 & 0.158823529 & 1 \\
\hline 82 & 0.160784314 & 1 \\
\hline 83 & 0.162745098 & 1 \\
\hline 84 & 0.164705882 & 1 \\
\hline 85 & 0.166666667 & 1 \\
\hline 86 & 0.168627451 & 1 \\
\hline 87 & 0.170588235 & 1 \\
\hline 88 & 0.17254902 & 1 \\
\hline 89 & 0.174509804 & 1 \\
\hline 90 & 0.176470588 & 1 \\
\hline 91 & 0.178431373 & 2 \\
\hline 92 & 0.180392157 & 2 \\
\hline 93 & 0.182352941 & 2 \\
\hline 94 & 0.184313725 & 2 \\
\hline 95 & 0.18627451 & 2 \\
\hline
\end{tabular}




\begin{tabular}{|c|c|}
\hline & 93 \\
\hline CHK 1 (h) & 97 \\
\hline & 97 \\
\hline & 97 \\
\hline & 97 \\
\hline & 97 \\
\hline & 97 \\
\hline CHK2(h) & 88 \\
\hline & 88 \\
\hline & 88 \\
\hline & 88 \\
\hline & 88 \\
\hline & 88 \\
\hline CKI(y) & 87 \\
\hline & 87 \\
\hline & 87 \\
\hline & 87 \\
\hline & 87 \\
\hline & 87 \\
\hline CK2(h) & 96 \\
\hline & 96 \\
\hline & 96 \\
\hline & 96 \\
\hline & 96 \\
\hline & 96 \\
\hline c-RAF(h) & 72 \\
\hline & 72 \\
\hline & 72 \\
\hline & 72 \\
\hline & 72 \\
\hline & 72 \\
\hline CSK(h) & 75 \\
\hline & 75 \\
\hline & 75 \\
\hline & 75 \\
\hline & 75 \\
\hline & 75 \\
\hline cSRC(h) & 6 \\
\hline & 6 \\
\hline & 6 \\
\hline & 6 \\
\hline & 6 \\
\hline & 6 \\
\hline Fes(h) & 70 \\
\hline & 70 \\
\hline & 70 \\
\hline & 70 \\
\hline & 70 \\
\hline & 70 \\
\hline FGFR3(h) & 91 \\
\hline & 91 \\
\hline & 91 \\
\hline & 91 \\
\hline & 91 \\
\hline & 91 \\
\hline $\mathrm{Fll} 3(\mathrm{~h})$ & 89 \\
\hline & 89 \\
\hline & 89 \\
\hline & 89 \\
\hline & 89 \\
\hline & 89 \\
\hline Fyn(h) & 5 \\
\hline & 5 \\
\hline & 5 \\
\hline & 5 \\
\hline & 5 \\
\hline & 5 \\
\hline GSK3 dh) & 89 \\
\hline & 89 \\
\hline & 89 \\
\hline & 89 \\
\hline & 89 \\
\hline & 89 \\
\hline GSK3B(h) & 52 \\
\hline & 52 \\
\hline & 52 \\
\hline & 52 \\
\hline & 52 \\
\hline & 52 \\
\hline IGF-1R(h) & 118 \\
\hline & 118 \\
\hline & 118 \\
\hline & 118 \\
\hline & 118 \\
\hline & 118 \\
\hline $\mathrm{IKK} \alpha(\mathrm{h})$ & 82 \\
\hline & 82 \\
\hline & 82 \\
\hline & 82 \\
\hline & 82 \\
\hline & 82 \\
\hline IKKB(h) & 93 \\
\hline & 93 \\
\hline & 93 \\
\hline & 93 \\
\hline & 93 \\
\hline & 93 \\
\hline
\end{tabular}




\begin{tabular}{|c|c|}
\hline $\mathrm{IR}(\mathrm{h})$ & 105 \\
\hline & 105 \\
\hline & 105 \\
\hline & 105 \\
\hline & 105 \\
\hline & 105 \\
\hline JNK Ia1(h) & 79 \\
\hline & 79 \\
\hline & 79 \\
\hline & 79 \\
\hline & 79 \\
\hline & 79 \\
\hline JNK2a2(h) & 89 \\
\hline & 89 \\
\hline & 89 \\
\hline & 89 \\
\hline & 89 \\
\hline & 89 \\
\hline JNK3(r) & 60 \\
\hline & 60 \\
\hline & 60 \\
\hline & 60 \\
\hline & 60 \\
\hline & 60 \\
\hline Lck(h) & 24 \\
\hline & 24 \\
\hline & 24 \\
\hline & 24 \\
\hline & 24 \\
\hline & 24 \\
\hline Lym(h) & 74 \\
\hline & 74 \\
\hline & 74 \\
\hline & 74 \\
\hline & 74 \\
\hline & 74 \\
\hline Lyn(m) & 5 \\
\hline & 5 \\
\hline & 5 \\
\hline & 5 \\
\hline & 5 \\
\hline & 5 \\
\hline MAPK1(h) & 85 \\
\hline & 85 \\
\hline & 85 \\
\hline & 85 \\
\hline & 85 \\
\hline & 85 \\
\hline MAPK2(h) & 113 \\
\hline & 113 \\
\hline & 113 \\
\hline & 113 \\
\hline & 113 \\
\hline & 113 \\
\hline $\operatorname{MAPK2} 2(\mathrm{~m})$ & 93 \\
\hline & 93 \\
\hline & 93 \\
\hline & 93 \\
\hline & 93 \\
\hline & 93 \\
\hline MAPKAP-K2(h) & 90 \\
\hline & 90 \\
\hline & 90 \\
\hline & 90 \\
\hline & 90 \\
\hline & 90 \\
\hline MEKI(h) & 88 \\
\hline & 88 \\
\hline & 88 \\
\hline & 88 \\
\hline & 88 \\
\hline & 88 \\
\hline MKK4(m) & 88 \\
\hline & 88 \\
\hline & 88 \\
\hline & 88 \\
\hline & 88 \\
\hline & 88 \\
\hline МКК $6(\mathrm{~h})$ & 86 \\
\hline & 86 \\
\hline & 86 \\
\hline & 86 \\
\hline & 86 \\
\hline & 86 \\
\hline MKK7B(h) & 116 \\
\hline & 116 \\
\hline & 116 \\
\hline & 116 \\
\hline & 116 \\
\hline & 116 \\
\hline MSK1(h) & 92 \\
\hline & 92 \\
\hline & 92 \\
\hline & 92 \\
\hline & 92 \\
\hline & 92 \\
\hline $\mathrm{p} 70 \mathrm{~S} 6 \mathrm{~K}(\mathrm{~h})$ & 36 \\
\hline
\end{tabular}




\begin{tabular}{|c|c|}
\hline & 36 \\
\hline & 36 \\
\hline & 36 \\
\hline & 36 \\
\hline & 36 \\
\hline PAK2(h) & 98 \\
\hline & 98 \\
\hline & 98 \\
\hline & 98 \\
\hline & 98 \\
\hline & 98 \\
\hline PDGFRa(h) & 88 \\
\hline & 88 \\
\hline & 88 \\
\hline & 88 \\
\hline & 88 \\
\hline & 88 \\
\hline PDGFRB(h) & 67 \\
\hline & 67 \\
\hline & 67 \\
\hline & 67 \\
\hline & 67 \\
\hline & 67 \\
\hline PDK I(h) & 84 \\
\hline & 84 \\
\hline & 84 \\
\hline & 84 \\
\hline & 84 \\
\hline & 84 \\
\hline PKA(b) & 74 \\
\hline & 74 \\
\hline & 74 \\
\hline & 74 \\
\hline & 74 \\
\hline & 74 \\
\hline PKA(h) & 107 \\
\hline & 107 \\
\hline & 107 \\
\hline & 107 \\
\hline & 107 \\
\hline & 107 \\
\hline PKBu(h) & 99 \\
\hline & 99 \\
\hline & 99 \\
\hline & 99 \\
\hline & 99 \\
\hline & 99 \\
\hline PKBB(h) & 90 \\
\hline & 90 \\
\hline & 90 \\
\hline & 90 \\
\hline & 90 \\
\hline & 90 \\
\hline PKB $\{(\mathrm{h})$ & 91 \\
\hline & 91 \\
\hline & 91 \\
\hline & 91 \\
\hline & 91 \\
\hline & 91 \\
\hline PKC $a(h)$ & 87 \\
\hline & 87 \\
\hline & 87 \\
\hline & 87 \\
\hline & 87 \\
\hline & 87 \\
\hline РКСВII(h) & 99 \\
\hline & 99 \\
\hline & 99 \\
\hline & 99 \\
\hline & 99 \\
\hline & 99 \\
\hline PKC $\{(\mathrm{h})$ & 105 \\
\hline & 105 \\
\hline & 105 \\
\hline & 105 \\
\hline & 105 \\
\hline & 105 \\
\hline PKC $\delta(h)$ & 107 \\
\hline & 107 \\
\hline & 107 \\
\hline & 107 \\
\hline & 107 \\
\hline & 107 \\
\hline PKC ah) & 78 \\
\hline & 78 \\
\hline & 78 \\
\hline & 78 \\
\hline & 78 \\
\hline & 78 \\
\hline PKC $\eta(1)$ & 96 \\
\hline & 96 \\
\hline & 96 \\
\hline & 96 \\
\hline & 96 \\
\hline & 96 \\
\hline P KC (1) & 90 \\
\hline & 90 \\
\hline
\end{tabular}




\begin{tabular}{|c|c|}
\hline & 90 \\
\hline & 90 \\
\hline & 90 \\
\hline & 90 \\
\hline PKC $\mu(\mathrm{h})$ & 86 \\
\hline & 86 \\
\hline & 86 \\
\hline & 86 \\
\hline & 86 \\
\hline & 86 \\
\hline РКС日() & 91 \\
\hline & 91 \\
\hline & 91 \\
\hline & 91 \\
\hline & 91 \\
\hline & 91 \\
\hline PKD2(h) & 73 \\
\hline & 73 \\
\hline & 73 \\
\hline & 73 \\
\hline & 73 \\
\hline & 73 \\
\hline PRAK(h) & 76 \\
\hline & 76 \\
\hline & 76 \\
\hline & 76 \\
\hline & 76 \\
\hline & 76 \\
\hline PRK2(h) & 88 \\
\hline & 88 \\
\hline & 88 \\
\hline & 88 \\
\hline & 88 \\
\hline & 88 \\
\hline ROCK-II(h) & 23 \\
\hline & 23 \\
\hline & 23 \\
\hline & 23 \\
\hline & 23 \\
\hline & 23 \\
\hline ROCK-II(r) & 32 \\
\hline & 32 \\
\hline & 32 \\
\hline & 32 \\
\hline & 32 \\
\hline & 32 \\
\hline Rsk1(h) & 89 \\
\hline & 89 \\
\hline & 89 \\
\hline & 89 \\
\hline & 89 \\
\hline & 89 \\
\hline Rskl(r) & 72 \\
\hline & 72 \\
\hline & 72 \\
\hline & 72 \\
\hline & 72 \\
\hline & 72 \\
\hline Rsk2(h) & 58 \\
\hline & 58 \\
\hline & 58 \\
\hline & 58 \\
\hline & 58 \\
\hline & 58 \\
\hline Rsk3(h) & 104 \\
\hline & 104 \\
\hline & 104 \\
\hline & 104 \\
\hline & 104 \\
\hline & 104 \\
\hline SAPK2a(h) & 99 \\
\hline & 99 \\
\hline & 99 \\
\hline & 99 \\
\hline & 99 \\
\hline & 99 \\
\hline $\mathrm{SAPK} 2 \mathrm{~b}(\mathrm{~h})$ & 103 \\
\hline & 103 \\
\hline & 103 \\
\hline & 103 \\
\hline & 103 \\
\hline & 103 \\
\hline SAPK3(h) & 84 \\
\hline & 84 \\
\hline & 84 \\
\hline & 84 \\
\hline & 84 \\
\hline & 84 \\
\hline SAPK4(h) & 94 \\
\hline & 94 \\
\hline & 94 \\
\hline & 94 \\
\hline & 94 \\
\hline & 94 \\
\hline SGK(h) & 63 \\
\hline & 63 \\
\hline & 63 \\
\hline
\end{tabular}


AG1024; $10 \mu \mathrm{M}$; $10 \mu \mathrm{M}$ ATP; sample of 400 kinases

\begin{tabular}{|c|c|c|c|}
\hline position & inhibition\% & rand & cumulative sample fraction \\
\hline 1 & 12 & 0.798495088 & 0.0025 \\
\hline 2 & 28 & 0.517119145 & 0.005 \\
\hline 3 & 12 & 0.364377937 & 0.0075 \\
\hline 4 & 4 & 0.028979306 & 0.01 \\
\hline 5 & 76 & 0.571515371 & 0.0125 \\
\hline 6 & 76 & 0.266047091 & 0.015 \\
\hline 7 & 95 & 0.327293542 & 0.0175 \\
\hline 8 & 0 & 0.077252078 & 0.02 \\
\hline 9 & 12 & 0.512077961 & 0.0225 \\
\hline 10 & 0 & 0.426626425 & 0.025 \\
\hline 11 & 7 & 0.420978818 & 0.0275 \\
\hline 12 & 10 & 0.948997001 & 0.03 \\
\hline 13 & 2 & 0.286146585 & 0.0325 \\
\hline 14 & 13 & 0.903207761 & 0.035 \\
\hline 15 & 1 & 0.343592037 & 0.0375 \\
\hline 16 & 14 & 0.339260508 & 0.04 \\
\hline 17 & 95 & 0.434903587 & 0.0425 \\
\hline 18 & 0 & 0.427286416 & 0.045 \\
\hline 19 & 8 & 0.653929639 & 0.0475 \\
\hline 20 & 33 & 0.008453739 & 0.05 \\
\hline 21 & 1 & 0.341914014 & 0.0525 \\
\hline 22 & 16 & 0.57856488 & 0.055 \\
\hline 23 & 94 & 0.181197592 & 0.0575 \\
\hline 24 & 12 & 0.595716538 & 0.06 \\
\hline 25 & 12 & 0.07758184 & 0.0625 \\
\hline 26 & 48 & 0.202746006 & 0.065 \\
\hline 27 & 28 & 0.800768107 & 0.0675 \\
\hline 28 & 10 & 0.822224855 & 0.07 \\
\hline 29 & 0 & 0.101254022 & 0.0725 \\
\hline 30 & 7 & 0.915706307 & 0.075 \\
\hline 31 & 95 & 0.693263628 & 0.0775 \\
\hline 32 & 0 & 0.721777617 & 0.08 \\
\hline 33 & 1 & 0.961297626 & 0.0825 \\
\hline 34 & 94 & 0.603435981 & 0.085 \\
\hline 35 & 1 & 0.875081728 & 0.0875 \\
\hline 36 & 40 & 0.598579598 & 0.09 \\
\hline 37 & 64 & 0.689807345 & 0.0925 \\
\hline 38 & 10 & 0.72473393 & 0.095 \\
\hline 39 & 22 & 0.852246802 & 0.0975 \\
\hline 40 & 26 & 0.85047942 & 0.1 \\
\hline 41 & 16 & 0.922480869 & 0.1025 \\
\hline 42 & 13 & 0.755666692 & 0.105 \\
\hline 43 & 68 & 0.013625855 & 0.1075 \\
\hline 44 & 64 & 0.838188262 & 0.11 \\
\hline 45 & 40 & 0.682617691 & 0.1125 \\
\hline 46 & 9 & 0.945850893 & 0.115 \\
\hline 47 & 13 & 0.430845974 & 0.1175 \\
\hline 48 & 8 & 0.012421393 & 0.12 \\
\hline 49 & 19 & 0.662377885 & 0.1225 \\
\hline 50 & 0 & 0.013229472 & 0.125 \\
\hline 51 & 0 & 0.912558322 & 0.1275 \\
\hline 52 & 94 & 0.752174048 & 0.13 \\
\hline 53 & 4 & 0.958705096 & 0.1325 \\
\hline 54 & 12 & 0.402206852 & 0.135 \\
\hline 55 & 11 & 0.235805693 & 0.1375 \\
\hline 56 & 1 & 0.217010005 & 0.14 \\
\hline 57 & 0 & 0.205131462 & 0.1425 \\
\hline 58 & 33 & 0.561936572 & 0.145 \\
\hline 59 & 1 & 0.364858432 & 0.1475 \\
\hline 60 & 95 & 0.984243697 & 0.15 \\
\hline 61 & 3 & 0.043470671 & 0.1525 \\
\hline 62 & 96 & 0.055190257 & 0.155 \\
\hline 63 & 11 & 0.926728429 & 0.1575 \\
\hline 64 & 64 & 0.937701541 & 0.16 \\
\hline 65 & 12 & 0.833132627 & 0.1625 \\
\hline 66 & 13 & 0.57755009 & 0.165 \\
\hline 67 & 14 & 0.070962994 & 0.1675 \\
\hline 68 & 48 & 0.006074391 & 0.17 \\
\hline 69 & 94 & 0.397547136 & 0.1725 \\
\hline 70 & 11 & 0.87138515 & 0.175 \\
\hline 71 & 0 & 0.634674354 & 0.1775 \\
\hline 72 & 8 & 0.765132573 & 0.18 \\
\hline 73 & 0 & 0.227473857 & 0.1825 \\
\hline 74 & 16 & 0.314416453 & 0.185 \\
\hline 75 & 11 & 0.673549842 & 0.1875 \\
\hline 76 & 0 & 0.012201598 & 0.19 \\
\hline 77 & 13 & 0.520789394 & 0.1925 \\
\hline 78 & 13 & 0.674772245 & 0.195 \\
\hline 79 & 22 & 0.250722051 & 0.1975 \\
\hline 80 & 26 & 0.124972379 & 0.2 \\
\hline 81 & 33 & 0.5795552 & 0.2025 \\
\hline 82 & 14 & 0.228279171 & 0.205 \\
\hline 83 & 33 & 0.201881955 & 0.2075 \\
\hline 84 & 10 & 0.541674942 & 0.21 \\
\hline 85 & 0 & 0.031290621 & 0.2125 \\
\hline 86 & 22 & 0.249362592 & 0.215 \\
\hline 87 & 95 & 0.815517044 & 0.2175 \\
\hline 88 & 5 & 0.346084285 & 0.22 \\
\hline 89 & 16 & 0.405910442 & 0.2225 \\
\hline 90 & 9 & 0.110264793 & 0.225 \\
\hline 91 & 13 & 0.779671755 & 0.2275 \\
\hline 92 & 0 & 0.866325386 & 0.23 \\
\hline 93 & 42 & 0.492925555 & 0.2325 \\
\hline 94 & 33 & 0.252069166 & 0.235 \\
\hline
\end{tabular}




\begin{tabular}{|c|c|c|}
\hline 95 & 2 & 0.82204052 \\
\hline 96 & 21 & 0.192166035 \\
\hline 97 & 0 & 0.302988989 \\
\hline 98 & 8 & 0.354532206 \\
\hline 99 & 10 & 0.691754643 \\
\hline 100 & 0 & 0.55939314 \\
\hline 101 & 0 & 0.155097239 \\
\hline 102 & 11 & 0.330780398 \\
\hline 103 & 12 & 0.169523989 \\
\hline 104 & 0 & 0.814991579 \\
\hline 105 & 22 & 0.175153546 \\
\hline 106 & 99 & 0.015760903 \\
\hline 107 & 25 & 0.978526429 \\
\hline 108 & 16 & 0.932069163 \\
\hline 109 & 0 & 0.203509665 \\
\hline 110 & 28 & 0.647912195 \\
\hline 111 & 4 & 0.211436127 \\
\hline 112 & 5 & 0.208471916 \\
\hline 113 & 40 & 0.986308573 \\
\hline 114 & 24 & 0.037904734 \\
\hline 115 & 8 & 0.611523987 \\
\hline 116 & 10 & 0.315123661 \\
\hline 117 & 21 & 0.111099382 \\
\hline 118 & 0 & 0.825304358 \\
\hline 119 & 96 & 0.59523178 \\
\hline 120 & 14 & 0.783927064 \\
\hline 121 & 2 & 0.72297974 \\
\hline 122 & 13 & 0.098555246 \\
\hline 123 & 25 & 0.93030389 \\
\hline 124 & 7 & 0.16885361 \\
\hline 125 & 12 & 0.377444226 \\
\hline 126 & 25 & 0.733345474 \\
\hline 127 & 24 & 0.079738373 \\
\hline 128 & 0 & 0.268245035 \\
\hline 129 & 11 & 0.499673909 \\
\hline 130 & 91 & 0.696939125 \\
\hline 131 & 14 & 0.188429162 \\
\hline 132 & 0 & 0.544190849 \\
\hline 133 & 10 & 0.8411961 \\
\hline 134 & 4 & 0.366947192 \\
\hline 135 & 10 & 0.112004678 \\
\hline 136 & 4 & 0.535659787 \\
\hline 137 & 11 & 0.073094056 \\
\hline 138 & 14 & 0.75916017 \\
\hline 139 & 22 & 0.696993167 \\
\hline 140 & 14 & 0.357737074 \\
\hline 141 & 10 & 0.550533328 \\
\hline 142 & 18 & 0.520251288 \\
\hline 143 & 0 & 0.422034522 \\
\hline 144 & 12 & 0.056852244 \\
\hline 145 & 12 & 0.6088404 \\
\hline 146 & 0 & 0.278693773 \\
\hline 147 & 22 & 0.905682381 \\
\hline 148 & 4 & 0.291822552 \\
\hline 149 & 13 & 0.046232937 \\
\hline 150 & 19 & 0.019614688 \\
\hline 151 & 37 & 0.68177416 \\
\hline 152 & 37 & 0.388418036 \\
\hline 153 & 16 & 0.263689511 \\
\hline 154 & 15 & 0.051138185 \\
\hline 155 & 40 & 0.257031742 \\
\hline 156 & 3 & 0.162513909 \\
\hline 157 & 14 & 0.881646884 \\
\hline 158 & 12 & 0.188582018 \\
\hline 159 & 48 & 0.943866783 \\
\hline 160 & 30 & 0.803943901 \\
\hline 161 & 28 & 0.55089425 \\
\hline 162 & 96 & 0.60582832 \\
\hline 163 & 12 & 0.97840054 \\
\hline 164 & 0 & 0.248747559 \\
\hline 165 & 48 & 0.447433823 \\
\hline 166 & 0 & 0.954289123 \\
\hline 167 & 40 & 0.495738289 \\
\hline 168 & 6 & 0.597822533 \\
\hline 169 & 77 & 0.96693457 \\
\hline 170 & 22 & 0.182334445 \\
\hline 171 & 33 & 0.304696528 \\
\hline 172 & 1 & 0.878491416 \\
\hline 173 & 27 & 0.735924177 \\
\hline 174 & 7 & 0.336871872 \\
\hline 175 & 1 & 0.616069011 \\
\hline 176 & 64 & 0.395441328 \\
\hline 177 & 19 & 0.39085075 \\
\hline 178 & 94 & 0.029694592 \\
\hline 179 & 68 & 0.384095925 \\
\hline 180 & 91 & 0.355593823 \\
\hline 181 & 76 & 0.087771595 \\
\hline 182 & 31 & 0.301117595 \\
\hline 183 & 11 & 0.065838864 \\
\hline 184 & 11 & 0.441686802 \\
\hline 185 & 0 & 0.987440518 \\
\hline 186 & 77 & 0.105907112 \\
\hline 187 & 0 & 0.427594187 \\
\hline 188 & 9 & 0.780573381 \\
\hline 189 & 10 & 0.393752098 \\
\hline 190 & 1 & 0.695874457 \\
\hline
\end{tabular}

\begin{tabular}{|c|c|c|}
\hline 2 & 5 & 0.000537634 \\
\hline 21 & 5 & 0.000537634 \\
\hline 0 & 5 & 0.000537634 \\
\hline 8 & 5 & 0.000537634 \\
\hline 10 & 6 & 0.000645161 \\
\hline 0 & 6 & 0.000645161 \\
\hline 0 & 6 & 0.000645161 \\
\hline 11 & 6 & 0.000645161 \\
\hline 12 & 7 & 0.000752688 \\
\hline 0 & 7 & 0.000752688 \\
\hline 22 & 7 & 0.000752688 \\
\hline 99 & 7 & 0.000752688 \\
\hline 25 & 7 & 0.000752688 \\
\hline 16 & 7 & 0.000752688 \\
\hline 0 & 7 & 0.000752688 \\
\hline 28 & 7 & 0.000752688 \\
\hline 4 & 7 & 0.000752688 \\
\hline 5 & 7 & 0.000752688 \\
\hline 40 & 7 & 0.000752688 \\
\hline 24 & 7 & 0.000752688 \\
\hline 8 & 7 & 0.000752688 \\
\hline 10 & 7 & 0.000752688 \\
\hline 21 & 8 & 0.000860215 \\
\hline 0 & 8 & 0.000860215 \\
\hline 96 & 8 & 0.000860215 \\
\hline 14 & 8 & 0.000860215 \\
\hline 2 & 8 & 0.000860215 \\
\hline 13 & 8 & 0.000860215 \\
\hline 25 & 8 & 0.000860215 \\
\hline 7 & 8 & 0.000860215 \\
\hline 12 & 8 & 0.000860215 \\
\hline 25 & 8 & 0.000860215 \\
\hline 24 & 9 & 0.000967742 \\
\hline 0 & 9 & 0.000967742 \\
\hline 11 & 9 & 0.000967742 \\
\hline 91 & 9 & 0.000967742 \\
\hline 14 & 9 & 0.000967742 \\
\hline 0 & 9 & 0.000967742 \\
\hline 10 & 9 & 0.000967742 \\
\hline 4 & 9 & 0.000967742 \\
\hline 10 & 10 & 0.001075269 \\
\hline 4 & 10 & 0.001075269 \\
\hline 11 & 10 & 0.001075269 \\
\hline 14 & 10 & 0.001075269 \\
\hline 22 & 10 & 0.001075269 \\
\hline 14 & 10 & 0.001075269 \\
\hline 10 & 10 & 0.001075269 \\
\hline 18 & 10 & 0.001075269 \\
\hline 0 & 10 & 0.001075269 \\
\hline 12 & 10 & 0.001075269 \\
\hline 12 & 10 & 0.001075269 \\
\hline 0 & 10 & 0.001075269 \\
\hline 22 & 10 & 0.001075269 \\
\hline 4 & 10 & 0.001075269 \\
\hline 13 & 10 & 0.001075269 \\
\hline 19 & 11 & 0.001182796 \\
\hline 37 & 11 & 0.001182796 \\
\hline 37 & 11 & 0.001182796 \\
\hline 16 & 11 & 0.001182796 \\
\hline 15 & 11 & 0.001182796 \\
\hline 40 & 11 & 0.001182796 \\
\hline 3 & 11 & 0.001182796 \\
\hline 14 & 11 & 0.001182796 \\
\hline 12 & 11 & 0.001182796 \\
\hline 48 & 11 & 0.001182796 \\
\hline 30 & 11 & 0.001182796 \\
\hline 28 & 11 & 0.001182796 \\
\hline 96 & 11 & 0.001182796 \\
\hline 12 & 11 & 0.001182796 \\
\hline 0 & 11 & 0.001182796 \\
\hline 48 & 11 & 0.001182796 \\
\hline 0 & 11 & 0.001182796 \\
\hline 40 & 11 & 0.001182796 \\
\hline 6 & 11 & 0.001182796 \\
\hline 77 & 11 & 0.001182796 \\
\hline 22 & 11 & 0.001182796 \\
\hline 33 & 11 & 0.001182796 \\
\hline 1 & 11 & 0.001182796 \\
\hline 27 & 12 & 0.001290323 \\
\hline 7 & 12 & 0.001290323 \\
\hline 1 & 12 & 0.001290323 \\
\hline 64 & 12 & 0.001290323 \\
\hline 19 & 12 & 0.001290323 \\
\hline 94 & 12 & 0.001290323 \\
\hline 68 & 12 & 0.001290323 \\
\hline 91 & 12 & 0.001290323 \\
\hline 76 & 12 & 0.001290323 \\
\hline 31 & 12 & 0.001290323 \\
\hline 11 & 12 & 0.001290323 \\
\hline 11 & 12 & 0.001290323 \\
\hline 0 & 12 & 0.001290323 \\
\hline 77 & 12 & 0.001290323 \\
\hline 0 & 12 & 0.001290323 \\
\hline 9 & 12 & 0.001290323 \\
\hline 10 & 12 & 0.001290323 \\
\hline 1 & 12 & 0.001290323 \\
\hline
\end{tabular}

$0.009784946 \quad 2.38 \mathrm{E}-05$ $0.010322581 \quad 2.51 \mathrm{E}-05$ $0.010860215 \quad 2.65 \mathrm{E}-05$ $0.011397849 \quad 2.78 \mathrm{E}-05$ $0.012043011 \quad 2.93 \mathrm{E}-05$ $0.0126881723 .09 \mathrm{E}-05$ $0.013333333 \quad 3.25 \mathrm{E}-05$ $0.013978495 \quad 3.41 \mathrm{E}-05$ $0.014731183 \quad 3.59 \mathrm{E}-05$ $\begin{array}{ll}0.015483871 & 3.78 \mathrm{E}-05 \\ 0.016236559 & 3.97 \mathrm{E}-05\end{array}$ $0.016989247 \quad 4.15 \mathrm{E}-05$ 0.017741935 4.34E-05 $\begin{array}{ll}0.017741935 & 4.34 \mathrm{E}-05 \\ 0.018494624 & 4.53 \mathrm{E}-05\end{array}$ $\begin{array}{rr}0.018494624 & 4.53 \mathrm{E}-05 \\ 0.019247312 & 4.72 \mathrm{E}-05\end{array}$ $0.02 \quad 4.91 \mathrm{E}-05$ $0.020752688 \quad 5.09 \mathrm{E}-05$ $0.021505376 \quad 5.28 \mathrm{E}-05$ $\begin{array}{ll}0.022258065 & 5.47 \mathrm{E}-05 \\ 0.023010753 & 5.66 \mathrm{E}-05\end{array}$ $\begin{array}{ll}0.023010753 & 5.66 \mathrm{E}-05 \\ 0.023763441 & 5.85 \mathrm{E}-05\end{array}$ $0.024516129 \quad 6.03 \mathrm{E}-05$ $0.025376344 \quad 6.24 \mathrm{E}-05$ $\begin{array}{ll}0.025376344 & 6.24 \mathrm{E}-05 \\ 0.026236559 & 6.45 \mathrm{E}-05\end{array}$ $\begin{array}{ll}0.026236559 & 6.45 \mathrm{E}-05 \\ 0.027096774 & 6.67 \mathrm{E}-05\end{array}$ $\begin{array}{ll}0.027096774 & 6.67 \mathrm{E}-05 \\ 0.027956989 & 6.88 \mathrm{E}-05 \\ 0.02881204 & 7.1 \mathrm{E}-05\end{array}$ $\begin{array}{rr}0.027956989 & 6.88 \mathrm{E}-05 \\ 0.028817204 & 7.1 \mathrm{E}-05\end{array}$ $\begin{array}{rr}0.028817204 & 7.1 \mathrm{E}-05 \\ 0.029677419 & 7.31 \mathrm{E}-05\end{array}$ $0.030537634 \quad 7.53 \mathrm{E}-05$ $0.031397849 \quad 7.74 \mathrm{E}-05$ 0.032258065 7.96E-05 $0.03311828 \quad 8.17 \mathrm{E}-05$ $0.034086022 \quad 8.4 \mathrm{E}-05$ $0.035053763 \quad 8.64 \mathrm{E}-05$ $0.036021505 \quad 8.88 \mathrm{E}-05$ $\begin{array}{ll}0.036989247 & 9.13 \mathrm{E}-05 \\ 0.037956989 & 9.37 \mathrm{E}-05\end{array}$ $\begin{array}{ll}0.037956989 & 9.37 \mathrm{E}-05 \\ 0.038924731 & 9.61 \mathrm{E}-05\end{array}$ $0.039892473 \quad 9.85 \mathrm{E}-05$ $0.040860215 \quad 0.000101$ $0.041935484 \quad 0.000103$ $\begin{array}{lll}0.043010753 & 0.000106\end{array}$ $\begin{array}{lll}0.044086022 & 0.000109\end{array}$ $\begin{array}{ll}0.04516129 & 0.000112\end{array}$ $\begin{array}{ll}0.04516129 & 0.000112 \\ 0.046236559 & 0.000114\end{array}$ $\begin{array}{ll}0.046236559 & 0.000114 \\ 0.047311828 & 0.000117\end{array}$ $\begin{array}{lr}0.047311828 & 0.000117 \\ 0.048387097 & 0.00012\end{array}$ $\begin{array}{lr}0.048387097 & 0.00012 \\ 0.049462366 & 0.000122\end{array}$ $\begin{array}{ll}0.049462366 & 0.000122 \\ 0.050537634 & 0.000125\end{array}$ $\begin{array}{ll}0.050537634 & 0.000125 \\ 0.051612903 & 0.000128\end{array}$ $\begin{array}{ll}0.052688172 & 0.00013\end{array}$ $\begin{array}{ll}0.053763441 & 0.000133\end{array}$ $\begin{array}{lll}0.05483871 & 0.000136\end{array}$ $\begin{array}{lll}0.055913978 & 0.000138\end{array}$ $\begin{array}{ll}0.055913978 & 0.000138 \\ 0.056989247 & 0.000141\end{array}$ $\begin{array}{ll}0.056989247 & 0.000141 \\ 0.058172043 & 0.000144\end{array}$ $\begin{array}{ll}0.059354839 & 0.000147\end{array}$ $\begin{array}{rr}0.059354839 & 0.000147 \\ 0.060537634 & 0.00015\end{array}$ $0.06172043 \quad 0.000153$ 0.0629032260 .000156 $\begin{array}{lll}0.064086022 & 0.000159\end{array}$ $\begin{array}{lll}0.065268817 & 0.000162\end{array}$ $\begin{array}{lll}0.066451613 & 0.000165\end{array}$ $\begin{array}{lll}0.067634409 & 0.000168\end{array}$ $\begin{array}{rr}0.067634409 & 0.000168 \\ 0.068817204 & 0.000171\end{array}$ $\begin{array}{ll}0.07 & 0.000174\end{array}$ $0.071182796 \quad 0.000176$ $\begin{array}{ll}0.072365591 & 0.000179\end{array}$ $0.073548387 \quad 0.000182$ $0.074731183 \quad 0.000185$ $\begin{array}{lll}0.075913978 & 0.000188\end{array}$ $\begin{array}{lll}0.077096774 & 0.000191\end{array}$ $\begin{array}{ll}0.07827957 & 0.000194 \\ 0.079662366 & 0.000197\end{array}$ $\begin{array}{ll}0.079462366 & 0.000197\end{array}$ $\begin{array}{ll}0.080645161 & 0.0002\end{array}$ $\begin{array}{lr}0.081827957 & 0.000203\end{array}$ $0.083010753 \quad 0.000206$ $\begin{array}{lll}0.084193548 & 0.000209\end{array}$ $0.085483871 \quad 0.000212$ $0.086774194 \quad 0.000215$ $\begin{array}{lll}0.088064516 & 0.000219\end{array}$ 0.0893548390 .000222 $\begin{array}{lll}0.090645161 & 0.000225\end{array}$ $\begin{array}{lll}0.091935484 & 0.000228\end{array}$ $0.093225806 \quad 0.000231$ $0.094516129 \quad 0.000235$ $0.095806452 \quad 0.000238$ $\begin{array}{lll}0.097096774 & 0.000241\end{array}$ $\begin{array}{lll}0.098387097 & 0.000244\end{array}$ 0.0996774190 .000248 $\begin{array}{lll}0.100967742 & 0.000251\end{array}$ $0.102258065 \quad 0.000254$ $0.103548387 \quad 0.000257$ $\begin{array}{ll}0.10483871 & 0.00026\end{array}$ $\begin{array}{ll}0.106129032 & 0.000264\end{array}$ 


\begin{tabular}{|c|c|c|}
\hline 191 & 28 & 0.589803092 \\
\hline 192 & 42 & 0.250778579 \\
\hline 193 & 14 & 0.77501997 \\
\hline 194 & 16 & 0.441482049 \\
\hline 195 & 11 & 0.474826591 \\
\hline 196 & 2 & 0.936389097 \\
\hline 197 & 1 & 0.330278103 \\
\hline 198 & 16 & 0.853475439 \\
\hline 199 & 9 & 0.788870861 \\
\hline 200 & 10 & 0.174597308 \\
\hline 201 & 7 & 0.113444337 \\
\hline 202 & 1 & 0.695781648 \\
\hline 203 & 0 & 0.030759739 \\
\hline 204 & 7 & 0.224449172 \\
\hline 205 & 30 & 0.350431551 \\
\hline 206 & 0 & 0.978074288 \\
\hline 207 & 3 & 0.384564016 \\
\hline 208 & 0 & 0.425482443 \\
\hline 209 & 16 & 0.141656029 \\
\hline 210 & 30 & 0.279114091 \\
\hline 211 & 4 & 0.595826551 \\
\hline 212 & 95 & 0.168112859 \\
\hline 213 & 14 & 0.313464206 \\
\hline 214 & 1 & 0.691902014 \\
\hline 215 & 68 & 0.673292269 \\
\hline 216 & 0 & 0.077818326 \\
\hline 217 & 2 & 0.485913475 \\
\hline 218 & 12 & 0.953806126 \\
\hline 219 & 10 & 0.835606841 \\
\hline 220 & 3 & 0.800222035 \\
\hline 221 & 28 & 0.79301763 \\
\hline 222 & 19 & 0.734608469 \\
\hline 223 & 15 & 0.960733213 \\
\hline 224 & 28 & 0.878430159 \\
\hline 225 & 15 & 0.753007528 \\
\hline 226 & 26 & 0.391560643 \\
\hline 227 & 7 & 0.414303081 \\
\hline 228 & 8 & 0.065587414 \\
\hline 229 & 11 & 0.623948218 \\
\hline 230 & 8 & 0.893391252 \\
\hline 231 & 8 & 0.049461827 \\
\hline 232 & 28 & 0.397029508 \\
\hline 233 & 11 & 0.073106456 \\
\hline 234 & 16 & 0.891744192 \\
\hline 235 & 15 & 0.377927417 \\
\hline 236 & 13 & 0.123440144 \\
\hline 237 & 76 & 0.628054599 \\
\hline 238 & 76 & 0.732646531 \\
\hline 239 & 33 & 0.476800188 \\
\hline 240 & 0 & 0.576850043 \\
\hline 241 & 14 & 0.917729796 \\
\hline 242 & 95 & 0.177867327 \\
\hline 243 & 0 & 0.625253773 \\
\hline 244 & 11 & 0.82598721 \\
\hline 245 & 0 & 0.141855117 \\
\hline 246 & 31 & 0.779308002 \\
\hline 247 & 26 & 0.290902295 \\
\hline 248 & 30 & 0.056692315 \\
\hline 249 & 0 & 0.444582456 \\
\hline 250 & 94 & 0.340856052 \\
\hline 251 & 94 & 0.190998644 \\
\hline 252 & 95 & 0.686343639 \\
\hline 253 & 99 & 0.593287036 \\
\hline 254 & 0 & 0.680180879 \\
\hline 255 & 7 & 0.14200117 \\
\hline 256 & 11 & 0.428290848 \\
\hline 257 & 4 & 0.595911161 \\
\hline 258 & 95 & 0.14189174 \\
\hline 259 & 99 & 0.760168406 \\
\hline 260 & 13 & 0.298894245 \\
\hline 261 & 9 & 0.043395057 \\
\hline 262 & 26 & 0.725738737 \\
\hline 263 & 7 & 0.743234752 \\
\hline 264 & 0 & 0.656480324 \\
\hline 265 & 96 & 0.22711289 \\
\hline 266 & 42 & 0.125166601 \\
\hline 267 & 37 & 0.10088081 \\
\hline 268 & 1 & 0.645056683 \\
\hline 269 & 0 & 0.88228514 \\
\hline 270 & 10 & 0.082262455 \\
\hline 271 & 12 & 0.801366063 \\
\hline 272 & 0 & 0.177730815 \\
\hline 273 & 19 & 0.001763038 \\
\hline 274 & 26 & 0.765689023 \\
\hline 275 & 31 & 0.137663389 \\
\hline 276 & 14 & 0.493870166 \\
\hline 277 & 5 & 0.038628621 \\
\hline 278 & 0 & 0.661438317 \\
\hline 279 & 10 & 0.176345128 \\
\hline 280 & 33 & 0.69691953 \\
\hline 281 & 0 & 0.091261686 \\
\hline 282 & 12 & 0.695814283 \\
\hline 283 & 11 & 0.62835087 \\
\hline 284 & 9 & 0.446235308 \\
\hline 285 & 19 & 0.306060691 \\
\hline 286 & 5 & 0.343296308 \\
\hline
\end{tabular}

0.001290323

0.001290323

0.001290323

0.001397849
0.001397849

0.001397849

0.001397849

0.001397849

0.001397849

0.001397849

0.001397849

0.001397849

0.001397849

0.001397849

0.001397849

0.001397849

0.001505376
0.001505376

0.001505376

0.001505376

0.001505376

0.001505376

0.001505376

0.001505376

0.001505376

0.001505376

0.001505376

0.001612903

0.001612903

0.001612903

0.001612903

0.00172043

0.00172043

0.00172043

0.00172043

0.00172043

0.00172043

0.00172043

0.00172043

0.00172043

0.00172043

0.00172043

0.00172043

0.00172043

0.00172043

0.00172043

0.00172043

0.001935484

0.001935484

0.001935484

0.002043011

0.00204301

0.00204301

0.002043011

0.00204301

0.002258065

0.002258065

0.002258065

0.002258065

0.002365591

0.002365591

0.002365591

0.002365591

0.002365591

0.002365591

0.002365591

0.002365591

0.002365591

0.002580645

0.002580645

0.002580645

0.002580645

0.002580645

0.002688172

0.002688172

0.002688172

0.002795699

0.002795699

0.002795699

0.002795699

0.002795699

0.002795699

0.002795699

0.002795699

0.002795699

0.002903226 $\begin{array}{lll}0.108709677 & 0.00027\end{array}$

$\begin{array}{ll}0.11 & 0.000273\end{array}$

$\begin{array}{ll}0.111290323 & 0.000277\end{array}$

$\begin{array}{ll}0.112580645 & 0.00028\end{array}$

$\begin{array}{lll}0.113978495 & 0.000283\end{array}$

$\begin{array}{lll}0.115376344 & 0.000287\end{array}$

$\begin{array}{ll}0.116774194 & 0.00029\end{array}$

$\begin{array}{ll}0.118172043 & 0.000294 \\ 0.119569892 & 0.000297\end{array}$

$\begin{array}{lll}0.120967742 & 0.000301\end{array}$

$0.122365591 \quad 0.000304$

$0.123763441 \quad 0.000308$

$\begin{array}{lll}0.12516129 & 0.000311\end{array}$

0.126559140 .000315

0.1279569890 .000318

$\begin{array}{ll}0.129354839 & 0.000322 \\ 0.130752688 & 0.000325\end{array}$

$0.132150538 \quad 0.000329$

$\begin{array}{ll}0.133548387 & 0.000332\end{array}$

$0.135053763 \quad 0.000336$

$\begin{array}{ll}0.13655914 & 0.00034\end{array}$

$0.138064516 \quad 0.000343$

$0.139569892 \quad 0.000347$

$\begin{array}{ll}0.141075269 & 0.000351\end{array}$

$\begin{array}{ll}0.142580645 & 0.000355 \\ 0.144086022 & 0.000358\end{array}$

$0.145591398 \quad 0.000362$

$\begin{array}{lll}0.147096774 & 0.000366\end{array}$

$\begin{array}{ll}0.148602151 & 0.00037\end{array}$

$0.150107527 \quad 0.000373$

$\begin{array}{lll}0.151612903 & 0.000377\end{array}$

$\begin{array}{lll}0.153225806 & 0.000381\end{array}$

$\begin{array}{lll}0.15483871 & 0.000385\end{array}$

$\begin{array}{lll}0.156451613 & 0.000389\end{array}$

$\begin{array}{ll}0.158064516 & 0.000393\end{array}$

$\begin{array}{lll}0.159677419 & 0.000397\end{array}$

$\begin{array}{lll}0.161397849 & 0.000401\end{array}$

0.163118280 .000406

$\begin{array}{ll}0.16483871 & 0.00041\end{array}$

$\begin{array}{lll}0.16655914 & 0.000414\end{array}$

$\begin{array}{lll}0.16827957 & 0.000419\end{array}$

$\begin{array}{ll}0.17 & 0.000423\end{array}$

$\begin{array}{ll}0.17172043 & 0.000427\end{array}$

$0.17344086 \quad 0.000431$

0.175161290 .000436

$\begin{array}{ll}0.17688172 & 0.00044\end{array}$

$\begin{array}{lll}0.178602151 & 0.000444\end{array}$

$0.180322581 \quad 0.000449$

0.1820430110 .000453

0.1837634410 .000457

0.1854838710 .000462

$\begin{array}{lll}0.187204301 & 0.000466\end{array}$

$\begin{array}{ll}0.188924731 & 0.00047\end{array}$

$\begin{array}{lll}0.190860215 & 0.000475\end{array}$

$\begin{array}{ll}0.192795699 & 0.00048\end{array}$

$\begin{array}{lll}0.194731183 & 0.000484\end{array}$

$\begin{array}{lll}0.196666667 & 0.000489\end{array}$

$\begin{array}{lll}0.198709677 & 0.000494\end{array}$

$0.200752688 \quad 0.000499$

$0.202795699 \quad 0.000504$

$\begin{array}{ll}0.20483871 & 0.00051\end{array}$

0.206881720 .000515

$\begin{array}{ll}0.208924731 & 0.00052\end{array}$

$\begin{array}{lll}0.211182796 & 0.000525\end{array}$

$0.21344086 \quad 0.000531$

$\begin{array}{lll}0.215698925 & 0.000536\end{array}$

0.2179569890 .000542

$\begin{array}{lll}0.220322581 & 0.000548\end{array}$

$\begin{array}{ll}0.222688172 & 0.000554\end{array}$

$\begin{array}{ll}0.225053763 & 0.00056\end{array}$

$\begin{array}{ll}0.227419355 & 0.000566\end{array}$

$\begin{array}{lll}0.229784946 & 0.000572\end{array}$

$0.232150538 \quad 0.000577$

$0.234516129 \quad 0.000583$

$\begin{array}{lll}0.23688172 & 0.000589\end{array}$

0.23681720 .000589

$\begin{array}{lll}0.239247312 & 0.000595\end{array}$

$\begin{array}{lll}0.244193548 & 0.000607\end{array}$

$\begin{array}{lll}0.246774194 & 0.000614\end{array}$

0.2493548390 .00062

$\begin{array}{lll}0.251935484 & 0.000627\end{array}$

0.2545161290 .000633

$\begin{array}{ll}0.257204301 & 0.00064\end{array}$

$\begin{array}{lll}0.259892473 & 0.000646\end{array}$

$\begin{array}{lll}0.262580645 & 0.000653\end{array}$

$0.265376344 \quad 0.00066$

$\begin{array}{lll}0.265376344 & 0.00066\end{array}$

$\begin{array}{ll}0.270967742 & 0.000674\end{array}$

$0.273763441 \quad 0.000681$

$0.27655914 \quad 0.000688$

$\begin{array}{lll}0.279354839 & 0.000695\end{array}$

$0.282150538 \quad 0.000702$

$0.284946237 \quad 0.000709$

$0.287741935 \quad 0.000716$

$0.290645161 \quad 0.000723$

$\begin{array}{ll}0.293548387 & 0.00073\end{array}$ 


\begin{tabular}{|c|c|c|}
\hline 287 & 8 & 0.021011572 \\
\hline 288 & 42 & 0.503483508 \\
\hline 289 & 26 & 0.008470266 \\
\hline 290 & 11 & 0.895119299 \\
\hline 291 & 42 & 0.181621804 \\
\hline 292 & 33 & 0.501718151 \\
\hline 293 & 28 & 0.950737445 \\
\hline 294 & 94 & 0.590921956 \\
\hline 295 & 16 & 0.696062747 \\
\hline 296 & 95 & 0.576405065 \\
\hline 297 & 26 & 0.012915697 \\
\hline 298 & 0 & 0.713354013 \\
\hline 299 & 37 & 0.009952816 \\
\hline 300 & 0 & 0.188075975 \\
\hline 301 & 16 & 0.124029285 \\
\hline 302 & 0 & 0.576512308 \\
\hline 303 & 0 & 0.528907891 \\
\hline 304 & 24 & 0.255052694 \\
\hline 305 & 7 & 0.103169989 \\
\hline 306 & 8 & 0.808754865 \\
\hline 307 & 31 & 0.653513708 \\
\hline 308 & 0 & 0.480888524 \\
\hline 309 & 4 & 0.581544841 \\
\hline 310 & 42 & 0.273561428 \\
\hline 311 & 33 & 0.276330295 \\
\hline 312 & 0 & 0.84086016 \\
\hline 313 & 16 & 0.153853847 \\
\hline 314 & 12 & 0.460194549 \\
\hline 315 & 0 & 0.452105569 \\
\hline 316 & 11 & 0.699861662 \\
\hline 317 & 0 & 0.293452428 \\
\hline 318 & 91 & 0.686648714 \\
\hline 319 & 64 & 0.12391828 \\
\hline 320 & 13 & 0.852965392 \\
\hline 321 & 13 & 0.204479979 \\
\hline 322 & 30 & 0.843456863 \\
\hline 323 & 27 & 0.118338474 \\
\hline 324 & 21 & 0.779737909 \\
\hline 325 & 1 & 0.538252786 \\
\hline 326 & 12 & 0.259036681 \\
\hline 327 & 18 & 0.768974217 \\
\hline 328 & 13 & 0.675651104 \\
\hline 329 & 6 & 0.916341325 \\
\hline 330 & 16 & 0.263065504 \\
\hline 331 & 6 & 0.184990825 \\
\hline 332 & 64 & 0.367187458 \\
\hline 333 & 12 & 0.568641957 \\
\hline 334 & 4 & 0.233136353 \\
\hline 335 & 0 & 0.71894078 \\
\hline 336 & 42 & 0.198944408 \\
\hline 337 & 0 & 0.172424667 \\
\hline 338 & 22 & 0.381965537 \\
\hline 339 & 16 & 0.807948247 \\
\hline 340 & 96 & 0.312456914 \\
\hline 341 & 30 & 0.663066664 \\
\hline 342 & 33 & 0.008990328 \\
\hline 343 & 77 & 0.326188732 \\
\hline 344 & 0 & 0.442435382 \\
\hline 345 & 33 & 0.27798358 \\
\hline 346 & 11 & 0.691231974 \\
\hline 347 & 0 & 0.995110504 \\
\hline 348 & 11 & 0.089557165 \\
\hline 349 & 26 & 0.040041698 \\
\hline 350 & 42 & 0.946681907 \\
\hline 351 & 31 & 0.628829792 \\
\hline 352 & 0 & 0.581688645 \\
\hline 353 & 16 & 0.819294772 \\
\hline 354 & 40 & 0.629452343 \\
\hline 355 & 0 & 0.763901992 \\
\hline 356 & 13 & 0.28149729 \\
\hline 357 & 28 & 0.560298097 \\
\hline 358 & 24 & 0.578991004 \\
\hline 359 & 9 & 0.158667796 \\
\hline 360 & 6 & 0.140644551 \\
\hline 361 & 7 & 0.618278464 \\
\hline 362 & 11 & 0.548827947 \\
\hline 363 & 11 & 0.330586836 \\
\hline 364 & 5 & 0.920750128 \\
\hline 365 & 0 & 0.641585668 \\
\hline 366 & 99 & 0.6625827 \\
\hline 367 & 22 & 0.744653681 \\
\hline 368 & 10 & 0.259083141 \\
\hline 369 & 0 & 0.249327271 \\
\hline 370 & 11 & 0.267572489 \\
\hline 371 & 12 & 0.071513474 \\
\hline 372 & 28 & 0.781184723 \\
\hline 373 & 11 & 0.430588197 \\
\hline 374 & 22 & 0.436516846 \\
\hline 375 & 12 & 0.932034504 \\
\hline 376 & 0 & 0.45853662 \\
\hline 377 & 24 & 0.809359363 \\
\hline 378 & 42 & 0.616196649 \\
\hline 379 & 18 & 0.405599247 \\
\hline 380 & 99 & 0.286120855 \\
\hline 381 & 33 & 0.097070092 \\
\hline 382 & 7 & 0.722955222 \\
\hline
\end{tabular}

\begin{tabular}{|c|c|c|}
\hline 8 & 27 & 0.002903226 \\
\hline 42 & 27 & 0.002903226 \\
\hline 26 & 28 & 0.003010753 \\
\hline 11 & 28 & 0.003010753 \\
\hline 42 & 28 & 0.003010753 \\
\hline 33 & 28 & 0.003010753 \\
\hline 28 & 28 & 0.003010753 \\
\hline 94 & 28 & 0.003010753 \\
\hline 16 & 28 & 0.003010753 \\
\hline 95 & 28 & 0.003010753 \\
\hline 26 & 28 & 0.003010753 \\
\hline 0 & 28 & 0.003010753 \\
\hline 37 & 28 & 0.003010753 \\
\hline 0 & 30 & 0.003225806 \\
\hline 16 & 30 & 0.003225806 \\
\hline 0 & 30 & 0.003225806 \\
\hline 0 & 30 & 0.003225806 \\
\hline 24 & 30 & 0.003225806 \\
\hline 7 & 30 & 0.003225806 \\
\hline 8 & 31 & 0.003333333 \\
\hline 31 & 31 & 0.003333333 \\
\hline 0 & 31 & 0.003333333 \\
\hline 4 & 31 & 0.003333333 \\
\hline 42 & 31 & 0.003333333 \\
\hline 33 & 33 & 0.003548387 \\
\hline 0 & 33 & 0.003548387 \\
\hline 16 & 33 & 0.003548387 \\
\hline 12 & 33 & 0.003548387 \\
\hline 0 & 33 & 0.003548387 \\
\hline 11 & 33 & 0.003548387 \\
\hline 0 & 33 & 0.003548387 \\
\hline 91 & 33 & 0.003548387 \\
\hline 64 & 33 & 0.003548387 \\
\hline 13 & 33 & 0.003548387 \\
\hline 13 & 33 & 0.003548387 \\
\hline 30 & 33 & 0.003548387 \\
\hline 27 & 33 & 0.003548387 \\
\hline 21 & 33 & 0.003548387 \\
\hline 1 & 33 & 0.003548387 \\
\hline 12 & 33 & 0.003548387 \\
\hline 18 & 37 & 0.003978495 \\
\hline 13 & 37 & 0.003978495 \\
\hline 6 & 37 & 0.003978495 \\
\hline 16 & 37 & 0.003978495 \\
\hline 6 & 40 & 0.004301075 \\
\hline 64 & 40 & 0.004301075 \\
\hline 12 & 40 & 0.004301075 \\
\hline 4 & 40 & 0.004301075 \\
\hline 0 & 40 & 0.004301075 \\
\hline 42 & 40 & 0.004301075 \\
\hline 0 & 42 & 0.004516129 \\
\hline 22 & 42 & 0.004516129 \\
\hline 16 & 42 & 0.004516129 \\
\hline 96 & 42 & 0.004516129 \\
\hline 30 & 42 & 0.004516129 \\
\hline 33 & 42 & 0.004516129 \\
\hline 77 & 42 & 0.004516129 \\
\hline 0 & 42 & 0.004516129 \\
\hline 33 & 42 & 0.004516129 \\
\hline 11 & 48 & 0.00516129 \\
\hline 0 & 48 & 0.00516129 \\
\hline 11 & 48 & 0.00516129 \\
\hline 26 & 48 & 0.00516129 \\
\hline 42 & 48 & 0.00516129 \\
\hline 31 & 64 & 0.00688172 \\
\hline 0 & 64 & 0.00688172 \\
\hline 16 & 64 & 0.00688172 \\
\hline 40 & 64 & 0.00688172 \\
\hline 0 & 64 & 0.00688172 \\
\hline 13 & 64 & 0.00688172 \\
\hline 28 & 68 & 0.007311828 \\
\hline 24 & 68 & 0.007311828 \\
\hline 9 & 68 & 0.007311828 \\
\hline 6 & 76 & 0.008172043 \\
\hline 7 & 76 & 0.008172043 \\
\hline 11 & 76 & 0.008172043 \\
\hline 11 & 76 & 0.008172043 \\
\hline 5 & 76 & 0.008172043 \\
\hline 0 & 77 & 0.00827957 \\
\hline 99 & 77 & 0.00827957 \\
\hline 22 & 77 & 0.00827957 \\
\hline 10 & 91 & 0.009784946 \\
\hline 0 & 91 & 0.009784946 \\
\hline 11 & 91 & 0.009784946 \\
\hline 12 & 94 & 0.010107527 \\
\hline 28 & 94 & 0.010107527 \\
\hline 11 & 94 & 0.010107527 \\
\hline 22 & 94 & 0.010107527 \\
\hline 12 & 94 & 0.010107527 \\
\hline 0 & 94 & 0.010107527 \\
\hline 24 & 94 & 0.010107527 \\
\hline 42 & 94 & 0.010107527 \\
\hline 18 & 95 & 0.010215054 \\
\hline 99 & 95 & 0.010215054 \\
\hline 33 & 95 & 0.010215054 \\
\hline 7 & 95 & 0.010215054 \\
\hline
\end{tabular}

$0.296451613 \quad 0.000738$ $\begin{array}{lll}0.299354839 & 0.000745\end{array}$ $\begin{array}{ll}0.302365591 & 0.000752\end{array}$ $0.305376344 \quad 0.00076$ $\begin{array}{lll}0.308387097 & 0.000767\end{array}$ $\begin{array}{lll}0.311397849 & 0.000775\end{array}$ $\begin{array}{lll}0.314408602 & 0.000782\end{array}$ $\begin{array}{lll}0.317419355 & 0.00079\end{array}$ $\begin{array}{ll}0.320430108 & 0.000797 \\ 0.32344086 & 0.000805\end{array}$ $\begin{array}{ll}0.32344086 & 0.000805 \\ 0.326451613 & 0.000812\end{array}$ $0.326451613 \quad 0.000812$ $\begin{array}{ll}0.329462366 & 0.00082\end{array}$ 0.3324731180 .000827 $0.335698925 \quad 0.000835$ $\begin{array}{ll}0.338924731 & 0.000843\end{array}$ $\begin{array}{ll}0.342150538 & 0.000851 \\ 0.345376344 & 0.000859\end{array}$ $\begin{array}{ll}0.345376344 & 0.000859 \\ 0.348602151 & 0.000867\end{array}$ $\begin{array}{ll}0.348602151 & 0.000867 \\ 0.351827957 & 0.000876\end{array}$ $0.35516129 \quad 0.000884$ $\begin{array}{ll}0.358494624 & 0.000892\end{array}$ $\begin{array}{lll}0.361827957 & 0.0009\end{array}$ $0.36516129 \quad 0.000909$ $0.368494624 \quad 0.000917$ $\begin{array}{lll}0.372043011 & 0.000926\end{array}$ $\begin{array}{ll}0.375591398 & 0.000935 \\ 0.379139785 & 0.000943\end{array}$ $\begin{array}{ll}0.379139785 & 0.000943 \\ 0.382688172 & 0.000952\end{array}$ 0.3862365590 .000961 $\begin{array}{ll}0.389784946 & 0.00097\end{array}$ 0.3933333330 .000979 $\begin{array}{ll}0.39688172 & 0.000988\end{array}$ $\begin{array}{lll}0.400430108 & 0.000997\end{array}$ $0.403978495 \quad 0.001006$ $\begin{array}{lll}0.407526882 & 0.001014 \\ 0.411075269 & 0.00102\end{array}$ $\begin{array}{ll}0.411075269 & 0.001023 \\ 0.414623656 & 0.001032\end{array}$ $\begin{array}{ll}0.414623656 & 0.001032 \\ 0.418172043 & 0.001041\end{array}$ $\begin{array}{ll}0.42172043 & 0.00105\end{array}$ $0.425268817 \quad 0.001059$ $\begin{array}{ll}0.429247312 & 0.001068 \\ 0.433225806 & 0.001078\end{array}$ $\begin{array}{lll}0.433225806 & 0.001078\end{array}$ $\begin{array}{lll}0.437204301 & 0.001088\end{array}$ $\begin{array}{lll}0.441182796 & 0.001098\end{array}$ $\begin{array}{lll}0.445483871 & 0.001108\end{array}$ $\begin{array}{lll}0.449784946 & 0.001119\end{array}$ $\begin{array}{ll}0.454086022 & 0.00113\end{array}$ $\begin{array}{lll}0.458387097 & 0.001141\end{array}$ $\begin{array}{lll}0.462688172 & 0.001151\end{array}$ $0.466989247 \quad 0.001162$ $\begin{array}{lll}0.471505376 & 0.001173\end{array}$ $\begin{array}{lll}0.476021505 & 0.001184\end{array}$ $\begin{array}{lll}0.480537634 & 0.001196\end{array}$ $\begin{array}{lll}0.485053763 & 0.001207\end{array}$ $\begin{array}{ll}0.485053763 & 0.001207 \\ 0.489569892 & 0.001218\end{array}$ $\begin{array}{rr}0.489569892 & 0.001218 \\ 0.494086022 & 0.00123\end{array}$ $\begin{array}{ll}0.498602151 & 0.001241\end{array}$ $\begin{array}{rr}0.498602151 & 0.001241 \\ 0.50311828 & 0.001252\end{array}$ 0.5076344090 .001263 $\begin{array}{lll}0.512795699 & 0.001276\end{array}$ $\begin{array}{lll}0.517956989 & 0.001288\end{array}$ $\begin{array}{ll}0.52311828 & 0.001301 \\ 0.52827957 & 0.001314\end{array}$ $\begin{array}{ll}0.52827957 & 0.001314 \\ 0.5334086 & 0.001327\end{array}$ $\begin{array}{lll}0.53344086 & 0.001327\end{array}$ $\begin{array}{lll}0.540322581 & 0.001342\end{array}$ $\begin{array}{ll}0.540322581 & 0.001342 \\ 0.547204301 & 0.001359\end{array}$ $\begin{array}{ll}0.547204301 & 0.001359 \\ 0.554086022 & 0.001377\end{array}$ $\begin{array}{ll}0.560967742 & 0.001394\end{array}$ $\begin{array}{ll}0.567849462 & 0.001411\end{array}$ $\begin{array}{lll}0.574731183 & 0.001428\end{array}$ 0.5820430110 .001446 $\begin{array}{lll}0.589354839 & 0.001464\end{array}$ $\begin{array}{ll}0.596666667 & 0.001483\end{array}$ $\begin{array}{ll}0.60483871 & 0.001502\end{array}$ $\begin{array}{lll}0.613010753 & 0.001522\end{array}$ $\begin{array}{ll}0.621182796 & 0.001543\end{array}$ $\begin{array}{lll}0.629354839 & 0.001563\end{array}$ $\begin{array}{ll}0.637526882 & 0.001584\end{array}$ 0.6458064520 .001604 0.6540860220 .001625 0.6623655910 .001646 $\begin{array}{ll}0.672150538 & 0.001668\end{array}$ $\begin{array}{ll}0.681935484 & 0.001693 \\ 0.69172043 & 0.001717\end{array}$ $\begin{array}{ll}0.69172043 & 0.001717 \\ 0.701827957 & 0.001742\end{array}$ $\begin{array}{ll}0.701827957 & 0.001742 \\ 0.711935484 & 0.001767\end{array}$ $\begin{array}{ll}0.711935484 & 0.001767 \\ 0.722043011 & 0.001792\end{array}$ $\begin{array}{ll}0.722043011 & 0.001792 \\ 0.732150538 & 0.001818\end{array}$ $\begin{array}{ll}0.732150538 & 0.001818 \\ 0.742258065 & 0.001843\end{array}$ $\begin{array}{ll}0.742258065 & 0.001843 \\ 0.752365591 & 0.001868\end{array}$ $\begin{array}{ll}0.752365591 & 0.001868 \\ 0.762473118 & 0.001894\end{array}$ $\begin{array}{ll}0.762473118 & 0.001894 \\ 0.772580645 & 0.001919\end{array}$ $0.782795699 \quad 0.001944$ $\begin{array}{ll}0.793010753 & 0.00197\end{array}$ $\begin{array}{lll}0.803225806 & 0.001995\end{array}$ $0.81344086 \quad 0.002021$ 


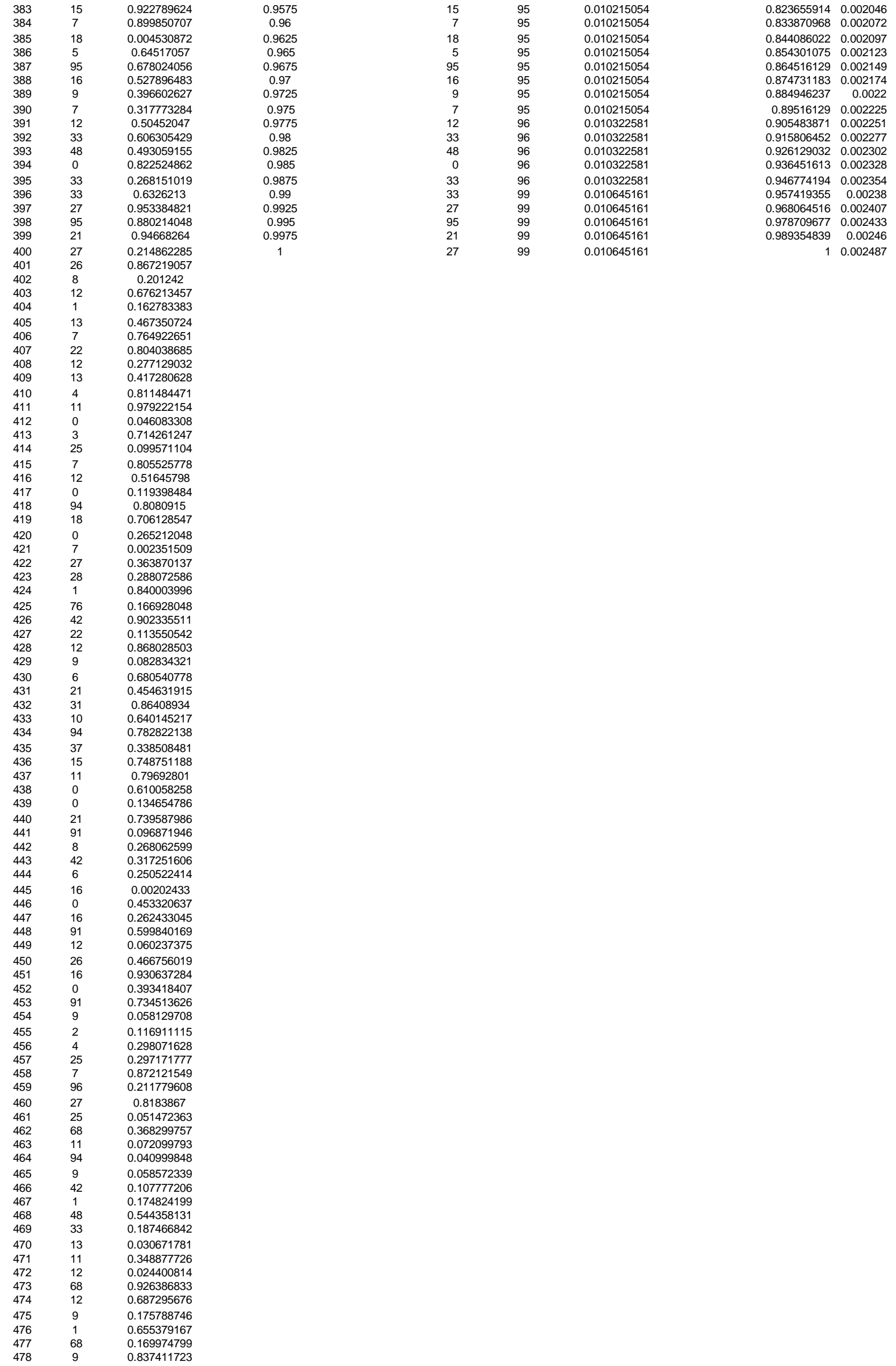




$\begin{array}{lcc}479 & 37 & 0.449236806 \\ 480 & 0 & 0.085206153 \\ 481 & 3 & 0.559639564 \\ 482 & 12 & 0.314254425 \\ 483 & 10 & 0.752213258 \\ 484 & 11 & 0.089931972 \\ 485 & 0 & 0.929851235 \\ 486 & 77 & 0.526185358 \\ 487 & 0 & 0.22761854 \\ 488 & 11 & 0.538876205 \\ 489 & 16 & 0.825439592 \\ 490 & 18 & 0.70646685 \\ 491 & 9 & 0.432694748 \\ 492 & 26 & 0.690326299 \\ 493 & 16 & 0.679338146 \\ 494 & 9 & 0.098361094 \\ 495 & 77 & 0.312415899 \\ 496 & 9 & 0.15225266 \\ 497 & 33 & 0.0485911 \\ 498 & 94 & 0.106260578 \\ 499 & 77 & 0.314224472 \\ 500 & 9 & 0.377271951 \\ 501 & 16 & 0.852851271 \\ 502 & 0 & 0.389426875 \\ 503 & 99 & 0.916512113 \\ 504 & 16 & 0.674222942 \\ 505 & 24 & 0.333321511 \\ 506 & 0 & 0.504593874 \\ 507 & 0 & 0.851295126 \\ 508 & 9 & 0.066994933 \\ 509 & 10 & 0.544978766 \\ 510 & 11 & 0.1450492\end{array}$

total inhibition

9300

Total area $\quad 0.215729$

Gini 0.568542 
AG1024; $10 \mu \mathrm{M} ; 10 \mu \mathrm{M}$ ATP; sample of 300 kinases

\begin{tabular}{|c|c|c|c|}
\hline position & inhibition \% & rand & cumulative sample fraction \\
\hline 1 & 0 & 0.272615576 & 0.003333333 \\
\hline 2 & 0 & 0.034219926 & 0.0066666667 \\
\hline 3 & 40 & 0.971256154 & 0.01 \\
\hline 4 & 24 & 0.883066374 & 0.013333333 \\
\hline 5 & 12 & 0.705135004 & 0.016666666 \\
\hline 6 & 28 & 0.399842322 & 0.02 \\
\hline 7 & 26 & 0.102147465 & 0.023333333 \\
\hline 8 & 3 & 0.411023687 & 0.0266666667 \\
\hline 9 & 0 & 0.240553446 & 0.03 \\
\hline 10 & 19 & 0.535599308 & 0.033333333 \\
\hline 11 & 12 & 0.646559543 & 0.036666667 \\
\hline 12 & 24 & 0.233813878 & 0.04 \\
\hline 13 & 22 & 0.915119699 & 0.043333333 \\
\hline 14 & 12 & 0.26692418 & 0.046666667 \\
\hline 15 & 4 & 0.611322774 & 0.05 \\
\hline 16 & 7 & 0.623954219 & 0.053333333 \\
\hline 17 & 10 & 0.981097783 & 0.056666667 \\
\hline 18 & 33 & 0.142392524 & 0.06 \\
\hline 19 & 19 & 0.904253364 & 0.063333333 \\
\hline 20 & 11 & 0.004221264 & 0.0666666667 \\
\hline 21 & 99 & 0.095021704 & 0.07 \\
\hline 22 & 40 & 0.166561152 & 0.073333333 \\
\hline 23 & 77 & 0.359169193 & 0.076666667 \\
\hline 24 & 95 & 0.469347073 & 0.08 \\
\hline 25 & 11 & 0.385622739 & 0.083333333 \\
\hline 26 & 11 & 0.658597141 & 0.0866666667 \\
\hline 27 & 7 & 0.568392385 & 0.09 \\
\hline 28 & 2 & 0.335282948 & 0.093333333 \\
\hline 29 & 77 & 0.037341008 & 0.096666667 \\
\hline 30 & 4 & 0.87748236 & 0.1 \\
\hline 31 & 5 & 0.558593023 & 0.103333333 \\
\hline 32 & 28 & 0.448528432 & 0.106666667 \\
\hline 33 & 33 & 0.387491874 & 0.11 \\
\hline 34 & 22 & 0.97424925 & 0.113333333 \\
\hline 35 & 10 & 0.424678393 & 0.1166666667 \\
\hline 36 & 12 & 0.884243045 & 0.12 \\
\hline 37 & 9 & 0.364646399 & 0.123333333 \\
\hline 38 & 30 & 0.02627458 & 0.126666667 \\
\hline 39 & 12 & 0.407239439 & 0.13 \\
\hline 40 & 14 & 0.938279136 & 0.133333333 \\
\hline 41 & 26 & 0.793241116 & 0.136666667 \\
\hline 42 & 33 & 0.388230108 & 0.14 \\
\hline 43 & 0 & 0.846250662 & 0.143333333 \\
\hline 44 & 9 & 0.071954088 & 0.146666667 \\
\hline 45 & 37 & 0.527187808 & 0.15 \\
\hline 46 & 7 & 0.172111279 & 0.153333333 \\
\hline 47 & 42 & 0.771513149 & 0.156666667 \\
\hline 48 & 33 & 0.308005766 & 0.16 \\
\hline 49 & 0 & 0.891412858 & 0.163333333 \\
\hline 50 & 12 & 0.467452381 & 0.166666667 \\
\hline 51 & 6 & 0.914881652 & 0.17 \\
\hline 52 & 8 & 0.474536507 & 0.173333333 \\
\hline 53 & 95 & 0.901063278 & 0.176666667 \\
\hline 54 & 16 & 0.16869233 & 0.18 \\
\hline 55 & 16 & 0.157443537 & 0.183333333 \\
\hline 56 & 5 & 0.925563842 & 0.186666667 \\
\hline 57 & 42 & 0.898008869 & 0.19 \\
\hline 58 & 16 & 0.107413879 & 0.193333333 \\
\hline 59 & 9 & 0.755786983 & 0.196666667 \\
\hline 60 & 94 & 0.169625825 & 0.2 \\
\hline 61 & 13 & 0.085051752 & 0.203333333 \\
\hline 62 & 7 & 0.088394762 & 0.206666667 \\
\hline 63 & 1 & 0.669961305 & 0.21 \\
\hline 64 & 91 & 0.29045423 & 0.213333333 \\
\hline 65 & 0 & 0.642817529 & 0.216666667 \\
\hline 66 & 0 & 0.257464089 & 0.22 \\
\hline 67 & 4 & 0.209975852 & 0.223333333 \\
\hline 68 & 12 & 0.85715015 & 0.226666667 \\
\hline 69 & 77 & 0.515115967 & 0.23 \\
\hline 70 & 15 & 0.898382484 & 0.233333333 \\
\hline 71 & 15 & 0.056748119 & 0.236666667 \\
\hline 72 & 64 & 0.468443096 & 0.24 \\
\hline 73 & 7 & 0.483963706 & 0.243333333 \\
\hline 74 & 4 & 0.681516132 & 0.2466666667 \\
\hline 75 & 10 & 0.253675935 & 0.25 \\
\hline 76 & 7 & 0.607310908 & 0.253333333 \\
\hline 77 & 68 & 0.352271655 & 0.256666667 \\
\hline 78 & 7 & 0.144685032 & 0.26 \\
\hline 79 & 4 & 0.529146155 & 0.263333333 \\
\hline 80 & 11 & 0.80159376 & 0.2666666667 \\
\hline 81 & 4 & 0.073467767 & 0.27 \\
\hline 82 & 13 & 0.732054065 & 0.273333333 \\
\hline 83 & 1 & 0.24159102 & 0.276666667 \\
\hline 84 & 28 & 0.344615356 & 0.28 \\
\hline 85 & 11 & 0.445673 & 0.283333333 \\
\hline 86 & 42 & 0.962842706 & 0.286666667 \\
\hline 87 & 94 & 0.509714088 & 0.29 \\
\hline 88 & 0 & 0.242191493 & 0.293333333 \\
\hline 89 & 95 & 0.899087083 & 0.2966666667 \\
\hline 90 & 13 & 0.479217155 & 0.3 \\
\hline 91 & 33 & 0.549615614 & 0.303333333 \\
\hline 92 & 76 & 0.168614346 & 0.306666667 \\
\hline 93 & 99 & 0.158634254 & 0.31 \\
\hline 94 & 8 & 0.080822507 & 0.3133333333 \\
\hline
\end{tabular}




\begin{tabular}{|c|c|c|}
\hline 95 & 8 & 0.177255232 \\
\hline 96 & 0 & 0.80259816 \\
\hline 97 & 22 & 0.284834097 \\
\hline 98 & 15 & 0.854331829 \\
\hline 99 & 0 & 0.022612818 \\
\hline 100 & 2 & 0.452534207 \\
\hline 101 & 19 & 0.980960999 \\
\hline 102 & 0 & 0.862789514 \\
\hline 103 & 37 & 0.247188156 \\
\hline 104 & 10 & 0.371348135 \\
\hline 105 & 9 & 0.987340905 \\
\hline 106 & 0 & 0.575758664 \\
\hline 107 & 0 & 0.426143451 \\
\hline 108 & 0 & 0.781969606 \\
\hline 109 & 0 & 0.557753806 \\
\hline 110 & 0 & 0.224253076 \\
\hline 111 & 16 & 0.854325448 \\
\hline 112 & 33 & 0.975233204 \\
\hline 113 & 76 & 0.895691859 \\
\hline 114 & 0 & 0.57856636 \\
\hline 115 & 42 & 0.147907377 \\
\hline 116 & 4 & 0.149498549 \\
\hline 117 & 64 & 0.214388276 \\
\hline 118 & 33 & 0.345814783 \\
\hline 119 & 5 & 0.972604706 \\
\hline 120 & 94 & 0.224497767 \\
\hline 121 & 76 & 0.733302386 \\
\hline 122 & 68 & 0.397707272 \\
\hline 123 & 1 & 0.696354384 \\
\hline 124 & 27 & 0.759078521 \\
\hline 125 & 26 & 0.824567792 \\
\hline 126 & 96 & 0.506951177 \\
\hline 127 & 42 & 0.395509738 \\
\hline 128 & 27 & 0.231091284 \\
\hline 129 & 91 & 0.79646769 \\
\hline 130 & 0 & 0.209012306 \\
\hline 131 & 0 & 0.023646092 \\
\hline 132 & 9 & 0.947313803 \\
\hline 133 & 7 & 0.593770619 \\
\hline 134 & 0 & 0.318283233 \\
\hline 135 & 18 & 0.706383757 \\
\hline 136 & 1 & 0.320159406 \\
\hline 137 & 24 & 0.657314241 \\
\hline 138 & 64 & 0.461514069 \\
\hline 139 & 11 & 0.103537791 \\
\hline 140 & 14 & 0.831114541 \\
\hline 141 & 28 & 0.590526559 \\
\hline 142 & 8 & 0.793735028 \\
\hline 143 & 18 & 0.524697559 \\
\hline 144 & 24 & 0.402494337 \\
\hline 145 & 0 & 0.120800934 \\
\hline 146 & 28 & 0.801584107 \\
\hline 147 & 99 & 0.855406832 \\
\hline 148 & 14 & 0.626285591 \\
\hline 149 & 9 & 0.11228047 \\
\hline 150 & 40 & 0.553415103 \\
\hline 151 & 0 & 0.974110107 \\
\hline 152 & 0 & 0.036500492 \\
\hline 153 & 6 & 0.123081492 \\
\hline 154 & 11 & 0.491700185 \\
\hline 155 & 16 & 0.537137209 \\
\hline 156 & 8 & 0.042748383 \\
\hline 157 & 28 & 0.271809751 \\
\hline 158 & 4 & 0.131829613 \\
\hline 159 & 91 & 0.409422989 \\
\hline 160 & 91 & 0.547598143 \\
\hline 161 & 9 & 0.694076973 \\
\hline 162 & 16 & 0.633187264 \\
\hline 163 & 0 & 0.990158128 \\
\hline 164 & 5 & 0.143367839 \\
\hline 165 & 0 & 0.948936082 \\
\hline 166 & 4 & 0.350659749 \\
\hline 167 & 27 & 0.242787823 \\
\hline 168 & 33 & 0.695086073 \\
\hline 169 & 33 & 0.158905769 \\
\hline 170 & 22 & 0.4055333395 \\
\hline 171 & 9 & 0.795794518 \\
\hline 172 & 16 & 0.414387028 \\
\hline 173 & 48 & 0.630726597 \\
\hline 174 & 33 & 0.467352001 \\
\hline 175 & 12 & 0.695182233 \\
\hline 176 & 11 & 0.162466309 \\
\hline 177 & 8 & 0.297163316 \\
\hline 178 & 9 & 0.232619275 \\
\hline 179 & 76 & 0.05047873 \\
\hline 180 & 26 & 0.108878806 \\
\hline 181 & 3 & 0.559147968 \\
\hline 182 & 1 & 0.315109968 \\
\hline 183 & 26 & 0.792351336 \\
\hline 184 & 16 & 0.412148292 \\
\hline 185 & 22 & 0.255471835 \\
\hline 186 & 13 & 0.178005821 \\
\hline 187 & 42 & 0.149585591 \\
\hline 188 & 25 & 0.043480077 \\
\hline 189 & 11 & 0.927557484 \\
\hline 190 & 22 & 0.991661406 \\
\hline
\end{tabular}

$0.03547392 \quad 0.000116$ $\begin{array}{ll}0.036735838 & 0.00012\end{array}$ $\begin{array}{ll}0.037997757 & 0.000125\end{array}$ $\begin{array}{lll}0.039259675 & 0.000129\end{array}$ $0.040521593 \quad 0.000133$ $\begin{array}{lll}0.041783511 & 0.000137\end{array}$ $0.043045429 \quad 0.000141$ $0.044307347 \quad 0.000146$ $\begin{array}{ll}0.045569265 & 0.00015\end{array}$ $\begin{array}{lll}0.046831183 & 0.000154\end{array}$ $0.048093102 \quad 0.000158$ $\begin{array}{ll}0.04935502 & 0.000162\end{array}$ $\begin{array}{lll}0.050616938 & 0.000167\end{array}$ $\begin{array}{lll}0.052019069 & 0.000171\end{array}$ 0.05342120 .000176 $0.054823331 \quad 0.00018$ $\begin{array}{ll}0.056225463 & 0.000185\end{array}$ $\begin{array}{ll}0.057627594 & 0.00019\end{array}$ $0.059029725 \quad 0.000194$ $\begin{array}{lll}0.060431856 & 0.000199\end{array}$ $\begin{array}{lll}0.061833988 & 0.000204\end{array}$ $\begin{array}{lll}0.063376332 & 0.000209\end{array}$ $\begin{array}{lll}0.064918676 & 0.000214\end{array}$ $\begin{array}{ll}0.066461021 & 0.000219\end{array}$ $\begin{array}{lll}0.068003365 & 0.000224\end{array}$ $\begin{array}{ll}0.069545709 & 0.000229 \\ 0.071088054 & 0.000234\end{array}$ $\begin{array}{rr}0.071088054 & 0.000234 \\ 0.072630398 & 0.00024\end{array}$ $0.074172743 \quad 0.000245$ $\begin{array}{ll}0.075715087 & 0.00025\end{array}$ $\begin{array}{lll}0.077257431 & 0.000255\end{array}$ $\begin{array}{lll}0.078799776 & 0.00026\end{array}$ 0.080342120 .000265 $\begin{array}{ll}0.081884464 & 0.00027\end{array}$ $0.083426809 \quad 0.000276$ $\begin{array}{lll}0.084969153 & 0.000281\end{array}$ $\begin{array}{lll}0.086511497 & 0.000286\end{array}$ $\begin{array}{ll}0.088053842 & 0.000291\end{array}$ $0.089596186 \quad 0.000296$ $0.091138531 \quad 0.000301$ $0.092680875 \quad 0.000306$ $\begin{array}{lll}0.094363432 & 0.000312\end{array}$ $\begin{array}{lll}0.09604599 & 0.000317\end{array}$ $\begin{array}{lll}0.097728547 & 0.000323\end{array}$ $\begin{array}{lll}0.099411105 & 0.000329\end{array}$ $\begin{array}{lll}0.101093662 & 0.000334\end{array}$ $\begin{array}{ll}0.10277622 & 0.00034\end{array}$ $\begin{array}{ll}0.104458777 & 0.000345\end{array}$ 0.1061413350 .000351 $\begin{array}{ll}0.107823892 & 0.000357\end{array}$ $0.10950645 \quad 0.000362$ $\begin{array}{lll}0.111189007 & 0.000368\end{array}$ $\begin{array}{lll}0.112871565 & 0.000373\end{array}$ $0.114554122 \quad 0.000379$ $\begin{array}{ll}0.114554122 & 0.000379 \\ 0.116376893 & 0.000385\end{array}$ $\begin{array}{ll}0.116376893 & 0.000385 \\ 0.118199663 & 0.000391\end{array}$ $\begin{array}{ll}0.120022434 & 0.000397\end{array}$ $\begin{array}{lll}0.121845205 & 0.000403\end{array}$ $\begin{array}{lll}0.123667975 & 0.000409\end{array}$ $\begin{array}{ll}0.125490746 & 0.000415\end{array}$ $0.127313517 \quad 0.000421$ $\begin{array}{lll}0.129136287 & 0.000427\end{array}$ $0.130959058 \quad 0.000433$ $0.132922042 \quad 0.00044$ $\begin{array}{ll}0.134885025 & 0.000446\end{array}$ $\begin{array}{ll}0.134885025 & 0.000446 \\ 0.136848009 & 0.000453\end{array}$ $\begin{array}{lll}0.138810993 & 0.000459\end{array}$ $\begin{array}{ll}0.140773976 & 0.000466\end{array}$ $0.14273696 \quad 0.000473$ $0.144699944 \quad 0.000479$ 0.1468031410 .000486 0.1489063380 .000493 $\begin{array}{ll}0.151009534 & 0.0005\end{array}$ $\begin{array}{lll}0.153112731 & 0.000507\end{array}$ $\begin{array}{lll}0.155215928 & 0.000514\end{array}$ $\begin{array}{ll}0.157319125 & 0.000521\end{array}$ $\begin{array}{lll}0.159562535 & 0.000528\end{array}$ $0.161805945 \quad 0.000536$ $0.164049355 \quad 0.000543$ $0.166292765 \quad 0.000551$ $0.168536175 \quad 0.000558$ 0.1707795850 .000566 $0.173022995 \quad 0.000573$ $\begin{array}{lll}0.175266405 & 0.00058\end{array}$ $\begin{array}{lll}0.177509815 & 0.000588\end{array}$ $0.179753225 \quad 0.000595$ 0.1819966350 .000603 $\begin{array}{ll}0.184240045 & 0.00061\end{array}$ $\begin{array}{ll}0.186483455 & 0.000618\end{array}$ 0.1890072910 .000626 $0.191531127 \quad 0.000634$ $\begin{array}{lll}0.194054964 & 0.000643\end{array}$ $0.196719013 \quad 0.000651$ $0.199383062 \quad 0.00066$ $0.202047112 \quad 0.000669$ $\begin{array}{lll}0.204991587 & 0.000678\end{array}$ 


\begin{tabular}{|c|c|c|}
\hline 191 & 77 & 0.203523901 \\
\hline 192 & 9 & 0.455525283 \\
\hline 193 & 48 & 0.368730629 \\
\hline 194 & 1 & 0.260273929 \\
\hline 195 & 12 & 0.004653055 \\
\hline 196 & 31 & 0.483159393 \\
\hline 197 & 12 & 0.658601944 \\
\hline 198 & 11 & 0.792237501 \\
\hline 199 & 11 & 0.522600439 \\
\hline 200 & 0 & 0.113499706 \\
\hline 201 & 99 & 0.687906539 \\
\hline 202 & 25 & 0.0298597 \\
\hline 203 & 99 & 0.281260048 \\
\hline 204 & 7 & 0.767636778 \\
\hline 205 & 40 & 0.991126666 \\
\hline 206 & 11 & 0.332588981 \\
\hline 207 & 0 & 0.242958565 \\
\hline 208 & 30 & 0.185210848 \\
\hline 209 & 11 & 0.136752356 \\
\hline 210 & 33 & 0.538288853 \\
\hline 211 & 13 & 0.876528653 \\
\hline 212 & 9 & 0.254117339 \\
\hline 213 & 13 & 0.134602944 \\
\hline 214 & 3 & 0.837747208 \\
\hline 215 & 33 & 0.339020531 \\
\hline 216 & 16 & 0.777057072 \\
\hline 217 & 15 & 0.049082013 \\
\hline 218 & 11 & 0.970617419 \\
\hline 219 & 9 & 0.597370482 \\
\hline 220 & 14 & 0.105639292 \\
\hline 221 & 96 & 0.145533526 \\
\hline 222 & 18 & 0.649581496 \\
\hline 223 & 91 & 0.88364867 \\
\hline 224 & 7 & 0.306561318 \\
\hline 225 & 96 & 0.924740917 \\
\hline 226 & 27 & 0.734940842 \\
\hline 227 & 33 & 0.862144529 \\
\hline 228 & 0 & 0.363108352 \\
\hline 229 & 15 & 0.114021574 \\
\hline 230 & 91 & 0.479864027 \\
\hline 231 & 0 & 0.160701257 \\
\hline 232 & 0 & 0.540159613 \\
\hline 233 & 26 & 0.837618182 \\
\hline 234 & 26 & 0.796469563 \\
\hline 235 & 33 & 0.467091108 \\
\hline 236 & 94 & 0.810017728 \\
\hline 237 & 14 & 0.655651952 \\
\hline 238 & 94 & 0.038950506 \\
\hline 239 & 11 & 0.321346178 \\
\hline 240 & 13 & 0.458103302 \\
\hline 241 & 10 & 0.972650624 \\
\hline 242 & 22 & 0.870046162 \\
\hline 243 & 0 & 0.32158419 \\
\hline 244 & 16 & 0.209048984 \\
\hline 245 & 11 & 0.911353039 \\
\hline 246 & 16 & 0.753071957 \\
\hline 247 & 64 & 0.56779446 \\
\hline 248 & 11 & 0.041324357 \\
\hline 249 & 10 & 0.009483434 \\
\hline 250 & 95 & 0.380422768 \\
\hline 251 & 31 & 0.311968016 \\
\hline 252 & 99 & 0.457592099 \\
\hline 253 & 0 & 0.750656822 \\
\hline 254 & 7 & 0.793675086 \\
\hline 255 & 0 & 0.659712777 \\
\hline 256 & 7 & 0.236339437 \\
\hline 257 & 10 & 0.367213455 \\
\hline 258 & 11 & 0.533369536 \\
\hline 259 & 7 & 0.778400868 \\
\hline 260 & 1 & 0.534470815 \\
\hline 261 & 0 & 0.847777384 \\
\hline 262 & 13 & 0.157150101 \\
\hline 263 & 48 & 0.710505601 \\
\hline 264 & 4 & 0.603995117 \\
\hline 265 & 0 & 0.483701126 \\
\hline 266 & 1 & 0.128060938 \\
\hline 267 & 76 & 0.914519943 \\
\hline 268 & 12 & 0.78881306 \\
\hline 269 & 6 & 0.131721148 \\
\hline 270 & 14 & 0.96070535 \\
\hline 271 & 0 & 0.382845768 \\
\hline 272 & 22 & 0.76018262 \\
\hline 273 & 11 & 0.092572691 \\
\hline 274 & 37 & 0.136718071 \\
\hline 275 & 10 & 0.576065528 \\
\hline 276 & 0 & 0.485429842 \\
\hline 277 & 11 & 0.111077556 \\
\hline 278 & 95 & 0.67106122 \\
\hline 279 & 15 & 0.276362372 \\
\hline 280 & 0 & 0.306681995 \\
\hline 281 & 9 & 0.120848911 \\
\hline 282 & 0 & 0.539795849 \\
\hline 283 & 1 & 0.400053003 \\
\hline 284 & 0 & 0.909692481 \\
\hline 285 & 68 & 0.701893362 \\
\hline 286 & 37 & 0.496440857 \\
\hline
\end{tabular}

$0.208076276 \quad 0.000688$ $\begin{array}{lll}0.211160965 & 0.000699\end{array}$ $\begin{array}{ll}0.214245653 & 0.000709\end{array}$ $\begin{array}{ll}0.217330342 & 0.000719\end{array}$ $\begin{array}{ll}0.220415031 & 0.00073\end{array}$ $\begin{array}{lll}0.22349972 & 0.00074\end{array}$ $\begin{array}{ll}0.226584408 & 0.00075\end{array}$ $0.229669097 \quad 0.00076$ $\begin{array}{lll}0.233034212 & 0.000771\end{array}$ $\begin{array}{lll}0.236399327 & 0.000782\end{array}$ $0.239764442 \quad 0.000794$ $0.243129557 \quad 0.000805$ $\begin{array}{lll}0.246494672 & 0.000816\end{array}$ $\begin{array}{ll}0.25 & 0.000827\end{array}$ $0.253505328 \quad 0.000839$ $\begin{array}{lll}0.257150869 & 0.000851\end{array}$ $\begin{array}{lll}0.260796411 & 0.000863\end{array}$ $\begin{array}{lll}0.264441952 & 0.000875\end{array}$ $0.268087493 \quad 0.000888$ $\begin{array}{lr}0.271733034 & 0.0009\end{array}$ $\begin{array}{ll}0.275378575 & 0.000912\end{array}$ $\begin{array}{lll}0.279024117 & 0.000924\end{array}$ $0.282669658 \quad 0.000936$ 0.2864554120 .000949 $\begin{array}{lll}0.290241167 & 0.000961\end{array}$ $\begin{array}{ll}0.294026921 & 0.000974 \\ 0.297812675 & 0.000986\end{array}$ $\begin{array}{ll}0.297812675 & 0.000986 \\ 0.301738643 & 0.000999\end{array}$ $\begin{array}{ll}0.30566461 & 0.001012\end{array}$ $\begin{array}{ll}0.309590578 & 0.001025 \\ 0.313516545 & 0.001039\end{array}$ $\begin{array}{ll}0.313516545 & 0.001039 \\ 0.317442513 & 0.001052\end{array}$ $0.32136848 \quad 0.001065$ $\begin{array}{rr}0.32136848 & 0.001065 \\ 0.325574874 & 0.001078\end{array}$ $\begin{array}{ll}0.325574874 & 0.001078 \\ 0.329781268 & 0.001092\end{array}$ $\begin{array}{ll}0.329781268 & 0.001092 \\ 0.334127874 & 0.001107\end{array}$ $\begin{array}{ll}0.334127874 & 0.001107 \\ 0.338474481 & 0.001121\end{array}$ $\begin{array}{ll}0.338474481 & 0.001121 \\ 0.342821088 & 0.001135\end{array}$ $\begin{array}{ll}0.347448121 & 0.00115\end{array}$ $0.352075154 \quad 0.001166$ $\begin{array}{ll}0.356702187 & 0.001181\end{array}$ $0.36132922 \quad 0.001197$ $\begin{array}{lll}0.365956254 & 0.001212\end{array}$ 0.3705832870 .001228 0.375210320 .001243 $\begin{array}{ll}0.379837353 & 0.001258 \\ 0.384464386 & 0.001274\end{array}$ $\begin{array}{ll}0.384464386 & 0.001274 \\ 0.389091419 & 0.001289\end{array}$ 0.3937184520 .001305 $0.398345485 \quad 0.00132$ $0.402972518 \quad 0.001336$ $\begin{array}{ll}0.407599551 & 0.001351\end{array}$ $\begin{array}{lll}0.412787437 & 0.001367\end{array}$ $0.417975322 \quad 0.001385$ $\begin{array}{ll}0.423163208 & 0.001402\end{array}$ $\begin{array}{ll}0.423163208 & 0.001402 \\ 0.428351094 & 0.001419\end{array}$ $\begin{array}{ll}0.428351094 & 0.001419 \\ 0.433959619 & 0.001437\end{array}$ $\begin{array}{lll}0.439568144 & 0.001456\end{array}$ $\begin{array}{lll}0.445176669 & 0.001475\end{array}$ $\begin{array}{ll}0.450785193 & 0.001493\end{array}$ $\begin{array}{ll}0.456674145 & 0.001512\end{array}$ $0.462563096 \quad 0.001532$ $\begin{array}{lll}0.468452047 & 0.001552\end{array}$ $\begin{array}{lll}0.474340998 & 0.001571\end{array}$ 0.480229950 .001591 $\begin{array}{rr}0.48022995 & 0.001591 \\ 0.486118901 & 0.001611\end{array}$ $\begin{array}{ll}0.486118901 & 0.001611 \\ 0.492849131 & 0.001632\end{array}$ $\begin{array}{ll}0.492849131 & 0.001632 \\ 0.499579361 & 0.001654\end{array}$ 0.5063095910 .001676 $\begin{array}{lll}0.515283231 & 0.001703\end{array}$ $0.52425687 \quad 0.001733$ $0.53323051 \quad 0.001762$ $\begin{array}{ll}0.54220415 & 0.001792\end{array}$ \begin{tabular}{lll}
0.551738643 & 0.001823 \\
\hline
\end{tabular} $\begin{array}{lll}0.561273135 & 0.001855\end{array}$ $\begin{array}{ll}0.570807628 & 0.001887\end{array}$ $\begin{array}{ll}0.581463825 & 0.00192\end{array}$ $\begin{array}{rr}0.581463825 & 0.00192 \\ 0.592120022 & 0.001956\end{array}$ $\begin{array}{rr}0.592120022 & 0.001956 \\ 0.60277622 & 0.001991\end{array}$ $\begin{array}{rr}0.60277622 & 0.001991 \\ 0.613432417 & 0.002027\end{array}$ $\begin{array}{ll}0.624088615 & 0.002063\end{array}$ $\begin{array}{lll}0.634885025 & 0.002098\end{array}$ $\begin{array}{lll}0.645681436 & 0.002134\end{array}$ $\begin{array}{ll}0.656477846 & 0.00217\end{array}$ $0.667274257 \quad 0.002206$ $0.680033651 \quad 0.002246$ $0.692793045 \quad 0.002288$ $0.70555244 \quad 0.002331$ $0.718311834 \quad 0.002373$ $0.731071228 \quad 0.002416$ $\begin{array}{ll}0.743830623 & 0.002458\end{array}$ $\begin{array}{rl}0.757010656 & 0.002501\end{array}$ $\begin{array}{lll}0.77019069 & 0.002545\end{array}$ $0.783370723 \quad 0.002589$ $0.796550757 \quad 0.002633$ $\begin{array}{lll}0.809730791 & 0.002677\end{array}$ 


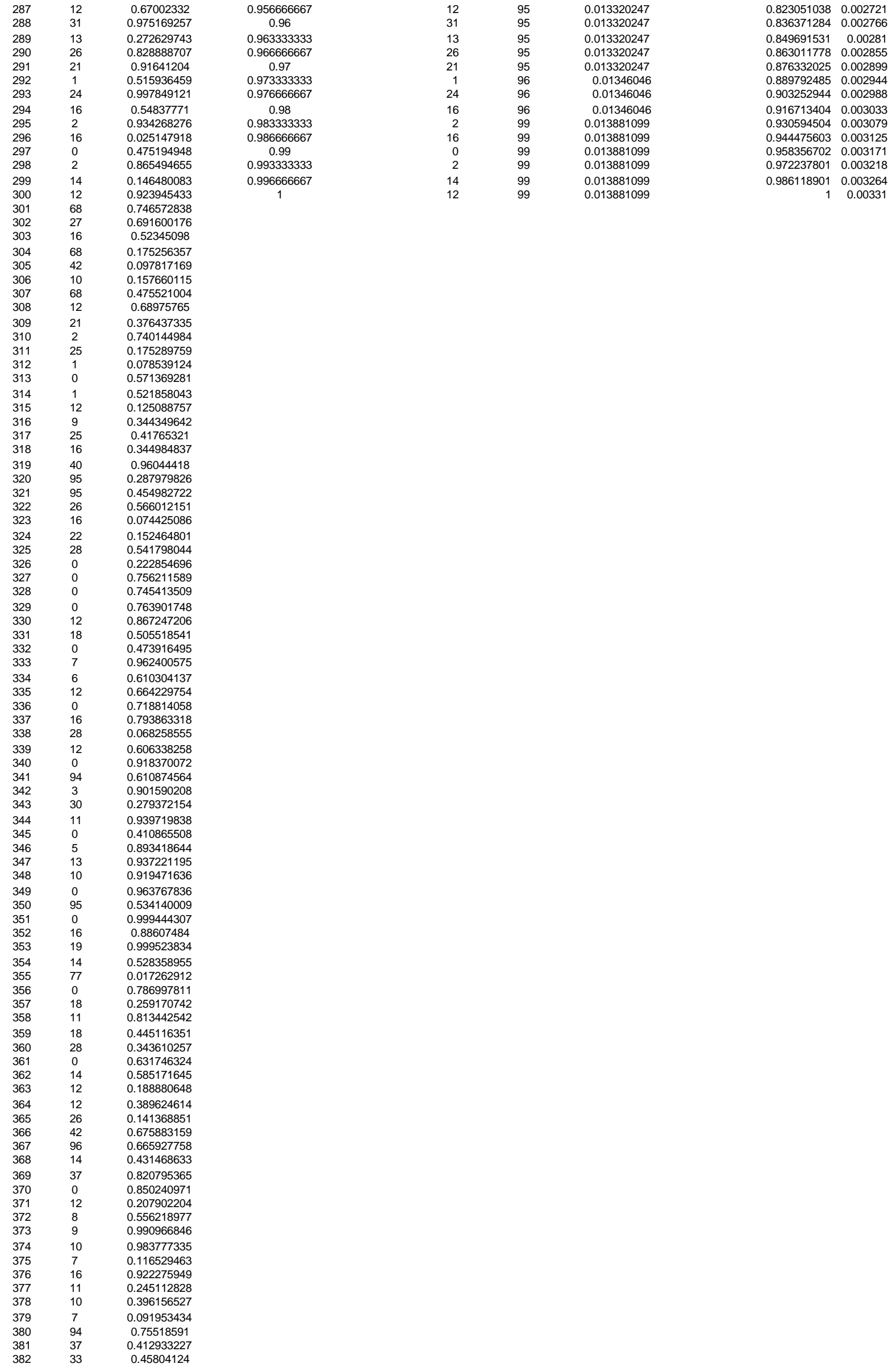




\begin{tabular}{|c|c|c|}
\hline 383 & 0 & 0.794057816 \\
\hline 384 & 7 & 0.744344108 \\
\hline 385 & 13 & 0.824689459 \\
\hline 386 & 13 & 0.991562615 \\
\hline 387 & 33 & 0.591455781 \\
\hline 388 & 94 & 0.675352441 \\
\hline 389 & 0 & 0.006885606 \\
\hline 390 & 0 & 0.885050295 \\
\hline 391 & 28 & 0.945087288 \\
\hline 392 & 21 & 0.705328634 \\
\hline 393 & 13 & 0.371003919 \\
\hline 394 & 8 & 0.751380253 \\
\hline 395 & 0 & 0.184427625 \\
\hline 396 & 48 & 0.794258405 \\
\hline 397 & 33 & 0.964461889 \\
\hline 398 & 10 & 0.758815959 \\
\hline 399 & 21 & 0.478518592 \\
\hline 400 & 25 & 0.609969163 \\
\hline 401 & 64 & 0.418223675 \\
\hline 402 & 9 & 0.971651318 \\
\hline 403 & 1 & 0.916306729 \\
\hline 404 & 10 & 0.085681615 \\
\hline 405 & 12 & 0.417701253 \\
\hline 406 & 19 & 0.95562666 \\
\hline 407 & 31 & 0.504596805 \\
\hline 408 & 24 & 0.413311646 \\
\hline 409 & 95 & 0.129928094 \\
\hline 410 & 0 & 0.888686898 \\
\hline 411 & 0 & 0.755171315 \\
\hline 412 & 76 & 0.278993572 \\
\hline 413 & 42 & 0.328451515 \\
\hline 414 & 9 & 0.177704075 \\
\hline 415 & 96 & 0.566163302 \\
\hline 416 & 5 & 0.854095403 \\
\hline 417 & 30 & 0.360275024 \\
\hline 418 & 11 & 0.371305961 \\
\hline 419 & 0 & 0.323134298 \\
\hline 420 & 28 & 0.441487753 \\
\hline 421 & 96 & 0.07419251 \\
\hline 422 & 22 & 0.497580541 \\
\hline 423 & 95 & 0.170430457 \\
\hline 424 & 11 & 0.215223102 \\
\hline 425 & 94 & 0.731549803 \\
\hline 426 & 7 & 0.561531947 \\
\hline 427 & 10 & 0.092834067 \\
\hline 428 & 6 & 0.153549969 \\
\hline 429 & 94 & 0.938047424 \\
\hline 430 & 0 & 0.048700832 \\
\hline 431 & 0 & 0.022950603 \\
\hline 432 & 14 & 0.915039032 \\
\hline 433 & 12 & 0.080567473 \\
\hline 434 & 25 & 0.98228303 \\
\hline 435 & 10 & 0.619354647 \\
\hline 436 & 4 & 0.603063469 \\
\hline 437 & 14 & 0.151400074 \\
\hline 438 & 31 & 0.978824683 \\
\hline 439 & 11 & 0.246516324 \\
\hline 440 & 0 & 0.563784602 \\
\hline 441 & 13 & 0.964209111 \\
\hline 442 & 1 & 0.900328407 \\
\hline 443 & 1 & 0.746196082 \\
\hline 444 & 0 & 0.825809265 \\
\hline 445 & 30 & 0.936812821 \\
\hline 446 & 13 & 0.662405442 \\
\hline 447 & 28 & 0.508224555 \\
\hline 448 & 22 & 0.019751751 \\
\hline 449 & 19 & 0.819128948 \\
\hline 450 & 21 & 0.513842313 \\
\hline 451 & 12 & 0.263919582 \\
\hline 452 & 11 & 0.724429059 \\
\hline 453 & 94 & 0.203320399 \\
\hline 454 & 48 & 0.318117921 \\
\hline 455 & 48 & 0.973373465 \\
\hline 456 & 3 & 0.315808424 \\
\hline 457 & 12 & 0.966651971 \\
\hline 458 & 0 & 0.339382013 \\
\hline 459 & 11 & 0.815403742 \\
\hline 460 & 12 & 0.658927379 \\
\hline 461 & 6 & 0.303027787 \\
\hline 462 & 21 & 0.535771631 \\
\hline 463 & 12 & 0.478909911 \\
\hline 464 & 26 & 0.305719323 \\
\hline 465 & 16 & 0.678424522 \\
\hline 466 & 40 & 0.26264837 \\
\hline 467 & 30 & 0.819107742 \\
\hline 468 & 12 & 0.043808996 \\
\hline 469 & 11 & 0.928777853 \\
\hline 470 & 8 & 0.630105001 \\
\hline 471 & 16 & 0.80394442 \\
\hline 472 & 27 & 0.823590865 \\
\hline 473 & 0 & 0.911688242 \\
\hline 474 & 1 & 0.161713087 \\
\hline 475 & 22 & 0.542709761 \\
\hline 476 & 95 & 0.365159335 \\
\hline 477 & 42 & 0.942215881 \\
\hline 478 & 0 & 0.960279716 \\
\hline
\end{tabular}


0.790308517

0.764617892

0.479683207

0.181563632

0.436320429

0.434519485

0.290960917

0.312940273

0.009765083

0.941487553

total inhibition

7132

Total area $\quad 0.214829$

.891340797

0.779825412

0.779825412

0.509699466

0.693683813

0.391868061

0.616082778

0.563490677

0.726462713

0.242041079

0.488148837

0.715676203

0.757008311

0.064217962

0.879201626

0.031346096

0.780306201

0.447683386

0.800860056

0.870616814

0.619397308

0.456109302

Gini 0.570341

\begin{tabular}{c}
\multicolumn{1}{c}{ Gini } \\
\hline 0.5728161 \\
0.56988854 \\
0.57157604 \\
0.56569438 \\
0.56426408 \\
0.57500979 \\
0.55788613 \\
0.56618506 \\
0.56666989 \\
0.57253552 \\
0.56620572 \\
0.57127378 \\
0.57034119 \\
\hline
\end{tabular}

$\begin{array}{ll}\text { mean } & 0.568488171 \\ \text { st dev } & 0.004584432\end{array}$ 
AG1024; $10 \mu \mathrm{M} ; 10 \mu \mathrm{M}$ ATP; sample of 200 kinases

\begin{tabular}{|c|c|c|c|}
\hline position & inhibition \% & rand & cumulative sample fraction \\
\hline 1 & 30 & 0.269466505 & 0.005 \\
\hline 2 & 68 & 0.327554866 & 0.01 \\
\hline 3 & 2 & 0.912333241 & 0.015 \\
\hline 4 & 28 & 0.956427019 & 0.02 \\
\hline 5 & 10 & 0.901593584 & 0.025 \\
\hline 6 & 11 & 0.032189902 & 0.03 \\
\hline 7 & 11 & 0.039475937 & 0.035 \\
\hline 8 & 12 & 0.77565286 & 0.04 \\
\hline 9 & 12 & 0.060914638 & 0.045 \\
\hline 10 & 12 & 0.460846197 & 0.05 \\
\hline 11 & 0 & 0.820267895 & 0.055 \\
\hline 12 & 0 & 0.742193436 & 0.06 \\
\hline 13 & 1 & 0.869280166 & 0.065 \\
\hline 14 & 27 & 0.330853416 & 0.07 \\
\hline 15 & 16 & 0.301803263 & 0.075 \\
\hline 16 & 22 & 0.164490822 & 0.08 \\
\hline 17 & 28 & 0.396808968 & 0.085 \\
\hline 18 & 8 & 0.320408615 & 0.09 \\
\hline 19 & 12 & 0.153751056 & 0.095 \\
\hline 20 & 64 & 0.727779187 & 0.1 \\
\hline 21 & 0 & 0.635806897 & 0.105 \\
\hline 22 & 16 & 0.25928735 & 0.11 \\
\hline 23 & 30 & 0.687929882 & 0.115 \\
\hline 24 & 0 & 0.48249578 & 0.12 \\
\hline 25 & 26 & 0.223504377 & 0.125 \\
\hline 26 & 0 & 0.018192663 & 0.13 \\
\hline 27 & 0 & 0.880843217 & 0.135 \\
\hline 28 & 10 & 0.90098242 & 0.14 \\
\hline 29 & 22 & 0.625578157 & 0.145 \\
\hline 30 & 12 & 0.22280694 & 0.15 \\
\hline 31 & 10 & 0.7746986 & 0.155 \\
\hline 32 & 77 & 0.522749683 & 0.16 \\
\hline 33 & 11 & 0.723590503 & 0.165 \\
\hline 34 & 11 & 0.851398026 & 0.17 \\
\hline 35 & 0 & 0.988258693 & 0.175 \\
\hline 36 & 0 & 0.008619398 & 0.18 \\
\hline 37 & 14 & 0.251675442 & 0.185 \\
\hline 38 & 95 & 0.921617742 & 0.19 \\
\hline 39 & 13 & 0.598532309 & 0.195 \\
\hline 40 & 11 & 0.487047208 & 0.2 \\
\hline 41 & 95 & 0.436449896 & 0.205 \\
\hline 42 & 33 & 0.892887274 & 0.21 \\
\hline 43 & 16 & 0.718554227 & 0.215 \\
\hline 44 & 13 & 0.817243922 & 0.22 \\
\hline 45 & 10 & 0.116994935 & 0.225 \\
\hline 46 & 25 & 0.574061671 & 0.23 \\
\hline 47 & 94 & 0.240757468 & 0.235 \\
\hline 48 & 14 & 0.635087493 & 0.24 \\
\hline 49 & 1 & 0.215832811 & 0.245 \\
\hline 50 & 7 & 0.654950071 & 0.25 \\
\hline 51 & 31 & 0.121069734 & 0.255 \\
\hline 52 & 42 & 0.080913793 & 0.26 \\
\hline 53 & 4 & 0.904653375 & 0.265 \\
\hline 54 & 11 & 0.810913335 & 0.27 \\
\hline 55 & 22 & 0.397466705 & 0.275 \\
\hline 56 & 99 & 0.273512049 & 0.28 \\
\hline 57 & 24 & 0.004801745 & 0.285 \\
\hline 58 & 16 & 0.312452093 & 0.29 \\
\hline 59 & 91 & 0.122214521 & 0.295 \\
\hline 60 & 94 & 0.14094932 & 0.3 \\
\hline 61 & 42 & 0.734712303 & 0.305 \\
\hline 62 & 0 & 0.368893645 & 0.31 \\
\hline 63 & 77 & 0.589150785 & 0.315 \\
\hline 64 & 11 & 0.05232653 & 0.32 \\
\hline 65 & 25 & 0.440810415 & 0.325 \\
\hline 66 & 16 & 0.443156088 & 0.33 \\
\hline 67 & 4 & 0.813038988 & 0.335 \\
\hline 68 & 1 & 0.259883366 & 0.34 \\
\hline 69 & 26 & 0.798460394 & 0.345 \\
\hline 70 & 42 & 0.949560709 & 0.35 \\
\hline 71 & 14 & 0.944426866 & 0.355 \\
\hline 72 & 10 & 0.888300244 & 0.36 \\
\hline 73 & 10 & 0.577393422 & 0.365 \\
\hline 74 & 9 & 0.683919284 & 0.37 \\
\hline 75 & 8 & 0.401657746 & 0.375 \\
\hline 76 & 10 & 0.229724904 & 0.38 \\
\hline 77 & 26 & 0.465095063 & 0.385 \\
\hline 78 & 12 & 0.602637795 & 0.39 \\
\hline 79 & 12 & 0.4473421 & 0.395 \\
\hline 80 & 22 & 0.733413893 & 0.4 \\
\hline 81 & 0 & 0.901925078 & 0.405 \\
\hline 82 & 24 & 0.54235509 & 0.41 \\
\hline 83 & 9 & 0.989641024 & 0.415 \\
\hline 84 & 48 & 0.544284046 & 0.42 \\
\hline 85 & 18 & 0.441409495 & 0.425 \\
\hline 86 & 22 & 0.357656572 & 0.43 \\
\hline 87 & 7 & 0.45172508 & 0.435 \\
\hline 88 & 76 & 0.592170318 & 0.44 \\
\hline 89 & 0 & 0.561502566 & 0.445 \\
\hline 90 & 40 & 0.93751051 & 0.45 \\
\hline 91 & 25 & 0.004730774 & 0.455 \\
\hline 92 & 37 & 0.992723492 & 0.46 \\
\hline 93 & 28 & 0.686374117 & 0.465 \\
\hline 94 & 16 & 0.397304574 & 0.47 \\
\hline
\end{tabular}




\begin{tabular}{|c|c|c|}
\hline 95 & 13 & 0.105614054 \\
\hline 96 & 11 & 0.738321184 \\
\hline 97 & 7 & 0.29483271 \\
\hline 98 & 12 & 0.913001498 \\
\hline 99 & 12 & 0.022674211 \\
\hline 100 & 3 & 0.545557483 \\
\hline 101 & 15 & 0.716266374 \\
\hline 102 & 91 & 0.846537632 \\
\hline 103 & 0 & 0.156965612 \\
\hline 104 & 96 & 0.292016213 \\
\hline 105 & 40 & 0.350930748 \\
\hline 106 & 42 & 0.737754956 \\
\hline 107 & 0 & 0.216729001 \\
\hline 108 & 26 & 0.827490918 \\
\hline 109 & 0 & 0.330198297 \\
\hline 110 & 14 & 0.697946191 \\
\hline 111 & 13 & 0.859272238 \\
\hline 112 & 68 & 0.891100334 \\
\hline 113 & 96 & 0.631040397 \\
\hline 114 & 0 & 0.503382219 \\
\hline 115 & 5 & 0.435633608 \\
\hline 116 & 10 & 0.35271447 \\
\hline 117 & 11 & 0.936259966 \\
\hline 118 & 0 & 0.830964366 \\
\hline 119 & 0 & 0.959371052 \\
\hline 120 & 13 & 0.973105011 \\
\hline 121 & 0 & 0.445175132 \\
\hline 122 & 8 & 0.21107231 \\
\hline 123 & 19 & 0.585774537 \\
\hline 124 & 9 & 0.447181502 \\
\hline 125 & 10 & 0.780564647 \\
\hline 126 & 8 & 0.861822593 \\
\hline 127 & 0 & 0.990365856 \\
\hline 128 & 7 & 0.30739954 \\
\hline 129 & 7 & 0.527364562 \\
\hline 130 & 11 & 0.899499668 \\
\hline 131 & 1 & 0.848421404 \\
\hline 132 & 37 & 0.204234014 \\
\hline 133 & 11 & 0.543576676 \\
\hline 134 & 18 & 0.250539026 \\
\hline 135 & 12 & 0.207051126 \\
\hline 136 & 95 & 0.765761809 \\
\hline 137 & 33 & 0.534774384 \\
\hline 138 & 0 & 0.946078089 \\
\hline 139 & 3 & 0.522460183 \\
\hline 140 & 11 & 0.864905646 \\
\hline 141 & 21 & 0.928688237 \\
\hline 142 & 22 & 0.553293456 \\
\hline 143 & 9 & 0.856496674 \\
\hline 144 & 0 & 0.600366629 \\
\hline 145 & 28 & 0.02027855 \\
\hline 146 & 0 & 0.603729147 \\
\hline 147 & 9 & 0.678266441 \\
\hline 148 & 18 & 0.319677765 \\
\hline 149 & 0 & 0.144353396 \\
\hline 150 & 5 & 0.201835103 \\
\hline 151 & 99 & 0.077162308 \\
\hline 152 & 0 & 0.923231123 \\
\hline 153 & 37 & 0.639189016 \\
\hline 154 & 27 & 0.386383352 \\
\hline 155 & 94 & 0.971030322 \\
\hline 156 & 10 & 0.776317498 \\
\hline 157 & 26 & 0.891849083 \\
\hline 158 & 16 & 0.078646872 \\
\hline 159 & 26 & 0.94200839 \\
\hline 160 & 10 & 0.223101803 \\
\hline 161 & 22 & 0.085411089 \\
\hline 162 & 0 & 0.519026526 \\
\hline 163 & 10 & 0.593108719 \\
\hline 164 & 24 & 0.973041796 \\
\hline 165 & 96 & 0.687729203 \\
\hline 166 & 12 & 0.007443135 \\
\hline 167 & 11 & 0.444072969 \\
\hline 168 & 8 & 0.723406627 \\
\hline 169 & 16 & 0.165910693 \\
\hline 170 & 6 & 0.03089017 \\
\hline 171 & 11 & 0.345350559 \\
\hline 172 & 95 & 0.772269102 \\
\hline 173 & 16 & 0.92223374 \\
\hline 174 & 99 & 0.747728972 \\
\hline 175 & 2 & 0.665519707 \\
\hline 176 & 1 & 0.732416553 \\
\hline 177 & 0 & 0.749881127 \\
\hline 178 & 0 & 0.969408377 \\
\hline 179 & 12 & 0.312411207 \\
\hline 180 & 95 & 0.397829977 \\
\hline 181 & 16 & 0.056957844 \\
\hline 182 & 12 & 0.573406328 \\
\hline 183 & 15 & 0.697001264 \\
\hline 184 & 7 & 0.220462813 \\
\hline 185 & 25 & 0.160265706 \\
\hline 186 & 33 & 0.855973707 \\
\hline 187 & 7 & 0.813747011 \\
\hline 188 & 11 & 0.349208789 \\
\hline 189 & 16 & 0.179489197 \\
\hline 190 & 0 & 0.731213835 \\
\hline
\end{tabular}

\begin{tabular}{|c|c|c|}
\hline 13 & 12 & 0.002691186 \\
\hline 11 & 12 & 0.002691186 \\
\hline 7 & 12 & 0.002691186 \\
\hline 12 & 12 & 0.002691186 \\
\hline 12 & 12 & 0.002691186 \\
\hline 3 & 12 & 0.002691186 \\
\hline 15 & 12 & 0.002691186 \\
\hline 91 & 12 & 0.002691186 \\
\hline 0 & 12 & 0.002691186 \\
\hline 96 & 12 & 0.002691186 \\
\hline 40 & 13 & 0.002915452 \\
\hline 42 & 13 & 0.002915452 \\
\hline 0 & 13 & 0.002915452 \\
\hline 26 & 13 & 0.002915452 \\
\hline 0 & 13 & 0.002915452 \\
\hline 14 & 13 & 0.002915452 \\
\hline 13 & 14 & 0.003139717 \\
\hline 68 & 14 & 0.003139717 \\
\hline 96 & 14 & 0.003139717 \\
\hline 0 & 14 & 0.003139717 \\
\hline 5 & 15 & 0.003363983 \\
\hline 10 & 15 & 0.003363983 \\
\hline 11 & 16 & 0.003588248 \\
\hline 0 & 16 & 0.003588248 \\
\hline 0 & 16 & 0.003588248 \\
\hline 13 & 16 & 0.003588248 \\
\hline 0 & 16 & 0.003588248 \\
\hline 8 & 16 & 0.003588248 \\
\hline 19 & 16 & 0.003588248 \\
\hline 9 & 16 & 0.003588248 \\
\hline 10 & 16 & 0.003588248 \\
\hline 8 & 16 & 0.003588248 \\
\hline 0 & 16 & 0.003588248 \\
\hline 7 & 18 & 0.00403678 \\
\hline 7 & 18 & 0.00403678 \\
\hline 11 & 18 & 0.00403678 \\
\hline 1 & 19 & 0.004261045 \\
\hline 37 & 21 & 0.004709576 \\
\hline 11 & 22 & 0.004933842 \\
\hline 18 & 22 & 0.004933842 \\
\hline 12 & 22 & 0.004933842 \\
\hline 95 & 22 & 0.004933842 \\
\hline 33 & 22 & 0.004933842 \\
\hline 0 & 22 & 0.004933842 \\
\hline 3 & 22 & 0.004933842 \\
\hline 11 & 24 & 0.005382373 \\
\hline 21 & 24 & 0.005382373 \\
\hline 22 & 24 & 0.005382373 \\
\hline 9 & 25 & 0.005606638 \\
\hline 0 & 25 & 0.005606638 \\
\hline 28 & 25 & 0.005606638 \\
\hline 0 & 25 & 0.005606638 \\
\hline 9 & 26 & 0.005830904 \\
\hline 18 & 26 & 0.005830904 \\
\hline 0 & 26 & 0.005830904 \\
\hline 5 & 26 & 0.005830904 \\
\hline 99 & 26 & 0.005830904 \\
\hline 0 & 26 & 0.005830904 \\
\hline 37 & 26 & 0.005830904 \\
\hline 27 & 27 & 0.006055169 \\
\hline 94 & 27 & 0.006055169 \\
\hline 10 & 28 & 0.006279435 \\
\hline 26 & 28 & 0.006279435 \\
\hline 16 & 28 & 0.006279435 \\
\hline 26 & 28 & 0.006279435 \\
\hline 10 & 30 & 0.006727966 \\
\hline 22 & 30 & 0.006727966 \\
\hline 0 & 31 & 0.006952231 \\
\hline 10 & 33 & 0.007400763 \\
\hline 24 & 33 & 0.007400763 \\
\hline 96 & 33 & 0.007400763 \\
\hline 12 & 33 & 0.007400763 \\
\hline 11 & 37 & 0.008297825 \\
\hline 8 & 37 & 0.008297825 \\
\hline 16 & 37 & 0.008297825 \\
\hline 6 & 40 & 0.008970621 \\
\hline 11 & 40 & 0.008970621 \\
\hline 95 & 42 & 0.009419152 \\
\hline 16 & 42 & 0.009419152 \\
\hline 99 & 42 & 0.009419152 \\
\hline 2 & 42 & 0.009419152 \\
\hline 1 & 48 & 0.010764745 \\
\hline 0 & 64 & 0.014352994 \\
\hline 0 & 64 & 0.014352994 \\
\hline 12 & 68 & 0.015250056 \\
\hline 95 & 68 & 0.015250056 \\
\hline 16 & 76 & 0.01704418 \\
\hline 12 & 77 & 0.017268446 \\
\hline 15 & 77 & 0.017268446 \\
\hline 7 & 77 & 0.017268446 \\
\hline 25 & 91 & 0.020408163 \\
\hline 33 & 91 & 0.020408163 \\
\hline 7 & 94 & 0.02108096 \\
\hline 11 & 94 & 0.02108096 \\
\hline 16 & 94 & 0.02108096 \\
\hline 0 & 95 & 0.021305225 \\
\hline
\end{tabular}

$\begin{array}{lll}0.116169545 & 0.000574\end{array}$ $\begin{array}{ll}0.118860731 & 0.000588\end{array}$ $\begin{array}{lll}0.121551917 & 0.000601\end{array}$ $\begin{array}{lll}0.124243104 & 0.000614\end{array}$ 0.126934290 .000628 $\begin{array}{lll}0.129625477 & 0.000641\end{array}$ $\begin{array}{lll}0.132316663 & 0.000655\end{array}$ 0.1350078490 .000668 0.1376990360 .000682 $0.140390222 \quad 0.000695$ $\begin{array}{lll}0.143305674 & 0.000709\end{array}$ $\begin{array}{lll}0.146221126 & 0.000724\end{array}$ $\begin{array}{lll}0.149136578 & 0.000738\end{array}$ $\begin{array}{lll}0.15205203 & 0.000753\end{array}$ $\begin{array}{lll}0.154967481 & 0.000768\end{array}$ $\begin{array}{ll}0.157882933 & 0.000782 \\ 0.161022651 & 0.000797\end{array}$ $0.164162368 \quad 0.000813$ $\begin{array}{lll}0.167302086 & 0.000829\end{array}$ $\begin{array}{lll}0.170441803 & 0.000844\end{array}$ $\begin{array}{lll}0.173805786 & 0.000861\end{array}$ $0.177169769 \quad 0.000877$ $\begin{array}{lll}0.180758017 & 0.000895\end{array}$ $\begin{array}{lll}0.184346266 & 0.000913\end{array}$ $\begin{array}{lll}0.187934514 & 0.000931\end{array}$ 0.1915227630 .000949 $\begin{array}{lll}0.195111011 & 0.000967\end{array}$ $\begin{array}{ll}0.19869926 & 0.000985\end{array}$ $0.202287508 \quad 0.001002$ $\begin{array}{ll}0.205875757 & 0.00102\end{array}$ $0.209464005 \quad 0.001038$ $0.213052254 \quad 0.001056$ $\begin{array}{lll}0.216640502 & 0.001074\end{array}$ $0.220677282 \quad 0.001093$ $0.224714061 \quad 0.001113$ $\begin{array}{ll}0.228750841 & 0.001134\end{array}$ $\begin{array}{lll}0.233011886 & 0.001154\end{array}$ $\begin{array}{lll}0.237721462 & 0.001177\end{array}$ $\begin{array}{lll}0.242655304 & 0.001201\end{array}$ $0.247589146 \quad 0.001226$ $0.252522987 \quad 0.00125$ 0.2574568290 .001275 $\begin{array}{ll}0.262390671 & 0.0013\end{array}$ $\begin{array}{ll}0.267324512 & 0.001324\end{array}$ $\begin{array}{ll}0.272258354 & 0.001349\end{array}$ $\begin{array}{ll}0.272258354 & 0.001349 \\ 0.277640727 & 0.001375\end{array}$ $\begin{array}{ll}0.283023099 & 0.001402\end{array}$ $\begin{array}{ll}0.283023099 & 0.001402 \\ 0.288405472 & 0.001429\end{array}$ 0.294012110 .001456 $\begin{array}{lll}0.299618749 & 0.001484\end{array}$ $\begin{array}{lll}0.305225387 & 0.001512\end{array}$ $\begin{array}{ll}0.310832025 & 0.00154\end{array}$ $\begin{array}{lll}0.316662929 & 0.001569\end{array}$ 0.3224938330 .001598 $\begin{array}{ll}0.322493833 & 0.001598 \\ 0.328324736 & 0.001627\end{array}$ $\begin{array}{rr}0.328324736 & 0.001627 \\ 0.33415564 & 0.001656\end{array}$ $\begin{array}{lll}0.339986544 & 0.001685\end{array}$ $\begin{array}{lll}0.345817448 & 0.001715\end{array}$ $\begin{array}{lll}0.351648352 & 0.001744\end{array}$ $0.357703521 \quad 0.001773$ $\begin{array}{ll}0.36375869 & 0.001804 \\ 0.370038125 & 0.001834\end{array}$ $\begin{array}{rr}0.370038125 & 0.001834 \\ 0.37631756 & 0.001866\end{array}$ $\begin{array}{ll}0.382596995 & 0.001897\end{array}$ $\begin{array}{rr}0.38887643 & 0.001929\end{array}$ $\begin{array}{ll}0.395604396 & 0.001961\end{array}$ $\begin{array}{ll}0.395604396 & 0.001961 \\ 0.402332362 & 0.001995\end{array}$ $\begin{array}{ll}0.409284593 & 0.002029\end{array}$ $0.416685355 \quad 0.002065$ $\begin{array}{ll}0.424086118 & 0.002102\end{array}$ $\begin{array}{ll}0.43148688 & 0.002139\end{array}$ $0.438887643 \quad 0.002176$ \begin{tabular}{lll}
0.447185468 & 0.002215 \\
\hline .455483292 & 0.002257
\end{tabular} $\begin{array}{ll}0.455483292 & 0.002257\end{array}$ $\begin{array}{lll}0.463781117 & 0.002298\end{array}$ $\begin{array}{lll}0.472751738 & 0.002341\end{array}$ $\begin{array}{lll}0.481722359 & 0.002386\end{array}$ $\begin{array}{lll}0.491141512 & 0.002432\end{array}$ $\begin{array}{ll}0.500560664 & 0.002479\end{array}$ 0.5099798160 .002526 $0.519398968 \quad 0.002573$ $\begin{array}{lll}0.530163714 & 0.002624\end{array}$ $\begin{array}{ll}0.544516708 & 0.002687 \\ 0.55889702 & 0.002758\end{array}$ $\begin{array}{lll}0.558869702 & 0.002758\end{array}$ $\begin{array}{lll}0.574119758 & 0.002832\end{array}$ $\begin{array}{lll}0.589369814 & 0.002909\end{array}$ $\begin{array}{ll}0.589369814 & 0.002909 \\ 0.606413994 & 0.002989\end{array}$ $0.62368244 \quad 0.003075$ 0.6409508860 .003162 $\begin{array}{lll}0.658219332 & 0.003248\end{array}$ $\begin{array}{lll}0.678627495 & 0.003342\end{array}$ $\begin{array}{lll}0.699035658 & 0.003444\end{array}$ $0.720116618 \quad 0.003548$ $0.741197578 \quad 0.003653$ $\begin{array}{lll}0.762278538 & 0.003759\end{array}$ $0.783583763 \quad 0.003865$ 


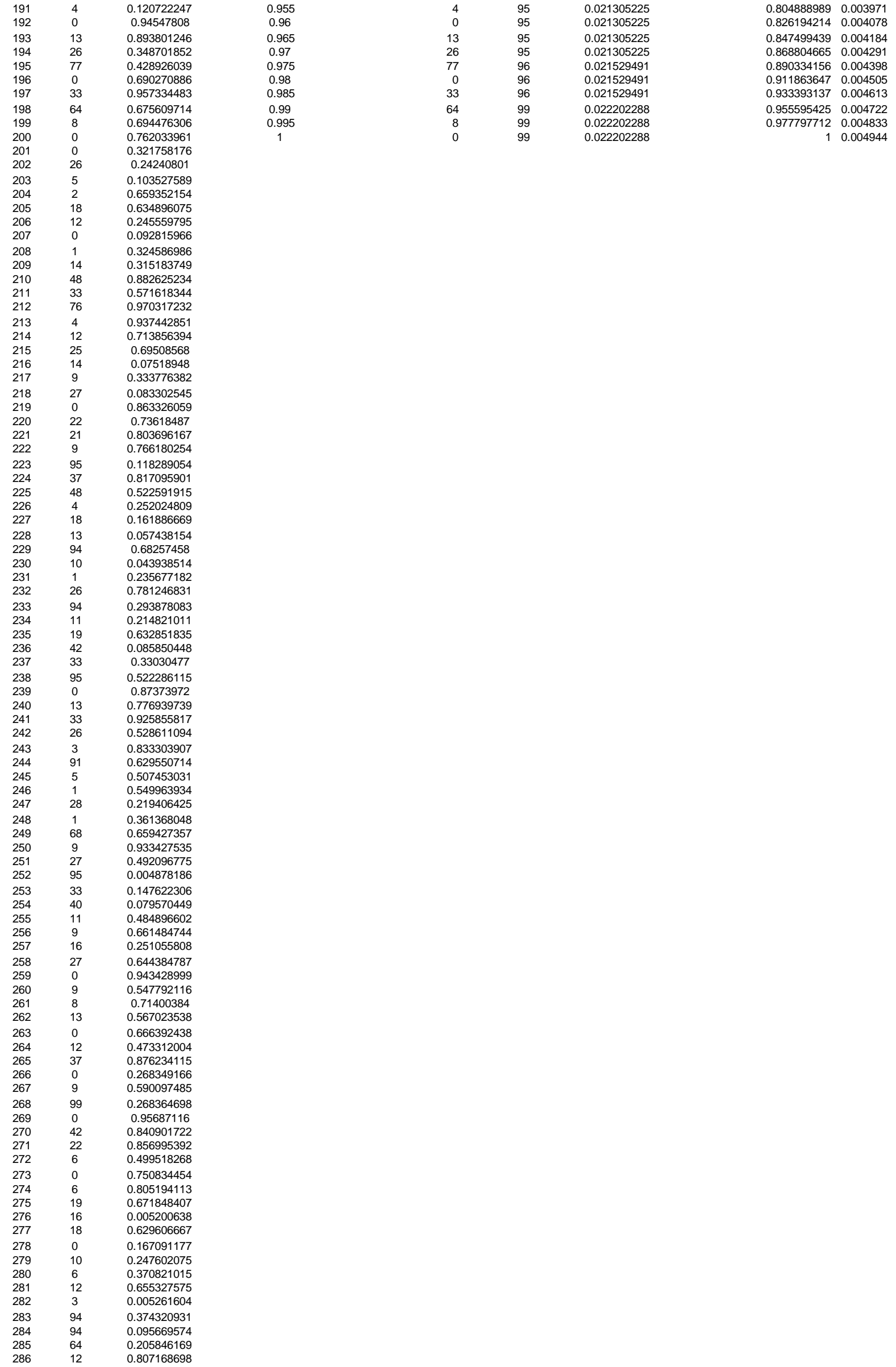




\begin{tabular}{|c|c|c|}
\hline 287 & 0 & 0.838356994 \\
\hline 288 & 16 & 0.364150191 \\
\hline 289 & 11 & 0.552013607 \\
\hline 290 & 0 & 0.466168611 \\
\hline 291 & 9 & 0.243936727 \\
\hline 292 & 33 & 0.158071748 \\
\hline 293 & 1 & 0.472459735 \\
\hline 294 & 0 & 0.880364232 \\
\hline 295 & 42 & 0.380101908 \\
\hline 296 & 16 & 0.65018294 \\
\hline 297 & 9 & 0.828008383 \\
\hline 298 & 33 & 0.810214302 \\
\hline 299 & 96 & 0.219070982 \\
\hline 300 & 0 & 0.830325698 \\
\hline 301 & 9 & 0.352229002 \\
\hline 302 & 15 & 0.385998837 \\
\hline 303 & 40 & 0.857440098 \\
\hline 304 & 68 & 0.86543682 \\
\hline 305 & 76 & 0.56875984 \\
\hline 306 & 77 & 0.14534627 \\
\hline 307 & 7 & 0.27631442 \\
\hline 308 & 6 & 0.525468709 \\
\hline 309 & 4 & 0.603979568 \\
\hline 310 & 77 & 0.354792359 \\
\hline 311 & 48 & 0.284083117 \\
\hline 312 & 12 & 0.123017985 \\
\hline 313 & 11 & 0.57704359 \\
\hline 314 & 0 & 0.959781252 \\
\hline 315 & 31 & 0.375754148 \\
\hline 316 & 0 & 0.879308777 \\
\hline 317 & 0 & 0.624934976 \\
\hline 318 & 28 & 0.79895658 \\
\hline 319 & 16 & 0.721642376 \\
\hline 320 & 28 & 0.443351853 \\
\hline 321 & 0 & 0.320291554 \\
\hline 322 & 12 & 0.372327386 \\
\hline 323 & 7 & 0.42854256 \\
\hline 324 & 30 & 0.771164516 \\
\hline 325 & 0 & 0.307806102 \\
\hline 326 & 11 & 0.259977711 \\
\hline 327 & 0 & 0.017969373 \\
\hline 328 & 11 & 0.002044114 \\
\hline 329 & 1 & 0.003298622 \\
\hline 330 & 2 & 0.526608261 \\
\hline 331 & 13 & 0.083889131 \\
\hline 332 & 14 & 0.030484673 \\
\hline 333 & 0 & 0.380574434 \\
\hline 334 & 1 & 0.206483807 \\
\hline 335 & 77 & 0.268000215 \\
\hline 336 & 5 & 0.512268446 \\
\hline 337 & 28 & 0.453495125 \\
\hline 338 & 30 & 0.105899201 \\
\hline 339 & 33 & 0.501816407 \\
\hline 340 & 37 & 0.659448472 \\
\hline 341 & 16 & 0.167561688 \\
\hline 342 & 0 & 0.484559129 \\
\hline 343 & 0 & 0.877923296 \\
\hline 344 & 4 & 0.623233744 \\
\hline 345 & 9 & 0.703849327 \\
\hline 346 & 8 & 0.640957347 \\
\hline 347 & 0 & 0.649360413 \\
\hline 348 & 13 & 0.863603691 \\
\hline 349 & 16 & 0.109504933 \\
\hline 350 & 16 & 0.938529877 \\
\hline 351 & 76 & 0.50743323 \\
\hline 352 & 12 & 0.675202327 \\
\hline 353 & 3 & 0.231041697 \\
\hline 354 & 4 & 0.275841614 \\
\hline 355 & 42 & 0.960645832 \\
\hline 356 & 12 & 0.323509034 \\
\hline 357 & 16 & 0.833612506 \\
\hline 358 & 16 & 0.530956844 \\
\hline 359 & 68 & 0.760945627 \\
\hline 360 & 64 & 0.815932199 \\
\hline 361 & 22 & 0.862162948 \\
\hline 362 & 11 & 0.396277817 \\
\hline 363 & 42 & 0.229538025 \\
\hline 364 & 1 & 0.953112946 \\
\hline 365 & 0 & 0.364289371 \\
\hline 366 & 28 & 0.464040218 \\
\hline 367 & 31 & 0.428938263 \\
\hline 368 & 7 & 0.328969966 \\
\hline 369 & 13 & 0.77643888 \\
\hline 370 & 4 & 0.551447668 \\
\hline 371 & 0 & 0.240660349 \\
\hline 372 & 1 & 0.056430742 \\
\hline 373 & 33 & 0.775710807 \\
\hline 374 & 0 & 0.60692394 \\
\hline 375 & 33 & 0.602519544 \\
\hline 376 & 94 & 0.95387084 \\
\hline 377 & 31 & 0.347671684 \\
\hline 378 & 64 & 0.571584902 \\
\hline 379 & 16 & 0.538123793 \\
\hline 380 & 7 & 0.663037702 \\
\hline 381 & 0 & 0.236591489 \\
\hline 382 & 7 & 0.776516945 \\
\hline
\end{tabular}




\begin{tabular}{|c|c|c|}
\hline 383 & 8 & 0.754649979 \\
\hline 384 & 7 & 0.817067557 \\
\hline 385 & 0 & 0.365935252 \\
\hline 386 & 15 & 0.773890747 \\
\hline 387 & 94 & 0.224401524 \\
\hline 388 & 24 & 0.120136323 \\
\hline 389 & 12 & 0.513930392 \\
\hline 390 & 24 & 0.933238494 \\
\hline 391 & 40 & 0.0149254 \\
\hline 392 & 10 & 0.29851393 \\
\hline 393 & 16 & 0.276287102 \\
\hline 394 & 22 & 0.239424467 \\
\hline 395 & 1 & 0.545431719 \\
\hline 396 & 11 & 0.927657088 \\
\hline 397 & 99 & 0.504691679 \\
\hline 398 & 99 & 0.710015316 \\
\hline 399 & 8 & 0.007181574 \\
\hline 400 & 91 & 0.688554118 \\
\hline 401 & 30 & 0.35863399 \\
\hline 402 & 9 & 0.255807359 \\
\hline 403 & 19 & 0.837795064 \\
\hline 404 & 8 & 0.687593437 \\
\hline 405 & 2 & 0.631939479 \\
\hline 406 & 10 & 0.973544999 \\
\hline 407 & 33 & 0.296213963 \\
\hline 408 & 7 & 0.585455276 \\
\hline 409 & 76 & 0.379256681 \\
\hline 410 & 5 & 0.371327848 \\
\hline 411 & 13 & 0.756749554 \\
\hline 412 & 11 & 0.568348805 \\
\hline 413 & 15 & 0.07242829 \\
\hline 414 & 11 & 0.42464707 \\
\hline 415 & 91 & 0.211230073 \\
\hline 416 & 19 & 0.272052691 \\
\hline 417 & 12 & 0.710903535 \\
\hline 418 & 30 & 0.06164383 \\
\hline 419 & 31 & 0.662356426 \\
\hline 420 & 7 & 0.568032823 \\
\hline 421 & 0 & 0.231628535 \\
\hline 422 & 11 & 0.967574889 \\
\hline 423 & 0 & 0.287575514 \\
\hline 424 & 76 & 0.344316152 \\
\hline 425 & 0 & 0.497373676 \\
\hline 426 & 4 & 0.377199868 \\
\hline 427 & 12 & 0.126698113 \\
\hline 428 & 1 & 0.644137855 \\
\hline 429 & 14 & 0.594351723 \\
\hline 430 & 8 & 0.66552254 \\
\hline 431 & 12 & 0.254599447 \\
\hline 432 & 12 & 0.546160074 \\
\hline 433 & 64 & 0.041519116 \\
\hline 434 & 22 & 0.970584917 \\
\hline 435 & 26 & 0.69221939 \\
\hline 436 & 21 & 0.16148939 \\
\hline 437 & 13 & 0.421359169 \\
\hline 438 & 14 & 0.872057495 \\
\hline 439 & 0 & 0.250783363 \\
\hline 440 & 0 & 0.950316591 \\
\hline 441 & 14 & 0.452422529 \\
\hline 442 & 95 & 0.22403238 \\
\hline 443 & 10 & 0.613828346 \\
\hline 444 & 33 & 0.530622104 \\
\hline 445 & 12 & 0.068421236 \\
\hline 446 & 24 & 0.49068663 \\
\hline 447 & 33 & 0.154057041 \\
\hline 448 & 94 & 0.183385104 \\
\hline 449 & 11 & 0.294693048 \\
\hline 450 & 21 & 0.209577812 \\
\hline 451 & 11 & 0.647314024 \\
\hline 452 & 4 & 0.140746153 \\
\hline 453 & 15 & 0.550762842 \\
\hline 454 & 0 & 0.051383835 \\
\hline 455 & 13 & 0.502910024 \\
\hline 456 & 13 & 0.345005074 \\
\hline 457 & 0 & 0.52674255 \\
\hline 458 & 68 & 0.247561633 \\
\hline 459 & 10 & 0.452779281 \\
\hline 460 & 13 & 0.92590256 \\
\hline 461 & 6 & 0.436210604 \\
\hline 462 & 11 & 0.156231488 \\
\hline 463 & 0 & 0.233563416 \\
\hline 464 & 31 & 0.013564794 \\
\hline 465 & 14 & 0.944874796 \\
\hline 466 & 7 & 0.179446466 \\
\hline 467 & 25 & 0.329809088 \\
\hline 468 & 96 & 0.4279886655 \\
\hline 469 & 96 & 0.104313255 \\
\hline 470 & 21 & 0.026075492 \\
\hline 471 & 91 & 0.65654315 \\
\hline 472 & 33 & 0.615175522 \\
\hline 473 & 28 & 0.437982995 \\
\hline 474 & 28 & 0.416283887 \\
\hline 475 & 13 & 0.72576111 \\
\hline 476 & 7 & 0.432838357 \\
\hline 477 & 0 & 0.382495108 \\
\hline 478 & 28 & 0.650626616 \\
\hline
\end{tabular}


0.265318429

0.576389621

0.438418807

0.702135108

0.243534689

total inhibition

4459

Total area $\quad 0.216495$

.820357852

0.326522453

0.241053899
0.342282296

0.658543111

0.044954767

0.584604192

0.678348555

0.729574497

0.751840039

.446377597

0.048488019

0.850630015

0.511914571

0.57911585
0.923328056

0.923328056
0.730428119

0.026130611

0.49225572

0.729612565

0.746201012

Gini 0.567009 $\begin{array}{r}\multicolumn{1}{c}{\text { Gini }} \\ \hline 0.55015896 \\ 0.56288053 \\ 0.5514082 \\ 0.58027615 \\ 0.56532478 \\ 0.57523373 \\ 0.56690737 \\ 0.56647154 \\ 0.5567786 \\ 0.57039275 \\ 0.57422558 \\ 0.59087035 \\ 0.56700942 \\ \hline\end{array}$ 
AG1024; $10 \mu \mathrm{M} ; 10 \mu \mathrm{M}$ ATP; sample of 100 kinases

\begin{tabular}{|c|c|c|c|}
\hline position & inhibition\% & rand & cumulative sample fraction \\
\hline 1 & 95 & 0.181743154 & 0.01 \\
\hline 2 & 95 & 0.108353509 & 0.02 \\
\hline 3 & 6 & 0.271430913 & 0.03 \\
\hline 4 & 4 & 0.997232207 & 0.04 \\
\hline 5 & 1 & 0.777272253 & 0.05 \\
\hline 6 & 16 & 0.154943938 & 0.06 \\
\hline 7 & 11 & 0.336430595 & 0.07 \\
\hline 8 & 0 & 0.869828825 & 0.08 \\
\hline 9 & 0 & 0.870895223 & 0.09 \\
\hline 10 & 9 & 0.433450705 & 0.1 \\
\hline 11 & 16 & 0.276728353 & 0.11 \\
\hline 12 & 0 & 0.871262365 & 0.12 \\
\hline 13 & 0 & 0.425135429 & 0.13 \\
\hline 14 & 11 & 0.814842381 & 0.14 \\
\hline 15 & 4 & 0.225169473 & 0.15 \\
\hline 16 & 33 & 0.535988728 & 0.16 \\
\hline 17 & 7 & 0.033500624 & 0.17 \\
\hline 18 & 1 & 0.895153457 & 0.18 \\
\hline 19 & 19 & 0.647894303 & 0.19 \\
\hline 20 & 99 & 0.029526643 & 0.2 \\
\hline 21 & 68 & 0.570635909 & 0.21 \\
\hline 22 & 48 & 0.448861409 & 0.22 \\
\hline 23 & 12 & 0.514705192 & 0.23 \\
\hline 24 & 10 & 0.37313277 & 0.24 \\
\hline 25 & 5 & 0.589465728 & 0.25 \\
\hline 26 & 48 & 0.948380141 & 0.26 \\
\hline 27 & 10 & 0.670014227 & 0.27 \\
\hline 28 & 12 & 0.530377574 & 0.28 \\
\hline 29 & 13 & 0.921666598 & 0.29 \\
\hline 30 & 94 & 0.313748872 & 0.3 \\
\hline 31 & 22 & 0.620497342 & 0.31 \\
\hline 32 & 0 & 0.108527476 & 0.32 \\
\hline 33 & 0 & 0.304298691 & 0.33 \\
\hline 34 & 40 & 0.810381983 & 0.34 \\
\hline 35 & 14 & 0.252997837 & 0.35 \\
\hline 36 & 0 & 0.281633902 & 0.36 \\
\hline 37 & 22 & 0.78939186 & 0.37 \\
\hline 38 & 12 & 0.25703438 & 0.38 \\
\hline 39 & 21 & 0.076295254 & 0.39 \\
\hline 40 & 11 & 0.310196959 & 0.4 \\
\hline 41 & 0 & 0.345443001 & 0.41 \\
\hline 42 & 12 & 0.183080287 & 0.42 \\
\hline 43 & 30 & 0.479798229 & 0.43 \\
\hline 44 & 16 & 0.548175381 & 0.44 \\
\hline 45 & 95 & 0.745783144 & 0.45 \\
\hline 46 & 2 & 0.192340352 & 0.46 \\
\hline 47 & 26 & 0.305068881 & 0.47 \\
\hline 48 & 91 & 0.741653985 & 0.48 \\
\hline 49 & 7 & 0.83021832 & 0.49 \\
\hline 50 & 9 & 0.092055614 & 0.5 \\
\hline 51 & 16 & 0.196058866 & 0.51 \\
\hline 52 & 16 & 0.635071069 & 0.52 \\
\hline 53 & 0 & 0.645307726 & 0.53 \\
\hline 54 & 0 & 0.596522871 & 0.54 \\
\hline 55 & 12 & 0.466969664 & 0.55 \\
\hline 56 & 0 & 0.359295347 & 0.56 \\
\hline 57 & 10 & 0.659994997 & 0.57 \\
\hline 58 & 94 & 0.619540677 & 0.58 \\
\hline 59 & 33 & 0.055816255 & 0.59 \\
\hline 60 & 5 & 0.994013395 & 0.6 \\
\hline 61 & 16 & 0.093188075 & 0.61 \\
\hline 62 & 22 & 0.079020532 & 0.62 \\
\hline 63 & 94 & 0.847540587 & 0.63 \\
\hline 64 & 12 & 0.605559781 & 0.64 \\
\hline 65 & 12 & 0.669262605 & 0.65 \\
\hline 66 & 40 & 0.159531561 & 0.66 \\
\hline 67 & 7 & 0.064938883 & 0.67 \\
\hline 68 & 2 & 0.618262533 & 0.68 \\
\hline 69 & 33 & 0.203010864 & 0.69 \\
\hline 70 & 27 & 0.697072929 & 0.7 \\
\hline 71 & 10 & 0.746828962 & 0.71 \\
\hline 72 & 8 & 0.986533044 & 0.72 \\
\hline 73 & 37 & 0.050314045 & 0.73 \\
\hline 74 & 7 & 0.705660107 & 0.74 \\
\hline 75 & 0 & 0.182885697 & 0.75 \\
\hline 76 & 94 & 0.313199047 & 0.76 \\
\hline 77 & 10 & 0.346929107 & 0.77 \\
\hline 78 & 11 & 0.724943567 & 0.78 \\
\hline 79 & 1 & 0.276510444 & 0.79 \\
\hline 80 & 96 & 0.65867453 & 0.8 \\
\hline 81 & 16 & 0.665025306 & 0.81 \\
\hline 82 & 77 & 0.567144557 & 0.82 \\
\hline 83 & 99 & 0.955067061 & 0.83 \\
\hline 84 & 15 & 0.053649559 & 0.84 \\
\hline 85 & 30 & 0.111065536 & 0.85 \\
\hline 86 & 13 & 0.395200871 & 0.86 \\
\hline 87 & 0 & 0.281187845 & 0.87 \\
\hline 88 & 1 & 0.546336917 & 0.88 \\
\hline 89 & 4 & 0.780216242 & 0.89 \\
\hline 90 & 2 & 0.852969282 & 0.9 \\
\hline 91 & 15 & 0.361597467 & 0.91 \\
\hline 92 & 25 & 0.73641061 & 0.92 \\
\hline 93 & 3 & 0.028735787 & 0.93 \\
\hline 94 & 64 & 0.183741478 & 0.94 \\
\hline
\end{tabular}




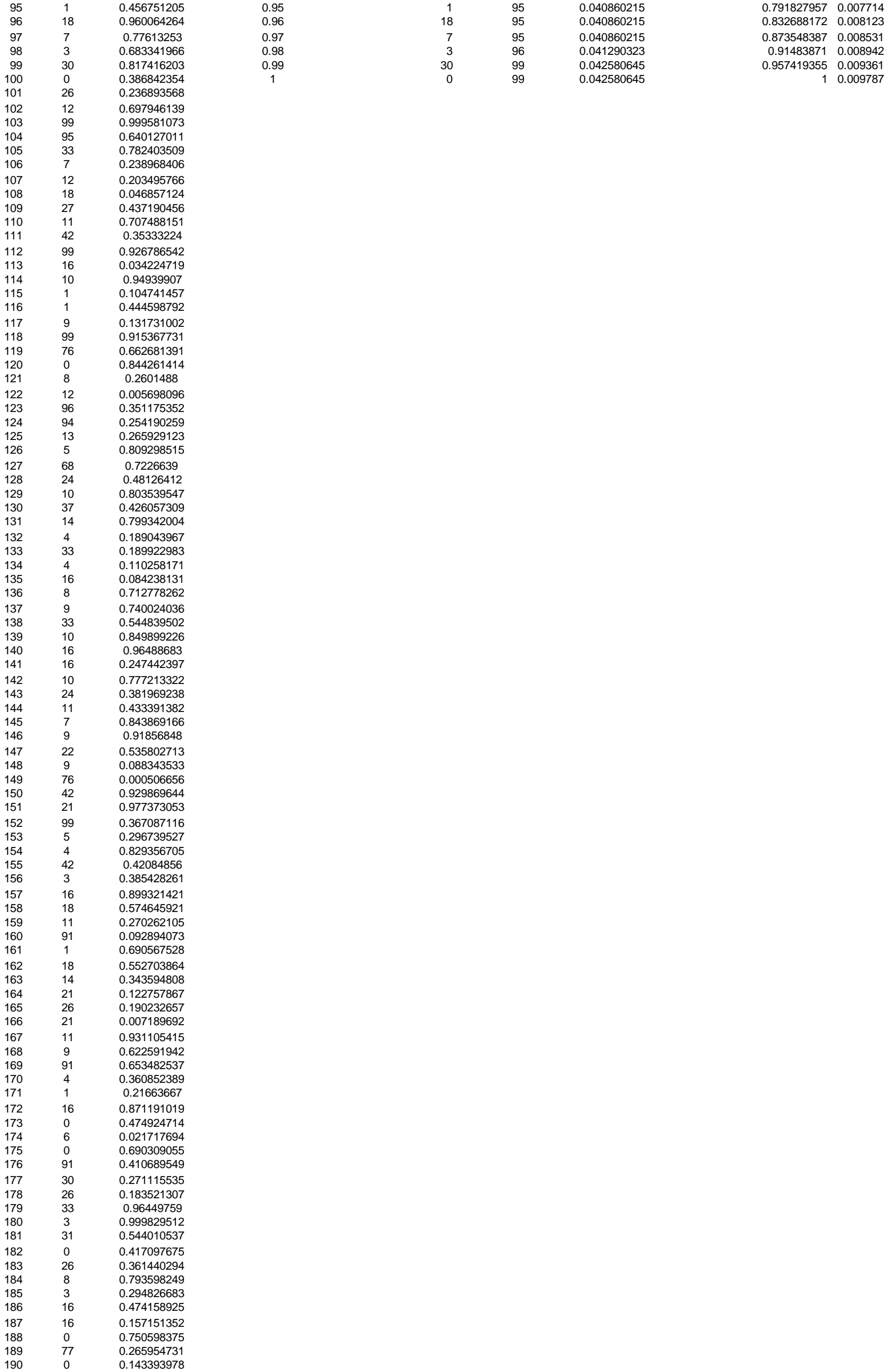




\begin{tabular}{|c|c|c|}
\hline 191 & 0 & 0.970832126 \\
\hline 192 & 76 & 0.854034708 \\
\hline 193 & 13 & 0.996923294 \\
\hline 194 & 0 & 0.13712629 \\
\hline 195 & 28 & 0.123864891 \\
\hline 196 & 24 & 0.52754915 \\
\hline 197 & 26 & 0.84436774 \\
\hline 198 & 0 & 0.373952497 \\
\hline 199 & 0 & 0.702741993 \\
\hline 200 & 11 & 0.037095784 \\
\hline 201 & 77 & 0.773455634 \\
\hline 202 & 0 & 0.264936549 \\
\hline 203 & 94 & 0.707020368 \\
\hline 204 & 6 & 0.273277543 \\
\hline 205 & 96 & 0.174803565 \\
\hline 206 & 12 & 0.963731686 \\
\hline 207 & 16 & 0.282960024 \\
\hline 208 & 0 & 0.211080307 \\
\hline 209 & 16 & 0.495999561 \\
\hline 210 & 22 & 0.361335294 \\
\hline 211 & 0 & 0.452803258 \\
\hline 212 & 9 & 0.815544508 \\
\hline 213 & 13 & 0.43962499 \\
\hline 214 & 0 & 0.199340234 \\
\hline 215 & 13 & 0.152356142 \\
\hline 216 & 30 & 0.141588223 \\
\hline 217 & 9 & 0.390001573 \\
\hline 218 & 22 & 0.928129836 \\
\hline 219 & 0 & 0.271176447 \\
\hline 220 & 11 & 0.988068703 \\
\hline 221 & 25 & 0.024081135 \\
\hline 222 & 12 & 0.15575321 \\
\hline 223 & 7 & 0.664640952 \\
\hline 224 & 10 & 0.192515563 \\
\hline 225 & 13 & 0.717296813 \\
\hline 226 & 13 & 0.46930945 \\
\hline 227 & 14 & 0.765482389 \\
\hline 228 & 8 & 0.929382197 \\
\hline 229 & 42 & 0.0064073 \\
\hline 230 & 10 & 0.895443252 \\
\hline 231 & 1 & 0.988867437 \\
\hline 232 & 68 & 0.214438424 \\
\hline 233 & 7 & 0.715101897 \\
\hline 234 & 9 & 0.15348709 \\
\hline 235 & 7 & 0.820642301 \\
\hline 236 & 13 & 0.387288967 \\
\hline 237 & 13 & 0.140807532 \\
\hline 238 & 68 & 0.795489935 \\
\hline 239 & 40 & 0.977091711 \\
\hline 240 & 11 & 0.404631374 \\
\hline 241 & 33 & 0.175890062 \\
\hline 242 & 11 & 0.065316131 \\
\hline 243 & 4 & 0.638734491 \\
\hline 244 & 9 & 0.575831082 \\
\hline 245 & 12 & 0.630729948 \\
\hline 246 & 12 & 0.778465145 \\
\hline 247 & 0 & 0.223344018 \\
\hline 248 & 19 & 0.424670883 \\
\hline 249 & 11 & 0.751396657 \\
\hline 250 & 0 & 0.742098032 \\
\hline 251 & 76 & 0.734741672 \\
\hline 252 & 11 & 0.598166942 \\
\hline 253 & 9 & 0.435699548 \\
\hline 254 & 14 & 0.373001074 \\
\hline 255 & 8 & 0.799140332 \\
\hline 256 & 19 & 0.806861759 \\
\hline 257 & 42 & 0.129264916 \\
\hline 258 & 12 & 0.723336572 \\
\hline 259 & 68 & 0.368184418 \\
\hline 260 & 10 & 0.40608085 \\
\hline 261 & 33 & 0.680452194 \\
\hline 262 & 0 & 0.708225118 \\
\hline 263 & 48 & 0.147856227 \\
\hline 264 & 28 & 0.705653812 \\
\hline 265 & 30 & 0.252935527 \\
\hline 266 & 21 & 0.137766571 \\
\hline 267 & 11 & 0.356777922 \\
\hline 268 & 8 & 0.275431073 \\
\hline 269 & 28 & 0.833743261 \\
\hline 270 & 9 & 0.481400548 \\
\hline 271 & 10 & 0.099990833 \\
\hline 272 & 31 & 0.869267556 \\
\hline 273 & 11 & 0.160178531 \\
\hline 274 & 12 & 0.569040604 \\
\hline 275 & 6 & 0.596233829 \\
\hline 276 & 42 & 0.849708831 \\
\hline 277 & 95 & 0.355248596 \\
\hline 278 & 4 & 0.075500233 \\
\hline 279 & 5 & 0.625040401 \\
\hline 280 & 0 & 0.516655396 \\
\hline 281 & 4 & 0.244573385 \\
\hline 282 & 3 & 0.644896506 \\
\hline 283 & 0 & 0.246086626 \\
\hline 284 & 0 & 0.429727119 \\
\hline 285 & 0 & 0.678916128 \\
\hline 286 & 0 & 0.523134514 \\
\hline
\end{tabular}




\begin{tabular}{|c|c|c|}
\hline 287 & 10 & 0.296137014 \\
\hline 288 & 8 & 0.749212949 \\
\hline 289 & 64 & 0.615658169 \\
\hline 290 & 0 & 0.761167148 \\
\hline 291 & 14 & 0.510310502 \\
\hline 292 & 77 & 0.655762521 \\
\hline 293 & 11 & 0.622557126 \\
\hline 294 & 8 & 0.620430621 \\
\hline 295 & 42 & 0.219504087 \\
\hline 296 & 25 & 0.56470116 \\
\hline 297 & 13 & 0.104419002 \\
\hline 298 & 33 & 0.369242116 \\
\hline 299 & 31 & 0.041286807 \\
\hline 300 & 12 & 0.522909276 \\
\hline 301 & 12 & 0.004046732 \\
\hline 302 & 9 & 0.825430712 \\
\hline 303 & 28 & 0.181016665 \\
\hline 304 & 1 & 0.59082539 \\
\hline 305 & 8 & 0.858203977 \\
\hline 306 & 1 & 0.390167468 \\
\hline 307 & 12 & 0.672069535 \\
\hline 308 & 25 & 0.128802883 \\
\hline 309 & 21 & 0.963111331 \\
\hline 310 & 11 & 0.414782816 \\
\hline 311 & 2 & 0.735413631 \\
\hline 312 & 11 & 0.051457946 \\
\hline 313 & 12 & 0.631842093 \\
\hline 314 & 15 & 0.242807012 \\
\hline 315 & 26 & 0.970534388 \\
\hline 316 & 42 & 0.204413817 \\
\hline 317 & 94 & 0.151903554 \\
\hline 318 & 37 & 0.517174049 \\
\hline 319 & 14 & 0.84166433 \\
\hline 320 & 33 & 0.746821286 \\
\hline 321 & 95 & 0.392619848 \\
\hline 322 & 11 & 0.044926362 \\
\hline 323 & 11 & 0.640575718 \\
\hline 324 & 7 & 0.355986579 \\
\hline 325 & 0 & 0.354064735 \\
\hline 326 & 13 & 0.057041491 \\
\hline 327 & 22 & 0.577037631 \\
\hline 328 & 7 & 0.255356087 \\
\hline 329 & 14 & 0.349209758 \\
\hline 330 & 19 & 0.187622407 \\
\hline 331 & 15 & 0.276097762 \\
\hline 332 & 26 & 0.140797912 \\
\hline 333 & 95 & 0.91939574 \\
\hline 334 & 15 & 0.396664386 \\
\hline 335 & 7 & 0.342344128 \\
\hline 336 & 16 & 0.886384663 \\
\hline 337 & 64 & 0.091360942 \\
\hline 338 & 95 & 0.713105668 \\
\hline 339 & 28 & 0.00884558 \\
\hline 340 & 37 & 0.240784119 \\
\hline 341 & 7 & 0.513954818 \\
\hline 342 & 48 & 0.719244337 \\
\hline 343 & 26 & 0.013455937 \\
\hline 344 & 0 & 0.579470144 \\
\hline 345 & 11 & 0.271855995 \\
\hline 346 & 0 & 0.031701757 \\
\hline 347 & 14 & 0.151685874 \\
\hline 348 & 12 & 0.67635353 \\
\hline 349 & 0 & 0.051823158 \\
\hline 350 & 0 & 0.847016727 \\
\hline 351 & 28 & 0.34552364 \\
\hline 352 & 2 & 0.143611441 \\
\hline 353 & 9 & 0.675792442 \\
\hline 354 & 96 & 0.509699187 \\
\hline 355 & 14 & 0.209827919 \\
\hline 356 & 11 & 0.021965422 \\
\hline 357 & 0 & 0.721731592 \\
\hline 358 & 64 & 0.450147649 \\
\hline 359 & 9 & 0.617697623 \\
\hline 360 & 28 & 0.922187125 \\
\hline 361 & 94 & 0.761955383 \\
\hline 362 & 33 & 0.915682732 \\
\hline 363 & 42 & 0.208452709 \\
\hline 364 & 16 & 0.75840221 \\
\hline 365 & 94 & 0.542231857 \\
\hline 366 & 10 & 0.887460157 \\
\hline 367 & 12 & 0.142422976 \\
\hline 368 & 0 & 0.609152899 \\
\hline 369 & 31 & 0.273481811 \\
\hline 370 & 37 & 0.377024956 \\
\hline 371 & 0 & 0.283578047 \\
\hline 372 & 7 & 0.289516031 \\
\hline 373 & 28 & 0.055215856 \\
\hline 374 & 42 & 0.831552242 \\
\hline 375 & 6 & 0.394699923 \\
\hline 376 & 77 & 0.738924186 \\
\hline 377 & 0 & 0.177530798 \\
\hline 378 & 0 & 0.18479596 \\
\hline 379 & 0 & 0.45029595 \\
\hline 380 & 9 & 0.48558335 \\
\hline 381 & 0 & 0.070836036 \\
\hline 382 & 5 & 0.527469034 \\
\hline
\end{tabular}




\begin{tabular}{|c|c|c|}
\hline 383 & 14 & 0.154803638 \\
\hline 384 & 24 & 0.656056733 \\
\hline 385 & 18 & 0.781566888 \\
\hline 386 & 27 & 0.417394025 \\
\hline 387 & 96 & 0.891748114 \\
\hline 388 & 12 & 0.619565311 \\
\hline 389 & 64 & 0.633663705 \\
\hline 390 & 0 & 0.766651098 \\
\hline 391 & 31 & 0.164537889 \\
\hline 392 & 22 & 0.432563832 \\
\hline 393 & 0 & 0.432560769 \\
\hline 394 & 4 & 0.824142813 \\
\hline 395 & 25 & 0.010127513 \\
\hline 396 & 11 & 0.996987247 \\
\hline 397 & 95 & 0.021346608 \\
\hline 398 & 10 & 0.08352201 \\
\hline 399 & 95 & 0.511823464 \\
\hline 400 & 0 & 0.30429141 \\
\hline 401 & 25 & 0.461420966 \\
\hline 402 & 33 & 0.143349211 \\
\hline 403 & 0 & 0.859907372 \\
\hline 404 & 31 & 0.867965735 \\
\hline 405 & 11 & 0.293175261 \\
\hline 406 & 1 & 0.317708606 \\
\hline 407 & 24 & 0.467858514 \\
\hline 408 & 16 & 0.800916648 \\
\hline 409 & 28 & 0.0947197 \\
\hline 410 & 77 & 0.991709294 \\
\hline 411 & 0 & 0.750159516 \\
\hline 412 & 1 & 0.688388665 \\
\hline 413 & 16 & 0.725815897 \\
\hline 414 & 40 & 0.038634749 \\
\hline 415 & 48 & 0.459629199 \\
\hline 416 & 94 & 0.467151497 \\
\hline 417 & 24 & 0.145125957 \\
\hline 418 & 0 & 0.844108937 \\
\hline 419 & 28 & 0.948084935 \\
\hline 420 & 12 & 0.268900295 \\
\hline 421 & 2 & 0.013215052 \\
\hline 422 & 19 & 0.19751377 \\
\hline 423 & 11 & 0.458244806 \\
\hline 424 & 95 & 0.694150082 \\
\hline 425 & 1 & 0.232548146 \\
\hline 426 & 22 & 0.701226687 \\
\hline 427 & 0 & 0.401532603 \\
\hline 428 & 11 & 0.898358856 \\
\hline 429 & 33 & 0.333487461 \\
\hline 430 & 13 & 0.550216809 \\
\hline 431 & 1 & 0.334178678 \\
\hline 432 & 91 & 0.239776631 \\
\hline 433 & 27 & 0.437484481 \\
\hline 434 & 7 & 0.016307082 \\
\hline 435 & 0 & 0.998643139 \\
\hline 436 & 0 & 0.743731474 \\
\hline 437 & 26 & 0.207965235 \\
\hline 438 & 22 & 0.97777889 \\
\hline 439 & 11 & 0.074597383 \\
\hline 440 & 13 & 0.125640526 \\
\hline 441 & 16 & 0.774767482 \\
\hline 442 & 11 & 0.221170204 \\
\hline 443 & 13 & 0.926974312 \\
\hline 444 & 26 & 0.662010315 \\
\hline 445 & 13 & 0.55393866 \\
\hline 446 & 6 & 0.099286581 \\
\hline 447 & 76 & 0.701549394 \\
\hline 448 & 1 & 0.036277691 \\
\hline 449 & 0 & 0.421982552 \\
\hline 450 & 48 & 0.896683806 \\
\hline 451 & 27 & 0.371504477 \\
\hline 452 & 33 & 0.916760394 \\
\hline 453 & 10 & 0.46125975 \\
\hline 454 & 42 & 0.571965568 \\
\hline 455 & 40 & 0.458690863 \\
\hline 456 & 0 & 0.451660529 \\
\hline 457 & 16 & 0.282485214 \\
\hline 458 & 0 & 0.498324065 \\
\hline 459 & 94 & 0.659463423 \\
\hline 460 & 91 & 0.812026335 \\
\hline 461 & 27 & 0.831054914 \\
\hline 462 & 8 & 0.358549392 \\
\hline 463 & 7 & 0.811991146 \\
\hline 464 & 28 & 0.985547119 \\
\hline 465 & 12 & 0.898606252 \\
\hline 466 & 94 & 0.378266345 \\
\hline 467 & 0 & 0.505135806 \\
\hline 468 & 0 & 0.485913351 \\
\hline 469 & 37 & 0.311382796 \\
\hline 470 & 22 & 0.637592323 \\
\hline 471 & 8 & 0.279663832 \\
\hline 472 & 12 & 0.028647537 \\
\hline 473 & 0 & 0.300898199 \\
\hline 474 & 0 & 0.002500897 \\
\hline 475 & 10 & 0.272823193 \\
\hline 476 & 12 & 0.783707599 \\
\hline 477 & 96 & 0.030503967 \\
\hline 478 & 1 & 0.051061296 \\
\hline
\end{tabular}


0.221042148

0.700611492

0.328429244

0.515846446

0.10741887

0.851997508

0.941041013

0.664443756

0.217949132

0.308855138

0.067036481

0.579369548

0.722096915

0.640460242

0.661937364

0.716826604
0.872658999

0.874097918

0.835040738

0.311112307

0.096398656

0.72468728

0.010737148

0.327466486

.207268415

0.151858842

0.456431579

0.694094537

0.457579509

0.860499421

0.750588313

0.106924822 total inhibition

2325

Total area $\quad 0.197869$

Gini 0.604262 \begin{tabular}{c} 
Gini \\
\hline 0.57145775 \\
0.56864333 \\
0.53838318 \\
0.55086818 \\
0.52963621 \\
0.59178044 \\
0.54229404 \\
0.52677676 \\
0.57670927 \\
0.57489437 \\
0.58103321 \\
0.5250305 \\
0.55502773 \\
\hline
\end{tabular}

mean $\quad 0.556348842$ 
AG1024; $10 \mu \mathrm{M} ; 10 \mu \mathrm{M}$ ATP; sample of 50 kinases

\begin{tabular}{|c|c|c|c|c|c|c|c|c|}
\hline position & inhibition \% & rand & cumulative sample fraction & sample inhibition & sorted & fraction of inhibition & cumulative fraction of inhibition & area \\
\hline 1 & 68 & 0.106016966 & 0.02 & 68 & 0 & 0 & 0 & 0 \\
\hline 2 & 12 & 0.189613338 & 0.04 & 12 & 0 & 0 & 0 & 0 \\
\hline 3 & 33 & 0.65019279 & 0.06 & 33 & 0 & 0 & 0 & 0 \\
\hline 4 & 5 & 0.658420586 & 0.08 & 5 & 0 & 0 & 0 & 0 \\
\hline 5 & 64 & 0.839861847 & 0.1 & 64 & 0 & 0 & 0 & 0 \\
\hline 6 & 11 & 0.057271696 & 0.12 & 11 & 0 & 0 & 0 & 0 \\
\hline 7 & 16 & 0.407577005 & 0.14 & 16 & 0 & 0 & 0 & 0 \\
\hline 8 & 14 & 0.963676816 & 0.16 & 14 & 1 & 0.000979432 & 0.000979432 & $9.79 \mathrm{E}-06$ \\
\hline 9 & 1 & 0.572185043 & 0.18 & 1 & 1 & 0.000979432 & 0.001958864 & 2.94E-05 \\
\hline 10 & 12 & 0.77449029 & 0.2 & 12 & 2 & 0.001958864 & 0.003917728 & $5.88 \mathrm{E}-05$ \\
\hline 11 & 21 & 0.954013748 & 0.22 & 21 & 4 & 0.003917728 & 0.007835455 & 0.000118 \\
\hline 12 & 18 & 0.775107615 & 0.24 & 18 & 5 & 0.00489716 & 0.012732615 & 0.000206 \\
\hline 13 & 0 & 0.665675609 & 0.26 & 0 & 9 & 0.008814887 & 0.021547502 & 0.000343 \\
\hline 14 & 28 & 0.676496457 & 0.28 & 28 & 9 & 0.008814887 & 0.03036239 & 0.000519 \\
\hline 15 & 10 & 0.2879048 & 0.3 & 10 & 10 & 0.009794319 & 0.040156709 & 0.000705 \\
\hline 16 & 19 & 0.89106251 & 0.32 & 19 & 10 & 0.009794319 & 0.049951028 & 0.000901 \\
\hline 17 & 11 & 0.211174997 & 0.34 & 11 & 10 & 0.009794319 & 0.059745348 & 0.001097 \\
\hline 18 & 0 & 0.536566315 & 0.36 & 0 & 11 & 0.010773751 & 0.070519099 & 0.001303 \\
\hline 19 & 0 & 0.752691091 & 0.38 & 0 & 11 & 0.010773751 & 0.08129285 & 0.001518 \\
\hline 20 & 31 & 0.829906192 & 0.4 & 31 & 11 & 0.010773751 & 0.092066601 & 0.001734 \\
\hline 21 & 2 & 0.280757934 & 0.42 & 2 & 12 & 0.011753183 & 0.103819785 & 0.001959 \\
\hline 22 & 10 & 0.980994637 & 0.44 & 10 & 12 & 0.011753183 & 0.115572968 & 0.002194 \\
\hline 23 & 9 & 0.54894241 & 0.46 & 9 & 12 & 0.011753183 & 0.127326151 & 0.002429 \\
\hline 24 & 0 & 0.884990213 & 0.48 & 0 & 12 & 0.011753183 & 0.139079334 & 0.002664 \\
\hline 25 & 0 & 0.129146739 & 0.5 & 0 & 12 & 0.011753183 & 0.150832517 & 0.002899 \\
\hline 26 & 12 & 0.326042104 & 0.52 & 12 & 14 & 0.013712047 & 0.164544564 & 0.003154 \\
\hline 27 & 10 & 0.597183691 & 0.54 & 10 & 14 & 0.013712047 & 0.178256611 & 0.003428 \\
\hline 28 & 31 & 0.108334333 & 0.56 & 31 & 16 & 0.015670911 & 0.193927522 & 0.003722 \\
\hline 29 & 9 & 0.076661161 & 0.58 & 9 & 16 & 0.015670911 & 0.209598433 & 0.004035 \\
\hline 30 & 4 & 0.820920558 & 0.6 & 4 & 18 & 0.017629775 & 0.227228208 & 0.004368 \\
\hline 31 & 1 & 0.157953327 & 0.62 & 1 & 19 & 0.018609207 & 0.245837414 & 0.004731 \\
\hline 32 & 48 & 0.426914017 & 0.64 & 48 & 21 & 0.020568071 & 0.266405485 & 0.005122 \\
\hline 33 & 77 & 0.665683699 & 0.66 & 77 & 21 & 0.020568071 & 0.286973555 & 0.005534 \\
\hline 34 & 30 & 0.774337519 & 0.68 & 30 & 25 & 0.024485798 & 0.311459354 & 0.005984 \\
\hline 35 & 48 & 0.963678591 & 0.7 & 48 & 27 & 0.026444662 & 0.337904016 & 0.006494 \\
\hline 36 & 21 & 0.525322021 & 0.72 & 21 & 28 & 0.027424094 & 0.36532811 & 0.007032 \\
\hline 37 & 77 & 0.886397779 & 0.74 & 77 & 30 & 0.029382958 & 0.394711068 & 0.0076 \\
\hline 38 & 11 & 0.054187815 & 0.76 & 11 & 31 & 0.03036239 & 0.425073457 & 0.008198 \\
\hline 39 & 14 & 0.766589404 & 0.78 & 14 & 31 & 0.03036239 & 0.455435847 & 0.008805 \\
\hline 40 & 27 & 0.513726471 & 0.8 & 27 & 33 & 0.032321254 & 0.487757101 & 0.009432 \\
\hline 41 & 25 & 0.38681337 & 0.82 & 25 & 33 & 0.032321254 & 0.520078355 & 0.010078 \\
\hline 42 & 0 & 0.439843913 & 0.84 & 0 & 33 & 0.032321254 & 0.552399608 & 0.010725 \\
\hline 43 & 0 & 0.706754913 & 0.86 & 0 & 33 & 0.032321254 & 0.584720862 & 0.011371 \\
\hline 44 & 33 & 0.586315312 & 0.88 & 33 & 42 & 0.041136141 & 0.625857003 & 0.012106 \\
\hline 45 & 33 & 0.995996318 & 0.9 & 33 & 48 & 0.047012733 & 0.672869736 & 0.012987 \\
\hline 46 & 12 & 0.569304658 & 0.92 & 12 & 48 & 0.047012733 & 0.719882468 & 0.013928 \\
\hline 47 & 42 & 0.840912976 & 0.94 & 42 & 64 & 0.062683643 & 0.782566112 & 0.015024 \\
\hline 48 & 33 & 0.960370967 & 0.96 & 33 & 68 & 0.066601371 & 0.849167483 & 0.016317 \\
\hline 49 & 16 & 0.056567745 & 0.98 & 16 & 77 & 0.075416259 & 0.924583741 & 0.017738 \\
\hline 50 & 12 & 0.850834757 & 1 & 12 & 77 & 0.075416259 & 1 & 0.019246 \\
\hline
\end{tabular}

0.829956838

0.638292078

0.772402133

0.435742341

0.954726

0.954726

0.157088001
0.568135551

0.568135551
0.510312645

0.184371013

0.739138339

0.104917052

0.931360529

0.384562725

0.05536336

0.327880307

0.397676085

0.411004073

0.349447597

0.318819717

0.797417053

0.675313509
0.113659426

0.669527069

0.153388217

0.755070077

0.224965376

0.258425919
0.376399803

0.112971856

0.739872797

0.957798957

0.193683059

0.276705344

0.37160304
0.964809696

0.875369422

0.819008962

0.571175332

0.733171106

0.529958005

$\begin{array}{ll}1 & 0.019246\end{array}$ 


\begin{tabular}{|c|c|}
\hline 96 & 13 \\
\hline 97 & 22 \\
\hline 98 & 10 \\
\hline 99 & 91 \\
\hline 100 & 1 \\
\hline 101 & 0 \\
\hline 102 & 68 \\
\hline 103 & 0 \\
\hline 104 & 0 \\
\hline 105 & 0 \\
\hline 106 & 64 \\
\hline 107 & 0 \\
\hline 108 & 7 \\
\hline 109 & 7 \\
\hline 110 & 16 \\
\hline 111 & 33 \\
\hline 112 & 12 \\
\hline 113 & 15 \\
\hline 114 & 1 \\
\hline 115 & 28 \\
\hline 116 & 8 \\
\hline 117 & 11 \\
\hline 118 & 76 \\
\hline 119 & 6 \\
\hline 120 & 77 \\
\hline 121 & 48 \\
\hline 122 & 16 \\
\hline 123 & 26 \\
\hline 124 & 33 \\
\hline 125 & 24 \\
\hline 126 & 19 \\
\hline 127 & 48 \\
\hline 128 & 37 \\
\hline 129 & 96 \\
\hline 130 & 26 \\
\hline 131 & 0 \\
\hline 132 & 16 \\
\hline 133 & 1 \\
\hline 134 & 0 \\
\hline 135 & 18 \\
\hline 136 & 42 \\
\hline 137 & 13 \\
\hline 138 & 95 \\
\hline 139 & 0 \\
\hline 140 & 4 \\
\hline 141 & 99 \\
\hline 142 & 9 \\
\hline 143 & 18 \\
\hline 144 & 94 \\
\hline 145 & 13 \\
\hline 146 & 13 \\
\hline 147 & 0 \\
\hline 148 & 16 \\
\hline 149 & 16 \\
\hline 150 & 40 \\
\hline 151 & 26 \\
\hline 152 & 12 \\
\hline 153 & 94 \\
\hline 154 & 27 \\
\hline 155 & 9 \\
\hline 156 & 12 \\
\hline 157 & 27 \\
\hline 158 & 0 \\
\hline 159 & 13 \\
\hline 160 & 10 \\
\hline 161 & 28 \\
\hline 162 & 7 \\
\hline 163 & 0 \\
\hline 164 & 21 \\
\hline 165 & 1 \\
\hline 166 & 0 \\
\hline 167 & 1 \\
\hline 168 & 99 \\
\hline 169 & 11 \\
\hline 170 & 24 \\
\hline 171 & 0 \\
\hline 172 & 1 \\
\hline 173 & 0 \\
\hline 174 & 11 \\
\hline 175 & 9 \\
\hline 176 & 31 \\
\hline 177 & 0 \\
\hline 178 & 9 \\
\hline 179 & 11 \\
\hline 180 & 10 \\
\hline 181 & 9 \\
\hline 182 & 4 \\
\hline 183 & 8 \\
\hline 184 & 7 \\
\hline 185 & 10 \\
\hline 186 & 22 \\
\hline 187 & 11 \\
\hline 188 & 11 \\
\hline 189 & 5 \\
\hline 190 & 13 \\
\hline 191 & 14 \\
\hline 192 & 28 \\
\hline
\end{tabular}




\begin{tabular}{|c|c|}
\hline 193 & 11 \\
\hline 194 & 95 \\
\hline 195 & 12 \\
\hline 196 & 9 \\
\hline 197 & 18 \\
\hline 198 & 8 \\
\hline 199 & 26 \\
\hline 200 & 16 \\
\hline 201 & 26 \\
\hline 202 & 7 \\
\hline 203 & 8 \\
\hline 204 & 9 \\
\hline 205 & 12 \\
\hline 206 & 13 \\
\hline 207 & 64 \\
\hline 208 & 5 \\
\hline 209 & 95 \\
\hline 210 & 0 \\
\hline 211 & 0 \\
\hline 212 & 0 \\
\hline 213 & 33 \\
\hline 214 & 22 \\
\hline 215 & 10 \\
\hline 216 & 7 \\
\hline 217 & 2 \\
\hline 218 & 3 \\
\hline 219 & 0 \\
\hline 220 & 40 \\
\hline 221 & 25 \\
\hline 222 & 48 \\
\hline 223 & 9 \\
\hline 224 & 33 \\
\hline 225 & 13 \\
\hline 226 & 26 \\
\hline 227 & 5 \\
\hline 228 & 12 \\
\hline 229 & 40 \\
\hline 230 & 95 \\
\hline 231 & 0 \\
\hline 232 & 0 \\
\hline 233 & 6 \\
\hline 234 & 0 \\
\hline 235 & 0 \\
\hline 236 & 26 \\
\hline 237 & 0 \\
\hline 238 & 0 \\
\hline 239 & 8 \\
\hline 240 & 48 \\
\hline 241 & 1 \\
\hline 242 & 68 \\
\hline 243 & 77 \\
\hline 244 & 95 \\
\hline 245 & 19 \\
\hline 246 & 95 \\
\hline 247 & 16 \\
\hline 248 & 12 \\
\hline 249 & 12 \\
\hline 250 & 11 \\
\hline 251 & 40 \\
\hline 252 & 12 \\
\hline 253 & 11 \\
\hline 254 & 22 \\
\hline 255 & 76 \\
\hline 256 & 16 \\
\hline 257 & 16 \\
\hline 258 & 33 \\
\hline 259 & 33 \\
\hline 260 & 68 \\
\hline 261 & 21 \\
\hline 262 & 10 \\
\hline 263 & 16 \\
\hline 264 & 7 \\
\hline 265 & 40 \\
\hline 266 & 33 \\
\hline 267 & 15 \\
\hline 268 & 14 \\
\hline 269 & 12 \\
\hline 270 & 42 \\
\hline 271 & 6 \\
\hline 272 & 76 \\
\hline 273 & 13 \\
\hline 274 & 94 \\
\hline 275 & 10 \\
\hline 276 & 26 \\
\hline 277 & 8 \\
\hline 278 & 22 \\
\hline 279 & 4 \\
\hline 280 & 28 \\
\hline 281 & 42 \\
\hline 282 & 30 \\
\hline 283 & 11 \\
\hline 284 & 1 \\
\hline 285 & 2 \\
\hline 286 & 13 \\
\hline 287 & 0 \\
\hline 288 & 9 \\
\hline 289 & 10 \\
\hline
\end{tabular}




\begin{tabular}{|c|c|}
\hline 290 & 11 \\
\hline 291 & 11 \\
\hline 292 & 0 \\
\hline 293 & 14 \\
\hline 294 & 95 \\
\hline 295 & 0 \\
\hline 296 & 0 \\
\hline 297 & 13 \\
\hline 298 & 4 \\
\hline 299 & 0 \\
\hline 300 & 0 \\
\hline 301 & 4 \\
\hline 302 & 12 \\
\hline 303 & 27 \\
\hline 304 & 1 \\
\hline 305 & 37 \\
\hline 306 & 0 \\
\hline 307 & 12 \\
\hline 308 & 25 \\
\hline 309 & 14 \\
\hline 310 & 94 \\
\hline 311 & 21 \\
\hline 312 & 91 \\
\hline 313 & 11 \\
\hline 314 & 95 \\
\hline 315 & 12 \\
\hline 316 & 0 \\
\hline 317 & 22 \\
\hline 318 & 0 \\
\hline 319 & 4 \\
\hline 320 & 64 \\
\hline 321 & 91 \\
\hline 322 & 16 \\
\hline 323 & 11 \\
\hline 324 & 0 \\
\hline 325 & 95 \\
\hline 326 & 1 \\
\hline 327 & 31 \\
\hline 328 & 11 \\
\hline 329 & 9 \\
\hline 330 & 33 \\
\hline 331 & 16 \\
\hline 332 & 11 \\
\hline 333 & 1 \\
\hline 334 & 99 \\
\hline 335 & 11 \\
\hline 336 & 4 \\
\hline 337 & 9 \\
\hline 338 & 12 \\
\hline 339 & 22 \\
\hline 340 & 7 \\
\hline 341 & 96 \\
\hline 342 & 0 \\
\hline 343 & 26 \\
\hline 344 & 8 \\
\hline 345 & 25 \\
\hline 346 & 3 \\
\hline 347 & 14 \\
\hline 348 & 91 \\
\hline 349 & 0 \\
\hline 350 & 14 \\
\hline 351 & 96 \\
\hline 352 & 11 \\
\hline 353 & 22 \\
\hline 354 & 30 \\
\hline 355 & 7 \\
\hline 356 & 26 \\
\hline 357 & 15 \\
\hline 358 & 7 \\
\hline 359 & 16 \\
\hline 360 & 76 \\
\hline 361 & 7 \\
\hline 362 & 0 \\
\hline 363 & 9 \\
\hline 364 & 94 \\
\hline 365 & 26 \\
\hline 366 & 8 \\
\hline 367 & 9 \\
\hline 368 & 0 \\
\hline 369 & 95 \\
\hline 370 & 10 \\
\hline 371 & 18 \\
\hline 372 & 12 \\
\hline 373 & 5 \\
\hline 374 & 0 \\
\hline 375 & 99 \\
\hline 376 & 42 \\
\hline 377 & 16 \\
\hline 378 & 95 \\
\hline 379 & 3 \\
\hline 380 & 12 \\
\hline 381 & 94 \\
\hline 382 & 19 \\
\hline 383 & 3 \\
\hline 384 & 11 \\
\hline 385 & 12 \\
\hline 386 & 1 \\
\hline
\end{tabular}




\begin{tabular}{|c|c|}
\hline 387 & 18 \\
\hline 388 & 37 \\
\hline 389 & 77 \\
\hline 390 & 0 \\
\hline 391 & 8 \\
\hline 392 & 26 \\
\hline 393 & 11 \\
\hline 394 & 28 \\
\hline 395 & 0 \\
\hline 396 & 42 \\
\hline 397 & 76 \\
\hline 398 & 42 \\
\hline 399 & 2 \\
\hline 400 & 21 \\
\hline 401 & 2 \\
\hline 402 & 24 \\
\hline 403 & 22 \\
\hline 404 & 11 \\
\hline 405 & 1 \\
\hline 406 & 10 \\
\hline 407 & 0 \\
\hline 408 & 8 \\
\hline 409 & 10 \\
\hline 410 & 1 \\
\hline 411 & 11 \\
\hline 412 & 0 \\
\hline 413 & 16 \\
\hline 414 & 7 \\
\hline 415 & 99 \\
\hline 416 & 33 \\
\hline 417 & 0 \\
\hline 418 & 16 \\
\hline 419 & 10 \\
\hline 420 & 94 \\
\hline 421 & 13 \\
\hline 422 & 33 \\
\hline 423 & 30 \\
\hline 424 & 0 \\
\hline 425 & 16 \\
\hline 426 & 12 \\
\hline 427 & 25 \\
\hline 428 & 0 \\
\hline 429 & 96 \\
\hline 430 & 96 \\
\hline 431 & 0 \\
\hline 432 & 28 \\
\hline 433 & 8 \\
\hline 434 & 33 \\
\hline 435 & 76 \\
\hline 436 & 22 \\
\hline 437 & 12 \\
\hline 438 & 5 \\
\hline 439 & 3 \\
\hline 440 & 33 \\
\hline 441 & 13 \\
\hline 442 & 7 \\
\hline 443 & 22 \\
\hline 444 & 7 \\
\hline 445 & 10 \\
\hline 446 & 6 \\
\hline 447 & 16 \\
\hline 448 & 9 \\
\hline 449 & 64 \\
\hline 450 & 12 \\
\hline 451 & 19 \\
\hline 452 & 15 \\
\hline 453 & 10 \\
\hline 454 & 22 \\
\hline 455 & 4 \\
\hline 456 & 94 \\
\hline 457 & 14 \\
\hline 458 & 28 \\
\hline 459 & 28 \\
\hline 460 & 27 \\
\hline 461 & 94 \\
\hline 462 & 31 \\
\hline 463 & 91 \\
\hline 464 & 19 \\
\hline 465 & 64 \\
\hline 466 & 42 \\
\hline 467 & 15 \\
\hline 468 & 12 \\
\hline 469 & 68 \\
\hline 470 & 14 \\
\hline 471 & 1 \\
\hline 472 & 14 \\
\hline 473 & 0 \\
\hline 474 & 4 \\
\hline 475 & 0 \\
\hline 476 & 4 \\
\hline 477 & 9 \\
\hline 478 & 40 \\
\hline 479 & 12 \\
\hline 480 & 27 \\
\hline 481 & 42 \\
\hline 482 & 12 \\
\hline 483 & 0 \\
\hline
\end{tabular}




$\begin{array}{lcl}484 & 0 & 0.542030534 \\ 485 & 0 & 0.041655282 \\ 486 & 0 & 0.51246208 \\ 487 & 0 & 0.731579742 \\ 488 & 68 & 0.305530167 \\ 489 & 30 & 0.377652431 \\ 490 & 95 & 0.633150554 \\ 491 & 16 & 0.926016046 \\ 492 & 6 & 0.865855543 \\ 493 & 16 & 0.573160397 \\ 494 & 13 & 0.346674117 \\ 495 & 4 & 0.127412649 \\ 496 & 0 & 0.801575739 \\ 497 & 42 & 0.842277972 \\ 498 & 91 & 0.546346632 \\ 499 & 42 & 0.816921816 \\ 500 & 24 & 0.235078384 \\ 501 & 8 & 0.749287462 \\ 502 & 0 & 0.110990367 \\ 503 & 12 & 0.344961852 \\ 504 & 42 & 0.907420929 \\ 505 & 16 & 0.11421931 \\ 506 & 13 & 0.800607594 \\ 507 & 9 & 0.029363653 \\ 508 & 28 & 0.465284221 \\ 509 & 7 & 0.557705072 \\ 510 & 7 & 0.806538259\end{array}$
0.5043095

$\begin{array}{lr}\text { mean } & 0.565651383 \\ \text { st dev } & 0.03331947\end{array}$

0.565651383 


\begin{tabular}{|c|c|c|c|c|c|c|}
\hline $\begin{array}{r}100 \mu \mathrm{M} \text { ATP } \\
\text { normalized } \\
100\end{array}$ & position & $\begin{array}{c}\text { cumulative sample fraction } \\
0.001960784\end{array}$ & $\begin{array}{l}\text { inhibition } \% \\
0\end{array}$ & $\begin{array}{c}\text { fraction of total inhibition } \\
0\end{array}$ & $\begin{array}{c}\text { cumulative inhibition } \\
0\end{array}$ & area \\
\hline 100 & 2 & 0.003921569 & 0 & 0 & 0 & 0 \\
\hline $\begin{array}{l}100 \\
100\end{array}$ & $\begin{array}{l}3 \\
4\end{array}$ & $\begin{array}{l}0.005882353 \\
0.007843137\end{array}$ & $\begin{array}{l}0 \\
0\end{array}$ & $\begin{array}{l}0 \\
0\end{array}$ & $\begin{array}{l}0 \\
0\end{array}$ & $\begin{array}{l}0 \\
0\end{array}$ \\
\hline 100 & 5 & 0.009803922 & 0 & 0 & 0 & 0 \\
\hline 100 & 6 & 0.011764706 & 0 & 0 & 0 & 0 \\
\hline 97 & 7 & 0.01372549 & 3 & $7.07214 \mathrm{E}-05$ & $7.07214 \mathrm{E}-05$ & $6.93347 \mathrm{E}-08$ \\
\hline 97 & 8 & 0.015686275 & 3 & 7.07214E-05 & 0.000141443 & $2.08004 \mathrm{E}-07$ \\
\hline 97 & 9 & 0.017647059 & 3 & $7.07214 \mathrm{E}-05$ & 0.000212164 & 3.46673E-07 \\
\hline 97 & 10 & 0.019607843 & 3 & 7.07214E-05 & 0.000282885 & 4.85343E-07 \\
\hline 97 & 11 & 0.021568627 & 3 & 7.07214E-05 & 0.000353607 & $6.24012 \mathrm{E}-07$ \\
\hline 97 & 12 & 0.023529412 & 3 & 7.07214E-05 & 0.000424328 & $7.62681 \mathrm{E}-07$ \\
\hline 92 & 13 & 0.025490196 & 8 & 0.00018859 & 0.000612918 & $1.01691 \mathrm{E}-06$ \\
\hline 92 & 14 & 0.02745098 & 8 & 0.00018859 & 0.000801509 & 1.38669E-06 \\
\hline 92 & 15 & 0.029411765 & 8 & 0.00018859 & 0.000990099 & $1.75648 \mathrm{E}-06$ \\
\hline 92 & 16 & 0.031372549 & 8 & 0.00018859 & 0.001178689 & $2.12626 \mathrm{E}-06$ \\
\hline 92 & 17 & 0.033333333 & 8 & 0.00018859 & 0.00136728 & $2.49605 \mathrm{E}-06$ \\
\hline 92 & 18 & 0.035294118 & 8 & 0.00018859 & 0.00155587 & 2.86583E-06 \\
\hline 84 & 19 & 0.037254902 & 16 & 0.000377181 & 0.00193305 & $3.42051 \mathrm{E}-06$ \\
\hline 84 & 20 & 0.039215686 & 16 & 0.000377181 & 0.002310231 & $4.16008 \mathrm{E}-06$ \\
\hline 84 & 21 & 0.041176471 & 16 & 0.000377181 & 0.002687412 & $4.89965 \mathrm{E}-06$ \\
\hline 84 & 22 & 0.043137255 & 16 & 0.000377181 & 0.003064592 & 5.63922E-06 \\
\hline 84 & 23 & 0.045098039 & 16 & 0.000377181 & 0.003441773 & 6.37879E-06 \\
\hline 84 & 24 & 0.047058824 & 16 & 0.000377181 & 0.003818953 & $7.11836 \mathrm{E}-06$ \\
\hline 82 & 25 & 0.049019608 & 18 & 0.000424328 & 0.004243281 & 7.90415E-06 \\
\hline 82 & 26 & 0.050980392 & 18 & 0.000424328 & 0.00466761 & 8.73617E-06 \\
\hline 82 & 27 & 0.052941176 & 18 & 0.000424328 & 0.005091938 & $9.56818 \mathrm{E}-06$ \\
\hline 82 & 28 & 0.054901961 & 18 & 0.000424328 & 0.005516266 & $1.04002 \mathrm{E}-05$ \\
\hline 82 & 29 & 0.056862745 & 18 & 0.000424328 & 0.005940594 & $1.12322 \mathrm{E}-05$ \\
\hline 82 & 30 & 0.058823529 & 18 & 0.000424328 & 0.006364922 & $1.20642 \mathrm{E}-05$ \\
\hline 80 & 31 & 0.060784314 & 20 & 0.000471476 & 0.006836398 & 1.29425E-05 \\
\hline 80 & 32 & 0.062745098 & 20 & 0.000471476 & 0.007307874 & $1.38669 \mathrm{E}-05$ \\
\hline 80 & 33 & 0.064705882 & 20 & 0.000471476 & 0.007779349 & $1.47914 \mathrm{E}-05$ \\
\hline 80 & 34 & 0.066666667 & 20 & 0.000471476 & 0.008250825 & 1.57159E-05 \\
\hline 80 & 35 & 0.068627451 & 20 & 0.000471476 & 0.008722301 & 1.66403E-05 \\
\hline 80 & 36 & 0.070588235 & 20 & 0.000471476 & 0.009193777 & $1.75648 \mathrm{E}-05$ \\
\hline 71 & 37 & 0.07254902 & 29 & 0.00068364 & 0.009877416 & $1.86972 \mathrm{E}-05$ \\
\hline 71 & 38 & 0.074509804 & 29 & 0.00068364 & 0.010561056 & $2.00377 \mathrm{E}-05$ \\
\hline 71 & 39 & 0.076470588 & 29 & 0.00068364 & 0.011244696 & 2.13782E-05 \\
\hline 71 & 40 & 0.078431373 & 29 & 0.00068364 & 0.011928336 & 2.27187E-05 \\
\hline 71 & 41 & 0.080392157 & 29 & 0.00068364 & 0.012611975 & $2.40591 \mathrm{E}-05$ \\
\hline 71 & 42 & 0.082352941 & 29 & 0.00068364 & 0.013295615 & 2.53996E-05 \\
\hline 69 & 43 & 0.084313725 & 31 & 0.000730787 & 0.014026403 & 2.67863E-05 \\
\hline 69 & 44 & 0.08627451 & 31 & 0.000730787 & 0.01475719 & 2.82192E-05 \\
\hline 69 & 45 & 0.088235294 & 31 & 0.000730787 & 0.015487977 & $2.96521 \mathrm{E}-05$ \\
\hline 69 & 46 & 0.090196078 & 31 & 0.000730787 & 0.016218765 & $3.1085 \mathrm{E}-05$ \\
\hline 69 & 47 & 0.092156863 & 31 & 0.000730787 & 0.016949552 & $3.2518 \mathrm{E}-05$ \\
\hline 69 & 48 & 0.094117647 & 31 & 0.000730787 & 0.017680339 & 3.39509E-05 \\
\hline 64 & 49 & 0.096078431 & 36 & 0.000848656 & 0.018528996 & 3.54993E-05 \\
\hline 64 & 50 & 0.098039216 & 36 & 0.000848656 & 0.019377652 & $3.71634 \mathrm{E}-05$ \\
\hline 64 & 51 & 0.1 & 36 & 0.000848656 & 0.020226308 & 3.88274E-05 \\
\hline 64 & 52 & 0.101960784 & 36 & 0.000848656 & 0.021074965 & 4.04914E-05 \\
\hline 64 & 53 & 0.103921569 & 36 & 0.000848656 & 0.021923621 & 4.21555E-05 \\
\hline 64 & 54 & 0.105882353 & 36 & 0.000848656 & 0.022772277 & 4.38195E-05 \\
\hline 59 & 55 & 0.107843137 & 41 & 0.000966525 & 0.023738802 & 4.55991E-05 \\
\hline 59 & 56 & 0.109803922 & 41 & 0.000966525 & 0.024705328 & 4.74942E-05 \\
\hline 59 & 57 & 0.111764706 & 41 & 0.000966525 & 0.025671853 & 4.93894E-05 \\
\hline 59 & $\begin{array}{l}58 \\
59\end{array}$ & 0.11372549 & 41 & 0.000966525 & & $\begin{array}{l}5.12845 \mathrm{E}-05 \\
5.1797 \mathrm{~F}-05\end{array}$ \\
\hline $\begin{array}{l}59 \\
59\end{array}$ & $\begin{array}{l}59 \\
60\end{array}$ & $\begin{array}{l}0.115686275 \\
0.117647059\end{array}$ & $\begin{array}{l}41 \\
41\end{array}$ & $\begin{array}{l}0.000966525 \\
0.000966525\end{array}$ & $\begin{array}{l}0.027604903 \\
0.028571429\end{array}$ & $\begin{array}{l}5.31797 \mathrm{E}-05 \\
5.50748 \mathrm{E}-05\end{array}$ \\
\hline 56 & 61 & 0.119607843 & 44 & 0.001037247 & 0.029608675 & 5.70393E-05 \\
\hline 56 & 62 & 0.121568627 & 44 & 0.001037247 & 0.030645922 & $5.90731 \mathrm{E}-05$ \\
\hline 56 & 63 & 0.123529412 & 44 & 0.001037247 & 0.031683168 & $6.1107 \mathrm{E}-05$ \\
\hline 56 & 64 & 0.125490196 & 44 & 0.001037247 & 0.032720415 & $6.31408 \mathrm{E}-05$ \\
\hline 56 & 65 & 0.12745098 & 44 & 0.001037247 & 0.033757661 & $6.51746 \mathrm{E}-05$ \\
\hline 56 & 66 & 0.129411765 & 44 & 0.001037247 & 0.034794908 & $6.72084 \mathrm{E}-05$ \\
\hline 54 & 67 & 0.131372549 & 46 & 0.001084394 & 0.035879302 & 6.92884E-05 \\
\hline 54 & 68 & 0.133333333 & 46 & 0.001084394 & 0.036963696 & 7.14147E-05 \\
\hline 54 & 69 & 0.135294118 & 46 & 0.001084394 & 0.038048091 & $7.3541 \mathrm{E}-05$ \\
\hline 54 & 70 & 0.137254902 & 46 & 0.001084394 & 0.039132485 & 7.56672E-05 \\
\hline 54 & 71 & 0.139215686 & 46 & 0.001084394 & 0.040216879 & $7.77935 \mathrm{E}-05$ \\
\hline 54 & 72 & 0.141176471 & 46 & 0.001084394 & 0.041301273 & $7.99198 \mathrm{E}-05$ \\
\hline 53 & 73 & 0.143137255 & 47 & 0.001107968 & 0.042409241 & $8.20691 \mathrm{E}-05$ \\
\hline 53 & 74 & 0.145098039 & 47 & 0.001107968 & 0.043517209 & $8.42416 \mathrm{E}-05$ \\
\hline 53 & 75 & 0.147058824 & 47 & 0.001107968 & 0.044625177 & $8.64141 \mathrm{E}-05$ \\
\hline 53 & 76 & 0.149019608 & 47 & 0.001107968 & 0.045733145 & $8.85866 \mathrm{E}-05$ \\
\hline 53 & 77 & 0.150980392 & 47 & 0.001107968 & 0.046841113 & $9.07591 \mathrm{E}-05$ \\
\hline 53 & 78 & 0.152941176 & 47 & 0.001107968 & 0.047949081 & $9.29316 \mathrm{E}-05$ \\
\hline 47 & 79 & 0.154901961 & 53 & 0.001249411 & 0.049198491 & $9.52427 \mathrm{E}-05$ \\
\hline 47 & 80 & 0.156862745 & 53 & 0.001249411 & 0.050447902 & $9.76925 \mathrm{E}-05$ \\
\hline 47 & 81 & 0.158823529 & 53 & 0.001249411 & 0.051697313 & 0.000100142 \\
\hline 47 & 82 & 0.160784314 & 53 & 0.001249411 & 0.052946723 & 0.000102592 \\
\hline 47 & 83 & 0.162745098 & 53 & 0.001249411 & 0.054196134 & 0.000105042 \\
\hline 47 & 84 & 0.164705882 & 53 & 0.001249411 & 0.055445545 & 0.000107492 \\
\hline 46 & 85 & 0.166666667 & 54 & 0.001272984 & 0.056718529 & 0.000109965 \\
\hline 46 & 86 & 0.168627451 & 54 & 0.001272984 & 0.057991513 & 0.000112461 \\
\hline 46 & 87 & 0.170588235 & 54 & 0.001272984 & 0.059264498 & 0.000114957 \\
\hline 46 & 88 & 0.17254902 & 54 & 0.001272984 & 0.060537482 & 0.000117453 \\
\hline 46 & 89 & 0.174509804 & 54 & 0.001272984 & 0.061810467 & 0.000119949 \\
\hline 46 & 90 & 0.176470588 & 54 & 0.001272984 & 0.063083451 & 0.000122445 \\
\hline 39 & 91 & 0.178431373 & 61 & 0.001438001 & 0.064521452 & 0.000125103 \\
\hline 39 & 92 & 0180392157 & 61 & 0.001438001 & 0065959453 & 0000127922 \\
\hline
\end{tabular}




\begin{tabular}{|c|c|c|c|c|c|c|}
\hline 39 & 93 & 0.182352941 & 61 & 0.001438001 & 0.067397454 & 0.000130742 \\
\hline $\begin{array}{l}39 \\
39\end{array}$ & $\begin{array}{l}94 \\
95\end{array}$ & $\begin{array}{l}0.184313725 \\
0.18627451\end{array}$ & $\begin{array}{l}61 \\
61\end{array}$ & $\begin{array}{l}0.001438001 \\
0.001438001\end{array}$ & $\begin{array}{l}0.068835455 \\
0.070273456\end{array}$ & $\begin{array}{l}0.000133562 \\
0.000136381\end{array}$ \\
\hline $\begin{array}{l}39 \\
34 \\
34\end{array}$ & $\begin{array}{l}96 \\
97 \\
98\end{array}$ & $\begin{array}{l}0.188235294 \\
0.190196078 \\
0.192156863\end{array}$ & $\begin{array}{l}61 \\
66 \\
66\end{array}$ & $\begin{array}{c}0.001438001 \\
0.00155587 \\
0.00155587\end{array}$ & $\begin{array}{l}0.071711457 \\
0.073267327 \\
0.074823197\end{array}$ & $\begin{array}{l}0.000139201 \\
0.000142136 \\
0.000145187\end{array}$ \\
\hline $\begin{array}{l}34 \\
34\end{array}$ & $\begin{array}{r}99 \\
100\end{array}$ & $\begin{array}{l}0.194117647 \\
0.196078431\end{array}$ & $\begin{array}{l}66 \\
66\end{array}$ & $\begin{array}{l}0.00155587 \\
0.00155587\end{array}$ & $\begin{array}{l}0.076379066 \\
0.077934936\end{array}$ & $\begin{array}{l}0.000148238 \\
0.000151288\end{array}$ \\
\hline $\begin{array}{l}34 \\
34\end{array}$ & $\begin{array}{l}101 \\
102\end{array}$ & $\begin{array}{c}0.198039216 \\
0.2\end{array}$ & $\begin{array}{l}66 \\
66\end{array}$ & $\begin{array}{l}0.00155587 \\
0.00155587\end{array}$ & $\begin{array}{l}0.079490806 \\
0.081046676\end{array}$ & $\begin{array}{r}0.000154339 \\
0.00015739\end{array}$ \\
\hline 31 & 103 & 0.201960784 & 69 & 0.001626591 & 0.082673267 & 0.00016051 \\
\hline 31 & 104 & 0.203921569 & 69 & 0.001626591 & 0.084299859 & 0.000163699 \\
\hline 31 & 105 & 0.205882353 & 69 & 0.001626591 & 0.08592645 & 0.000166889 \\
\hline 31 & 106 & 0.207843137 & 69 & 0.001626591 & 0.087553041 & 0.000170078 \\
\hline 31 & 107 & 0.209803922 & 69 & 0.001626591 & 0.089179632 & 0.000173267 \\
\hline 31 & 108 & 0.211764706 & 69 & 0.001626591 & 0.090806223 & 0.000176457 \\
\hline 27 & 109 & 0.21372549 & 73 & 0.001720886 & 0.09252711 & 0.000179739 \\
\hline 27 & 110 & 0.215686275 & 73 & 0.001720886 & 0.094247996 & 0.000183113 \\
\hline 27 & 111 & 0.217647059 & 73 & 0.001720886 & 0.095968883 & 0.000186487 \\
\hline $\begin{array}{l}27 \\
27\end{array}$ & $\begin{array}{l}112 \\
113\end{array}$ & $\begin{array}{l}0.219607843 \\
0.221568627\end{array}$ & $\begin{array}{l}73 \\
73\end{array}$ & $\begin{array}{l}0.001720886 \\
0.001720886\end{array}$ & $\begin{array}{l}0.097689769 \\
0.099410655\end{array}$ & $\begin{array}{l}0.000189861 \\
0.000193236\end{array}$ \\
\hline 27 & 114 & 0.223529412 & 73 & 0.001720886 & 0.101131542 & 0.00019661 \\
\hline 25 & 115 & 0.225490196 & 75 & 0.001768034 & 0.102899576 & 0.000200031 \\
\hline 25 & 116 & 0.22745098 & 75 & 0.001768034 & 0.10466761 & 0.000203497 \\
\hline 25 & 117 & 0.229411765 & 75 & 0.001768034 & 0.106435644 & 0.000206964 \\
\hline 25 & 118 & 0.231372549 & 75 & 0.001768034 & 0.108203678 & 0.000210431 \\
\hline 25 & 119 & 0.233333333 & 75 & 0.001768034 & 0.109971711 & 0.000213897 \\
\hline 25 & 120 & 0.235294118 & 75 & 0.001768034 & 0.111739745 & 0.000217364 \\
\hline $\begin{array}{l}22 \\
22\end{array}$ & $\begin{array}{l}121 \\
122\end{array}$ & $\begin{array}{l}0.237254902 \\
0.239215686\end{array}$ & $\begin{array}{l}78 \\
78\end{array}$ & $\begin{array}{l}0.001838755 \\
0.001838755\end{array}$ & $\begin{array}{l}0.113578501 \\
0.115417256\end{array}$ & $\begin{array}{r}0.0002209 \\
0.000224506\end{array}$ \\
\hline 22 & 123 & 0.241176471 & 78 & 0.001838755 & 0.117256011 & 0.000228111 \\
\hline 22 & 124 & 0.243137255 & 78 & 0.001838755 & 0.119094767 & 0.000231716 \\
\hline 22 & 125 & 0.245098039 & 78 & 0.001838755 & 0.120933522 & 0.000235322 \\
\hline 22 & 126 & 0.247058824 & 78 & 0.001838755 & 0.122772277 & 0.000238927 \\
\hline 19 & 127 & 0.249019608 & 81 & 0.001909477 & 0.124681754 & 0.000242602 \\
\hline 19 & 128 & 0.250980392 & 81 & 0.001909477 & 0.126591231 & 0.000246346 \\
\hline 19 & 129 & 0.252941176 & 81 & 0.001909477 & 0.128500707 & 0.00025009 \\
\hline 19 & 130 & 0.254901961 & 81 & 0.001909477 & 0.130410184 & 0.000253834 \\
\hline 19 & 131 & 0.256862745 & 81 & 0.001909477 & 0.132319661 & 0.000257578 \\
\hline 19 & 132 & 0.258823529 & 81 & 0.001909477 & 0.134229137 & 0.000261322 \\
\hline 17 & 133 & 0.260784314 & 83 & 0.001956624 & 0.136185761 & 0.000265113 \\
\hline 17 & 134 & 0.262745098 & 83 & 0.001956624 & 0.138142386 & 0.000268949 \\
\hline 17 & 135 & 0.264705882 & 83 & 0.001956624 & 0.14009901 & 0.000272786 \\
\hline 17 & 136 & 0.266666667 & 83 & 0.001956624 & 0.142055634 & 0.000276622 \\
\hline 17 & 137 & 0.268627451 & 83 & 0.001956624 & 0.144012258 & 0.000280459 \\
\hline 17 & 138 & 0.270588235 & 83 & 0.001956624 & 0.145968883 & 0.000284295 \\
\hline 17 & 139 & 0.27254902 & 83 & 0.001956624 & 0.147925507 & 0.000288132 \\
\hline 17 & 140 & 0.274509804 & 83 & 0.001956624 & 0.149882131 & 0.000291968 \\
\hline 17 & 141 & 0.276470588 & 83 & 0.001956624 & 0.151838755 & 0.000295805 \\
\hline 17 & 142 & 0.278431373 & 83 & 0.001956624 & 0.15379538 & 0.000299641 \\
\hline 17 & 143 & 0.280392157 & 83 & 0.001956624 & 0.155752004 & 0.000303478 \\
\hline 17 & 144 & 0.282352941 & 83 & 0.001956624 & 0.157708628 & 0.000307314 \\
\hline 15 & 145 & 0.284313725 & 85 & 0.002003772 & 0.1597124 & 0.000311197 \\
\hline 15 & 146 & 0.28627451 & 85 & 0.002003772 & 0.161716172 & 0.000315126 \\
\hline 15 & 147 & 0.288235294 & 85 & 0.002003772 & 0.163719943 & 0.000319055 \\
\hline 15 & 148 & 0.290196078 & 85 & 0.002003772 & 0.165723715 & 0.000322984 \\
\hline 15 & 149 & 0.292156863 & 85 & 0.002003772 & 0.167727487 & 0.000326913 \\
\hline 15 & 150 & 0.294117647 & 85 & 0.002003772 & 0.169731259 & 0.000330842 \\
\hline 15 & 151 & 0.296078431 & 85 & 0.002003772 & 0.171735031 & 0.000334771 \\
\hline 15 & 152 & 0.298039216 & 85 & 0.002003772 & 0.173738802 & 0.0003387 \\
\hline 15 & 153 & 0.3 & 85 & 0.002003772 & 0.175742574 & 0.000342629 \\
\hline 15 & 154 & 0.301960784 & 85 & 0.002003772 & 0.177746346 & 0.000346558 \\
\hline 15 & 155 & 0.303921569 & 85 & 0.002003772 & 0.179750118 & 0.000350487 \\
\hline 15 & 156 & 0.305882353 & 85 & 0.002003772 & 0.18175389 & 0.000354416 \\
\hline 14 & 157 & 0.307843137 & 86 & 0.002027346 & 0.183781235 & 0.000358368 \\
\hline 14 & 158 & 0.309803922 & 86 & 0.002027346 & 0.185808581 & 0.000362343 \\
\hline 14 & 159 & 0.311764706 & 86 & 0.002027346 & 0.187835926 & 0.000366318 \\
\hline 14 & 160 & 0.31372549 & 86 & 0.002027346 & 0.189863272 & 0.000370293 \\
\hline 14 & 161 & 0.315686275 & 86 & 0.002027346 & 0.191890618 & 0.000374269 \\
\hline 14 & 162 & 0.317647059 & 86 & 0.002027346 & 0.193917963 & 0.000378244 \\
\hline 12 & 163 & 0.319607843 & 88 & 0.002074493 & 0.195992456 & 0.000382265 \\
\hline 12 & 164 & 0.321568627 & 88 & 0.002074493 & 0.19806695 & 0.000386333 \\
\hline 12 & 165 & 0.323529412 & 88 & 0.002074493 & 0.200141443 & 0.0003904 \\
\hline 12 & 166 & 0.325490196 & 88 & 0.002074493 & 0.202215936 & 0.000394468 \\
\hline 12 & 167 & 0.32745098 & 88 & 0.002074493 & 0.204290429 & 0.000398536 \\
\hline 12 & 168 & 0.329411765 & 88 & 0.002074493 & 0.206364922 & 0.000402603 \\
\hline 9 & 169 & 0.331372549 & 91 & 0.002145215 & 0.208510137 & 0.00040674 \\
\hline 9 & 170 & 0.333333333 & 91 & 0.002145215 & 0.210655351 & 0.000410947 \\
\hline 9 & 171 & 0.335294118 & 91 & 0.002145215 & 0.212800566 & 0.000415153 \\
\hline 9 & 172 & 0.337254902 & 91 & 0.002145215 & 0.21494578 & 0.000419359 \\
\hline 9 & 173 & 0.339215686 & 91 & 0.002145215 & 0.217090995 & 0.000423565 \\
\hline 9 & 174 & 0.341176471 & 91 & 0.002145215 & 0.219236209 & 0.000427772 \\
\hline 8 & 175 & 0.343137255 & 92 & 0.002168788 & 0.221404998 & 0.000432001 \\
\hline 8 & 176 & 0.345098039 & 92 & 0.002168788 & 0.223573786 & 0.000436254 \\
\hline 8 & 177 & 0.347058824 & 92 & 0.002168788 & 0.225742574 & 0.000440506 \\
\hline 8 & 178 & 0.349019608 & 92 & 0.002168788 & 0.227911363 & 0.000444759 \\
\hline 8 & 179 & 0.350980392 & 92 & 0.002168788 & 0.230080151 & 0.000449011 \\
\hline 8 & 180 & 0.352941176 & 92 & 0.002168788 & 0.232248939 & 0.000453264 \\
\hline 8 & 181 & 0.354901961 & 92 & 0.002168788 & 0.234417727 & 0.000457516 \\
\hline 8 & 182 & 0.356862745 & 92 & 0.002168788 & 0.236586516 & 0.000461769 \\
\hline 8 & 183 & 0.358823529 & 92 & 0.002168788 & 0.238755304 & 0.000466021 \\
\hline 8 & 184 & 0.360784314 & 92 & 0.002168788 & 0.240924092 & 0.000470274 \\
\hline 8 & 185 & 0.362745098 & 92 & 0.002168788 & 0.243092881 & 0.000474526 \\
\hline 8 & 186 & 0.364705882 & 92 & 0.002168788 & 0.245261669 & 0.000478779 \\
\hline
\end{tabular}




\begin{tabular}{|c|c|c|}
\hline 7 & 187 & 0.366666667 \\
\hline $\begin{array}{l}7 \\
7\end{array}$ & $\begin{array}{l}188 \\
189\end{array}$ & $\begin{array}{l}0.368627451 \\
0.370588235\end{array}$ \\
\hline $\begin{array}{l}7 \\
7\end{array}$ & 190 & $\begin{array}{l}0.37254902 \\
0.374509804\end{array}$ \\
\hline 7 & 192 & 0.376470588 \\
\hline 6 & 193 & 0.378431373 \\
\hline 6 & 194 & 0.380392157 \\
\hline 6 & 195 & 0.382352941 \\
\hline 6 & 196 & 0.384313725 \\
\hline 6 & 197 & 0.38627451 \\
\hline 6 & 198 & 0.388235294 \\
\hline 5 & 199 & 0.390196078 \\
\hline 5 & 200 & 0.392156863 \\
\hline 5 & 201 & 0.394117647 \\
\hline 5 & 202 & 0.396078431 \\
\hline 5 & 203 & 0.398039216 \\
\hline 5 & 204 & 0.4 \\
\hline 5 & 205 & 0.401960784 \\
\hline $\begin{array}{l}5 \\
5\end{array}$ & $\begin{array}{l}206 \\
207\end{array}$ & $\begin{array}{l}0.403921569 \\
0.405882353\end{array}$ \\
\hline 5 & 208 & 0.407843137 \\
\hline 5 & 209 & 0.409803922 \\
\hline 5 & 210 & 0.411764706 \\
\hline 4 & 211 & 0.41372549 \\
\hline 4 & 212 & 0.415686275 \\
\hline 4 & 213 & 0.417647059 \\
\hline 4 & 214 & 0.419607843 \\
\hline 4 & 215 & 0.421568627 \\
\hline 4 & 216 & 0.423529412 \\
\hline 4 & 217 & 0.425490196 \\
\hline 4 & 218 & 0.42745098 \\
\hline 4 & 219 & 0.429411765 \\
\hline 4 & 220 & 0.431372549 \\
\hline 4 & 221 & 0.433333333 \\
\hline 4 & 222 & 0.435294118 \\
\hline 4 & 223 & 0.437254902 \\
\hline 4 & 224 & 0.439215686 \\
\hline 4 & 225 & 0.441176471 \\
\hline 4 & 226 & 0.443137255 \\
\hline 4 & 227 & 0.445098039 \\
\hline 4 & 228 & 0.447058824 \\
\hline 3 & 229 & 0.449019608 \\
\hline 3 & 230 & 0.450980392 \\
\hline 3 & 231 & 0.452941176 \\
\hline 3 & 232 & 0.454901961 \\
\hline 3 & 233 & 0.456862745 \\
\hline 3 & 234 & 0.458823529 \\
\hline 3 & 235 & 0.460784314 \\
\hline 3 & 236 & 0.462745098 \\
\hline 3 & 237 & 0.464705882 \\
\hline 3 & 238 & 0.466666667 \\
\hline 3 & 239 & 0.468627451 \\
\hline 3 & 240 & 0.470588235 \\
\hline 3 & 241 & 0.47254902 \\
\hline 3 & 242 & 0.474509804 \\
\hline 3 & 243 & 0.476470588 \\
\hline 3 & 244 & 0.478431373 \\
\hline 3 & 245 & 0.480392157 \\
\hline 3 & 246 & 0.482352941 \\
\hline 3 & 247 & 0.484313725 \\
\hline 3 & 248 & 0.48627451 \\
\hline 3 & 249 & 0.488235294 \\
\hline 3 & 250 & 0.490196078 \\
\hline 3 & 251 & 0.492156863 \\
\hline 3 & 252 & 0.494117647 \\
\hline 2 & 253 & 0.496078431 \\
\hline 2 & 254 & 0.498039216 \\
\hline 2 & 255 & 0.5 \\
\hline 2 & 256 & 0.501960784 \\
\hline 2 & 257 & 0.503921569 \\
\hline 2 & 258 & 0.505882353 \\
\hline 2 & 259 & 0.507843137 \\
\hline 2 & 260 & 0.509803922 \\
\hline 2 & 261 & 0.511764706 \\
\hline 2 & 262 & 0.51372549 \\
\hline 2 & 263 & 0.515686275 \\
\hline 2 & 264 & 0.517647059 \\
\hline 2 & 265 & 0.519607843 \\
\hline 2 & 266 & 0.521568627 \\
\hline 2 & 267 & 0.523529412 \\
\hline 2 & 268 & 0.525490196 \\
\hline 2 & 269 & 0.52745098 \\
\hline 2 & 270 & 0.529411765 \\
\hline 2 & 271 & 0.531372549 \\
\hline 2 & 272 & 0.533333333 \\
\hline 2 & 273 & 0.535294118 \\
\hline 2 & 274 & 0.537254902 \\
\hline 2 & 275 & 0.539215686 \\
\hline 2 & 276 & 0.541176471 \\
\hline 1 & 277 & 0.543137255 \\
\hline 1 & 278 & 0.545098039 \\
\hline 1 & 279 & 0.547058824 \\
\hline & & \\
\hline
\end{tabular}

\subsection{2}

0.002192362

0.002192362

0.002192362

0.002192362

0.002215936
0.002215936

0.002215936

0.002215936

0.002215936

.002215936

0.00223951

0.00223951

0.00223951

0.00223951

0.00223951

0.00223951

0.00223951

0.00223951
0.00223951

0.00223951

0.00223951

0.00223951

0.002263083

0.002263083

0.002263083

0.002263083

0.002263083

0.002263083

0.002263083

0.002263083

0.002263083

.002263083

0.002263083

0.002263083

0.002263083

0.002263083

0.002263083

0.002286657

0.002286657

0.002286657

0.002286657

0.002286657

0.002286657

0.002286657

002286657

0.002286657
0.002286657

0.002286657

0.002286657

0.002286657

0.002286657

0.002286657

002286657

0.002286657

0.002286657

0.002286657

0.002286657

0.002286657

0.002286657

.002310231

0.002310231

0.002310231

0.00231023

0.002310231

.002310231

.00231023

0.002310231
0.002310231

002310231

0.002310231

0.002310231

0.00231023

0.002310231

0.002310231
0.002310231

0.002310231

0.002310231

0.002310231

0.002310231

0.002310231

0.002310231
0.002310231

0.002333805

0.002333805

0.002333805

0.002333805
0.247454031

0.249646393

1838755

0.254031117

0.256223479
0.258415842

0.260631777

0.262847713

0.265063649

0.267279585

0.269495521
0.271711457

0.273950967

0.276190476

0.278429986

0.280669496

0.282909005

0.285148515

0.289627534

0.291867044

0.294106554

0.298585573

0.300848656

0.30311174

0.305374823

0.307637907

0.30990099

0.312164074
0.314427157

0.31669024

0.318953324

0.321216407

0.325742574
0.328005658

0.330268741

0.332531825

0.334794908

0.337057992

0.339321075

0.341607732

0.343894389

0.346181047
0.348467704

0.350754361

0.353041018

0.355327676

0.357614333

0.35990099

0.362187647

0.364474305

0.366760962

0.369047619

0.371334276

0.373620934

0.378194248

0.380480905

0.382767562

0.38505422

0.387340877

0.389627534

0.391914191

0.394200849

0.39651108

0.398821311
0.401131542

0.403441773

0.405752004

0.408062235

0.410372466

0.412682697

0.414992928
0.417303159

0.41961339

0.421923621

0.424233852

0.426544083

0.428854314

0.431164545

0.433474776

0.435785007

0.438095238

0.440405469

0.4427157

0.445025931

0.447336162

0.449646393

0.451980198

0.454314003

0.456647808

0.458981612

0.000483055

0.000487353

0.000491652

0.000495951

0.00050025

0.000504548

0.00050887

0.000513215

0.00051756

0.000521905

0.00052625

0.000530595

0.000534963

0.000539354

0.000543746

0.000548137
0.000552528

0.000556919

0.00565919

.000565702

0.000570093

0.000574484

0.000578875

0.000583266

0.000587681

0.000592118

0.000596555

0.000600993

0.00060543

0.000609868

0.000614305

0.000618743

0.00062318

0.000627617

0.000632055

0.000636492

0.00064093

0.000645367

0.000649804

0.000654242

0.000658679

0.000663117

0.000667577

0.000672061

0.000676545

0.000681028

0.000685512

0.000689995

0.000698963

0.000703446

0.00070793

0.000712414

0.000716897

0.000721381 


\begin{tabular}{|c|c|c|c|c|c|c|}
\hline 1 & 281 & 0.550980392 & 99 & 0.002333805 & 0.461315417 & 0.000902252 \\
\hline $\begin{array}{l}1 \\
1\end{array}$ & $\begin{array}{l}282 \\
283\end{array}$ & $\begin{array}{l}0.552941176 \\
0.554901961\end{array}$ & $\begin{array}{l}99 \\
99\end{array}$ & $\begin{array}{l}0.002333805 \\
0.002333805\end{array}$ & $\begin{array}{l}0.463649222 \\
0.465983027\end{array}$ & $\begin{array}{l}0.000906828 \\
0.000911404\end{array}$ \\
\hline $\begin{array}{l}1 \\
1 \\
1\end{array}$ & $\begin{array}{l}284 \\
285 \\
286\end{array}$ & $\begin{array}{l}0.556862745 \\
0.558823529 \\
0.560784314\end{array}$ & $\begin{array}{l}99 \\
99 \\
99\end{array}$ & $\begin{array}{l}0.002333805 \\
0.002333805 \\
0.002333805\end{array}$ & $\begin{array}{l}0.468316832 \\
0.470650636 \\
0.472984441\end{array}$ & $\begin{array}{r}0.00091598 \\
0.000920556 \\
0.000925132\end{array}$ \\
\hline $\begin{array}{l}1 \\
1\end{array}$ & $\begin{array}{l}287 \\
288\end{array}$ & $\begin{array}{l}0.562745098 \\
0.564705882\end{array}$ & $\begin{array}{l}99 \\
99\end{array}$ & $\begin{array}{l}0.002333805 \\
0.002333805\end{array}$ & $\begin{array}{l}0.475318246 \\
0.477652051\end{array}$ & $\begin{array}{l}0.000929709 \\
0.000934285\end{array}$ \\
\hline $\begin{array}{l}1 \\
1\end{array}$ & $\begin{array}{l}289 \\
290\end{array}$ & $\begin{array}{l}0.566666667 \\
0.568627451\end{array}$ & $\begin{array}{l}99 \\
99\end{array}$ & $\begin{array}{l}0.002333805 \\
0.002333805\end{array}$ & $\begin{array}{l}0.479985856 \\
0.482319661\end{array}$ & $\begin{array}{l}0.000938861 \\
0.000943437\end{array}$ \\
\hline 1 & 291 & 0.570588235 & 99 & 0.002333805 & 0.484653465 & 0.000948013 \\
\hline 1 & 292 & 0.57254902 & 99 & 0.002333805 & 0.48698727 & 0.000952589 \\
\hline 1 & 293 & 0.574509804 & 99 & 0.002333805 & 0.489321075 & 0.000957165 \\
\hline 1 & 294 & 0.576470588 & 99 & 0.002333805 & 0.49165488 & 0.000961741 \\
\hline 1 & 295 & 0.578431373 & 99 & 0.002333805 & 0.493988685 & 0.000966317 \\
\hline 1 & 296 & 0.580392157 & 99 & 0.002333805 & 0.496322489 & 0.000970893 \\
\hline 1 & 297 & 0.582352941 & 99 & 0.002333805 & 0.498656294 & 0.000975469 \\
\hline $\begin{array}{l}1 \\
1\end{array}$ & $\begin{array}{l}298 \\
299\end{array}$ & $\begin{array}{l}0.584313725 \\
0.58627451\end{array}$ & $\begin{array}{l}99 \\
99\end{array}$ & $\begin{array}{l}0.002333805 \\
0.002333805\end{array}$ & $\begin{array}{l}0.500990099 \\
0.503323904\end{array}$ & $\begin{array}{l}0.000980045 \\
0.000984622\end{array}$ \\
\hline 1 & 300 & 0.588235294 & 99 & 0.002333805 & 0.505657709 & 0.000989198 \\
\hline 1 & 301 & 0.590196078 & 99 & 0.002333805 & 0.507991513 & 0.000993774 \\
\hline 1 & 302 & 0.592156863 & 99 & 0.002333805 & 0.510325318 & 0.00099835 \\
\hline 1 & 303 & 0.594117647 & 99 & 0.002333805 & 0.512659123 & 0.001002926 \\
\hline 1 & 304 & 0.596078431 & 99 & 0.002333805 & 0.514992928 & 0.001007502 \\
\hline 1 & 305 & 0.598039216 & 99 & 0.002333805 & 0.517326733 & 0.001012078 \\
\hline 1 & 306 & 0.6 & 99 & 0.002333805 & 0.519660537 & 0.001016654 \\
\hline 1 & 307 & 0.601960784 & 99 & 0.002333805 & 0.521994342 & 0.00102123 \\
\hline 1 & 308 & 0.603921569 & 99 & 0.002333805 & 0.524328147 & 0.001025806 \\
\hline 1 & 309 & 0.605882353 & 99 & 0.002333805 & 0.526661952 & 0.001030382 \\
\hline 1 & 310 & 0.607843137 & 99 & 0.002333805 & 0.528995757 & 0.001034959 \\
\hline 1 & 311 & 0.609803922 & 99 & 0.002333805 & 0.531329562 & 0.001039535 \\
\hline 1 & 312 & 0.611764706 & 99 & 0.002333805 & 0.533663366 & 0.001044111 \\
\hline 1 & 313 & 0.61372549 & 99 & 0.002333805 & 0.535997171 & 0.001048687 \\
\hline 1 & 314 & 0.615686275 & 99 & 0.002333805 & 0.538330976 & 0.001053263 \\
\hline 1 & 315 & 0.617647059 & 99 & 0.002333805 & 0.540664781 & 0.001057839 \\
\hline 1 & 316 & 0.619607843 & 99 & 0.002333805 & 0.542998586 & 0.001062415 \\
\hline 1 & 317 & 0.621568627 & 99 & 0.002333805 & 0.54533239 & 0.001066991 \\
\hline 1 & 318 & 0.623529412 & 99 & 0.002333805 & 0.547666195 & 0.001071567 \\
\hline 1 & 319 & 0.625490196 & 99 & 0.002333805 & 0.55 & 0.001076143 \\
\hline 1 & 320 & 0.62745098 & 99 & 0.002333805 & 0.552333805 & 0.001080719 \\
\hline 1 & 321 & 0.629411765 & 99 & 0.002333805 & 0.55466761 & 0.001085296 \\
\hline 1 & 322 & 0.631372549 & 99 & 0.002333805 & 0.557001414 & 0.001089872 \\
\hline 1 & 323 & 0.633333333 & 99 & 0.002333805 & 0.559335219 & 0.001094448 \\
\hline 1 & 324 & 0.635294118 & 99 & 0.002333805 & 0.561669024 & 0.001099024 \\
\hline 1 & 325 & 0.637254902 & 99 & 0.002333805 & 0.564002829 & 0.0011036 \\
\hline 1 & 326 & 0.639215686 & 99 & 0.002333805 & 0.566336634 & 0.001108176 \\
\hline 1 & 327 & 0.641176471 & 99 & 0.002333805 & 0.568670438 & 0.001112752 \\
\hline 1 & 328 & 0.643137255 & 99 & 0.002333805 & 0.571004243 & 0.001117328 \\
\hline 1 & 329 & 0.645098039 & 99 & 0.002333805 & 0.573338048 & 0.001121904 \\
\hline 1 & 330 & 0.647058824 & 99 & 0.002333805 & 0.575671853 & 0.00112648 \\
\hline 0 & 331 & 0.649019608 & 100 & 0.002357379 & 0.578029231 & 0.001131079 \\
\hline 0 & 332 & 0.650980392 & 100 & 0.002357379 & 0.58038661 & 0.001135702 \\
\hline 0 & 333 & 0.652941176 & 100 & 0.002357379 & 0.582743989 & 0.001140324 \\
\hline 0 & 334 & 0.654901961 & 100 & 0.002357379 & 0.585101367 & 0.001144946 \\
\hline 0 & 335 & 0.656862745 & 100 & 0.002357379 & 0.587458746 & 0.001149569 \\
\hline 0 & 336 & 0.658823529 & 100 & 0.002357379 & 0.589816124 & 0.001154191 \\
\hline 0 & 337 & 0.660784314 & 100 & 0.002357379 & 0.592173503 & 0.001158813 \\
\hline 0 & 338 & 0.662745098 & 100 & 0.002357379 & 0.594530882 & 0.001163436 \\
\hline 0 & 339 & 0.664705882 & 100 & 0.002357379 & 0.59688826 & 0.001168058 \\
\hline 0 & 340 & 0.666666667 & 100 & 0.002357379 & 0.599245639 & 0.00117268 \\
\hline 0 & 341 & 0.668627451 & 100 & 0.002357379 & 0.601603017 & 0.001177303 \\
\hline 0 & 342 & 0.670588235 & 100 & 0.002357379 & 0.603960396 & 0.001181925 \\
\hline 0 & 343 & 0.67254902 & 100 & 0.002357379 & 0.606317775 & 0.001186547 \\
\hline 0 & 344 & 0.674509804 & 100 & 0.002357379 & 0.608675153 & 0.00119117 \\
\hline 0 & 345 & 0.676470588 & 100 & 0.002357379 & 0.611032532 & 0.001195792 \\
\hline 0 & 346 & 0.678431373 & 100 & 0.002357379 & 0.61338991 & 0.001200414 \\
\hline 0 & 347 & 0.680392157 & 100 & 0.002357379 & 0.615747289 & 0.001205036 \\
\hline 0 & 348 & 0.682352941 & 100 & 0.002357379 & 0.618104668 & 0.001209659 \\
\hline 0 & 349 & 0.684313725 & 100 & 0.002357379 & 0.620462046 & 0.001214281 \\
\hline 0 & 350 & 0.68627451 & 100 & 0.002357379 & 0.622819425 & 0.001218903 \\
\hline 0 & 351 & 0.688235294 & 100 & 0.002357379 & 0.625176803 & 0.001223526 \\
\hline 0 & 352 & 0.690196078 & 100 & 0.002357379 & 0.627534182 & 0.001228148 \\
\hline 0 & 353 & 0.692156863 & 100 & 0.002357379 & 0.629891561 & 0.00123277 \\
\hline 0 & 354 & 0.694117647 & 100 & 0.002357379 & 0.632248939 & 0.001237393 \\
\hline 0 & 355 & 0.696078431 & 100 & 0.002357379 & 0.634606318 & 0.001242015 \\
\hline 0 & 356 & 0.698039216 & 100 & 0.002357379 & 0.636963696 & 0.001246637 \\
\hline 0 & 357 & 0.7 & 100 & 0.002357379 & 0.639321075 & 0.00125126 \\
\hline 0 & 358 & 0.701960784 & 100 & 0.002357379 & 0.641678454 & 0.001255882 \\
\hline 0 & 359 & 0.703921569 & 100 & 0.002357379 & 0.644035832 & 0.001260504 \\
\hline 0 & 360 & 0.705882353 & 100 & 0.002357379 & 0.646393211 & 0.001265127 \\
\hline 0 & 361 & 0.707843137 & 100 & 0.002357379 & 0.648750589 & 0.001269749 \\
\hline 0 & 362 & 0.709803922 & 100 & 0.002357379 & 0.651107968 & 0.001274371 \\
\hline 0 & 363 & 0.711764706 & 100 & 0.002357379 & 0.653465347 & 0.001278993 \\
\hline 0 & 364 & 0.71372549 & 100 & 0.002357379 & 0.655822725 & 0.001283616 \\
\hline 0 & 365 & 0.715686275 & 100 & 0.002357379 & 0.658180104 & 0.001288238 \\
\hline 0 & 366 & 0.717647059 & 100 & 0.002357379 & 0.660537482 & 0.00129286 \\
\hline 0 & 367 & 0.719607843 & 100 & 0.002357379 & 0.662894861 & 0.001297483 \\
\hline 0 & 368 & 0.721568627 & 100 & 0.002357379 & 0.66525224 & 0.001302105 \\
\hline 0 & 369 & 0.723529412 & 100 & 0.002357379 & 0.667609618 & 0.001306727 \\
\hline 0 & 370 & 0.725490196 & 100 & 0.002357379 & 0.669966997 & 0.00131135 \\
\hline 0 & 371 & 0.72745098 & 100 & 0.002357379 & 0.672324375 & 0.001315972 \\
\hline 0 & 372 & 0.729411765 & 100 & 0.002357379 & 0.674681754 & 0.001320594 \\
\hline 0 & 373 & 0.731372549 & 100 & 0.002357379 & 0.677039132 & 0.001325217 \\
\hline 0 & 374 & 0.733333333 & 100 & 0.002357379 & 0.679396511 & 0.001329839 \\
\hline
\end{tabular}




\begin{tabular}{|c|c|c|c|c|c|}
\hline 0 & 375 & 0.735294118 & 100 & 0.002357379 & 0.68175389 \\
\hline $\begin{array}{l}0 \\
0\end{array}$ & $\begin{array}{l}376 \\
377\end{array}$ & $\begin{array}{l}0.737254902 \\
0.739215686\end{array}$ & $\begin{array}{l}100 \\
100\end{array}$ & $\begin{array}{l}0.002357379 \\
0.002357379\end{array}$ & $\begin{array}{l}0.684111268 \\
0.686468647\end{array}$ \\
\hline 0 & 378 & 0.741176471 & 100 & 0.002357379 & 0.688826025 \\
\hline 0 & 379 & 0.743137255 & 100 & 0.002357379 & 0.691183404 \\
\hline 0 & 380 & 0.745098039 & 100 & 0.002357379 & 0.693540783 \\
\hline 0 & 381 & 0.747058824 & 100 & 0.002357379 & 0.695898161 \\
\hline 0 & 382 & 0.749019608 & 100 & 0.002357379 & 0.69825554 \\
\hline 0 & 383 & 0.750980392 & 100 & 0.002357379 & 0.700612918 \\
\hline 0 & 384 & 0.752941176 & 100 & 0.002357379 & 0.702970297 \\
\hline 0 & 385 & 0.754901961 & 100 & 0.002357379 & 0.705327676 \\
\hline 0 & 386 & 0.756862745 & 100 & 0.002357379 & 0.707685054 \\
\hline 0 & 387 & 0.758823529 & 100 & 0.002357379 & 0.710042433 \\
\hline 0 & 388 & 0.760784314 & 100 & 0.002357379 & 0.712399811 \\
\hline 0 & 389 & 0.762745098 & 100 & 0.002357379 & 0.71475719 \\
\hline 0 & 390 & 0.764705882 & 100 & 0.002357379 & 0.717114569 \\
\hline 0 & 391 & 0.766666667 & 100 & 0.002357379 & 0.719471947 \\
\hline 0 & 392 & 0.768627451 & 100 & 0.002357379 & 0.721829326 \\
\hline 0 & 393 & 0.770588235 & 100 & 0.002357379 & 0.724186704 \\
\hline 0 & 394 & 0.77254902 & 100 & 0.002357379 & 0.726544083 \\
\hline 0 & 395 & 0.774509804 & 100 & 0.002357379 & 0.728901462 \\
\hline 0 & 396 & 0.776470588 & 100 & 0.002357379 & 0.73125884 \\
\hline 0 & 397 & 0.778431373 & 100 & 0.002357379 & 0.733616219 \\
\hline 0 & 398 & 0.780392157 & 100 & 0.002357379 & 0.735973597 \\
\hline 0 & 399 & 0.782352941 & 100 & 0.002357379 & 0.738330976 \\
\hline 0 & 400 & 0.784313725 & 100 & 0.002357379 & 0.740688355 \\
\hline 0 & 401 & 0.78627451 & 100 & 0.002357379 & 0.743045733 \\
\hline 0 & 402 & 0.788235294 & 100 & 0.002357379 & 0.745403112 \\
\hline 0 & 403 & 0.790196078 & 100 & 0.002357379 & 0.74776049 \\
\hline 0 & 404 & 0.792156863 & 100 & 0.002357379 & 0.750117869 \\
\hline 0 & 405 & 0.794117647 & 100 & 0.002357379 & 0.752475248 \\
\hline 0 & 406 & 0.796078431 & 100 & 0.002357379 & 0.754832626 \\
\hline 0 & 407 & 0.798039216 & 100 & 0.002357379 & 0.757190005 \\
\hline 0 & 408 & 0.8 & 100 & 0.002357379 & 0.759547383 \\
\hline 0 & 409 & 0.801960784 & 100 & 0.002357379 & 0.761904762 \\
\hline 0 & 410 & 0.803921569 & 100 & 0.002357379 & 0.76426214 \\
\hline 0 & 411 & 0.805882353 & 100 & 0.002357379 & 0.766619519 \\
\hline 0 & 412 & 0.807843137 & 100 & 0.002357379 & 0.768976898 \\
\hline 0 & 413 & 0.809803922 & 100 & 0.002357379 & 0.771334276 \\
\hline 0 & 414 & 0.811764706 & 100 & 0.002357379 & 0.773691655 \\
\hline 0 & 415 & 0.81372549 & 100 & 0.002357379 & 0.776049033 \\
\hline 0 & 416 & 0.815686275 & 100 & 0.002357379 & 0.778406412 \\
\hline 0 & 417 & 0.817647059 & 100 & 0.002357379 & 0.780763791 \\
\hline 0 & 418 & 0.819607843 & 100 & 0.002357379 & 0.783121169 \\
\hline 0 & 419 & 0.821568627 & 100 & 0.002357379 & 0.785478548 \\
\hline 0 & 420 & 0.823529412 & 100 & 0.002357379 & 0.787835926 \\
\hline 0 & 421 & 0.825490196 & 100 & 0.002357379 & 0.790193305 \\
\hline 0 & 422 & 0.82745098 & 100 & 0.002357379 & 0.792550684 \\
\hline 0 & 423 & 0.829411765 & 100 & 0.002357379 & 0.794908062 \\
\hline 0 & 424 & 0.831372549 & 100 & 0.002357379 & 0.797265441 \\
\hline 0 & 425 & 0.833333333 & 100 & 0.002357379 & 0.799622819 \\
\hline 0 & 426 & 0.835294118 & 100 & 0.002357379 & 0.801980198 \\
\hline 0 & 427 & 0.837254902 & 100 & 0.002357379 & 0.804337577 \\
\hline 0 & 428 & 0.839215686 & 100 & 0.002357379 & 0.806694955 \\
\hline 0 & 429 & 0.841176471 & 100 & 0.002357379 & 0.809052334 \\
\hline 0 & 430 & 0.843137255 & 100 & 0.002357379 & 0.811409712 \\
\hline 0 & 431 & 0.845098039 & 100 & 0.002357379 & 0.813767091 \\
\hline 0 & 432 & 0.847058824 & 100 & 0.002357379 & 0.81612447 \\
\hline 0 & 433 & 0.849019608 & 100 & 0.002357379 & 0.818481848 \\
\hline 0 & 434 & 0.850980392 & 100 & 0.002357379 & 0.820839227 \\
\hline 0 & 435 & 0.852941176 & 100 & 0.002357379 & 0.823196605 \\
\hline 0 & 436 & 0.854901961 & 100 & 0.002357379 & 0.825553984 \\
\hline 0 & 437 & 0.856862745 & 100 & 0.002357379 & 0.827911363 \\
\hline 0 & 438 & 0.858823529 & 100 & 0.002357379 & 0.830268741 \\
\hline 0 & 439 & 0.860784314 & 100 & 0.002357379 & 0.83262612 \\
\hline 0 & 440 & 0.862745098 & 100 & 0.002357379 & 0.834983498 \\
\hline 0 & 441 & 0.864705882 & 100 & 0.002357379 & 0.837340877 \\
\hline 0 & 442 & 0.866666667 & 100 & 0.002357379 & 0.839698256 \\
\hline 0 & 443 & 0.868627451 & 100 & 0.002357379 & 0.842055634 \\
\hline 0 & 444 & 0.870588235 & 100 & 0.002357379 & 0.844413013 \\
\hline 0 & 445 & 0.87254902 & 100 & 0.002357379 & 0.846770391 \\
\hline 0 & 446 & 0.874509804 & 100 & 0.002357379 & 0.84912777 \\
\hline 0 & 447 & 0.876470588 & 100 & 0.002357379 & 0.851485149 \\
\hline 0 & 448 & 0.878431373 & 100 & 0.002357379 & 0.853842527 \\
\hline 0 & 449 & 0.880392157 & 100 & 0.002357379 & 0.856199906 \\
\hline 0 & 450 & 0.882352941 & 100 & 0.002357379 & 0.858557284 \\
\hline 0 & 451 & 0.884313725 & 100 & 0.002357379 & 0.860914663 \\
\hline 0 & 452 & 0.88627451 & 100 & 0.002357379 & 0.863272041 \\
\hline 0 & 453 & 0.888235294 & 100 & 0.002357379 & 0.86562942 \\
\hline 0 & 454 & 0.890196078 & 100 & 0.002357379 & 0.867986799 \\
\hline 0 & 455 & 0.892156863 & 100 & 0.002357379 & 0.870344177 \\
\hline 0 & 456 & 0.894117647 & 100 & 0.002357379 & 0.872701556 \\
\hline 0 & 457 & 0.896078431 & 100 & 0.002357379 & 0.875058934 \\
\hline 0 & 458 & 0.898039216 & 100 & 0.002357379 & 0.877416313 \\
\hline 0 & 459 & 0.9 & 100 & 0.002357379 & 0.879773692 \\
\hline 0 & 460 & 0.901960784 & 100 & 0.002357379 & 0.88213107 \\
\hline 0 & 461 & 0.903921569 & 100 & 0.002357379 & 0.8844884449 \\
\hline 0 & 462 & 0.905882353 & 100 & 0.002357379 & 0.886845827 \\
\hline 0 & 463 & 0.907843137 & 100 & 0.002357379 & 0.889203206 \\
\hline 0 & 464 & 0.909803922 & 100 & 0.002357379 & 0.891560585 \\
\hline 0 & 465 & 0.911764706 & 100 & 0.002357379 & 0.893917963 \\
\hline 0 & 466 & 0.91372549 & 100 & 0.002357379 & 0.896275342 \\
\hline 0 & 467 & 0.915686275 & 100 & 0.002357379 & 0.89863272 \\
\hline 0 & 468 & 0.917647059 & 100 & 0.002357379 & 0.900990099 \\
\hline
\end{tabular}




\begin{tabular}{|c|c|c|c|c|c|c|}
\hline 0 & 469 & 0.919607843 & 100 & 0.002357379 & 0.903347478 & 0.001768958 \\
\hline 0 & 470 & 0.921568627 & 100 & 0.002357379 & 0.905704856 & 0.001773581 \\
\hline 0 & 471 & 0.923529412 & 100 & 0.002357379 & 0.908062235 & 0.001778203 \\
\hline 0 & 472 & 0.925490196 & 100 & 0.002357379 & 0.910419613 & 0.001782825 \\
\hline 0 & 473 & 0.92745098 & 100 & 0.002357379 & 0.912776992 & 0.001787448 \\
\hline 0 & 474 & 0.929411765 & 100 & 0.002357379 & 0.915134371 & 0.00179207 \\
\hline 0 & 475 & 0.931372549 & 100 & 0.002357379 & 0.917491749 & 0.001796692 \\
\hline 0 & 476 & 0.933333333 & 100 & 0.002357379 & 0.919849128 & 0.001801315 \\
\hline 0 & 477 & 0.935294118 & 100 & 0.002357379 & 0.922206506 & 0.001805937 \\
\hline $\begin{array}{l}0 \\
0\end{array}$ & $\begin{array}{l}478 \\
479\end{array}$ & $\begin{array}{r}0.937254902 \\
0.939215686\end{array}$ & 100 & $\begin{array}{r}0.002357379 \\
0.02357379\end{array}$ & $\begin{array}{l}0.924563885 \\
0.926921264\end{array}$ & $\begin{array}{l}0.001810559 \\
0.001815182\end{array}$ \\
\hline 0 & 479 & 0.939215686 & 100 & 0.002357379 & 0.926921264 & 0.001815182 \\
\hline 0 & 480 & 0.941176471 & 100 & 0.002357379 & 0.929278642 & $\begin{array}{l}0.001819804 \\
0.001824426\end{array}$ \\
\hline 0 & 481 & 0.943137255 & 100 & 0.002357379 & 0.931636021 & 0.001824426 \\
\hline 0 & 482 & 0.945098039 & 100 & 0.002357379 & 0.933993399 & 0.001829048 \\
\hline 0 & 483 & 0.947058824 & 100 & 0.002357379 & 0.936350778 & 0.001833671 \\
\hline 0 & 484 & 0.949019608 & 100 & 0.002357379 & 0.938708157 & 0.001838293 \\
\hline 0 & 485 & 0.950980392 & 100 & 0.002357379 & 0.941065535 & 0.001842915 \\
\hline 0 & 486 & 0.952941176 & 100 & 0.002357379 & 0.943422914 & 0.001847538 \\
\hline 0 & 487 & 0.954901961 & 100 & 0.002357379 & 0.945780292 & 0.00185216 \\
\hline 0 & 488 & 0.956862745 & 100 & 0.002357379 & 0.948137671 & 0.001856782 \\
\hline 0 & 489 & 0.958823529 & 100 & 0.002357379 & 0.95049505 & 0.001861405 \\
\hline 0 & 490 & 0.960784314 & 100 & 0.002357379 & 0.952852428 & 0.001866027 \\
\hline 0 & 491 & 0.962745098 & 100 & 0.002357379 & 0.955209807 & 0.001870649 \\
\hline 0 & 492 & 0.964705882 & 100 & 0.002357379 & 0.957567185 & 0.001875272 \\
\hline 0 & 493 & 0.966666667 & 100 & 0.002357379 & 0.959924564 & 0.001879894 \\
\hline 0 & 494 & 0.968627451 & 100 & 0.002357379 & 0.962281942 & 0.001884516 \\
\hline 0 & 495 & 0.970588235 & 100 & 0.002357379 & 0.964639321 & 0.001889138 \\
\hline 0 & 496 & 0.97254902 & 100 & 0.002357379 & 0.9669967 & 0.001893761 \\
\hline 0 & 497 & 0.974509804 & 100 & 0.002357379 & 0.969354078 & 0.001898383 \\
\hline 0 & 498 & 0.976470588 & 100 & 0.002357379 & 0.971711457 & 0.001903005 \\
\hline 0 & 499 & 0.978431373 & 100 & 0.002357379 & 0.974068835 & 0.001907628 \\
\hline 0 & 500 & 0.980392157 & 100 & 0.002357379 & 0.976426214 & 0.00191225 \\
\hline 0 & 501 & 0.982352941 & 100 & 0.002357379 & 0.978783593 & 0.001916872 \\
\hline 0 & 502 & 0.984313725 & 100 & 0.002357379 & 0.981140971 & 0.001921495 \\
\hline 0 & 503 & 0.98627451 & 100 & 0.002357379 & 0.98349835 & 0.001926117 \\
\hline 0 & 504 & 0.988235294 & 100 & 0.002357379 & 0.985855728 & 0.001930739 \\
\hline 0 & 505 & 0.990196078 & 100 & 0.002357379 & 0.988213107 & 0.001935362 \\
\hline 0 & 506 & 0.992156863 & 100 & 0.002357379 & 0.990570486 & 0.001939984 \\
\hline 0 & 507 & 0.994117647 & 100 & 0.002357379 & 0.992927864 & 0.001944606 \\
\hline 0 & 508 & 0.996078431 & 100 & 0.002357379 & 0.995285243 & 0.001949229 \\
\hline 0 & 509 & 0.998039216 & 100 & 0.002357379 & 0.997642621 & 0.001953851 \\
\hline \multirow[t]{3}{*}{0} & 510 & 1 & 100 & 0.002357379 & 1 & 0.001958473 \\
\hline & & \multirow[t]{2}{*}{ Total } & 42420 & & Total area & 0.425141859 \\
\hline & & & & & Gini & 0.149716283 \\
\hline
\end{tabular}




\begin{tabular}{|c|c|c|}
\hline $\begin{array}{l}\text { Staurospor } \\
\text { position }\end{array}$ & $\begin{array}{l}\text { rine; } 1 \mu \mathrm{M} ; 100 \\
\text { inhibition } \%\end{array}$ & $\begin{array}{c}\text { AM ATP; samp } \\
\text { rand }\end{array}$ \\
\hline 1 & 99 & 0.447106714 \\
\hline 2 & 100 & 0.749149374 \\
\hline 3 & 97 & 0.279319111 \\
\hline 4 & 93 & 0.736840922 \\
\hline 5 & 96 & 0.513144992 \\
\hline 6 & 100 & 0.669669046 \\
\hline 7 & 100 & 0.303857308 \\
\hline 8 & 100 & 0.31835067 \\
\hline 9 & 83 & 0.917542473 \\
\hline 10 & 100 & 0.702154281 \\
\hline 11 & 99 & 0.658642005 \\
\hline 12 & 97 & 0.542653157 \\
\hline 13 & 96 & 0.260763399 \\
\hline 14 & 100 & 0.839249319 \\
\hline 15 & 99 & 0.901132583 \\
\hline 16 & 100 & 0.460827822 \\
\hline 17 & 53 & 0.196723272 \\
\hline 18 & 88 & 0.024878739 \\
\hline 19 & 98 & 0.385753718 \\
\hline 20 & 100 & 0.695321271 \\
\hline 21 & 83 & 0.498769917 \\
\hline 22 & 97 & 0.556549401 \\
\hline 23 & 98 & 0.542343032 \\
\hline 24 & 18 & 0.148687494 \\
\hline 25 & 73 & 0.304127364 \\
\hline 26 & 98 & 0.679730971 \\
\hline 27 & 100 & 0.684568686 \\
\hline 28 & 100 & 0.354158388 \\
\hline 29 & 36 & 0.933298637 \\
\hline 30 & 98 & 0.07589444 \\
\hline 31 & 100 & 0.539655382 \\
\hline 32 & 100 & 0.060322926 \\
\hline 33 & 96 & 0.324560652 \\
\hline 34 & 100 & 0.923181789 \\
\hline 35 & 100 & 0.225603523 \\
\hline 36 & 95 & 0.230464543 \\
\hline 37 & 100 & 0.607934347 \\
\hline 38 & 85 & 0.465638987 \\
\hline 39 & 54 & 0.145749776 \\
\hline 40 & 99 & 0.013293522 \\
\hline 41 & 94 & 0.804428689 \\
\hline 42 & 99 & 0.70729524 \\
\hline 43 & 100 & 0.461226408 \\
\hline 44 & 86 & 0.445204068 \\
\hline 45 & 100 & 0.333883594 \\
\hline 46 & 100 & 0.304794196 \\
\hline 47 & 100 & 0.671506318 \\
\hline 48 & 75 & 0.743975559 \\
\hline 49 & 100 & 0.05465542 \\
\hline 50 & 16 & 0.972799742 \\
\hline 51 & 100 & 0.271393823 \\
\hline 52 & 20 & 0.761863508 \\
\hline 53 & 81 & 0.667081082 \\
\hline 54 & 100 & 0.977400033 \\
\hline 55 & 100 & 0.016617717 \\
\hline 56 & 100 & 0.08418316 \\
\hline 57 & 83 & 0.568682817 \\
\hline 58 & 100 & 0.157499926 \\
\hline 59 & 99 & 0.265274005 \\
\hline 60 & 85 & 0.099708688 \\
\hline 61 & 95 & 0.454693962 \\
\hline 62 & 98 & 0.806867184 \\
\hline 63 & 100 & 0.62752414 \\
\hline 64 & 66 & 0.052836902 \\
\hline 65 & 100 & 0.42388493 \\
\hline 66 & 100 & 0.576755676 \\
\hline 67 & 83 & 0.769116123 \\
\hline 68 & 100 & 0.956003571 \\
\hline 69 & 88 & 0.012641672 \\
\hline 70 & 100 & 0.065896613 \\
\hline 71 & 85 & 0.317522949 \\
\hline 72 & 8 & 0.556673125 \\
\hline 73 & 100 & 0.323826817 \\
\hline 74 & 95 & 0.118587823 \\
\hline 75 & 41 & 0.102636914 \\
\hline 76 & 86 & 0.734082809 \\
\hline 77 & 66 & 0.94861569 \\
\hline 78 & 100 & 0.825563142 \\
\hline 79 & 100 & 0.793701556 \\
\hline 80 & 97 & 0.792151598 \\
\hline 81 & 61 & 0.503397515 \\
\hline 82 & 99 & 0.93312249 \\
\hline 83 & 54 & 0.611015829 \\
\hline 84 & 99 & 0.651760222 \\
\hline 85 & 0 & 0.971390925 \\
\hline 86 & 100 & 0.47449113 \\
\hline 87 & 31 & 0.728949106 \\
\hline 88 & 73 & 0.715852709 \\
\hline 89 & 100 & 0.971637007 \\
\hline 90 & 91 & 0.373888265 \\
\hline 91 & 100 & 0.883493796 \\
\hline 92 & 95 & 0.490893174 \\
\hline 93 & 99 & 0.160860097 \\
\hline 94 & 41 & 0.804029857 \\
\hline 95 & 92 & 0.531553255 \\
\hline
\end{tabular}




\begin{tabular}{|c|c|}
\hline 96 & 31 \\
\hline 97 & 100 \\
\hline 98 & 83 \\
\hline 99 & 0 \\
\hline 100 & 3 \\
\hline 101 & 100 \\
\hline 102 & 16 \\
\hline 103 & 86 \\
\hline 104 & 100 \\
\hline 105 & 29 \\
\hline 106 & 69 \\
\hline 107 & 100 \\
\hline 108 & 98 \\
\hline 109 & 99 \\
\hline 110 & 100 \\
\hline 111 & 88 \\
\hline 112 & 100 \\
\hline 113 & 100 \\
\hline 114 & 99 \\
\hline 115 & 29 \\
\hline 116 & 99 \\
\hline 117 & 99 \\
\hline 118 & 96 \\
\hline 119 & 100 \\
\hline 120 & 96 \\
\hline 121 & 100 \\
\hline 122 & 91 \\
\hline 123 & 99 \\
\hline 124 & 100 \\
\hline 125 & 18 \\
\hline 126 & 91 \\
\hline 127 & 61 \\
\hline 128 & 96 \\
\hline 129 & 100 \\
\hline 130 & 94 \\
\hline 131 & 98 \\
\hline 132 & 100 \\
\hline 133 & 99 \\
\hline 134 & 41 \\
\hline 135 & 100 \\
\hline 136 & 99 \\
\hline 137 & 93 \\
\hline 138 & 85 \\
\hline 139 & 31 \\
\hline 140 & 100 \\
\hline 141 & 92 \\
\hline 142 & 95 \\
\hline 143 & 31 \\
\hline 144 & 3 \\
\hline 145 & 100 \\
\hline 146 & 46 \\
\hline 147 & 100 \\
\hline 148 & 83 \\
\hline 149 & 97 \\
\hline 150 & 100 \\
\hline 151 & 93 \\
\hline 152 & 100 \\
\hline 153 & 100 \\
\hline 154 & 100 \\
\hline 155 & 69 \\
\hline 156 & 100 \\
\hline 157 & 66 \\
\hline 158 & 69 \\
\hline 159 & 85 \\
\hline 160 & 20 \\
\hline 161 & 99 \\
\hline 162 & 97 \\
\hline 163 & 16 \\
\hline 164 & 100 \\
\hline 165 & 100 \\
\hline 166 & 97 \\
\hline 167 & 36 \\
\hline 168 & 100 \\
\hline 169 & 92 \\
\hline 170 & 98 \\
\hline 171 & 100 \\
\hline 172 & 75 \\
\hline 173 & 16 \\
\hline 174 & 98 \\
\hline 175 & 18 \\
\hline 176 & 95 \\
\hline 177 & 100 \\
\hline 178 & 18 \\
\hline 179 & 75 \\
\hline 180 & 69 \\
\hline 181 & 100 \\
\hline 182 & 20 \\
\hline 183 & 18 \\
\hline 184 & 99 \\
\hline 185 & 100 \\
\hline 186 & 100 \\
\hline 187 & 92 \\
\hline 188 & 100 \\
\hline 189 & 100 \\
\hline 190 & 98 \\
\hline 191 & 99 \\
\hline 192 & 100 \\
\hline
\end{tabular}

\begin{tabular}{|c|c|}
\hline 31 & 78 \\
\hline 100 & 78 \\
\hline 83 & 78 \\
\hline 0 & 81 \\
\hline 3 & 81 \\
\hline 100 & 83 \\
\hline 16 & 83 \\
\hline 86 & 83 \\
\hline 100 & 83 \\
\hline 29 & 83 \\
\hline 69 & 83 \\
\hline 100 & 83 \\
\hline 98 & 83 \\
\hline 99 & 83 \\
\hline 100 & 83 \\
\hline 88 & 85 \\
\hline 100 & 85 \\
\hline 100 & 85 \\
\hline 99 & 85 \\
\hline 29 & 85 \\
\hline 99 & 85 \\
\hline 99 & 85 \\
\hline 96 & 85 \\
\hline 100 & 85 \\
\hline 96 & 86 \\
\hline 100 & 86 \\
\hline 91 & 86 \\
\hline 99 & 86 \\
\hline 100 & 88 \\
\hline 18 & 88 \\
\hline 91 & 88 \\
\hline 61 & 88 \\
\hline 96 & 88 \\
\hline 100 & 88 \\
\hline 94 & 91 \\
\hline 98 & 91 \\
\hline 100 & 91 \\
\hline 99 & 91 \\
\hline 41 & 91 \\
\hline 100 & 92 \\
\hline 99 & 92 \\
\hline 93 & 92 \\
\hline 85 & 92 \\
\hline 31 & 92 \\
\hline 100 & 92 \\
\hline 92 & 92 \\
\hline 95 & 92 \\
\hline 31 & 92 \\
\hline 3 & 92 \\
\hline 100 & 92 \\
\hline 46 & 93 \\
\hline 100 & 93 \\
\hline 83 & 93 \\
\hline 97 & 93 \\
\hline 100 & 93 \\
\hline 93 & 94 \\
\hline 100 & 94 \\
\hline 100 & 94 \\
\hline 100 & 95 \\
\hline 69 & 95 \\
\hline 100 & 95 \\
\hline 66 & 95 \\
\hline 69 & 95 \\
\hline 85 & 95 \\
\hline 20 & 95 \\
\hline 99 & 95 \\
\hline 97 & 95 \\
\hline 16 & 96 \\
\hline 100 & 96 \\
\hline 100 & 96 \\
\hline 97 & 96 \\
\hline 36 & 96 \\
\hline 100 & 96 \\
\hline 92 & 96 \\
\hline 98 & 96 \\
\hline 100 & 96 \\
\hline 75 & 96 \\
\hline 16 & 96 \\
\hline 98 & 96 \\
\hline 18 & 96 \\
\hline 95 & 96 \\
\hline 100 & 97 \\
\hline 18 & 97 \\
\hline 75 & 97 \\
\hline 69 & 97 \\
\hline 100 & 97 \\
\hline 20 & 97 \\
\hline 18 & 97 \\
\hline 99 & 97 \\
\hline 100 & 97 \\
\hline 100 & 97 \\
\hline 92 & 97 \\
\hline 100 & 97 \\
\hline 100 & 97 \\
\hline 98 & 97 \\
\hline 99 & 97 \\
\hline 100 & 97 \\
\hline
\end{tabular}

$\begin{array}{rr}0.113214931 & 0.00028 \\ 0.115562914 & 0.000286\end{array}$

$\begin{array}{lll}0.117910897 & 0.000292\end{array}$ $\begin{array}{lll}0.117910897 & 0.000292\end{array}$ $\begin{array}{lll}0.120349187 & 0.000298\end{array}$ 0.1227874770 .000304 $\begin{array}{lr}0.125285972 & 0.00031 \\ 0.127784467 & 0.000316\end{array}$ $\begin{array}{lll}0.127784467 & 0.000316\end{array}$ $\begin{array}{ll}0.130282962 & 0.000323 \\ 0.132781457 & 0.000329 \\ 0.135279952 & 0.000335\end{array}$ $\begin{array}{lll}0.132781457 & 0.000329\end{array}$ $\begin{array}{ll}0.135279952 & 0.000335 \\ 0.137778447 & 0.000341\end{array}$ 0.1402769420 .000348 $\begin{array}{lll}0.142775436 & 0.000354\end{array}$ $\begin{array}{ll}0.145273931 & 0.00036\end{array}$ $0.147772426 \quad 0.000366$ $\begin{array}{ll}0.147772426 & 0.000366 \\ 0.150331126 & 0.000373\end{array}$ $\begin{array}{lll}0.152889825 & 0.000379\end{array}$ 0.1554485250 .000385 $\begin{array}{lll}0.158007225 & 0.000392\end{array}$ 0.1605659240 .000398 $0.163124624 \quad 0.000405$ $0.165683323 \quad 0.000411$ $0.168242023 \quad 0.000417$ $0.170800722 \quad 0.000424$ $0.173389524 \quad 0.00043$ $\begin{array}{ll}0.175978326 & 0.000437\end{array}$ $\begin{array}{ll}0.178567128 & 0.000443\end{array}$

$\begin{array}{ll}0.18115593 & 0.00045\end{array}$ $0.183804937 \quad 0.000456$ 0.1864539430 .000463 $0.18910295 \quad 0.000469$ $0.191751957 \quad 0.000476$ $0.194400963 \quad 0.000483$ $\begin{array}{lll}0.19704997 & 0.000489\end{array}$ $0.199789284 \quad 0.000496$ $0.202528597 \quad 0.000503$ $\begin{array}{ll}0.205267911 & 0.00051\end{array}$ $0.208007225 \quad 0.000517$ $\begin{array}{ll}0.210746538 & 0.000523\end{array}$ $\begin{array}{ll}0.213515954 & 0.0005\end{array}$ $\begin{array}{lll}0.21628537 & 0.00053\end{array}$ $0.219054786 \quad 0.000544$ $0.221824202 \quad 0.000551$ $0.224593618 \quad 0.000558$ $0.227363034 \quad 0.000565$ $0.23013245 \quad 0.000572$ $0.232901866 \quad 0.000579$ $0.235671282 \quad 0.000586$ $0.238440698 \quad 0.00059$ $\begin{array}{ll}0.241210114 & 0.0006\end{array}$ $0.244009633 \quad 0.000607$ $0.246809151 \quad 0.000614$ $\begin{array}{lll}0.249608669 & 0.000621\end{array}$ $0.252408188 \quad 0.000628$ $0.255207706 \quad 0.000635$ $0.258037327 \quad 0.000642$ $0.263696568 \quad 0.000656$ $0.266556291 \quad 0.000663$ $\begin{array}{lll}0.269416014 & 0.00067\end{array}$ $0.272275738 \quad 0.000677$ $\begin{array}{lll}0.275135461 & 0.000684\end{array}$ $0.277995184 \quad 0.000691$ $\begin{array}{lll}0.280854907 & 0.000699\end{array}$ $\begin{array}{lll}0.28371463 & 0.000706\end{array}$ $0.286574353 \quad 0.000713$ $0.289434076 \quad 0.00072$ $\begin{array}{lll}0.292323901 & 0.000727\end{array}$ $0.295213727 \quad 0.000734$ 0.2981035520 .000742 $\begin{array}{lll}0.300993377 & 0.000749\end{array}$ $0.303883203 \quad 0.000756$ $0.306773028 \quad 0.000763$ $0.309662854 \quad 0.000771$ $\begin{array}{ll}0.312552679 & 0.000778\end{array}$ $0.315442505 \quad 0.000785$ $0.31833233 \quad 0.000792$ $0.321222155 \quad 0.000799$ $0.324111981 \quad 0.000807$ $0.327001806 \quad 0.000814$ $\begin{array}{ll}0.329891632 & 0.000821 \\ 0.332811559 & 0.000828\end{array}$ $\begin{array}{ll}0.332811559 & 0.000828 \\ 0.335731487 & 0.00083\end{array}$ $\begin{array}{ll}0.335731487 & 0.000836 \\ 0.338651415 & 0.000843\end{array}$ $\begin{array}{rr}0.338651415 & 0.000843 \\ 0.341571343 & 0.00085\end{array}$ $\begin{array}{lll}0.34449127 & 0.000858\end{array}$ $0.347411198 \quad 0.000865$ $0.350331126 \quad 0.000872$ $\begin{array}{lll}0.353251054 & 0.000879\end{array}$ $\begin{array}{lll}0.356170981 & 0.000887\end{array}$ $0.359090909 \quad 0.000894$ $\begin{array}{lll}0.362010837 & 0.000901\end{array}$ 0.3649307650 .00090 $0.367850692 \quad 0.000916$ $0.37077062 \quad 0.000923$ $0.373690548 \quad 0.000931$ 0.3766104760 .000938 


\begin{tabular}{|c|c|c|}
\hline $\begin{array}{l}193 \\
194\end{array}$ & $\begin{array}{l}88 \\
100\end{array}$ & $\begin{array}{c}0.53626506 \\
0.631277431\end{array}$ \\
\hline 195 & 99 & 0.248307063 \\
\hline 196 & 96 & 0.818655733 \\
\hline 197 & 100 & 0.884154465 \\
\hline 198 & 83 & 0.969518233 \\
\hline 199 & 100 & 0.171278746 \\
\hline 200 & 100 & 0.977715952 \\
\hline 201 & 100 & 0.388167755 \\
\hline 202 & 100 & 0.154150945 \\
\hline 203 & 99 & 0.426527473 \\
\hline 204 & 100 & 0.061416732 \\
\hline 205 & 99 & 0.043544022 \\
\hline 206 & 97 & 0.742141431 \\
\hline 207 & 100 & 0.598954732 \\
\hline 208 & 98 & 0.696555654 \\
\hline 209 & 78 & 0.877043468 \\
\hline 210 & 100 & 0.944309615 \\
\hline 211 & 99 & 0.221788134 \\
\hline 212 & 98 & 0.110081613 \\
\hline 213 & 91 & 0.437352316 \\
\hline 214 & 100 & 0.482932673 \\
\hline 215 & 93 & 0.410737836 \\
\hline 216 & 44 & 0.072610494 \\
\hline 217 & 100 & 0.655402523 \\
\hline 218 & 53 & 0.477718075 \\
\hline 219 & 0 & 0.989865925 \\
\hline 220 & 0 & 0.346703073 \\
\hline 221 & 66 & 0.670026894 \\
\hline 222 & 41 & 0.063869123 \\
\hline 223 & 92 & 0.719717133 \\
\hline 224 & 98 & 0.422323315 \\
\hline 225 & 20 & 0.425820762 \\
\hline 226 & 73 & 0.959680274 \\
\hline 227 & 31 & 0.901107821 \\
\hline 228 & 47 & 0.547008472 \\
\hline 229 & 100 & 0.376496912 \\
\hline 230 & 54 & 0.76149401 \\
\hline 231 & 99 & 0.763659181 \\
\hline 232 & 96 & 0.665604549 \\
\hline 233 & 91 & 0.182811759 \\
\hline 234 & 88 & 0.29164348 \\
\hline 235 & 100 & 0.056958981 \\
\hline 236 & 47 & 0.000412393 \\
\hline 237 & 100 & 0.534839381 \\
\hline 238 & 96 & 0.183097958 \\
\hline 239 & 100 & 0.189475831 \\
\hline 240 & 100 & 0.889620404 \\
\hline 241 & 100 & 0.634619815 \\
\hline 242 & 99 & 0.316528596 \\
\hline 243 & 20 & 0.534284558 \\
\hline 244 & 97 & 0.165456211 \\
\hline 245 & 97 & 0.050228162 \\
\hline 246 & 16 & 0.589027447 \\
\hline 247 & 8 & 0.547606131 \\
\hline 248 & 44 & 0.401361031 \\
\hline 249 & 78 & 0.397549003 \\
\hline 250 & 100 & 0.012345066 \\
\hline 251 & 100 & 0.231404191 \\
\hline 252 & 97 & 0.254752442 \\
\hline 253 & 8 & 0.733563588 \\
\hline 254 & 100 & 0.310958235 \\
\hline 255 & 100 & 0.498623551 \\
\hline 256 & 99 & 0.346088754 \\
\hline 257 & 99 & 0.036835854 \\
\hline 258 & 8 & 0.957672403 \\
\hline 259 & 95 & 0.345767557 \\
\hline 260 & 86 & 0.001482972 \\
\hline 261 & 100 & 0.285810455 \\
\hline 262 & 100 & 0.29961456 \\
\hline 263 & 100 & 0.91729512 \\
\hline 264 & 100 & 0.833260887 \\
\hline 265 & 61 & 0.775571712 \\
\hline 266 & 99 & 0.018631712 \\
\hline 267 & 100 & 0.440423261 \\
\hline 268 & 100 & 0.668545605 \\
\hline 269 & 81 & 0.991856191 \\
\hline 270 & 97 & 0.304538217 \\
\hline 271 & 100 & 0.910527951 \\
\hline 272 & 99 & 0.378196381 \\
\hline 273 & 85 & 0.364416034 \\
\hline 274 & 92 & 0.905161829 \\
\hline 275 & 100 & 0.740434989 \\
\hline 276 & 98 & 0.132154552 \\
\hline 277 & 54 & 0.573555324 \\
\hline 278 & 100 & 0.803638931 \\
\hline 279 & 100 & 0.856599982 \\
\hline 280 & 92 & 0.025539802 \\
\hline 281 & 97 & 0.226875962 \\
\hline 282 & 100 & 0.87486839 \\
\hline 283 & 18 & 0.503521452 \\
\hline 284 & 46 & 0.256324412 \\
\hline 285 & 100 & 0.039785174 \\
\hline 286 & 100 & 0.214182739 \\
\hline 287 & 100 & 0.7808805 \\
\hline 288 & 8 & 0.241625354 \\
\hline 289 & 98 & 0.804348553 \\
\hline
\end{tabular}

\begin{tabular}{|c|c|}
\hline 88 & 97 \\
\hline 100 & 97 \\
\hline 99 & 97 \\
\hline 96 & 98 \\
\hline 100 & 98 \\
\hline 83 & 98 \\
\hline 100 & 98 \\
\hline 100 & 98 \\
\hline 100 & 98 \\
\hline 100 & 98 \\
\hline 99 & 98 \\
\hline 100 & 98 \\
\hline 99 & 98 \\
\hline 97 & 98 \\
\hline 100 & 98 \\
\hline 98 & 98 \\
\hline 78 & 98 \\
\hline 100 & 98 \\
\hline 99 & 98 \\
\hline 98 & 98 \\
\hline 91 & 98 \\
\hline 100 & 98 \\
\hline 93 & 98 \\
\hline 44 & 99 \\
\hline 100 & 99 \\
\hline 53 & 99 \\
\hline 0 & 99 \\
\hline 0 & 99 \\
\hline 66 & 99 \\
\hline 41 & 99 \\
\hline 92 & 99 \\
\hline 98 & 99 \\
\hline 20 & 99 \\
\hline 73 & 99 \\
\hline 31 & 99 \\
\hline 47 & 99 \\
\hline 100 & 99 \\
\hline 54 & 99 \\
\hline 99 & 99 \\
\hline 96 & 99 \\
\hline 91 & 99 \\
\hline 88 & 99 \\
\hline 100 & 99 \\
\hline 47 & 99 \\
\hline 100 & 99 \\
\hline 96 & 99 \\
\hline 100 & 99 \\
\hline 100 & 99 \\
\hline 100 & 99 \\
\hline 99 & 99 \\
\hline 20 & 99 \\
\hline 97 & 99 \\
\hline 97 & 99 \\
\hline 16 & 99 \\
\hline 8 & 99 \\
\hline 44 & 99 \\
\hline 78 & 99 \\
\hline 100 & 99 \\
\hline 100 & 99 \\
\hline 97 & 99 \\
\hline 8 & 99 \\
\hline 100 & 99 \\
\hline 100 & 99 \\
\hline 99 & 99 \\
\hline 99 & 99 \\
\hline 8 & 100 \\
\hline 95 & 100 \\
\hline 86 & 100 \\
\hline 100 & 100 \\
\hline 100 & 100 \\
\hline 100 & 100 \\
\hline 100 & 100 \\
\hline 61 & 100 \\
\hline 99 & 100 \\
\hline 100 & 100 \\
\hline 100 & 100 \\
\hline 81 & 100 \\
\hline 97 & 100 \\
\hline 100 & 100 \\
\hline 99 & 100 \\
\hline 85 & 100 \\
\hline 92 & 100 \\
\hline 100 & 100 \\
\hline 98 & 100 \\
\hline 54 & 100 \\
\hline 100 & 100 \\
\hline 100 & 100 \\
\hline 92 & 100 \\
\hline 97 & 100 \\
\hline 100 & 100 \\
\hline 18 & 100 \\
\hline 46 & 100 \\
\hline 100 & 100 \\
\hline 100 & 100 \\
\hline 100 & 100 \\
\hline 8 & 100 \\
\hline 98 & 100 \\
\hline
\end{tabular}

$\begin{array}{ll}0.379530403 & 0.000945 \\ 0.382450331 & 0.000952\end{array}$

$\begin{array}{ll}0.382450331 & 0.0009\end{array}$ 0.00096 0.3883202890 .000967 $\begin{array}{lll}0.391270319 & 0.000974\end{array}$ $\begin{array}{ll}0.394220349 & 0.000982 \\ 0.397170379 & 0.000989\end{array}$ $\begin{array}{lll}0.397170379 & 0.000989 \\ 0.400120409 & 0.000997\end{array}$ $\begin{array}{ll}0.400120409 & 0.000997 \\ 0.403070439 & 0.001004 \\ 0.40602047 & 0.001011\end{array}$ $0.40602047 \quad 0.001011$ $0.4089705 \quad 0.001019$ $\begin{array}{lll}0.41192053 & 0.001026\end{array}$ $0.41487056 \quad 0.001033$ $\begin{array}{ll}0.41782059 & 0.001041\end{array}$ $0.42077062 \quad 0.001048$ $0.42372065 \quad 0.001056$ $0.42667068 \quad 0.001063$ $\begin{array}{ll}0.42962071 & 0.00107\end{array}$ $\begin{array}{lll}0.432570741 & 0.001078\end{array}$ 0.4355207710 .001085 $0.438470801 \quad 0.001092$ $\begin{array}{ll}0.441420831 & 0.0011\end{array}$ $\begin{array}{lll}0.444370861 & 0.001107\end{array}$ $0.447350993 \quad 0.001115$ $\begin{array}{lll}0.450331126 & 0.001122\end{array}$ $0.453311258 \quad 0.00113$ $\begin{array}{lll}0.459271523 & 0.001144\end{array}$ $\begin{array}{ll}0.459271523 & 0.001144 \\ 0.462251656 & 0.001152 \\ 0.465231788 & 0.001159\end{array}$ $\begin{array}{ll}0.462251656 & 0.001152 \\ 0.465231788 & 0.001159\end{array}$ $0.468211921 \quad 0.001167$ $0.471192053 \quad 0.001174$ $0.474172185 \quad 0.001182$ $0.477152318 \quad 0.001189$ $\begin{array}{lll}0.48013245 & 0.001197\end{array}$ $0.483112583 \quad 0.001204$ 0.4860927150 .001212 $0.489072848 \quad 0.001219$ $0.49205298 \quad 0.001226$ 0.4950331130 .001234 $0.498013245 \quad 0.00124$ $\begin{array}{lll}0.500993377 & 0.001249\end{array}$ $0.50397351 \quad 0.001256$ $0.506953642 \quad 0.001264$ $\begin{array}{lll}0.509933775 & 0.001271\end{array}$ $0.512913907 \quad 0.001279$ $0.51589404 \quad 0.001286$ 0.5188741720 .001293 $\begin{array}{lll}0.521854305 & 0.00130\end{array}$ 0.5248344370 .001308 $0.52781457 \quad 0.001316$ $0.530794702 \quad 0.001323$ $\begin{array}{lll}0.533774834 & 0.001331 \\ 0.536754967 & 0.001338\end{array}$ $0.536754967 \quad 0.001338$ $\begin{array}{lll}0.539735099 & 0.001346\end{array}$ $\begin{array}{lll}0.542715232 & 0.001353 \\ 0.545695364 & 0.001361\end{array}$ $\begin{array}{lll}0.548675497 & 0.001368\end{array}$ $\begin{array}{ll}0.548675497 & 0.001368 \\ 0.551655629 & 0.001375\end{array}$ $\begin{array}{ll}0.551655629 & 0.001375 \\ 0.554635762 & 0.001383\end{array}$ $\begin{array}{ll}0.554635762 & 0.001383 \\ 0.557615894 & 0.00139\end{array}$ $\begin{array}{rr}0.557615894 & 0.00139 \\ 0.560596026 & 0.001398\end{array}$ $\begin{array}{lll}0.563576159 & 0.001405\end{array}$ $0.566556291 \quad 0.001413$ $0.569536424 \quad 0.00142$ 0.5725466590 .001428 $\begin{array}{lll}0.575556893 & 0.001435\end{array}$ $0.578567128 \quad 0.001443$ $0.581577363 \quad 0.00145$ $\begin{array}{ll}0.584587598 & 0.001458 \\ 0.587597833 & 0.001465\end{array}$ $0.587597833 \quad 0.001465$ $\begin{array}{lll}0.590608067 & 0.001473\end{array}$ $0.593618302 \quad 0.00148$ $\begin{array}{lll}0.596628537 & 0.001488\end{array}$ 0.5996387720 .001495 0.6026490070 .001503 $0.608669476 \quad 0.001518$ $\begin{array}{ll}0.608669476 & 0.001518 \\ 0.611679711 & 0.001525\end{array}$ $0.614689946 \quad 0.001533$ 0.6177001810 .00154 0.6207104150 .001548 $0.62372065 \quad 0.001556$ 0.6267308850 .001563 $\begin{array}{lll}0.62974112 & 0.001571\end{array}$ $0.632751355 \quad 0.001578$ 0.6357615890 .001586 $0.638771824 \quad 0.001593$ 0.6417820590 .001601 0.6447922940 .001608 0.6478025290 .001616 $0.650812763 \quad 0.001623$ $\begin{array}{ll}0.653822998 & 0.001631 \\ 0.656833233 & 0.001638\end{array}$ 0.6568332330 .001638 $\begin{array}{ll}0.659843468 & 0.001646 \\ 0.662853703 & 0.001653\end{array}$ $0.665863937 \quad 0.00166$ 


\begin{tabular}{|c|c|}
\hline 290 & 100 \\
\hline 291 & 100 \\
\hline 292 & 92 \\
\hline 293 & 100 \\
\hline 294 & 96 \\
\hline 295 & 100 \\
\hline 296 & 100 \\
\hline 297 & 36 \\
\hline 298 & 100 \\
\hline 299 & 53 \\
\hline 300 & 95 \\
\hline 301 & 31 \\
\hline 302 & 99 \\
\hline 303 & 99 \\
\hline 304 & 78 \\
\hline 305 & 100 \\
\hline 306 & 100 \\
\hline 307 & 100 \\
\hline 308 & 66 \\
\hline 309 & 99 \\
\hline 310 & 99 \\
\hline 311 & 99 \\
\hline 312 & 97 \\
\hline 313 & 75 \\
\hline 314 & 100 \\
\hline 315 & 100 \\
\hline 316 & 100 \\
\hline 317 & 99 \\
\hline 318 & 92 \\
\hline 319 & 100 \\
\hline 320 & 100 \\
\hline 321 & 100 \\
\hline 322 & 100 \\
\hline 323 & 98 \\
\hline 324 & 100 \\
\hline 325 & 100 \\
\hline 326 & 100 \\
\hline 327 & 92 \\
\hline 328 & 36 \\
\hline 329 & 100 \\
\hline 330 & 99 \\
\hline 331 & 92 \\
\hline 332 & 85 \\
\hline 333 & 93 \\
\hline 334 & 98 \\
\hline 335 & 3 \\
\hline 336 & 47 \\
\hline 337 & 99 \\
\hline 338 & 100 \\
\hline 339 & 85 \\
\hline 340 & 100 \\
\hline 341 & 97 \\
\hline 342 & 29 \\
\hline 343 & 46 \\
\hline 344 & 100 \\
\hline 345 & 100 \\
\hline 346 & 96 \\
\hline 347 & 99 \\
\hline 348 & 73 \\
\hline 349 & 44 \\
\hline 350 & 41 \\
\hline 351 & 97 \\
\hline 352 & 78 \\
\hline 353 & 61 \\
\hline 354 & 20 \\
\hline 355 & 98 \\
\hline 356 & 97 \\
\hline 357 & 96 \\
\hline 358 & 96 \\
\hline 359 & 100 \\
\hline 360 & 100 \\
\hline 361 & 3 \\
\hline 362 & 53 \\
\hline 363 & 95 \\
\hline 364 & 99 \\
\hline 365 & 99 \\
\hline 366 & 100 \\
\hline 367 & 97 \\
\hline 368 & 83 \\
\hline 369 & 100 \\
\hline 370 & 44 \\
\hline 371 & 54 \\
\hline 372 & 100 \\
\hline 373 & 88 \\
\hline 374 & 100 \\
\hline 375 & 100 \\
\hline 376 & 98 \\
\hline 377 & 99 \\
\hline 378 & 29 \\
\hline 379 & 16 \\
\hline 380 & 73 \\
\hline 381 & 83 \\
\hline 382 & 96 \\
\hline 383 & 98 \\
\hline 384 & 100 \\
\hline 385 & 44 \\
\hline & \\
\hline
\end{tabular}

\begin{tabular}{|c|c|}
\hline 100 & 100 \\
\hline 100 & 100 \\
\hline 92 & 100 \\
\hline 100 & 100 \\
\hline 96 & 100 \\
\hline 100 & 100 \\
\hline 100 & 100 \\
\hline 36 & 100 \\
\hline 100 & 100 \\
\hline 53 & 100 \\
\hline 95 & 100 \\
\hline 31 & 100 \\
\hline 99 & 100 \\
\hline 99 & 100 \\
\hline 78 & 100 \\
\hline 100 & 100 \\
\hline 100 & 100 \\
\hline 100 & 100 \\
\hline 66 & 100 \\
\hline 99 & 100 \\
\hline 99 & 100 \\
\hline 99 & 100 \\
\hline 97 & 100 \\
\hline 75 & 100 \\
\hline 100 & 100 \\
\hline 100 & 100 \\
\hline 100 & 100 \\
\hline 99 & 100 \\
\hline 92 & 100 \\
\hline 100 & 100 \\
\hline 100 & 100 \\
\hline 100 & 100 \\
\hline 100 & 100 \\
\hline 98 & 100 \\
\hline 100 & 100 \\
\hline 100 & 100 \\
\hline 100 & 100 \\
\hline 92 & 100 \\
\hline 36 & 100 \\
\hline 100 & 100 \\
\hline 99 & 100 \\
\hline 92 & 100 \\
\hline 85 & 100 \\
\hline 93 & 100 \\
\hline 98 & 100 \\
\hline 3 & 100 \\
\hline 47 & 100 \\
\hline 99 & 100 \\
\hline 100 & 100 \\
\hline 85 & 100 \\
\hline 100 & 100 \\
\hline 97 & 100 \\
\hline 29 & 100 \\
\hline 46 & 100 \\
\hline 100 & 100 \\
\hline 100 & 100 \\
\hline 96 & 100 \\
\hline 99 & 100 \\
\hline 73 & 100 \\
\hline 44 & 100 \\
\hline 41 & 100 \\
\hline 97 & 100 \\
\hline 78 & 100 \\
\hline 61 & 100 \\
\hline 20 & 100 \\
\hline 98 & 100 \\
\hline 97 & 100 \\
\hline 96 & 100 \\
\hline 96 & 100 \\
\hline 100 & 100 \\
\hline 100 & 100 \\
\hline 3 & 100 \\
\hline 53 & 100 \\
\hline 95 & 100 \\
\hline 99 & 100 \\
\hline 99 & 100 \\
\hline 100 & 100 \\
\hline 97 & 100 \\
\hline 83 & 100 \\
\hline 100 & 100 \\
\hline 44 & 100 \\
\hline 54 & 100 \\
\hline 100 & 100 \\
\hline 88 & 100 \\
\hline 100 & 100 \\
\hline 100 & 100 \\
\hline 98 & 100 \\
\hline 99 & 100 \\
\hline 29 & 100 \\
\hline 16 & 100 \\
\hline 73 & 100 \\
\hline 83 & 100 \\
\hline 96 & 100 \\
\hline 98 & 100 \\
\hline 100 & 100 \\
\hline 44 & 100 \\
\hline & \\
\hline
\end{tabular}

$\begin{array}{ll}0.668874172 & 0.001668 \\ 0.671884407 & 0.001676\end{array}$ $\begin{array}{lll}0.674894642 & 0.001683\end{array}$ $\begin{array}{lll}0.674894642 & 0.001683\end{array}$ $\begin{array}{lll}0.677904877 & 0.001691\end{array}$ $\begin{array}{lll}0.680915111 & 0.001699\end{array}$ $\begin{array}{ll}0.683925346 & 0.001706 \\ 0.686935581 & 0.001714\end{array}$ $\begin{array}{ll}0.686935581 & 0.001714 \\ 0.689945816 & 0.001721\end{array}$ $\begin{array}{ll}0.689945816 & 0.001721 \\ 0.692956051 & 0.001729 \\ 0.695966285 & 0.001736\end{array}$ $\begin{array}{lll}0.695966285 & 0.001736\end{array}$ $0.69897652 \quad 0.001744$ $0.701986755 \quad 0.001751$

$\begin{array}{lll}0.70499699 & 0.001759\end{array}$ $0.708007225 \quad 0.001766$ $0.711017459 \quad 0.001774$ .7170379290 .001789 $0.720048164 \quad 0.001796$ $0.723058399 \quad 0.001804$ $\begin{array}{lll}0.726068633 & 0.001811\end{array}$ $0.729078868 \quad 0.001819$ $0.732089103 \quad 0.001826$ $0.735099338 \quad 0.001834$ $\begin{array}{lll}0.738109573 & 0.001842\end{array}$ $\begin{array}{lll}0.741119807 & 0.001849\end{array}$ $\begin{array}{lll}0.744130042 & 0.001857\end{array}$ $\begin{array}{ll}0.747140277 & 0.001864\end{array}$ $0.750150512 \quad 0.001872$ $\begin{array}{ll}0.753160747 & 0.001879\end{array}$ $\begin{array}{ll}0.756170981 & 0.001887\end{array}$ $0.759181216 \quad 0.001894$ $0.762191451 \quad 0.001902$ $0.765201686 \quad 0.001909$ $\begin{array}{lll}0.768211921 & 0.001917\end{array}$ $0.771222155 \quad 0.001924$ $0.77423239 \quad 0.001932$ $0.777242625 \quad 0.001939$ $\begin{array}{ll}0.78025286 & 0.001947\end{array}$ $\begin{array}{lll}0.783263095 & 0.001\end{array}$ $0.786273329 \quad 0.001962$ $0.789283564 \quad 0.001969$ $0.792293799 \quad 0.001977$ $0.795304034 \quad 0.001984$ 0.7983142690 .001992 $\begin{array}{ll}0.801324503 & 0.002\end{array}$ $\begin{array}{lr}0.801324503 & 0.002 \\ 0.804334738 & 0.002007\end{array}$ $0.807344973 \quad 0.002015$ $0.810355208 \quad 0.002022$ $0.813365443 \quad 0.00203$ $0.816375677 \quad 0.002037$ 0.8193859120 .002045 $0.822396147 \quad 0.002052$ $\begin{array}{ll}0.825406382 & 0.00206\end{array}$ $0.828416616 \quad 0.002067$ $\begin{array}{lll}0.831426851 & 0.002075\end{array}$ $\begin{array}{lll}0.834437086 & 0.002082\end{array}$ $\begin{array}{lr}0.834447321 & 0.00209\end{array}$ $\begin{array}{lll}0.840457556 & 0.002097\end{array}$ 0.843467790 .002105 $0.846478025 \quad 0.002112$ $\begin{array}{ll}0.84948826 & 0.00212\end{array}$ $\begin{array}{lll}0.852498495 & 0.002127\end{array}$ $0.85550873 \quad 0.002135$ $0.858518964 \quad 0.002143$ $0.861529199 \quad 0.00215$ 0.8645394340 .002158 $\begin{array}{lll}0.867549669 & 0.002165\end{array}$ $0.870559904 \quad 0.002173$ $0.873570138 \quad 0.00218$ $\begin{array}{lll}0.876580373 & 0.002188\end{array}$ 0.8795906080 .002195 $0.882600843 \quad 0.002203$ $0.885611078 \quad 0.00221$ 0.8886213120 .002218 $\begin{array}{lll}0.891631547 & 0.002225\end{array}$ 0.8946417820 .002233 $\begin{array}{lll}0.897652017 & 0.00224\end{array}$ $0.900662252 \quad 0.002248$ $\begin{array}{lll}0.903672486 & 0.002255\end{array}$ $0.906682721 \quad 0.002263$ $\begin{array}{lll}0.909692956 & 0.00227\end{array}$ $\begin{array}{lll}0.912703191 & 0.002278\end{array}$ $\begin{array}{rr}0.915713426 & 0.002286 \\ 0.91872366 & 0.002293\end{array}$ $\begin{array}{lll}0.921733895 & 0.002301\end{array}$ $\begin{array}{lll}0.92474413 & 0.002308\end{array}$ $\begin{array}{ll}0.927754365 & 0.002316\end{array}$

$\begin{array}{ll}0.9307646 & 0.002323\end{array}$ 0.00233 $\begin{array}{lll}0.936785069 & 0.002338\end{array}$ $0.939795304 \quad 0.002346$ $\begin{array}{lll}0.942805539 & 0.002353 \\ 0.945815774 & 0.002361\end{array}$ $\begin{array}{ll}0.945815774 & 0.002361 \\ 0.948826008 & 0.002368\end{array}$ $\begin{array}{ll}0.948826008 & 0.002368 \\ 0.951836243 & 0.002376\end{array}$ $\begin{array}{lll}0.954846478 & 0.002383\end{array}$ 0.9578567130 .00239 


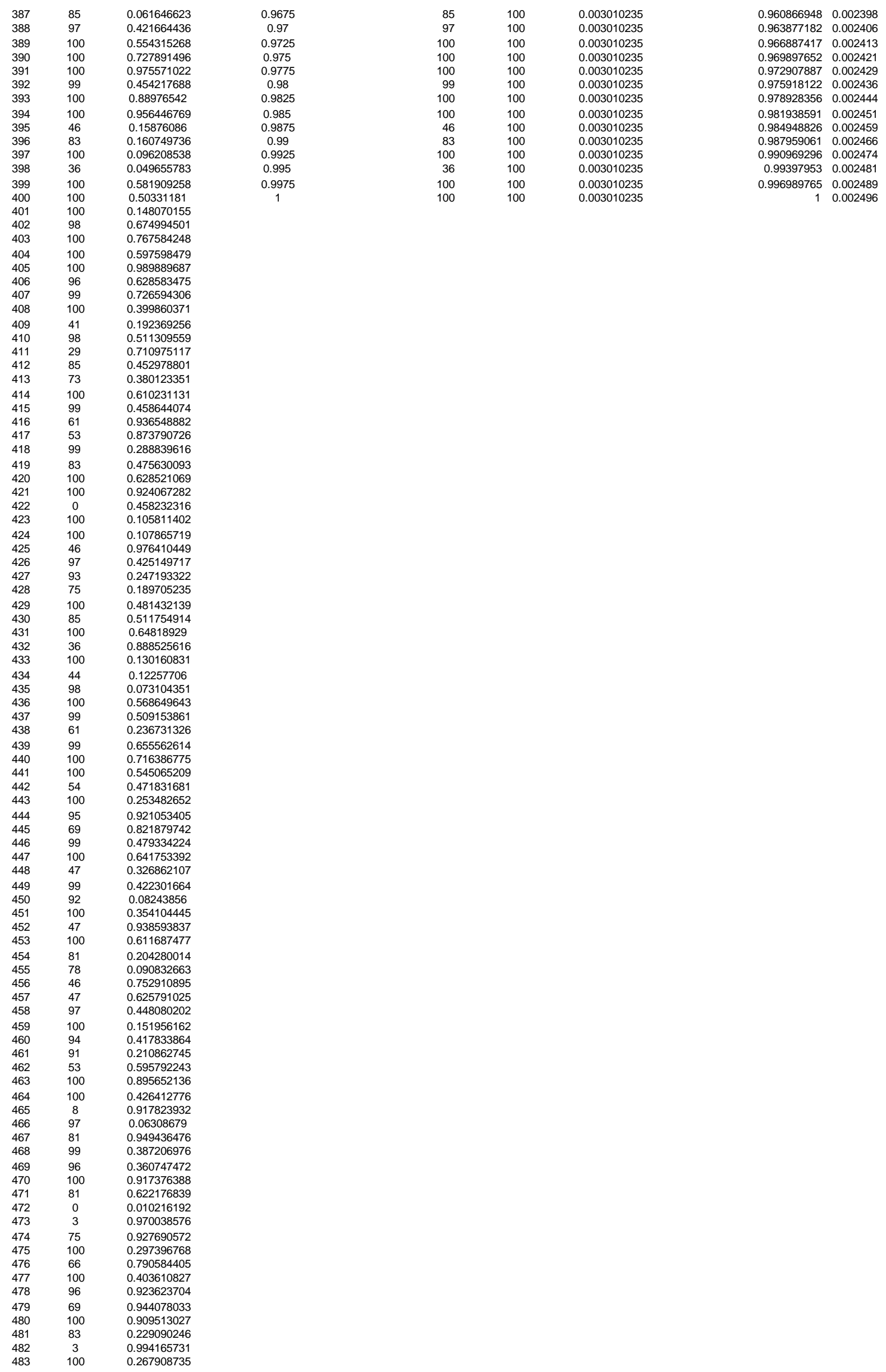




$\begin{array}{lcl}484 & 96 & 0.965408517 \\ 485 & 100 & 0.300134942 \\ 486 & 95 & 0.924783976 \\ 487 & 100 & 0.63819629 \\ 488 & 94 & 0.704292426 \\ 489 & 99 & 0.842273187 \\ 490 & 100 & 0.016478467 \\ 491 & 97 & 0.386939114 \\ 492 & 99 & 0.825514193 \\ 493 & 100 & 0.410870015 \\ 494 & 94 & 0.526064839 \\ 495 & 100 & 0.847782668 \\ 496 & 100 & 0.261306173 \\ 497 & 99 & 0.764000126 \\ 498 & 99 & 0.472868328 \\ 499 & 85 & 0.45761065 \\ 500 & 100 & 0.79159022 \\ 501 & 95 & 0.844321261 \\ 502 & 81 & 0.29547572 \\ 503 & 100 & 0.726032827 \\ 504 & 86 & 0.631474388 \\ 505 & 100 & 0.62036328 \\ 506 & 97 & 0.740334361 \\ 507 & 98 & 0.460750925 \\ 508 & 78 & 0.803967809 \\ 509 & 29 & 0.093181578 \\ 510 & 86 & 0.946591221\end{array}$




\begin{tabular}{|c|c|c|}
\hline $\begin{array}{l}\text { Staurospor } \\
\text { position }\end{array}$ & $\begin{array}{r}\text { rine; } 1 \mu \mathrm{M} ; 100 \\
\text { inhibition }\end{array}$ & $\begin{array}{l}\text { M ATP; sampl } \\
\text { rand }\end{array}$ \\
\hline 1 & 61 & 0.222107504 \\
\hline 2 & 100 & 0.916756176 \\
\hline 3 & 53 & 0.168383221 \\
\hline 4 & 100 & 0.674489094 \\
\hline 5 & 100 & 0.465110153 \\
\hline 6 & 100 & 0.776684749 \\
\hline 7 & 97 & 0.28464812 \\
\hline 8 & 99 & 0.183854843 \\
\hline 9 & 97 & 0.677832677 \\
\hline 10 & 83 & 0.39874837 \\
\hline 11 & 94 & 0.039150435 \\
\hline 12 & 85 & 0.857097068 \\
\hline 13 & 100 & 0.876521149 \\
\hline 14 & 100 & 0.710206713 \\
\hline 15 & 97 & 0.789368965 \\
\hline 16 & 98 & 0.606292064 \\
\hline 17 & 69 & 0.628126653 \\
\hline 18 & 88 & 0.651361383 \\
\hline 19 & 100 & 0.290940441 \\
\hline 20 & 3 & 0.352707248 \\
\hline 21 & 100 & 0.380876139 \\
\hline 22 & 92 & 0.276913144 \\
\hline 23 & 47 & 0.561070727 \\
\hline 24 & 54 & 0.042583265 \\
\hline 25 & 100 & 0.869116558 \\
\hline 26 & 97 & 0.885430604 \\
\hline 27 & 75 & 0.794771308 \\
\hline 28 & 99 & 0.540938317 \\
\hline 29 & 44 & 0.910091933 \\
\hline 30 & 97 & 0.028330572 \\
\hline 31 & 85 & 0.571290041 \\
\hline 32 & 100 & 0.585526018 \\
\hline 33 & 100 & 0.688545258 \\
\hline 34 & 85 & 0.49316693 \\
\hline 35 & 98 & 0.133823274 \\
\hline 36 & 100 & 0.241219219 \\
\hline 37 & 100 & 0.420833135 \\
\hline 38 & 92 & 0.902776054 \\
\hline 39 & 100 & 0.454249913 \\
\hline 40 & 41 & 0.462085719 \\
\hline 41 & 88 & 0.531122156 \\
\hline 42 & 93 & 0.802446349 \\
\hline 43 & 54 & 0.579771082 \\
\hline 44 & 91 & 0.227070719 \\
\hline 45 & 73 & 0.526728582 \\
\hline 46 & 53 & 0.668244773 \\
\hline 47 & 100 & 0.655112345 \\
\hline 48 & 73 & 0.440136718 \\
\hline 49 & 100 & 0.558272668 \\
\hline 50 & 61 & 0.014045764 \\
\hline 51 & 95 & 0.607435847 \\
\hline 52 & 44 & 0.661323707 \\
\hline 53 & 100 & 0.011119003 \\
\hline 54 & 100 & 0.115168148 \\
\hline 55 & 20 & 0.977680456 \\
\hline 56 & 81 & 0.639474335 \\
\hline 57 & 100 & 0.162423862 \\
\hline 58 & 99 & 0.231779634 \\
\hline 59 & 99 & 0.111424093 \\
\hline 60 & 36 & 0.674554561 \\
\hline 61 & 96 & 0.524604876 \\
\hline 62 & 98 & 0.307497015 \\
\hline 63 & 100 & 0.710531899 \\
\hline 64 & 98 & 0.263916807 \\
\hline 65 & 66 & 0.109878979 \\
\hline 66 & 100 & 0.926367348 \\
\hline 67 & 100 & 0.924859005 \\
\hline 68 & 78 & 0.609620758 \\
\hline 69 & 53 & 0.809592222 \\
\hline 70 & 92 & 0.937724089 \\
\hline 71 & 99 & 0.967617123 \\
\hline 72 & 36 & 0.517826472 \\
\hline 73 & 44 & 0.80986999 \\
\hline 74 & 46 & 0.677402082 \\
\hline 75 & 98 & 0.817648739 \\
\hline 76 & 95 & 0.470397105 \\
\hline 77 & 98 & 0.99447986 \\
\hline 78 & 95 & 0.271968706 \\
\hline 79 & 99 & 0.551756055 \\
\hline 80 & 100 & 0.697941058 \\
\hline 81 & 61 & 0.690209134 \\
\hline 82 & 85 & 0.011008958 \\
\hline 83 & 36 & 0.413477327 \\
\hline 84 & 100 & 0.080758199 \\
\hline 85 & 100 & 0.203965527 \\
\hline 86 & 99 & 0.839613791 \\
\hline 87 & 66 & 0.204932678 \\
\hline 88 & 100 & 0.856597262 \\
\hline 89 & 8 & 0.8862471 \\
\hline 90 & 100 & 0.07947065 \\
\hline 91 & 100 & 0.758261001 \\
\hline 92 & 31 & 0.790791403 \\
\hline 93 & 100 & 0.60281152 \\
\hline 94 & 100 & 0.907451416 \\
\hline 95 & 20 & 0.084227657 \\
\hline
\end{tabular}




\begin{tabular}{|c|c|}
\hline 96 & 100 \\
\hline 97 & 94 \\
\hline 98 & 54 \\
\hline 99 & 100 \\
\hline 100 & 100 \\
\hline 101 & 95 \\
\hline 102 & 99 \\
\hline 103 & 100 \\
\hline 104 & 97 \\
\hline 105 & 100 \\
\hline 106 & 100 \\
\hline 107 & 100 \\
\hline 108 & 69 \\
\hline 109 & 100 \\
\hline 110 & 100 \\
\hline 111 & 99 \\
\hline 112 & 100 \\
\hline 113 & 92 \\
\hline 114 & 100 \\
\hline 115 & 97 \\
\hline 116 & 100 \\
\hline 117 & 99 \\
\hline 118 & 98 \\
\hline 119 & 99 \\
\hline 120 & 36 \\
\hline 121 & 46 \\
\hline 122 & 96 \\
\hline 123 & 97 \\
\hline 124 & 100 \\
\hline 125 & 100 \\
\hline 126 & 98 \\
\hline 127 & 98 \\
\hline 128 & 100 \\
\hline 129 & 96 \\
\hline 130 & 85 \\
\hline 131 & 100 \\
\hline 132 & 100 \\
\hline 133 & 97 \\
\hline 134 & 18 \\
\hline 135 & 100 \\
\hline 136 & 75 \\
\hline 137 & 99 \\
\hline 138 & 66 \\
\hline 139 & 83 \\
\hline 140 & 100 \\
\hline 141 & 100 \\
\hline 142 & 100 \\
\hline 143 & 99 \\
\hline 144 & 96 \\
\hline 145 & 41 \\
\hline 146 & 41 \\
\hline 147 & 97 \\
\hline 148 & 100 \\
\hline 149 & 98 \\
\hline 150 & 81 \\
\hline 151 & 99 \\
\hline 152 & 100 \\
\hline 153 & 99 \\
\hline 154 & 99 \\
\hline 155 & 98 \\
\hline 156 & 81 \\
\hline 157 & 100 \\
\hline 158 & 100 \\
\hline 159 & 100 \\
\hline 160 & 46 \\
\hline 161 & 88 \\
\hline 162 & 31 \\
\hline 163 & 99 \\
\hline 164 & 88 \\
\hline 165 & 75 \\
\hline 166 & 96 \\
\hline 167 & 99 \\
\hline 168 & 99 \\
\hline 169 & 99 \\
\hline 170 & 8 \\
\hline 171 & 95 \\
\hline 172 & 73 \\
\hline 173 & 96 \\
\hline 174 & 100 \\
\hline 175 & 3 \\
\hline 176 & 100 \\
\hline 177 & 29 \\
\hline 178 & 88 \\
\hline 179 & 100 \\
\hline 180 & 100 \\
\hline 181 & 95 \\
\hline 182 & 31 \\
\hline 183 & 100 \\
\hline 184 & 47 \\
\hline 185 & 100 \\
\hline 186 & 94 \\
\hline 187 & 85 \\
\hline 188 & 16 \\
\hline 189 & 99 \\
\hline 190 & 100 \\
\hline 191 & 100 \\
\hline 192 & 29 \\
\hline
\end{tabular}

$0.207394227 \quad 0.000686$ $\begin{array}{lll}0.210873863 & 0.000697\end{array}$ $\begin{array}{lll}0.214472123 & 0.000709\end{array}$ $\begin{array}{lll}0.218070384 & 0.000721\end{array}$ $\begin{array}{ll}0.22168644 & 0.000733\end{array}$ $\begin{array}{lll}0.225306445 & 0.000745\end{array}$ $0.228944247 \quad 0.000757$ $\begin{array}{ll}0.232582048 & 0.000769\end{array}$ $0.23621985 \quad 0.000781$ $0.239857651 \quad 0.000793$ $0.243495453 \quad 0.000806$ $0.247133254 \quad 0.000818$ $\begin{array}{lll}0.250810597 & 0.00083\end{array}$ $\begin{array}{lll}0.25448794 & 0.000842\end{array}$ $0.258165283 \quad 0.000854$ $\begin{array}{lll}0.261842626 & 0.000867\end{array}$ $0.26555951 \quad 0.000879$ $0.269276394 \quad 0.00089$ $0.272993278 \quad 0.000904$ 0.2767101620 .000916 $0.280427046 \quad 0.000929$ 0.2841834720 .000941 $0.287939897 \quad 0.000954$ $0.291696323 \quad 0.000966$ $\begin{array}{lll}0.295452748 & 0.000979\end{array}$ $0.299209174 \quad 0.000991$ $0.302965599 \quad 0.001004$ 0.3067220250 .001016 $0.31047845 \quad 0.001029$ $0.314274417 \quad 0.00104$ $\begin{array}{lll}0.318070384 & 0.001054\end{array}$ $\begin{array}{lll}0.32186635 & 0.001067\end{array}$ $0.325662317 \quad 0.001079$ $0.329458284 \quad 0.001092$ $0.333254251 \quad 0.001105$ $\begin{array}{ll}0.337050217 & 0.001117\end{array}$ $\begin{array}{ll}0.344642151 & 0.001142\end{array}$ $0.348438118 \quad 0.00115$ 0.3522340850 .001168 $\begin{array}{ll}0.356030051 & 0.00118\end{array}$ $0.35986556 \quad 0.001193$ $0.363701068 \quad 0.001206$ $\begin{array}{lll}0.367536576 & 0.001219\end{array}$ $0.371372084 \quad 0.001232$ $0.375207592 \quad 0.001244$ $\begin{array}{ll}0.3790431 & 0.001257\end{array}$ $0.382878608 \quad 0.00127$ $0.386714116 \quad 0.00128$ $0.390549624 \quad 0.001295$ 0.3943851320 .001308 $0.398220641 \quad 0.001321$ $\begin{array}{ll}0.402056149 & 0.001334 \\ 0.405891657 & 0.001347\end{array}$ $\begin{array}{lll}0.409727165 & 0.001359\end{array}$ $0.413562673 \quad 0.001372$ $0.417398181 \quad 0.001385$ 0.4212336890 .001398 0.4251087390 .00141 $0.428983788 \quad 0.001423$ $0.432858837 \quad 0.001436$ 0.4367338870 .001449 $0.440608936 \quad 0.001462$ $0.444483986 \quad 0.001475$ $0.448359035 \quad 0.001488$ $\begin{array}{ll}0.452234085 & 0.00150 \\ 0.456109134 & 0.001514\end{array}$ $0.459984183 \quad 0.001527$ $\begin{array}{rr}0.459984183 & 0.001527 \\ 0.463859233 & 0.00154\end{array}$ $\begin{array}{rr}0.463859233 & 0.00154 \\ 0.467734282 & 0.001553\end{array}$ $\begin{array}{ll}0.467734282 & 0.001553 \\ 0.471609332 & 0.001566\end{array}$ $\begin{array}{ll}0.471609332 & 0.001566 \\ 0.475484381 & 0.001578\end{array}$ $\begin{array}{lll}0.479359431 & 0.001591\end{array}$ $0.48323448 \quad 0.001604$ $\begin{array}{ll}0.487109529 & 0.001617\end{array}$ $0.490984579 \quad 0.00163$ 0.4987742190 .001656 $\begin{array}{rr}0.50268881 & 0.001669 \\ 0.506603401 & 0.001682\end{array}$ $\begin{array}{ll}0.506603401 & 0.001682 \\ 0.510517991 & 0.001695\end{array}$ $\begin{array}{ll}0.510517991 & 0.001695 \\ 0.514432582 & 0.001708\end{array}$ $\begin{array}{ll}0.514432582 & 0.001708 \\ 0.518347173 & 0.001721\end{array}$ $\begin{array}{ll}0.518347173 & 0.00172 \\ 0.522261764 & 0.00173\end{array}$ $\begin{array}{ll}0.526176354 & 0.001747\end{array}$ $0.530090945 \quad 0.00176$ $0.534005536 \quad 0.001773$ $\begin{array}{lll}0.537920127 & 0.001787\end{array}$ $0.541834717 \quad 0.0018$ $\begin{array}{ll}0.545749308 & 0.001813\end{array}$ 0.5496638990 .00182 0.553578490 .00 $0.55749308 \quad 0.001852$ $\begin{array}{lll}0.561407671 & 0.001865\end{array}$ 0.5653222620 .001878 $\begin{array}{ll}0.569236853 & 0.00189\end{array}$ $0.573151443 \quad 0.001904$ 


\begin{tabular}{|c|c|}
\hline 193 & 91 \\
\hline 194 & 47 \\
\hline 195 & 98 \\
\hline 196 & 100 \\
\hline 197 & 97 \\
\hline 198 & 100 \\
\hline 199 & 100 \\
\hline 200 & 96 \\
\hline 201 & 100 \\
\hline 202 & 91 \\
\hline 203 & 98 \\
\hline 204 & 100 \\
\hline 205 & 73 \\
\hline 206 & 8 \\
\hline 207 & 86 \\
\hline 208 & 100 \\
\hline 209 & 96 \\
\hline 210 & 81 \\
\hline 211 & 100 \\
\hline 212 & 61 \\
\hline 213 & 100 \\
\hline 214 & 83 \\
\hline 215 & 98 \\
\hline 216 & 46 \\
\hline 217 & 8 \\
\hline 218 & 98 \\
\hline 219 & 94 \\
\hline 220 & 83 \\
\hline 221 & 86 \\
\hline 222 & 99 \\
\hline 223 & 100 \\
\hline 224 & 83 \\
\hline 225 & 100 \\
\hline 226 & 96 \\
\hline 227 & 100 \\
\hline 228 & 100 \\
\hline 229 & 97 \\
\hline 230 & 99 \\
\hline 231 & 100 \\
\hline 232 & 97 \\
\hline 233 & 98 \\
\hline 234 & 97 \\
\hline 235 & 95 \\
\hline 236 & 100 \\
\hline 237 & 100 \\
\hline 238 & 100 \\
\hline 239 & 100 \\
\hline 240 & 41 \\
\hline 241 & 95 \\
\hline 242 & 99 \\
\hline 243 & 100 \\
\hline 244 & 93 \\
\hline 245 & 61 \\
\hline 246 & 96 \\
\hline 247 & 16 \\
\hline 248 & 97 \\
\hline 249 & 100 \\
\hline 250 & 100 \\
\hline 251 & 96 \\
\hline 252 & 46 \\
\hline 253 & 20 \\
\hline 254 & 44 \\
\hline 255 & 100 \\
\hline 256 & 100 \\
\hline 257 & 100 \\
\hline 258 & 81 \\
\hline 259 & 96 \\
\hline 260 & 100 \\
\hline 261 & 78 \\
\hline 262 & 98 \\
\hline 263 & 100 \\
\hline 264 & 3 \\
\hline 265 & 92 \\
\hline 266 & 100 \\
\hline 267 & 93 \\
\hline 268 & 99 \\
\hline 269 & 100 \\
\hline 270 & 86 \\
\hline 271 & 100 \\
\hline 272 & 18 \\
\hline 273 & 73 \\
\hline 274 & 53 \\
\hline 275 & 100 \\
\hline 276 & 100 \\
\hline 277 & 94 \\
\hline 278 & 100 \\
\hline 279 & 93 \\
\hline 280 & 66 \\
\hline 281 & 100 \\
\hline 282 & 98 \\
\hline 283 & 97 \\
\hline 284 & 100 \\
\hline 285 & 92 \\
\hline 286 & 98 \\
\hline 287 & 100 \\
\hline 288 & 100 \\
\hline 289 & 100 \\
\hline
\end{tabular}

$\begin{array}{rr}0.577066034 & 0.001917 \\ 0.580980625 & 0.00193\end{array}$ $\begin{array}{ll}0.584895216 & 0.001943\end{array}$ $\begin{array}{ll}0.588809806 & 0.001956\end{array}$ $\begin{array}{ll}0.592724397 & 0.001969\end{array}$ 0.5966785290 .001982 $0.604586793 \quad 0.002009$ $0.608540925 \quad 0.002022$ $0.612495057 \quad 0.002035$ 0.6164491890 .002048 0.6204033210 .002061 $\begin{array}{lll}0.624357454 & 0.002075\end{array}$ $\begin{array}{lll}0.628311586 & 0.002088\end{array}$ $\begin{array}{lll}0.632265718 & 0.002101\end{array}$ $\begin{array}{rr}0.636173982 & 0.002127\end{array}$ $0.644128114 \quad 0.00214$ $0.648082246 \quad 0.002154$ 0.6520363780 .002167 $\begin{array}{ll}0.65599051 & 0.00218\end{array}$ $0.659944642 \quad 0.002193$ $0.663898774 \quad 0.002206$ $\begin{array}{lll}0.667852906 & 0.00222\end{array}$ $0.671807038 \quad 0.002233$ $\begin{array}{lll}0.67576117 & 0.002246\end{array}$ $0.679715302 \quad 0.002259$ 0.6836694350 .002272 $0.687623567 \quad 0.002285$ 0.6915776990 .002299 0.6955318310 .002312 $0.699485963 \quad 0.002325$ $0.703440095 \quad 0.002338$ $\begin{array}{ll}0.707394227 & 0.002351\end{array}$ 0.7113483590 .002365 $\begin{array}{lll}0.715302491 & 0.002378\end{array}$ $0.719256623 \quad 0.002391$ $0.723210755 \quad 0.002404$ $0.727164887 \quad 0.00241$ $0.731119019 \quad 0.00243$ $0.735073151 \quad 0.00244$ $0.739027284 \quad 0.002457$ $\begin{array}{ll}0.742981416 & 0.00247\end{array}$ $0.746935548 \quad 0.002483$ 0.750889680 .002496 $\begin{array}{ll}0.754843812 & 0.00251\end{array}$ $\begin{array}{ll}0.758797944 & 0.002523\end{array}$ $0.762752076 \quad 0.002536$ $\begin{array}{lll}0.766706208 & 0.002549\end{array}$ $0.77066034 \quad 0.002562$ 0.7746144720 .002575 $0.778568604 \quad 0.002589$ $\begin{array}{lll}0.782522736 & 0.002602\end{array}$ $0.786476868 \quad 0.002615$ $0.790431-0.002628$ $0.798339265 \quad 0.002655$ $0.802293397 \quad 0.002668$ $\begin{array}{ll}0.802293397 & 0.002668 \\ 0.806247529 & 0.002681\end{array}$ $\begin{array}{ll}0.806247529 & 0.002681 \\ 0.810201661 & 0.002694\end{array}$ $\begin{array}{lll}0.818109925 & 0.00272\end{array}$ $0.822064057 \quad 0.002734$ 0.8260181890 .002747 $\begin{array}{ll}0.829972321 & 0.00276\end{array}$ $0.833926453 \quad 0.002773$ 0.8378805850 .002786 $\begin{array}{ll}0.841834717 & 0.0028\end{array}$ $\begin{array}{ll}0.845788849 & 0.002813\end{array}$ 0.8497429810 .002826 $\begin{array}{ll}0.853697113 & 0.002839 \\ 0.857651246 & 0.002852\end{array}$ $\begin{array}{ll}0.857651246 & 0.002852 \\ 0.861605378 & 0.002865\end{array}$ $\begin{array}{rr}0.861605378 & 0.002865 \\ 0.86555951 & 0.002879\end{array}$ $\begin{array}{ll}0.86555951 & 0.002879\end{array}$ $0.873467774 \quad 0.002905$ $0.877421906 \quad 0.002918$ 0.8813760380 .002931 $\begin{array}{lll}0.88533017 & 0.002945\end{array}$ 0.8892843020 .002958 $\begin{array}{ll}0.893238434 & 0.002971 \\ 0.897192566 & 0.002984\end{array}$ $\begin{array}{lll}0.901146698 & 0.002997\end{array}$ $\begin{array}{lll}0.90510083 & 0.00301\end{array}$ 0.9090549620 .003024 $0.913009095 \quad 0.003037$ $\begin{array}{ll}0.916963227 & 0.00305\end{array}$ 0.9209173590 .003063 $\begin{array}{lll}0.924871491 & 0.00307\end{array}$ $0.928825623 \quad 0.003089$ $\begin{array}{lll}0.932779755 & 0.003103\end{array}$ $\begin{array}{ll}0.936733887 & 0.003116 \\ 0.940688019 & 0.003129\end{array}$ $\begin{array}{ll}0.940688019 & 0.003129 \\ 0.944642151 & 0.003142\end{array}$ $0.948596283 \quad 0.003155$ $\begin{array}{lll}0.952550415 & 0.003169\end{array}$ $0.956504547 \quad 0.003182$ 


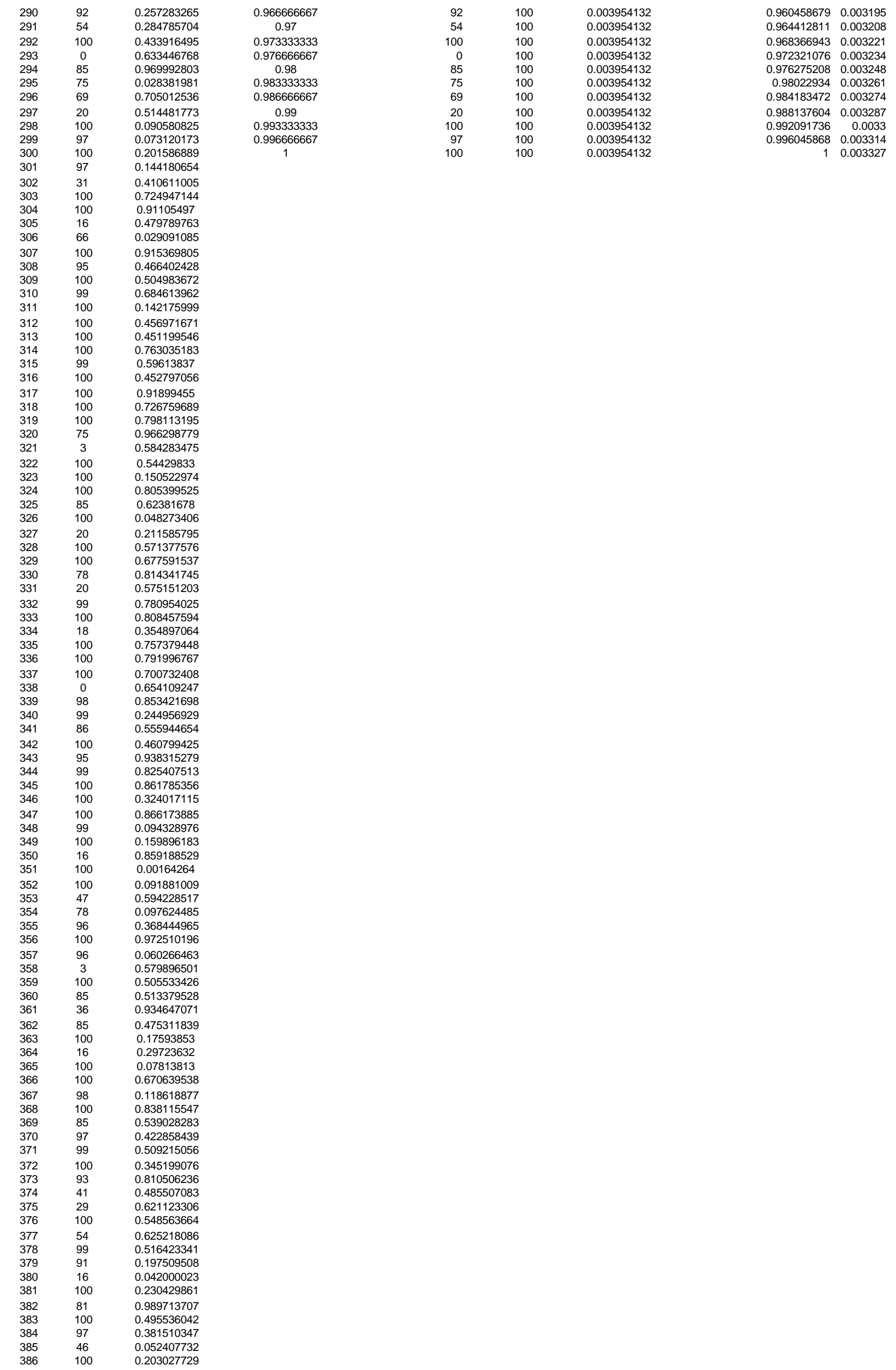




\begin{tabular}{|c|c|}
\hline 387 & 99 \\
\hline 388 & 99 \\
\hline 389 & 100 \\
\hline 390 & 83 \\
\hline 391 & 100 \\
\hline 392 & 99 \\
\hline 393 & 94 \\
\hline 394 & 99 \\
\hline 395 & 95 \\
\hline 396 & 78 \\
\hline 397 & 100 \\
\hline 398 & 75 \\
\hline 399 & 69 \\
\hline 400 & 99 \\
\hline 401 & 92 \\
\hline 402 & 18 \\
\hline 403 & 18 \\
\hline 404 & 83 \\
\hline 405 & 99 \\
\hline 406 & 100 \\
\hline 407 & 100 \\
\hline 408 & 29 \\
\hline 409 & 69 \\
\hline 410 & 18 \\
\hline 411 & 98 \\
\hline 412 & 97 \\
\hline 413 & 97 \\
\hline 414 & 36 \\
\hline 415 & 53 \\
\hline 416 & 100 \\
\hline 417 & 99 \\
\hline 418 & 47 \\
\hline 419 & 0 \\
\hline 420 & 100 \\
\hline 421 & 83 \\
\hline 422 & 83 \\
\hline 423 & 100 \\
\hline 424 & 91 \\
\hline 425 & 66 \\
\hline 426 & 99 \\
\hline 427 & 100 \\
\hline 428 & 86 \\
\hline 429 & 92 \\
\hline 430 & 100 \\
\hline 431 & 93 \\
\hline 432 & 96 \\
\hline 433 & 8 \\
\hline 434 & 100 \\
\hline 435 & 100 \\
\hline 436 & 41 \\
\hline 437 & 95 \\
\hline 438 & 99 \\
\hline 439 & 100 \\
\hline 440 & 100 \\
\hline 441 & 96 \\
\hline 442 & 100 \\
\hline 443 & 0 \\
\hline 444 & 100 \\
\hline 445 & 97 \\
\hline 446 & 44 \\
\hline 447 & 88 \\
\hline 448 & 100 \\
\hline 449 & 100 \\
\hline 450 & 3 \\
\hline 451 & 92 \\
\hline 452 & 98 \\
\hline 453 & 47 \\
\hline 454 & 99 \\
\hline 455 & 44 \\
\hline 456 & 100 \\
\hline 457 & 61 \\
\hline 458 & 100 \\
\hline 459 & 100 \\
\hline 460 & 100 \\
\hline 461 & 99 \\
\hline 462 & 92 \\
\hline 463 & 31 \\
\hline 464 & 100 \\
\hline 465 & 0 \\
\hline 466 & 91 \\
\hline 467 & 86 \\
\hline 468 & 54 \\
\hline 469 & 96 \\
\hline 470 & 100 \\
\hline 471 & 92 \\
\hline 472 & 100 \\
\hline 473 & 97 \\
\hline 474 & 99 \\
\hline 475 & 29 \\
\hline 476 & 100 \\
\hline 477 & 99 \\
\hline 478 & 100 \\
\hline 479 & 100 \\
\hline 480 & 99 \\
\hline 481 & 0 \\
\hline 482 & 100 \\
\hline 483 & 83 \\
\hline
\end{tabular}




$\begin{array}{lcl}484 & 85 & 0.695229915 \\ 485 & 31 & 0.534878379 \\ 486 & 99 & 0.486880832 \\ 487 & 83 & 0.206408035 \\ 488 & 100 & 0.777987774 \\ 489 & 96 & 0.183886162 \\ 490 & 73 & 0.274122836 \\ 491 & 53 & 0.718749288 \\ 492 & 83 & 0.444614773 \\ 493 & 78 & 0.239165966 \\ 494 & 8 & 0.477903205 \\ 495 & 98 & 0.017065316 \\ 496 & 99 & 0.353106192 \\ 497 & 100 & 0.566561849 \\ 498 & 100 & 0.858733942 \\ 499 & 99 & 0.474654591 \\ 500 & 100 & 0.223966249 \\ 501 & 100 & 0.079557573 \\ 502 & 100 & 0.221965094 \\ 503 & 29 & 0.269882274 \\ 504 & 99 & 0.740489162 \\ 505 & 99 & 0.483181511 \\ 506 & 99 & 0.30494578 \\ 507 & 99 & 0.313970665 \\ 508 & 69 & 0.870653019 \\ 509 & 100 & 0.144068852 \\ 510 & 100 & 0.350207103\end{array}$




\begin{tabular}{|c|c|c|}
\hline $\begin{array}{l}\text { Staurospor } \\
\text { position }\end{array}$ & $\begin{array}{l}\text { rine; } 1 \mu \mathrm{M} ; 100 \\
\text { inhibition\% }\end{array}$ & $\begin{array}{l}\text { M ATP; sampl } \\
\text { rand }\end{array}$ \\
\hline 1 & 16 & 0.460947923 \\
\hline 2 & 100 & 0.69632412 \\
\hline 3 & 96 & 0.658855556 \\
\hline 4 & 81 & 0.937674663 \\
\hline 5 & 83 & 0.469806259 \\
\hline 6 & 99 & 0.661459519 \\
\hline 7 & 100 & 0.411871424 \\
\hline 8 & 66 & 0.855415753 \\
\hline 9 & 100 & 0.907259314 \\
\hline 10 & 96 & 0.275902571 \\
\hline 11 & 100 & 0.186310416 \\
\hline 12 & 96 & 0.588803181 \\
\hline 13 & 20 & 0.446237265 \\
\hline 14 & 100 & 0.984165036 \\
\hline 15 & 100 & 0.943971796 \\
\hline 16 & 46 & 0.476153101 \\
\hline 17 & 98 & 0.633550805 \\
\hline 18 & 46 & 0.346290516 \\
\hline 19 & 100 & 0.755608023 \\
\hline 20 & 92 & 0.419617906 \\
\hline 21 & 100 & 0.181399467 \\
\hline 22 & 66 & 0.678384091 \\
\hline 23 & 29 & 0.441327643 \\
\hline 24 & 100 & 0.745835044 \\
\hline 25 & 99 & 0.768035456 \\
\hline 26 & 99 & 0.265236636 \\
\hline 27 & 100 & 0.674707655 \\
\hline 28 & 97 & 0.026101131 \\
\hline 29 & 100 & 0.285621088 \\
\hline 30 & 75 & 0.758538891 \\
\hline 31 & 91 & 0.053561591 \\
\hline 32 & 99 & 0.196737262 \\
\hline 33 & 78 & 0.225497832 \\
\hline 34 & 98 & 0.297622472 \\
\hline 35 & 85 & 0.884144467 \\
\hline 36 & 36 & 0.289742971 \\
\hline 37 & 99 & 0.205323095 \\
\hline 38 & 100 & 0.465091811 \\
\hline 39 & 92 & 0.414175439 \\
\hline 40 & 100 & 0.886381609 \\
\hline 41 & 31 & 0.364383734 \\
\hline 42 & 100 & 0.339267352 \\
\hline 43 & 100 & 0.739655621 \\
\hline 44 & 99 & 0.108842053 \\
\hline 45 & 100 & 0.160436751 \\
\hline 46 & 95 & 0.289332717 \\
\hline 47 & 100 & 0.700970426 \\
\hline 48 & 100 & 0.995473588 \\
\hline 49 & 91 & 0.08942722 \\
\hline 50 & 69 & 0.451567126 \\
\hline 51 & 83 & 0.775606089 \\
\hline 52 & 96 & 0.815260016 \\
\hline 53 & 81 & 0.78529931 \\
\hline 54 & 100 & 0.299314618 \\
\hline 55 & 69 & 0.633485867 \\
\hline 56 & 100 & 0.38972206 \\
\hline 57 & 100 & 0.240083016 \\
\hline 58 & 99 & 0.318703568 \\
\hline 59 & 54 & 0.148788786 \\
\hline 60 & 99 & 0.425730608 \\
\hline 61 & 97 & 0.409541403 \\
\hline 62 & 85 & 0.617927093 \\
\hline 63 & 100 & 0.187672192 \\
\hline 64 & 46 & 0.479859247 \\
\hline 65 & 3 & 0.880748165 \\
\hline 66 & 44 & 0.369185747 \\
\hline 67 & 100 & 0.365523021 \\
\hline 68 & 100 & 0.620420625 \\
\hline 69 & 31 & 0.920863482 \\
\hline 70 & 100 & 0.706067603 \\
\hline 71 & 96 & 0.579888134 \\
\hline 72 & 18 & 0.37546928 \\
\hline 73 & 97 & 0.424469368 \\
\hline 74 & 100 & 0.443704869 \\
\hline 75 & 73 & 0.903761695 \\
\hline 76 & 100 & 0.945200845 \\
\hline 77 & 100 & 0.873901416 \\
\hline 78 & 69 & 0.071599189 \\
\hline 79 & 3 & 0.217695238 \\
\hline 80 & 99 & 0.417919912 \\
\hline 81 & 100 & 0.664238258 \\
\hline 82 & 100 & 0.507136825 \\
\hline 83 & 93 & 0.280874708 \\
\hline 84 & 100 & 0.693399947 \\
\hline 85 & 98 & 0.177623151 \\
\hline 86 & 100 & 0.954212324 \\
\hline 87 & 54 & 0.859463102 \\
\hline 88 & 96 & 0.975601211 \\
\hline 89 & 8 & 0.418937694 \\
\hline 90 & 83 & 0.23367855 \\
\hline 91 & 16 & 0.199629471 \\
\hline 92 & 100 & 0.481381275 \\
\hline 93 & 99 & 0.871779196 \\
\hline 94 & 92 & 0.311132927 \\
\hline 95 & 100 & 0.943849453 \\
\hline
\end{tabular}




\begin{tabular}{|c|c|c|}
\hline 96 & 100 & 0.182921245 \\
\hline 97 & 100 & 0.376446168 \\
\hline $\begin{array}{l}98 \\
99\end{array}$ & 83 & 0.985109812 \\
\hline $\begin{array}{r}99 \\
100\end{array}$ & 96 & 0.64372979 \\
\hline 101 & $\begin{array}{l}90 \\
100\end{array}$ & $\begin{array}{l}0.6 / 2996919 \\
0.429057319\end{array}$ \\
\hline 102 & 100 & 0.819349223 \\
\hline 103 & 100 & 0.574304455 \\
\hline 104 & 100 & 0.827673716 \\
\hline 105 & 98 & 0.780585254 \\
\hline 106 & 100 & 0.107047399 \\
\hline 107 & 100 & 0.291412175 \\
\hline 108 & 97 & 0.338602706 \\
\hline 109 & 100 & 0.354039711 \\
\hline 110 & 100 & 0.183808756 \\
\hline 111 & 85 & 0.979999855 \\
\hline 112 & 53 & 0.297626597 \\
\hline 113 & 92 & 0.784106053 \\
\hline 114 & 47 & 0.048973017 \\
\hline 115 & 94 & 0.258352043 \\
\hline 116 & 98 & 0.135013479 \\
\hline 117 & 66 & 0.847773229 \\
\hline 118 & 100 & 0.308948897 \\
\hline 119 & 99 & 0.162389941 \\
\hline 120 & 100 & 0.066188507 \\
\hline 121 & 100 & 0.153628019 \\
\hline 122 & 100 & 0.603057615 \\
\hline 123 & 100 & 0.95540466 \\
\hline 124 & 29 & 0.884210078 \\
\hline 125 & 69 & 0.310708134 \\
\hline 126 & 100 & 0.665978687 \\
\hline 127 & 16 & 0.844894785 \\
\hline 128 & 100 & 0.384409973 \\
\hline 129 & 100 & 0.678911307 \\
\hline 130 & 95 & 0.556176892 \\
\hline 131 & 46 & 0.474582942 \\
\hline 132 & 61 & 0.286601616 \\
\hline 133 & 53 & 0.938648502 \\
\hline 134 & 99 & 0.11721221 \\
\hline 135 & 36 & 0.953855895 \\
\hline 136 & 100 & 0.023278399 \\
\hline 137 & 97 & 0.349611116 \\
\hline 138 & 47 & 0.332635618 \\
\hline 139 & 97 & 0.08959475 \\
\hline 140 & 93 & 0.407371813 \\
\hline 141 & 100 & 0.721776564 \\
\hline 142 & 100 & 0.1553787 \\
\hline 143 & 20 & 0.973117184 \\
\hline 144 & 18 & 0.863629291 \\
\hline 145 & 100 & 0.66947559 \\
\hline 146 & 98 & 0.062565318 \\
\hline 147 & 41 & 0.831376815 \\
\hline 148 & 83 & 0.69772019 \\
\hline 149 & 86 & 0.122242251 \\
\hline 150 & 100 & 0.138533196 \\
\hline 151 & 100 & 0.102184725 \\
\hline 152 & 100 & 0.977461016 \\
\hline 153 & 100 & 0.414736434 \\
\hline 154 & 100 & 0.423130035 \\
\hline 155 & 100 & 0.383143592 \\
\hline 156 & 98 & 0.639534669 \\
\hline 157 & 31 & 0.648342209 \\
\hline 158 & 97 & 0.045254171 \\
\hline 159 & 96 & 0.621490736 \\
\hline 160 & 99 & 0.523612391 \\
\hline 161 & 61 & 0.544800488 \\
\hline 162 & 99 & 0.019366518 \\
\hline 163 & 100 & 0.346471135 \\
\hline 164 & 100 & 0.811339819 \\
\hline 165 & 100 & 0.7401394 \\
\hline 166 & 100 & 0.694328403 \\
\hline 167 & 100 & 0.874527007 \\
\hline 168 & 100 & 0.885713821 \\
\hline 169 & 100 & 0.477204272 \\
\hline 170 & 88 & 0.475061219 \\
\hline 171 & 100 & 0.884192456 \\
\hline 172 & 66 & 0.371253401 \\
\hline 173 & 100 & 0.195174133 \\
\hline 174 & 54 & 0.292928814 \\
\hline 175 & 92 & 0.226394222 \\
\hline 176 & 98 & 0.922269263 \\
\hline 177 & 53 & 0.110468795 \\
\hline 178 & 100 & 0.4529898 \\
\hline 179 & 100 & 0.646116347 \\
\hline 180 & 100 & 0.310102146 \\
\hline 181 & 100 & 0.551534443 \\
\hline 182 & 73 & 0.138618094 \\
\hline 183 & 92 & 0.840445022 \\
\hline 184 & 98 & 0.674431933 \\
\hline 185 & 83 & 0.783655042 \\
\hline 186 & 98 & 0.074731758 \\
\hline 187 & 36 & 0.217072651 \\
\hline 188 & 99 & 0.268050024 \\
\hline 189 & 41 & 0.444517906 \\
\hline 190 & 98 & 0.811584062 \\
\hline 191 & 99 & 0.422554065 \\
\hline 192 & 100 & 0.99340081 \\
\hline
\end{tabular}

\begin{tabular}{|c|c|}
\hline 100 & 98 \\
\hline 100 & 98 \\
\hline 83 & 98 \\
\hline 96 & 98 \\
\hline 96 & 98 \\
\hline 100 & 98 \\
\hline 100 & 98 \\
\hline 100 & 98 \\
\hline 100 & 98 \\
\hline 98 & 98 \\
\hline 100 & 98 \\
\hline 100 & 99 \\
\hline 97 & 99 \\
\hline 100 & 99 \\
\hline 100 & 99 \\
\hline 85 & 99 \\
\hline 53 & 99 \\
\hline 92 & 99 \\
\hline 47 & 99 \\
\hline 94 & 99 \\
\hline 98 & 99 \\
\hline 66 & 99 \\
\hline 100 & 99 \\
\hline 99 & 99 \\
\hline 100 & 99 \\
\hline 100 & 99 \\
\hline 100 & 99 \\
\hline 100 & 99 \\
\hline 29 & 100 \\
\hline 69 & 100 \\
\hline 100 & 100 \\
\hline 16 & 100 \\
\hline 100 & 100 \\
\hline 100 & 100 \\
\hline 95 & 100 \\
\hline 46 & 100 \\
\hline 61 & 100 \\
\hline 53 & 100 \\
\hline 99 & 100 \\
\hline 36 & 100 \\
\hline 100 & 100 \\
\hline 97 & 100 \\
\hline 47 & 100 \\
\hline 97 & 100 \\
\hline 93 & 100 \\
\hline 100 & 100 \\
\hline 100 & 100 \\
\hline 20 & 100 \\
\hline 18 & 100 \\
\hline 100 & 100 \\
\hline 98 & 100 \\
\hline 41 & 100 \\
\hline 83 & 100 \\
\hline 86 & 100 \\
\hline 100 & 100 \\
\hline 100 & 100 \\
\hline 100 & 100 \\
\hline 100 & 100 \\
\hline 100 & 100 \\
\hline 100 & 100 \\
\hline 98 & 100 \\
\hline 31 & 100 \\
\hline 97 & 100 \\
\hline 96 & 100 \\
\hline 99 & 100 \\
\hline 61 & 100 \\
\hline 99 & 100 \\
\hline 100 & 100 \\
\hline 100 & 100 \\
\hline 100 & 100 \\
\hline 100 & 100 \\
\hline 100 & 100 \\
\hline 100 & 100 \\
\hline 100 & 100 \\
\hline 88 & 100 \\
\hline 100 & 100 \\
\hline 66 & 100 \\
\hline 100 & 100 \\
\hline 54 & 100 \\
\hline 92 & 100 \\
\hline 98 & 100 \\
\hline 53 & 100 \\
\hline 100 & 100 \\
\hline 100 & 100 \\
\hline 100 & 100 \\
\hline 100 & 100 \\
\hline 73 & 100 \\
\hline 92 & 100 \\
\hline 98 & 100 \\
\hline 83 & 100 \\
\hline 98 & 100 \\
\hline 36 & 100 \\
\hline 99 & 100 \\
\hline 41 & 100 \\
\hline 98 & 100 \\
\hline 99 & 100 \\
\hline 100 & 100 \\
\hline
\end{tabular}

$\begin{array}{ll}0.383374985 & 0.001902 \\ 0.389206236 & 0.001931\end{array}$ $\begin{array}{ll}0.389206236 & 0.001931 \\ 0.4008687 & 0.00196\end{array}$ $\begin{array}{ll}0.400868737 & 0.0019 \\ 0.406\end{array}$ 0.4066999880 .002019 $\begin{array}{rr}0.412531239 & 0.002048 \\ 0.41836249 & 0.002077\end{array}$ $\begin{array}{lll}0.41836249 & 0.002077 \\ 0.42419374 & 0.002106\end{array}$ $\begin{array}{ll}0.42419374 & 0.002106 \\ 0.430024991 & 0.002136\end{array}$ 0.4300249910 .002136 $\begin{array}{ll}0.435856242 & 0.002165 \\ 0.441687493 & 0.002194\end{array}$ $\begin{array}{lll}0.447578246 & 0.002223\end{array}$ 0.4534689990 .002253 0.4593597520 .002282 $\begin{array}{lll}0.465250506 & 0.002312\end{array}$ $\begin{array}{lll}0.465250506 & 0.002312\end{array}$ $\begin{array}{lr}0.477032012 & 0.00237\end{array}$ $\begin{array}{lr}0.477032012 & 0.00237 \\ 0.482922766 & 0.0024\end{array}$ $\begin{array}{rr}0.482922766 & 0.0024 \\ 0.488813519 & 0.002429\end{array}$ 0.4947042720 .002459 $0.500595026 \quad 0.002488$ 0.5064857790 .002518 0.5123765320 .002547 $\begin{array}{lll}0.518267285 & 0.002577\end{array}$ $\begin{array}{lll}0.524158039 & 0.002606\end{array}$ $\begin{array}{lll}0.530048792 & 0.002636\end{array}$ $0.535939545 \quad 0.002665$ 0.5418302990 .002694 $0.547780555 \quad 0.002724$ 0.553730810 .002754 $0.559681066 \quad 0.002784$ $0.565631322 \quad 0.002813$ $\begin{array}{lll}0.571581578 & 0.002843\end{array}$ $0.577531834 \quad 0.002873$ $\begin{array}{lll}0.58348209 & 0.002903\end{array}$ $\begin{array}{lll}0.589432346 & 0.002932\end{array}$ $\begin{array}{lll}0.595382601 & 0.002962\end{array}$ $0.601332857 \quad 0.002992$ 0.6072831130 .00302 0.6132333690 .00305 0.6191836250 .00308 $\begin{array}{lll}0.625133881 & 0.003111\end{array}$ $0.631084137 \quad 0.003141$ $0.637034392 \quad 0.00317$ $\begin{array}{ll}0.642984648 & 0.0032\end{array}$ $\begin{array}{ll}0.648934904 & 0.00323\end{array}$ $\begin{array}{rr}0.65488516 & 0.00326\end{array}$ 0.6608354160 .003289 0.6667856720 .003319 $0.672735928 \quad 0.003349$ $0.678686184 \quad 0.003379$ 0.6846364390 .003408 $\begin{array}{lll}0.690586695 & 0.003438 \\ 0.696536951 & 0.003468\end{array}$ $0.696536951 \quad 0.003468$ $\begin{array}{lll}0.702487207 & 0.003498\end{array}$ $\begin{array}{lll}0.708437463 & 0.003527 \\ 0.714387719 & 0.003557\end{array}$ $\begin{array}{lll}0.720337975 & 0.003587\end{array}$ $\begin{array}{lll}0.72628823 & 0.003617\end{array}$ $0.732238486 \quad 0.003646$ 0.7381887420 .003676 $0.744138998 \quad 0.003706$ $0.750089254 \quad 0.003736$ $\begin{array}{ll}0.75603951 & 0.003765 \\ 0.761989766 & 0.003795\end{array}$ $\begin{array}{lll}0.761989766 & 0.003795 \\ 0.767940021 & 0.003825\end{array}$ $\begin{array}{lll}0.767380027 & 0.003825\end{array}$ $\begin{array}{lll}0.779840533 & 0.003884\end{array}$ $\begin{array}{lll}0.785790789 & 0.00391\end{array}$ $0.791741045 \quad 0.003944$ $\begin{array}{lll}0.797691301 & 0.003974\end{array}$ $\begin{array}{lll}0.803641557 & 0.004003\end{array}$ 0.8095918120 .004033 $0.815542068 \quad 0.004063$ $0.821492324 \quad 0.004093$ $0.82744258 \quad 0.004122$ $\begin{array}{lll}0.833392836 & 0.004152\end{array}$ $0.839343092 \quad 0.004182$ $\begin{array}{lll}0.845293348 & 0.004212\end{array}$ $\begin{array}{lll}0.851243603 & 0.00424\end{array}$ $\begin{array}{ll}0.857193859 & 0.004271 \\ 0.863144115 & 0.004301\end{array}$ $\begin{array}{ll}0.863144115 & 0.004301 \\ 0.869094371 & 0.004331\end{array}$ $\begin{array}{rr}0.869094371 & 0.004331 \\ 0.875044627 & 0.00436\end{array}$ $0.880994883 \quad 0.00439$ $0.886945139 \quad 0.00442$ $\begin{array}{ll}0.892895395 & 0.00445\end{array}$ $\begin{array}{ll}0.89884565 & 0.004479\end{array}$ $\begin{array}{ll}0.904795906 & 0.004509\end{array}$ $\begin{array}{lll}0.910746162 & 0.004539\end{array}$ $\begin{array}{lll}0.916696418 & 0.004569\end{array}$ 0.9226466740 .004598 $\begin{array}{lll}0.92859693 & 0.004628\end{array}$ $\begin{array}{lll}0.934547186 & 0.004658\end{array}$ $\begin{array}{ll}0.940497441 & 0.004688 \\ 0.946447697 & 0.004717\end{array}$ $\begin{array}{lll}0.946447697 & 0.004717\end{array}$ 


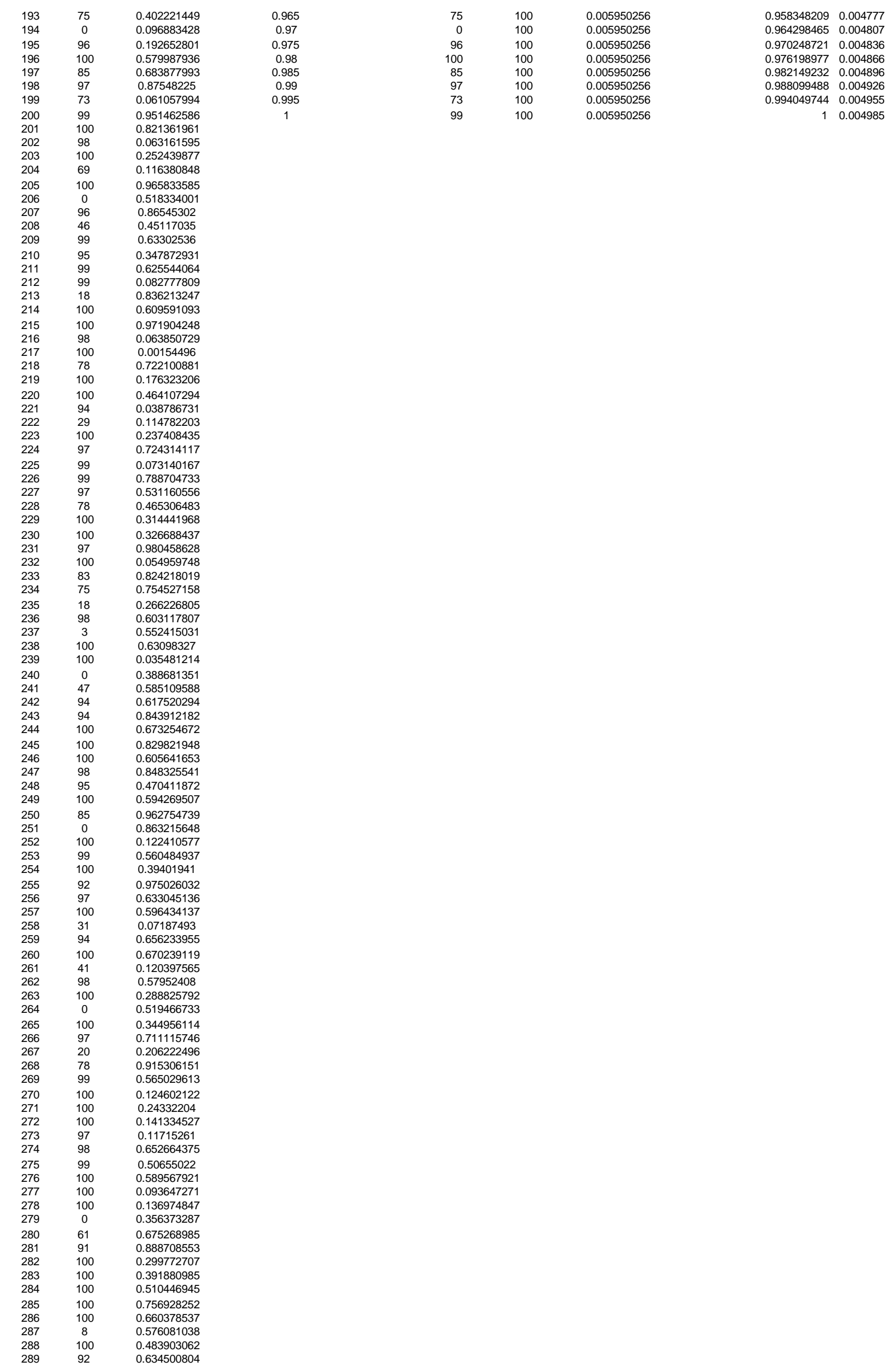




\begin{tabular}{|c|c|}
\hline 290 & 99 \\
\hline 291 & 88 \\
\hline 292 & 31 \\
\hline 293 & 93 \\
\hline 294 & 31 \\
\hline 295 & 53 \\
\hline 296 & 98 \\
\hline 297 & 100 \\
\hline 298 & 8 \\
\hline 299 & 99 \\
\hline 300 & 100 \\
\hline 301 & 61 \\
\hline 302 & 99 \\
\hline 303 & 29 \\
\hline 304 & 96 \\
\hline 305 & 20 \\
\hline 306 & 100 \\
\hline 307 & 98 \\
\hline 308 & 99 \\
\hline 309 & 54 \\
\hline 310 & 100 \\
\hline 311 & 100 \\
\hline 312 & 95 \\
\hline 313 & 100 \\
\hline 314 & 85 \\
\hline 315 & 100 \\
\hline 316 & 86 \\
\hline 317 & 73 \\
\hline 318 & 83 \\
\hline 319 & 100 \\
\hline 320 & 44 \\
\hline 321 & 16 \\
\hline 322 & 85 \\
\hline 323 & 96 \\
\hline 324 & 100 \\
\hline 325 & 8 \\
\hline 326 & 86 \\
\hline 327 & 100 \\
\hline 328 & 100 \\
\hline 329 & 95 \\
\hline 330 & 83 \\
\hline 331 & 100 \\
\hline 332 & 100 \\
\hline 333 & 99 \\
\hline 334 & 18 \\
\hline 335 & 100 \\
\hline 336 & 29 \\
\hline 337 & 100 \\
\hline 338 & 98 \\
\hline 339 & 93 \\
\hline 340 & 44 \\
\hline 341 & 100 \\
\hline 342 & 100 \\
\hline 343 & 100 \\
\hline 344 & 97 \\
\hline 345 & 99 \\
\hline 346 & 99 \\
\hline 347 & 96 \\
\hline 348 & 99 \\
\hline 349 & 99 \\
\hline 350 & 99 \\
\hline 351 & 97 \\
\hline 352 & 95 \\
\hline 353 & 100 \\
\hline 354 & 99 \\
\hline 355 & 100 \\
\hline 356 & 16 \\
\hline 357 & 73 \\
\hline 358 & 100 \\
\hline 359 & 88 \\
\hline 360 & 18 \\
\hline 361 & 78 \\
\hline 362 & 83 \\
\hline 363 & 100 \\
\hline 364 & 16 \\
\hline 365 & 78 \\
\hline 366 & 95 \\
\hline 367 & 95 \\
\hline 368 & 100 \\
\hline 369 & 100 \\
\hline 370 & 99 \\
\hline 371 & 100 \\
\hline 372 & 100 \\
\hline 373 & 99 \\
\hline 374 & 91 \\
\hline 375 & 94 \\
\hline 376 & 100 \\
\hline 377 & 81 \\
\hline 378 & 100 \\
\hline 379 & 99 \\
\hline 380 & 99 \\
\hline 381 & 36 \\
\hline 382 & 99 \\
\hline 383 & 96 \\
\hline 384 & 97 \\
\hline 385 & 46 \\
\hline 386 & 100 \\
\hline
\end{tabular}




\begin{tabular}{|c|c|}
\hline 387 & 100 \\
\hline 388 & 99 \\
\hline 389 & 96 \\
\hline 390 & 100 \\
\hline 391 & 99 \\
\hline 392 & 100 \\
\hline 393 & 100 \\
\hline 394 & 81 \\
\hline 395 & 100 \\
\hline 396 & 95 \\
\hline 397 & 96 \\
\hline 398 & 83 \\
\hline 399 & 88 \\
\hline 400 & 75 \\
\hline 401 & 100 \\
\hline 402 & 92 \\
\hline 403 & 83 \\
\hline 404 & 100 \\
\hline 405 & 92 \\
\hline 406 & 99 \\
\hline 407 & 86 \\
\hline 408 & 100 \\
\hline 409 & 41 \\
\hline 410 & 93 \\
\hline 411 & 47 \\
\hline 412 & 100 \\
\hline 413 & 100 \\
\hline 414 & 85 \\
\hline 415 & 99 \\
\hline 416 & 100 \\
\hline 417 & 85 \\
\hline 418 & 99 \\
\hline 419 & 36 \\
\hline 420 & 66 \\
\hline 421 & 36 \\
\hline 422 & 100 \\
\hline 423 & 100 \\
\hline 424 & 100 \\
\hline 425 & 100 \\
\hline 426 & 97 \\
\hline 427 & 100 \\
\hline 428 & 66 \\
\hline 429 & 100 \\
\hline 430 & 75 \\
\hline 431 & 85 \\
\hline 432 & 100 \\
\hline 433 & 100 \\
\hline 434 & 96 \\
\hline 435 & 93 \\
\hline 436 & 97 \\
\hline 437 & 8 \\
\hline 438 & 100 \\
\hline 439 & 100 \\
\hline 440 & 97 \\
\hline 441 & 99 \\
\hline 442 & 100 \\
\hline 443 & 100 \\
\hline 444 & 98 \\
\hline 445 & 99 \\
\hline 446 & 95 \\
\hline 447 & 81 \\
\hline 448 & 3 \\
\hline 449 & 100 \\
\hline 450 & 99 \\
\hline 451 & 53 \\
\hline 452 & 100 \\
\hline 453 & 100 \\
\hline 454 & 97 \\
\hline 455 & 88 \\
\hline 456 & 97 \\
\hline 457 & 85 \\
\hline 458 & 3 \\
\hline 459 & 41 \\
\hline 460 & 8 \\
\hline 461 & 99 \\
\hline 462 & 20 \\
\hline 463 & 99 \\
\hline 464 & 41 \\
\hline 465 & 100 \\
\hline 466 & 69 \\
\hline 467 & 100 \\
\hline 468 & 100 \\
\hline 469 & 44 \\
\hline 470 & 100 \\
\hline 471 & 100 \\
\hline 472 & 92 \\
\hline 473 & 85 \\
\hline 474 & 98 \\
\hline 475 & 54 \\
\hline 476 & 100 \\
\hline 477 & 20 \\
\hline 478 & 86 \\
\hline 479 & 61 \\
\hline 480 & 99 \\
\hline 481 & 97 \\
\hline 482 & 100 \\
\hline 483 & 47 \\
\hline
\end{tabular}




$\begin{array}{lcl}484 & 98 & 0.947730817 \\ 485 & 86 & 0.974211753 \\ 486 & 91 & 0.070448089 \\ 487 & 29 & 0.261774247 \\ 488 & 100 & 0.767878655 \\ 489 & 44 & 0.975327232 \\ 490 & 100 & 0.270659626 \\ 491 & 88 & 0.279858106 \\ 492 & 81 & 0.307433897 \\ 493 & 97 & 0.587421823 \\ 494 & 53 & 0.252730227 \\ 495 & 61 & 0.490119739 \\ 496 & 75 & 0.022087663 \\ 497 & 54 & 0.269266467 \\ 498 & 99 & 0.517672 \\ 499 & 100 & 0.617534199 \\ 500 & 95 & 0.321102621 \\ 501 & 99 & 0.740577377 \\ 502 & 98 & 0.39280242 \\ 503 & 100 & 0.757991441 \\ 504 & 3 & 0.650364417 \\ 505 & 91 & 0.384350948 \\ 506 & 44 & 0.455071431 \\ 507 & 100 & 0.899054834 \\ 508 & 73 & 0.582252356 \\ 509 & 47 & 0.544368418 \\ 510 & 92 & 0.000917301\end{array}$

0.13641117

0.13426411

0.14587674

0.15169711

0.16538813

0.17414426

0.12779634

0.1708401

0.14079733

$\begin{array}{lr}\text { mean } & 0.14905709 \\ \text { st dev } & 0.020064509\end{array}$ 


\begin{tabular}{|c|c|c|}
\hline $\begin{array}{l}\text { Staurospor } \\
\text { position }\end{array}$ & $\begin{array}{l}\text { ine; } 1 \mu \mathrm{M} ; 100 \\
\text { inhibition\% }\end{array}$ & $\begin{array}{l}\text { M ATP; sampl } \\
\text { rand }\end{array}$ \\
\hline 1 & 16 & 0.266199022 \\
\hline 2 & 20 & 0.226125157 \\
\hline 3 & 81 & 0.08898558 \\
\hline 4 & 100 & 0.502867933 \\
\hline 5 & 99 & 0.208710348 \\
\hline 6 & 46 & 0.074360801 \\
\hline 7 & 98 & 0.907601077 \\
\hline 8 & 83 & 0.321593056 \\
\hline 9 & 96 & 0.738485992 \\
\hline 10 & 93 & 0.640493108 \\
\hline 11 & 47 & 0.472071319 \\
\hline 12 & 83 & 0.658074703 \\
\hline 13 & 100 & 0.868735493 \\
\hline 14 & 94 & 0.97367671 \\
\hline 15 & 100 & 0.092474996 \\
\hline 16 & 100 & 0.282376413 \\
\hline 17 & 100 & 0.321739995 \\
\hline 18 & 100 & 0.422243293 \\
\hline 19 & 96 & 0.338408406 \\
\hline 20 & 97 & 0.405500861 \\
\hline 21 & 29 & 0.222195785 \\
\hline 22 & 100 & 0.03432233 \\
\hline 23 & 31 & 0.169357353 \\
\hline 24 & 100 & 0.889786936 \\
\hline 25 & 100 & 0.481306139 \\
\hline 26 & 100 & 0.601853348 \\
\hline 27 & 99 & 0.486533945 \\
\hline 28 & 36 & 0.989407053 \\
\hline 29 & 75 & 0.664641427 \\
\hline 30 & 96 & 0.068163824 \\
\hline 31 & 54 & 0.318856038 \\
\hline 32 & 100 & 0.119494388 \\
\hline 33 & 29 & 0.807467876 \\
\hline 34 & 92 & 0.085807609 \\
\hline 35 & 99 & 0.815620361 \\
\hline 36 & 94 & 0.11405443 \\
\hline 37 & 97 & 0.033548113 \\
\hline 38 & 92 & 0.732156839 \\
\hline 39 & 85 & 0.065520505 \\
\hline 40 & 97 & 0.351375528 \\
\hline 41 & 85 & 0.106995262 \\
\hline 42 & 54 & 0.493037802 \\
\hline 43 & 20 & 0.402070569 \\
\hline 44 & 0 & 0.876398225 \\
\hline 45 & 98 & 0.510940272 \\
\hline 46 & 100 & 0.753554002 \\
\hline 47 & 73 & 0.940391152 \\
\hline 48 & 99 & 0.034805147 \\
\hline 49 & 95 & 0.021847338 \\
\hline 50 & 100 & 0.490405542 \\
\hline 51 & 29 & 0.82014146 \\
\hline 52 & 61 & 0.691581005 \\
\hline 53 & 8 & 0.711873032 \\
\hline 54 & 100 & 0.876120529 \\
\hline 55 & 53 & 0.804940499 \\
\hline 56 & 99 & 0.857266491 \\
\hline 57 & 100 & 0.640868891 \\
\hline 58 & 100 & 0.599358484 \\
\hline 59 & 31 & 0.886400019 \\
\hline 60 & 100 & 0.330900546 \\
\hline 61 & 69 & 0.125552785 \\
\hline 62 & 100 & 0.474597063 \\
\hline 63 & 54 & 0.37952267 \\
\hline 64 & 8 & 0.700166883 \\
\hline 65 & 100 & 0.448317668 \\
\hline 66 & 53 & 0.907683476 \\
\hline 67 & 92 & 0.593505376 \\
\hline 68 & 75 & 0.878974032 \\
\hline 69 & 41 & 0.434575409 \\
\hline 70 & 85 & 0.040052031 \\
\hline 71 & 100 & 0.844122433 \\
\hline 72 & 100 & 0.876429871 \\
\hline 73 & 36 & 0.559039599 \\
\hline 74 & 31 & 0.736809927 \\
\hline 75 & 31 & 0.261272128 \\
\hline 76 & 99 & 0.34380872 \\
\hline 77 & 100 & 0.891311006 \\
\hline 78 & 88 & 0.439516552 \\
\hline 79 & 73 & 0.11838364 \\
\hline 80 & 100 & 0.824656682 \\
\hline 81 & 98 & 0.976906963 \\
\hline 82 & 18 & 0.530791228 \\
\hline 83 & 16 & 0.738880315 \\
\hline 84 & 100 & 0.092257644 \\
\hline 85 & 99 & 0.434161707 \\
\hline 86 & 100 & 0.648809675 \\
\hline 87 & 100 & 0.659606191 \\
\hline 88 & 100 & 0.92628987 \\
\hline 89 & 47 & 0.772117087 \\
\hline 90 & 100 & 0.502104545 \\
\hline 91 & 83 & 0.408869826 \\
\hline 92 & 46 & 0.343192196 \\
\hline 93 & 99 & 0.527383192 \\
\hline 94 & 97 & 0.902736061 \\
\hline 95 & 100 & 0.085157223 \\
\hline
\end{tabular}




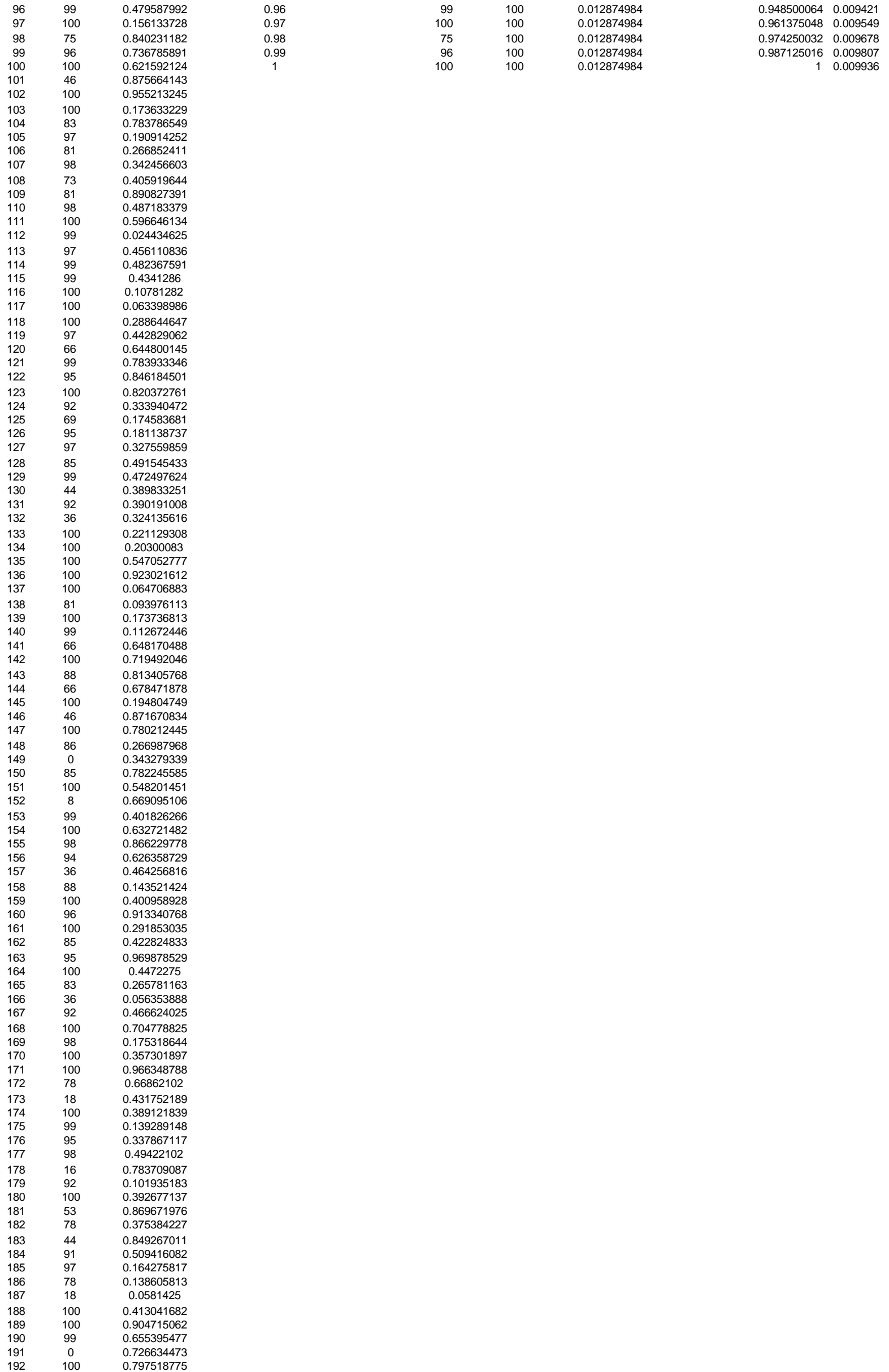




\begin{tabular}{|c|c|}
\hline 193 & 100 \\
\hline 194 & 20 \\
\hline 195 & 99 \\
\hline 196 & 100 \\
\hline 197 & 83 \\
\hline 198 & 47 \\
\hline 199 & 99 \\
\hline 200 & 61 \\
\hline 201 & 93 \\
\hline 202 & 92 \\
\hline 203 & 100 \\
\hline 204 & 97 \\
\hline 205 & 98 \\
\hline 206 & 36 \\
\hline 207 & 99 \\
\hline 208 & 61 \\
\hline 209 & 73 \\
\hline 210 & 97 \\
\hline 211 & 97 \\
\hline 212 & 88 \\
\hline 213 & 95 \\
\hline 214 & 99 \\
\hline 215 & 100 \\
\hline 216 & 0 \\
\hline 217 & 85 \\
\hline 218 & 100 \\
\hline 219 & 3 \\
\hline 220 & 100 \\
\hline 221 & 86 \\
\hline 222 & 97 \\
\hline 223 & 99 \\
\hline 224 & 100 \\
\hline 225 & 99 \\
\hline 226 & 100 \\
\hline 227 & 99 \\
\hline 228 & 100 \\
\hline 229 & 100 \\
\hline 230 & 98 \\
\hline 231 & 41 \\
\hline 232 & 16 \\
\hline 233 & 54 \\
\hline 234 & 98 \\
\hline 235 & 99 \\
\hline 236 & 100 \\
\hline 237 & 100 \\
\hline 238 & 75 \\
\hline 239 & 100 \\
\hline 240 & 81 \\
\hline 241 & 97 \\
\hline 242 & 92 \\
\hline 243 & 100 \\
\hline 244 & 100 \\
\hline 245 & 61 \\
\hline 246 & 100 \\
\hline 247 & 41 \\
\hline 248 & 100 \\
\hline 249 & 100 \\
\hline 250 & 99 \\
\hline 251 & 100 \\
\hline 252 & 99 \\
\hline 253 & 97 \\
\hline 254 & 86 \\
\hline 255 & 3 \\
\hline 256 & 100 \\
\hline 257 & 100 \\
\hline 258 & 100 \\
\hline 259 & 3 \\
\hline 260 & 95 \\
\hline 261 & 99 \\
\hline 262 & 99 \\
\hline 263 & 100 \\
\hline 264 & 94 \\
\hline 265 & 95 \\
\hline 266 & 66 \\
\hline 267 & 88 \\
\hline 268 & 100 \\
\hline 269 & 47 \\
\hline 270 & 98 \\
\hline 271 & 98 \\
\hline 272 & 8 \\
\hline 273 & 100 \\
\hline 274 & 100 \\
\hline 275 & 100 \\
\hline 276 & 100 \\
\hline 277 & 99 \\
\hline 278 & 20 \\
\hline 279 & 69 \\
\hline 280 & 47 \\
\hline 281 & 31 \\
\hline 282 & 91 \\
\hline 283 & 100 \\
\hline 284 & 100 \\
\hline 285 & 97 \\
\hline 286 & 100 \\
\hline 287 & 100 \\
\hline 288 & 98 \\
\hline 289 & 99 \\
\hline
\end{tabular}




\begin{tabular}{|c|c|}
\hline 290 & 100 \\
\hline 291 & 99 \\
\hline 292 & 54 \\
\hline 293 & 100 \\
\hline 294 & 99 \\
\hline 295 & 100 \\
\hline 296 & 93 \\
\hline 297 & 93 \\
\hline 298 & 100 \\
\hline 299 & 78 \\
\hline 300 & 44 \\
\hline 301 & 0 \\
\hline 302 & 100 \\
\hline 303 & 99 \\
\hline 304 & 100 \\
\hline 305 & 66 \\
\hline 306 & 100 \\
\hline 307 & 61 \\
\hline 308 & 61 \\
\hline 309 & 99 \\
\hline 310 & 69 \\
\hline 311 & 100 \\
\hline 312 & 97 \\
\hline 313 & 18 \\
\hline 314 & 86 \\
\hline 315 & 96 \\
\hline 316 & 100 \\
\hline 317 & 100 \\
\hline 318 & 69 \\
\hline 319 & 100 \\
\hline 320 & 99 \\
\hline 321 & 96 \\
\hline 322 & 29 \\
\hline 323 & 100 \\
\hline 324 & 100 \\
\hline 325 & 100 \\
\hline 326 & 44 \\
\hline 327 & 29 \\
\hline 328 & 96 \\
\hline 329 & 44 \\
\hline 330 & 100 \\
\hline 331 & 98 \\
\hline 332 & 100 \\
\hline 333 & 100 \\
\hline 334 & 98 \\
\hline 335 & 100 \\
\hline 336 & 100 \\
\hline 337 & 96 \\
\hline 338 & 92 \\
\hline 339 & 100 \\
\hline 340 & 47 \\
\hline 341 & 100 \\
\hline 342 & 41 \\
\hline 343 & 100 \\
\hline 344 & 100 \\
\hline 345 & 98 \\
\hline 346 & 75 \\
\hline 347 & 95 \\
\hline 348 & 99 \\
\hline 349 & 93 \\
\hline 350 & 98 \\
\hline 351 & 97 \\
\hline 352 & 83 \\
\hline 353 & 99 \\
\hline 354 & 96 \\
\hline 355 & 91 \\
\hline 356 & 100 \\
\hline 357 & 100 \\
\hline 358 & 100 \\
\hline 359 & 100 \\
\hline 360 & 96 \\
\hline 361 & 29 \\
\hline 362 & 73 \\
\hline 363 & 100 \\
\hline 364 & 88 \\
\hline 365 & 99 \\
\hline 366 & 81 \\
\hline 367 & 100 \\
\hline 368 & 100 \\
\hline 369 & 98 \\
\hline 370 & 100 \\
\hline 371 & 100 \\
\hline 372 & 31 \\
\hline 373 & 83 \\
\hline 374 & 100 \\
\hline 375 & 85 \\
\hline 376 & 100 \\
\hline 377 & 16 \\
\hline 378 & 100 \\
\hline 379 & 99 \\
\hline 380 & 100 \\
\hline 381 & 100 \\
\hline 382 & 100 \\
\hline 383 & 97 \\
\hline 384 & 96 \\
\hline 385 & 100 \\
\hline 386 & 100 \\
\hline
\end{tabular}




\begin{tabular}{|c|c|}
\hline 387 & 83 \\
\hline 388 & 98 \\
\hline 389 & 100 \\
\hline 390 & 3 \\
\hline 391 & 100 \\
\hline 392 & 93 \\
\hline 393 & 100 \\
\hline 394 & 100 \\
\hline 395 & 95 \\
\hline 396 & 53 \\
\hline 397 & 100 \\
\hline 398 & 99 \\
\hline 399 & 78 \\
\hline 400 & 98 \\
\hline 401 & 100 \\
\hline 402 & 100 \\
\hline 403 & 73 \\
\hline 404 & 69 \\
\hline 405 & 100 \\
\hline 406 & 86 \\
\hline 407 & 100 \\
\hline 408 & 92 \\
\hline 409 & 100 \\
\hline 410 & 100 \\
\hline 411 & 8 \\
\hline 412 & 100 \\
\hline 413 & 99 \\
\hline 414 & 98 \\
\hline 415 & 100 \\
\hline 416 & 99 \\
\hline 417 & 100 \\
\hline 418 & 91 \\
\hline 419 & 99 \\
\hline 420 & 100 \\
\hline 421 & 96 \\
\hline 422 & 97 \\
\hline 423 & 100 \\
\hline 424 & 96 \\
\hline 425 & 96 \\
\hline 426 & 91 \\
\hline 427 & 0 \\
\hline 428 & 99 \\
\hline 429 & 100 \\
\hline 430 & 96 \\
\hline 431 & 97 \\
\hline 432 & 99 \\
\hline 433 & 92 \\
\hline 434 & 44 \\
\hline 435 & 100 \\
\hline 436 & 100 \\
\hline 437 & 16 \\
\hline 438 & 99 \\
\hline 439 & 41 \\
\hline 440 & 85 \\
\hline 441 & 83 \\
\hline 442 & 46 \\
\hline 443 & 96 \\
\hline 444 & 53 \\
\hline 445 & 100 \\
\hline 446 & 85 \\
\hline 447 & 46 \\
\hline 448 & 100 \\
\hline 449 & 100 \\
\hline 450 & 75 \\
\hline 451 & 100 \\
\hline 452 & 8 \\
\hline 453 & 100 \\
\hline 454 & 95 \\
\hline 455 & 97 \\
\hline 456 & 97 \\
\hline 457 & 100 \\
\hline 458 & 85 \\
\hline 459 & 91 \\
\hline 460 & 18 \\
\hline 461 & 100 \\
\hline 462 & 100 \\
\hline 463 & 100 \\
\hline 464 & 3 \\
\hline 465 & 97 \\
\hline 466 & 100 \\
\hline 467 & 99 \\
\hline 468 & 78 \\
\hline 469 & 20 \\
\hline 470 & 54 \\
\hline 471 & 100 \\
\hline 472 & 100 \\
\hline 473 & 3 \\
\hline 474 & 95 \\
\hline 475 & 100 \\
\hline 476 & 100 \\
\hline 477 & 18 \\
\hline 478 & 100 \\
\hline 479 & 100 \\
\hline 480 & 100 \\
\hline 481 & 100 \\
\hline 482 & 99 \\
\hline 483 & 100 \\
\hline
\end{tabular}




$\begin{array}{lll}484 & 100 & 0.113937419 \\ 485 & 100 & 0.762852397 \\ 486 & 83 & 0.081600859 \\ 487 & 100 & 0.795400999 \\ 488 & 100 & 0.352178721 \\ 489 & 100 & 0.125681669 \\ 490 & 53 & 0.869788811 \\ 491 & 100 & 0.87638627 \\ 492 & 99 & 0.071273623 \\ 493 & 100 & 0.106999563 \\ 494 & 98 & 0.452531593 \\ 495 & 96 & 0.123393926 \\ 496 & 41 & 0.281119437 \\ 497 & 99 & 0.685973702 \\ 498 & 98 & 0.654124091 \\ 499 & 100 & 0.53822245 \\ 500 & 83 & 0.28695734 \\ 501 & 20 & 0.848437003 \\ 502 & 100 & 0.780834092 \\ 503 & 85 & 0.360701796 \\ 504 & 86 & 0.41894737 \\ 505 & 94 & 0.795184441 \\ 506 & 100 & 0.349033632 \\ 507 & 66 & 0.038744117 \\ 508 & 99 & 0.012246413 \\ 509 & 94 & 0.203504298 \\ 510 & 99 & 0.926485269\end{array}$


Staurosporine; $1 \mu \mathrm{M} ; 100 \mu \mathrm{M}$ ATP; sample of $\mathbf{5 0}$ kinases

position inhibition \% rand cumulative sample fraction sample inhibition 0.942256641 0.765407607 0.53437369

0.530271356

0.858080721

0.259210816

0.757111328

0.381568845

0.478031756

0.47031756

0.275762588

0.248667092

0.513034274
0.059547804

0.059547804
0.317235799

0.082586776

0.872787568

0.272366151

0.063971359

0.997919563

.105558166

0.450774285

0.113325723
0.321029858

0.525815771

0.796226236

0.192122725
0.361551956

0.221630108

0.276726893

0.646140501

0.553818137

0.093543808

0.160141208

0.206259287

0.313459655

0.245711037

0.9199174

0.021288974

0.478725772

0.537277989

0.439732886

0.439732886
0.22891297

0.22891297
0.013162043

0.013162043

0.079416416

0.193466452

0.726265797

0.050752683

0.167165254

0.450824084

0.957598274

0.22872702

0.829573598

0.065759894

0.577499184

0.062691212

0.016209217

0.584837229

0.926177845

0.73568907

0.059091998

0.897026181

0.369317032

0.334833216

0.901723877

0.546528447

0.620555426

0.220298875

0.886326409

0.724104678

0.898981097

0.640229319
0.977736861

1104124

0.238813806

0.567674752

0.014300572

0.996564456

0.097973179

0.713667115

0.412940007
0.772356417

0.772356417
0.766066199

0.314478437

0.009203453

0.578302783

0.271303335
0.06

0.1

0.12

0.14

0.16

0.2

0.2
0.22
0.24

0.2

0.32

0.34

0.36

0.38

0.4

0.42

0.44

0.46

0.5

0.52

0.54

0.56

0.58
0.6

0.62

0.64

0.66

0.68

0.72

0.72
0.74

0.76

0.78 action of inhibition cumulative fraction of inhibition area

$\begin{array}{llll}0.006755183 & 0.006755183 & 6.76 \mathrm{E}-05\end{array}$ $\begin{array}{lll}0.007221058 & 0.01397624 & 0.000207\end{array}$ $\begin{array}{llll}0.007221058 & 0.021197298 & 0.000352\end{array}$ $\begin{array}{llll}0.008385744 & 0.029583042 & 0.000508\end{array}$ $\begin{array}{llll}0.009550431 & 0.039133473 & 0.000687\end{array}$ $\begin{array}{llll}0.009550431 & 0.048683904 & 0.000878\end{array}$ $\begin{array}{lll}0.010715118 & 0.059399022 & 0.001081\end{array}$ $\begin{array}{lll}0.010948055 & 0.070347077 & 0.001297\end{array}$ $0.012578616 \quad 0.0829256930 .001533$ $\begin{array}{lll}0.014209178 & 0.097134871 & 0.001801\end{array}$ $\begin{array}{lll}0.018169113 & 0.115303983 & 0.002124\end{array}$ $\begin{array}{lll}0.019799674 & 0.135103657 & 0.002504\end{array}$ $\begin{array}{lll}0.019799674 & 0.154903331 & 0.0029\end{array}$ $0.020498486-0.1754018170 .003303$ 0.021430235

0.021430235

0.021430235

0.021663173

0.022361985

0.022361985

0.022361985

0.023060797

0.023060797

0.023060797

.023060797

0.023060797

0.023293734

0.023293734

0.023293734

0.023293734

0.023293734

0.023293734

0.023293734

0.023293734

0.023293734

0.023293734

0.023293734

0.023293734

0.023293734

0.023293734

0.023293734

0.023293734

0.023293734

0.023293734

0.023293734

0.023293734

0.023293734

$\begin{array}{lll}0.175401817 & 0.003\end{array}$

$\begin{array}{ll}0.196832052 & 0.003722 \\ 0.218262287 & 0.004151\end{array}$

$\begin{array}{rr}0.218262287 & 0.004151 \\ 0.239692523 & 0.00458\end{array}$

$\begin{array}{ll}0.239692523 & 0.00458 \\ 0.261355695 & 0.00501 \\ 0.283484743 & 0.005448\end{array}$

$\begin{array}{ll}0.261355695 & 0.00501 \\ 0.283484743 & 0.005448\end{array}$

$0.305846727 \quad 0.005893$

$\begin{array}{lll}0.328208712 & 0.006341\end{array}$

$\begin{array}{lll}0.350570696 & 0.006788\end{array}$

$\begin{array}{lll}0.372932681 & 0.007235\end{array}$

$0.395527603 \quad 0.007685$

$0.4185884 \quad 0.008141$

$0.441649196 \quad 0.008602$

$0.464709993 \quad 0.009064$

$\begin{array}{lll}0.48777079 & 0.009525\end{array}$

$\begin{array}{lll}0.510831586 & 0.009986\end{array}$

$\begin{array}{ll}0.53412532 & 0.01045\end{array}$

$0.557419054 \quad 0.010915$

$\begin{array}{lll}0.580712788 & 0.011381\end{array}$

$\begin{array}{lll}0.604006522 & 0.011847\end{array}$

$0.627300256 \quad 0.012313$

$0.65059399 \quad 0.012779$

$0.673887724 \quad 0.013245$

$0.697181458 \quad 0.013711$

$0.720475192 \quad 0.014177$

$\begin{array}{ll}0.743768926 & 0.014642\end{array}$

$\begin{array}{lll}0.76706266 & 0.015108\end{array}$

$0.790356394 \quad 0.015574$

$0.813650128 \quad 0.01604$

0.8369438620 .016506

$\begin{array}{lll}0.836943862 & 0.016506\end{array}$

$\begin{array}{lll}0.860237596 & 0.016972 \\ 0.88353133 & 0.017438\end{array}$

0.9068250640 .017904

$0.930118798 \quad 0.01836$

0.9534125320 .018835

$0.976706266 \quad 0.019301$ $\begin{array}{ll}1 & 0.019767\end{array}$ 


\begin{tabular}{|c|c|}
\hline 96 & 97 \\
\hline 97 & 29 \\
\hline 98 & 100 \\
\hline 99 & 100 \\
\hline 100 & 95 \\
\hline 101 & 100 \\
\hline 102 & 97 \\
\hline 103 & 29 \\
\hline 104 & 100 \\
\hline 105 & 85 \\
\hline 106 & 100 \\
\hline 107 & 100 \\
\hline 108 & 100 \\
\hline 109 & 100 \\
\hline 110 & 97 \\
\hline 111 & 99 \\
\hline 112 & 100 \\
\hline 113 & 46 \\
\hline 114 & 100 \\
\hline 115 & 53 \\
\hline 116 & 99 \\
\hline 117 & 97 \\
\hline 118 & 31 \\
\hline 119 & 99 \\
\hline 120 & 100 \\
\hline 121 & 100 \\
\hline 122 & 18 \\
\hline 123 & 97 \\
\hline 124 & 99 \\
\hline 125 & 100 \\
\hline 126 & 100 \\
\hline 127 & 99 \\
\hline 128 & 100 \\
\hline 129 & 98 \\
\hline 130 & 83 \\
\hline 131 & 99 \\
\hline 132 & 100 \\
\hline 133 & 100 \\
\hline 134 & 98 \\
\hline 135 & 97 \\
\hline 136 & 100 \\
\hline 137 & 81 \\
\hline 138 & 8 \\
\hline 139 & 92 \\
\hline 140 & 69 \\
\hline 141 & 98 \\
\hline 142 & 100 \\
\hline 143 & 94 \\
\hline 144 & 95 \\
\hline 145 & 93 \\
\hline 146 & 99 \\
\hline 147 & 83 \\
\hline 148 & 99 \\
\hline 149 & 98 \\
\hline 150 & 100 \\
\hline 151 & 66 \\
\hline 152 & 97 \\
\hline 153 & 99 \\
\hline 154 & 44 \\
\hline 155 & 0 \\
\hline 156 & 100 \\
\hline 157 & 16 \\
\hline 158 & 94 \\
\hline 159 & 100 \\
\hline 160 & 99 \\
\hline 161 & 100 \\
\hline 162 & 100 \\
\hline 163 & 69 \\
\hline 164 & 54 \\
\hline 165 & 100 \\
\hline 166 & 47 \\
\hline 167 & 98 \\
\hline 168 & 100 \\
\hline 169 & 100 \\
\hline 170 & 100 \\
\hline 171 & 98 \\
\hline 172 & 100 \\
\hline 173 & 91 \\
\hline 174 & 86 \\
\hline 175 & 0 \\
\hline 176 & 85 \\
\hline 177 & 85 \\
\hline 178 & 99 \\
\hline 179 & 100 \\
\hline 180 & 92 \\
\hline 181 & 98 \\
\hline 182 & 100 \\
\hline 183 & 83 \\
\hline 184 & 61 \\
\hline 185 & 81 \\
\hline 186 & 99 \\
\hline 187 & 94 \\
\hline 188 & 92 \\
\hline 189 & 99 \\
\hline 190 & 100 \\
\hline 191 & 100 \\
\hline 192 & 92 \\
\hline
\end{tabular}




\begin{tabular}{|c|c|}
\hline 193 & 100 \\
\hline 194 & 83 \\
\hline 195 & 88 \\
\hline 196 & 100 \\
\hline 197 & 100 \\
\hline 198 & 16 \\
\hline 199 & 83 \\
\hline 200 & 100 \\
\hline 201 & 99 \\
\hline 202 & 97 \\
\hline 203 & 98 \\
\hline 204 & 97 \\
\hline 205 & 100 \\
\hline 206 & 100 \\
\hline 207 & 100 \\
\hline 208 & 31 \\
\hline 209 & 100 \\
\hline 210 & 54 \\
\hline 211 & 100 \\
\hline 212 & 81 \\
\hline 213 & 100 \\
\hline 214 & 94 \\
\hline 215 & 88 \\
\hline 216 & 100 \\
\hline 217 & 99 \\
\hline 218 & 36 \\
\hline 219 & 98 \\
\hline 220 & 100 \\
\hline 221 & 92 \\
\hline 222 & 100 \\
\hline 223 & 100 \\
\hline 224 & 97 \\
\hline 225 & 81 \\
\hline 226 & 97 \\
\hline 227 & 99 \\
\hline 228 & 61 \\
\hline 229 & 100 \\
\hline 230 & 53 \\
\hline 231 & 95 \\
\hline 232 & 98 \\
\hline 233 & 75 \\
\hline 234 & 100 \\
\hline 235 & 100 \\
\hline 236 & 100 \\
\hline 237 & 3 \\
\hline 238 & 41 \\
\hline 239 & 93 \\
\hline 240 & 99 \\
\hline 241 & 61 \\
\hline 242 & 69 \\
\hline 243 & 69 \\
\hline 244 & 96 \\
\hline 245 & 100 \\
\hline 246 & 41 \\
\hline 247 & 81 \\
\hline 248 & 85 \\
\hline 249 & 100 \\
\hline 250 & 99 \\
\hline 251 & 100 \\
\hline 252 & 97 \\
\hline 253 & 95 \\
\hline 254 & 100 \\
\hline 255 & 97 \\
\hline 256 & 3 \\
\hline 257 & 99 \\
\hline 258 & 98 \\
\hline 259 & 86 \\
\hline 260 & 100 \\
\hline 261 & 36 \\
\hline 262 & 91 \\
\hline 263 & 100 \\
\hline 264 & 20 \\
\hline 265 & 98 \\
\hline 266 & 100 \\
\hline 267 & 66 \\
\hline 268 & 86 \\
\hline 269 & 85 \\
\hline 270 & 100 \\
\hline 271 & 36 \\
\hline 272 & 75 \\
\hline 273 & 66 \\
\hline 274 & 98 \\
\hline 275 & 53 \\
\hline 276 & 100 \\
\hline 277 & 100 \\
\hline 278 & 100 \\
\hline 279 & 75 \\
\hline 280 & 47 \\
\hline 281 & 97 \\
\hline 282 & 73 \\
\hline 283 & 98 \\
\hline 284 & 97 \\
\hline 285 & 88 \\
\hline 286 & 98 \\
\hline 287 & 100 \\
\hline 288 & 20 \\
\hline 289 & 99 \\
\hline
\end{tabular}




\begin{tabular}{|c|c|}
\hline 290 & 100 \\
\hline 291 & 8 \\
\hline 292 & 100 \\
\hline 293 & 97 \\
\hline 294 & 100 \\
\hline 295 & 83 \\
\hline 296 & 100 \\
\hline 297 & 86 \\
\hline 298 & 95 \\
\hline 299 & 100 \\
\hline 300 & 99 \\
\hline 301 & 16 \\
\hline 302 & 91 \\
\hline 303 & 99 \\
\hline 304 & 100 \\
\hline 305 & 8 \\
\hline 306 & 100 \\
\hline 307 & 100 \\
\hline 308 & 16 \\
\hline 309 & 100 \\
\hline 310 & 91 \\
\hline 311 & 100 \\
\hline 312 & 85 \\
\hline 313 & 18 \\
\hline 314 & 99 \\
\hline 315 & 100 \\
\hline 316 & 47 \\
\hline 317 & 99 \\
\hline 318 & 100 \\
\hline 319 & 97 \\
\hline 320 & 36 \\
\hline 321 & 98 \\
\hline 322 & 96 \\
\hline 323 & 20 \\
\hline 324 & 97 \\
\hline 325 & 96 \\
\hline 326 & 100 \\
\hline 327 & 20 \\
\hline 328 & 3 \\
\hline 329 & 85 \\
\hline 330 & 99 \\
\hline 331 & 54 \\
\hline 332 & 31 \\
\hline 333 & 47 \\
\hline 334 & 85 \\
\hline 335 & 18 \\
\hline 336 & 100 \\
\hline 337 & 97 \\
\hline 338 & 29 \\
\hline 339 & 36 \\
\hline 340 & 100 \\
\hline 341 & 92 \\
\hline 342 & 100 \\
\hline 343 & 100 \\
\hline 344 & 75 \\
\hline 345 & 96 \\
\hline 346 & 99 \\
\hline 347 & 97 \\
\hline 348 & 99 \\
\hline 349 & 46 \\
\hline 350 & 100 \\
\hline 351 & 88 \\
\hline 352 & 73 \\
\hline 353 & 100 \\
\hline 354 & 100 \\
\hline 355 & 83 \\
\hline 356 & 20 \\
\hline 357 & 100 \\
\hline 358 & 83 \\
\hline 359 & 93 \\
\hline 360 & 66 \\
\hline 361 & 69 \\
\hline 362 & 96 \\
\hline 363 & 96 \\
\hline 364 & 100 \\
\hline 365 & 100 \\
\hline 366 & 83 \\
\hline 367 & 100 \\
\hline 368 & 100 \\
\hline 369 & 18 \\
\hline 370 & 94 \\
\hline 371 & 88 \\
\hline 372 & 99 \\
\hline 373 & 100 \\
\hline 374 & 16 \\
\hline 375 & 99 \\
\hline 376 & 100 \\
\hline 377 & 97 \\
\hline 378 & 100 \\
\hline 379 & 78 \\
\hline 380 & 100 \\
\hline 381 & 99 \\
\hline 382 & 96 \\
\hline 383 & 100 \\
\hline 384 & 54 \\
\hline 385 & 61 \\
\hline 386 & 100 \\
\hline
\end{tabular}




\begin{tabular}{|c|c|c|}
\hline 387 & 0 & 0.578785536 \\
\hline 388 & 96 & 0.318639234 \\
\hline $\begin{array}{l}389 \\
390\end{array}$ & 73 & 0.763658838 \\
\hline 391 & 44 & 0.097504162 \\
\hline 392 & 100 & 0.844823345 \\
\hline 393 & 100 & 0.316571717 \\
\hline 394 & 99 & 0.900245688 \\
\hline 395 & 95 & 0.870740951 \\
\hline 396 & 100 & 0.132154985 \\
\hline 397 & 98 & 0.025024279 \\
\hline 398 & 98 & 0.120526821 \\
\hline 399 & 3 & 0.824869379 \\
\hline 400 & 18 & 0.400510271 \\
\hline 401 & 97 & 0.285008493 \\
\hline 402 & 100 & 0.040961676 \\
\hline 403 & 99 & 0.236147953 \\
\hline 404 & 96 & 0.73684051 \\
\hline 405 & 99 & 0.962002573 \\
\hline 406 & 86 & 0.530644302 \\
\hline 407 & 100 & 0.899401791 \\
\hline 408 & 100 & 0.122639312 \\
\hline 409 & 85 & 0.327788665 \\
\hline 410 & 29 & 0.903975373 \\
\hline 411 & 8 & 0.237447913 \\
\hline 412 & 78 & 0.361057058 \\
\hline 413 & 100 & 0.915841353 \\
\hline 414 & 100 & 0.365178184 \\
\hline 415 & 99 & 0.031069897 \\
\hline 416 & 99 & 0.925943811 \\
\hline 417 & 100 & 0.137764064 \\
\hline 418 & 73 & 0.674725005 \\
\hline 419 & 100 & 0.790605545 \\
\hline 420 & 100 & 0.697022048 \\
\hline 421 & 100 & 0.032127225 \\
\hline 422 & 100 & 0.889066026 \\
\hline 423 & 41 & 0.42191803 \\
\hline 424 & 44 & 0.569825383 \\
\hline 425 & 46 & 0.527550283 \\
\hline 426 & 73 & 0.918727103 \\
\hline 427 & 100 & 0.257706647 \\
\hline 428 & 92 & 0.714379289 \\
\hline 429 & 100 & 0.51114166 \\
\hline 430 & 99 & 0.011401473 \\
\hline 431 & 96 & 0.012734619 \\
\hline 432 & 100 & 0.018816348 \\
\hline 433 & 100 & 0.285169838 \\
\hline 434 & 100 & 0.775145198 \\
\hline 435 & 83 & 0.98588353 \\
\hline 436 & 100 & 0.320092592 \\
\hline 437 & 99 & 0.127019462 \\
\hline 438 & 100 & 0.595323627 \\
\hline 439 & 100 & 0.275197434 \\
\hline 440 & 54 & 0.367501512 \\
\hline 441 & 100 & 0.555752108 \\
\hline 442 & 8 & 0.527889579 \\
\hline 443 & 47 & 0.014279088 \\
\hline 444 & 100 & 0.392805265 \\
\hline 445 & 78 & 0.921583271 \\
\hline 446 & 100 & 0.130376447 \\
\hline 447 & 97 & 0.278243172 \\
\hline 448 & 100 & 0.051763149 \\
\hline 449 & 95 & 0.78695047 \\
\hline 450 & 100 & 0.466253747 \\
\hline 451 & 44 & 0.062742392 \\
\hline 452 & 100 & 0.248357707 \\
\hline 453 & 41 & 0.92893664 \\
\hline 454 & 16 & 0.311276562 \\
\hline 455 & 75 & 0.65397925 \\
\hline 456 & 100 & 0.251472637 \\
\hline 457 & 100 & 0.861911701 \\
\hline 458 & 100 & 0.791263784 \\
\hline 459 & 100 & 0.266116334 \\
\hline 460 & 100 & 0.331572164 \\
\hline 461 & 99 & 0.232738744 \\
\hline 462 & 99 & 0.274341346 \\
\hline 463 & 93 & 0.474888419 \\
\hline 464 & 98 & 0.26570772 \\
\hline 465 & 69 & 0.646863813 \\
\hline 466 & 44 & 0.823992357 \\
\hline 467 & 92 & 0.889001812 \\
\hline 468 & 94 & 0.517480245 \\
\hline 469 & 99 & 0.263049574 \\
\hline 470 & 61 & 0.422420068 \\
\hline 471 & 100 & 0.865402227 \\
\hline 472 & 8 & 0.385323281 \\
\hline 473 & 29 & 0.629282453 \\
\hline 474 & 95 & 0.672436887 \\
\hline 475 & 46 & 0.735674952 \\
\hline 476 & 53 & 0.393286025 \\
\hline 477 & 73 & 0.004058528 \\
\hline 478 & 18 & 0.659379906 \\
\hline 479 & 98 & 0.247954752 \\
\hline 480 & 100 & 0.778179593 \\
\hline 481 & 99 & 0.694282227 \\
\hline 482 & 98 & 0.170459923 \\
\hline 483 & 81 & 0.48459425 \\
\hline
\end{tabular}




$\begin{array}{lcc}484 & 100 & 0.807078477 \\ 485 & 100 & 0.326313388 \\ 486 & 46 & 0.421837603 \\ 487 & 100 & 0.845377735 \\ 488 & 66 & 0.12177759 \\ 489 & 100 & 0.416883049 \\ 490 & 92 & 0.976289536 \\ 491 & 83 & 0.313943917 \\ 492 & 100 & 0.705815192 \\ 493 & 93 & 0.264574564 \\ 494 & 100 & 0.796297235 \\ 495 & 100 & 0.202220797 \\ 496 & 53 & 0.116396905 \\ 497 & 100 & 0.819953974 \\ 498 & 95 & 0.263701849 \\ 499 & 98 & 0.154571486 \\ 500 & 99 & 0.1720101 \\ 501 & 99 & 0.014546293 \\ 502 & 0 & 0.985615049 \\ 503 & 78 & 0.532120814 \\ 504 & 53 & 0.38253868 \\ 505 & 100 & 0.149660897 \\ 506 & 96 & 0.799575904 \\ 507 & 100 & 0.476435895 \\ 508 & 100 & 0.544022066 \\ 509 & 78 & 0.885010395 \\ 510 & 3 & 0.812226419\end{array}$

0.09986456

0.17340583

0.13569852

0.18376207

0.19248105

0.19411646

0.14707846

0.17705868

0.12262287

$\begin{array}{ll}\text { mean } & 0.150675376 \\ \text { st dev } & 0.033848803\end{array}$ 


\begin{tabular}{|c|c|}
\hline & $10 \mu \mathrm{M}$ ATP \\
\hline Kinase & PD184352@ @ $10 \mu \mathrm{M}$ \\
\hline $\mathrm{Ab}(\mathrm{m})$ & 99 \\
\hline & 99 \\
\hline & 99 \\
\hline & 99 \\
\hline & 99 \\
\hline & 99 \\
\hline $\mathrm{AMPK}(\mathrm{r})$ & 100 \\
\hline & 100 \\
\hline & 100 \\
\hline & 100 \\
\hline & 100 \\
\hline & 100 \\
\hline $\operatorname{Arg}(\mathrm{m})$ & 105 \\
\hline & 105 \\
\hline & 105 \\
\hline & 105 \\
\hline & 105 \\
\hline & 105 \\
\hline Aurora-A(h) & 106 \\
\hline & 106 \\
\hline & 106 \\
\hline & 106 \\
\hline & 106 \\
\hline & 106 \\
\hline Axl(h) & 119 \\
\hline & 119 \\
\hline & 119 \\
\hline & 119 \\
\hline & 119 \\
\hline & 119 \\
\hline $\mathrm{Blk}(\mathrm{m})$ & 105 \\
\hline & 105 \\
\hline & 105 \\
\hline & 105 \\
\hline & 105 \\
\hline & 105 \\
\hline $\operatorname{Bmx}(\mathrm{h})$ & 104 \\
\hline & 104 \\
\hline & 104 \\
\hline & 104 \\
\hline & 104 \\
\hline & 104 \\
\hline CaMKII(r) & 95 \\
\hline & 95 \\
\hline & 95 \\
\hline & 95 \\
\hline & 95 \\
\hline & 95 \\
\hline CaMKIV(h) & 106 \\
\hline & 106 \\
\hline & 106 \\
\hline & 106 \\
\hline & 106 \\
\hline & 106 \\
\hline CDK1/cyclinB(h) & 101 \\
\hline & 101 \\
\hline & 101 \\
\hline & 101 \\
\hline & 101 \\
\hline & 101 \\
\hline $\mathrm{CDK} 2 / \mathrm{cyc} \operatorname{lin} \mathrm{A}(\mathrm{h})$ & 102 \\
\hline & 102 \\
\hline & 102 \\
\hline & 102 \\
\hline & 102 \\
\hline & 102 \\
\hline $\mathrm{CDK} 2 / \mathrm{cyclin} \mathrm{E}(\mathrm{h})$ & 93 \\
\hline & 93 \\
\hline & 93 \\
\hline & 93 \\
\hline & 93 \\
\hline & 93 \\
\hline CDK3/cyclinE(h) & 101 \\
\hline & 101 \\
\hline & 101 \\
\hline & 101 \\
\hline & 101 \\
\hline & 101 \\
\hline CDK5/p35(h) & 106 \\
\hline & 106 \\
\hline & 106 \\
\hline & 106 \\
\hline & 106 \\
\hline & 106 \\
\hline CDK6/cyclinD3(h) & 95 \\
\hline & 95 \\
\hline & 95 \\
\hline & 95 \\
\hline & 95 \\
\hline & 95 \\
\hline CDK7//yclinH/MAT1(h) & 100 \\
\hline & 100 \\
\hline & 100 \\
\hline & 100 \\
\hline & 100 \\
\hline
\end{tabular}

position

$\begin{array}{ccccc}\text { cumulative sample fraction } & \text { inhibition\% } & \text { fraction of total inhibition } & \text { cumulative inhibition } & \text { area } \\ 0.001960784 & 0 & 0 & 0 & \end{array}$ 0.003921569

0.007843137

0.009803922

0.011764706

0.01372549

0.015686275

0.017647059

0.019607843

0.023529412

0.02745098

0.029411765

0.033333333

0.035294118

0.037254902

0.039215686

0.041176471

0.043137255

0.045098039

0.047058824

0.049019608

0.050980392

0.052941176

0.054901961

0.056862745

0.058823529

0.060784314

0.062745098

0.064705882

0.066666667

0.068627451

0.070588235

0.07254902

0.074509804

0.076470588

0.078431373

0.080392157

0.082352941

0.084313725

0.08627451

0.088235294

0.090196078

0.092156863
0.094117647

0.096078431

0.098039216

0.1

0.101960784

0.105882353

0.107843137

0.109803922

0.111764706

0.11372549

0.115686275

0.117647059

0.119607843

0.121568627
0.123529412

0.125490196

0.12745098

0.129411765

0.131372549

0.133333333

0.135294118

0.137254902

0.139215686

0.141176471

0.143137255

0.145098039

0.147058824

0.149019608

0.150980392

0.152941176
0.154901961

0.154901961

0.156862745

0.158823529
0.160784314

0.162745098

0.164705882

0.166666667

0.168627451

0.170588235

0.17254902

0.174509804

0.176470588

0.178431373

0.180392157

0.182352941

0.184313725

0.18627451

$\begin{array}{ll}0 & 0 \\ 0 & 0\end{array}$

0

0
0
0
0

0

0
0
0

0
0




\begin{tabular}{|c|c|}
\hline & 100 \\
\hline CHK1(h) & 115 \\
\hline & 115 \\
\hline & 115 \\
\hline & 115 \\
\hline & 115 \\
\hline & 115 \\
\hline CHK2(h) & 98 \\
\hline & 98 \\
\hline & 98 \\
\hline & 98 \\
\hline & 98 \\
\hline & 98 \\
\hline CK1(y) & 97 \\
\hline & 97 \\
\hline & 97 \\
\hline & 97 \\
\hline & 97 \\
\hline & 97 \\
\hline CK2(h) & 109 \\
\hline & 109 \\
\hline & 109 \\
\hline & 109 \\
\hline & 109 \\
\hline & 109 \\
\hline c-RAF(h) & 98 \\
\hline & 98 \\
\hline & 98 \\
\hline & 98 \\
\hline & 98 \\
\hline & 98 \\
\hline $\operatorname{CSK}(\mathrm{h})$ & 76 \\
\hline & 76 \\
\hline & 76 \\
\hline & 76 \\
\hline & 76 \\
\hline & 76 \\
\hline $\operatorname{csRC}(\mathrm{h})$ & 103 \\
\hline & 103 \\
\hline & 103 \\
\hline & 103 \\
\hline & 103 \\
\hline & 103 \\
\hline Fes(h) & 108 \\
\hline & 108 \\
\hline & 108 \\
\hline & 108 \\
\hline & 108 \\
\hline & 108 \\
\hline FGFR3(h) & 109 \\
\hline & 109 \\
\hline & 109 \\
\hline & 109 \\
\hline & 109 \\
\hline & 109 \\
\hline Fll3(h) & 105 \\
\hline & 105 \\
\hline & 105 \\
\hline & 105 \\
\hline & 105 \\
\hline & 105 \\
\hline Fynn(h) & 94 \\
\hline & 94 \\
\hline & 94 \\
\hline & 94 \\
\hline & 94 \\
\hline & 94 \\
\hline GSK3a(h) & 91 \\
\hline & 91 \\
\hline & 91 \\
\hline & 91 \\
\hline & 91 \\
\hline & 91 \\
\hline GSK3B(h) & 75 \\
\hline & 75 \\
\hline & 75 \\
\hline & 75 \\
\hline & 75 \\
\hline & 75 \\
\hline IGF-IR(h) & 124 \\
\hline & 124 \\
\hline & 124 \\
\hline & 124 \\
\hline & 124 \\
\hline & 124 \\
\hline IKKa(h) & 99 \\
\hline & 99 \\
\hline & 99 \\
\hline & 99 \\
\hline & 99 \\
\hline & 99 \\
\hline IKKB(h) & 104 \\
\hline & 104 \\
\hline & 104 \\
\hline & 104 \\
\hline & 104 \\
\hline & 104 \\
\hline
\end{tabular}




\begin{tabular}{|c|c|}
\hline $\mathrm{IR}(\mathrm{h})$ & 93 \\
\hline & 93 \\
\hline & 93 \\
\hline & 93 \\
\hline & 93 \\
\hline & 93 \\
\hline JNK Ial(h) & 101 \\
\hline & 101 \\
\hline & 101 \\
\hline & 101 \\
\hline & 101 \\
\hline & 101 \\
\hline JNK2a2(h) & 92 \\
\hline & 92 \\
\hline & 92 \\
\hline & 92 \\
\hline & 92 \\
\hline & 92 \\
\hline JNK3(r) & 87 \\
\hline & 87 \\
\hline & 87 \\
\hline & 87 \\
\hline & 87 \\
\hline & 87 \\
\hline Lck(h) & 119 \\
\hline & 119 \\
\hline & 119 \\
\hline & 119 \\
\hline & 119 \\
\hline & 119 \\
\hline Lynn(h) & 96 \\
\hline & 96 \\
\hline & 96 \\
\hline & 96 \\
\hline & 96 \\
\hline & 96 \\
\hline Lynn(m) & 91 \\
\hline & 91 \\
\hline & 91 \\
\hline & 91 \\
\hline & 91 \\
\hline & 91 \\
\hline MAPK1(h) & 98 \\
\hline & 98 \\
\hline & 98 \\
\hline & 98 \\
\hline & 98 \\
\hline & 98 \\
\hline MAPK2(h) & 120 \\
\hline & 120 \\
\hline & 120 \\
\hline & 120 \\
\hline & 120 \\
\hline & 120 \\
\hline $\operatorname{MAPK2(m)~}$ & 123 \\
\hline & 123 \\
\hline & 123 \\
\hline & 123 \\
\hline & 123 \\
\hline & 123 \\
\hline MAPKAP-K2(h) & 110 \\
\hline & 110 \\
\hline & 110 \\
\hline & 110 \\
\hline & 110 \\
\hline & 110 \\
\hline MEKI(h) & 25 \\
\hline & 25 \\
\hline & 25 \\
\hline & 25 \\
\hline & 25 \\
\hline & 25 \\
\hline MKK4(m) & 116 \\
\hline & 116 \\
\hline & 116 \\
\hline & 116 \\
\hline & 116 \\
\hline & 116 \\
\hline MKK6(h) & 104 \\
\hline & 104 \\
\hline & 104 \\
\hline & 104 \\
\hline & 104 \\
\hline & 104 \\
\hline MKK7B(h) & 108 \\
\hline & 108 \\
\hline & 108 \\
\hline & 108 \\
\hline & 108 \\
\hline & 108 \\
\hline MSK1(h) & 104 \\
\hline & 104 \\
\hline & 104 \\
\hline & 104 \\
\hline & 104 \\
\hline & 104 \\
\hline p70s6K(h) & 101 \\
\hline
\end{tabular}




\begin{tabular}{|c|c|}
\hline & 101 \\
\hline & 101 \\
\hline & 101 \\
\hline & 101 \\
\hline & 101 \\
\hline PAK2(h) & 107 \\
\hline & 107 \\
\hline & 107 \\
\hline & 107 \\
\hline & 107 \\
\hline & 107 \\
\hline PDGFRa(h) & 96 \\
\hline & 96 \\
\hline & 96 \\
\hline & 96 \\
\hline & 96 \\
\hline & 96 \\
\hline PDGFRB(h) & 107 \\
\hline & 107 \\
\hline & 107 \\
\hline & 107 \\
\hline & 107 \\
\hline & 107 \\
\hline PDK1(h) & 97 \\
\hline & 97 \\
\hline & 97 \\
\hline & 97 \\
\hline & 97 \\
\hline & 97 \\
\hline PKA(b) & 117 \\
\hline & 117 \\
\hline & 117 \\
\hline & 117 \\
\hline & 117 \\
\hline & 117 \\
\hline PKA(h) & 92 \\
\hline & 92 \\
\hline & 92 \\
\hline & 92 \\
\hline & 92 \\
\hline & 92 \\
\hline PKB $\alpha(h)$ & 97 \\
\hline & 97 \\
\hline & 97 \\
\hline & 97 \\
\hline & 97 \\
\hline & 97 \\
\hline PKBB(h) & 132 \\
\hline & 132 \\
\hline & 132 \\
\hline & 132 \\
\hline & 132 \\
\hline & 132 \\
\hline $\mathrm{PKB}_{f}(\mathrm{~h})$ & 87 \\
\hline & 87 \\
\hline & 87 \\
\hline & 87 \\
\hline & 87 \\
\hline & 87 \\
\hline PKCa(h) & 94 \\
\hline & 94 \\
\hline & 94 \\
\hline & 94 \\
\hline & 94 \\
\hline & 94 \\
\hline РКСBI(h) & 110 \\
\hline & 110 \\
\hline & 110 \\
\hline & 110 \\
\hline & 110 \\
\hline & 110 \\
\hline $\mathrm{PKC} \gamma(\mathrm{h})$ & 91 \\
\hline & 91 \\
\hline & 91 \\
\hline & 91 \\
\hline & 91 \\
\hline & 91 \\
\hline PKC $\delta(\mathrm{h})$ & 96 \\
\hline & 96 \\
\hline & 96 \\
\hline & 96 \\
\hline & 96 \\
\hline & 96 \\
\hline $\operatorname{PKC} \varepsilon(\mathrm{h})$ & 100 \\
\hline & 100 \\
\hline & 100 \\
\hline & 100 \\
\hline & 100 \\
\hline & 100 \\
\hline $\mathrm{P} \mathrm{KC \eta \eta (h)}$ & 91 \\
\hline & 91 \\
\hline & 91 \\
\hline & 91 \\
\hline & 91 \\
\hline & 91 \\
\hline РКС (1) & 101 \\
\hline & 101 \\
\hline
\end{tabular}




\begin{tabular}{|c|c|}
\hline & 101 \\
\hline & 101 \\
\hline & 101 \\
\hline & 101 \\
\hline PKCu(h) & 96 \\
\hline & 96 \\
\hline & 96 \\
\hline & 96 \\
\hline & 96 \\
\hline & 96 \\
\hline P KC $\theta(\mathrm{h})$ & 97 \\
\hline & 97 \\
\hline & 97 \\
\hline & 97 \\
\hline & 97 \\
\hline & 97 \\
\hline PKD2(h) & 102 \\
\hline & 102 \\
\hline & 102 \\
\hline & 102 \\
\hline & 102 \\
\hline & 102 \\
\hline PRAK(h) & 112 \\
\hline & 112 \\
\hline & 112 \\
\hline & 112 \\
\hline & 112 \\
\hline & 112 \\
\hline PRK2(h) & 92 \\
\hline & 92 \\
\hline & 92 \\
\hline & 92 \\
\hline & 92 \\
\hline & 92 \\
\hline ROCK-II(h) & 98 \\
\hline & 98 \\
\hline & 98 \\
\hline & 98 \\
\hline & 98 \\
\hline & 98 \\
\hline ROCK-II(r) & 83 \\
\hline & 83 \\
\hline & 83 \\
\hline & 83 \\
\hline & 83 \\
\hline & 83 \\
\hline Rskl(h) & 111 \\
\hline & 111 \\
\hline & 111 \\
\hline & 111 \\
\hline & 111 \\
\hline & 111 \\
\hline Rskl(r) & 67 \\
\hline & 67 \\
\hline & 67 \\
\hline & 67 \\
\hline & 67 \\
\hline & 67 \\
\hline Rsk2(h) & 93 \\
\hline & 93 \\
\hline & 93 \\
\hline & 93 \\
\hline & 93 \\
\hline & 93 \\
\hline Rsk3(h) & 99 \\
\hline & 99 \\
\hline & 99 \\
\hline & 99 \\
\hline & 99 \\
\hline & 99 \\
\hline SAPK2a(h) & 105 \\
\hline & 105 \\
\hline & 105 \\
\hline & 105 \\
\hline & 105 \\
\hline & 105 \\
\hline SAPK2b(h) & 100 \\
\hline & 100 \\
\hline & 100 \\
\hline & 100 \\
\hline & 100 \\
\hline & 100 \\
\hline SAPK3(h) & 112 \\
\hline & 112 \\
\hline & 112 \\
\hline & 112 \\
\hline & 112 \\
\hline & 112 \\
\hline SAPK4(h) & 112 \\
\hline & 112 \\
\hline & 112 \\
\hline & 112 \\
\hline & 112 \\
\hline & 112 \\
\hline SGK(h) & 104 \\
\hline & 104 \\
\hline & 104 \\
\hline
\end{tabular}




\begin{tabular}{|c|c|c|c|c|c|c|c|c|c|}
\hline & 104 & 83 & 83 & 484 & 0.949019608 & 17 & 0.0082846 & 0.524366472 & 0.001020047 \\
\hline & 104 & 83 & 83 & 485 & 0.950980392 & 17 & 0.0082846 & 0.532651072 & 0.001036292 \\
\hline & 104 & 83 & 83 & 486 & 0.952941176 & 17 & 0.0082846 & 0.540935673 & 0.001052536 \\
\hline \multirow[t]{6}{*}{ Syk(h) } & 105 & 76 & 76 & 487 & 0.954901961 & 24 & 0.011695906 & 0.552631579 & 0.001072125 \\
\hline & 105 & 76 & 76 & 488 & 0.956862745 & 24 & 0.011695906 & 0.564327485 & 0.001095058 \\
\hline & 105 & 76 & 76 & 489 & 0.958823529 & 24 & 0.011695906 & 0.576023392 & 0.001117991 \\
\hline & 105 & 76 & 76 & 490 & 0.960784314 & 24 & 0.011695906 & 0.587719298 & 0.001140924 \\
\hline & 105 & 76 & 76 & 491 & 0.962745098 & 24 & 0.011695906 & 0.599415205 & 0.001163857 \\
\hline & 105 & 76 & 76 & 492 & 0.964705882 & 24 & 0.011695906 & 0.6111111111 & 0.001186791 \\
\hline \multirow[t]{6}{*}{ TrkB(h) } & 111 & 75 & 75 & 493 & 0.966666667 & 25 & 0.012183236 & 0.623294347 & 0.001210201 \\
\hline & 111 & 75 & 75 & 494 & 0.968627451 & 25 & 0.012183236 & 0.635477583 & 0.00123409 \\
\hline & 111 & 75 & 75 & 495 & 0.970588235 & 25 & 0.012183236 & 0.647660819 & 0.001257979 \\
\hline & 111 & 75 & 75 & 496 & 0.97254902 & 25 & 0.012183236 & 0.659844055 & 0.001281868 \\
\hline & 111 & 75 & 75 & 497 & 0.974509804 & 25 & 0.012183236 & 0.67202729 & 0.001305756 \\
\hline & 111 & 75 & 75 & 498 & 0.976470588 & 25 & 0.012183236 & 0.684210526 & 0.001329645 \\
\hline \multirow[t]{6}{*}{ Yes(h) } & 109 & 67 & 67 & 499 & 0.978431373 & 33 & 0.016081871 & 0.700292398 & 0.001357356 \\
\hline & 109 & 67 & 67 & 500 & 0.980392157 & 33 & 0.016081871 & 0.716374269 & 0.001388889 \\
\hline & 109 & 67 & 67 & 501 & 0.982352941 & 33 & 0.016081871 & 0.73245614 & 0.001420422 \\
\hline & 109 & 67 & 67 & 502 & 0.984313725 & 33 & 0.016081871 & 0.748538012 & 0.001451955 \\
\hline & 109 & 67 & 67 & 503 & 0.98627451 & 33 & 0.016081871 & 0.764619883 & 0.001483488 \\
\hline & 109 & 67 & 67 & 504 & 0.988235294 & 33 & 0.016081871 & 0.780701754 & 0.001515021 \\
\hline \multirow[t]{7}{*}{ ZAP-70(h) } & 106 & 25 & 25 & 505 & 0.990196078 & 75 & 0.036549708 & 0.817251462 & 0.001566621 \\
\hline & 106 & 25 & 25 & 506 & 0.992156863 & 75 & 0.036549708 & 0.85380117 & 0.001638287 \\
\hline & 106 & 25 & 25 & 507 & 0.994117647 & 75 & 0.036549708 & 0.890350877 & 0.001709953 \\
\hline & 106 & 25 & 25 & 508 & 0.996078431 & 75 & 0.036549708 & 0.926900585 & 0.001781619 \\
\hline & 106 & 25 & 25 & 509 & 0.998039216 & 75 & 0.036549708 & 0.963450292 & 0.001853285 \\
\hline & 106 & 25 & 25 & 510 & 1 & 75 & 0.036549708 & 1 & 0.001924951 \\
\hline & & & & & Total inhibition & 2052 & & Total area & 0.098899209 \\
\hline
\end{tabular}


PD184352; $10 \mu \mathrm{M} ; 10 \mu \mathrm{M}$ ATP; sample of 400 kinases

position inhibition\% rand cumulative sample fraction sample inhibition

$$
0.708136056
$$
0.708136056 0.0025 $0.458797656 \quad 0.0075$ $\begin{array}{ll}0.776963294 & 0.01\end{array}$

0.0125

$0.453909378 \quad 0.015$

$\begin{array}{ll}0.745596311 & 0.0175\end{array}$

$\begin{array}{lc}0.74596311 & 0.02 \\ 0.910040109 & 0.0225\end{array}$

$0.198246533 \quad 0.025$

$0.819649262 \quad 0.0275$

$0.119238589 \quad 0.03$

$0.080175801 \quad 0.0325$

$\begin{array}{ll}0.942718512 & 0.035 \\ 0.065259053 & 0.0375\end{array}$

$0.936269706 \quad 0.04$

$0.408771186 \quad 0.0425$

$\begin{array}{ll}0.553112056 & 0.045 \\ 0.734710091 & 0.0475\end{array}$

$\begin{array}{lc}0.734710091 & 0.0475 \\ 0.085184568 & 0.05\end{array}$

$0.164635929 \quad 0.0525$

$0.101635344 \quad 0.055$

$0.362005917-0.0575$

$\begin{array}{ll}0.534530015 & 0.06\end{array}$

$\begin{array}{ll}0.671387065 & 0.0625 \\ 0.998953417 & 0.065\end{array}$

$\begin{array}{ll}0.998953417 & 0.065 \\ 0.356260869 & 0.0675\end{array}$

$0.944222711 \quad 0.07$

$\begin{array}{ll}0.46982353 & 0.0725 \\ 0.228259291 & 0.075\end{array}$

$0.039112751 \quad 0.0775$

$\begin{array}{ll}0.804366771 & 0.08\end{array}$

$0.507184034 \quad 0.0825$

$0.211136975 \quad 0.085$

$0.05479716 \quad 0.0875$

$\begin{array}{ll}0.439021817 & 0.09\end{array}$

$\begin{array}{ll}0.116253237 & 0.0925 \\ 0.707646755 & 0.095\end{array}$

0.0975

$0.383935708 \quad 0.1$

$\begin{array}{ll}0.325768044 & 0.1025 \\ 0.37420956 & 0.105\end{array}$

0.374420956

$0.723254033-0.11$

$\begin{array}{ll}0.723254033 & 0.11 \\ 0.905495236 & 0.1125\end{array}$

$0.324920455 \quad 0.115$

$\begin{array}{ll}0.004772546 & 0.1175 \\ 0.994548459 & 0.12\end{array}$

$\begin{array}{lc}0.994548459 & 0.12 \\ 0.693319709 & 0.1225\end{array}$

$\begin{array}{ll}0.693319709 & 0.1225 \\ 0.676695844 & 0.125\end{array}$

$0.202304599 \quad 0.1275$

$0.948633222 \quad 0.13$

$0.595728793 \quad 0.1325$

$0.078969419 \quad 0.135$

$0.82523686 \quad 0.1375$

$0.55083277 \quad 0.14$

$\begin{array}{ll}0.522169846 & 0.1425 \\ 0.459097532 & 0.145\end{array}$

$\begin{array}{ll}0.459097532 & 0.145 \\ 0.05645536 & 0.1475\end{array}$

$\begin{array}{ll}0.878696479 & 0.15\end{array}$

$\begin{array}{ll}0.517997522 & 0.1525\end{array}$

$\begin{array}{ll}0.787430272 & 0.1525 \\ 0.853518999 & 0.155 \\ 0.073119128 & 0.1575\end{array}$

$0.073119128 \quad 0.16$

$0.74755445 \quad 0.1625$

$0.145060415 \quad 0.165$

$\begin{array}{lc}0.962453074 & 0.1675 \\ 0.072833559 & 0.17\end{array}$

$\begin{array}{ll}0.072833559 & 0.17 \\ 0.868571641 & 0.1725\end{array}$

$\begin{array}{ll}0.868571641 & 0.1725 \\ 0.092994734 & 0.175\end{array}$

$\begin{array}{ll}0.553972923 & 0.1775\end{array}$

$\begin{array}{ll}0.543337809 & 0.18\end{array}$

$\begin{array}{ll}0.5328960715 & 0.1825 \\ 0.603181357 & 0.185\end{array}$

$0.633238392 \quad 0.1875$

$0.572208563 \quad 0.19$

0.56431938

$\begin{array}{ll}0.029706241 & 0.195 \\ 0.2336964 & 0.1975\end{array}$

$\begin{array}{cc}0.2336964 & 0.1975 \\ 0.479576586 & 0.2\end{array}$

$0.913918938 \quad 0.2025$

$\begin{array}{ll}0.630695193 & 0.205 \\ 0.467189412 & 0.2075\end{array}$

$0.467189412 \quad 0.2075$

$\begin{array}{ll}0.567189412 & 0.21 \\ 0.45906282 & 0.2125\end{array}$

$0.583459134 \quad 0.215$

$0.904380868 \quad 0.2175$

$0.755554756 \quad 0.22$

$\begin{array}{ll}0.7508969074 & 0.2225 \\ 0.749744381 & 0.225\end{array}$

$\begin{array}{ll}0.749744381 & 0.225 \\ 0.318964396 & 0.2275\end{array}$

$0.119466763 \quad 0.23$

$\begin{array}{ll}0.793128825 & 0.2325 \\ 0.545318824 & 0.235\end{array}$

$\begin{array}{ll}0.54378824 & 0.235 \\ 0.54787903 & 0.2375\end{array}$

sorted fraction of inhibition cumulative fraction of inhibition

0

0

(n)




\begin{tabular}{|c|c|}
\hline 96 & 0 \\
\hline 97 & 0 \\
\hline 98 & 0 \\
\hline 99 & 6 \\
\hline 100 & 8 \\
\hline 101 & 13 \\
\hline 102 & 0 \\
\hline 103 & 1 \\
\hline 104 & 75 \\
\hline 105 & 0 \\
\hline 106 & 2 \\
\hline 107 & 0 \\
\hline 108 & 8 \\
\hline 109 & 0 \\
\hline 110 & 0 \\
\hline 111 & 0 \\
\hline 112 & 4 \\
\hline 113 & 0 \\
\hline 114 & 0 \\
\hline 115 & 0 \\
\hline 116 & 0 \\
\hline 117 & 0 \\
\hline 118 & 3 \\
\hline 119 & 0 \\
\hline 120 & 24 \\
\hline 121 & 3 \\
\hline 122 & 0 \\
\hline 123 & 24 \\
\hline 124 & 0 \\
\hline 125 & 9 \\
\hline 126 & 0 \\
\hline 127 & 0 \\
\hline 128 & 0 \\
\hline 129 & 0 \\
\hline 130 & 0 \\
\hline 131 & 9 \\
\hline 132 & 3 \\
\hline 133 & 0 \\
\hline 134 & 0 \\
\hline 135 & 3 \\
\hline 136 & 0 \\
\hline 137 & 0 \\
\hline 138 & 0 \\
\hline 139 & 75 \\
\hline 140 & 8 \\
\hline 141 & 0 \\
\hline 142 & 2 \\
\hline 143 & 5 \\
\hline 144 & 0 \\
\hline 145 & 2 \\
\hline 146 & 0 \\
\hline 147 & 1 \\
\hline 148 & 4 \\
\hline 149 & 75 \\
\hline 150 & 1 \\
\hline 151 & 7 \\
\hline 152 & 0 \\
\hline 153 & 0 \\
\hline 154 & 9 \\
\hline 155 & 24 \\
\hline 156 & 17 \\
\hline 157 & 3 \\
\hline 158 & 0 \\
\hline 159 & 7 \\
\hline 160 & 9 \\
\hline 161 & 3 \\
\hline 162 & 1 \\
\hline 163 & 9 \\
\hline 164 & 13 \\
\hline 165 & 8 \\
\hline 166 & 0 \\
\hline 167 & 17 \\
\hline 168 & 8 \\
\hline 169 & 4 \\
\hline 170 & 6 \\
\hline 171 & 13 \\
\hline 172 & 5 \\
\hline 173 & 5 \\
\hline 174 & 7 \\
\hline 175 & 0 \\
\hline 176 & 0 \\
\hline 177 & 3 \\
\hline 178 & 17 \\
\hline 179 & 0 \\
\hline 180 & 8 \\
\hline 181 & 0 \\
\hline 182 & 4 \\
\hline 183 & 3 \\
\hline 184 & 13 \\
\hline 185 & 3 \\
\hline 186 & 6 \\
\hline 187 & 75 \\
\hline 188 & 0 \\
\hline 189 & 0 \\
\hline 190 & 0 \\
\hline 191 & 0 \\
\hline 192 & 0 \\
\hline
\end{tabular}

\begin{tabular}{|c|c|}
\hline 0 & 0 \\
\hline 0 & 0 \\
\hline 0 & 0 \\
\hline 6 & 0 \\
\hline 8 & 0 \\
\hline 13 & 0 \\
\hline 0 & 0 \\
\hline 1 & 0 \\
\hline 75 & 0 \\
\hline 0 & 0 \\
\hline 2 & 0 \\
\hline 0 & 0 \\
\hline 8 & 0 \\
\hline 0 & 0 \\
\hline 0 & 0 \\
\hline 0 & 0 \\
\hline 4 & 0 \\
\hline 0 & 0 \\
\hline 0 & 0 \\
\hline 0 & 0 \\
\hline 0 & 0 \\
\hline 0 & 0 \\
\hline 3 & 0 \\
\hline 0 & 0 \\
\hline 24 & 0 \\
\hline 3 & 0 \\
\hline 0 & 0 \\
\hline 24 & 0 \\
\hline 0 & 0 \\
\hline 9 & 0 \\
\hline 0 & 0 \\
\hline 0 & 0 \\
\hline 0 & 0 \\
\hline 0 & 0 \\
\hline 0 & 0 \\
\hline 9 & 0 \\
\hline 3 & 0 \\
\hline 0 & 0 \\
\hline 0 & 0 \\
\hline 3 & 0 \\
\hline 0 & 0 \\
\hline 0 & 0 \\
\hline 0 & 0 \\
\hline 75 & 0 \\
\hline 8 & 0 \\
\hline 0 & 0 \\
\hline 2 & 0 \\
\hline 5 & 0 \\
\hline 0 & 0 \\
\hline 2 & 0 \\
\hline 0 & 0 \\
\hline 1 & 0 \\
\hline 4 & 0 \\
\hline 75 & 0 \\
\hline 1 & 0 \\
\hline 7 & 0 \\
\hline 0 & 0 \\
\hline 0 & 0 \\
\hline 9 & 0 \\
\hline 24 & 0 \\
\hline 17 & 0 \\
\hline 3 & 0 \\
\hline 0 & 0 \\
\hline 7 & 0 \\
\hline 9 & 0 \\
\hline 3 & 0 \\
\hline 1 & 0 \\
\hline 9 & 0 \\
\hline 13 & 0 \\
\hline 8 & 0 \\
\hline 0 & 0 \\
\hline 17 & 0 \\
\hline 8 & 0 \\
\hline 4 & 0 \\
\hline 6 & 0 \\
\hline 13 & 0 \\
\hline 5 & 0 \\
\hline 5 & 0 \\
\hline 7 & 0 \\
\hline 0 & 0 \\
\hline 0 & 0 \\
\hline 3 & 0 \\
\hline 17 & 0 \\
\hline 0 & 0 \\
\hline 8 & 0 \\
\hline 0 & 0 \\
\hline 4 & 0 \\
\hline 3 & 0 \\
\hline 13 & 0 \\
\hline 3 & 0 \\
\hline 6 & 0 \\
\hline 75 & 0 \\
\hline 0 & 0 \\
\hline 0 & 0 \\
\hline 0 & 0 \\
\hline 0 & 0 \\
\hline 0 & 0 \\
\hline
\end{tabular}




\begin{tabular}{|c|c|}
\hline 193 & 0 \\
\hline 194 & 0 \\
\hline 195 & 0 \\
\hline 196 & 5 \\
\hline 197 & 9 \\
\hline 198 & 0 \\
\hline 199 & 0 \\
\hline 200 & 0 \\
\hline 201 & 0 \\
\hline 202 & 2 \\
\hline 203 & 0 \\
\hline 204 & 0 \\
\hline 205 & 0 \\
\hline 206 & 0 \\
\hline 207 & 0 \\
\hline 208 & 2 \\
\hline 209 & 0 \\
\hline 210 & 0 \\
\hline 211 & 3 \\
\hline 212 & 0 \\
\hline 213 & 0 \\
\hline 214 & 0 \\
\hline 215 & 0 \\
\hline 216 & 0 \\
\hline 217 & 0 \\
\hline 218 & 0 \\
\hline 219 & 9 \\
\hline 220 & 0 \\
\hline 221 & 25 \\
\hline 222 & 0 \\
\hline 223 & 9 \\
\hline 224 & 75 \\
\hline 225 & 0 \\
\hline 226 & 0 \\
\hline 227 & 13 \\
\hline 228 & 0 \\
\hline 229 & 0 \\
\hline 230 & 0 \\
\hline 231 & 0 \\
\hline 232 & 1 \\
\hline 233 & 0 \\
\hline 234 & 2 \\
\hline 235 & 0 \\
\hline 236 & 0 \\
\hline 237 & 1 \\
\hline 238 & 0 \\
\hline 239 & 0 \\
\hline 240 & 13 \\
\hline 241 & 0 \\
\hline 242 & 5 \\
\hline 243 & 13 \\
\hline 244 & 0 \\
\hline 245 & 0 \\
\hline 246 & 7 \\
\hline 247 & 0 \\
\hline 248 & 9 \\
\hline 249 & 7 \\
\hline 250 & 8 \\
\hline 251 & 0 \\
\hline 252 & 0 \\
\hline 253 & 2 \\
\hline 254 & 0 \\
\hline 255 & 0 \\
\hline 256 & 2 \\
\hline 257 & 0 \\
\hline 258 & 0 \\
\hline 259 & 0 \\
\hline 260 & 0 \\
\hline 261 & 7 \\
\hline 262 & 0 \\
\hline 263 & 9 \\
\hline 264 & 0 \\
\hline 265 & 0 \\
\hline 266 & 0 \\
\hline 267 & 2 \\
\hline 268 & 0 \\
\hline 269 & 1 \\
\hline 270 & 0 \\
\hline 271 & 0 \\
\hline 272 & 0 \\
\hline 273 & 0 \\
\hline 274 & 5 \\
\hline 275 & 0 \\
\hline 276 & 3 \\
\hline 277 & 0 \\
\hline 278 & 0 \\
\hline 279 & 33 \\
\hline 280 & 6 \\
\hline 281 & 3 \\
\hline 282 & 7 \\
\hline 283 & 6 \\
\hline 284 & 0 \\
\hline 285 & 4 \\
\hline 286 & 0 \\
\hline 287 & 17 \\
\hline 288 & 0 \\
\hline 289 & 25 \\
\hline
\end{tabular}

\begin{tabular}{|c|c|}
\hline 0 & 0 \\
\hline 0 & 0 \\
\hline 0 & 0 \\
\hline 5 & 0 \\
\hline 9 & 0 \\
\hline 0 & 0 \\
\hline 0 & 0 \\
\hline 0 & 0 \\
\hline 0 & 0 \\
\hline 2 & 0 \\
\hline 0 & 0 \\
\hline 0 & 0 \\
\hline 0 & 0 \\
\hline 0 & 0 \\
\hline 0 & 0 \\
\hline 2 & 0 \\
\hline 0 & 0 \\
\hline 0 & 0 \\
\hline 3 & 0 \\
\hline 0 & 0 \\
\hline 0 & 0 \\
\hline 0 & 0 \\
\hline 0 & 0 \\
\hline 0 & 0 \\
\hline 0 & 0 \\
\hline 0 & 0 \\
\hline 9 & 0 \\
\hline 0 & 0 \\
\hline 25 & 0 \\
\hline 0 & 0 \\
\hline 9 & 0 \\
\hline 75 & 0 \\
\hline 0 & 0 \\
\hline 0 & 0 \\
\hline 13 & 0 \\
\hline 0 & 1 \\
\hline 0 & 1 \\
\hline 0 & 1 \\
\hline 0 & 1 \\
\hline 1 & 1 \\
\hline 0 & 1 \\
\hline 2 & 1 \\
\hline 0 & 1 \\
\hline 0 & 1 \\
\hline 1 & 1 \\
\hline 0 & 1 \\
\hline 0 & 1 \\
\hline 13 & 1 \\
\hline 0 & 1 \\
\hline 5 & 2 \\
\hline 13 & 2 \\
\hline 0 & 2 \\
\hline 0 & 2 \\
\hline 7 & 2 \\
\hline 0 & 2 \\
\hline 9 & 2 \\
\hline 7 & 2 \\
\hline 8 & 2 \\
\hline 0 & 2 \\
\hline 0 & 2 \\
\hline 2 & 2 \\
\hline 0 & 2 \\
\hline 0 & 2 \\
\hline 2 & 2 \\
\hline 0 & 2 \\
\hline 0 & 2 \\
\hline 0 & 2 \\
\hline 0 & 2 \\
\hline 7 & 2 \\
\hline 0 & 3 \\
\hline 9 & 3 \\
\hline 0 & 3 \\
\hline 0 & 3 \\
\hline 0 & 3 \\
\hline 2 & 3 \\
\hline 0 & 3 \\
\hline 1 & 3 \\
\hline 0 & 3 \\
\hline 0 & 3 \\
\hline 0 & 3 \\
\hline 0 & 3 \\
\hline 5 & 3 \\
\hline 0 & 3 \\
\hline 3 & 3 \\
\hline 0 & 3 \\
\hline 0 & 3 \\
\hline 33 & 3 \\
\hline 6 & 3 \\
\hline 3 & 3 \\
\hline 7 & 3 \\
\hline 6 & 4 \\
\hline 0 & 4 \\
\hline 4 & 4 \\
\hline 0 & 4 \\
\hline 17 & 4 \\
\hline 0 & 4 \\
\hline 25 & 4 \\
\hline
\end{tabular}

\begin{tabular}{|c|c|}
\hline 0 & 0 \\
\hline 0 & 0 \\
\hline 0 & 0 \\
\hline 0 & 0 \\
\hline 0 & 0 \\
\hline 0 & 0 \\
\hline 0 & 0 \\
\hline 0 & 0 \\
\hline 0 & 0 \\
\hline 0 & 0 \\
\hline 0 & 0 \\
\hline 0 & 0 \\
\hline 0 & 0 \\
\hline 0 & 0 \\
\hline 0 & 0 \\
\hline 0 & 0 \\
\hline 0 & 0 \\
\hline 0 & 0 \\
\hline 0 & 0 \\
\hline 0 & 0 \\
\hline 0 & 0 \\
\hline 0 & 0 \\
\hline 0 & 0 \\
\hline 0 & 0 \\
\hline 0 & 0 \\
\hline 0 & 0 \\
\hline 0 & 0 \\
\hline 0 & 0 \\
\hline 0 & 0 \\
\hline 0 & 0 \\
\hline 0 & 0 \\
\hline 0 & 0 \\
\hline 0 & 0 \\
\hline 0 & 0 \\
\hline 0 & 0 \\
\hline 0.000565291 & 7.07E-07 \\
\hline 0.001130582 & $2.12 \mathrm{E}-06$ \\
\hline $\begin{array}{l}0.001695873 \\
0.002261164\end{array}$ & $\begin{array}{l}3.53 \mathrm{E}-06 \\
4.95 \mathrm{E}-06\end{array}$ \\
\hline $\begin{array}{l}0.002261164 \\
0.002826456\end{array}$ & $\begin{array}{l}4.95 \mathrm{E}-06 \\
6.36 \mathrm{E}-06\end{array}$ \\
\hline 0.003391747 & 7.77E-06 \\
\hline 0.003957038 & $9.19 \mathrm{E}-06$ \\
\hline 0.004522329 & 1.06E-05 \\
\hline 0.00508762 & 1.2E-05 \\
\hline 0.005652911 & $1.34 \mathrm{E}-05$ \\
\hline 0.006218202 & $1.48 \mathrm{E}-05$ \\
\hline 0.006783493 & 1.63E-05 \\
\hline 0.007348785 & 1.77E-05 \\
\hline 0.007914076 & $1.91 \mathrm{E}-05$ \\
\hline 0.009044658 & 2.12E-05 \\
\hline 0.01017524 & 2.4E-05 \\
\hline 0.011305822 & 2.69E- 05 \\
\hline 0.012436405 & 2.97E-05 \\
\hline 0.013566987 & 3.25E-05 \\
\hline 0.014697569 & 3.53E-05 \\
\hline 0.015828151 & 3.82E-05 \\
\hline 0.016958734 & 4.1E-05 \\
\hline 0.018089316 & 4.38E-05 \\
\hline 0.019219898 & 4.66E-05 \\
\hline 0.02035048 & 4.95E-05 \\
\hline 0.021481063 & $5.23 \mathrm{E}-05$ \\
\hline 0.022611645 & 5.51E-05 \\
\hline 0.023742227 & $5.79 \mathrm{E}-05$ \\
\hline 0.024872809 & $6.08 \mathrm{E}-05$ \\
\hline 0.026003392 & $6.36 \mathrm{E}-05$ \\
\hline 0.027133974 & $6.64 \mathrm{E}-05$ \\
\hline 0.028264556 & $6.92 \mathrm{E}-05$ \\
\hline 0.029395138 & 7.21E-05 \\
\hline 0.030525721 & 7.49E-05 \\
\hline 0.032221594 & $7.84 \mathrm{E}-05$ \\
\hline 0.033917467 & 8.27E-05 \\
\hline 0.035613341 & 8.69E-05 \\
\hline 0.037309214 & $9.12 \mathrm{E}-05$ \\
\hline 0.039005088 & 9.54E-05 \\
\hline 0.040700961 & $9.96 \mathrm{E}-05$ \\
\hline 0.042396834 & 0.000104 \\
\hline 0.044092708 & 0.000108 \\
\hline 0.045788581 & 0.000112 \\
\hline 0.047484454 & 0.000117 \\
\hline 0.049180328 & 0.000121 \\
\hline 0.050876201 & 0.000125 \\
\hline 0.052572075 & 0.000129 \\
\hline 0.054267948 & 0.000134 \\
\hline 0.055963821 & 0.000138 \\
\hline 0.057659695 & 0.000142 \\
\hline 0.059355568 & 0.000146 \\
\hline 0.061051441 & 0.000151 \\
\hline 0.062747315 & 0.000155 \\
\hline 0.064443188 & 0.000159 \\
\hline 0.066139062 & 0.000163 \\
\hline 0.068400226 & 0.000168 \\
\hline 0.070661391 & 0.000174 \\
\hline 0.072922555 & 0.000179 \\
\hline 0.07518372 & 0.000185 \\
\hline 0.077444884 & 0.000191 \\
\hline 0.079706049 & 0.000196 \\
\hline 0.081967213 & 0.000202 \\
\hline
\end{tabular}




\begin{tabular}{|c|c|}
\hline 290 & 0 \\
\hline 291 & 4 \\
\hline 292 & 25 \\
\hline 293 & 8 \\
\hline 294 & 0 \\
\hline 295 & 6 \\
\hline 296 & 0 \\
\hline 297 & 0 \\
\hline 298 & 4 \\
\hline 299 & 0 \\
\hline 300 & 0 \\
\hline 301 & 0 \\
\hline 302 & 8 \\
\hline 303 & 0 \\
\hline 304 & 1 \\
\hline 305 & 3 \\
\hline 306 & 0 \\
\hline 307 & 4 \\
\hline 308 & 0 \\
\hline 309 & 0 \\
\hline 310 & 0 \\
\hline 311 & 0 \\
\hline 312 & 0 \\
\hline 313 & 9 \\
\hline 314 & 1 \\
\hline 315 & 7 \\
\hline 316 & 6 \\
\hline 317 & 25 \\
\hline 318 & 0 \\
\hline 319 & 17 \\
\hline 320 & 33 \\
\hline 321 & 13 \\
\hline 322 & 0 \\
\hline 323 & 0 \\
\hline 324 & 2 \\
\hline 325 & 2 \\
\hline 326 & 0 \\
\hline 327 & 0 \\
\hline 328 & 0 \\
\hline 329 & 0 \\
\hline 330 & 0 \\
\hline 331 & 0 \\
\hline 332 & 0 \\
\hline 333 & 0 \\
\hline 334 & 0 \\
\hline 335 & 0 \\
\hline 336 & 0 \\
\hline 337 & 2 \\
\hline 338 & 4 \\
\hline 339 & 0 \\
\hline 340 & 9 \\
\hline 341 & 1 \\
\hline 342 & 0 \\
\hline 343 & 0 \\
\hline 344 & 3 \\
\hline 345 & 0 \\
\hline 346 & 3 \\
\hline 347 & 0 \\
\hline 348 & 0 \\
\hline 349 & 17 \\
\hline 350 & 3 \\
\hline 351 & 9 \\
\hline 352 & 25 \\
\hline 353 & 0 \\
\hline 354 & 6 \\
\hline 355 & 4 \\
\hline 356 & 0 \\
\hline 357 & 5 \\
\hline 358 & 0 \\
\hline 359 & 0 \\
\hline 360 & 2 \\
\hline 361 & 0 \\
\hline 362 & 0 \\
\hline 363 & 0 \\
\hline 364 & 0 \\
\hline 365 & 1 \\
\hline 366 & 0 \\
\hline 367 & 0 \\
\hline 368 & 0 \\
\hline 369 & 0 \\
\hline 370 & 0 \\
\hline 371 & 4 \\
\hline 372 & 0 \\
\hline 373 & 9 \\
\hline 374 & 2 \\
\hline 375 & 0 \\
\hline 376 & 0 \\
\hline 377 & 0 \\
\hline 378 & 13 \\
\hline 379 & 0 \\
\hline 380 & 0 \\
\hline 381 & 0 \\
\hline 382 & 0 \\
\hline 383 & 2 \\
\hline 384 & 0 \\
\hline 385 & 0 \\
\hline 386 & \\
\hline
\end{tabular}

\begin{tabular}{|c|c|}
\hline 0 & 4 \\
\hline 4 & 4 \\
\hline 25 & 4 \\
\hline 8 & 4 \\
\hline 0 & 4 \\
\hline 6 & 4 \\
\hline 0 & 5 \\
\hline 0 & 5 \\
\hline 4 & 5 \\
\hline 0 & 5 \\
\hline 0 & 5 \\
\hline 0 & 5 \\
\hline 8 & 5 \\
\hline 0 & 5 \\
\hline 1 & 5 \\
\hline 3 & 5 \\
\hline 0 & 5 \\
\hline 4 & 6 \\
\hline 0 & 6 \\
\hline 0 & 6 \\
\hline 0 & 6 \\
\hline 0 & 6 \\
\hline 0 & 6 \\
\hline 9 & 6 \\
\hline 1 & 6 \\
\hline 7 & 6 \\
\hline 6 & 6 \\
\hline 25 & 7 \\
\hline 0 & 7 \\
\hline 17 & 7 \\
\hline 33 & 7 \\
\hline 13 & 7 \\
\hline 0 & 7 \\
\hline 0 & 7 \\
\hline 2 & 7 \\
\hline 2 & 7 \\
\hline 0 & 7 \\
\hline 0 & 7 \\
\hline 0 & 7 \\
\hline 0 & 8 \\
\hline 0 & 8 \\
\hline 0 & 8 \\
\hline 0 & 8 \\
\hline 0 & 8 \\
\hline 0 & 8 \\
\hline 0 & 8 \\
\hline 0 & 8 \\
\hline 2 & 8 \\
\hline 4 & 8 \\
\hline 0 & 8 \\
\hline 9 & 8 \\
\hline 1 & 8 \\
\hline 0 & 8 \\
\hline 0 & 9 \\
\hline 3 & 9 \\
\hline 0 & 9 \\
\hline 3 & 9 \\
\hline 0 & 9 \\
\hline 0 & 9 \\
\hline 17 & 9 \\
\hline 3 & 9 \\
\hline 9 & 9 \\
\hline 25 & 9 \\
\hline 0 & 9 \\
\hline 6 & 9 \\
\hline 4 & 9 \\
\hline 0 & 9 \\
\hline 5 & 9 \\
\hline 0 & 9 \\
\hline 0 & 9 \\
\hline 2 & 9 \\
\hline 0 & 9 \\
\hline 0 & 13 \\
\hline 0 & 13 \\
\hline 0 & 13 \\
\hline 1 & 13 \\
\hline 0 & 13 \\
\hline 0 & 13 \\
\hline 0 & 13 \\
\hline 0 & 13 \\
\hline 0 & 13 \\
\hline 4 & 13 \\
\hline 0 & 13 \\
\hline 9 & 13 \\
\hline 2 & 17 \\
\hline 0 & 17 \\
\hline 0 & 17 \\
\hline 0 & 17 \\
\hline 13 & 17 \\
\hline 0 & 17 \\
\hline 0 & 24 \\
\hline 0 & 24 \\
\hline 0 & 24 \\
\hline 2 & 24 \\
\hline 0 & 24 \\
\hline 0 & 25 \\
\hline & \\
\hline
\end{tabular}

$\begin{array}{ll}0.084228378 & 0.000208 \\ 0.086489542 & 0.000213\end{array}$ $\begin{array}{lll}0.088750707 & 0.000219\end{array}$ $\begin{array}{lll}0.091011871 & 0.000225\end{array}$ $\begin{array}{ll}0.093273036 & 0.00023\end{array}$ $0.0955342 \quad 0.000236$ 000242 0.1011871110 .000249 $0.104013567 \quad 0.000257$ $0.106840023 \quad 0.000264$ $\begin{array}{ll}0.109666478 & 0.000271\end{array}$ 0.1124929340 .000278 $\begin{array}{lll}0.115319389 & 0.000285\end{array}$ $\begin{array}{ll}0.118145845 & 0.000292\end{array}$ $0.120972301 \quad 0.000299$ $\begin{array}{ll}0.120972301 & 0.000299 \\ 0.123798756 & 0.000306\end{array}$ $\begin{array}{ll}0.123798756 & 0.000306 \\ 0.126625212 & 0.000313\end{array}$ 0.1300169590 .000321 $\begin{array}{ll}0.133408705 & 0.000329\end{array}$ 0.1368004520 .000338 $0.140192199 \quad 0.000346$ 0.1435839460 .000355 0.1469756920 .000363 $\begin{array}{lll}0.150367439 & 0.000372\end{array}$ $\begin{array}{ll}0.153759186 & 0.00038\end{array}$ $\begin{array}{ll}0.153759186 & 0.00038 \\ 0.157150933 & 0.000389\end{array}$ $\begin{array}{ll}0.160542679 & 0.000397\end{array}$ $0.164499717 \quad 0.000406$ 0.1684567550 .000416 $0.172413793 \quad 0.000426$ 0.1763708310 .000436 $\begin{array}{ll}0.180327869 & 0.000446\end{array}$ $\begin{array}{ll}0.184284907 & 0.000456\end{array}$ $\begin{array}{lll}0.188241945 & 0.000466\end{array}$ 0.1921989820 .000476 $\begin{array}{rr}0.19615602 & 0.000485\end{array}$ $\begin{array}{lll}0.200113058 & 0.000495\end{array}$ $0.204070096 \quad 0.000505$ $0.208027134 \quad 0.000515$ 0.2125494630 .000526 $\begin{array}{lll}0.217071792 & 0.000537\end{array}$ $\begin{array}{lll}0.221594121 & 0.000548\end{array}$ $\begin{array}{ll}0.22611645 & 0.00056\end{array}$ $\begin{array}{lll}0.230638779 & 0.000571\end{array}$ $\begin{array}{lll}0.235161108 & 0.000582\end{array}$ $\begin{array}{ll}0.239683437 & 0.000594\end{array}$ $\begin{array}{ll}0.244205766 & 0.000605\end{array}$ $0.248728095 \quad 0.000616$ $\begin{array}{lll}0.253250424 & 0.000627\end{array}$ $\begin{array}{ll}0.257772753 & 0.000639\end{array}$ $0.262295082 \quad 0.00065$ $\begin{array}{lll}0.266817411 & 0.000661\end{array}$ $\begin{array}{ll}0.27133974 & 0.000673 \\ 0.27642736 & 0.000685\end{array}$ $\begin{array}{ll}0.28151498 & 0.000697\end{array}$ $\begin{array}{rr}0.28151498 & 0.000697 \\ 0.2866026 & 0.00071\end{array}$ $\begin{array}{rr}0.29169022 & 0.000723\end{array}$ $0.296777841 \quad 0.000736$ 0.3018654610 .000748 0.3069530810 .000761 $\begin{array}{ll}0.312040701 & 0.000774\end{array}$ 0.3171283210 .000786 0.3222159410 .000799 $\begin{array}{ll}0.327303561 & 0.000812 \\ 0.332391181 & 0.000825\end{array}$ $\begin{array}{ll}0.332391181 & 0.000825\end{array}$ $\begin{array}{lll}0.337478802 & 0.000837\end{array}$ $\begin{array}{ll}0.347654042 & 0.000863\end{array}$ $\begin{array}{ll}0.347654042 & 0.000863 \\ 0.352741662 & 0.000875\end{array}$ $\begin{array}{ll}0.352741662 & 0.000875 \\ 0.357829282 & 0.000888\end{array}$ $\begin{array}{ll}0.357829282 & 0.000888 \\ 0.362916902 & 0.000901\end{array}$ $0.368004522 \quad 0.000914$ $0.375353307 \quad 0.000929$ $\begin{array}{ll}0.382702092 & 0.000948 \\ 0.390050876 & 0.000966\end{array}$ $\begin{array}{ll}0.397399661 & 0.000984\end{array}$ $\begin{array}{ll}0.404748445 & 0.001003\end{array}$ $0.41209723 \quad 0.001021$ 0.001039
-15 $\begin{array}{ll}0.426794799 & 0.001058 \\ 0.434143584 & 0.001076\end{array}$ 0.4341435840 .001076 $\begin{array}{ll}0.441492369 & 0.001095\end{array}$ $0.448841153 \quad 0.001113$ $0.456189938 \quad 0.001131$ $\begin{array}{ll}0.465799887 & 0.001152 \\ 0.475409836 & 0.001177\end{array}$ $\begin{array}{ll}0.475409836 & 0.001177 \\ 0.485019785 & 0.001201\end{array}$ $0.494629734 \quad 0.001225$ $\begin{array}{ll}0.494629734 & 0.001225 \\ 0.504239683 & 0.001249\end{array}$ $\begin{array}{ll}0.513849633 & 0.001273\end{array}$ $\begin{array}{rr}0.513849633 & 0.001273 \\ 0.52741662 & 0.001302\end{array}$ $\begin{array}{rr}0.52741662 & 0.001302 \\ 0.540983607 & 0.001336\end{array}$ $\begin{array}{ll}0.540983607 & 0.001336 \\ 0.554550594 & 0.001369\end{array}$ $\begin{array}{lll}0.568117581 & 0.001403\end{array}$ $\begin{array}{lll}0.581684568 & 0.001437\end{array}$ $\begin{array}{ll}0.595816846 & 0.001472\end{array}$ 


\begin{tabular}{|c|c|}
\hline 387 & 33 \\
\hline 388 & 0 \\
\hline 389 & 8 \\
\hline 390 & 0 \\
\hline 391 & 9 \\
\hline 392 & 0 \\
\hline 393 & 0 \\
\hline 394 & 0 \\
\hline 395 & 6 \\
\hline 396 & 8 \\
\hline 397 & 0 \\
\hline 398 & 0 \\
\hline 399 & 0 \\
\hline 400 & 0 \\
\hline 401 & 33 \\
\hline 402 & 0 \\
\hline 403 & 0 \\
\hline 404 & 8 \\
\hline 405 & 0 \\
\hline 406 & 0 \\
\hline 407 & 0 \\
\hline 408 & 0 \\
\hline 409 & 0 \\
\hline 410 & 0 \\
\hline 411 & 4 \\
\hline 412 & 0 \\
\hline 413 & 4 \\
\hline 414 & 4 \\
\hline 415 & 0 \\
\hline 416 & 1 \\
\hline 417 & 9 \\
\hline 418 & 0 \\
\hline 419 & 0 \\
\hline 420 & 0 \\
\hline 421 & 0 \\
\hline 422 & 0 \\
\hline 423 & 0 \\
\hline 424 & 0 \\
\hline 425 & 2 \\
\hline 426 & 7 \\
\hline 427 & 2 \\
\hline 428 & 3 \\
\hline 429 & 0 \\
\hline 430 & 0 \\
\hline 431 & 0 \\
\hline 432 & 3 \\
\hline 433 & 0 \\
\hline 434 & 7 \\
\hline 435 & 0 \\
\hline 436 & 0 \\
\hline 437 & 0 \\
\hline 438 & 0 \\
\hline 439 & 9 \\
\hline 440 & 24 \\
\hline 441 & 1 \\
\hline 442 & 0 \\
\hline 443 & 4 \\
\hline 444 & 9 \\
\hline 445 & 4 \\
\hline 446 & 0 \\
\hline 447 & 7 \\
\hline 448 & 6 \\
\hline 449 & 0 \\
\hline 450 & 0 \\
\hline 451 & 0 \\
\hline 452 & 8 \\
\hline 453 & 1 \\
\hline 454 & 0 \\
\hline 455 & 2 \\
\hline 456 & 0 \\
\hline 457 & 0 \\
\hline 458 & 7 \\
\hline 459 & 4 \\
\hline 460 & 2 \\
\hline 461 & 0 \\
\hline 462 & 0 \\
\hline 463 & 4 \\
\hline 464 & 1 \\
\hline 465 & 0 \\
\hline 466 & 4 \\
\hline 467 & 0 \\
\hline 468 & 0 \\
\hline 469 & 0 \\
\hline 470 & 0 \\
\hline 471 & 0 \\
\hline 472 & 0 \\
\hline 473 & 0 \\
\hline 474 & 7 \\
\hline 475 & 0 \\
\hline 476 & 0 \\
\hline 477 & 0 \\
\hline 478 & 0 \\
\hline 479 & 4 \\
\hline 480 & 0 \\
\hline 481 & 0 \\
\hline 482 & 0 \\
\hline 483 & 4 \\
\hline
\end{tabular}

0.014132278
0.014132278

0.014132278

0.018654607

0.018654607

0.018654607

0.042396834

0.042396834

0.042396834

0.042396834

0.042396834

0.042396834
$0.624081402 \quad 0.001543$

$0.63821368 \quad 0.001578$

$0.652345958 \quad 0.001613$ $\begin{array}{lll}0.671000565 & 0.001654\end{array}$ $\begin{array}{ll}0.689655172 & 0.001701\end{array}$

$\begin{array}{lll}0.70830978 & 0.001747\end{array}$

$0.726964387 \quad 0.001794$

$\begin{array}{lll}0.745618994 & 0.001841\end{array}$

$0.788015828 \quad 0.001917$

$\begin{array}{lll}0.830412663 & 0.002023\end{array}$

$\begin{array}{lll}0.872809497 & 0.002129\end{array}$

$\begin{array}{lll}0.915206331 & 0.002235\end{array}$

$\begin{array}{lll}0.957603166 & 0.002341\end{array}$ 


$\begin{array}{lcl}484 & 4 & 0.970125757 \\ 485 & 0 & 0.043582931 \\ 486 & 8 & 0.513951908 \\ 487 & 9 & 0.139757147 \\ 488 & 7 & 0.521637933 \\ 489 & 25 & 0.493144316 \\ 490 & 0 & 0.483187193 \\ 491 & 9 & 0.600830196 \\ 492 & 0 & 0.87591112 \\ 493 & 0 & 0.340702219 \\ 494 & 0 & 0.73412333 \\ 495 & 0 & 0.086708119 \\ 496 & 3 & 0.708682749 \\ 497 & 0 & 0.941254953 \\ 498 & 0 & 0.230814774 \\ 499 & 8 & 0.798826947 \\ 500 & 0 & 0.128576283 \\ 501 & 0 & 0.903925665 \\ 502 & 0 & 0.201798486 \\ 503 & 0 & 0.153498744 \\ 504 & 0 & 0.92582291 \\ 505 & 0 & 0.231470847 \\ 506 & 0 & 0.44370013 \\ 507 & 0 & 0.787068178 \\ 508 & 5 & 0.693494357 \\ 509 & 6 & 0.811000435 \\ 510 & 0 & 0.190630259\end{array}$


PD184352; $10 \mu \mathrm{M} ; 10 \mu \mathrm{M}$ ATP; sample of 300 kinases

\begin{tabular}{|c|c|c|}
\hline position & inhibition\% & rand \\
\hline 1 & 0 & 0.052690239 \\
\hline 2 & 3 & 0.949927824 \\
\hline 3 & 0 & 0.175207605 \\
\hline 4 & 24 & 0.882924266 \\
\hline 5 & 0 & 0.195667842 \\
\hline 6 & 0 & 0.377968813 \\
\hline 7 & 4 & 0.266381313 \\
\hline 8 & 13 & 0.179341683 \\
\hline 9 & 9 & 0.159772193 \\
\hline 10 & 0 & 0.664738695 \\
\hline 11 & 0 & 0.446723265 \\
\hline 12 & 4 & 0.803015504 \\
\hline 13 & 1 & 0.213499173 \\
\hline 14 & 33 & 0.689027684 \\
\hline 15 & 4 & 0.486956324 \\
\hline 16 & 0 & 0.146541058 \\
\hline 17 & 0 & 0.123498246 \\
\hline 18 & 3 & 0.301339615 \\
\hline 19 & 0 & 0.386552361 \\
\hline 20 & 8 & 0.832946762 \\
\hline 21 & 3 & 0.476848266 \\
\hline 22 & 0 & 0.787585352 \\
\hline 23 & 6 & 0.359573941 \\
\hline 24 & 0 & 0.005417106 \\
\hline 25 & 25 & 0.784525267 \\
\hline 26 & 0 & 0.445798396 \\
\hline 27 & 0 & 0.638033658 \\
\hline 28 & 0 & 0.909536799 \\
\hline 29 & 9 & 0.742395578 \\
\hline 30 & 4 & 0.668737136 \\
\hline 31 & 0 & 0.135561554 \\
\hline 32 & 6 & 0.098923508 \\
\hline 33 & 25 & 0.57746258 \\
\hline 34 & 2 & 0.442157166 \\
\hline 35 & 5 & 0.511534252 \\
\hline 36 & 0 & 0.52485806 \\
\hline 37 & 24 & 0.386438695 \\
\hline 38 & 0 & 0.354891913 \\
\hline 39 & 0 & 0.815781781 \\
\hline 40 & 0 & 0.988532296 \\
\hline 41 & 0 & 0.347867967 \\
\hline 42 & 2 & 0.615143392 \\
\hline 43 & 0 & 0.528556048 \\
\hline 44 & 0 & 0.910799618 \\
\hline 45 & 7 & 0.126753653 \\
\hline 46 & 0 & 0.472742586 \\
\hline 47 & 7 & 0.584222638 \\
\hline 48 & 3 & 0.614541137 \\
\hline 49 & 0 & 0.940803045 \\
\hline 50 & 0 & 0.736407198 \\
\hline 51 & 9 & 0.767752051 \\
\hline 52 & 9 & 0.012503704 \\
\hline 53 & 0 & 0.85089508 \\
\hline 54 & 0 & 0.026921385 \\
\hline 55 & 4 & 0.284965092 \\
\hline 56 & 0 & 0.424529152 \\
\hline 57 & 0 & 0.441734837 \\
\hline 58 & 24 & 0.311503943 \\
\hline 59 & 9 & 0.807730467 \\
\hline 60 & 17 & 0.191315625 \\
\hline 61 & 0 & 0.653833299 \\
\hline 62 & 13 & 0.442341591 \\
\hline 63 & 0 & 0.592247071 \\
\hline 64 & 0 & 0.210521296 \\
\hline 65 & 0 & 0.519710957 \\
\hline 66 & 1 & 0.530479905 \\
\hline 67 & 3 & 0.085867922 \\
\hline 68 & 0 & 0.388100833 \\
\hline 69 & 0 & 0.518174992 \\
\hline 70 & 4 & 0.680038325 \\
\hline 71 & 9 & 0.319625914 \\
\hline 72 & 0 & 0.731607167 \\
\hline 73 & 0 & 0.978823693 \\
\hline 74 & 4 & 0.969704298 \\
\hline 75 & 0 & 0.647821748 \\
\hline 76 & 0 & 0.329418507 \\
\hline 77 & 0 & 0.041209688 \\
\hline 78 & 7 & 0.537545461 \\
\hline 79 & 0 & 0.007919642 \\
\hline 80 & 0 & 0.719273458 \\
\hline 81 & 6 & 0.212729353 \\
\hline 82 & 7 & 0.463387675 \\
\hline 83 & 0 & 0.139391501 \\
\hline 84 & 3 & 0.296709452 \\
\hline 85 & 0 & 0.548624204 \\
\hline 86 & 4 & 0.297314384 \\
\hline 87 & 0 & 0.883911638 \\
\hline 88 & 9 & 0.300489277 \\
\hline 89 & 2 & 0.117436581 \\
\hline 90 & 7 & 0.607149873 \\
\hline 91 & 4 & 0.406446191 \\
\hline 92 & 0 & 0.296752964 \\
\hline 93 & 7 & 0.089007797 \\
\hline 94 & 0 & 0.836166437 \\
\hline & & \\
\hline
\end{tabular}




\begin{tabular}{|c|c|}
\hline 96 & 0 \\
\hline 97 & 0 \\
\hline 98 & 8 \\
\hline 99 & 24 \\
\hline 100 & 0 \\
\hline 101 & 0 \\
\hline 102 & 0 \\
\hline 103 & 33 \\
\hline 104 & 4 \\
\hline 105 & 0 \\
\hline 106 & 0 \\
\hline 107 & 7 \\
\hline 108 & 0 \\
\hline 109 & 0 \\
\hline 110 & 0 \\
\hline 111 & 0 \\
\hline 112 & 0 \\
\hline 113 & 5 \\
\hline 114 & 6 \\
\hline 115 & 2 \\
\hline 116 & 9 \\
\hline 117 & 0 \\
\hline 118 & 0 \\
\hline 119 & 0 \\
\hline 120 & 5 \\
\hline 121 & 0 \\
\hline 122 & 4 \\
\hline 123 & 0 \\
\hline 124 & 0 \\
\hline 125 & 2 \\
\hline 126 & 1 \\
\hline 127 & 0 \\
\hline 128 & 9 \\
\hline 129 & 8 \\
\hline 130 & 2 \\
\hline 131 & 4 \\
\hline 132 & 9 \\
\hline 133 & 0 \\
\hline 134 & 0 \\
\hline 135 & 9 \\
\hline 136 & 8 \\
\hline 137 & 0 \\
\hline 138 & 0 \\
\hline 139 & 0 \\
\hline 140 & 0 \\
\hline 141 & 1 \\
\hline 142 & 7 \\
\hline 143 & 4 \\
\hline 144 & 0 \\
\hline 145 & 0 \\
\hline 146 & 0 \\
\hline 147 & 8 \\
\hline 148 & 0 \\
\hline 149 & 0 \\
\hline 150 & 0 \\
\hline 151 & 0 \\
\hline 152 & 0 \\
\hline 153 & 0 \\
\hline 154 & 0 \\
\hline 155 & 8 \\
\hline 156 & 0 \\
\hline 157 & 24 \\
\hline 158 & 0 \\
\hline 159 & 2 \\
\hline 160 & 3 \\
\hline 161 & 2 \\
\hline 162 & 13 \\
\hline 163 & 0 \\
\hline 164 & 0 \\
\hline 165 & 0 \\
\hline 166 & 1 \\
\hline 167 & 0 \\
\hline 168 & 0 \\
\hline 169 & 0 \\
\hline 170 & 0 \\
\hline 171 & 0 \\
\hline 172 & 0 \\
\hline 173 & 0 \\
\hline 174 & 4 \\
\hline 175 & 2 \\
\hline 176 & 0 \\
\hline 177 & 0 \\
\hline 178 & 3 \\
\hline 179 & 1 \\
\hline 180 & 9 \\
\hline 181 & 5 \\
\hline 182 & 0 \\
\hline 183 & 8 \\
\hline 184 & 0 \\
\hline 185 & 0 \\
\hline 186 & 24 \\
\hline 187 & 8 \\
\hline 188 & 0 \\
\hline 189 & 0 \\
\hline 190 & 9 \\
\hline 191 & 0 \\
\hline & \\
\hline
\end{tabular}

\begin{tabular}{|c|c|}
\hline 0 & 0 \\
\hline 0 & 0 \\
\hline 8 & 0 \\
\hline 24 & 0 \\
\hline 0 & 0 \\
\hline 0 & 0 \\
\hline 0 & 0 \\
\hline 33 & 0 \\
\hline 4 & 0 \\
\hline 0 & 0 \\
\hline 0 & 0 \\
\hline 7 & 0 \\
\hline 0 & 0 \\
\hline 0 & 0 \\
\hline 0 & 0 \\
\hline 0 & 0 \\
\hline 0 & 0 \\
\hline 5 & 0 \\
\hline 6 & 0 \\
\hline 2 & 0 \\
\hline 9 & 0 \\
\hline 0 & 0 \\
\hline 0 & 0 \\
\hline 0 & 0 \\
\hline 5 & 0 \\
\hline 0 & 0 \\
\hline 4 & 0 \\
\hline 0 & 0 \\
\hline 0 & 0 \\
\hline 2 & 0 \\
\hline 1 & 0 \\
\hline 0 & 0 \\
\hline 9 & 0 \\
\hline 8 & 0 \\
\hline 2 & 0 \\
\hline 4 & 0 \\
\hline 9 & 0 \\
\hline 0 & 0 \\
\hline 0 & 0 \\
\hline 9 & 0 \\
\hline 8 & 0 \\
\hline 0 & 0 \\
\hline 0 & 0 \\
\hline 0 & 0 \\
\hline 0 & 0 \\
\hline 1 & 0 \\
\hline 7 & 0 \\
\hline 4 & 0 \\
\hline 0 & 0 \\
\hline 0 & 0 \\
\hline 0 & 0 \\
\hline 8 & 0 \\
\hline 0 & 0 \\
\hline 0 & 0 \\
\hline 0 & 0 \\
\hline 0 & 0 \\
\hline 0 & 0 \\
\hline 0 & 0 \\
\hline 0 & 0 \\
\hline 8 & 0 \\
\hline 0 & 0 \\
\hline 24 & 0 \\
\hline 0 & 0 \\
\hline 2 & 0 \\
\hline 3 & 0 \\
\hline 2 & 0 \\
\hline 13 & 0 \\
\hline 0 & 0 \\
\hline 0 & 0 \\
\hline 0 & 0 \\
\hline 1 & 0 \\
\hline 0 & 0 \\
\hline 0 & 0 \\
\hline 0 & 0 \\
\hline 0 & 1 \\
\hline 0 & 1 \\
\hline 0 & 1 \\
\hline 0 & 1 \\
\hline 4 & 1 \\
\hline 2 & 1 \\
\hline 0 & 1 \\
\hline 0 & 1 \\
\hline 3 & 1 \\
\hline 1 & 1 \\
\hline 9 & 2 \\
\hline 5 & 2 \\
\hline 0 & 2 \\
\hline 8 & 2 \\
\hline 0 & 2 \\
\hline 0 & 2 \\
\hline 24 & 2 \\
\hline 8 & 2 \\
\hline 0 & 2 \\
\hline 0 & 2 \\
\hline 9 & 2 \\
\hline 0 & 2 \\
\hline & 3 \\
\hline
\end{tabular}

\begin{tabular}{|c|c|}
\hline 0 & 0 \\
\hline 0 & 0 \\
\hline 0 & 0 \\
\hline 0 & 0 \\
\hline 0 & 0 \\
\hline 0 & 0 \\
\hline 0 & 0 \\
\hline 0 & 0 \\
\hline 0 & 0 \\
\hline 0 & 0 \\
\hline 0 & 0 \\
\hline 0 & 0 \\
\hline 0 & 0 \\
\hline 0 & 0 \\
\hline 0 & 0 \\
\hline 0 & 0 \\
\hline 0 & 0 \\
\hline 0 & 0 \\
\hline 0 & 0 \\
\hline 0 & 0 \\
\hline 0 & 0 \\
\hline 0 & 0 \\
\hline 0 & 0 \\
\hline 0 & 0 \\
\hline 0 & 0 \\
\hline 0 & 0 \\
\hline 0 & 0 \\
\hline 0 & 0 \\
\hline 0 & 0 \\
\hline 0 & 0 \\
\hline 0 & 0 \\
\hline 0 & 0 \\
\hline 0 & 0 \\
\hline 0 & 0 \\
\hline 0 & 0 \\
\hline 0 & 0 \\
\hline 0 & 0 \\
\hline 0 & 0 \\
\hline 0 & 0 \\
\hline 0 & 0 \\
\hline 0 & 0 \\
\hline 0 & 0 \\
\hline 0 & 0 \\
\hline 0 & 0 \\
\hline 0 & 0 \\
\hline 0 & 0 \\
\hline 0 & 0 \\
\hline 0 & 0 \\
\hline 0 & 0 \\
\hline 0 & 0 \\
\hline 0 & 0 \\
\hline 0 & 0 \\
\hline 0 & 0 \\
\hline 0 & 0 \\
\hline 0 & 0 \\
\hline 0 & 0 \\
\hline 0 & 0 \\
\hline 0 & 0 \\
\hline 0 & 0 \\
\hline 0 & 0 \\
\hline 0 & 0 \\
\hline 0 & 0 \\
\hline 0 & 0 \\
\hline 0 & 0 \\
\hline 0 & 0 \\
\hline 0 & 0 \\
\hline 0 & 0 \\
\hline 0 & 0 \\
\hline 0 & 0 \\
\hline 0 & 0 \\
\hline 0 & 0 \\
\hline 0 & 0 \\
\hline 0 & 0 \\
\hline 0 & 0 \\
\hline 000772201 & $1.29 \mathrm{E}-06$ \\
\hline 001544402 & 3.86E-06 \\
\hline .002316602 & $6.44 \mathrm{E}-06$ \\
\hline .003088803 & $9.01 \mathrm{E}-06$ \\
\hline .003861004 & $1.16 \mathrm{E}-05$ \\
\hline .004633205 & $1.42 \mathrm{E}-05$ \\
\hline .005405405 & 1.67E-05 \\
\hline .006177606 & $1.93 \mathrm{E}-05$ \\
\hline .006949807 & 2.19E-05 \\
\hline .007722008 & 2.45E-05 \\
\hline .009266409 & 2.83E-05 \\
\hline .010810811 & 3.35E-05 \\
\hline .012355212 & $3.86 \mathrm{E}-05$ \\
\hline .013899614 & $4.38 \mathrm{E}-05$ \\
\hline .015444015 & $4.89 \mathrm{E}-05$ \\
\hline .016988417 & $5.41 \mathrm{E}-05$ \\
\hline .018532819 & 5.92E-05 \\
\hline 0.02007722 & 6.44E-05 \\
\hline .021621622 & $6.95 \mathrm{E}-05$ \\
\hline .023166023 & 7.46E-05 \\
\hline .024710425 & 7.98E-05 \\
\hline $\begin{array}{l}.026254826 \\
.028571429\end{array}$ & $\begin{array}{l}8.49 \mathrm{E}-05 \\
9.14 \mathrm{E}-05\end{array}$ \\
\hline
\end{tabular}




\begin{tabular}{|c|c|}
\hline 193 & 5 \\
\hline 194 & 75 \\
\hline 195 & 13 \\
\hline 196 & 0 \\
\hline 197 & 0 \\
\hline 198 & 0 \\
\hline 199 & 0 \\
\hline 200 & 3 \\
\hline 201 & 0 \\
\hline 202 & 8 \\
\hline 203 & 2 \\
\hline 204 & 0 \\
\hline 205 & 1 \\
\hline 206 & 4 \\
\hline 207 & 0 \\
\hline 208 & 75 \\
\hline 209 & 5 \\
\hline 210 & 0 \\
\hline 211 & 5 \\
\hline 212 & 17 \\
\hline 213 & 0 \\
\hline 214 & 0 \\
\hline 215 & 0 \\
\hline 216 & 0 \\
\hline 217 & 9 \\
\hline 218 & 0 \\
\hline 219 & 0 \\
\hline 220 & 3 \\
\hline 221 & 0 \\
\hline 222 & 0 \\
\hline 223 & 4 \\
\hline 224 & 8 \\
\hline 225 & 33 \\
\hline 226 & 0 \\
\hline 227 & 0 \\
\hline 228 & 0 \\
\hline 229 & 0 \\
\hline 230 & 3 \\
\hline 231 & 0 \\
\hline 232 & 0 \\
\hline 233 & 7 \\
\hline 234 & 0 \\
\hline 235 & 0 \\
\hline 236 & 75 \\
\hline 237 & 0 \\
\hline 238 & 0 \\
\hline 239 & 0 \\
\hline 240 & 13 \\
\hline 241 & 0 \\
\hline 242 & 9 \\
\hline 243 & 0 \\
\hline 244 & 0 \\
\hline 245 & 0 \\
\hline 246 & 0 \\
\hline 247 & 0 \\
\hline 248 & 6 \\
\hline 249 & 0 \\
\hline 250 & 0 \\
\hline 251 & 0 \\
\hline 252 & 1 \\
\hline 253 & 75 \\
\hline 254 & 6 \\
\hline 255 & 0 \\
\hline 256 & 0 \\
\hline 257 & 7 \\
\hline 258 & 0 \\
\hline 259 & 2 \\
\hline 260 & 1 \\
\hline 261 & 0 \\
\hline 262 & 2 \\
\hline 263 & 3 \\
\hline 264 & 0 \\
\hline 265 & 0 \\
\hline 266 & 9 \\
\hline 267 & 0 \\
\hline 268 & 17 \\
\hline 269 & 0 \\
\hline 270 & 0 \\
\hline 271 & 1 \\
\hline 272 & 0 \\
\hline 273 & 0 \\
\hline 274 & 0 \\
\hline 275 & 0 \\
\hline 276 & 0 \\
\hline 277 & 0 \\
\hline 278 & 25 \\
\hline 279 & 0 \\
\hline 280 & 25 \\
\hline 281 & 5 \\
\hline 282 & 0 \\
\hline 283 & 7 \\
\hline 284 & 0 \\
\hline 285 & 0 \\
\hline 286 & 0 \\
\hline 287 & 4 \\
\hline 288 & 9 \\
\hline 289 & 3 \\
\hline
\end{tabular}

$\begin{array}{ll}0.030888031 & 9.91 \mathrm{E}-05 \\ 0.033204633 & 0.000107\end{array}$ $\begin{array}{lll}0.035521236 & 0.000115\end{array}$ $\begin{array}{ll}0.037837838 & 0.000122\end{array}$ $\begin{array}{ll}0.04015444 & 0.00013\end{array}$ $0.042471042 \quad 0.000138$ $\begin{array}{lll}0.044787645 & 0.000145\end{array}$ $0.047104247 \quad 0.000153$ 0.0494208490 .00016 0.0517374520 .000169 $0.054054054 \quad 0.000176$ $0.056370656 \quad 0.000184$ $0.058687259 \quad 0.000192$ $\begin{array}{lll}0.061776062 & 0.000201\end{array}$ $\begin{array}{lll}0.064864865 & 0.00021\end{array}$ $\begin{array}{lll}0.067953668 & 0.000221\end{array}$ $0.071042471 \quad 0.000232$ $\begin{array}{lll}0.074131274 & 0.00024\end{array}$ $0.077220077 \quad 0.000252$ $0.08030888 \quad 0.000263$ $0.083397683 \quad 0.000273$ $\begin{array}{ll}0.086486486 & 0.000283\end{array}$

$0.08957529 \quad 0.000293$ $\begin{array}{lll}0.092664093 & 0.000304\end{array}$ $\begin{array}{lll}0.095752896 & 0.000314\end{array}$ $0.098841699 \quad 0.000324$ $0.101930502 \quad 0.000335$ $0.105019305 \quad 0.000345$ $0.108108108 \quad 0.000355$ 0.1111969110 .000366 0.1150579150 .000377 $0.118918919 \quad 0.00039$ $0.122779923 \quad 0.000403$ $0.126640927 \quad 0.000416$ $0.130501931 \quad 0.000429$ $0.134362934 \quad 0.000441$ $0.138223938 \quad 0.000454$ 0.1420849420 .000467 $\begin{array}{lll}0.146718147 & 0.00048\end{array}$ $\begin{array}{lll}0.151351351 & 0.00049\end{array}$ $0.155984556 \quad 0.00051$ $0.160617761 \quad 0.000528$ $\begin{array}{ll}0.165250965 & 0.000543 \\ 0.16988417 & 0.000559\end{array}$ $\begin{array}{ll}0.16988417 & 0.000559\end{array}$ $0.174517375 \quad 0.000574$ $0.17992278 \quad 0.000591$ $\begin{array}{lll}0.185328185 & 0.000609\end{array}$ $0.190733591 \quad 0.000627$ $0.196138996 \quad 0.00064$ 0.2015444020 .000663 $0.206949807 \quad 0.00068$ 0.2123552120 .000699 $\begin{array}{ll}0.217760618 & 0.000717\end{array}$ $0.223166023 \quad 0.000735$ 0.2285714290 .000753 $0.233976834 \quad 0.000771$ $0.24015444 \quad 0.00079$ $\begin{array}{lll}0.246332046 & 0.000811\end{array}$ .2525096530 .00083 0.2586872590 .000852 $0.264864865 \quad 0.000873$ $\begin{array}{lll}0.271042471 & 0.000893\end{array}$ $0.277220077 \quad 0.000914$ $0.283397683 \quad 0.000934$ $\begin{array}{lll}0.28957529 & 0.000955\end{array}$ $0.295752896 \quad 0.000976$ $0.302702703 \quad 0.000997$ $\begin{array}{lll}0.30965251 & 0.001021\end{array}$ $\begin{array}{lll}0.316602317 & 0.00104\end{array}$ 0.3235521240 .00106 $\begin{array}{ll}0.330501931 & 0.00109\end{array}$ $\begin{array}{lll}0.337451737 & 0.001113\end{array}$ $0.344401544 \quad 0.001136$ $\begin{array}{ll}0.351351351 & 0.00116\end{array}$ $0.358301158 \quad 0.001183$ $0.365250965 \quad 0.001206$ 0.3722007720 .001229 0.3791505790 .001252 $\begin{array}{ll}0.3786100386 & 0.001275\end{array}$ 0.3930501930 .001299 $0.4 \quad 0.00132$ $\begin{array}{ll}0.406949807 & 0.001345 \\ 0.413899614 & 0.001368\end{array}$ $\begin{array}{ll}0.413899614 & 0.001368 \\ 0.423938224 & 0.001396 \\ 0.433976834 & 0.00143\end{array}$ $\begin{array}{rr}0.423938224 & 0.001396 \\ 0.433976834 & 0.00143\end{array}$ $0.444015444 \quad 0.001463$ $\begin{array}{lll}0.454054054 & 0.001497\end{array}$ $\begin{array}{lll}0.464092664 & 0.00153\end{array}$ $0.477220077 \quad 0.001569$ $\begin{array}{ll}0.49034749 & 0.001613\end{array}$ $0.503474903 \quad 0.00165$ $\begin{array}{ll}0.540540541 & 0.001771\end{array}$ $0.559073359 \quad 0.001833$ $\begin{array}{ll}0.577606178 & 0.001894 \\ 0.596138996 & 0.001956\end{array}$ $0.614671815 \quad 0.002018$ 


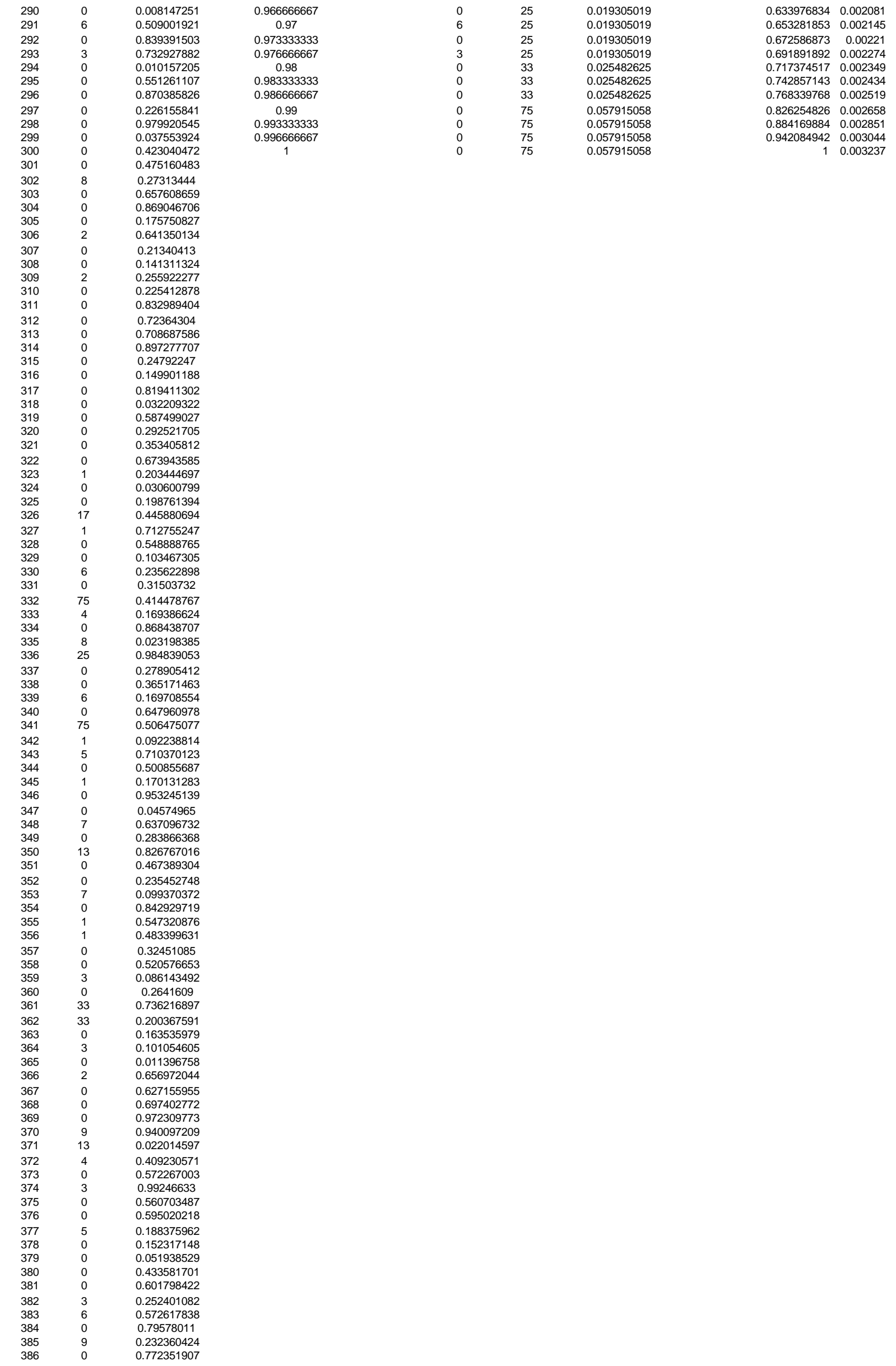




\begin{tabular}{|c|c|}
\hline 387 & 0 \\
\hline 388 & 8 \\
\hline 389 & 0 \\
\hline 390 & 25 \\
\hline 391 & 0 \\
\hline 392 & 5 \\
\hline 393 & 0 \\
\hline 394 & 0 \\
\hline 395 & 9 \\
\hline 396 & 17 \\
\hline 397 & 0 \\
\hline 398 & 2 \\
\hline 399 & 4 \\
\hline 400 & 5 \\
\hline 401 & 13 \\
\hline 402 & 2 \\
\hline 403 & 0 \\
\hline 404 & 13 \\
\hline 405 & 6 \\
\hline 406 & 0 \\
\hline 407 & 8 \\
\hline 408 & 0 \\
\hline 409 & 0 \\
\hline 410 & 0 \\
\hline 411 & 7 \\
\hline 412 & 0 \\
\hline 413 & 0 \\
\hline 414 & 0 \\
\hline 415 & 0 \\
\hline 416 & 3 \\
\hline 417 & 17 \\
\hline 418 & 0 \\
\hline 419 & 0 \\
\hline 420 & 2 \\
\hline 421 & 8 \\
\hline 422 & 0 \\
\hline 423 & 9 \\
\hline 424 & 0 \\
\hline 425 & 0 \\
\hline 426 & 0 \\
\hline 427 & 0 \\
\hline 428 & 3 \\
\hline 429 & 0 \\
\hline 430 & 0 \\
\hline 431 & 0 \\
\hline 432 & 8 \\
\hline 433 & 0 \\
\hline 434 & 2 \\
\hline 435 & 9 \\
\hline 436 & 8 \\
\hline 437 & 0 \\
\hline 438 & 4 \\
\hline 439 & 8 \\
\hline 440 & 2 \\
\hline 441 & 4 \\
\hline 442 & 0 \\
\hline 443 & 0 \\
\hline 444 & 7 \\
\hline 445 & 0 \\
\hline 446 & 13 \\
\hline 447 & 0 \\
\hline 448 & 0 \\
\hline 449 & 0 \\
\hline 450 & 7 \\
\hline 451 & 0 \\
\hline 452 & 0 \\
\hline 453 & 0 \\
\hline 454 & 2 \\
\hline 455 & 0 \\
\hline 456 & 33 \\
\hline 457 & 0 \\
\hline 458 & 0 \\
\hline 459 & 0 \\
\hline 460 & 0 \\
\hline 461 & 0 \\
\hline 462 & 0 \\
\hline 463 & 0 \\
\hline 464 & 0 \\
\hline 465 & 0 \\
\hline 466 & 2 \\
\hline 467 & 0 \\
\hline 468 & 0 \\
\hline 469 & 0 \\
\hline 470 & 9 \\
\hline 471 & 7 \\
\hline 472 & 0 \\
\hline 473 & 0 \\
\hline 474 & 0 \\
\hline 475 & 0 \\
\hline 476 & 0 \\
\hline 477 & 13 \\
\hline 478 & 0 \\
\hline 479 & 0 \\
\hline 480 & 3 \\
\hline 481 & 4 \\
\hline 482 & 0 \\
\hline 483 & 0 \\
\hline
\end{tabular}




$\begin{array}{lll}484 & 2 & 0.029035981 \\ 485 & 0 & 0.555369562 \\ 486 & 3 & 0.010095372 \\ 487 & 3 & 0.649693084 \\ 488 & 3 & 0.17413717 \\ 489 & 0 & 0.626878868 \\ 490 & 7 & 0.588693165 \\ 491 & 0 & 0.689141517 \\ 492 & 2 & 0.441123445 \\ 493 & 0 & 0.192086848 \\ 494 & 9 & 0.476846674 \\ 495 & 0 & 0.620566645 \\ 496 & 0 & 0.192068395 \\ 497 & 0 & 0.548628374 \\ 498 & 0 & 0.802791764 \\ 499 & 13 & 0.425179349 \\ 500 & 0 & 0.973296151 \\ 501 & 0 & 0.302806831 \\ 502 & 0 & 0.38382644 \\ 503 & 0 & 0.073857173 \\ 504 & 6 & 0.07319745 \\ 505 & 0 & 0.574095762 \\ 506 & 0 & 0.283800879 \\ 507 & 1 & 0.160478894 \\ 508 & 4 & 0.40386053 \\ 509 & 1 & 0.055687243 \\ 510 & 0 & 0.556975664\end{array}$


PD184352; $10 \mu \mathrm{M} ; 10 \mu \mathrm{M}$ ATP; sample of 200 kinases

position inhibition \% rand cumulative sample fraction sample inhibition

$\begin{array}{ll}0.672002779 & 0.005\end{array}$

$\begin{array}{ll}0.418946221 & 0.015\end{array}$

$\begin{array}{ll}0.319258712 & 0.02\end{array}$

0.025

$\begin{array}{ll}0.127393232 & 0.03 \\ 0.087798308 & 0.035\end{array}$

$\begin{array}{ll}0.964771381 & 0.04 \\ 0.916160822 & 0.045\end{array}$

$\begin{array}{ll}0.916160822 & 0.045 \\ 0.089957892 & 0.05\end{array}$

$0.843036476 \quad 0.055$

$0.633514308 \quad 0.06$

$0.653573816 \quad 0.065$

$\begin{array}{lc}0.255391447 & 0.07 \\ 0.816469906 & 0.075\end{array}$

$0.542513788-0.08$

$0.055629314 \quad 0.085$

$\begin{array}{lr}0.673310161 & 0.09 \\ 0.637798401 & 0.095\end{array}$

$0.139049014-0.1$

$\begin{array}{ll}0.769648318 & 0.105 \\ 0.227670317 & 0.11\end{array}$

$0.591524707-0.115$

$0.914938877 \quad 0.12$

$0.016240402-0.125$

$0.697658821 \quad 0.13$

$0.327238453-0.135$

$\begin{array}{ll}0.26129846 & 0.14 \\ 0.755043283 & 0.145\end{array}$

$0.150854344 \quad 0.15$

$\begin{array}{ll}0.914235481 & 0.155\end{array}$

$0.16563911-0.16$

$0.758036499 \quad 0.165$

$\begin{array}{lr}0.273060012 & 0.17 \\ 0.647250023 & 0.175\end{array}$

$0.935297333 \quad 0.18$

$\begin{array}{ll}0.803591977 & 0.185 \\ 0.734130928 & 0.19\end{array}$

$\begin{array}{lr}0.734130928 & 0.19 \\ 0.702571662 & 0.195\end{array}$

$\begin{array}{ll}0.776493928 & 0.2\end{array}$

$\begin{array}{ll}0.374337447 & 0.205 \\ 0.12793619 & 0.21\end{array}$

$0.909524602 \quad 0.215$

$\begin{array}{ll}0.129306279 & 0.22\end{array}$

$\begin{array}{ll}0.129306279 & 0.22 \\ 0.085237537 & 0.225\end{array}$

$\begin{array}{ll}0.496830629 & 0.23\end{array}$

$\begin{array}{cc}0.6882778 & 0.235 \\ 0.432206364 & 0.24\end{array}$

$0.446698546 \quad 0.245$

$\begin{array}{lr}0.355953234 & 0.25 \\ 0.189991627 & 0.255\end{array}$

$0.189991627 \quad 0.255$

$0.235264719 \quad 0.265$

$\begin{array}{ll}0.758563234 & 0.27\end{array}$

$\begin{array}{lc}0.753103919 & 0.275 \\ 0.411036073 & 0.28\end{array}$

$\begin{array}{lr}0.411036073 & 0.28 \\ 0.401104725 & 0.285\end{array}$

$0.286483921 \quad 0.29$

$0.590829269 \quad 0.295$

$\begin{array}{cc}0.847162349 & 0.3 \\ 0.957952698 & 0.305\end{array}$

$0.019609588 \quad 0.31$

$\begin{array}{ll}0.134331249 & 0.315\end{array}$

$\begin{array}{lr}0.436710062 & 0.32 \\ 0.877616502 & 0.325\end{array}$

$\begin{array}{ll}0.877616502 & 0.325 \\ 0.94035752 & 0.33\end{array}$

$\begin{array}{lr}0.94035752 & 0.33 \\ 0.905372179 & 0.335\end{array}$

$0.932503364 \quad 0.34$

$\begin{array}{ll}0.509393222 & 0.345 \\ 0.882599863 & 0.35\end{array}$

$\begin{array}{ll}0.44726239 & 0.355 \\ 0.265106043 & 0.36\end{array}$

$\begin{array}{ll}0.265106043 & 0.36 \\ 0.200973707 & 0.365\end{array}$

$\begin{array}{ll}0.68614858 & 0.37\end{array}$

$0.950730716 \quad 0.375$

$0.090138073 \quad 0.38$

0.806961394

$\begin{array}{lr}0.249021408 & 0.39 \\ 0.380355159 & 0.395\end{array}$

$0.162730554 \quad 0.4$

$\begin{array}{ll}0.597601971 & 0.405 \\ 0.014305816 & 0.41\end{array}$

$\begin{array}{ll}0.014305816 & 0.41 \\ 0.952417541 & 0.415\end{array}$

$\begin{array}{ll}0.083208186 & 0.42 \\ 0.94394236 & 0.425\end{array}$

$0.943944236-0.425$

$\begin{array}{ll}0.014828152 & 0.43\end{array}$

$\begin{array}{ll}0.784946287 & 0.435 \\ 0.543164224 & 0.44\end{array}$

$\begin{array}{ll}0.267480824 & 0.445\end{array}$

$0.018054953 \quad 0.45$

$\begin{array}{cc}0.342775082 & 0.455 \\ 0.401303816 & 0.46\end{array}$

$\begin{array}{ll}0.447285724 & 0.465 \\ 0.751163959 & 0.47\end{array}$

$\begin{array}{ll}0.751163959 & 0.47 \\ 0.242845494 & 0.475\end{array}$

sorted fraction of inhibition cumulative fraction of inhibition$$
0
$$$$
0
$$$$
0
$$$$
\begin{aligned}
& 0 \\
& 0 \\
& 0
\end{aligned}
$$$$
\begin{array}{lll}
0 & 0 & 0 \\
0 & 0 & 0 \\
0 & 0 & 0
\end{array}
$$ 


\begin{tabular}{|c|c|}
\hline 96 & 0 \\
\hline 97 & 0 \\
\hline 98 & 6 \\
\hline 99 & 7 \\
\hline 100 & 2 \\
\hline 101 & 0 \\
\hline 102 & 0 \\
\hline 103 & 7 \\
\hline 104 & 0 \\
\hline 105 & 9 \\
\hline 106 & 0 \\
\hline 107 & 6 \\
\hline 108 & 0 \\
\hline 109 & 5 \\
\hline 110 & 0 \\
\hline 111 & 0 \\
\hline 112 & 0 \\
\hline 113 & 3 \\
\hline 114 & 0 \\
\hline 115 & 3 \\
\hline 116 & 0 \\
\hline 117 & 25 \\
\hline 118 & 24 \\
\hline 119 & 6 \\
\hline 120 & 0 \\
\hline 121 & 5 \\
\hline 122 & 0 \\
\hline 123 & 0 \\
\hline 124 & 3 \\
\hline 125 & 0 \\
\hline 126 & 25 \\
\hline 127 & 0 \\
\hline 128 & 33 \\
\hline 129 & 0 \\
\hline 130 & 0 \\
\hline 131 & 0 \\
\hline 132 & 33 \\
\hline 133 & 1 \\
\hline 134 & 0 \\
\hline 135 & 0 \\
\hline 136 & 13 \\
\hline 137 & 0 \\
\hline 138 & 0 \\
\hline 139 & 0 \\
\hline 140 & 0 \\
\hline 141 & 0 \\
\hline 142 & 9 \\
\hline 143 & 0 \\
\hline 144 & 6 \\
\hline 145 & 2 \\
\hline 146 & 4 \\
\hline 147 & 0 \\
\hline 148 & 0 \\
\hline 149 & 0 \\
\hline 150 & 4 \\
\hline 151 & 0 \\
\hline 152 & 0 \\
\hline 153 & 0 \\
\hline 154 & 17 \\
\hline 155 & 0 \\
\hline 156 & 0 \\
\hline 157 & 13 \\
\hline 158 & 7 \\
\hline 159 & 0 \\
\hline 160 & 0 \\
\hline 161 & 0 \\
\hline 162 & 0 \\
\hline 163 & 0 \\
\hline 164 & 6 \\
\hline 165 & 0 \\
\hline 166 & 0 \\
\hline 167 & 0 \\
\hline 168 & 17 \\
\hline 169 & 0 \\
\hline 170 & 13 \\
\hline 171 & 1 \\
\hline 172 & 0 \\
\hline 173 & 0 \\
\hline 174 & 25 \\
\hline 175 & 0 \\
\hline 176 & 5 \\
\hline 177 & 0 \\
\hline 178 & 13 \\
\hline 179 & 3 \\
\hline 180 & 9 \\
\hline 181 & 0 \\
\hline 182 & 0 \\
\hline 183 & 0 \\
\hline 184 & 4 \\
\hline 185 & 4 \\
\hline 186 & 0 \\
\hline 187 & 3 \\
\hline 188 & 0 \\
\hline 189 & 6 \\
\hline 190 & 5 \\
\hline 191 & 13 \\
\hline 192 & 0 \\
\hline
\end{tabular}

\begin{tabular}{|c|c|}
\hline 0 & 0 \\
\hline 0 & 0 \\
\hline 6 & 0 \\
\hline 7 & 0 \\
\hline 2 & 0 \\
\hline 0 & 0 \\
\hline 0 & 0 \\
\hline 7 & 0 \\
\hline 0 & 0 \\
\hline 9 & 0 \\
\hline 0 & 0 \\
\hline 6 & 0 \\
\hline 0 & 0 \\
\hline 5 & 0 \\
\hline 0 & 0 \\
\hline 0 & 0 \\
\hline 0 & 0 \\
\hline 3 & 1 \\
\hline 0 & 1 \\
\hline 3 & 1 \\
\hline 0 & 1 \\
\hline 25 & 1 \\
\hline 24 & 2 \\
\hline 6 & 2 \\
\hline 0 & 2 \\
\hline 5 & 2 \\
\hline 0 & 2 \\
\hline 0 & 2 \\
\hline 3 & 2 \\
\hline 0 & 3 \\
\hline 25 & 3 \\
\hline 0 & 3 \\
\hline 33 & 3 \\
\hline 0 & 3 \\
\hline 0 & 3 \\
\hline 0 & 3 \\
\hline 33 & 3 \\
\hline 1 & 4 \\
\hline 0 & 4 \\
\hline 0 & 4 \\
\hline 13 & 4 \\
\hline 0 & 4 \\
\hline 0 & 4 \\
\hline 0 & 4 \\
\hline 0 & 4 \\
\hline 0 & 4 \\
\hline 9 & 5 \\
\hline 0 & 5 \\
\hline 6 & 5 \\
\hline 2 & 5 \\
\hline 4 & 5 \\
\hline 0 & 5 \\
\hline 0 & 5 \\
\hline 0 & 5 \\
\hline 4 & 6 \\
\hline 0 & 6 \\
\hline 0 & 6 \\
\hline 0 & 6 \\
\hline 17 & 6 \\
\hline 0 & 6 \\
\hline 0 & 6 \\
\hline 13 & 6 \\
\hline 7 & 7 \\
\hline 0 & 7 \\
\hline 0 & 7 \\
\hline 0 & 7 \\
\hline 0 & 7 \\
\hline 0 & 7 \\
\hline 6 & 7 \\
\hline 0 & 7 \\
\hline 0 & 7 \\
\hline 0 & 8 \\
\hline 17 & 8 \\
\hline 0 & 8 \\
\hline 13 & 9 \\
\hline 1 & 9 \\
\hline 0 & 9 \\
\hline 0 & 9 \\
\hline 25 & 9 \\
\hline 0 & 9 \\
\hline 5 & 9 \\
\hline 0 & 9 \\
\hline 13 & 9 \\
\hline 3 & 9 \\
\hline 9 & 9 \\
\hline 0 & 13 \\
\hline 0 & 13 \\
\hline 0 & 13 \\
\hline 4 & 13 \\
\hline 4 & 13 \\
\hline 0 & 13 \\
\hline 3 & 17 \\
\hline 0 & 17 \\
\hline 6 & 24 \\
\hline 5 & 25 \\
\hline 13 & 25 \\
\hline 0 & 25 \\
\hline
\end{tabular}

$0.001160093 \quad 2.9 \mathrm{E}-06$ $0.002320186 \quad 8.7 \mathrm{E}-06$ $0.003480278 \quad 1.45 \mathrm{E}-05$ $0.0046403712 .03 \mathrm{E}-05$ $0.0058004642 .61 \mathrm{E}-05$

$0.00812065 \quad 3.48 \mathrm{E}-05$ $0.010440835 \quad 4.64 \mathrm{E}-05$ $0.012761021 \quad 5.8 \mathrm{E}-05$ $\begin{array}{rr}0.012761021 & 5.8 \mathrm{E}-05 \\ 0.015081206 & 6.96 \mathrm{E}-05\end{array}$ $0.017401392 \quad 8.12 \mathrm{E}-05$ $0.019721578 \quad 9.28 \mathrm{E}-05$ 0.0220417630 .00010 0.0255220420 .000119 0.029002320 .000136 $0.032482599 \quad 0.000154$ $0.035962877 \quad 0.000171$ $0.039443155 \quad 0.000189$ 0.0429234340 .000206 $\begin{array}{ll}0.046403712 & 0.000223\end{array}$ $\begin{array}{ll}0.046403712 & 0.000223 \\ 0.049883991 & 0.000241\end{array}$ 0.0545243620 .000261 0.0591647330 .00028 $\begin{array}{ll}0.063805104 & 0.000307\end{array}$ $\begin{array}{lll}0.068445476 & 0.00033\end{array}$ $\begin{array}{lll}0.073085847 & 0.000354\end{array}$ $0.077726218 \quad 0.000377$ $\begin{array}{ll}0.082366589 & 0.0004\end{array}$ $0.087006961 \quad 0.000423$ $\begin{array}{lll}0.091647332 & 0.000447\end{array}$ $0.097447796 \quad 0.000473$ $\begin{array}{lll}0.10324826 & 0.000502\end{array}$ $0.109048724 \quad 0.00053$ $0.114849188 \quad 0.00056$ 0.1206496520 .000589 $\begin{array}{ll}0.126450116 & 0.000618\end{array}$ $\begin{array}{lll}0.13225058 & 0.000647\end{array}$ $0.138051044 \quad 0.000676$ $0.145011601 \quad 0.000708$ $\begin{array}{lll}0.151972158 & 0.000742\end{array}$ $\begin{array}{ll}0.158932715 & 0.000777\end{array}$ $0.165893271 \quad 0.000812$ $0.172853828 \quad 0.00084$ 0.1798143850 .000882 0.1867749420 .000916 0.1937354990 .000951 $0.201856148 \quad 0.000989$ $0.209976798 \quad 0.00103$ $\begin{array}{ll}0.218097448 & 0.00107\end{array}$ $0.226218097 \quad 0.001111$ $0.234338747 \quad 0.001151$ $\begin{array}{lll}0.242459397 & 0.001192\end{array}$ $0.250580046 \quad 0.001233$ $\begin{array}{lll}0.258700696 & 0.001273\end{array}$ $0.266821346 \quad 0.001314$ 0.2761020880 .001357 $0.285382831 \quad 0.001404$ $0.294663573 \quad 0.00145$ $\begin{array}{ll}0.305104408 & 0.001499\end{array}$ $0.315545244 \quad 0.001552$ $0.336426914 \quad 0.001656$ 0.3468677490 .001708 $0.357308585 \quad 0.00176$ $\begin{array}{rr}0.36774942 & 0.001813 \\ 0.378190255 & 0.001865\end{array}$ $\begin{array}{lll}0.38863109 & 0.001917\end{array}$ $\begin{array}{lll}0.399071926 & 0.001969\end{array}$ $0.409512761 \quad 0.002021$ $0.424593968 \quad 0.002085$ $\begin{array}{lll}0.439675174 & 0.002161\end{array}$ $\begin{array}{lll}0.454756381 & 0.002236\end{array}$ $\begin{array}{lll}0.469837587 & 0.002311\end{array}$ $0.484918794 \quad 0.002387$ $0.5 \quad 0.002462$ $\begin{array}{lll}0.519721578 & 0.002549\end{array}$ $0.539443155 \quad 0.002648$ $\begin{array}{lll}0.567285383 & 0.002767\end{array}$ $\begin{array}{lll}0.596287703 & 0.002909 \\ 0.625290023 & 0.003054\end{array}$ $0.654292343 \quad 0.00319$ 


\begin{tabular}{|c|c|}
\hline 193 & 7 \\
\hline 194 & 0 \\
\hline 195 & 0 \\
\hline 196 & 0 \\
\hline 197 & 0 \\
\hline 198 & 0 \\
\hline 199 & 0 \\
\hline 200 & 9 \\
\hline 201 & 1 \\
\hline 202 & 4 \\
\hline 203 & 0 \\
\hline 204 & 0 \\
\hline 205 & 0 \\
\hline 206 & 0 \\
\hline 207 & 7 \\
\hline 208 & 2 \\
\hline 209 & 0 \\
\hline 210 & 0 \\
\hline 211 & 0 \\
\hline 212 & 0 \\
\hline 213 & 8 \\
\hline 214 & 4 \\
\hline 215 & 25 \\
\hline 216 & 4 \\
\hline 217 & 0 \\
\hline 218 & 0 \\
\hline 219 & 9 \\
\hline 220 & 17 \\
\hline 221 & 1 \\
\hline 222 & 2 \\
\hline 223 & 0 \\
\hline 224 & 0 \\
\hline 225 & 3 \\
\hline 226 & 0 \\
\hline 227 & 0 \\
\hline 228 & 0 \\
\hline 229 & 0 \\
\hline 230 & 8 \\
\hline 231 & 0 \\
\hline 232 & 25 \\
\hline 233 & 6 \\
\hline 234 & 9 \\
\hline 235 & 0 \\
\hline 236 & 0 \\
\hline 237 & 0 \\
\hline 238 & 2 \\
\hline 239 & 0 \\
\hline 240 & 3 \\
\hline 241 & 1 \\
\hline 242 & 0 \\
\hline 243 & 0 \\
\hline 244 & 2 \\
\hline 245 & 0 \\
\hline 246 & 0 \\
\hline 247 & 0 \\
\hline 248 & 8 \\
\hline 249 & 4 \\
\hline 250 & 0 \\
\hline 251 & 5 \\
\hline 252 & 0 \\
\hline 253 & 7 \\
\hline 254 & 4 \\
\hline 255 & 2 \\
\hline 256 & 75 \\
\hline 257 & 0 \\
\hline 258 & 0 \\
\hline 259 & 2 \\
\hline 260 & 75 \\
\hline 261 & 0 \\
\hline 262 & 8 \\
\hline 263 & 13 \\
\hline 264 & 0 \\
\hline 265 & 2 \\
\hline 266 & 0 \\
\hline 267 & 8 \\
\hline 268 & 0 \\
\hline 269 & 0 \\
\hline 270 & 9 \\
\hline 271 & 0 \\
\hline 272 & 0 \\
\hline 273 & 0 \\
\hline 274 & 1 \\
\hline 275 & 0 \\
\hline 276 & 0 \\
\hline 277 & 9 \\
\hline 278 & 0 \\
\hline 279 & 0 \\
\hline 280 & 0 \\
\hline 281 & 9 \\
\hline 282 & 2 \\
\hline 283 & 0 \\
\hline 284 & 0 \\
\hline 285 & 0 \\
\hline 286 & 9 \\
\hline 287 & 4 \\
\hline 288 & 0 \\
\hline 289 & 0 \\
\hline
\end{tabular}

$\begin{array}{lll}7 & 25 & 0.02900232 \\ 0 & 33 & 0.038283063 \\ 0 & 33 & 0.038283063 \\ 0 & 33 & 0.038283063 \\ 0 & 33 & 0.038283063 \\ 0 & 33 & 0.038283063 \\ 0 & 33 & 0.038283063 \\ 9 & 75 & 0.087006961\end{array}$




\begin{tabular}{|c|c|}
\hline 290 & 0 \\
\hline 291 & 0 \\
\hline 292 & 0 \\
\hline 293 & 0 \\
\hline 294 & 0 \\
\hline 295 & 0 \\
\hline 296 & 3 \\
\hline 297 & 0 \\
\hline 298 & 2 \\
\hline 299 & 0 \\
\hline 300 & 0 \\
\hline 301 & 0 \\
\hline 302 & 6 \\
\hline 303 & 3 \\
\hline 304 & 7 \\
\hline 305 & 0 \\
\hline 306 & 0 \\
\hline 307 & 0 \\
\hline 308 & 0 \\
\hline 309 & 0 \\
\hline 310 & 0 \\
\hline 311 & 17 \\
\hline 312 & 3 \\
\hline 313 & 0 \\
\hline 314 & 0 \\
\hline 315 & 0 \\
\hline 316 & 0 \\
\hline 317 & 75 \\
\hline 318 & 0 \\
\hline 319 & 13 \\
\hline 320 & 4 \\
\hline 321 & 6 \\
\hline 322 & 0 \\
\hline 323 & 7 \\
\hline 324 & 9 \\
\hline 325 & 8 \\
\hline 326 & 0 \\
\hline 327 & 17 \\
\hline 328 & 8 \\
\hline 329 & 0 \\
\hline 330 & 0 \\
\hline 331 & 0 \\
\hline 332 & 0 \\
\hline 333 & 0 \\
\hline 334 & 0 \\
\hline 335 & 4 \\
\hline 336 & 5 \\
\hline 337 & 2 \\
\hline 338 & 7 \\
\hline 339 & 0 \\
\hline 340 & 2 \\
\hline 341 & 0 \\
\hline 342 & 0 \\
\hline 343 & 1 \\
\hline 344 & 2 \\
\hline 345 & 0 \\
\hline 346 & 0 \\
\hline 347 & 7 \\
\hline 348 & 0 \\
\hline 349 & 24 \\
\hline 350 & 0 \\
\hline 351 & 8 \\
\hline 352 & 0 \\
\hline 353 & 0 \\
\hline 354 & 0 \\
\hline 355 & 0 \\
\hline 356 & 6 \\
\hline 357 & 0 \\
\hline 358 & 0 \\
\hline 359 & 0 \\
\hline 360 & 0 \\
\hline 361 & 0 \\
\hline 362 & 3 \\
\hline 363 & 13 \\
\hline 364 & 0 \\
\hline 365 & 0 \\
\hline 366 & 24 \\
\hline 367 & 0 \\
\hline 368 & 0 \\
\hline 369 & 0 \\
\hline 370 & 0 \\
\hline 371 & 9 \\
\hline 372 & 0 \\
\hline 373 & 0 \\
\hline 374 & 0 \\
\hline 375 & 0 \\
\hline 376 & 24 \\
\hline 377 & 0 \\
\hline 378 & 7 \\
\hline 379 & 3 \\
\hline 380 & 3 \\
\hline 381 & 9 \\
\hline 382 & 1 \\
\hline 383 & 0 \\
\hline 384 & 0 \\
\hline 385 & 4 \\
\hline 386 & 5 \\
\hline
\end{tabular}




\begin{tabular}{|c|c|}
\hline 387 & 0 \\
\hline 388 & 0 \\
\hline 389 & 0 \\
\hline 390 & 8 \\
\hline 391 & 0 \\
\hline 392 & 0 \\
\hline 393 & 0 \\
\hline 394 & 0 \\
\hline 395 & 0 \\
\hline 396 & 9 \\
\hline 397 & 3 \\
\hline 398 & 75 \\
\hline 399 & 2 \\
\hline 400 & 0 \\
\hline 401 & 0 \\
\hline 402 & 0 \\
\hline 403 & 0 \\
\hline 404 & 0 \\
\hline 405 & 0 \\
\hline 406 & 9 \\
\hline 407 & 3 \\
\hline 408 & 2 \\
\hline 409 & 0 \\
\hline 410 & 5 \\
\hline 411 & 0 \\
\hline 412 & 0 \\
\hline 413 & 3 \\
\hline 414 & 0 \\
\hline 415 & 0 \\
\hline 416 & 0 \\
\hline 417 & 8 \\
\hline 418 & 1 \\
\hline 419 & 0 \\
\hline 420 & 0 \\
\hline 421 & 9 \\
\hline 422 & 3 \\
\hline 423 & 1 \\
\hline 424 & 0 \\
\hline 425 & 0 \\
\hline 426 & 0 \\
\hline 427 & 0 \\
\hline 428 & 0 \\
\hline 429 & 0 \\
\hline 430 & 75 \\
\hline 431 & 4 \\
\hline 432 & 4 \\
\hline 433 & 0 \\
\hline 434 & 3 \\
\hline 435 & 0 \\
\hline 436 & 0 \\
\hline 437 & 4 \\
\hline 438 & 24 \\
\hline 439 & 8 \\
\hline 440 & 1 \\
\hline 441 & 3 \\
\hline 442 & 0 \\
\hline 443 & 0 \\
\hline 444 & 0 \\
\hline 445 & 0 \\
\hline 446 & 0 \\
\hline 447 & 13 \\
\hline 448 & 0 \\
\hline 449 & 0 \\
\hline 450 & 0 \\
\hline 451 & 0 \\
\hline 452 & 0 \\
\hline 453 & 4 \\
\hline 454 & 0 \\
\hline 455 & 0 \\
\hline 456 & 7 \\
\hline 457 & 24 \\
\hline 458 & 0 \\
\hline 459 & 13 \\
\hline 460 & 2 \\
\hline 461 & 0 \\
\hline 462 & 0 \\
\hline 463 & 0 \\
\hline 464 & 0 \\
\hline 465 & 2 \\
\hline 466 & 1 \\
\hline 467 & 0 \\
\hline 468 & 0 \\
\hline 469 & 13 \\
\hline 470 & 2 \\
\hline 471 & 0 \\
\hline 472 & 7 \\
\hline 473 & 0 \\
\hline 474 & 4 \\
\hline 475 & 9 \\
\hline 476 & 0 \\
\hline 477 & 0 \\
\hline 478 & 4 \\
\hline 479 & 0 \\
\hline 480 & 0 \\
\hline 481 & 0 \\
\hline 482 & 8 \\
\hline 483 & 0 \\
\hline
\end{tabular}




$\begin{array}{lll}484 & 0 & 0.600869045 \\ 485 & 0 & 0.991330719 \\ 486 & 8 & 0.963591395 \\ 487 & 8 & 0.204365837 \\ 488 & 3 & 0.086046937 \\ 489 & 0 & 0.622644773 \\ 490 & 17 & 0.97745051 \\ 491 & 3 & 0.965387728 \\ 492 & 0 & 0.183796384 \\ 493 & 0 & 0.566543161 \\ 494 & 0 & 0.062711604 \\ 495 & 0 & 0.219486443 \\ 496 & 0 & 0.938051925 \\ 497 & 1 & 0.383099295 \\ 498 & 0 & 0.253439377 \\ 499 & 1 & 0.369873723 \\ 500 & 0 & 0.74506426 \\ 501 & 0 & 0.926918944 \\ 502 & 8 & 0.730312648 \\ 503 & 0 & 0.856097848 \\ 504 & 0 & 0.622255646 \\ 505 & 0 & 0.274456755 \\ 506 & 0 & 0.935649452 \\ 507 & 1 & 0.231315771 \\ 508 & 0 & 0.167194438 \\ 509 & 0 & 0.288390894 \\ 510 & 0 & 0.256765797\end{array}$

total inhibition

Total area $\quad 0.11319$

Gini 0.773619

\begin{tabular}{rr}
\multicolumn{1}{c}{ Gini } & \\
\hline 0.76747727 & \\
0.80034214 & \\
0.81365613 & \\
0.788789 & \\
0.81365747 & \\
0.77361949 & \\
0.82812373 & \\
0.78290173 & \\
0.80092044 & \\
0.80683197 & \\
0.785075 & \\
0.80056671 & \\
0.77361949 & \\
mean & \\
st dev & 0.79504466 \\
&
\end{tabular}


PD184352; $10 \mu \mathrm{M} ; 10 \mu \mathrm{M}$ ATP; sample of 100 kinases

position inhibition\% rand cumulative sample fraction sample inhibition 0.862895106

0.01 $\begin{array}{ll}0.219048473 & 0.02 \\ 0.567072653 & 0.03\end{array}$ $\begin{array}{ll}0.567072653 & 0.03 \\ 0.575984458 & 0.04\end{array}$

$0.874327171 \quad 0.05$

$\begin{array}{ll}0.013411235 & 0.06 \\ 0.747443094 & 0.07\end{array}$

$\begin{array}{ll}0.486006129 & 0.08 \\ 0.803862784 & 0.09\end{array}$

$0.096309096 \quad 0.1$

$\begin{array}{ll}0.216172136 & 0.11 \\ 0.731742183 & 0.12\end{array}$

$\begin{array}{ll}0.731742183 & 0.12 \\ 0.397449166 & 0.13\end{array}$

$\begin{array}{ll}0.718200213 & 0.14\end{array}$

$\begin{array}{ll}0.577099008 & 0.15 \\ 0.407425376 & 0.16\end{array}$

$0.09269019 \quad 0.17$

$\begin{array}{ll}0.837917547 & 0.18 \\ 0.186981017 & 0.19\end{array}$

$0.865124841-0.2$

$0.710900566 \quad 0.21$

$0.708665398 \quad 0.22$

$\begin{array}{ll}0.302125359 & 0.23 \\ 0.320403555 & 0.24\end{array}$

$0.823198216 \quad 0.25$

$0.792778402 \quad 0.26$

$\begin{array}{ll}0.724232839 & 0.27 \\ 0.763815598 & 0.28\end{array}$

$\begin{array}{ll}0.704735067 & 0.29\end{array}$

$0.705913764 \quad 0.3$

$\begin{array}{ll}0.988454177 & 0.31 \\ 0.501647022 & 0.32\end{array}$

$\begin{array}{ll}0.501647022 & 0.32 \\ 0.130959079 & 0.33\end{array}$

0.35

$\begin{array}{ll}0.856218489 & 0.35 \\ 0.278899716 & 0.36\end{array}$

$\begin{array}{ll}0.278899716 & 0.36 \\ 0.389918553 & 0.37\end{array}$

$0.679684027 \quad 0.38$

$\begin{array}{cc}0.296941129 & 0.39 \\ 0.575202448 & 0.4\end{array}$

$\begin{array}{ll}0.295502201 & 0.41\end{array}$

$0.868431711-0.43$

$\begin{array}{ll}0.127736409 & 0.43 \\ 0.544935062 & 0.44\end{array}$

$\begin{array}{ll}0.127736409 & 0.44 \\ 0.544935062 & 0.45\end{array}$

$0.169019101 \quad 0.46$

$\begin{array}{ll}0.789358711 & 0.47 \\ 0.270853439 & 0.48\end{array}$

$\begin{array}{ll}0.22041059 & 0.49\end{array}$

$0.123924758 \quad 0.5$

$\begin{array}{ll}0.237826692 & 0.51 \\ 0.977599243 & 0.52\end{array}$

$0.89435229 \quad 0.53$

$\begin{array}{ll}0.932392678 & 0.54 \\ 0.826242941 & 0.55\end{array}$

$\begin{array}{ll}0.826242941 & 0.55 \\ 0.01427807 & 0.56\end{array}$

$0.980664109 \quad 0.57$

$\begin{array}{ll}0.884728336 & 0.58 \\ 0.80157872 & 0.59\end{array}$

$\begin{array}{ll}0.80157872 & 0.59 \\ 0.871907602 & 0.6\end{array}$

$0.28390891 \quad 0.61$

$0.145132742 \quad 0.62$

$0.551283929 \quad 0.64$

$\begin{array}{ll}0.649820655 & 0.65\end{array}$

$0.28683332 \quad 0.66$

$0.685557413 \quad 0.67$

$\begin{array}{ll}0.819527163 & 0.68 \\ 0.957393096 & 0.69\end{array}$

$\begin{array}{ll}0.825926844 & 0.7\end{array}$

$\begin{array}{ll}0.93624326 & 0.71 \\ 0.020006386 & 0.72\end{array}$

$0.543210751 \quad 0.73$

$\begin{array}{ll}0.42634009 & 0.74 \\ 0.035753836 & 0.75\end{array}$

$\begin{array}{ll}0.172886896 & 0.76\end{array}$

0.77

$\begin{array}{ll}0.634186691 & 0.78 \\ 0.537152993 & 0.79\end{array}$

$\begin{array}{ll}0.537152993 & 0.79 \\ 0.256016057 & 0.8\end{array}$

$\begin{array}{ll}0.426502199 & 0.81 \\ 0.135401998 & 0.82\end{array}$

$\begin{array}{ll}0.135401998 & 0.82 \\ 0.504515487 & 0.83\end{array}$

$0.952643377 \quad 0.84$

$0.782664242-0.85$

$0.20321567 \quad 0.86$

$\begin{array}{ll}0.304526249 & 0.87 \\ 0.833041436 & 0.88\end{array}$

$0.72216723 \quad 0.89$

$\begin{array}{lc}0.88782544 & 0.9 \\ 0.647442094 & 0.91\end{array}$

$0.233712654 \quad 0.92$

$\begin{array}{ll}0.973486476 & 0.93\end{array}$

0.592389104

0.95

fraction of inhibition cumulative fraction of inhibition

(




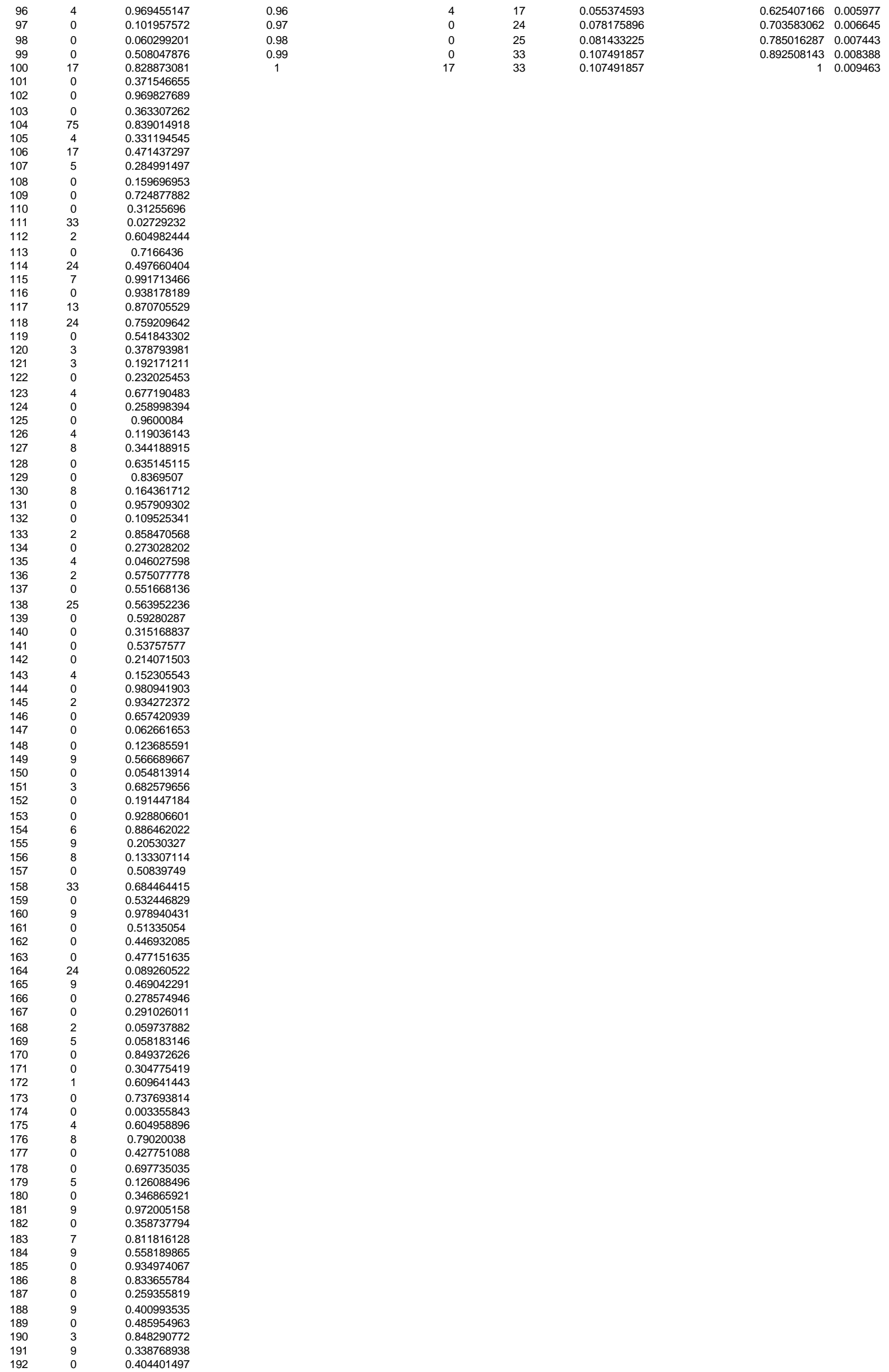




\begin{tabular}{|c|c|}
\hline 193 & 0 \\
\hline 194 & 3 \\
\hline 195 & 1 \\
\hline 196 & 9 \\
\hline 197 & 5 \\
\hline 198 & 2 \\
\hline 199 & 0 \\
\hline 200 & 0 \\
\hline 201 & 0 \\
\hline 202 & 5 \\
\hline 203 & 13 \\
\hline 204 & 8 \\
\hline 205 & 8 \\
\hline 206 & 0 \\
\hline 207 & 0 \\
\hline 208 & 0 \\
\hline 209 & 2 \\
\hline 210 & 17 \\
\hline 211 & 8 \\
\hline 212 & 0 \\
\hline 213 & 4 \\
\hline 214 & 2 \\
\hline 215 & 3 \\
\hline 216 & 0 \\
\hline 217 & 3 \\
\hline 218 & 7 \\
\hline 219 & 17 \\
\hline 220 & 0 \\
\hline 221 & 0 \\
\hline 222 & 5 \\
\hline 223 & 0 \\
\hline 224 & 1 \\
\hline 225 & 25 \\
\hline 226 & 0 \\
\hline 227 & 0 \\
\hline 228 & 2 \\
\hline 229 & 0 \\
\hline 230 & 7 \\
\hline 231 & 4 \\
\hline 232 & 0 \\
\hline 233 & 0 \\
\hline 234 & 0 \\
\hline 235 & 0 \\
\hline 236 & 7 \\
\hline 237 & 1 \\
\hline 238 & 7 \\
\hline 239 & 0 \\
\hline 240 & 0 \\
\hline 241 & 1 \\
\hline 242 & 6 \\
\hline 243 & 0 \\
\hline 244 & 0 \\
\hline 245 & 24 \\
\hline 246 & 0 \\
\hline 247 & 0 \\
\hline 248 & 0 \\
\hline 249 & 0 \\
\hline 250 & 2 \\
\hline 251 & 0 \\
\hline 252 & 0 \\
\hline 253 & 0 \\
\hline 254 & 7 \\
\hline 255 & 0 \\
\hline 256 & 25 \\
\hline 257 & 0 \\
\hline 258 & 9 \\
\hline 259 & 0 \\
\hline 260 & 0 \\
\hline 261 & 0 \\
\hline 262 & 0 \\
\hline 263 & 0 \\
\hline 264 & 0 \\
\hline 265 & 0 \\
\hline 266 & 13 \\
\hline 267 & 1 \\
\hline 268 & 0 \\
\hline 269 & 2 \\
\hline 270 & 0 \\
\hline 271 & 17 \\
\hline 272 & 0 \\
\hline 273 & 0 \\
\hline 274 & 0 \\
\hline 275 & 4 \\
\hline 276 & 0 \\
\hline 277 & 9 \\
\hline 278 & 25 \\
\hline 279 & 0 \\
\hline 280 & 2 \\
\hline 281 & 8 \\
\hline 282 & 0 \\
\hline 283 & 0 \\
\hline 284 & 0 \\
\hline 285 & 6 \\
\hline 286 & 0 \\
\hline 287 & 0 \\
\hline 288 & 0 \\
\hline 289 & 13 \\
\hline
\end{tabular}




\begin{tabular}{|c|c|}
\hline 290 & 0 \\
\hline 291 & 0 \\
\hline 292 & 6 \\
\hline 293 & 6 \\
\hline 294 & 0 \\
\hline 295 & 4 \\
\hline 296 & 0 \\
\hline 297 & 3 \\
\hline 298 & 0 \\
\hline 299 & 5 \\
\hline 300 & 0 \\
\hline 301 & 2 \\
\hline 302 & 4 \\
\hline 303 & 2 \\
\hline 304 & 0 \\
\hline 305 & 0 \\
\hline 306 & 9 \\
\hline 307 & 4 \\
\hline 308 & 0 \\
\hline 309 & 0 \\
\hline 310 & 3 \\
\hline 311 & 0 \\
\hline 312 & 0 \\
\hline 313 & 0 \\
\hline 314 & 0 \\
\hline 315 & 0 \\
\hline 316 & 0 \\
\hline 317 & 9 \\
\hline 318 & 0 \\
\hline 319 & 3 \\
\hline 320 & 0 \\
\hline 321 & 1 \\
\hline 322 & 0 \\
\hline 323 & 0 \\
\hline 324 & 0 \\
\hline 325 & 6 \\
\hline 326 & 8 \\
\hline 327 & 0 \\
\hline 328 & 2 \\
\hline 329 & 0 \\
\hline 330 & 4 \\
\hline 331 & 0 \\
\hline 332 & 13 \\
\hline 333 & 0 \\
\hline 334 & 0 \\
\hline 335 & 0 \\
\hline 336 & 0 \\
\hline 337 & 1 \\
\hline 338 & 75 \\
\hline 339 & 6 \\
\hline 340 & 5 \\
\hline 341 & 0 \\
\hline 342 & 0 \\
\hline 343 & 0 \\
\hline 344 & 0 \\
\hline 345 & 0 \\
\hline 346 & 0 \\
\hline 347 & 24 \\
\hline 348 & 13 \\
\hline 349 & 75 \\
\hline 350 & 4 \\
\hline 351 & 0 \\
\hline 352 & 7 \\
\hline 353 & 3 \\
\hline 354 & 0 \\
\hline 355 & 0 \\
\hline 356 & 0 \\
\hline 357 & 6 \\
\hline 358 & 0 \\
\hline 359 & 0 \\
\hline 360 & 33 \\
\hline 361 & 0 \\
\hline 362 & 7 \\
\hline 363 & 3 \\
\hline 364 & 7 \\
\hline 365 & 0 \\
\hline 366 & 1 \\
\hline 367 & 0 \\
\hline 368 & 0 \\
\hline 369 & 0 \\
\hline 370 & 0 \\
\hline 371 & 0 \\
\hline 372 & 2 \\
\hline 373 & 0 \\
\hline 374 & 0 \\
\hline 375 & 0 \\
\hline 376 & 7 \\
\hline 377 & 0 \\
\hline 378 & 0 \\
\hline 379 & 9 \\
\hline 380 & 0 \\
\hline 381 & 8 \\
\hline 382 & 0 \\
\hline 383 & 0 \\
\hline 384 & 2 \\
\hline 385 & 0 \\
\hline 386 & 0 \\
\hline
\end{tabular}




\begin{tabular}{|c|c|}
\hline 387 & 1 \\
\hline 388 & 0 \\
\hline 389 & 9 \\
\hline 390 & 0 \\
\hline 391 & 0 \\
\hline 392 & 1 \\
\hline 393 & 8 \\
\hline 394 & 0 \\
\hline 395 & 2 \\
\hline 396 & 0 \\
\hline 397 & 0 \\
\hline 398 & 2 \\
\hline 399 & 0 \\
\hline 400 & 0 \\
\hline 401 & 75 \\
\hline 402 & 0 \\
\hline 403 & 0 \\
\hline 404 & 8 \\
\hline 405 & 2 \\
\hline 406 & 4 \\
\hline 407 & 0 \\
\hline 408 & 0 \\
\hline 409 & 0 \\
\hline 410 & 0 \\
\hline 411 & 0 \\
\hline 412 & 0 \\
\hline 413 & 0 \\
\hline 414 & 0 \\
\hline 415 & 9 \\
\hline 416 & 0 \\
\hline 417 & 2 \\
\hline 418 & 0 \\
\hline 419 & 5 \\
\hline 420 & 0 \\
\hline 421 & 13 \\
\hline 422 & 4 \\
\hline 423 & 0 \\
\hline 424 & 0 \\
\hline 425 & 1 \\
\hline 426 & 75 \\
\hline 427 & 75 \\
\hline 428 & 0 \\
\hline 429 & 0 \\
\hline 430 & 7 \\
\hline 431 & 0 \\
\hline 432 & 0 \\
\hline 433 & 0 \\
\hline 434 & 0 \\
\hline 435 & 0 \\
\hline 436 & 0 \\
\hline 437 & 8 \\
\hline 438 & 0 \\
\hline 439 & 0 \\
\hline 440 & 4 \\
\hline 441 & 1 \\
\hline 442 & 0 \\
\hline 443 & 9 \\
\hline 444 & 3 \\
\hline 445 & 0 \\
\hline 446 & 0 \\
\hline 447 & 0 \\
\hline 448 & 0 \\
\hline 449 & 0 \\
\hline 450 & 0 \\
\hline 451 & 5 \\
\hline 452 & 7 \\
\hline 453 & 4 \\
\hline 454 & 8 \\
\hline 455 & 0 \\
\hline 456 & 8 \\
\hline 457 & 0 \\
\hline 458 & 0 \\
\hline 459 & 4 \\
\hline 460 & 0 \\
\hline 461 & 0 \\
\hline 462 & 0 \\
\hline 463 & 4 \\
\hline 464 & 0 \\
\hline 465 & 0 \\
\hline 466 & 9 \\
\hline 467 & 0 \\
\hline 468 & 0 \\
\hline 469 & 0 \\
\hline 470 & 0 \\
\hline 471 & 6 \\
\hline 472 & 5 \\
\hline 473 & 0 \\
\hline 474 & 0 \\
\hline 475 & 0 \\
\hline 476 & 0 \\
\hline 477 & 0 \\
\hline 478 & 0 \\
\hline 479 & 0 \\
\hline 480 & 3 \\
\hline 481 & 9 \\
\hline 482 & 1 \\
\hline 483 & 0 \\
\hline
\end{tabular}




$\begin{array}{lcl}484 & 0 & 0.210786074 \\ 485 & 13 & 0.510184708 \\ 486 & 4 & 0.487940227 \\ 487 & 3 & 0.650186856 \\ 488 & 0 & 0.803103003 \\ 489 & 0 & 0.403274802 \\ 490 & 33 & 0.759653851 \\ 491 & 0 & 0.537957433 \\ 492 & 3 & 0.415850135 \\ 493 & 17 & 0.312173505 \\ 494 & 0 & 0.924501082 \\ 495 & 0 & 0.974885314 \\ 496 & 13 & 0.695173185 \\ 497 & 0 & 0.972998537 \\ 498 & 0 & 0.346371245 \\ 499 & 0 & 0.654223869 \\ 500 & 7 & 0.411999293 \\ 501 & 3 & 0.15150364 \\ 502 & 0 & 0.192107874 \\ 503 & 2 & 0.980172862 \\ 504 & 6 & 0.95389705 \\ 505 & 8 & 0.337712703 \\ 506 & 0 & 0.980413708 \\ 507 & 25 & 0.307764025 \\ 508 & 0 & 0.758136749 \\ 509 & 0 & 0.925548967 \\ 510 & 3 & 0.446528368\end{array}$


PD184352; $10 \mu \mathrm{M} ; 10 \mu \mathrm{M}$ ATP; sample of 50 kinases

position inhibition\% rand cumulative sample fraction sample inhibition

sorted

fraction of inhibition cumulative fraction of inhibition

0.725752545

0.02

$0.736237148-0.06$

$0.271078996 \quad 0.08$

0.112232263

0.006311528

0.582764159

0.29661883

0.86384846

0.268090161

0.964091753

0.90571296

0.715141656

0.188594292
0.091865632

0.091865632
0.416234251

0.038422172

0.471241523
0.048265943

0.090332712

0.665742152

0.665742152
0.73743861

0.768849308

0.559097152

0.910071467

0.920535765
0.368953934

0.145581101

0.926449762

0.314262421

0.331575364

0.115344665

0.900679199

0.470438995
0.219255763

0.219255763
0.494704122

0.446392689

0.215704818

0.899381946

0.66322041

0.880323519

0.362355309

0.201053106

0.341767484

0.241141495
0.843376509

0.089827396

0.311288321

0.138669088

0.48998599

0.778986809

0.259561076

0.377364838

0.996015638

0.034236277
0.570732351

0.01004359

0.76717673

0.308154156

0.206065437

0.112970729

0.617382742

0.27005694

0.760728461
0.056619639

0.056619639
0.743861461

0.82347103

0.968312822

0.537896065

0.547757804

0.525174421

0.941152321

0.180937111
0.456543526

0.456543526
0.635461556

0.537946898

0.755538008

0.235070965

0.193664118

0.355821788

0.725487876

0.581497386

0.122423897
0.617746198

0.617746198
0.249023676

0.249023676
0.481785037

0.72335498

0.247625275

0.649245801 


\begin{tabular}{|c|c|}
\hline 96 & 4 \\
\hline 97 & 8 \\
\hline 98 & 0 \\
\hline 99 & 0 \\
\hline 100 & 0 \\
\hline 101 & 0 \\
\hline 102 & 0 \\
\hline 103 & 6 \\
\hline 104 & 0 \\
\hline 105 & 9 \\
\hline 106 & 9 \\
\hline 107 & 0 \\
\hline 108 & 0 \\
\hline 109 & 0 \\
\hline 110 & 0 \\
\hline 111 & 17 \\
\hline 112 & 75 \\
\hline 113 & 1 \\
\hline 114 & 0 \\
\hline 115 & 0 \\
\hline 116 & 0 \\
\hline 117 & 75 \\
\hline 118 & 3 \\
\hline 119 & 0 \\
\hline 120 & 0 \\
\hline 121 & 2 \\
\hline 122 & 2 \\
\hline 123 & 0 \\
\hline 124 & 33 \\
\hline 125 & 0 \\
\hline 126 & 0 \\
\hline 127 & 0 \\
\hline 128 & 0 \\
\hline 129 & 9 \\
\hline 130 & 0 \\
\hline 131 & 0 \\
\hline 132 & 0 \\
\hline 133 & 0 \\
\hline 134 & 6 \\
\hline 135 & 9 \\
\hline 136 & 0 \\
\hline 137 & 8 \\
\hline 138 & 8 \\
\hline 139 & 17 \\
\hline 140 & 0 \\
\hline 141 & 0 \\
\hline 142 & 0 \\
\hline 143 & 2 \\
\hline 144 & 8 \\
\hline 145 & 0 \\
\hline 146 & 2 \\
\hline 147 & 0 \\
\hline 148 & 13 \\
\hline 149 & 13 \\
\hline 150 & 6 \\
\hline 151 & 0 \\
\hline 152 & 9 \\
\hline 153 & 3 \\
\hline 154 & 7 \\
\hline 155 & 0 \\
\hline 156 & 0 \\
\hline 157 & 4 \\
\hline 158 & 0 \\
\hline 159 & 0 \\
\hline 160 & 0 \\
\hline 161 & 4 \\
\hline 162 & 3 \\
\hline 163 & 0 \\
\hline 164 & 0 \\
\hline 165 & 0 \\
\hline 166 & 9 \\
\hline 167 & 0 \\
\hline 168 & 3 \\
\hline 169 & 0 \\
\hline 170 & 25 \\
\hline 171 & 17 \\
\hline 172 & 5 \\
\hline 173 & 17 \\
\hline 174 & 9 \\
\hline 175 & 2 \\
\hline 176 & 0 \\
\hline 177 & 7 \\
\hline 178 & 0 \\
\hline 179 & 0 \\
\hline 180 & 3 \\
\hline 181 & 3 \\
\hline 182 & 0 \\
\hline 183 & 9 \\
\hline 184 & 0 \\
\hline 185 & 3 \\
\hline 186 & 0 \\
\hline 187 & 0 \\
\hline 188 & 7 \\
\hline 189 & 0 \\
\hline 190 & 0 \\
\hline 191 & 8 \\
\hline 192 & \\
\hline
\end{tabular}




\begin{tabular}{|c|c|}
\hline 193 & 0 \\
\hline 194 & 24 \\
\hline 195 & 0 \\
\hline 196 & 0 \\
\hline 197 & 33 \\
\hline 198 & 0 \\
\hline 199 & 0 \\
\hline 200 & 0 \\
\hline 201 & 0 \\
\hline 202 & 1 \\
\hline 203 & 0 \\
\hline 204 & 4 \\
\hline 205 & 75 \\
\hline 206 & 8 \\
\hline 207 & 9 \\
\hline 208 & 2 \\
\hline 209 & 0 \\
\hline 210 & 0 \\
\hline 211 & 0 \\
\hline 212 & 0 \\
\hline 213 & 0 \\
\hline 214 & 0 \\
\hline 215 & 0 \\
\hline 216 & 0 \\
\hline 217 & 2 \\
\hline 218 & 0 \\
\hline 219 & 0 \\
\hline 220 & 0 \\
\hline 221 & 0 \\
\hline 222 & 1 \\
\hline 223 & 0 \\
\hline 224 & 0 \\
\hline 225 & 0 \\
\hline 226 & 0 \\
\hline 227 & 0 \\
\hline 228 & 2 \\
\hline 229 & 0 \\
\hline 230 & 0 \\
\hline 231 & 0 \\
\hline 232 & 0 \\
\hline 233 & 0 \\
\hline 234 & 0 \\
\hline 235 & 0 \\
\hline 236 & 0 \\
\hline 237 & 0 \\
\hline 238 & 4 \\
\hline 239 & 3 \\
\hline 240 & 2 \\
\hline 241 & 0 \\
\hline 242 & 4 \\
\hline 243 & 0 \\
\hline 244 & 0 \\
\hline 245 & 0 \\
\hline 246 & 0 \\
\hline 247 & 9 \\
\hline 248 & 0 \\
\hline 249 & 0 \\
\hline 250 & 0 \\
\hline 251 & 6 \\
\hline 252 & 3 \\
\hline 253 & 0 \\
\hline 254 & 0 \\
\hline 255 & 0 \\
\hline 256 & 3 \\
\hline 257 & 1 \\
\hline 258 & 3 \\
\hline 259 & 0 \\
\hline 260 & 0 \\
\hline 261 & 0 \\
\hline 262 & 4 \\
\hline 263 & 0 \\
\hline 264 & 2 \\
\hline 265 & 2 \\
\hline 266 & 13 \\
\hline 267 & 0 \\
\hline 268 & 2 \\
\hline 269 & 0 \\
\hline 270 & 0 \\
\hline 271 & 0 \\
\hline 272 & 0 \\
\hline 273 & 0 \\
\hline 274 & 0 \\
\hline 275 & 5 \\
\hline 276 & 0 \\
\hline 277 & 4 \\
\hline 278 & 0 \\
\hline 279 & 0 \\
\hline 280 & 0 \\
\hline 281 & 6 \\
\hline 282 & 0 \\
\hline 283 & 0 \\
\hline 284 & 0 \\
\hline 285 & 0 \\
\hline 286 & 0 \\
\hline 287 & 0 \\
\hline 288 & 0 \\
\hline 289 & 5 \\
\hline
\end{tabular}




\begin{tabular}{|c|c|}
\hline 290 & 0 \\
\hline 291 & 0 \\
\hline 292 & 7 \\
\hline 293 & 0 \\
\hline 294 & 5 \\
\hline 295 & 9 \\
\hline 296 & 8 \\
\hline 297 & 7 \\
\hline 298 & 0 \\
\hline 299 & 13 \\
\hline 300 & 9 \\
\hline 301 & 6 \\
\hline 302 & 0 \\
\hline 303 & 0 \\
\hline 304 & 0 \\
\hline 305 & 24 \\
\hline 306 & 8 \\
\hline 307 & 1 \\
\hline 308 & 0 \\
\hline 309 & 0 \\
\hline 310 & 0 \\
\hline 311 & 5 \\
\hline 312 & 25 \\
\hline 313 & 0 \\
\hline 314 & 0 \\
\hline 315 & 0 \\
\hline 316 & 1 \\
\hline 317 & 0 \\
\hline 318 & 0 \\
\hline 319 & 0 \\
\hline 320 & 0 \\
\hline 321 & 4 \\
\hline 322 & 0 \\
\hline 323 & 0 \\
\hline 324 & 0 \\
\hline 325 & 3 \\
\hline 326 & 0 \\
\hline 327 & 9 \\
\hline 328 & 0 \\
\hline 329 & 8 \\
\hline 330 & 7 \\
\hline 331 & 0 \\
\hline 332 & 1 \\
\hline 333 & 0 \\
\hline 334 & 4 \\
\hline 335 & 0 \\
\hline 336 & 0 \\
\hline 337 & 0 \\
\hline 338 & 0 \\
\hline 339 & 0 \\
\hline 340 & 0 \\
\hline 341 & 0 \\
\hline 342 & 0 \\
\hline 343 & 0 \\
\hline 344 & 0 \\
\hline 345 & 3 \\
\hline 346 & 1 \\
\hline 347 & 0 \\
\hline 348 & 0 \\
\hline 349 & 9 \\
\hline 350 & 1 \\
\hline 351 & 0 \\
\hline 352 & 0 \\
\hline 353 & 0 \\
\hline 354 & 6 \\
\hline 355 & 0 \\
\hline 356 & 2 \\
\hline 357 & 0 \\
\hline 358 & 0 \\
\hline 359 & 0 \\
\hline 360 & 0 \\
\hline 361 & 0 \\
\hline 362 & 0 \\
\hline 363 & 4 \\
\hline 364 & 8 \\
\hline 365 & 13 \\
\hline 366 & 0 \\
\hline 367 & 4 \\
\hline 368 & 0 \\
\hline 369 & 0 \\
\hline 370 & 3 \\
\hline 371 & 0 \\
\hline 372 & 0 \\
\hline 373 & 3 \\
\hline 374 & 0 \\
\hline 375 & 0 \\
\hline 376 & 4 \\
\hline 377 & 0 \\
\hline 378 & 0 \\
\hline 379 & 7 \\
\hline 380 & 0 \\
\hline 381 & 0 \\
\hline 382 & 8 \\
\hline 383 & 0 \\
\hline 384 & 9 \\
\hline 385 & 5 \\
\hline 386 & 0 \\
\hline
\end{tabular}




\begin{tabular}{|c|c|}
\hline 387 & 2 \\
\hline 388 & 5 \\
\hline 389 & 17 \\
\hline 390 & 9 \\
\hline 391 & 0 \\
\hline 392 & 0 \\
\hline 393 & 0 \\
\hline 394 & 0 \\
\hline 395 & 8 \\
\hline 396 & 9 \\
\hline 397 & 1 \\
\hline 398 & 7 \\
\hline 399 & 13 \\
\hline 400 & 0 \\
\hline 401 & 0 \\
\hline 402 & 0 \\
\hline 403 & 0 \\
\hline 404 & 0 \\
\hline 405 & 25 \\
\hline 406 & 3 \\
\hline 407 & 1 \\
\hline 408 & 24 \\
\hline 409 & 5 \\
\hline 410 & 7 \\
\hline 411 & 2 \\
\hline 412 & 9 \\
\hline 413 & 3 \\
\hline 414 & 0 \\
\hline 415 & 75 \\
\hline 416 & 0 \\
\hline 417 & 33 \\
\hline 418 & 0 \\
\hline 419 & 0 \\
\hline 420 & 8 \\
\hline 421 & 4 \\
\hline 422 & 2 \\
\hline 423 & 0 \\
\hline 424 & 13 \\
\hline 425 & 0 \\
\hline 426 & 0 \\
\hline 427 & 0 \\
\hline 428 & 0 \\
\hline 429 & 2 \\
\hline 430 & 0 \\
\hline 431 & 0 \\
\hline 432 & 0 \\
\hline 433 & 0 \\
\hline 434 & 0 \\
\hline 435 & 7 \\
\hline 436 & 4 \\
\hline 437 & 25 \\
\hline 438 & 8 \\
\hline 439 & 1 \\
\hline 440 & 0 \\
\hline 441 & 7 \\
\hline 442 & 3 \\
\hline 443 & 0 \\
\hline 444 & 0 \\
\hline 445 & 5 \\
\hline 446 & 6 \\
\hline 447 & 7 \\
\hline 448 & 0 \\
\hline 449 & 0 \\
\hline 450 & 75 \\
\hline 451 & 0 \\
\hline 452 & 4 \\
\hline 453 & 0 \\
\hline 454 & 0 \\
\hline 455 & 0 \\
\hline 456 & 0 \\
\hline 457 & 4 \\
\hline 458 & 7 \\
\hline 459 & 4 \\
\hline 460 & 0 \\
\hline 461 & 24 \\
\hline 462 & 0 \\
\hline 463 & 13 \\
\hline 464 & 0 \\
\hline 465 & 0 \\
\hline 466 & 0 \\
\hline 467 & 0 \\
\hline 468 & 9 \\
\hline 469 & 0 \\
\hline 470 & 0 \\
\hline 471 & 7 \\
\hline 472 & 2 \\
\hline 473 & 24 \\
\hline 474 & 0 \\
\hline 475 & 0 \\
\hline 476 & 0 \\
\hline 477 & 0 \\
\hline 478 & 0 \\
\hline 479 & 0 \\
\hline 480 & 3 \\
\hline 481 & 0 \\
\hline 482 & 13 \\
\hline 483 & 0 \\
\hline
\end{tabular}




$\begin{array}{lcl}484 & 17 & 0.072753453 \\ 485 & 13 & 0.803965391 \\ 486 & 0 & 0.862782782 \\ 487 & 0 & 0.330894729 \\ 488 & 0 & 0.268650466 \\ 489 & 0 & 0.151801123 \\ 490 & 4 & 0.699957172 \\ 491 & 0 & 0.798126877 \\ 492 & 33 & 0.991531694 \\ 493 & 24 & 0.249960595 \\ 494 & 0 & 0.713146829 \\ 495 & 0 & 0.663770179 \\ 496 & 0 & 0.581542934 \\ 497 & 0 & 0.78849267 \\ 498 & 0 & 0.363024776 \\ 499 & 1 & 0.136921156 \\ 500 & 6 & 0.908276522 \\ 501 & 7 & 0.496717327 \\ 502 & 0 & 0.280213831 \\ 503 & 0 & 0.523389284 \\ 504 & 0 & 0.674888915 \\ 505 & 0 & 0.419459296 \\ 506 & 4 & 0.618760484 \\ 507 & 8 & 0.339724742 \\ 508 & 0 & 0.40299441 \\ 509 & 0 & 0.090159109 \\ 510 & 0 & 0.790869944\end{array}$


Distribution testing based on Gini coefficients calculated for a subset of 50 kinases inhibited by $10 \mu \mathrm{M}$ AG1024 at $10 \mu \mathrm{M}$ ATP

List of the calculated Gini coefficients as a bar graph and a table with +/-SD and +/-2SD ranges marked

Spreadsheet for calculation of the Gini values

S218-S223

Macro used in the spreadsheet

S224-S225

Analysis of distribution using the GenStat statistical package

S226-S228 
Distribution testing based on Gini coefficients calculated for a subset of 50 kinases inhibited by $10 \mu \mathrm{M}$ AG1024 at $10 \mu \mathrm{M}$ ATP

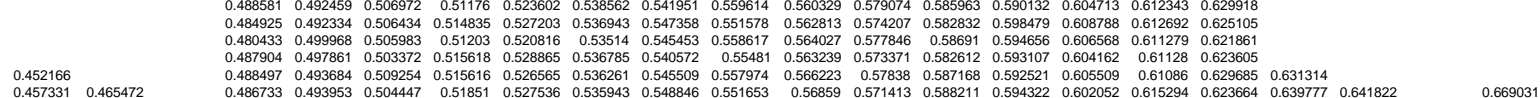

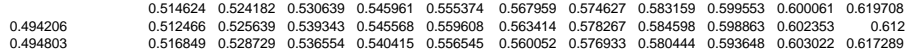

$\begin{array}{llllllllllll}0.491396 & 0.512219 & 0.523195 & 0.533714 & 0.54083 & 0.552 & 0.569164 & 0.570441 & 0.589486 & 0.597278 & 0.600548 & 0.618019 \\ 0.498417 & 0.514648 & 0.520268 & 0.538702 & 0.540761 & 0.551794 & 0.561907 & 0.5799 & 0.583781 & 0.59833 & 0.601558 & 0.610408\end{array}$

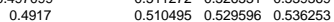

$\begin{array}{lllllllllllllll}0.4917 & 0.510495 & 0.529596 & 0.536253 & 0.542938 & 0.555781 & 0.569718 & 0.571769 & 0.5838577 & 0.594393 & 0.607323 & 0.613827 & 0.625777 \\ 0.496109 & 0.50431 & 0.513775 & 0.528869 & 0.53028 & 0.546302 & 0.55963 & 0.569335 & 0.570632 & 0.586711 & 0.591648 & 0.603345 & 0.612261 & 0.624735\end{array}$

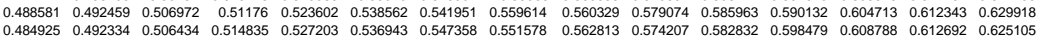

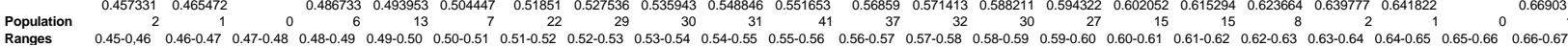

\begin{tabular}{|c|c|c|c|c|c|c|}
\hline $\begin{array}{l}0.452166 \\
0.457331 \\
0.465472 \\
0.480433 \\
0.484925 \\
\end{array}$ & $\begin{array}{l}0.51878 \\
0.5220268 \\
0.520349 \\
0.520816 \\
0.521576\end{array}$ & $\begin{array}{l}0.533785 \\
0.536943 \\
0.533562 \\
0.538678 \\
0.538702\end{array}$ & $\begin{array}{l}0.553598 \\
0.553601 \\
0.553824 \\
0.554281 \\
.5544417\end{array}$ & $\begin{array}{l}0.5665575 \\
0.565918 \\
0.566223 \\
0.566278 \\
0.566362\end{array}$ & $\begin{array}{l}0.5799 \\
0.580316 \\
0.580444 \\
0.582612 \\
0.582621\end{array}$ & $\begin{array}{c}0 \\
0 \\
0.5 \\
0.5\end{array}$ \\
\hline & & & & & & \\
\hline 88904 & & & $\begin{array}{l}0.554536 \\
0.554552\end{array}$ & & & \\
\hline & & & & & & \\
\hline & & & & & & \\
\hline 2334 & 24834 & & & & & \\
\hline & 0.524868 & & & & & \\
\hline & 0.524983 & & & & & \\
\hline & & & & & & \\
\hline & & & & & & \\
\hline & & & & & & \\
\hline & 0.527203 & & & & & \\
\hline & 0.5 & & & & & \\
\hline & & & & & & \\
\hline & & & & & & \\
\hline & & & & & & \\
\hline & & & & & & \\
\hline & 0. & & & & & \\
\hline & & & & & & \\
\hline & & & & & & \\
\hline & & & & & & \\
\hline & & & & & & \\
\hline & & & & & & \\
\hline & & & & & & \\
\hline & & & & & & \\
\hline & & & & & & \\
\hline & & & & & & \\
\hline & & & & & & \\
\hline & & & & & & \\
\hline & & & & & & \\
\hline & & & & & & \\
\hline & & & & & & \\
\hline & & & & & & \\
\hline & & & & & & \\
\hline & & & & & & \\
\hline & & & & & & \\
\hline & & & & & $\begin{array}{l}0.597278 \\
0.57925\end{array}$ & \\
\hline
\end{tabular}

$\begin{array}{lr}\text { Mean } & \text { J.55818 } \\ \text { St. } & 0.035706\end{array}$

$\begin{array}{cc}\text { mean+SD } & 0.593886 \\ \text { mean-SD } & 0.522474\end{array}$

mean+2SD 0.629592
mean-2SD 0.486768

\begin{tabular}{lrr} 
datapoints & number & percentage \\
within $+<-1$ SD & 350 & 100 \\
within +-2 SD & 207 & 59 \\
\hline & 340 & 97
\end{tabular} 
AG1024; $10 \mu \mathrm{M}$; $10 \mu \mathrm{M}$ ATP; sample of 50 kinases

position inhibition\% rand cumulative sample fraction sample inhibition

sorted

fraction of inhibition cumulative fraction of inhibition area $0.462121398-0.06$

$0.271516182 \quad 0.08$

0.682221017

0.961148247

0.744478083

0.948151759

0.06

0.1

0.12

0.14

$0.491302435-0.22$

0.724

$0.743422679-0.26$

$\begin{array}{ll}0.36079054 & 0.28 \\ 0.740391126 & 0.3\end{array}$

$0.1654795 \quad 0.32$

$\begin{array}{ll}0.369696425 & 0.34 \\ 0.205549758 & 0.36\end{array}$

$\begin{array}{ll}0.205549758 & 0.36 \\ 0.303704405 & 0.38\end{array}$

$0.563814158 \quad 0.4$

$0.631889426 \quad 0.42$

$\begin{array}{ll}0.236875919 & 0.44 \\ 0.617305017 & 0.46\end{array}$

$\begin{array}{ll}0.617305017 & 0.46 \\ 0.650050029 & 0.48\end{array}$

$0.892700985 \quad 0.5$

$0.269955994 \quad 0.52$

$\begin{array}{ll}0.511820583 & 0.54 \\ 0.665805383 & 0.56\end{array}$

$\begin{array}{ll}0.574706022 & 0.58\end{array}$

$0.25438952 \quad 0.6$

$\begin{array}{ll}0.724835772 & 0.62 \\ 0.781896734 & 0.64\end{array}$

$\begin{array}{ll}0.400472743 & 0.64 \\ 0.23261132 & 0.66\end{array}$

$\begin{array}{ll}0.400472743 & 0.66 \\ 0.232681132 & 0.68\end{array}$

$0.264574376 \quad 0.7$

$\begin{array}{ll}0.272273227 & 0.72 \\ 0.174341023 & 0.74\end{array}$

$\begin{array}{ll}0.174341023 & 0.74 \\ 0.03239866 & 0.76\end{array}$

$\begin{array}{ll}0.755669001 & 0.78\end{array}$

$\begin{array}{ll}0.293361516 & 0.8\end{array}$

$\begin{array}{ll}0.801988973 & 0.82 \\ 0.231315189 & 0.84\end{array}$

$0.251524821 \quad 0.86$

$0.401669402 \quad 0.88$

$\begin{array}{ll}0.675061256 & 0.9 \\ 0.056502252 & 0.92\end{array}$

$0.603179107 \quad 0.94$

$0.32500089 \quad 0.96$

$\begin{array}{lc}0.63901262 & 0.98 \\ 0.030855491 & 1\end{array}$

0.866353497

0.276452188

0.775675388

0.423693313

0.71433326
0.487433218

0.487433218
0.033453516

0.033453516
0.027305053

0.027305053

0.023586688

0.156524546

0.035208881

0.442693794

0.784990811

0.903228795

0.532943937

0.531175937
0.335189776

0.335189776

0.926665036

0.896479074

0.000736338

0.060977503

0.445192109

0.750512397

0.452793258

0.288257879

0.627502164

0.511850639

0.862682578

0.765559908

0.275813679

0.875183432

0.29385039

0.667323398

0.000801307

0.275311795

0.761291208
0.032189022

0.032189022
0.702750814

0.12528224

0.656410803

0.008301887

0.008301887

0.009056604

0.009811321

.012075472

0.012075472

0.012075472

0.013584906

0.018113208

0.018113208

0.018113208

0.018867925

0.018867925

0.019622642

0.020377358

0.022641509

0.02490566

0.02490566

0.030188679

0.030188679

0.036226415

0.048301887

0.068679245

0.071698113

0.071698113

0.07245283

0.07245283
0.074716981

$0.000754717 \quad 7.55 \mathrm{E}-06$ $0.003773585 \quad 4.53 \mathrm{E}-05$ $\begin{array}{ll}0.006792453 & 0.000106 \\ 0.012075472 & 0.000189\end{array}$ $\begin{array}{ll}0.012075472 & 0.000189 \\ 0.017358491 & 0.000294\end{array}$ $\begin{array}{ll}0.017358491 & 0.000294 \\ 0.024150943 & 0.000415\end{array}$ $\begin{array}{ll}0.030943396 & 0.000551\end{array}$ $0.038490566 \quad 0.000694$ $\begin{array}{lll}0.046037736 & 0.000845\end{array}$ $\begin{array}{ll}0.054339623 & 0.001004\end{array}$ $\begin{array}{ll}0.062641509 & 0.00117\end{array}$ $\begin{array}{ll}0.071698113 & 0.001343\end{array}$ $\begin{array}{ll}0.071698113 & 0.001343 \\ 0.081509434 & 0.001532\end{array}$ $\begin{array}{ll}0.081509434 & 0.001532 \\ 0.093584906 & 0.001751\end{array}$ 0.1056603770 .001992 $\begin{array}{lll}0.117735849 & 0.002234\end{array}$ $\begin{array}{ll}0.131320755 & 0.002491\end{array}$ $\begin{array}{ll}0.147924528 & 0.002792\end{array}$ $\begin{array}{ll}0.166037736 & 0.00314\end{array}$ 0.1841509430 .003502 $\begin{array}{lll}0.202264151 & 0.003864\end{array}$ $\begin{array}{lll}0.221132075 & 0.004234\end{array}$ $\begin{array}{rr}0.221132075 & 0.004234 \\ 0.24 & 0.004611\end{array}$ $\begin{array}{rr}0.24 & 0.004611 \\ 0.259622642 & 0.004996\end{array}$ $0.28 \quad 0.005396$ 0.3026415090 .005826 $0.32754717 \quad 0.006302$ $\begin{array}{ll}0.35245283 & 0.0068\end{array}$ 0.3826415090 .007351 $\begin{array}{ll}0.412830189 & 0.007955 \\ 0.449056604 & 0.008619\end{array}$ $\begin{array}{lll}0.449056604 & 0.008619\end{array}$ $\begin{array}{lll}0.497358491 & 0.009464\end{array}$ $\begin{array}{ll}0.466037736 & 0.010634\end{array}$ $\begin{array}{lr}0.636981132 & 0.01203\end{array}$ 0.7086792450 .013457 $0.780377358 \quad 0.014891$ 0.8528301890 .016332 $\begin{array}{rr}0.925283019 & 0.017781 \\ 1 & 0.019253\end{array}$ 


\begin{tabular}{|c|c|}
\hline 96 & 11 \\
\hline 97 & 21 \\
\hline 98 & 24 \\
\hline 99 & 0 \\
\hline 100 & 0 \\
\hline 101 & 7 \\
\hline 102 & 77 \\
\hline 103 & 0 \\
\hline 104 & 12 \\
\hline 105 & 0 \\
\hline 106 & 18 \\
\hline 107 & 9 \\
\hline 108 & 13 \\
\hline 109 & 11 \\
\hline 110 & 27 \\
\hline 111 & 48 \\
\hline 112 & 13 \\
\hline 113 & 26 \\
\hline 114 & 10 \\
\hline 115 & 10 \\
\hline 116 & 30 \\
\hline 117 & 94 \\
\hline 118 & 13 \\
\hline 119 & 15 \\
\hline 120 & 11 \\
\hline 121 & 13 \\
\hline 122 & 26 \\
\hline 123 & 12 \\
\hline 124 & 14 \\
\hline 125 & 12 \\
\hline 126 & 11 \\
\hline 127 & 0 \\
\hline 128 & 9 \\
\hline 129 & 19 \\
\hline 130 & 26 \\
\hline 131 & 16 \\
\hline 132 & 22 \\
\hline 133 & 16 \\
\hline 134 & 0 \\
\hline 135 & 91 \\
\hline 136 & 30 \\
\hline 137 & 3 \\
\hline 138 & 16 \\
\hline 139 & 6 \\
\hline 140 & 10 \\
\hline 141 & 14 \\
\hline 142 & 14 \\
\hline 143 & 31 \\
\hline 144 & 42 \\
\hline 145 & 94 \\
\hline 146 & 0 \\
\hline 147 & 4 \\
\hline 148 & 4 \\
\hline 149 & 11 \\
\hline 150 & 28 \\
\hline 151 & 91 \\
\hline 152 & 37 \\
\hline 153 & 13 \\
\hline 154 & 11 \\
\hline 155 & 26 \\
\hline 156 & 6 \\
\hline 157 & 8 \\
\hline 158 & 11 \\
\hline 159 & 12 \\
\hline 160 & 19 \\
\hline 161 & 21 \\
\hline 162 & 68 \\
\hline 163 & 28 \\
\hline 164 & 16 \\
\hline 165 & 91 \\
\hline 166 & 0 \\
\hline 167 & 11 \\
\hline 168 & 3 \\
\hline 169 & 0 \\
\hline 170 & 4 \\
\hline 171 & 11 \\
\hline 172 & 0 \\
\hline 173 & 11 \\
\hline 174 & 4 \\
\hline 175 & 96 \\
\hline 176 & 33 \\
\hline 177 & 95 \\
\hline 178 & 0 \\
\hline 179 & 8 \\
\hline 180 & 11 \\
\hline 181 & 42 \\
\hline 182 & 42 \\
\hline 183 & 68 \\
\hline 184 & 42 \\
\hline 185 & 40 \\
\hline 186 & 4 \\
\hline 187 & 9 \\
\hline 188 & 13 \\
\hline 189 & 13 \\
\hline 190 & 77 \\
\hline 191 & 94 \\
\hline 192 & 30 \\
\hline
\end{tabular}




\begin{tabular}{|c|c|}
\hline 193 & 9 \\
\hline 194 & 91 \\
\hline 195 & 28 \\
\hline 196 & 14 \\
\hline 197 & 42 \\
\hline 198 & 21 \\
\hline 199 & 4 \\
\hline 200 & 14 \\
\hline 201 & 10 \\
\hline 202 & 12 \\
\hline 203 & 1 \\
\hline 204 & 25 \\
\hline 205 & 4 \\
\hline 206 & 16 \\
\hline 207 & 3 \\
\hline 208 & 0 \\
\hline 209 & 9 \\
\hline 210 & 13 \\
\hline 211 & 94 \\
\hline 212 & 99 \\
\hline 213 & 95 \\
\hline 214 & 7 \\
\hline 215 & 0 \\
\hline 216 & 1 \\
\hline 217 & 27 \\
\hline 218 & 14 \\
\hline 219 & 6 \\
\hline 220 & 12 \\
\hline 221 & 31 \\
\hline 222 & 11 \\
\hline 223 & 18 \\
\hline 224 & 11 \\
\hline 225 & 16 \\
\hline 226 & 30 \\
\hline 227 & 9 \\
\hline 228 & 2 \\
\hline 229 & 8 \\
\hline 230 & 48 \\
\hline 231 & 12 \\
\hline 232 & 96 \\
\hline 233 & 11 \\
\hline 234 & 7 \\
\hline 235 & 11 \\
\hline 236 & 0 \\
\hline 237 & 42 \\
\hline 238 & 0 \\
\hline 239 & 27 \\
\hline 240 & 77 \\
\hline 241 & 0 \\
\hline 242 & 99 \\
\hline 243 & 11 \\
\hline 244 & 96 \\
\hline 245 & 33 \\
\hline 246 & 0 \\
\hline 247 & 0 \\
\hline 248 & 68 \\
\hline 249 & 42 \\
\hline 250 & 94 \\
\hline 251 & 22 \\
\hline 252 & 0 \\
\hline 253 & 48 \\
\hline 254 & 0 \\
\hline 255 & 0 \\
\hline 256 & 33 \\
\hline 257 & 7 \\
\hline 258 & 9 \\
\hline 259 & 7 \\
\hline 260 & 12 \\
\hline 261 & 40 \\
\hline 262 & 1 \\
\hline 263 & 37 \\
\hline 264 & 8 \\
\hline 265 & 14 \\
\hline 266 & 33 \\
\hline 267 & 4 \\
\hline 268 & 28 \\
\hline 269 & 16 \\
\hline 270 & 0 \\
\hline 271 & 31 \\
\hline 272 & 0 \\
\hline 273 & 1 \\
\hline 274 & 64 \\
\hline 275 & 28 \\
\hline 276 & 8 \\
\hline 277 & 10 \\
\hline 278 & 10 \\
\hline 279 & 33 \\
\hline 280 & 13 \\
\hline 281 & 14 \\
\hline 282 & 0 \\
\hline 283 & 2 \\
\hline 284 & 40 \\
\hline 285 & 3 \\
\hline 286 & 76 \\
\hline 287 & 1 \\
\hline 288 & 7 \\
\hline 289 & 28 \\
\hline
\end{tabular}




\begin{tabular}{|c|c|}
\hline 290 & 8 \\
\hline 291 & 77 \\
\hline 292 & 16 \\
\hline 293 & 19 \\
\hline 294 & 16 \\
\hline 295 & 3 \\
\hline 296 & 0 \\
\hline 297 & 0 \\
\hline 298 & 10 \\
\hline 299 & 0 \\
\hline 300 & 22 \\
\hline 301 & 33 \\
\hline 302 & 0 \\
\hline 303 & 37 \\
\hline 304 & 4 \\
\hline 305 & 0 \\
\hline 306 & 10 \\
\hline 307 & 15 \\
\hline 308 & 0 \\
\hline 309 & 64 \\
\hline 310 & 12 \\
\hline 311 & 0 \\
\hline 312 & 0 \\
\hline 313 & 12 \\
\hline 314 & 12 \\
\hline 315 & 12 \\
\hline 316 & 1 \\
\hline 317 & 26 \\
\hline 318 & 11 \\
\hline 319 & 25 \\
\hline 320 & 76 \\
\hline 321 & 0 \\
\hline 322 & 64 \\
\hline 323 & 14 \\
\hline 324 & 0 \\
\hline 325 & 10 \\
\hline 326 & 99 \\
\hline 327 & 31 \\
\hline 328 & 11 \\
\hline 329 & 11 \\
\hline 330 & 9 \\
\hline 331 & 22 \\
\hline 332 & 5 \\
\hline 333 & 42 \\
\hline 334 & 10 \\
\hline 335 & 68 \\
\hline 336 & 10 \\
\hline 337 & 16 \\
\hline 338 & 33 \\
\hline 339 & 15 \\
\hline 340 & 1 \\
\hline 341 & 1 \\
\hline 342 & 95 \\
\hline 343 & 33 \\
\hline 344 & 96 \\
\hline 345 & 0 \\
\hline 346 & 0 \\
\hline 347 & 33 \\
\hline 348 & 15 \\
\hline 349 & 22 \\
\hline 350 & 18 \\
\hline 351 & 1 \\
\hline 352 & 0 \\
\hline 353 & 12 \\
\hline 354 & 13 \\
\hline 355 & 9 \\
\hline 356 & 0 \\
\hline 357 & 68 \\
\hline 358 & 22 \\
\hline 359 & 0 \\
\hline 360 & 11 \\
\hline 361 & 11 \\
\hline 362 & 9 \\
\hline 363 & 7 \\
\hline 364 & 16 \\
\hline 365 & 16 \\
\hline 366 & 9 \\
\hline 367 & 2 \\
\hline 368 & 95 \\
\hline 369 & 37 \\
\hline 370 & 8 \\
\hline 371 & 76 \\
\hline 372 & 8 \\
\hline 373 & 5 \\
\hline 374 & 42 \\
\hline 375 & 42 \\
\hline 376 & 42 \\
\hline 377 & 22 \\
\hline 378 & 3 \\
\hline 379 & 10 \\
\hline 380 & 7 \\
\hline 381 & 6 \\
\hline 382 & 5 \\
\hline 383 & 1 \\
\hline 384 & 13 \\
\hline 385 & 12 \\
\hline 386 & 94 \\
\hline
\end{tabular}




\begin{tabular}{|c|c|}
\hline 387 & 5 \\
\hline 388 & 33 \\
\hline 389 & 12 \\
\hline 390 & 12 \\
\hline 391 & 22 \\
\hline 392 & 10 \\
\hline 393 & 8 \\
\hline 394 & 16 \\
\hline 395 & 27 \\
\hline 396 & 10 \\
\hline 397 & 21 \\
\hline 398 & 12 \\
\hline 399 & 16 \\
\hline 400 & 0 \\
\hline 401 & 11 \\
\hline 402 & 28 \\
\hline 403 & 12 \\
\hline 404 & 31 \\
\hline 405 & 37 \\
\hline 406 & 95 \\
\hline 407 & 12 \\
\hline 408 & 5 \\
\hline 409 & 2 \\
\hline 410 & 12 \\
\hline 411 & 18 \\
\hline 412 & 48 \\
\hline 413 & 24 \\
\hline 414 & 22 \\
\hline 415 & 76 \\
\hline 416 & 14 \\
\hline 417 & 13 \\
\hline 418 & 22 \\
\hline 419 & 0 \\
\hline 420 & 16 \\
\hline 421 & 8 \\
\hline 422 & 12 \\
\hline 423 & 27 \\
\hline 424 & 7 \\
\hline 425 & 26 \\
\hline 426 & 13 \\
\hline 427 & 0 \\
\hline 428 & 16 \\
\hline 429 & 0 \\
\hline 430 & 0 \\
\hline 431 & 28 \\
\hline 432 & 6 \\
\hline 433 & 33 \\
\hline 434 & 19 \\
\hline 435 & 16 \\
\hline 436 & 7 \\
\hline 437 & 25 \\
\hline 438 & 28 \\
\hline 439 & 64 \\
\hline 440 & 0 \\
\hline 441 & 0 \\
\hline 442 & 26 \\
\hline 443 & 30 \\
\hline 444 & 13 \\
\hline 445 & 1 \\
\hline 446 & 94 \\
\hline 447 & 31 \\
\hline 448 & 0 \\
\hline 449 & 1 \\
\hline 450 & 7 \\
\hline 451 & 0 \\
\hline 452 & 26 \\
\hline 453 & 9 \\
\hline 454 & 1 \\
\hline 455 & 13 \\
\hline 456 & 1 \\
\hline 457 & 1 \\
\hline 458 & 2 \\
\hline 459 & 0 \\
\hline 460 & 0 \\
\hline 461 & 33 \\
\hline 462 & 77 \\
\hline 463 & 15 \\
\hline 464 & 68 \\
\hline 465 & 33 \\
\hline 466 & 12 \\
\hline 467 & 48 \\
\hline 468 & 94 \\
\hline 469 & 0 \\
\hline 470 & 0 \\
\hline 471 & 95 \\
\hline 472 & 9 \\
\hline 473 & 33 \\
\hline 474 & 12 \\
\hline 475 & 0 \\
\hline 476 & 9 \\
\hline 477 & 11 \\
\hline 478 & 37 \\
\hline 479 & 11 \\
\hline 480 & 94 \\
\hline 481 & 33 \\
\hline 482 & 95 \\
\hline 483 & 0 \\
\hline
\end{tabular}




$\begin{array}{lcl}484 & 7 & 0.944811107 \\ 485 & 22 & 0.732856339 \\ 486 & 11 & 0.375459851 \\ 487 & 16 & 0.728027307 \\ 488 & 9 & 0.95757677 \\ 489 & 16 & 0.681200774 \\ 490 & 99 & 0.591249594 \\ 491 & 10 & 0.430758991 \\ 492 & 21 & 0.106262628 \\ 493 & 16 & 0.933935529 \\ 494 & 12 & 0.925898415 \\ 495 & 99 & 0.951475831 \\ 496 & 12 & 0.054138212 \\ 497 & 21 & 0.947474367 \\ 498 & 11 & 0.842643707 \\ 499 & 0 & 0.339902457 \\ 500 & 0 & 0.511117918 \\ 501 & 9 & 0.409143329 \\ 502 & 25 & 0.644311675 \\ 503 & 2 & 0.995377167 \\ 504 & 7 & 0.265395138 \\ 505 & 26 & 0.729733317 \\ 506 & 0 & 0.792416722 \\ 507 & 28 & 0.096781934 \\ 508 & 11 & 0.562095345 \\ 509 & 10 & 0.42608767 \\ 510 & 14 & 0.655969639\end{array}$

Gini $\quad 0.588211$

Press CTRL-E for a new Gini 


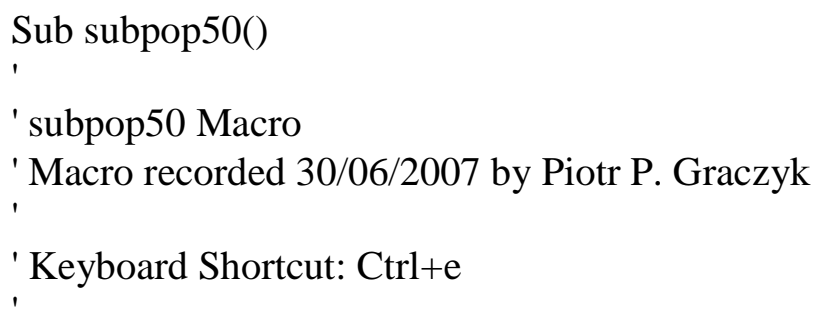

Range("B3:C512").Select

Selection.Sort Key1:=Range("C3"), Order1:=xlAscending, Header:=xlGuess, _ OrderCustom:=1, MatchCase:=False, Orientation:=xlTopToBottom, _ DataOption 1:=x1SortNormal

Range("E3:E52").Select

Selection.Copy

Range("F3").Select

Selection.PasteSpecial Paste:=xlPasteValues, Operation:=xlNone, SkipBlanks _ $:=$ False, Transpose:=False

Application .CutCopyMode $=$ False

Selection.Sort Key1:=Range("F3"), Order1:=xlAscending, Header:=xlGuess, OrderCustom:=1, MatchCase:=False, Orientation:=xlTopToBottom, _

DataOption $1:=x 1$ SortNormal

ActiveWindow. ScrollRow $=2$

ActiveWindow. ScrollRow $=7$

ActiveWindow. ScrollRow $=14$

ActiveWindow .ScrollRow $=22$

ActiveWindow. ScrollRow $=31$

ActiveWindow. ScrollRow $=41$

ActiveWindow . ScrollRow $=50$

ActiveWindow.ScrollRow $=59$

ActiveWindow .ScrollRow $=69$

ActiveWindow . ScrollRow $=79$

ActiveWindow. ScrollRow $=91$

ActiveWindow. ScrollRow $=106$

ActiveWindow. ScrollRow $=119$

ActiveWindow. ScrollRow $=134$

Active Window .ScrollRow $=151$

ActiveWindow .ScrollRow $=169$

Active Window. ScrollRow $=188$

ActiveWindow .ScrollRow $=206$

ActiveWindow. ScrollRow $=225$

ActiveWindow .ScrollRow $=241$

ActiveWindow .ScrollRow $=251$

ActiveWindow. ScrollRow $=261$

ActiveWindow. ScrollRow $=269$

ActiveWindow .ScrollRow $=274$ 
ActiveWindow .ScrollRow $=279$

ActiveWindow .ScrollRow $=282$

ActiveWindow. ScrollRow $=284$

ActiveWindow .ScrollRow $=286$

ActiveWindow .ScrollRow $=289$

ActiveWindow. ScrollRow $=292$

ActiveWindow .ScrollRow $=296$

ActiveWindow .ScrollRow $=301$

ActiveWindow.ScrollRow $=307$

ActiveWindow. ScrollRow $=313$

Active Window. ScrollRow $=320$

ActiveWindow .ScrollRow $=328$

Active Window. ScrollRow $=336$

ActiveWindow. ScrollRow $=343$

ActiveWindow. ScrollRow $=354$

ActiveWindow. ScrollRow $=362$

ActiveWindow. ScrollRow $=370$

ActiveWindow. ScrollRow $=377$

ActiveWindow. ScrollRow $=385$

ActiveWindow. ScrollRow $=392$

Active Window. ScrollRow $=399$

Active Window. ScrollRow $=406$

ActiveWindow .ScrollRow $=411$

ActiveWindow. ScrollRow $=416$

ActiveWindow. ScrollRow $=420$

ActiveWindow. ScrollRow $=425$

ActiveWindow. ScrollRow $=429$

ActiveWindow. ScrollRow $=433$

ActiveWindow. ScrollRow $=437$

ActiveWindow. ScrollRow $=439$

ActiveWindow. ScrollRow $=442$

ActiveWindow. ScrollRow $=444$

Active Window. ScrollRow $=445$

ActiveWindow. ScrollRow $=447$

ActiveWindow. ScrollRow $=448$

ActiveWindow. ScrollRow $=450$

ActiveWindow. ScrollRow $=452$

ActiveWindow. ScrollRow $=454$

ActiveWindow. ScrollRow $=457$

ActiveWindow. ScrollRow $=461$

ActiveWindow. ScrollRow $=464$

Range("I492").Select

End Sub 
GenStat Release 8.1 ( PC/Windows XP) 05 July 2007 13:34:16

Copyright 2005, Lawes Agricultural Trust (Rothamsted Experimental Station) Registered to: Eisai London Research Laboratories

GenStat Eighth Edition

GenStat Procedure Library Release PL16

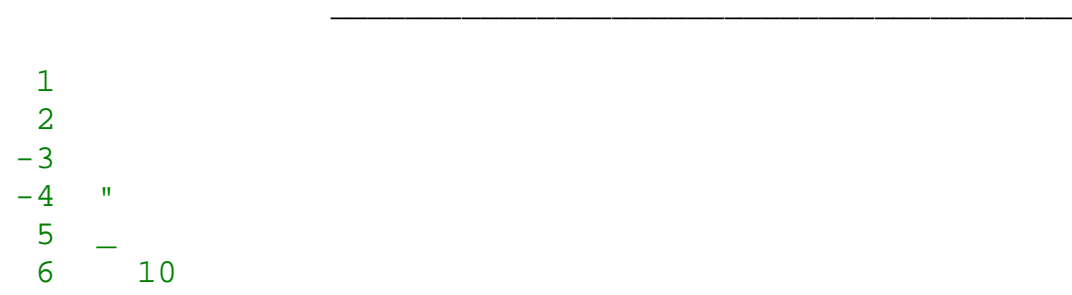

Data imported from Excel file: $\mathrm{H}: \mathrm{I}$

on: 5-Jul-2007 13:34:46

taken from sheet "'Sheet1"', cells A1:A350

11 DELETE [redefine=yes] C1

12 UNITS [NVALUES=* ]

13 VARIATE [nvalues=350] C1

14 READ C1

$\begin{array}{rrrrrr}\text { Identifier } & \text { Minimum } & \text { Mean } & \text { Maximum } & \text { Values } & \text { Missing } \\ \text { C1 } & 0.4522 & 0.5582 & 0.6690 & 350 & 0\end{array}$

102

103 DISTRIBUTION [PRINT=parameters, samplestatistics, fittedvalues;

DISTRIBUTION=Normal ] C1 


\section{Fit continuous distribution}

\section{Sample statistics}

\begin{tabular}{|c|c|}
\hline Sample Size & 350 \\
\hline Mean & 0.56 \\
\hline Variance & 0.00 \\
\hline Skewness & -0.08 \\
\hline Kurtosis & -0.21 \\
\hline Quartiles: & \\
\hline $25 \%$ & $50 \%$ \\
\hline 0.5 & 0.6 \\
\hline
\end{tabular}

\section{Summary of analysis}

Observations: $\mathrm{C} 1$

Parameter estimates from individual data values

Distribution: Normal (Gaussian)

$X$ distributed as $\operatorname{Normal}\left(m, s^{\star *} 2\right)$

Deviance: 11.50 on 16 d.f.

\section{Estimates of parameters}

$\begin{array}{lllll} & \text { estimate } & \text { s.e. } & \text { correlations } & \\ \mathrm{m} & 0.5582 & 0.0019 & 1.0000 & \\ \mathrm{~s} & 0.0357 & 0.0013 & 0.0000 & 1.0000\end{array}$

Fitted quartiles

$\begin{array}{rrr}25 \% & 50 \% & 75 \% \\ 0.534 & 0.558 & 0.582\end{array}$

Fitted values (expected frequencies) and residuals

$\begin{array}{lrrr}x & \begin{array}{r}\text { Number } \\ \text { observed }\end{array} & \begin{array}{r}\text { Number } \\ \text { expected }\end{array} & \begin{array}{r}\text { Weighted } \\ \text { residual }\end{array} \\ <0.497 & 18 & 14.73 & 0.82 \\ <0.512 & 18 & 20.02 & -0.46 \\ <0.522 & 19 & 18.80 & 0.05 \\ <0.529 & 18 & 17.86 & 0.03 \\ <0.535 & 19 & 17.91 & 0.25 \\ <0.540 & 18 & 18.60 & -0.14 \\ <0.546 & 18 & 19.42 & -0.33 \\ <0.552 & 19 & 24.01 & -1.06 \\ <0.557 & 18 & 17.16 & 0.20 \\ <0.560 & 19 & 14.02 & 1.26 \\ <0.566 & 18 & 23.11 & -1.11\end{array}$




$\begin{array}{rrrr}<0.571 & 19 & 16.79 & 0.53 \\ <0.577 & 18 & 22.60 & -1.00 \\ <0.583 & 18 & 19.83 & -0.42 \\ <0.587 & 19 & 11.60 & 1.99 \\ <0.595 & 18 & 19.62 & -0.37 \\ <0.603 & 19 & 16.82 & 0.52 \\ <0.613 & 18 & 15.67 & 0.58 \\ >0.613 & 19 & 21.42 & -0.53\end{array}$


Testing sensitivity of the Gini coefficient to $10 \%$ (relative) changes in inhibition data using AG1024; Staurosporine and PD184352

Summary

AG1024; $10 \mu \mathrm{M} ; 10 \mu \mathrm{M}$ ATP

Staurosporine; $1 \mu \mathrm{M} ; 100 \mu \mathrm{M}$ ATP

PD184352; $10 \mu \mathrm{M} ; 10 \mu \mathrm{M}$ ATP
$\mathrm{S} 230$

S231-S236

S237-S242

S243-S248 
Results of testing sensitivity of the Gini coefficient to $10 \%$ (relative) changes in inhibition data using AG1024;

Staurosporine and PD184352

\begin{tabular}{|c|c|c|c|c|}
\hline & $\begin{array}{l}\text { activity } \\
\text { before }\end{array}$ & & & Change [\%] \\
\hline AG1024; 10 & $10 \mu \mathrm{M} \mathrm{AT}$ & & & \\
\hline no change & & & 0.5680 & \\
\hline SAPK2a(h) & 99 & 89 & 0.5626 & -0.94 \\
\hline GSK3B(h) & 52 & 47 & 0.5684 & 0.07 \\
\hline & 52 & 57 & 0.5676 & -0.07 \\
\hline Yes(h) & 9 & 8 & 0.5681 & 0.02 \\
\hline & 9 & 10 & 0.5678 & -0.02 \\
\hline Staurospori & $\overline{\mu \mathrm{M} ; 100 \mu}$ & $\overline{\text { ATF }}$ & & \\
\hline no change & & & 0.1497 & \\
\hline CK2(h) & 97 & 87 & 0.1482 & -1.04 \\
\hline JNK3(r) & 53 & 48 & 0.1491 & -0.40 \\
\hline & 53 & 58 & 0.1503 & 0.42 \\
\hline GSK3ß(h) & 9 & 8 & 0.1496 & -0.05 \\
\hline & 9 & 7 & 0.1498 & 0.05 \\
\hline PD184352; & $\overline{\mathrm{M} ; 10 \mu \mathrm{M}}$ & & & \\
\hline no change & & & 0.8022 & \\
\hline AMPK(r) & 100 & 90 & 0.7933 & -1.11 \\
\hline Rsk1(r) & 67 & 60 & 0.8055 & 0.41 \\
\hline & & 74 & 0.7988 & -0.42 \\
\hline MEK1(h) & 25 & 22 & 0.8038 & 0.20 \\
\hline & & 28 & 0.8006 & -0.21 \\
\hline
\end{tabular}




\begin{tabular}{|c|c|c|c|c|c|c|c|c|c|}
\hline Kinase & $\begin{array}{l}10 \mu \mathrm{M} \text { ATP } \\
\text { AG1024 @ } 10 \mu \mathrm{M}\end{array}$ & $\begin{array}{c}\text { AG1024; } 10 \mu \mathrm{M} ; 10 \\
\text { sorted }\end{array}$ & $\begin{array}{l}\text { uM ATP } \\
\text { normalized }\end{array}$ & position & cumulative sample fraction & inhibition\% & fraction of total inhibition & cumulative inhibition & area \\
\hline $\mathrm{Abl}(\mathrm{m})$ & 1 & 131 & 100 & 1 & 0.011764706 & 0 & 0 & 0 & 0 \\
\hline AMPK(r) & 84 & 118 & 100 & 2 & 0.023529412 & 0 & 0 & 0 & 0 \\
\hline $\operatorname{Arg}(\mathrm{m})$ & 6 & 116 & 100 & 3 & 0.035294118 & 0 & 0 & 0 & 0 \\
\hline Aurora-A(h) & 104 & 113 & 100 & 4 & 0.047058824 & 0 & 0 & 0 & 0 \\
\hline Axl(h) & 58 & 107 & 100 & 5 & 0.058823529 & 0 & 0 & 0 & 0 \\
\hline $\mathrm{Blk}(\mathrm{m})$ & 4 & 107 & 100 & 6 & 0.070588235 & 0 & 0 & 0 & 0 \\
\hline Bnxx(h) & 84 & 105 & 100 & 7 & 0.082352941 & 0 & 0 & 0 & 0 \\
\hline CaMKII(r) & 89 & 105 & 100 & 8 & 0.094117647 & 0 & 0 & 0 & 0 \\
\hline CaMKIV(h) & 81 & 104 & 100 & 9 & 0.105882353 & 0 & 0 & 0 & 0 \\
\hline CDK1/cyclinB(h) & 67 & 104 & 100 & 10 & 0.117647059 & 0 & 0 & 0 & 0 \\
\hline CDK2/cyclinA(h) & 101 & 103 & 100 & 11 & 0.129411765 & 0 & 0 & 0 & 0 \\
\hline CDK2/cyclinE(h) & 95 & 101 & 100 & 12 & 0.141176471 & 0 & 0 & 0 & 0 \\
\hline CDK3/cyclinE(h) & 87 & 99 & 99 & 13 & 0.152941176 & 1 & 0.000503525 & 0.000503525 & $2.96191 \mathrm{E}-06$ \\
\hline CDK5/p35(h) & 78 & 99 & 99 & 14 & 0.164705882 & 1 & 0.000503525 & 0.001007049 & 8.88573E-06 \\
\hline CDK6/cyclinD3(h) & 92 & 99 & 99 & 15 & 0.176470588 & 1 & 0.000503525 & 0.001510574 & 1.48095E-05 \\
\hline CDK7/cyclinHMATI(h) & 93 & 98 & 98 & 16 & 0.188235294 & 2 & 0.001007049 & 0.002517623 & 2.36953E-05 \\
\hline CHK 1 (h) & 97 & 97 & 97 & 17 & 0.2 & 3 & 0.001510574 & 0.004028197 & $3.85048 \mathrm{E}-05$ \\
\hline СНK2(h) & 88 & 96 & 96 & 18 & 0.211764706 & 4 & 0.002014099 & 0.006042296 & 5.92382E-05 \\
\hline CK1(y) & 87 & 96 & 96 & 19 & 0.223529412 & 4 & 0.002014099 & 0.008056395 & 8.29335E-05 \\
\hline CK2(h) & 96 & 95 & 95 & 20 & 0.235294118 & 5 & 0.002517623 & 0.010574018 & 0.000109591 \\
\hline c.RAF(h) & 72 & 94 & 94 & 21 & 0.247058824 & 6 & 0.003021148 & 0.013595166 & 0.000142172 \\
\hline $\operatorname{CSK}(\mathfrak{h})$ & 75 & 93 & 93 & 22 & 0.258823529 & 7 & 0.003524673 & 0.017119839 & 0.000180677 \\
\hline cSRC(h) & 6 & 93 & 93 & 23 & 0.270588235 & 7 & 0.003524673 & 0.020644512 & 0.000222143 \\
\hline Fes(h) & 70 & 93 & 93 & 24 & 0.282352941 & 7 & 0.003524673 & 0.024169184 & 0.00026361 \\
\hline FGFR3(h) & 91 & 92 & 92 & 25 & 0.294117647 & 8 & 0.004028197 & 0.028197382 & 0.000308039 \\
\hline Fll3(h) & 89 & 92 & 92 & 26 & 0.305882353 & 8 & 0.004028197 & 0.032225579 & 0.000355429 \\
\hline Fyn(h) & 5 & 91 & 91 & 27 & 0.317647059 & 9 & 0.004531722 & 0.036757301 & 0.000405782 \\
\hline GSK3 ah) & 89 & 91 & 91 & 28 & 0.329411765 & 9 & 0.004531722 & 0.041289023 & 0.000459096 \\
\hline GSK3B(h) & 52 & 91 & 91 & 29 & 0.341176471 & 9 & 0.004531722 & 0.045820745 & 0.00051241 \\
\hline IGF-IR(h) & 118 & 90 & 90 & 30 & 0.352941176 & 10 & 0.005035247 & 0.050855992 & 0.000568687 \\
\hline IKK $\alpha(\mathrm{h})$ & 82 & 90 & 90 & 31 & 0.364705882 & 10 & 0.005035247 & 0.055891239 & 0.000627925 \\
\hline IKKB(h) & 93 & 90 & 90 & 32 & 0.376470588 & 10 & 0.005035247 & 0.060926485 & 0.000687163 \\
\hline $\mathrm{IR}(\mathrm{h})$ & 105 & 89 & 89 & 33 & 0.388235294 & 11 & 0.005538771 & 0.066465257 & 0.000749363 \\
\hline JNK1al(h) & 79 & 89 & 89 & 34 & 0.4 & 11 & 0.005538771 & 0.072004028 & 0.000814525 \\
\hline JNK2@2(h) & 89 & 89 & 89 & 35 & 0.411764706 & 11 & 0.005538771 & 0.0775428 & 0.000879687 \\
\hline JNK3(r) & 60 & 89 & 89 & 36 & 0.423529412 & 11 & 0.005538771 & 0.083081571 & 0.000944849 \\
\hline Lck(h) & 24 & 89 & 89 & 37 & 0.435294118 & 11 & 0.005538771 & 0.088620342 & 0.001010011 \\
\hline Lynn(h) & 74 & 88 & 88 & 38 & 0.447058824 & 12 & 0.006042296 & 0.094662638 & 0.001078135 \\
\hline Lyn(m) & 5 & 88 & 88 & 39 & 0.458823529 & 12 & 0.006042296 & 0.100704935 & 0.001149221 \\
\hline MAPK1(h) & 85 & 88 & 88 & 40 & 0.470588235 & 12 & 0.006042296 & 0.106747231 & 0.001220307 \\
\hline MAPK2(h) & 113 & 88 & 88 & 41 & 0.482352941 & 12 & 0.006042296 & 0.112789527 & 0.001291393 \\
\hline MAPK2(m) & 93 & 88 & 88 & 42 & 0.494117647 & 12 & 0.006042296 & 0.118831823 & 0.001362479 \\
\hline MAPKAP-K2(h) & 90 & 87 & 87 & 43 & 0.505882353 & 13 & 0.006545821 & 0.125377644 & 0.001436526 \\
\hline MEK I(h) & 88 & 87 & 87 & 44 & 0.517647059 & 13 & 0.006545821 & 0.131923464 & 0.001513536 \\
\hline MKK4(m) & 88 & 87 & 87 & 45 & 0.529411765 & 13 & 0.006545821 & 0.138469285 & 0.001590546 \\
\hline MKKG(h) & 86 & 86 & 86 & 46 & 0.541176471 & 14 & 0.007049345 & 0.14551863 & 0.001670517 \\
\hline MKK 7 (h) & 116 & 86 & 86 & 47 & 0.552941176 & 14 & 0.007049345 & 0.152567976 & 0.001753451 \\
\hline MSK1(h) & 92 & 85 & 85 & 48 & 0.564705882 & 15 & 0.00755287 & 0.160120846 & 0.001839346 \\
\hline p70s6K(h) & 36 & 84 & 84 & 49 & 0.576470588 & 16 & 0.008056395 & 0.168177241 & 0.001931165 \\
\hline PAK2(h) & 98 & 84 & 84 & 50 & 0.588235294 & 16 & 0.008056395 & 0.176233635 & 0.002025946 \\
\hline PDGFRa(h) & 88 & 84 & 84 & 51 & 0.6 & 16 & 0.008056395 & 0.18429003 & 0.002120727 \\
\hline PDGFRB(h) & 67 & 84 & 84 & 52 & 0.611764706 & 16 & 0.008056395 & 0.192346425 & 0.002215509 \\
\hline PDK 1(h) & 84 & 82 & 82 & 53 & 0.623529412 & 18 & 0.009063444 & 0.201409869 & 0.002316213 \\
\hline PKA(b) & 74 & 81 & 81 & 54 & 0.635294118 & 19 & 0.009566969 & 0.210976838 & 0.002425804 \\
\hline PKA(h) & 107 & 79 & 79 & 55 & 0.647058824 & 21 & 0.010574018 & 0.221550856 & 0.002544281 \\
\hline PKBu(h) & 99 & 78 & 78 & 56 & 0.658823529 & 22 & 0.011077543 & 0.232628399 & 0.002671643 \\
\hline PKBB(h) & 90 & 78 & 78 & 57 & 0.670588235 & 22 & 0.011077543 & 0.243705942 & 0.002801967 \\
\hline PKB $\gamma(\mathfrak{h l})$ & 91 & 76 & 76 & 58 & 0.682352941 & 24 & 0.012084592 & 0.255790534 & 0.002938215 \\
\hline PKC $\alpha(\mathrm{h})$ & 87 & 75 & 75 & 59 & 0.694117647 & 25 & 0.012588117 & 0.268378651 & 0.003083348 \\
\hline PKCBII(h) & 99 & 74 & 74 & 60 & 0.705882353 & 26 & 0.013091641 & 0.281470292 & 0.003234406 \\
\hline PKC $\{$ (h) & 105 & 74 & 74 & 61 & 0.717647059 & 26 & 0.013091641 & 0.294561934 & 0.003388425 \\
\hline $\operatorname{PKC} \delta(\mathrm{h})$ & 107 & 73 & 73 & 62 & 0.729411765 & 27 & 0.013595166 & 0.3081571 & 0.003545406 \\
\hline PKC \&h) & 78 & 72 & 72 & 63 & 0.741176471 & 28 & 0.014098691 & 0.322255791 & 0.003708311 \\
\hline PKC $\eta(i)$ & 96 & 72 & 72 & 64 & 0.752941176 & 28 & 0.014098691 & 0.336354481 & 0.003874178 \\
\hline P KC (1) & 90 & 70 & 70 & 65 & 0.764705882 & 30 & 0.01510574 & 0.351460222 & 0.004045969 \\
\hline PKC $\mu(\mathrm{h})$ & 86 & 69 & 69 & 66 & 0.776470588 & 31 & 0.015609265 & 0.367069486 & 0.004226645 \\
\hline PKC $\theta$ (l) & 91 & 67 & 67 & 67 & 0.788235294 & 33 & 0.016616314 & 0.383685801 & 0.004416208 \\
\hline PKD2(h) & 73 & 67 & 67 & 68 & 0.8 & 33 & 0.016616314 & 0.400302115 & 0.004611694 \\
\hline PRAK(h) & 76 & 67 & 67 & 69 & 0.811764706 & 33 & 0.016616314 & 0.416918429 & 0.00480718 \\
\hline PRK2(h) & 88 & 63 & 63 & 70 & 0.823529412 & 37 & 0.018630413 & 0.435548842 & 0.005014513 \\
\hline ROCK-II(h) & 23 & 60 & 60 & 71 & 0.835294118 & 40 & 0.020140987 & 0.455689829 & 0.00524258 \\
\hline ROCK-II(r) & 32 & 58 & 58 & 72 & 0.847058824 & 42 & 0.021148036 & 0.476837865 & 0.005485457 \\
\hline Rsk1(h) & 89 & 58 & 58 & 73 & 0.858823529 & 42 & 0.021148036 & 0.497985901 & 0.005734257 \\
\hline Rskl(r) & 72 & 52 & 52 & 74 & 0.870588235 & 48 & 0.024169184 & 0.522155086 & 0.006000829 \\
\hline Rsk2(h) & 58 & 36 & 36 & 75 & 0.882352941 & 64 & 0.032225579 & 0.554380665 & 0.006332563 \\
\hline Rsk3(h) & 104 & 32 & 32 & 76 & 0.894117647 & 68 & 0.034239678 & 0.588620342 & 0.006723535 \\
\hline SAPK2a(h) & 99 & 24 & 24 & 77 & 0.905882353 & 76 & 0.038267875 & 0.626888218 & 0.00715005 \\
\hline SAPK2b(h) & 103 & 23 & 23 & 78 & 0.917647059 & 77 & 0.0387714 & 0.665659617 & 0.007603223 \\
\hline SAPK3(h) & 84 & 9 & 9 & 79 & 0.929411765 & 91 & 0.045820745 & 0.711480363 & 0.008100823 \\
\hline SAPK 4(h) & 94 & 6 & 6 & 80 & 0.941176471 & 94 & 0.047331319 & 0.758811682 & 0.008648777 \\
\hline SGK(h) & 63 & 6 & 6 & 81 & 0.952941176 & 94 & 0.047331319 & 0.806143001 & 0.009205616 \\
\hline Syk(h) & 67 & 5 & 5 & 82 & 0.964705882 & 95 & 0.047834844 & 0.853977845 & 0.009765417 \\
\hline TrkB(h) & 131 & 5 & 5 & 83 & 0.976470588 & 95 & 0.047834844 & 0.901812689 & 0.01032818 \\
\hline Yes(h) & 9 & 4 & 4 & 84 & 0.988235294 & 96 & 0.048338369 & 0.950151057 & 0.010893904 \\
\hline ZAP-70(h) & 69 & 1 & 1 & 85 & 1 & 99 & 0.049848943 & 1 & 0.011471477 \\
\hline & & & & & Total inhibition & 1986 & & Total area & 0.216012085 \\
\hline & & & & & & & & Gini & 0.567975831 \\
\hline
\end{tabular}




\begin{tabular}{|c|c|c|c|c|c|c|c|c|c|}
\hline & $10 \mu \mathrm{M}$ ATP & AG1024; $10 \mu \mathrm{M} ; 10$ & $\mu \mathrm{M}$ ATP & & & & & & \\
\hline Kinase & AG1024@10 $10 \mathrm{M}$ & sorted & normalized & position & cumulative sample fraction & inhibition\% & fraction of total inhibition & cumulative inhibition & area \\
\hline $\mathrm{Abl}(\mathrm{m})$ & 1 & 131 & 100 & 1 & 0.011764706 & 0 & 0 & 0 & 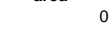 \\
\hline $\mathrm{AMPK}(\mathrm{r})$ & 84 & 118 & 100 & 2 & 0.023529412 & 0 & 0 & 0 & 0 \\
\hline $\operatorname{Arg}(\mathrm{m})$ & 6 & 116 & 100 & 3 & 0.035294118 & 0 & 0 & 0 & 0 \\
\hline Aurora-A(h) & 104 & 113 & 100 & 4 & 0.047058824 & 0 & 0 & 0 & 0 \\
\hline Axx(h) & 58 & 107 & 100 & 5 & 0.058823529 & 0 & 0 & 0 & 0 \\
\hline $\mathrm{Bk}(\mathrm{m})$ & 4 & 107 & 100 & 6 & 0.070588235 & 0 & 0 & 0 & 0 \\
\hline Bmx(h) & 84 & 105 & 100 & 7 & 0.082352941 & 0 & 0 & 0 & 0 \\
\hline CaMKII(r) & 89 & 105 & 100 & 8 & 0.094117647 & 0 & 0 & 0 & 0 \\
\hline CaMKIV(h) & 81 & 104 & 100 & 9 & 0.105882353 & 0 & 0 & 0 & 0 \\
\hline CDK1/cyclinB(h) & 67 & 104 & 100 & 10 & 0.117647059 & 0 & 0 & 0 & 0 \\
\hline $\mathrm{CDK} 2 /$ cyclinA(h) & 101 & 103 & 100 & 11 & 0.129411765 & 0 & 0 & 0 & 0 \\
\hline $\mathrm{CDK} 2 /$ cyclinE(h) & 95 & 101 & 100 & 12 & 0.141176471 & 0 & 0 & 0 & 0 \\
\hline CDK3/cyclinE(h) & 87 & 99 & 99 & 13 & 0.152941176 & 1 & 0.000501002 & 0.000501002 & 2.94707E-06 \\
\hline CDK5 5 355(h) & 78 & 99 & 99 & 14 & 0.164705882 & 1 & 0.000501002 & 0.001002004 & $8.84121 \mathrm{E}-06$ \\
\hline CDK6/cyclinD3(h) & 92 & 98 & 98 & 15 & 0.176470588 & 2 & 0.001002004 & 0.002004008 & $1.76824 \mathrm{E}-05$ \\
\hline CDK7/cyclinHMATI(h) & 93 & 97 & 97 & 16 & 0.188235294 & 3 & 0.001503006 & 0.003507014 & $3.24178 \mathrm{E}-05$ \\
\hline СнK1(h) & 97 & 96 & 96 & 17 & 0.2 & 4 & 0.002004008 & 0.005511022 & $5.30473 \mathrm{E}-05$ \\
\hline СHK2(h) & 88 & 96 & 96 & 18 & 0.211764706 & 4 & 0.002004008 & 0.00751503 & 7.66238E-05 \\
\hline $\mathrm{CKK}(\mathrm{y})$ & 87 & 95 & 95 & 19 & 0.223529412 & 5 & 0.00250501 & 0.01002004 & 0.000103147 \\
\hline СК2(h) & 96 & 94 & 94 & 20 & 0.235294118 & 6 & 0.003006012 & 0.013026052 & 0.000135565 \\
\hline$c \cdot \operatorname{RAF(h)}$ & 72 & 93 & 93 & 21 & 0.247058824 & 7 & 0.003507014 & 0.016533066 & 0.000173877 \\
\hline $\operatorname{csK}(\mathrm{h})$ & 75 & 93 & 93 & 22 & 0.258823529 & 7 & 0.003507014 & 0.02004008 & 0.000215136 \\
\hline $\operatorname{cSRC(h)}$ & 6 & 93 & 93 & 23 & 0.270588235 & 7 & 0.003507014 & 0.023547094 & 0.000256395 \\
\hline Fes(h) & 70 & 92 & 92 & 24 & 0.282352941 & 8 & 0.004008016 & 0.02755511 & 0.000300601 \\
\hline FGFR3(h) & 91 & 92 & 92 & 25 & 0.294117647 & 8 & 0.004008016 & 0.031563126 & 0.000347754 \\
\hline $\mathrm{FH}$ 3(h) & 89 & 91 & 91 & 26 & 0.305882353 & 9 & 0.004509018 & 0.036072144 & 0.000397855 \\
\hline Fyn(h) & 5 & 91 & 91 & 27 & 0.317647059 & 9 & 0.004509018 & 0.040581162 & 0.000450902 \\
\hline GSK3 dh) & 89 & 91 & 91 & 28 & 0.329411765 & 9 & 0.004509018 & 0.04509018 & 0.000503949 \\
\hline GSK3B(h) & 52 & 90 & 90 & 29 & 0.341176471 & 10 & 0.00501002 & 0.0501002 & 0.000559943 \\
\hline IGF-IR(h) & 118 & 90 & 90 & 30 & 0.352941176 & 10 & 0.00501002 & 0.05511022 & 0.000618885 \\
\hline IKKa(h) & 82 & 90 & 90 & 31 & 0.364705882 & 10 & 0.00501002 & 0.06012024 & 0.000677826 \\
\hline $\operatorname{IKKB(h)}$ & 93 & 89 & 89 & 32 & 0.376470588 & 11 & 0.005511022 & 0.065631263 & 0.000739715 \\
\hline $\mathrm{IR}(\mathrm{h})$ & 105 & 89 & 89 & 33 & 0.388235294 & 11 & 0.005511022 & 0.071142285 & 0.00080455 \\
\hline JNKIal(h) & 79 & 89 & 89 & 34 & 0.4 & 11 & 0.005511022 & 0.076653307 & 0.000869386 \\
\hline JNK202(h) & 89 & 89 & 89 & 35 & 0.411764706 & 11 & 0.005511022 & 0.082164329 & 0.000934221 \\
\hline JNK3(r) & 60 & 89 & 89 & 36 & 0.423529412 & 11 & 0.005511022 & 0.087675351 & 0.000999057 \\
\hline Lck(h) & 24 & 89 & 89 & 37 & 0.435294118 & 11 & 0.005511022 & 0.093186373 & 0.001063892 \\
\hline Lym(h) & 74 & 88 & 88 & 38 & 0.447058824 & 12 & 0.006012024 & 0.099198397 & 0.001131675 \\
\hline Lyn(m) & 5 & 88 & 88 & 39 & 0.458823529 & 12 & 0.006012024 & 0.105210421 & 0.001202405 \\
\hline MAPK1(h) & 85 & 88 & 88 & 40 & 0.470588235 & 12 & 0.006012024 & 0.111222445 & 0.001273135 \\
\hline MAPK $2(\mathrm{~h})$ & 113 & 88 & 88 & 41 & 0.482352941 & 12 & 0.006012024 & 0.117234469 & 0.001343864 \\
\hline MAPK2(m) & 93 & 88 & 88 & 42 & 0.494117647 & 12 & 0.006012024 & 0.123246493 & 0.001414594 \\
\hline MAPKAP-K2(h) & 90 & 87 & 87 & 43 & 0.505882353 & 13 & 0.006513026 & 0.129759519 & 0.001488271 \\
\hline MEK I(h) & 88 & 87 & 87 & 44 & 0.517647059 & 13 & 0.006513026 & 0.136272545 & 0.001564894 \\
\hline $\mathrm{MKK} 4(\mathrm{~m})$ & 88 & 87 & 87 & 45 & 0.529411765 & 13 & 0.006513026 & 0.142785571 & 0.001641518 \\
\hline MKK6(h) & 86 & 86 & 86 & 46 & 0.541176471 & 14 & 0.007014028 & 0.149799599 & 0.001721089 \\
\hline MKКㄱ⒣ & 116 & 86 & 86 & 47 & 0.552941176 & 14 & 0.007014028 & 0.156813627 & 0.001803607 \\
\hline MSK1(h) & 92 & 85 & 85 & 48 & 0.564705882 & 15 & 0.00751503 & 0.164328657 & 0.001889072 \\
\hline $\mathrm{p} 70 \mathrm{~S} 6 \mathrm{~K}(\mathrm{~h})$ & 36 & 84 & 84 & 49 & 0.576470588 & 16 & 0.008016032 & 0.172344689 & 0.001980431 \\
\hline PAK2(h) & 98 & 84 & 84 & 50 & 0.588235294 & 16 & 0.008016032 & 0.180360721 & 0.002074738 \\
\hline PDGFRa(h) & 88 & 84 & 84 & 51 & 0.6 & 16 & 0.008016032 & 0.188376754 & 0.002169044 \\
\hline PDGFRB(h) & 67 & 84 & 84 & 52 & 0.611764706 & 16 & 0.008016032 & 0.196392786 & 0.00226335 \\
\hline PDK I(h) & 84 & 82 & 82 & 53 & 0.623529412 & 18 & 0.009018036 & 0.205410822 & 0.002363551 \\
\hline PKA(b) & 74 & 81 & 81 & 54 & 0.635294118 & 19 & 0.009519038 & 0.21492986 & 0.002472592 \\
\hline PKA(h) & 107 & 79 & 79 & 55 & 0.647058824 & 21 & 0.010521042 & 0.225450902 & 0.002590475 \\
\hline PKBa(h) & 99 & 78 & 78 & 56 & 0.658823529 & 22 & 0.011022044 & 0.236472946 & 0.002717199 \\
\hline PKBB(h) & 90 & 78 & 78 & 57 & 0.670588235 & 22 & 0.011022044 & 0.24749499 & 0.00284687 \\
\hline PKB $\times(h)$ & 91 & 76 & 76 & 58 & 0.682352941 & 24 & 0.012024048 & 0.259519038 & 0.002982435 \\
\hline PKC $a(\mathrm{~h})$ & 87 & 75 & 75 & 59 & 0.694117647 & 25 & 0.01252505 & 0.272044088 & 0.003126842 \\
\hline РКСВI(h) & 99 & 74 & 74 & 60 & 0.705882353 & 26 & 0.013026052 & 0.28507014 & 0.003277143 \\
\hline PKC $($ hh) & 105 & 74 & 74 & 61 & 0.717647059 & 26 & 0.013026052 & 0.298096192 & 0.00343039 \\
\hline PKC $\delta(h)$ & 107 & 73 & 73 & 62 & 0.729411765 & 27 & 0.013527054 & 0.311623246 & 0.003586585 \\
\hline PKC ah) & 78 & 72 & 72 & 63 & 0.741176471 & 28 & 0.014028056 & 0.325651303 & 0.003748674 \\
\hline PKC $\eta($ (i) & 96 & 72 & 72 & 64 & 0.752941176 & 28 & 0.014028056 & 0.339679359 & 0.00391371 \\
\hline P KC, (h) & 90 & 70 & 70 & 65 & 0.764705882 & 30 & 0.01503006 & 0.354709419 & 0.00408464 \\
\hline РКСц(h) & 86 & 69 & 69 & 66 & 0.776470588 & 31 & 0.015531062 & 0.370240481 & 0.004264411 \\
\hline PKC & 91 & 67 & 67 & 67 & 0.788235294 & 33 & 0.016533066 & 0.386773547 & 0.004453024 \\
\hline PKD2(h) & 73 & 67 & 67 & 68 & 0.8 & 33 & 0.016533066 & 0.403306613 & 0.00464753 \\
\hline $\begin{array}{l}\text { PRAK(h) } \\
\text { (n) }\end{array}$ & 76 & 67 & 67 & 69 & 0.811764706 & 33 & 0.016533066 & 0.419839679 & 0.004842037 \\
\hline PRK2(h) & 88 & 63 & 63 & 70 & 0.823529412 & 37 & 0.018537074 & 0.438376754 & 0.005048332 \\
\hline ROCK-III(h) & 23 & 60 & 60 & 71 & 0.835294118 & 40 & 0.02004008 & 0.458416834 & 0.005275256 \\
\hline ROCK-II(r) & 32 & 58 & 58 & 72 & 0.847058824 & 42 & 0.021042084 & 0.479458918 & 0.005516916 \\
\hline Rsk1(h) & 89 & 58 & 58 & 73 & 0.858823529 & 42 & 0.021042084 & 0.500501002 & 0.00576447 \\
\hline Rskl(r) & 72 & 52 & 52 & 74 & 0.870588235 & 48 & 0.024048096 & 0.524549098 & 0.006029706 \\
\hline Rsk2(h) & 58 & 36 & 36 & 75 & 0.882352941 & 64 & 0.032064128 & 0.556613226 & 0.006359778 \\
\hline Rsk3(h) & 104 & 32 & 32 & 76 & 0.894117647 & 68 & 0.034068136 & 0.590681363 & 0.006748792 \\
\hline SAPK2a(h) & 89 & 24 & 24 & 77 & 0.905882353 & 76 & 0.038076152 & 0.628757515 & 0.00717317 \\
\hline SAPK2b(h) & 103 & 23 & 23 & 78 & 0.917647059 & 77 & 0.038577154 & 0.667334669 & 0.007624072 \\
\hline SAPK3(h) & 84 & 9 & 9 & 79 & 0.929411765 & 91 & 0.045591182 & 0.712925852 & 0.00811918 \\
\hline SAPK4(h) & 94 & 6 & 6 & 80 & 0.941176471 & 94 & 0.047094188 & 0.76002004 & 0.008664388 \\
\hline $\mathrm{SGK}(\mathrm{h})$ & 63 & 6 & 6 & 81 & 0.952941176 & 94 & 0.047094188 & 0.807114228 & 0.009218437 \\
\hline Syk(h) & 67 & 5 & 5 & 82 & 0.964705882 & 95 & 0.04759519 & 0.854709419 & 0.009775433 \\
\hline $\operatorname{TrkB(h)}$ & 131 & 5 & 5 & 83 & 0.976470588 & 95 & 0.04759519 & 0.902304609 & 0.010335377 \\
\hline Yes(h) & 9 & 4 & 4 & 84 & 0.988235294 & 96 & 0.048096192 & 0.950400802 & 0.010898267 \\
\hline ZAP-70(h) & 69 & 1 & 1 & 85 & 1 & 99 & 0.049599198 & 1 & 0.011472946 \\
\hline & & & & & Total inhibition & 1996 & & Total area & 0.218678534 \\
\hline & & & & & & & & Gini & 0.562642933 \\
\hline
\end{tabular}




\begin{tabular}{|c|c|c|c|c|c|c|c|c|c|}
\hline & $10 \mu \mathrm{M}$ ATP & AG1024; $10 \mu \mathrm{M} ; 10$ & $\mu \mathrm{M}$ АTP & & & & & & \\
\hline Kinase & AG1024@ @ $10 \mu \mathrm{M}$ & sorted & normalized & position & cumulative sample fraction & inhibition\% & fraction of total inhibition & cumulative inhibition & area \\
\hline $\mathrm{Abl}(\mathrm{m})$ & 1 & 131 & 100 & 1 & 0.011764706 & 0 & 0 & 0 & 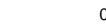 \\
\hline $\operatorname{AMPK}(r)$ & 84 & 118 & 100 & 2 & 0.023529412 & 0 & 0 & 0 & 0 \\
\hline $\operatorname{Arg}(\mathrm{m})$ & 6 & 116 & 100 & 3 & 0.035294118 & 0 & 0 & 0 & 0 \\
\hline Aurora-A(h) & 104 & 113 & 100 & 4 & 0.047058824 & 0 & 0 & 0 & 0 \\
\hline $\operatorname{Ax}(\mathrm{h})$ & 58 & 107 & 100 & 5 & 0.058823529 & 0 & 0 & 0 & 0 \\
\hline $\mathrm{Bl}(\mathrm{m})$ & 4 & 107 & 100 & 6 & 0.070588235 & 0 & 0 & 0 & \\
\hline Bmx(h) & 84 & 105 & 100 & 7 & 0.082352941 & 0 & 0 & 0 & 0 \\
\hline CaMKII(r) & 89 & 105 & 100 & 8 & 0.094117647 & 0 & 0 & 0 & 0 \\
\hline CaMKIV(h) & 81 & 104 & 100 & 9 & 0.105882353 & 0 & 0 & 0 & 0 \\
\hline CDK1/cyclinB(h) & 67 & 104 & 100 & 10 & 0.117647059 & 0 & 0 & 0 & 0 \\
\hline $\mathrm{CDK} 2 /$ cyclinA(h) & 101 & 103 & 100 & 11 & 0.129411765 & 0 & 0 & 0 & 0 \\
\hline $\mathrm{CDK} 2 /$ cyclinE(h) & 95 & 101 & 100 & 12 & 0.141176471 & 0 & 0 & 0 & 0 \\
\hline CDK3/cyclinE(h) & 87 & 99 & 99 & 13 & 0.152941176 & 1 & 0.00050226 & 0.00050226 & $2.95447 \mathrm{E}-06$ \\
\hline CDK5/p35(h) & 78 & 99 & 99 & 14 & 0.164705882 & 1 & 0.00050226 & 0.00100452 & $8.86341 \mathrm{E}-06$ \\
\hline CDK6//cyclinD3(h) & 92 & 99 & 99 & 15 & 0.176470588 & 1 & 0.00050226 & 0.001506781 & $1.47724 \mathrm{E}-05$ \\
\hline CDK7/cyclinHMATI(h) & 93 & 98 & 98 & 16 & 0.188235294 & 2 & 0.00100452 & 0.002511301 & $2.36358 \mathrm{E}-05$ \\
\hline CHK1(h) & 97 & 97 & 97 & 17 & 0.2 & 3 & 0.001506781 & 0.004018081 & $3.84081 \mathrm{E}-05$ \\
\hline СHK2(h) & 88 & 96 & 96 & 18 & 0.211764706 & 4 & 0.002009041 & 0.006027122 & $5.90894 \mathrm{E}-05$ \\
\hline $\mathrm{CKI}(\mathrm{y})$ & 87 & 96 & 96 & 19 & 0.223529412 & 4 & 0.002009041 & 0.008036163 & $8.27252 \mathrm{E}-05$ \\
\hline CK2(h) & 96 & 95 & 95 & 20 & 0.235294118 & 5 & 0.002511301 & 0.010547464 & 0.000109315 \\
\hline c-RAF(h) & 72 & 94 & 94 & 21 & 0.247058824 & 6 & 0.003013561 & 0.013561025 & 0.000141815 \\
\hline CSK(h) & 75 & 93 & 93 & 22 & 0.258823529 & 7 & 0.003515821 & 0.017076846 & 0.000180223 \\
\hline cSRC(h) & 6 & 93 & 93 & 23 & 0.270588235 & 7 & 0.003515821 & 0.020592667 & 0.000221585 \\
\hline Fes(h) & 70 & 93 & 93 & 24 & 0.282352941 & 7 & 0.003515821 & 0.024108488 & 0.000262948 \\
\hline FGFR3(h) & 91 & 92 & 92 & 25 & 0.294117647 & 8 & 0.004018081 & 0.02812657 & 0.000307265 \\
\hline $\mathrm{F} \mathfrak{l} 3$ (h) & 89 & 92 & 92 & 26 & 0.305882353 & 8 & 0.004018081 & 0.032144651 & 0.000354537 \\
\hline Fyn(h) & 5 & 91 & 91 & 27 & 0.317647059 & 9 & 0.004520342 & 0.036664992 & 0.000404763 \\
\hline GSK3 ah) & 89 & 91 & 91 & 28 & 0.329411765 & 9 & 0.004520342 & 0.041185334 & 0.000457943 \\
\hline $\operatorname{GSK3B(h)}$ & 47 & 91 & 91 & 29 & 0.341176471 & 9 & 0.004520342 & 0.045705676 & 0.000511124 \\
\hline IGF-IR(h) & 118 & 90 & 90 & 30 & 0.352941176 & 10 & 0.005022602 & 0.050728277 & 0.000567259 \\
\hline $\operatorname{IKK} \alpha(\mathrm{h})$ & 82 & 90 & 90 & 31 & 0.364705882 & 10 & 0.005022602 & 0.055750879 & 0.000626348 \\
\hline IKKB(h) & 93 & 90 & 90 & 32 & 0.376470588 & 10 & 0.005022602 & 0.060773481 & 0.000685437 \\
\hline $\mathrm{IR}(\mathrm{h})$ & 105 & 89 & 89 & 33 & 0.388235294 & 11 & 0.005524862 & 0.066298343 & 0.000747481 \\
\hline JNKIal(h) & 79 & 89 & 89 & 34 & 0.4 & 11 & 0.005524862 & 0.071823204 & 0.00081248 \\
\hline $\mathrm{JNK} 2 \alpha 2(\mathrm{~h})$ & 89 & 89 & 89 & 35 & 0.411764706 & 11 & 0.005524862 & 0.077348066 & 0.000877478 \\
\hline JNK3(r) & 60 & 89 & 89 & 36 & 0.423529412 & 11 & 0.005524862 & 0.082872928 & 0.000942476 \\
\hline Lck(h) & 24 & 89 & 89 & 37 & 0.435294118 & 11 & 0.005524862 & 0.08839779 & 0.001007475 \\
\hline Lyn(h) & 74 & 88 & 88 & 38 & 0.447058824 & 12 & 0.006027122 & 0.094424912 & 0.001075428 \\
\hline Lyn(m) & 5 & 88 & 88 & 39 & 0.458823529 & 12 & 0.006027122 & 0.100452034 & 0.001146335 \\
\hline MAPKI(h) & 85 & 88 & 88 & 40 & 0.470588235 & 12 & 0.006027122 & 0.106479156 & 0.001217242 \\
\hline MAPK $2(\mathrm{~h})$ & 113 & 88 & 88 & 41 & 0.482352941 & 12 & 0.006027122 & 0.112506278 & 0.00128815 \\
\hline MAPK2(m) & 93 & 88 & 88 & 42 & 0.494117647 & 12 & 0.006027122 & 0.1185334 & 0.001359057 \\
\hline MAPKAP-K2(h) & 90 & 87 & 87 & 43 & 0.505882353 & 13 & 0.006529382 & 0.125062783 & 0.001432919 \\
\hline MEK1(h) & 88 & 87 & 87 & 44 & 0.517647059 & 13 & 0.006529382 & 0.131592165 & 0.001509735 \\
\hline MKK4(m) & 88 & 87 & 87 & 45 & 0.529411765 & 13 & 0.006529382 & 0.138121547 & 0.001586551 \\
\hline MKK6(h) & 86 & 86 & 86 & 46 & 0.541176471 & 14 & 0.007031642 & 0.145153189 & 0.001666322 \\
\hline MKK $78(h)$ & 116 & 86 & 86 & 47 & 0.552941176 & 14 & 0.007031642 & 0.152184832 & 0.001749047 \\
\hline MSKI(h) & 92 & 85 & 85 & 48 & 0.564705882 & 15 & 0.007533903 & 0.159718734 & 0.001834727 \\
\hline p7056K(h) & 36 & 84 & 84 & 49 & 0.576470588 & 16 & 0.008036163 & 0.167754897 & 0.001926315 \\
\hline PAK2(h) & 98 & 84 & 84 & 50 & 0.588235294 & 16 & 0.008036163 & 0.17579106 & 0.002020859 \\
\hline PDGFRa(h) & 88 & 84 & 84 & 51 & 0.6 & 16 & 0.008036163 & 0.183827223 & 0.002115402 \\
\hline PDGFRB(h) & 67 & 84 & 84 & 52 & 0.611764706 & 16 & 0.008036163 & 0.191863385 & 0.002209945 \\
\hline PDK1(h) & 84 & 82 & 82 & 53 & 0.623529412 & 18 & 0.009040683 & 0.200904068 & 0.002310397 \\
\hline PKA(b) & 74 & 81 & 81 & 54 & 0.635294118 & 19 & 0.009542943 & 0.210447012 & 0.002419712 \\
\hline PKA(h) & 107 & 79 & 79 & 55 & 0.647058824 & 21 & 0.010547464 & 0.220994475 & 0.002537891 \\
\hline PKBa(h) & 99 & 78 & 78 & 56 & 0.658823529 & 22 & 0.011049724 & 0.232044199 & 0.002664933 \\
\hline PKBB(h) & 90 & 78 & 78 & 57 & 0.670588235 & 22 & 0.011049724 & 0.243093923 & 0.00279493 \\
\hline PKB $\{(\mathrm{h})$ & 91 & 76 & 76 & 58 & 0.682352941 & 24 & 0.012054244 & 0.255148167 & 0.002930836 \\
\hline PKCa $(\mathrm{h})$ & 87 & 75 & 75 & 59 & 0.694117647 & 25 & 0.012556504 & 0.267704671 & 0.003075605 \\
\hline РКСВI(h) & 99 & 74 & 74 & 60 & 0.705882353 & 26 & 0.013058764 & 0.280763435 & 0.003226283 \\
\hline PKC (h) & 105 & 74 & 74 & 61 & 0.717647059 & 26 & 0.013058764 & 0.2938222 & 0.003379916 \\
\hline PKCố(h) & 107 & 73 & 73 & 62 & 0.729411765 & 27 & 0.013561025 & 0.307383225 & 0.003536502 \\
\hline PKCałh & 78 & 72 & 72 & 63 & 0.741176471 & 28 & 0.014063285 & 0.321446509 & 0.003698998 \\
\hline PKC & 96 & 72 & 72 & 64 & 0.752941176 & 28 & 0.014063285 & 0.335509794 & 0.003864449 \\
\hline $\mathrm{P} \mathrm{KC}_{\text {(h) }}$ & 90 & 70 & 70 & 65 & 0.764705882 & 30 & 0.015067805 & 0.350577599 & 0.004035808 \\
\hline РКС $\mu(\mathrm{h})$ & 86 & 69 & 69 & 66 & 0.776470588 & 31 & 0.015570065 & 0.366147664 & 0.004216031 \\
\hline PKC $\theta$ (i) & 91 & 67 & 67 & 67 & 0.788235294 & 33 & 0.016574586 & 0.38272225 & 0.004405117 \\
\hline PKD2(h) & 73 & 67 & 67 & 68 & 0.8 & 33 & 0.016574586 & 0.399296836 & 0.004600112 \\
\hline PRAK(h) & 76 & 67 & 67 & 69 & 0.811764706 & 33 & 0.016574586 & 0.415871421 & 0.004795107 \\
\hline PRK2(h) & 88 & 63 & 63 & 70 & 0.823529412 & 37 & 0.018583626 & 0.434455048 & 0.00500192 \\
\hline ROCK-II(h) & 23 & 60 & 60 & 71 & 0.835294118 & 40 & 0.020090407 & 0.454545455 & 0.005229415 \\
\hline ROCK-II(r) & 32 & 58 & 58 & 72 & 0.847058824 & 42 & 0.021094927 & 0.475640382 & 0.005471681 \\
\hline Rsk1(h) & 89 & 58 & 58 & 73 & 0.858823529 & 42 & 0.021094927 & 0.496735309 & 0.005719857 \\
\hline Rskl(r) & 72 & 47 & 47 & 74 & 0.870588235 & 53 & 0.026619789 & 0.523355098 & 0.006000532 \\
\hline Rsk2(h) & 58 & 36 & 36 & 75 & 0.882352941 & 64 & 0.032144651 & 0.555499749 & 0.006346205 \\
\hline Rsk3(h) & 104 & 32 & 32 & 76 & 0.894117647 & 68 & 0.034153692 & 0.58965344 & 0.006736195 \\
\hline SAPK2a(h) & 99 & 24 & 24 & 77 & 0.905882353 & 76 & 0.038171773 & 0.627825213 & 0.007161639 \\
\hline SAPK2b(h) & 103 & 23 & 23 & 78 & 0.917647059 & 77 & 0.038674033 & 0.666499247 & 0.007613673 \\
\hline SAPK3(h) & 84 & 9 & 9 & 79 & 0.929411765 & 91 & 0.045705676 & 0.712204922 & 0.008110025 \\
\hline SAPK4(h) & 94 & 6 & 6 & 80 & 0.941176471 & 94 & 0.047212456 & 0.759417378 & 0.008656602 \\
\hline SGK(h) & 63 & 6 & 6 & 81 & 0.952941176 & 94 & 0.047212456 & 0.806629834 & 0.009212042 \\
\hline Syk(h) & 67 & 5 & 5 & 82 & 0.964705882 & 95 & 0.047714716 & 0.85434455 & 0.009770438 \\
\hline TrkB(h) & 131 & 5 & 5 & 83 & 0.976470588 & 95 & 0.047714716 & 0.902059267 & 0.010331787 \\
\hline Yes(h) & 9 & 4 & 4 & 84 & 0.988235294 & 96 & 0.048216976 & 0.950276243 & 0.010896091 \\
\hline ZAP-70(h) & 69 & 1 & 1 & 85 & 1 & 99 & 0.049723757 & 1 & 0.011472213 \\
\hline & & & & & Total inhibition & 1991 & & Total area & 0.215809377 \\
\hline & & & & & & & & Gini & 0.568381245 \\
\hline
\end{tabular}




\begin{tabular}{|c|c|c|c|c|c|c|c|c|c|}
\hline & $10 \mu \mathrm{M}$ ATP & AG1024; $10 \mu \mathrm{M} ; 10$ & $\mu \mathrm{M}$ ATP & & & & & & \\
\hline Kinase & AG1024@ $10 \mu \mathrm{M}$ & sorted & normalized & position & cumulative sample fraction & inhibition\% & fraction of total inhibition & cumulative inhibition & area \\
\hline $\mathrm{Abl}(\mathrm{m})$ & 1 & 131 & 100 & 1 & 0.011764706 & 0 & 0 & 0 & 0 \\
\hline $\mathrm{AMPK}(\mathrm{r})$ & 84 & 118 & 100 & 2 & 0.023529412 & 0 & 0 & 0 & 0 \\
\hline $\operatorname{Arg}(\mathrm{m})$ & 6 & 116 & 100 & 3 & 0.035294118 & 0 & 0 & 0 & 0 \\
\hline Aurora-A(h) & 104 & 113 & 100 & 4 & 0.047058824 & 0 & 0 & 0 & 0 \\
\hline Axl(h) & 58 & 107 & 100 & 5 & 0.058823529 & 0 & 0 & 0 & 0 \\
\hline $\operatorname{Bk}(\mathrm{m})$ & 4 & 107 & 100 & 6 & 0.070588235 & 0 & 0 & 0 & 0 \\
\hline $\operatorname{Bmx}(\mathrm{h})$ & 84 & 105 & 100 & 7 & 0.082352941 & 0 & 0 & 0 & 0 \\
\hline CaMKII(r) & 89 & 105 & 100 & 8 & 0.094117647 & 0 & 0 & 0 & 0 \\
\hline CaMKIV(h) & 81 & 104 & 100 & 9 & 0.105882353 & 0 & 0 & 0 & 0 \\
\hline $\mathrm{CDK} 1 /$ cyclinB(h) & 67 & 104 & 100 & 10 & 0.117647059 & 0 & 0 & 0 & 0 \\
\hline CDK2/cyclina(h) & 101 & 103 & 100 & 11 & 0.129411765 & 0 & 0 & 0 & 0 \\
\hline CDK2/cyclinE(h) & 95 & 101 & 100 & 12 & 0.141176471 & 0 & 0 & 0 & 0 \\
\hline CDK3/cyclinE(h) & 87 & 99 & 99 & 13 & 0.152941176 & 1 & 0.000504796 & 0.000504796 & 2.96939E- 06 \\
\hline CDK5/p35(h) & 78 & 99 & 99 & 14 & 0.164705882 & 1 & 0.000504796 & 0.001009591 & $8.90816 \mathrm{E}-06$ \\
\hline CDK6/(yclinD3(h) & 92 & 99 & 99 & 15 & 0.176470588 & 1 & 0.000504796 & 0.001514387 & $1.48469 \mathrm{E}-05$ \\
\hline CDK7/cyclinHMATI(h) & 93 & 98 & 98 & 16 & 0.188235294 & 2 & 0.001009591 & 0.002523978 & $2.37551 \mathrm{E}-05$ \\
\hline CHK1(h) & 97 & 97 & 97 & 17 & 0.2 & 3 & 0.001514387 & 0.004038364 & $3.8602 \mathrm{E}-05$ \\
\hline СНК2(h) & 88 & 96 & 96 & 18 & 0.211764706 & 4 & 0.002019182 & 0.006057547 & 5.93877E- 05 \\
\hline CKl(y) & 87 & 96 & 96 & 19 & 0.223529412 & 4 & 0.002019182 & 0.008076729 & $8.31428 \mathrm{E}-05$ \\
\hline CK2(h) & 96 & 95 & 95 & 20 & 0.235294118 & 5 & 0.002523978 & 0.010600707 & 0.000109867 \\
\hline$c-\operatorname{RAF(h)}$ & 72 & 94 & 94 & 21 & 0.247058824 & 6 & 0.003028773 & 0.01362948 & 0.000142531 \\
\hline $\operatorname{CSK}(\mathrm{h})$ & 75 & 93 & 93 & 22 & 0.258823529 & 7 & 0.003533569 & 0.017163049 & 0.000181133 \\
\hline cSRC(h) & 6 & 93 & 93 & 23 & 0.270588235 & 7 & 0.003533569 & 0.020696618 & 0.000222704 \\
\hline Fes(h) & 70 & 93 & 93 & 24 & 0.282352941 & 7 & 0.003533569 & 0.024230187 & 0.000264275 \\
\hline FGFR3(h) & 91 & 92 & 92 & 25 & 0.294117647 & 8 & 0.004038364 & 0.028268551 & 0.000308816 \\
\hline Fll3(h) & 89 & 92 & 92 & 26 & 0.305882353 & 8 & 0.004038364 & 0.032306916 & 0.000356326 \\
\hline Fyn(h) & 5 & 91 & 91 & 27 & 0.317647059 & 9 & 0.00454316 & 0.036850076 & 0.000406806 \\
\hline GSK3 ah) & 89 & 91 & 91 & 28 & 0.329411765 & 9 & 0.00454316 & 0.041393236 & 0.000460255 \\
\hline $\operatorname{GSK3B}(h)$ & 57 & 91 & 91 & 29 & 0.341176471 & 9 & 0.00454316 & 0.045936396 & 0.000513704 \\
\hline IGF-IR(h) & 118 & 90 & 90 & 30 & 0.352941176 & 10 & 0.005047956 & 0.050984351 & 0.000570122 \\
\hline $\mathrm{IKK} \alpha(\mathrm{h})$ & 82 & 90 & 90 & 31 & 0.364705882 & 10 & 0.005047956 & 0.056032307 & 0.00062951 \\
\hline IKKB(h) & 93 & 90 & 90 & 32 & 0.376470588 & 10 & 0.005047956 & 0.061080262 & 0.000688897 \\
\hline $\mathrm{IR}(\mathrm{h})$ & 105 & 89 & 89 & 33 & 0.388235294 & 11 & 0.005552751 & 0.066633014 & 0.000751255 \\
\hline JNKIal(h) & 79 & 89 & 89 & 34 & 0.4 & 11 & 0.005552751 & 0.072185765 & 0.000816581 \\
\hline JNK2a2(h) & 89 & 89 & 89 & 35 & 0.411764706 & 11 & 0.005552751 & 0.077738516 & 0.000881908 \\
\hline JNK3(r) & 60 & 89 & 89 & 36 & 0.423529412 & 11 & 0.005552751 & 0.083291267 & 0.000947234 \\
\hline Lck(h) & 24 & 89 & 89 & 37 & 0.435294118 & 11 & 0.005552751 & 0.088844018 & 0.001012561 \\
\hline Lyn(h) & 74 & 88 & 88 & 38 & 0.447058824 & 12 & 0.006057547 & 0.094901565 & 0.001080856 \\
\hline Lyn(m) & 5 & 88 & 88 & 39 & 0.458823529 & 12 & 0.006057547 & 0.100959112 & 0.001152122 \\
\hline MAPK I(h) & 85 & 88 & 88 & 40 & 0.470588235 & 12 & 0.006057547 & 0.107016658 & 0.001223387 \\
\hline MAPK $2(\mathrm{~h})$ & 113 & 88 & 88 & 41 & 0.482352941 & 12 & 0.006057547 & 0.113074205 & 0.001294652 \\
\hline MAPK2(m) & 93 & 88 & 88 & 42 & 0.494117647 & 12 & 0.006057547 & 0.119131752 & 0.001365917 \\
\hline MAPKAP-K2(h) & 90 & 87 & 87 & 43 & 0.505882353 & 13 & 0.006562342 & 0.125694094 & 0.001440152 \\
\hline MEK1(h) & 88 & 87 & 87 & 44 & 0.517647059 & 13 & 0.006562342 & 0.132256436 & 0.001517356 \\
\hline MKK4(m) & 88 & 87 & 87 & 45 & 0.529411765 & 13 & 0.006562342 & 0.138818778 & 0.00159456 \\
\hline МKK6(h) & 86 & 86 & 86 & 46 & 0.541176471 & 14 & 0.007067138 & 0.145885916 & 0.001674733 \\
\hline MKK7в(h) & 116 & 86 & 86 & 47 & 0.552941176 & 14 & 0.007067138 & 0.152953054 & 0.001757876 \\
\hline MSK1(h) & 92 & 85 & 85 & 48 & 0.564705882 & 15 & 0.007571933 & 0.160524987 & 0.001843988 \\
\hline p7056K(h) & 36 & 84 & 84 & 49 & 0.576470588 & 16 & 0.008076729 & 0.168601716 & 0.001936039 \\
\hline PAK2(h) & 98 & 84 & 84 & 50 & 0.588235294 & 16 & 0.008076729 & 0.176678445 & 0.00203106 \\
\hline PDGFRa(h) & 88 & 84 & 84 & 51 & 0.6 & 16 & 0.008076729 & 0.184755174 & 0.00212608 \\
\hline PDGFRB(h) & 67 & 84 & 84 & 52 & 0.611764706 & 16 & 0.008076729 & 0.192831903 & 0.0022211 \\
\hline PDKI(h) & 84 & 82 & 82 & 53 & 0.623529412 & 18 & 0.00908632 & 0.201918223 & 0.00232206 \\
\hline PKA(b) & 74 & 81 & 81 & 54 & 0.635294118 & 19 & 0.009591116 & 0.211509339 & 0.002431927 \\
\hline PKA(h) & 107 & 79 & 79 & 55 & 0.647058824 & 21 & 0.010600707 & 0.222110045 & 0.002550702 \\
\hline PKBa(h) & 99 & 78 & 78 & 56 & 0.658823529 & 22 & 0.011105502 & 0.233215548 & 0.002678386 \\
\hline PKBB(h) & 90 & 78 & 78 & 57 & 0.670588235 & 22 & 0.011105502 & 0.24432105 & 0.002809039 \\
\hline PKB $\gamma(\mathrm{h})$ & 91 & 76 & 76 & 58 & 0.682352941 & 24 & 0.012115093 & 0.256436143 & 0.002945631 \\
\hline PKCa $a(\mathrm{~h})$ & 87 & 75 & 75 & 59 & 0.694117647 & 25 & 0.012619889 & 0.269056032 & 0.00309113 \\
\hline РКСBI(h) & 99 & 74 & 74 & 60 & 0.705882353 & 26 & 0.013124685 & 0.282180717 & 0.003242569 \\
\hline PKC $(\mathrm{h})$ & 105 & 74 & 74 & 61 & 0.717647059 & 26 & 0.013124685 & 0.295305401 & 0.003396977 \\
\hline PKCố(h) & 107 & 73 & 73 & 62 & 0.729411765 & 27 & 0.01362948 & 0.308934881 & 0.003554355 \\
\hline PKCąh) & 78 & 72 & 72 & 63 & 0.741176471 & 28 & 0.014134276 & 0.323069157 & 0.003717671 \\
\hline 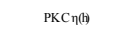 & 96 & 72 & 72 & 64 & 0.752941176 & 28 & 0.014134276 & 0.337203433 & 0.003883956 \\
\hline $\mathrm{PKC}_{\text {(h) }}$ & 90 & 70 & 70 & 65 & 0.764705882 & 30 & 0.015143867 & 0.352347299 & 0.004056181 \\
\hline PKC(ph) & 86 & 69 & 69 & 66 & 0.776470588 & 31 & 0.015648662 & 0.367995962 & 0.004237313 \\
\hline PKC $\theta$ (1) & 91 & 67 & 67 & 67 & 0.788235294 & 33 & 0.016658253 & 0.384654215 & 0.004427354 \\
\hline PKD2(h) & 73 & 67 & 67 & 68 & 0.8 & 33 & 0.016658253 & 0.401312468 & 0.004623333 \\
\hline PRAK(h) & 76 & 67 & 67 & 69 & 0.811764706 & 33 & 0.016658253 & 0.417970722 & 0.004819313 \\
\hline PRK2(h) & 88 & 63 & 63 & 70 & 0.823529412 & 37 & 0.018677436 & 0.436648157 & 0.00502717 \\
\hline ROCK-II(h) & 23 & 60 & 60 & 71 & 0.835294118 & 40 & 0.020191822 & 0.45683998 & 0.005255813 \\
\hline ROCK-II(r) & 32 & 58 & 58 & 72 & 0.847058824 & 42 & 0.021201413 & 0.478041393 & 0.005499302 \\
\hline Rskl(h) & 89 & 58 & 58 & 73 & 0.858823529 & 42 & 0.021201413 & 0.499242807 & 0.005748731 \\
\hline Rsk1(r) & 72 & 57 & 57 & 74 & 0.870588235 & 43 & 0.021706209 & 0.520949016 & 0.006001128 \\
\hline Rsk2(h) & 58 & 36 & 36 & 75 & 0.882352941 & 64 & 0.032306916 & 0.553255931 & 0.006318853 \\
\hline Rsk3(h) & 104 & 32 & 32 & 76 & 0.894117647 & 68 & 0.034326098 & 0.587582029 & 0.006710812 \\
\hline SAPK2a(h) & 99 & 24 & 24 & 77 & 0.905882353 & 76 & 0.038364462 & 0.625946492 & 0.007138403 \\
\hline SAPK2b(h) & 103 & 23 & 23 & 78 & 0.917647059 & 77 & 0.038869258 & 0.66481575 & 0.007592719 \\
\hline SAPK3(h) & 84 & 9 & 9 & 79 & 0.929411765 & 91 & 0.045936396 & 0.710752145 & 0.008091576 \\
\hline $\mathrm{SAPK} 4(\mathrm{~h})$ & 94 & 6 & 6 & 80 & 0.941176471 & 94 & 0.047450782 & 0.758202928 & 0.008640912 \\
\hline SGK(h) & 63 & 6 & 6 & 81 & 0.952941176 & 94 & 0.047450782 & 0.80565371 & 0.009199157 \\
\hline Syk(h) & 67 & 5 & 5 & 82 & 0.964705882 & 95 & 0.047955578 & 0.853609288 & 0.009760371 \\
\hline TrkB(h) & 131 & 5 & 5 & 83 & 0.976470588 & 95 & 0.047955578 & 0.901564866 & 0.010324554 \\
\hline Yes(h) & 9 & 4 & 4 & 84 & 0.988235294 & 96 & 0.048460374 & 0.95002524 & 0.010891707 \\
\hline $\mathrm{ZAP}-70(\mathrm{~h})$ & 69 & 1 & 1 & 85 & 1 & 99 & 0.04997476 & 1 & 0.011470737 \\
\hline & & & & & Total inhibition & 1981 & & Total area & 0.216215815 \\
\hline & & & & & & & & Gini & 0.56756837 \\
\hline
\end{tabular}




\begin{tabular}{|c|c|c|c|}
\hline & $10 \mu \mathrm{M}$ ATP & AG1024; $10 \mu \mathrm{M} ; 10$ & यM ATP \\
\hline Kinase & AG1024@ @ $10 \mu \mathrm{M}$ & sorted & normalized \\
\hline $\mathrm{Abl}(\mathrm{m})$ & 1 & 131 & 100 \\
\hline AMPK(r) & 84 & 118 & 100 \\
\hline $\operatorname{Arg}(\mathrm{m})$ & 6 & 116 & 100 \\
\hline Aurora-A(h) & 104 & 113 & 100 \\
\hline $\operatorname{Ax}(\mathrm{h})$ & 58 & 107 & 100 \\
\hline $\operatorname{Bk}(\mathrm{m})$ & 4 & 107 & 100 \\
\hline Bmxx(h) & 84 & 105 & 100 \\
\hline CaMKII(r) & 89 & 105 & 100 \\
\hline CaMKIV(h) & 81 & 104 & 100 \\
\hline CDK1/cyclinB(h) & 67 & 104 & 100 \\
\hline $\mathrm{CDK} 2 / \mathrm{cyclin} \mathrm{A}(\mathrm{h})$ & 101 & 103 & 100 \\
\hline $\mathrm{CDK} 2 / \mathrm{cyclinE}(\mathrm{h})$ & 95 & 101 & 100 \\
\hline CDK3/cyclinE(h) & 87 & 99 & 99 \\
\hline CDK5/p35(h) & 78 & 99 & 99 \\
\hline CDK6/cyclinD3(h) & 92 & 99 & 99 \\
\hline CDK7/cyclinHMATI(h) & 93 & 98 & 98 \\
\hline CHK1(h) & 97 & 97 & 97 \\
\hline СНК2(h) & 88 & 96 & 96 \\
\hline CKI(y) & 87 & 96 & 96 \\
\hline СK2(h) & 96 & 95 & 95 \\
\hline $\mathrm{c} \cdot \mathrm{RAF}(\mathrm{h})$ & 72 & 94 & 94 \\
\hline $\operatorname{CSK}(\mathrm{h})$ & 75 & 93 & 93 \\
\hline $\operatorname{cSRC(h)}$ & 6 & 93 & 93 \\
\hline Fes(h) & 70 & 93 & 93 \\
\hline FGFR3(h) & 91 & 92 & 92 \\
\hline $\mathrm{FH} 3(\mathrm{~h})$ & 89 & 92 & 92 \\
\hline Fyn(h) & 5 & 91 & 91 \\
\hline GSK3 ah) & 89 & 91 & 91 \\
\hline GSK3B(h) & 52 & 91 & 91 \\
\hline IGF-IR(h) & 118 & 90 & 90 \\
\hline $\mathrm{IKK} \alpha(\mathrm{h})$ & 82 & 90 & 90 \\
\hline IKKB(h) & 93 & 90 & 90 \\
\hline $\mathrm{IR}(\mathrm{h})$ & 105 & 89 & 89 \\
\hline JNKI 1 1(h) & 79 & 89 & 89 \\
\hline JNK2 $22(\mathrm{~h})$ & 89 & 89 & 89 \\
\hline JNK3(r) & 60 & 89 & 89 \\
\hline Lck(h) & 24 & 89 & 89 \\
\hline Lyynhlh) & 74 & 88 & 88 \\
\hline Lyn(m) & 5 & 88 & 88 \\
\hline MAPK1(h) & 85 & 88 & 88 \\
\hline MAPK2(h) & 113 & 88 & 88 \\
\hline MAPK2(m) & 93 & 88 & 88 \\
\hline MAPKAP-K2(h) & 90 & 87 & 87 \\
\hline MEK 1(h) & 88 & 87 & 87 \\
\hline MKK $4(\mathrm{~m})$ & 88 & 87 & 87 \\
\hline MKK6(h) & 86 & 86 & 86 \\
\hline MKK $7 B(\mathrm{~h})$ & 116 & 86 & 86 \\
\hline MSK1(h) & 92 & 85 & 85 \\
\hline $\mathrm{p} 70 \mathrm{~s} 6 \mathrm{~K}(\mathrm{~h})$ & 36 & 84 & 84 \\
\hline PAK2(h) & 98 & 84 & 84 \\
\hline PDGFRa(h) & 88 & 84 & 84 \\
\hline PDGFRß(h) & 67 & 84 & 84 \\
\hline PDK1(h) & 84 & 82 & 82 \\
\hline PKA(b) & 74 & 81 & 81 \\
\hline PKA(h) & 107 & 79 & 79 \\
\hline PKBa(h) & 99 & 78 & 78 \\
\hline РКBВ(h) & 90 & 78 & 78 \\
\hline PKB $\gamma(\mathrm{h})$ & 91 & 76 & 76 \\
\hline PKC $\alpha(\mathrm{h})$ & 87 & 75 & 75 \\
\hline РКСВII(h) & 99 & 74 & 74 \\
\hline PKC $\{(\mathbf{l})$ & 105 & 74 & 74 \\
\hline $\operatorname{PKC} \delta(\mathrm{h})$ & 107 & 73 & 73 \\
\hline PKCah) & 78 & 72 & 72 \\
\hline $\mathrm{PKC \eta}($ (i) & 96 & 72 & 72 \\
\hline P KC (hi) & 90 & 70 & 70 \\
\hline PKCu(h) & 86 & 69 & 69 \\
\hline PKC & 91 & 67 & 67 \\
\hline PKD2(h) & 73 & 67 & 67 \\
\hline PRAK(h) & 76 & 67 & 67 \\
\hline PRK2(h) & 88 & 63 & 63 \\
\hline ROCK-II(h) & 23 & 60 & 60 \\
\hline ROCK-II(r) & 32 & 58 & 58 \\
\hline Rskl(h) & 89 & 58 & 58 \\
\hline Rskl(r) & 72 & 52 & 52 \\
\hline Rsk2(h) & 58 & 36 & 36 \\
\hline Rsk3(h) & 104 & 32 & 32 \\
\hline SAPK2a(h) & 99 & 24 & 24 \\
\hline SAPK2b(h) & 103 & 23 & 23 \\
\hline SAPK3(h) & 84 & 8 & 8 \\
\hline SAPK 4(h) & 94 & 6 & 6 \\
\hline SGK(h) & 63 & 6 & 6 \\
\hline Syk(h) & 67 & 5 & 5 \\
\hline $\operatorname{TrkB(h)}$ & 131 & 5 & 5 \\
\hline Yes(h) & 8 & 4 & 4 \\
\hline ZAP-70(h) & 69 & 1 & 1 \\
\hline
\end{tabular}

\begin{tabular}{|c|c|c|c|c|c|}
\hline position & cumulative sample fraction & inhibition\% & fraction of total inhibition & cumulative inhibition & area \\
\hline 1 & 0.011764706 & 0 & 0 & 0 & 0 \\
\hline 2 & 0.023529412 & 0 & 0 & 0 & 0 \\
\hline 3 & 0.035294118 & 0 & 0 & 0 & 0 \\
\hline 4 & 0.047058824 & 0 & 0 & 0 & 0 \\
\hline 5 & 0.058823529 & 0 & 0 & 0 & 0 \\
\hline 6 & 0.070588235 & 0 & 0 & 0 & 0 \\
\hline 7 & 0.082352941 & 0 & 0 & 0 & 0 \\
\hline 8 & 0.094117647 & 0 & 0 & 0 & 0 \\
\hline 9 & 0.105882353 & 0 & 0 & 0 & 0 \\
\hline 10 & 0.117647059 & 0 & 0 & 0 & 0 \\
\hline 11 & 0.129411765 & 0 & 0 & 0 & 0 \\
\hline 12 & 0.141176471 & 0 & 0 & 0 & 0 \\
\hline 13 & 0.152941176 & 1 & 0.000503271 & 0.000503271 & 2.96042E-06 \\
\hline 14 & 0.164705882 & 1 & 0.000503271 & 0.001006543 & $8.88126 \mathrm{E}-06$ \\
\hline 15 & 0.176470588 & 1 & 0.000503271 & 0.001509814 & $1.48021 \mathrm{E}-05$ \\
\hline 16 & 0.188235294 & 2 & 0.001006543 & 0.002516356 & $2.36834 \mathrm{E}-05$ \\
\hline 17 & 0.2 & 3 & 0.001509814 & 0.00402617 & $3.84854 \mathrm{E}-05$ \\
\hline 18 & 0.211764706 & 4 & 0.002013085 & 0.006039255 & $5.92084 \mathrm{E}-05$ \\
\hline 19 & 0.223529412 & 4 & 0.002013085 & 0.00805234 & $8.28917 \mathrm{E}-05$ \\
\hline 20 & 0.235294118 & 5 & 0.002516356 & 0.010568697 & 0.000109536 \\
\hline 21 & 0.247058824 & 6 & 0.003019628 & 0.013588324 & 0.0001421 \\
\hline 22 & 0.258823529 & 7 & 0.003522899 & 0.017111223 & 0.000180586 \\
\hline 23 & 0.270588235 & 7 & 0.003522899 & 0.020634122 & 0.000222031 \\
\hline 24 & 0.282352941 & 7 & 0.003522899 & 0.024157021 & 0.000263477 \\
\hline 25 & 0.294117647 & 8 & 0.00402617 & 0.028183191 & 0.000307884 \\
\hline 26 & 0.305882353 & 8 & 0.00402617 & 0.032209361 & 0.00035525 \\
\hline 27 & 0.317647059 & 9 & 0.004529441 & 0.036738802 & 0.000405577 \\
\hline 28 & 0.329411765 & 9 & 0.004529441 & 0.041268244 & 0.000458865 \\
\hline 29 & 0.341176471 & 9 & 0.004529441 & 0.045797685 & 0.000512153 \\
\hline 30 & 0.352941176 & 10 & 0.005032713 & 0.050830398 & 0.0005684 \\
\hline 31 & 0.364705882 & 10 & 0.005032713 & 0.05586311 & 0.000627609 \\
\hline 32 & 0.376470588 & 10 & 0.005032713 & 0.060895823 & 0.000686817 \\
\hline 33 & 0.388235294 & 11 & 0.005535984 & 0.066431807 & 0.000748986 \\
\hline 34 & 0.4 & 11 & 0.005535984 & 0.071967791 & 0.000814115 \\
\hline 35 & 0.411764706 & 11 & 0.005535984 & 0.077503775 & 0.000879245 \\
\hline 36 & 0.423529412 & 11 & 0.005535984 & 0.083039758 & 0.000944374 \\
\hline 37 & 0.435294118 & 11 & 0.005535984 & 0.088575742 & 0.001009503 \\
\hline 38 & 0.447058824 & 12 & 0.006039255 & 0.094614997 & 0.001077593 \\
\hline 39 & 0.458823529 & 12 & 0.006039255 & 0.100654253 & 0.001148643 \\
\hline 40 & 0.470588235 & 12 & 0.006039255 & 0.106693508 & 0.001219693 \\
\hline 41 & 0.482352941 & 12 & 0.006039255 & 0.112732763 & 0.001290743 \\
\hline 42 & 0.494117647 & 12 & 0.006039255 & 0.118772018 & 0.001361793 \\
\hline 43 & 0.505882353 & 13 & 0.006542526 & 0.125314545 & 0.001435803 \\
\hline 44 & 0.517647059 & 13 & 0.006542526 & 0.131857071 & 0.001512774 \\
\hline 45 & 0.529411765 & 13 & 0.006542526 & 0.138399597 & 0.001589745 \\
\hline 46 & 0.541176471 & 14 & 0.007045798 & 0.145445395 & 0.001669676 \\
\hline 47 & 0.552941176 & 14 & 0.007045798 & 0.152491193 & 0.001752568 \\
\hline 48 & 0.564705882 & 15 & 0.007549069 & 0.160040262 & 0.00183842 \\
\hline 49 & 0.576470588 & 16 & 0.00805234 & 0.168092602 & 0.001930193 \\
\hline 50 & 0.588235294 & 16 & 0.00805234 & 0.176144942 & 0.002024927 \\
\hline 51 & 0.6 & 16 & 0.00805234 & 0.184197282 & 0.00211966 \\
\hline 52 & 0.611764706 & 16 & 0.00805234 & 0.192249623 & 0.002214394 \\
\hline 53 & 0.623529412 & 18 & 0.009058883 & 0.201308505 & 0.002315048 \\
\hline 54 & 0.635294118 & 19 & 0.009562154 & 0.210870659 & 0.002424583 \\
\hline 55 & 0.647058824 & 21 & 0.010568697 & 0.221439356 & 0.002543 \\
\hline 56 & 0.658823529 & 22 & 0.011071968 & 0.232511324 & 0.002670298 \\
\hline 57 & 0.670588235 & 22 & 0.011071968 & 0.243583291 & 0.002800557 \\
\hline 58 & 0.682352941 & 24 & 0.01207851 & 0.255661802 & 0.002936736 \\
\hline 59 & 0.694117647 & 25 & 0.012581782 & 0.268243583 & 0.003081796 \\
\hline 60 & 0.705882353 & 26 & 0.013085053 & 0.281328636 & 0.003232778 \\
\hline 61 & 0.717647059 & 26 & 0.013085053 & 0.294413689 & 0.00338672 \\
\hline 62 & 0.729411765 & 27 & 0.013588324 & 0.308002013 & 0.003543622 \\
\hline 63 & 0.741176471 & 28 & 0.014091595 & 0.322093608 & 0.003706445 \\
\hline 64 & 0.752941176 & 28 & 0.014091595 & 0.336185204 & 0.003872228 \\
\hline 65 & 0.764705882 & 30 & 0.015098138 & 0.351283342 & 0.004043933 \\
\hline 66 & 0.776470588 & 31 & 0.015601409 & 0.366884751 & 0.004224518 \\
\hline 67 & 0.788235294 & 33 & 0.016607952 & 0.383492703 & 0.004413985 \\
\hline 68 & 0.8 & 33 & 0.016607952 & 0.400100654 & 0.004609373 \\
\hline 69 & 0.811764706 & 33 & 0.016607952 & 0.416708606 & 0.00480476 \\
\hline 70 & 0.823529412 & 37 & 0.018621037 & 0.435329643 & 0.00501199 \\
\hline 71 & 0.835294118 & 40 & 0.020130851 & 0.455460493 & 0.005239942 \\
\hline 72 & 0.847058824 & 42 & 0.021137393 & 0.476597886 & 0.005482696 \\
\hline 73 & 0.858823529 & 42 & 0.021137393 & 0.497735279 & 0.005731372 \\
\hline 74 & 0.870588235 & 48 & 0.024157021 & 0.5218923 & 0.005997809 \\
\hline 75 & 0.882352941 & 64 & 0.032209361 & 0.554101661 & 0.006329376 \\
\hline 76 & 0.894117647 & 68 & 0.034222446 & 0.588324107 & 0.006720152 \\
\hline 77 & 0.905882353 & 76 & 0.038248616 & 0.626572723 & 0.007146452 \\
\hline 78 & 0.917647059 & 77 & 0.038751887 & 0.66532461 & 0.007599396 \\
\hline 79 & 0.929411765 & 92 & 0.046300956 & 0.711625566 & 0.008099707 \\
\hline 80 & 0.941176471 & 94 & 0.047307499 & 0.758933065 & 0.008650345 \\
\hline 81 & 0.952941176 & 94 & 0.047307499 & 0.806240564 & 0.009206904 \\
\hline 82 & 0.964705882 & 95 & 0.04781077 & 0.854051334 & 0.009766423 \\
\hline 83 & 0.976470588 & 95 & 0.04781077 & 0.901862104 & 0.010328903 \\
\hline \multirow{3}{*}{$\begin{array}{l}84 \\
85\end{array}$} & 0.988235294 & 96 & 0.048314041 & 0.950176145 & 0.010894343 \\
\hline & 1 & 99 & 0.049823855 & 1 & 0.011471624 \\
\hline & Total inhibition & 1987 & & Total area & 0.215941857 \\
\hline
\end{tabular}

Gini

0.568116285 


\begin{tabular}{|c|c|c|c|}
\hline & $10 \mu \mathrm{M}$ ATP & AG1024; $10 \mu \mathrm{M} ; 10$ & यM ATP \\
\hline Kinase & AG1024@ @ $10 \mu \mathrm{M}$ & sorted & normalized \\
\hline $\mathrm{Abl}(\mathrm{m})$ & 1 & 131 & 100 \\
\hline AMPK(r) & 84 & 118 & 100 \\
\hline $\operatorname{Arg}(\mathrm{m})$ & 6 & 116 & 100 \\
\hline Aurora-A(h) & 104 & 113 & 100 \\
\hline $\operatorname{Ax}(\mathrm{h})$ & 58 & 107 & 100 \\
\hline $\operatorname{Bk}(\mathrm{m})$ & 4 & 107 & 100 \\
\hline Bmxx(h) & 84 & 105 & 100 \\
\hline CaMKII(r) & 89 & 105 & 100 \\
\hline CaMKIV(h) & 81 & 104 & 100 \\
\hline CDK1/cyclinB(h) & 67 & 104 & 100 \\
\hline $\mathrm{CDK} 2 / \mathrm{cyclin} \mathrm{A}(\mathrm{h})$ & 101 & 103 & 100 \\
\hline $\mathrm{CDK} 2 / \mathrm{cyclinE}(\mathrm{h})$ & 95 & 101 & 100 \\
\hline CDK3/cyclinE(h) & 87 & 99 & 99 \\
\hline CDK5/p35(h) & 78 & 99 & 99 \\
\hline CDK6/cyclinD3(h) & 92 & 99 & 99 \\
\hline CDK7/cyclinHMATI(h) & 93 & 98 & 98 \\
\hline CHK1(h) & 97 & 97 & 97 \\
\hline СНК2(h) & 88 & 96 & 96 \\
\hline CKI(y) & 87 & 96 & 96 \\
\hline СK2(h) & 96 & 95 & 95 \\
\hline $\mathrm{c} \cdot \mathrm{RAF}(\mathrm{h})$ & 72 & 94 & 94 \\
\hline $\operatorname{CSK}(\mathrm{h})$ & 75 & 93 & 93 \\
\hline $\operatorname{cSRC(h)}$ & 6 & 93 & 93 \\
\hline Fes(h) & 70 & 93 & 93 \\
\hline FGFR3(h) & 91 & 92 & 92 \\
\hline $\mathrm{FH} 3(\mathrm{~h})$ & 89 & 92 & 92 \\
\hline Fyn(h) & 5 & 91 & 91 \\
\hline GSK3 ah) & 89 & 91 & 91 \\
\hline GSK3B(h) & 52 & 91 & 91 \\
\hline IGF-IR(h) & 118 & 90 & 90 \\
\hline $\mathrm{IKK} \alpha(\mathrm{h})$ & 82 & 90 & 90 \\
\hline IKKB(h) & 93 & 90 & 90 \\
\hline $\mathrm{IR}(\mathrm{h})$ & 105 & 89 & 89 \\
\hline JNKI 1 1(h) & 79 & 89 & 89 \\
\hline JNK2 $22(\mathrm{~h})$ & 89 & 89 & 89 \\
\hline JNK3(r) & 60 & 89 & 89 \\
\hline Lck(h) & 24 & 89 & 89 \\
\hline Lyynhlh) & 74 & 88 & 88 \\
\hline Lyn(m) & 5 & 88 & 88 \\
\hline MAPK1(h) & 85 & 88 & 88 \\
\hline MAPK2(h) & 113 & 88 & 88 \\
\hline MAPK2(m) & 93 & 88 & 88 \\
\hline MAPKAP-K2(h) & 90 & 87 & 87 \\
\hline MEK 1(h) & 88 & 87 & 87 \\
\hline MKK $4(\mathrm{~m})$ & 88 & 87 & 87 \\
\hline MKK6(h) & 86 & 86 & 86 \\
\hline MKK $7 B(\mathrm{~h})$ & 116 & 86 & 86 \\
\hline MSK1(h) & 92 & 85 & 85 \\
\hline $\mathrm{p} 70 \mathrm{~s} 6 \mathrm{~K}(\mathrm{~h})$ & 36 & 84 & 84 \\
\hline PAK2(h) & 98 & 84 & 84 \\
\hline PDGFRa(h) & 88 & 84 & 84 \\
\hline PDGFRß(h) & 67 & 84 & 84 \\
\hline PDK1(h) & 84 & 82 & 82 \\
\hline PKA(b) & 74 & 81 & 81 \\
\hline PKA(h) & 107 & 79 & 79 \\
\hline PKBa(h) & 99 & 78 & 78 \\
\hline РКBВ(h) & 90 & 78 & 78 \\
\hline PKB $\gamma(\mathrm{h})$ & 91 & 76 & 76 \\
\hline PKC $\alpha(\mathrm{h})$ & 87 & 75 & 75 \\
\hline РКСВII(h) & 99 & 74 & 74 \\
\hline PKC $\{(\mathbf{l})$ & 105 & 74 & 74 \\
\hline $\operatorname{PKC} \delta(\mathrm{h})$ & 107 & 73 & 73 \\
\hline PKCah) & 78 & 72 & 72 \\
\hline $\mathrm{PKC \eta}($ (i) & 96 & 72 & 72 \\
\hline P KC (hi) & 90 & 70 & 70 \\
\hline PKCu(h) & 86 & 69 & 69 \\
\hline PKC & 91 & 67 & 67 \\
\hline PKD2(h) & 73 & 67 & 67 \\
\hline PRAK(h) & 76 & 67 & 67 \\
\hline PRK2(h) & 88 & 63 & 63 \\
\hline ROCK-II(h) & 23 & 60 & 60 \\
\hline ROCK-II(r) & 32 & 58 & 58 \\
\hline Rskl(h) & 89 & 58 & 58 \\
\hline Rskl(r) & 72 & 52 & 52 \\
\hline Rsk2(h) & 58 & 36 & 36 \\
\hline Rsk3(h) & 104 & 32 & 32 \\
\hline SAPK2a(h) & 99 & 24 & 24 \\
\hline SAPK2b(h) & 103 & 23 & 23 \\
\hline SAPK3(h) & 84 & 10 & 10 \\
\hline SAPK4(h) & 94 & 6 & 6 \\
\hline SGK(h) & 63 & 6 & 6 \\
\hline Syk(h) & 67 & 5 & 5 \\
\hline $\operatorname{TrkB(h)}$ & 131 & 5 & 5 \\
\hline Yes(h) & 10 & 4 & 4 \\
\hline ZAP-70(h) & 69 & 1 & 1 \\
\hline
\end{tabular}

\begin{tabular}{|c|c|c|c|c|c|}
\hline position & cumulative sample fraction & inhibition\% & fraction of total inhibition & cumulative inhibition & area \\
\hline 1 & 0.011764706 & 0 & 0 & 0 & 0 \\
\hline 2 & 0.023529412 & 0 & 0 & 0 & 0 \\
\hline 3 & 0.035294118 & 0 & 0 & 0 & 0 \\
\hline 4 & 0.047058824 & 0 & 0 & 0 & 0 \\
\hline 5 & 0.058823529 & 0 & 0 & 0 & 0 \\
\hline 6 & 0.070588235 & 0 & 0 & 0 & 0 \\
\hline 7 & 0.082352941 & 0 & 0 & 0 & 0 \\
\hline 8 & 0.094117647 & 0 & 0 & 0 & 0 \\
\hline 9 & 0.105882353 & 0 & 0 & 0 & 0 \\
\hline 10 & 0.117647059 & 0 & 0 & 0 & 0 \\
\hline 11 & 0.129411765 & 0 & 0 & 0 & 0 \\
\hline 12 & 0.141176471 & 0 & 0 & 0 & 0 \\
\hline 13 & 0.152941176 & 1 & 0.000503778 & 0.000503778 & $2.9634 \mathrm{E}-06$ \\
\hline 14 & 0.164705882 & 1 & 0.000503778 & 0.001007557 & $8.89021 \mathrm{E}-06$ \\
\hline 15 & 0.176470588 & 1 & 0.000503778 & 0.001511335 & 1.4817E-05 \\
\hline 16 & 0.188235294 & 2 & 0.001007557 & 0.002518892 & $2.37072 \mathrm{E}-05$ \\
\hline 17 & 0.2 & 3 & 0.001511335 & 0.004030227 & $3.85242 \mathrm{E}-05$ \\
\hline 18 & 0.211764706 & 4 & 0.002015113 & 0.00604534 & $5.9268 \mathrm{E}-05$ \\
\hline 19 & 0.223529412 & 4 & 0.002015113 & 0.008060453 & $8.29753 \mathrm{E}-05$ \\
\hline 20 & 0.235294118 & 5 & 0.002518892 & 0.010579345 & 0.000109646 \\
\hline 21 & 0.247058824 & 6 & 0.00302267 & 0.013602015 & 0.000142243 \\
\hline 22 & 0.258823529 & 7 & 0.003526448 & 0.017128463 & 0.000180768 \\
\hline 23 & 0.270588235 & 7 & 0.003526448 & 0.020654912 & 0.000222255 \\
\hline 24 & 0.282352941 & 7 & 0.003526448 & 0.02418136 & 0.000263743 \\
\hline 25 & 0.294117647 & 8 & 0.004030227 & 0.028211587 & 0.000308194 \\
\hline 26 & 0.305882353 & 8 & 0.004030227 & 0.032241814 & 0.000355608 \\
\hline 27 & 0.317647059 & 9 & 0.004534005 & 0.036775819 & 0.000405986 \\
\hline 28 & 0.329411765 & 9 & 0.004534005 & 0.041309824 & 0.000459327 \\
\hline 29 & 0.341176471 & 9 & 0.004534005 & 0.045843829 & 0.000512669 \\
\hline 30 & 0.352941176 & 10 & 0.005037783 & 0.050881612 & 0.000568973 \\
\hline 31 & 0.364705882 & 10 & 0.005037783 & 0.055919395 & 0.000628241 \\
\hline 32 & 0.376470588 & 10 & 0.005037783 & 0.060957179 & 0.000687509 \\
\hline 33 & 0.388235294 & 11 & 0.005541562 & 0.066498741 & 0.000749741 \\
\hline 34 & 0.4 & 11 & 0.005541562 & 0.072040302 & 0.000814936 \\
\hline 35 & 0.411764706 & 11 & 0.005541562 & 0.077581864 & 0.00088013 \\
\hline 36 & 0.423529412 & 11 & 0.005541562 & 0.083123426 & 0.000945325 \\
\hline 37 & 0.435294118 & 11 & 0.005541562 & 0.088664987 & 0.00101052 \\
\hline 38 & 0.447058824 & 12 & 0.00604534 & 0.094710327 & 0.001078678 \\
\hline 39 & 0.458823529 & 12 & 0.00604534 & 0.100755668 & 0.0011498 \\
\hline 40 & 0.470588235 & 12 & 0.00604534 & 0.106801008 & 0.001220922 \\
\hline 41 & 0.482352941 & 12 & 0.00604534 & 0.112846348 & 0.001292043 \\
\hline 42 & 0.494117647 & 12 & 0.00604534 & 0.118891688 & 0.001363165 \\
\hline 43 & 0.505882353 & 13 & 0.006549118 & 0.125440806 & 0.00143725 \\
\hline 44 & 0.517647059 & 13 & 0.006549118 & 0.131989924 & 0.001514298 \\
\hline 45 & 0.529411765 & 13 & 0.006549118 & 0.138539043 & 0.001591347 \\
\hline 46 & 0.541176471 & 14 & 0.007052897 & 0.14559194 & 0.001671359 \\
\hline 47 & 0.552941176 & 14 & 0.007052897 & 0.152644836 & 0.001754334 \\
\hline 48 & 0.564705882 & 15 & 0.007556675 & 0.160201511 & 0.001840273 \\
\hline 49 & 0.576470588 & 16 & 0.008060453 & 0.168261965 & 0.001932138 \\
\hline 50 & 0.588235294 & 16 & 0.008060453 & 0.176322418 & 0.002026967 \\
\hline 51 & 0.6 & 16 & 0.008060453 & 0.184382872 & 0.002121796 \\
\hline 52 & 0.611764706 & 16 & 0.008060453 & 0.192443325 & 0.002216625 \\
\hline 53 & 0.623529412 & 18 & 0.00906801 & 0.201511335 & 0.00231738 \\
\hline 54 & 0.635294118 & 19 & 0.009571788 & 0.211083123 & 0.002427026 \\
\hline 55 & 0.647058824 & 21 & 0.010579345 & 0.221662469 & 0.002545562 \\
\hline 56 & 0.658823529 & 22 & 0.011083123 & 0.232745592 & 0.002672989 \\
\hline 57 & 0.670588235 & 22 & 0.011083123 & 0.243828715 & 0.002803378 \\
\hline 58 & 0.682352941 & 24 & 0.01209068 & 0.255919395 & 0.002939695 \\
\hline 59 & 0.694117647 & 25 & 0.012594458 & 0.268513854 & 0.003084901 \\
\hline 60 & 0.705882353 & 26 & 0.013098237 & 0.281612091 & 0.003236035 \\
\hline 61 & 0.717647059 & 26 & 0.013098237 & 0.294710327 & 0.003390132 \\
\hline 62 & 0.729411765 & 27 & 0.013602015 & 0.308312343 & 0.003547192 \\
\hline 63 & 0.741176471 & 28 & 0.014105793 & 0.322418136 & 0.003710179 \\
\hline 64 & 0.752941176 & 28 & 0.014105793 & 0.336523929 & 0.00387613 \\
\hline 65 & 0.764705882 & 30 & 0.01511335 & 0.35163728 & 0.004048007 \\
\hline 66 & 0.776470588 & 31 & 0.015617128 & 0.367254408 & 0.004228775 \\
\hline 67 & 0.788235294 & 33 & 0.016624685 & 0.383879093 & 0.004418432 \\
\hline 68 & 0.8 & 33 & 0.016624685 & 0.400503778 & 0.004614017 \\
\hline 69 & 0.811764706 & 33 & 0.016624685 & 0.417128463 & 0.004809601 \\
\hline 70 & 0.823529412 & 37 & 0.018639798 & 0.435768262 & 0.00501704 \\
\hline 71 & 0.835294118 & 40 & 0.020151134 & 0.455919395 & 0.005245222 \\
\hline 72 & 0.847058824 & 42 & 0.02115869 & 0.477078086 & 0.00548822 \\
\hline 73 & 0.858823529 & 42 & 0.02115869 & 0.498236776 & 0.005737146 \\
\hline 74 & 0.870588235 & 48 & 0.02418136 & 0.522418136 & 0.006003852 \\
\hline 75 & 0.882352941 & 64 & 0.032241814 & 0.55465995 & 0.006335753 \\
\hline 76 & 0.894117647 & 68 & 0.034256927 & 0.588916877 & 0.006726923 \\
\hline 77 & 0.905882353 & 76 & 0.038287154 & 0.62720403 & 0.007153652 \\
\hline 78 & 0.917647059 & 77 & 0.038790932 & 0.665994962 & 0.007607053 \\
\hline 79 & 0.929411765 & 90 & 0.04534005 & 0.711335013 & 0.008101941 \\
\hline 80 & 0.941176471 & 94 & 0.047355164 & 0.758690176 & 0.008647207 \\
\hline 81 & 0.952941176 & 94 & 0.047355164 & 0.80604534 & 0.009204327 \\
\hline 82 & 0.964705882 & 95 & 0.047858942 & 0.853904282 & 0.00976441 \\
\hline 83 & 0.976470588 & 95 & 0.047858942 & 0.901763224 & 0.010327456 \\
\hline \multirow{3}{*}{$\begin{array}{l}84 \\
85\end{array}$} & 0.988235294 & 96 & 0.04836272 & 0.950125945 & 0.010893466 \\
\hline & 1 & 99 & 0.049874055 & 1 & 0.011471329 \\
\hline & Total inhibition & 1985 & & Total area & 0.216082383 \\
\hline
\end{tabular}

Gini

0.567835235 

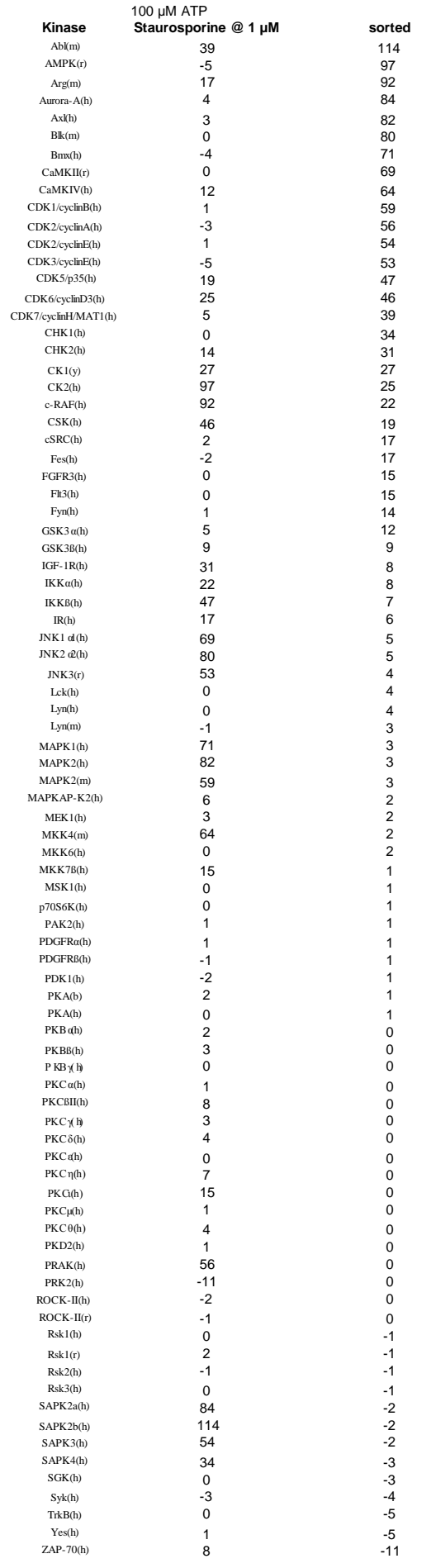

0.129411765

0.141176471
0.152941176

0.164705882

0.176470588

0.188235294
0.2

0.211764706

0.223529412

0.235294118

0.258823529

0.270588235

0.282352941

0.294117647

. 305882353

0.317647059

0.34117647

0.352941176

0.364705882
0.376470588

0.388235294

0.4
0.411764706

0.423529412

0.435294118

0.458823529

0.48235294

0.494117647

0.505882353

0.517647059

0.529411765

0.541176471
0.552941176

0.57647058

0.588235294
0.6

0.6
0.611764706

0.623529412

0.635294118
0.647058824

0.647058824
0.658823529

0.658823529
0.670588235

0.682352941

0.70588235

0.729411765

0.741176471

0.752941176

0.764705882

0.776470588
0.788235294

0.811764706
0.823529412

0.823529412
0.835294118

0.84705882

0.858823529
0.870588235

0.870588235
0.882352941

0.882352941
0.894117647

0.905882353

0.929411765
0.941176471

0.952941176

0.976470588

1

Total inhibition

$\begin{array}{cr} & \\ 0 & \\ 3 & \\ 8 & 0.000 \\ 16 & 0.001 \\ 18 & 0.002\end{array}$

of total inhibition cum
0
0.000424328
0.001131542

cumulative inhibition
0
0.000424328
0.00155587
0.003818953

area

.49605E-06

3.16166E-05

$\begin{array}{lll}0.002263083 & 0.003818953 & 3.16166 \mathrm{E}-05 \\ 0.002545969 & 0.006364922 & 5.99052 \mathrm{E}-05\end{array}$

$\begin{array}{lll}0.002828854 & 0.009193777 & 9.15218 \mathrm{E}-05\end{array}$

0.004101839

$\begin{array}{lll}0.004384724 & 0.017680339 & 0.000182211 \\ 0.005091938 & 0.022772277 & 0.000237957 \\ 0.005799151 & 0.02857429 & 0.00030222\end{array}$

$\begin{array}{lll}0.005799151 & 0.028571429 & 0.000302022 \\ 0.006223479 & 0.034794908 & 0.000372743\end{array}$

$0.041301273 \quad 0.000447625$

$\begin{array}{lll}0.006647808 & 0.047949081 & 0.000525002\end{array}$

$\begin{array}{lll}0.007496464 & 0.055445545 & 0.000608204 \\ 0.007637907 & 0.063083451 & 0.000697229\end{array}$

$\begin{array}{lll}0.008628006 & 0.071711457 & 0.000792911\end{array}$

0.00089857

$\begin{array}{lll}0.009759547 & 0.090806223 & 0.001010899\end{array}$

$\begin{array}{lll}0.010253204 & 0.101131542 & 0.001129046\end{array}$

$\begin{array}{lll}0.011032532 & 0.122772277 & 0.001379482\end{array}$

$\begin{array}{lll}0.01145686 & 0.134229137 & 0.001511773\end{array}$

$0.011739745-0.145968883 \quad 0.001648224$

$\begin{array}{lll}0.011739745 & 0.157708628 & 0.001648224 \\ 0.00176338\end{array}$

$\begin{array}{rrr}0.012022631 & 0.169731259 & 0.001926117 \\ 0.012022631 & 0.18175389 & 0.00206756\end{array}$

$\begin{array}{lll}0.0193917963 & 0.002209834\end{array}$

$\begin{array}{lll}0.012446959 & 0.206364922 & 0.002354605\end{array}$

$\begin{array}{lll}0.012871287 & 0.219236209 & 0.002503536\end{array}$

$\begin{array}{lll}0.01301273 & 0.232248939 & 0.002655795\end{array}$

$\begin{array}{lll}0.01301273 & 0.245261669 & 0.002808886\end{array}$

$\begin{array}{lll}0.013154173 & 0.258415842 & 0.002962809\end{array}$

$\begin{array}{lll}0.013295615 & 0.271711457 & 0.003118396\end{array}$

$\begin{array}{llr}0.013437058 & 0.298585573 & 0.003275647 \\ 0.0135850143373\end{array}$

$\begin{array}{lll}0.013578501 & 0.312164074 & 0.003592645 \\ 0.013578501 & 0.325742574 & 0.003752392\end{array}$

$\begin{array}{lll}0.013578501 & 0.325742574 & 0.003752392 \\ 0.013578501 & 0.339321075 & 0.003912139\end{array}$

0.353041018

$\begin{array}{lll}0.013719943 & 0.353041018 & 0.004072718 \\ 0.013719943 & 0.360760962 & 0.004234129\end{array}$

$0.013719943 \quad 0.380480905-0.00439554$

$\begin{array}{lll}0.013719943 & 0.394200849 & 0.004556951\end{array}$

$0.408062235-0.004719195$

$\begin{array}{lll}0.013861386 & 0.40802235 & 0.004719195 \\ 0.013861386 & 0.421923621 & 0.00488227\end{array}$

$\begin{array}{lll}0.013861386 & 0.435785007 & 0.005045345\end{array}$

$\begin{array}{llr}0.013861386 & 0.449646393 & 0.00520842 \\ 0.014002829 & 0.463649222 & 0.005372327\end{array}$

$\begin{array}{lll}0.014002829 & 0.477652051 & 0.005537066\end{array}$

$\begin{array}{lll}0.014002829 & 0.49165488 & 0.005701805\end{array}$

$\begin{array}{lll}0.014002829 & 0.505657709 & 0.005866545\end{array}$

$\begin{array}{lll}0.014002829 & 0.519660537 & 0.006031284\end{array}$

$\begin{array}{lll}0.014002829 & 0.533663366 & 0.006196023\end{array}$

$\begin{array}{lll}0.014002829 & 0.537666195 & 0.006360762 \\ 0.014002829 & 0.561669024 & 0.006525501\end{array}$

$\begin{array}{ll}0.014002829 & 0.575671853\end{array}$

$\begin{array}{lll}0.014144272 & 0.589816124 & 0.006855812\end{array}$

$\begin{array}{lll}0.014144272 & 0.603960396 & 0.007022215 \\ 0.01414272 & 0.618104668 & 0.007180618\end{array}$

$0.014144272=0.632248939-0.007355021$

$0.646393211-0.007521424$

$\begin{array}{lll}0.014144272 & 0.632248939 & 0.00735521 \\ 0.01414272 & 0.646393211 & 0.007521424 \\ 0.014144272 & 0.660537482 & 0.007687828\end{array}$

$0.674681754-0.007854231$

$\begin{array}{lll}0.014144272 & 0.688826025 & 0.008020634\end{array}$

$\begin{array}{lll}0.014144272 & 0.702970297 & 0.008187037\end{array}$

$\begin{array}{lll}0.01414272 & 0.717114569 & 0.00835344\end{array}$

$\begin{array}{lll}0.014144272 & 0.73125884 & 0.008519844 \\ 0.014144272 & 0.745403112 & 0.008686247\end{array}$

$\begin{array}{lll}0.014144272 & 0.759547383 & 0.00885265 \\ 0.014144272 & 0.773691655 & 0.009019053\end{array}$

$\begin{array}{lll}0.014144272 & 0.773691655 & 0.009019053 \\ 0.014144272 & 0.787835926 & 0.009185456\end{array}$

$\begin{array}{llr}0.01444272 & 0.787835926 & 0.009185456 \\ 0.014144272 & 0.801980198 & 0.00935186 \\ 0.0144272 & 0.861247 & 0.00958263\end{array}$

$\begin{array}{lll}0.014144272 & 0.81612447 & 0.009518263\end{array}$

$\begin{array}{lll}0.014144272 & 0.830268741 & 0.009684666 \\ 0.014144272 & 0.844413013 & 0.009851069\end{array}$

$\begin{array}{lll}0.014144272 & 0.844413013 & 0.009851069 \\ 0.014144272 & 0.858557284 & 0.010017472\end{array}$

$\begin{array}{lll}0.01414427272 & 0.858557284 & 0.010017472 \\ 0.014144272 & 0.872701556 & 0.010183876\end{array}$

$\begin{array}{lll}0.014144272 & 0.886845827 & 0.010350279\end{array}$

$\begin{array}{lll}0.014144272 & 0.900990099 & 0.010516682 \\ 0.014144272 & 0.915134371 & 0.010683085\end{array}$

$\begin{array}{lll}0.014144272 & 0.915134371 & 0.010683085 \\ 0.014144272 & 0.929278642 & 0.010849488\end{array}$

$\begin{array}{lll}0.014144272 & 0.943422914 & 0.011015892\end{array}$

$\begin{array}{lll}0.014144272 & 0.943422914 & 0.011015892 \\ 0.014144272 & 0.957567185 & 0.011182295\end{array}$

$\begin{array}{lll}0.014144272 & 0.957567185 & 0.011182295 \\ 0.014144272 & 0.971711457 & 0.011348698 \\ 0.014144272 & 0.985855728 & 0.011515101\end{array}$

$\begin{array}{ccc}0.014144272 & 1 & 0.01151510 \\ & & 0.911681504\end{array}$

Total area $\quad 0.425141859$ 

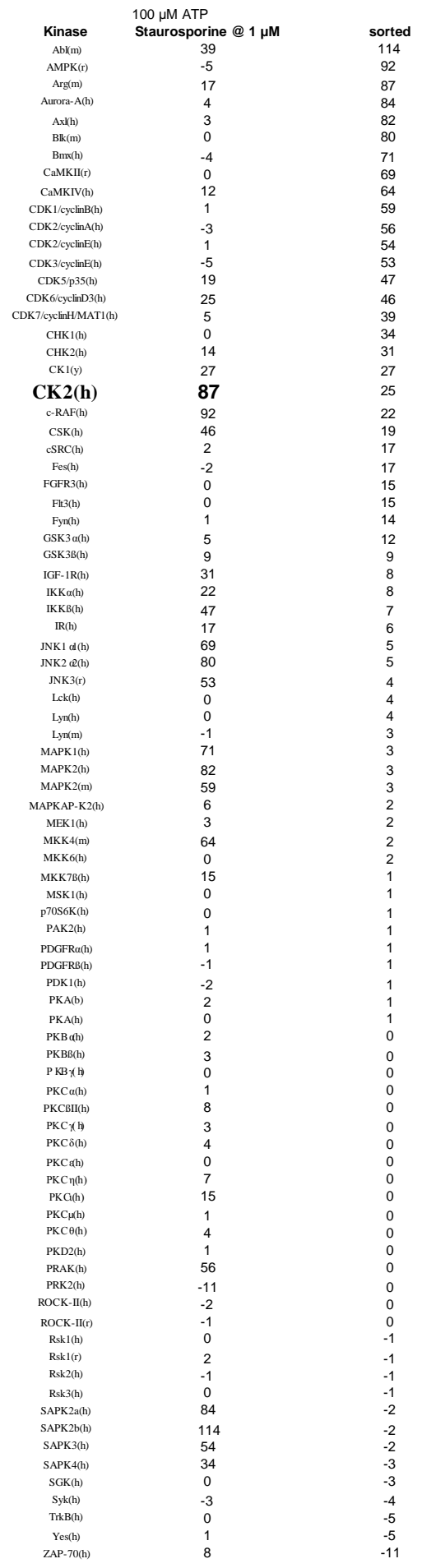

\begin{tabular}{|c|c|c|c|c|c|c|}
\hline normalized & position & cumulative sample fraction & inhibition\% & fraction of total inhibition & cumulative inhibition & area \\
\hline 100 & 1 & 0.011764706 & 0 & 0 & 0 & 0 \\
\hline 92 & 2 & 0.023529412 & 8 & 0.001129944 & 0.001129944 & $6.64673 \mathrm{E}-06$ \\
\hline 87 & 3 & 0.035294118 & 13 & 0.001836158 & 0.002966102 & 2.40944E-05 \\
\hline 84 & 4 & 0.047058824 & 16 & 0.002259887 & 0.005225989 & $4.81888 \mathrm{E}-05$ \\
\hline 82 & 5 & 0.058823529 & 18 & 0.002542373 & 0.007768362 & $7.64374 \mathrm{E}-05$ \\
\hline 80 & 6 & 0.070588235 & 20 & 0.002824859 & 0.01059322 & 0.000108009 \\
\hline 71 & 7 & 0.082352941 & 29 & 0.004096045 & 0.014689266 & 0.000148721 \\
\hline 69 & 8 & 0.094117647 & 31 & 0.004378531 & 0.019067797 & 0.000198571 \\
\hline 64 & 9 & 0.105882353 & 36 & 0.005084746 & 0.024152542 & 0.000254237 \\
\hline 59 & 10 & 0.117647059 & 41 & 0.00579096 & 0.029943503 & 0.000318212 \\
\hline 56 & 11 & 0.129411765 & 44 & 0.006214689 & 0.036158192 & 0.000388833 \\
\hline 54 & 12 & 0.141176471 & 46 & 0.006497175 & 0.042655367 & 0.000463609 \\
\hline 53 & 13 & 0.152941176 & 47 & 0.006638418 & 0.049293785 & 0.000540877 \\
\hline 47 & 14 & 0.164705882 & 53 & 0.007485876 & 0.056779661 & 0.000623961 \\
\hline 46 & 15 & 0.176470588 & 54 & 0.007627119 & 0.06440678 & 0.000712861 \\
\hline 39 & 16 & 0.188235294 & 61 & 0.008615819 & 0.073022599 & 0.000808408 \\
\hline 34 & 17 & 0.2 & 66 & 0.009322034 & 0.082344633 & 0.000913925 \\
\hline 31 & 18 & 0.211764706 & 69 & 0.009745763 & 0.092090395 & 0.001026088 \\
\hline 27 & 19 & 0.223529412 & 73 & 0.010310734 & 0.10240113 & 0.001144068 \\
\hline 25 & 20 & 0.235294118 & 75 & 0.01059322 & 0.11299435 & 0.001267032 \\
\hline 22 & 21 & 0.247058824 & 78 & 0.011016949 & 0.124011299 & 0.001394151 \\
\hline 19 & 22 & 0.258823529 & 81 & 0.011440678 & 0.135451977 & 0.001526255 \\
\hline 17 & 23 & 0.270588235 & 83 & 0.011723164 & 0.147175141 & 0.001662512 \\
\hline 17 & 24 & 0.282352941 & 83 & 0.011723164 & 0.158898305 & 0.001800432 \\
\hline 15 & 25 & 0.294117647 & 85 & 0.01200565 & 0.170903955 & 0.001940013 \\
\hline 15 & 26 & 0.305882353 & 85 & 0.01200565 & 0.182909605 & 0.002081256 \\
\hline 14 & 27 & 0.317647059 & 86 & 0.012146893 & 0.195056497 & 0.00222333 \\
\hline 12 & 28 & 0.329411765 & 88 & 0.012429379 & 0.207485876 & 0.002367896 \\
\hline 9 & 29 & 0.341176471 & 91 & 0.012853107 & 0.220338983 & 0.002516617 \\
\hline 8 & 30 & 0.352941176 & 92 & 0.01299435 & 0.233333333 & 0.002668661 \\
\hline 8 & 31 & 0.364705882 & 92 & 0.01299435 & 0.246327684 & 0.002821535 \\
\hline 7 & 32 & 0.376470588 & 93 & 0.013135593 & 0.259463277 & 0.002975241 \\
\hline 6 & 33 & 0.388235294 & 94 & 0.013276836 & 0.272740113 & 0.003130608 \\
\hline 5 & 34 & 0.4 & 95 & 0.013418079 & 0.286158192 & 0.003287637 \\
\hline 5 & 35 & 0.411764706 & 95 & 0.013418079 & 0.299576271 & 0.003445497 \\
\hline 4 & 36 & 0.423529412 & 96 & 0.013559322 & 0.313135593 & 0.003604187 \\
\hline 4 & 37 & 0.435294118 & 96 & 0.013559322 & 0.326694915 & 0.003763709 \\
\hline 4 & 38 & 0.447058824 & 96 & 0.013559322 & 0.340254237 & 0.00392323 \\
\hline 3 & 39 & 0.458823529 & 97 & 0.013700565 & 0.353954802 & 0.004083583 \\
\hline 3 & 40 & 0.470588235 & 97 & 0.013700565 & 0.367655367 & 0.004244766 \\
\hline 3 & 41 & 0.482352941 & 97 & 0.013700565 & 0.381355932 & 0.004405949 \\
\hline 3 & 42 & 0.494117647 & 97 & 0.013700565 & 0.395056497 & 0.004567132 \\
\hline 2 & 43 & 0.505882353 & 98 & 0.013841808 & 0.408898305 & 0.004729146 \\
\hline 2 & 44 & 0.517647059 & 98 & 0.013841808 & 0.422740113 & 0.004891991 \\
\hline 2 & 45 & 0.529411765 & 98 & 0.013841808 & 0.436581921 & 0.005054835 \\
\hline 2 & 46 & 0.541176471 & 98 & 0.013841808 & 0.450423729 & 0.00521768 \\
\hline 1 & 47 & 0.552941176 & 99 & 0.013983051 & 0.46440678 & 0.005381356 \\
\hline 1 & 48 & 0.564705882 & 99 & 0.013983051 & 0.478389831 & 0.005545862 \\
\hline 1 & 49 & 0.576470588 & 99 & 0.013983051 & 0.492372881 & 0.005710369 \\
\hline 1 & 50 & 0.588235294 & 99 & 0.013983051 & 0.506355932 & 0.005874875 \\
\hline 1 & 51 & 0.6 & 99 & 0.013983051 & 0.520338983 & 0.006039382 \\
\hline 1 & 52 & 0.611764706 & 99 & 0.013983051 & 0.534322034 & 0.006203888 \\
\hline 1 & 53 & 0.623529412 & 99 & 0.013983051 & 0.548305085 & 0.006368395 \\
\hline 1 & 54 & 0.635294118 & 99 & 0.013983051 & 0.562288136 & 0.006532901 \\
\hline 1 & 55 & 0.647058824 & 99 & 0.013983051 & 0.576271186 & 0.006697408 \\
\hline 0 & 56 & 0.658823529 & 100 & 0.014124294 & 0.59039548 & 0.006862745 \\
\hline 0 & 57 & 0.670588235 & 100 & 0.014124294 & 0.604519774 & 0.007028913 \\
\hline 0 & 58 & 0.682352941 & 100 & 0.014124294 & 0.618644068 & 0.007195081 \\
\hline 0 & 59 & 0.694117647 & 100 & 0.014124294 & 0.632768362 & 0.00736125 \\
\hline 0 & 60 & 0.705882353 & 100 & 0.014124294 & 0.646892655 & 0.007527418 \\
\hline 0 & 61 & 0.717647059 & 100 & 0.014124294 & 0.661016949 & 0.007693586 \\
\hline 0 & 62 & 0.729411765 & 100 & 0.014124294 & 0.675141243 & 0.007859754 \\
\hline 0 & 63 & 0.741176471 & 100 & 0.014124294 & 0.689265537 & 0.008025922 \\
\hline 0 & 64 & 0.752941176 & 100 & 0.014124294 & 0.703389831 & 0.00819209 \\
\hline 0 & 65 & 0.764705882 & 100 & 0.014124294 & 0.717514124 & 0.008358259 \\
\hline 0 & 66 & 0.776470588 & 100 & 0.014124294 & 0.731638418 & 0.008524427 \\
\hline 0 & 67 & 0.788235294 & 100 & 0.014124294 & 0.745762712 & 0.008690595 \\
\hline 0 & 68 & 0.8 & 100 & 0.014124294 & 0.759887006 & 0.008856763 \\
\hline 0 & 69 & 0.811764706 & 100 & 0.014124294 & 0.774011299 & 0.009022931 \\
\hline 0 & 70 & 0.823529412 & 100 & 0.014124294 & 0.788135593 & 0.009189099 \\
\hline 0 & 71 & 0.835294118 & 100 & 0.014124294 & 0.802259887 & 0.009355268 \\
\hline 0 & 72 & 0.847058824 & 100 & 0.014124294 & 0.816384181 & 0.009521436 \\
\hline 0 & 73 & 0.858823529 & 100 & 0.014124294 & 0.830508475 & 0.009687604 \\
\hline 0 & 74 & 0.870588235 & 100 & 0.014124294 & 0.844632768 & 0.009853772 \\
\hline 0 & 75 & 0.882352941 & 100 & 0.014124294 & 0.858757062 & 0.01001994 \\
\hline 0 & 76 & 0.894117647 & 100 & 0.014124294 & 0.872881356 & 0.010186108 \\
\hline 0 & 77 & 0.905882353 & 100 & 0.014124294 & 0.88700565 & 0.010352277 \\
\hline 0 & 78 & 0.917647059 & 100 & 0.014124294 & 0.901129944 & 0.010518445 \\
\hline 0 & 79 & 0.929411765 & 100 & 0.014124294 & 0.915254237 & 0.010684613 \\
\hline 0 & 80 & 0.941176471 & 100 & 0.014124294 & 0.929378531 & 0.010850781 \\
\hline 0 & 81 & 0.952941176 & 100 & 0.014124294 & 0.943502825 & 0.011016949 \\
\hline 0 & 82 & 0.964705882 & 100 & 0.014124294 & 0.957627119 & 0.011183117 \\
\hline 0 & 83 & 0.976470588 & 100 & 0.014124294 & 0.971751412 & 0.011349285 \\
\hline \multirow{3}{*}{0} & 84 & 0.988235294 & 100 & 0.014124294 & 0.985875706 & 0.011515454 \\
\hline & 85 & 1 & 100 & 0.014124294 & 1 & 0.011681622 \\
\hline & & Total inhibition & 7080 & & Total area & 0.425920572 \\
\hline
\end{tabular}

Gini

0.148158857 


\begin{tabular}{|c|c|}
\hline & $100 \mu \mathrm{M}$ ATP \\
\hline Kinase & Staurosporine @ $1 \mu \mathrm{M}$ \\
\hline $\mathrm{Ab}(\mathrm{m})$ & 39 \\
\hline AMPK(r) & -5 \\
\hline $\operatorname{Arg}(\mathrm{m})$ & 17 \\
\hline Aurora-A(h) & 4 \\
\hline Axl(h) & 3 \\
\hline $\mathrm{Blk}(\mathrm{m})$ & 0 \\
\hline Bmxxh) & -4 \\
\hline CaMKII(r) & 0 \\
\hline CaMKIV(h) & 12 \\
\hline $\mathrm{CDK} 1 / \mathrm{cyclinB}(\mathrm{h})$ & 1 \\
\hline $\mathrm{CDK} 2 / \mathrm{cyclin} \mathrm{A}(\mathrm{h})$ & -3 \\
\hline $\mathrm{CDK} 2 /$ cyclinE(h) & 1 \\
\hline CDK3/cyclinE(h) & -5 \\
\hline CDK5/p35(h) & 19 \\
\hline CDK6/cyclinD3(h) & 25 \\
\hline CDK7/cyclinH/MAT1(h) & 5 \\
\hline CHKI(h) & 0 \\
\hline СHK2(h) & 14 \\
\hline CKI(y) & 27 \\
\hline CK2(h) & 97 \\
\hline c-RAF(h) & 92 \\
\hline CSK(h) & 46 \\
\hline $\operatorname{cSRC}(\mathrm{h})$ & 2 \\
\hline Fes(h) & -2 \\
\hline FGFR3(h) & 0 \\
\hline $\mathrm{Fll} 3(\mathrm{~h})$ & 0 \\
\hline Fyn(h) & 1 \\
\hline GSK3a(h) & 5 \\
\hline GSK3B(h) & 9 \\
\hline IGF-IR(h) & 31 \\
\hline $\mathrm{IKK} \alpha(\mathrm{h})$ & 22 \\
\hline ІККВ(h) & 47 \\
\hline $\mathrm{IR}(\mathrm{h})$ & 17 \\
\hline JNK1 d (h) & 69 \\
\hline JNK2 $2(h)$ & 80 \\
\hline JNK3(r) & 48 \\
\hline Lck(h) & 0 \\
\hline Lynn(h) & 0 \\
\hline $\operatorname{Lyn}(\mathrm{m})$ & -1 \\
\hline MAPK1(h) & 71 \\
\hline MAPK2(h) & 82 \\
\hline MAPK2(m) & 59 \\
\hline MAPKAP-K2(h) & 6 \\
\hline MEK1(h) & 3 \\
\hline MKK4(m) & 64 \\
\hline MKK6(h) & 0 \\
\hline MKK7B(h) & 15 \\
\hline MSK1(h) & 0 \\
\hline $\mathrm{p} 70 \mathrm{~S} 6 \mathrm{~K}(\mathrm{~h})$ & 0 \\
\hline PAK2(h) & 1 \\
\hline PDGFRa(h) & 1 \\
\hline PDGFRB(h) & -1 \\
\hline PDK1(h) & -2 \\
\hline PKA(b) & 2 \\
\hline PKA(h) & 0 \\
\hline PKB ah) & 2 \\
\hline PKBB(h) & 3 \\
\hline P KB $($ (b) & 0 \\
\hline $\mathrm{PKC} a(\mathrm{~h})$ & 1 \\
\hline РКСBI(h) & 8 \\
\hline PKC $($ hi) & 3 \\
\hline PKCớ(h) & 4 \\
\hline PKCąh) & 0 \\
\hline PKC $\eta(h)$ & 7 \\
\hline PKG(h) & 15 \\
\hline PKC $\mu(\mathrm{h})$ & 1 \\
\hline PKC $\theta(h)$ & 4 \\
\hline PKD2(h) & 1 \\
\hline PRAK(h) & 56 \\
\hline PRK2(h) & -11 \\
\hline ROCK-II(h) & -2 \\
\hline ROCK-III(r) & -1 \\
\hline Rskl(h) & 0 \\
\hline Rskl(r) & 2 \\
\hline Rsk2(h) & -1 \\
\hline Rsk3(h) & 0 \\
\hline SAPK2a(h) & 84 \\
\hline SAPK2b(h) & 114 \\
\hline SAPK3(h) & 54 \\
\hline $\mathrm{SAPK} 4(\mathrm{~h})$ & 34 \\
\hline SGK(h) & 0 \\
\hline Syk(h) & -3 \\
\hline TrkB(h) & 0 \\
\hline $\begin{array}{c}\text { Yes(h) } \\
\text { ZAP-70(h) }\end{array}$ & $\begin{array}{l}1 \\
8\end{array}$ \\
\hline ZAP-70(h) & 8 \\
\hline
\end{tabular}

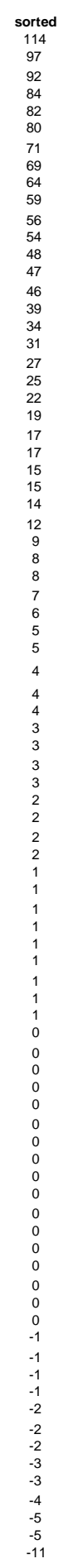

$\begin{array}{cc}\text { normalized } & \text { positio } \\ 100 & \\ 97 & \\ 92 & \\ 84 & \\ 82 & \\ 80 & \\ 71 & \\ 69 & \\ 64 & \\ 59 & \\ 56 & \\ 54 & \\ 48 & \\ 47 & \\ 46 & \\ 39 & \\ 34 & \\ 31 & \\ 27 & \\ 25 & \\ 22 & \\ 19 & \\ 17 & \\ 17 & \\ 15 & \\ 15 & \\ 14 & \\ 12 & \\ 9 & \\ 8 & \\ 8 & \\ 7 & \\ 6 & \\ 5 & \\ 5 & \\ 4 & \\ 4 & \end{array}$

$\begin{array}{lcl} & \text { cumulative sample fraction } & \text { inhi } \\ 1 & 0.011764706 \\ 2 & 0.023529412 \\ 3 & 0.035294118 \\ 4 & 0.047058824 \\ 5 & 0.058823529\end{array}$

0.047058824

0.070588235

0.082352941

0.094117647

0.105882353

0.117647059

0.141176471

0.152941176

0.176470588

0.188235294

0.188235294
0.2

0.211764706
0.223529412

0.235294118

0.235294118

0.258823529
0.270588235

0.282352941
0.294117647

0.305882353
0.317647059

0.329411765

0.341176471

0.352941176

0.364705882
0.376470588

0.388235294

0.4
0.41176470

0.423529412

0.435294118

0.447058824

0.458823529

0.470588235

0.4235294

0.505882353

0.517647059

0.529411765

0.541176471

0.552941176

0.576470588

0.588235294

0.6
0.611764706
0.623529412

0.647058824

0.658823529
0.670588235

0.682352941

0.69411764

0.705882353
0.717647059

0.729411765

.752941716

0.764705882

0.776470588

0.788235294

0.8
0.811764706
0.823529412

0.835294118

0.847058824

0.858823529
0.870588235

0.882352941

0.894117647

0.905882353
0.917647059

0.929411765
0.941176471

0.952941176

0.964705882

0.976470588

0.988235294
1

Total inhibition

$\begin{array}{cc}\text { Mhibition\% } & \text { fraction of total inhibition } \\ 0 & 0 \\ 3 & 0.000424028 \\ 8 & 0.001130742 \\ 16 & 0.002261484 \\ 18 & 0.00254417 \\ 20 & 0.002826855 \\ 29 & 0.000409894 \\ 31 & 0.004381625 \\ 36 & 0.005088339 \\ 41 & 0.005795053 \\ 44 & 0.006219081 \\ 46 & 0.006501767 \\ 52 & 0.007349823 \\ 53 & 0.007491166 \\ 54 & 0.007632509 \\ 61 & 0.008621908\end{array}$

cumulative inhibition
0
0.000424028
0.00155477
0.003816254
0.006360424

2.49428E-06

1.164E-05

$0.006360424 \quad 5.98628 \mathrm{E}-05$

$0.009187279 \quad 9.14571 \mathrm{E}-05$

$0.013286219 \quad 0.00013219$

$0.017667845 \quad 0.000182083$

$0.022756184 \quad 0.000237788$

$\begin{array}{ll}0.028551237 & 0.000301808 \\ 0.034770318 & 0.00037248\end{array}$

$0.041272085 \quad 0.000447308$

$\begin{array}{ll}0.048621908 & 0.00052878 \\ 0.056113074 & 0.000610088\end{array}$

$0.063745583-0.000705051$

$0.072367491 \quad 0.000800665$

$\begin{array}{ll}0.081696133 & 0.000906256 \\ 0.091448763 & 0.001018499\end{array}$

$0.101766784 \quad 0.001136562$

$0.112367491 \quad 0.001259613$

$\begin{array}{ll}0.123392226 & 0.001259613 \\ 0.13396820 & 0.001386822\end{array}$

$0.134840989-0.001519019$

$0.146572438 \quad 0.001655373$

$\begin{array}{ll}0.158303887 & 0.00179339 \\ 0.170318021 & 0.00193307\end{array}$

$\begin{array}{rr}0.170318021 & 0.00193307 \\ 0.183332155 & 0.002074413\end{array}$

$\begin{array}{ll}0.182332155 & 0.002074413 \\ 0.194487633 & 0.002216587\end{array}$

$0.194487633 \quad 0.002216587$

$0.219787986 \quad 0.002510081$

$0.232791519 \quad 0.002662232$

$0.245795053-0.002815215$

$\begin{array}{lrr}0.013286219 & 0.272226148 & 0.003124506 \\ 0.013427562 & 0.28565371 & 0.003281666 \\ 0.013427562 & 0.299081272 & 0.003439618\end{array}$

$\begin{array}{lll}0.013286219 & 0.272226148 & 0.003124506 \\ 0.013427562 & 0.28565371 & 0.003281646 \\ 0.013427562 & 0.299081272 & 0.003439618\end{array}$

$\begin{array}{llr}0.013427562 & 0.299081272 & 0.003439618 \\ 0.013568905 & 0.312650177 & 0.00359842\end{array}$

$\begin{array}{lll}0.013568905 & 0.326219081 & 0.003758054\end{array}$

$\begin{array}{lll}0.013568905 & 0.339787986 & 0.003917689\end{array}$

$\begin{array}{lll}0.013710247 & 0.353498233 & 0.004078154 \\ 0.013710247 & 0.0367208481 & 0.0023951\end{array}$

$0.013710247-0.36720848100 .004239451$

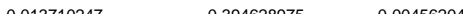

$\begin{array}{lll}0.013710247 & 0.380918728 & 0.004400748 \\ 0.013710247 & 0.394628975 & 0.004562045 \\ 0.01385159 & 0.408480565 & 0.004724174\end{array}$

$\begin{array}{lll}0.01385159 & 0.422332155 & 0.0047247144\end{array}$

$\begin{array}{lll}0.01385159 & 0.436183746 & 0.005050094\end{array}$

$0.450035336-0.005213053$

$\begin{array}{lll}0.013992933 & 0.464028269 & 0.005376845\end{array}$

$\begin{array}{llr}0.013992933 & 0.478021201 & 0.005541467 \\ 0.013992933 & 0.492014134 & 0.00570609\end{array}$

$\begin{array}{lll}0.013992933 & 0.506007067 & 0.005870713\end{array}$

$\begin{array}{lll}0.013992933 & 0.52 & 0.006035336\end{array}$

$\begin{array}{lll}0.013992933 & 0.533992933 & 0.006199958\end{array}$

$\begin{array}{lll}0.013992933 & 0.547985866 & 0.006364581\end{array}$

$\begin{array}{lll}0.013992933 & 0.561978799 & 0.006529204\end{array}$

$\begin{array}{lll}0.013992933 & 0.575971731 & 0.006693827\end{array}$

$\begin{array}{lll}0.014134276 & 0.590106007 & 0.006859281\end{array}$

$\begin{array}{lll}0.014134276 & 0.618374558 & 0.007191852\end{array}$

$\begin{array}{lll}0.014134276 & 0.632508834 & 0.007358138 \\ 0.01413476 & 0.6460311 & 0.00752443\end{array}$

$\begin{array}{lll}0.014134276 & 0.64664311 & 0.007524423 \\ 0.014134276 & 0.660777385 & 0.007690709\end{array}$

$\begin{array}{lll}0.014134276 & 0.674911661 & 0.007856994\end{array}$

$\begin{array}{lll}0.014134276 & 0.689045936 & 0.00802328 \\ 0.014134276 & 0.703180212 & 0.008189566\end{array}$

$\begin{array}{lll}0.014134276 & 0.7890459212 & 0.008189566 \\ 0.014134276 & 0.717314488 & 0.008355851\end{array}$

$\begin{array}{lll}0.014134276 & 0.731448763 & 0.008522137\end{array}$

$\begin{array}{lll}0.014134276 & 0.745583039 & 0.008688422\end{array}$

$\begin{array}{lll}0.014134276 & 0.759717314 & 0.008854708\end{array}$

$\begin{array}{lll}0.014134276 & 0.77385159 & 0.009020994 \\ 0.014134276 & 0.787985866 & 0.009187279\end{array}$

$\begin{array}{lll}0.014134276 & 0.802120141 & 0.009353565 \\ 0.014134276 & 0.816254417 & 0.00951985\end{array}$

$\begin{array}{lll}0.014134276 & 0.816254417 & 0.00951985 \\ 0.014134276 & 0.3038693 & 0.009686136\end{array}$

$\begin{array}{lll}0.014134276 & 0.830388693 & 0.009686136 \\ 0.014134276 & 0.844522968 & 0.009852422\end{array}$

$\begin{array}{lll}0.014134276 & 0.858657244 & 0.010018707\end{array}$

$\begin{array}{lll}0.014134276 & 0.872791519 & 0.010184993 \\ 0.0143476 & 0.8695795 & 0.0151764\end{array}$

$\begin{array}{lll}0.014134276 & 0.886925795 & 0.010351278\end{array}$

$\begin{array}{lll}0.014134276 & 0.915194346 & 0.01068385\end{array}$

$0.014134276-0.029328622-0.010850135$

$\begin{array}{lll}0.014134276 & 0.943462898 & 0.01101642 \\ 0.014134276 & 0.957597173 & 0.011182706\end{array}$

$0.014134276-0.971731449=0.011348992$

$\begin{array}{ccc}0.014134276 & 0.971731449 & 0.011348992 \\ 0.014134276 & 0.985865724 & 0.011515277\end{array}$

0.42544419

Total area

0.149111619 


\begin{tabular}{|c|c|}
\hline & $100 \mu \mathrm{M}$ ATP \\
\hline Kinase & Staurosporine @ $1 \mu \mathrm{M}$ \\
\hline $\mathrm{Ab}(\mathrm{m})$ & 39 \\
\hline AMPK(r) & -5 \\
\hline $\operatorname{Arg}(\mathrm{m})$ & 17 \\
\hline Aurora-A(h) & 4 \\
\hline Axl(h) & 3 \\
\hline $\mathrm{Blk}(\mathrm{m})$ & 0 \\
\hline Bmxxh) & -4 \\
\hline CaMKII(r) & 0 \\
\hline CaMKIV(h) & 12 \\
\hline $\mathrm{CDK} 1 / \mathrm{cyclinB}(\mathrm{h})$ & 1 \\
\hline $\mathrm{CDK} 2 / \mathrm{cyclin} \mathrm{A}(\mathrm{h})$ & -3 \\
\hline $\mathrm{CDK} 2 /$ cyclinE(h) & 1 \\
\hline CDK3/cyclinE(h) & -5 \\
\hline CDK5/p35(h) & 19 \\
\hline CDK6/cyclinD3(h) & 25 \\
\hline CDK7/cyclinH/MAT1(h) & 5 \\
\hline CHKI(h) & 0 \\
\hline СHK2(h) & 14 \\
\hline CKI(y) & 27 \\
\hline CK2(h) & 97 \\
\hline c-RAF(h) & 92 \\
\hline CSK(h) & 46 \\
\hline $\operatorname{cSRC}(\mathrm{h})$ & 2 \\
\hline Fes(h) & -2 \\
\hline FGFR3(h) & 0 \\
\hline $\mathrm{Fll} 3(\mathrm{~h})$ & 0 \\
\hline Fyn(h) & 1 \\
\hline GSK3a(h) & 5 \\
\hline GSK3B(h) & 9 \\
\hline IGF-IR(h) & 31 \\
\hline $\mathrm{IKK} \alpha(\mathrm{h})$ & 22 \\
\hline ІККВ(h) & 47 \\
\hline $\mathrm{IR}(\mathrm{h})$ & 17 \\
\hline JNK1 d (h) & 69 \\
\hline JNK2 $2(h)$ & 80 \\
\hline JNK3(r) & 58 \\
\hline Lck(h) & 0 \\
\hline Lynn(h) & 0 \\
\hline $\operatorname{Lyn}(\mathrm{m})$ & -1 \\
\hline MAPK1(h) & 71 \\
\hline MAPK2(h) & 82 \\
\hline MAPK2(m) & 59 \\
\hline MAPKAP-K2(h) & 6 \\
\hline MEK1(h) & 3 \\
\hline MKK4(m) & 64 \\
\hline MKK6(h) & 0 \\
\hline MKK7B(h) & 15 \\
\hline MSK1(h) & 0 \\
\hline $\mathrm{p} 70 \mathrm{~S} 6 \mathrm{~K}(\mathrm{~h})$ & 0 \\
\hline $\operatorname{PAK} 2(\mathrm{~h})$ & 1 \\
\hline PDGFRa(h) & 1 \\
\hline PDGFRB(h) & -1 \\
\hline PDK1(h) & -2 \\
\hline PKA(b) & 2 \\
\hline PKA(h) & 0 \\
\hline PKB ah) & 2 \\
\hline PKBB(h) & 3 \\
\hline P KB $($ (b) & 0 \\
\hline $\mathrm{PKC} a(\mathrm{~h})$ & 1 \\
\hline РКСBI(h) & 8 \\
\hline PKC $($ hi) & 3 \\
\hline PKCớ(h) & 4 \\
\hline PKCąh) & 0 \\
\hline PKC $\eta(h)$ & 7 \\
\hline PKG(h) & 15 \\
\hline PKC $\mu(\mathrm{h})$ & 1 \\
\hline PKC $\theta(h)$ & 4 \\
\hline PKD2(h) & 1 \\
\hline PRAK(h) & 56 \\
\hline PRK2(h) & -11 \\
\hline ROCK-II(h) & -2 \\
\hline ROCK-III(r) & -1 \\
\hline Rskl(h) & 0 \\
\hline Rskl(r) & 2 \\
\hline Rsk2(h) & -1 \\
\hline Rsk3(h) & 0 \\
\hline SAPK2a(h) & 84 \\
\hline SAPK2b(h) & 114 \\
\hline SAPK3(h) & 54 \\
\hline $\mathrm{SAPK} 4(\mathrm{~h})$ & 34 \\
\hline SGK(h) & 0 \\
\hline Syk(h) & -3 \\
\hline TrkB(h) & 0 \\
\hline $\begin{array}{c}\text { Yes(h) } \\
\text { ZAP-70(h) }\end{array}$ & $\begin{array}{l}1 \\
8\end{array}$ \\
\hline ZAP-70(h) & 8 \\
\hline
\end{tabular}

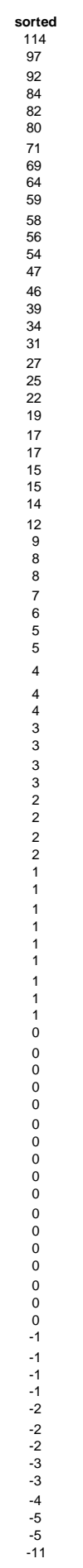

$\begin{array}{cc}\text { normalized } & \text { positio } \\ 100 & \\ 97 & \\ 92 & \\ 84 & \\ 82 & \\ 80 & \\ 71 & \\ 69 & \\ 64 & \\ 59 & \\ 58 & \\ 56 & \\ 54 & \\ 47 & \\ 46 & \\ 39 & \\ 34 & \\ 31 & \\ 27 & \\ 25 & \\ 22 & \\ 19 & \\ 17 & \\ 17 & \\ 15 & \\ 15 & \\ 14 & \\ 12 & \\ 9 & \\ 8 & \\ 8 & \\ 7 & \\ 6 & \\ 5 & \\ 5 & \\ 4 & \\ 4 & \end{array}$

$\begin{array}{lcl} & \text { cumulative sample fraction } & \text { inhi } \\ 1 & 0.011764706 \\ 2 & 0.023529412 \\ 3 & 0.035294118 \\ 4 & 0.047058824 \\ 5 & 0.058823529\end{array}$

0.047058824

0.070588235

0.082352941

0.094117647

0.105882353

0.117647059
0.129411765

0.141176471

0.152941176

0.176470588

0.188235294

0.188235294
0.2

0.211764706
0.223529412

0.235294118

0.235294118

0.258823529
0.270588235

0.282352941
0.294117647

0.305882353
0.317647059

0.329411765

0.341176471

0.352941176

0.364705882
0.376470588

0.388235294

0.4
0.41176470

0.423529412

0.435294118

0.447058824

0.458823529

0.470588235

0.494117647

0.505882353

0.517647059

0.529411765

0.541176471

0.552941176

0.576470588

0.588235294

0.6
0.611764706
0.623529412

0.647058824

0.658823529
0.670588235

0.682352941

0.69411764

0.705882353
0.717647059

0.729411765

.752941716

0.764705882

0.776470588

0.788235294

0.8
0.811764706
0.823529412

0.835294118

0.847058824

0.858823529
0.870588235

0.882352941

0.894117647

0.905882353
0.917647059

0.929411765
0.941176471

0.952941176

0.964705882

0.976470588

0.98823529
1

Total inhibition

$\begin{array}{cc}0 & \\ 3 & \\ 8 & \\ 16 & \\ 18 & \end{array}$

of tota

0.000424628

0.000424628
0.001132343

0.002264685

0.002547771

0.002830856
0.004104742

0.004387827

0.005095541
0.005803255

0.005803255
0.005944798

0.006227884

0.00651097
0.007501769

0.007501769
0.007643312

0.008634112
0.009341826

0.009341826

0.010332626

0.010615711

0.01104034

0.011464968
0.011748054

0.011748054

0.012031139

0.012031139
0.012172682

0.012455768

0.012880396

0.013021939
0.013021939

0.013163482

0.013305025

0.013446568
0.013446568

0.01358811

0.01358811

0.01358811

0.013729653
0.013729653

0.013729653

0.013729653
0.013871196

0.013871196

0.013871196

0.013871196

0.014012739

0.014012739
0.014012739

0.014012739

0.014012739

0.014012739
0.014012739

0.014012739
0.014012739

0.014012739
0.014012739

0.014154282
0.014154282

0.014154282

0.014154282
0.014154282

0.014154282
0.014154282

0.014154282

0.014154282

0.014154282

0.014154282

0.014154282
0.014154282

0.014154282

0.014154282

0.014154282

0.014154282

0.014154282
0.014154282

0.014154282

0.014154282

0.014154282

0.014154282

0.014154282

0.014154282
0.014154282

0.014154282

0.014154282
0.014154282

0.014154282
0.014154282

cumulative inhibition ar

0.00042462

$\begin{array}{ll}0.000424628 & 2.49781 \mathrm{E}-06 \\ 0.001556971 & 1.16565 \mathrm{E}-05\end{array}$

$0.003821656 \quad 3.1639 \mathrm{E}-05$

$0.006369427 \quad 5.99475 \mathrm{E}-05$

$\begin{array}{ll}0.009200283 & 9.15865 \mathrm{E}-05 \\ 0.013305025 & 0.000132384 \\ 0.01769252 & 0.00018234\end{array}$

$\begin{array}{ll}0.017692852 & 0.00018234\end{array}$

$0.022788393 \quad 0.000238125$

$\begin{array}{ll}0.028591649 & 0.000302236 \\ 0.034536447 & 0.000371342\end{array}$

$0.040764331 \quad 0.000442946$

$\begin{array}{ll}0.047275301 & 0.00051788 \\ 0.05477707 & 0.00060308\end{array}$

$0.062420382-0.000000307$

$\begin{array}{ll}0.071054494 & 0.000785146\end{array}$

0.090162774

$0.1004954 \quad 0.001121519$

$0.111111111 \quad 0.00124474$

$\begin{array}{ll}0.122151451 & 0.001244744 \\ 0.13372133\end{array}$

$0.133616419 \quad 0.001504517$

$\begin{array}{ll}0.145364473 & 0.001641064\end{array}$

$\begin{array}{ll}0.157112527 & 0.001779276 \\ 0.169143666 & 0.001919154\end{array}$

$\begin{array}{ll}0.159143666 & 0.001919154 \\ 0.181174805 & 0.002060697\end{array}$

$\begin{array}{ll}0.18934805 & 0.002060697 \\ 0.193347488 & 0.002203072\end{array}$

$.205803255 \quad 0.00234794$

$\begin{array}{ll}0.218683652 & 0.002496982 \\ 0.231705591 & 0.00249358\end{array}$

$0.231705591-0.002649348$

$0.24472753-0.002802548$

$0.271196037 \quad 0.003112277$

0.00326963

$0.311677282 \quad 0.003586861$

$\begin{array}{ll}0.325265393 & 0.003746722 \\ 0.33855303 & 0.003065274\end{array}$

$\begin{array}{ll}0.338853503 & 0.003906582 \\ 0.352503156 & 0.000672\end{array}$

$0.352583156 \quad 0.004067274$

$0.36631281-0.004228$

$\begin{array}{ll}0.393772116 & 0.00455185\end{array}$

$\begin{array}{ll}0.421514508 & 0.00471420\end{array}$

$0.435385704 \quad 0.004877399$

$0.4492569 \quad 0.00520378$

$\begin{array}{ll}0.463269639 & 0.00520378 \\ 0.472639 & 0.00536783\end{array}$

$0.477282378 \quad 0.005532659$

$\begin{array}{ll}0.491295117 & 0.005697515\end{array}$

$0.505307856 \quad 0.00586237$

$0.519320594 \quad 0.006027226$

$\begin{array}{ll}0.5333333333 & 0.006192082 \\ 0.547346072 & 0.006356938\end{array}$

$0.561358811-0.006521793$

$\begin{array}{ll}0.57537155 & 0.006686649\end{array}$

$\begin{array}{ll}0.589525832 & 0.006852338 \\ 0 & 0.003680113\end{array}$

$0.617834395 \quad 0.007185379$

$\begin{array}{ll}0.631988677 & 0.0073519\end{array}$

$0.646142958-0.00751842$

$0.674451522 \quad 0.007851463$

$0.702760085-0.008184505$

$0.716914367 \quad 0.008351026$

$\begin{array}{ll}0.731068648 & 0.008517547\end{array}$

$0.74522293 \quad 0.008684068$

$\begin{array}{ll}0.731068648 & 0.0085684068 \\ 0.759377212 & 0.008850589\end{array}$

$0.773531493 \quad 0.000901711$

$\begin{array}{ll}0.787685775 & 0.009183631\end{array}$

$\begin{array}{ll}0.801840057 & 0.009350152 \\ 0.815994338 & 0.009516673\end{array}$

$0.815994338 \quad 0.009516673$

$\begin{array}{ll}0.83014862 & 0.009683194 \\ 0.844302902 & 0.009849715\end{array}$

$\begin{array}{ll}0.858457183 & 0.010016236\end{array}$

$0.872611465 \quad 0.010182757$

$0.886765747-0.010349278$

$\begin{array}{ll}0.91507431 & 0.01068232 \\ 0.94228592 & 0.01015312\end{array}$

$0.029228592-0.010848841$

$\begin{array}{ll}0.943882873 & 0.011015362 \\ 0.957537155 & 0.011181883\end{array}$

$0.971691437 \quad 0.011348403$

$\begin{array}{ll}0.971691437 & 0.011348403 \\ 0.985845718 & 0.011514924\end{array}$

Total area $\quad 0.424829108$

Gini

0.150341784 


\begin{tabular}{|c|c|c|c|c|c|c|c|c|c|}
\hline & & & & & & & & & \\
\hline Kinase & Staurosporine @ $1 \mu \mathrm{M}$ & sorted & normalized & position & cumulative sample fraction & inhibition\% & fraction of total inhibition & cumulative inhibition & area \\
\hline $\mathrm{Ab}(\mathrm{m})$ & 39 & 114 & 100 & 1 & 0.011764706 & 0 & 0 & 0 & 0 \\
\hline $\mathrm{AMPK}(\mathrm{r})$ & -5 & 97 & 97 & 2 & 0.023529412 & 3 & 0.000424268 & 0.000424268 & $2.49569 \mathrm{E}-06$ \\
\hline $\operatorname{Arg}(\mathrm{m})$ & 17 & 92 & 92 & 3 & 0.035294118 & 8 & 0.001131382 & 0.00155565 & $1.16466 \mathrm{E}-05$ \\
\hline Aurora-A(h) & 4 & 84 & 84 & 4 & 0.047058824 & 16 & 0.002262763 & 0.003818413 & $3.16121 \mathrm{E}-05$ \\
\hline Axl(h) & 3 & 82 & 82 & 5 & 0.058823529 & 18 & 0.002545609 & 0.006364022 & $5.98967 \mathrm{E}-05$ \\
\hline $\mathrm{Bk}(\mathrm{m})$ & 0 & 80 & 80 & 6 & 0.070588235 & 20 & 0.002828454 & 0.009192476 & $9.15088 \mathrm{E}-05$ \\
\hline Bmx(h) & -4 & 71 & 71 & 7 & 0.082352941 & 29 & 0.004101259 & 0.013293735 & 0.000132272 \\
\hline CaMKII(r) & 0 & 69 & 69 & 8 & 0.094117647 & 31 & 0.004384104 & 0.017677839 & 0.000182186 \\
\hline CaMKIV(h) & 12 & 64 & 64 & 9 & 0.105882353 & 36 & 0.005091218 & 0.022769057 & 0.000237923 \\
\hline CDK1/cyclinB(h) & 1 & 59 & 59 & 10 & 0.117647059 & 41 & 0.005798331 & 0.028567388 & 0.000301979 \\
\hline $\mathrm{CDK} 2 /$ cyclina(h) & -3 & 56 & 56 & 11 & 0.129411765 & 44 & 0.006222599 & 0.034789987 & 0.00037269 \\
\hline CDK2/(cyclinE(h) & 1 & 54 & 54 & 12 & 0.141176471 & 46 & 0.006505445 & 0.041295432 & 0.000447561 \\
\hline CDK3/cyclinE(h) & -5 & 53 & 53 & 13 & 0.152941176 & 47 & 0.006646867 & 0.0479423 & 0.000524928 \\
\hline CDK5/p35(h) & 19 & 47 & 47 & 14 & 0.164705882 & 53 & 0.007495404 & 0.055437703 & 0.000608118 \\
\hline $\mathrm{CDK} / / \mathrm{cyc} \operatorname{cin} \mathrm{D} 3(\mathrm{~h})$ & 25 & 46 & 46 & 15 & 0.176470588 & 54 & 0.007636826 & 0.06307453 & 0.000697131 \\
\hline CDK7/cyclinH/MATI(h) & 5 & 39 & 39 & 16 & 0.188235294 & 61 & 0.008626785 & 0.071701315 & 0.000792799 \\
\hline CHKI(h) & 0 & 34 & 34 & 17 & 0.2 & 66 & 0.009333899 & 0.081035214 & 0.00089845 \\
\hline СНK2(h) & 14 & 31 & 31 & 18 & 0.211764706 & 69 & 0.009758167 & 0.090793381 & 0.001010756 \\
\hline CKI(y) & 27 & 27 & 27 & 19 & 0.223529412 & 73 & 0.010323858 & 0.101117239 & 0.001128886 \\
\hline СК2(h) & 97 & 25 & 25 & 20 & 0.235294118 & 75 & 0.010606703 & 0.111723943 & 0.001252007 \\
\hline$c-\operatorname{RAF}(\mathrm{h})$ & 92 & 22 & 22 & 21 & 0.247058824 & 78 & 0.011030972 & 0.122754914 & 0.001379287 \\
\hline CSK(h) & 46 & 19 & 19 & 22 & 0.258823529 & 81 & 0.01145524 & 0.134210154 & 0.001511559 \\
\hline $\operatorname{cSRC(h)}$ & 2 & 17 & 17 & 23 & 0.270588235 & 83 & 0.011738085 & 0.145948239 & 0.001647991 \\
\hline Fes(h) & -2 & 17 & 17 & 24 & 0.282352941 & 83 & 0.011738085 & 0.157686324 & 0.001786086 \\
\hline FGFR3(h) & 0 & 15 & 15 & 25 & 0.294117647 & 85 & 0.012020931 & 0.169707255 & 0.001925845 \\
\hline $\mathrm{Flls}(\mathrm{h})$ & 0 & 15 & 15 & 26 & 0.305882353 & 85 & 0.012020931 & 0.181728186 & 0.002067267 \\
\hline Fyn(h) & 1 & 14 & 14 & 27 & 0.317647059 & 86 & 0.012162353 & 0.193890539 & 0.002209522 \\
\hline GSK3a(h) & 5 & 12 & 12 & 28 & 0.329411765 & 88 & 0.012445199 & 0.206335738 & 0.002354272 \\
\hline GSK3B(h) & 8 & 8 & 8 & 29 & 0.341176471 & 92 & 0.01301089 & 0.219346627 & 0.002504014 \\
\hline IGF-IR(h) & 31 & 8 & 8 & 30 & 0.352941176 & 92 & 0.01301089 & 0.232357517 & 0.002657083 \\
\hline IKKa(h) & 22 & 8 & 8 & 31 & 0.364705882 & 92 & 0.01301089 & 0.245368406 & 0.002810152 \\
\hline IKKB(h) & 47 & 7 & 7 & 32 & 0.376470588 & 93 & 0.013152312 & 0.258520718 & 0.002964054 \\
\hline $\mathrm{IR}(\mathrm{h})$ & 17 & 6 & 6 & 33 & 0.388235294 & 94 & 0.013293735 & 0.271814453 & 0.003119619 \\
\hline JNK1 d (h) & 69 & 5 & 5 & 34 & 0.4 & 95 & 0.013435158 & 0.285249611 & 0.003276847 \\
\hline JNK2 (2(1) & 80 & 5 & 5 & 35 & 0.411764706 & 95 & 0.013435158 & 0.298684769 & 0.003434908 \\
\hline JNK3(r) & 53 & 4 & 4 & 36 & 0.423529412 & 96 & 0.01357658 & 0.312261349 & 0.003593801 \\
\hline Lck(h) & 0 & 4 & 4 & 37 & 0.435294118 & 96 & 0.01357658 & 0.32583793 & 0.003753525 \\
\hline Lym(h) & 0 & 4 & 4 & 38 & 0.447058824 & 96 & 0.01357658 & 0.33941451 & 0.00391325 \\
\hline Lyn(m) & -1 & 3 & 3 & 39 & 0.458823529 & 97 & 0.013718003 & 0.353132513 & 0.004073806 \\
\hline MAPKI(h) & 71 & 3 & 3 & 40 & 0.470588235 & 97 & 0.013718003 & 0.366850516 & 0.004235194 \\
\hline MAPK $2(\mathrm{~h})$ & 82 & 3 & 3 & 41 & 0.482352941 & 97 & 0.013718003 & 0.380568519 & 0.004396583 \\
\hline MAPK2(m) & 59 & 3 & 3 & 42 & 0.494117647 & 97 & 0.013718003 & 0.394286522 & 0.004557971 \\
\hline MAPKAP-K2(h) & 6 & 2 & 2 & 43 & 0.505882353 & 98 & 0.013859426 & 0.408145948 & 0.004720191 \\
\hline MEKI(h) & 3 & 2 & 2 & 44 & 0.517647059 & 98 & 0.013859426 & 0.422005374 & 0.004883243 \\
\hline MKK4(m) & 64 & 2 & 2 & 45 & 0.529411765 & 98 & 0.013859426 & 0.4358648 & 0.005046295 \\
\hline MKK $6(\mathrm{~h})$ & 0 & 2 & 2 & 46 & 0.541176471 & 98 & 0.013859426 & 0.449724226 & 0.005209347 \\
\hline MKK 7 (h) & 15 & 1 & 1 & 47 & 0.552941176 & 99 & 0.014000849 & 0.463725074 & 0.005373231 \\
\hline MSK1(h) & 0 & 1 & 1 & 48 & 0.564705882 & 99 & 0.014000849 & 0.477725923 & 0.005537947 \\
\hline $\mathrm{p} 7056 \mathrm{~K}(\mathrm{~h})$ & 0 & 1 & 1 & 49 & 0.576470588 & 99 & 0.014000849 & 0.491726771 & 0.005702663 \\
\hline PAK2(h) & 1 & 1 & 1 & 50 & 0.588235294 & 99 & 0.014000849 & 0.50572762 & 0.005867379 \\
\hline PDGFRa(h) & 1 & 1 & 1 & 51 & 0.6 & 99 & 0.014000849 & 0.519728468 & 0.006032095 \\
\hline PDGFRB(h) & -1 & 1 & 1 & 52 & 0.611764706 & 99 & 0.014000849 & 0.533729317 & 0.006196811 \\
\hline PDK1(h) & -2 & 1 & 1 & 53 & 0.623529412 & 99 & 0.014000849 & 0.547730165 & 0.006361526 \\
\hline PKA(b) & 2 & 1 & 1 & 54 & 0.635294118 & 99 & 0.014000849 & 0.561731014 & 0.006526242 \\
\hline PKA(h) & 0 & 1 & 1 & 55 & 0.647058824 & 99 & 0.014000849 & 0.575731863 & 0.006690958 \\
\hline PKB (h) & 2 & 0 & 0 & 56 & 0.658823529 & 100 & 0.014142271 & 0.589874134 & 0.006856506 \\
\hline PKBB(h) & 3 & 0 & 0 & 57 & 0.670588235 & 100 & 0.014142271 & 0.604016405 & 0.007022886 \\
\hline P КB $(\mathrm{ll}$ & 0 & 0 & 0 & 58 & 0.682352941 & 100 & 0.014142271 & 0.618158676 & 0.007189265 \\
\hline $\mathrm{PKC} \alpha(\mathrm{h})$ & 1 & 0 & 0 & 59 & 0.694117647 & 100 & 0.014142271 & 0.632300948 & 0.007355645 \\
\hline PKCBII(h) & 8 & 0 & 0 & 60 & 0.705882353 & 100 & 0.014142271 & 0.646443219 & 0.007522025 \\
\hline PKC (1) & 3 & 0 & 0 & 61 & 0.717647059 & 100 & 0.014142271 & 0.66058549 & 0.007688404 \\
\hline PKC $\delta(\mathrm{h})$ & 4 & 0 & 0 & 62 & 0.729411765 & 100 & 0.014142271 & 0.674727761 & 0.007854784 \\
\hline PKC ah) & 0 & 0 & 0 & 63 & 0.741176471 & 100 & 0.014142271 & 0.688870033 & 0.008021163 \\
\hline $\begin{array}{l}\text { PKC }(\text { (h) } \\
\text { Pal }\end{array}$ & 7 & 0 & 0 & 64 & 0.752941176 & 100 & 0.014142271 & 0.703012304 & 0.008187543 \\
\hline PKG(h) & 15 & 0 & 0 & 65 & 0.764705882 & 100 & 0.014142271 & 0.717154575 & 0.008353923 \\
\hline PKC $(\mathrm{h}(\mathrm{h})$ & 1 & 0 & 0 & 66 & 0.776470588 & 100 & 0.014142271 & 0.731296846 & 0.008520302 \\
\hline PKC $\theta(\mathrm{h})$ & 4 & 0 & 0 & 67 & 0.788235294 & 100 & 0.014142271 & 0.745439118 & 0.008686682 \\
\hline PKD2(h) & 1 & 0 & 0 & 68 & 0.8 & 100 & 0.014142271 & 0.759581389 & 0.008853062 \\
\hline PRAK(h) & 56 & 0 & 0 & 69 & 0.811764706 & 100 & 0.014142271 & 0.77372366 & 0.009019441 \\
\hline PRK2(h) & -11 & 0 & 0 & 70 & 0.823529412 & 100 & 0.014142271 & 0.787865931 & 0.009185821 \\
\hline ROCK-II(h) & -2 & 0 & 0 & 71 & 0.835294118 & 100 & 0.014142271 & 0.802008203 & 0.009352201 \\
\hline ROCK-II(r) & -1 & 0 & 0 & 72 & 0.847058824 & 100 & 0.014142271 & 0.816150474 & 0.00951858 \\
\hline Rskl (h) & 0 & -1 & 0 & 73 & 0.858823529 & 100 & 0.014142271 & 0.830292745 & 0.00968496 \\
\hline Rskl(r) & 2 & -1 & 0 & 74 & 0.870588235 & 100 & 0.014142271 & 0.844435016 & 0.00985134 \\
\hline Rsk2(h) & $\begin{array}{c}2 \\
-1\end{array}$ & -1 & 0 & 75 & 0.882352941 & 100 & 0.014142271 & 0.858577288 & 0.010017719 \\
\hline Rsk3(h) & 0 & -1 & 0 & 76 & 0.894117647 & 100 & 0.014142271 & 0.872719559 & 0.010184099 \\
\hline $\begin{array}{l}\text { SAPK(n) } \\
\text { SAPKa(h) }\end{array}$ & 84 & -2 & 0 & 77 & 0.905882353 & 100 & 0.014142271 & 0.88686183 & 0.010350479 \\
\hline SAPK2b(h) & 114 & -2 & 0 & 78 & 0.917647059 & 100 & 0.014142271 & 0.901004101 & 0.010516858 \\
\hline SAPK3(h) & 54 & -2 & 0 & 79 & 0.929411765 & 100 & 0.014142271 & 0.915146373 & 0.010683238 \\
\hline SAPK4(h) & 34 & -3 & 0 & 80 & 0.941176471 & 100 & 0.014142271 & 0.929288644 & 0.010849618 \\
\hline SGK(h) & 0 & -3 & 0 & 81 & 0.952941176 & 100 & 0.014142271 & 0.943430915 & 0.011015997 \\
\hline Syk(h) & -3 & -4 & 0 & 82 & 0.964705882 & 100 & 0.014142271 & 0.957573186 & 0.011182377 \\
\hline TrkB(h) & 0 & -5 & 0 & 83 & 0.976470588 & 100 & 0.014142271 & 0.971715458 & 0.011348757 \\
\hline Yes(h) & 1 & -5 & 0 & 84 & 0.988235294 & 100 & 0.014142271 & 0.985857729 & 0.011515136 \\
\hline ZAP-70(h) & 8 & -11 & 0 & 85 & 1 & 100 & 0.014142271 & 1 & 0.011681516 \\
\hline & & & & & Total inhibition & 7071 & & Total area & 0.425175739 \\
\hline & & & & & & & & Gini & 0.149648523 \\
\hline
\end{tabular}




\begin{tabular}{|c|c|c|c|c|c|c|c|c|c|}
\hline & $100 \mu \mathrm{M}$ ATP & & & & & & & & \\
\hline Kinase & Staurosporine@1 $1 \mu \mathrm{M}$ & sorted & normalized & position & cumulative sample fraction & inhibition\% & fraction of total inhibition & cumulative inhibition & area \\
\hline $\mathrm{Abl}(\mathrm{m})$ & 39 & 114 & 100 & 1 & 0.011764706 & 0 & 0 & 0 & 0 \\
\hline AMPK(r) & -5 & 97 & 97 & 2 & 0.023529412 & 3 & 0.000424388 & 0.000424388 & $2.4964 \mathrm{E}-06$ \\
\hline $\operatorname{Arg}(\mathrm{m})$ & 17 & 92 & 92 & 3 & 0.035294118 & 8 & 0.001131702 & 0.00155609 & 1.16499E-05 \\
\hline Aurora-A(h) & 4 & 84 & 84 & 4 & 0.047058824 & 16 & 0.002263404 & 0.003819494 & 3.16211E-05 \\
\hline Axl(h) & 3 & 82 & 82 & 5 & 0.058823529 & 18 & 0.002546329 & 0.006365823 & $5.99136 \mathrm{E}-05$ \\
\hline $\mathrm{Bk}(\mathrm{m})$ & 0 & 80 & 80 & 6 & 0.070588235 & 20 & 0.002829254 & 0.009195077 & $9.15347 \mathrm{E}-05$ \\
\hline Bmx(h) & -4 & 71 & 71 & 7 & 0.082352941 & 29 & 0.004102419 & 0.013297496 & 0.000132309 \\
\hline CaMKIII(r) & 0 & 69 & 69 & 8 & 0.094117647 & 31 & 0.004385344 & 0.017682841 & 0.000182237 \\
\hline CaMKIV(h) & 12 & 64 & 64 & 9 & 0.105882353 & 36 & 0.005092658 & 0.022775499 & 0.00023799 \\
\hline CDK1/cyclinB(h) & 1 & 59 & 59 & 10 & 0.117647059 & 41 & 0.005799972 & 0.02857547 & 0.000302065 \\
\hline $\mathrm{CDK} 2 /$ cyclinA(h) & -3 & 56 & 56 & 11 & 0.129411765 & 44 & 0.00622436 & 0.03479983 & 0.000372796 \\
\hline CDK2/cyclinE(h) & 1 & 54 & 54 & 12 & 0.141176471 & 46 & 0.006507285 & 0.041307116 & 0.000447688 \\
\hline CDK3/cyclinE(h) & -5 & 53 & 53 & 13 & 0.152941176 & 47 & 0.006648748 & 0.047955864 & 0.000525076 \\
\hline CDK5/p35(h) & 19 & 47 & 47 & 14 & 0.164705882 & 53 & 0.007497524 & 0.055453388 & 0.00060829 \\
\hline $\mathrm{CDK} 6 / \mathrm{cyc} \operatorname{cin} \mathrm{n} 3(\mathrm{~h})$ & 25 & 46 & 46 & 15 & 0.176470588 & 54 & 0.007638987 & 0.063092375 & 0.000697328 \\
\hline CDK7/cyclinH/MATI(h) & 5 & 39 & 39 & 16 & 0.188235294 & 61 & 0.008629226 & 0.071721601 & 0.000793023 \\
\hline CHKI(h) & 0 & 34 & 34 & 17 & 0.2 & 66 & 0.00933654 & 0.081058141 & 0.000898704 \\
\hline CHK2(h) & 14 & 31 & 31 & 18 & 0.211764706 & 69 & 0.009760928 & 0.090819069 & 0.001011042 \\
\hline CKI(y) & 27 & 27 & 27 & 19 & 0.223529412 & 73 & 0.010326779 & 0.101145848 & 0.001129205 \\
\hline СК2(h) & 97 & 25 & 25 & 20 & 0.235294118 & 75 & 0.010609704 & 0.111755552 & 0.001252361 \\
\hline $\mathrm{c}-\mathrm{RAF}(\mathrm{h})$ & 92 & 22 & 22 & 21 & 0.247058824 & 78 & 0.011034093 & 0.122789645 & 0.001379678 \\
\hline CSK(h) & 46 & 19 & 19 & 22 & 0.258823529 & 81 & 0.011458481 & 0.134248126 & 0.001511987 \\
\hline $\operatorname{cSRC(h)}$ & 2 & 17 & 17 & 23 & 0.270588235 & 83 & 0.011741406 & 0.145989532 & 0.001648457 \\
\hline Fes(h) & -2 & 17 & 17 & 24 & 0.282352941 & 83 & 0.011741406 & 0.157730938 & 0.001786591 \\
\hline FGFR3(h) & 0 & 15 & 15 & 25 & 0.294117647 & 85 & 0.012024332 & 0.169755269 & 0.001926389 \\
\hline Flls(h) & 0 & 15 & 15 & 26 & 0.305882353 & 85 & 0.012024332 & 0.181779601 & 0.002067852 \\
\hline Fynn(h) & 1 & 14 & 14 & 27 & 0.317647059 & 86 & 0.012165794 & 0.193945395 & 0.002210147 \\
\hline GSK3a(h) & 5 & 12 & 12 & 28 & 0.329411765 & 88 & 0.01244872 & 0.206394115 & 0.002354938 \\
\hline $\operatorname{GSK3B(h)}$ & 10 & 10 & 10 & 29 & 0.341176471 & 90 & 0.012731645 & 0.21912576 & 0.002503058 \\
\hline IGF-IR(h) & 31 & 8 & 8 & 30 & 0.352941176 & 92 & 0.013014571 & 0.232140331 & 0.002654506 \\
\hline IKKa(h) & 22 & 8 & 8 & 31 & 0.364705882 & 92 & 0.013014571 & 0.245154902 & 0.002807619 \\
\hline IKKB(h) & 47 & 7 & 7 & 32 & 0.376470588 & 93 & 0.013156033 & 0.258310935 & 0.002961564 \\
\hline $\mathrm{IR}(\mathrm{h})$ & 17 & 6 & 6 & 33 & 0.388235294 & 94 & 0.013297496 & 0.271608431 & 0.003117173 \\
\hline JNK1 d d (h) & 69 & 5 & 5 & 34 & 0.4 & 95 & 0.013438959 & 0.28504739 & 0.003274446 \\
\hline JNK2 (2)(h) & 80 & 5 & 5 & 35 & 0.411764706 & 95 & 0.013438959 & 0.298486349 & 0.003432551 \\
\hline JNK3(r) & 53 & 4 & 4 & 36 & 0.423529412 & 96 & 0.013580422 & 0.31206677 & 0.003591489 \\
\hline Lck(h) & 0 & 4 & 4 & 37 & 0.435294118 & 96 & 0.013580422 & 0.325647192 & 0.003751259 \\
\hline Lyn(h) & 0 & 4 & 4 & 38 & 0.447058824 & 96 & 0.013580422 & 0.339227614 & 0.003911028 \\
\hline Lyn(m) & -1 & 3 & 3 & 39 & 0.458823529 & 97 & 0.013721884 & 0.352949498 & 0.00407163 \\
\hline MAPKI (h) & 71 & 3 & 3 & 40 & 0.470588235 & 97 & 0.013721884 & 0.366671382 & 0.004233064 \\
\hline MAPK2(h) & 82 & 3 & 3 & 41 & 0.482352941 & 97 & 0.013721884 & 0.380393266 & 0.004394498 \\
\hline MAPK2(m) & 59 & 3 & 3 & 42 & 0.494117647 & 97 & 0.013721884 & 0.394115151 & 0.004555932 \\
\hline MAPKAP-K2(h) & 6 & 2 & 2 & 43 & 0.505882353 & 98 & 0.013863347 & 0.407978498 & 0.004718198 \\
\hline MEKI(h) & 3 & 2 & 2 & 44 & 0.517647059 & 98 & 0.013863347 & 0.421841845 & 0.004881296 \\
\hline MKK4(m) & 64 & 2 & 2 & 45 & 0.529411765 & 98 & 0.013863347 & 0.435705192 & 0.005044394 \\
\hline MKK6(h) & 0 & 2 & 2 & 46 & 0.541176471 & 98 & 0.013863347 & 0.449568539 & 0.005207493 \\
\hline МКK7B(h) & 15 & 1 & 1 & 47 & 0.552941176 & 99 & 0.01400481 & 0.463573348 & 0.005371423 \\
\hline MSK1(h) & 0 & 1 & 1 & 48 & 0.564705882 & 99 & 0.01400481 & 0.477578158 & 0.005536185 \\
\hline $\mathrm{p} 7056 \mathrm{~K}(\mathrm{~h})$ & 0 & 1 & 1 & 49 & 0.576470588 & 99 & 0.01400481 & 0.491582968 & 0.005700948 \\
\hline PAK2(h) & 1 & 1 & 1 & 50 & 0.588235294 & 99 & 0.01400481 & 0.505587778 & 0.00586571 \\
\hline PDGFRa(h) & 1 & 1 & 1 & 51 & 0.6 & 99 & 0.01400481 & 0.519592587 & 0.006030473 \\
\hline PDGFRB(h) & -1 & 1 & 1 & 52 & 0.611764706 & 99 & 0.01400481 & 0.533597397 & 0.006195235 \\
\hline PDK1(h) & -2 & 1 & 1 & 53 & 0.623529412 & 99 & 0.01400481 & 0.547602207 & 0.006359998 \\
\hline PKA(b) & 2 & 1 & 1 & 54 & 0.635294118 & 99 & 0.01400481 & 0.561607017 & 0.00652476 \\
\hline PKA(h) & 0 & 1 & 1 & 55 & 0.647058824 & 99 & 0.01400481 & 0.575611826 & 0.006689523 \\
\hline PKB (ah) & 2 & 0 & 0 & 56 & 0.658823529 & 100 & 0.014146272 & 0.589758099 & 0.006855117 \\
\hline 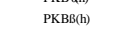 & 3 & 0 & 0 & 57 & 0.670588235 & 100 & 0.014146272 & 0.603904371 & 0.007021544 \\
\hline $\mathrm{P} \mathrm{KB} \times(\mathrm{B})$ & 0 & 0 & 0 & 58 & 0.682352941 & 100 & 0.014146272 & 0.618050644 & 0.007187971 \\
\hline PKC $a(\mathrm{~h})$ & 1 & 0 & 0 & 59 & 0.694117647 & 100 & 0.014146272 & 0.632196916 & 0.007354397 \\
\hline PKCBII(h) & 8 & 0 & 0 & 60 & 0.705882353 & 100 & 0.014146272 & 0.646343189 & 0.007520824 \\
\hline PKC ( (i) & 3 & 0 & 0 & 61 & 0.717647059 & 100 & 0.014146272 & 0.660489461 & 0.007687251 \\
\hline PKCố(h) & 4 & 0 & 0 & 62 & 0.729411765 & 100 & 0.014146272 & 0.674635733 & 0.007853678 \\
\hline PKC \&h) & 0 & 0 & 0 & 63 & 0.741176471 & 100 & 0.014146272 & 0.688782006 & 0.008020104 \\
\hline $\begin{array}{l}\text { PKC n(h) } \\
\text { (h) }\end{array}$ & 7 & 0 & 0 & 64 & 0.752941176 & 100 & 0.014146272 & 0.702928278 & 0.008186531 \\
\hline PKG(h) & 15 & 0 & 0 & 65 & 0.764705882 & 100 & 0.014146272 & 0.717074551 & 0.008352958 \\
\hline PKC & 1 & 0 & 0 & 66 & 0.776470588 & 100 & 0.014146272 & 0.731220823 & 0.008519385 \\
\hline PKC $\theta(h)$ & 4 & 0 & 0 & 67 & 0.788235294 & 100 & 0.014146272 & 0.745367096 & 0.008685811 \\
\hline PKD2(h) & 1 & 0 & 0 & 68 & 0.8 & 100 & 0.014146272 & 0.759513368 & 0.008852238 \\
\hline $\operatorname{PRAK}(\mathrm{h})$ & 56 & 0 & 0 & 69 & 0.811764706 & 100 & 0.014146272 & 0.773659641 & 0.009018665 \\
\hline PRK2(h) & -11 & 0 & 0 & 70 & 0.823529412 & 100 & 0.014146272 & 0.787805913 & 0.009185091 \\
\hline ROCK-II(h) & -2 & 0 & 0 & 71 & 0.835294118 & 100 & 0.014146272 & 0.801952186 & 0.009351518 \\
\hline ROCK-II(r) & -1 & 0 & 0 & 72 & 0.847058824 & 100 & 0.014146272 & 0.816098458 & 0.009517945 \\
\hline Rskl(h) & 0 & -1 & 0 & 73 & 0.858823529 & 100 & 0.014146272 & 0.830244731 & 0.009684372 \\
\hline Rsk1(r) & 2 & -1 & 0 & 74 & 0.870588235 & 100 & 0.014146272 & 0.844391003 & 0.009850798 \\
\hline Rsk2(h) & -1 & -1 & 0 & 75 & 0.882352941 & 100 & 0.014146272 & 0.858537275 & 0.010017225 \\
\hline Rsk3(h) & 0 & -1 & 0 & 76 & 0.894117647 & 100 & 0.014146272 & 0.872683548 & 0.010183652 \\
\hline $\begin{array}{l}\text { RAKK(7) } \\
\text { SAPK2a(h) }\end{array}$ & 84 & -2 & 0 & 77 & 0.905882353 & 100 & 0.014146272 & 0.88682982 & 0.010350079 \\
\hline SAPK2b(h) & 114 & -2 & 0 & 78 & 0.917647059 & 100 & 0.014146272 & 0.900976093 & 0.010516505 \\
\hline SAPK3(h) & 54 & -2 & 0 & 79 & 0.929411765 & 100 & 0.014146272 & 0.915122365 & 0.010682932 \\
\hline SAPK $4(\mathrm{~h})$ & 34 & -3 & 0 & 80 & 0.941176471 & 100 & 0.014146272 & 0.929268638 & 0.010849359 \\
\hline SGK(h) & 0 & -3 & 0 & 81 & 0.952941176 & 100 & 0.014146272 & 0.94341491 & 0.011015786 \\
\hline Syk(h) & -3 & -4 & 0 & 82 & 0.964705882 & 100 & 0.014146272 & 0.957561183 & 0.011182212 \\
\hline $\operatorname{TrkB(h)}$ & 0 & -5 & 0 & 83 & 0.976470588 & 100 & 0.014146272 & 0.971707455 & 0.011348639 \\
\hline Yes(h) & 1 & -5 & 0 & 84 & 0.988235294 & 100 & 0.014146272 & 0.985853728 & 0.011515066 \\
\hline ZAP-70(h) & 8 & -11 & 0 & 85 & 1 & 100 & 0.014146272 & 1 & 0.011681493 \\
\hline & & & & & Total inhibition & 7069 & & Total area & 0.425107969 \\
\hline & & & & & & & & Gini & 0.149784061 \\
\hline
\end{tabular}




\begin{tabular}{|c|c|}
\hline & $10 \mu \mathrm{M}$ ATP \\
\hline Kinase & PD184352@ @ $10 \mu \mathrm{M}$ \\
\hline $\mathrm{Abl}(\mathrm{m})$ & 99 \\
\hline AMPK(r) & 100 \\
\hline $\operatorname{Arg}(\mathrm{m})$ & 105 \\
\hline Aurora-A(h) & 106 \\
\hline Axl(h) & 119 \\
\hline $\mathrm{Blk}(\mathrm{m})$ & 105 \\
\hline Bmx(h) & 104 \\
\hline CaMKII(r) & 95 \\
\hline CaMKIV(h) & 106 \\
\hline $\mathrm{CDK} 1 /$ cyclinB(h) & 101 \\
\hline $\mathrm{CDK} 2 / \mathrm{cyclin} \mathrm{A}(\mathrm{h})$ & 102 \\
\hline $\mathrm{CDK} 2$ /cyclinE(h) & 93 \\
\hline CDK3/(cyctinE(h) & 101 \\
\hline $\mathrm{CDK} 5 / \mathrm{p} 35(\mathrm{~h})$ & 106 \\
\hline CDK6/cyclinD3(h) & 95 \\
\hline CDK7/yyclinHMATI(h) & 100 \\
\hline CHK $1(\mathrm{~h})$ & 115 \\
\hline СHK2(h) & 98 \\
\hline $\mathrm{CK} 1(y)$ & 97 \\
\hline CK2(h) & 109 \\
\hline c-RAF(h) & 98 \\
\hline $\operatorname{CsK}(\mathrm{h})$ & 76 \\
\hline $\operatorname{cSRC}(\mathrm{h})$ & 103 \\
\hline Fes(h) & 108 \\
\hline FGFR3(h) & 109 \\
\hline $\mathrm{Fll3( \textrm {h } )}$ & 105 \\
\hline Fyn(h) & 94 \\
\hline GSK3a(h) & 91 \\
\hline GSK3B(h) & 75 \\
\hline IGF- $1 \mathrm{R}(\mathrm{h})$ & 124 \\
\hline $\mathrm{IKK} \alpha(\mathrm{h})$ & 99 \\
\hline ІККВ(h) & 104 \\
\hline $\mathbb{R}(\mathrm{R})$ & 93 \\
\hline JNKIal(h) & 101 \\
\hline JNK2a2(h) & 92 \\
\hline JNK3(r) & 87 \\
\hline Lck(h) & 119 \\
\hline Lyyn(h) & 96 \\
\hline Lyn(m) & 91 \\
\hline MAPKI(h) & 98 \\
\hline MAPK2(h) & 120 \\
\hline MAPK2(m) & 123 \\
\hline MAPKAP-K2(h) & 110 \\
\hline MEK1(h) & 25 \\
\hline MKK $4(\mathrm{~m})$ & 116 \\
\hline MKK $6(\mathrm{~h})$ & 104 \\
\hline MKК7B(h) & 108 \\
\hline MSK1(h) & 104 \\
\hline p70s6K(h) & 101 \\
\hline PAK2(h) & 107 \\
\hline PDGFRa(h) & 96 \\
\hline PDGFRB(h) & 107 \\
\hline PDK1(h) & 97 \\
\hline PKA(b) & 117 \\
\hline PKA(h) & 92 \\
\hline PKBr(h) & 97 \\
\hline PKBB(h) & 132 \\
\hline $\mathrm{PKB}_{(\mathrm{h} / \mathrm{h})}$ & 87 \\
\hline PKCa(h) & 94 \\
\hline РКСBI(h) & 110 \\
\hline PKCy(h) & 91 \\
\hline PKG(b) & 96 \\
\hline PKG:(h) & 100 \\
\hline PKG)(h) & 91 \\
\hline PKC (h) & 101 \\
\hline $\begin{array}{l}\text { PKC } \mu(\mathrm{hl}) \\
\end{array}$ & 96 \\
\hline РКС日(h) & 97 \\
\hline PKD2(h) & 102 \\
\hline PRAK(h) & 112 \\
\hline PRK2(h) & 92 \\
\hline ROCK-II(h) & 98 \\
\hline ROCK-II(r) & 83 \\
\hline Rskl(h) & 111 \\
\hline Rskl(r) & 67 \\
\hline Rsk2(h) & 93 \\
\hline Rsk3(h) & 99 \\
\hline SAPK2a(h) & 105 \\
\hline SAPK2b(h) & 100 \\
\hline SAPK3(h) & 112 \\
\hline $\operatorname{SAPK} 4(\mathrm{~h})$ & 112 \\
\hline SGK (h) & 104 \\
\hline Syk(h) & 105 \\
\hline ThkB(h) & 111 \\
\hline 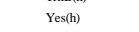 & 109 \\
\hline ZAP-70(h) & 106 \\
\hline
\end{tabular}

\begin{tabular}{|c|c|}
\hline sorted & normalized \\
\hline 132 & 100 \\
\hline 124 & 100 \\
\hline 123 & 100 \\
\hline 120 & 100 \\
\hline 119 & 100 \\
\hline 119 & 100 \\
\hline 117 & 100 \\
\hline 116 & 100 \\
\hline 115 & 100 \\
\hline 112 & 100 \\
\hline 112 & 100 \\
\hline 112 & 100 \\
\hline 111 & 100 \\
\hline 111 & 100 \\
\hline 110 & 100 \\
\hline 110 & 100 \\
\hline 109 & 100 \\
\hline 109 & 100 \\
\hline 109 & 100 \\
\hline 108 & 100 \\
\hline 108 & 100 \\
\hline 107 & 100 \\
\hline 107 & 100 \\
\hline 106 & 100 \\
\hline 106 & 100 \\
\hline 106 & 100 \\
\hline 106 & 100 \\
\hline 105 & 100 \\
\hline 105 & 100 \\
\hline 105 & 100 \\
\hline 105 & 100 \\
\hline 105 & 100 \\
\hline 104 & 100 \\
\hline 104 & 100 \\
\hline 104 & 100 \\
\hline 104 & 100 \\
\hline 104 & 100 \\
\hline 103 & 100 \\
\hline 102 & 100 \\
\hline 102 & 100 \\
\hline 101 & 100 \\
\hline 101 & 100 \\
\hline 101 & 100 \\
\hline 101 & 100 \\
\hline 101 & 100 \\
\hline 100 & 100 \\
\hline 100 & 100 \\
\hline 100 & 100 \\
\hline 100 & 100 \\
\hline 99 & 99 \\
\hline 99 & 99 \\
\hline 99 & 99 \\
\hline 98 & 98 \\
\hline 98 & 98 \\
\hline 98 & 98 \\
\hline 98 & 98 \\
\hline $\begin{array}{l}50 \\
97\end{array}$ & 97 \\
\hline 97 & 97 \\
\hline 97 & 97 \\
\hline 97 & 97 \\
\hline 96 & 96 \\
\hline 96 & 96 \\
\hline 96 & 96 \\
\hline 96 & 96 \\
\hline 95 & 95 \\
\hline 95 & 95 \\
\hline 94 & 94 \\
\hline 94 & 94 \\
\hline 93 & 93 \\
\hline 93 & 93 \\
\hline 93 & 93 \\
\hline 92 & 92 \\
\hline 92 & 92 \\
\hline 92 & 92 \\
\hline 91 & 91 \\
\hline 91 & 91 \\
\hline 91 & 91 \\
\hline 91 & 91 \\
\hline 87 & 87 \\
\hline 87 & 87 \\
\hline 83 & 83 \\
\hline 76 & 76 \\
\hline 75 & 75 \\
\hline 67 & 67 \\
\hline 25 & 25 \\
\hline
\end{tabular}

position

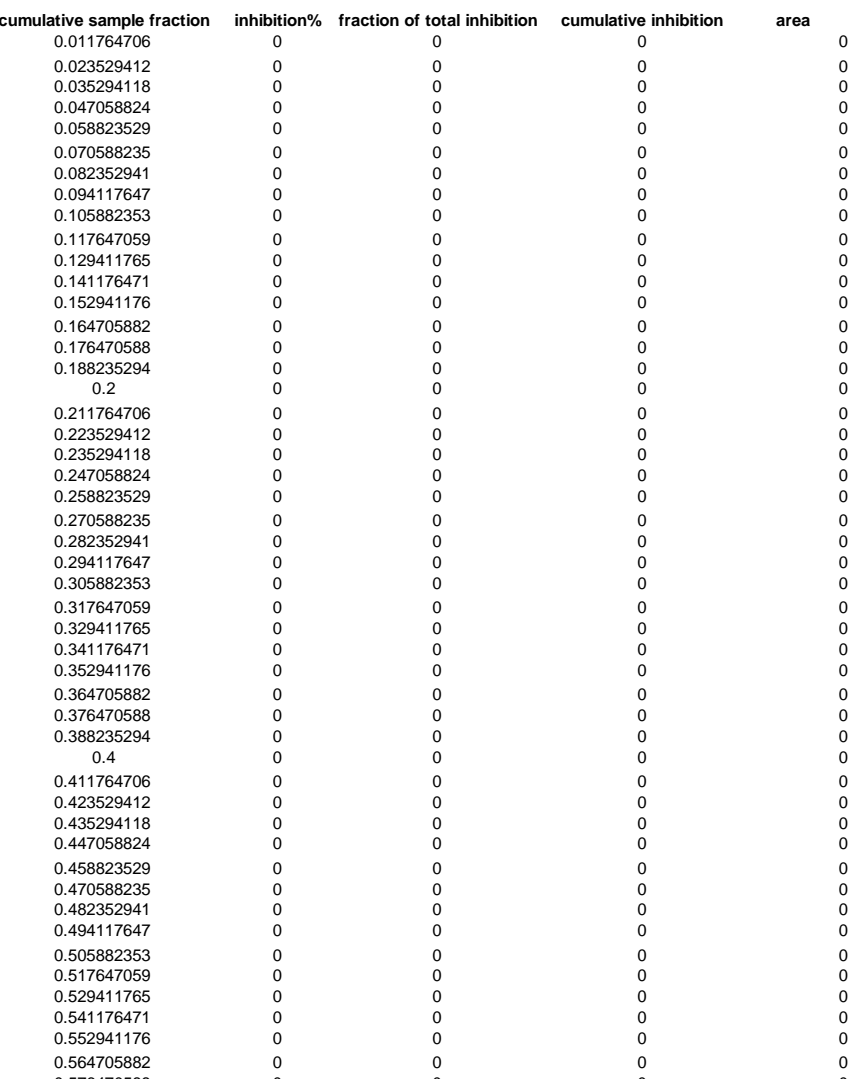

0.564705882

0.576470588
0.588235294

0.588235294
0.6

0.611764706

0.62352941

0.635294118

0.647058824

0.658823529

0.682352941

0.682352941
0.694117647

0.705882353

0.717647059

0.717647059
0.729411765
0.741176471

0.764705882

0.776470588
0.788235294

0.8
0.811764706

0.811764706
0.823529412

0.83529411

0.847058824

0.858823529
0.870588235

0.870588235
0.882352941

0.894117647

0.905882353

0.91764705

0.941176471

0.952941176

0.964705882

0.976470588

88823

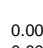

$\begin{array}{lll}0.002923977 & 0.002923977 & 1.71999 \mathrm{E}-05 \\ 0.022923977 & 0.005847953 & 5.15996 \mathrm{E}-05 \\ 0.002923977 & 0.00877193 & 8.59993 \mathrm{E}-05\end{array}$

$\begin{array}{lrr}0.002923977 & 0.002923977 & 1.71999 \mathrm{E}-05 \\ 0.002923977 & 0.005847953 & 5.15996 \mathrm{E}-05 \\ 0.002923977 & 0.00877193 & 8.59993 \mathrm{E}-05\end{array}$

$\begin{array}{lll}0.002923977 & 0.00877193 & 8.59993 \mathrm{E}-05 \\ 0.005847953 & 0.014619883 & 0.000137599\end{array}$

$\begin{array}{lll}0.005847953 & 0.014619883 & 0.000137599 \\ 0.005847953 & 0.020467836 & 0.000206398 \\ 0.0584753 & 0.02631589 & 0.00027598\end{array}$

$\begin{array}{lll}0.005847953 & 0.026467836 & 0.000206398 \\ 0 & 0.026315789 & 0.000275198\end{array}$

$\begin{array}{lll}0.005847953 & 0.032163743 & 0.000343997\end{array}$

$\begin{array}{lll}0.00877953 & 0.040935673 & 0.000429997 \\ 0.00877193 & 0.049707602 & 0.000533196\end{array}$

$0.00877193 \quad 0.058479532-0.000636395$

$0.00877193 \quad-0.067251462 \quad 0.000739594$

$0.011695906-0.072514368-0.000739594$

$0.090643275-0.000997592$

$\begin{array}{lll}0.011695906 & 0.102339181 & 0.001135191\end{array}$

$\begin{array}{lll}0.011695906 & 0.114035088 & 0.00127279\end{array}$

$\begin{array}{lll}0.014619883 & 0.128654971 & 0.001427589 \\ 0 & 0.01434854 & 0.00159958\end{array}$

$\begin{array}{lll}0.014619883 & 0.143274854 & 0.001599587 \\ 0.01754386 & 0.160818713 & 0.001788786\end{array}$

001788786

$\begin{array}{lll}0.020467836 & 0.198830409 & 0.002218782\end{array}$

$\begin{array}{lll}0.020467836 & 0.219298246 & 0.00245958\end{array}$

$\begin{array}{lll}0.020467836 & 0.239766082 & 0.002700378\end{array}$

$0.263157895-0.002958376$

$\begin{array}{lll}0.023391813 & 0.286549708 & 0.003233574\end{array}$

$\begin{array}{lll}0.02339813 & 0.30994152 & 0.003508742 \\ 0.026315789 & 0.33625731 & 0.00380117\end{array}$

$\begin{array}{lll}0.026315789 & 0.30925731 & 0.00380117\end{array}$

$\begin{array}{lll}0.026315789 & 0.388888889 & 0.004420365\end{array}$

$\begin{array}{lll}0.026315789 & 0.38888889 & 0.00420365 \\ 0.038011696 & 0.453216374 & 0.004729962\end{array}$

$\begin{array}{lll}0.03801696 & 0.453212874 & 0.0052985 \\ 0.038011696 & 0.49122807 & 0.005555556\end{array}$

$\begin{array}{lll}0.049707602 & 0.540935673 & 0.006071551\end{array}$

$\begin{array}{lll}0.040175439 & 0.61111111 & 0.006776546\end{array}$

$0.684210526-0.007619539$

$\begin{array}{ccc}0.096491228 & 0.784210526 & 0.007619539 \\ 0.219298246 & 1 & 0.008617131\end{array}$

Total inhibition $\quad 342$

Total area

0.098899209

Gini

0.802201582 


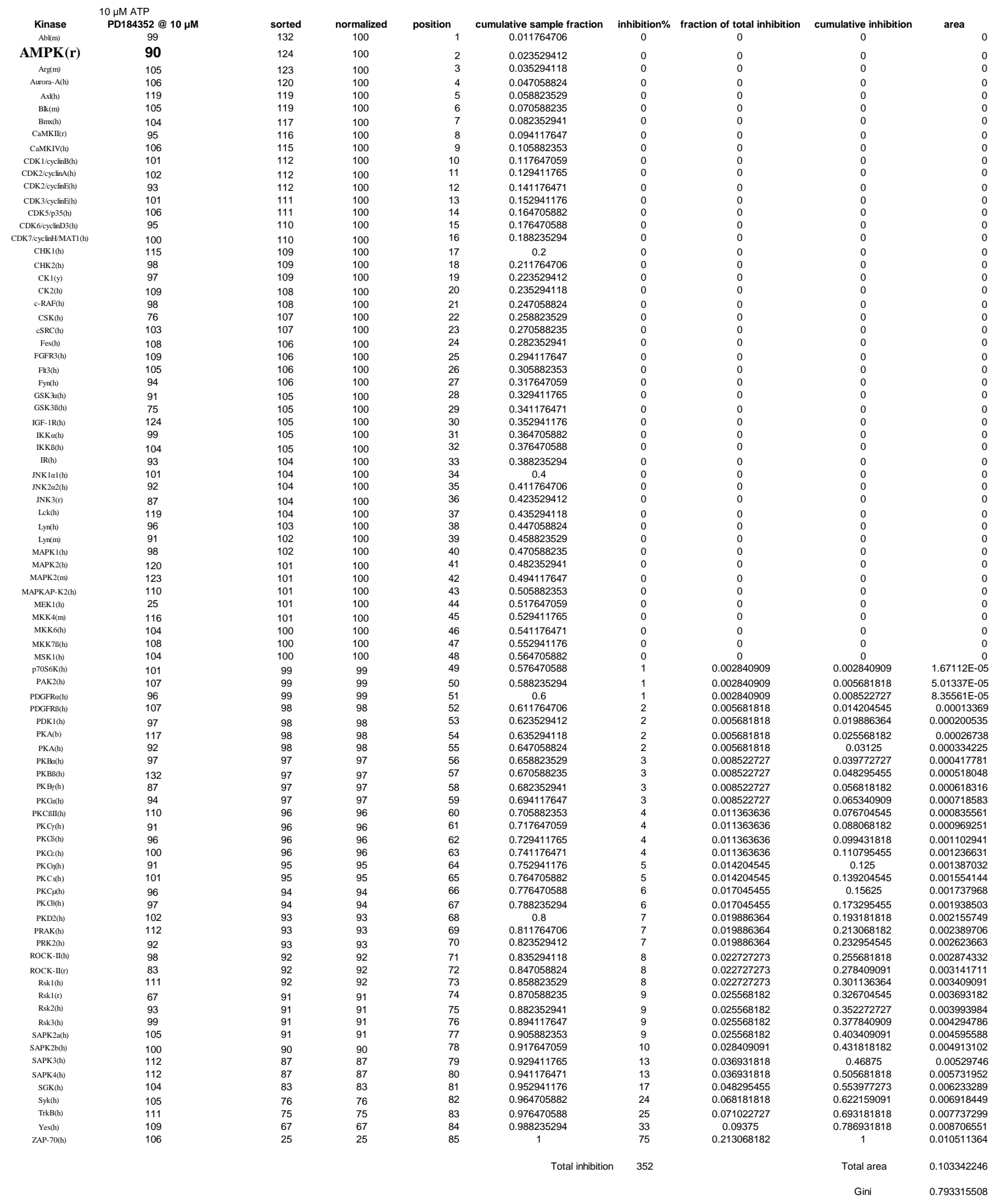




\begin{tabular}{|c|c|}
\hline & $10 \mu \mathrm{M}$ ATP \\
\hline Kinase & PD184352 @ $10 \mu \mathrm{M}$ \\
\hline $\mathrm{Abl}(\mathrm{m})$ & 99 \\
\hline AMPK(r) & 100 \\
\hline $\operatorname{Arg}(m)$ & 105 \\
\hline Aurora-A(h) & 106 \\
\hline Axl(h) & 119 \\
\hline $\operatorname{Blk}(\mathrm{m})$ & 105 \\
\hline Bmx(h) & 104 \\
\hline CaMKII(r) & 95 \\
\hline CaMKIV(h) & 106 \\
\hline $\mathrm{CDK} 1 / \mathrm{cyclin} \mathrm{B}(\mathrm{h})$ & 101 \\
\hline CDK2/cyclinA(h) & 102 \\
\hline CDK2/cyclinE(h) & 93 \\
\hline CDK3/yclinE(h) & 101 \\
\hline CDK5/p35(h) & 106 \\
\hline CDK6/cyclinD3(h) & 95 \\
\hline CDK7//yclinH/MATI(h) & 100 \\
\hline СHK1(h) & 115 \\
\hline СНК2(h) & 98 \\
\hline CKI(y) & 97 \\
\hline CK2(h) & 109 \\
\hline $\mathrm{c} \cdot \mathrm{RAF}(\mathrm{h})$ & 98 \\
\hline $\operatorname{CSK}(\mathrm{h})$ & 76 \\
\hline $\operatorname{cSRC}(\mathrm{h})$ & 103 \\
\hline Fes(h) & 108 \\
\hline FGFR3(h) & 109 \\
\hline Fll3(h) & 105 \\
\hline Fyn(h) & 94 \\
\hline GSK3a(h) & 91 \\
\hline GSK3B(h) & 75 \\
\hline IGF-1R(h) & 124 \\
\hline $\mathrm{IKK} \alpha(\mathrm{h})$ & 99 \\
\hline IKKB(h) & 104 \\
\hline $\mathrm{IR}(\mathrm{h})$ & 93 \\
\hline JNK Ial(h) & 101 \\
\hline JNK2a2(h) & 92 \\
\hline JNK3(r) & 87 \\
\hline Lck(h) & 119 \\
\hline Lynn(h) & 96 \\
\hline Lyn(m) & 91 \\
\hline MAPK1(h) & 98 \\
\hline MAPK2(h) & 120 \\
\hline MAPK2(m) & 123 \\
\hline MAPKAP-K2(h) & 110 \\
\hline MEK1(h) & 25 \\
\hline MKK4(m) & 116 \\
\hline MKK6(h) & 104 \\
\hline MKK7B(h) & 108 \\
\hline MSK1(h) & 104 \\
\hline $\mathrm{p} 70 \mathrm{~s} 6 \mathrm{~K}(\mathrm{~h})$ & 101 \\
\hline PAK2(h) & 107 \\
\hline PDGFRa(h) & 96 \\
\hline PDGFRB(h) & 107 \\
\hline PDK1(h) & 97 \\
\hline PKA(b) & 117 \\
\hline PKA(h) & 92 \\
\hline $\operatorname{PKBa}(\mathrm{h})$ & 97 \\
\hline PKBB(h) & 132 \\
\hline $\mathrm{PKB}_{f}(\mathrm{~h})$ & 87 \\
\hline PKCa(h) & 94 \\
\hline PKCBII(h) & 110 \\
\hline PKCy(h) & 91 \\
\hline РКСర(h) & 96 \\
\hline $\mathrm{PKG}:(\mathrm{h})$ & 100 \\
\hline PKCh(h) & 91 \\
\hline PKC (h) & 101 \\
\hline PKC $\mu(\mathrm{h})$ & 96 \\
\hline РКС®(h) & 97 \\
\hline PKD2(h) & 102 \\
\hline PRAK(h) & 112 \\
\hline PRK2(h) & 92 \\
\hline ROCK-II(h) & 98 \\
\hline ROCK-II(r) & 83 \\
\hline Rskl(h) & 111 \\
\hline Rsk1(r) & 60 \\
\hline Rsk2(h) & 93 \\
\hline Rsk3(h) & $\begin{array}{c}99 \\
105\end{array}$ \\
\hline SAPK2a(h) & 105 \\
\hline SAPK2b(h) & 100 \\
\hline SAPK3(h) & 112 \\
\hline $\operatorname{SAPK4(h)}$ & 112 \\
\hline $\operatorname{SGK}(\mathrm{h})$ & 104 \\
\hline Syk(h) & 105 \\
\hline TikB(h) & 111 \\
\hline Yes(h) & 109 \\
\hline ZAP-70(h) & 106 \\
\hline
\end{tabular}

\begin{tabular}{|c|c|c|c|c|c|c|c|}
\hline sorted & normalized & position & cumulative sample fraction & inhibition\% & fraction of total inhibition & cumulative inhibition & area \\
\hline 132 & 100 & 1 & 0.011764706 & 0 & 0 & 0 & 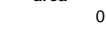 \\
\hline 124 & 100 & 2 & 0.023529412 & 0 & 0 & 0 & 0 \\
\hline 123 & 100 & 3 & 0.035294118 & 0 & 0 & 0 & 0 \\
\hline 120 & 100 & 4 & 0.047058824 & 0 & 0 & 0 & 0 \\
\hline 119 & 100 & 5 & 0.058823529 & 0 & 0 & 0 & 0 \\
\hline 119 & 100 & 6 & 0.070588235 & 0 & 0 & 0 & 0 \\
\hline 117 & 100 & 7 & 0.082352941 & 0 & 0 & 0 & 0 \\
\hline 116 & 100 & 8 & 0.094117647 & 0 & 0 & 0 & 0 \\
\hline 115 & 100 & 9 & 0.105882353 & 0 & 0 & 0 & 0 \\
\hline 112 & 100 & 10 & 0.117647059 & 0 & 0 & 0 & 0 \\
\hline 112 & 100 & 11 & 0.129411765 & 0 & 0 & 0 & 0 \\
\hline 112 & 100 & 12 & 0.141176471 & 0 & 0 & 0 & 0 \\
\hline 111 & 100 & 13 & 0.152941176 & 0 & 0 & 0 & 0 \\
\hline 111 & 100 & 14 & 0.164705882 & 0 & 0 & 0 & 0 \\
\hline 110 & 100 & 15 & 0.176470588 & 0 & 0 & 0 & 0 \\
\hline 110 & 100 & 16 & 0.188235294 & 0 & 0 & 0 & 0 \\
\hline 109 & 100 & 17 & 0.2 & 0 & 0 & 0 & 0 \\
\hline 109 & 100 & 18 & 0.211764706 & 0 & 0 & 0 & 0 \\
\hline 109 & 100 & 19 & 0.223529412 & 0 & 0 & 0 & 0 \\
\hline 108 & 100 & 20 & 0.235294118 & 0 & 0 & 0 & 0 \\
\hline 108 & 100 & 21 & 0.247058824 & 0 & 0 & 0 & 0 \\
\hline 107 & 100 & 22 & 0.258823529 & 0 & 0 & 0 & 0 \\
\hline 107 & 100 & 23 & 0.270588235 & 0 & 0 & 0 & 0 \\
\hline 106 & 100 & 24 & 0.282352941 & 0 & 0 & 0 & 0 \\
\hline 106 & 100 & 25 & 0.294117647 & 0 & 0 & 0 & 0 \\
\hline 106 & 100 & 26 & 0.305882353 & 0 & 0 & 0 & 0 \\
\hline 106 & 100 & 27 & 0.317647059 & 0 & 0 & 0 & 0 \\
\hline 105 & 100 & 28 & 0.329411765 & 0 & 0 & 0 & 0 \\
\hline 105 & 100 & 29 & 0.341176471 & 0 & 0 & 0 & 0 \\
\hline 105 & 100 & 30 & 0.352941176 & 0 & 0 & 0 & 0 \\
\hline 105 & 100 & 31 & 0.364705882 & 0 & 0 & 0 & 0 \\
\hline 105 & 100 & 32 & 0.376470588 & 0 & 0 & 0 & 0 \\
\hline 104 & 100 & 33 & 0.388235294 & 0 & 0 & 0 & 0 \\
\hline 104 & 100 & 34 & 0.4 & 0 & 0 & 0 & 0 \\
\hline 104 & 100 & 35 & 0.411764706 & 0 & 0 & 0 & 0 \\
\hline 104 & 100 & 36 & 0.423529412 & 0 & 0 & 0 & 0 \\
\hline 104 & 100 & 37 & 0.435294118 & 0 & 0 & 0 & 0 \\
\hline 103 & 100 & 38 & 0.447058824 & 0 & 0 & 0 & 0 \\
\hline 102 & 100 & 39 & 0.458823529 & 0 & 0 & 0 & 0 \\
\hline 102 & 100 & 40 & 0.470588235 & 0 & 0 & 0 & 0 \\
\hline 101 & 100 & 41 & 0.482352941 & 0 & 0 & 0 & 0 \\
\hline 101 & 100 & 42 & 0.494117647 & 0 & 0 & 0 & 0 \\
\hline 101 & 100 & 43 & 0.505882353 & 0 & 0 & 0 & 0 \\
\hline 101 & 100 & 44 & 0.517647059 & 0 & 0 & 0 & 0 \\
\hline 101 & 100 & 45 & 0.529411765 & 0 & 0 & 0 & 0 \\
\hline 100 & 100 & 46 & 0.541176471 & 0 & 0 & 0 & 0 \\
\hline 100 & 100 & 47 & 0.552941176 & 0 & 0 & 0 & 0 \\
\hline 100 & 100 & 48 & 0.564705882 & 0 & 0 & 0 & 0 \\
\hline 100 & 100 & 49 & 0.576470588 & 0 & 0 & 0 & 0 \\
\hline 99 & 99 & 50 & 0.588235294 & 1 & 0.00286533 & 0.00286533 & $1.68549 \mathrm{E}-05$ \\
\hline 99 & 99 & 51 & 0.6 & 1 & 0.00286533 & 0.005730659 & 5.05646E-05 \\
\hline 99 & 99 & 52 & 0.611764706 & 1 & 0.00286533 & 0.008595989 & 8.42744E-05 \\
\hline 98 & 98 & 53 & 0.623529412 & 2 & 0.005730659 & 0.014326648 & 0.000134839 \\
\hline 98 & 98 & 54 & 0.635294118 & 2 & 0.005730659 & 0.020057307 & 0.000202259 \\
\hline 98 & 98 & 55 & 0.647058824 & 2 & 0.005730659 & 0.025787966 & 0.000269678 \\
\hline 98 & 98 & 56 & 0.658823529 & 2 & 0.005730659 & 0.031518625 & 0.000337098 \\
\hline 97 & 97 & 57 & 0.670588235 & 3 & 0.008595989 & 0.040114613 & 0.000421372 \\
\hline 97 & 97 & 58 & 0.682352941 & 3 & 0.008595989 & 0.048710602 & 0.000522501 \\
\hline 97 & 97 & 59 & 0.694117647 & 3 & 0.008595989 & 0.05730659 & 0.000623631 \\
\hline 97 & 97 & 60 & 0.705882353 & 3 & 0.008595989 & 0.065902579 & 0.00072476 \\
\hline 96 & 96 & 61 & 0.717647059 & 4 & 0.011461318 & 0.077363897 & 0.000842744 \\
\hline 96 & 96 & 62 & 0.729411765 & 4 & 0.011461318 & 0.088825215 & 0.000977583 \\
\hline 96 & 96 & 63 & 0.741176471 & 4 & 0.011461318 & 0.100286533 & 0.001112422 \\
\hline 96 & 96 & 64 & 0.752941176 & 4 & 0.011461318 & 0.111747851 & 0.001247261 \\
\hline 95 & 95 & 65 & 0.764705882 & 5 & 0.014326648 & 0.126074499 & 0.001398955 \\
\hline 95 & 95 & 66 & 0.776470588 & 5 & 0.014326648 & 0.140401146 & 0.001567504 \\
\hline 94 & 94 & 67 & 0.788235294 & 6 & 0.017191977 & 0.157593123 & 0.001752907 \\
\hline 94 & 94 & 68 & 0.8 & 6 & 0.017191977 & 0.1747851 & 0.001955166 \\
\hline 93 & 93 & 69 & 0.811764706 & 7 & 0.020057307 & 0.194842407 & 0.002174279 \\
\hline 93 & 93 & 70 & 0.823529412 & 7 & 0.020057307 & 0.214899713 & 0.002410248 \\
\hline 93 & 93 & 71 & 0.835294118 & 7 & 0.020057307 & 0.23495702 & 0.002646216 \\
\hline 92 & 92 & 72 & 0.847058824 & 8 & 0.022922636 & 0.257879656 & 0.002899039 \\
\hline 92 & 92 & 73 & 0.858823529 & 8 & 0.022922636 & 0.280802292 & 0.003168717 \\
\hline 92 & 92 & 74 & 0.870588235 & 8 & 0.022922636 & 0.303724928 & 0.003438395 \\
\hline 91 & 91 & 75 & 0.882352941 & 9 & 0.025787966 & 0.329512894 & 0.003724928 \\
\hline 91 & 91 & 76 & 0.894117647 & 9 & 0.025787966 & 0.35530086 & 0.004028316 \\
\hline 91 & 91 & 77 & 0.905882353 & 9 & 0.025787966 & 0.381088825 & 0.004331704 \\
\hline 91 & 91 & 78 & 0.917647059 & 9 & 0.025787966 & 0.406876791 & 0.004635092 \\
\hline 87 & 87 & 79 & 0.929411765 & 13 & 0.037249284 & 0.444126074 & 0.005005899 \\
\hline 87 & 87 & 80 & 0.941176471 & 13 & 0.037249284 & 0.481375358 & 0.005444126 \\
\hline 83 & 83 & 81 & 0.952941176 & 17 & 0.048710602 & 0.53008596 & 0.005949772 \\
\hline 76 & 76 & 82 & 0.964705882 & 24 & 0.068767908 & 0.598853868 & 0.006640823 \\
\hline 75 & 75 & 83 & 0.976470588 & 25 & 0.071633238 & 0.670487106 & 0.007466712 \\
\hline 60 & 60 & 84 & 0.988235294 & 40 & 0.114613181 & 0.785100287 & 0.008562279 \\
\hline \multirow[t]{3}{*}{25} & 25 & 85 & 1 & 75 & 0.214899713 & 1 & 0.01050059 \\
\hline & & & Total inhibition & 349 & & Total area & 0.09726951 \\
\hline & & & & & & Gini & 0.805460981 \\
\hline
\end{tabular}




\begin{tabular}{|c|c|}
\hline \multicolumn{2}{|c|}{$10 \mu \mathrm{M}$ ATP } \\
\hline Kinase & 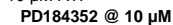 \\
\hline $\mathrm{Ab}(\mathrm{m})$ & 99 \\
\hline AMPK(r) & 100 \\
\hline $\operatorname{Arg}(\mathrm{m})$ & 105 \\
\hline Aurora-A(h) & 106 \\
\hline $\operatorname{Ax}(\mathrm{h})$ & 119 \\
\hline $\operatorname{Blk}(\mathrm{m})$ & 105 \\
\hline Bmx(h) & 104 \\
\hline CaMKI(r) & 95 \\
\hline CaMKIV(h) & 106 \\
\hline $\mathrm{CDK} 1 / \mathrm{cyclin} \mathrm{B}(\mathrm{h})$ & 101 \\
\hline $\operatorname{CDK} 2 /$ cyclinA(h) & 102 \\
\hline $\mathrm{CDK} 2 /$ cyclinE(h) & 93 \\
\hline $\mathrm{CDK} 3 / \mathrm{cyclin} \mathrm{E}(\mathrm{h})$ & 101 \\
\hline CDK5/p35(h) & 106 \\
\hline $\mathrm{CDK} 6 /$ cyclinD3(h) & 95 \\
\hline CDK7/cyclinH/MATI(h) & 100 \\
\hline СнK1(h) & 115 \\
\hline СНК2(h) & 98 \\
\hline $\mathrm{CK} 1(y)$ & 97 \\
\hline CK2(h) & 109 \\
\hline$c-\operatorname{RAF}(\mathrm{h})$ & 98 \\
\hline $\operatorname{CsK}(\mathrm{h})$ & 76 \\
\hline $\operatorname{cSRC(h)}$ & 103 \\
\hline Fes(h) & 108 \\
\hline FGFR3(h) & 109 \\
\hline Fll3(h) & 105 \\
\hline Fyn(h) & 94 \\
\hline GSK3a(h) & 91 \\
\hline GSK3B(h) & 75 \\
\hline IGF-1R(h) & 124 \\
\hline IKKa(h) & 99 \\
\hline IKKB(h) & 104 \\
\hline $\mathbb{R}(\mathrm{h})$ & 93 \\
\hline JNK $1 \alpha 1(\mathrm{~h})$ & 101 \\
\hline JNK2 $22(\mathrm{~h})$ & 92 \\
\hline JNK3(r) & 87 \\
\hline Lck(h) & 119 \\
\hline Lynn(h) & 96 \\
\hline Lyn(m) & 91 \\
\hline MAPKI(h) & 98 \\
\hline MAPK $2(\mathrm{~h})$ & 120 \\
\hline $\operatorname{MAPK} 2(\mathrm{~m})$ & 123 \\
\hline MAPKAP-K2(h) & 110 \\
\hline MEK1(h) & 25 \\
\hline MKK $4(\mathrm{~m})$ & 116 \\
\hline MKK6(h) & 104 \\
\hline MKK $7($ (h) & 108 \\
\hline MSK1(h) & 104 \\
\hline $\mathrm{p} 70 \mathrm{~s} 6 \mathrm{~K}(\mathrm{~h})$ & 101 \\
\hline PAK2(h) & 107 \\
\hline PDGFRa(h) & 96 \\
\hline PDGFRB(h) & 107 \\
\hline PDK1(h) & 97 \\
\hline PKA(b) & 117 \\
\hline PKA(h) & 92 \\
\hline PKBa(h) & 97 \\
\hline PKBB(h) & 132 \\
\hline $\mathrm{PKB}_{/}(\mathrm{h})$ & 87 \\
\hline PKCa(h) & 94 \\
\hline PKCBII(h) & 110 \\
\hline PKCy(h) & 91 \\
\hline PKCऽ(h) & 96 \\
\hline $\mathrm{PKG}(\mathrm{h})$ & 100 \\
\hline PKG(h(h) & 91 \\
\hline PKC (h) & 101 \\
\hline $\mathrm{PKC} \mu(\mathrm{h})$ & 96 \\
\hline РКС®(h) & 97 \\
\hline PKD2(h) & 102 \\
\hline PRAK(h) & 112 \\
\hline PRK2(h) & 92 \\
\hline ROCK-II(h) & 98 \\
\hline ROCK-III(r) & 83 \\
\hline Rskl(h) & 111 \\
\hline Rsk1(r) & 74 \\
\hline Rsk2(h) & 93 \\
\hline Rsk3(h) & 99 \\
\hline SAPK2a(h) & 105 \\
\hline SAPK2b(h) & 100 \\
\hline SAPK3(h) & 112 \\
\hline SAPK 4(h) & 112 \\
\hline SGK(h) & 104 \\
\hline Syk(h) & 105 \\
\hline TrkB(h) & 111 \\
\hline Yes(h) & 109 \\
\hline ZAP-70(h) & 106 \\
\hline
\end{tabular}

\begin{tabular}{|c|c|c|c|c|c|c|c|}
\hline sorted & normalized & position & cumulative sample fraction & inhibition\% & fraction of total inhibition & cumulative inhibition & area \\
\hline 132 & 100 & 1 & 0.011764706 & 0 & 0 & 0 & 0 \\
\hline 124 & 100 & 2 & 0.023529412 & 0 & 0 & 0 & 0 \\
\hline 123 & 100 & 3 & 0.035294118 & 0 & 0 & 0 & 0 \\
\hline 120 & 100 & 4 & 0.047058824 & 0 & 0 & 0 & 0 \\
\hline 119 & 100 & 5 & 0.058823529 & 0 & 0 & 0 & 0 \\
\hline 119 & 100 & 6 & 0.070588235 & 0 & 0 & 0 & 0 \\
\hline 117 & 100 & 7 & 0.082352941 & 0 & 0 & 0 & 0 \\
\hline 116 & 100 & 8 & 0.094117647 & 0 & 0 & 0 & 0 \\
\hline 115 & 100 & 9 & 0.105882353 & 0 & 0 & 0 & 0 \\
\hline 112 & 100 & 10 & 0.117647059 & 0 & 0 & 0 & 0 \\
\hline 112 & 100 & 11 & 0.129411765 & 0 & 0 & 0 & 0 \\
\hline 112 & 100 & 12 & 0.141176471 & 0 & 0 & 0 & 0 \\
\hline 111 & 100 & 13 & 0.152941176 & 0 & 0 & 0 & 0 \\
\hline 111 & 100 & 14 & 0.164705882 & 0 & 0 & 0 & 0 \\
\hline 110 & 100 & 15 & 0.176470588 & 0 & 0 & 0 & 0 \\
\hline 110 & 100 & 16 & 0.188235294 & 0 & 0 & 0 & 0 \\
\hline 109 & 100 & 17 & 0.2 & 0 & 0 & 0 & 0 \\
\hline 109 & 100 & 18 & 0.211764706 & 0 & 0 & 0 & 0 \\
\hline 109 & 100 & 19 & 0.223529412 & 0 & 0 & 0 & 0 \\
\hline 108 & 100 & 20 & 0.235294118 & 0 & 0 & 0 & 0 \\
\hline 108 & 100 & 21 & 0.247058824 & 0 & 0 & 0 & 0 \\
\hline 107 & 100 & 22 & 0.258823529 & 0 & 0 & 0 & 0 \\
\hline 107 & 100 & 23 & 0.270588235 & 0 & 0 & 0 & 0 \\
\hline 106 & 100 & 24 & 0.282352941 & 0 & 0 & 0 & 0 \\
\hline 106 & 100 & 25 & 0.294117647 & 0 & 0 & 0 & 0 \\
\hline 106 & 100 & 26 & 0.305882353 & 0 & 0 & 0 & 0 \\
\hline 106 & 100 & 27 & 0.317647059 & 0 & 0 & 0 & 0 \\
\hline 105 & 100 & 28 & 0.329411765 & 0 & 0 & 0 & 0 \\
\hline 105 & 100 & 29 & 0.341176471 & 0 & 0 & 0 & 0 \\
\hline 105 & 100 & 30 & 0.352941176 & 0 & 0 & 0 & 0 \\
\hline 105 & 100 & 31 & 0.364705882 & 0 & 0 & 0 & 0 \\
\hline 105 & 100 & 32 & 0.376470588 & 0 & 0 & 0 & 0 \\
\hline 104 & 100 & 33 & 0.388235294 & 0 & 0 & 0 & 0 \\
\hline 104 & 100 & 34 & 0.4 & 0 & 0 & 0 & 0 \\
\hline 104 & 100 & 35 & 0.411764706 & 0 & 0 & 0 & 0 \\
\hline 104 & 100 & 36 & 0.423529412 & 0 & 0 & 0 & 0 \\
\hline 104 & 100 & 37 & 0.435294118 & 0 & 0 & 0 & 0 \\
\hline 103 & 100 & 38 & 0.447058824 & 0 & 0 & 0 & 0 \\
\hline 102 & 100 & 39 & 0.458823529 & 0 & 0 & 0 & 0 \\
\hline 102 & 100 & 40 & 0.470588235 & 0 & 0 & 0 & 0 \\
\hline 101 & 100 & 41 & 0.482352941 & 0 & 0 & 0 & 0 \\
\hline 101 & 100 & 42 & 0.494117647 & 0 & 0 & 0 & 0 \\
\hline 101 & 100 & 43 & 0.505882353 & 0 & 0 & 0 & 0 \\
\hline 101 & 100 & 44 & 0.517647059 & 0 & 0 & 0 & 0 \\
\hline 101 & 100 & 45 & 0.529411765 & 0 & 0 & 0 & 0 \\
\hline 100 & 100 & 46 & 0.541176471 & 0 & 0 & 0 & 0 \\
\hline 100 & 100 & 47 & 0.552941176 & 0 & 0 & 0 & 0 \\
\hline 100 & 100 & 48 & 0.564705882 & 0 & 0 & 0 & 0 \\
\hline 100 & 100 & 49 & 0.576470588 & 0 & 0 & 0 & 0 \\
\hline 99 & 99 & 50 & 0.588235294 & 1 & 0.002985075 & 0.002985075 & $1.75593 \mathrm{E}-05$ \\
\hline 99 & 99 & 51 & 0.6 & 1 & 0.002985075 & 0.005970149 & 5.26778E-05 \\
\hline 99 & 99 & 52 & 0.611764706 & 1 & 0.002985075 & 0.008955224 & 8.77963E-05 \\
\hline 98 & 98 & 53 & 0.623529412 & 2 & 0.005970149 & 0.014925373 & 0.000140474 \\
\hline 98 & 98 & 54 & 0.635294118 & 2 & 0.005970149 & 0.020895522 & 0.000210711 \\
\hline 98 & 98 & 55 & 0.647058824 & 2 & 0.005970149 & 0.026865672 & 0.000280948 \\
\hline 98 & 98 & 56 & 0.658823529 & 2 & 0.005970149 & 0.032835821 & 0.000351185 \\
\hline 97 & 97 & 57 & 0.670588235 & 3 & 0.008955224 & 0.041791045 & 0.000438982 \\
\hline 97 & 97 & 58 & 0.682352941 & 3 & 0.008955224 & 0.050746269 & 0.000544337 \\
\hline 97 & 97 & 59 & 0.694117647 & 3 & 0.008955224 & 0.059701493 & 0.000649693 \\
\hline 97 & 97 & 60 & 0.705882353 & 3 & 0.008955224 & 0.068656716 & 0.000755048 \\
\hline 96 & 96 & 61 & 0.717647059 & 4 & 0.011940299 & 0.080597015 & 0.000877963 \\
\hline 96 & 96 & 62 & 0.729411765 & 4 & 0.011940299 & 0.092537313 & 0.001018437 \\
\hline 96 & 96 & 63 & 0.741176471 & 4 & 0.011940299 & 0.104477612 & 0.001158911 \\
\hline 96 & 96 & 64 & 0.752941176 & 4 & 0.011940299 & 0.11641791 & 0.001299385 \\
\hline 95 & 95 & 65 & 0.764705882 & 5 & 0.014925373 & 0.131343284 & 0.001457419 \\
\hline 95 & 95 & 66 & 0.776470588 & 5 & 0.014925373 & 0.146268657 & 0.001633011 \\
\hline 94 & 94 & 67 & 0.788235294 & 6 & 0.017910448 & 0.164179104 & 0.001826163 \\
\hline 94 & 94 & 68 & 0.8 & 6 & 0.017910448 & 0.182089552 & 0.002036874 \\
\hline 93 & 93 & 69 & 0.811764706 & 7 & 0.020895522 & 0.202985075 & 0.002265145 \\
\hline 93 & 93 & 70 & 0.823529412 & 7 & 0.020895522 & 0.223880597 & 0.002510975 \\
\hline 93 & 93 & 71 & 0.835294118 & 7 & 0.020895522 & 0.244776119 & 0.002756804 \\
\hline 92 & 92 & 72 & 0.847058824 & 8 & 0.023880597 & 0.268656716 & 0.003020193 \\
\hline 92 & 92 & 73 & 0.858823529 & 8 & 0.023880597 & 0.292537313 & 0.003301141 \\
\hline 92 & 92 & 74 & 0.870588235 & 8 & 0.023880597 & 0.31641791 & 0.00358209 \\
\hline 91 & 91 & 75 & 0.882352941 & 9 & 0.026865672 & 0.343283582 & 0.003880597 \\
\hline 91 & 91 & 76 & 0.894117647 & 9 & 0.026865672 & 0.370149254 & 0.004196664 \\
\hline 91 & 91 & 77 & 0.905882353 & 9 & 0.026865672 & 0.397014925 & 0.00451273 \\
\hline 91 & 91 & 78 & 0.917647059 & 9 & 0.026865672 & 0.423880597 & 0.004828797 \\
\hline 87 & 87 & 79 & 0.929411765 & 13 & 0.03880597 & 0.462686567 & 0.005215101 \\
\hline 87 & 87 & 80 & 0.941176471 & 13 & 0.03880597 & 0.501492537 & 0.005671642 \\
\hline 83 & 83 & 81 & 0.952941176 & 17 & 0.050746269 & 0.552238806 & 0.00619842 \\
\hline 76 & 76 & 82 & 0.964705882 & 24 & 0.071641791 & 0.623880597 & 0.006918349 \\
\hline 75 & 75 & 83 & 0.976470588 & 25 & 0.074626866 & 0.698507463 & 0.007778753 \\
\hline 74 & 74 & 84 & 0.988235294 & 26 & 0.07761194 & 0.776119403 & 0.008674276 \\
\hline \multirow{2}{*}{$\begin{array}{l}4 \\
25\end{array}$} & 25 & 85 & 1 & 75 & 0.223880597 & 1 & 0.010447761 \\
\hline & & & Total inhibition & 335 & & Total area & 0.100597015 \\
\hline
\end{tabular}




\begin{tabular}{|c|c|}
\hline & $10 \mu \mathrm{M}$ ATP \\
\hline Kinase & PD184352@ @ $10 \mu \mathrm{M}$ \\
\hline $\mathrm{Abl}(\mathrm{m})$ & 99 \\
\hline AMPK(r) & 100 \\
\hline $\operatorname{Arg}(\mathrm{m})$ & 105 \\
\hline Aurora-A(h) & 106 \\
\hline Axl(h) & 119 \\
\hline $\operatorname{Blk}(\mathrm{m})$ & 105 \\
\hline Bmx(h) & 104 \\
\hline CaMKII(r) & 95 \\
\hline CaMKIV(h) & 106 \\
\hline $\mathrm{CDK} 1 / \mathrm{cyc} \ln \mathrm{B}(\mathrm{h})$ & 101 \\
\hline $\mathrm{CDK} 2 / \mathrm{cyclin} A(\mathrm{~h})$ & 102 \\
\hline $\mathrm{CDK} 2 /$ cyclinE(h) & 93 \\
\hline CDK3/yclinE(h) & 101 \\
\hline CDK5/p35(h) & 106 \\
\hline CDK6/cyclinD3(h) & 95 \\
\hline CDK7/cyclinH/MAT1(h) & 100 \\
\hline CHK $1(\mathrm{~h})$ & 115 \\
\hline СнК2(h) & 98 \\
\hline $\mathrm{CKI}(\mathrm{y})$ & 97 \\
\hline СK2(h) & 109 \\
\hline$c-\operatorname{RAF}(\mathrm{h})$ & 98 \\
\hline $\operatorname{CSK}(\mathrm{h})$ & 76 \\
\hline $\operatorname{csRC(h)}$ & 103 \\
\hline Fes(h) & 108 \\
\hline FGFR3(h) & 109 \\
\hline Fll3(h) & 105 \\
\hline Fyn(h) & 94 \\
\hline GSK3a(h) & 91 \\
\hline GSK3B(h) & 75 \\
\hline $\operatorname{IGF}-1 \mathrm{R}(\mathrm{h})$ & 124 \\
\hline $\mathrm{IKK} \alpha(\mathrm{h})$ & 99 \\
\hline IKKB(h) & 104 \\
\hline $\mathrm{IR}(\mathrm{h})$ & 93 \\
\hline JNK1 1 1(h) & 101 \\
\hline JNK2a2(h) & 92 \\
\hline JNK3(r) & 87 \\
\hline Lck(h) & 119 \\
\hline Lyn(h) & 96 \\
\hline $\operatorname{Lyn}(\mathrm{m})$ & 91 \\
\hline MAPKI(h) & 98 \\
\hline MAPK $2(\mathrm{~h})$ & 120 \\
\hline MAPK2(m) & 123 \\
\hline MAPKAP-K2(h) & 110 \\
\hline MEK1(h) & 22 \\
\hline MKK4(m) & 116 \\
\hline MKK6(h) & 104 \\
\hline MKK & 108 \\
\hline MSK1(h) & 104 \\
\hline p7006K(h) & 101 \\
\hline PAK2(h) & 107 \\
\hline PDGFRa(h) & 96 \\
\hline $\begin{array}{l}\text { PDGFRB(h) } \\
\text { Path }\end{array}$ & 107 \\
\hline PDK1(h) & 97 \\
\hline PKA(b) & 117 \\
\hline PKA(h) & 92 \\
\hline PKBr(h) & 97 \\
\hline PKBB(h) & 132 \\
\hline $\mathrm{PKB}_{/}(\mathrm{h})$ & 87 \\
\hline PKCa(h) & 94 \\
\hline PKCBII(h) & 110 \\
\hline PKCy(h) & 91 \\
\hline PKC(h) & 96 \\
\hline $\mathrm{PKG}(\mathrm{h})$ & $\begin{array}{l}50 \\
100\end{array}$ \\
\hline PKC(Yh) & 91 \\
\hline $\begin{array}{l}\text { PKC (l(t) } \\
\text { (n) }\end{array}$ & 101 \\
\hline PKC $\mu(\mathrm{h})$ & 96 \\
\hline $\mathrm{PKCP(h)}$ & 97 \\
\hline PKD2(h) & 102 \\
\hline $\begin{array}{l}\text { PRAK(h) } \\
\text { (h) }\end{array}$ & 112 \\
\hline PRK2(h) & 92 \\
\hline ROCK-II(h) & 98 \\
\hline ROCK-II(r) & 83 \\
\hline Rskl(h) & 111 \\
\hline Rskl(r) & 67 \\
\hline Rsk2(h) & 93 \\
\hline Rsk3(h) & 99 \\
\hline SAPK2a(h) & 105 \\
\hline SAPK2b(h) & 100 \\
\hline SAPK3(h) & 112 \\
\hline SAPK4(h) & 112 \\
\hline SGK(h) & 104 \\
\hline Syk(h) & 105 \\
\hline TrkB(h) & 111 \\
\hline Yes(h) & 109 \\
\hline ZAP-70(h) & 106 \\
\hline
\end{tabular}

\begin{tabular}{|c|c|c|c|c|c|c|c|}
\hline sorted & normalized & position & cumulative sample fraction & inhibition\% & fraction of total inhibition & cumulative inhibition & area \\
\hline 132 & 100 & 1 & 0.011764706 & 0 & 0 & 0 & 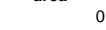 \\
\hline 124 & 100 & 2 & 0.023529412 & 0 & 0 & 0 & 0 \\
\hline 123 & 100 & 3 & 0.035294118 & 0 & 0 & 0 & 0 \\
\hline 120 & 100 & 4 & 0.047058824 & 0 & 0 & 0 & 0 \\
\hline 119 & 100 & 5 & 0.058823529 & 0 & 0 & 0 & 0 \\
\hline 119 & 100 & 6 & 0.070588235 & 0 & 0 & 0 & 0 \\
\hline 117 & 100 & 7 & 0.082352941 & 0 & 0 & 0 & 0 \\
\hline 116 & 100 & 8 & 0.094117647 & 0 & 0 & 0 & 0 \\
\hline 115 & 100 & 9 & 0.105882353 & 0 & 0 & 0 & 0 \\
\hline 112 & 100 & 10 & 0.117647059 & 0 & 0 & 0 & 0 \\
\hline 112 & 100 & 11 & 0.129411765 & 0 & 0 & 0 & 0 \\
\hline 112 & 100 & 12 & 0.141176471 & 0 & 0 & 0 & 0 \\
\hline 111 & 100 & 13 & 0.152941176 & 0 & 0 & 0 & 0 \\
\hline 111 & 100 & 14 & 0.164705882 & 0 & 0 & 0 & 0 \\
\hline 110 & 100 & 15 & 0.176470588 & 0 & 0 & 0 & 0 \\
\hline 110 & 100 & 16 & 0.188235294 & 0 & 0 & 0 & 0 \\
\hline 109 & 100 & 17 & 0.2 & 0 & 0 & 0 & 0 \\
\hline 109 & 100 & 18 & 0.211764706 & 0 & 0 & 0 & 0 \\
\hline 109 & 100 & 19 & 0.223529412 & 0 & 0 & 0 & 0 \\
\hline 108 & 100 & 20 & 0.235294118 & 0 & 0 & 0 & 0 \\
\hline 108 & 100 & 21 & 0.247058824 & 0 & 0 & 0 & 0 \\
\hline 107 & 100 & 22 & 0.258823529 & 0 & 0 & 0 & 0 \\
\hline 107 & 100 & 23 & 0.270588235 & 0 & 0 & 0 & 0 \\
\hline 106 & 100 & 24 & 0.282352941 & 0 & 0 & 0 & 0 \\
\hline 106 & 100 & 25 & 0.294117647 & 0 & 0 & 0 & 0 \\
\hline 106 & 100 & 26 & 0.305882353 & 0 & 0 & 0 & 0 \\
\hline 106 & 100 & 27 & 0.317647059 & 0 & 0 & 0 & 0 \\
\hline 105 & 100 & 28 & 0.329411765 & 0 & 0 & 0 & 0 \\
\hline 105 & 100 & 29 & 0.341176471 & 0 & 0 & 0 & 0 \\
\hline 105 & 100 & 30 & 0.352941176 & 0 & 0 & 0 & 0 \\
\hline 105 & 100 & 31 & 0.364705882 & 0 & 0 & 0 & 0 \\
\hline 105 & 100 & 32 & 0.376470588 & 0 & 0 & 0 & 0 \\
\hline 104 & 100 & 33 & 0.388235294 & 0 & 0 & 0 & 0 \\
\hline 104 & 100 & 34 & 0.4 & 0 & 0 & 0 & 0 \\
\hline 104 & 100 & 35 & 0.411764706 & 0 & 0 & 0 & 0 \\
\hline 104 & 100 & 36 & 0.423529412 & 0 & 0 & 0 & 0 \\
\hline 104 & 100 & 37 & 0.435294118 & 0 & 0 & 0 & 0 \\
\hline 103 & 100 & 38 & 0.447058824 & 0 & 0 & 0 & 0 \\
\hline 102 & 100 & 39 & 0.458823529 & 0 & 0 & 0 & 0 \\
\hline 102 & 100 & 40 & 0.470588235 & 0 & 0 & 0 & 0 \\
\hline 101 & 100 & 41 & 0.482352941 & 0 & 0 & 0 & 0 \\
\hline 101 & 100 & 42 & 0.494117647 & 0 & 0 & 0 & 0 \\
\hline 101 & 100 & 43 & 0.505882353 & 0 & 0 & 0 & 0 \\
\hline 101 & 100 & 44 & 0.517647059 & 0 & 0 & 0 & 0 \\
\hline 101 & 100 & 45 & 0.529411765 & 0 & 0 & 0 & 0 \\
\hline 100 & 100 & 46 & 0.541176471 & 0 & 0 & 0 & 0 \\
\hline 100 & 100 & 47 & 0.552941176 & 0 & 0 & 0 & 0 \\
\hline 100 & 100 & 48 & 0.564705882 & 0 & 0 & 0 & 0 \\
\hline 100 & 100 & 49 & 0.576470588 & 0 & 0 & 0 & 0 \\
\hline 99 & 99 & 50 & 0.588235294 & 1 & 0.002898551 & 0.002898551 & $1.70503 \mathrm{E}-05$ \\
\hline 99 & 99 & 51 & 0.6 & 1 & 0.002898551 & 0.005797101 & $5.11509 \mathrm{E}-05$ \\
\hline 99 & 99 & 52 & 0.611764706 & 1 & 0.002898551 & 0.008695652 & $8.52515 \mathrm{E}-05$ \\
\hline 98 & 98 & 53 & 0.623529412 & 2 & 0.005797101 & 0.014492754 & 0.000136402 \\
\hline 98 & 98 & 54 & 0.635294118 & 2 & 0.005797101 & 0.020289855 & 0.000204604 \\
\hline 98 & 98 & 55 & 0.647058824 & 2 & 0.005797101 & 0.026086957 & 0.000272805 \\
\hline 98 & 98 & 56 & 0.658823529 & 2 & 0.005797101 & 0.031884058 & 0.000341006 \\
\hline 97 & 97 & 57 & 0.670588235 & 3 & 0.008695652 & 0.04057971 & 0.000426257 \\
\hline 97 & 97 & 58 & 0.682352941 & 3 & 0.008695652 & 0.049275362 & 0.000528559 \\
\hline 97 & 97 & 59 & 0.694117647 & 3 & 0.008695652 & 0.057971014 & 0.000630861 \\
\hline 97 & 97 & 60 & 0.705882353 & 3 & 0.008695652 & 0.066666667 & 0.000733163 \\
\hline 96 & 96 & 61 & 0.717647059 & 4 & 0.011594203 & 0.07826087 & 0.000852515 \\
\hline 96 & 96 & 62 & 0.729411765 & 4 & 0.011594203 & 0.089855072 & 0.000988917 \\
\hline 96 & 96 & 63 & 0.741176471 & 4 & 0.011594203 & 0.101449275 & 0.00112532 \\
\hline 96 & 96 & 64 & 0.752941176 & 4 & 0.011594203 & 0.113043478 & 0.001261722 \\
\hline 95 & 95 & 65 & 0.764705882 & 5 & 0.014492754 & 0.127536232 & 0.001415175 \\
\hline 95 & 95 & 66 & 0.776470588 & 5 & 0.014492754 & 0.142028986 & 0.001585678 \\
\hline 94 & 94 & 67 & 0.788235294 & 6 & 0.017391304 & 0.15942029 & 0.001773231 \\
\hline 94 & 94 & 68 & 0.8 & 6 & 0.017391304 & 0.176811594 & 0.001977835 \\
\hline 93 & 93 & 69 & 0.811764706 & 7 & 0.020289855 & 0.197101449 & 0.002199488 \\
\hline 93 & 93 & 70 & 0.823529412 & 7 & 0.020289855 & 0.217391304 & 0.002438193 \\
\hline 93 & 93 & 71 & 0.835294118 & 7 & 0.020289855 & 0.237681159 & 0.002676897 \\
\hline 92 & 92 & 72 & 0.847058824 & 8 & 0.023188406 & 0.260869565 & 0.002932651 \\
\hline 92 & 92 & 73 & 0.858823529 & 8 & 0.023188406 & 0.284057971 & 0.003205456 \\
\hline 92 & 92 & 74 & 0.870588235 & 8 & 0.023188406 & 0.307246377 & 0.003478261 \\
\hline 91 & 91 & 75 & 0.882352941 & 9 & 0.026086957 & 0.333333333 & 0.003768116 \\
\hline 91 & 91 & 76 & 0.894117647 & 9 & 0.026086957 & 0.35942029 & 0.004075021 \\
\hline 91 & 91 & 77 & 0.905882353 & 9 & 0.026086957 & 0.385507246 & 0.004381927 \\
\hline 91 & 91 & 78 & 0.917647059 & 9 & 0.026086957 & 0.411594203 & 0.004688832 \\
\hline 87 & 87 & 79 & 0.929411765 & 13 & 0.037681159 & 0.449275362 & 0.005063939 \\
\hline 87 & 87 & 80 & 0.941176471 & 13 & 0.037681159 & 0.486956522 & 0.005507246 \\
\hline 83 & 83 & 81 & 0.952941176 & 17 & 0.049275362 & 0.536231884 & 0.006018755 \\
\hline 76 & 76 & 82 & 0.964705882 & 24 & 0.069565217 & 0.605797101 & 0.006717818 \\
\hline 75 & 75 & 83 & 0.976470588 & 25 & 0.072463768 & 0.67826087 & 0.007553282 \\
\hline 67 & 67 & 84 & 0.988235294 & 33 & 0.095652174 & 0.773913043 & 0.008542199 \\
\hline \multirow[t]{3}{*}{22} & 22 & 85 & 1 & 78 & 0.226086957 & 1 & 0.010434783 \\
\hline & & & Total inhibition & 345 & & Total area & 0.098090367 \\
\hline & & & & & & Gini & 0.803819267 \\
\hline
\end{tabular}




\begin{tabular}{|c|c|}
\hline & $10 \mu \mathrm{M}$ ATP \\
\hline Kinase & PD184352@ $10 \mu \mathrm{M}$ \\
\hline $\mathrm{Abl}(\mathrm{m})$ & $\begin{array}{c}99 \\
100\end{array}$ \\
\hline AMPK(r) & 100 \\
\hline $\operatorname{Arg}(\mathrm{m})$ & 105 \\
\hline Aurora-A(h) & 106 \\
\hline $\operatorname{Ax}(\mathrm{h})$ & 119 \\
\hline $\mathrm{Blk}(\mathrm{m})$ & 105 \\
\hline Bmxx(h) & 104 \\
\hline CaMKI(r) & 95 \\
\hline CaMKIV(h) & 106 \\
\hline $\mathrm{CDK} 1 /$ cyclinB(h) & 101 \\
\hline $\mathrm{CDK} 2 / \mathrm{cyclin} \mathrm{A}(\mathrm{h})$ & 102 \\
\hline $\mathrm{CDK} 2 /$ (yclinE(h) & 93 \\
\hline CDK3/cyclinE(h) & 101 \\
\hline CDK5/p35(h) & 106 \\
\hline CDK6/cyclinD3(h) & 95 \\
\hline CDK $7 /$ yclinH/MATl(h) & 100 \\
\hline СHK1(h) & 115 \\
\hline СHK2(h) & 98 \\
\hline $\mathrm{CK} 1(\mathrm{y})$ & 97 \\
\hline CK2(h) & 109 \\
\hline $\mathrm{c} \cdot \mathrm{RAF}(\mathrm{h})$ & 98 \\
\hline $\operatorname{CSK}(\mathrm{l})$ & 76 \\
\hline $\operatorname{csRC(h)}$ & 103 \\
\hline Fes(h) & 108 \\
\hline FGFR3(h) & 109 \\
\hline Fll3(h) & 105 \\
\hline Fyn(h) & 94 \\
\hline GSK3a(h) & 91 \\
\hline GSK3B(h) & 75 \\
\hline IGF-1R(h) & 124 \\
\hline IKKa(h) & 99 \\
\hline IKKB(h) & 104 \\
\hline $\mathbb{R}(\mathrm{h})$ & 93 \\
\hline JNKIal(h) & 101 \\
\hline JNK2a2(h) & 92 \\
\hline JNK3(r) & 87 \\
\hline Lck(h) & 119 \\
\hline Lynn(h) & 96 \\
\hline Lyn(m) & 91 \\
\hline MAPKI(h) & 98 \\
\hline MAPK2(h) & 120 \\
\hline MAPK2(m) & 123 \\
\hline MAPKAP-K2(h) & 110 \\
\hline MEK1(h) & 28 \\
\hline MKK4(m) & 116 \\
\hline MKKG(h) & 104 \\
\hline MKKᄀ७(h) & 108 \\
\hline MSK1(h) & 104 \\
\hline $\mathrm{p} 70 \mathrm{~s} 6 \mathrm{~K}(\mathrm{~h})$ & 101 \\
\hline PAK2(h) & 107 \\
\hline PDGFRa(h) & 96 \\
\hline PDGFRG(h) & 107 \\
\hline PDK1(h) & 97 \\
\hline PKA(b) & 117 \\
\hline PKA(h) & 92 \\
\hline PKBa (h) & 97 \\
\hline PKBB(h) & 132 \\
\hline $\mathrm{PKB}_{\gamma}(\mathrm{h})$ & 87 \\
\hline PKCa(h) & 94 \\
\hline РКСBI(h) & 110 \\
\hline PKGy/(h) & 91 \\
\hline PKCळ(h) & 96 \\
\hline $\mathrm{PKG}(\mathrm{h})$ & 100 \\
\hline PKG (h) & 91 \\
\hline $\begin{array}{l}\text { PKC (h) } \\
\text { (h) }\end{array}$ & 101 \\
\hline PKC $\mu(\mathrm{h})$ & 96 \\
\hline PKCO(h) & 97 \\
\hline PKD2(h) & 102 \\
\hline PRAK(h) & 112 \\
\hline PRK2(h) & 92 \\
\hline ROCK-II(h) & 98 \\
\hline ROCK-II(r) & 83 \\
\hline Rsk1(h) & 111 \\
\hline Rskl(r) & 67 \\
\hline Rsk2(h) & 93 \\
\hline Rsk3(h) & 99 \\
\hline SAPK2a(h) & 105 \\
\hline SAPK2b(h) & 100 \\
\hline $\mathrm{SAPK} 3(\mathrm{~h})$ & 112 \\
\hline $\mathrm{SAPK} 4(\mathrm{~h})$ & 112 \\
\hline SGK(h) & 104 \\
\hline Syk(h) & 105 \\
\hline TrkB(h) & 111 \\
\hline Yes(h) & 109 \\
\hline ZAP-70(h) & 106 \\
\hline
\end{tabular}

\begin{tabular}{|c|c|c|c|c|c|c|c|}
\hline sorted & normalized & position & cumulative sample fraction & inhibition\% & fraction of total inhibition & cumulative inhibition & area \\
\hline 132 & 100 & 1 & 0.011764706 & 0 & 0 & 0 & 0 \\
\hline 124 & 100 & 2 & 0.023529412 & 0 & 0 & 0 & 0 \\
\hline 123 & 100 & 3 & 0.035294118 & 0 & 0 & 0 & 0 \\
\hline 120 & 100 & 4 & 0.047058824 & 0 & 0 & 0 & 0 \\
\hline 119 & 100 & 5 & 0.058823529 & 0 & 0 & 0 & 0 \\
\hline 119 & 100 & 6 & 0.070588235 & 0 & 0 & 0 & 0 \\
\hline 117 & 100 & 7 & 0.082352941 & 0 & 0 & 0 & 0 \\
\hline 116 & 100 & 8 & 0.094117647 & 0 & 0 & 0 & 0 \\
\hline 115 & 100 & 9 & 0.105882353 & 0 & 0 & 0 & 0 \\
\hline 112 & 100 & 10 & 0.117647059 & 0 & 0 & 0 & 0 \\
\hline 112 & 100 & 11 & 0.129411765 & 0 & 0 & 0 & 0 \\
\hline 112 & 100 & 12 & 0.141176471 & 0 & 0 & 0 & 0 \\
\hline 111 & 100 & 13 & 0.152941176 & 0 & 0 & 0 & 0 \\
\hline 111 & 100 & 14 & 0.164705882 & 0 & 0 & 0 & 0 \\
\hline 110 & 100 & 15 & 0.176470588 & 0 & 0 & 0 & 0 \\
\hline 110 & 100 & 16 & 0.188235294 & 0 & 0 & 0 & 0 \\
\hline 109 & 100 & 17 & 0.2 & 0 & 0 & 0 & 0 \\
\hline 109 & 100 & 18 & 0.211764706 & 0 & 0 & 0 & 0 \\
\hline 109 & 100 & 19 & 0.223529412 & 0 & 0 & 0 & 0 \\
\hline 108 & 100 & 20 & 0.235294118 & 0 & 0 & 0 & 0 \\
\hline 108 & 100 & 21 & 0.247058824 & 0 & 0 & 0 & 0 \\
\hline 107 & 100 & 22 & 0.258823529 & 0 & 0 & 0 & 0 \\
\hline 107 & 100 & 23 & 0.270588235 & 0 & 0 & 0 & 0 \\
\hline 106 & 100 & 24 & 0.282352941 & 0 & 0 & 0 & 0 \\
\hline 106 & 100 & 25 & 0.294117647 & 0 & 0 & 0 & 0 \\
\hline 106 & 100 & 26 & 0.305882353 & 0 & 0 & 0 & 0 \\
\hline 106 & 100 & 27 & 0.317647059 & 0 & 0 & 0 & 0 \\
\hline 105 & 100 & 28 & 0.329411765 & 0 & 0 & 0 & 0 \\
\hline 105 & 100 & 29 & 0.341176471 & 0 & 0 & 0 & 0 \\
\hline 105 & 100 & 30 & 0.352941176 & 0 & 0 & 0 & 0 \\
\hline 105 & 100 & 31 & 0.364705882 & 0 & 0 & 0 & 0 \\
\hline 105 & 100 & 32 & 0.376470588 & 0 & 0 & 0 & 0 \\
\hline 104 & 100 & 33 & 0.388235294 & 0 & 0 & 0 & 0 \\
\hline 104 & 100 & 34 & 0.4 & 0 & 0 & 0 & 0 \\
\hline 104 & 100 & 35 & 0.411764706 & 0 & 0 & 0 & 0 \\
\hline 104 & 100 & 36 & 0.423529412 & 0 & 0 & 0 & 0 \\
\hline 104 & 100 & 37 & 0.435294118 & 0 & 0 & 0 & 0 \\
\hline 103 & 100 & 38 & 0.447058824 & 0 & 0 & 0 & 0 \\
\hline 102 & 100 & 39 & 0.458823529 & 0 & 0 & 0 & 0 \\
\hline 102 & 100 & 40 & 0.470588235 & 0 & 0 & 0 & 0 \\
\hline 101 & 100 & 41 & 0.482352941 & 0 & 0 & 0 & 0 \\
\hline 101 & 100 & 42 & 0.494117647 & 0 & 0 & 0 & 0 \\
\hline 101 & 100 & 43 & 0.505882353 & 0 & 0 & 0 & 0 \\
\hline 101 & 100 & 44 & 0.517647059 & 0 & 0 & 0 & 0 \\
\hline 101 & 100 & 45 & 0.529411765 & 0 & 0 & 0 & 0 \\
\hline 100 & 100 & 46 & 0.541176471 & 0 & 0 & 0 & 0 \\
\hline 100 & 100 & 47 & 0.552941176 & 0 & 0 & 0 & 0 \\
\hline 100 & 100 & 48 & 0.564705882 & 0 & 0 & 0 & 0 \\
\hline 100 & 100 & 49 & 0.576470588 & 0 & 0 & 0 & 0 \\
\hline 99 & 99 & 50 & 0.588235294 & 1 & 0.002949853 & 0.002949853 & $1.73521 \mathrm{E}-05$ \\
\hline 99 & 99 & 51 & 0.6 & 1 & 0.002949853 & 0.005899705 & $5.20562 \mathrm{E}-05$ \\
\hline 99 & 99 & 52 & 0.611764706 & 1 & 0.002949853 & 0.008849558 & $8.67604 \mathrm{E}-05$ \\
\hline 98 & 98 & 53 & 0.623529412 & 2 & 0.005899705 & 0.014749263 & 0.000138817 \\
\hline 98 & 98 & 54 & 0.635294118 & 2 & 0.005899705 & 0.020648968 & 0.000208225 \\
\hline 98 & 98 & 55 & 0.647058824 & 2 & 0.005899705 & 0.026548673 & 0.000277633 \\
\hline 98 & 98 & 56 & 0.658823529 & 2 & 0.005899705 & 0.032448378 & 0.000347041 \\
\hline 97 & 97 & 57 & 0.670588235 & 3 & 0.008849558 & 0.041297935 & 0.000433802 \\
\hline 97 & 97 & 58 & 0.682352941 & 3 & 0.008849558 & 0.050147493 & 0.000537914 \\
\hline 97 & 97 & 59 & 0.694117647 & 3 & 0.008849558 & 0.05899705 & 0.000642027 \\
\hline 97 & 97 & 60 & 0.705882353 & 3 & 0.008849558 & 0.067846608 & 0.000746139 \\
\hline 96 & 96 & 61 & 0.717647059 & 4 & 0.01179941 & 0.079646018 & 0.000867604 \\
\hline 96 & 96 & 62 & 0.729411765 & 4 & 0.01179941 & 0.091445428 & 0.00100642 \\
\hline 96 & 96 & 63 & 0.741176471 & 4 & 0.01179941 & 0.103244838 & 0.001145237 \\
\hline 96 & 96 & 64 & 0.752941176 & 4 & 0.01179941 & 0.115044248 & 0.001284053 \\
\hline 95 & 95 & 65 & 0.764705882 & 5 & 0.014749263 & 0.12979351 & 0.001440222 \\
\hline 95 & 95 & 66 & 0.776470588 & 5 & 0.014749263 & 0.144542773 & 0.001613743 \\
\hline 94 & 94 & 67 & 0.788235294 & 6 & 0.017699115 & 0.162241888 & 0.001804616 \\
\hline 94 & 94 & 68 & 0.8 & 6 & 0.017699115 & 0.179941003 & 0.002012841 \\
\hline 93 & 93 & 69 & 0.811764706 & 7 & 0.020648968 & 0.200589971 & 0.002238417 \\
\hline 93 & 93 & 70 & 0.823529412 & 7 & 0.020648968 & 0.221238938 & 0.002481347 \\
\hline 93 & 93 & 71 & 0.835294118 & 7 & 0.020648968 & 0.241887906 & 0.002724276 \\
\hline 92 & 92 & 72 & 0.847058824 & 8 & 0.02359882 & 0.265486726 & 0.002984557 \\
\hline 92 & 92 & 73 & 0.858823529 & 8 & 0.02359882 & 0.289085546 & 0.00326219 \\
\hline 92 & 92 & 74 & 0.870588235 & 8 & 0.02359882 & 0.312684366 & 0.003539823 \\
\hline 91 & 91 & 75 & 0.882352941 & 9 & 0.026548673 & 0.339233038 & 0.003834808 \\
\hline 91 & 91 & 76 & 0.894117647 & 9 & 0.026548673 & 0.365781711 & 0.004147146 \\
\hline 91 & 91 & 77 & 0.905882353 & 9 & 0.026548673 & 0.392330383 & 0.004459483 \\
\hline 91 & 91 & 78 & 0.917647059 & 9 & 0.026548673 & 0.418879056 & 0.00477182 \\
\hline 87 & 87 & 79 & 0.929411765 & 13 & 0.038348083 & 0.457227139 & 0.005153566 \\
\hline 87 & 87 & 80 & 0.941176471 & 13 & 0.038348083 & 0.495575221 & 0.00560472 \\
\hline 83 & 83 & 81 & 0.952941176 & 17 & 0.050147493 & 0.545722714 & 0.006125282 \\
\hline 76 & 76 & 82 & 0.964705882 & 24 & 0.07079646 & 0.616519174 & 0.006836717 \\
\hline 75 & 75 & 83 & 0.976470588 & 25 & 0.073746313 & 0.690265487 & 0.007686969 \\
\hline \multirow{4}{*}{$\begin{array}{l}67 \\
28\end{array}$} & 67 & 84 & 0.988235294 & 33 & 0.097345133 & 0.787610619 & 0.008693389 \\
\hline & 28 & 85 & 1 & 72 & 0.212389381 & 1 & 0.010515357 \\
\hline & & & Total inhibition & 339 & & Total area & 0.099722367 \\
\hline & & & & & & Gini & 0.800555266 \\
\hline
\end{tabular}




\section{Effect of concentration on the Gini coefficient}

Gini coefficient values for $S=1, a=10$ and $100 \mathrm{nM}, \mathrm{b}=0.5,0.8,1.0,1.2$ and 1.5 at concentrations ranging from $0.0001 \mathrm{nM}$ to $10000 \mathrm{nM}$ with no limitation on experimental accuracy

Example of calculations for $S=1, a=10 n M, b=1.2$ at concentration of $100 \mathrm{nM}$ with no limitation on experimental accuracy

Gini coefficient values for $S=1, a=10$ and $100 \mathrm{nM}, b=0.5,0.8,1.0,1.2$ and 1.5 at concentrations ranging from $0.0001 \mathrm{nM}$ to $10000 \mathrm{nM}$ for experimental accuracy $\mathrm{r}=0,1$ and $2 \%$

Example of calculations for $S=1, a=10 n M, b=1.2$ at concentration of $100 \mathrm{nM}$ with experimental accuracy $r=2 \%$ 
Gini coefficient values for $S=1, a=10$ and $100 \mathrm{nM}, \mathrm{b}=0.5,0.8,1.0,1.2$ and 1.5 at concentrations ranging from $0.0001 \mathrm{nM}$ to $10000 \mathrm{nM}$ with no limitation on experimental accuracy

\begin{tabular}{|c|c|c|c|c|c|c|}
\hline Assumptions: & $S=1$ & $a=10$ & & & & \\
\hline Concentration [nM] & $b=0.5$ & $b=0.8$ & $b=1$ & $b=1.2$ & $b=1.5$ & max inhibition [\%] \\
\hline 0.0001 & 0.312531 & 0.554638 & 0.709025 & 0.830271 & 0.934514 & 0.00 \\
\hline 0.001 & 0.312525 & 0.554632 & 0.709019 & 0.830266 & 0.934511 & 0.01 \\
\hline 0.01 & 0.31247 & 0.55457 & 0.708959 & 0.830217 & 0.934484 & 0.10 \\
\hline 0.1 & 0.31192 & 0.553955 & 0.708363 & 0.82973 & 0.934219 & 0.99 \\
\hline 1 & 0.306634 & 0.548089 & 0.702686 & 0.825083 & 0.931672 & 9.09 \\
\hline $\begin{array}{r}10 \\
100\end{array}$ & $\begin{array}{l}0.267342 \\
0.138255\end{array}$ & $\begin{array}{l}0.506296 \\
0.363657\end{array}$ & $\begin{array}{l}0.662743 \\
0.530114\end{array}$ & $\begin{array}{l}0.791999 \\
0.680595\end{array}$ & $\begin{array}{l}0.912795 \\
0.843794\end{array}$ & $\begin{array}{l}50.00 \\
90.91\end{array}$ \\
\hline 1000 & 0.026779 & 0.139289 & 0.287397 & 0.460633 & 0.690917 & 99.01 \\
\hline 10000 & 0.002988 & 0.021874 & 0.069336 & 0.175456 & 0.424487 & 99.90 \\
\hline IC50 & 0.312531 & 0.554639 & 0.709026 & 0.830272 & 0.934514 & \\
\hline
\end{tabular}
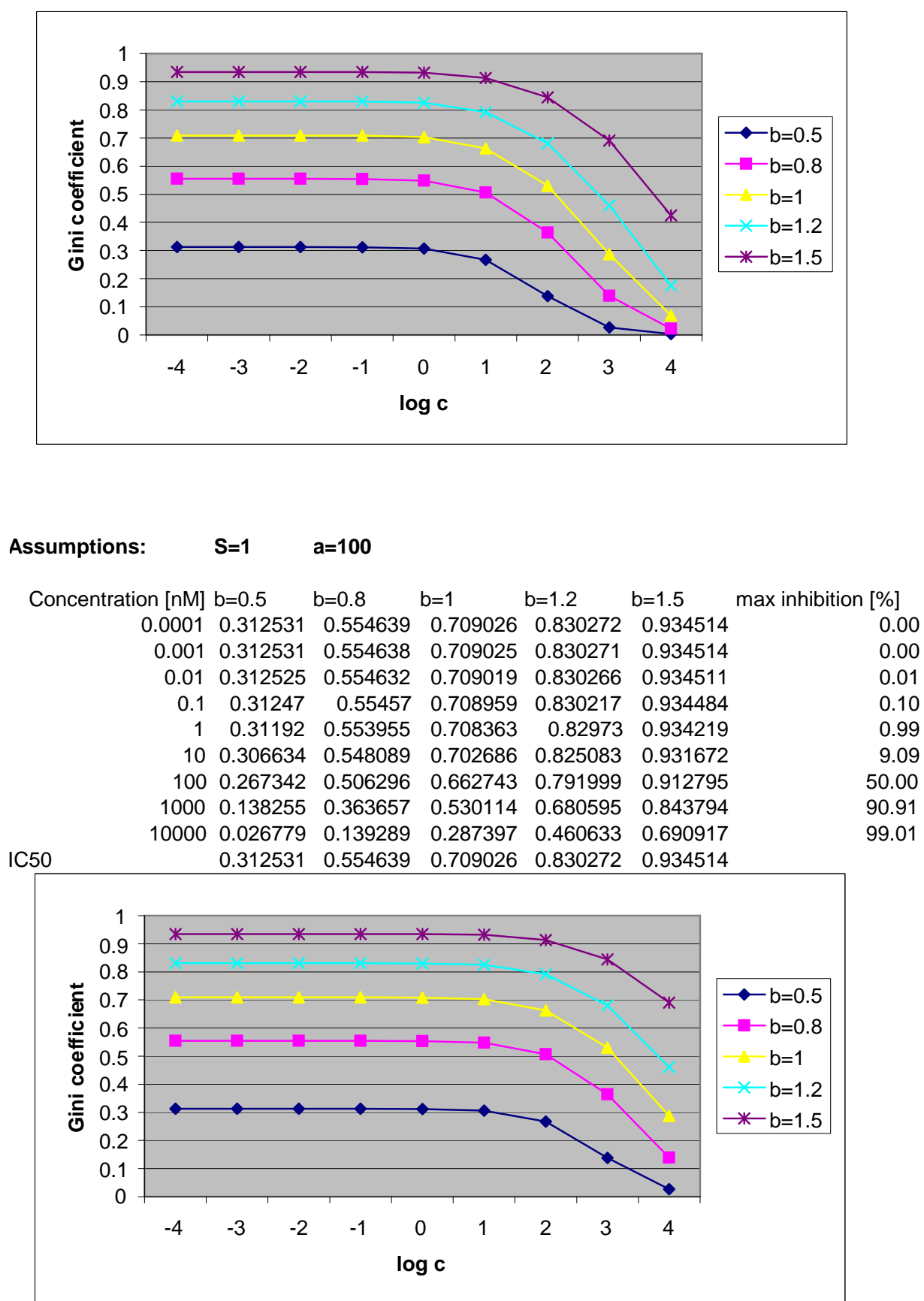


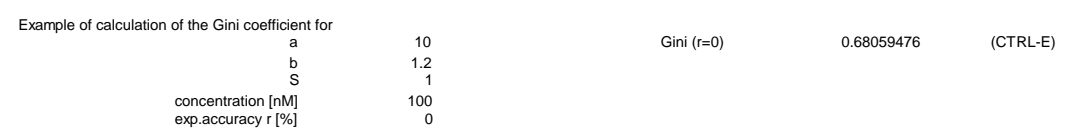

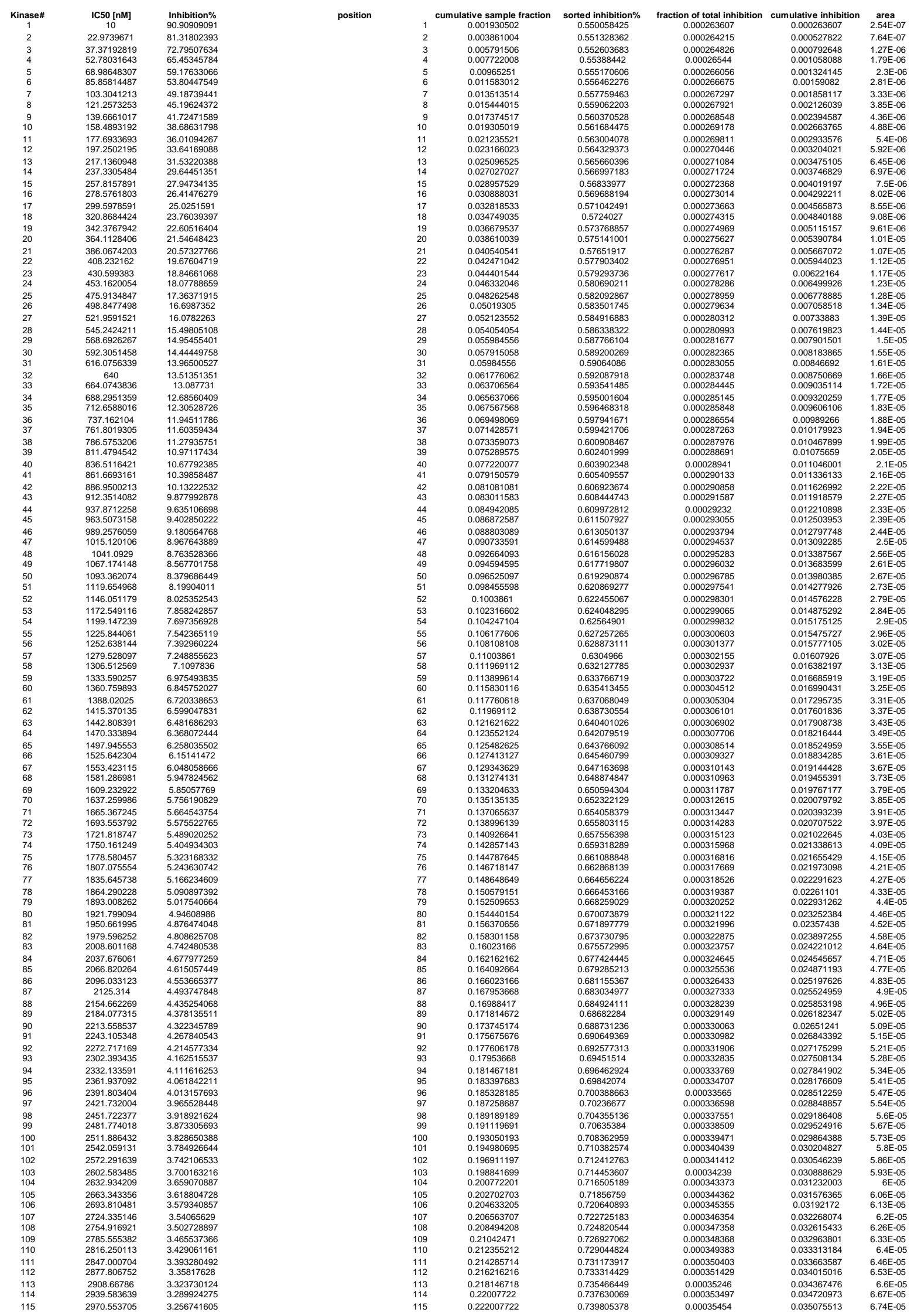




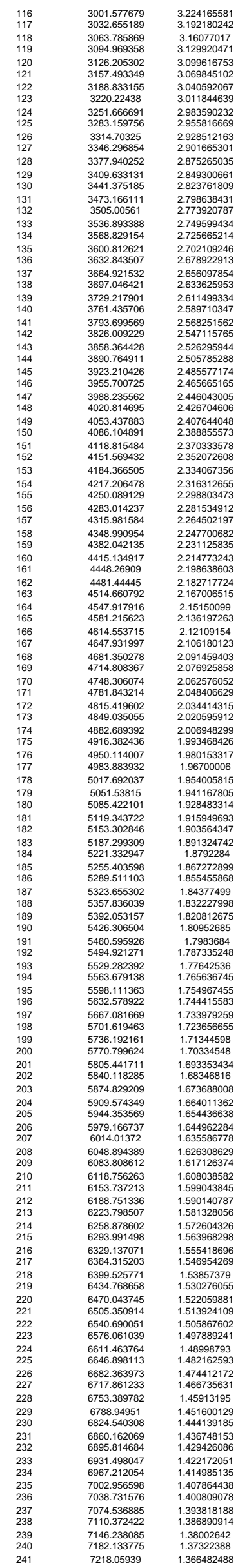

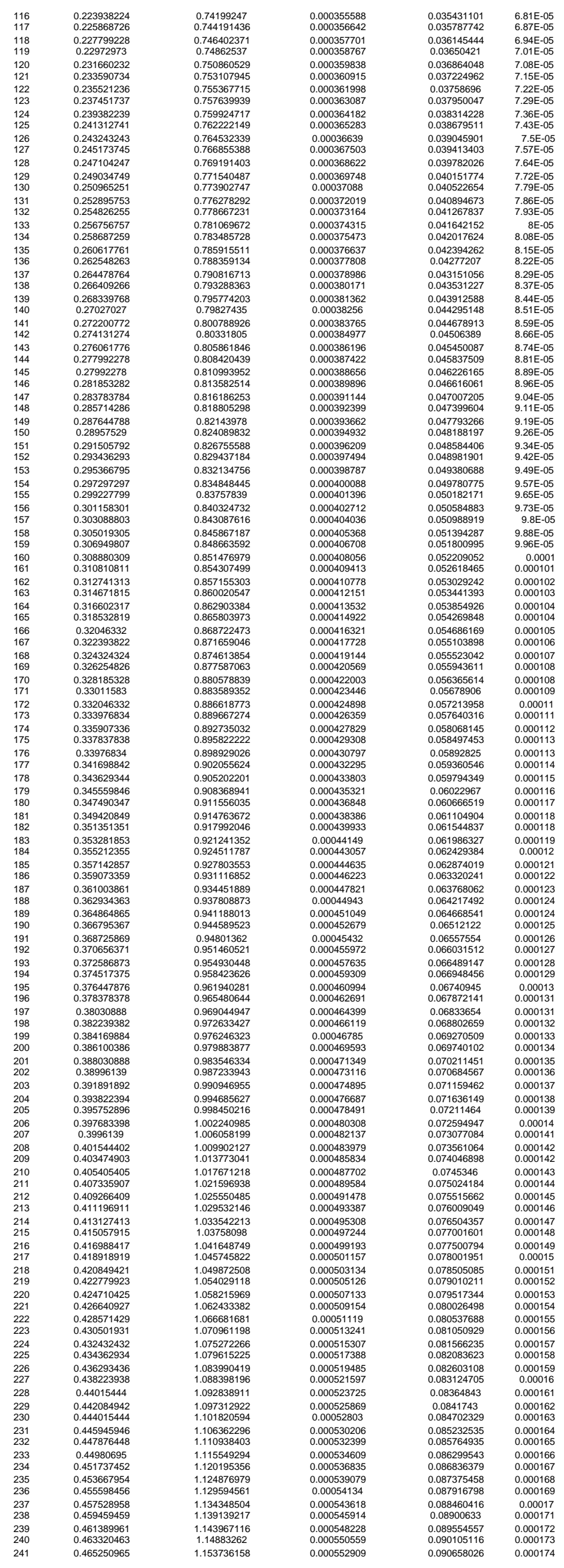




\begin{tabular}{|c|c|c|}
\hline${ }_{243}^{242}$ & $\begin{array}{c}7254.014831 \\
7290\end{array}$ & $\begin{array}{l}1.3598014446 \\
1.353179973\end{array}$ \\
\hline $\begin{array}{l}244 \\
245 \\
245\end{array}$ & $\begin{array}{r}7326.014799 \\
7 \\
762505913\end{array}$ & $\begin{array}{r}1.3466173 \\
\text {.1.340117267 }\end{array}$ \\
\hline 24 & $\begin{array}{l}7398.1328997 \\
7\end{array}$ & $\begin{array}{l}1.1333665545 \\
1.327274\end{array}$ \\
\hline & $\begin{array}{l}74434.263004 \\
7470.368357\end{array}$ & $\begin{array}{r}1.3272727599 \\
1.320939686\end{array}$ \\
\hline & $\begin{array}{r}75560.528966 \\
7542.720421\end{array}$ & $\begin{array}{l}1.3146559928 \\
1.308434621\end{array}$ \\
\hline & & $\begin{array}{r}1.308434621 \\
1.302263082\end{array}$ \\
\hline & $\begin{array}{r}7615.188342 \\
765514651\end{array}$ & $\begin{array}{l}1.2966144638 \\
1.29007682\end{array}$ \\
\hline & 76887.7713858 & $\begin{array}{r}.299408628 \\
1.284064401\end{array}$ \\
\hline & $\begin{array}{l}7724.105848 \\
77664682\end{array}$ & $\begin{array}{l}1.278101319 \\
1.27218875\end{array}$ \\
\hline & 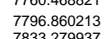 & 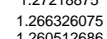 \\
\hline & $\begin{array}{l}78333.279937 \\
7869.727904\end{array}$ & $\begin{array}{r}1.2650512686 \\
1.25474798\end{array}$ \\
\hline 260 & 7906.204028 & $\begin{array}{l}1.249031372 \\
1.25\end{array}$ \\
\hline $\begin{array}{l}261 \\
262\end{array}$ & $\begin{array}{l}79442.70822 \\
7997.240397\end{array}$ & $\begin{array}{l}1.243362276 \\
1.237740123\end{array}$ \\
\hline & $\begin{array}{l}8015.80007 \\
805238837\end{array}$ & $\begin{array}{l}1.232164349 \\
1.2663434\end{array}$ \\
\hline & $\begin{array}{l}8089.003973 \\
80305\end{array}$ & $\begin{array}{l}1.221149731 \\
\end{array}$ \\
\hline & 8125.647233 & 1.215709806 \\
\hline & $\begin{array}{l}81262.318056 \\
8199.016357\end{array}$ & $\begin{array}{l}.1 .1203144095 \\
1.204962079\end{array}$ \\
\hline & $\begin{array}{l}8235.742056 \\
827249507\end{array}$ & $\begin{array}{l}1.199653244 \\
1.19438703\end{array}$ \\
\hline & & $\begin{array}{l}1.1994387087 \\
1.149163111\end{array}$ \\
\hline & $\begin{array}{r}8399.275318 \\
8346.082721\end{array}$ & $\begin{array}{l}\begin{array}{l}1.1889163111 \\
1.183980826\end{array}\end{array}$ \\
\hline & $\begin{array}{r}8382.917198 \\
841977867\end{array}$ & $\begin{array}{l}1.178839751 \\
1.17337941\end{array}$ \\
\hline & 845.667058 & $\begin{array}{l}1.168679339 \\
\end{array}$ \\
\hline & 8493.582284 & 1.163659074 \\
\hline & $\begin{array}{r}8530.52426269 \\
8567.429298\end{array}$ & $\begin{array}{l}1.1158678162 \\
1.153736158\end{array}$ \\
\hline & $\begin{array}{l}8604.488212 \\
8\end{array}$ & $\begin{array}{l}1.14883262 \\
\end{array}$ \\
\hline & $\begin{array}{l}8841.5150015 \\
8668.585272\end{array}$ & $\begin{array}{l}1.143967116 \\
1.13913921\end{array}$ \\
\hline & $\begin{array}{l}8715.632908 \\
875273894\end{array}$ & $\begin{array}{l}1.1343448504 \\
.192504\end{array}$ \\
\hline & $\begin{array}{l}87252.733847 \\
8789.86101\end{array}$ & $\begin{array}{l}1.1259995461 \\
1.124876979\end{array}$ \\
\hline & 8827.014338 & 1.120195356 \\
\hline & $\begin{array}{l}8864.199742 \\
8901.399156\end{array}$ & $\begin{array}{l}1.11155499294 \\
1.110938403\end{array}$ \\
\hline & $\begin{array}{r}8938.630506 \\
897588772\end{array}$ & $\begin{array}{l}1.106362296 \\
1.101820596\end{array}$ \\
\hline & $\begin{array}{r}8957.88772 \\
9013.170726\end{array}$ & $\begin{array}{l}1.1018182505942 \\
1.097312922\end{array}$ \\
\hline & 9050.479454 & $\begin{array}{r}1.0993712922 \\
1.092838911\end{array}$ \\
\hline & $\begin{array}{l}9087.813832 \\
91251717792\end{array}$ & 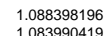 \\
\hline & 9162.55926 & \\
\hline & 9199.97017 & 1.075272266 \\
\hline & $\begin{array}{r}9237.406452 \\
9274.868037\end{array}$ & $\begin{array}{l}.0709661198 \\
1.0666816161\end{array}$ \\
\hline & $\begin{array}{r}9312.354858 \\
\text { 934486684 }\end{array}$ & $\begin{array}{l}1.0624333882 \\
1058 m\end{array}$ \\
\hline & $\begin{array}{l}93498.866846 \\
9387.403934\end{array}$ & $\begin{array}{l}1.055821195699 \\
1.05402918\end{array}$ \\
\hline & $\begin{array}{l}9424.966055 \\
9\end{array}$ & $\begin{array}{r}1.049872508 \\
1\end{array}$ \\
\hline & $\begin{array}{r}9462.5553142 \\
9500.165131\end{array}$ & $\begin{array}{l}1.0457445822 \\
1.041648749\end{array}$ \\
\hline & 9537.801953 & $\begin{array}{l}0.03758098 \\
\end{array}$ \\
\hline $\begin{array}{l}30 \\
30\end{array}$ & $\begin{array}{l}9575.463545 \\
9613.149845\end{array}$ & $\begin{array}{l}1.033342213 \\
1.029532146\end{array}$ \\
\hline & 9650.860778 & $\begin{array}{l}1.0250500485 \\
1.02550\end{array}$ \\
\hline & 9688.596289 & 1.021596938 \\
\hline & $\begin{array}{l}9726.356312 \\
9764.140783\end{array}$ & $\begin{array}{l}.1 .017671218 \\
1.0177773041\end{array}$ \\
\hline & 9801.949639 & 1.009902127 \\
\hline & 9839.782817 & $\begin{array}{r}1.006058199 \\
1.00224909\end{array}$ \\
\hline & $\begin{array}{r}9877.640255 \\
9915.521891\end{array}$ & $\begin{array}{r}.00022409855 \\
0.998450216\end{array}$ \\
\hline & 9953.427663 & $\begin{array}{l}0.9944685627 \\
\end{array}$ \\
\hline & $\begin{array}{r}9991.35751 \\
100293111\end{array}$ & 0.9909469555 \\
\hline $\begin{array}{l}\begin{array}{l}317 \\
318\end{array} \\
3\end{array}$ & $\begin{array}{l}10029.31137 \\
10067.28918\end{array}$ & $\begin{array}{l}0.9887233943 \\
0.98354634\end{array}$ \\
\hline & $\begin{array}{l}10105.29089 \\
10143.31643\end{array}$ & $\begin{array}{l}0.979883877 \\
0.976243623\end{array}$ \\
\hline & 10181.36575 & 0.972633427 \\
\hline & $\begin{array}{l}10219.43877 \\
1057554\end{array}$ & 0.969044947 \\
\hline & $\begin{array}{l}102527.555446 \\
10295.65573\end{array}$ & $\begin{array}{l}0.9655860644 \\
0.96194028\end{array}$ \\
\hline $\begin{array}{l}325 \\
326\end{array}$ & 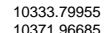 & $\begin{array}{l}0.958423626 \\
0.95493044\end{array}$ \\
\hline & $\begin{array}{l}1037.1 .96685 \\
10410.15757\end{array}$ & \\
\hline & 10448.37166 & $\begin{array}{l}.0394801361 \\
0.9480162\end{array}$ \\
\hline & 10486.60905 & 0.944589523 \\
\hline & $\begin{array}{l}105224.8697 \\
10563.15354\end{array}$ & $\begin{array}{l}0.941188013 \\
0.937808873\end{array}$ \\
\hline & 10601.46052 & 0.934451889 \\
\hline & & 0.931116852 \\
\hline & $\begin{array}{l}10678.14368 \\
1071651974\end{array}$ & $\begin{array}{l}0.9287803353 \\
0.92451178\end{array}$ \\
\hline & 10754.91873 & 0.921241352 \\
\hline & 10793.34057 & 0.917992046 \\
\hline & $\begin{array}{l}10831178523 \\
18877025264\end{array}$ & $\begin{array}{l}0.914763672 \\
0.911556035\end{array}$ \\
\hline $\begin{array}{l}344 \\
34 \\
34\end{array}$ & $\begin{array}{l}10908.74275 \\
10947.25555\end{array}$ & $\begin{array}{l}0.908368941 \\
0.90520220\end{array}$ \\
\hline & $\begin{array}{l}10985.79087 \\
\end{array}$ & 0.902055624 \\
\hline & & 0.898929026 \\
\hline & $\begin{array}{l}11062.929215 \\
11101.133197\end{array}$ & $\begin{array}{l}0.8958222222 \\
0.892735032\end{array}$ \\
\hline & $\begin{array}{l}11140.15718 \\
118\end{array}$ & $\begin{array}{l}0.8996067274 \\
0.8896\end{array}$ \\
\hline & $\begin{array}{l}11178.80472 \\
1 \\
17174445\end{array}$ & $\begin{array}{l}0.8866187773 \\
0.883589352\end{array}$ \\
\hline & $\begin{array}{l}11217.4455 \\
11256.1666\end{array}$ & $\begin{array}{l}0.8835839352 \\
0.88057889\end{array}$ \\
\hline & $\begin{array}{l}11294.88083 \\
113336711\end{array}$ & 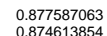 \\
\hline & 11372.37563 & 0.871659046 \\
\hline & 114111.1561 & 0.868722473 \\
\hline & 11449.95855 & 0.865803973 \\
\hline & $\begin{array}{l}14888878292 \\
1152782918\end{array}$ & $\begin{array}{l}0.8629003384 \\
0.860020547\end{array}$ \\
\hline & $\begin{array}{l}11566.49726 \\
1\end{array}$ & 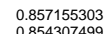 \\
\hline & $\begin{array}{l}\begin{array}{l}116055.38713 \\
11644.29872\end{array}\end{array}$ & $\begin{array}{l}0.85340074999 \\
0.851476979\end{array}$ \\
\hline & 11683.232 & 0.848663592 \\
\hline & $\begin{array}{l}11722.18692 \\
11161.113444\end{array}$ & $\begin{array}{l}0.8458677187 \\
0.84308761\end{array}$ \\
\hline & 11800.16147 & 0.840324732 \\
\hline & $\begin{array}{r}11839.181 \\
11878201\end{array}$ & $\begin{array}{l}0.83757839 \\
0.83484445\end{array}$ \\
\hline & 11917.28437 & 0.832134756 \\
\hline & 11956.3681 & 0.829437184 \\
\hline
\end{tabular}

\begin{tabular}{|c|c|c|c|c|c|}
\hline 2425 & 0.467181467 & 1.158678162 & 0.000555278 & 0.091213303 & 0.000176 \\
\hline $\begin{array}{l}243 \\
244\end{array}$ & $\begin{array}{l}0.469111969 \\
0.47104241\end{array}$ & $\begin{array}{l}1.163659074 \\
1.1188679399\end{array}$ & $\begin{array}{l}0.000557665 \\
0.000560077\end{array}$ & $\begin{array}{l}0.091770968 \\
0.092331039\end{array}$ & $\begin{array}{l}0.000177 \\
0.000178\end{array}$ \\
\hline 45 & $\begin{array}{l}0.471042471729297 \\
0.4729\end{array}$ & $\begin{array}{l}1.1686693399 \\
1.173739411\end{array}$ & $\begin{array}{l}0.0000562460 \\
0.0056\end{array}$ & $\begin{array}{l}0.0923289353939 \\
0.0925\end{array}$ & $\begin{array}{l}0.00001 / 8 \\
0.000179\end{array}$ \\
\hline 246 & $\begin{array}{l}0.4749034745 \\
0.476833977\end{array}$ & $\begin{array}{l}1.178839751 \\
1.1183980026\end{array}$ & $\begin{array}{l}0.000564944 \\
0.000567404\end{array}$ & $\begin{array}{l}0.0993458474 \\
0.099402887\end{array}$ & $\begin{array}{l}0.00018 \\
0.000181\end{array}$ \\
\hline $\begin{array}{l}248 \\
249\end{array}$ & $\begin{array}{l}0.4687358717 \\
0.47876479 \\
0.480699891\end{array}$ & 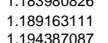 & 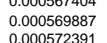 & $\begin{array}{l}0.09902587 / 74 \\
0.094595764 \\
0.095168155\end{array}$ & $\begin{array}{l}0.000182 \\
0.000182 \\
0.000183\end{array}$ \\
\hline & 0.482625483 & 1.1.199653244 & 0.000574914 & 0.095743069 & 0.000184 \\
\hline & 0.4845559855 & 1.204962079 & 0.000577458 & 0.096320528 & 0.000185 \\
\hline 25 & $\begin{array}{l}0.48648684666 \\
0.4884698\end{array}$ & $\begin{array}{l}1.21033140955 \\
1.215709806\end{array}$ & $\begin{array}{l}0.00050580023 \\
0.000582609\end{array}$ & $\begin{array}{l}0.0996900551 \\
0.099748316\end{array}$ & $\begin{array}{l}0.000187 \\
0.000188\end{array}$ \\
\hline 25 & 0.49034749 & 1.221149731 & 0.000585211 & 0.098068376 & 0.000189 \\
\hline 256 & $\begin{array}{l}0.4992779929 \\
0.4942089494\end{array}$ & $\begin{array}{r}1.22663444 \\
1.232643449\end{array}$ & $\begin{array}{l}0.00050587844 \\
0.000590495\end{array}$ & $\begin{array}{l}0.0998656221 \\
0.099246716\end{array}$ & $\begin{array}{l}0.00019 \\
0.000191\end{array}$ \\
\hline 257 & $\begin{array}{l}0.496138996 \\
.09089649\end{array}$ & $\begin{array}{l}1.237740123 \\
.22332527\end{array}$ & $\begin{array}{l}0.00059316767 \\
0.0059586\end{array}$ & 0.0998398822 & 0.000192 \\
\hline 259 & 0.40 .5 & $\begin{array}{l}1.2433622276 \\
1.249031372\end{array}$ & $\begin{array}{l}0.0005595861 \\
0.00059878\end{array}$ & $\begin{array}{l}0.100435744 \\
0.101034321\end{array}$ & $\begin{array}{l}0.0000193 \\
0.000194\end{array}$ \\
\hline & 0.501930502 & $\begin{array}{l}1.254747981 \\
1.20551280\end{array}$ & 0.000601318 & 0.1016356399 & 0.000196 \\
\hline & $\begin{array}{l}0.5038616004 \\
0.505791506\end{array}$ & $\begin{array}{l}1.2605512686 \\
1.266326075\end{array}$ & $\begin{array}{l}0.0000600088 \\
0.000606866\end{array}$ & $\begin{array}{l}0.1022337919 \\
0.102846585\end{array}$ & $\begin{array}{l}0.000197 \\
0.000198\end{array}$ \\
\hline & $\begin{array}{l}0.50077220088 \\
0.50965251\end{array}$ & $\begin{array}{l}1.27218875 \\
1.2781011919\end{array}$ & $\begin{array}{l}0.0000009676 \\
0.0006612509\end{array}$ & $\begin{array}{l}0.103456261 \\
0.10406877\end{array}$ & $\begin{array}{r}0.000199 \\
0.00002\end{array}$ \\
\hline & $\begin{array}{l}0.511583012 \\
0.512512514\end{array}$ & $\begin{array}{l}1.284064401 \\
1.20007902\end{array}$ & 0.0000615367 & $\begin{array}{l}0.104684137 \\
0.1530239\end{array}$ & 0.000201 \\
\hline & & $\begin{array}{l}1.2900078628 \\
1.2064144638\end{array}$ & $\begin{array}{l}0.00066182494 \\
0.00062115\end{array}$ & $\begin{array}{l}0.1053023866 \\
0.159923543\end{array}$ & $\begin{array}{l}0.000203 \\
0.000204\end{array}$ \\
\hline & $\begin{array}{l}0.5514444015 \\
0.517374517\end{array}$ & $\begin{array}{l}1.26914446388 \\
1.302263082\end{array}$ & $\begin{array}{l}0.0006211156 \\
0.000624088\end{array}$ & $\begin{array}{l}0.1059235343 \\
0.106547631\end{array}$ & $\begin{array}{l}0.000204 \\
0.000205\end{array}$ \\
\hline 269 & $\begin{array}{l}0.519305019 \\
0.52125551\end{array}$ & $\begin{array}{l}1.308434621 \\
1.31465921\end{array}$ & $\begin{array}{l}0.0000627046 \\
0.00633020\end{array}$ & $\begin{array}{l}0.107174677 \\
0108078\end{array}$ & $\begin{array}{l}0.000206 \\
0.00206\end{array}$ \\
\hline & 0.523166023 & 1.3209396868 & 0.000633039 & 0.108437745 & $\begin{array}{l}0.000208 \\
0.000209\end{array}$ \\
\hline & 0.525096525 & 1.32727459 & 0.000636075 & $\begin{array}{l}0.10907382 \\
0.12\end{array}$ & 0.00021 \\
\hline (1) & $\begin{array}{l}0.52702702727 \\
0.52895729\end{array}$ & $\begin{array}{l}1.33366565345 \\
1.3401121272\end{array}$ & $\begin{array}{l}0.00063913737 \\
0.000662227\end{array}$ & $\begin{array}{l}0.1097129555 \\
0.1110355185\end{array}$ & $\begin{array}{l}0.000211 \\
0.000212\end{array}$ \\
\hline 年7 & $\begin{array}{l}0.530888031 \\
.0532819531\end{array}$ & $\begin{array}{l}1.34666773 \\
1.351179797\end{array}$ & 0.000645344 & $\begin{array}{l}0.1111000529 \\
0111640018\end{array}$ & $\begin{array}{l}0.000214 \\
0.00215\end{array}$ \\
\hline & $\begin{array}{l}0.5328878533 \\
0.534749035\end{array}$ & $\begin{array}{l}1.353198013 \\
1.3598143\end{array}$ & $\begin{array}{l}0.00066484899 \\
0.000551663\end{array}$ & $\begin{array}{l}0.1116490088 \\
0.11230068\end{array}$ & $\begin{array}{l}0.000215 \\
0.000216\end{array}$ \\
\hline & 0.536679537 & 1.366482488 & 0.000654864 & 0.112955546 & 0.000217 \\
\hline & 0.538610039 & 1.37322388 & 0.000658095 & 0.113613641 & 0.000219 \\
\hline & $\begin{array}{l}0.54050405441 \\
0.542471042\end{array}$ & $\begin{array}{r}1.38802642 \\
1.386890914\end{array}$ & $\begin{array}{l}0.0006661355 \\
0.000664645\end{array}$ & $\begin{array}{l}0.1114274996 \\
0.114993641\end{array}$ & $\begin{array}{r}0.00022 \\
0.000221\end{array}$ \\
\hline & 0.544401544 & 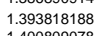 & $\begin{array}{l}0.0006679656 \\
.0006725\end{array}$ & $\begin{array}{l}0.1115607606 \\
0.1607006\end{array}$ & $\begin{array}{l}0.000223 \\
0\end{array}$ \\
\hline & $\begin{array}{l}0.544332840646 \\
0.54826248\end{array}$ & $\begin{array}{r}1.400895018 \\
1.407864438\end{array}$ & $\begin{array}{l}0.00066773151 \\
0.000674696\end{array}$ & $\begin{array}{l}0.111628789827 \\
0.11693617\end{array}$ & $\begin{array}{l}0.000224 \\
0.002225\end{array}$ \\
\hline 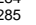 & $\begin{array}{l}0.5842025018 \\
0.55019305\end{array}$ & $\begin{array}{l}.4018044386 \\
1.414985135\end{array}$ & $\begin{array}{l}0.0000677869 \\
0.006109\end{array}$ & 0.117631725 & $\begin{array}{l}0.000025 \\
0.000226\end{array}$ \\
\hline 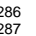 & $\begin{array}{l}0.5521235552 \\
0.55400445044\end{array}$ & $\begin{array}{l}1.422172051 \\
1.4492426806\end{array}$ & $\begin{array}{l}0.000681553 \\
0.000605029\end{array}$ & $\begin{array}{l}0.118313278 \\
0111999380\end{array}$ & $\begin{array}{l}0.0000228 \\
0.00229\end{array}$ \\
\hline 288 & 0.555984556 & 1.436748153 & 0.000688538 & 0.119686845 & $\begin{array}{l}0.000025 \\
0.00023\end{array}$ \\
\hline & 0.557915058 & $\begin{array}{l}1.444139185 \\
1.45160\end{array}$ & 0.00069208 & 0.120378926 & 0.000232 \\
\hline 25 & $\begin{array}{l}0.555844556 \\
0.561776062\end{array}$ & $\begin{array}{l}\text { 1.4556001929 } \\
1.459113195\end{array}$ & $\begin{array}{l}0.00066956565 \\
0.000699265\end{array}$ & $\begin{array}{l}0.12101074581 \\
0.121773846\end{array}$ & $\begin{array}{l}0.0000233 \\
0.000234\end{array}$ \\
\hline 29 & 0.56370656464 & $\begin{array}{l}1.466735631 \\
1.4571\end{array}$ & $\begin{array}{l}0.000702909 \\
0.000\end{array}$ & $\begin{array}{l}0.122476756 \\
0.1226\end{array}$ & $\begin{array}{l}0.000236 \\
0.000\end{array}$ \\
\hline & $\begin{array}{l}0.56666736066 \\
0.56757568\end{array}$ & $\begin{array}{l}1.474412172 \\
1.482162593\end{array}$ & $\begin{array}{l}0.0007065588 \\
0.000710302\end{array}$ & $\begin{array}{l}0.1221383344 \\
0.123893646\end{array}$ & $\begin{array}{l}0.000237 \\
0.000238\end{array}$ \\
\hline 29 & 0.569498069 & $\begin{array}{l}1.48998793 \\
1\end{array}$ & $\begin{array}{l}0.00077103020 \\
0.000714052\end{array}$ & $\begin{array}{l}0.1238936646 \\
0.124607698\end{array}$ & $\begin{array}{l}0.000238 \\
0.00024\end{array}$ \\
\hline 2969 & $\begin{array}{l}0.571428571 \\
0.573359073\end{array}$ & $\begin{array}{l}1.4978892441 \\
1.550867602\end{array}$ & $\begin{array}{l}0.000717839 \\
0.0007216363\end{array}$ & $\begin{array}{l}0.125325537 \\
0.1260472\end{array}$ & $\begin{array}{l}0.000241 \\
0.000243\end{array}$ \\
\hline 29 & 0.575289575 & 1.513924109 & 0.000725523 & 0.126772723 & 0.000244 \\
\hline & 0.577220077 & 1.522059881 & 0.000729422 & 0.127502146 & 0.000245 \\
\hline 301 & $\begin{array}{l}0.57719055979 \\
0.581081081\end{array}$ & $\begin{array}{l}1.5302026055 \\
1.53857379\end{array}$ & $\begin{array}{l}0.000733366 \\
0.000737336\end{array}$ & $\begin{array}{l}0.1228233506 \\
0.128972842\end{array}$ & $\begin{array}{l}0.000247 \\
0.000248\end{array}$ \\
\hline 302 & $\begin{array}{l}0.583011583 \\
0.58942085\end{array}$ & $\begin{array}{l}1.5469542699 \\
1.554418096\end{array}$ & $\begin{array}{l}0.000744353 \\
0.00757540\end{array}$ & 0.129714195 & $\begin{array}{r}0.00025 \\
0.00251\end{array}$ \\
\hline 304 & $\begin{array}{l}0.58494248085 \\
0.586872587\end{array}$ & $\begin{array}{l}1.5554486966 \\
1.563968298\end{array}$ & $\begin{array}{l}0.00077544499 \\
0.000749506\end{array}$ & $\begin{array}{l}0.1304095604 \\
0.131209011\end{array}$ & $\begin{array}{l}0.000251 \\
0.000253\end{array}$ \\
\hline 30 & $\begin{array}{l}0.5888030898 \\
0.50773559\end{array}$ & $\begin{array}{l}1.572604326 \\
1.581328505\end{array}$ & 0.000753645 & $\begin{array}{l}0.131962755 \\
0.13727558\end{array}$ & $\begin{array}{l}0.000254 \\
0.00255\end{array}$ \\
\hline & $\begin{array}{l}0.5097733551 \\
0.592664091\end{array}$ & $\begin{array}{l}1.5581328056 \\
1.59014076\end{array}$ & $\begin{array}{l}0.0007757862 \\
0.000762049\end{array}$ & $\begin{array}{l}0.1327205581 \\
0.13348263\end{array}$ & $\begin{array}{l}0.000255 \\
0.000257\end{array}$ \\
\hline & 0.594594595 & 1.599043845 & 0.000766316 & 0.134248946 & 0.000258 \\
\hline 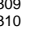 & $\begin{array}{l}0.5965525097 \\
0.598455598\end{array}$ & $\begin{array}{l}1.6080083582 \\
1.6171726374\end{array}$ & $\begin{array}{l}0.00007776626 \\
0.00077491\end{array}$ & $\begin{array}{l}0.13350195727 \\
0.135794554\end{array}$ & $\begin{array}{r}0.00026 \\
0.000261\end{array}$ \\
\hline${ }^{31}$ & 0.6003861 & 1.626308629 & $\begin{array}{l}0.000779382 \\
0\end{array}$ & 0.1365739366 & $\begin{array}{l}0.000263 \\
0\end{array}$ \\
\hline & 0.602316602 & $\begin{array}{l}1.635586778 \\
1640202\end{array}$ & 0.000783828 & 0.137357764 & 0.000264 \\
\hline . & $\begin{array}{l}0.600242411040 \\
0.606177706\end{array}$ & $\begin{array}{l}1.6449628284 \\
1.654436638\end{array}$ & $\begin{array}{l}0.0007883221 \\
0.000792862\end{array}$ & $\begin{array}{l}0.1381460858 \\
0.138938947\end{array}$ & $\begin{array}{l}0.000266 \\
0.000267\end{array}$ \\
\hline 31 & $\begin{array}{l}0.608108108 \\
0.61003861\end{array}$ & $\begin{array}{r}1.664011362 \\
1.6673688008\end{array}$ & $\begin{array}{l}0.00079775 \\
0.000802088\end{array}$ & $\begin{array}{l}0.1397363997 \\
0.144538485\end{array}$ & $\begin{array}{l}0.000269 \\
0.0026271\end{array}$ \\
\hline 31 & 0.6119691112 & 1.68346816 & $\begin{array}{l}0.000806775 \\
0\end{array}$ & 0.14134526 & $\begin{array}{l}0.000272 \\
0.0027\end{array}$ \\
\hline & & 1.693353434 & 0.000811512 & 0.142156772 & 0.000274 \\
\hline 年 & $\begin{array}{l}0.658303016 \\
0.617760616\end{array}$ & $\begin{array}{l}\begin{array}{l}1.703344448 \\
1.71344598\end{array} \\
1\end{array}$ & $\begin{array}{l}0.0008616301 \\
0.000821114\end{array}$ & $\begin{array}{l}0.1429273027 \\
0.143794214\end{array}$ & $\begin{array}{l}0.000275 \\
0.000277\end{array}$ \\
\hline 32 & 0.61969112 & 1.723656655 & $\begin{array}{l}0.000826034 \\
.\end{array}$ & $\begin{array}{l}0.144620248 \\
0.1446\end{array}$ & 0.000278 \\
\hline & 0.621621622 & $\begin{array}{l}1.733979259 \\
1.7441558\end{array}$ & 0.000830981 & & 0.00028 \\
\hline 324 & $\begin{array}{l}0.623555212424 \\
0.625482625\end{array}$ & $\begin{array}{l}1.74444155538 \\
1.774967455\end{array}$ & $\begin{array}{l}0.0008339983 \\
0.00084404\end{array}$ & $\begin{array}{l}0.14628721212 \\
0.1471728252\end{array}$ & $\begin{array}{l}0.000282 \\
0.000283\end{array}$ \\
\hline 32. & 0.627413127 & 1.7656636745 & 0.0008461535 & 0.147974404 & 0.000285 \\
\hline & & $\begin{array}{r}1.77642536 \\
1.78735294\end{array}$ & $\begin{array}{l}0.00085513225 \\
0.00085551\end{array}$ & 0.148825727 & \\
\hline 然 & $\begin{array}{l}0.6631274111 \\
0.63320461\end{array}$ & $\begin{array}{r}1.1873535248 \\
1.778684\end{array}$ & $\begin{array}{l}0.0008565555 \\
0.000861839\end{array}$ & $\begin{array}{l}0.149682279 \\
0.150544117\end{array}$ & $\begin{array}{c}0.0000288 \\
0.00029\end{array}$ \\
\hline & 0.635135135 & 1.80952685 & 0.000867186 & 0.151411304 & 0.000291 \\
\hline & $\begin{array}{l}0.637065637 \\
.663896913\end{array}$ & $\begin{array}{l}1.820812675 \\
1.832272909\end{array}$ & 0.000872595 & $\begin{array}{l}0.152283898 \\
0.1516196\end{array}$ & 0.000293 \\
\hline & $\begin{array}{l}0.6389969139 \\
0.6409641\end{array}$ & $\begin{array}{l}1.8322279989 \\
1.84777498\end{array}$ & $\begin{array}{l}0.000878065 \\
0.000883599\end{array}$ & & 0.000295 \\
\hline (3) & $\begin{array}{l}0.6449626641 \\
0.642857143\end{array}$ & $\begin{array}{l}1.843777499 \\
1.855455868\end{array}$ & $\begin{array}{l}0.0008885999 \\
0.000889199\end{array}$ & $\begin{array}{l}0.1540445633 \\
0.15493476\end{array}$ & $\begin{array}{l}0.000297 \\
0.000298\end{array}$ \\
\hline 等3 & $\begin{array}{l}0.6447876445 \\
0.646718147\end{array}$ & $\begin{array}{c}1.8677272899 \\
1.17892284\end{array}$ & $\begin{array}{l}0.000894866 \\
0.00090059\end{array}$ & $\begin{array}{l}0.155829622 \\
0.05763021\end{array}$ & $\begin{array}{r}0.0003 \\
0.000302\end{array}$ \\
\hline & $\begin{array}{l}0.6467878171 \\
0.64864864\end{array}$ & $\begin{array}{l}1.87892284 \\
1.891324742\end{array}$ & $\begin{array}{l}0.000900638387 \\
0.009\end{array}$ & $\begin{array}{l}0.1566732121 \\
0.157636596\end{array}$ & $\begin{array}{l}0.000302 \\
0.000303\end{array}$ \\
\hline & 0.650579151 & 1.903564347 & 0.0009112252 & 0.158548849 & 0.000305 \\
\hline & $\begin{array}{l}0.6555090653 \\
0.654440154\end{array}$ & $\begin{array}{l}1.9159496963) \\
1.928483314\end{array}$ & $\begin{array}{l}0.000918188 \\
0.000924194\end{array}$ & $\begin{array}{l}0.159467036 \\
0.16039123\end{array}$ & $\begin{array}{l}0.000307 \\
0.000309\end{array}$ \\
\hline & 0.656370656 & $\begin{array}{l}1.9441678005 \\
1.95405851\end{array}$ & $\begin{array}{l}0.00009302773 \\
0 \\
0\end{array}$ & $\begin{array}{l}0.161321504 \\
01625902\end{array}$ & 0.000311 \\
\hline & 0.658301158 & $\begin{array}{l}1.954005815 \\
1.96070000\end{array}$ & $\begin{array}{l}0.0009936425 \\
0.00992653\end{array}$ & $\begin{array}{l}0.162257929 \\
0.16320558\end{array}$ & 0.000312 \\
\hline 343 & $\begin{array}{l}0.66023366 \\
0.662162162\end{array}$ & $\begin{array}{r}1.9677000066 \\
1.980153317\end{array}$ & $\begin{array}{l}0.0009442653 \\
0.000948956\end{array}$ & $\begin{array}{l}0.163200582 \\
0.164149538\end{array}$ & $\begin{array}{l}0.000314 \\
0.000316\end{array}$ \\
\hline & 0.664092664 & $\begin{array}{l}1.993468426 \\
.19030200\end{array}$ & 0.0009553337 & 0.165104875 & 0.000318 \\
\hline & 0.66600231666 & $\begin{array}{r}2.0609482999 \\
20202555912\end{array}$ & $\begin{array}{l}0.000961797 \\
0 \\
0\end{array}$ & $\begin{array}{l}0.166066673 \\
0.1635035\end{array}$ & $\begin{array}{r}0.00032 \\
0.000322\end{array}$ \\
\hline & 0.66988417 & 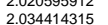 & $\begin{array}{l}\begin{array}{l}0.0009686338 \\
0.00097496\end{array} \\
0\end{array}$ & $\begin{array}{l}0.1677035010 \\
0.16800997\end{array}$ & $\begin{array}{l}0.000322 \\
0.000323\end{array}$ \\
\hline & 0.671814672 & 2.048406629 & 0.000981666 & $\begin{array}{l}0.168991636 \\
0.1690936\end{array}$ & $\begin{array}{l}0.000325 \\
0.00327\end{array}$ \\
\hline & 0.673745174 & $\begin{array}{l}2.062576055 \\
2\end{array}$ & $\begin{array}{l}0.000988456 \\
0 \\
0\end{array}$ & 0.169980092 & 0.000327 \\
\hline 35 & $\begin{array}{l}0.67565756676 \\
0.677606178\end{array}$ & $\begin{array}{r}2.0769255588 \\
2.091459403\end{array}$ & $\begin{array}{l}0.0009993333 \\
0.00102298\end{array}$ & $\begin{array}{l}0.1779975425 \\
0.171977722\end{array}$ & $\begin{array}{l}0.000329 \\
0.000331\end{array}$ \\
\hline & $\begin{array}{l}0.67953668 \\
0.691467119\end{array}$ & $\begin{array}{l}2.106180123 \\
2112109154\end{array}$ & 0.0010093535 & $\begin{array}{l}0.172987075 \\
0.1700505\end{array}$ & 0.000333 \\
\hline & $\begin{array}{l}0.68814678781 \\
0.68397868\end{array}$ & $\begin{array}{l}2.1211095154 \\
2.136197263\end{array}$ & $\begin{array}{l}0.01010164999 \\
0.001023738\end{array}$ & $\begin{array}{l}0.174003574 \\
0.17502731\end{array}$ & $\begin{array}{l}0.0000335 \\
0.000337\end{array}$ \\
\hline & $\begin{array}{l}0.685328185 \\
0.685\end{array}$ & 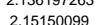 & 0.001031072 & $\begin{array}{l}0.175027311 \\
0.17058383\end{array}$ & $\begin{array}{l}0.000337 \\
0.000339\end{array}$ \\
\hline & 0.687258687 & $\begin{array}{l}2.1 .17006515 \\
2\end{array}$ & 0.001038503 & $\begin{array}{l}0.1770968806 \\
0\end{array}$ & $\begin{array}{l}0.000339 \\
0.000341\end{array}$ \\
\hline & 0.689189189 & 2.182717724 & 0.001046032 & 0.178142918 & 0.000343 \\
\hline & 119691 & 2.1986386003 & $\begin{array}{l}.0010536662 \\
.\end{array}$ & 0.179196579 & 0.000345 \\
\hline & $\begin{array}{l}0.6934980063 \\
0.694065\end{array}$ & $\begin{array}{l}2.2 .21 / 7 / 34243 \\
2.23112583\end{array}$ & $\begin{array}{l}0.010010613344 \\
0.010169231\end{array}$ & $\begin{array}{l}0.1882575973 \\
0.181327204\end{array}$ & $\begin{array}{l}0.000347 \\
0.000349\end{array}$ \\
\hline & 0.696911197 & $\begin{array}{l}2.247700682 \\
\end{array}$ & 0.0010771774 & 0.182404378 & $\begin{array}{l}0.000351 \\
0.0035\end{array}$ \\
\hline & 0.698841699 & 2.264502197 & $\begin{array}{l}0.001085226 \\
0.001093388\end{array}$ & 0.183489604 & 0.000353 \\
\hline 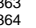 & $\begin{array}{l}0.7007722010 \\
0.702702703\end{array}$ & $\begin{array}{l}2.2815154491212 \\
2.298803473\end{array}$ & $\begin{array}{l}0.01001093388 \\
0.001101664\end{array}$ & $\begin{array}{l}0.184582992 \\
0.185864656\end{array}$ & $\begin{array}{l}0.000355 \\
0.000357\end{array}$ \\
\hline & $\begin{array}{l}0.7046332005 \\
0.7065367707\end{array}$ & $\begin{array}{l}2.316312655 \\
.2340675\end{array}$ & $\begin{array}{l}0.001110055 \\
0.0011118554\end{array}$ & $\begin{array}{l}0.18679471212 \\
0.1879713275\end{array}$ & $\begin{array}{l}0.00036 \\
0.000362\end{array}$ \\
\hline & 0.7084944208 & 2.352072608 & 0.001127192 & 0.189040468 & 0.000364 \\
\hline
\end{tabular}




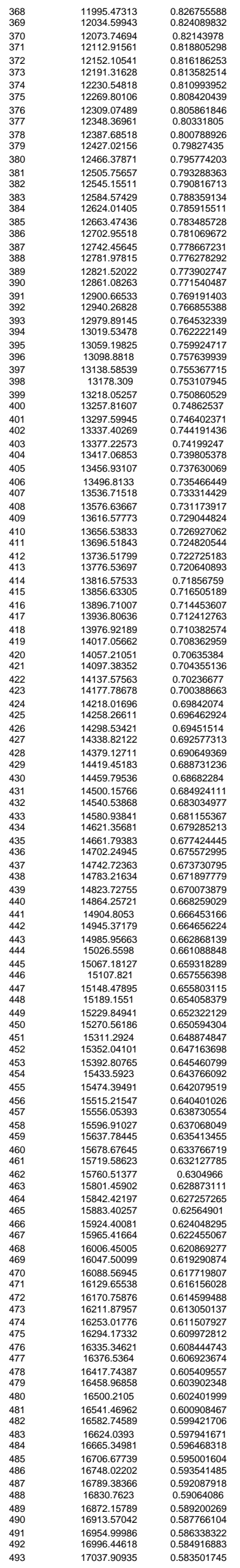

\begin{tabular}{|c|c|c|c|c|c|}
\hline 608 & 0.71042471 & 2.370333578 & 0.001135944 & 0.190176412 & 0.000366 \\
\hline 370 & $\begin{array}{l}0.71123552121 \\
0.714285714\end{array}$ & $\begin{array}{r}2.3888555773 \\
240764404\end{array}$ & $\begin{array}{l}0.00114482 \\
0.001143824\end{array}$ & $\begin{array}{l}0.191321232 \\
0.192475056\end{array}$ & $\begin{array}{r}0.000368 \\
0.00037\end{array}$ \\
\hline (31) & 0.716216216 & 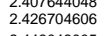 & $\begin{array}{l}0.00116584 \\
0.00116294\end{array}$ & $\begin{array}{l}0.19363806 \\
0.19635\end{array}$ & $\begin{array}{l}0.0000373 \\
0.00373\end{array}$ \\
\hline 373 & $\begin{array}{l}0.718146718 \\
0.72007722\end{array}$ & $\begin{array}{l}2.4460430005 \\
2.465656165\end{array}$ & $\begin{array}{l}0.001172226 \\
0.0011818163\end{array}$ & $\begin{array}{l}0.194810241 \\
0.199991871\end{array}$ & $\begin{array}{l}0.0000375 \\
0.000377\end{array}$ \\
\hline $\begin{array}{ll}374 \\
375\end{array}$ & $\begin{array}{l}0.7220077222 \\
0.723938224\end{array}$ & $\begin{array}{l}2.4855771744 \\
2.550758288\end{array}$ & $\begin{array}{l}0.0011911727 \\
0.000120827\end{array}$ & $\begin{array}{l}0.1971838043 \\
0.1988383\end{array}$ & $\begin{array}{l}0.00038 \\
0.000382 \\
0.0038\end{array}$ \\
\hline & 0.725868726 & 2.526295944 & 0.001210686 & 0.199594586 & 0.000384 \\
\hline & 0.727799228 & 2.547115765 & 0.001220664 & 0.20081525 & 0.000386 \\
\hline 37 & $\begin{array}{l}0.72972973) \\
0.73166023\end{array}$ & $\begin{array}{r}2.56682551562 \\
2.589710347\end{array}$ & $\begin{array}{l}0.001233793 \\
0.001241077\end{array}$ & $\begin{array}{l}0.202044043 \\
0.203287119\end{array}$ & $\begin{array}{l}0.0000389 \\
0.000391\end{array}$ \\
\hline 38 & 0.733590734 & 2.611499334 & 0.001251519 & 0.204538638 & 0.000394 \\
\hline${ }_{38}$ & $\begin{array}{l}0.73352121263 \\
0.737451737\end{array}$ & $\begin{array}{r}2.6336259553 \\
2.656097954\end{array}$ & $\begin{array}{l}0.001262122 \\
0.0127292\end{array}$ & $\begin{array}{l}0.20550076 \\
0.207073652\end{array}$ & $\begin{array}{l}0.000396 \\
0.000399\end{array}$ \\
\hline & $\begin{array}{l}0.739382239 \\
0.7431274\end{array}$ & $\begin{array}{l}2.678922911 \\
.2701020\end{array}$ & $\begin{array}{l}0.00128383 \\
0.0212904\end{array}$ & 0.208357482 & $\begin{array}{l}0.000401 \\
0.00401\end{array}$ \\
\hline 385 & $\begin{array}{l}0.743243243 \\
0.4\end{array}$ & $\begin{array}{l}2.7021209246 \\
2.72565214\end{array}$ & $\begin{array}{l}0.00012999424 \\
0.001306231\end{array}$ & $\begin{array}{l}0.20206524242 \\
0.210958654\end{array}$ & $\begin{array}{l}0.00040303 \\
0.000406\end{array}$ \\
\hline & 0.745173745 & $\begin{array}{l}2.7495994344 \\
.7770270\end{array}$ & 0.001317701 & 0.212276355 & 0.000409 \\
\hline & $\begin{array}{l}0.74711042477 \\
0.74903474\end{array}$ & $\begin{array}{l}2.77793920787 \\
2.798638431\end{array}$ & $\begin{array}{l}0.00113293566 \\
0.001341202\end{array}$ & $\begin{array}{l}0.213605712 \\
0.214946913\end{array}$ & $\begin{array}{l}0.000011 \\
0.000414\end{array}$ \\
\hline 年 & $\begin{array}{l}0.750965251 \\
0.75285953\end{array}$ & $\begin{array}{r}2.8237661809 \\
289430661\end{array}$ & $\begin{array}{l}0.001353242 \\
0.00136458\end{array}$ & $\begin{array}{l}0.216300155 \\
0.2176565635\end{array}$ & $\begin{array}{l}0.000416 \\
0.000419\end{array}$ \\
\hline & $\begin{array}{l}0.7528855253 \\
0.75482625\end{array}$ & $\begin{array}{l}2.8493006661 \\
2.875265035\end{array}$ & $\begin{array}{l}0.01361374924 \\
0.0013794\end{array}$ & $\begin{array}{l}0.21 / 656636 \\
0.21904356\end{array}$ & $\begin{array}{l}0.000419 \\
0.000422\end{array}$ \\
\hline & 0.756756757 & 2.901665301 & 0.001390576 & 0.220434136 & $\begin{array}{l}0.000422 \\
0.000424\end{array}$ \\
\hline 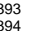 & $\begin{array}{l}0.758687259 \\
0.760617761\end{array}$ & $\begin{array}{l}2.9285111663 \\
2.955866699\end{array}$ & $\begin{array}{l}0.0014014342 \\
0.001416527\end{array}$ & $\begin{array}{l}0.221837578 \\
0.2232551105\end{array}$ & $\begin{array}{c}0.0000427 \\
0.00043\end{array}$ \\
\hline 列 & $\begin{array}{l}0.762548263 \\
076448764\end{array}$ & $\begin{array}{l}2.98359023232 \\
3.01184462\end{array}$ & $\begin{array}{l}0.001429837 \\
014437\end{array}$ & $\begin{array}{l}0.2246839442 \\
02261739\end{array}$ & $\begin{array}{l}0.0000432 \\
0.00432\end{array}$ \\
\hline & $\begin{array}{l}0.766409266 \\
0.70\end{array}$ & $\begin{array}{r}3.01818446939 \\
3.040592067\end{array}$ & $\begin{array}{l}0.000144353 \\
0.001457154\end{array}$ & $\begin{array}{l}0.2227125819 \\
0.2254444\end{array}$ & $\begin{array}{l}0.000435 \\
0.000438\end{array}$ \\
\hline & 0.768339768 & 3.069845102 & 0.001471173 & 0.229055647 & 0.000441 \\
\hline 399 & $\begin{array}{l}0.777027027 \\
0.772200772\end{array}$ & $\begin{array}{r}3.09961676537 \\
3.1 .129920471\end{array}$ & $\begin{array}{l}0.001485441 \\
0.001499963\end{array}$ & $\begin{array}{l}0.230544088 \\
0.23204104\end{array}$ & $\begin{array}{l}0.0000444 \\
0.000447\end{array}$ \\
\hline & $\begin{array}{l}0.774131274 \\
0776061776\end{array}$ & $\begin{array}{l}3.16077017 \\
3.192180272\end{array}$ & $\begin{array}{l}0.00151474748 \\
0.015298\end{array}$ & $\begin{array}{l}0.2335557999 \\
0.23508559\end{array}$ & 0.000449 \\
\hline & 0.777992278 & $\begin{array}{l}3.224165558 \\
3.265\end{array}$ & $\begin{array}{l}0.00151545129 \\
0.00159\end{array}$ & $\begin{array}{l}0.2330585599 \\
0.236630728\end{array}$ & 0.000455 \\
\hline & 0.77992278 & $\begin{array}{l}3.256744605 \\
3\end{array}$ & 0.00156074 & 0.238191468 & 0.000458 \\
\hline & 0.781853282 & 3.289924275 & 0.001576643 & 0.239768111 & 0.000461 \\
\hline $\begin{array}{l}40-1 \\
40\end{array}$ & $\begin{array}{l}0.7887383784 \\
0.785714286\end{array}$ & $\begin{array}{l}\begin{array}{l}3.3237301244 \\
3.35817628\end{array} \\
3\end{array}$ & $\begin{array}{l}0.0001592844 \\
0.001609351\end{array}$ & $\begin{array}{l}0.241360955 \\
0.242970306\end{array}$ & $\begin{array}{l}0.000464 \\
0.000468\end{array}$ \\
\hline 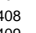 & 0.787644788 & $\begin{array}{l}3.393280492 \\
.3200619\end{array}$ & $\begin{array}{l}0.001626174 \\
0\end{array}$ & $\begin{array}{l}0.24459648 \\
0.245098\end{array}$ & $\begin{array}{l}0.000471 \\
0.0040\end{array}$ \\
\hline & $\begin{array}{l}0.7895 / 529 \\
0.791505792\end{array}$ & $\begin{array}{l}3.429006611616 \\
3.44557366\end{array}$ & & & $\begin{array}{l}0.000474 \\
0.00477\end{array}$ \\
\hline 411 & $\begin{array}{l}0.793436292 \\
0.0350\end{array}$ & $\begin{array}{l}3.4505272860 \\
3.5027897\end{array}$ & $\begin{array}{l}0.001666802 \\
0.001678626\end{array}$ & $\begin{array}{l}0.249006004 \\
0.24957923\end{array}$ & $\begin{array}{c}0.0000477 \\
0.00048\end{array}$ \\
\hline 41 & $\begin{array}{l}0.795366795 \\
0797297297\end{array}$ & $\begin{array}{l}3.540056629 \\
3.579340857\end{array}$ & $\begin{array}{l}0.001696802 \\
0 \\
000711534\end{array}$ & $\begin{array}{l}0.251276032 \\
025292991372\end{array}$ & $\begin{array}{l}0.000483 \\
0.00487\end{array}$ \\
\hline 414 & 0.799227799 & 3.618804728 & $\begin{array}{l}0.001734253 \\
0.03\end{array}$ & 0.254725626 & 0.00049 \\
\hline & 0.801158301 & $\begin{array}{r}3.659070887 \\
37012021\end{array}$ & 0.00175355 & $\begin{array}{l}0.256479176 \\
\end{array}$ & 0.000493 \\
\hline 41 & $\begin{array}{l}0.80300888030 \\
0.805019305\end{array}$ & $\begin{array}{r}3.700163216 \\
3.742106533\end{array}$ & $\begin{array}{l}0.0001773243 \\
0.00179334\end{array}$ & $\begin{array}{l}0.25258252419 \\
0.260045763\end{array}$ & $\begin{array}{l}0.0000497 \\
0.00005\end{array}$ \\
\hline 418 & 0.8069498007 & 3.7.784926644 & $\begin{array}{l}0.001813864 \\
0.04\end{array}$ & 0.261859627 & $\begin{array}{l}0.000504 \\
0\end{array}$ \\
\hline 41 & 0.808880309 & & 0.001834818 & & 0.000507 \\
\hline 420 & $\begin{array}{l}0.81080808111 \\
0.812741313\end{array}$ & $\begin{array}{l}3.87733056693 \\
3.918921624\end{array}$ & $\begin{array}{l}0.0001856219 \\
0.001878079\end{array}$ & $\begin{array}{l}0.2655556664 \\
0.267428744\end{array}$ & $\begin{array}{l}0.000511 \\
0.000514\end{array}$ \\
\hline 422 & $\begin{array}{l}0.814671815 \\
081660237\end{array}$ & $\begin{array}{r}3.9655284448 \\
4011157693\end{array}$ & $\begin{array}{l}0.001900415 \\
0.00192324\end{array}$ & $\begin{array}{l}0.269329159 \\
0.271252399\end{array}$ & $\begin{array}{l}0.000518 \\
0.005522\end{array}$ \\
\hline 42. & 0.818532819 & 4.061842211 & $\begin{array}{l}0.001946572 \\
0.07\end{array}$ & 0.273198971 & 0.000526 \\
\hline & 0.82046332 & 4.111616253 & 0.001970425 & 0.275169396 & 0.000529 \\
\hline 42. & $\begin{array}{l}0.82233938222 \\
0.82432424\end{array}$ & $\begin{array}{l}4.1665255537 \\
4.214577334\end{array}$ & $\begin{array}{l}0.001999818 \\
0.002019768\end{array}$ & $\begin{array}{l}0.2777646214 \\
0.279183981\end{array}$ & $\begin{array}{l}0.0000533 \\
0.000537\end{array}$ \\
\hline 42 & $\begin{array}{l}0.826254826 \\
0828153238\end{array}$ & $\begin{array}{r}4.267840543 \\
4.32235479\end{array}$ & 0.002045293 & 0.281229275 & 0.000541 \\
\hline 43 & $\begin{array}{l}0.828185338 \\
0.83011583\end{array}$ & $\begin{array}{l}4.32232447898 \\
4.378135511\end{array}$ & $\begin{array}{l}0.00020771414 \\
0.00209815\end{array}$ & $\begin{array}{l}0.2883300688 \\
0.28539839\end{array}$ & $\begin{array}{l}0.000545 \\
0.000549\end{array}$ \\
\hline 431 & 0.832046332 & 4.435254068 & $\begin{array}{l}0.0021255232 \\
0.021525\end{array}$ & $\begin{array}{l}0.2875243652 \\
0.250\end{array}$ & $\begin{array}{l}0.000549 \\
0.000553\end{array}$ \\
\hline & $\begin{array}{l}0.8339968834 \\
0.83599736\end{array}$ & $\begin{array}{l}4.49937478484 \\
4.53565377\end{array}$ & $\begin{array}{l}0.0001535555 \\
0.02218227\end{array}$ & $\begin{array}{l}0.2896779177 \\
0.291860187\end{array}$ & $\begin{array}{l}0.000557 \\
0.000561\end{array}$ \\
\hline 列 & 0.837837838 & $\begin{array}{l}4.53565653717 \\
4.61505749\end{array}$ & 0.002211691 & $\begin{array}{l}0.298601878 \\
0.294071878\end{array}$ & $\begin{array}{l}0.000561 \\
0.000566\end{array}$ \\
\hline 年5 & $\begin{array}{l}0.83976834 \\
084469892\end{array}$ & $\begin{array}{r}4.6779772599 \\
4774248538\end{array}$ & $\begin{array}{l}0.002244844 \\
0.0227275\end{array}$ & $\begin{array}{l}0.296313723 \\
029586479\end{array}$ & $\begin{array}{r}0.00057 \\
0.005774\end{array}$ \\
\hline 43 & 0.843629344 & 4.808625700 & 0.002304456 & 0.300890935 & $\begin{array}{l}0.0005 / 4 \\
0.00579\end{array}$ \\
\hline & 0.845559846 & 4.876474048 & 0.002336971 & 0.303227906 & 0.000583 \\
\hline 44 & $\begin{array}{l}0.84449030347 \\
0.84942049\end{array}$ & $\begin{array}{l}4.946089866 \\
5.01750664\end{array}$ & $\begin{array}{l}0.0023703333 \\
0.02440457\end{array}$ & $\begin{array}{l}0.305598239 \\
0.30802894\end{array}$ & $\begin{array}{l}0.000588 \\
0.000592\end{array}$ \\
\hline 44 & 0.851351351 & $\begin{array}{r}5.0900997392 \\
5.16220602\end{array}$ & $\begin{array}{l}0.00243973 \\
0.024750\end{array}$ & 0.310442543 & $\begin{array}{l}0.000597 \\
0.0057\end{array}$ \\
\hline & $\begin{array}{l}0.8532881853 \\
0.855212535\end{array}$ & 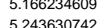 & $\begin{array}{l}0.0024578344 \\
0.022512925\end{array}$ & $\begin{array}{l}0.3129183877 \\
0.31543130\end{array}$ & \\
\hline 4 & $\begin{array}{l}0.85711425857 \\
0\end{array}$ & 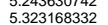 & $\begin{array}{l}0.00252510252 \\
0.00\end{array}$ & $\begin{array}{l}0.351382323 \\
0.3179234\end{array}$ & $\begin{array}{l}0.000607 \\
0.000611\end{array}$ \\
\hline 44 & 0.8590733595 & 5.404934303 & 0.002590227 & 0.32057257 & 0.000616 \\
\hline & 0.861003861 & 5.489020252 & 0.002630524 & 0.323203094 & 0.000621 \\
\hline t4 & $\begin{array}{l}0.86693436363 \\
0.864864865\end{array}$ & $\begin{array}{l}5.575552272765 \\
5.664543754\end{array}$ & $\begin{array}{l}0.00026771978 \\
0.00271464\end{array}$ & $\begin{array}{l}0.3258775072 \\
0.3285897113\end{array}$ & $\begin{array}{l}0.000627 \\
0.000632\end{array}$ \\
\hline 44 & 0.8667953677 & 5.756190829 & 0.002758561 & 0.331348273 & 0.000637 \\
\hline & $\begin{array}{l}0.8687258696 \\
0.870656731\end{array}$ & $\begin{array}{r}5.850577769 \\
5.947824562\end{array}$ & $\begin{array}{l}0.002803794 \\
0.028850398\end{array}$ & $\begin{array}{l}0.334452067 \\
0.33702465\end{array}$ & $\begin{array}{l}0.000642 \\
0.000648\end{array}$ \\
\hline 45 & $\begin{array}{l}0.87725868771 \\
0.878\end{array}$ & $\begin{array}{l}5.944784256666 \\
6.048058666\end{array}$ & $\begin{array}{l}0.0028503988 \\
0.002898434\end{array}$ & $\begin{array}{l}0.33370024655 \\
0.339900899\end{array}$ & $\begin{array}{l}0.000648 \\
0.000653\end{array}$ \\
\hline 45 & 0.874517375 & 6.15141472 & 0.002947965 & 0.342848864 & 0.000659 \\
\hline & $\begin{array}{l}0.876447876 \\
0.87837873\end{array}$ & $\begin{array}{l}6.25803035020 \\
6.36807244\end{array}$ & $\begin{array}{l}0.002999062 \\
0.00301592\end{array}$ & & 0.000665 \\
\hline & $\begin{array}{l}0.8 / 7387838 \\
0.88030888\end{array}$ & $\begin{array}{l}6.36808 / 24444 \\
6.48168293\end{array}$ & $\begin{array}{l}0.00030551795 \\
0.003106243\end{array}$ & $\begin{array}{l}0.348899721 \\
0.352005964\end{array}$ & $\begin{array}{l}0.000671 \\
0.000677\end{array}$ \\
\hline 45 & 0.882239382 & 6.599047831 & $\begin{array}{l}0.003316243 \\
0.003162486\end{array}$ & 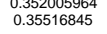 & 0.000683 \\
\hline 45 & $\begin{array}{l}0.8884169888 \\
0.8681000836\end{array}$ & $\begin{array}{l}6.7203386552 \\
6.845752027\end{array}$ & $\begin{array}{l}0.003220613 \\
0.03280715\end{array}$ & $\begin{array}{l}0.358389063 \\
0.361696778\end{array}$ & $\begin{array}{l}0.0000699 \\
0.0006995\end{array}$ \\
\hline 46 & 0.888030888 & 6.9754938353 & $\begin{array}{l}0.0033428282 \\
0.00\end{array}$ & $\begin{array}{l}0.36501267 \\
0.367\end{array}$ & $\begin{array}{l}0.000701 \\
0.000\end{array}$ \\
\hline & $\begin{array}{l}0.889966139 \\
0.89189189\end{array}$ & $\begin{array}{r}7.10998366 \\
7.24885623\end{array}$ & $\begin{array}{l}0.00034072748 \\
0.003473896\end{array}$ & $\begin{array}{l}0.368419918 \\
0.37189314\end{array}$ & $\begin{array}{l}0.000708 \\
0.000715\end{array}$ \\
\hline & 0.893822394 & $\begin{array}{l}7.392960224 \\
75+535611\end{array}$ & 0.003542956 & $\begin{array}{l}0.37543677 \\
0.370955325\end{array}$ & 0.000721 \\
\hline & $\begin{array}{l}0.89575289696 \\
0.897683398\end{array}$ & $\begin{array}{l}7.5423365119 \\
7.697359928\end{array}$ & $\begin{array}{l}0.0003641556 \\
0.00368833\end{array}$ & $\begin{array}{l}\begin{array}{l}0.3770955325 \\
0.38274015\end{array} \\
0\end{array}$ & $\begin{array}{l}0.000728 \\
0.000735\end{array}$ \\
\hline 46 & $\begin{array}{l}0.8996139 \\
0.901544402\end{array}$ & $\begin{array}{l}7.8582428257 \\
8.8252553\end{array}$ & 0.003765935 & 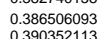 & 0.000743 \\
\hline & $\begin{array}{l}0.9015444402 \\
0.90344903\end{array}$ & $\begin{array}{l}8.025352525434 \\
8.199044011\end{array}$ & $\begin{array}{l}0.000384002 \\
0.003929256\end{array}$ & $\begin{array}{l}0.390352113 \\
0.39428133\end{array}$ & $\begin{array}{r}0.00075 \\
0.000757\end{array}$ \\
\hline 46 & 0.905405405 & 8.3796866449 & 0.004015828 & $\begin{array}{l}0.3942883699 \\
0.398297198\end{array}$ & $\begin{array}{l}0.000757 \\
0.000765\end{array}$ \\
\hline & 0.907335907 & 8.567701758 & 0.004105932 & 0.402403129 & 0.000773 \\
\hline & 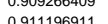 & $\begin{array}{l}8.7635283366 \\
8996763889\end{array}$ & 0.004199778 & $\begin{array}{l}0.4066002907 \\
0.41090050\end{array}$ & $\begin{array}{l}0.000781 \\
0.000789\end{array}$ \\
\hline & 0.913127411 & 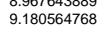 & $\begin{array}{l}0.0004299597 \\
0.00439636\end{array}$ & $\begin{array}{l}0.4109000505 \\
0.415300141\end{array}$ & $\begin{array}{l}0.000789 \\
0.000797\end{array}$ \\
\hline & 0.915057915 & $\begin{array}{l}9.4028502222 \\
.9535062\end{array}$ & $\begin{array}{l}0.004506163 \\
0.006617468\end{array}$ & 0.419806304 & $\begin{array}{l}0.000806 \\
0.06\end{array}$ \\
\hline & 0.916988417 & 9.635106698 & $\begin{array}{l}0.004617468 \\
0\end{array}$ & 0.424423772 & 0.000815 \\
\hline 年 & $\begin{array}{l}0.91898189919 \\
0.920849421\end{array}$ & $\begin{array}{l}9.8779928878 \\
10.13222532\end{array}$ & $\begin{array}{l}0.0004733867 \\
0.004857704\end{array}$ & $\begin{array}{l}0.4291515639 \\
0.434013343\end{array}$ & $\begin{array}{l}0.000024 \\
0.000833\end{array}$ \\
\hline 47 & 0.922779923 & 10.398584877 & 0.004983352 & 0.4389966955 & 0.000843 \\
\hline & $0.924710425)$ & 10.67792385 & $\begin{array}{l}0.0055117221 \\
0.00525775\end{array}$ & 0.444113917 & 0.000852 \\
\hline 48 & 0.928571429 & $\begin{array}{l}11.27935751 \\
1\end{array}$ & $\begin{array}{l}0.005405448 \\
0.053\end{array}$ & $\begin{array}{l}0.4454777122 \\
0.02\end{array}$ & $\begin{array}{l}0.000862 \\
0.000873\end{array}$ \\
\hline & 0.930501931 & 11.60359434 & 0.005560834 & 0.460337955 & 0.000883 \\
\hline & $\begin{array}{l}0.932432432 \\
0.934362934\end{array}$ & $\begin{array}{l}11.94511786 \\
1230582726\end{array}$ & $\begin{array}{l}0.005724503 \\
0.05897190\end{array}$ & $\begin{array}{l}0.466062459 \\
0.471959567\end{array}$ & $\begin{array}{l}0.0000994 \\
0.009095\end{array}$ \\
\hline & $\begin{array}{l}0.936293434 \\
0.936234\end{array}$ & $\begin{array}{l}12.685650400 \\
12\end{array}$ & 0.006079369 & $\begin{array}{l}0.477955567 \\
0.478038936\end{array}$ & $\begin{array}{l}0.000905 \\
0.000917\end{array}$ \\
\hline & 0.938223938 & 13.087731 & 0.006272082 & 0.484311018 & 0.000929 \\
\hline & $\begin{array}{l}0.944154444 \\
0.942084942\end{array}$ & $\begin{array}{l}13.5 .53551355 \\
13.96500527\end{array}$ & $\begin{array}{l}0.0064761313 \\
0.000662501\end{array}$ & $\begin{array}{l}0.490787715 \\
0.497479651\end{array}$ & $\begin{array}{l}0.000941 \\
0.000954\end{array}$ \\
\hline & 0.944015444 & $\begin{array}{l}14.44449758 \\
\end{array}$ & $\begin{array}{l}0.00692229 \\
\end{array}$ & $\begin{array}{l}0.504401941 \\
0.504514\end{array}$ & $\begin{array}{l}0.000967 \\
0.0096\end{array}$ \\
\hline & $\begin{array}{l}0.99459454946 \\
0.94787648\end{array}$ & $\begin{array}{l}1.45544554401 \\
15.49805108\end{array}$ & $\begin{array}{l}0.00074627189 \\
0.0747\end{array}$ & $\begin{array}{l}0.5115868686 \\
0.518995856\end{array}$ & 0.000981 \\
\hline & 980695 & 16.0782263 & 0.007705228 & 0.526701085 & \\
\hline & 0.9517777452 & 16.6987352 & 0.008002597 & 0.534703681 & 0.001025 \\
\hline
\end{tabular}




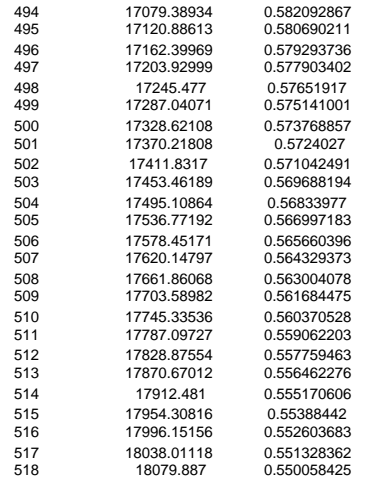

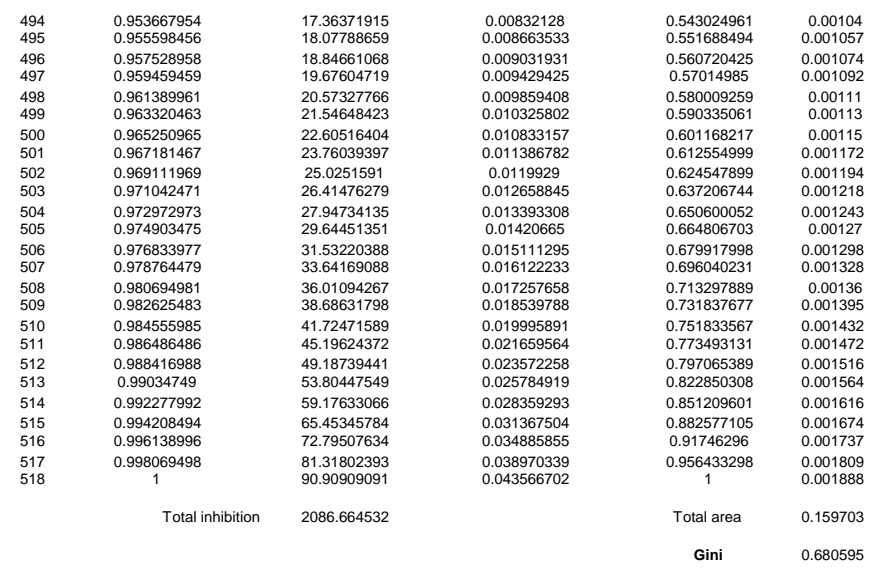


Gini coefficient values for $S=1, a=10$ and $100 \mathrm{nM}, b=0.5,0.8,1.0,1.2$ and 1.5 at concentrations ranging from $0.0001 \mathrm{nM}$ to $10000 \mathrm{nM}$ for experimental accuracy $r=0,1$ and $2 \%$

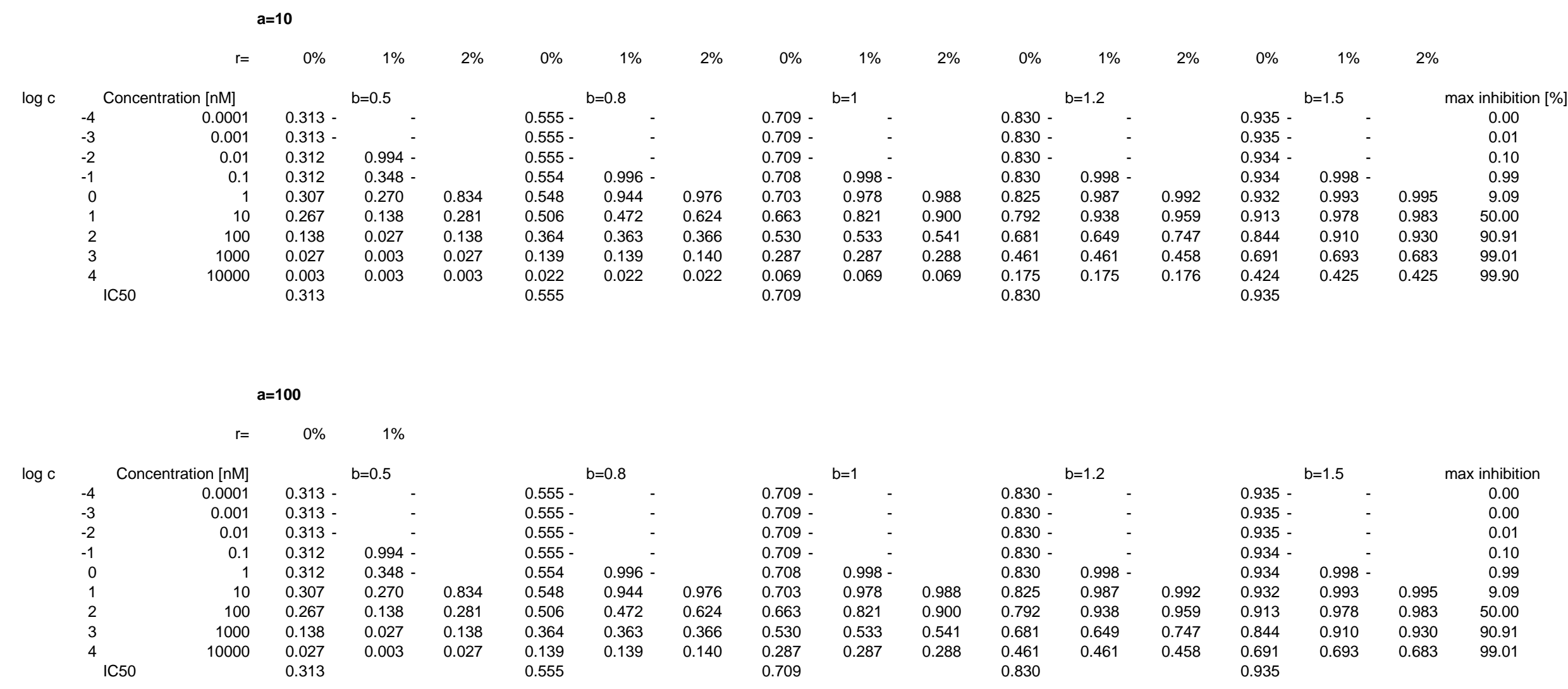




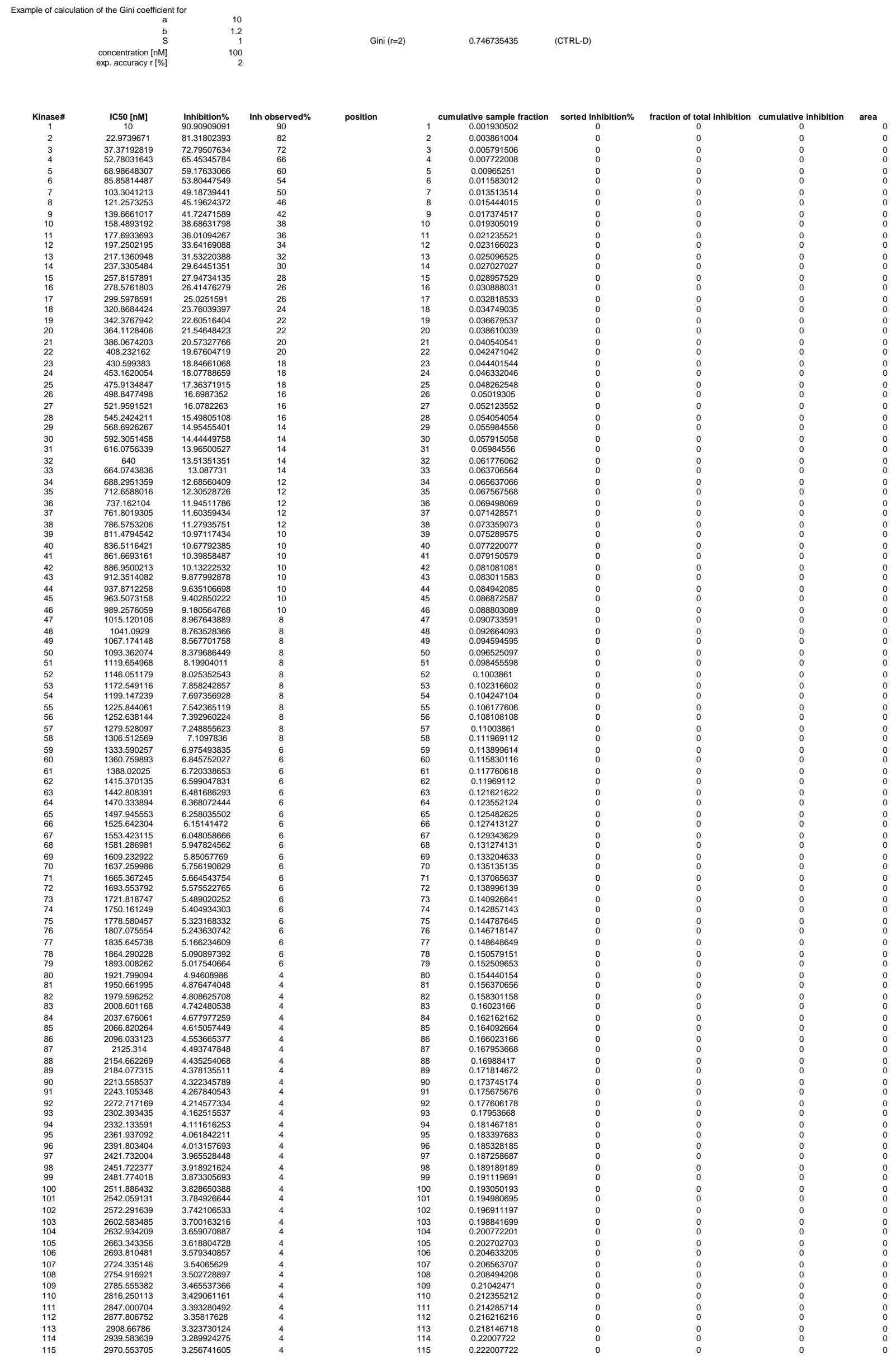




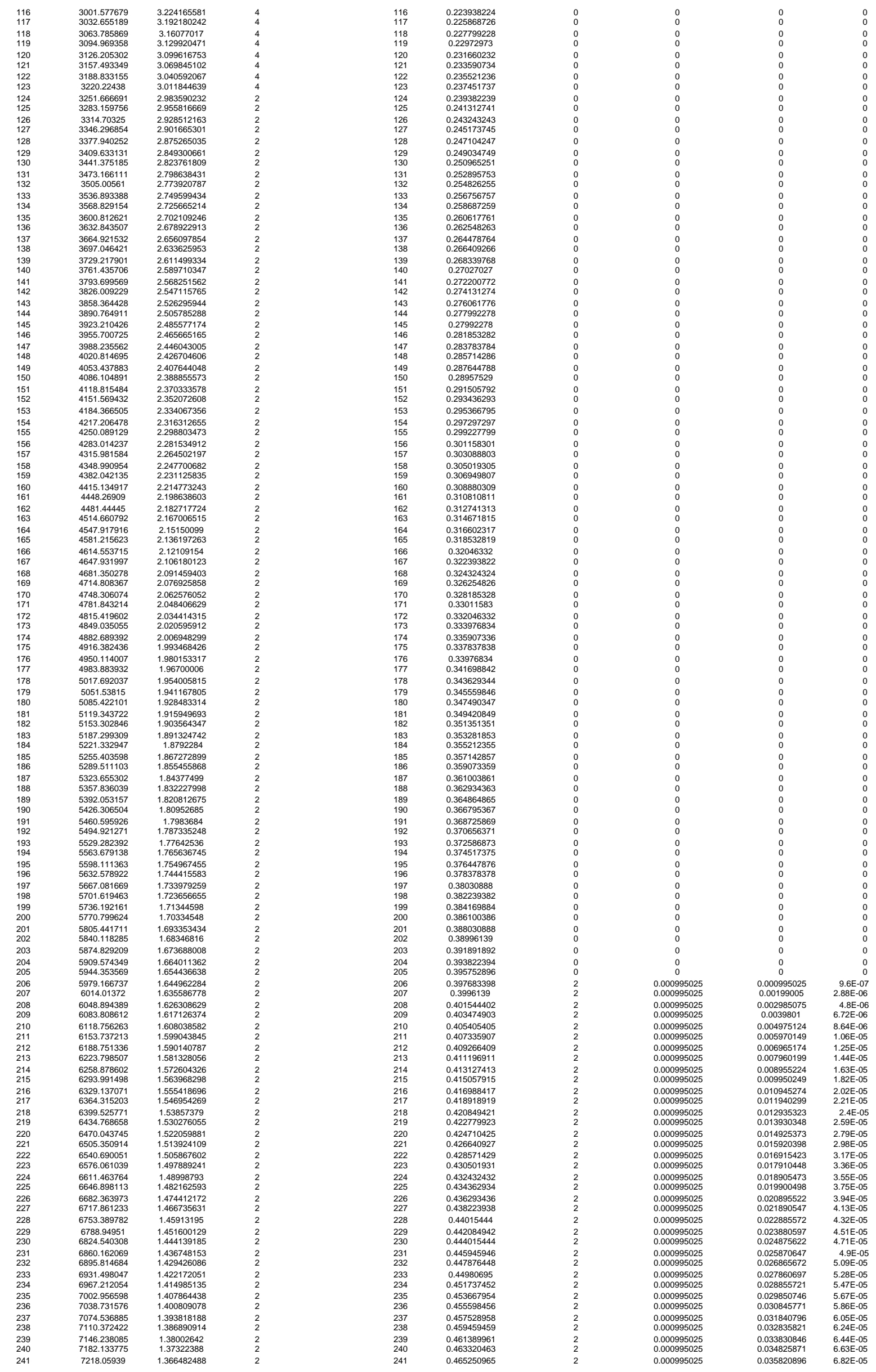




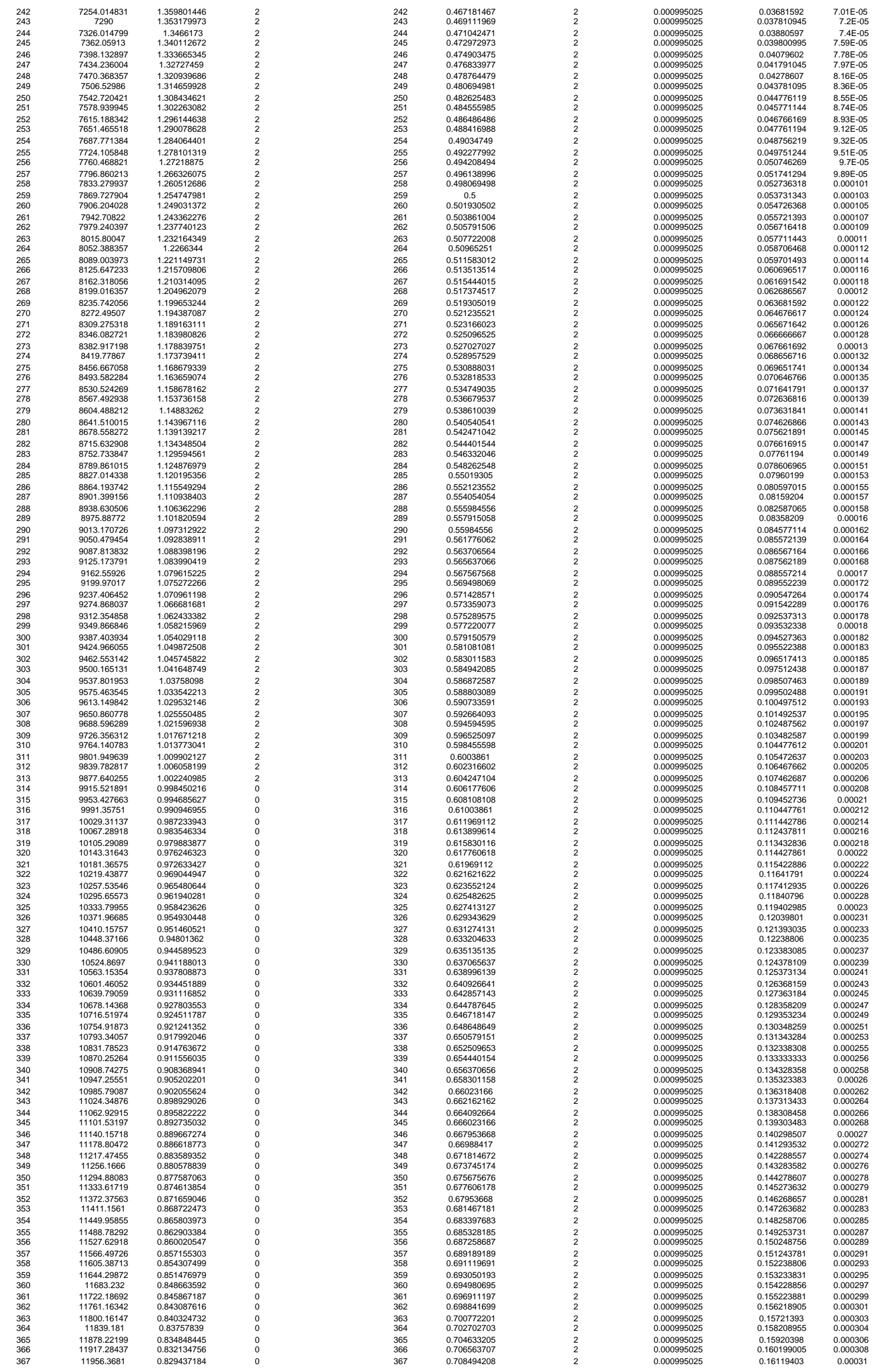




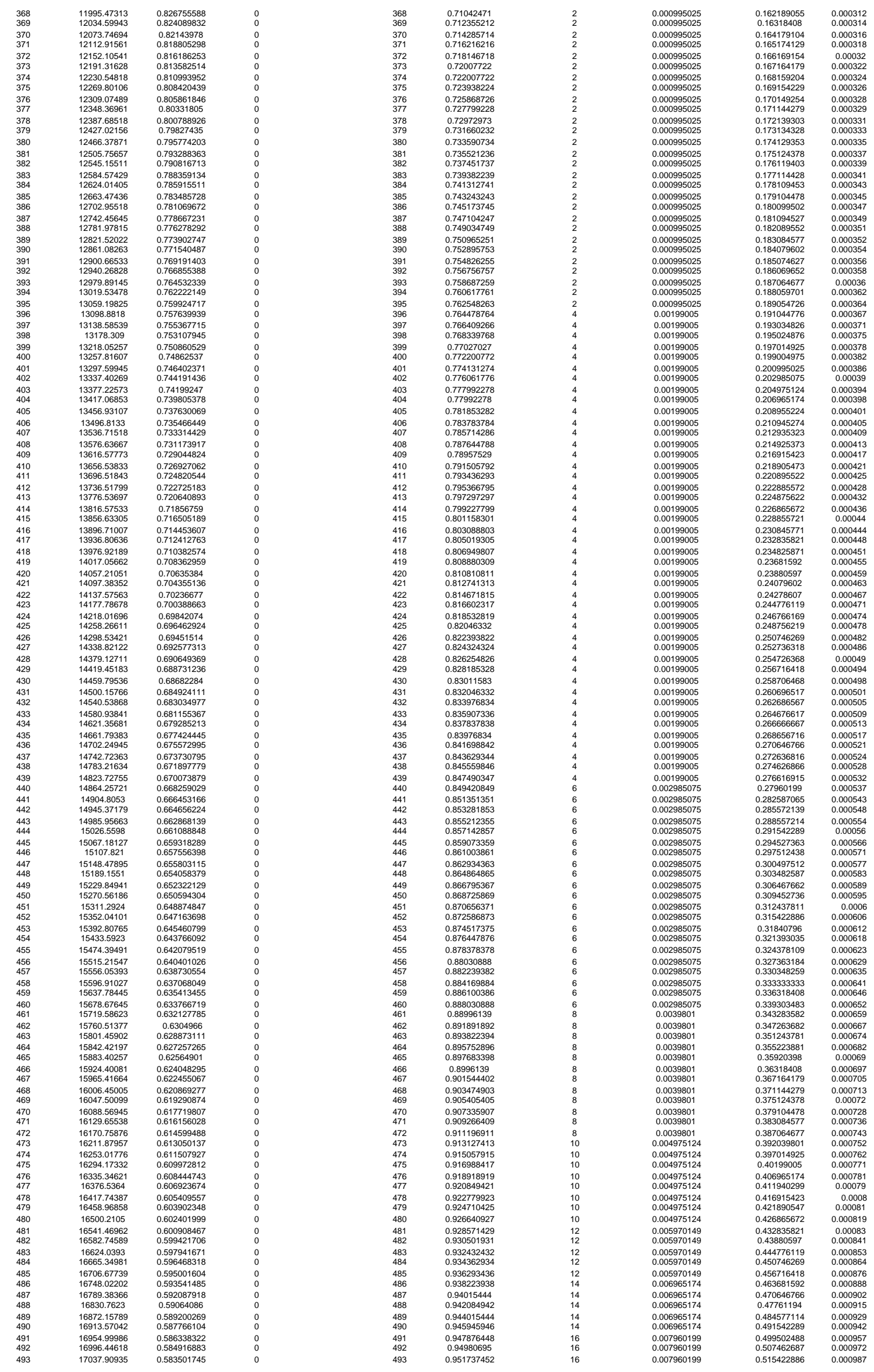




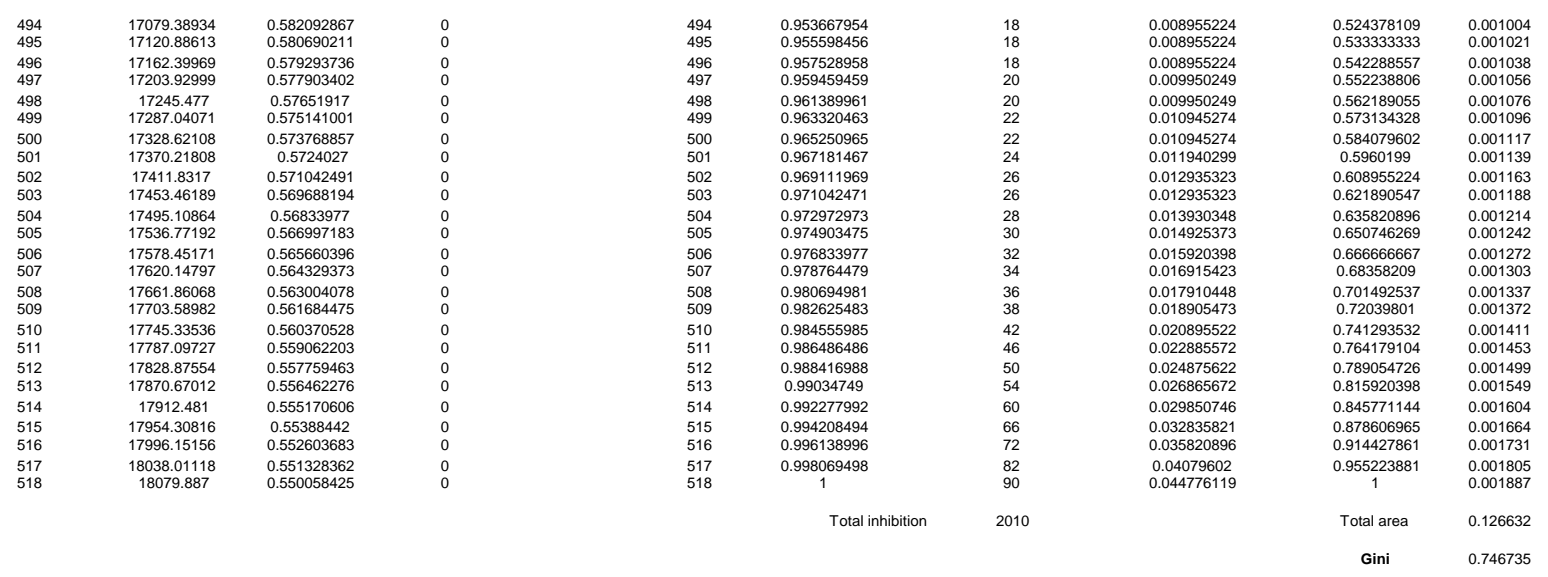




\section{Example of calculation of the Gini coefficient using 1/IC50}


$\begin{array}{rr}\text { a } & 10 \\ \text { b } & 1.2 \\ \text { slope } & 1 \\ \text { concentration [nM] } & 100\end{array}$

Inhib IC50

0.830271771

(CTRL-B)

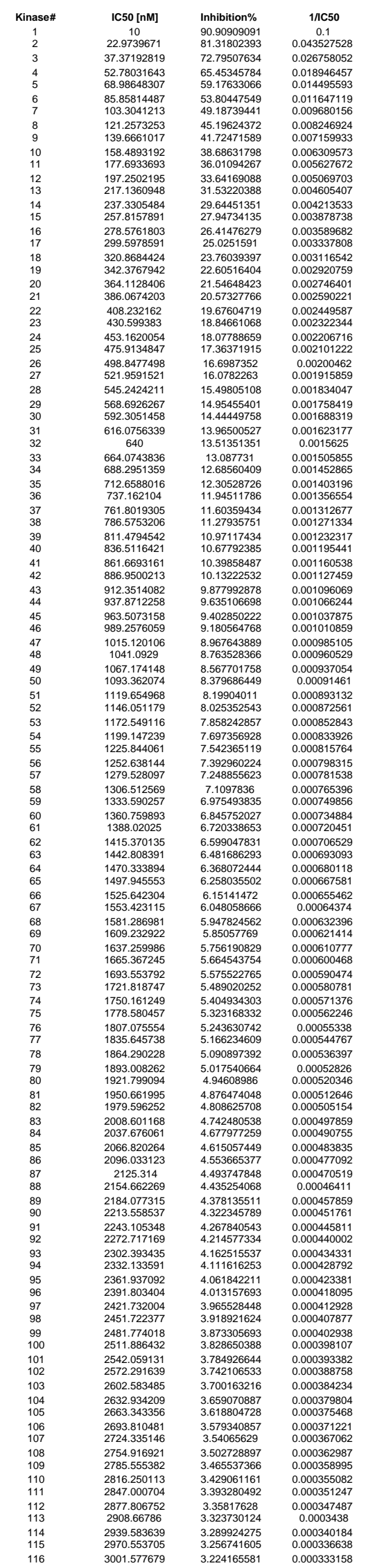

\begin{tabular}{cc} 
position & \multicolumn{1}{c}{ cumulative sample fraction } \\
1 & 0.001930502 \\
2 & 0.003861004 \\
3 & 0.005791506 \\
4 & 0.007722008 \\
5 & 0.00965251 \\
6 & 0.011583012 \\
7 & 0.013513514 \\
8 & 0.015444015 \\
9 & 0.017374517 \\
10 & 0.019305019 \\
11 & 0.021235521 \\
12 & 0.023366023 \\
13 & 0.025096525 \\
14 & 0.027027027 \\
15 & 0.028957529 \\
16 & 0.030888031 \\
17 & 0.032818533 \\
18 & 0.034749035 \\
19 & 0.036679537 \\
20 & 0.038610039 \\
21 & 0.04545451
\end{tabular}

sorted 1/1C50

$5.53101 \mathrm{E}-05$
$5.54385 \mathrm{E}-05$
$5.55674 \mathrm{E}-05$

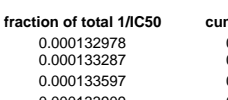

cumulative 1//C50

area

$\begin{array}{ll}0.000399863 & 3.85 \mathrm{E}-07 \\ 0.000533771 & 6.07\end{array}$

$\begin{array}{llll}5.5827 \mathrm{E}-05 & 0.000134221 & 0.000667992 & 9.01 \mathrm{E}-07 \\ 5 & 0.16 \mathrm{E}-06 \\ 5.50888 \mathrm{E} & 0.0053525 & 1.26 \mathrm{E}-06\end{array}$

$\begin{array}{llll}5.59576 \mathrm{E}-05 & 0.000134535 & 0.000802528 & 1.42 \mathrm{E}-06 \\ 5.60888 \mathrm{E}-05 & 0.000134851 & 0.000937378 & 1.68 \mathrm{E}-06\end{array}$

$\begin{array}{lllr}5.62205 \mathrm{E}-05 & 0.000135167 & 0.001072546 & 1.94 \mathrm{E}-06 \\ 5.63528 \mathrm{E}-05 & 0.000135485 & 0.001208031 & 2.2 \mathrm{E}-06 \\ 5 & 0.00013565 & 0.00134386 & 2.27 \mathrm{E}-06\end{array}$

$\begin{array}{llll}5.64857 \mathrm{E}-05 & 0.000135805 & 0.001343836 & 2.46 \mathrm{E}-06 \\ 5.66192 \mathrm{E}-05 & 0.000136126 & 0.001479962 & 2.73 \mathrm{E}-06 \\ 5 & 0.000136772 & 0.00175318 & 3.25 \mathrm{E}-06\end{array}$

$\begin{array}{llll}5.67532 \mathrm{E}-05 & 0.000136448 & 0.00161641 & 2.99 \mathrm{E}-06 \\ 5.68878 \mathrm{E}-05 & 0.000136772 & 0.001753181 & 3.25 \mathrm{E}-06\end{array}$

$\begin{array}{llll}5.7023 E-05 & 0.000137097 & 0.001890278 & 3.52 \mathrm{E}-06 \\ 5.71588 \mathrm{E}-05 & 0.000137423 & 0.002027701 & 3.78 \mathrm{E}-06\end{array}$

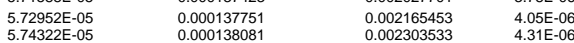

$\begin{array}{llll}5.75698 \mathrm{E}-05 & 0.000138411 & 0.002441944 & 4.58 \mathrm{E}-06 \\ 5.7708 \mathrm{E}-05 & 0.000138744 & 0.002580688 & 4.85 \mathrm{E}-06\end{array}$

$\begin{array}{llll}5.7708 \mathrm{E}-05 & 0.000138744 & 0.002580688 & 4.85 \mathrm{E}-06 \\ 5.78468 \mathrm{E}-05 & 0.000139077 & 0.002719765 & 5.12 \mathrm{E}-\mathrm{C} \\ 5.79862 \mathrm{E}-05 & 0.000139412 & 0.002859178 & 5.39 \mathrm{E}-06\end{array}$

$\begin{array}{lllll}0.042471042 & 5.81226 \mathrm{E}-05 & 0.000139749 & 0.002998927 & 5.55 \mathrm{EE}-06 \\ 0.044401544 & 5.82669 \mathrm{E}-05 & 0.000140087 & 0.003139014 & 5.92 \mathrm{E}-06\end{array}$

$\begin{array}{llllr}0.046332046 & 5.84082 \mathrm{E}-05 & 0.000140427 & 0.003279441 & 6.2 \mathrm{E}-06 \\ 0.048262548 & 5.85501 \mathrm{E}-05 & 0.000140768 & 0.003420209 & 6.47 \mathrm{E}-06\end{array}$

$\begin{array}{lllll}0.05019305 & 5.86926 \mathrm{E}-05 & 0.000141111 & 0.00356132 & 6.74 \mathrm{E}-06 \\ 0.052123552 & 5.88358 \mathrm{E}-05 & 0.000141455 & 0.003702775 & 7.01 \mathrm{E}-06\end{array}$

$0.052123552 \quad 5.88358 \mathrm{E}-05$

$\begin{array}{lllll}0.054054054 & 5.80797 \mathrm{E}-05 & 0.000141801 & 0.003844576 & 7.29 \mathrm{E}-06 \\ 0.05598456 & 5.91241 \mathrm{E}-05 & 0.000142148 & 0.003986725 & 7.56 \mathrm{E}-06 \\ 0.057915058 & 5.92692 \mathrm{E}-05 & 0.000142497 & 0.004129222 & 7.83 \mathrm{E}-06\end{array}$

$\begin{array}{llccc}0.05984556 & 5.9415 \mathrm{E}-05 & 0.000142848 & 0.004272069 & 8.11 \mathrm{E}-06 \\ 0.061776062 & 5.95614 \mathrm{E}-05 & 0.0001432 & 0.004415269 & 8.39 \mathrm{E}-06\end{array}$

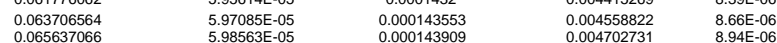

$\begin{array}{llllr}0.067567568 & 6.00047 \mathrm{E}-05 & 0.000144265 & 0.004846997 & 9.22 \mathrm{E}-06 \\ 0.069498069 & 6.01539 \mathrm{E}-05 & 0.000144624 & 0.004991621 & 9.5 \mathrm{E}-06\end{array}$

$\begin{array}{lllll}0.071428571 & 6.03036 \mathrm{E}-05 & 0.000144984 & 0.005136605 & 9.78 \mathrm{E}-06 \\ 0.073359073 & 6.04541 \mathrm{E}-05 & 0.000145346 & 0.005281951 & 1.01 \mathrm{E}-05\end{array}$

$\begin{array}{lllll}0.073359073 & 6.04541 \mathrm{E}-05 & 0.000145346 & 0.005281951 & 1.01 \mathrm{E}-05 \\ 0.075280575 & 6.06053 \mathrm{E}-05 & 0.000145709 & 0.00542766 & 1.03 \mathrm{E}-05 \\ 0.77220077 & 6.07571 \mathrm{E}-05 & 0.000146074 & 0.05573734 & 1.06 E-05\end{array}$

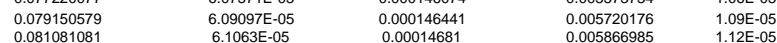

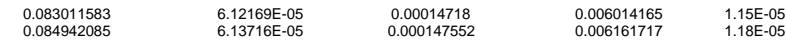

$\begin{array}{lllrr}0.086872587 & 6.1527 \mathrm{E}-05 & 0.000147552 & 0.000161717 & 1.18 \mathrm{E}-05 \\ 0.088803089 & 6.16832 \mathrm{E}-05 & 0.00014925 & 0.006309642 & 1.2 \mathrm{E}-05 \\ 0.05014301 & 0.006457943 & 1.23 \mathrm{E}-05\end{array}$

$\begin{array}{lllll}0.090733591 & 6.184 \mathrm{E}-\mathrm{-05} & 0.000148678 & 0.006606621 & 1.2 \mathrm{EE}-\mathrm{C} 5 \\ 0.092654093 & 6.19976 \mathrm{E}-05 & 0.000189057 & 0.006755678 & 1.29 \mathrm{E}-05\end{array}$

$\begin{array}{lllll}0.00264993 & 6.19976 \mathrm{E}-05 & 0.000149057 & 0.00679578 & 1.25 \mathrm{E}-5 \\ 0.094594595 & 6.21559 \mathrm{E}-05 & 0.000149437 & 0.006905115 & 1.32 \mathrm{E}-05 \\ 0.096525097 & 6.2315 \mathrm{E}-05 & 0.00014982 & 0.007054935 & 1.35 \mathrm{E}-05\end{array}$

$\begin{array}{lllll}0.096525097 & 6.2315 \mathrm{E}-05 & 0.00014982 & 0.007054935 & 1.35 \mathrm{E}-05 \\ 0.098455598 & 6.24748 \mathrm{E}-05 & 0.000150204 & 0.007205139 & 1.38 \mathrm{E}-05 \\ 0.1003861 & 6.26354 \mathrm{E}-05 & 0.00015059 & 0.007355729 & 1.41 \mathrm{E}-05\end{array}$

$\begin{array}{lllll}0.102316002 & 6.27967 \mathrm{E}-05 & 0.001507 & 0.007506708 & 1.43 \mathrm{E}-05 \\ 0.104247104 & 6.29588 \mathrm{E}-05 & 0.000151368 & 0.007658075 & 1.46 \mathrm{E}-05 \\ 0.106177606 & 6.31217 \mathrm{E}-05 & 0.000151759 & 0.007809835 & 1.49 \mathrm{E}-05\end{array}$

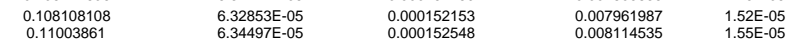

$\begin{array}{lllll}0.11003861 & 6.34497 \mathrm{E}-05 & 0.000152548 & 0.008114535 & 1.55 \mathrm{E}-05 \\ 0.111969112 & 6.36149 \mathrm{E}-05 & 0.000152945 & 0.00826748 & 1.58 \mathrm{E}-05 \\ 0.113899614 & 6.37809 \mathrm{E}-05 & 0.000153344 & 0.008420825 & 1.61 \mathrm{E}-05\end{array}$

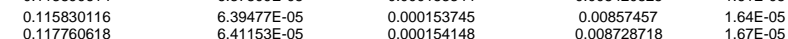

$\begin{array}{llllr}0.11969112 & 6.42837 \mathrm{E}-05 & 0.000154553 & 0.008883271 & 1.7 \mathrm{E}-05 \\ 0.121621622 & 6.44529 \mathrm{E}-05 & 0.00015496 & 0.009038231 & 1.73 \mathrm{E}-05\end{array}$

$\begin{array}{lllll}0.121621622 & 6.44529 \mathrm{E}-05 & 0.00015496 & 0.009038231 & 1.73 \mathrm{E}-05 \\ 0.123552124 & 6.46229 \mathrm{E}-05 & 0.000155369 & 0.00919399 & 1.76 \mathrm{E}-05 \\ 0.125482625 & 6.47937 \mathrm{E}-05 & 0.000155779 & 0.009349379 & 1.79 \mathrm{E}-05\end{array}$

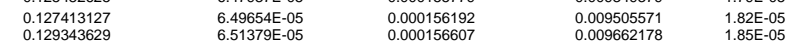

$\begin{array}{lllll}0.131274131 & 6.53113 \mathrm{E}-05 & 0.000157024 & 0.009819201 & 1.88 \mathrm{E}-05 \\ 0.133204633 & 6.54855 \mathrm{E}-05 & 0.000157442 & 0.009976644 & 1.91 \mathrm{E}-05\end{array}$

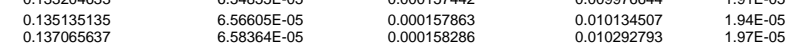

$\begin{array}{lllrr}0.137065637 & 6.58364 \mathrm{E}-05 & 0.000158286 & 0.010292793 & 1.97 \mathrm{E}-05 \\ 0.138996139 & 6.60132 \mathrm{E}-05 & 0.000158711 & 0.010451505 & 2 \mathrm{E}-05 \\ 0.140926641 & 661909 \mathrm{E}-05 & 0.000159138 & 0.010610643 & 203 \mathrm{E}-05\end{array}$

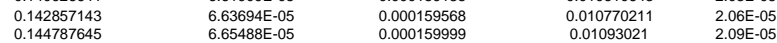

$\begin{array}{lllll}0.144787645 & 6.65488 \mathrm{E}-05 & 0.000159999 & 0.01093021 & 2.09 \mathrm{E}-05 \\ 0.146718147 & 6.67291 \mathrm{E}-05 & 0.000160433 & 0.011090642 & 2.13 \mathrm{E}-05 \\ 0.148648649 & 6.69103 \mathrm{E}-05 & 0.00160868 & 0.1125151 & 2.16 \mathrm{E}-05\end{array}$

$\begin{array}{lllll}0.148648649 & 6.6902 \mathrm{E}-05 & 0.000160868 & 0.01125151 & 2.16 \mathrm{E}-05 \\ 0.150579151 & 6.70925 \mathrm{E}-05 & 0.000161306 & 0.011412816 & 2.19 \mathrm{E}-05 \\ 0.152516 & 67595 & 0.01736751 & 2.25-05\end{array}$

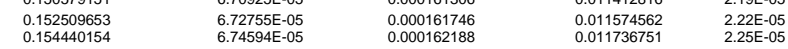

$\begin{array}{lllll}0.156370656 & 6.76443 E-05 & 0.000162633 & 0.011899383 & 2.28 E-05 \\ 0.158301158 & 6.78301 E-05 & 0.000163079 & 0.012062463 & 2.31 E-05\end{array}$

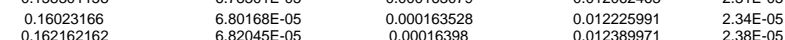

$\begin{array}{lllll}0.162162162 & 6.82045 \mathrm{E}-05 & 0.00016398 & 0.012389971 & 2.38 \mathrm{E}-05 \\ 0.164092664 & 6.83931 \mathrm{E}-05 & 0.000164433 & 0.012554404 & 2.41 \mathrm{E}-05 \\ 0.1662023166 & 6.85827 \mathrm{E}-05 & 0.000164889 & 0.012719293 & 2.44 \mathrm{E}-05\end{array}$

$\begin{array}{llllr}0.167953668 & 6.87732 \mathrm{E}-05 & 0.000165347 & 0.01288464 & 2.47 \mathrm{E}-05 \\ 0.16988417 & 6.89648 \mathrm{E}-05 & 0.000165807 & 0.013050447 & 2.5 \mathrm{E}-05\end{array}$

$\begin{array}{llllr}0.16988417 & 6.89648 \mathrm{E}-05 & 0.000165807 & 0.013050447 & 2.5 \mathrm{E}-05 \\ 0.171814672 & 6.91573 \mathrm{E}-05 & 0.00016627 & 0.013216717 & 2.54 \mathrm{E}-05 \\ 0.173745174 & 6.93508 \mathrm{E}-05 & 0.000166736 & 0.013383453 & 257 \mathrm{E}-05\end{array}$

$\begin{array}{llllr}0.175675676 & 6.95453 \mathrm{E}-05 & 0.000167203 & 0.013550656 & 2.6 \mathrm{E}-05 \\ 0.177606178 & 6.97407 \mathrm{E}-05 & 0.000167673 & 0.013718329 & 2.63 \mathrm{E}-05\end{array}$

$\begin{array}{llllr}0.17953668 & 6.99372 \mathrm{E}-05 & 0.000168146 & 0.013886475 & 2.66 \mathrm{E}-05 \\ 0.181467181 & 7.01348 \mathrm{E}-05 & 0.00016862 & 0.014055095 & 2.7 \mathrm{E}-05 \\ 0.185328155 & 7.05329 \mathrm{E} & 0.000169578 & 0.0143937 & 27 \mathrm{E}-05\end{array}$

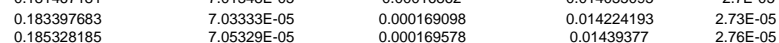

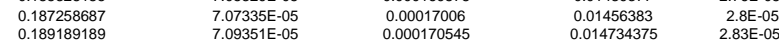

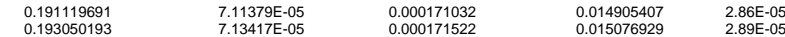

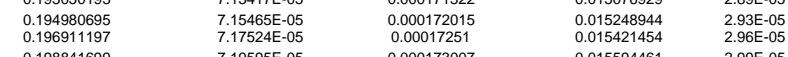

$\begin{array}{lllll}0.198841699 & 7.19595 \mathrm{E}-05 & 0.000173007 & 0.015594461 & 2.99 \mathrm{E}-05 \\ 0 & 7.2266 \mathrm{E}-05 & 0.0017309 & 0.0159469 & 3.03 E\end{array}$

$\begin{array}{lllll}0.200772201 & 7.21676 \mathrm{E}-05 & 0.000173508 & 0.015767969 & 3.03 \mathrm{E}-05 \\ 0.202702703 & 7.23768 \mathrm{E}-05 & 0.000174011 & 0.01594198 & 3.06 \mathrm{E}-05\end{array}$

$\begin{array}{lllll}0.204633205 & 7.25872 \mathrm{E}-05 & 0.000174517 & 0.016116496 & 3.09 \mathrm{E}-05 \\ 0.206563707 & 7.27987 \mathrm{E}-05 & 0.000175025 & 0.016291521 & 3.13 \mathrm{E}-05\end{array}$

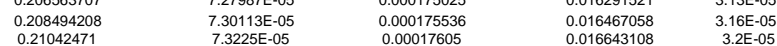

$\begin{array}{lllll}0.212355212 & 7.34399 \mathrm{E}-05 & 0.000176567 & 0.016819674 & 3.23 \mathrm{E}-05 \\ 0.214285714 & 7.36559 \mathrm{E}-05 & 0.000177086 & 0.016996761 & 3.26 \mathrm{E}-05\end{array}$

$\begin{array}{llllr}0.216216216 & 7.38732 \mathrm{E}-05 & 0.000177608 & 0.017174369 & 3.3 \mathrm{E}-05 \\ 0.218146718 & 7.40916 \mathrm{E}-05 & 0.000178134 & 0.017352503 & 3.33 \mathrm{E}-05\end{array}$

$\begin{array}{llllr}0.218146718 & 7.40916 \mathrm{E}-05 & 0.000178134 & 0.017352503 & 3.33 \mathrm{E}-05 \\ 0.22007722 & 7.4311 \mathrm{E}-05 & 0.000178661 & 0.017531164 & 3.37 \mathrm{E}-05 \\ 0.222007722 & 7.45319 \mathrm{E}-05 & 0.000179192 & 0.017710356 & 3.4 \mathrm{E}-05 \\ 0.22303824 & 7.4753 \mathrm{E}-05 & 0.000179726 & 0.0178008 & 3.44 \mathrm{E}-05\end{array}$ 


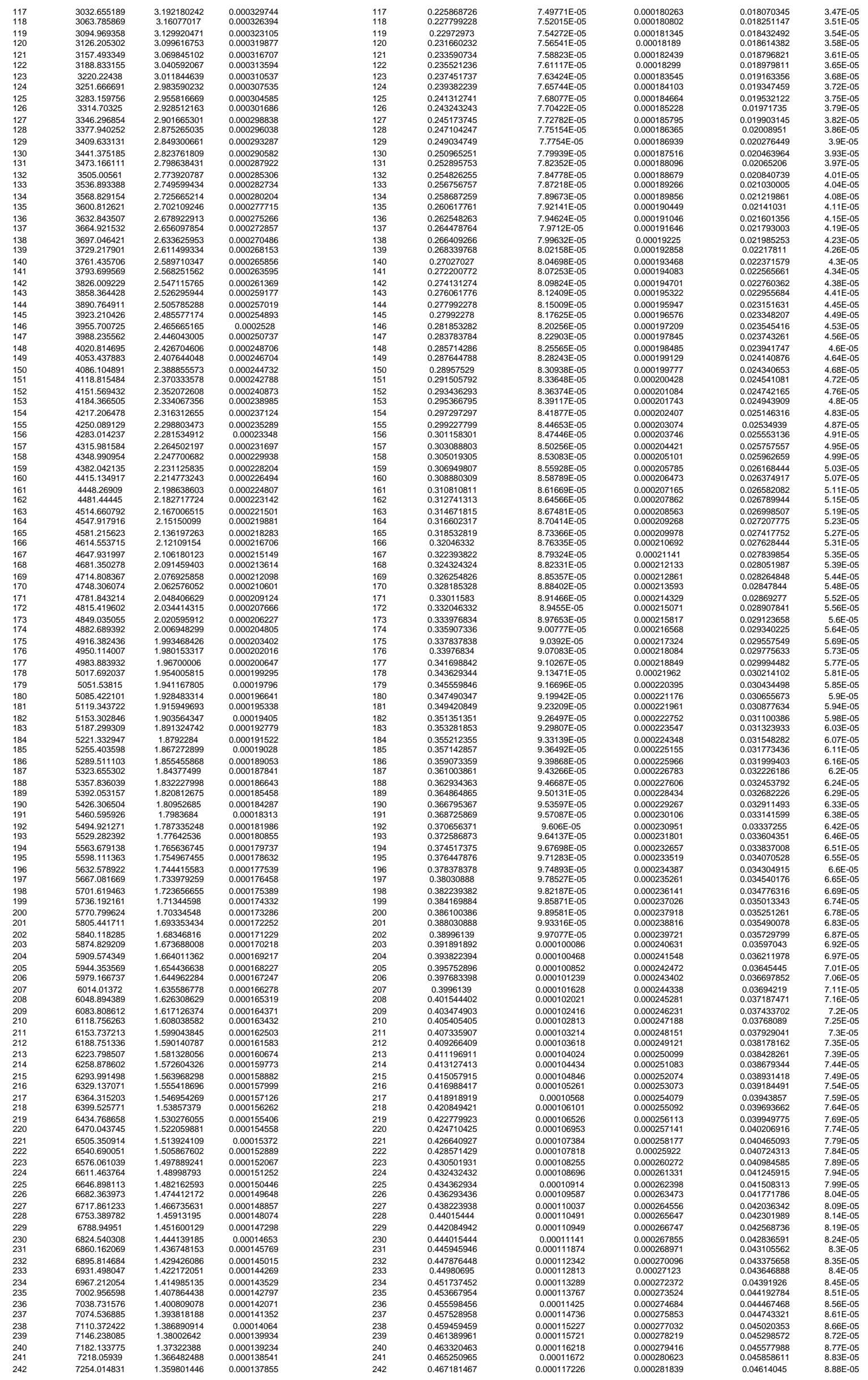




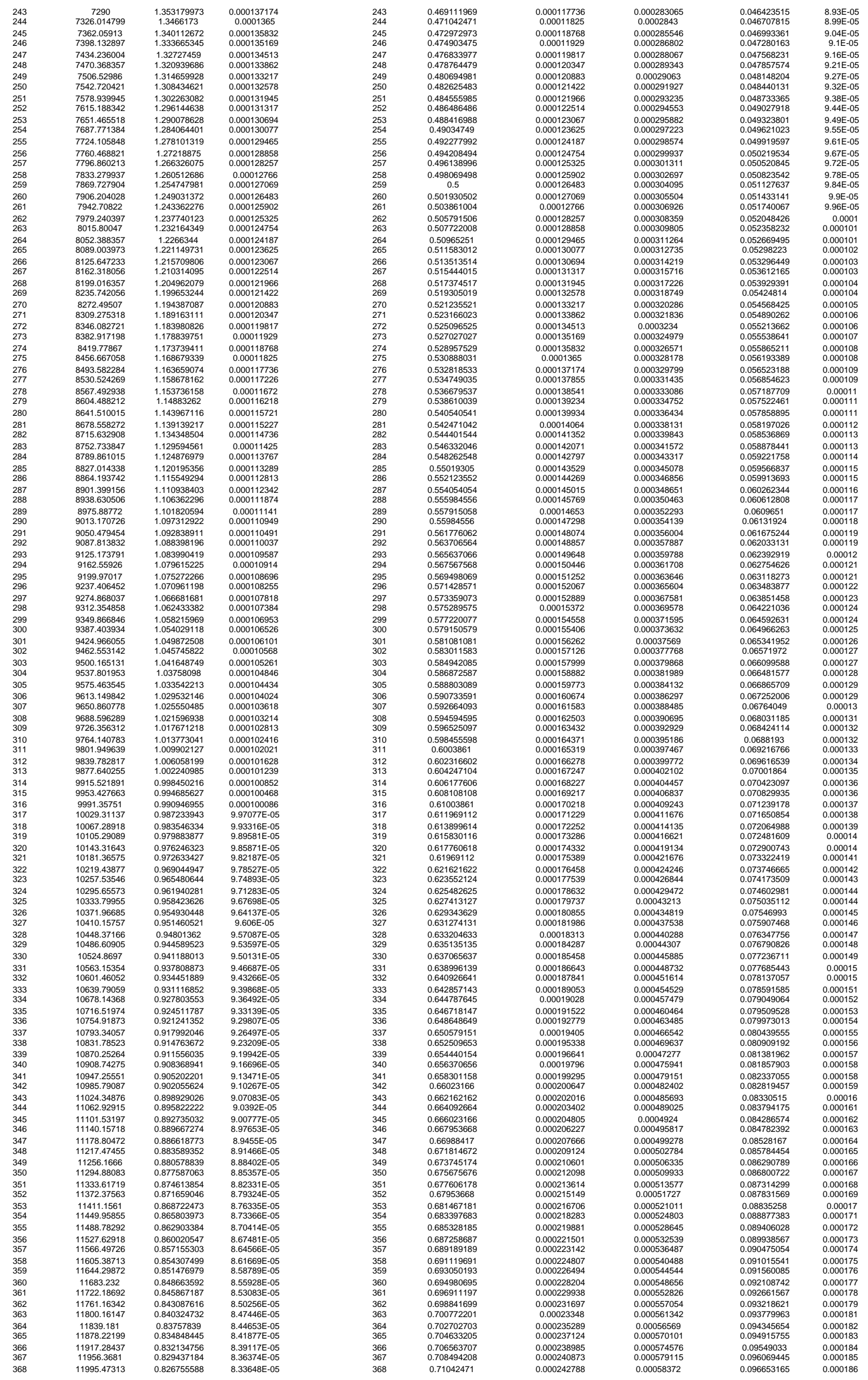




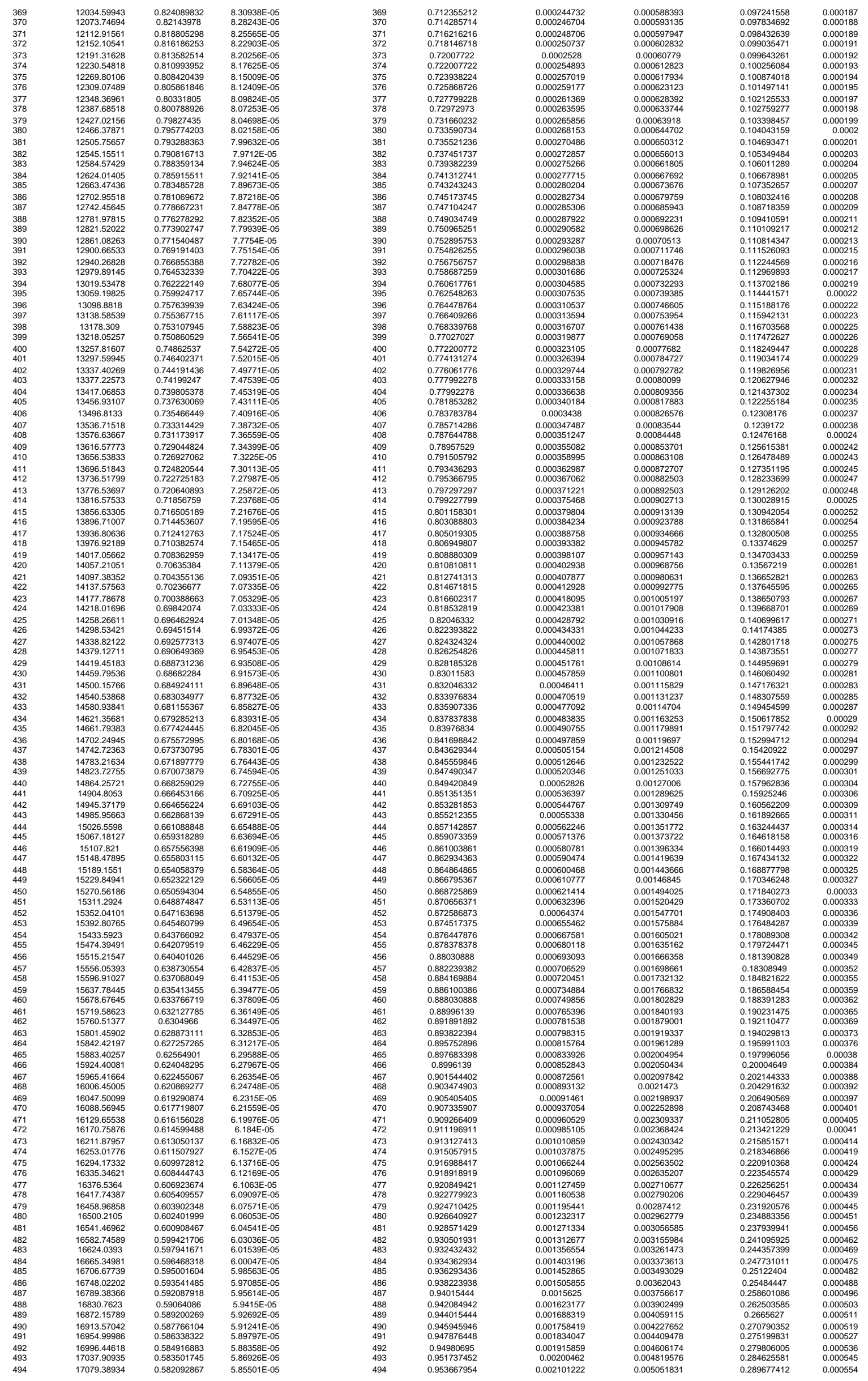




\begin{tabular}{|c|c|c|c|}
\hline 495 & 17120.88613 & 0.580690211 & $5.84082 \mathrm{E}-05$ \\
\hline 496 & 17162.39969 & 0.579293736 & $\begin{array}{l}5.82669 \mathrm{E}-05 \\
58123 \mathrm{~F}-05\end{array}$ \\
\hline 497 & 17203.92999 & 0.577903402 & $5.81263 \mathrm{E}-05$ \\
\hline 498 & 17245.477 & 0.57651917 & $5.79862 \mathrm{E}-05$ \\
\hline 499 & 17287.04071 & 0.575141001 & $5.78468 \mathrm{E}-05$ \\
\hline 500 & 17328.62108 & 0.573768857 & $5.7708 \mathrm{E}-05$ \\
\hline 501 & 17370.21808 & 0.5724027 & $5.75698 \mathrm{E}-05$ \\
\hline 502 & 17411.8317 & 0.571042491 & $5.74322 \mathrm{E}-05$ \\
\hline 503 & 17453.46189 & 0.569688194 & $5.72952 \mathrm{E}-05$ \\
\hline 504 & 17495.10864 & 0.56833977 & $5.71588 \mathrm{E}-05$ \\
\hline 505 & 17536.77192 & 0.566997183 & $5.7023 \mathrm{E}-05$ \\
\hline 506 & 17578.45171 & 0.565660396 & $5.68878 \mathrm{E}-05$ \\
\hline 507 & 17620.14797 & 0.564329373 & $5.67532 \mathrm{E}-05$ \\
\hline 508 & 17661.86068 & 0.563004078 & $5.66192 \mathrm{E}-05$ \\
\hline 509 & 17703.58982 & 0.561684475 & $5.64857 \mathrm{E}-05$ \\
\hline 510 & 17745.33536 & 0.560370528 & $5.63528 \mathrm{E}-05$ \\
\hline 511 & 17787.09727 & 0.559062203 & $5.62205 \mathrm{E}-05$ \\
\hline 512 & 17828.87554 & 0.557759463 & $5.60888 \mathrm{E}-05$ \\
\hline 513 & 17870.67012 & 0.556462276 & $5.59576 \mathrm{E}-05$ \\
\hline 514 & 17912.481 & 0.555170606 & $5.5827 \mathrm{E}-05$ \\
\hline 515 & 17954.30816 & 0.55388442 & $5.56969 \mathrm{E}-05$ \\
\hline 516 & 17996.15156 & 0.552603683 & $5.55674 \mathrm{E}-05$ \\
\hline 517 & 18038.01118 & 0.551328362 & $5.54385 \mathrm{E}-05$ \\
\hline 518 & 18079.887 & 0.550058425 & $5.53101 \mathrm{E}-05$ \\
\hline
\end{tabular}

\begin{tabular}{|c|c|c|}
\hline 495 & 0.955598456 & 0.002206716 \\
\hline 496 & 0.957528958 & 0.002322344 \\
\hline 497 & 0.959459459 & 0.002449587 \\
\hline 498 & 0.961389961 & 0002590221 \\
\hline 499 & 0.963320463 & 0.002746401 \\
\hline 500 & 0.965250965 & 0.002920759 \\
\hline & 0.967181467 & 0.003116542 \\
\hline 502 & 0.969111969 & 0.003337808 \\
\hline & 0.971042471 & 0.003589682 \\
\hline 504 & 0.972972973 & 0.003878738 \\
\hline 505 & 0.974903475 & 0.004213533 \\
\hline 506 & 0.976833977 & 0.004605407 \\
\hline 507 & 0.978764479 & 0.005069703 \\
\hline 508 & 0.980694981 & 0.005627672 \\
\hline 509 & 0.982625483 & 0.006309573 \\
\hline 510 & 0.984555985 & 0.007159933 \\
\hline & 0.986486486 & 0.008246924 \\
\hline 512 & 0.988416988 & 0.009680156 \\
\hline 513 & 0.99034749 & 0.011647119 \\
\hline 514 & 0.992277992 & 0.014495593 \\
\hline 515 & 0.994208494 & 0.018946457 \\
\hline 516 & 0.996138996 & 0.026758052 \\
\hline \multirow{3}{*}{518} & 0.998069498 & 0.043527528 \\
\hline & & 0.1 \\
\hline & Total & 0.415932781 \\
\hline
\end{tabular}

\begin{tabular}{|c|c|c|}
\hline & & \\
\hline 0.005305464 & 0.294982876 & 0.000564 \\
\hline $\begin{array}{l}0.005583346 \\
0.005889381\end{array}$ & $\begin{array}{l}0.300566336 \\
0.306455717\end{array}$ & $\begin{array}{l}0.000575 \\
0.000586\end{array}$ \\
\hline 0.006227499 & 0.312683217 & 0.000598 \\
\hline 0.006602993 & 0.31928621 & 0.00061 \\
\hline 0.007022189 & 0.326308399 & 0.000623 \\
\hline 0.007492898 & 0.333801297 & 0.000637 \\
\hline $\begin{array}{l}0.008024873 \\
0.008630439\end{array}$ & $\begin{array}{c}0.34182617 \\
0.350456609\end{array}$ & $\begin{array}{l}0.000662 \\
0.000668\end{array}$ \\
\hline $\begin{array}{l}0.00863040399 \\
0.009325397\end{array}$ & 0.359782006 & 0.000686 \\
\hline 0.010130321 & 0.369912327 & 0.000704 \\
\hline 0.011072478 & 0.380984805 & 0.000725 \\
\hline 0.012188755 & $\begin{array}{l}0.39317356 \\
0\end{array}$ & 0.000747 \\
\hline 0.013530244 & 0.406703804 & 0.000772 \\
\hline $\begin{array}{l}0.015169695 \\
0.01721416\end{array}$ & $\begin{array}{l}0.4218734499 \\
0.439087659\end{array}$ & $\begin{array}{r}0.0008 \\
0.000831\end{array}$ \\
\hline 0.019827541 & 0.458915201 & 0.000867 \\
\hline 0.023273366 & 0.482188567 & 0.000908 \\
\hline $\begin{array}{l}0.028002406 \\
0.034850807\end{array}$ & $\begin{array}{l}0.510190973 \\
0.545041781\end{array}$ & $\begin{array}{l}0.000958 \\
0.001019\end{array}$ \\
\hline 0.045551728 & 0.590593509 & 0.001096 \\
\hline 0.064332636 & 0.654926145 & 0.001202 \\
\hline $\begin{array}{l}0.104650391 \\
0.240423464\end{array}$ & 0.759576536 & $\begin{array}{l}0.001365 \\
0.001698\end{array}$ \\
\hline & Total area & 0.084864 \\
\hline & & \\
\hline & Gini & 0.830272 \\
\hline
\end{tabular}


Testing sensitivity of the Gini coefficient to changes in the value of slope $S$

\begin{tabular}{llll}
\hline \multicolumn{1}{c}{ Slope $\mathrm{S}=$} & 0.9 & 1.0 & 1.1 \\
\hline $\begin{array}{l}\text { Parameters } \\
\mathrm{a}=10 \mathrm{~b}=0.5 \quad \mathrm{r}=2 \mathrm{c}=100\end{array}$ & 0.124 & 0.138 & 0.153 \\
$\mathrm{a}=10 \mathrm{~b}=0.5 \quad \mathrm{r}=2 \mathrm{c}=1000$ & 0.028 & 0.027 & 0.025 \\
$\mathrm{a}=10 \mathrm{~b}=1 \mathrm{r}=2 \mathrm{c}=100$ & 0.463 & 0.541 & 0.568 \\
$\mathrm{a}=10 \mathrm{~b}=1 \mathrm{r}=2 \mathrm{c}=1000$ & 0.258 & 0.288 & 0.317 \\
$\mathrm{a}=10 \mathrm{~b}=1.5 \mathrm{r}=2 \mathrm{c}=100$ & 0.896 & 0.930 & 0.949 \\
$\mathrm{a}=10 \mathrm{~b}=1.5 \mathrm{r}=2 \mathrm{c}=1000$ & 0.621 & 0.683 & 0.770 \\
\hline
\end{tabular}




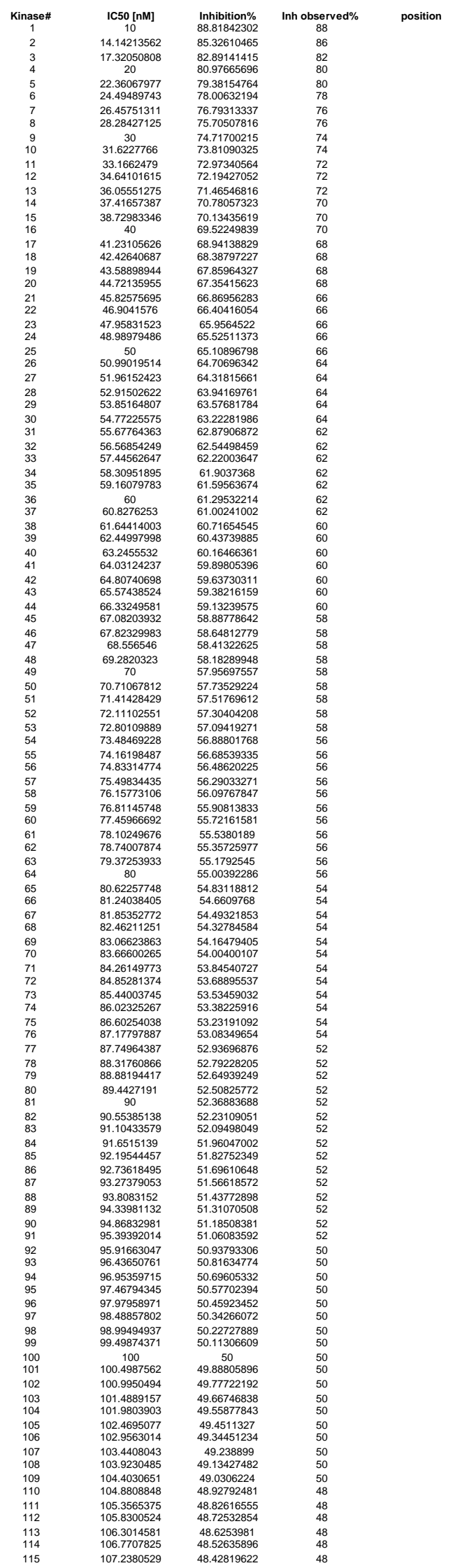

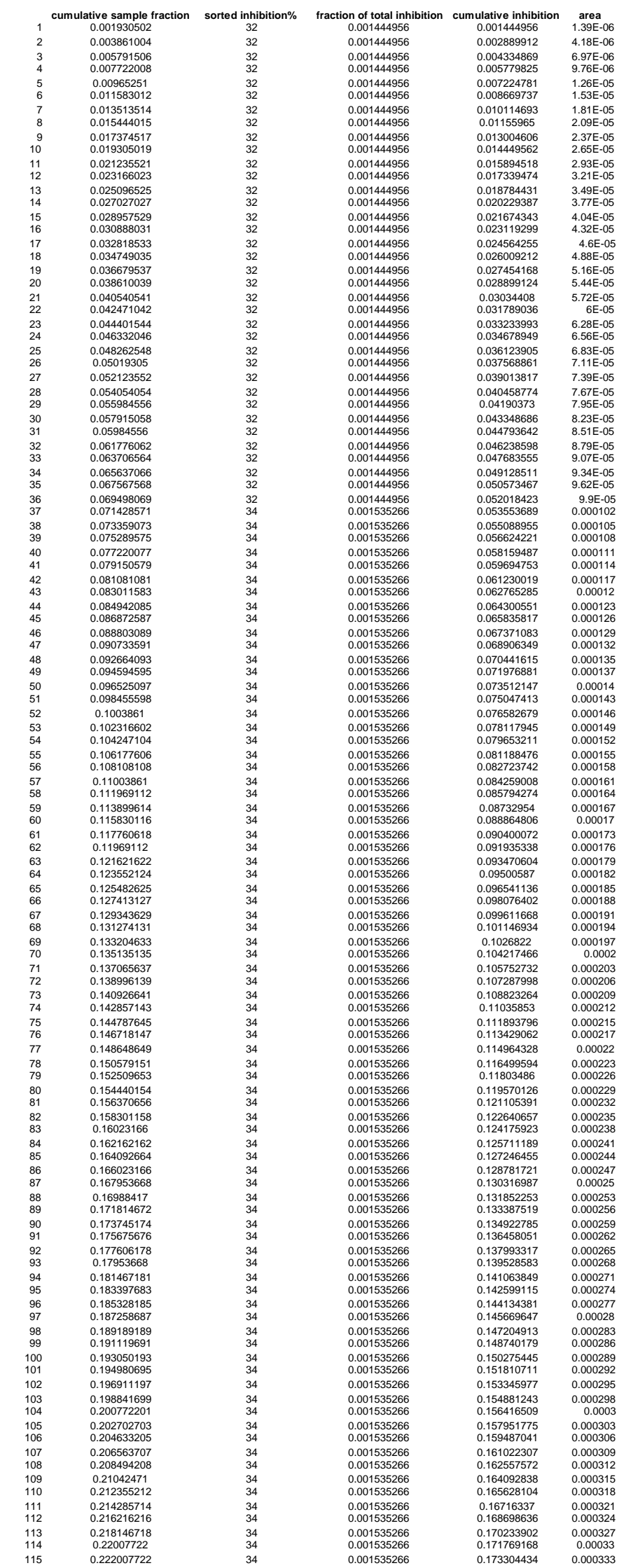




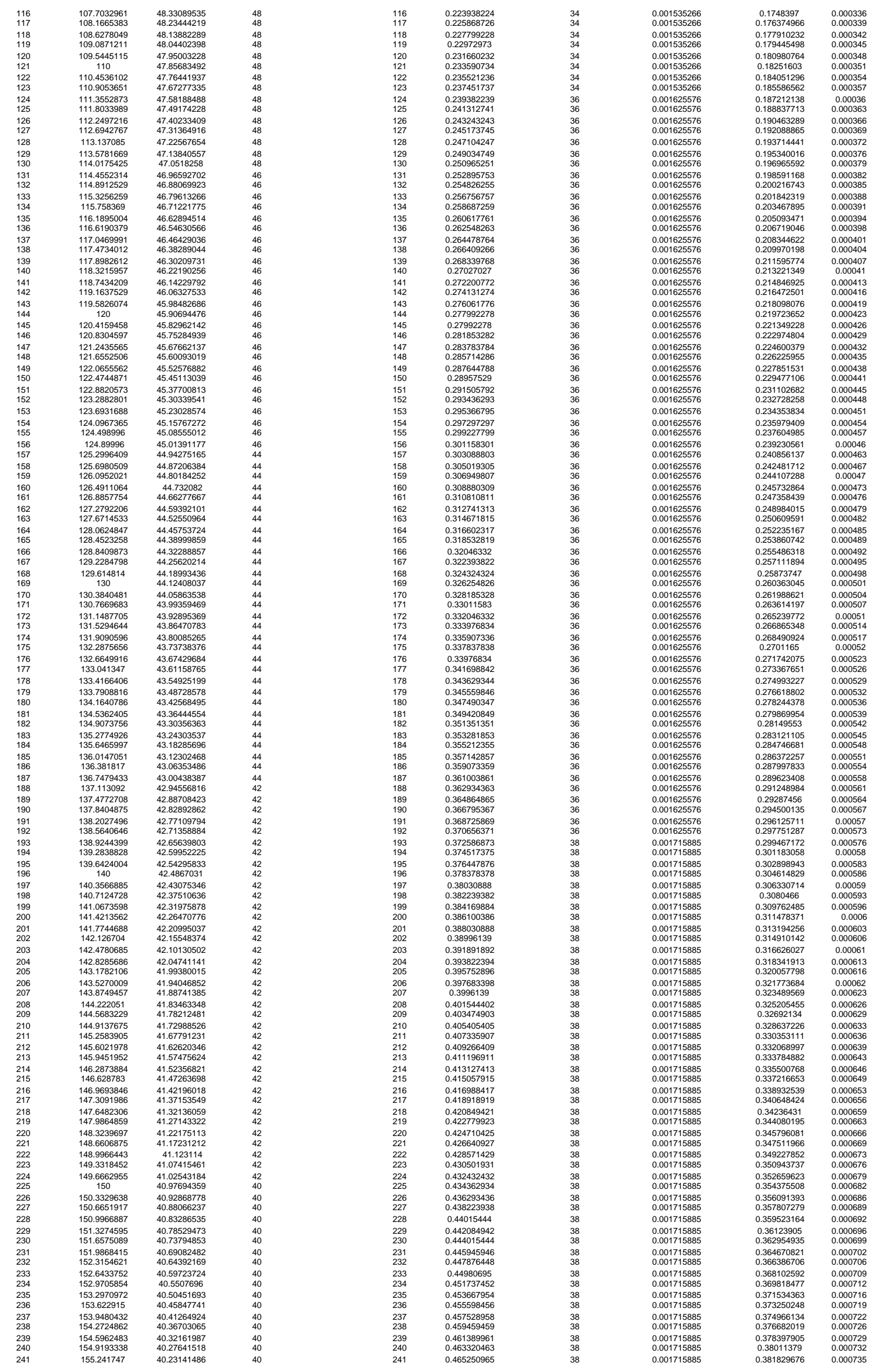




\begin{tabular}{|c|c|c|c|c|c|}
\hline 242 & 155.5634919 & 40.186661722 & 40 & 242 & 0.467181467 \\
\hline 243 & 155.8845727 & 40.14202059 & 40 & 243 & 0.469111969 \\
\hline $\begin{array}{l}244 \\
245\end{array}$ & $\begin{array}{l}156.20499933 \\
156.5247584\end{array}$ & $\begin{array}{l}\begin{array}{l}40.099623333 \\
40.0534233\end{array}\end{array}$ & $\begin{array}{l}40 \\
40\end{array}$ & $\begin{array}{l}244 \\
245\end{array}$ & $\begin{array}{l}0.474104424711 \\
0.47292973\end{array}$ \\
\hline 246 & $\begin{array}{l}156.84388714 \\
15762335\end{array}$ & 40.00942038 & 40 & $\begin{array}{l}246 \\
227\end{array}$ & $\begin{array}{l}0.474903475 \\
047639397\end{array}$ \\
\hline $\begin{array}{l}248 \\
248\end{array}$ & $\begin{array}{l}15.7 .163236656 \\
157.4801575\end{array}$ & $\begin{array}{l}39.96656115 \\
39.9219957\end{array}$ & $\begin{array}{l}40 \\
40\end{array}$ & $\begin{array}{l}248 \\
248\end{array}$ & $\begin{array}{l}0.47768339777 \\
0.47876447\end{array}$ \\
\hline & $\begin{array}{l}157.79733884 \\
158113883\end{array}$ & $\begin{array}{r}39.87857105 \\
398535353\end{array}$ & 40 & $\begin{array}{l}249 \\
250\end{array}$ & 0.4806949811 \\
\hline $\begin{array}{l}250 \\
251\end{array}$ & $\begin{array}{r}158.1 .13883 \\
158.4297952\end{array}$ & $\begin{array}{r}39.833336339 \\
39.7922900\end{array}$ & $\begin{array}{l}40 \\
40\end{array}$ & $\begin{array}{l}250 \\
251\end{array}$ & $\begin{array}{l}0.4828252583 \\
0.48545985\end{array}$ \\
\hline 252 & $\begin{array}{l}158.7450787 \\
\end{array}$ & 39.74943062 & 40 & 252 & 0.486486486 \\
\hline 253 & $\begin{array}{l}159.05973772 \\
1599737745\end{array}$ & $\begin{array}{r}39.70675652 \\
39.6462632\end{array}$ & $\begin{array}{l}40 \\
40\end{array}$ & $\begin{array}{l}253 \\
254\end{array}$ & $\begin{array}{l}0.488416988 \\
0.09094749\end{array}$ \\
\hline $\begin{array}{l}254 \\
255\end{array}$ & $\begin{array}{l}159.6871942 \\
\end{array}$ & $\begin{array}{r}39.66426633 \\
39.6219589\end{array}$ & 40 & $\begin{array}{l}254 \\
255\end{array}$ & $\begin{array}{l}. .4902474759 \\
0.49277992\end{array}$ \\
\hline 256 & 160 & 39.57983187 & 40 & 256 & 0.494208494 \\
\hline $\begin{array}{l}257 \\
258\end{array}$ & $\begin{array}{l}160.312191954 \\
160.623784\end{array}$ & 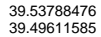 & $\begin{array}{l}40 \\
40\end{array}$ & $\begin{array}{l}257 \\
258\end{array}$ & $\begin{array}{l}0.4961389996 \\
0.4998099498\end{array}$ \\
\hline 259 & 160.9347694 & $\begin{array}{l}39.45452377 \\
\end{array}$ & 40 & 259 & $\begin{array}{l}0.4505 \\
0.5\end{array}$ \\
\hline & & & & & \\
\hline $\begin{array}{l}261 \\
262\end{array}$ & $\begin{array}{l}166.1 .554944442 \\
161.8641406\end{array}$ & $\begin{array}{r}39.37786464 \\
39.3070949\end{array}$ & $\begin{array}{l}40 \\
40\end{array}$ & $\begin{array}{l}261 \\
262\end{array}$ & $\begin{array}{l}0.5038366004 \\
0.505791506\end{array}$ \\
\hline 263 & $\begin{array}{l}162.1727474 \\
628707684\end{array}$ & $\begin{array}{r}39.28989659 \\
39201969\end{array}$ & 40 & 263 & 0.507722000 \\
\hline 265 & $\begin{array}{l}162.788206 \\
1\end{array}$ & $\begin{array}{r}39.24910844 \\
39.20860913\end{array}$ & 40 & $\begin{array}{l}264 \\
265\end{array}$ & 0.511583012 \\
\hline 266 & 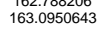 & 39.1682174 & $\begin{array}{l}40 \\
40\end{array}$ & $\begin{array}{l}260 \\
266\end{array}$ & $\begin{array}{l}0.513513514 \\
0.5135\end{array}$ \\
\hline $\begin{array}{l}267 \\
268\end{array}$ & $\begin{array}{l}163.4013464 \\
163.7070554\end{array}$ & $\begin{array}{l}39.12799197 \\
39.0793161\end{array}$ & $\begin{array}{l}40 \\
40\end{array}$ & $\begin{array}{l}267 \\
268\end{array}$ & $\begin{array}{l}0.515444415 \\
0.517374511\end{array}$ \\
\hline $\begin{array}{l}269 \\
270\end{array}$ & $\begin{array}{l}164.0121947 \\
1643146673\end{array}$ & $\begin{array}{l}39.04803507 \\
390003301\end{array}$ & $\begin{array}{l}40 \\
40\end{array}$ & $\begin{array}{l}269 \\
270\end{array}$ & $\begin{array}{l}0.519305019 \\
0522123552\end{array}$ \\
\hline 271 & 164.6207763 & 38.96872858 & 38 & 271 & 0.523166023 \\
\hline 272 & 164.924225 & 38.92931623 & 38 & 272 & 0.525096525 \\
\hline $\begin{array}{l}273 \\
274\end{array}$ & $\begin{array}{l}165.2271164 \\
165.5294536\end{array}$ & $\begin{array}{r}38.890060292 \\
38.85096741\end{array}$ & $\begin{array}{l}38 \\
38 \\
38\end{array}$ & $\begin{array}{l}273 \\
274\end{array}$ & $\begin{array}{l}0.527027027 \\
0.52892757529\end{array}$ \\
\hline 275 & $\begin{array}{l}165.83212305 \\
\end{array}$ & $\begin{array}{l}30.038 .8120286 \\
38.8208\end{array}$ & 38 & 275 & 0.530888031 \\
\hline 276 & $\begin{array}{l}166.13247770 \\
1\end{array}$ & $\begin{array}{r}38.773245344 \\
38773461649\end{array}$ & $\begin{array}{ll}38 \\
38\end{array}$ & $\begin{array}{l}276 \\
277\end{array}$ & $\begin{array}{l}0.5328185333 \\
0.5374400325\end{array}$ \\
\hline $\begin{array}{l}277 \\
278\end{array}$ & $\begin{array}{c}16.643316988 \\
166.73332\end{array}$ & $\begin{array}{r}38.734661649 \\
38.69614093\end{array}$ & $\begin{array}{l}38 \\
38 \\
38\end{array}$ & $\begin{array}{l}277 \\
278\end{array}$ & $\begin{array}{l}0.5334449035 \\
0.536679537\end{array}$ \\
\hline 279 & 167.0329309 & 38.65781754 & 38 & 279 & 0.538610039 \\
\hline $\begin{array}{l}280 \\
281\end{array}$ & $\begin{array}{l}167.3320053 \\
1676350546\end{array}$ & $\begin{array}{l}38.61964524 \\
38.58162929\end{array}$ & $\begin{array}{l}38 \\
38\end{array}$ & $\begin{array}{l}2800 \\
281\end{array}$ & $\begin{array}{l}0.540540541 \\
0.54247104\end{array}$ \\
\hline 282 & 167.9285562 & 38.543774956 & 38 & 282 & 0.544401544 \\
\hline 283 & & & & 283 & 0.546332046 \\
\hline $\begin{array}{l}284 \\
285\end{array}$ & $\begin{array}{l}166.82229955 \\
168.8194302\end{array}$ & $\begin{array}{l}\begin{array}{l}38.46644454 \\
38.4310124\end{array}\end{array}$ & $\begin{array}{l}38 \\
38 \\
38\end{array}$ & $\begin{array}{l}284 \\
285\end{array}$ & $\begin{array}{l}0.548262548 \\
0.055019305\end{array}$ \\
\hline 286 & 169.11534553 & $\begin{array}{l}38.393724242 \\
383555797\end{array}$ & 38 & $\begin{array}{l}286 \\
207\end{array}$ & 0.5521235522 \\
\hline & $\begin{array}{l}169.941707435 \\
169.7056275\end{array}$ & $\begin{array}{r}38.3555597272 \\
38.31957776\end{array}$ & $\begin{array}{l}38 \\
38\end{array}$ & $\begin{array}{l}287 \\
288\end{array}$ & \\
\hline 289 & 169.70502/5 & 38.2827170 & ${ }_{38}$ & $\begin{array}{l}288 \\
289\end{array}$ & $\begin{array}{l}0.557915058 \\
0\end{array}$ \\
\hline 290 & $\begin{array}{l}170.2938637 \\
17058231\end{array}$ & 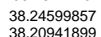 & $\begin{array}{l}38 \\
38\end{array}$ & $\begin{array}{l}2900 \\
229\end{array}$ & $\begin{array}{l}0.55984556 \\
0.56176066\end{array}$ \\
\hline 292 & 170.8800749 & 38.17297818 & 38 & $\begin{array}{l}291 \\
292\end{array}$ & $\begin{array}{l}0.5667760662 \\
0.563706564\end{array}$ \\
\hline 293 & 171.1724277 & 38.1366752 & 38 & 293 & 0.565637066 \\
\hline $\begin{array}{l}294 \\
295\end{array}$ & $\begin{array}{l}171.4642828 \\
177.7556404\end{array}$ & $\begin{array}{l}38.10050908 \\
38.0644789\end{array}$ & $\begin{array}{l}38 \\
38\end{array}$ & $\begin{array}{l}294 \\
295\end{array}$ & $\begin{array}{l}0.567567568 \\
0.569490696\end{array}$ \\
\hline 296 & $\begin{array}{l}172.0465053 \\
172023670\end{array}$ & $\begin{array}{l}38.028583772 \\
3\end{array}$ & 38 & 296 & 0.571428571 \\
\hline 297 & $\begin{array}{l}172.33687794 \\
172626764\end{array}$ & $\begin{array}{l}37.99282263 \\
37.9571947\end{array}$ & $\begin{array}{l}38 \\
38 \\
38\end{array}$ & 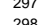 & $\begin{array}{l}0.5733559073 \\
0.575289575\end{array}$ \\
\hline 2999 & $\begin{array}{r}172.2626665 \\
172.9161647\end{array}$ & $\begin{array}{l}33.9577947 \\
37.9216995\end{array}$ & 势 & $\begin{array}{l}2909 \\
299\end{array}$ & $\begin{array}{l}0.575289575 \\
0.577220077\end{array}$ \\
\hline 300 & 173.2050808 & 37.88633477 & 38 & 300 & 0.579150579 \\
\hline 301 & 173.4935157 & 37.85110098 & 38 & 301 & 0.581081081 \\
\hline $\begin{array}{l}302 \\
303 \\
3\end{array}$ & $\begin{array}{r}173.7814722 \\
174.0869519\end{array}$ & $\begin{array}{l}37.8159998119 \\
37.71010139\end{array}$ & $\begin{array}{l}38 \\
38 \\
38\end{array}$ & $\begin{array}{l}302 \\
303\end{array}$ & $\begin{array}{l}0.583011583 \\
0.588942083\end{array}$ \\
\hline 304 & 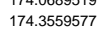 & 37.74617385 & $\begin{array}{l}38 \\
38\end{array}$ & $\begin{array}{l}305 \\
304\end{array}$ & $\begin{array}{l}0.58844470 \\
0.58872587\end{array}$ \\
\hline 305 & $\begin{array}{l}174.642492 \\
17492985568\end{array}$ & $\begin{array}{l}37.71145336 \\
376768590\end{array}$ & 38 & $\begin{array}{l}305 \\
306\end{array}$ & $\begin{array}{l}0.588803089 \\
050725259\end{array}$ \\
\hline 307 & $\begin{array}{l}177.92855568 \\
175.2141547\end{array}$ & $\begin{array}{l}37.676859507 \\
377.64239014\end{array}$ & $\begin{array}{l}38 \\
38\end{array}$ & $\begin{array}{l}\begin{array}{l}306 \\
307\end{array}\end{array}$ & $\begin{array}{l}0.5907335991 \\
0.59264093\end{array}$ \\
\hline 308 & $\begin{array}{l}175.4992877 \\
\end{array}$ & $\begin{array}{r}77.645014 \\
37.60804575\end{array}$ & 38 & 308 & 0.594594595 \\
\hline $\begin{array}{l}309 \\
310\end{array}$ & $\begin{array}{l}175.78395833 \\
170688168\end{array}$ & $\begin{array}{l}37.57382508 \\
37.53972732\end{array}$ & $\begin{array}{l}38 \\
38\end{array}$ & $\begin{array}{l}309 \\
330\end{array}$ & $\begin{array}{l}0.596525097 \\
0.599455598\end{array}$ \\
\hline 311 & 176.3519209 & 37.50575167 & 38 & 311 & 0.6003861 \\
\hline 312 & 176.6352173 & 37.47189733 & 38 & 312 & 0.602316602 \\
\hline $\begin{array}{l}\begin{array}{l}313 \\
314\end{array} \\
3\end{array}$ & 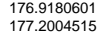 & $\begin{array}{r}\begin{array}{l}37.438163652 \\
37740454946\end{array}\end{array}$ & $\begin{array}{l}38 \\
38 \\
38\end{array}$ & $\begin{array}{l}313 \\
314\end{array}$ & $\begin{array}{l}0.06024247104 \\
0.606177606\end{array}$ \\
\hline $\begin{array}{l}315 \\
316\end{array}$ & $\begin{array}{l}177.4823935 \\
1777683883\end{array}$ & $\begin{array}{l}37.37105438 \\
3373376775\end{array}$ & $\begin{array}{l}38 \\
38\end{array}$ & $\begin{array}{l}315 \\
316\end{array}$ & $\begin{array}{l}0.6088108108 \\
0.61003861\end{array}$ \\
\hline 317 & $\begin{array}{l}1 / 7.166888838 \\
178.04938\end{array}$ & $\begin{array}{l}37.336 / 67 / 5 \\
37.30441808\end{array}$ & $\begin{array}{l}50 \\
38\end{array}$ & 317 & $\begin{array}{l}0.6600303871 \\
0.611969112\end{array}$ \\
\hline 318 & 178.325545 & $\begin{array}{l}37.27127536 \\
\end{array}$ & 38 & 318 & 0.613899614 \\
\hline 319 & $\begin{array}{l}178.6057111 \\
178055028\end{array}$ & $\begin{array}{l}37.2382486 \\
3720232\end{array}$ & $\begin{array}{l}38 \\
{ }_{38}\end{array}$ & $\begin{array}{l}319 \\
330\end{array}$ & 0.615830116 \\
\hline & $\begin{array}{l}1 / 8.888458888 \\
179.1647287\end{array}$ & $\begin{array}{l}37.20533706 \\
37.1725402\end{array}$ & 38 & ${ }_{321}^{20}$ & $\begin{array}{l}0.6177660618 \\
0.61969112\end{array}$ \\
\hline $\begin{array}{l}321 \\
322\end{array}$ & 179.44358844 & 37.13985674 & 38 & 322 & $\begin{array}{l}0.61969611212 \\
0.621621622\end{array}$ \\
\hline 323 & 179.7220076 & $\begin{array}{l}37.10728652 \\
3707020\end{array}$ & 38 & 323 & 0.623552124 \\
\hline & & & 海 & $\begin{array}{l}324 \\
325\end{array}$ & $\begin{array}{l}0.6252582625 \\
0.627471312\end{array}$ \\
\hline 年25 & 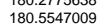 & $\begin{array}{r}37.040248424 \\
37.0102471\end{array}$ & 38 & 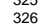 & $\begin{array}{l}0.6253934629 \\
0.62\end{array}$ \\
\hline $\begin{array}{l}327 \\
328\end{array}$ & $\begin{array}{l}180.83141322 \\
1811077028\end{array}$ & $\begin{array}{l}36.97812205 \\
369461505\end{array}$ & $\begin{array}{l}36 \\
36\end{array}$ & $\begin{array}{l}327 \\
328\end{array}$ & $\begin{array}{l}0.631274131 \\
06332014631\end{array}$ \\
\hline 320 & $\begin{array}{l}181.10770282 \\
181.3835715\end{array}$ & $\begin{array}{r}36.94610658 \\
36.91419999\end{array}$ & $\begin{array}{l}36 \\
36\end{array}$ & $\begin{array}{l}328 \\
329\end{array}$ & $\begin{array}{l}0.63323026433 \\
0.635135135\end{array}$ \\
\hline 330 & 181.6590212 & 36.88240162 & 36 & 330 & 0.637065637 \\
\hline & & 36.85071081 & & 331 & 0.638996139 \\
\hline $\begin{array}{c}\begin{array}{c}332 \\
333\end{array} \\
-\end{array}$ & $\begin{array}{l}188.20886716 \\
182.4828759\end{array}$ & $\begin{array}{l}36.81912688 \\
36.7876492\end{array}$ & $\begin{array}{l}36 \\
36 \\
36\end{array}$ & $\begin{array}{l}\begin{array}{l}332 \\
333\end{array} \\
33\end{array}$ & $\begin{array}{l}0.6490926641 \\
0.642857143\end{array}$ \\
\hline 334 & 182.7566688 & 36.75627711 & 36 & 334 & 0.644787645 \\
\hline 335 & 183.0300522 & 36.72500996 & 36 & 335 & 0.646718147 \\
\hline 萠366 & $\begin{array}{l}183.3030278 \\
1835575997\end{array}$ & $\begin{array}{r}36.69384713 \\
3666278798\end{array}$ & $\begin{array}{l}36 \\
36\end{array}$ & $\begin{array}{l}336 \\
337\end{array}$ & $\begin{array}{l}0.648648649 \\
0.655597915\end{array}$ \\
\hline 338 & 183.8477631 & $\begin{array}{r}30.060 \\
36.63183198\end{array}$ & $\begin{array}{l}30 \\
36\end{array}$ & 338 & $\begin{array}{l}0.652509653 \\
0.650\end{array}$ \\
\hline 339 & 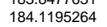 & $\begin{array}{l}30.0300978 \\
36.600782\end{array}$ & 36 & 339 & 0 \\
\hline 340 & 184.39088991 & 36.57022638 & 36 & 340 & 0.656370656 \\
\hline & $\begin{array}{l}184.66683531 \\
\end{array}$ & 36.53957575 & & 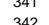 & 0.658301158 \\
\hline $\begin{array}{l}342 \\
343\end{array}$ & $\begin{array}{r}188.4332420101 \\
185.2025918\end{array}$ & $\begin{array}{l}36.5090025773 \\
36.47857572\end{array}$ & $\begin{array}{l}36 \\
36 \\
36\end{array}$ & $\begin{array}{l}342 \\
343\end{array}$ & $\begin{array}{l}0.066023166 \\
0.662162162\end{array}$ \\
\hline 344 & 185.4723699 & 36.44822513 & 36 & 344 & 0.664092664 \\
\hline 345 & 185.7417562 & 36.41797336 & 36 & 345 & 0.666023166 \\
\hline $\begin{array}{l}346 \\
347\end{array}$ & $\begin{array}{l}188.017075224 \\
186.2793601\end{array}$ & $\begin{array}{r}36.38781984 \\
36.375776999\end{array}$ & $\begin{array}{l}36 \\
36 \\
36\end{array}$ & $\begin{array}{l}346 \\
347\end{array}$ & $\begin{array}{l}0.6679953668 \\
0.066988417\end{array}$ \\
\hline 348 & $\begin{array}{l}180.67 .5475811 \\
1\end{array}$ & 36.32780523 & 36 & 348 & $\begin{array}{l}0.6659884717 \\
0.671814672\end{array}$ \\
\hline 349 & 186.8154169 & 36.29794299 & 36 & 349 & $\begin{array}{l}0.673745174 \\
0.67\end{array}$ \\
\hline 350 & 187.0828693 & 36.26817672 & 36 & $\begin{array}{l}350 \\
251\end{array}$ & 0.675675676 \\
\hline 351 & 187.34994 & 36.23850586 & 年 & $\begin{array}{l}351 \\
{ }_{320}\end{array}$ & 0.677606178 \\
\hline $\begin{array}{l}352 \\
353 \\
353\end{array}$ & $\begin{array}{l}187.61663604 \\
187.8829423\end{array}$ & $\begin{array}{r}36.208929894 \\
36.1794812\end{array}$ & $\begin{array}{l}36 \\
36\end{array}$ & $\begin{array}{l}532 \\
353 \\
353\end{array}$ & $\begin{array}{l}0.679556668 \\
0.681467181\end{array}$ \\
\hline 354 & 188.1488772 & 36.15006017 & 36 & 354 & 0.683397683 \\
\hline 355 & 188.4144368 & $\begin{array}{l}36.12076542 \\
2\end{array}$ & 36 & $\begin{array}{l}355 \\
556\end{array}$ & 0.685328185 \\
\hline & $\begin{array}{l}188.67964226 \\
189844363\end{array}$ & $\begin{array}{l}36.091566366 \\
3.0624535\end{array}$ & 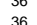 & $\begin{array}{l}356 \\
357\end{array}$ & $\begin{array}{l}0.6877258687 \\
0.689189189\end{array}$ \\
\hline 年 & 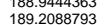 & 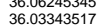 & 36 & $\begin{array}{l}350 \\
358\end{array}$ & $\begin{array}{l}0.0691196999 \\
0.69196\end{array}$ \\
\hline 359 & 189.4729532 & $\begin{array}{l}36.00450799 \\
0.07567\end{array}$ & 36 & 359 & 0.693050193 \\
\hline & & 35.97567139 & 36 & 360 & 0.694980695 \\
\hline 361 & 190750 & $\begin{array}{r}35.94692486 \\
35.919260\end{array}$ & 每6 & $\begin{array}{l}361 \\
360\end{array}$ & $\begin{array}{l}0.696911197 \\
0.69884469\end{array}$ \\
\hline 363 & 190.5255888 & $\begin{array}{r}35.91826679 \\
35.88969999\end{array}$ & 36 & 363 & 0.0500772201 \\
\hline 364 & 190.7878403 & 35.86122064 & 36 & 364 & $\begin{array}{l}0.702702703 \\
0.03\end{array}$ \\
\hline $\begin{array}{l}365 \\
366 \\
36\end{array}$ & $\begin{array}{l}191.04973717 \\
191.1112647\end{array}$ & $\begin{array}{r}35.83282934 \\
358045256\end{array}$ & $\begin{array}{l}36 \\
36\end{array}$ & $\begin{array}{l}365 \\
366\end{array}$ & $\begin{array}{l}0.704633205 \\
0.7065637707\end{array}$ \\
\hline 367 & 191.5724406 & 35.77630897 & 36 & 367 & 0.708494208 \\
\hline
\end{tabular}




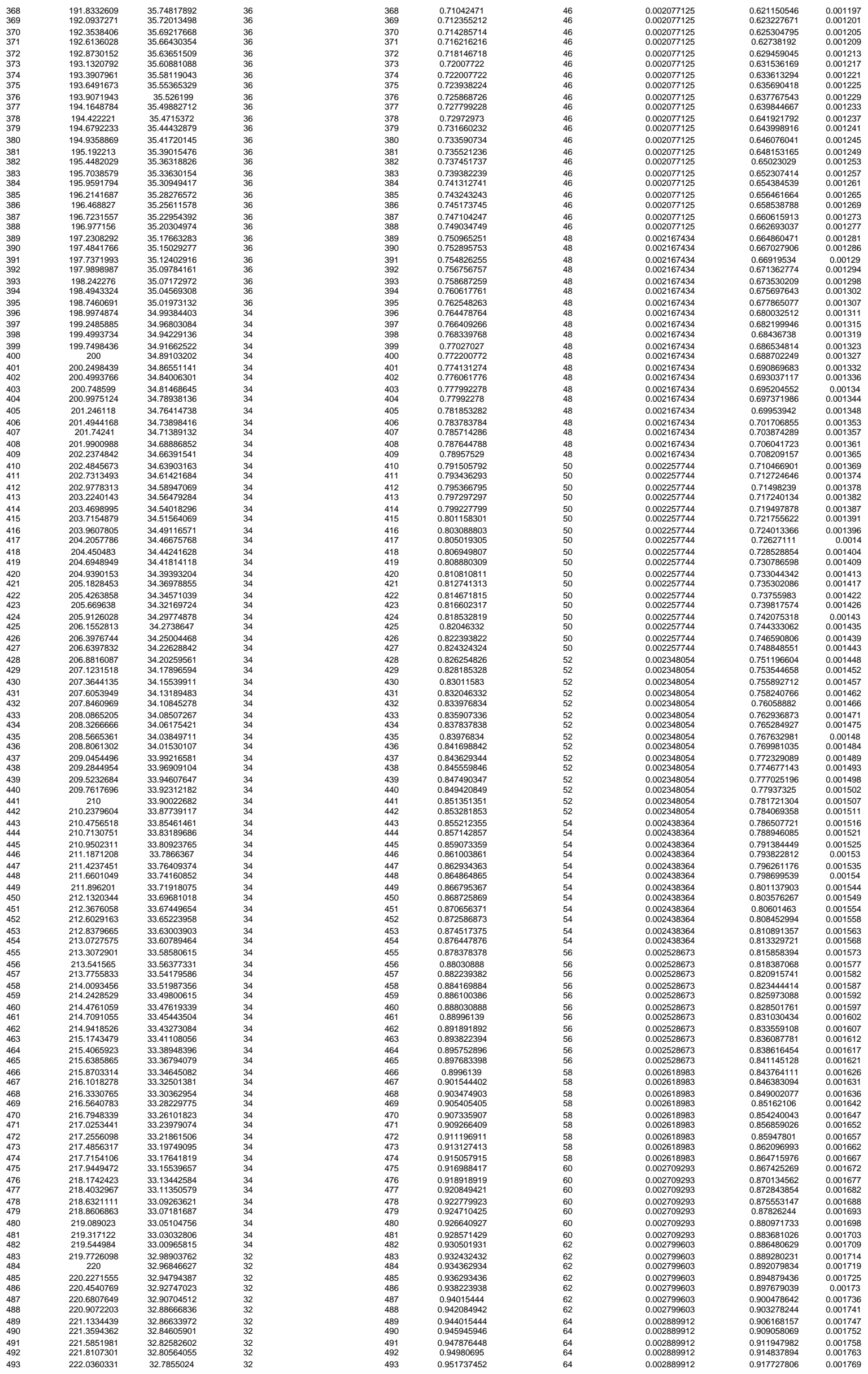




\begin{tabular}{|c|c|c|}
\hline 494 & 222.2611077 & 32.76541138 \\
\hline 495 & 222.4859546 & 32.74536727 \\
\hline 496 & 222.7105745 & 32.7253699 \\
\hline 497 & 222.9349681 & 32.70541905 \\
\hline 498 & 223.159136 & 32.68551455 \\
\hline 499 & 223.383079 & 32.66565618 \\
\hline 500 & 223.6067977 & 32.64584377 \\
\hline 501 & 223.8302929 & 32.62607712 \\
\hline 502 & 224.053565 & 32.60635604 \\
\hline 503 & 224.2766149 & 32.58668035 \\
\hline 504 & 224.4994432 & 32.56704984 \\
\hline 505 & 224.7220505 & 32.54746435 \\
\hline 506 & 224.9444376 & 32.52792368 \\
\hline 507 & 225.166605 & 32.50842765 \\
\hline 508 & 225.3885534 & 32.48897608 \\
\hline 509 & 225.6102835 & 32.46956878 \\
\hline 510 & 225.8317958 & 32.45020557 \\
\hline 511 & 226.0530911 & 32.43088628 \\
\hline 512 & 226.27417 & 32.41161073 \\
\hline 513 & 226.4950331 & 32.39237873 \\
\hline 514 & 226.715681 & 32.37319011 \\
\hline 515 & 226.9361144 & 32.3540447 \\
\hline $\begin{array}{l}516 \\
517\end{array}$ & $\begin{array}{r}227.1563338 \\
272764\end{array}$ & 32.33494232 \\
\hline $\begin{array}{l}517 \\
518\end{array}$ & $\begin{array}{r}227.376634 \\
227.5961335\end{array}$ & $\begin{array}{r}32.31588828 \\
32.29686596\end{array}$ \\
\hline
\end{tabular}

$\begin{array}{lcc}494 & 0.953667954 & 66 \\ 495 & 0.955598456 & 66 \\ 496 & 0.957528598 & 66 \\ 497 & 0.959459459 & 66 \\ 498 & 0.961389961 & 66 \\ 499 & 0.963320463 & 68 \\ 500 & 0.965250965 & 68 \\ 501 & 0.967181467 & 68 \\ 502 & 0.969111669 & 68 \\ 503 & 0.971042471 & 70 \\ 504 & 0.972972973 & 70 \\ 505 & 0.979403475 & 70 \\ 506 & 0.976833977 & 72 \\ 507 & 0.978764479 & 72 \\ 508 & 0.980694981 & 72 \\ 509 & 0.982625483 & 74 \\ 510 & 0.984555985 & 74 \\ 511 & 0.986486486 & 76 \\ 512 & 0.988416988 & 76 \\ 513 & 0.99034749 & 78 \\ 514 & 0.992277992 & 80 \\ 515 & 0.994208494 & 80 \\ 516 & 0.996138996 & 82 \\ 517 & 0.998069498 & 86 \\ 518 & 1 & 88 \\ & & \\ & & \end{array}$

\begin{tabular}{|c|c|c|}
\hline 0.002980222 & 0.920708029 & 0.001775 \\
\hline & 0.923688251 & 0.00178 \\
\hline 0.002980222 & 0.926668473 & 0.001786 \\
\hline 0.002980222 & 0.929648695 & \\
\hline $\begin{array}{r}0.0029802222 \\
0\end{array}$ & $\begin{array}{l}0.932628917 \\
0.935699449\end{array}$ & $\begin{array}{l}0.001798 \\
0.001803\end{array}$ \\
\hline $\begin{array}{l}0.003070532 \\
0.003070532\end{array}$ & $\begin{array}{l}0.935699449 \\
0.938769981\end{array}$ & $\begin{array}{l}0.001803 \\
0.001809\end{array}$ \\
\hline $\begin{array}{l}0.003070532 \\
0.03052\end{array}$ & 0.941840513 & 0.001815 \\
\hline 0.003070532 & 0.944911045 & 0.001821 \\
\hline 0.003160842 & 0.948071887 & 0.001827 \\
\hline 0.003160842 & 0.951232728 & 0.001833 \\
\hline 0.003160842 & 0.95439357 & 0.001839 \\
\hline 0.003251151 & 0.957644721 & 0.001846 \\
\hline 0.003251151 & 0.960895873 & 0.001852 \\
\hline 0.003251151 & 0.964147024 & 0.001858 \\
\hline 0.003341461 & 0.967488486 & 0.001865 \\
\hline 0.003341461 & 0.970829947 & 0.001871 \\
\hline 0.003431771 & 0.974261718 & 0.001878 \\
\hline 0.003431771 & 0.977693489 & 0.001884 \\
\hline 0.003522081 & 0.981215569 & 0.001891 \\
\hline 0.00361239 & 0.98482796 & 0.001898 \\
\hline 0.00361239 & 0.98844035 & 0.001905 \\
\hline 0.0037027 & 0.992143051 & 0.001912 \\
\hline \multirow{4}{*}{$\begin{array}{l}0.00388332 \\
0.00397363\end{array}$} & 0.99602637 & 0.001919 \\
\hline & & \\
\hline & Total area & \\
\hline & & 0.123781 \\
\hline
\end{tabular}

